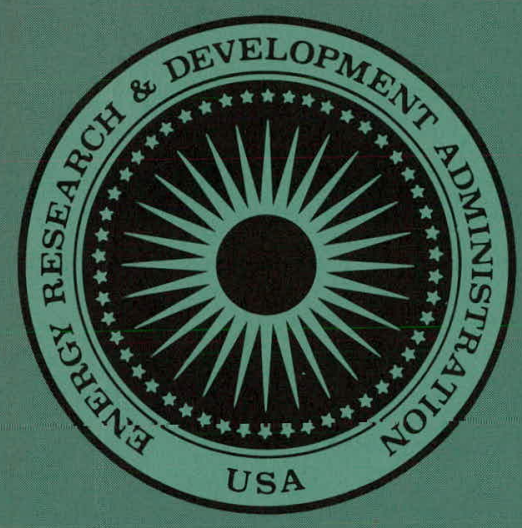

BERC/TPR-75/1

EL DORADO MICELLAR-POLYMER DEMONSTRATION PROJECT

By

Cities Service Oil Company

Date Published - October 1975

Bartlesville Energy Research Center

Energy Research and Development Administration

Bartlesville, Oklahoma 


\section{DISCLAIMER}

This report was prepared as an account of work sponsored by an agency of the United States Government. Neither the United States Government nor any agency Thereof, nor any of their employees, makes any warranty, express or implied, or assumes any legal liability or responsibility for the accuracy, completeness, or usefulness of any information, apparatus, product, or process disclosed, or represents that its use would not infringe privately owned rights. Reference herein to any specific commercial product, process, or service by trade name, trademark, manufacturer, or otherwise does not necessarily constitute or imply its endorsement, recommendation, or favoring by the United States Government or any agency thereof. The views and opinions of authors expressed herein do not necessarily state or reflect those of the United States Government or any agency thereof. 


\section{DISCLAIMER}

Portions of this document may be illegible in electronic image products. Images are produced from the best available original document. 


\section{NOTICE}

This report was prepared as an account of work sponsored by the United States Government. Neither the United States nor the United States Energy Research and Development Administration, nor any of their employees, nor any of their contractors, subcontractors, or their employees, makes any warranty, express or implied, or assumes any legal liability or responsibility for the accuracy, completeness or usefulness of any information, apparatus, product or process disclosed, or represents that its use would not infringe privately owned rights.

This report has been reproduced directly from the best available copy.

Available from the National Technical Information Service, U. S. Department of Commerce, Springfield, Virginia 22161

Price: Paper Copy $\$ 10.60$

Microfiche $\$ 2.25$ (domestic)

$\$ 3.75$ (foreign) 


\section{EL DORADO MICELLAR-POLYMER DEMONSTRATION PROJECT}

C. L. Coffman

Principal Investigator

Cities Service Oil Company

W. D. Howell

Technical Project Officer.

Bartlesville Energy Research Center

Energy Research \& Development Administration

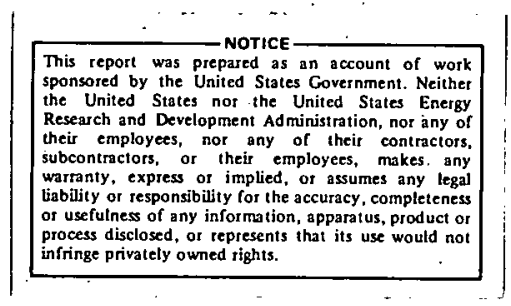

Date Published - October 1975

UNITED STATES ENERGY RESEARCH AND DEVELOPMENT ADMINISTRATION

OFFICE OF PIIRIII . AFFAIRS

TECHNICAL INFORMATION CENTER 


\section{THIS PAGE \\ WAS INTENTIONALLY \\ LEFT BLANK}


EL DORADO MIICELLAR-POLYMER

DEMONSTRATION PROJECT

FIRST ANNUAL REPORT

January, 1974 - June, 1975

\section{CITIES SERVICE OIL COMPANY}

G. W. Rosenwald, Editor

R. J. Miller, Assistant Editor

Contributing Editors

H. I. S.hang

C. L. Coffman

R. P. Nurray

J. Vairogs 


\section{ACKNOWLEDGEMENTS}

The authors and editors wish to express their sincere appreciation to all who contributed to the information contained herein. We wish to express a very special thank you to the Technicians, Typists, and Drafting and Reproduction personnel who spent many hard hours in the preparation of this report. 
FOREWORD

The ERDA program for enhanced recovery of $0 i 1$ and gas is aimed at demonstrating new recovery technology and transferring it to the private sector. The program is designed to maximize the participation of private industry by awarding cost-sharing contracts to companies which have capability in enhanced oil and gas recovery.

Data generated from these ERDA-industry field demonstration programs will be used to evaluate the technical and economic feasibility of an advanced recovery technique and should hasten the use of such techniques as one of the immediate measures that can be taken to ease the Nation's energy problem. Although much research has been done in both industrial and Government laboratories, additional testing is needed to provide information to reduce the risks associated with these high-cost processes. The ERDA demonstration projects, with full and timely public description of the results, will aid all oil and gas producers.

The El Dorado Micellar-Polymer Flood is one of several ERDAindustry demonstration projects now in progress. Data generated during the first year under this contract are described in this report. 
THIS PAGE

\section{WAS INTENTIONALLY \\ LEFT BLANK}




\section{SUMPIARY}

The El Dorado Nicellar-Polymer Demonstration Project is a cooperative venture between Cities Service 0il Company and the United States Energy Research and Development Administration (ERDA). The cooperative project is a result of the response by Cities Service $0 i 1$ Company to a Request for Proposals from the United States Bureau of Mines (The Bureau of Mines' responsibilities for the project are now under the jurisdiction of ERDA). Although beginning about one-half year earlier, the project officially became a cooperative venture with the completion of the cost-sharing contract on June 17, 1974. This project is governed by ERDA Contract Number E(34-1)=0003 (formerly, Contract Number H0242035). This report covers project activities from January, 1974, through June, 1975.

The primary objectives of this project are to determine the economic feasibility of improved oil recovery using micellar-polymer processes and to determine the associated benefits and problems of each system tested. Since the 650-foot Sand reservoir had been abandoned, any $0 i 1$ that is recovered will truly be tertiary oil.

The El Dorado Demonstration Project is designed to allow a side-by-side comparison of two distinct micellar-polymer processes in the same field so that the reservoir conditions for the two floods are as nearly alike as possible. Each of the floods is to involve multiple 
contiguous patterns. Each process is to be tested on 25.6 acres (a total of 51.2 acres for the two floods).

Following a comprehensive search for competent vendors of micellar-polymer oil recovery processes, Union 0il Company of California and Shell 0 il Company were selected to design the processes to be used. Suitable agreements were negotiated. Union submitted the specifications for its process in May, 1975. Injection of fluids into the pattern using the Union process is expected to begin within two months. The She 11 design is expected in August, 1975. Well logs, reservoir rock, and fluid samples were sent to Union and Shell for extensive testing of their processes. These data will be used to design the composition and size of each oil recovery system. Cities has been conducting comparable laboratory tests for confirmation.

Test wells were drilled early in the project to obtain reservoir data in the area of the demonstration floods. Discovery of what had apparently been a gas cap, now filled with water, resulted in a small shift in the orientation of the test area. Pattern wells were subsequently drilled. Well logs were run for each well. During this reporting period, open-hole logs have been evaluated for 49 pattern wells and five test wells; results are included. A total of 31 cores were obtained from new wells in the project area. The section on coring and core analyses summarizes geological findings as well as giving the results of routine and special core analyses.

During the summer of 1974, field injectivity and interference tests were run. Fluids were injected into the most centrally located. test well, and pressure responses were monitored in offset wells. The fluids used were a complete sequence (preflush, micellar fluids, polymer, 
and drive water) similar to those of the Union process. The tests indicated no major problems with injection of the various fluids although some difficulty was encountered with the preflush. Variations in directional permeability detectable with the test well spacing were sufficiently small that no change in the location of the test area was required. The results of these tests were prime reasons for the "go" decision made at the first project decision point in October, 1974.

Pressure transient tests are now being conducted using the pattern wells. Since the analyses of these tests are not complete, conclusions cannot be given at this time. The finalization of the decision on optimum pattern choice is dependent on the results of these tests.

Two major 1 iterature reviews have been conducted in support of this project. A report on the survey of polymer flooding has been submitted to ERDA. The second survey is concerned with surfactant, micellar, and alkaline waterfilooding. This study is nearing completion.

Performance predictions have been made for the demonstration floods under various modes of operation and during several phases of the injection sequence. The decision concerning the optimum pattern configuration has relied heavily on mathematical modeling. Several models have been written for use in evaluating various patterns and operational schemes. Performance of the previous waterflood in the El Dorado field has been examined to gain information that might be applied to predict the results of the demonstration flood.

The primary activities related to engineering operations have been, (1) selection of the test site, (2) development of the optimum pattern, (3) drilling of the test wells, and (4) drilling of the pattern 
wells. The Admire Sand (locally named the 650-foot Sand) was chosen in an area of the El Dorado Field, Butler County, Kansas, as the test site for this demonstration project. The initial pattern chosen to conduct the demonstration flood was a 6.4 acre nine-spot. However, additional study has led to the conclusion that a five-spot configuration will provide the optimum pattern.

The drilling program for the rest of the 50 pattern wells was initiated in December, 1974, and completed in May, 1975. The test site was drilled with a density of almost one well per acre in order to assure better reservoir definition, provide flexibility for the pattern selection, and reduce the time needed for project completion.

Project construction undertaken during this reporting period includes developing a fresh water system, building the injection plant, and installing battery facilities. The El Dorado City Lake was selected as the best water source for the project. The five-mile pipeline from the lake to the project site was essentially complete by June 30,1975 . The injection plant construction was delayed somewhat by severe wind damage to several tanks and the two buildings. The estimated completion date for the plant is August, 1975. The battery facilities for collecting, monitoring, and metering produced fluids were installed in June, 1975. 
TABLE OF CONTENTS

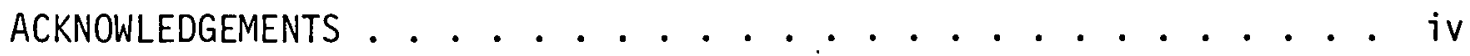

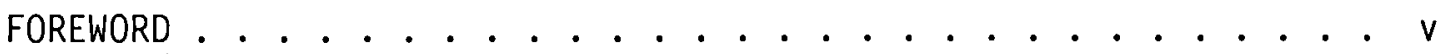

SUMMARY. .................................. vii RESEARCH SUPPORT ................. I-1

CHEMICAL SELECTION AND SUPPORT. ............. I-2

CORING AND CORE ANALYSES. ................. . . I-22

WELL LOGGING. . . . . . . . . . . . . I-31

FORMATION INJECTIVITY . . . . . . . . . . . . I-37

PATTERN PRESSURE TRANSIENT TESTS. . . . . . . . . . . . I-45

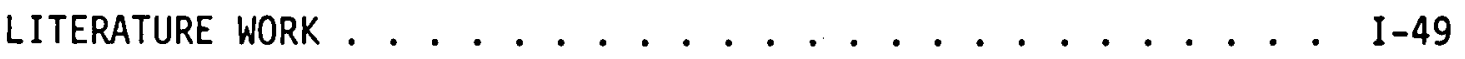

PERFORMANCE PREDICTION. . . . . . . . . . . . . I-57

ENGINEERING AND OPERATIONS . . . . . . . . . . . . I-77

SELECT TEST SITE. . . . . . . . . . . . . . I-78

DEVELOP OPTIMUM PATTERN . . . . . . . . . . . . . . I-81

DRILL TEST WELLS. . . . . . . . . . . . . I-84

DRILL PATTERN WELLS ................. I-87

CONSTRUCTION ............................ I-89

DEVELOP FRESH WATER SYSTEM. . . . . . . . . . . I-90

BUILD INJECTION PLANT . . . . . . . . . . . . . . I-94

INSTALL BATTERY FACILITIES. . . . . . . . . . . . I-97 
$x i i$

TABLE OF CONTENTS, Continued

PAGE

APPENDIX A: CHEMICAL SELECTION AND SUPPORT. . . . . . . II-I

APPENDIX B: CORING AND CORE ANALYSES. .......... II-31

APPENDIX C: WELL LOGGING. . . . . . . . . . . . II-109

APPENDIX D: FORMATION INJECTIVITY . . . . . . . . . II-253

APPENDIX E: LITERATURE WORK . . . . . . . . . . II-263

APPENDIX F: PERFORMANCE PREDICTION. . . . . . . . . . II-271

APPENDIX G: ENGINEERING AND OPERATIONS. . . . . . . . II-311

APPENDIX H: CONSTRUCTION. . . . . . . . . . . . II-315 
A-1 Summary of 0 il Recovery Flow Tests for the Union Process--Berea Cores... . . . . . . . . . II-2

$A=2$ Summary of 011 Recovery Flow Tests for the Union Process--650-Foot Sand Cores. . . . . . . . . II-3

A-3 Mobility Measurements in Berea Sand Cores for Polymer Dissolved in El Dorado Lake Water . . . . . . . II-4

A-4 Mobility Measurements in 650-Foot Sand Cores for Polymer Dissolved in El Dorado Lake Water . . . . . II-5

A-5 Characterization of 600 PPM* Polymer Solutions Prepared From Various Stock Solutions ... . . . . . . II-6

A-6 Effect of Salinity and Preservatives on Viscosities of 600 PPM Polyacrylamide Solutions . . . . . . . . II-7

A-7 Effect of Salinity and Preservatives on Screen Factor of 600 PPM Polyacrylamide Solutions. . . . . . . II-8

A-8 Composition of Chesney Produced Water and El Dorado Lake Water. . . . . . . . . . . . . II-9

A-9 Resident Water Composition. . . . . . . . . . . . II-10

A-10 Characterization of Emulsions, Crude $0 i 1$ Mixed wi th Aqueous Solutions ............. II-1l

A-11 Characterization of Emulsions, Soluble 0il Mixed with Aqueous Solutions............. . II-12

A-12 Characterization of Emulsions, Ten Percent. Soluble 011 in lirude 0il Mixed with Aqueous Solutions...... II-13

A-13 Characterization of Emulsions, One Percent Soluble $0 i 1$ in Crude $0 i 1$ Mixed with Aqueous Solutions . . . . . II-14

B-1 Routine Core Analysis Results vs Depth for We11 MP-101.................. . . II-32

B-2 Routine Core Analysis Results vs Depth for Wel1 MP-102.................. II-33

B-3 Routine Core Analysis Results vs Depth for Wel1 MP-103............... . . II-34

B-4 Routine Core Analysis Results vs Depth for Well MP-104.................. II-35 
B-5 Routine Core Analysis Results vs Depth for Well MP-105................ II-36

B-6 Routine Core Analysis Results vs Depth for Well MP-106................. . . II-37

B-7 Routine Core Analysis Results vs Depth for Well MP-110... . . . . . . . . . . . II -38

B-8 Routine Core Analysis Results vs Depth for Well MP-116.............. . . II-39

B-9 Routine Core Analysis Results vs Depth for Well MP-120................ II 4 . . . . .

B-10 Routine Core Analysis Results vs Depth for Wel1 MP-122.................. . II-41

B-11 Routine Core Analysis Results vs Depth for Wel1 MP-130................. II-42

B-12 Routine Core Analysis Results vs Depth for Well MP-201.................. II-43

B-13 Routine Core Analysis Results vs Depth for Wel] MP-203 ................ II - . . . . . .

B-14 Routine Core Analysis Results vs Depth for

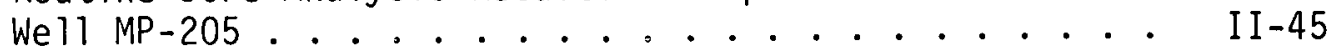

B-15 Routine Core Analysis Results vs Depth for

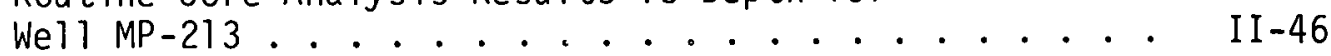

B-16 Routine Core Analysis Results vs Depth for Wel1 MP-215................ II-47

B-17 Rüutine Corc Nnalysis Results vs Depth for Wel1 MP -221.................. I1-48

B-18 Routine Core Analysis Results vs Depth for We11 MP-223.................. II-49

B-19 Routine Core Analysis Results vs Depth for Well MP-225.................. II-50

B-20 Summary of Routine Core Analyses El Dorado Micellar-Polymer Project. . . . . . . . . . . II-5I

B-21 Wettability Determination from Relative Permeability Data................. II-52

B-22 Wettability Determination from Capillary Pressure Curves................ II-53 
B-23 Compressive Strength of Admire Sandstone

PAGE

Cores . . . . . . . . . . . . . II-54

B-24 X-Ray Diffraction Analysis for Clay Fraction

of Samples from Well MP-221 .......... II-55

B-25 X-Ray Diffraction Analyses for Clay Fraction

of Samples from Well MP-225.......... II-56

C-1 Log Analysis Results for the Test Wells . . . . . . II-110

C-2 Core Analysis Results for the Test Wells...... II-111

C-3 Comparison of Core and Log Results for Test

Wells Based on Core Interval Only ........ II-112

C-4 Arithmetic and Weighted Averages of Well Log

Determined Properties for Five Test Wel1s ..... II-113

C-5 Geometric Mean of We11 Log Determined Properties

for. Five Test Wells ............ II-114

C-6 Arithmetic and Weighted Averages of Well Log

Determined Properties for Chesney Wells . . . . . II-115

C-7 Geometric Mean of Well Log Determined

Properties for Chesney We11s......... II-116

C-8 Arithmetic and Weighted Averages of Well Log

Determined Properties for Hegberg Wells ...... II-117

C-9 Geometric Mean of Well Log Determined

Properties for Hegberg Wells......... II-118

C-10 Arithmetic and Weighted Averages of Well Log

Determined Properties for Chesney and Hegberg Wells . . II-119

C-11 Geometric Mean of Well Log Determined Properties

for Chesney and Hegberg Wells......... II-120

C-12 Well Log Determined Properties vs Depth

for Wel1 MP-101................. II-121

C-13 Well Log Determined Properties vs Depth

for We11 MP-102............. II-122

C-14 We11 Log Determined Properties vs Depth

for Well MP-103 ............... II-123

C-15 We11 Log Determined Properties vs Depth

for well MP-104 .............. II-124

C-16 We11 Log Determined Properties vs Depth

for Well MP-105................ II-125 
$x \vee i$

C-17 Wel1 Log Determined Properties vs Depth

for Well 106.................. II-126

C-18 Well Log Determined Properties vs Depth

for Well MP-107............... . . II-127

C-19 Wel1 Log Determined Properties vs Depth

for Wel1 MP-108............... . . II-128

C-20 Wel1 Log Determined Properties vs Depth

for Well MP-109............... . . II-129

C-21 Wel1 Log Determined Properties vs Depth

for Well MP-110................ II-130

C-22 Well Log Determined Properties vs Depth

for Well MP-111................ II-131

C-23 We11 Log Determined Properties vs Depth

for Well MP-112.............. . . II-132

C-24 We11 Log Determined Properties vs Depth

for Wel1 MP-113................. II-133

C-25 Wel1 Log Determined Properties vs Depth

for Wel1 MP-114................. II-134

C-26 Well Log Determined Properties v:s Depth

for Well MP-115................. II-135

C-27 Wel1 Log Determined Properties vs Depth
for Well MP-116........................ II-136

C-28 Wel1 Log Determined Properties vs Depth
for Well MP-117.......... . . . . . II-137

W-2911 Log Determined Properties vs Depth
for Wel1 MP-118.................... II-138

C-30 We11 Log Determined Properties vs Depth

for Wel1 MP-119................ II-139

C-31 Wel1 Log Determined Properties vs Depth

for Well MP-120................. II-140

C-32 Well Log Determined Properties vs Depth

for Well MP-121.................. II-141

C-33 Well Log Determined Properties vs Depth

for Well MP-122............... II-142

C-34 Wel1 Log Determined Properties vs Depth
for Wel1 MP-123...................... II-143 
$x \vee i j$

TABLES

PAGE

C-35 Well Log Determined Properties vs Depth for Well MP-124................... . II-144

C-36 Wel1 Log Determined Properties vs Depth for Well MP-125................ . . II-145

C-37 We11 Log Determined Properties vs Depth

for Wel1 MP-126... . . . . . . . . . . . . II-146

C-38 We11 Log Determined Properties vs Depth

for Wel1 MP-127............... . . II-147

C-39 Well Log Determined Properties vs Depth

for Well MP-128................ . . II-148

C-40 Well Log Determined Properties vs Depth

for Wel1 MP-129................ II-149

C-41 Wel1 Log Determined Properties vs Depth

for Wel1 MP-130................ . II-150

C-42 Well Log Determined Properties vs Depth

for Wel1 MP-201.................. II-151

C-43 Well Log Determined Properties vs Depth

for Well MP-202................ . . II-152

C-44 Well Log Determined Properties vs Depth

for Well MP-203 . . . . . . . . . . . . . . II-153

C-45 Wel1 Log Determined Properties vs Depth

for Well MP-204................ . . . II-154

C-46 Well Log Determined Properties vs Depth

for Wel1 MP-205................. . . II-155

C-47 Well Log Determined Properties vs Depth

for Well MP-206............... II-156

C-48 Well Log Determined Properties vs Deptli

for Well MP-207................ . . II-157

C-49 Well Log Determined Properties vs Depth

for Well MP-208................. II-158

C-50 Well Log Determined Properties vs Depth

for Well MP-209............... . . II-159

C-51 We11 Log Determined Properties vs Depth

for Well MP-210................... II-160

C-52 Well Log Determined Properties vs Depth

for Well MP-211................. II-16] 
C-53 Well Log Determined Properties vs Depth for Well MP-212................ II-162

C-54 We11 Log Determined Properties vs Depth for Well MP-213.................. II-163

C-55 Wel1 Log Determined Properties vs Depth for Wel1 MP-214................ . . II-164

C-56 Well Log Determined Properties vs Depth for Well MP-215................. . . II-165

C-57 We11 Log Determined Properties vs Depth for Well MP-216................ II-166

C-58 We11 Log Determined Properties vs Depth for Well MP-217................ II-167

C-59 Well Log Determined Properties vs Depth for Well MP-218................ II-168

C-60 Wel1 Log Determined Properties vs Depth for Well MP-220................ . . II-169

C-61 Well Log Determined Properties vs Depth for Wel1 MP-221................. II I-170

C-62 Wel1 Log Determined Properties vs Depth for Well MP-222................. . . II-171

C-63 We1l Log Determined Properties vs Depth for Wel1 MP-223................ . . II-172

C-64 We1] Log Determined Properties vs Depth for Wel1 MP-224............... II-173

C-65 We11 Log Determined Froperties ve Depth for Well MP-225................ II-174

D-1 Static Pressure Comparison for El Dorado Test Wells and Chesney Well 190............. II-254

D-2 Summary of Injectivity for Test Using Well MP-104 . . II-255

D-3 Summary of Falloff Tests for Well MP-104. . . . . . II-256

E-1 Disclosure Keys for Analysis of the Scientific and Technical Contents of a Patent. . . . . . . . I I-264

F-1 Breakthrough Sweep Efficiency at Various Reservoir Conditions............... . . . II-272

F-2 Data Used in Preflush Simulation. . . . . . . . . II-273 
F-3 Linear Displacement Data. . . . . . . . . . . II-274

F-4 Injection. Rates and Slug Sizes El Dorado Micellar-Polymer Project. . . . . . . . . . II-275

F-5 Parameters Used in Simulation for Pattern Comparison. - II-276

F-6 Pore Volumes Injected at Breakthrough for El Dorado Micellar-Polymer Flood Mobility Ratio: = 1. . . II-277

F-7 Estimated Stabilized Injection Rates for El Dorado Micellar Polymer Flood . . . . . . . . . . . II-278

F-8 Comparison of 6.4 Acre And 3.2 Acre Five-Spots and 6.4 Acre Nine-Spots.............. II-279

F-9 Four 6.4 Acre Five-Spot Patterns with Overinjection............... . . II-280

F-10 Twinned Central Injection Wells for 6.4 Acre Five-Spot Patterns. .............. . . II-281

G-1 Chesney-Hegberg Unit MP Well Data . . . . . . . II-312

G-2 Chesney-Hegberg Unit MP Well Data.......... II-313 


\section{LIST OF FIGURES}

FIGURE

PAGE

A-1 0 il Recovery Flow Test No.1............. II-15

A-2 0 il Recovery Flow Test No.2............. II-15

A-3 0 il Recovery Flow Test No. 3. . . . . . . . . . II-16

A-4 011 Recovery Flow Test No.4............. II-16

A-5 0 il Recovery Flow Test No. 5. . . . . . . . . . . II-17a

A-6 0 il Recovery Flow Test No.6............... II-17a

A-7 0 il Recovery Flow Test No.7............. II-17b

A-8 Effect of Chesney Brine on Viscosity of 30 percent

Active Liquid Polymer................ II-18

A-9 Effect of Chesney Brine on Viscosity of Dry Polymer . . . . II-19

A-10 Viscosity vs Concentration of Liquid Polymer in

Supply Water. ................ . II-20

A-11 Effect of Temperature on Viscosity of $1000 \mathrm{ppm}$

(Active Basis) Liquid Polymer ........ . . . . . II-21

A-12 Screen Factor Loss After Shearing at Different

Frontal Velocities.............. II-22

A-13 Viscosity Loss After Shearing at Different

Frontal Velocities. .............. II-22

A-14 Water Sensitivity Flow Tests - Permeability

Unaffected by Salinity. . . . . . . . . . . . II-23

A-15 Water Sensitivity Flow Tests - Lower Permeability

to Fresh Water. . . . . . . . . . . . . . II-24

A-16 Water Sensitivity Flow Tests Constant Loss of

Permeability to Either Fresh or Salt Water. . . . . . . II-25

A-17 Viscosity vs Pressure for Bottom-Hole Crude 0il Sample

From Chesney 195............... . . II-26

A-18 Salinity Change in Initial Water Produced from

Well MP-124................... II-27 
FIGURE

PAGE

A-19 Polymer Concentrate Flow Rate vs Differential Pressure at Various Temperatures . . . . . . . . . . . . II-28

A-20 Polymer Concentrate Flow Rate vs Differential Pressure for Various Lengths of 1/8-inch I. D. Tubing. . . . . . . II-29

B-1 Project Layout Showing Locations and Types of Cores . . . II-57

B-2 Routine Core Analysis for Well MP-101 . . . . . . . . II-58

B-3 Routine Core Analysis for Well MP-702 . . . . . . . II-59

B-4 Routine Core Analysis for Well MP-103 . . . . . . . . II-60

B-5 Routine Core Analysis for Well MP-104 . . . . . . . . . II-6]

B-6 Routine Core Analysis for Well MP-105 . . . . . . . . II-62

B-7 Routine Core Analysis for Well MP-106 .......... II-63

B-8 Routine Core Analysis for Well MP-110 . . . . . . . . . II-64

B-9 Routine Core Analysis for Well MP-116.......... II-65

B-10 Routine Core Analysis for Well MP-120 . . . . . . . . II-66

B-11 Routine Core Analysis for Well MP-122 . . . . . . . . II-67

B-12 Routine Core Analysis for Well MP-130 . . . . . . . II-68

B-13 Routine Core Analysis for Well MP-201 .......... II-69

B-14 Routine Core Analysis for Well MP-203 . . . . . . . . . II-70

B-15 Routine Core Analysis for Well MP-205 . . . . . . . . II-71

B-16 Routine Core Analysis for Well MP-213 . . . . . . . . II-72

B-17 Routine Core Analysis for Well MP-215 . . . . . . . II 73

B-18 Routine Core Analysis for Well MP-221 . . . . . . . . II-74

B-19 Routine Core Analysis for Well MP-223 . . . . . . . II-75.

B-20 Routine Core Analysis for Well MP-225 ......... II-76

B-21 Contour Map of Permeability (from Core Analyses) for

El Dorado Project Area............. . . II-77

B-22 Contour Map of Porosity (from Core Analyses) for

El Dorado Project Area. . . . . . . . . . . . II-78

B-23 Contour Map of $0 i 1$ Saturation (from Core Analyses) for

El Dorado Project Area. . . . . . . . . . . . II-79 
FIGURE PAGE

B-24 Contour Map of Gross Thickness (from Core and Well Logging Analyses) for El Dorado Project Area . . . . . . . II-80

B-25 Contour Map of 0il In Place (from Core Analyses) for El Dorado Project Area ............. II-81

B-26 Logarithm of Permeability vs Standard Deviation We11 MP-101....................... II-82

B-27 Logarithm of Permeability vs Standard Deviation Well MP-102. ................... II -82

B-28 Logarithm of Permeability vs Standard Deviation We11 MP-103.................. II-83

B-29 Logarithm of Permeability vs Standard Deviation Wel1 MP-104..................... II-83

B-30 Logarithm of Permeability vs Standard Deviation Well MP-105.................. II-84

B-31 Logarithm of Permeability vs Standard Deviation Well MP-106. ................. II-84

B-32 Logarithm of Permeability vs Standard Deviation Well MP-110....................... II-85

B-33 Logarithm of Permeability vs Standard Deviation We11 MP-116...................... II-85

B-34 Logarithm of Permeability vs Standard Deviation Well MP-120.................... II-86

B-35 Logarithm of Permeability vs Standard Deviation Wel1 MP-122................... II-86

B-36 Logarithm of Permeability vs Standard Deviation Wel1 MP-130................... II-87

B-37 Logarithm of Permeability vs Standard Deviation We11 MP-201..................... II-87

B-38 Logarithim of Permeability vs Standard Deviation Well MP-203................. II-88

B-39 Logarithm of Permeability vs Standard Deviation Well MP-205.................... II-88

B-40 Logarithm of Permeability vs Standard Deviation Well MP-213................... II-89

B-41 Logarithm of Permeability vs Standard Deviation Well MP-215....................... II-89 
B-43 Logarithm of Permeability vs Standard Deviation Well MP-223..................... . . II-90

B-44 Logarithm of Permeability vs Standard Deviation Well MP-225. . . . . . . . . . . . . . . .

B-45 $\quad K_{W} / K_{0}$ vs Water Saturation We11 MP- 104

B-46 0il-Water Relative Permeabilities Well MP-104.

B-47 Relative Permeability Ratio vs Water Saturation for Sample 14, Well MP-104 . . . . . . . . . . . II-94

B-48 Relative Permeability Fraction vs Water Saturation for Sample 14, Well MP-104............ . II-95

B-49 Relative Permeability Ratio vs Water Saturation for Sample 17, Well MP-104............. . II-96

B-50 Relative Permeability Fraction vs Water Saturation for Sample 17, Well MP-104............

B-51 Relative Permeability Ratio vs Water Saturation for Sample 11, Well MP-104 . . . . . . . . . . . II-98

B-52 Relative Permeability Fraction vs Water Saturation

for Sample 11, Well MP-104.............

B-53 Relative Permeability Ratio vs Water Saturation

for Sample 6, Well MP-104.

B-54 Relative Permeability Fraction vs Water Saturation for Sample 6, Well MP-104.

B-55 Relative Permeability Ratio vs Water Saturation for Sample 21 , Well MP-104............. IT-1ח?

B-56 Relative Permeability Fraction vs, Water Saturation

B-57 Relative Permeability Ratio vs Water Saturation for Sample 3, Well MP-104.

B-58 Relative Permeability Fraction vs Water Saturation

for Sample 3, Well MP-104.............. II-105

B-59 Relative Permeability Ratio vs Water Saturation

for Sample 2, Well MP-124.

B-60 Relative Permeability Fraction vs Water Saturation

for Sample 2, Well MP-124. 
xxiv

FIGURES

PAGE

C-1 Dual Induction Focused Log for Well MP-208. . . . . . . . II-175

C-2 Proximity Log-Minilog with Caliper. . . . . . . . . . II-176

C-3 Compensated Densilog for Well MP-208. . . . . . . . . II-177

C-4 Compensated Neutron Log for Well MP-208 ......... . II-178

C-5 Compensated Acoustilog for Well MP-208. . . . . . . . . II-179

C-6 Example Epilog. . . . . . . . . . . . . . II-180

C-7 Epilog for Well MP-101................. II-181

C-8 Epilog for Well MP-102. . . . . . . . . . . . . . . II-182

C-9 Epilog for Well MP-103................ II-183

C-10 Epilog for Well MP-104................... II-184

C-11 Epilog for Well MP-105................ II-185

C-12 Epilog for Well MP-106................. II-186

C-13 Epilog for Well MP-107................. II-187

C-14 Epilog for Well MP-108................ II-188

C-15 Epilog for Well MP-109............... II-189

C-16 Epilog for Well MP-110................ II-190

C-17 Epilog for Well MP-111...................... II-191

C-18, Epilog for Well MP-112................ II-192

C-19 Epilog for Well MP-113................. II-193

C-20 Epilog for Well MP-114................ . II-194

C-21 Epilog for Well MP-115.๑............... II-195

C-22 Epilog for Well MP-116................ II-196

C-23 Epilog for Well MP-117................ . II-197

C-24 Epilog for Well MP-118. . . . . . . . . . . . . II-198

C-25 Epilog for Well MP-119................ II-199

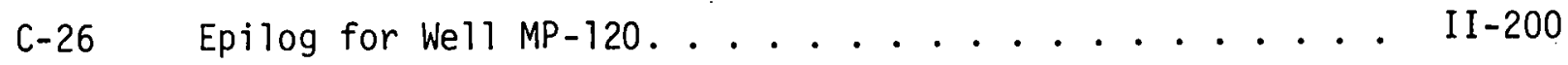

C-27 Epilog for Well MP-121................ II-201

C-28 Epilog for Well MP-122. . . . . . . . . . . . . II-202 
C-29 Epilog for Well MP-123............ II-203

C-30 Epilog for Well MP-124............. II-204

C-31 Epilog for Well MP-125................ II-205

C-32 Epilog for Well MP-126............ II-206

C-33 Epilog for Well MP-127............. II-207

C-34 Epilog for Well MP-128.............. II-208

C-35 Epilog for Well MP-129........... II-209

C-36 Epilog for Well MP-130............ II-210

C-37 Epilog for Well MP-201............ II-211

C-38 Epilog for Well MP-202. . . . . . . . . II-212

C-39 Epilog for Well MP-203............ II-213

C-40 Epilog for Well MP-204. . . . . . . . . . II-214

C-41 Epilog for Well MP-205................. II-215

C-42 Epilog for Well MP-206............ II-216

C-43 Epilog for Well MP-207. . . . . . . . . . II-217

C-44 Epilog for Well MP-208. . . . . . . . . II-218

C-45 Epilog for Well MP-209............ II-219

C-46 Epilog for Well MP-210............ II-220

C-47 Epilog for Well MP-211............ II-221

C-48 Epilog for Well MP-212............ II-222

C-49 Epilog for Well MP-213............. II-223

C-50 Epilog for Well MP-214............ II-224

C-51 Epilog for Well MP-215............ II-225

C-52 Epilog for Well MP-216............ TT-226

C-53 Epilog for Well MP-217............. II-227

C-54 Epilog for Well MP-218............. II-228

C-55 Epilog for Well MP-220. . . . . . . . . . II-229

C-56 Epilog for Well MP-221............ II-230

C-57 Epilog for Well MP-222............ II-231 
$x x v i$

FIGURES

PAGE

C-58 Epilog for Well MP-223

I I -232

C-59 Epilog for Well MP-224

I I -233

C-co Epilog for Well MP-225

I I -234

C-61 Top of Pay

I I -235

C-62 Gross Pay.

II -236

C-63 Net Pay.

II -237

C-64 Porosity

II -238

C-65 Permeability Index

I I -239

C-66 Porosity-Thickness.

I I -240

C-67 Permeability Index-Thickness . . . . . . . . . . . . II-24I

C-68 0 il Saturation . . . . . . . . . . . . . . . II-242

C-69 Res. 0 il Sat. . . . . . . . . . . . . . . . . II-243

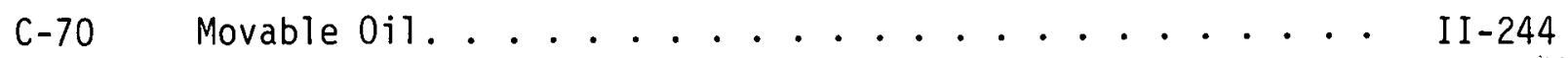

C-71 Hydrocarbon P.V. . . . . . . . . . . . . . . . II-245

C-72 \% Clay...................... . . II-246

C-73 Gamma Radiation. . . . . . . . . . . . . . II-247

C-74 Water Saturation . . . . . . . . . . . . . II-248

C-75 Mud Filtrate Saturation, Flushed Zone. . . . . . . . . II-249

C-76 True Resistivity . . . . . . . . . . . . . . II-250

C-77 Resistivity, Flushed Zone. . . . . . . . . . . . II-25I

D-1 Summary of Interference Test Data. . . . . . . . . . II-257

Injectivity (Q/DP) vs Cumulative Injection for
Well MP-104 Tests, Aug.-Sept., 1974. . . . . . . II-258

D-3 Test Well Interference Data for Well MP-104. . . . . . . II-259

D-4 Multi-Rate Injection Tests for Well MP-104 . . . . . . . II-260

D-5 Multi-Rate Injection Tests for Wel1s MP-1.01 and MP-103. . II-261

E-1 Viscosity Behavior of Hydrolyzed Polyacrylamide

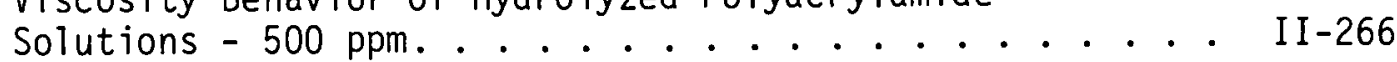


E-2 Viscosity Behavior of Xanthomonas Polysaccharide -

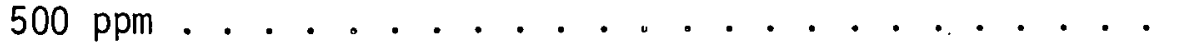

E-3 Adsorption of Polymer from 10 Percent Sodium Chloride Solution. ............ II-268

E-4 Viscosity vs Shear Rate, 600 ppm Polyacrylamide in $300 \mathrm{ppm} \mathrm{NaCl}$ Solution. ............

F-1 Waterflood Performance of Pattern Centered at Chesney 17................. II-282

F-2 Waterflood Performance of Pattern Centered at Chesney 19................... II-283

F-3 Waterflood Performance of Pattern Centered at Chesney 40.................. II-284

F-4 Relative Permeability and Capillary Pressure for Preflush Calculations . . . . . . . . . . II-285

F-5 Model Dimensions and Grids. . . . . . . . II-286

F-6 Displacement of Brine with Fresh Water in a Core. . . . II-287

F-7 Chesney Pattern - Estimated Preflush Injection vs Time (Case 1)................ II-288

F-8 Chesney Pattern - Estimated Preflush Injection vs Time (Case 2)............... II-289

F-9 Chesney Pattern - Estimated Preflush Injection vs Time (Case 3). . . . . . . . . . II-290

F-10 Hegberg Pattern - Estimated Preflush Injection vs Time (Case 1).

F-11 Hegberg Pattern - Estimated Preflush Injection vs Time (Case 2).

F-12 Hegberg Pattern - Estimated Preflush Injection vs Time (Case 3 ).

F-13 Hegberg Pattern - Fresh Water Saturation Maps for Quarter of a Nine-Spot - Time $=10$ Months ......

F-14 Hegberg Pattern - Fresh Water Saturation Maps for Quarter of a Nine-Spot - Time $=10$ Months ......

F-15 Hegberg Pattern - Comparison of Displacement Efficiencies. . . . . . . . . . . . .

F-16 El Dorado Composite Fractional Flow Curve Based on Restored State Cores. 
F-17 Estimated Production Schedule - Chesney Lease . . . . II-298

F-18 Estimated Production Schedule - Hegberg Lease. . . . . II-299

F-19 Composite E1 Dorado $k_{W} / k_{0}$ Curve for Chesney MP-104 Native-State Core................ II-300

F-20 Fractional Flow Curve Based on Core Laboratories, Inc., Permeability Ratio Curve for Native-State Core From Well Chesney MP-104.............. II-301

F-21 0i1 Recovery vs Cumulative Injection for Demonstration Flood .................... II -302

F-22 WOR vs Cumulative Injection for Demonstration Flood .......................... II

F-23 0 il Recovery vs Time for Demonstration Flood...... II-304

F-24 WOR vs Time for Demonstration Flood ........ II-305

F-25 0 il Rate vs Time for Demonstration Flood. . . . . II-306

F-26 Pattern Nomenclature. . . . . . . . . II-307

F-27 Four 6.4 Acre Nine-Spots on Each Lease. . . . . . . II-308

F-28 Four 6.4 Acre Five-Spots on Each Lease. . . . . . II-309

F-29 Five 3.2 Acre Five-Spots Plus Four Extra

Producers on Each Lease .......... II-310

G-1 Project Layout. ................ II-314

H-l Simplified Flow Diagram ............... II-316

H-2 Battery Farilitips. .............. II-317 
I-1

RESEARCH SUPPORT 


\section{CHEMICAL SELECTION AND SUPPORT}

by

J. W. Biles

with

Contributing Authors H. M. Al-Rikabi, R. S. Fulford, G. E. Kellerhals, S. A. Hobarak, W. H. Pusch, and E. F. Riedel

\section{Summary}

Following a comprehensive search for competent and available vendors of micellar-polymer oil recovery techniques, Union 0il Company and She11 Development Company were selected to design processes for the 650-foot Sand reservoir in Butler County, Kansas. Union 0il Company submitted its design in May, 1975. The final Shell design is expected in August, 1975.

$0 i 1$ recovery flow tests using fluids and slug sizes recommended by Union in ten-inch long Berea and 650-foot Sand cores resulted in final oil saturations of about ten percent pore volume. Most of the tertiary oil was recovered at a low water-oil ratio.

Testing has indicated that permeability of the 650 -foot Sand is slightly less to fresh water than to salt water in some localized areas. 
A preliminary test indicated that mobility control with polyacrylamide polymer solution should reduce viscous fingering and help maintain the integrity of the produced oil bank.

Use of a high wax content crude oil in the soluble oil recommended by Union will make it necessary to heat the oil before injection to prevent plugging of the formation.

Simultaneous use of a wide variety of waterflood tracers requires special schemes of analysis to avoid interference among analytical methods of identification.

Use of surfactants could result in production of stable emulsions, depending upon the salinity of the produced fluid. Adding field brine. to emulsions may be a practical method to separate the oil and water. 


\section{Introduction}

The objectives of this work are to (1) select two vendors of micellar-polymer processes to design different demonstration floods, (2) provide checks on chemical aspects of applying process designs to the 650-foot Sand reservoir, and (3) provide chemical support as needed in all phases of the project. Although many other disciplines are needed, chemical technology is essential in carrying out a successful large scale demonstration of any micellar-polymer process. Chemicals selected must be compatible with reservoir rock and fluids to prevent reduced permeability near injection and producing wells. Some chemicals require specialized handling or mixing techniques. Specifications for purchase and analysis of injected fluids require special analytical techniques. Enhanced oil recovery by micellar-polymer flooding is basically a chemical process and requires a concentrated application of chemical expertise, especially in the early stages of a project.

\section{Dis.cussion}

Selection of Process Vendors

An effort was made to contact all oil companies in the United States considered to have an advanced research position in the field of micellar-polymer flooding. Contacts were made with 13 companies. Early contacts by telephone and mail were sufficiently encouraging to arrange visits of the project technical personnel with technical experts from six interested oil companies. Some companies were interested in the micellar-polymer demonstration project, but either did not have enough personnel to work on an additional project or were not quite 
ready to take their process to the field. Interested prospective process vendors were then evaluated for the El Dorado project using the following criteria:

1. Technical competence based on publications and discussions with technical experts.

2. Field test experience.

3. Desire and ability to participate.

4. Degree of restrictions in proposed contracts.

5. Ability to meet the required time schedule.

An agreement between Union $0 i 1$ Company and Cities Service $0 i 1$ Company was signed in December, 1974. A contract between Shell 0il Company and Cities Service 0il Company has also been finalized.

\section{0il Recovery Flow Tests--Union Process}

Five oil recovery flow tests were conducted in the cities Service Laboratory using Berea Sand cores 2.0 inches in diameter and 10.0 inches long. Properties of these cores are shown in Table Al in Appendix A.* Cores were evacuated and saturated with brine produced from the 650-foot Sand to determine pore volume. Chesney lease crude was then injected until brine was no longer produced, thus establishing a connate water saturatinn. Cores werc then waterrluoded using lhesney produced brine and a post-waterflood oil saturation was calculated.

The sequence of fluids injected for tertiary oil recovery was as follows: 0.10 pore volume (PV) preflush, 0.04 PV micellar *Almost all Tables and Figures are located in the Appendices in Volume II of this report. 
fluid (soluble oil and water), 0.60 PV of a polyacrylamide solution, and fresh water. Tertiary oil recovery flow tests were conducted at a frontal advance rate of 3.5 feet per day. As illustrated in Figures A-1 through A-5 (which are plots of tertiary oil recoveries versus fluid volumes injected), crude oil injected as part of the soluble oil slug was taken into consideration for determining net oil recovery and final oil saturation. Post-waterflood oil saturation, oil recovery, and final oil saturation for each test are summarized in Table Al.

Data from Flow Tests 1 and 2 are useful only to 111 ustrate the poor results obtained when the proper soluble oil composition or polymer is not used (see remarks in Table Al). In Flow Test 3, the soluble oil was the composition used in the field injectivity test (see the section on "Formation Injectivity"). This soluble oil composition yielded better oil recovery, but the costs would be too great for commercial use. The optimal mixture had not been seiected at the time the injectivity test was conducted. This mixture contained about 16 percent more active sulfonate ingredient than the mixture finally recommended.

In Flow Tests 4 and 5 , the final soluble oil composition recommended by Union was used. As shown in Figures A-4 and A-5, about 0.73 of the post-waterflood oil saturation was recovered at an oil cut of 40 to 50 percent. The final oil saturations were about 0.09 PV. Table Al shows that Flow Tests 1 and 3 utilized 0.1 PV of 800 parts per million (ppm) polymer slugs followed by 0.5 PV slugs of $400 \mathrm{ppm}$ polymer. This procedure was changed to use $0.6 \mathrm{PV}$ of $600 \mathrm{ppm}$ polymer in flow tests $2,4,5,6$, and: 7 . 
Flow Tests 6 and 7 were conducted on stacked 650 -foot Sand cores having a total length of 10.0 to 11.0 inches. Each core in the stack was 2.0 inches in diameter and about 1.8 inches 1ong. These tests were conducted in a manner similar to the Berea core tests. Core data, slug descriptions, and oil recoveries are given in Table A2. $0 i 1$ recovery as a function of injected volume is illustrated for Tests 6 and 7 in Figures A-6 and A-7. Waterflood recovery was very good in these flow tests, leaving about 0.20 PV of $0 i 1$ to be recovered by the Union process. The micellar flood left about 0.11 PV of oil in the stacked cores; this is slightly more than was left in Flow Tests 4 and 5 using the same fluids in Berea cores. $0 i 1$ recovery tests using four-foot cores are scheduled.

Polymer Viscosity Measurements

Union $0 i 1$ Company personnel indicated that either of two commercially available polyacrylamides could be used for their process. One is sold in the conventional powder form and the other is a 30 percent active liquid. Viscosity development by each of these products was measured in the fresh water (raw water or El Dorado lake water) to be used in the project and in mixtures of lake water and resident waters. Figures $A-8$ and $A-9$ show the effect of small amounts of Chesriey brine on the viscosity of each polymer solution prepared with raw water.

A tentative decision was made by Cities to. use the liquid polymer with the Union Process, partly due to greater ease in handling. Viscosity developed by varlous concentrations of the liquid polymer in the fresh supply water was measured. The data are shown in Figure A-10. The coincidence of reservoir temperature $\left(69^{\circ} \mathrm{F}\right)$ being about the same as 
normal room temperature greatly facilitated realistic experimental work. However, since there was some variation in room temperature, the effect of temperature on viscosity of $1000 \mathrm{ppm}$ polymer solution was determined. Viscosity variations are minor in the range of $66^{\circ} \mathrm{F}$ to $82^{\circ} \mathrm{F}\left(19^{\circ} \mathrm{C}\right.$ to $\left.28^{\circ} \mathrm{C}\right)$. Data are shown in Figure $\mathrm{A}-11$.

Mobility Control by Polymers

Mobility of polymer solutions was measured in both Berea and 650-foot Sand cores. In these mobility tests, polymer was dissolved in filtered El Dorado lake water. Data from tests using Berea cores are shown in Table A3. Two 1.0-inch diameter cores were used in Test No. 1. Each core was mounted in a Hassler holder, and the two holders were connected in series. In Test No. 2 , the mobilities of solutions with lower polymer concentrations were determined in another 1.0-inch diameter Berea core. The data show the expected lower mobility for higher polymer concentrations in a given core. In tests using a larger, less permeable core 2.0 inches in diameter, the mobility was in the same order of magnitude as that of other tests. This set of experiments indicated that (1) the reduction of water mobility in Berea cores (455 to $803 \mathrm{md}$ ) is in the range from ten to one hundred fold (see Table A3) with polymer concentrations ranging from 200 to $1000 \mathrm{ppm}$, and (2) the efficiency of mobility reduction with low concentration polymer (200 ppm) is not as great as at higher concentrations.

Table A4 presents data from mobility measurements using 650foot Sand cores. In Test No. 1, two cores were connected in series as described above. During this test, flow resistance continued to increase even after injecting $50 \mathrm{PV}$ of polymer. The cause of this problem was 
not determined. Mobility values obtained seem abnormally low. In Test No. 2, three PV of soluble oil were injected followed by 40 PV of fresh water before beginning polymer injection. Behavior similar to that of Test 1 was observed even after injecting 80 PV of polymer. Constant mobilities were obtained in Test No. 3 , in which two 2.0-inch diameter by 2.0-inch long cores were stacked in a single Hassler holder. Stabilized resistance to flow was established after five PV of injection. Mobilities obtained in Test No. 3 compare well with values supplied by Union $0 i 1$ Company.

\section{Degradation of Polyacrylamide}

Shear degradation of polyacrylamide solutions was studied in order to determine loss of mobility control resulting from mechanical shearing of polymer due to mixing or throttling (to control injection well rates). Several tests were conducted to simulate mixing the commercial 30 percent active liquid polymer concentrate. In these tests, concentrate and either 50 or 100-fold dilutions of concentrate were subjected to shear in a Waring blender, a Hamilton-Beach mixer, or a pacile mixer. The sheared polymer was then diluted to a concentration of $600 \mathrm{ppm}$. Viscosities and screen factors were measured. The $600 \mathrm{ppm}$ solutions were then sheared for three minutes in the Waring blender, and viscosities and screen factors were again measured. Results are shown in Table A5.

The data show that shearing of the 30 percent active liquid polymer had no adverse effect on the viscosity and screen factor of $600 \mathrm{ppm}$ solutions prepared from the concentrates. Shearing of the 50 and 100-fold dilutions of the concentrates resulted in loss of 12 to 
84 percent of Brookfield viscosity, depending upon the severity of shearing. (Severity of shearing is in the order Waring blender > HamiltonBeach mixer > paddle mixer.) Loss of screen factor was more severe than loss of viscosity. Shearing of the $600 \mathrm{ppm}$ solutions resulted in severe loss of both viscosity and screen factor.

Mechanical shearing of polymer during entry into the sandface at the wellbore was also expected to contribute to loss of polymer effectiveness. To simulate and measure degradation due to this effect during application of the Union process, Union preflush and soluble oil were injected into 650 -foot Sand cores followed by $600 \mathrm{ppm}$ polymer in El Dorado lake water. The cores were 1.0-inch in diameter and 1.5 to 2.0 inches long. The viscosity and screen factor of effluent polymer solutions were found to decrease with increasing injection rate. This was interpreted as breakdown of polymer structure due to mechanical shearing. Data are shown in Figures $A-12$ and $A-13$. Loss of viscosity and screen factor are plotted as functions of frontal advance rate on the bottom axis and equivalent barrels per day injection rate on the top axis. Equivalent barrels per day injection rate is calculated from a comparison of cross-sectional areas available to flow in the laboratory test and in the $77 / 8$ inch wellbore open-hole complelions of injection wells (it was assumed that 14 feet of sand would be taking fluid). Maximum viscosity losses were in the range of 20 to 30 percent. Maximum screen factor losses were in the range of 32 to 52 percent.

The effect of polymer aging was studied under various salinity conditions, with and without formaldehyde and chlorinated phenol biocides. Two polymers supplied as a powder and the 30 percent active liquid polymer concentrate were used in these tests. Solutions containing $600 \mathrm{ppm}$ 
(dry basis) polymer were stored in glass bottles containing minimum air space. Viscosity and screen factors were measured on the date of mixing and 31 and 66 days afterward. Results are shown in Tables A6 and A7. Viscosity and screen factors are higher for samples prepared from the liquid concentrate than from the powder; this is at least partly due to the higher percentage of inorganic salt present in the powder form. This effect would not be as noticeable if the polymers were prepared in brine rather than fresh water. The data show that 66 days of aging has little effect on viscosity under the conditions studied in the absence of formaldehyde. Severe loss of viscosity was shown for the liquid polymer and the lower molecular weight powdered polymer in the presence of $200 \mathrm{ppm}$ formaldehyde. The intermediate molecular weight powder was not affected. All three polymers studied sustained most of their original viscosity in the presence of $50 \mathrm{ppm}$ chlorinated phenol. The liquid polymer concentrate developed slightly higher viscosity in the presence of chlorinated phenol than without it.

Since the original project plans included perforating injection wells, a program was initiated to evaluate polymer degradation in flow through perforations. Arrangements were made with Dresser Industries for them to supply several perforated targets to be used for polymer flow tests. Degranation was to be detcrmined by comparing the polymer properties before and after flowing through the perforated target. This work has been relegated to a low priority due to the decision to use open-hole completions. Two injectors were completed by perforating before this decision was made; however, they may be redrilled using open-hole completions. 
Fresh Water Sensitivity

Numerous clay analyses were performed and flow tests were conducted for the purpose of determining if the permeability of the 650foot Sand is less for fresh water than for salt water. Clay analyses by $X$-ray diffraction run on cores from new wells indicated that swellingtype clays were present in a small proportion of the samples (see the section on "Coring and Core Analyses"). Flow tests were run on old cores obtained during previous waterflood development. New cores were not available at the time. The procedure used for flow tests was as

follows: (1) saturate the core with salt water (either ten percent sodium chloride or Chesney produced water), and (2) inject salt water, fresh water, then salt water again at a constant frontal advance rate of about 190 feet per day while observing upstream pressure.

Nearly all of the flow tests could be grouped into one of the three following categories.

1. Permeability was the same for both fresh and salt water (eight tests).

2. Permeability to fresh water was definitely lower (averaging 28 percent lower) than to salt water (six tests).

3. There was a continuous downward trend in permeability at the same rate of decline with either fresh or salt water (seven tests).

Figures $A-14, A-15$, and $A-16$ are typical examples of the data obtained from each group of tests. It appears from these results that fresh water sensitivity is a localized problem. 
Fluid Analysis

Most of the laboratory work requiring reservoir fluids was done with $0 i 1$ and water produced from we 11 Chesney 195 which is located about one-half mile north of the Chesney test pattern. $0 i 1$ stored in the laboratory for experimental work had a viscosity of 5.2 centipoises at reservoir temperature $\left(69^{\circ} \mathrm{F}\right)$. A bottom-hole sample was taken from well Chesney 195 on June 17, 1974, and analyzed in the laboratory. The sample was taken at 450 feet; the pressure and temperature at sampling depth were $59 \mathrm{psig}$ and $69^{\circ} \mathrm{F}$. Comparing the bubble point pressure of 17 psig with the reservoir pressure of $164 \mathrm{psig}$ indicates that the oil is undersaturated at reservoir temperature. The oil volume changed only slightly from reservoir to stock tank conditions, and only a small amount of gas evolved. The solution gas-oil ratio is 0.95 with an API gravity of 37.4. Variation of viscosity with: pressure is shown in Figure A-17. Acid number (determined using ASTM P664-58) was less than 0.2. Analyses of produced water from well Chesney 195 and El Dorado lake water (the fresh water supply) are shown in Table A8. Water samples were collected from wells MP-112, 114, 122, $124,207,209,217$, and 219. An attempt was made to produce enough fluid to thoroughly sweep mud filtrate from the wellbore and obtain water samples representative of the reservoir. To determine how much fluid must be produced to reach a constant composition (assumed to be that of the resident brine), the chloride content of the first 1250 barrels of water produced from wel1 MP-124 was determined at frequent intervals. These data, shown in Figure A-18, indicate little change in salinity after the first 100 barrels of water were produced. Subsequently, water samples taken after producing 200 barrels were 
assumed to be representative of the brine in the reservoir in the vicinity of the well being pumped. Results of analyses of water samples from each of these wells are shown in Table A9.

The data in Table A9 reveal inconsistencies in concentrations of barium, strontium, and sulfate. These inconsistencies may be due to the use of sodium sulfite in the drilling fluid used to obtain "nativestate" cores. Sulfite is oxidized to sulfate by dissolved oxygen, thus decreasing oxidation of crude oil with a resulting oil-wetting tendency. Sulfates and sulfites of barium and strontium have very low solubilities. Sulfite or sulfate from the drilling fluid may have caused precipitation of bariuil and strontium in the produced water. The best indication of the actual concentrations of barium, strontium, and sulfate in the resident brine is the composition of water produced from well Chesney 195 shown in Table A8. This is one of the few waterflood wells still pumping; the water from this well should not be affected by sulfite contamination.

\section{Emulsions}

The primary area of work in this Laboratory has been to create and classify the ilany types and combinations of emulsions that may be produced during the micellar-polymer flood and to perform preliminary work on effective treating methods for these emulsions.

Generally, each of the two processes to be tested in the El Dorado project includes injection of four solutions--preflush, micellar (surfactant) fluid, a mobility control agent (polymer solution), and water (to drive the chemicals through the reservoir). Portions of these injected fluids, and the resident brine, native crude oil, and formation 
rock are considered as potential emulsion constituents. The use of two distinct surfactant systems may also significantly alter the nature of emulsions that may be produced by the two processes. Furthermore, the composition of the produced mixture will vary as the flood progresses. Many complex combinations may be generated over a period of time.

The first phase of the study included the preparation and classification of emulsions consisting of various proportions of fluids that may be mixed during the application of the Union process. The mixtures investigated have included:

1. Soluble oil + formation water

2. Soluble oil + formation water + native crude

3. Soluble oil + formation water + preflush

4. Soluble oil + formation water + native crude

5. Soluble oil + polymer solution

6. Soluble oil + polymer solution + native crude

7. Soluble oil + drive water

8. Soluble oil + drive water + native crude

The first problem was to choose a mixing apparatus and/or procedure capable of producing mixtures or emulsions which would be somewhat representative of produced fluid in the field. If emulsions prepared by varying the consentration of each of the components in the above mixtures can be effectively treated in the laboratory; then the probability of successfully treating produced emulsions in the field should be substantially increased.

Several methods of mixing fluids were evaluated for the preparation of emulsions. These included shaking by hand, a small magnetic stirrer, a Brookfield rotating mixer, a Waring laboratory blender, a 
laboratory colloid mill, and ultrasonic mixing. Emulsions were prepared using each of the above methods. On the basis of emulsion stability (respect to time) the Waring blender was selected as an appropriate mixing device for the preparations of emulsions. Emulsion stability was judged by observing phase separation with time. The emulsion prepared by use of the Waring blender was stable for 48 hours.

In order to characterize the emulsions studied, it was necessary to standardize definitions and criteria. If the continuous phase of a microemulsion is transparent, the microemulsion itself is transparent since the displaced phase droplets are less than one micron in diameter, and one micron is the wavelength of visible light. llacroemulsions, on the other hand, are cloudy or opaque and have particle sizes in the range of one to 100 microns. If the continuous phase consists of a dark brown or black oil, clarity and/or brightness of a thin film of the emulsion can be used as a guide in determining whether the emulsion is better described as macro or micro. The continuous phase of an emulsion can be determined by its miscibility with oil or water. If a drop of emulsion is miscible with water, it is an oil-in-water emulsion (water external). A water-in-oil emulsion (oil external) will be miscible with oil. Ehlers red food coloring was used as a water soluble dye to determine water miscibility. A one percent solution of blue Calco 0il Soluble N (American Cyanamide) in benzene was used to determine 011 miscibility.

Some surfactant systems in the patent 1 iterature are miscible with both oil and water, and hence, are colored.by both the water soluble and oil soluble dye solutions. This type of emulsion, however, does not bleach the blue color from cobalt bromide paper. An oil-in-water emulsion 
will change the color of cobalt bromide paper from blue to pink. For the case of $0 i 1$-in-water $(0 / w)$ emulsions prepared from El Dorado crude oil, the pink color could only be distinguished at the edge of the wetted cobalt bromide paper. Using the dye solutions, the prepared emulsions were characterized as macro $0 / w$, macro $w / 0^{*}$, micro $0 / w$, or micro $w / 0$. Mixtures consisting of various proportions of the aforementioned fluids were prepared by mixing in a Waring blender for two minutes. These laboratory mixtures were examined at $1 / 4,1 / 2,1,2,4,24$ hours and at seven days after preparation. The character (macro or micro, $0 / w$ or $w / 0)$, color $(s)$, and amount (s) of the various phases present in the mixtures were recorded. An ultraviolet light was used to show oil phases which appeared to be homogeneous. Each mixture prepared was unique in composition. For the most part each mixture was also unique in color, viscosity, and in the amounts, colors, and compositions of the various layers which separated after standing. No viscosity measurements were made, but the viscous character of many of the emulsions was apparent when the emulsion was poured. The composition of the various mixtures, and in most cases, a short qualitative comment about the mixtures are given in Tables A10 through Al3.

The soluble oil formed stable emulsions with the preflush solution, 100 pinl pulymer solution, and drive water.' The emulsions separated into oil and aqueous phases within one to two hours when mixed with Chesney brine (containing approximately 93,000 ppm total dissolved solids of which about 4200 ppm are calcium and magnesium ions). Mixtures prepared by combining a 90 percent by volume crude *water-in-oil 
oil and ten percent by volume surfactant fluid with formation brine also. separated into $0 i 1$ and water phases. In general, the presence of Chesney brine resulted in separation of the surfactant-oil-water mixtures into two phases. The results indicate the possible utility of brine addition as a demulsification method.

The amount of Chesney brine required to cause phase separation for the following o/w emulsions was determined.

(1) A mixture consisting of 2.2 volume percent soluble $0 i 1$ and 97.8 volume percent water (water consisted of 1.1 volume percent Chesney brine and 98.9 volume percent El Dorado raw water).

(2) A mixture consisting of 5 volume percent soluble oil and 95 volume percent water (same water as used in \#1 above).

(3) A mixture consisting of 10 volume percent soluble oil and 90 volume percent water (same water as used in \#1 above).

In each of the experiments, addition of Chesney brine to a level of five to six volume percent resulted in the separation of $0 i l$ and aqueous phases.

Two laboratory flow tests using 3.0-inch long by 1.0 -inch diameter Chesney formation cores were conducted in an effort to generate emulsions which might be representative of fluid produced during part of the El Dorado project. Filtered Chesney brine, water (consisting of 1.1 volume percent Chesney brine and 98.9 volume percent El Dorado raw water), and soluble oil were injected alternately, and effluent samples were collected for each pore volume of fluid injected. Analysis of the 
effluent samples indicated that the pore volume of the core was too small to permit adequate mixing of injected fluids to produce a low oil cut o/w emulsion. (Low oil cut o/w emulsions are expected to be produced sometime during the El Dorado flood.)

\section{Miscellaneous}

Analysis of petroleum sulfonates is important to most micellar recovery tests for both quality control of purchased materials and monitoring of produced fluids. Hethods found most useful for analysis of purchased materials are based on ASTM D 855 and D 2548. Use of mixtures of oil-soluble and water-soluble sulfonates requires special modification of these techniques. Work has been done to show the utility of the modifications, but further work will be required.

A series of tests were made to determine the feasibility of measuring flow rates of liquid polymer concentrate by pressure drop across a length of $1 / 8$-inch diameter tubing. The data indicated that the flow rate-differential pressure relationship is 1 inear at $92^{\circ} \mathrm{F}$ and $70^{\circ} \mathrm{F}$ but varies from linearity at $32^{\circ} \mathrm{F}$ (see Figure A-19). When different lengths of tubing were tested, it was found that flow rate vs differential pressure data were linear (see Figure A-20). It was concluded from the data that this technique could be used for measuring pniymer flow rate if the polymer were kept at a fixed temperature or the effect of temperature were taken into consideration.

The crude oil chosen for the soluble oil process has a high wax content and when cooled below $70^{\circ} \mathrm{F}$ caused plugging of the filters. A flow test was conducted on a 650-foot Sand core to show the effect of cooling on injectivity of the soluble oil. In this test, injectivity 
of soluble oil which had been cooled to $0^{\circ} \mathrm{F}$ and allowed to return to room temperature was compared with injectivity of the same soluble oil which had not been subjected to the cooling-warming cycle. It was found that injectivity was constant for the soluble oil which had not been cooled. Injectivity of the cooled and rewarmed soluble oil dropped to two-thirds its original value after five pore volumes injection and was continuing to decrease. As a result of these observations, it was decided to prevent loss of injectivity by heating all of the crude oil used to $120^{\circ} \mathrm{F}$. To avoid reprecipitation of wax the soluble $0 i 1$ will be kept at $70^{\circ} \mathrm{F}$ or higher until it is injected.

Consideration has been given to various plans for use of chemical tracers to detect severe well-to-well channeling before injecting micellar slugs. In order to distinguish the source of channeling, it is desirable to use different tracers in all injection wells. Chemical tracers that can be detected at low concentrations were investigated. Qualitative tests for these materials show a few interferences which will require special analytical techniques if tracer pairs are used in the same area. In addition to the work on chemical tracers, consideration was given to use of commercially available radioactive isotope tracers. Work is still underway to decide whether to use chemical tracers, isotopes, or both.

\section{Future Work}

$0 i 1$ recovery flow tests using the Union process will be continued using four-foot long Berea core and stacks of 650-foot Sand core. Similar tests will be conducted using the Shell process in both ten-inch and four-foot long cores. 
Since microemulsion floods are processes which do not maintain miscibility throughout the reservoir, phase diagrams will be developed to investigate the effects of reservoir fluids on micellar solutions. These phase diagrams should be beneficial in evaluating the field performance of the two demonstration floods. Selected fluid combinations will be stored to determine their stability with time.

Criteria and testing procedures will be developed to check polymer quality. Polymer effectiveness will be checked when each shipment is received and again when dilute polymer reaches the injection wells. Polymer mobility is to be measured in cores subsequent to injection of micellar solutions.

Static and dynamic adsorption/retention tests will be conducted to determine loss of all chemicals used in preflush, micellar, and polymer slugs. These data are to provide checks on the prescribed quantities of chemicals recommended by the process vendors.

Studies in breaking produced emulsions using field brine and commercial demulsifiers will continue.

Adsorption and dispersion of injected chemicals will be determined from analysis of fluid samples collected at observation and producing wells, 
CORING AND CORE ANALYSES

by

W. H. Pusch

with

Contributing Authors G. A. Flesch and C. M. Royo

\section{Summary}

The results of core analyses show a residual oil saturation of 26 percent which indicates about 500,000 barrels of $0 i 1$ in place within the boundaries of the two patterns. Average dry air permeability was found to be 450 millidarcies, porosity to be 26 percent, and average pay thickness equal to 18 feet. In addition to these basic rock properties, the formation was determined to possess intermediate wettability and oil-water permeability data were measured. The sandstone mineral composition typically contains 67 percent quartz, seven percent feldspar, four percent granitic and metamorphic rock fragments, one percent organic materials, and 21 percent clays and/or cements. 


\section{Introduction}

Samples of the Admire sand were taken to determine reservoir properties that will control the movement and production of fluids during the project. In addition, factors that might affect the well completion techniques, chemical injection, and subsequent producing operations were also investigated. The core samples underwent routine core analyses, mineralogical analyses, special fluid flow tests, and other studies to understand the behavior of the rock. Samples are also being preserved for testing in the future as the project progresses and questions or problems arise.

\section{$\underline{\text { Discussion }}$}

$\underline{\text { Coring }}$

The coring program for the El Dorado Micellar-Polymer Project was designed to determine not only detailed reservoir parameters but also to investigate the effects of different drilling fluids. It is known that core flushing will reduce formation fluid saturations so that only remaining oil saturation can be measured. The extent of mud filtrate invasion and its effect on oil saturation was evaluated by the use of a low fluid loss drilling fluid. In addition, to preserve native formation wettability which is often altered during coring, several cores were cut with deaerated brine containing oxygen scavengers. All of these "native state" cores were preserved at the well site by wrapping in plastic wrap and metal foil and sealing in wax.

A total of 31 cores were eut from the project area. These were from five test wells and 26 pattern wells. The drilling fluid for all we11s except MP-105 was formation water. MP-105 was cored 
with a low fluid loss emulsion mud to inhibit filtrate penetration into the core. The mud contained $10 \mathrm{lb} / \mathrm{bbl}$ pregelatinized starch, $8 \mathrm{lb} / \mathrm{bbl}$ bentonite, and small amounts of caustic soda, biocide, and soda ash. There were nine "native state" cores in which an oxygen scavenger $(0.25 \mathrm{lb} / \mathrm{bbl}$ sodium sulfite) was added to the water. Figure B-1 shows the location and types of cores taken from the project area. The results from core and log analyses were very similar and no significant differences could be attributed to the coring technique. The core analysis results for MP-105 which was cored with the low. fluid loss mud indicated that the drilling fluid did not inhibit flushing of the core. All special core flow tests have been conducted on the native state cores; which should result in data more representative of reservoir flow than restored-state core data. No attempt was made to compare the two techniques.

Core Analyses

A total of 19 cores from the project area were analyzed to determine local reservoir porosity, permeability, pay thickness, and immobile oil saturation. The laboratory methods used for the analyses are defined in API-RP-40, Recommended Practice for Core Analysis Procedure. Results of individual core analyses are presented by tables and core graphs in Tables B1 to B19 and Figures B-2 to B-20. The tables display reservoir properties versus depth. The graphs show permeability in millidarcies and porosity in percent versus depth. A summary of the core data and contour maps generated from the average properties of individual wells are included in Table B20 and Figures $B-21$ to $B-25$. 
Native state cores from wel1s MP-114, 118, 124, 207, 209, and 219 are being stored with no analyses having been performed. The cores from. I:P-119 and MP-128 were shipped in their entirety to She11 $0 i 1$ and smaller core samples from other wells have been distributed to subcontractors for various special tests.

\section{Absolute Permeability Distribution}

A detailed analysis of the air permeability data from core analyses indicates that two distinct distributions or populations exist. Observation of the graphs (Figures B-26 to B-44) of the logarithm of permeability on probability paper results in two straight line segments. One straight line can be attributed to the sand intervals while the other is produced from the shale and shaley sand data. The conclusion of this study is that no consistent vertical variation can be determined from core data.

\section{Relative Permeability}

Unsteady state water-oil relative permeability curves were determined for core samples from MP-104 by both Core Laboratories and Cities Service Research. Subsequently, relative permeability curves were measured by the steady-state method on a native state sample from $\mathrm{AP}-124$. The results of these tests are shown on Figures $B-45$ to B-60. Since samples do not exhibit a strong wetting preference for either water or oil, the steady-state results are considered to be more accurate as illustrated by the fact that the steady-state water imbibition and drainage curves lie closer to each other. These results are to be used in the reservoir simulation of the waterflood and micellar-polymer flood. 
Wettability

The wettability of core plugs from well MP-104 was determined by two independent methods. The results from both methods indicate that the cores have intermediate wettability; that is, the samples did not exhibit a strong wetting preference for either water or oil. Wetting preference was established on the basis of water-oil relative permeability data as well as from capillary pressure curves determined with a centrifuge. Both techniques are considered reliable for determining the wettability of cores.

Determination of wetting preference from water-oil relative permeability data is based on three criteria; connate water saturation, the saturation at which $0 i 1$ and water relative permeabilities are equal, and the relative permeability to water at residual $0 i 1$. Using the above criteria, strongly water-wet cores are characterized by:

(a) connate water saturation is greater than 20 to 25 percent of the pore space, (b) water saturation at which $0 i 1$ and water relative permeabilities are equal is greater than 50 percent, and (c) relative permeability to water at residual oil is less than 30 percent. This latter value is frequently less than 15 percent. Strongly oil-wet cores, on the other hand, (a) normally have a connate water saturation less than 15 percent, (b) water saturation at which $0 i 1$ and water relative permeabilities are equal is less than 50 percent, and (c) relative permeability to water at residual $0 i 1$ is greater than 50 percent. Cores must satisfy all the criteria just mentioned to be classified as either water-wet or oilwet. Cores with intermediate wettability usually have some of the characteristics of both water-wet and oil-wet formations. 
Table 821 shows the results of the relative permeability tests run by Core Laboratories, Inc. Each of the samples tested showed intermediate wettability. Connate water saturation ranged from 24.8 to 45.1 percent of the pore space indicating water wetting preference, while relative permeability to water at residual oil ranged between 31.8 to 62.9 percent indicating intermediate to oil-wet behavior. In each test, the water saturation at which oil and water relative permeabilities are equal was close to 50 percent indicating intermediate wettability. Tests performed by ERDA on four other core plugs indicated that the samples had intermediate wettability. The method uses capillary pressure curves determined with a centrifuge to calculate a wettability number. On a scale ranging from - 1 (strongly oil-wet) to +1 (strongly water-wet), the samples had wettability numbers ranging from -0.08 to +0.01 (Table B22). Numbers between -0.15 to +0.15 indicate intermediate wettability.

\section{Compressive Strength}

Compressive strength tests were run on two samples from a weathered core to determine whether bullets or jets should be used for perforating in this formation. Bullets are more effective than jets in soft rocks (compressive strength less than $3000 \mathrm{psi}$ ), while jets have greater penetration in rocks having a higher compressive strength.

The two samples tested had compressive strengths of 3520 psi and 2450 psi for an average of 2985 psi as shown in Table B23. For this formation strength both bullet and jet perforators should penetrate about the same distance. 
Geological Study ${ }^{1}$

Analysis of seven slabbed cores from the Admire 650-foot Sandstone was undertaken to interpret the environment of deposition and to determine rock parameters controlling porosity and permeability of the reservoir sandstone. The vertical sequence consists of (in ascending order): Unit 1--A predominantly claystone unit (17-ft thick) with lenticular laminations of sandstone, siltstone, and carbonaceous materia1; Unit 2--Sandstone (Admire--the reservoir), medium grained, with trough cross-stratification, climbing ripples, claystone, and distorted claystone laminations; and Unit 3--Fossiliferous, finegrained carbonates interbedded with claystones and lignites.

Interpretation of the depositional environment is based upon (1) internal sedimentary and biogenic structures, (2) external unit geometry(s), (3) unit lithology, and (4) the vertical sequence. The environmental setting is deltaic with rapid intermixing of swamp, bay, and distributary channel subenvironments occurring in the vertical sequence. The three lithologic units have the following genetic interpretations: a basal (Unit 1) interdistributary bay overlain by (Unit 2) distributary channels with variations in the major channel positions, overlain by (Unit 3) a swamp or marsh and a marine openbay. Lateral and vertical changes in position of the distributary channels occurred through time causing variations in current flow and

TFlesch, G. A., "Environments of Deposition of the Admire (650!) Sandstone from the EI Dorado Micellar-Polymer Project Area, Butler County, Kansas," Cities Service 0il Company Exploration and Production Research Report G74-19 (Technical Report \#33), Tulsa,.0klahoma, October, 1974. 
in the resulting sedimentary structures. At times the depositional process may even have been the result of sandy intertidal, distributary bay sedimentation. These changes had a marked effect on the porosity and permeability of the Admire (650-foot) Sandstone.

Porosity and especially permeability of the reservoir were found to vary directly with the lithology and sedimentary structures, which in turn are dependent upon the depositional environment. Medium grained, trough cross-stratified sandstone is typical of the distributary channel facies and is the most favorable reservoir facies. Sandstone facies which originated as natural levees, subsidiary channels, channel margins, or possibly intertidal deposits locally contain abundant claystone laminations, climbing ripples and fine grained sandstone, all of which contributed to reduce permeability.

Petrographically, the sandstone typically contains 47 percent quartz, 5 percent feldspar, 3 percent granitic and metamorphic rock fragments, 1 percent organic material (al1 detrital components), 15 percent clays and/or cements (diagenetic components), and approximately 29 percent observed porosity. Diagenetic alteration by iron-oxide or calcite cementation overall had only a minor effect, however, locally high concentrations drastically reduce porosity and permeability. For example, the normally high air permeahility of the upper sandstone subunit is locally reduced by calcite cement to about $150 \mathrm{md}$. Diagenetic clays (matrix) are present and consist of illite, kaolinite, chlorite, minor amounts of montmorillonite and/or illite-montmorillonite. Small amounts or carbunate (in some cases siderite) feldspar and quartz were observed in the clay fraction of solid sample less than the five micron 
range. Tables $B 24$ and $B 25$ present the results of $X$-ray analyses on M:P-221 and M:P-225.

\section{Future Work}

Samples of cores will be used continually for flow tests to study rock-fluid interaction and oil recovery. As the project progresses and questions arise concerning local well anomalies, the preserved cores will be analyzed. Additional geological analysis of the project area including a study of the three oriented cores will be performed. 
WELL LOGGING

by

H. M. Al-Rikabi

\section{Summary}

The open-hole well logs were evaluated for 25 Chesney wells and 24 Hegberg wells. The oil in place for the Chesney lease amounts to 277,700 barrels, and the pore volume is 888,100 barrels. The oil in place for Hegberg lease is 245,100 barrels and the pore volume 826,700 barrels. The combined oil in place is 522,800 barrels, and the total pore volume is $1,714,800$ barrels. These values were obtained by computing the geometric mean of pore volume and hydrocarbon pore volume. Formation intervals having residual oil saturation less than five percent were not included in evaluation of net pay. 


\section{Introduction}

Various formation evaluation techniques have been used for the determination of oil in place. Improved methods for the analysis of logging data have been used to determine remaining mobile and residual oil. Both open and cased-hole well logging programs were available for these analyses. The initial test wells (outside the field patterns) were used to formulate the procedures for both open and cased-hole logging measurements. These techniques were used for determination of oil in place and its distribution within the reservoir and for monitoring the fluid conditions. In open-hole well logging the formation water was used for the drilling mud so that the mud filtrate and formation water would be of equal salinity. The primary logging tools used in the field were:

1. Dual Induction-Laterolog, Focused Log (Dil-LL-FL),

2. Compensated Neutron and Density $(\mathrm{CNL}-\mathrm{FDC})$,

3. Proximity Log and Caliper (PLC), and

4. Borehole Compensated Sonic Log (BHC).

\section{Discussion}

Figures $C-1$ to $C-5$ are the base logs for we 11 MP-208 and serve as an example of the logs obtained. From these types of logs, the Epilog* analysis was developed. The Epilog, a log analysis of hydrocarbon bearing formations by computer, provides a quick and complete look at the interval of interest. The Epilog system converts log measurements to formation parameters which can be rapidly evaluated.

*Copyright Dresser Atlas 
The advantages of the Epilog are: (1) complete analysis of the formation, (2) reliable values of porosity, (3) computed log plotted against depth and coded, (4) tabulated listing of reservoir parameters, (5) rapid appraisal of well to select pay intervals, and (6) the capability to select intervals of interest for computations, eliminating dense intervals and thick shale sections.

Figure $\mathrm{C}-6$ is a typical Epilog analysis. Track 1 shows the formation characteristics. Shale volume $\left(V_{s h}\right)$ is the bulk volume fraction of shale, both wet clay and silts, and is computed from neutron density data using. logic relating other shale indicators (SP, GR, and Resistivity) to the volume of shale. This is an excellent correlation curve which allows differentiation between sands, shales, and shaly sands.

Track 2 shows the hydrocarbon analysis. Water saturation $\left(S_{W}\right)$ is the fraction of pore volume filled with formation water. Hydrocarbon volume $\left(\phi S_{h r}\right)$ is the residual hydrocarbon per bulk volume, where $\phi$ is the porosity and $S_{h r}$ is the residual hydrocarbon saturation. Hydrocarbon weight $\left(\phi S_{h r} \rho_{h}\right)$ is the weight of residual hydrocarbon per bulk volume, where $\rho_{h}$ is the density of the hydrocarbon. The two curves $\phi S_{h r}$ and $\phi S_{h r} \rho_{h}$ converge in oil zones when the density of oil approaches that of water. In light (low density) hydrocarbon zones, the two curves diverge.

Track 3 shows the porosity volume analysis. Porosity $(\phi)$ is the formation porosity corrected for hydrocarbon and shale effects. Water-filled porosity $\left(\phi S_{W}\right)$ represents the formation water in the pore space as a percent of bulk volume. The area between the two curves corresponds to hydrocarbon-filled porosity. 
Track 4 shows the bulk volume analysis. Clay volume $\left(V_{c l a y}\right)$ represents only the bulk volume fraction of wet clay, whereas $V_{s h}$ of Track 1 represents the total shale bulk volume (clay plus silt). Matrix volume $\left(V_{\text {matrix }}\right)$ is the bulk volume fraction of non-clay solids (includes silts). Figures $C-7$ to $C-60$ are the Epilog presentations of the computed results for the individual wells.

Open-hole logging was completed for the first five test wells, 25 Chesney wells, and 24 Hegberg wells. Studies of the open-hole logs and core analyses results for the test wells Chesney-Hegberg MP-101, $102,103,104,105,221$, and 225 revealed that the logs indicate higher oil saturation and lower porosities than the cores. It is evident from the results that the cores were flushed to or nearly to residual oil saturation during coring. For this reason, it has been recommended that $\log$ saturations and porosities be used: to determine the oil in place.

The arithmetic and weighted average values for properties for the test wells as determined from the logs compare to those from core analysis as follows: porosities, log values 3.6 percent lower; $0 i 1$ in place saturations, log values 16-19 percent higher than core determined oil saturations; and log residual oil saturations, 9.7-12.7 percent higher than core determined oil saturations. The low fluid loss mud used to core the MP-105 apparently did not inhibit flushing.

The pay thickness indicated by core analys is (Table C2) does not agree with log analyses (Table Cl) in most cases. This is due to the fact that the upper part of the sand was not cored in several wells. Any correlation between the logs and cores should include only that 
portion of the log which was cored. Table C3 compares the results of core analyses with the log analyses for equivalent depths. Core depths were correlated with log depths by determining the bottom of the sand for each well. The results are similar to those presented in Tables $\mathrm{Cl}$ and $\mathrm{C} 2$.

Tables C4 and C5 show the results of open-hole log evaluation for the five test wells. The table summarizes the net pay, pore volume, porosity, hydrocarbon pore volume, residual oil saturation, oil in place, movable oil, clay percent, and their arithmetic, weighted averages, and geometric means.

Tables $\mathrm{C} 6$ and $\mathrm{C} 7$ show the results of open-hole log evaluation for 20 Chesney wells. The oil in place for the Chesney pattern amounts to 277,700 barrels. This was obtained by computing the geometric mean of the hydrocarbon pore volume from Table C7. The pore volume for the Chesney lease is 888,100 barrels. Formation intervals having residual oil saturation less than five percent were not included in evaluation of net pay.

Tables $C 8$ and $C 9$ show the results for 24 Hegberg wells. The oil in place for the Hegberg lease pattern amounts to 245,100 barrels. This was obtained in the same manner as for the Chesney lease. The pore volume for the Hegberg lease is 826,700 barrels.

Tables $\mathrm{ClO}$ and $\mathrm{Cll}$ are the results of open-hole log evaluation for the combined leases. The combined oil in place amounts to 522,800 barrels and the total pore volume is $1,714,800$ barrels. These numbers will be refined by integrating the area on the contour maps for hydrocarbon pore volume and pore volume. 
Tables $\mathrm{C} 12$ to $\mathrm{C} 65$ show the computed results of open-hole evaluation for the individual wells. Figures $\mathrm{C}-61$ through $\mathrm{C}-77$ are the contour maps of the geometric means for different parameters of the Chesney-Hegberg leases.

\section{Future Work}

The future work can be divided into the following sections:

1. Complete the current evaluation and contour mapping of the various parameters of the reservoir.

2. To look at various suitable logging systems for monitoring injectivity, oil sweep efficiency, and evaluating the effectiveness of micellar-polymer flooding. 


\section{FOR!IATION INJECTIVITY}

by

\section{S. C. Swift}

witin

Contributing Autiors J. H. Biles and V. W. Rinoades

\section{Summary}

A complete suite of chemicals similar to those of the union micellar-polymer process were injected into well !1P-104. Juring much of this injection, pressure responses in other wells in the area of the demonstration project were observeci. Tests were also made on some otiner project test wells. Three important conclusions which can be derived from the injectivity test conducted on well ilP-104 are: (1) injectivities into : 1 -1:J4 of all the various fluids (including preflush, micellar oil, micellar water, polymer, anj drive water) cumprissing the Union 0il micellarpolymer process are satisfactory. (2) pressure and rate data from the multirate injectivity tests indicate that IMP-104 was not fractured until the breakdown test was conducted, and (3) variations in directional permeability in the test area are sufficiently small that no alteration in location of the test area will he required. 


\section{Introüuction}

Injection of a complete suite of chemicals similar to those of the inion 0 il company micellar-polymer process into well :IP-104 was accomplished during the summer of 1974 . The fluids were injected in the following order: An aqueous chemical preflush, soluble oil and "micellar water" (an aajusteu salinity water), polymer of different concentrations, and cirive water. Some difficulties were experienced ciuring injection of tine preflusil anc soluble oil. Altinough tinese problems are intimately associated with proprietary inforllation received from Union, a general discussion of the chemical aspects of the injectivity test is included herein.

Juring the injection test, pressure responses at the offset wells Chesney $190(C-190)$ and $: 1 P-101,103,221$, and 225 were recorded in order to cietermine directional properties in the general project area. Additional associated testing included a breakdown test on $11 \mathrm{P}-103$ prior to injectivity testing (to establish a limit on the injection pressure) and a breakdown test on I.P-104 after completion of the injectivity test ( to insure accidental fracturing had not occurred).

\section{jiscussion}

\section{Chemical Problems}

Chemical slugs recommended by Union nil Company were injected into 65.j-foot Sand cores in the laboratory in preparation for the field injectivity test. The laboratory work indicated continuing loss of injectivity to all fluids. The fluids were not filtered, and subsequent tests showed face plugging. The field test confirmed the loss of injectivity during preflush, but injectivity increased during injection of the soluble oil and micellar water (see Figure D-2 in Appendix D). The injectivity of soluble oil, micellar water, and polymer into well M.P-104 did not correspond with 
the losses noted in the laboratory flow tests. Additional laboratory tests will be run to try to determine the reasons for the differences. A polymer solution was prepared continously by injecting liquid polymer concentrate downstream from the water pump in.a mixing loop containing a Kenics mixer. Since long-term stability to oxidation was not needed for the injectivity test, no oxygen scavenger was used. Fullopening valves weré used downstream from the polymer mixing loop to avoid shear degradation. A sample bypass loop was used to catch polymer samples without using a valve-cracking or bleeding method which would shear the polymer. Viscosity and screen factors of polymer sampled at the injection well-head were comparable to values obtained under laboratory concitions. Polymer concentrations. were somewhat variable due to operating difficulties associated with weather changes (which affected polymer concentrate viscosity), moisture condensation in the polymer concentrate reservoir (which caused formation of thick, non-pumpable globs), and variations in water rate. : then these factors were not interfering, it was possible to control polymer concentration witinin $\pm 100 \mathrm{ppm}$ for as long as 44 hours continuously.

\section{Static Pressure Conparisons}

ilative brine from the Admire Sand exhibits a pressure graciient of $0.49 \mathrm{psi} / \mathrm{ft}$. Because of the low reservoir pressure $(211$ psia at 700 feet above mean sea level), variations of pressure with depth in this reservoir are quite significant. Therefore, it is essential to know the depth datum for each pressure measurement. Table DI ill Appendix $u$ summarizes various static pressure measurements observed in the course of pressure transient testing in the reservoir. 


\section{Interference Jata}

The purpose of conducting interference tests was to determine if gross directional variations (directional channeling) were present in tile permeability throughout the pattern area. As can be seen in Figure $D-1$, variation was from a maximum of $61.9 \mathrm{md}$ to a low of $47.5 \mathrm{md}$. The most significant contributor to these variations is the presence of a high conductivity zone which had been a gas cap lying just east of the pattern area. (see the section "Select Test Site").

It should be noted that tipe low variation in directional permeability does not rule out the possibility of a highly conductive iorizontal layer. If such a layer existed and were directionally uniform, it would make all calculated permeabilities too high and would adversely affect sweep efficiency. However, there is no evidence to indicate that such a layer exists in the pattern area.

\section{Injectivity Jata}

Figure $0-2$ shows the variation of injectivity with the fluid being injected. Injectivities during logging operations indicated values in excess of $1.0 \mathrm{bbl} / \mathrm{day} / \mathrm{psi}(\mathrm{BPD} / \mathrm{psi}$ ) for water. Immediately upon injecting a pretreatment fluid into the reservoir, the injectivity dropped to approximately $0.2 \mathrm{BPi} / \mathrm{psi}$ and remained there upon injection of preflush.

Injectivity varied with each batch (tank) of fluid, even when no differences in mixing procedure were known. However, the following effects were noted during preflush injection:

1. Detrimental effects due to minute amounts of petroleum sulfonate accidentally mixed with the preflush.

2. Detrimental effects due to mixing preflush constituents with city water which was not completely softened. 
3. Questionable effect of adding a solid oxygen scavenger.

4. Favorable effect using a liquid oxygen scavenger.

5. General increase in injectivity when injecting fully concentrated preflush as compared to one-half strength preflush. Under the most favorable conditions, full-strength preflush injectivity averaged only $0.3 \mathrm{BPD} / \mathrm{psi}$.

Based on laboratory tests (as discussed above) and the experience of accidentally injecting sulfonate contaminated preflush, it was expected that soluble oil and micellar water injectivity would be very low. Such was not the case. Injectivities to soluble oil and micellar water were much better than with preflush and were stable (see Table 02).

Polymer injection was limited to $75 \mathrm{bbl} /$ day to avoid shear degradation. It is believed that the low polymer injectivity $(0.30 \mathrm{BPD} / \mathrm{psi})$ is due primarily to the high apparent viscosities of polymer at low shear rates. Subsequent shear degradation tests, while inconclusive as to whether or not degradation was occurring, did indicate that injectivities comparable to drive water could be obtained with polymer at rates of 150 bbl/day.

Drive water following polymer held a steady injectivity of 0.49 $\mathrm{BPJ} / \mathrm{psi}$. A follow-up injection of $200 \mathrm{bb} l$ of preflusin indicated that after conditioning of the rock with the soluble oil, injectivities as high as $0.53 \mathrm{BPJ} / \mathrm{psi}$ could be sustained with preflush.

\section{Fall-off Tests}

Fall-off tests were condus.terl on I.P- 104 on 11 separate occasions. The initial test followed fresin water injection and indicated a permeability of $42.2 \mathrm{md}$ with a skin factor of -2.46 . Based on radius of investigation vs radius of invasion considerations, it was concluded that the damage would appear on the fall-off tests as though it were only a very thin layer. 
Because of this, the ability of the well to accept fluid would be directly reflected in the skin factor.

In practice, the slope of the early fall-off data did deviate considerably due to the presence of radial variation in mobility. Some tests exhibited distinct straight lines indicating a different value of $\frac{k h}{\mu}$ near the well compared to far from the well. However, for the purpose of maintaining a uniform standard of comparison, the original permeability of $42.2 \mathrm{md}$ and an infinite shut-in pressure of 220 psi were used for all tests. The parallel lines of each fall-off test shown on Figure D-3 match the long term fall-off behavior quite well. Therefore, the skin factors reported in Table D3 reflect the damage done to the immediate vicinity of the wellbore by the fluid being injected.

Breakdown Tests

Prior to injectivity testing in I.P-104, a breakdown test was conducted on $: \mathbb{P}-103$. This test was used to set a ceiling on injection pressure for $\mathbb{1 P}-104$ and to provide a reference for evaluating the breakdown test which was to be conducted on IIP-104 after completing all normal injectivity testing. Results from the I.PP-103 breakdown test indicated a fracture gradient of $1.6 \mathrm{psi} / \mathrm{ft}$ and a fracture extension gradient of 1.0 psi/ft. Dala from this test is included in Appendix $D$ (see Figures $D-4$ and $D-5)$.

The breakdown test conducted on we11 :IP-104 December 19, 1974, was inconclusive. The fracture gradient was only $1.05 \mathrm{psi} / \mathrm{ft}$ and the prebreakdown injection behavior was abnormal. On the other hand, skin factors before and after fracturing were as would be expected in an unfractured well. Injection profiles determined before and after the breakdown test were also inconclusive because both profiles were essentially the same and 
both corresponded to the permeabilities determined by core analyses.

This indicated that the pre-breakdown profile was normal for an unfractured well (paralleling measured core permeabilities), but on the other hand, was not significantly different after breakdown.

In order to clarify the situation, four additional multi-rate tests were conducted, one on well MP-101, one on MP-103, and two on MP-104. Care was taken not to fracture I.P-101; in fact, the hole was never completely filled.

The first result of these tests was the immediate discovery of the reason the reported data was abnormal for the first breakdown test. The reported data had taken time equal to zero as the time when the hole was loaded rather than when the first fluid was pumped into the well. This gave the reported pre-breakdown an apparent slope (on a plot of log $\Delta p$ v's $\log q$ ) of less than one, which while not impossible, is very unusual. When the effects of loading the hole are considered, the response is completely normal.

Hore important, however, was the injection rate behavior after breakdown. In both I:P-103 and IIP-104 the initial breakdown test exhibited a period of decreasing $\Delta p$ with increasing rate immediately after breakdown. On the subsequent tests $\Delta p$ increased slightly with increasing rates above parting pressure. Also the parting pressure was markedly luwer for the multirate injection tests into a known fractured well. This evidence clearly indicated that I.P-104 was not fractured prior to December 19, 1974.

Finally, it is interesting to note that the low fracture extension pressure, as well as the profile data, seem to point to a vertical fracture in $\mathrm{IP}-104$ rather than a horizontal fracture as was indicated in IAP-103. 
Future Hork

For application of the above data to future operations the following guidelines should be respected.

1. For fully developed patterns, the presence of additional injectors and open-hole vs perforated completions must be considered when comparing pattern wells with the injectivity behavior of well MP-104.

2. The low observed fracture extension gradient in $\mathrm{IP}-104$ $(0.74 \mathrm{psi} / \mathrm{ft})$ makes the previousiy recommended maximum gradient for pattern injection $(1.00 \mathrm{psi} / \mathrm{ft})$ too high. It would be best to hold all injectors below a gradient of $0.75 \mathrm{psi} / \mathrm{ft}$ at the casing point. However, a value of $0.90 \mathrm{psi} / \mathrm{ft}$ should be respected as the absolute maximum if fracturing of injectors is to be avoided. 
PATTERN PRESSURE TRANSIENT TESTS

by

S. C. Swift

with

Contributing Authors L. P. Brown and V. W. Rhoades

Summary

Information from this testing will provide essential information

for a rational decision as to pattern selection as well as optimum

injection rates. However, since the tests are still in progress, any

definite conclusion would be premature at this time. 


\section{Introduction}

Extensive directional permeability tests on actual pattern wells are now in progress. Each test consists of pumping one of the pattern producers for a minimum of 48 hours and observing the drawdown in the eight offset injection wells. Following the production period, the well is shut in and the pressure buildup is recorded in the offset injectirs as well as the producer itself. Analysis of this data will determine the interwell directional permeability, which determines optimum pattern selection, as well as optimum rates in the various injectors.

\section{Discussion}

These tests are being conducted sequentially, centered on wells PIP-124, 114, 112, 122, 207, 217, 219, and 209. As of June 30, tests have been completed up to and including well MP-207. Significant variations in directional permeability have been noted in all patterns. This is reflected by the rate of response in the offset wells. Indeed, some wells have responded so slowly that one had to suspect that they were not connected very well with the formation. As a matter of fact, due to mechanical problems during completion, I:P-110 is separated from the formation by a rement sheath.

Allliough a scvere impediment to flow is required to affect the response of an observation well materially, four of the slowest responding wells were tested to insure the reliability of the data. These four wel1s were I.1P-119, 123, 121, and 117. Of the four, only IIP-123 showed sufficient damage to cast suspicion on the data obtained. Because of the three-out-of-four record among the slowest responding 
wells, it is believed that overall, the responses seen are accurately reflecting the ability of the formation to conduct fluid between the wells. Analysis of the data obtained is still in progress. As has already been indicated, the project area cannot be characterized as a homogeneous reservoir. This does not mean that the site is a poor selection. Since very few, if any, reservoirs have been this carefully evaluated by pressure transient methods, there exists little basis for comparison.

The only analytical solution for a non-homogeneous reservoir which is available for comparison with the data is that of an ideally anisotropic reservoir (one with maximum and minimum values of permeability at right angles to each other). This model fits the observed behavior much better than the homogeneous case; however, many wells fail to conform to even this model. Research is now being conducted on the best way to characterize the non-homogeneous nature of the reservoir. The simple model of othogonal maximum and minimum permeability axes is not sufficient.

Because all the information has not yet been gathered and because the best analytical techniques are just now being developed, it is impossible to predict the final result of these directional permeability tests. However, based on the tests which have been completed to date, putting injectors and producers on a common northsouth line would appear to be a mistake, at least on the Chesney pattern. 
Future Work

Completion of the field testing will be accomplished during July, 1975. Analysis of the data and recommendations based thereon should also be completed during that month. 
LITERATURE WORK

by

C. M. Royo

with

Contributing Authors J. Vairog̣s and D. F. Zetik

\section{Summary}

A report covering the technical literature on polymer flooding was completed. The advantages as weld as the problems relating to the use of polymer solutions are discussed in the report. The effect of the principal variables such as water salinity, oxygen induced degradation, and mechanical degradation on the fluid properties and recovery efficiency is illustrated. Also included is a list of screening parameters which serve as criteria for evaluating potential polymer flood prospects.

A second report summarizing the 1 iterature survey of surfactant floods, microemulsion floods, and alkaline waterfloods is 90 percent complete. For this survey emphasis has been placed on (1) developing parameters to screen reservoirs for potential applications, (2) formulating a detailed description of the physical process that will aid in understanding the effects of variations in process parameters, and (3) tabulating case histories and literature references.

In addition, a brief literature search was made to ascertain which physical forces are known to affect fluid displacement. 


\section{Introduction}

Literature surveys on polymer flooding and miscible type processes (surfactant floods, microemulsion floods, and alkaline waterfloods) were conducted to assess the current state of the technology of these processes. The advantages and disadvantages of each are discussed below.

\section{Discussion}

Technical Appraisal of Polymer Flooding

A report covering the technical literature on polymer flooding was completed. The advantages as well as the problems relating to the use of polymer solutions are discussed in the report. The effect of the principal variables such as water salinity, oxygen induced degradation, and mechanical degradation, on the fluid properties and recovery efficiency are illustrated.

Polymer flooding improves oil recovery over conventional waterflooding by decreasing the mobility of the water phase and thereby increasing the volumetric sweep efficiency. Some of the factors which affect recovery efficiency and economics can be controlled while others cannot. For cxample, water salinity, polymer adsorption, inaccessible pore volume and thermal degradation are controlled by the reservoir. A properly designed polymer flood is one which accounts for the adverse effect from each of these factors and selects the type of polymer, polymer molecular weight, slug size, and concentration necessary to achieve the optimum mobility reduction. Screening parameters which serve as criteria for evaluating potential polymer flood prospects are: mobility ratio, 
mobile oil saturation, permeability, temperature, reservoir depletion: stage, water salinity, formation type, initial water saturation, and permeability variation.

Two types of water-soluble polymers have found general application in improved oil recovery operations--polyacrylamides and polysaccharides. (Polysaccharides are also commonly referred to as biopolymers.) Polyacrylamides are synthetic polymers consisting of large chain-like molecules of high molecular weight having $\mathrm{COHN}_{2}, \mathrm{COOH}$, and COONa groups attached to every other carbon atom on a carbon backbone chain. The naturally occurring polysaccharides contain several cyclic carbohydrate monomers which alternate in the polymer structure. Both additives increase oil recovery by lowering the mobility of the flood water. However, they effect this lowering by distinctly different mechanisms. Mobility control with the polyacrylamides results from an increase in fluid viscosity accompanied by a reduction in the water phase permeability. Polysaccharide solutions serve only to increase the fluid viscosity.

A typical viscosity-shear rate relationship for low flow velocities of polyacrylamide solutions is shown in Figure E-l in Appendix E. Note that viscosity of the solution increases with increasing moleculdr welght and decreasing water salinity. A drastic reduction in viscosity occurs when small amounts of salt are added to the water, with divalent cations such as calcium having a more pronounced effect on the flow behavior than sodium. At low flow rates the apparent. solution viscosity decreases with increasing flow velocity. Figure E-2 illustrates the similar behavior of biopolymer solutions. At low 
salt concentrations polyacrylamides are more effective viscosifiers than biopolymers. However, biopolymers are less sensitive to high salt concentrations.

The removal of polymer from solution by adsorption onto the reservoir rock has been repeatedly observed in polymer flooding tests. The amount of polymer adsorbed depends on the nature of the polymer and the rock surface. Both physical adsorption of polymer onto solid surfaces and polymer retention by mechanical entrapment appear to play a role in the total polymer retention in a reservoir. Although there is disagreement among the various investigators as to the effect of salinity on adsorption, it is generally agreed that high water salinity is detrimental to the polymer flooding process. Figure E-3 illustrates that adsorption is greater on carbonate rocks than on sandstone and that small amounts of calcium can increase adsorption substantially. Polymers are sensitive to mechanical, chemical, and thermal degradation, but degradation can be prevented or minimized through the use of special equipment and techniques. Oxygen induced degradation is usually controlled by adding oxygen scavengers to the water. Mechanical (shear) degradation of polyacrylamides presents serious practical problems in field operations. Figure E-4 illustrates the permanent loss of polymer viscosity due to shear degradation that can occur if the injection rate is too high. Since the highest reservoir flow rates are at the injection well sandface, one way of minimizirig degradation without decreasing flow rate is to increase the injection sandface area.

Finally, the economics of polymer flooding are especially dependent on the pore volume, since polymer requirements are based on 
this volume. The chemical cost of the polymer solution is directly related to the polymer concentration and slug size. Slug volume and concentration are usually determined from laboratory flow tests on reservoir rock. Typical slug sizes range from seven to 35 percent pore volume with the larger slug sizes having a better chance for success. Polymer concentration usually varies from $250 \mathrm{ppm}$ to over 1,000 ppm, with $250 \mathrm{ppm}$ to $500 \mathrm{ppm}$ being the most common.

\section{Miscible Type Processes}

A literature survey of surfactant floods, microemulsion floods, and alkaline waterfloods is 90 percent complete. Emphasis has been placed on (1) developing parameters to screen reservoirs for potential applications, (2) formulating a detailed description of the physical process that will aid in understanding the effects of variations in process parameters, and (3) tabulating case histories and literature references.

Surfactant and micellar floods have several advantages over most other tertiary recovery processes: (a) the chemical slug that is used to sweep the remaining oil from the reservoir is capable of mobilizing almost 100 percent of the oil in the parts of the reservoir it contacts, (b) since the chemical slug has a density similar to oil or water, gravity segregation is minimal, and (c) the viscosity of the slug can be readily controlled and a favorable mobility ratio between the slug and the displaced oil bank can be obtained in most reservoirs. Thus, sweep efficiencies of these flooding processes are usually very good, and the processes are capable of recovering a large fraction of the remaining oil from many reservoirs. Disadvantages of surfactant 
and micellar floods include: (1) interactions between the chemical slug and many reservoir waters can cause deterioration of the slug and loss of recovery, (2) adsorption of chemicals on reservoir rock has adverse effects on these processes, and (3) the costs of micellar or surfactant floods are high compared to other improved recovery methods. New developments have sought to increase the advantages of micellar or surfactant flooding and to reduce the disadvantages.

The oil in some reservoirs contains chemical compounds that react with alkaline water to form surfactants in situ. Thus, alkaline waterfloods (which use this principle) are similar to surfactant floods but have the advantage of lower cost. Although only a small percentage of crude oils contain sufficient quantities of the surfactant forming compounds, the quantity of oil recoverable by this process is nevertheless significant. The lower cost of alkaline waterflooding may make this process generally more profitable than other tertiary recovery methods.

A computerized data file of patent information relevant to micellar-polymer flooding has been prepared. United States patents were searched for petroleum recovery inventions that involved the injection of fluids into the reservoir. Additional U. S. patents relevant to micellar-polymer flooding were located in the literature search and through references in other patents. A classification scheme was devised to permit computer storage, processing, and retrieval of the patent information. Data input forms were designed to separate the scientific and technical contents of the patents from the input of clerical data. This allowed professional personnel to devote full 
time to the analysis of the scientific and technical contents. The input form used to code these contents is presented as Table El (see Appendix E). Secretarial help coded the patent titles, issue dates, assignees, and inventor names. Approximately 800 patents have been processed. Approximately 450 of the patents have some relevance to micellar-polymer flooding--the rest of the patents pertain to other recovery processes. Newly issued patents are being monitored and added to the file if they pertain to micellar-polymer flooding. A number of computer programs were employed in an extensive error check of the data file.

An information retrieval program was written to search the file for patents meeting a set of user supplied selection criteria. These criteria may relate to issue date, expiration date, assignee, inventor name, or any of the items listed in Table El. An optional scoring system allows the user to assign a point value to each criterion. If the total points of all satisfied criteria are greater than a user specified minimum value, the patent is selected. This optional system simplifies the input of complex selection criteria. A report describing the data file and the data retrieval program is 50 percent complete.

\section{Forces Affecting Fluid Displacement}

A brief literature search was made to check what forces are known to affect fluid displacement. The literature sources seemed to indicate that the capillary number correlates with the post-flood residual oil rather well for both waterfloods and surfactant floods in cores. By definition, capillary number is the ratio of viscous to interfacial forces. The ratio of gravitational to interfacial forces, known as the Bond number, also should have an effect on recovery, but 
it would be minor in laboratory tests. Finally, the oil-water viscosity ratio affects recovery, especially in immiscible floods.

\section{Future Work}

The literature survey of the miscible type processes will be completed and a report written. The report describing the patent data file and data retrieval program is to be completed.

\section{References}

1. Hill, H. J., J. R. Brew, E. L. Claridge, J. R. Hite, and G. A. Pope, "The Behavior of Polymers in Porous liedia," Society of Petroleum Engineers of AIAIE Improved $0 i 1$ Recovery Symposium, Paper No. SPE 4748, Tulsa, OkTahoma, April 22-24, 1974.

2. Jennings, R. R., J. H. Rogers, and T. J. West, "Factors Influencing

- Mobility Control by Polymer Solutions," Journal of Petroleum Technology, 23:391-401, March, 1971.

3. Royo, C. M., "Technical Appraisal of Polymer Flooding," Cities Service $0 i 1$ Company Exploration and Production Research Report PR 74-04, Tulsa, Oklahoma, November, 1974.

4. Smith, F. W., "The Behavior of Partially Hydrolyzed Polyacrylamide Solutions in Porous Hedia," Journal of Petroleum Technology, 22:148-156, February, 1970. 


\title{
PERFORMANCE PREDICTION
}

\author{
by \\ J. Vairogs \\ with \\ Contributing Author D. F. Zetik
}

\section{Summary}

This section reports work on the performance of the old waterflood as well as estimates of the demonstration flood performance under various modes of operation and injection. Waterflood performance was examined to gain information that might be used to model the demonstration flood.

The conclusions are:

1. The four contiguous 6.4-acre nine-spot patterns specified in the technical proposal for the project are not the best well configuration for the demonstration flood. In the absence of extreme reservoir heterogeneity, five contiguous 3.2-acre five-spots with additional wells on the periphery would be the preferred pattern configuration.

2. Well log analyses, core analyses, and geologic descriptions do not indicate gross reservoir heterogeneity other than the existence of two layers with a possible two-fold contrast in permeability. This degree of heterogeneity will not affect pilot performance too adversely.

3. A reservoir with a directional permeability ratio of $7: 1$ (as preliminarily determined by pressure testing the 650-foot sand) will have breakthrough about twice as soon as a homogeneous reservoir. Examination 
of old waterflood data shows that in three patterns water breakthrough occurred at 0.24 to 0.34 displaceable pore volumes of injection. This information indicates that directional permeability may exist and affect patterns oriented in the same direction as in the waterflood.

4. An estimation procedure for predicting future production has been implemented. Its reliability in field applications is not known and needs to be confirmed. 


\section{Introduction}

This section includes a brief evaluation of the performance of the old waterflood, estimation of the flood performance during preflush injection, estimates of the demonstration flood oil recovery, and discussions of pattern selection and reservoir heterogeneity. Performance prediction has involved the use of both finite-difference models and simpler procedures. Except where otherwise stated, all calculations reported in this section were made with the assumption of a homogeneous, single-layered reservoir.

\section{Discussion}

!'aterflood Past Performance

An estimate of the remaining oil saturation after waterflooding was made prior to drilling the test wells. A volumetric estimate based on oil recovered and the original oil in place gave 43 percent oil saturation for the entire reservoir. Another estimate based on volumetric sweep in a liquid-filled reservoir gave 46 percent remaining oil saturation and a 67 percent volumetric sweep. The Dykstra-Parsons method for stratified systems with a permeability distribution factor of 0.72 was used for this purpose. The latest average value of remaining oil saturation determined from well logging measurements is 33 percent. Literature sources indicate that the measured uil saturation atter a waterflood is generally lower than volumetric estimates. An evaluation of the old waterflood in the vicinity of the El Dorado project was based upon individual well rate data. Monthly production and injection rates for the early part of the waterflood were obtained from the Region office. These data, which extend through December, 1963, were combined with data from the Tulsa office. The 
Tulsa data is from July, 1963, to the present. Discrepancies were found where these two data sets overlap. Both earlier and later data appear to be good.

Plots of $0 i 1$ rate, water rate, and water-oil ratio were prepared for each of the production wells in the project vicinity. These plots showed no anomalies indicative of conditions that would adversely affect the project. The oil production from well Hegberg 121 was notably greater than the production from the other wells that were plotted. A comparison of total production and injection rates for the Teases indicates that neither the Chesney nor the Hegberg waterflood were as well balanced as had been believed. Theoretical breakthrough sweep efficiencies were calculated for the 12.8 acre waterflood for various reservoir heterogeneities after it was learned that directional permeability was probable. Four cases were run to compare theoretical breakthrough efficiencies: a homogeneous reservoir case, one with a $7: 1$ orthogonal directional permeability contrast, one with a 2.65:1 permeability contrast, and a case with two homogeneous layers where one layer has twice the permeability of the other. The last case is a realistic possibility since all geological descriptions of cores identify two zones in the 650-foot Sand. If the directional permeability case were to show a much lower breakthrough sweep efficiency than the homogeneous case, then there would be a possibility of identifying this condition on the basis of field production data.

Table Fl in Appendix F shows the results of waterflood simulations starting with a 16 percent gas saturation in the reservoir and with a liquid-filled reservoir. Mobility ratio for these runs was. more favorable than in the field, but the relative effect of the 
results should be the same. That is, a 7:1 directional permeability case can be distinguished from the layered or the homogeneous case on the basis of production data provided the data are of reasonable accuracy. A finite difference black-oil simulator was used for the calculations. Figures $F-1, F-2$, and $F-3$ show the actual production data plotted as cumulative injection, water rate, and WOR versus time for patterns centered on wells 17 and 19 on the Chesney lease and well 40 on the Hegberg lease. It was assumed that an injector contributed one-fourth of its rate to each producer. The resulting water breakthrough is shown by the arrows on the figures. Breakthrough occurred at $0.24,0.34$, and 0.34 displaceable pore volumes injected, respectively. This would tend to confirm the existence of a marked directional permeability contrast. However, the directionality may vary over short distances, and there may be other causes giving similar results.

\section{Preflush Calculations}

At the start of the project it was thought that a 70 percent pore volume preflush slug would be used to displace the formation brine prior to injection of surfactants. The pumping of these fluids would take a significant amount of time. For this reason and to help visualize the fresh-water saturation distribution, a model study of the preflush injection phase was made. A black-oil finite-difference simulator was used for this purpose. A unit mobility ratio was assumed since the problem dealt with fresh water displacing brine. Relative permeability curves (Figures F-4) used in the simulation were straight lines as suggested by Lantz ${ }^{3, *}$ when an immiscible model is used to simulate

* Reference for this section are given on the last page of the section. 
miscible displacements.

One nine-spot pattern was simulated using two large blocks on each of the two unconfined sides to include the effect of a partially confined pattern (see Figure F-5). Homogeneity in the vertical direction was assumed. The results showed that the nine-spot pattern is production limited. This conclusion was drawn from the fact that the total injection rate could be increased by increasing the productivity index (stimulation) of the producing well.

The effect of different corner-to-side well rate ratios on sweep efficiency was also investigated. The results varied greatly with the rate ratios. However, the results also displayed a rather large numerical dispersion effect caused by using too few blocks between wells. Numerical dispersion in simulation can be reduced by increasing the number of blocks in the model. The number of blocks needed to reduce numerical dispersion to the level of physical dispersion (which is not actually modeled in an immiscible simulator) was determined as follows. Data were obtained on fresh water displacing saline water in a 650-foot Sand core plug. The produced water salinity was then computed using different numbers of blocks in a linear displacement simulated with the hlar.k-0il model. The results are shown in Figure F- 6 .

It was obvious that the small number of blocks previously used in the simulation was inadequate. Consequently, a quarter of a nine-spot pattern was modeled with a 23 by 23 block model which gives 20 blocks (two were outside the pattern area) between wells rather than six. A much better agreement between the calculated and laboratory curves was obtained. This many blocks may not be needed to simulate field performance. 
Hork with the 23 by 23 block model was completed in October, 1974. As before, the oil was considered immobile. Data used in the simulation are shown in Table F2. Simulation effort using this model was concentrated entirely on investigating the best way to operate a nine-spot pattern to obtain optimum sweep. The locations of unconfined and confined wells were taken into consideration by running three types of quarter nine-spots (an inside quarter, an outside quarter, and two identical side quarters) and combining the results. Five cases were run. Case la was constant rate injection at a 5:1 corner-toside well ratio. Case $1 \mathrm{~b}$ was the same as Case la but with a productivity index of three for the producer (stimulated). Case $2 a$ and Case $2 b$ simulated delaying injection in side wells for 30 days and for 90 days, respectively. Case 3 simulated injecting in corner wells while producing side wells until fresh water breakthrough occurred in the side wells; then side wells were converted to injectors. The results of these cases for both the Chesney and Hegberg leases are shown in Figures $\mathrm{F}-7$ through F-12. Figures $\mathrm{F}-13$ and $\mathrm{F}-14$ show some of the corresponding fresh water saturation maps, and Figure F-15 presents relative efficiencies of each mode of operation in terms of area contacted by a given minimum fresh water saturation.

As betore, the results showed that stimulation of the producer would give the best fluid throughput. It appeared that producing side injectors until fresh water breakthrough gave the best injected water distribution. Delaying injection in the side wells was not as good as either one of these other operational schemes. 
At this point in the study it was apparent that the nine-spot patterns would take some special handling if they were to be operated in a near-optimum fashion. None of the alternates investigated (fracturing producers, delaying injection in side wells, and producing side wells) was very appealing. Also, the companies supplying the micellar-polymer processes started questioning the use of nine-spot patterns. Hence, preflush work on the patterns was stopped and comparisons between nineand five-spot patterns were made with the potential result of changing the pattern configuration. That work is described later under Pattern Selection.

\section{Estimation of Recovery}

A future production schedule was required to permit an economic analysis of the demonstration flood and of potential field-wide expansion. At this time there is no reliable and tested procedure for estimating recovery from chemical floods reported in the literature. Consequently, the Davis and Jones' displacement mechanism for micellar flooding was combined with the areal sweep efficiency for a confined nine-spot to give a first approximation of the recovery. It was assumed that no mobile oil was present. This is a good assumption based on well logging 011 salurations.

The Davis and Jones mechanism is based on an oil fractional flow curve. Relative permeability data for three restored-state core plugs from wells outside the Chesney or Hegberg leases were used to calculate a fractional flow curve. It was recognized that the data did not strictly apply to the flood area, but these were the only data available in early 1974. The composite curve is shown in Figure F-16. 
The breakthrough times for $0 i 1$ and micellar slug were calculated for a linear displacement using the equations of Davis and Jones. The calculations were made for a four and an eight percent pore volume slug. Values taken from the fractional flow curve and used in the calculations are shown in Table F3. The table also includes results for the linear displacement calculation.

It was recognized that the producing oil cut would not be constant at 57 percent as predicted for linear displacement. The oil cut increases starting from zero. Then, after micellar slug breakthrough the oil cut decreases because of dilution from micellar and polymer slug materials. This effect was accounted for through use of an areal sweep efficiency curve and its related displacing-fluid cut (water cut) curve. The curves were calculated with an incompressible simulator since only the breakthrough value is available from the literature. The calculations were made for a unit mobility ratio displacement. The resulting performance curve was combined with the estimated production rates and slug sizes shown in Table F4 to give the production schedules shown in Figures F-17 and F-18. An identical procedure was used later to compare production schedules for 6.4 acre and 10 acre five-spots and nine-spots.

A more accurate forecast of the demonstration flood performance was made in June, 1975. The Davis and Jones displacement mechanism was used, but by this time a streamline flow program had been developed for use in sweep calculations of unconfined patterns. The development. work is discussed under Pattern Selection. This program permitted one to look at each streamline as a linear displacement with breakthrough for each streamline occurring at a different time. Thus, the breakthrough 
times of the oil bank and the micellar slug for each streamline were calculated, and the streamlines were summed to give the production schedule.

The fractional flow curve used in these latest calculations was slightly different from the previous one. It was calculated from the composite relative permeability ratio curve shown in Figure F-19. This curve is a composite of six core plugs taken from the native-state core of well MP-104. The unsteady-state imbibition curve is shown. The points show values for steady-state imbibition and drainage curves run on a single native-state plug from well MP-124. The difference between the native-state and restored-state ratio curves is not great, but a new fractional flow curve was computed and used in the calculations. The curve is shown in Figure F-20 along with the parameters needed in the displacement calculations.

Production curves for 6.4 acre five-spots, 6.4 acre ninespots, and 3.2 acre five-spots with four producers are shown in Figures F-21 through F-25. Injection and production rates used in the calculations were the same as discussed under Pattern Selection (Table F8).

\section{Pattern Selection}

Pattcrn selection work was approached in two ways. At first, only the 6.4 acre five-spots and nine-spots were compared using a blackoil finite-difference model. Subsequently, a streamline flow model similar to the one published by LeBlanc and Caudle ${ }^{2}$ was developed. This model and one developed by superposition of the line-source solution were used to investigate several different patterns. Both approaches as well as model development are described below. 
Comparisons using the black-oil model were made assuming a single layer, homogeneous reservoir with unit mobility ratio fluids. The reservoir description was essentially the same as that used in the preflush calculations. A few pertinent parameters are shown in Table F5. Injection at a constant and equal injection pressure in all wells was investigated for four cases: the five-spot, the nine-spot, and nine-spots with 90 days and 150 days delay of injection in the side wells. Injection at a constant rate was investigated for three cases: the five-spot, the nine-spot with a 5:1 corner-to-side well ratio and the nine-spot with a 5:1 ratio and a 90 day delay in side well injection.

The breakthrough sweep efficiency for these seven cases is shown in Table F6. Injection rates were also determined from the runs although it is recognized that finite difference models generally are not very accurate when used for that purpose. However the accuracy should be suitable for comparing patterns. The results for most of these cases are shown in Table F7. Minimum producing well pressure was 30 psi and maximum injection well pressure was 675 psi.

Results of this study were:

1. The partially confined five-spot gave better sweep efficiency than the nine-spot when both were operdled at constant and equal injection pressure. The same is true when both were operated at constant injection rate with injection and production balanced.

2. Operating at constant injection rate (with 5:1 ratio for the nine-spot) gave better sweep efficiencies than the corresponding constant injection pressure cases. 
3. In all cases, the total pattern injection rate in the five-spot pattern was less than in the nine-spot for the corresponding mode of operation.

After these results were reported, comparisons to other well configurations were initiated. Although the black-oil model is a good tool. for investigating effects of heterogeneity, programs were needed to rapidly compare the sweep efficiencies of confined and unconfined patterns of other well configurations. A computer program that would permit calculation of well pressures more accurately than the blackoil model was also desired. These two goals were achieved as described below.

Four computer programs were developed to assist in pattern selection. All of these models assume a constant thickness, homogeneous reservoir with isotropic permeability. They also assume a unit mobility ratio between the injected slug and the fluids it displaces. Image wells may be used to simulate reservoir boundaries. All wells are assumed to have zero skin. Modification of these programs to allow a uniform but anisotropic permeability is 50 percent complete.

The first program is a superposition-of-line-sources computer model that will compute and print contour maps of reservoir pressures. Superposition in space enables the program to include the effect of all wells in both the Chesney and Hegberg test patterns on the pressure at any point in the reservoir. If individual well pressures are given, this program allows computation of the individual production and injection well rates. One operating condition of particular interest has all production well pressures fixed at one value and all injection well pressures fixed at a second value. The total injection rate is a 
function of both the injection well pressure and the production well pressure. The total production rate also depends on both of these pressures. If only the production well pressure is set, the program can compute the pressure for the injection wells that will make the total injection rate equal to the total production rate. The term "stabilized rates" is used in this section for the individual well rates that occur under these operating conditions.

The second program is another superposition-of-line-sources model that includes superposition in time. This enables the program to calculate the effect of well rates that vary with time. If the wells are operated at constant pressure conditions other than the special conditions that result in stabilized rates, the well rates will vary with time. The program can calculate these changing well rates for all interacting wells in a reservoir.

The third program is a point tracking computer model that was written to investigate the areal sweep of fluids injected into the reservoir. An infinitesimal point of fluid is followed as it flows through the reservoir. The equations for the pressure gradient (at the point) yield differential equations for the movement of the point. The effect of all wells in both the Chesney and Hegberg patterns is - included in equations for the pressure gradient. A number of points are "injected" into the reservoir at the time slug injection starts. The points injected into a well will remain on the slug front surrounding that we17. The program computes the front as a smooth curve through the points. It automatically handles the cusp that forms when the front breaks through into a producing well. The program anticipates when expansion of the front or cusp formation will necessitate additional 
points for good front definition. This feature of the program has been very successful in interpolating additional points before they become necessary. Prior to breakthrough, the pore volume enclosed within the front can be compared with the volume injected to obtain a material balance. It was found that elementary integration techniques can cause large material balance errors. After incorporation of an integration technique with higher order accuracy and implementation of the feature for interpolation of additional points, the material balance errors were consistently less than 0.1 percent.

The program continues to compute the area swept by the front after breakthrough has occurred. The difference between the volume inside the front and the volume injected is equal to the total volume of slug produced. A technique is being developed for assigning this production to individual wells when the front breaks through into two or more producers. The front tracking model also computes the streamlines for the flow from injection to production wells. These streamlines are the trajectories followed by individual points.

The fourth program computes recovery schedules. The flow between injectors and producers is divided into a number of streamtubes that are computed by techniques similar to those used in the program for streamline calculation. A recovery schedule for each streamtube is computed from the equations presented by Davis and Jones. ' The contributions from all the streamtubes are summed to obtain a recovery schedule for an entire pattern (lease). Results from this program were presented under Estimation of Recovery.

These four programs were used to investigate a number of patterns and operating conditions. The patterns that were considered 
are exhibited in Figure F-26 together with the names that were assigned to each pattern. In all calculations, it was assumed that the same pattern would be used for both the Chesney and Hegberg test sites. Two of the patterns do not have north-south symmetry. In those cases Figure F-26 illustrates the Hegberg pattern. The Chesney pattern would be its north-south inversion. Stabilized rates for these patterns are exhibited in Table F8. The initial reservoir pressure was set to $200 \mathrm{psi}$. All patterns are production limited. Even with a very low production pressure of 30 psi, injection pressures are moderate. Neither the 6.4 acre nine-spot nor the 6.4 acre five-spot have sufficient throughput to meet the project timetable. The pressure contour maps for stabilized rate operating conditions showed negligible interaction between the Chesney and Hegberg patterns.

Figure F-27 is a plot of fronts and streamtubes for the 6.4 acre nine-spot with stabilized-rate operating conditions. The position of each front is plotted at six month intervals beginning with the start of slug injection. The fronts from the side injectors breakthrough to the production wells much sooner than the fronts from the corner injectors. This early breakthrough causes poor sweep efficiency. The injection rates into the side wells must be reduced to very low values in order to obtain simultancous breakthrough tillles for the corner and side injectors. With so little injection into the side wells, the 6.4 acre nine-spot has little or no advantage over a 6.4 acre five-spot pattern.

Figures F-28 and F-29 are plots of the fronts and streamlines for the 6.4 acre five-spot pattern and the 3.2 acre five-spot pattern with four extra producers. Both patterns have good sweep efficiency. 
Streamline and front plots were also calculated for the rest of the patterns in Figure F-26. Except for the 6.4 acre nine-spot pattern, the spread between the earliest and the latest breakthrough times is quite small when stabilized rates are employed.

A major deficiency of the 6.4 acre five-spot pattern is its low throughput caused by the low capacity of the production wells. Overinjection would cause an increasing average pressure in the pattern vicinity. This increasing pressure would cause the production rate to increase with time. If production and injection pressures were held constant, the injection rate would actually decrease with time. The superposition-of-line-source-solutions (in time and space) program was used to calculate the changing well rates for two operating conditions. In one case the injectors were held at $400 \mathrm{psi}$; the second case they were held at $500 \mathrm{psi}$. In both cases the central injection wells were twinned as discussed in the following paragraph. The separation between twin wells was ten feet. Results of these calculations are presented in Table F9.

Analysis of Individual Well Injectivity

Injectivity into the central well of the four 6.4 acre fivespot pattern may be a problem because the net pay at the well is only about half of the average net pay. The superposition-of-line-sourcesolutions program was used to compute the improved injectivity with twinned central injection wells in both north and south test sites. Table Flo illustrates how the total of the stabilized rates for the twinned wells in one pattern varies with the separation between these wells. The stabilized rate injection pressure for twinned wells is 
less than the stabilized rate injection pressure for a single central wel1. Thus, the percentage increase in injectivity is greater than the percentage increase in rate. The line marked "Base Case" contains data for a single central injection well.

An analysis was performed to determine the cause of the progressive loss of injectivity into well M:P-104 during testing with preflush solution. The creation of an expanding low-permeability ring around the wellbore could account for the observed loss of injectivity. This analysis showed that deposition of a skin on the sandface cannot account for the observed injectivities.

A radial flow reservoir model was used to study the permeability distribution in well MP-104. This model indicates that permeability in the vertical direction has almost no effect on the distribution of injection water in the vicinity of a well. This distribution is almost completely determined by variations of horizontal permeability with depth. The zones that had low tracer survey injectivities also had low core permeabilities; the zones that had high injectivities also had high core permeabilities. However, the numerical values for injectivity distribution predicted from core permeabilities were somewhat different from those obtained by the injectivity profile.

\section{Reservoir Heterogeneity}

When the five test wells were drilled, very low oil saturations were encountered in the top part of cores from wells S.P-102 and MP-103. The most likely possibility was that an old gas cap area was encountered with the gas saturation being replaced by water. Cross-sections were made to show that the low oil saturation occurred at a specific datum level in the test wells. Checking core analyses from old wells in the 
area confirmed that low oil saturations were being encountered above 771 feet above sea.level. This indicated an old gas cap. The resulting action taken was to move the four contiguous patterns from the eastern part of the Chesney Lease (as originally proposed) to the Hegberg Lease (see also, the section "Select Test Site").

Core analyses, well logging, and pressure transient tests are described in other sections of this report. When the geological descriptions of the cores are completed, a description of the reservoir heterogeneity will be developed.

\section{Future Work}

During the coming year the following objectives will be achieved.

1. The streamline model and the superposition-of-line-sourcesolutions programs will be modified to accommodate orthogonal anisotropic permeability.

2. The recovery estimation program incorporating the Davis and Jones displacement mechanism will be modified to include the final residual oil saturation as a part of the simulation of chemical flooding.

3. llsing the results from (1) and (2) above, future production schedules will be forecast.

4. The Higgins-Leighton model will be modified for use in history matching of the pilot performance if the technique developed under points 1,2 and 3 proves to be inadequate.

5. The finite-difference black-oil model will be used in an attempt to give estimates of pilot performance with heterogeneity included. 
I -75

References

1. Davis, J. A. and S. C. Jones, "Displacement Mechanisms of Micellar Solutions," Journal of Petroleum Technology, 20: 1415-1428, December, 1968.

2. LeBlanc, J. L. and B. H. Caudle, "A Streamline Model for Secondary Recovery," Society of Petroleum Engineers Journal, 11: 7-12, March, 1971.

3. Lantz, R. B., "Rigorous Calculation of Miscible Displacement Using Immiscible Reservoir Simulators," Society of Petroleum Engineers Journal, 10: 192-202, June, 1970. 


\section{THIS PAGE \\ WAS INTENTIONALLY \\ LEFT BLANK}




\section{SELECT TEST SITE}

by

R. J. Miller

\section{Summary}

A test site was selected for the demonstration of micellarpolymer flooding in the 650-foot Sand in the El Dorado Field, Butler county, Kansas. The site was chosen by comparing areas of the reservoir to a set of screening criteria. The final test site was chosen in the south half of Section 21, Township 25 South, Range 5 East and the north half of Section 28. Township 25 South, Range 5 East, Butler County, kallsas. 


\section{Introduction}

A reservoir that had been commercially waterflooded with good sweep efficiency was selected for this demonstration. Since the reservoir was abandoned, micellar-polymer flooding can be technically and economically evaluated for tertiary recovery. The test site was chosen from a representative area of the reservoir after researching data from approximately 1100 old wêlls. Test wells were drilled to obtain additional reservoir data to check the suitability of the site. The information obtained from these wells led to a shift in the site location.

\section{Discussion}

The original test site was located on the Chesney Lease in the south half of Section 21, Township 25 South, Range 5 East, Butler County, Kansas. After it was determined that the initially chosen east pattern on the Chesney Lease was probably in an original gas cap (now filled with water), it was decided to shift the pattern south to the Hegberg Lease (see Figure G-1 in Appendix G) to minimize potential fluid loss and maintain similar reservoir conditions for each pattern. Some of the reasons for this conclusion were that oil saturations of core from wells MP-102 and MP-103 at elevations above 771 feet averaged 6.1 percent compared to an average of 29.1 percent below this elevation. Tops of pay in wells MP-101, 104, and 105 were below this 771 -foot elevation. $0 i 1$ saturations in cores from these three wells averaged 27.0 percent. As a result of this analysis it was decided to relocate the eastern pattern south of the other pattern so that it is primarily on the Hegberg Lease (for additional information, see the section of "Performance Prediction"). 
The test site was selected on the basis of the following criteria:

1. The area should have had good response during primary and secondary oil recovery operations with no early water breakthrough and a minimum of 25 percent residual oil saturation.

2. The area should have maximum core data from previously drilled wells.

3. The reservoir should have a net thickness of at least ten feet.

4. The area should have a sweep efficiency greater than 50 percent.

5. The area should have permeability greater than $50 \mathrm{md}$ and preferably over $100 \mathrm{md}$.

6. The area should have porosity greater than 20 percent.

7. The reservoir should have a temperature between $50^{\circ} \mathrm{F}$ and $175^{\circ} \mathrm{F}$.

8. The viscosity of the reservoir oil should be less than $10 \mathrm{cp}$.

9. The reservoir should be relatively homogeneous.

\section{Future Work}

Selection of the test site was completed in March, 1974. There is no future work planned for this task. 
DEVELOP OPTIMUP: PATTERN

by

R. J. Miller

\section{Summary}

The preliminary pattern chosen for the demonstration project was a 6.4 acre nine-spot. However, it has now been concluded a fivespot pattern can be more easily controlled to provide a more uniform breakthrough. The two patterns that are presently under consideration are 6.4 acre and 3.2 acre five-spots. 


\section{Introduction}

Selection of the optimum pattern had to take into account both technical and economic aspects of a micellar-polymer flood. The pattern selected should provide the most efficient and expedient method for flooding the El Dorado 650-foot Sand. Since many of the variables (for example, injection rates, relative permeability effects, chemical adsorption, and mobility changes) are not well defined, it has been difficult to accurately model various patterns (see the section on "Yerformance Prediction" for additional details). The pattern selection will receive prime attention during the next reporting period.

\section{Discussion}

The preliminary pattern chosen for the demonstration project was a 6.4 acre nine-spot. The selection was based on the following parameters:

1. The reservoir should be as well defined as practical.

2. The producing wells should be confined in the pattern.

3. The spacing should be somewhat consistent with spacing that was used for primary and secondary production.

4. The length of time to complete the project should be IIinimal for spacing selerted.

Subsequent detailed modeling work indicated the optimum pattern should be a five-spot. A five-spot pattern would supply a more uniform breakthrough (without undue operating problems) which is imperative for a micellar-polymer flood. The five-spot pattern also gives a better sweep efficiency than the nine-spot (with reasonable injection rates in the side wells). Therefore, it has been concluded 
that a five-spot pattern will provide the best pattern for the demonstration flood. However, the size of the pattern has not been finalized. The two sizes that are presently under consideration are 6.4 acre and 3.2 acre five-spots (see the sections "Formation Injectivity," "Pattern Pressure Transient Testing," and "Performance Prediction" for additional information).

Some of the items being considered in choosing the optimum pattern size are:

1. The pattern should take into account any directional permeability problems.

2. The array of patterns should provide the repetition needed for scientific evaluation. The repetition is desired to minimize the possibility of erroneous results due to a lack of homogeneity in the reservoir.

3. The pattern should utilize chemicals as effectively as possible.

4. Scale-up problems that may be encountered in expanding to a fieldwide development should be considered and minimized.

5. The overall timing of the project should be practical.

6. Attention should be given to any differences in overall economics that are due to pattern size.

\section{Future Work}

Pulse testing the individual nine-spot patterns will furnish additional information that must be analyzed before finalizing a decision on pattern size. This testing is currently in progress (see the section, "Pattern Pressure Transient Tests"). 
DRILL TEST WELLS

by

R. J. Miller

Summary

The seven test wells were drilled and completed before the end of June, 1974. Although only five test wells were originally planned, two additional wells were necessary to further define the proposed test site. The two additional wells were drilled as potential injection wells in the shifted pattern. When the pattern was shifted to the Hegberg Lease, the project was unitized and named the ChesneyHegberg MP !nil. (in this report individual wells are denoted "well MP-101" rather than the formal name "Chesney-Hegberg MIP- Unit Well Number 101"). 


\section{Introduction}

Drilling the test wells provided information to help evaluate the first decision point in 0ctober, 1974. The test wells were logged and cored to allow evaluation of reservoir properties such as porosity, permeability; and oil saturations (see the sections on "Vell Logging" and "Coring and Core Analyses" for details).

\section{Discussion}

The initial seven wells were drilled before the pattern wells for five principal reasons:

1. To determine the existing oil saturation in the test area and to compare it with volumetric oil calculations. The test wells indicated an oil saturation of 33 percent, whereas volumetric calculations showed 43 percent.

2. To determine if the preliminarily selected site was adequate. The initial proposal was to drill only five test wells. Two additional wells were necessary in order to investigate the quality of the reservoir south of the initial west Chesney pattern. These two wells were located at the southeast and southwest corners of the southern pattern then under consideration. The data obtained from these wells was used to support the decision to shift the pattern location and avoid the original gas cap area (see the section "Develop Optimum Pattern").

3. To determine if there were any severe directional permeabilities that would require a change in location 
or pattern orientation. This was accomplished through pressure transient analysis. The tests indicated no severe directional permeability on a general basis (see the section on "Formation Injectivity").

4. To determine the fracture gradient of the Admire Sand. A fracture test was run on well MP-103 to determine the maximum pressure at which fluids could be injected without exceeding the parting pressure of the zone. The fracture gradient was calculated to be $1.6 \mathrm{psi} /$ foot. However, the fracture extension pressure was $1.0 \mathrm{psi} /$ foot (see the subsection on Breakdown Tests in the section on "Formation Injectivity").

5. To determine the injection rates of the various fluids involved in the project, an injectivity test was run using well MP-104. The fluid rates and pressures were monitored during the injection of the four fluids--preflush, micellar fluid, polymer, and drive water (see the section "Formation Injectivity" for details).

The test wells were rotary drilled with formation water (from another well producing from the 650-foot Sand) as the drilliny fluid. All seven wells were cored, logged, cased, and perforated. Detailed information for the test wells is shown in Table Gl, Appendix G.

\section{Future Work}

Drilling the test wells was completed on schedule. No future test wells are planned. 


\section{DRILL PATTERN WELLS}

by

R. J. Miller

\section{Summary}

The remainder of the 50 pattern wells were completed in . May, 1975. The pattern area was drilled with a high well density (almost one well per acre) in order to assure a good reservoir definition and to provide flexibility for pattern selection.

\section{Introduction}

Two types of pattern wells were drilled--producers and injectors. The eight producers were drilled in a manner similar to the test wells. The 40 injection wells were drilled and completed in a somewhat different manner. An additional two injection wells had already been drilled as test wells. 


\section{Discussion}

The first pattern well, MP-124, was spudded on December 5, 1974. Cullum and Brown Drilling Contractor was awarded the drilling contract for 48 of the pattern wells.

Eight wells were drilled as producers using air to a point about 50 feet above the Admire Sand. The rest of the well was drilled and cored with formation water. Except for wells MP-112 and MP-122, an oxygen scavenger ( 0.25 pounds of sodium sulfite per barrel) was added to the drilling water. Five and one-half inch casing was run in the borehole and cemented from the bottom of the well to the surface. Two and one-half inch tubing and conventional oil field pumping equipment will be used to produce all fluids.

The 40 injection wells were drilled in a manner similar to that used for the producers. Four and one-half inch casing was set at the top of the Admire Sand and open-hole completions were made. The injection wells will be connected to the distribution system during the next two months.

Additional pertinent information concerning the pattern wells is located in Tables G1 and G2. The wells denoted by the 100-series are located in Sertion 21, and the 200-series are located in Section 28, Township 25 South, Range 5 East, Butler County, Kansas.

\section{Future Work}

Drilling the pattern wells was completed on schedule. No future work is planned for this task. 
CONSTRUCTION 
DEVELOP FRESH WATER SYSTEY:

by

R. J. Miller

Summary

Two primary fresh water sources were extensively investigated

for use in the demonstration flood. The El Dorado City Lake was selected as the water source. In order to transport water from the City Lake to the project site, it was necessary to purchase right-of-way and lay approximately five miles of line. 


\section{Introduction,}

The objective of this work was to develop a continuous, high quality water source for the micellar-polymer flood. Many potential sources were investigated and eliminated for reasons of insufficient quality or quantity. The two remaining sources were investigated in detail.

\section{Discussion}

A fresh water source with sufficient quality and quantity was developed to ensure ample water for mixing and injection purposes. The supply water had to contain less than $5000 \mathrm{ppm}$ total dissolved solids and less than $500 \mathrm{ppm}$ calcium and magnesium in aggregate to meet the requirements of the contract with ERDA. A discussion of the two primary water sources that were investigated is given in the next paragraphs.

Murray lease fresh water wells. Two shallow fresh water wells located on the Murray lease approximately three miles west of the project were tested for use in the demonstration flood. The drawdown test indicated a combined capacity of about 3400 barrels per day. An estimated 6300 barrels per day were required. The water analys is showed a high amount of sulfates which would combine with the barium present in the formation water to form a barium sulfate scale. It was concluded the Murray lease could not supply the water quality or quantity necessary to conduct the demonstration test.

The El Dorado City Lake. This alternate source, located about five miles east of the project site, was investigated as a fresh water 
supply. Preliminary meetings with El Dorado City officials were held in June, 1974, to determine the feasibility of utilizing city treated or raw water for the demonstration flood. City water, either treated or raw, could be purchased in the desired quantity. Water analysis showed that filtered raw water would provide better water for use in the demonstration flood. When the decision to purchase raw water was made, it became necessary to purchase right-of-way and lay a six-inch PVC line to intersect the city water supply line from the El Dorado City Lake. Since the raw lake water contains a large amount of suspended solids, it is necessary to filter all water. An upflow, automatic backflush sand filter was chosen to provide the water quality necessary for the demonstration flood. A model of the upflow filter was tested in October, 1974, at the EI Dorado City water plant. The objectives of the test were to determine water quality and flush cycle time. The test was designed to determine the type and quantity of polymer necessary to aid in flocculation. The following conclusions were reached:

1. The upflow sand filter was capable of clarifying the El Dorado lake water to the desired quality. A water quality of 0.3 las.kson Turbidity Units (JTU) or less will be satisfactory for the demonstration test. The filter is capable of producing water quality of 0.1 to $0.2 \mathrm{JTU}$.

2. The upflow filter system efficiency is reduced with increased concentrations of suspended solids in the water.

3. Polymer concentration should be increased during cooler temperatures and decreased during warmer temperatures. 


\section{Future Work}

Construction of the fresh water 1 ine began March 12 . The pump station and line have been completed with the exception of a river crossing which should be finished in July, 1975. 
BUILD INJECTION PLANT

by

R. J. Miller

Preliminary plans to build the injection plant began in March, 1974. Most long-term procurement items were ordered at that time. Several modifications were made in the initial design to correspond with changes in chemical selection. The plant is approximately 45 percent complete now and should be 100 percent complete in August, 1975. 


\section{Introduction}

The purpose of the injection plant is to measure, mix, and deliver chemicals to the distribution system. The El Dorado MicellarPolymer plant is probably more complex than most injection plants since it must be capable and flexible enough to handle two distinct chemical processes.

\section{Discussion}

The injection plant must be capable of mixing chemical additives, fresh water, and other materials in proper proportions for delivery to the distribution system at pressures sufficient to allow injection into the reservoir.

In preparation for starting injection as scheduled on the original milestone chart, most items requiring long-range procurement were ordered from the preliminary design in March, 1974. Some orders had to be modified after selecting the chemical process vendors. The preliminary drawings and design have been updated and revised periodically. The initial flow diagram was completed in Mlay, 1974, (see Figure $\mathrm{H}-1$ in Appendix $H$ ). The injection plant will consist of the components discussed below:

Two metal buildings. The injection building will house the triplex injection pumps, mixing pumps, control panels, and on-site laboratory. The building has been erected, and the triplex pumps have been set in place. The mixing pumps, control panels, and on-site laboratory installation shoild be completed by August, 1975 .

The header building will include the distribution headers and the polymer mixing equipment. The building has been completed. 
The headers and mixing equipment will be installed subsequent to the final pattern selection and polymer design.

Mixing, storage, and suction tanks. Three fiberglass 300barrel suction tanks, two 500-barrel bolted steel tanks, and one 1000barrel bolted steel tank were padded and set June 13, 1975. However, the plant was damaged by high winds on June 16, 1975, and these six tanks were completely destroyed. The walls of the two buildings were also damaged. The walls will be replaced when the new material arrives.

The three fiberglass tanks were to be used to feed the triplex pumps with the various fluids that will be injected into the formation. The replacements for the tanks will be three welded steel tanks that were being held for possible use at the battery site.

The 1000-barrel bolted steel tank is a fresh water holding vessel. The two 500-barrel bolted steel tanks are for storage of the preflush and micellar fluids. The replacements for these tanks were ordered June 17, and delivered June 26, 1975.

Four temporary 500-barrel tanks will be installed prior to injection initiation. These tanks will provide additional storage of crude oil and micellar fluids.

Fresh water filter unit. The foundation for the upflow sand filter has been poured and the unit has been delivered.

\section{Future Work}

The tanks and filter unit should be erected in July. The plant should be completed and ready for operation in August, 1975. 
INSTALL BATTERY FACILITIES

by

R. J. Miller

Summary

Installation of the battery facilities was completed in June, 1975. The battery system consists of a heater-treater, a lease automatic custody transfer unit, individual pattern test units, and several oil-water holding vessels. 


\section{Introduction}

The purpose of the gathering system is to transport produced fluids to a central storage area. The battery is designed to handle 4000 barrels per day of produced fluids.

\section{Discussion}

Figure $H-2$ in Appendix $H$ is a diagram of the battery facility. The heater-treater vessel is to separate the oil and water in order to deliver pipeline oil to stock tanks. Each pattern will have a 100barrel test tank which will allow individual well tests on a periodic basis. All equipment is designed to assure continuous operations. A lease automatic custody transfer (LACT) unit will automatically sample, meter, and deliver oil to the pipeline. The LACT unit is able to handle at least 1000 barrels per day. Its capacity could be increased to 2000 barrels per day with minimum cost.

\section{Future Work}

The battery facilities are completed and ready for hookup to the gathering system. No future work is planned for this task. 
APPENDIX A

CHEYIICAL SELECTION AND SUPPORT

Tables and Figures 
TABLE $\wedge 1$

SUMMARY OF OIL RECOVERY FLOW TESTS FOR. THE

UNION PROCESS--BEREA CORES

\section{Flow Test No.}

Porosity

$K_{w}$, at post-waterflood

$\mathrm{S}_{\mathrm{O}}$, md

Post-waterflood oil

saturation, PV

Sequence of injected fluids after waterflood, PV

Fresh water

Union Preflush

Soluble oil

Polyacryiamide

\section{Fresh water}

0 il recovery, fraction of post-waterflood $\mathrm{S}_{\text {or }}$

Final $S_{\text {or }}$

Remarks

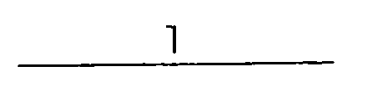

0.23

37

0.33

0.10

0.10

0.040

$0.10(800 \mathrm{ppm})$

0.50 (401 ppm)

0.56

0.48

0.58

0.17

0.15

Error in soluble oil composition

29

0.36

0.10

0.10

0.36

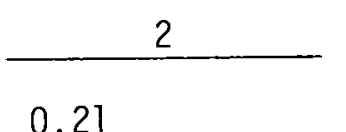

0.040

0.60 (600 ppm)

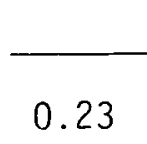

3

44

0.28

0.10

0.10

0.040

$0.10(800 \mathrm{ppm})$

$0.50(400 \mathrm{ppm})$

0.56

0.79

0.064

Injectivity test soluble oil

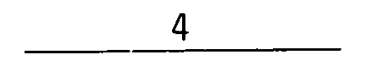

0.22

0.22

80

50

Note--A 17 cores were 2.0 inches in diameter and 10.0 inches long. 
TABLE A2

SUMMARY OF OIL RECOVERY FLOW TESTS FOR THE

UNION. PROCESS--650 FOOT SAND CORES

Flow Test No.

Length, $\mathrm{cm}$

Porosity

$K_{w}$, at post-waterflood $s_{0}$, md

Post-waterflood oil

saturation, PV

Sequence of injected fluids

after waterflood, PV

Fresh water

Union Preflush

Soluble oil

Polyacrylamide

Fresh water

$0 i 1$ recovery, fraction of post-waterflood $S_{\text {or }}$

Final $\mathrm{S}_{\text {or }}$

Remarks

0.10

0.10

0.040

$0.60(600 \mathrm{ppm})$

0.60

$6:$

27.9

0.28

60

0.22

0.18
55

\section{7}

26.3

0.26 
TABLE A3

MOBILITY HEASUREMENTS IN BEREA SAND CORES FOR

POLYPIER DISSOLVED IN EL DORADO LAKE WATER

Core

$\mathrm{K}_{\mathrm{w}}$,

Polymer Velocity,

Test No. Length, cm

md Concentration, ppm $\mathrm{ft} / \mathrm{day}$

$\lambda_{p}$

darcies/cp ${ }^{\lambda} p / \lambda_{\text {water }}^{*}$

1 - (7st core)

2.5

661

1000

3.7

0.007

0.011

1 (2nd core)

$2.5 \quad 803$

1000

3.7

0.010

0.012

2

2.8

622

600

3.0

0.004

0.006

2

2.8

622

400

2.5

0.006

0.010

2

$2.8 \quad 622$

200

2.5

0.068

0.109

3

8.4

455

600

3.0

0.005

0.011

Cores for tests 1 and 2 were $2.54 \mathrm{~cm}$ in diameter.

Core for test 3 was $5,08 \mathrm{~cm}$ in diameter.

*The viscosity of water at room temperature was assumed to be $1.0 \mathrm{cp}$. 
TABLE A4

MOBILITY IEEASURENENTS IN 650-FOOT SAND CORES FOR

POLYI:IER DISSOLVED IN EL DORADO LAKE WATER

\begin{tabular}{|c|c|c|c|c|c|c|}
\hline Test No. & $\begin{array}{c}\text { Core } \\
\text { Length, cm }\end{array}$ & $\begin{array}{l}K_{W}, \\
\underline{m d} \\
\end{array}$ & $\begin{array}{c}\text { Poljmer } \\
\text { Concentration, ppm }\end{array}$ & $\begin{array}{l}\text { Velocity, } \\
\text { ft/day }\end{array}$ & ${ }_{\mathrm{p}}^{\lambda_{\mathrm{darcies} / \mathrm{cp}}}$ & ${ }_{\mathrm{p}} /{ }^{*}{ }_{\text {water }}^{\star}$ \\
\hline 1 (1st core) & 6.9 & 165 & 300 & 3.7 & 0.0006 & 0.0036 \\
\hline 1 (2nd core) & 7.7 & 96 & 300 & 3.7 & 0.0005 & 0.0052 \\
\hline 1 (1st core) & 6.9 & 165 & 600 & 3.7 & 0.0002 & 0.0012 \\
\hline 1 (2nd core) & 7.1 & 96 & 600 & 3.7 & 0.0002 & 0.0021 \\
\hline 2 (1st core) & 6.4 & 47 & 300 & 3.7 & 0.0015 & 0.0319 \\
\hline 2 (2nd core) & 7.2 & 117 & 300 & 3.7 & 0.0018 & 0.0154 \\
\hline 3 & 10.2 & 85 & 600 & 9.3 & 0.0037 & 0.0435 \\
\hline 3 & 10.2 & 85 & 600 & 3.7 & 0.0034 & 0.0400 \\
\hline
\end{tabular}

Cores for tests 1 and 2 were $2.54 \mathrm{~cm}$ in diameter.

Core for test 3 was $5.08 \mathrm{~cm}$ in diameter.

*The viscosity of water at room temperature was assumed to be $1.0 \mathrm{cp}$. 
TABLE A5

CHARACTERIZATION OF 600 PPM* POLYMER SOLUTIONS

PREPARED FROM VARIOUS STOCK SOLUTIONS

Stock Solution From Which 600 ppm Polymer Was Prepared

1. $30 \%$ Liquid Concentrate

2. Same as No. 1. Sheared 3 minutes in Waring Blender

3. Same as No. 1, Sheared

16 minutes in Waring Blender

4. $30 \%$ Liquid Concentrate mixed at $2700 \mathrm{ppm}$ wi th paddle mixer

5. Same as. No. 4, Sheared 3 minutes in Waring $\mathrm{Blender}$

6. $30 \%$ Liquid Concentrate, mixed at 3000 ppm with HamiltonBeach mixer

7. Same as No. 6, Sheared

3 minutes in Waring Blender

8. $30 \%$ Liquid Concentrate, mixed at $6000 \mathrm{ppm}$ with HamiltonBeach mixer

*Dry basis

**Brookfield LV with No.l spindle

Viscosity at $6 \mathrm{rpm} * * /$ Screen Factor Before Shearing After Shearing 600 ppm Polymer 600 ppm Polymer

$75 / 13$

$9 / 3$

$75 / 14$

$9 / 3$

$87 / 17$

$11 / 2$

$91 / 15$

$15 / 2$

$42 / 6$

$10 / 3$

$66 / 8$

No Data

$14 / 4$

$6 / 2$

$38 / 7$

$7 / 3$ 
TABLE A6

EFFECT OF SALINITY AND PRESERVATIVES ON VISCOSITIES OF 600 PPM POLYACRYLAMIDE SOLUTIONS

(Viscosities in cp as Measured on Brookfield LV, No. I Spindle at 6 rpm)

Polymer

\section{$E D L W^{\star}$}

On date prepared

31 days

66 days

$\mathrm{EDLW}+1 \% \mathrm{NaCl}$

On date prepared

31 days

66 days

$E D L W+5 \% \mathrm{NaCl}$

On date prepared

31 days

66 days

EDLW + $7 \%$ CPW**

On date prepared

31 days

66 days

$E D L W+5 \% \mathrm{CPW}$

On date prepared

31 days

66 days

EDLW + 200 ppm formaldehyde

On date prepared

31 days

66 days

EDLW + 75 ppm commercial

chlorinated phenol

On date prepared

31 days

66 days
$30 \%$ Liquid Lower Molecular Concentrate Weight Solid
Intermediate Molecular Weight Solid
63

62

58

7

6

7

5

6

5

13

15

14

8

9

6

73

26

22

70

64

70
24

22

21

6

5

7

4

4

5

10

10

6

11

13

15

47

35

10

8

7

6

6
8

9

10
6

25

20

16

45

44

45

*El Dorado Lake Water, **Chesney Produced Water 
TABLE A7

EFFECT OF SALINITY AND PRESERVATIVES ON SCREEN FACTOR OF 600 PPM POLYACRYLAMIDE SOLUTIONS

Polymer

EDLW*

On date prepared

31 days

66 days

$\mathrm{EDLW}+1 \% \mathrm{NaCl}$

On date prepared

31 days

66 days

$\mathrm{EDLW}+5 \% \mathrm{NaCl}$

On date prepared

31 days

66 days

$E D L W+7 \%$ CPW**

On date prepared

31 days

66 days

$E D L W+5 \% \mathrm{CPW}$

On date prepared

31 days

66 days

EDLW + $200 \mathrm{ppm}$ formaldehyde

On date prepared

31 days

66 days

EDLW + 75 ppm commercial

chlorinated phenol

On date prepared

31 days

66 days
$30 \%$ Liquid Lower Molecular Concentrate Weight Solid
Intermediate Molecular Weight Solid

$\begin{array}{lll}17 & 14 & 27 \\ 18 & 10 & 18 \\ 20 & 10 & 18\end{array}$

14

16

17

12

10

10

32

18

15

7
16

11

10

15

10

17

15

14

13

11

15

9

28

21

18

13

16

13

13

10

13

27

18

18

16

13

13

11

25

22

13

21

*El Dorado Lake Water, ${ }^{\star \star}$ Chesney Produced Water 
TABLE A8

COMPOSITION OF CHESNEY PRODUCED WATER AND EL DORADO LAKE WATER

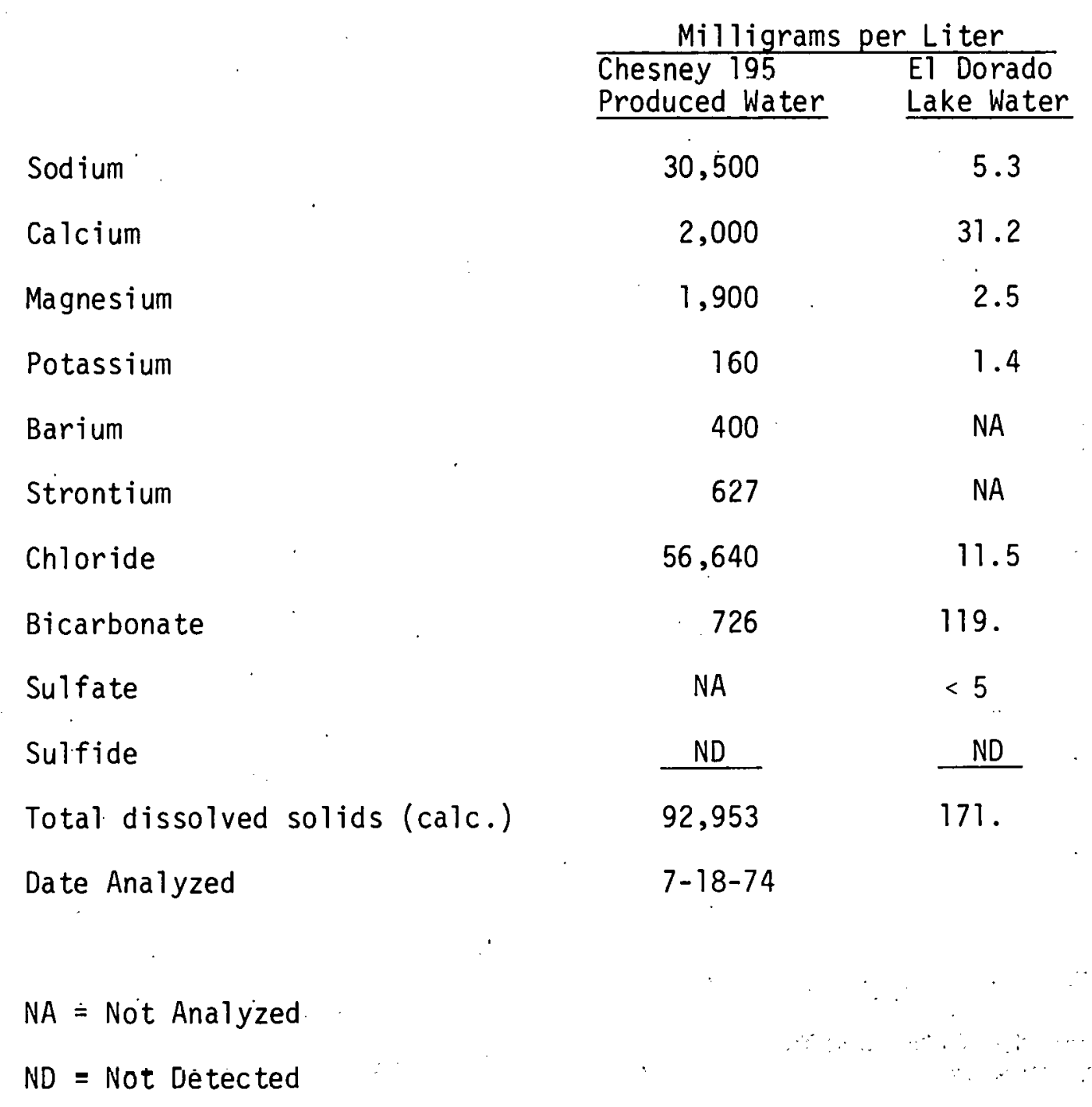




\section{TABLE A9}

RESIDENT WATER COMPOSITION

(Data in Milligrams per Liter)

Well Number

Sodium

Calcium

Magnesium

Potassium

Barium

Strontium

Chloride

Bicarbonate

Sulfate

Sulfide

Total dissolved solids (calc.)

Total dissolved solids (evaporation)

Well Number

Sodium

Calcium

Magnesium

Potassium

Barium

Strontium

Chloride

Bicarbonate

Sulfate

Sulfide

Total dissolved solids (calc.)

Total dissolved solids (evaporation)

$N A=$ Not analyzed

$N D=$ Not detected by odor
$\underline{M P-112} \quad \underline{M P-114} \quad \underline{M P-122} \quad \underline{M P-124}$

$28,900 \quad 27,200 \quad 30,500 \quad 29,500$

2,760

1,550

230

240

800

52,350

$\mathrm{NA}$

$<5$

ND

86,830

88,250

1,940

2,680

110

10

90

51,940

NA

2 1,000

ND

84,970

87,530

2,580

1,630

270

300

550

55,630

NA

$<5$

ND

91,400

93,300 2,640

1,630

210

300

710

54,930

NA

$<5$

ND

89,920

90,490

MP-207 MP-209 $\quad$ MP-217 $\quad$ MP-219

28,400

1,660

2,300

160

30

140

52,990

NA

90

ND

29,430

2,540

30,800

32,660

2,520

2,660

1,800

1,580

270

210

260

420

300

320

420

650

420

54,490

NA

$<5$

ND

56,800

NA

$<.5$

ND

59,700

NA

$<5$

ND

85,770

89,110

93,080

97,600

85,810

90,720

94,230

99,620 
TABLE A10

CHARACTERIZATION OF. EMULSIONS, CRUDE OIL

MIXED WITH AQUEOUS SOLUTIONS (NO SURFACTANTS)

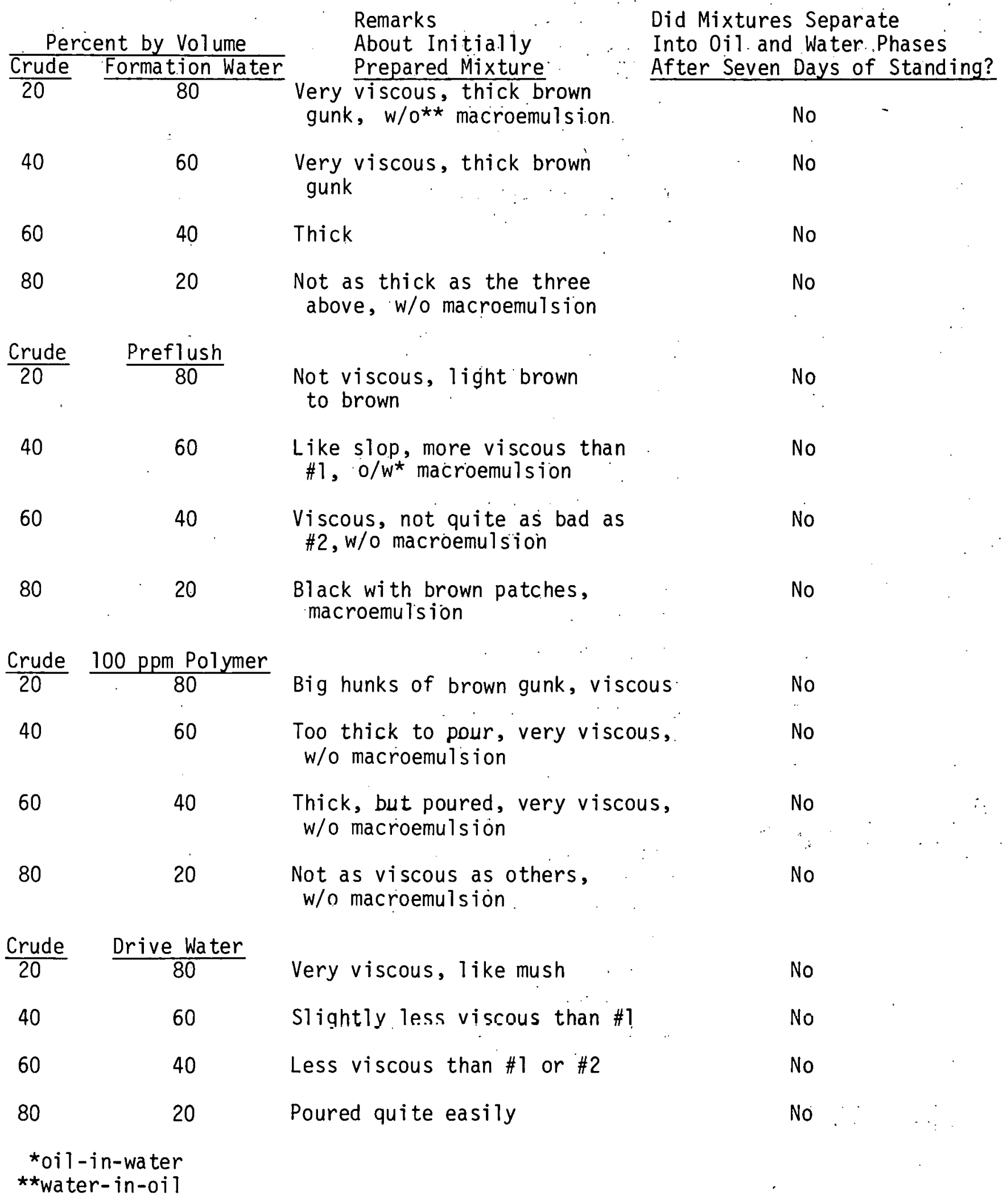


MIXED WITH AQUEOUS SOLUTIONS

\section{Soluble $\frac{0 i 1}{20} \quad \frac{\text { Formation water }}{80}$}

40

60

80

Soluble

$\frac{0 i 1}{20}$

$$
\frac{\text { Preflush }}{80}
$$

40

60

$$
60
$$

40

80

20

Remarks

About Initially

Prepared Mixture

Not stable enough to pour, $0 / w^{*}$ macroemulsion

Not stable enough to pour, $\mathrm{o} / \mathrm{w}$ macroemulsion

Not stable enough to pour, o/w macroemulsion

$0 / w$ macroemulsion
Did Mixtures Separate Into $0 i l$ and Water Phases After Seven Days of Standing?

Yes

Yes

Yes

Yes

No

$$
\text { o/w macroemulsion }
$$

No

microemulsion

Black mixture, no foam, stable

No

w/o** microemulsion

Black mixture, stable w/o

No

Soluble

$\frac{0 i 1}{20} \frac{100 \mathrm{ppm} \text { Polymer }}{80}$

Lot of foam, brown,

No

o/w macroemulsion

40

60

Lot of foam, o/w

No macroemulsion

60

40

Very little foam, black,

No w/ o microemulsion

80

20

No foam, black

No

Soluble

$\frac{0 i 1}{20}$

$\frac{\text { Drive Water }}{80}$

40

60

40

60

80

Light brown or gray, $0 / w$

No macroemulsion

Lot of foam, darker than \#1,

No $\mathrm{n} / \mathrm{w}$ macroemulsion

No

Less foam than \#2, darker than $\# 2$, microemulsion

No

\section{Essentially no foam, black} mixture, microemulsion 
Note: $90-10$ refers to a solution 90 percent by volume crude oil and ten percent by volume soluble oil.

Percent by Volume

$\begin{array}{cc}\frac{90-10}{20} & \frac{\text { Formation Water }}{80} \\ 40 & 60 \\ 60 & 40 \\ 80 & 20 \\ \frac{90-10}{20} & \frac{\text { Preflush }}{80} \\ 40 & 60 \\ 60 & 40 \\ 80 & 20 \\ \frac{90-10}{20} & \frac{100 \text { ppm Polymer }}{80} \\ 40 & 60 \\ 60 & 40 \\ 80 & 20\end{array}$

$\frac{90-10}{20} \quad \frac{\text { Drive Water }}{80}$

40

60

60

40

80 20
Remarks About Initially Prepared Mixture

No foam, not viscous, $0 / w^{*}$ macroemulsion

o/w macroemulsion

Black mixture, $0 / w$ macroemulsion

Black mixture, o/w macroemulsion

Light brown, $0 / w$ macroemulsion

Slightly darker than $\# 1,0 / w$ macroemulsion

Darker than \#2, microemulsion

Darker than \#3, microemulsion

Light brown or $\tan , 0 / w$ macroemulsion

Light brown or $\tan , 0 / w$ macroemulsion

Viscous but homogeneous, o/w macroemulsion

Not very viscous, darker than others, W/ $0^{\star \star}$ macroemulsion

Light brown or tan homogeneous mixture. like chncolate milk, o/w macroemulsion

Light brown or $\tan$ homogeneous mixture, like chocolate milk, o/w macroemulsion

Darker brown than \#1 or \#2, not viscous, w/o macroemulsion Dark brown, w/o microemulsion
Did Mixtures Separate Into $0 i 1$ and Water Phases After Seven Days of Standing? Yes

Yes

Yes

Yes

No

No

No

No

No

No

No

No

No

No

No

No 


\section{CHARACTERIZATION OF EMULSIONS; ONE PERCENT \\ SOLUBLE OIL IN CRUDE OIL MIXED WITH AQUEOUS SOLUTIONS}

Note: $99-1$ refers to a solution 99 percent by volume crude oil and one percent by volume soluble oil.

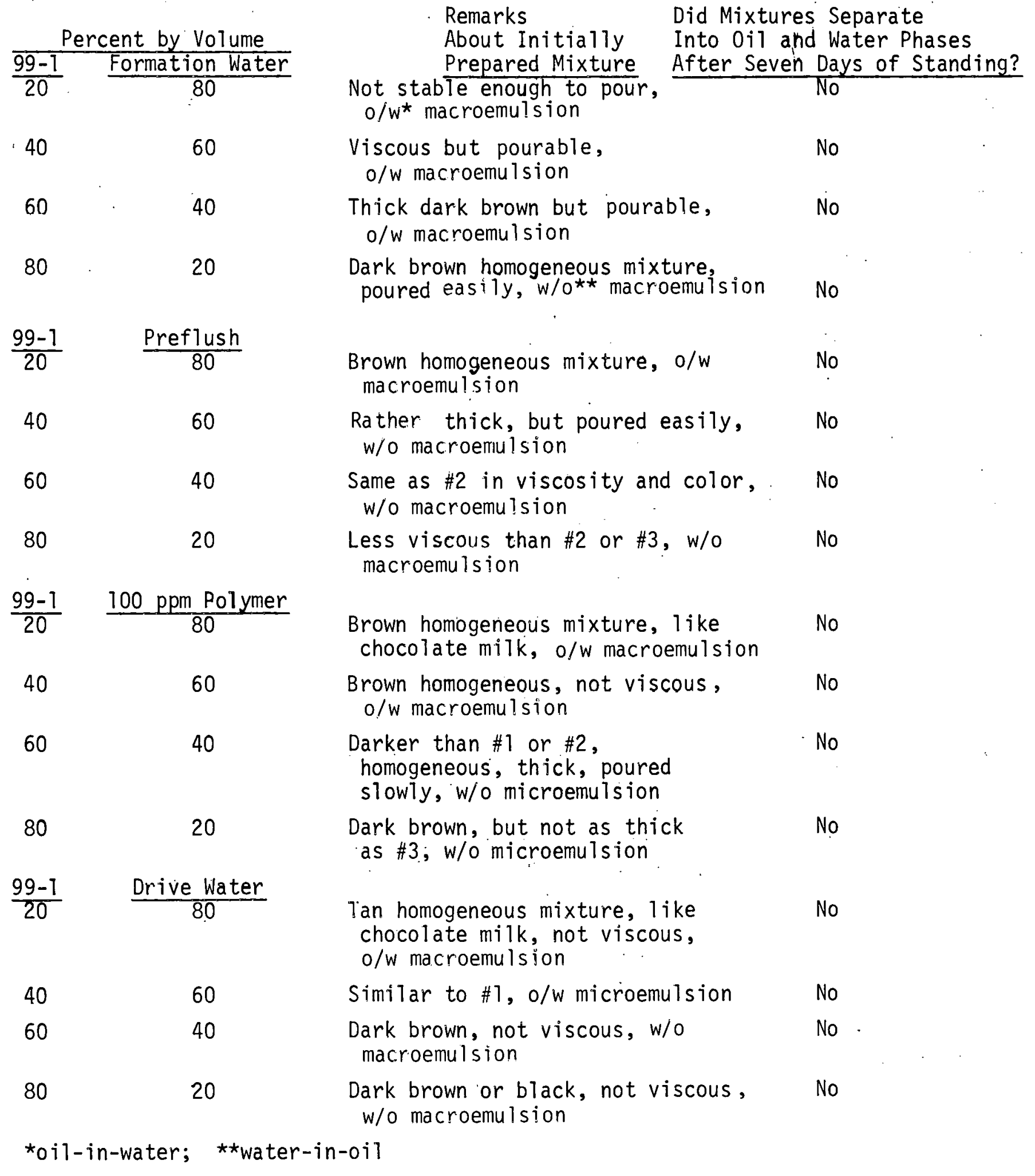


FIGURE $A-1$

OIL RECOVERY FLOW TEST NO. I

(BEREA CORE)

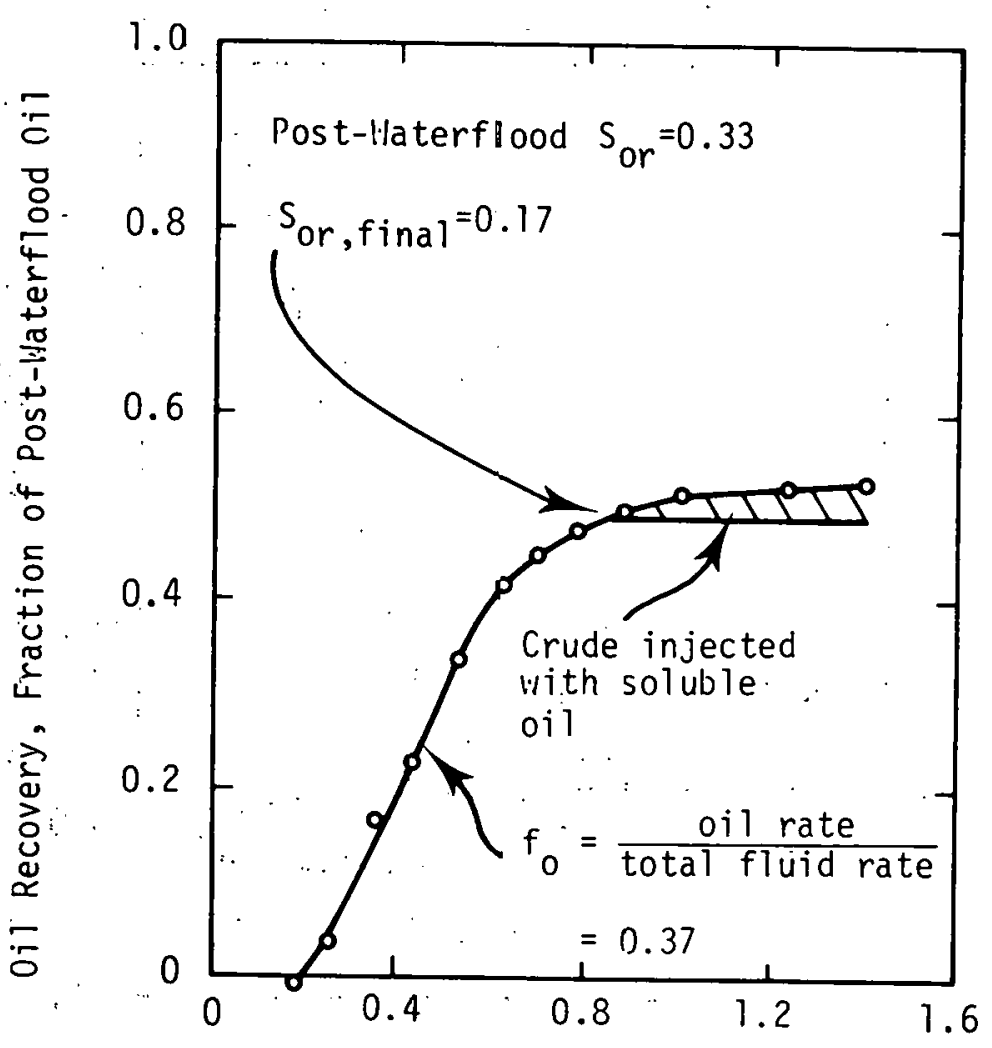

Pore Volune Injected After Soluble Dil Bank
FIGURE A-2

OIL RECOVERY FLOW TEST NO. 2

(BEREA CORE)

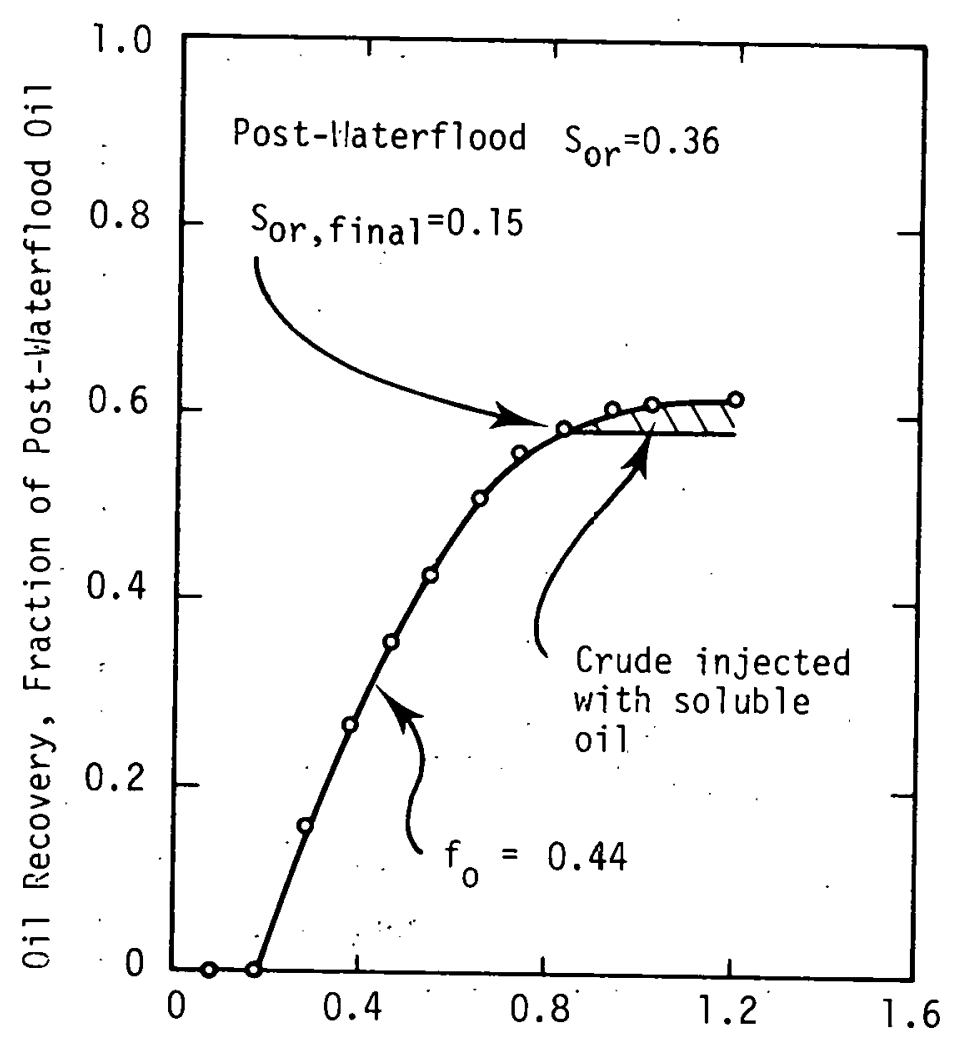

Pore Volume Injected After Soluble 0il Bank 
FIGURE $A-3$

OIL RECOVERY FLOW TEST NO. 3

(BEREA CORE)

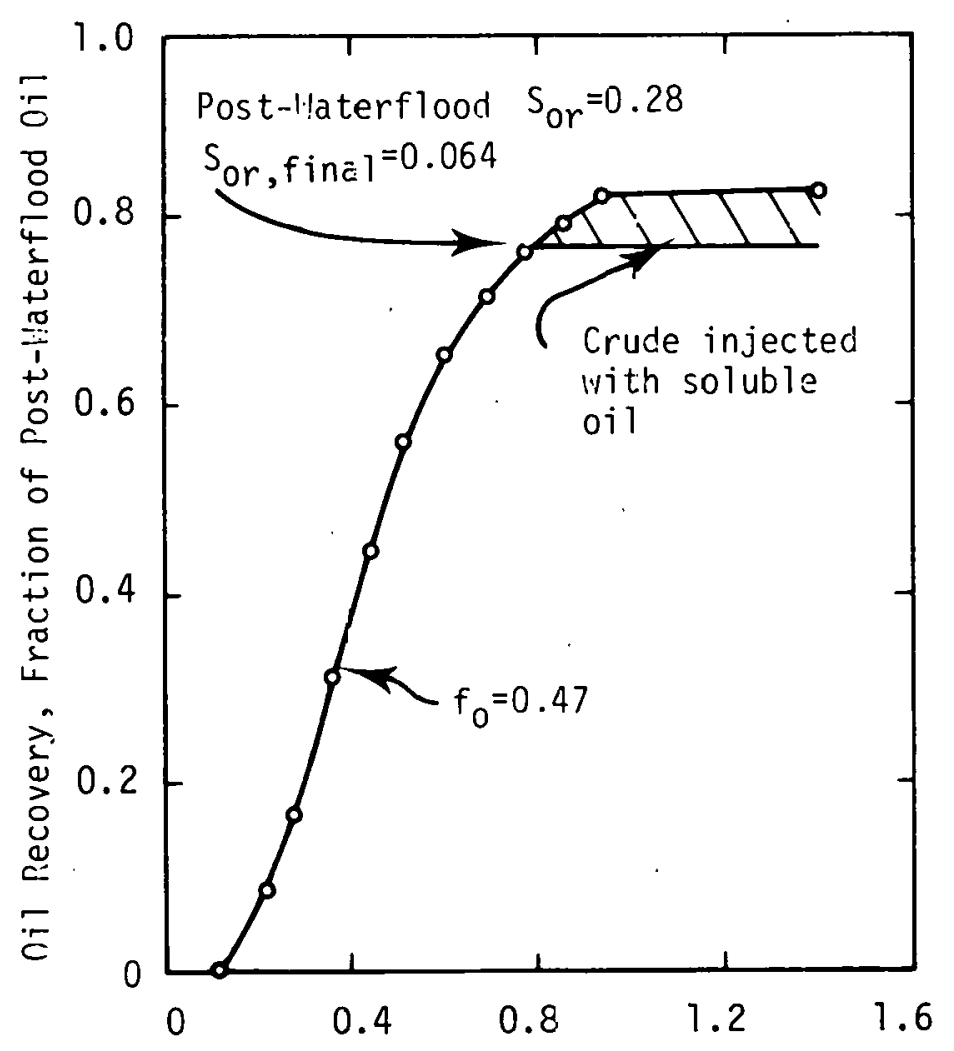

Fore Volume Injected After Soluble 0il Bank
FIGURE $A-4$

OIL RECOVERY FLOW TEST NO. 4

(BEREA CORE)

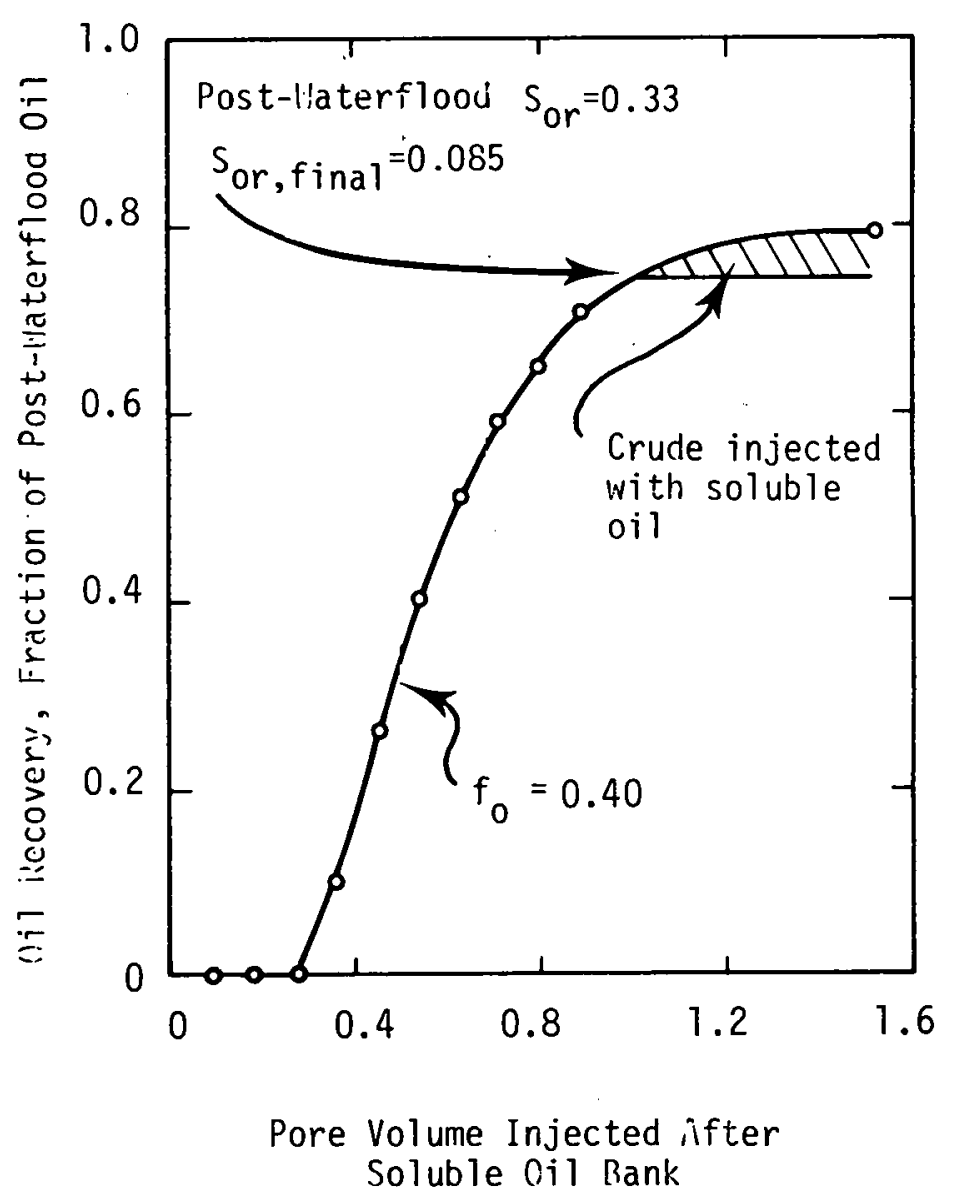


FIGURE $A-5$

OIL RECOVERY FLOW TEST NO. 5

(BEPEA CORE)

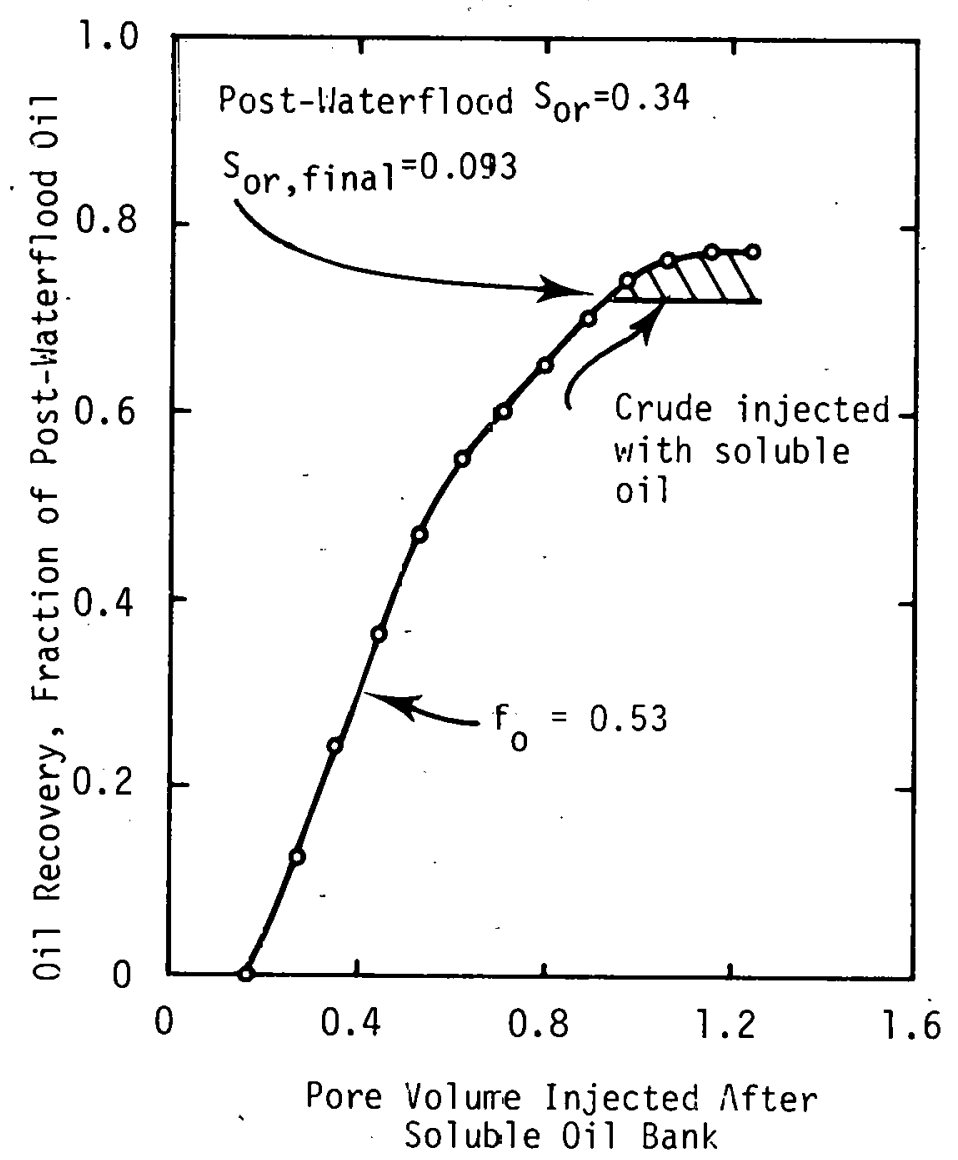

FIGURE $A-6$

OIL RECOVERY FLOW TEST NO. 6 (650-FOOT SAND CORE)

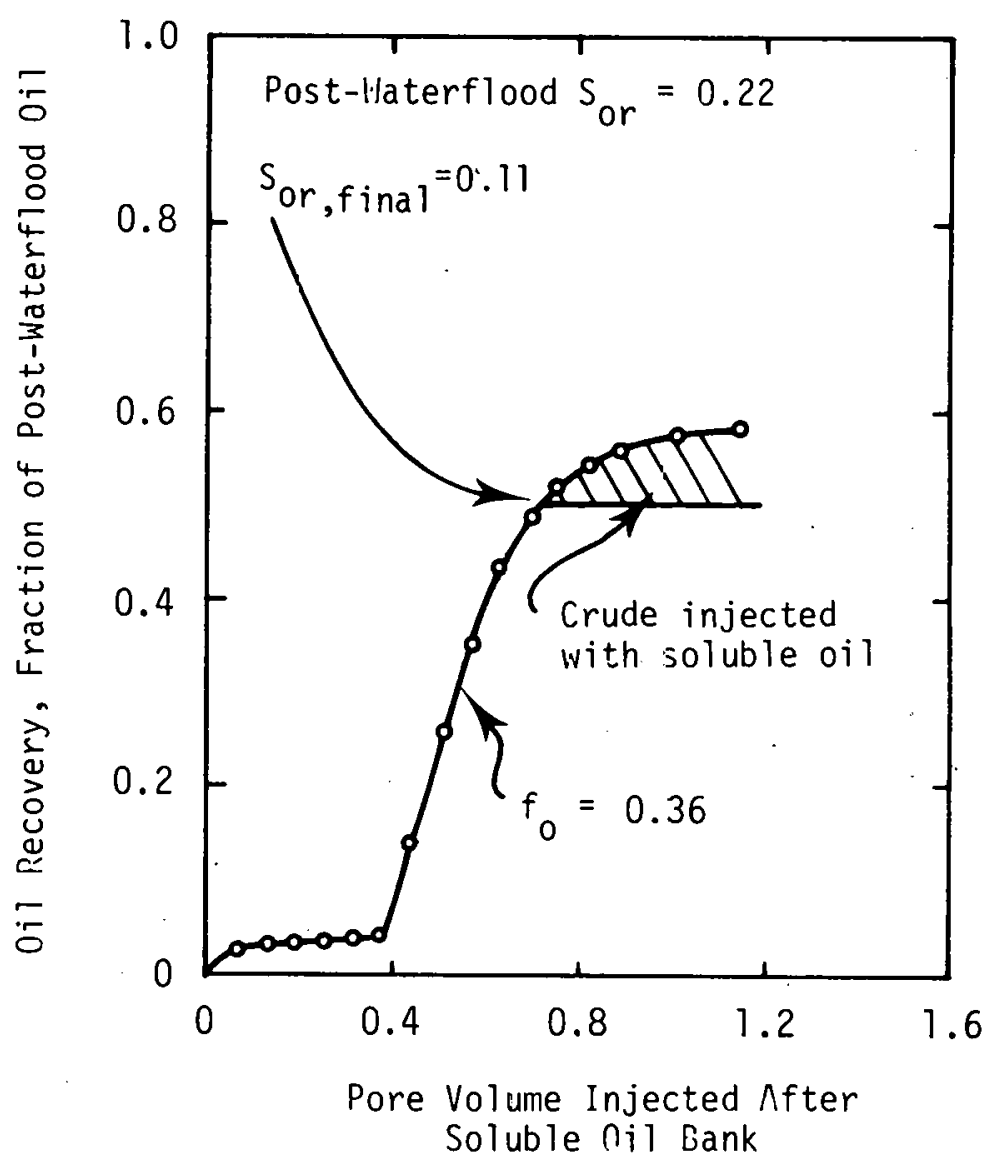


FIGURE A-7

OIL RECOVERY FLOW TEST NO. 7

(650-FOOT SAND CORE)

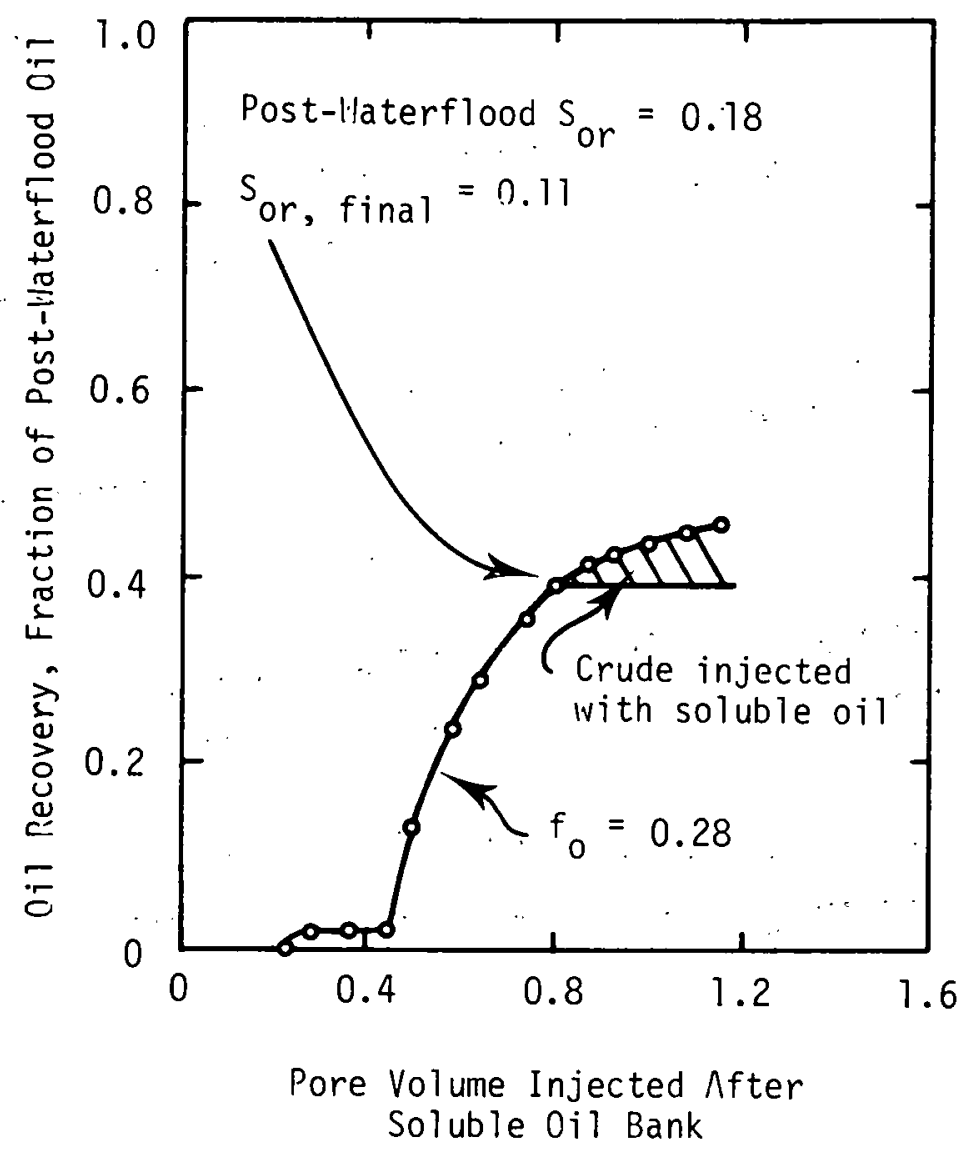




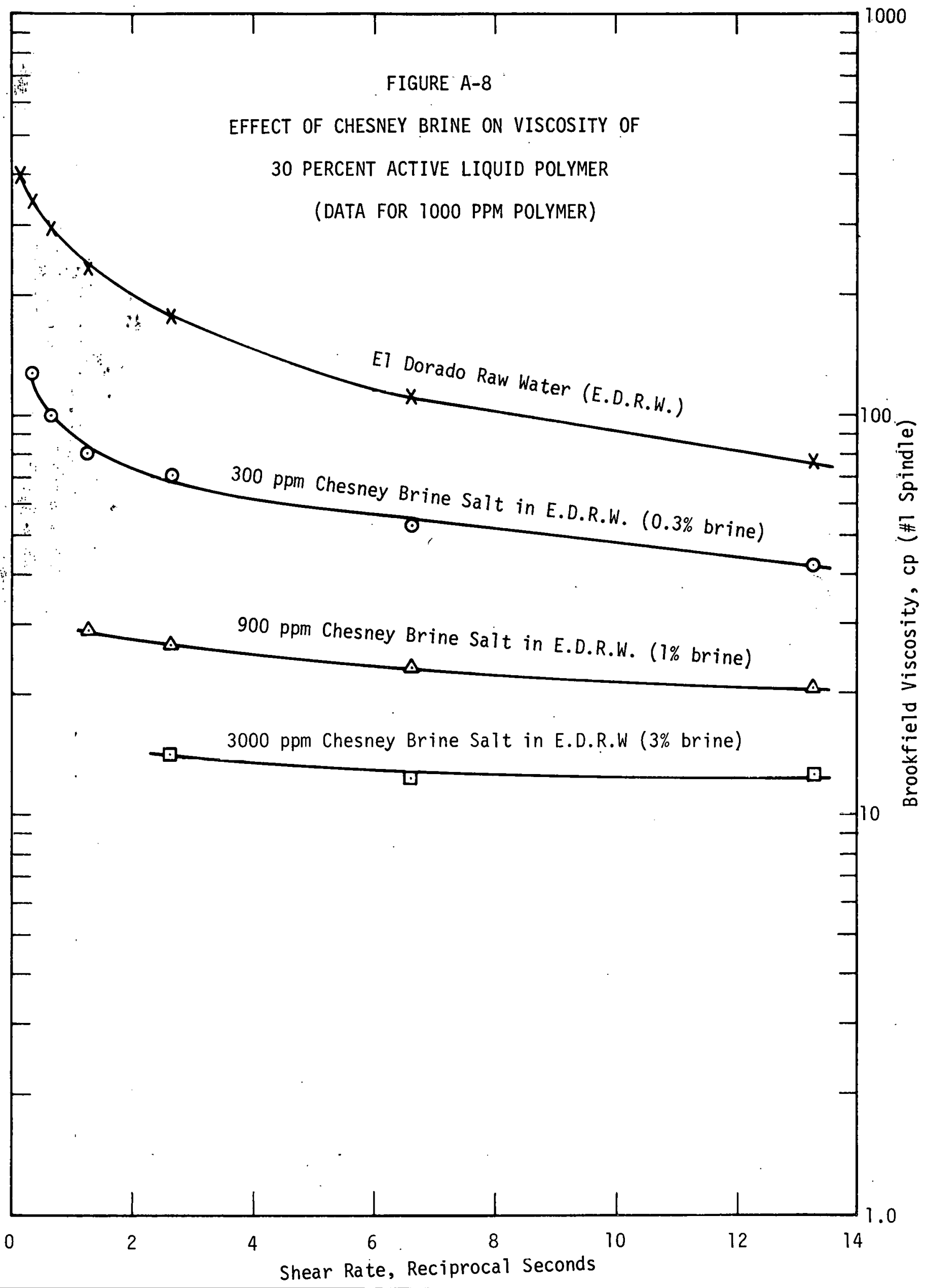


I I - 19

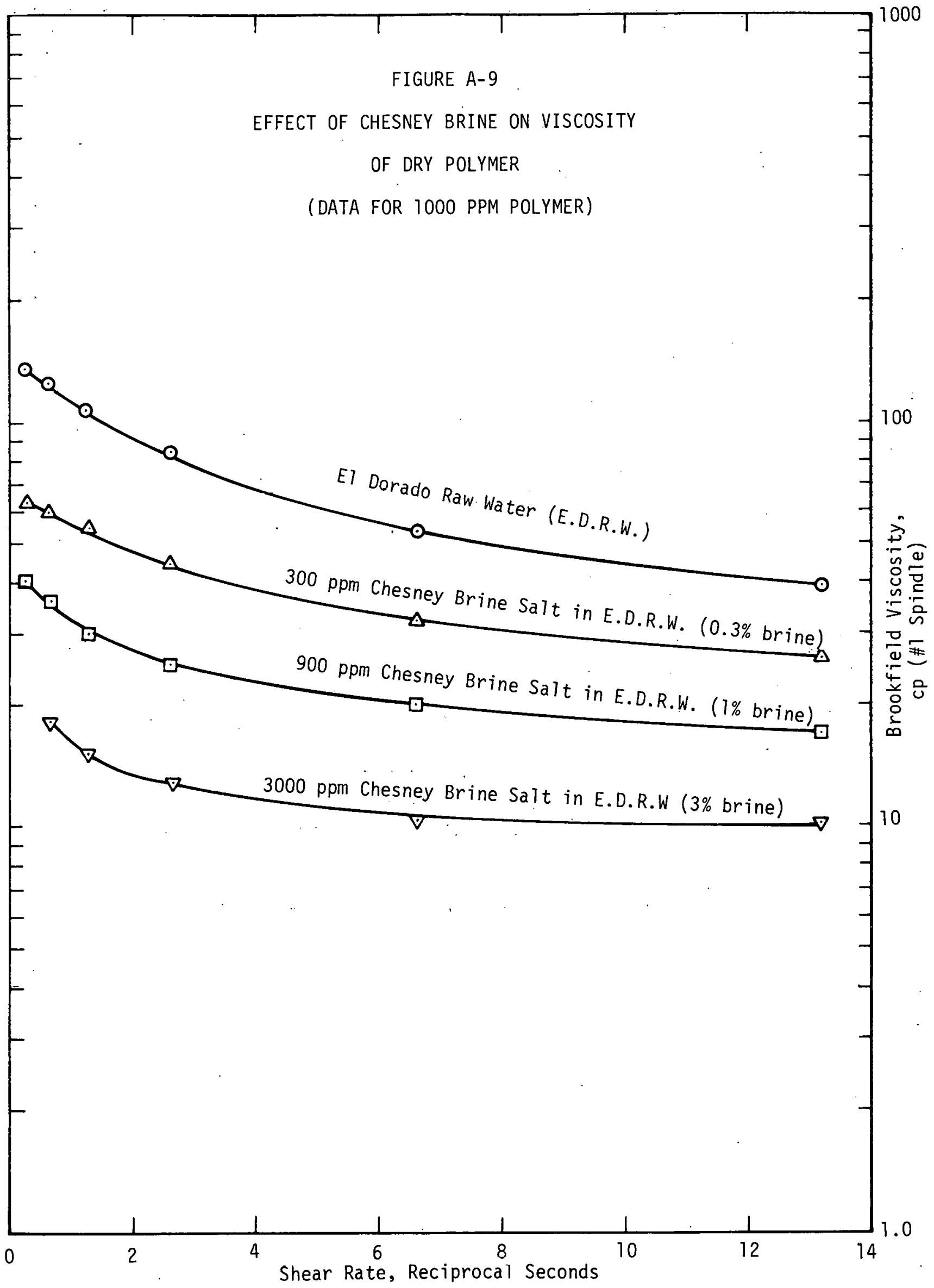




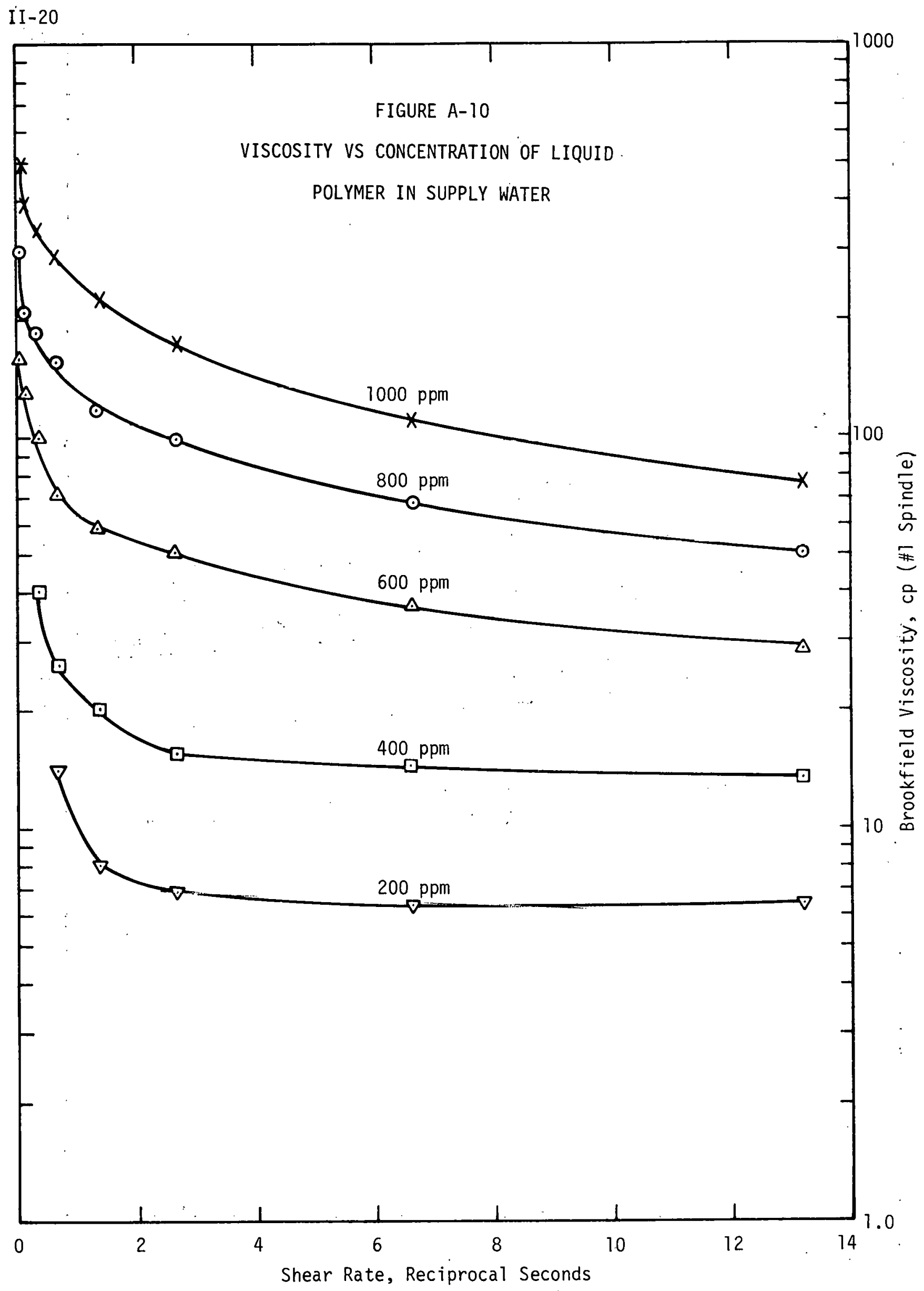




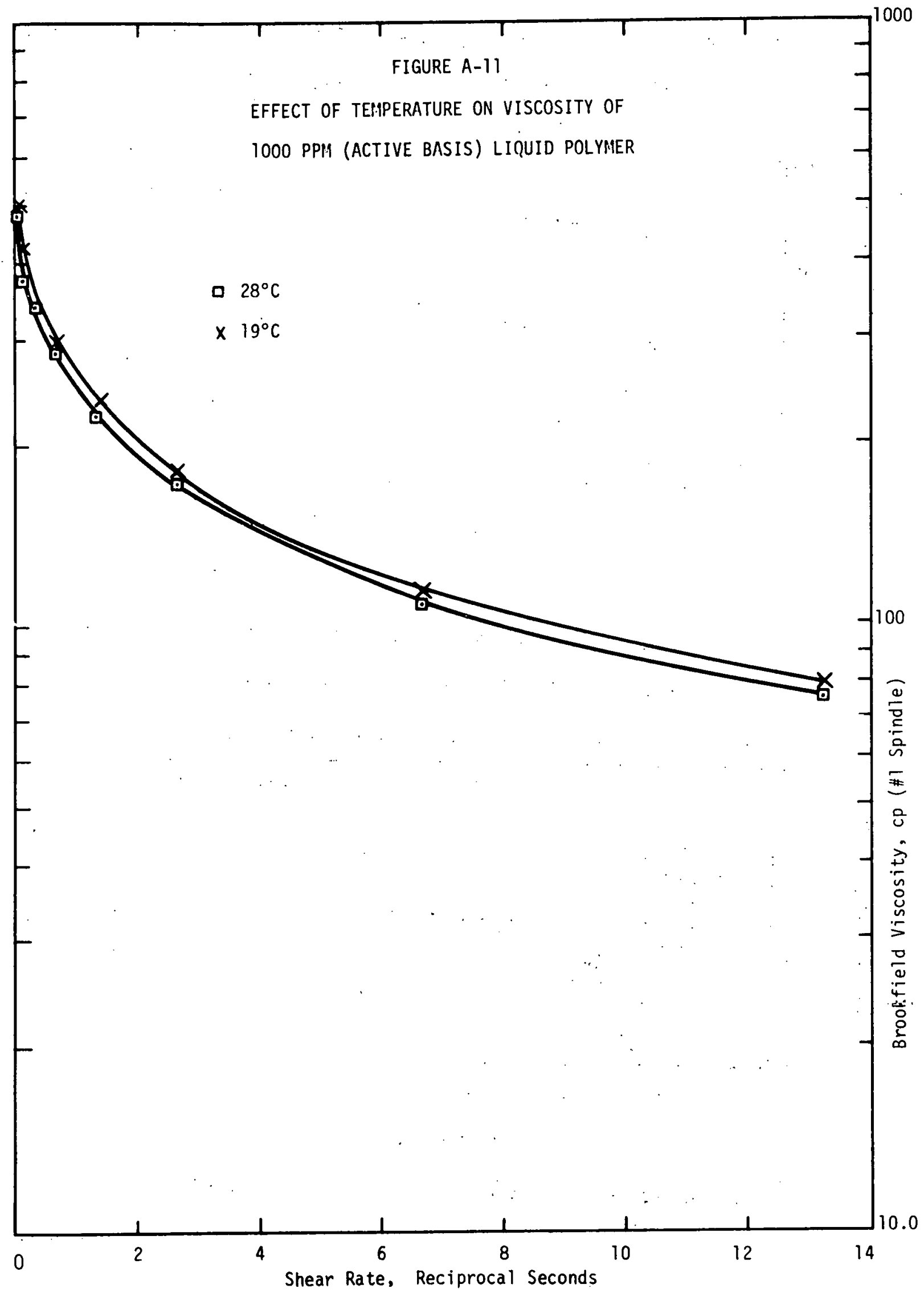


SCREEN FACTOR LOSS AFTER SHEARING AT DIFFERENT

FRONTAL VELOCITIES (650-F00T SAND CORE)

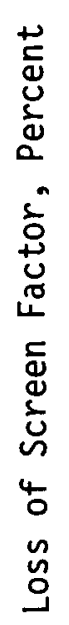

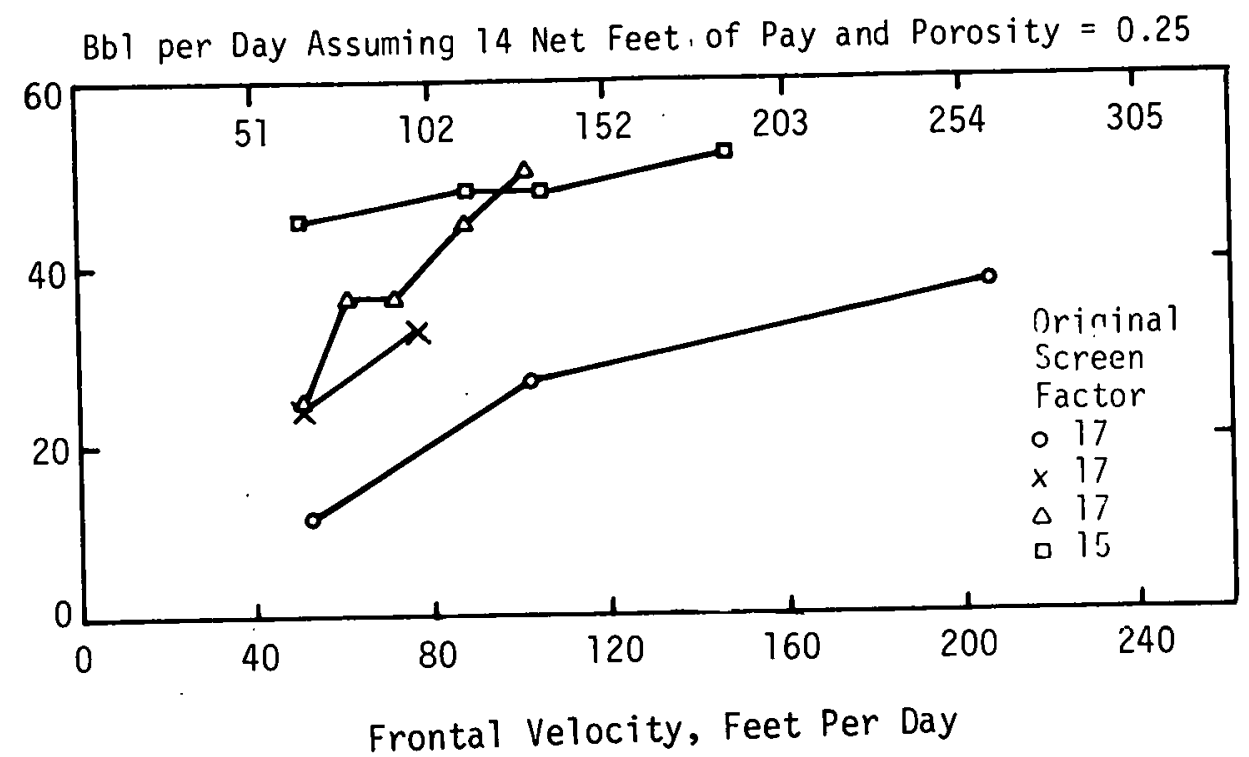

FIGURE $A-13$

VISCOSITY LOSS AFTER SHEARING AT DIFFERENT

FRONTAL VELOCITIES (650-FO0T SAND CORE)

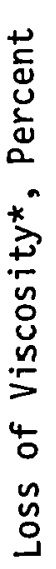

Bbl per Day Assuming 14 Net Feet of Pay and Porosity $=0.25$

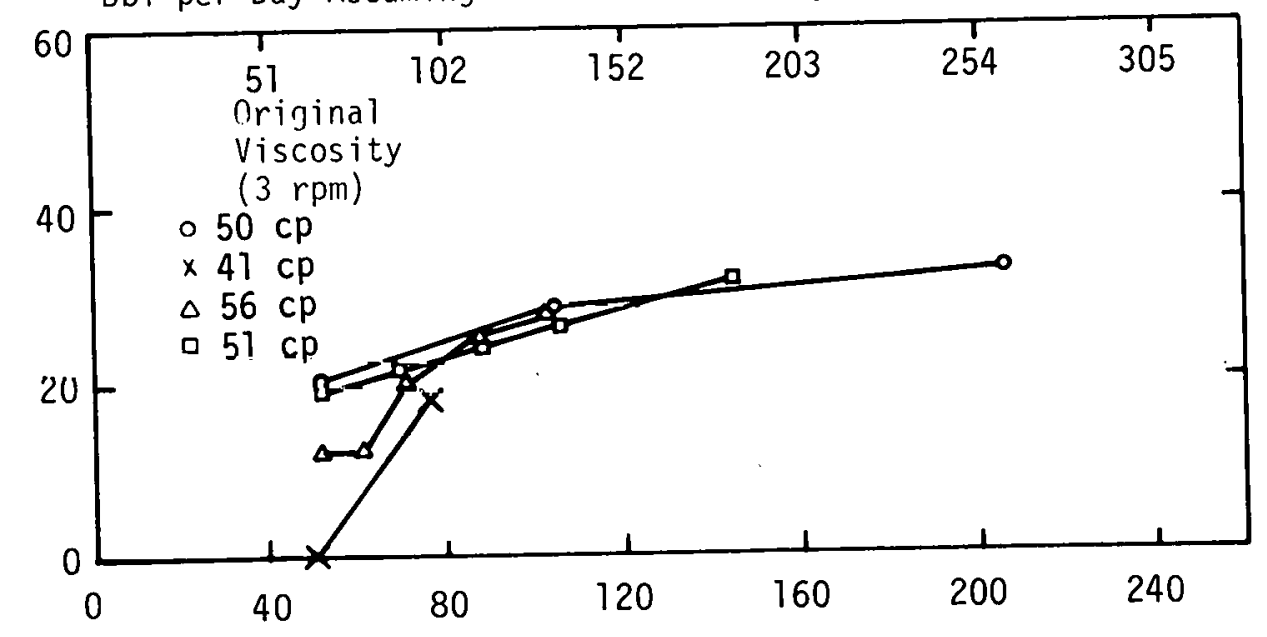

Frontal Velocity, Feet Per Day

*Evaluated at $3 \mathrm{rpm}$, Brookfield LV with UL adaptor 
FIGURE A-14

WATER SENSITIVITY FLOW TESTS - PERMEABILITY

UNAFFECTED BY SALINITY

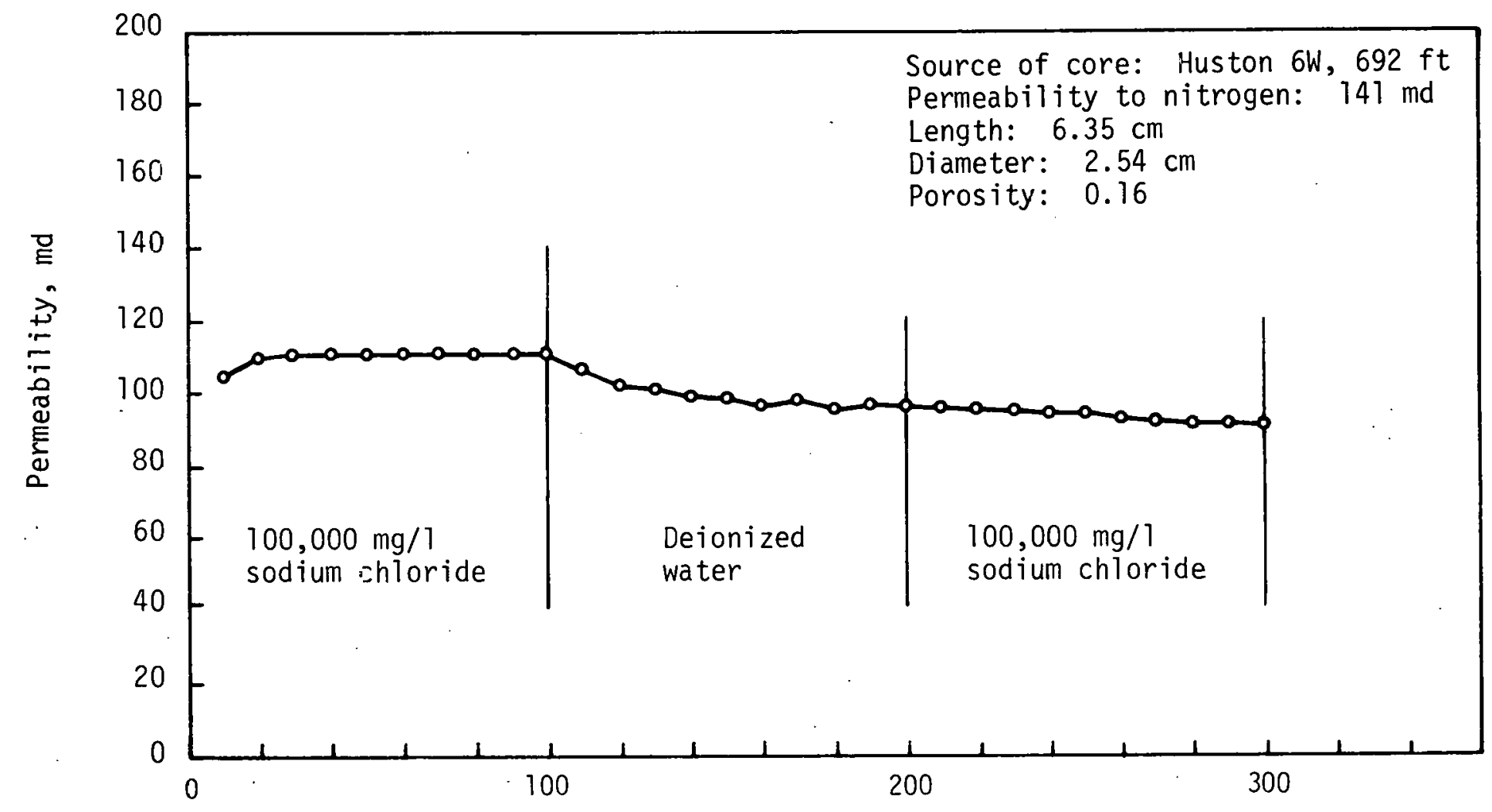

Pore Volumes Injected 
FIGURE A-15

WATER SENSITIVITY FLOW TESTS - LOWER PERMEABILITY TO FRESH WATER

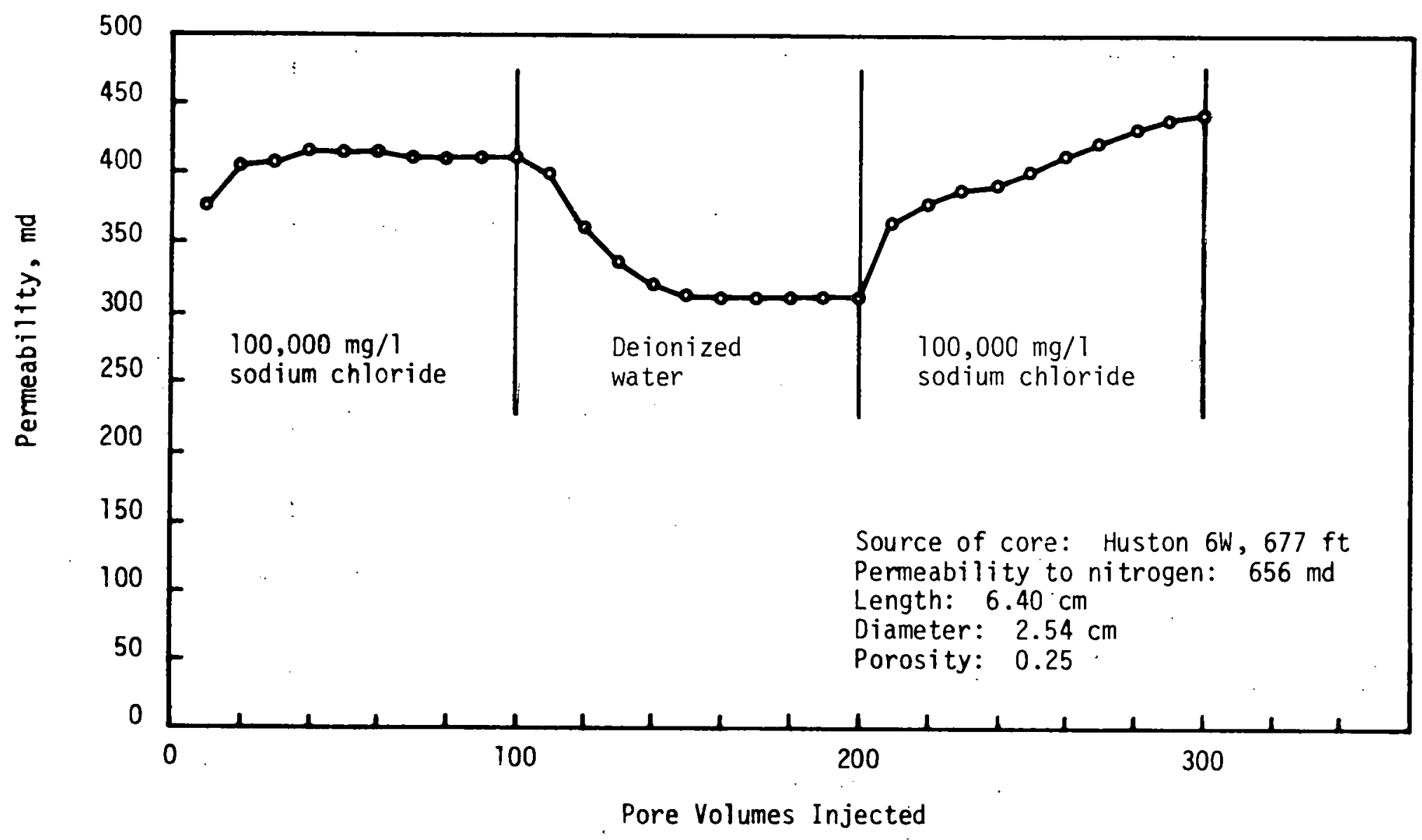


FIGURE $A-16$

WATER SENSITIVITY FLOW TESTS

CCINSTANT LOSS OF PERMEABILITY TO EITHER FRESH OR SALT WATER

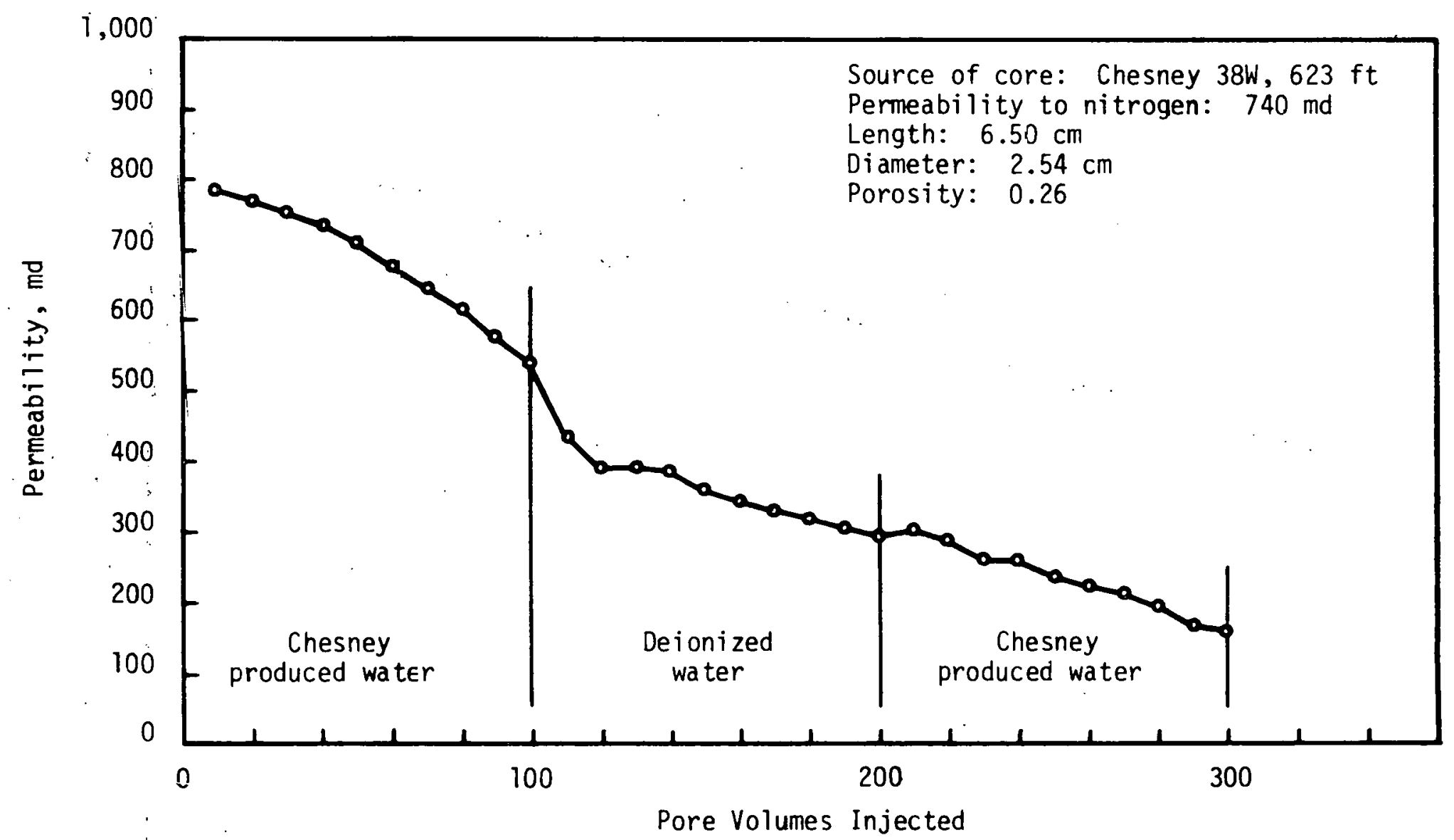


II -26

FIGURE $A-17$

VISCOSITY VS PRESSURE

FOR BOTTOM-HOLE CRUDE OIL SAMPLE

FROM CHESNEY 195

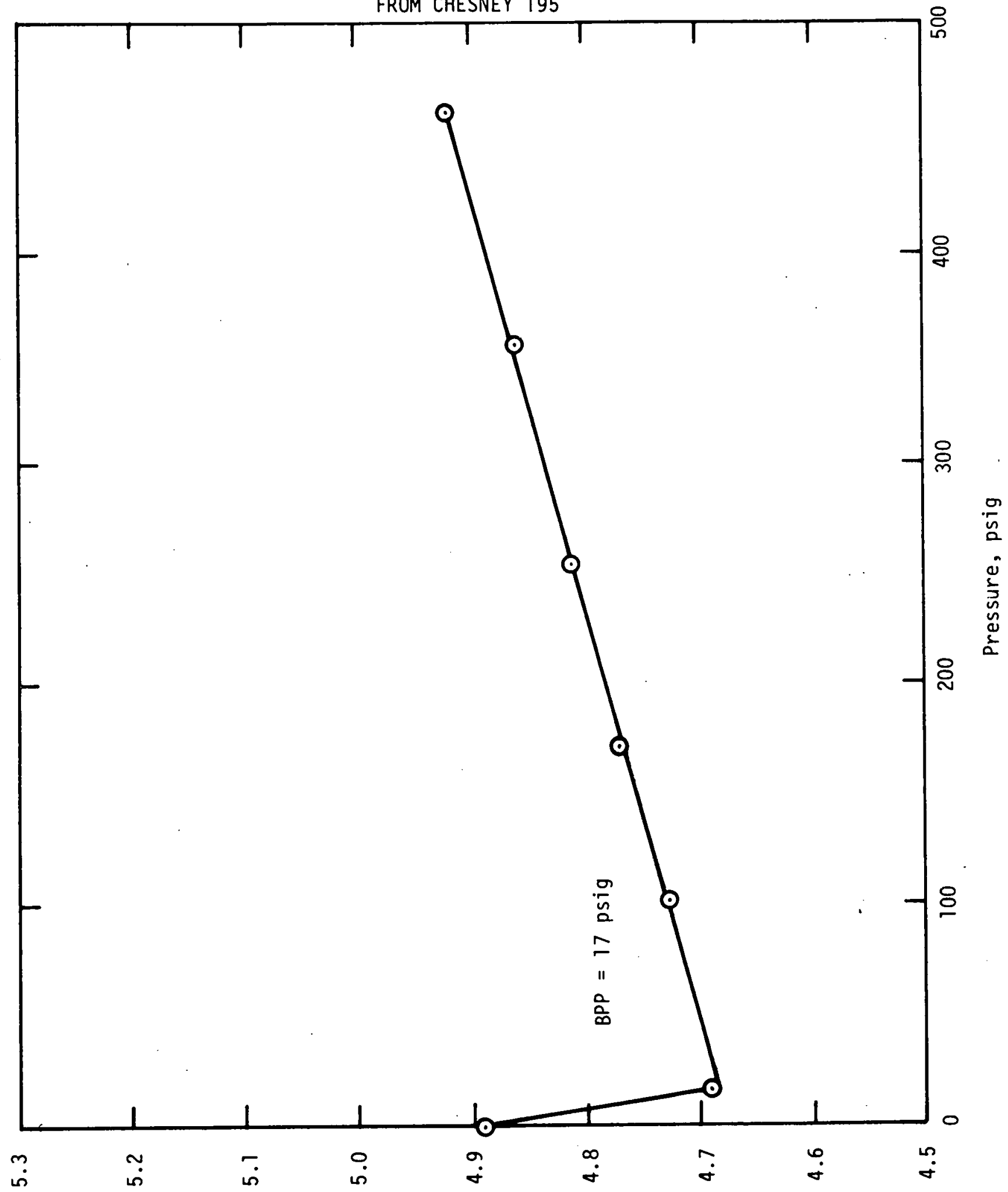

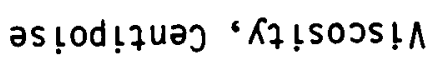


FIGURE A- 18

SALINITY CHANGE IN INITIAL

WATER PRODUCED FROM WELL IMP-124

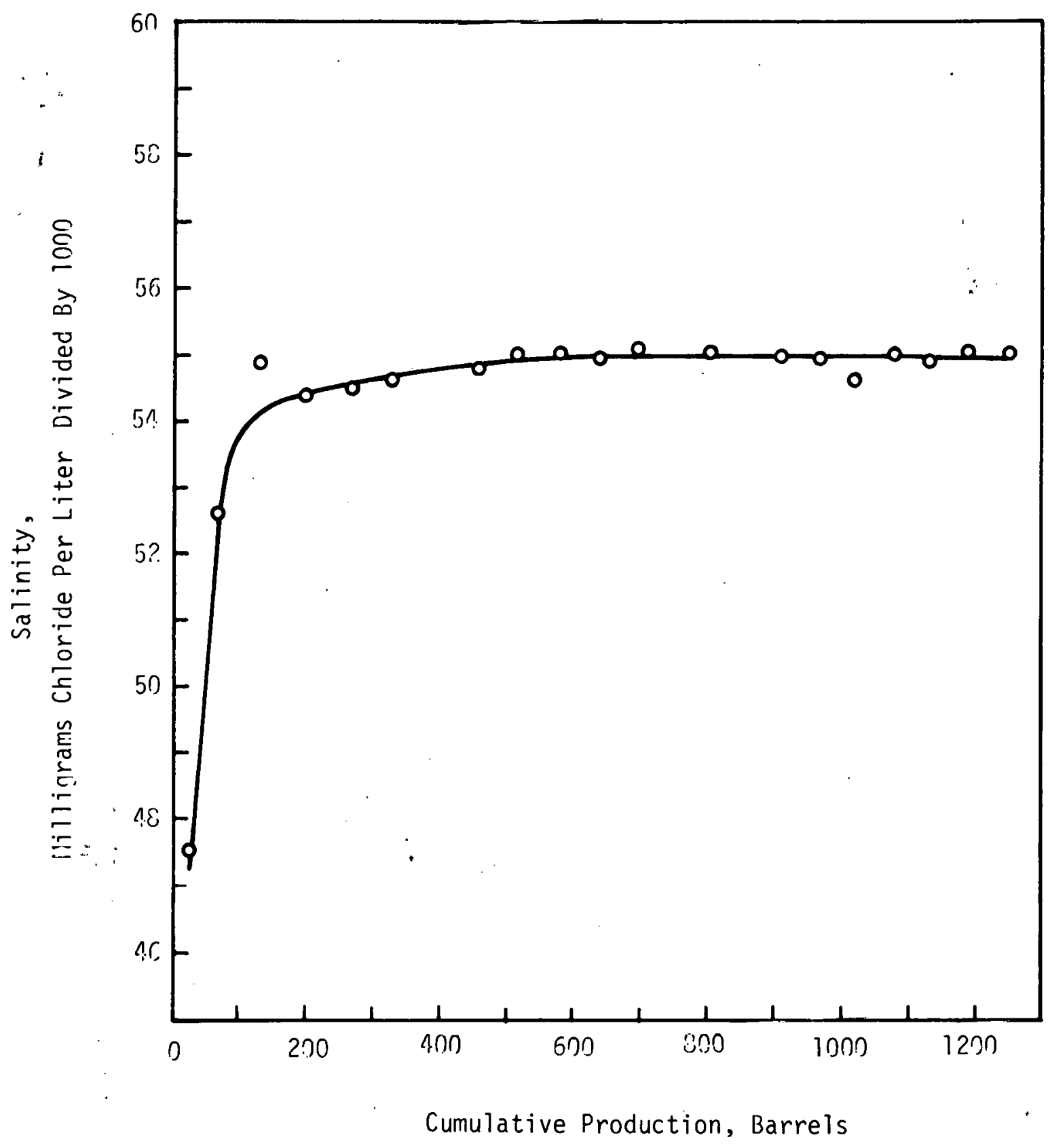




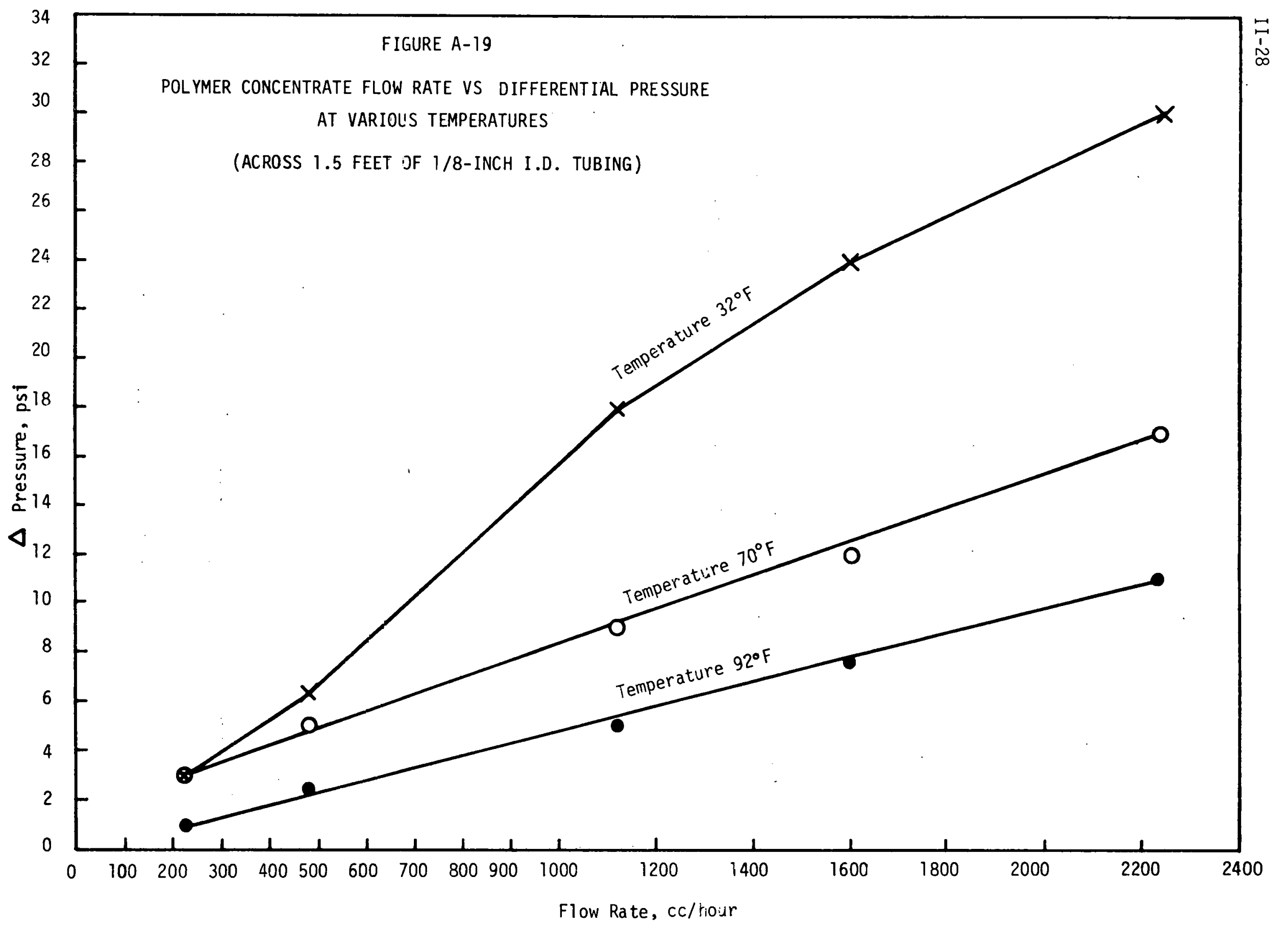




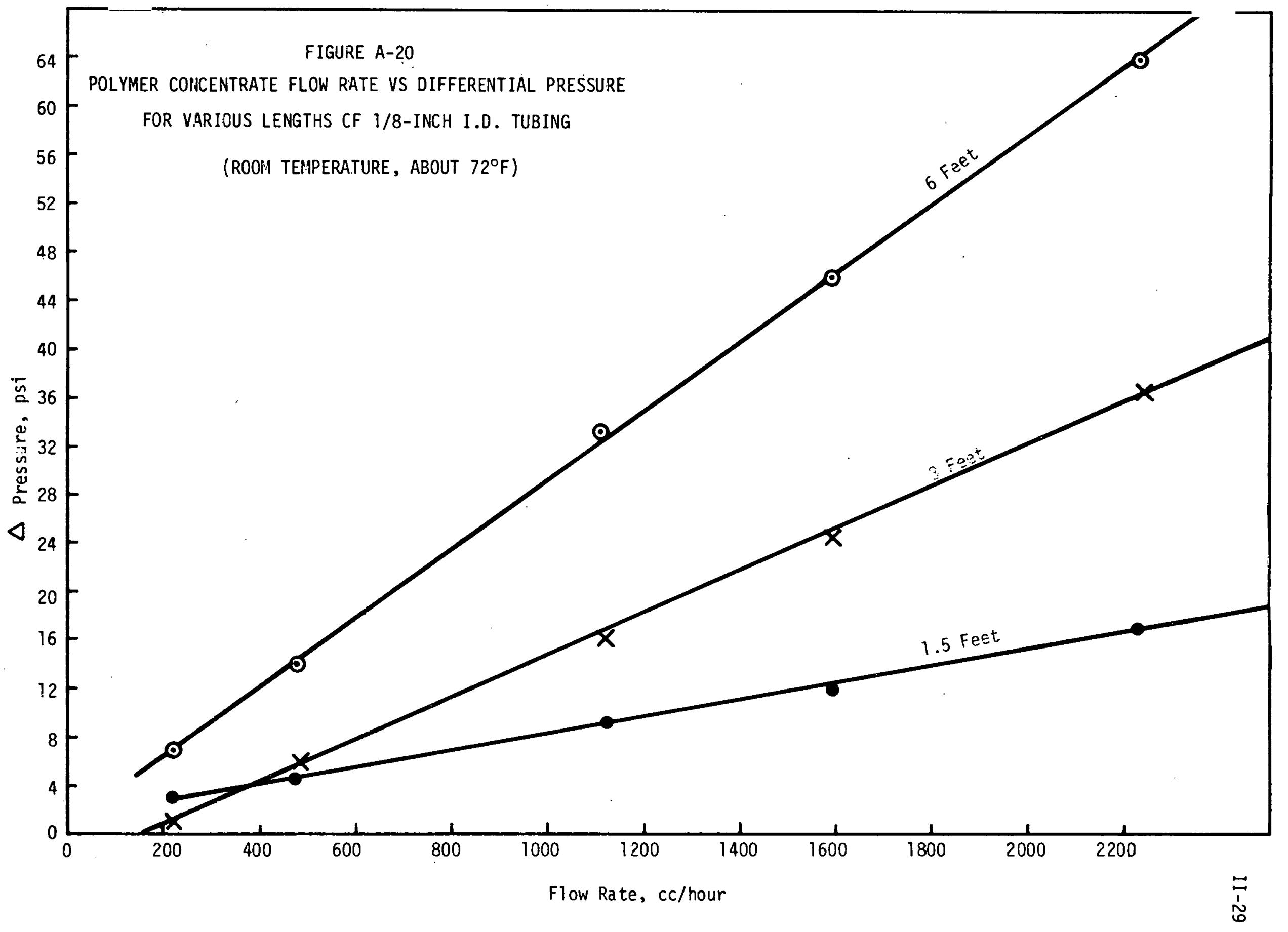


THIS PAGE

\section{WAS INTENTIONALLY \\ LEFT BLANK}


APPENDIX B

CORING AND CORE ANALYSES

Tables and Figures 
TABLE B1

ROUT INE CORE ANALYSIS RESULTS VS DEPTH

FOR WELL MP-101

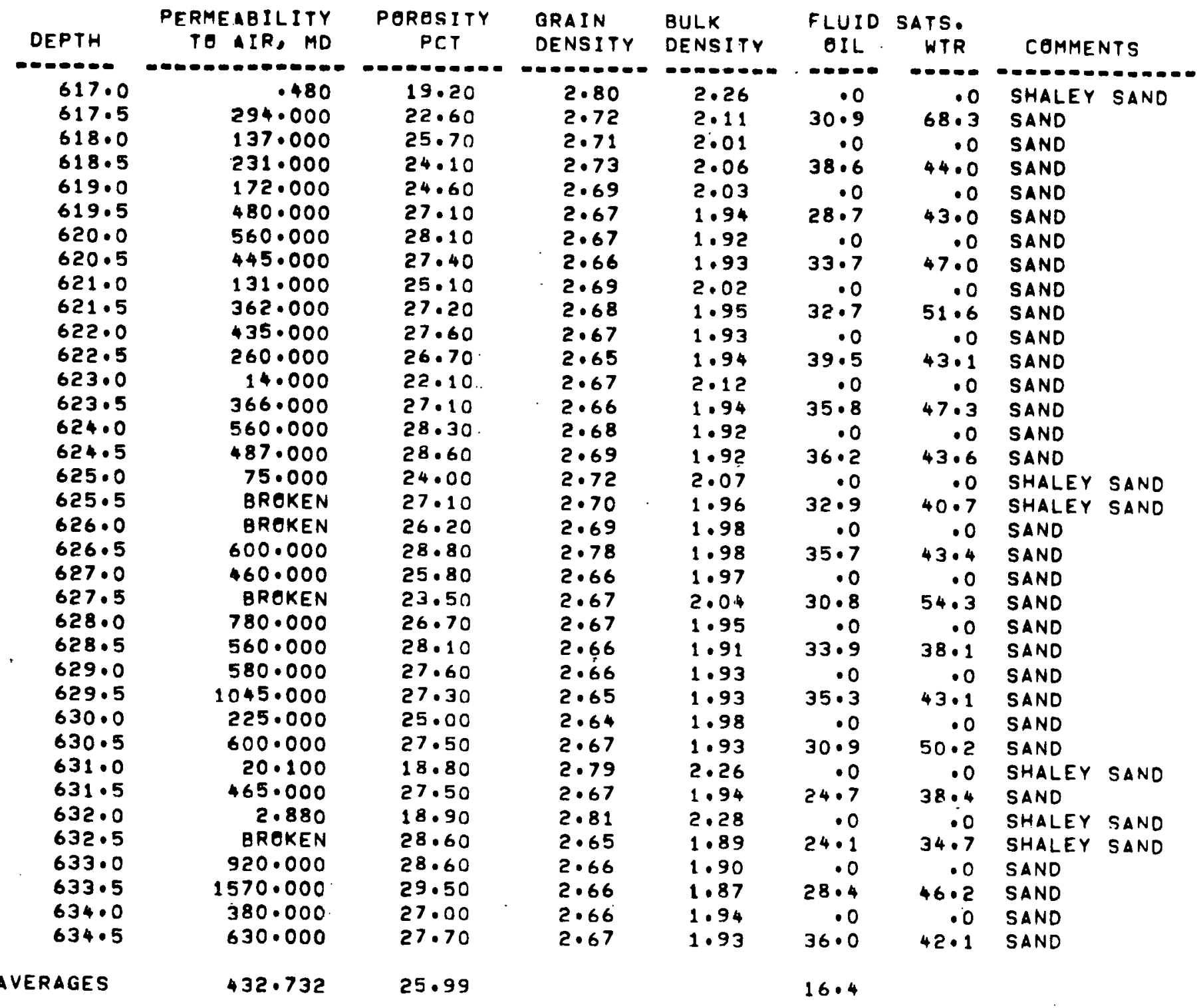


TABLE B2

ROUT INE CORE ANALYSIS RESULTS VS DEPTH

FOR WELL MP-102

\begin{tabular}{|c|c|c|c|c|c|c|c|c|}
\hline \multirow[t]{2}{*}{ DEPTH. } & $\begin{array}{c}\text { PERMEABILITY } \\
\text { TO AIR, MD }\end{array}$ & $\begin{array}{c}\text { POROSITY } \\
\text { PET }\end{array}$ & $\begin{array}{l}\text { GRAIN } \\
\text { DENSITY }\end{array}$ & $\begin{array}{l}\text { BULK } \\
\text { DENSITY }\end{array}$ & $\begin{array}{l}\text { FLUID } \\
\text { OIL }\end{array}$ & $\begin{array}{r}\text { SATS } \\
\text { WTR }\end{array}$ & \multicolumn{2}{|c|}{ COMMENTS } \\
\hline & $\cdot-\infty-0=0$ & $-\infty-0-0$ & $-\infty-0-\infty-0$ & 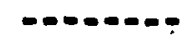 & - & $-\infty-\infty$ & & \\
\hline $\begin{array}{l}605 \cdot 5 \\
606: 0\end{array}$ & $\begin{array}{l}455.000 \\
525.000\end{array}$ & $\begin{array}{l}28.00 \\
28.00\end{array}$ & $\begin{array}{l}2.67 \\
2.68\end{array}$ & $\begin{array}{l}1.93 \\
1.93\end{array}$ & .0 & .0 & $\begin{array}{l}\text { SAND } \\
\text { SAND }\end{array}$ & \\
\hline 606.5 & 195.000 & 25.40 & 2.66 & $\begin{array}{l}1.73 \\
1.99\end{array}$ & $\begin{array}{r}3.1 \\
.0\end{array}$ & $\begin{array}{r}.30 \\
.0\end{array}$ & $\begin{array}{l}\text { SANU } \\
\text { SAND }\end{array}$ & \\
\hline 607.0 & $10: .000$ & 17.20 & $2 \cdot 71$ & 2.24 & 7.0 & $76 \cdot 3$ & SAND & \\
\hline $607 \cdot 5$ & 387.000 & 27.00 & 2.66 & 1.94 & .0 & .0 & SAND & \\
\hline 608.0 & 95.000 & $22 \cdot 10$ & 2.69 & $2 \cdot 10$ & $7 \cdot 9$ & $92 \cdot 1$ & SAND & \\
\hline $608: 5$ & 180.000 & 25.40 & 2.67 & 1.99 & .0 & .0 & SAND & \\
\hline 609.0 & 37.000 & $22 \cdot 90$ & 2.69 & 2.07 & $2 \cdot 9$ & $76 \cdot 3$ & SAND & \\
\hline $609 \cdot 5$ & 51.000 & 22.10 & 2.68 & 2.09 & .0 & .0 & SAND & \\
\hline $610 \cdot 0$ & $54 \mathrm{C} \cdot 000$ & $27 \cdot 30$ & $2 \cdot 67$ & $1 \cdot 94$ & $6 \cdot 4$ & $84 \cdot 1$ & SAND & \\
\hline $610 \cdot 5$ & 5.000 & 20.60 & $2 \cdot 72$ & $2 \cdot 16$ & $\cdot 0$ & .0 & SAND & \\
\hline 611.0 & 185.000 & 26.00 & 2.68 & $1 \cdot 98$ & $13 \cdot 1$ & 86.9 & SAND & \\
\hline $\begin{array}{l}611 \cdot 5 \\
612 \cdot 0\end{array}$ & $\begin{array}{l}660.000 \\
655.000\end{array}$ & $\begin{array}{l}27 \cdot 60 \\
28.20\end{array}$ & $\begin{array}{l}2.66 \\
2.67\end{array}$ & $\begin{array}{l}1.93 \\
1.91\end{array}$ & $\begin{array}{r}.0 \\
4.4\end{array}$ & $\begin{array}{r}.0 \\
65.8\end{array}$ & $\begin{array}{l}\text { SAND } \\
\text { SAND }\end{array}$ & \\
\hline $612 \cdot 5$ & $380: 000$ & 26.40 & 2.67 & 1.96 & .0 & .0 & SAND & \\
\hline 613.0 & 10.000 & $20 \cdot 4 c$ & 2.70 & $2 \cdot 15$ & 28.2 & 71.8 & SHALE & \\
\hline $613 \cdot 5$ & 265.000 & 26.50 & $2 \cdot 67$ & 1.96 & .0 & .0 & SAND & \\
\hline 614.0 & 26.000 & $20 \cdot 40$ & $2 \cdot 75$ & $2 \cdot 19$ & $20 \cdot 1$ & 62.9 & SHALEY & SAND \\
\hline $\begin{array}{l}614.5 \\
615.0\end{array}$ & $\begin{array}{r}1620.000 \\
185.000\end{array}$ & $\begin{array}{l}30 \cdot 10 \\
26.50\end{array}$ & $\begin{array}{l}2 \cdot 65 \\
2.68\end{array}$ & $\begin{array}{l}1.85 \\
1.97\end{array}$ & $\begin{array}{r}.0 \\
25 \cdot 4\end{array}$ & $\begin{array}{r}.0 \\
48.8\end{array}$ & $\begin{array}{l}\text { SAND } \\
\text { SHALEY }\end{array}$ & SANO \\
\hline 615.5 & 775.000 & 28.70 & 2.66 & 1.90 & .0 & .0 & SAND & \\
\hline $616 \cdot 0$ & 121.000 & $26 \cdot 30$ & $2 \cdot 66$ & 1.96 & $30 \cdot 4$ & $44 \cdot 1$ & SHALEY & SAND \\
\hline $616 \cdot 5$ & 345.000 & $25: 70$ & $2 \cdot 65$ & 1.97 & $\cdot 0$ & .0 & SAND & \\
\hline 617.0 & $2: 000$ & 17.80 & $2 \cdot 80$ & $2 \cdot 30$ & $3 \cdot 2$ & 86.5 & SHALEE & \\
\hline 617.5 & BRAKEN & $18: 60$ & $2 \cdot 72$ & $2 \cdot 21$ & .0 & .0 & SAND & \\
\hline $\begin{array}{l}618 \cdot 0 \\
618.5\end{array}$ & $\begin{array}{r}850.000 \\
\text { BREKEN }\end{array}$ & $\begin{array}{l}29: 40 \\
24.10\end{array}$ & $\begin{array}{l}2.68 \\
2.69\end{array}$ & $\begin{array}{l}1.89 \\
2.04\end{array}$ & $31 \cdot 7$ & $\begin{array}{r}50.7 \\
.0\end{array}$ & SAND & c \\
\hline 619.0 & 1450000 & 23.20 & 2.70 & 2.08 & $46 \cdot 5$ & 45.9 & SHALEY & SAND \\
\hline 619.5 & 800.000 & 27.50 & $2 \cdot 67$ & 1.94 & .0 & .0 & SANO & \\
\hline $620 \cdot 0$ & $980 \cdot 000$ & $29: 80$ & 2.69 & 1.89 & $8 \cdot 8$ & $72 \cdot 1$ & SANO & \\
\hline 620.5 & 850.000 & 29.00 & 2.66 & 1.89 & .0 & .0 & SAND & \\
\hline $621 \cdot 0$. & 885.000 & 30.00 & $2 \cdot 68$ & 1.88 & $25 \cdot 6$ & $58 \cdot 4$ & SAND & \\
\hline $621 \cdot 5$ & 810.000 & 23.10 & $2 \cdot 66$ & 1.91 & .0 & .0 & SAND. & \\
\hline $622 \cdot 0$ & 540.000 & 28.90 & 2.69 & 1.92 & $\therefore 26 \cdot 6$ & $54 \cdot 5$ & SAND & $:$ \\
\hline $\begin{array}{l}622.5 \\
623.0\end{array}$ & $\begin{array}{l}805.000 \\
380.000\end{array}$ & $\begin{array}{l}28.50 \\
23.90\end{array}$ & $\begin{array}{l}2.67 \\
2.75\end{array}$ & $\begin{array}{l}1.91 \\
2.09\end{array}$ & $\begin{array}{r}.0 \\
32.7\end{array}$ & $\begin{array}{r}.0 \\
63.2\end{array}$ & $\begin{array}{l}\text { SANO } \\
\text { SANO }\end{array}$ & \\
\hline $623 \cdot 5$ & 69.300 & 21.90 & 2.69 & 2.10 & .0 & .0 & SHALEY & SAND \\
\hline $624 \cdot 0$ & BROIREN & 24.90 & $2 \cdot 70$ & 2.03 & $27 \cdot 7$ & 53.0 & SHALEY & SANO \\
\hline $624 \cdot 5$ & $530 \cdot 1000$ & $27 \cdot 30$ & 2.67 & 1.94 & .0 & $\cdot 0$ & SAND & \\
\hline $625 \cdot C$ & $1120 \cdot 000$ & $28 \cdot 30$ & 2.69 & $1 \cdot 93$ & $28 \cdot 0$ & 57.6 & SAND & \\
\hline GES & 447.675 & 25.50 & & & $9 \cdot 5$ & & & \\
\hline
\end{tabular}


TABLE B3

ROUTINE CORE ANALYSIS RESULTS VS DEPTH

FOR WELL MP- 103

\begin{tabular}{|c|c|c|c|c|c|c|c|c|}
\hline DEPTH & $\begin{array}{r}\text { PERMEABILITY } \\
\text { TO AIR, MD }\end{array}$ & $\begin{array}{c}\text { PORESITY } \\
\text { PCT }\end{array}$ & $\begin{array}{l}\text { GRAIN } \\
\text { DENSITY }\end{array}$ & $\begin{array}{l}\text { BULK } \\
\text { DENSITY }\end{array}$ & $\begin{array}{l}\text { FLUID } \\
\text { OIL }\end{array}$ & $\begin{array}{r}\text { SATS P } \\
\text { WTR }\end{array}$ & \multicolumn{2}{|c|}{ COMMENTS } \\
\hline$-0-0-0$ & $-0-0.0-0,-0-\infty$ & $-0-0-0=-\infty$ & $---0-0-0$ & $---0-\infty-\infty$ & $-\infty-\infty$ & $-\infty-\infty$ & & 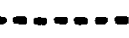 \\
\hline 608.5 & .500 & $26 \cdot 10$ & $2 \cdot 98$ & $2 \cdot 20$ & $3 \cdot 4$ & $52 \cdot 3$ & SHALE & \\
\hline 609.0 & .700 & 15.80 & $2 \cdot 79$ & $2 \cdot 35$ & .0 & .0 & SHALE & \\
\hline 609.5 & 143.000 & 25.20 & $2 \cdot 68$ & 2.01 & $4 \cdot 2$ & 77.4 & SAND & \\
\hline 610.0 & 253.000 & 26.70 & $2 \cdot 70$ & 1.98 & .0 & .0 & SAND & \\
\hline $610 \cdot 5$ & $110 \cdot 000$ & 21.80 & 2.69 & $2 \cdot 10$ & $3 \cdot 3$ & $37 \cdot 6$ & SAND & \\
\hline 611.0 & 75.500 & $24 \cdot 30$ & 2.68 & 2.03 & .0 & .0 & SHALEY & SAND \\
\hline $611 \cdot 5$ & 94.500 & 19.30 & $2 \cdot 71$ & $2 \cdot 19$ & $4 \cdot 4$ & $95 \cdot 6$ & SAND & \\
\hline $612 \cdot 0$ & 169.500 & 23.80 & 2.67 & 2.04 & .0 & .0 & SAND & \\
\hline $612 \cdot 5$ & 68.000 & 19.10 & $2 \cdot 70$ & $2 \cdot 18$ & $6 \cdot 9$ & $89 \cdot 5$ & SHALEY & SAND \\
\hline 613.0 & 155.000 & 23.40 & $2 \cdot 70$ & 2.07 & .0 & .0 & SAND & \\
\hline 613.5 & 137.000 & 25.60 & $2 \cdot 68$ & 1.99 & $4 \cdot 4$ & 73.8 & SAND & \\
\hline 614.0 & 350.000 & $26 \cdot 30$ & $2 \cdot 66$ & 1.96 & .0 & .0 & SAND & \\
\hline 614.5 & 64.000 & $22 \cdot 10$ & 2.66 & 2.08 & $7 \cdot 8$ & $75 \cdot 7$ & SHALEY & SANO \\
\hline 615.0 & $350 \cdot 000$ & 27.10 & 2.66 & 1.93 & $\cdot 0$ & .0 & SAND & \\
\hline $615 \cdot 5$ & 167.000 & 25.00 & $2 \cdot 66$ & 2.00 & $7 \cdot 0$ & $73 \cdot 2$ & SAND & \\
\hline 616.0 & BROKEN & 24.10 & 2.66 & 2.02 & .0 & .0 & SAND & \\
\hline 616.5 & $5.85 \cdot 000$ & 28.00 & $2 \cdot 65$ & 1.91 & $5 \cdot 6$ & $87 \cdot 3$ & SAND & \\
\hline 617.0 & .25 .000 & $27 \cdot 30$ & $2 \cdot 65$ & 1.92 & .0 & .0 & SAND & \\
\hline 617.5 & 315.000 & 27.20 & 2.66 & 1.94 & $7 \cdot 3$ & $80 \cdot 2$ & SAND & \\
\hline 618.0 & 220.000 & $26 \cdot 50$ & $2 \cdot 68$ & 1.97 & .0 & .0 & SAND & \\
\hline $618 \cdot 5$ & 310.000 & 27.20 & $2 \cdot 68$ & $i .95$ & $6 \cdot 8$ & $79 \cdot 6$ & SAND & \\
\hline 619.0 & BREKEN & 27.60 & 2.68 & 1.94 & .0 & .0 & SAND & \\
\hline 619.5 & 485.000 & 28.00 & 2.68 & 1.93 & $7 \cdot 6$ & 69.6 & SAND & \\
\hline $620 \cdot 0$ & $20 \cdot 200$ & 22.20 & $2 \cdot 77$ & $2 \cdot 15$ & .0 & .0 & SHALEY & SANO \\
\hline 620.5 & 1.14 .000 & 24.20 & $2 \cdot 73$ & 2.07 & $38 \cdot 1$ & $42 \cdot 5$ & SAND & \\
\hline $621 \cdot 0$ & 335.000 & $26 \cdot 20$ & $2 \cdot 68$ & 1.98 & .0 & .0 & SAND & \\
\hline $621 \cdot 5$ & 265.000 & 27.60 & $2 \cdot 67$ & 1.93 & $34 \cdot 6$ & $45 \cdot 5$ & SAND & \\
\hline $622 \cdot 0$ & 1450.000 & $30 \cdot 40$ & $2 \cdot 68$ & 1.87 & .0 & .0 & SAND & \\
\hline $622 \cdot 5$ & $2 \cdot 780$ & $20 \cdot 40$ & $2 \cdot 81$ & 2.24 & $39 \cdot 3$ & 60.7 & SHALEY & SAND \\
\hline 623.0 & 29.300 & $22 \cdot 40$ & 2.76 & $2 \cdot 15$ & .0 & .0 & SHALEY & SAND \\
\hline 623.5 & 18.000 & $21 \cdot 20$ & $2 \cdot 78$ & 2.19 & $16 \cdot 8$ & 63.4 & SHALEY & SAND \\
\hline $.624 \cdot 0$ & 1700.000 & 31.20 & $2 \cdot 68$ & 1.84. & .0 & .0 & SAND & \\
\hline $624 \cdot 5$ & 28.200 & $21 \cdot 90$ & $2 \cdot 76$ & $2 \cdot 15$ & $24 \cdot 6$ & $56 \cdot 5$ & SAND & \\
\hline $\begin{array}{l}625 \cdot 0 \\
625 \cdot 5\end{array}$ & $\begin{array}{r}330.000 \\
\text { BREKEN }\end{array}$ & $\begin{array}{l}27.30 \\
25.50\end{array}$ & $\begin{array}{l}2.70 \\
2.72\end{array}$ & $\begin{array}{l}1.96 \\
2.02\end{array}$ & $\begin{array}{r}.0 \\
46.6\end{array}$ & $\begin{array}{r}.0 \\
49.1\end{array}$ & $\begin{array}{l}\text { SAND } \\
\text { SAND }\end{array}$ & \\
\hline 626.0 & 510.000 & 27.40 & $2 \cdot 68$ & $1 \cdot 94$ & .0 & .0 & SAND & \\
\hline $626 \cdot 5$ & 550.000 & 27.80 & $2 \cdot 71$ & 1.95 & $36 \cdot 8$ & $44 \cdot 2$ & SAND & \\
\hline $627 \cdot 0$ & 650.000 & 28.80 & 2.69 & $1 \cdot 92$ & .0 & .0 & SAND & \\
\hline $627 \cdot 5$ & 770.000 & 27.80 & $2 \cdot 70$ & 1.95 & $24 \cdot 4$ & $47 \cdot 0$ & SAND & \\
\hline 628.0 & 267.000 & 24.50 & $2 \cdot 70$ & 2.04 & .0 & .0 & SANO & \\
\hline AGES & 311.275 & 25.11 & & & $8 \cdot 2$ & & & \\
\hline
\end{tabular}


TABLE B4

ROUTINE CORE ANALYSIS RESULTS VS DEPTH

, FOR WELL MP-104

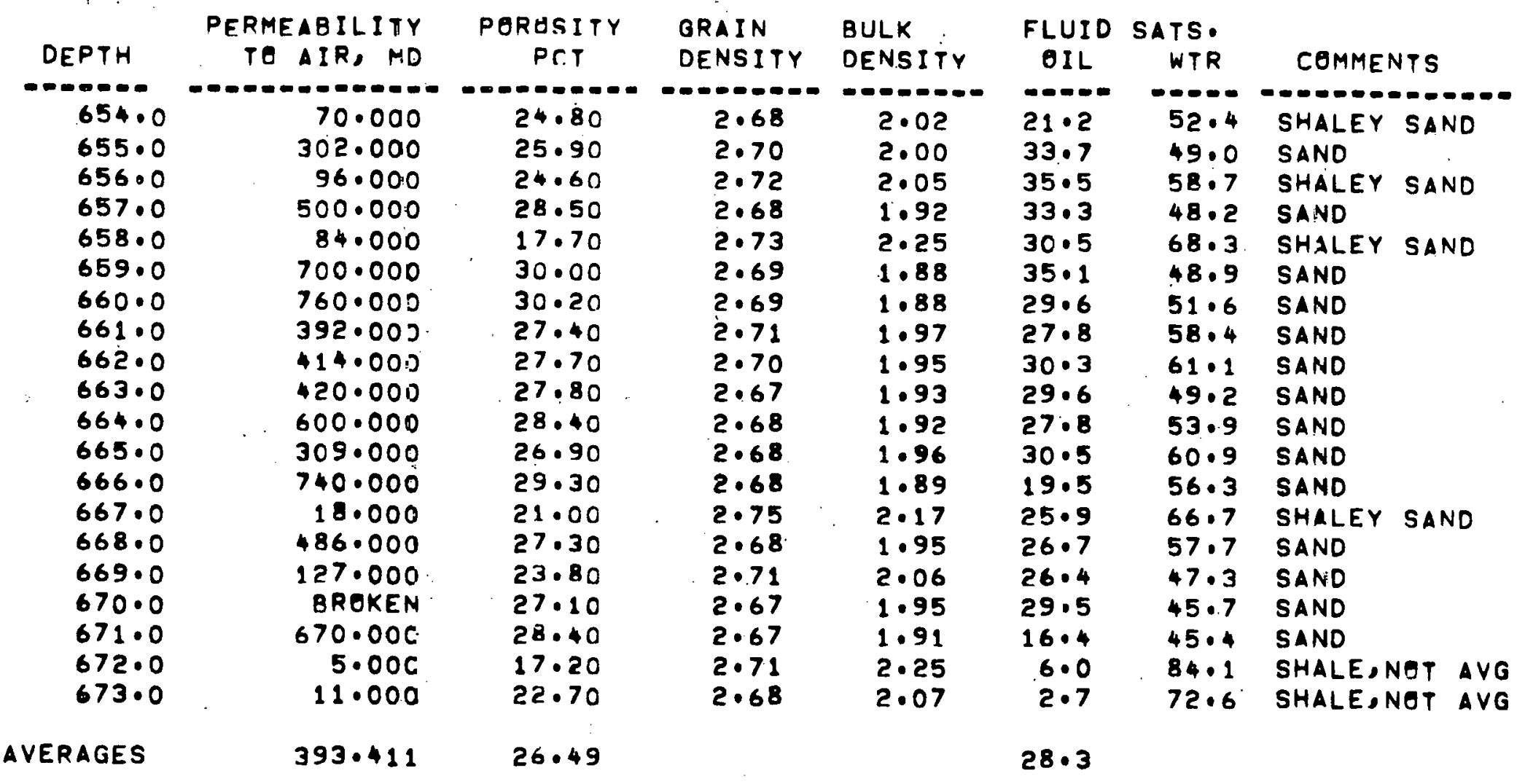


TABLE B5

ROUT INE CORE ANALYSIS RESULTS VS DEPTH

FOR WELL MP-105

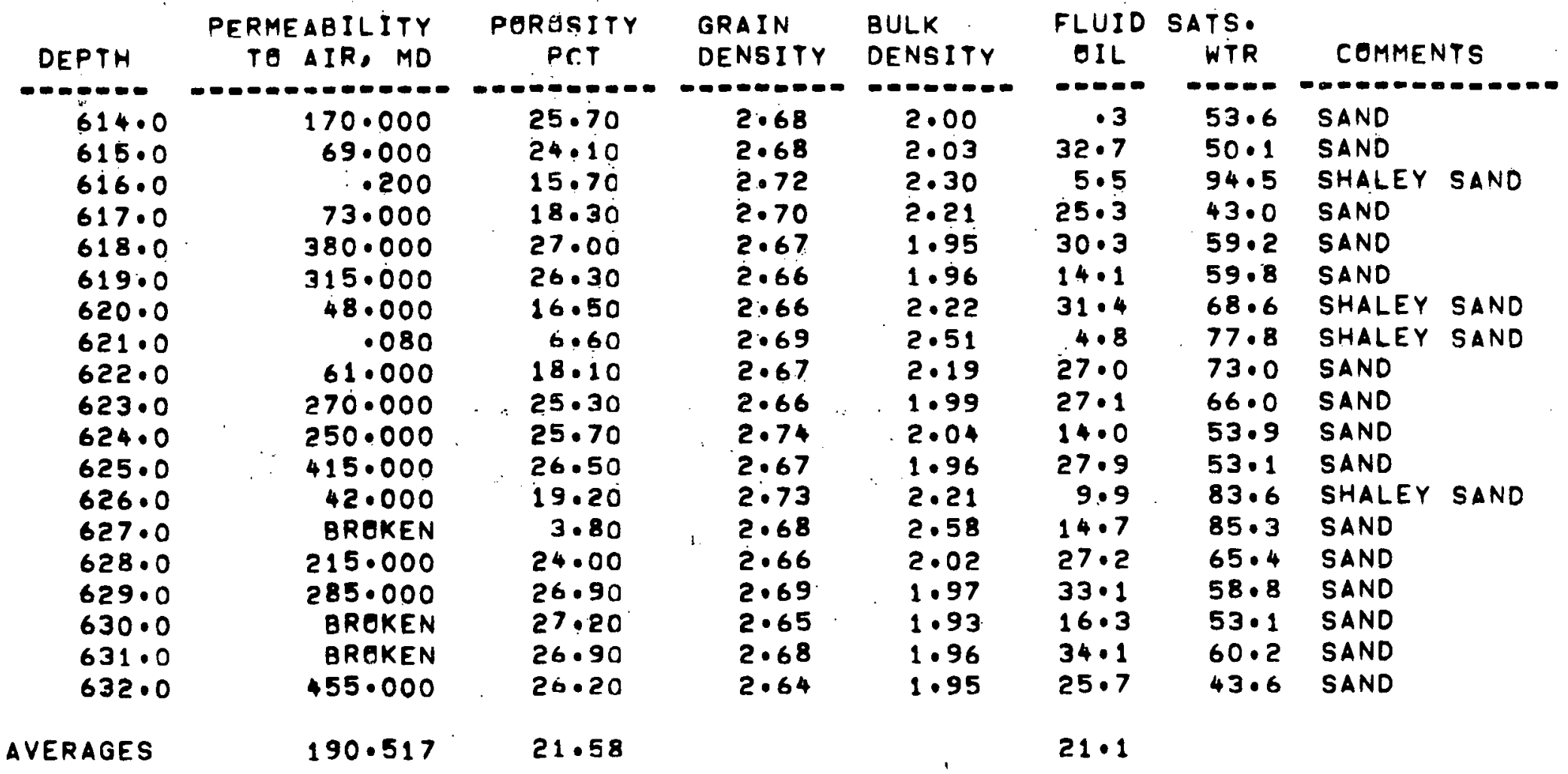


TABLE B6

ROUTINE CORE ANALYSIS RESULTS VS DEPTH

FOR WELL MP-106

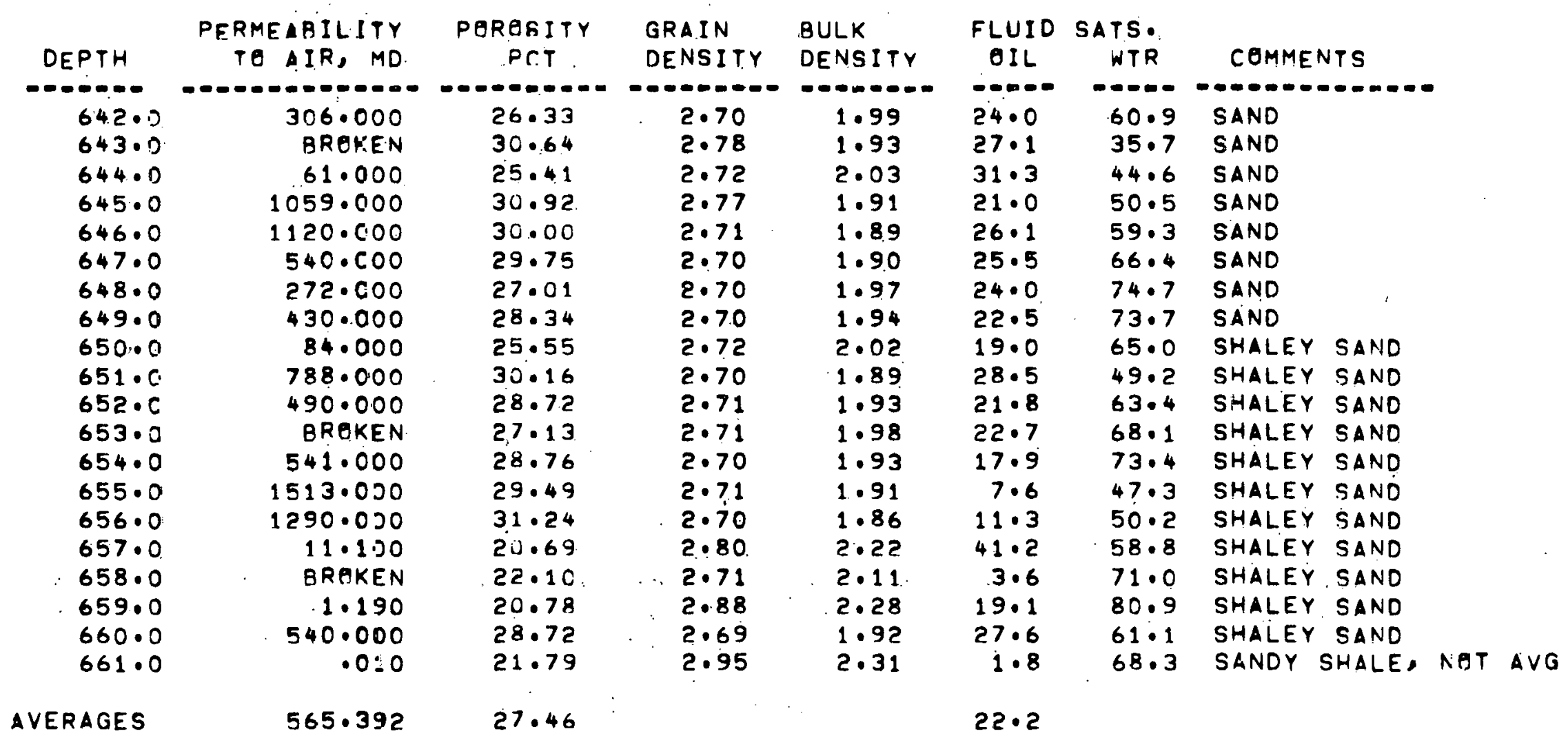


TABLE B7

ROUT INE CORE ANALYSIS RESULTS VS DEPTH

FOR WELL MP-110

\begin{tabular}{|c|c|c|c|c|c|c|c|c|c|}
\hline DEPTH & $\begin{array}{c}\text { PERMEABILITY } \\
\text { TE AIR, MD }\end{array}$ & $\begin{array}{c}\text { PORESITY } \\
\text { PCT }\end{array}$ & $\begin{array}{l}\text { GRAIN } \\
\text { DENSITY }\end{array}$ & $\begin{array}{l}\text { BULK } \\
\text { DENSITY }\end{array}$ & $\begin{array}{l}\text { FLUID } \\
\text { OIL }\end{array}$ & $\begin{array}{l}\text { SATS. } \\
\text { WTR }\end{array}$ & COMM & IENTS & \\
\hline$-\infty-\infty$ & 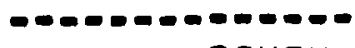 & $--\infty-0-0-0 .-0$ & $-\infty-0-\infty-\infty$ & $-\infty-\infty-\infty-\infty$ & ---0 & $-\infty-\infty$ & & & \\
\hline $\begin{array}{l}602 \cdot 0 \\
603 \cdot 0\end{array}$ & $\begin{array}{r}\text { BREKEN } \\
.260\end{array}$ & $\begin{array}{l}19 \cdot 96 \\
22.21\end{array}$ & $\begin{array}{l}3.05 \\
3.05\end{array}$ & $\begin{array}{l}2.44 \\
2.37\end{array}$ & $\begin{array}{l}2 \cdot 9 \\
2 \cdot 4\end{array}$ & $\begin{array}{l}55 \cdot 2 \\
61 \cdot 9\end{array}$ & $\begin{array}{l}\text { SAND } \\
\text { SAND }\end{array}$ & $\begin{array}{l}\text { NET } \\
\text { NET }\end{array}$ & $\begin{array}{l}A \vee G \\
\triangle \vee G\end{array}$ \\
\hline $604 \cdot 0$ & .010 & 11.69 & $2 \cdot 87$ & 2.53 & $4 \cdot 6$ & $46 \cdot 3$ & SAND & NOT & $A V G$ \\
\hline $\begin{array}{l}605.0 \\
606.0\end{array}$ & $\begin{array}{r}.560 \\
9.500\end{array}$ & $\begin{array}{l}18.10 \\
20.24\end{array}$ & $\begin{array}{l}2.90 \\
2.86\end{array}$ & $\begin{array}{l}2 \cdot 37 \\
2.28\end{array}$ & $\begin{array}{l}5 \cdot 6 \\
9.6\end{array}$ & $\begin{array}{l}89.5 \\
89.8\end{array}$ & $\begin{array}{l}\text { SAND } \\
\text { SAND }\end{array}$ & $\begin{array}{l}\text { NOT } \\
\text { NOT }\end{array}$ & $\begin{array}{l}\triangle V G \\
\triangle V G\end{array}$ \\
\hline $607 \cdot 0$ & 54.000 & 23.67 & 2.69 & 2.05 & $8 \cdot 6$ & 77.9 & SAND & NOT & $\Delta V G$ \\
\hline $608 \cdot 0$ & 450.000 & 27.93 & 2.69 & 1.94 & $5 \cdot 8$ & 87.6 & SAND & NOT & $A \vee G$ \\
\hline $609 \cdot 0$ & 283.000 & 27.25 & $2 \cdot 69$ & 1.95 & $14 \cdot .7$ & $55 \cdot 5$ & SAND & & \\
\hline $\begin{array}{l}610.0 \\
611.0\end{array}$ & $\begin{array}{l}407.000 \\
650.000\end{array}$ & $\begin{array}{l}25.93 \\
28.02\end{array}$ & $\begin{array}{l}2.69 \\
2.68\end{array}$ & $\begin{array}{l}1.99 \\
1.93\end{array}$ & $\begin{array}{l}38 \cdot 3 \\
28 \cdot 9\end{array}$ & $\begin{array}{l}50 \cdot 2 \\
54 \cdot 8\end{array}$ & $\begin{array}{l}\text { SAND } \\
\text { SAND }\end{array}$ & & \\
\hline $612 \cdot 0$ & $730 \cdot 000$ & 28.98 & $2 \cdot 70$ & 1.91 & $27 \cdot 2$ & $52 \cdot 5$ & SAND & & \\
\hline $\begin{array}{l}613.0 \\
61.4 .0\end{array}$ & $\begin{array}{l}430 \cdot 000 \\
900.000\end{array}$ & $\begin{array}{l}27.91 \\
30.03\end{array}$ & $\begin{array}{l}2.68 \\
2.69\end{array}$ & $\begin{array}{l}1.93 \\
1.88\end{array}$ & $\begin{array}{l}28 \cdot 1 \\
24 \cdot 9\end{array}$ & $\begin{array}{l}58 \cdot 4 \\
47 \cdot 3\end{array}$ & $\begin{array}{l}\text { SAND } \\
\text { SAND }\end{array}$ & & \\
\hline $615 \cdot 0$ & $500 \cdot 000$ & 28.97 & $2 \cdot 70$ & 1.92 & $24 \cdot 2$ & $41 \cdot 6$ & SAND & & \\
\hline 616.0 & 440.000 & 27.61 & $2 \cdot 68$ & $1 \cdot 94$ & $30 \cdot 0$ & $54 \cdot 1$ & SAND & & \\
\hline $617 \cdot 0$ & 420.000 & 27.57 & $2 \cdot 66$ & $1 \cdot .93$ & $22 \cdot 2$ & $43 \cdot 9$ & SAND & & \\
\hline $618 \cdot 0$ & BROKEN & 24.50 & $2 \cdot 70$ & $2 \cdot 04$ & $36 \cdot 2$ & 63.8 & SAND & & \\
\hline 619.0 & $381 \cdot 000$ & 26.76 & $2 \cdot 68$ & $1 \cdot 96$ & $29 \cdot 7$ & $51 \cdot 9$ & SAND & & \\
\hline $620 \cdot 0$ & BREKEN & 26.20 & $2 \cdot 69$ & $1 \cdot 98$ & $25 \cdot 8$ & $48 \cdot 6$ & SAND & & \\
\hline $621 \cdot 0$ & 324.000 & $27 \cdot 35$ & $2 \cdot 68$ & 1.95 & $31 \cdot 0$ & 60.9 & SAND & & \\
\hline $622 \cdot 0$ & $565 \cdot 000$ & 28.72 & 2.69 & 1.92 & $5 \cdot 7$ & $42 \cdot 7$ & SAND & & \\
\hline $62 \cdot 3 \cdot 0$ & 714.000 & $29 \cdot 17$ & $2 \cdot 69$ & $1 \cdot 91$ & $29 \cdot 7$ & $48 \cdot 5$ & SAND & & \\
\hline $624 \cdot 0$ & $1212 \cdot 000$ & 29.64 & 2.69 & 1.89 & $22 \cdot 5$ & $59 \cdot 8$ & SAND & & \\
\hline $625 \cdot 0$ & 315.000 & $25 \cdot 26$ & $2 \cdot 73$ & 2.04 & $6 \cdot 6$ & $46 \cdot 5$ & SAND & & \\
\hline $626 \cdot 0$ & 591.000 & $28 \cdot 70$ & $2 \cdot 69$ & 1.92 & $33 \cdot 4$ & $53 \cdot 0$ & SAND & & \\
\hline $627 \cdot 0$ & .000 & 19.43 & $2 \cdot 88$ & $2 \cdot 32$ & $16 \cdot 5$ & $54 \cdot 3$ & SAND & & \\
\hline IGES & $521 \cdot 293$ & 27.26 & & & $25 \cdot 0$ & & & & \\
\hline
\end{tabular}


TABLE B8

ROUT INE CORE ANALYSIS RESULTS VS DEPTH

FOR WELL MP-116

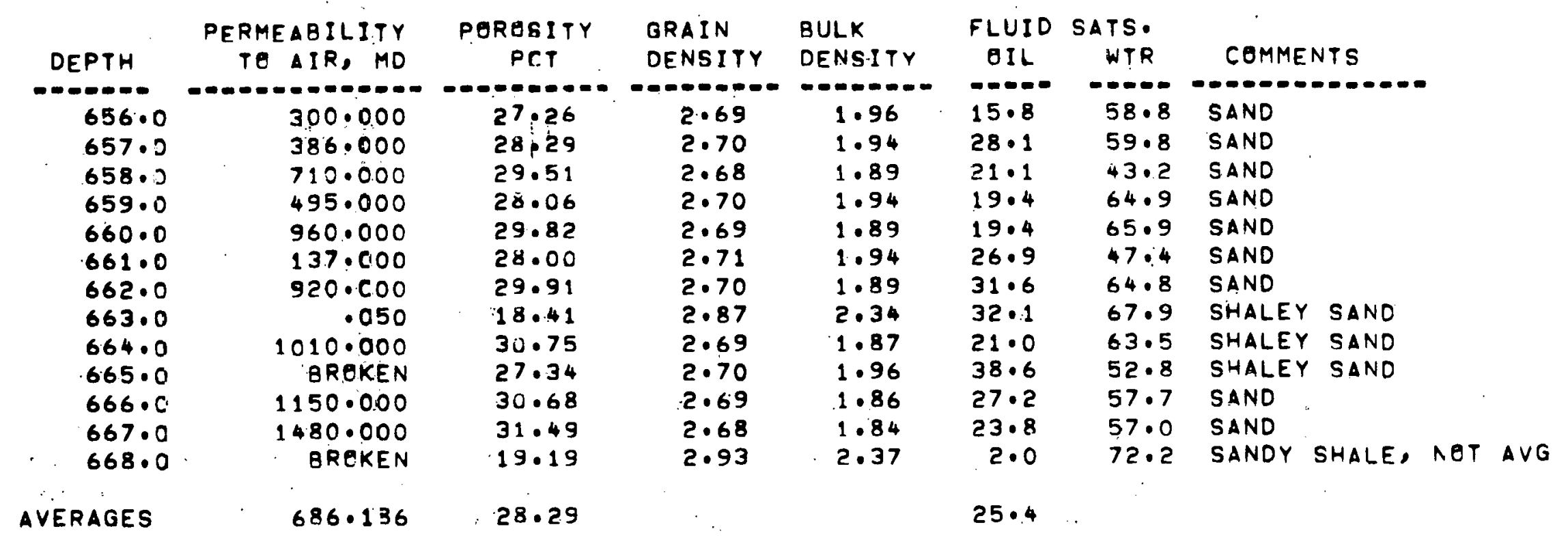


TABLE B9

RJUTINE CORE ANALYSIS RESULTS VS DEPTH

FOR WELL MP-120

\begin{tabular}{|c|c|c|c|c|c|c|c|c|c|}
\hline DEPTH & $\begin{array}{c}\text { PERME ABILITY } \\
\text { TA AIR, MD }\end{array}$ & $\begin{array}{c}\text { DERESITY } \\
\text { PC.T }\end{array}$ & $\begin{array}{l}\text { GRAIN } \\
\text { DENSITY }\end{array}$ & $\begin{array}{l}\text { BULK } \\
\text { DENSITY }\end{array}$ & $\begin{array}{c}\text { FLUID } \\
\text { OIL }\end{array}$ & $\begin{array}{r}\text { SATS } \\
\text { WTR }\end{array}$ & \multicolumn{3}{|c|}{ COMMENTS } \\
\hline$--\infty-\infty-0$ & $-\infty-0-0-0-0-0-\infty$ & $--0-0-0-0.0$ & $-0-0-0-0-0$ & $-0-0---0$ & $-0-0$ & $--\infty-0$ & $--0-0$ & $-\infty-\infty$ & $-\infty-\infty$ \\
\hline $\begin{array}{l}613 \cdot 0 \\
614 \cdot 0\end{array}$ & $\begin{array}{l}\text { BREKEN } \\
\text { BREKEN }\end{array}$ & $\begin{array}{l}19.60 \\
22.91\end{array}$ & $\begin{array}{l}2.86 \\
3.01\end{array}$ & $\begin{array}{l}2 \cdot 30 \\
2 \cdot 32\end{array}$ & $\begin{array}{l}6 \cdot 3 \\
3 \cdot 2\end{array}$ & $\begin{array}{l}67 \cdot 5 \\
62 \cdot 5\end{array}$ & $\begin{array}{l}\text { SAND } \\
\text { SAND }\end{array}$ & $\begin{array}{l}\text { NET } \\
\text { NET }\end{array}$ & $\begin{array}{l}\triangle \vee G \\
\triangle \vee G\end{array}$ \\
\hline $615 \cdot 0$ & BRGKEN & 20.84 & $2 \cdot 98$ & $2 \cdot 36$ & $2 \cdot 0$ & $65 \cdot 5$ & SAND & NOT & $\Delta V G$ \\
\hline $616 \cdot 0$ & .010 & $12 \cdot 28$ & $2 \cdot 85$ & $2 \cdot 50$ & $7 \cdot 4$ & $39 \cdot 4$ & SANO & -NET & $A \vee G$ \\
\hline $617 \cdot 0$ & .010 & 13.99 & $2 \cdot 82$ & $2 \cdot 43$ & $4 \cdot 3$ & $46 \cdot 1$ & SAND & NOT & $A \vee G$ \\
\hline $\begin{array}{l}618 \cdot 0 \\
619 \cdot 0\end{array}$ & $\begin{array}{l}10 \cdot 100 \\
13 \cdot 300\end{array}$ & $\begin{array}{r}21.27 \\
8.47\end{array}$ & $\begin{array}{l}2 \cdot 72 \\
2 \cdot 71\end{array}$ & $\begin{array}{l}2.14 \\
2.05\end{array}$ & $\begin{array}{l}16 \cdot 1 \\
50 \cdot 8\end{array}$ & $\begin{array}{l}55 \cdot 3 \\
49 \cdot 2\end{array}$ & $\begin{array}{l}\text { SAND } \\
\text { SAND }\end{array}$ & & \\
\hline $620 \cdot 0$ & 365.000 & $28 \cdot 44$ & $2 \cdot 69$ & 1.92 & $37 \cdot 8$ & $42 \cdot 7$ & SAND & & \\
\hline $621 \cdot 0$ & .000 & 3.19 & $2 \cdot 77$ & $2 \cdot 68$ & $56 \cdot 9$ & $43 \cdot 1$ & SAND & & \\
\hline $622 \cdot 0$ & $37 \cdot 700$ & $22 \cdot 67$ & $2 \cdot 67$ & 2.06 & $32 \cdot 5$ & $40 \cdot 4$ & SANND & & \\
\hline $623 \cdot 0$ & $1150 \cdot 000$ & $30 \cdot 28$ & $2 \cdot 66$ & $1 \cdot 85$ & $35 \cdot 4$ & $37 \cdot 7$ & SAND & & \\
\hline $624 \cdot 0$ & 210.000 & $28 \cdot 53$ & $2 \cdot 68$ & $1 \cdot 91$ & $30 \cdot 9$ & $46 \cdot 6$ & SAND & & \\
\hline $625 \cdot 0$ & 460.000 & 27.93 & $2 \cdot 66$ & $1 \cdot 92$ & $27 \cdot 8$ & $53 \cdot 7$ & SAND & & \\
\hline $\begin{array}{l}626.0 \\
627.0\end{array}$ & $\begin{array}{l}291.000 \\
630.000\end{array}$ & $\begin{array}{l}26 \cdot 26 \\
28 \cdot 78\end{array}$ & $\begin{array}{l}2 \cdot 66 \\
2 \cdot 6.5\end{array}$ & $\begin{array}{l}1.96 \\
1.89\end{array}$ & $\begin{array}{l}30 \cdot 1 \\
34 \cdot 9\end{array}$ & $\begin{array}{l}51 \cdot 2 \\
45 \cdot 0\end{array}$ & $\begin{array}{l}\text { SAND } \\
\text { SAND }\end{array}$ & & \\
\hline $\begin{array}{l}628.0 \\
629.0\end{array}$ & $\begin{array}{l}610.000 \\
740.000\end{array}$ & $\begin{array}{l}29.29 \\
28.87\end{array}$ & $\begin{array}{l}2 \cdot 68 \\
2 \cdot 66\end{array}$ & $\begin{array}{l}1.90 \\
1.89\end{array}$ & $\begin{array}{l}25 \cdot 0 \\
28 \cdot 9\end{array}$ & $\begin{array}{l}53 \cdot 1 \\
56 \cdot 6\end{array}$ & $\begin{array}{l}\text { SAND } \\
\text { SAND }\end{array}$ & & \\
\hline $\begin{array}{l}630 \cdot 0 \\
631 \cdot 0\end{array}$ & $\begin{array}{l}54.000 \\
\text { BREKEN }\end{array}$ & $\begin{array}{l}25.88 \\
19.50\end{array}$ & $\begin{array}{l}2.66 \\
2.81\end{array}$ & $\begin{array}{l}1 \cdot 97 \\
2 \cdot 26\end{array}$ & $\begin{array}{l}29 \cdot 7 \\
31 \cdot 9\end{array}$ & $\begin{array}{l}59 \cdot 0 \\
66.9\end{array}$ & $\begin{array}{l}\text { SAND } \\
\text { SAND }\end{array}$ & & \\
\hline $\begin{array}{l}632 \cdot 0 \\
633 \cdot 0\end{array}$ & $\begin{array}{r}\text { BREKEN } \\
1.600\end{array}$ & $\begin{array}{l}29.00 \\
19.67\end{array}$ & $\begin{array}{l}2.68 \\
2.82\end{array}$ & $\begin{array}{l}1 \cdot 90 \\
2 \cdot 26\end{array}$ & $\begin{array}{l}31 \cdot 5 \\
49 \cdot 3\end{array}$ & $\begin{array}{l}50 \cdot 0 \\
50 \cdot 7\end{array}$ & $\begin{array}{l}\text { SAND } \\
\text { SAND }\end{array}$ & & \\
\hline $\begin{array}{l}634 \cdot 0 \\
635 \cdot 0\end{array}$ & $\begin{array}{r}1380.000 \\
970.000\end{array}$ & $\begin{array}{l}30 \cdot 95 \\
32 \cdot 10\end{array}$ & $\begin{array}{l}2 \cdot 67 \\
2 \cdot 76\end{array}$ & $\begin{array}{l}1 \cdot 85 \\
1.88\end{array}$ & $\begin{array}{l}34 \cdot 1 \\
30 \cdot 5\end{array}$ & $\begin{array}{l}35 \cdot 1 \\
49 \cdot 2\end{array}$ & $\begin{array}{l}\text { SAND } \\
\text { SAND }\end{array}$ & & \\
\hline $636 \cdot 0$ & BREKEN & $28 \cdot 23$ & $2 \cdot 72$ & $1 \cdot 95$ & $20 \cdot 6$ & $38 \cdot 6$ & SAND & NOT & $\Delta V G$ \\
\hline $637 \cdot 0$ & .010 & 17.78 & $2 \cdot 82$ & $2 \cdot 32$ & $2 \cdot 7$ & $73 \cdot 5$ & SAND & NOT & $\triangle V G$ \\
\hline $638 \cdot 0$ & .010 & 18.88 & $2 \cdot 83$ & $2 \cdot 30$ & $1 \cdot 6$ & $87 \cdot 9$ & SAND & NET & $A \vee G$ \\
\hline GES & 432.667 & 24.50 & & & $34 \cdot 1$ & & & & \\
\hline
\end{tabular}


TABLE B10

ROUTINE CORE ANALYSIS RESULTS VS DEPTH

FOR WELL MP-122

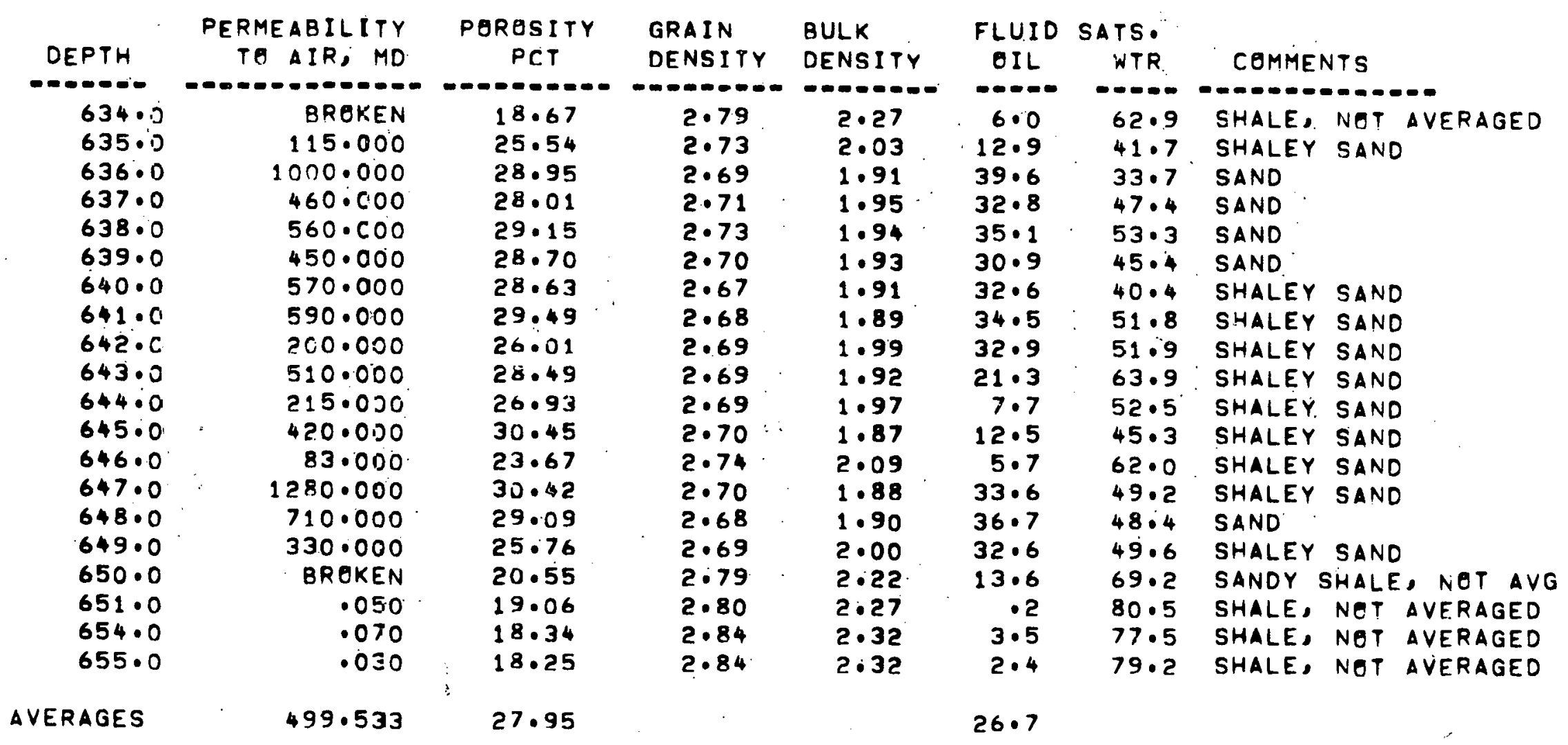


TABLE BII

ROUTINE CORE ANALYSIS RESULTS VS DEPTH

FOR WELL MP-130

\begin{tabular}{|c|c|c|c|c|c|c|c|c|c|}
\hline DEPTH & $\begin{array}{r}\text { PERMEABILITY } \\
\text { TE AIR, MD }\end{array}$ & $\begin{array}{c}\text { PERESITY } \\
\text { PCT }\end{array}$ & $\begin{array}{l}\text { GRAIN } \\
\text { DENSITY }\end{array}$ & $\begin{array}{l}\text { BULK } \\
\text { DENSITY }\end{array}$ & $\begin{array}{c}\text { FLUID } \\
\text { OIL }\end{array}$ & $\begin{array}{r}\text { SATS. } \\
\text { WTR }\end{array}$ & \multicolumn{3}{|c|}{ COMMENTS } \\
\hline$-\infty-\infty$ & $-\infty-\infty,-\infty-\infty,-\infty, \infty$ & $-\infty-\infty$ & $-\infty-\infty-\infty$ & $-\infty-\infty-\infty-\infty$ & $-\infty-\infty$ & $-\infty-\infty$ & & & $=$ \\
\hline $\begin{array}{l}628.0 \\
629.0 \\
630.0 \\
631.0\end{array}$ & $\begin{array}{r}\text { BREKEN } \\
.120 \\
\text { BROKEN } \\
2.060\end{array}$ & $\begin{array}{r}23.08 \\
23.70 \\
8.58 \\
17.01\end{array}$ & $\begin{array}{l}3.06 \\
3.08 \\
2.81 \\
2.86\end{array}$ & $\begin{array}{l}2.36 \\
2.35 \\
2.57 \\
2.37\end{array}$ & $\begin{array}{r}3.9 \\
3.2 \\
11.2 \\
4.8\end{array}$ & $\begin{array}{l}55.0 \\
51.9 \\
41.0 \\
76.0\end{array}$ & $\begin{array}{l}\text { SAND } \\
\text { SAND } \\
\text { SAND } \\
\text { SAND }\end{array}$ & $\begin{array}{l}\text { NeT } \\
\text { NET } \\
\text { NET } \\
\text { NOT }\end{array}$ & $\begin{array}{l}\triangle V G \\
\triangle V G \\
\triangle V G \\
A V G\end{array}$ \\
\hline $\begin{array}{l}632 \cdot 0 \\
633 \cdot 0\end{array}$ & $\begin{array}{r}400.000 \\
37.600\end{array}$ & $\begin{array}{l}28 \cdot 26 \\
25.15\end{array}$ & $\begin{array}{l}2 \cdot 71 \\
2 \cdot 74\end{array}$ & $\begin{array}{l}1 \cdot 94 \\
2 \cdot 05\end{array}$ & $\begin{array}{l}31 \cdot 7 \\
35 \cdot 7\end{array}$ & $\begin{array}{l}35 \cdot 4 \\
39 \cdot 9\end{array}$ & $\begin{array}{l}\text { SAND } \\
\text { SAND }\end{array}$ & & \\
\hline $\begin{array}{l}634 \cdot 0 \\
635 \cdot 0\end{array}$ & $\begin{array}{l}\text { BREKEN } \\
73.000\end{array}$ & $\begin{array}{l}24.82 \\
25.66\end{array}$ & $\begin{array}{l}2 \cdot 72 \\
2 \cdot 70\end{array}$ & $\begin{array}{l}2.04 \\
2.01\end{array}$ & $\begin{array}{l}13 \cdot 9 \\
30 \cdot 3\end{array}$ & $\begin{array}{l}55: 4 \\
50: 1\end{array}$ & $\begin{array}{l}\text { SAND } \\
\text { SAND }\end{array}$ & & \\
\hline $636 \cdot 0$ & 700.000 & 26.65 & $2 \cdot .71$ & 1.99 & $30 \cdot 1$ & 63.5 & SAND & & \\
\hline 637.0 & $500 \cdot 000$ & 29.62 & $2 \cdot 70$ & 1.90 & $24 \cdot 0$ & $62 \cdot 9$ & SAND & & \\
\hline $638 \cdot 0$ & 460.000 & $28 \cdot 70$ & $2 \cdot 70$ & $1 \cdot 93$ & $25 \cdot 7$ & $64 \cdot 6$ & SAND & & \\
\hline $\begin{array}{l}639.0 \\
640.0\end{array}$ & $\begin{array}{l}460.000 \\
630.000\end{array}$ & $\begin{array}{l}29.22 \\
28.26\end{array}$ & $\begin{array}{l}2.69 \\
2.69\end{array}$ & $\begin{array}{l}1.90 \\
1.93\end{array}$ & $\begin{array}{l}26 \cdot 7 \\
31 \cdot 7\end{array}$ & $\begin{array}{l}57 \cdot 3 \\
55 \cdot 1\end{array}$ & $\begin{array}{l}\text { SAND } \\
\text { SAND }\end{array}$ & & \\
\hline $641 \cdot 0$ & $200 \cdot 000$ & $27 \cdot 31$ & $2 \cdot 71$ & 1.97 & $30 \cdot 5$ & $56 \cdot 3$ & SAND & & \\
\hline $642 \cdot 0$ & $500 \cdot 000$ & 29.39 & $2 \cdot 70$ & $1 \cdot 90$ & $32 \cdot 1$ & $52 \cdot 4$ & SAND & & \\
\hline 643.0 & $190 \cdot 000$ & $26 \cdot 10$ & $2 \cdot 75$ & 2.03 & $31 \cdot 2$ & 63.1 & SAND & & \\
\hline 644.0 & 380.000 & 27.67 & $2 \cdot 70$ & $1 \cdot 95$ & $29 \cdot 6$ & $55 \cdot 5$ & SAND & & \\
\hline $645 \cdot 0$ & BREKEN & 26.98 & $2 \cdot 70$ & 1.97 & $36 \cdot 9$ & $59 \cdot 3$ & SAND & & \\
\hline $646 \cdot 0$ & 74.000 & $24 \cdot 13$ & 2.76 & $2 \cdot 10$ & $8 \cdot 9$ & $55 \cdot 4$ & SAND & & \\
\hline $647 \cdot 0$ & 410.000 & 28.51 & $2 \cdot 70$ & 1.93 & $19 \cdot 3$ & $53 \cdot 5$ & SAND & & \\
\hline $648 \cdot 0$ & $380 \cdot 000$ & $25 \cdot 11$ & 2.67 & 2.00 & $4 \cdot 4$ & $52 \cdot 4$ & SAND & & \\
\hline 649.0 & 690.000 & 27.00 & 2.66 & 1.94 & $26 \cdot 6$ & $52 \cdot 0$ & SAND & & \\
\hline $650 \cdot 0$ & $350 \cdot 000$ & $26 \cdot 44$ & $2 \cdot 66$ & $1 \cdot 96$ & $32 \cdot 6$ & 63.8 & SAND & & \\
\hline AVERAGES & 378.504 & 27.10 & & & $26 \cdot 4$ & & & & \\
\hline
\end{tabular}


TABLE B12

ROUTINE CORE ANALYSIS RESULTS VS DEPTH

FOR WELL MP-201

\begin{tabular}{|c|c|c|c|c|c|c|c|c|}
\hline $\begin{array}{c}\vdots \\
\text { DEPTH }\end{array}$ & $\begin{array}{c}\text { PERMEABILITY } \\
\text { TE } \triangle I R, M\end{array}$ & $\begin{array}{c}\text { PEROSITY } \\
\text { PCT }\end{array}$ & $\begin{array}{l}\text { GRAIN } \\
\text { DENSITY }\end{array}$ & $\begin{array}{l}\text { BULK } \\
\text { DENSITY }\end{array}$ & $\begin{array}{c}\text { FLUIO } \\
\text { OIL }\end{array}$ & $\begin{array}{r}\text { SATS } \\
\text { WTR }\end{array}$ & \multicolumn{2}{|c|}{ COMMENTS } \\
\hline$=-0-\infty-\infty$ & $-0-0-0-0.0-0-00$ & $-0-0-0=0$ & $-\infty-\infty-\infty-\infty$ & $-\infty-0-0-0$ & $-\infty-\infty$ & $-\infty-\infty$ & $-\infty-\infty-\infty-\infty$ & $-0-0-0$ \\
\hline $655 \cdot 0$ & .025 & $10 \cdot 88$ & $2 \cdot 78$ & $2 \cdot 48$ & $5 \cdot 2$ & $94 \cdot 8$ & SHALE, & NET AVG \\
\hline $656 \cdot 0$ & $20 \cdot 000$ & $25 \cdot 37$ & $2 \cdot 71$ & 2.02 & $45 \cdot 0$ & 37.0 & SAND & \\
\hline $657 \cdot 0$ & $220: 000$ & 28.04 & $2 \cdot 69$ & $1 \cdot 93$ & $61: 2$ & 23.7 & SAND & \\
\hline $658 \cdot 0$ & $340: 000$ & $28 \cdot 63$ & $2 \cdot 71$ & $1 \cdot 93$ & $38 \cdot 8$ & $43 \cdot 6$ & SAND & \\
\hline 659.0 & 115.000 & $22 \cdot 95$ & $2 \cdot 72$ & $2 \cdot 10$ & $4 \cdot 2$ & $15 \cdot 2$ & SAND & \\
\hline $660 \cdot 0$ & $107: 000$ & $34 \cdot 56$ & $2 \cdot 73$ & $2 \cdot 19$ & $24 \cdot 7$ & $29 \cdot 6$ & SAND & \\
\hline $\begin{array}{l}661 \cdot 0 \\
662.0\end{array}$ & $\begin{array}{l}530: 000 \\
870: 000\end{array}$ & $\begin{array}{l}26 \cdot 81 \\
31 \cdot 01\end{array}$ & $\begin{array}{l}2 \cdot 70 \\
2 \cdot 68\end{array}$ & $\begin{array}{l}1 \cdot 98 \\
1 \cdot 85\end{array}$ & $\begin{array}{l}23 \cdot 1 \\
28 \cdot 4\end{array}$ & $\begin{array}{l}37 \cdot 4 \\
55 \cdot 2\end{array}$ & $\begin{array}{l}\text { SAND } \\
\text { SAND }\end{array}$ & \\
\hline $\begin{array}{l}663.0 \\
664.0\end{array}$ & $\begin{array}{l}780.000 \\
320.000\end{array}$ & $\begin{array}{l}30 \cdot 37 \\
28 \cdot 36\end{array}$ & $\begin{array}{l}2 \cdot 69 \\
2 \cdot 69\end{array}$ & $\begin{array}{l}1 \cdot 87 \\
1 \cdot 93\end{array}$ & $\begin{array}{l}33 \cdot 2 \\
28 \cdot 1\end{array}$ & $\begin{array}{l}53 \cdot 3 \\
57 \cdot 8\end{array}$ & $\begin{array}{l}\text { SAND } \\
\text { SAND }\end{array}$ & \\
\hline $\begin{array}{l}665 \cdot 0 \\
666 \cdot 0\end{array}$ & $\begin{array}{r}1130.000 \\
156.000\end{array}$ & $\begin{array}{l}30 \cdot 18 \\
25 \cdot 22\end{array}$ & $\begin{array}{l}2 \cdot 67 \\
2 \cdot 69\end{array}$ & $\begin{array}{l}1 \cdot 87 \\
2.01\end{array}$ & $\begin{array}{l}22 \cdot 5 \\
17 \cdot 5\end{array}$ & $\begin{array}{l}62 \cdot 1 \\
78 \cdot 4\end{array}$ & $\begin{array}{l}\text { SAND } \\
\text { SAND }\end{array}$ & \\
\hline 667.0 & .280 & 8.23 & $2 \cdot 71$ & $2 \cdot 48$ & $6 \cdot 8$ & $61 \cdot 4$ & SHALEY & SAND \\
\hline $668 \cdot 0$ & 1030 & 5.55 & $2 \cdot 69$ & $2 \cdot 54$ & $8 \cdot 7$ & $67 \cdot 0$ & SHALEY & SAND \\
\hline 669.0 & 250.000 & $22 \cdot 53$ & 2.69 & $2 \cdot 08$ & $33 \cdot 2$ & $55 \cdot 7$ & SHALEY & SAND \\
\hline $\begin{array}{l}670 \cdot 0 \\
671 \cdot 0\end{array}$ & $\begin{array}{l}360.000 \\
810.000\end{array}$ & $\begin{array}{l}28.53 \\
29.71\end{array}$ & $\begin{array}{l}2 \cdot 69 \\
2 \cdot 67\end{array}$ & $\begin{array}{l}1.92 \\
1.88\end{array}$ & $\begin{array}{r}37 \cdot 2 \\
7 \cdot 6\end{array}$ & $\begin{array}{l}48 \cdot 5 \\
48.3\end{array}$ & $\begin{array}{l}\text { SHALEY } \\
\text { SHALEY }\end{array}$ & SAND \\
\hline $\begin{array}{l}672 \cdot 0 \\
673 \cdot 0\end{array}$ & $\begin{array}{r}7.900 \\
1270.000\end{array}$ & $\begin{array}{l}20 \cdot 85 \\
30.96\end{array}$ & $\begin{array}{l}2 \cdot 76 \\
2 \cdot 67\end{array}$ & $\begin{array}{l}2.18 \\
1.84\end{array}$ & $32 \cdot 7$ & $67 \cdot 3$ & SHALEY & SAND \\
\hline VERAGES & 404.789 & $25 \cdot 44$ & & & $27 \cdot 1$ & & & \\
\hline
\end{tabular}


TABLE $B 13$

ROUTINE CORE ANALYSIS RESULTS VS DEPTH

FOR WELL MP-203

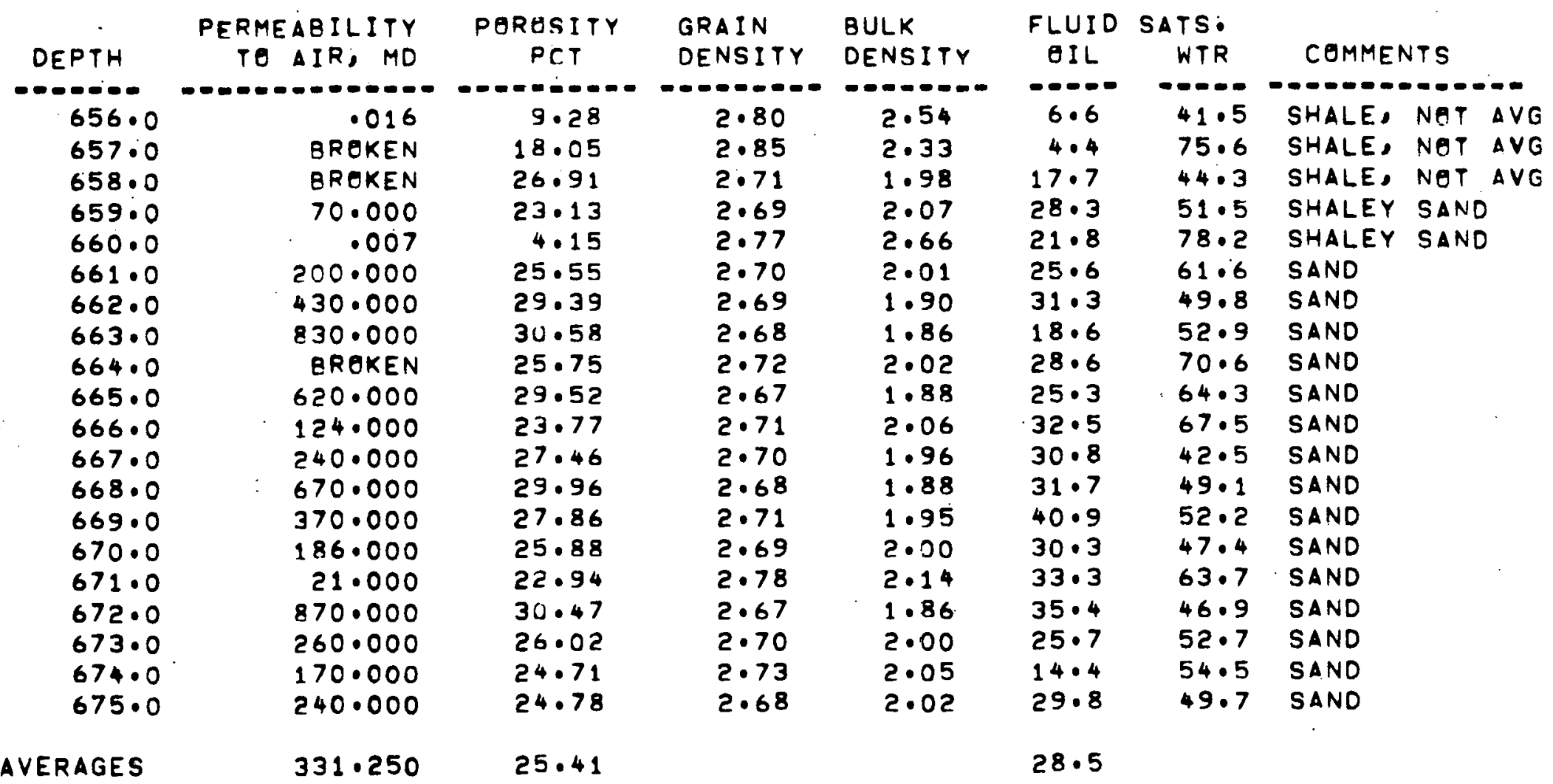


TABLE B14

ROUTINE CORE ANALYSIS RESULTS VS DEPTH

FOR WELL IIP-205

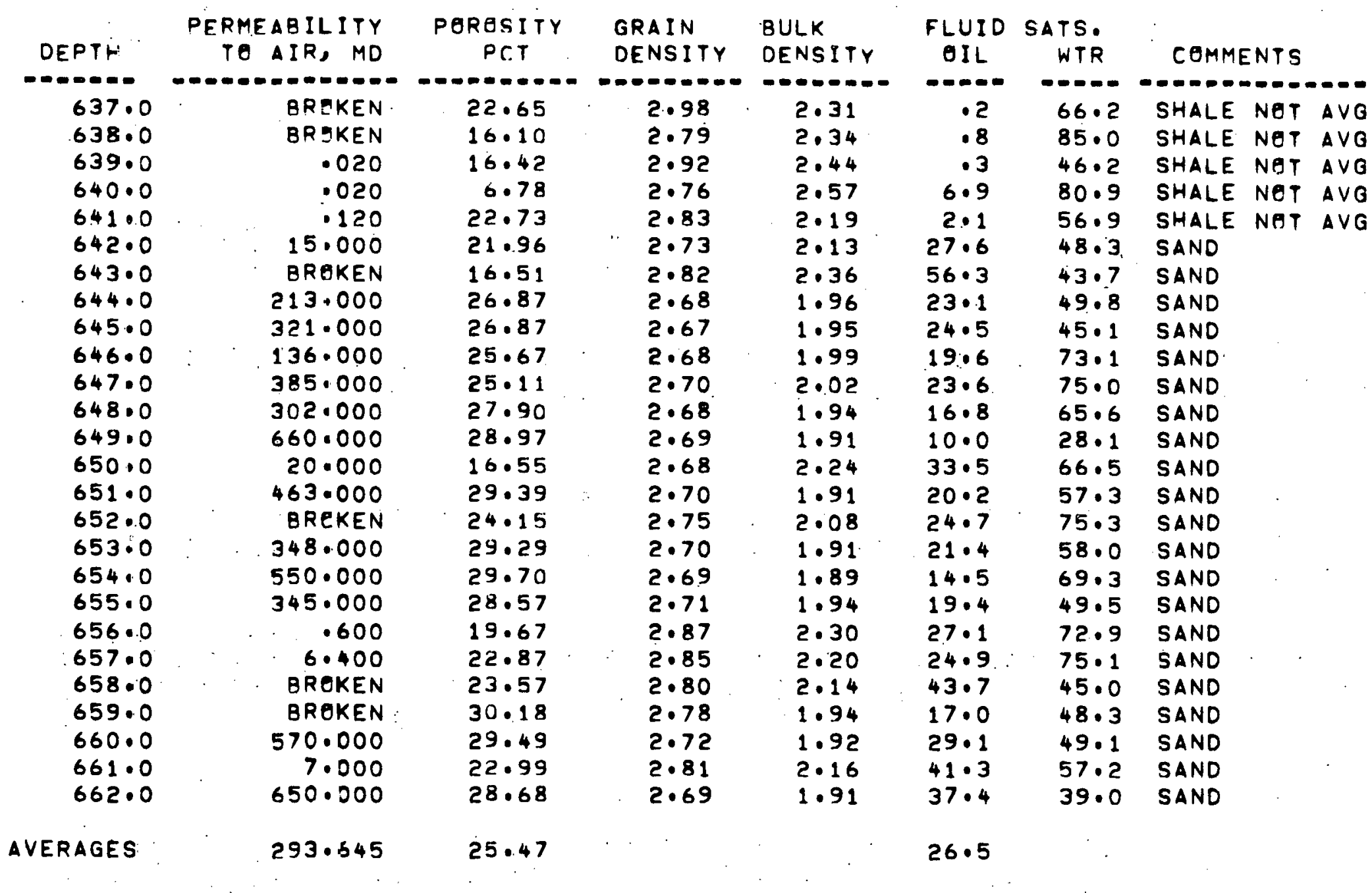


TABLE B15

ROUT INE CORE ANALYSIS RESULTS VS DEPTH

FOR WELL MP-213

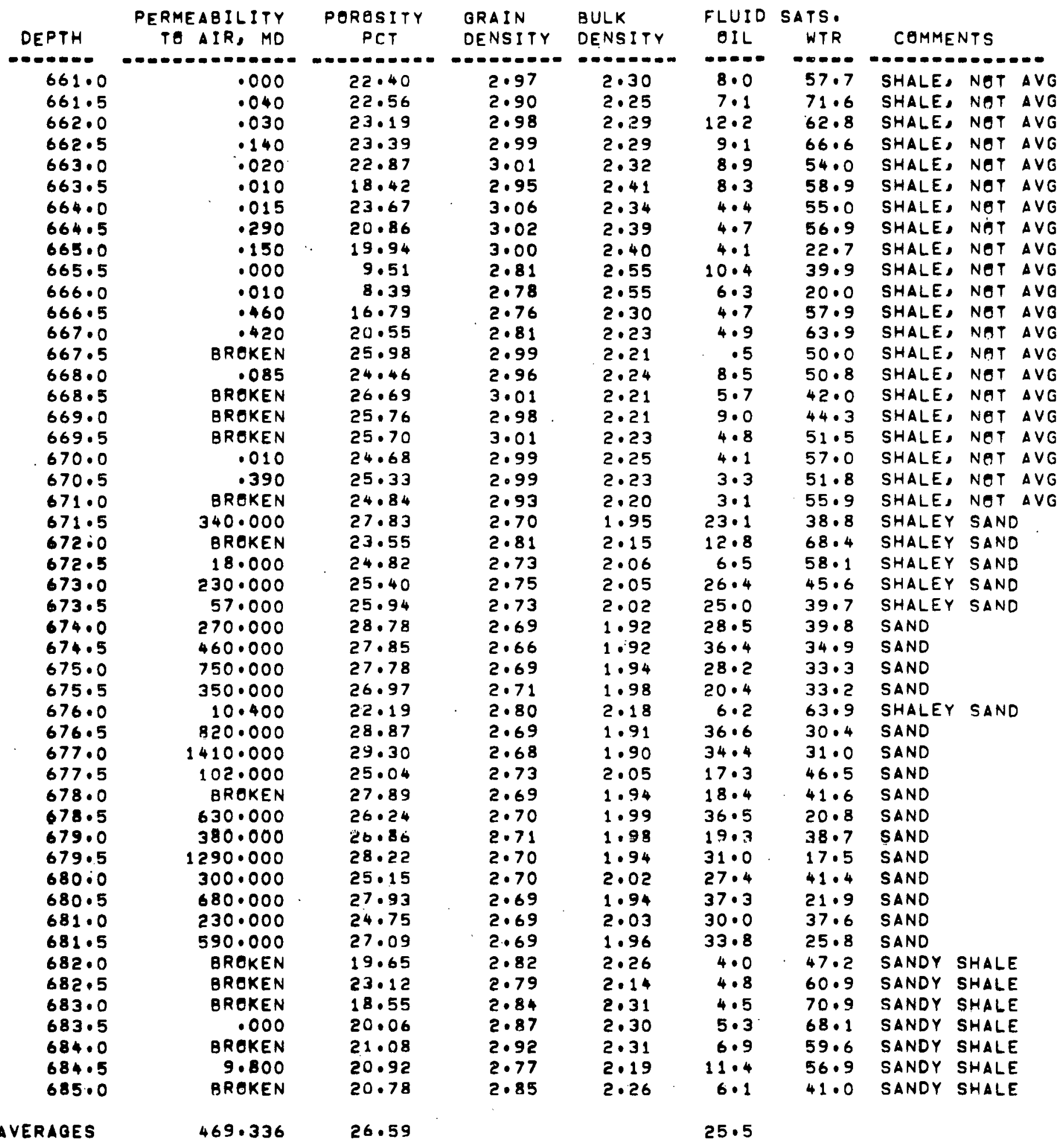


TABLE B16

ROUT INE CORE ANALYSIS RESULTS VS DEPTH

FOR WELL MP-215

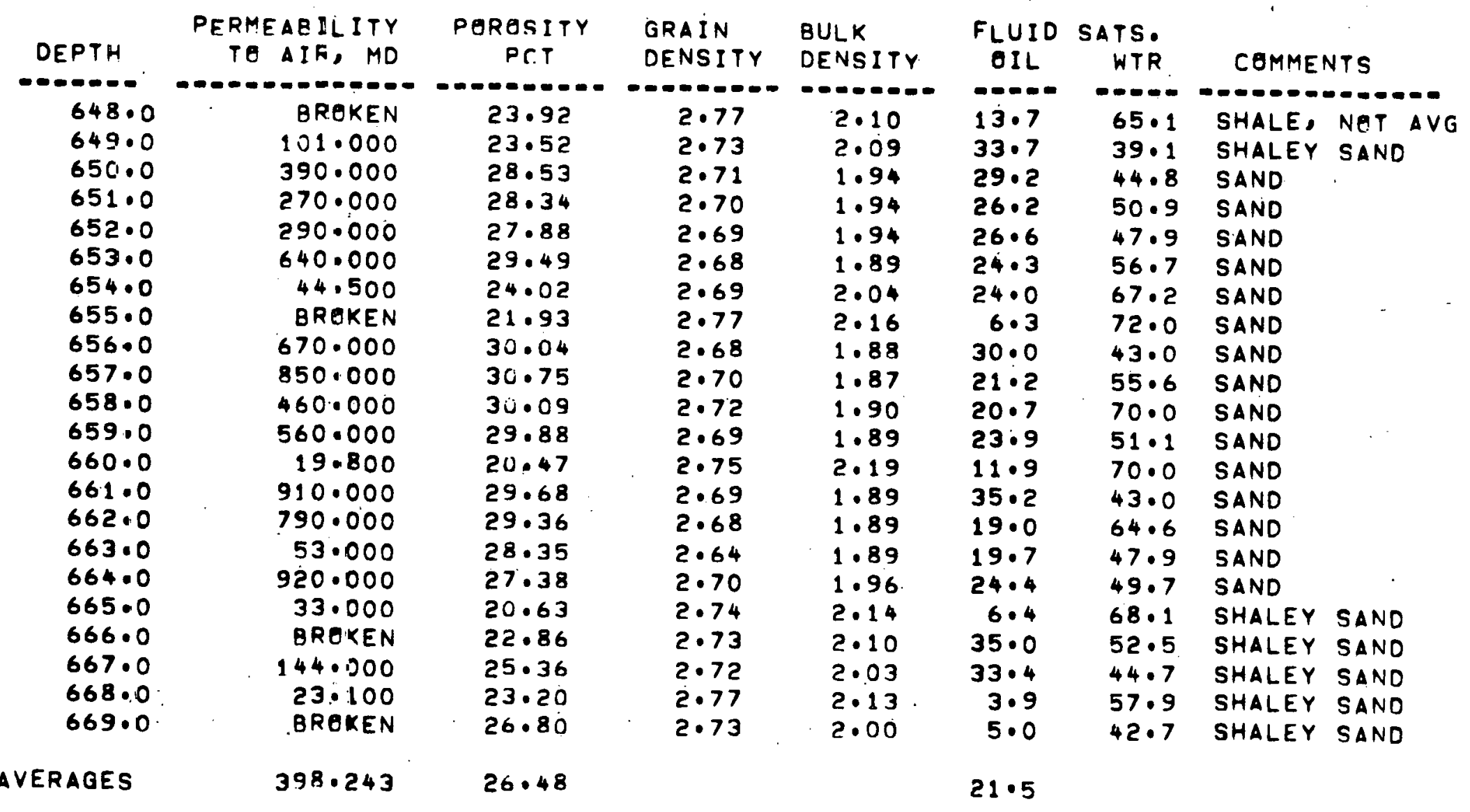


TABLE BI7

ROUTINE CORE ANALYSIS RESULTS VS DEPTH

FOR WELL MP-221

\begin{tabular}{|c|c|c|c|c|c|c|c|c|c|}
\hline DEPTH & $\begin{array}{l}\text { PERMEABILITY } \\
\text { TO AIR, MD }\end{array}$ & $\begin{array}{c}\text { PERESTTY } \\
\text { PC.T }\end{array}$ & $\begin{array}{l}\text { GRAIN: } \\
\text { DENSITY }\end{array}$ & $\begin{array}{l}\text { BULK } \\
\text { DENSITY }\end{array}$ & $\begin{array}{l}\text { FLUID } \\
\text { OIL }\end{array}$ & $\begin{array}{c}\text { SATS. } \\
\text { WTR. }\end{array}$ & COMME & NTS & \\
\hline . & - - - - - - - - & $-0-0-0.00$ & $=--0-0-00$ & $-0-0-\infty-\infty$ & $---\infty$ & $=\infty$ & $-0-0=0$ & - & $-\infty$ \\
\hline $\begin{array}{l}662 \cdot 0 \\
663 \cdot 0\end{array}$ & $\begin{array}{l}.006 \\
.200\end{array}$ & $\begin{array}{r}6.90 \\
17.00\end{array}$ & $\begin{array}{l}2 \cdot 76 \\
2 \cdot 84\end{array}$ & $\begin{array}{l}2.57 \\
2.36\end{array}$ & $\begin{array}{l}9.8 \\
4.6\end{array}$ & $\begin{array}{l}64 \cdot 6 \\
87 \cdot 8\end{array}$ & $\begin{array}{l}\text { SHALE } \\
\text { SHALE }\end{array}$ & $\begin{array}{l}\text { NET } \\
\text { NET }\end{array}$ & $\begin{array}{l}A \vee G \\
\triangle \vee G\end{array}$ \\
\hline $664 \cdot 0$ & $238 \cdot 000$ & $27 \cdot 50$ & $2 \cdot 73$ & $1 \cdot 98$ & $50 \cdot 6$ & 39.0 & SHALEY & SAN & VD \\
\hline $665 \cdot 0$ & 640.000 & $25 \cdot 95$ & $2 \cdot 69$ & $1 \cdot 99$ & $42 \cdot 2$ & $41 \cdot 1$ & SAND & & \\
\hline $666 \cdot 0$ & $400 \cdot 000$ & $27: 41$ & $2 \cdot 67$ & 1.94 & $36 \cdot 2$ & $43 \cdot ?$ & SAND & & \\
\hline $\begin{array}{l}667 \cdot 0 \\
668 \cdot 0\end{array}$ & $\begin{array}{l}530 \cdot 000 \\
211.000\end{array}$ & $\begin{array}{l}27 \cdot 17 \\
24 \cdot 24\end{array}$ & $\begin{array}{l}2 \cdot 68 \\
2 \cdot 72\end{array}$ & $\begin{array}{l}1 \cdot 95 \\
2 \cdot 06\end{array}$ & $\begin{array}{l}26 \cdot 4 \\
38 \cdot 9\end{array}$ & $\begin{array}{l}47 \cdot 1 \\
42 \cdot 9\end{array}$ & $\begin{array}{l}\text { SAND } \\
\text { SAND }\end{array}$ & & \\
\hline $669 \cdot 0$ & 360.000 & 28.74 & $2 \cdot 69$ & 1.92 & $36 \cdot 0$ & $45 \cdot 7$ & SAND & & \\
\hline $670 \cdot 0$ & $1100 \cdot 000$ & $31 \cdot 12$ & $2 \cdot 68$ & $1 \cdot 85$ & $26: 5$ & $39 \cdot 7$ & SAND & & \\
\hline $\begin{array}{l}671 \cdot 0 \\
672 \cdot 0\end{array}$ & 995.000 & $\begin{array}{l}31: 60 \\
28: 36\end{array}$ & $2 \cdot 69$ & 1.84 & $35 \cdot 0$ & $47 \cdot 6$ & SAND & & \\
\hline $\begin{array}{l}672 \cdot 0 \\
673.0\end{array}$ & $\begin{array}{r}590 \cdot 000 \\
1045.000\end{array}$ & $\begin{array}{l}28 \cdot 36 \\
26 \cdot 32\end{array}$ & $\begin{array}{l}2 \cdot 68 \\
2 \cdot 68\end{array}$ & $1 \cdot 92$ & $28 \cdot 3$ & $66 \cdot 6$ & SAND & & \\
\hline 674.0 & BROKEN & 26.92 & $\begin{array}{l}2.68 \\
2.69\end{array}$ & $\begin{array}{l}1.97 \\
1.97\end{array}$ & $\begin{array}{l}25 \cdot 3 \\
24 \cdot 5\end{array}$ & $\begin{array}{l}56 \cdot 8 \\
75 \cdot 0\end{array}$ & SAND & & \\
\hline $675 \cdot 0$ & 650.000 & 29.68 & $\begin{array}{l}2.69 \\
2.69\end{array}$ & 1.89 & $34 \cdot 3$ & $\begin{array}{l}75 \cdot 0 \\
46 \cdot 4\end{array}$ & $\begin{array}{l}\text { SAND } \\
\text { SAND }\end{array}$ & & \\
\hline $676 \cdot 0$ & $325 \cdot 000$ & 27.76 & $2 \cdot 70$ & 1.95 & $24 \cdot 7$ & $56 \cdot 3$ & SAND & & \\
\hline $677 \cdot 0$ & BROKEN & 19.56 & $2 \cdot 82$ & $2 \cdot 27$ & $34 \cdot 6$ & $65 \cdot 4$ & SAND. & & - \\
\hline $\begin{array}{l}678 \cdot 0 \\
679 \cdot 0\end{array}$ & $\begin{array}{r}72 \cdot 000 \\
590 \cdot 000\end{array}$ & $\begin{array}{l}22 \cdot 99 \\
29.09\end{array}$ & $\begin{array}{l}2 \cdot 75 \\
2 \cdot 68\end{array}$ & $\begin{array}{l}2 \cdot 12 \\
1 \cdot 90\end{array}$ & $\begin{array}{r}7 \cdot 1 \\
33 \cdot 1\end{array}$ & $\begin{array}{l}62 \cdot 5 \\
53 \cdot 1\end{array}$ & $\begin{array}{l}\text { SAND } \\
\text { SAND }\end{array}$ & & \\
\hline $\begin{array}{l}680 \cdot 0 \\
680 \cdot 5\end{array}$ & $\begin{array}{l}975 \cdot 000 \\
\text { BREKEN }\end{array}$ & $\begin{array}{l}28 \cdot 68 \\
26 \cdot 72\end{array}$ & $\begin{array}{l}2 \cdot 67 \\
2 \cdot 75\end{array}$ & $\begin{array}{l}1 \cdot 90 \\
2 \cdot 01\end{array}$ & $\begin{array}{l}26 \cdot 9 \\
36 \cdot 1\end{array}$ & $\begin{array}{l}51 \cdot 6 \\
50 \cdot 1\end{array}$ & $\begin{array}{l}\text { SAND } \\
\text { SHALE }\end{array}$ & NET & $A \vee G$ \\
\hline AVERAGES & $581 \cdot 399$ & $27 \cdot 24$ & & & $31 \cdot 2$ & & & & \\
\hline
\end{tabular}


TABLE B18

ROUTINE CORE ANALYSIS RESULTS VS DEPTH

FOR WELL MP-223

\begin{tabular}{|c|c|c|c|c|c|c|c|c|c|}
\hline DEPTH & $\begin{array}{c}\text { PERMEABILITY } \\
\text { TO AIR, MD }\end{array}$ & $\begin{array}{c}\text { POROSITY } \\
\text { PCT }\end{array}$ & $\begin{array}{l}\text { GRAIN } \\
\text { DENSITY }\end{array}$ & $\begin{array}{l}\text { BULK } \\
\text { DENSITY }\end{array}$ & $\begin{array}{l}\text { FLUID } \\
\text { OIL }\end{array}$ & $\begin{array}{r}\text { SATS. } \\
\text { WTR }\end{array}$ & \multicolumn{3}{|c|}{ COMMENTS } \\
\hline & $\infty \cdots$ & & $-0-0-0.0$ & $-0-0-000$ & $-\infty-\infty$ & $-\infty-\infty$ & & & \\
\hline 667.0 & 188.000 & 24.47 & 2.69 & 2.03 & 37.3 & $42 \cdot 4$ & SAND & & \\
\hline 668.0 & 74.000 & 23.57 & $2 \cdot 71$ & 2.07 & 29.0 & $48 \cdot 6$ & SAND & & \\
\hline 669.0 & 47.800 & 25.07 & $2 \cdot 70$ & 2.03 & $21 \cdot 6$ & $54 \cdot 8$ & SAND & & \\
\hline 670.0 & 97.000 & 15.24 & $2 \cdot 74$ & $2 \cdot 32$ & $44 \cdot 7$ & $55 \cdot 3$ & SAND & & \\
\hline 671.0 & BREKEN & 28.81 & 2.69 & 1.92 & $30 \cdot 9$ & 57.3 & SAND & & \\
\hline 672.0 & 790.000 & 30.47 & 2.67 & 1.86 & $25 \cdot 6$ & $50 \cdot 3$ & SAND & & \\
\hline $\begin{array}{l}673 \cdot 0 \\
674 \cdot 0\end{array}$ & $\begin{array}{r}230.000 \\
1.940\end{array}$ & $\begin{array}{l}23.76 \\
20.41\end{array}$ & $\begin{array}{l}2 \cdot 71 \\
2 \cdot 80\end{array}$ & $\begin{array}{l}2.07 \\
2.23\end{array}$ & $\begin{array}{r}24 \cdot 9 \\
5 \cdot 2\end{array}$ & $\begin{array}{l}49 \cdot 3 \\
74 \cdot 6\end{array}$ & $\begin{array}{l}\text { SAND } \\
\text { SHALEY }\end{array}$ & SANC & \\
\hline $\begin{array}{l}675.0 \\
676.0\end{array}$ & $\begin{array}{r}1 \cdot 640 \\
31 \cdot 600\end{array}$ & $\begin{array}{l}21.04 \\
24.17\end{array}$ & $\begin{array}{l}2 \cdot 77 \\
2 \cdot 73\end{array}$ & $\begin{array}{l}2 \cdot 19 \\
2 \cdot 07\end{array}$ & $\begin{array}{r}7 \cdot 1 \\
10 \cdot 4\end{array}$ & $\begin{array}{l}74 \cdot 1 \\
65 \cdot 1\end{array}$ & $\begin{array}{l}\text { SHALEY } \\
\text { SHALEY }\end{array}$ & $\begin{array}{l}\text { SAND } \\
\text { SAND }\end{array}$ & \\
\hline $\begin{array}{l}677.0 \\
678.0\end{array}$ & $\begin{array}{r}1300.000 \\
730.000\end{array}$ & $\begin{array}{l}31.76 \\
30.03\end{array}$ & $\begin{array}{l}2.67 \\
2.68\end{array}$ & $\begin{array}{l}1.82 \\
1.88\end{array}$ & $\begin{array}{l}28.9 \\
23.0\end{array}$ & $\begin{array}{l}54 \cdot 1 \\
56 \cdot 6\end{array}$ & $\begin{array}{l}\text { SAND } \\
\text { SAND }\end{array}$ & & \\
\hline $\begin{array}{l}679.0 \\
680: 0 \\
681.0\end{array}$ & $\begin{array}{r}1090 \cdot 000 \\
15.300 \\
1650.000\end{array}$ & $\begin{array}{l}30.04 \\
21.13 \\
29.96\end{array}$ & $\begin{array}{l}2 \cdot 67 \\
2 \cdot 76 \\
2.66\end{array}$ & $\begin{array}{l}1.87 \\
2.18 \\
1.86\end{array}$ & $\begin{array}{l}37 \cdot 2 \\
19 \cdot 4 \\
29.5\end{array}$ & $\begin{array}{l}43 \cdot 0 \\
67 \cdot 5\end{array}$ & $\begin{array}{l}\text { SAND } \\
\text { SAND }\end{array}$ & & \\
\hline $682 \cdot 0$ & 640.000 & 28.55 & 2.72 & $\begin{array}{l}1.86 \\
1.94\end{array}$ & $\begin{array}{l}29.5 \\
17.5\end{array}$ & $\begin{array}{l}49.3 \\
64.6\end{array}$ & $\begin{array}{l}\text { SAND } \\
\text { SAND }\end{array}$ & & \\
\hline 683.0 & 360.000 & 28.89 & 2.67 & 1.90 & $10 \cdot 1$ & 44.0 & SAND & & \\
\hline $684 \cdot 0$ & $980 \cdot 000$ & $29 \cdot 15$ & $2 \cdot 67$ & 1.89 & $32 \cdot 8$ & $55 \cdot 3$ & SAND & & \\
\hline $685 \cdot 0$ & 1.560 & 14.35 & $2 \cdot 71$ & $2 \cdot 32$ & $22 \cdot 3$ & 77.7 & SHALE, & NOT & $\Delta V G$ \\
\hline $686 \cdot 0$ & BREKEN & $19.9 \mathrm{C}$ & 2.77 & $2 \cdot 22$ & $2 \cdot 0$ & 73.8 & SHALE, & NAT & $\Delta V G$ \\
\hline GES & 513.310 & 25.92 & & & $24 \cdot 2$ & & & & \\
\hline
\end{tabular}


TABLE B19

ROUTINE CORE ANALYSIS RESULTS VS DEPTH

FOR WELL MP -225

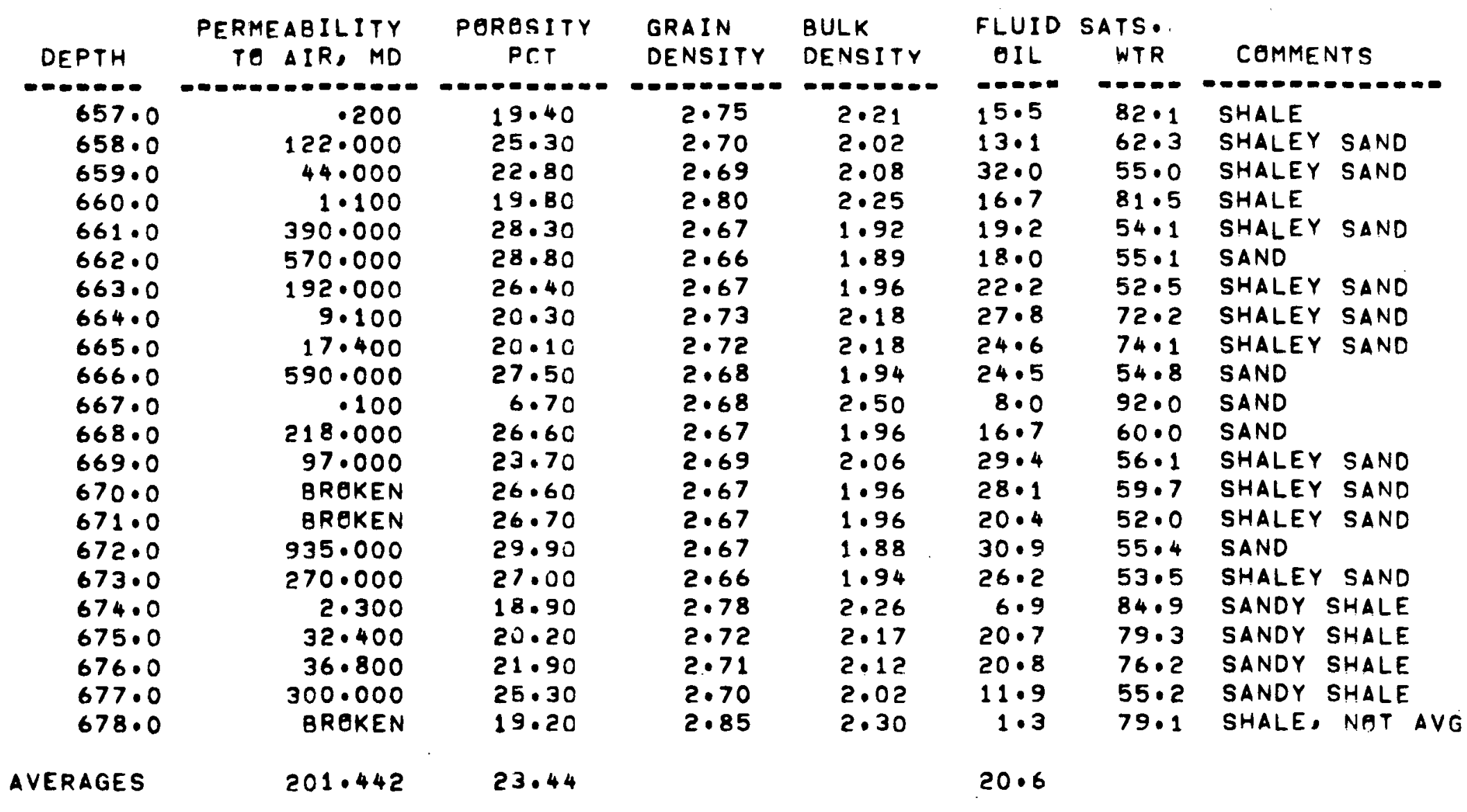


TABLE B20

SUMMARY OF ROUTINE CORE ANALYSES

EL DORADO MICELLAR-POL YMER PROJECT

Permeability Porosity 0 il Saturation Pay Thickness 0il-in-place Well Number millidarcies percent

percent

27.5

27.3

28.3

24.5

28.0

27.1
22.2

25.0

25.4

34.1

26.7

26.4 feet

19

19

19

18

15

19 barrels/acre

$M P-122$

379

MP-201

MP-203

MP-205

$M P-213$

$M P-215$

$M P-221$

$M P-223$

MP-225

$M P-101$

MP-102

MP-103

MP-104

MP- 105

405

331

294

469

398

581

513

201

432

448

311

393

191

25.4

25.4

25.5

26.6

26.5

27.2

26.0

23.4
27.1

28.5

26.5

25.5

21.5 :

31.5

24.2

20.6
18

17

21

11

21

17

18

21

Avg. $\quad 9,147$

Total 234,163

9,547

11,009

5,788

9,282

11,300

8,786

7,853
8,999

10,060

10,596

11,666

8,700

10,546

Avg. $\quad 10,095$

Total 258,432

9,612

334,

32.7

19.0

16.4

28.3

27.2
18

21

19

18 


\section{TABLE B21}

\section{WETTABILITY DETERMINATION FROM RELATIVE}

\section{PERMEABILITY DATA}

\begin{tabular}{|c|c|c|c|c|}
\hline Sample & Depth, ft & $\begin{array}{l}\text { Connate Water } \\
\text { Saturation, \% }\end{array}$ & $\begin{array}{l}\text { Saturation at Which } \\
\mathrm{K}_{r_{0}}=\mathrm{K}_{\mathrm{rW}}, \% \\
\end{array}$ & $\begin{array}{l}\text { Water Relative } \\
\text { Permeability at } \\
\text { Residual 0il, \% } \\
\end{array}$ \\
\hline .14 & 666 & $24.8(W W)^{*}$ & 54.0 (bth) & 31.8 (I) \\
\hline 17 & 667 & $25.3(b W)$ & $53.0(W(W)$ & 40.5 (I) \\
\hline 11 & 664 & 30.1 (WW) & 55.0 (WW) & 32.0 (I) \\
\hline 6 & 658 & 27.5 (WW) & 56.0 (WW) & 41.9 (I) \\
\hline 21 & 669 & 45.1 (WW) & 53.0 (WW) & $62.9(0 W)$ \\
\hline 3 & 657 & 27.1 (WW & 49.0 (OW) & 46.7 (I) \\
\hline \multicolumn{5}{|c|}{$\star \| N \mid=$ Water-wet } \\
\hline OW $=$ & wet & & & \\
\hline
\end{tabular}


TABLE B22

WETTABILITY DETERMINATION FROII CAPILLARY PRESSURE CURVES

\section{Core No. $5 a$}

\section{Length}

Initial Water

Saturation $3.34 \mathrm{~cm}$

0.3
$4.06 \mathrm{cc}$

Pore Volume

Water Displacing $0 i]$.

\begin{tabular}{cc} 
Differential & Water \\
Pressure, & Saturation, \\
psi & fraction \\
\hline
\end{tabular}

PS 1

1.05

1.73

3.01

4.37

6.02

8.32

11.79

19.21

26.00

0.487
0.610
0.660
0.704
0.726
0.746
0.766
0.785
0.805

Area under curve $(A 2)=$

$$
0.893
$$

Area under curve $(A])=$

0.743

$0 i 1$ density $0.84 \mathrm{gm} / \mathrm{cc}$
Brire density $1.065 \mathrm{gm} / \mathrm{cc}$

0il Displacing Water

Differential Water
Pressure, Saturation,

psi

0.83

1.23

1.99

2.94

3.90

5.30

12.24

15.38

0.578

0.507

0.443

0.413

0.386

0.362

0.342

0.327

0.312

Wettabiiity number $(\log A Y / A 2)=-0.08$
Core No. 13a

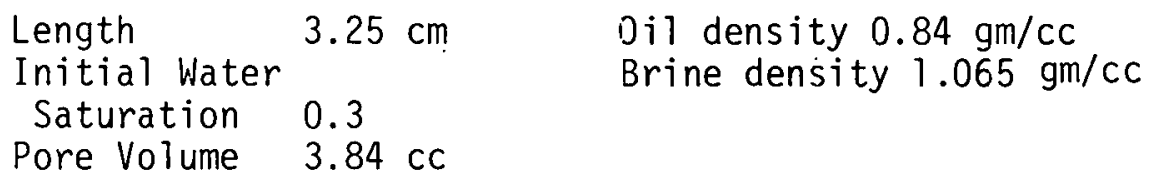

\begin{tabular}{|c|c|}
\hline $\begin{array}{c}\text { Water Disp } \\
\text { Differential } \\
\text { Pressure, } \\
\text { psi }\end{array}$ & $\begin{array}{l}\text { Water } \\
\text { Saturation, } \\
\text { fraction }\end{array}$ \\
\hline $\begin{array}{r}1.02 \\
1.68 \\
2.93 \\
4.25 \\
5.86 \\
8.09 \\
11.47 \\
18.69 \\
25.30\end{array}$ & $\begin{array}{l}0.498 \\
0.579 \\
0.613 \\
0.639 \\
0.644 \\
0.659 \\
0.672 \\
0.691 \\
0.717\end{array}$ \\
\hline
\end{tabular}

$\begin{array}{cc}\begin{array}{c}\text { Dil Displacing Water } \\ \text { Differential } \\ \text { Pressure, } \\ \text { psi }\end{array} & \begin{array}{c}\text { Water } \\ \text { Saturation, } \\ \text { fraction }\end{array} \\ 0.81 & 0.506 \\ 1.19 & 0.449 \\ 1.94 & 0.391 \\ 2.86 & 0.370 \\ 3.79 & 0.344 \\ 5.16 & 0.326 \\ 7.20 & 0.311 \\ 11.91 & 0.300 \\ 14.97 & 0.292\end{array}$

Area under curve to

$10 \mathrm{psi}(\mathrm{A} 2)=0.583$

Area under curve to

$10 \mathrm{psi}(\mathrm{Al})=0.600$

Wettability number $(\log A 1 / A 2)=0.01$ 
TABLE B23

COMPRESSIVE STRENGTH OF ADMIRE SANDSTONE CORES

\begin{tabular}{|c|c|c|c|c|}
\hline Specimen & $\begin{array}{c}\text { Average } \\
\text { Diameter, in }\end{array}$ & $\begin{array}{l}\text { Area, } \\
\text { sq in }\end{array}$ & Length, in & $\begin{array}{c}\text { Compressive } \\
\text { Strength, psi }\end{array}$ \\
\hline $\begin{array}{c}\text { Chesney } 21 \mathrm{~W} \\
650 \text {-feet }\end{array}$ & 3.01 & 7.11 & 5.7 & 3,520 \\
\hline $\begin{array}{c}\text { Chesney } 21 \mathrm{~W} \\
563-\text { feet }\end{array}$ & 3.02 & 7.15 & 2.82 & 2,450 \\
\hline
\end{tabular}


TABLE B24

$X$-RAY DIFFRACTION ANALYSES FOR

CLAY FRACTION OF SAMPLES FROM WELL MAP-221

\begin{tabular}{|c|c|c|c|c|c|c|c|}
\hline \multirow[b]{2}{*}{$\begin{array}{c}\text { Depth, } \\
\text { feet }\end{array}$} & \multirow[b]{2}{*}{$\begin{array}{l}\text { Percert of } \\
\text { Sample }<5 \mu\end{array}$} & \multicolumn{6}{|c|}{ Clay Type with Relative Percentages } \\
\hline & & Illite & $\begin{array}{c}\text { Illite- } \\
\text { Montmorillonite }\end{array}$ & Kaolinite & Chlorite & Calcite & Quartz \\
\hline 662 & 7.8 & 45 & 5 & 39 & 1 & 10 & 10 \\
\hline 663 & 30.0 & 30 & 10 & 40 & 15 & - & 5 \\
\hline $664-667$ & 5.7 & 35 & - & 45 & 10 & 5 & 5 \\
\hline $668-672$ & $6 . \overline{5}$ & 25 & 5 & 40 & 15 & 5 & 10 \\
\hline $6 ; 3-674$ & 3.4 & 30 & - & 50 & 10 & - & 10 \\
\hline 675 & 7.0 & 30 & - & 45 & 15 & - & 10 \\
\hline $677-680$ & 8.9 & 35 & - & 45 & - & 5 & 10 \\
\hline
\end{tabular}


TABLE B25

$X$-RAY DIFFRACTION ANALYSES FOR

CLAY FRACTION OF SAMPLES FROM WELL MP-225

\begin{tabular}{|c|c|c|c|c|c|c|c|}
\hline \multirow[b]{2}{*}{$\begin{array}{r}\text { Depth, } \\
\text { feet }\end{array}$} & \multirow[b]{2}{*}{$\begin{array}{l}\text { Percent of } \\
\text { Sample }<5 \mu\end{array}$} & \multicolumn{6}{|c|}{$\begin{array}{l}\text { Clay Type with Relative Percentages } \\
\text { Illite- }\end{array}$} \\
\hline & & Illite & $\begin{array}{c}\text { Il7ite- } \\
\text { Montmorillonite }\end{array}$ & Kaolinite & Chlorite & Calcite & Quartz \\
\hline $657-658$ & 13.6 & 45 & 5 & 20 & 10 & 10 & 10 \\
\hline $659^{\circ}$ & 6.8 & 40 & 15 & 20 & 10 & - & 15 \\
\hline 660 & 16.2 & 55 & 15 & 10 & 10 & - & 10 \\
\hline $660.4-662$ & 7.4 & 45 & 10 & 20 & 10 & 5 & 10 \\
\hline 664 & 7.1 & 40 & 15 & 25 & 10 & - & 10 \\
\hline $665-666$ & 8.7 & 50 & 10 & 25 & 10 & - & 5 \\
\hline 667 & 6.1 & 40 & 10 & 20 & 15 & - & 5 \\
\hline $668-669$ & 11.0 & 50 & 10 & 20 & 15 & - & 5 \\
\hline 669.8 & 5.8 & 40 & 10 & 25 & 15 & - & 10 \\
\hline $670-671$ & 6.3 & 35 & 15 & 20 & 15 & - & 15 \\
\hline $672-673$ & 5.7 & 4.0 & 10 & 20 & 15 & - & 15 \\
\hline $675-677$ & 15.2 & 50 & 10 & 15 & 20 & - & 5 \\
\hline 678 & 23.0 & 45 & 5 & 20 & 25 & - & 5 \\
\hline
\end{tabular}




\section{FIGURE B-1}

\section{PROJECT LAYOUT SHOWING LOCATIONS AND TYPES OF CORES}

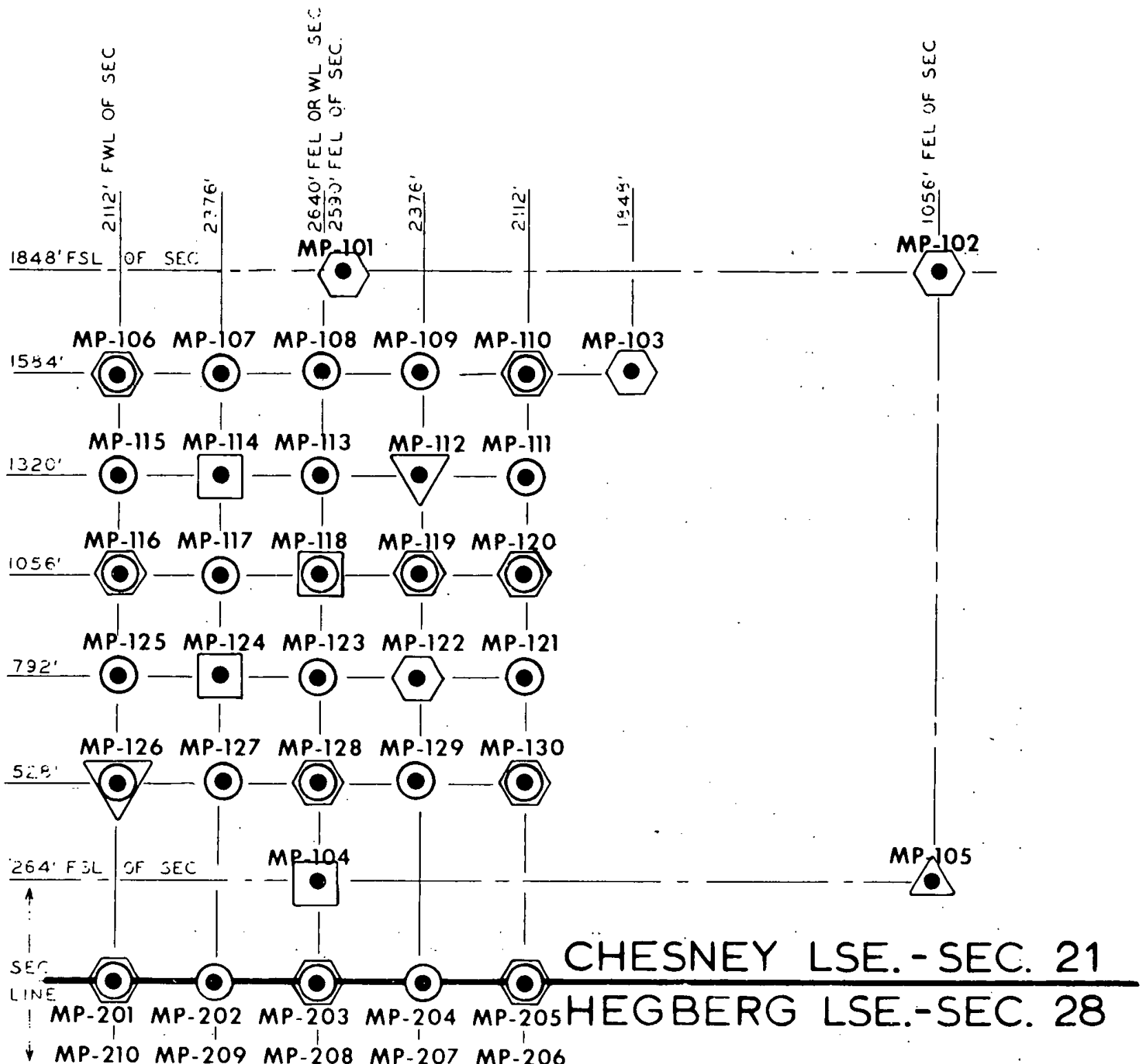

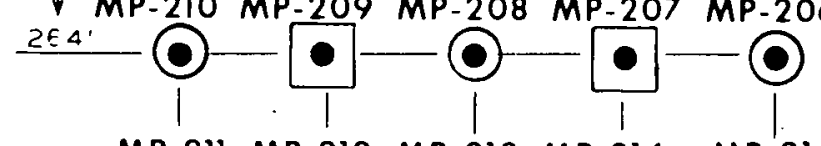

$O_{1}^{M P-211} M P-212 M P-213 \quad M P-214$

MF MP-220 MP-219 MP-218 MP-217 MP-216

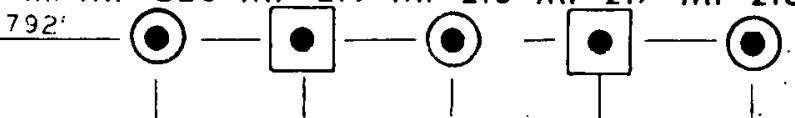

MP-221 MP-222 MP-223 MP-224 MP-225 $\frac{105 E^{\prime}}{F_{1 i}}(0-0-0$ OF JER

\section{LEGEND}

CONVENTIONAL CORES

$\square$ NATIVE-STATE CORES

$\nabla$ ORIENTED CORES

$\triangle$ LOW FLUID LOSS CORE

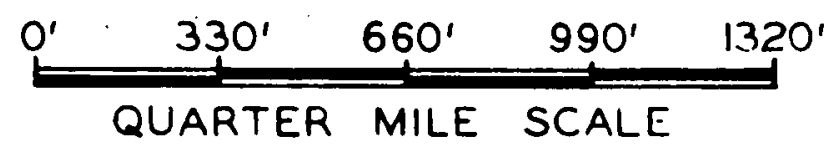


FIGURE B-2

ROUTINE CORE ANALYSIS FOR WELL IIP-101

PERMEABILITY X-.-X $\times 10^{-1}$

WATER SATURATION, \%PV $X---X$
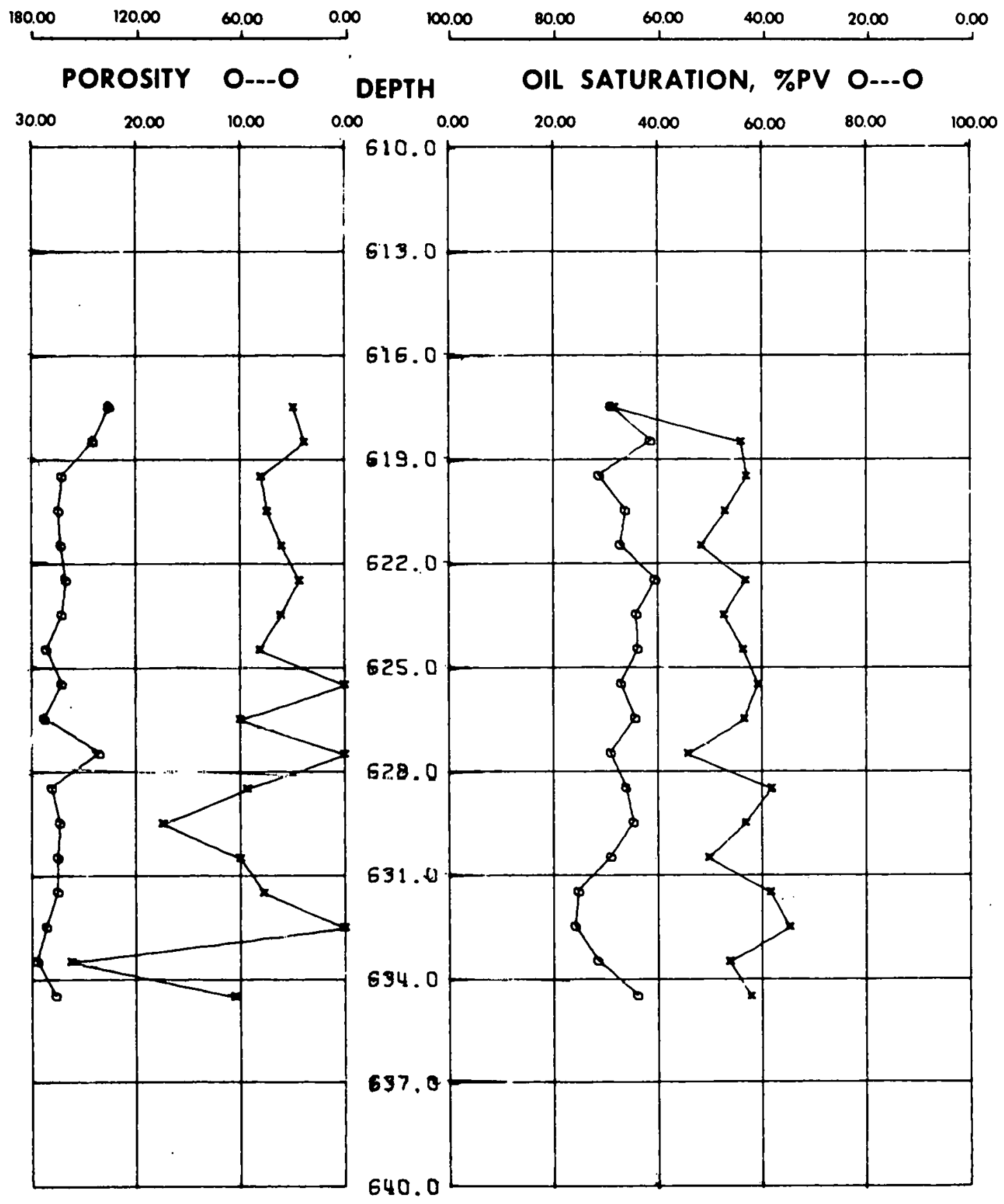
FIGURE $B-3$

ROUTINE CORE ANALYSIS FOR HELL HP-102
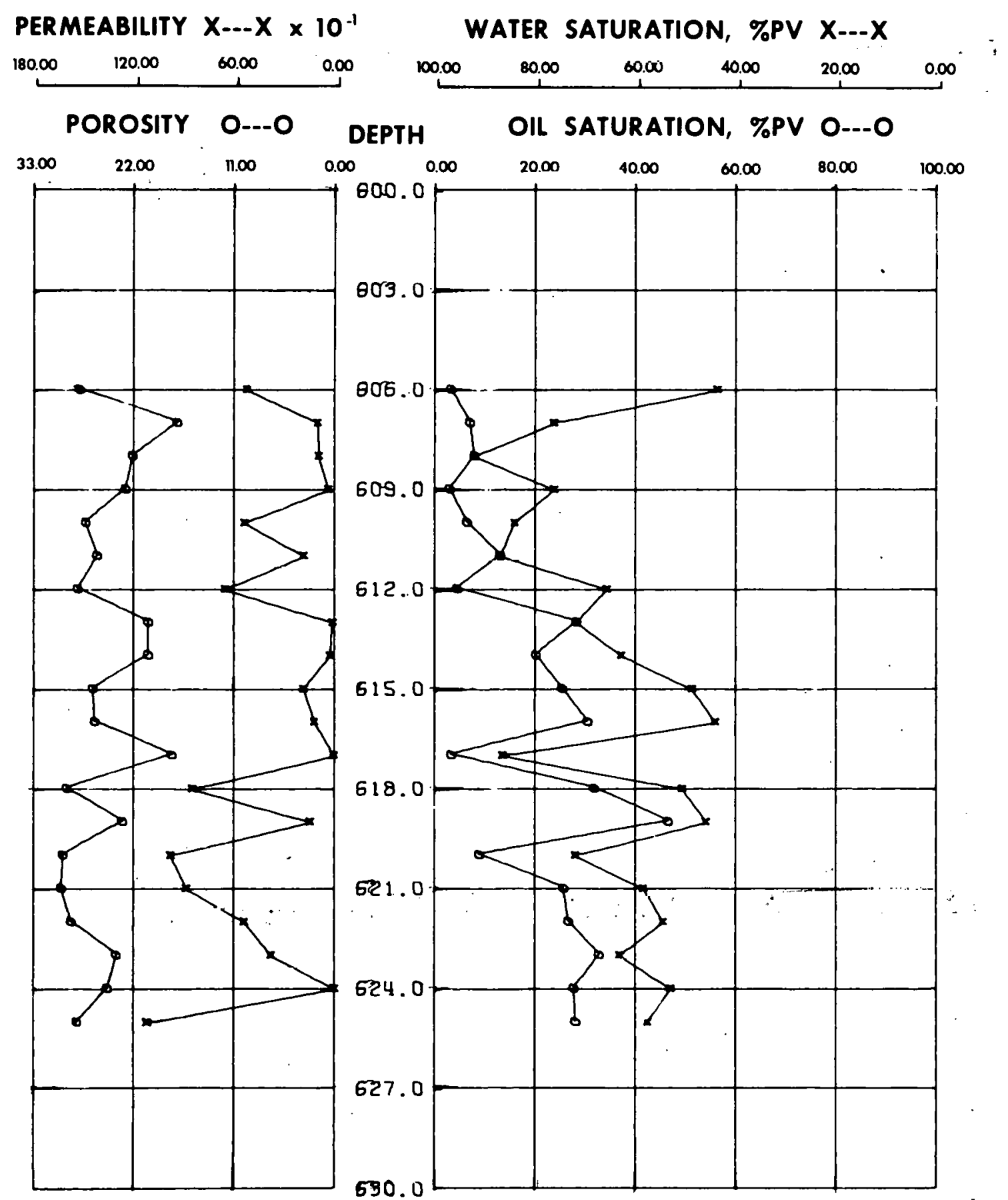
FIGURE B-4

ROUTINE CORE ANALYSIS FOR HELL IIP-103
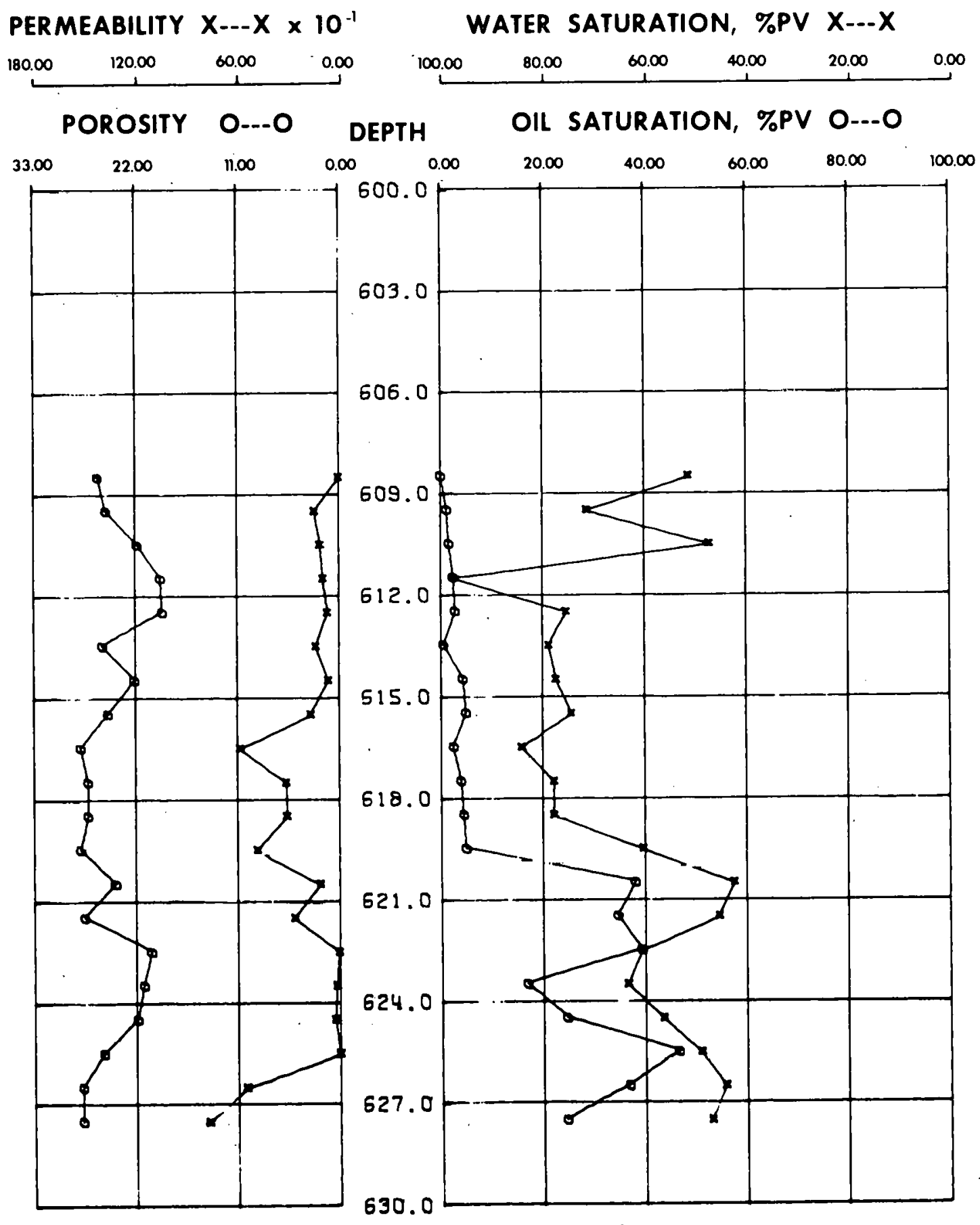
FIGURE B-5

ROUTINE CORE ANALYSIS FOR HELL IIP-104

PERMEABILITY $X---X \times 10^{-1}$

WATER SATURATION, \%PV $X---X$
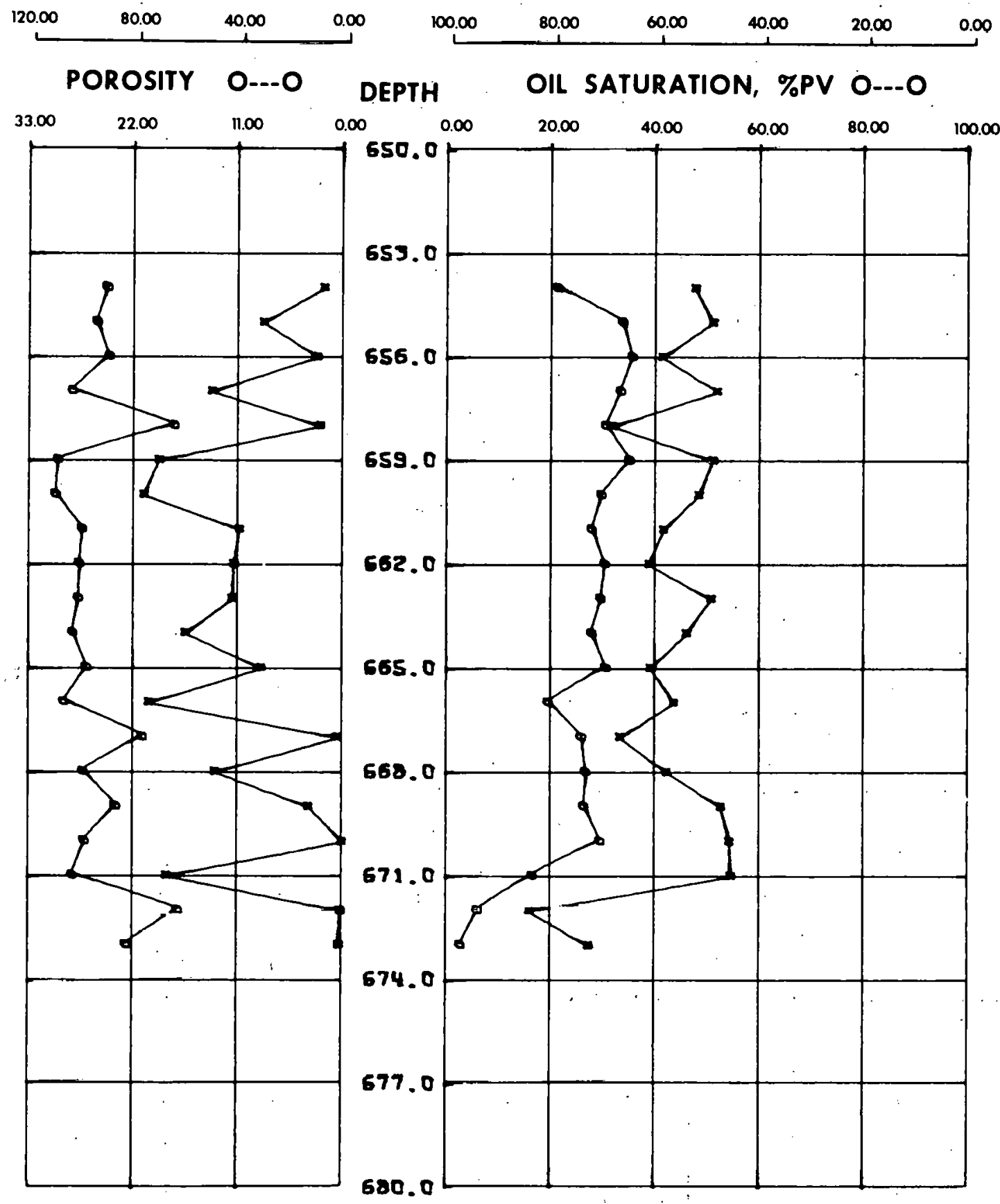
FIGURE B-6

ROUTINE CORE ANALYSIS FOR WELL MP-105

PERMEABILITY $X---X \times 10^{-1}$ WATER SATURATION, \%PV $X---X$

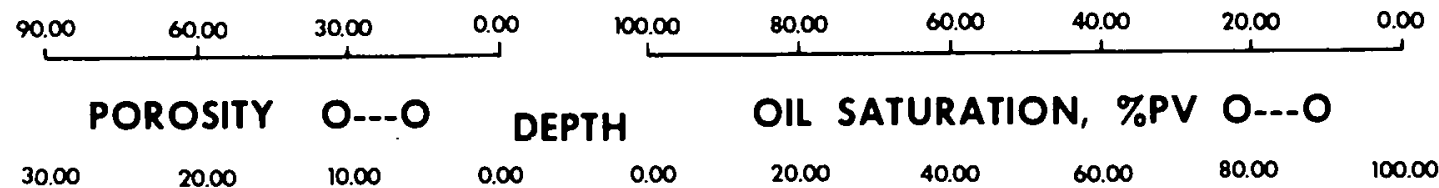

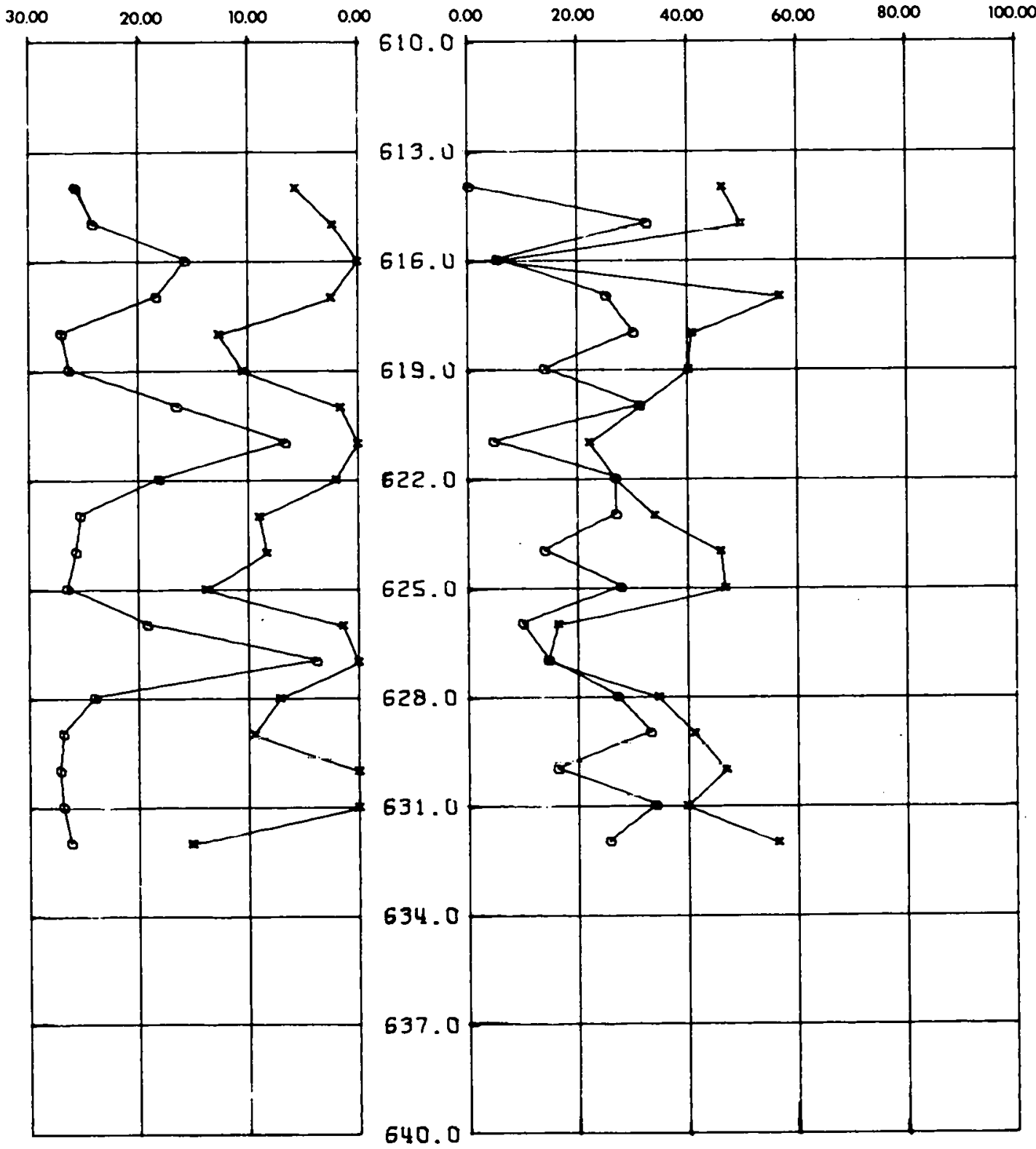


FIGURE $B-7$

ROUTINE CORE ANALYSIS FOR HELL I.P-106

PERMEABILITY $X-.-X \times 10^{-1}$

WATER SATURATION, \%PV $X---X$
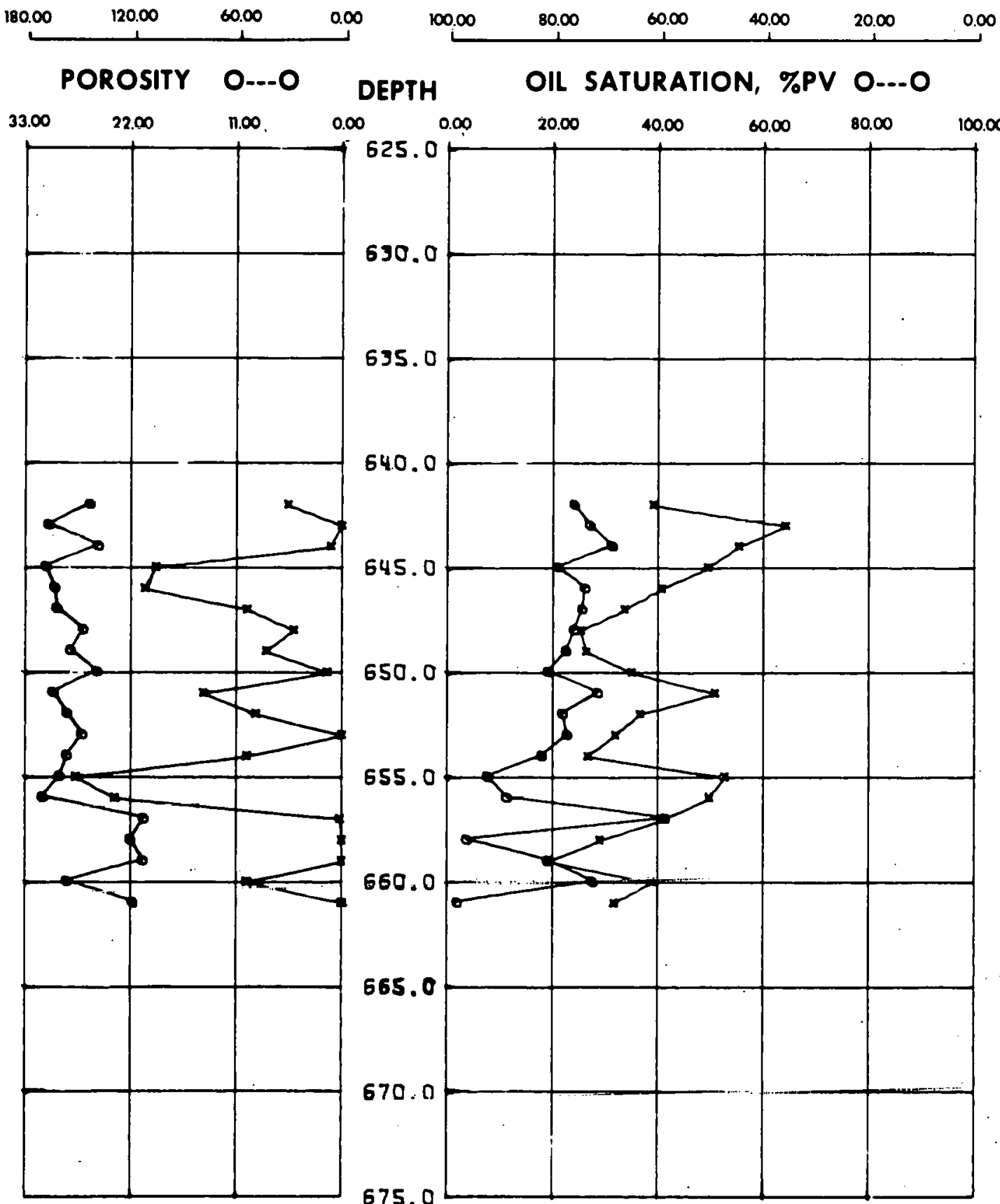
FIGURE B-8

ROUTINE CORE ANALYSIS FOR HELL IIP-IIn

PERMEABILITY $X---X \times 10^{-1}$ WATER SATURATION, \%PV $X---X$
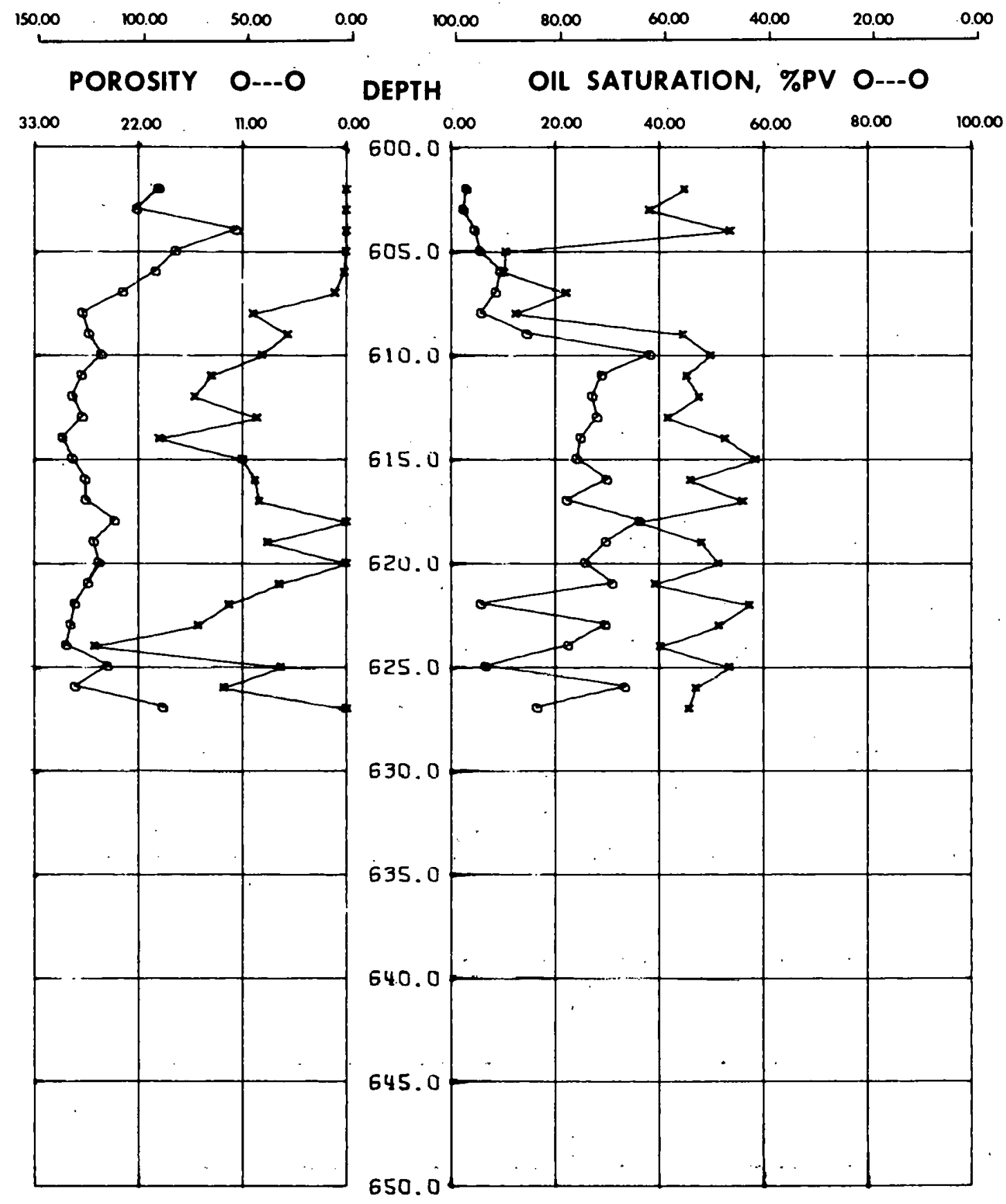
FIGURE B-9

ROUTINE CORE ANALYSIS FOR-HELL MP-116

PERMEABILITY $X---X \times 10^{-1}$

WATER SATURATION, \%PV $X---X$.

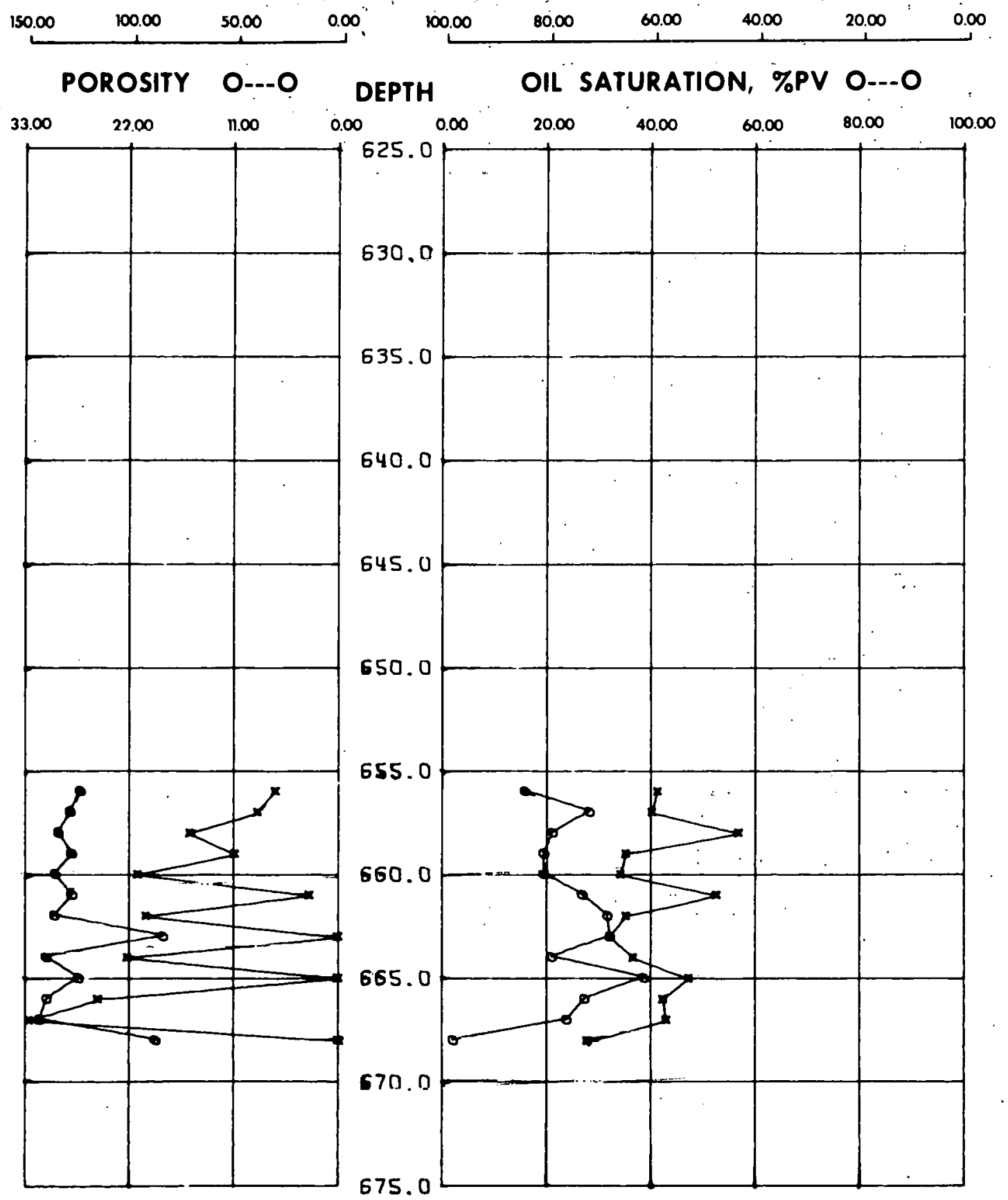


FIGURE B-10

ROUTINE CORE ANALYSIS FOR WELL MP-120

PERMEABILITY $X-\cdots X \times 10$

WATER SATURATION, \%PV $X---X$
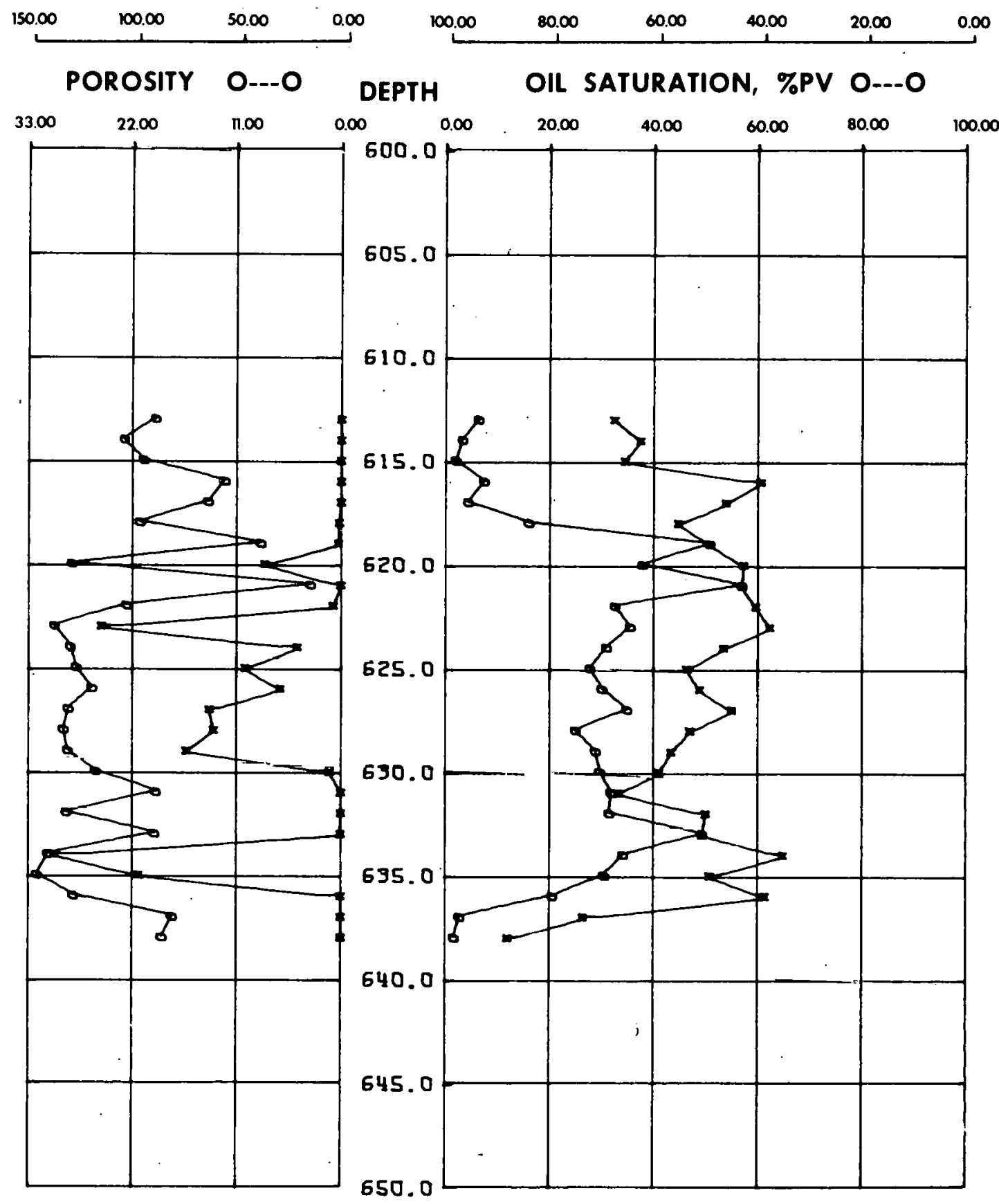
FIGURE B-11

ROUTINE CORE ANALYSIS FOR WELL IIP-122

PERMEABILITY $X---X \times 10^{-1}$

WATER SATURATION, \%PV $X---X$
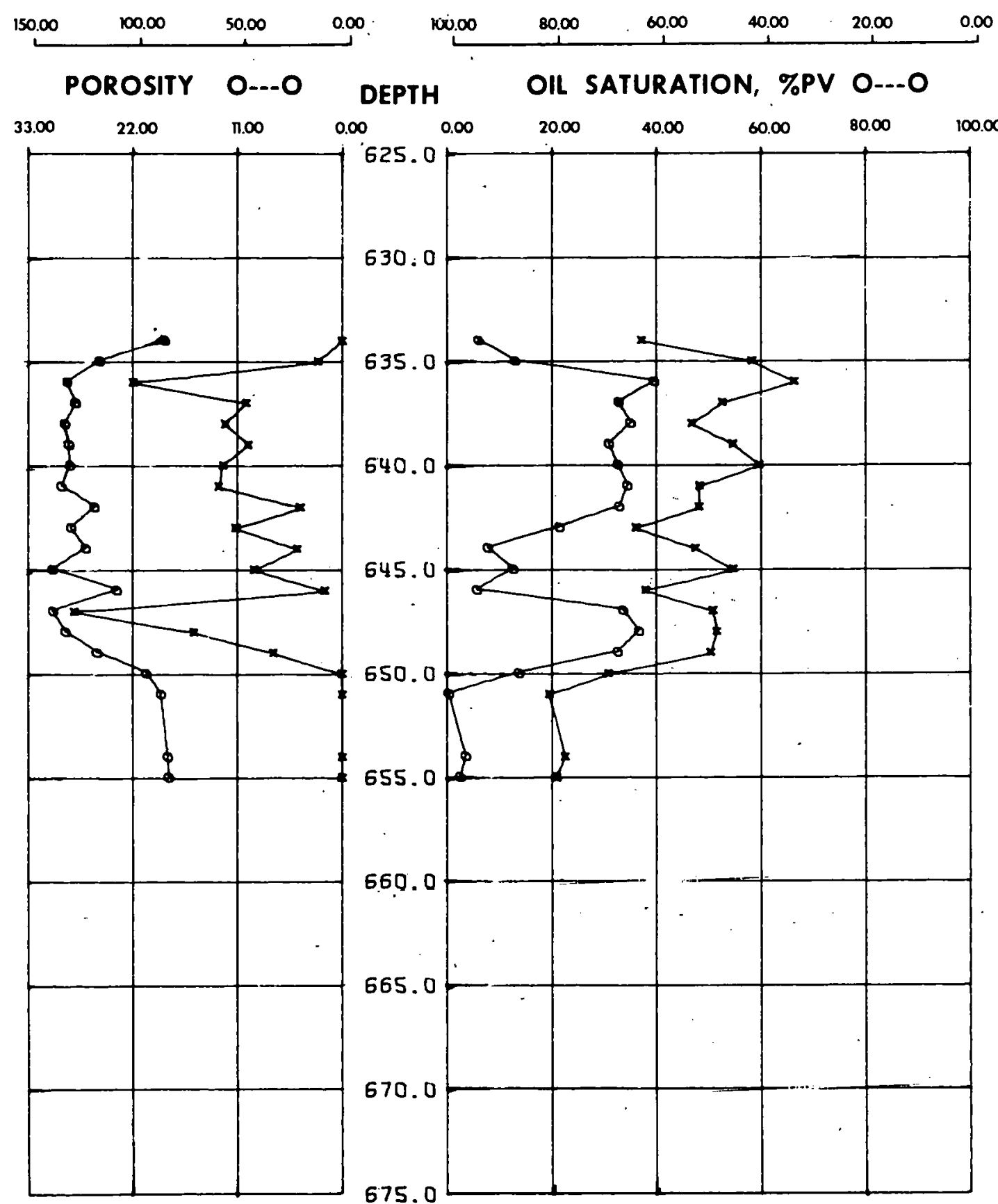
FIGURE B-1.?

ROUTINE CORE NNALYSIS FOR HELL IIP-130

PERMEABILITY $X---X \times 10^{-1}$ WATER SATURATION, \%PV $X---X$

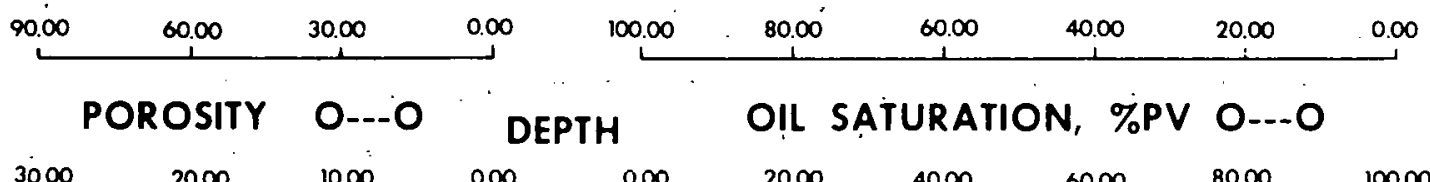
$\begin{array}{llllllllll}30.00 & 20.00 & 10.00 & 0.00 & 0.00 & 20.00 & 40.00 & 60.00 & 80.00 & 100.00\end{array}$

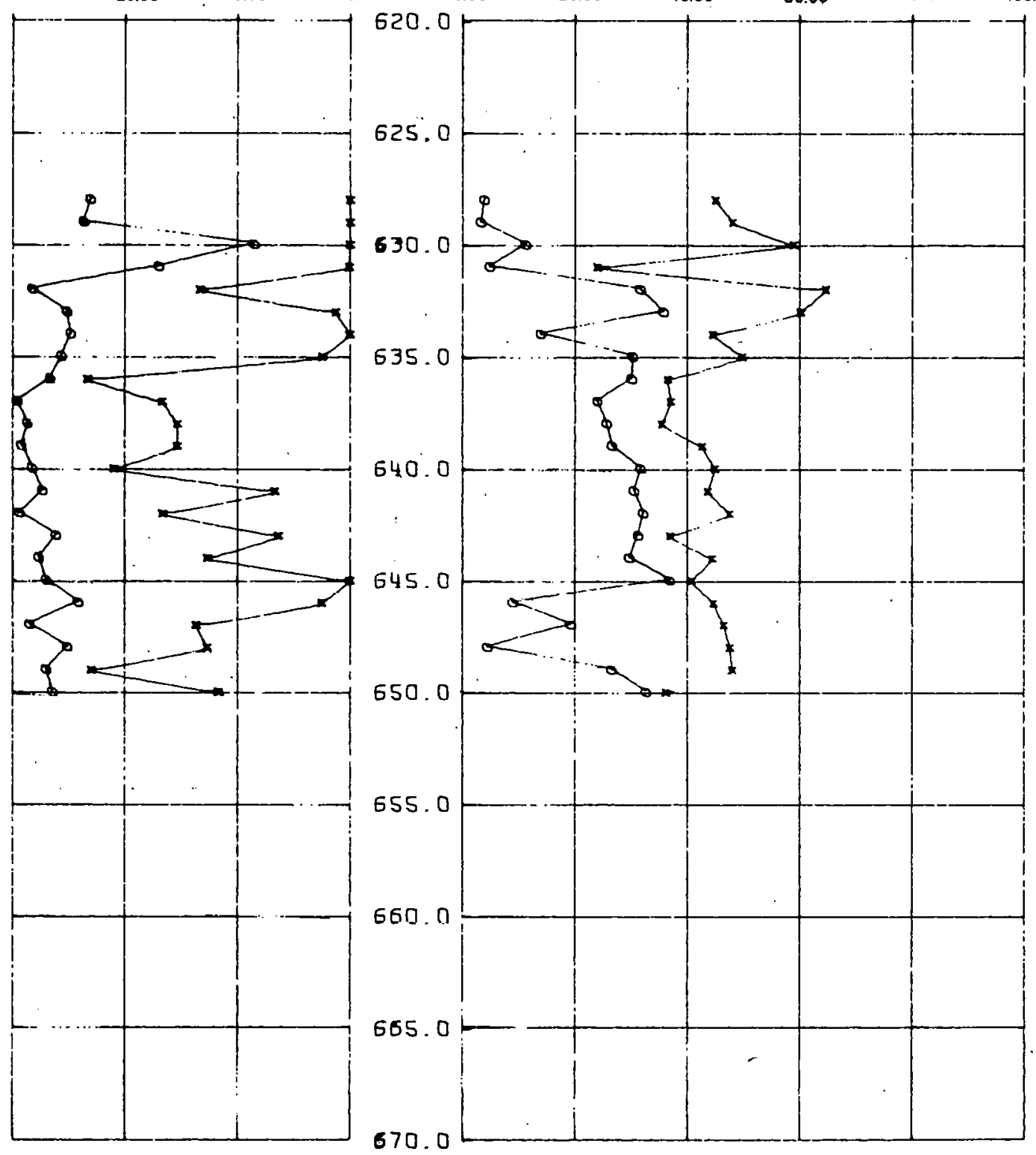


FIGURE, B-13

ROUTINE CORE ANALYSIS FOR HELL PIP-201

PERMEABILITY $X---X \times 10^{-1}$

WATER SATURATION, \%PV $X---X$
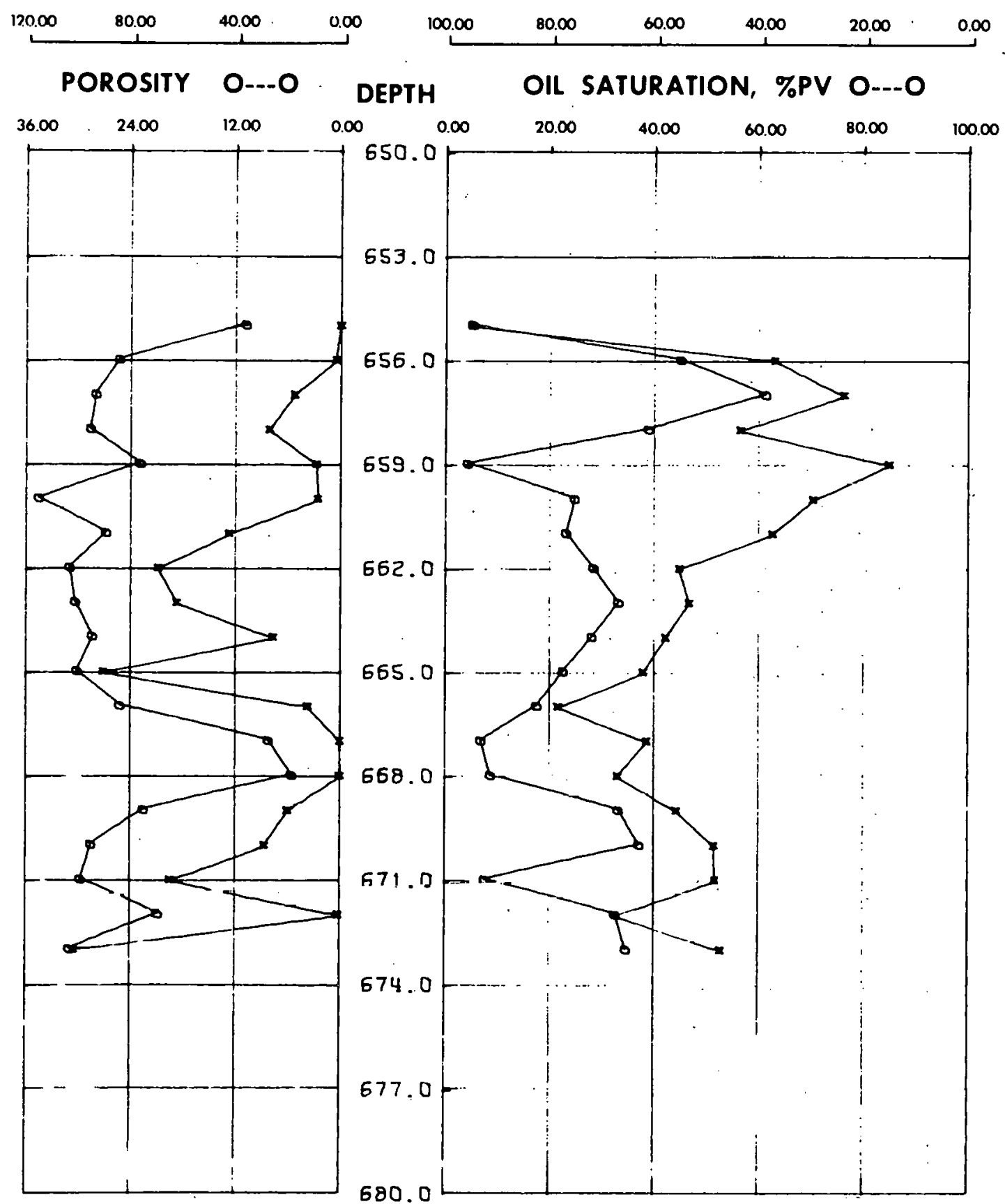
FIGURE B-14

ROUTIHE CORE ANALYSIS FOR HELL IIP-203

PERMEABILITY $X-\cdots \times 10^{-1}$

WATER SATURATION, \%PV $X$-.-X

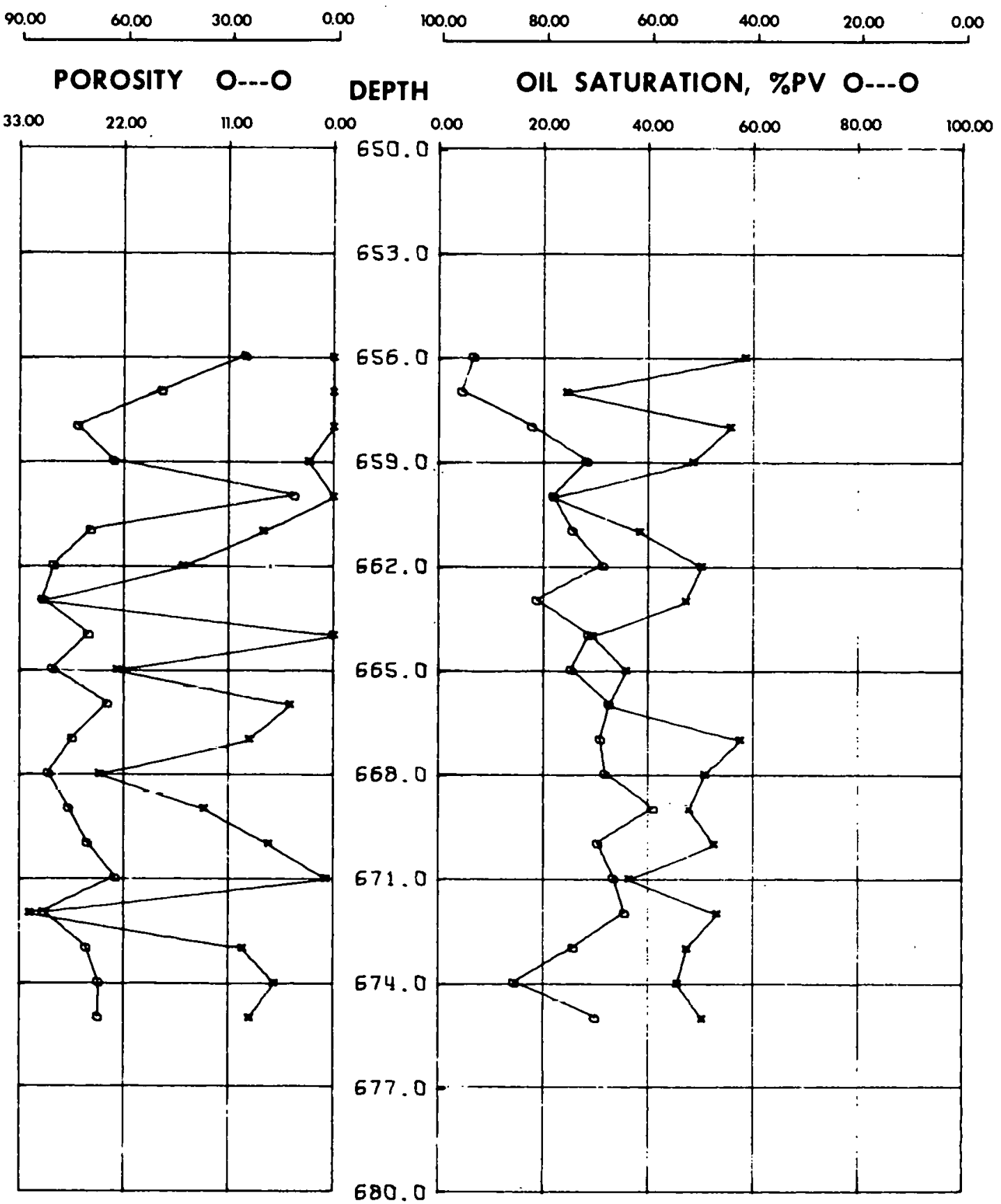


FIGURE B-15

ROUTINE CORE ANALYSIS FOR WELL MP-205

PERMEABILITY $X---X \times 10^{-1}$

WATER SATURATION, \%PV $X---X$
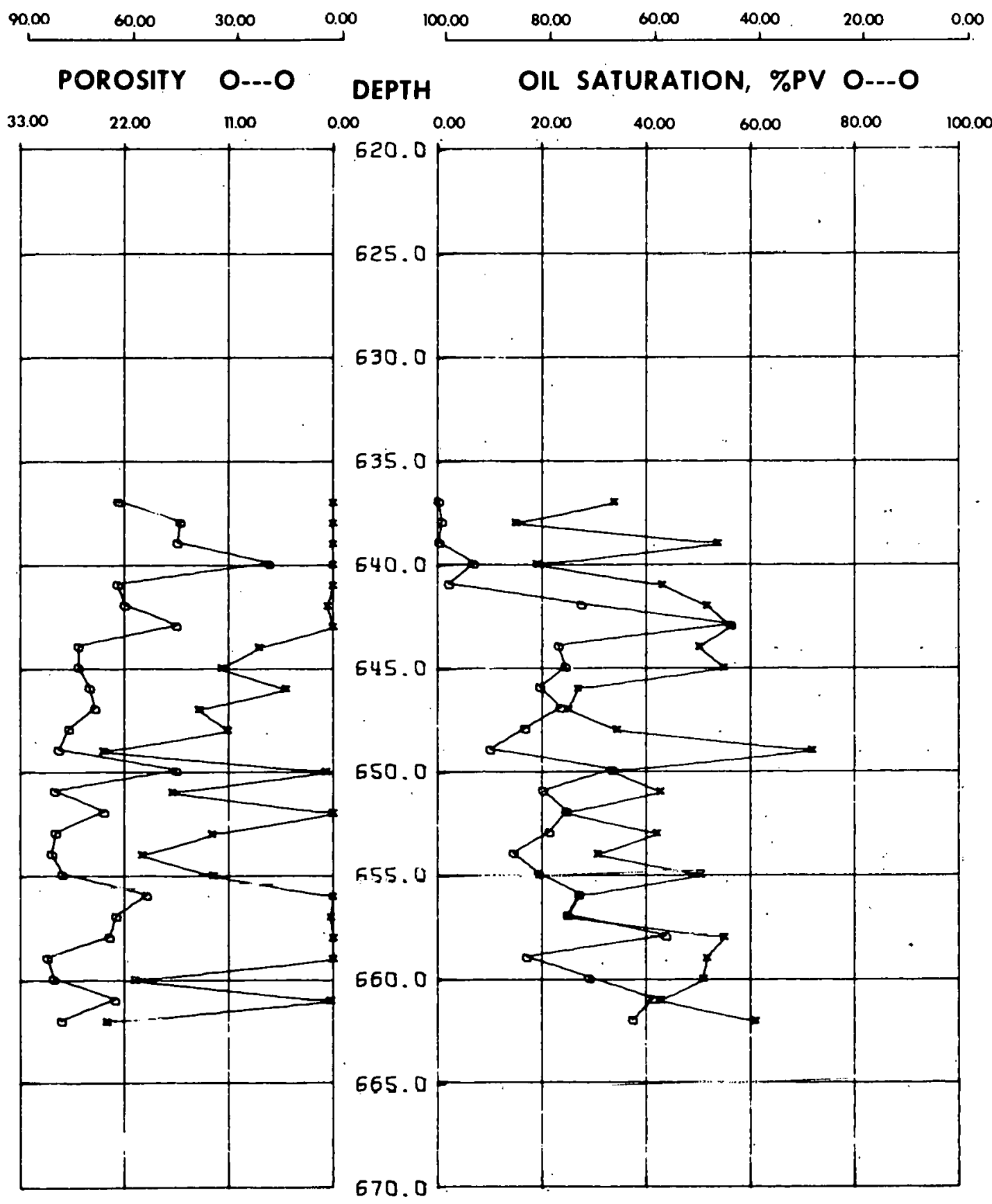
FIGURE B-16

ROUTINE CORE ANALYSIS FOR WELL IIP-213

PERMEABILITY $X---X \times 10^{-1}$

150.00 $100.00 \quad 50.00 \quad 0.00$

WATER SATURATION, \%PV $X---X$

DEPTH
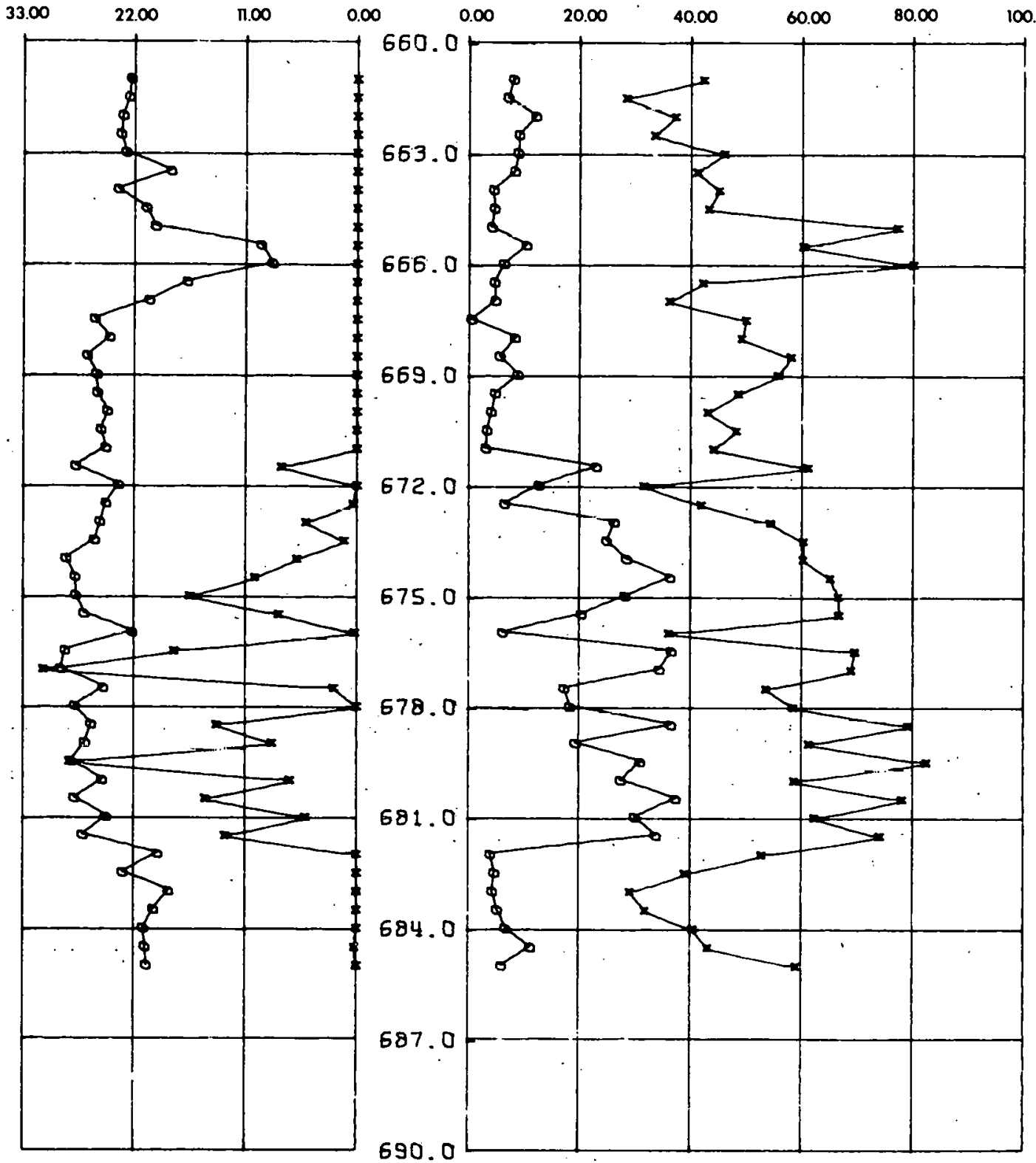
FIGURE B-17

ROUTINE CORE ANALYSIS FOR WELL MP-215
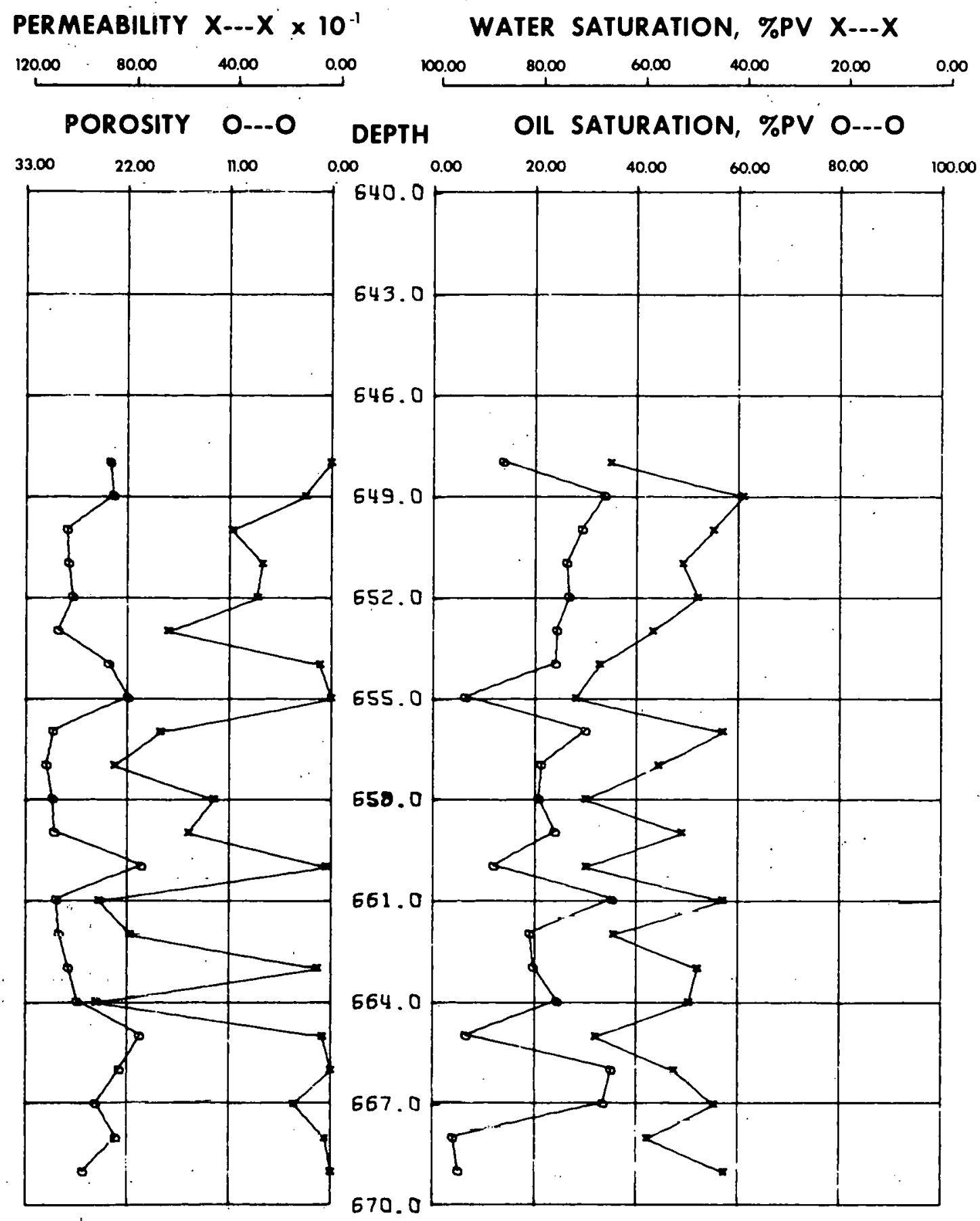
FIGURE B-18

ROUTIHE CORE NHALYSIS FOR HELL IIP-22.1

PERMEABBILITY $X---X \times 10^{-1}$

WATER SATURATION, \%PV $X---X$
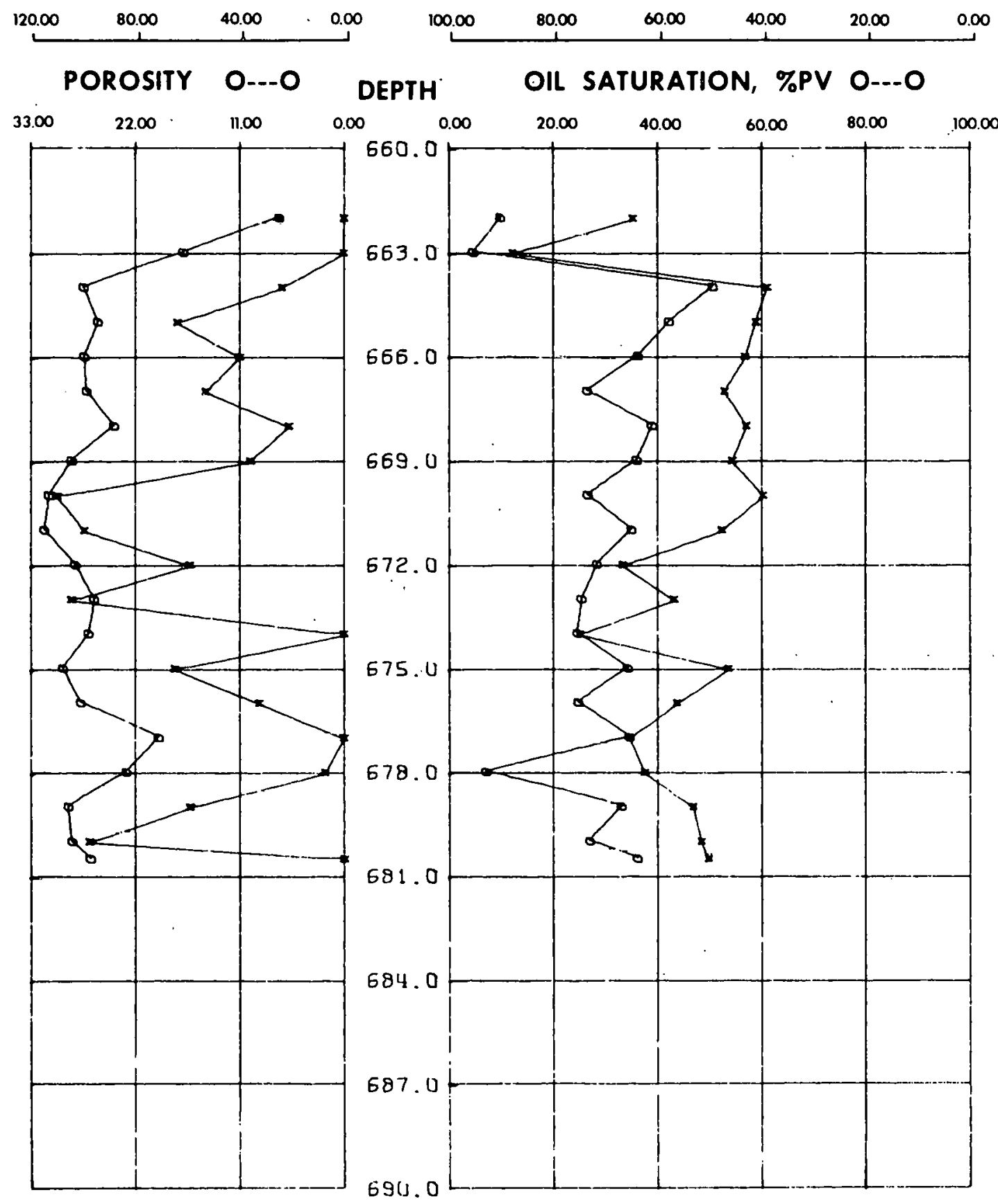
FIGURE B-19

ROUTINE CORE NIINLYSIS FOR WELL I.PP-223

PERMEABILITY $X---X \times 10^{-1}$

WATER SATURATION, \%PV $X---x$
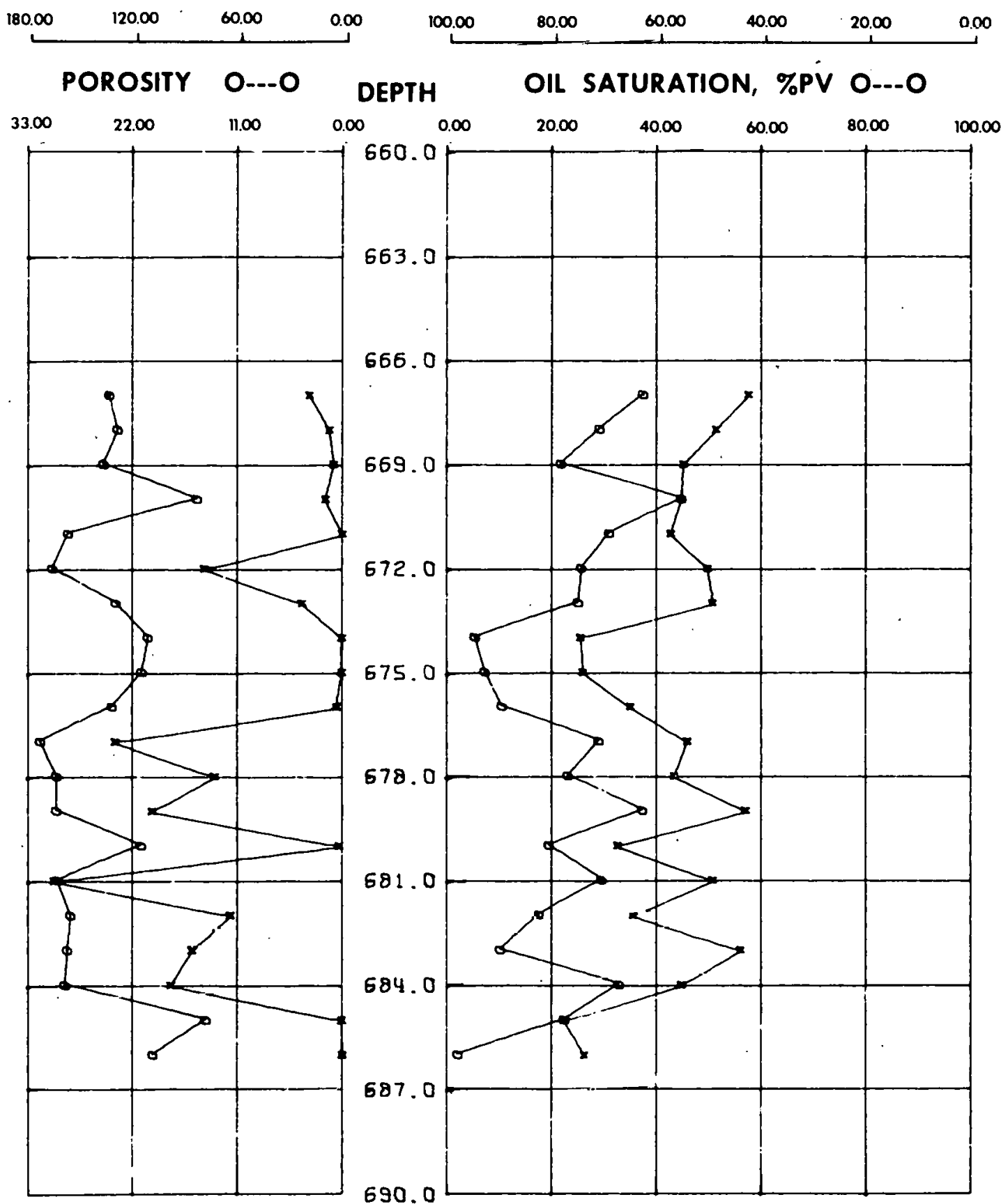
FIGURE $B \cdots 20$

ROUTINE CORE ANALYSIS FOR HELL MP-225

PERMEABILITY $X---X \times 10^{-1}$

WATER SATURATION, \%PV $X---X$
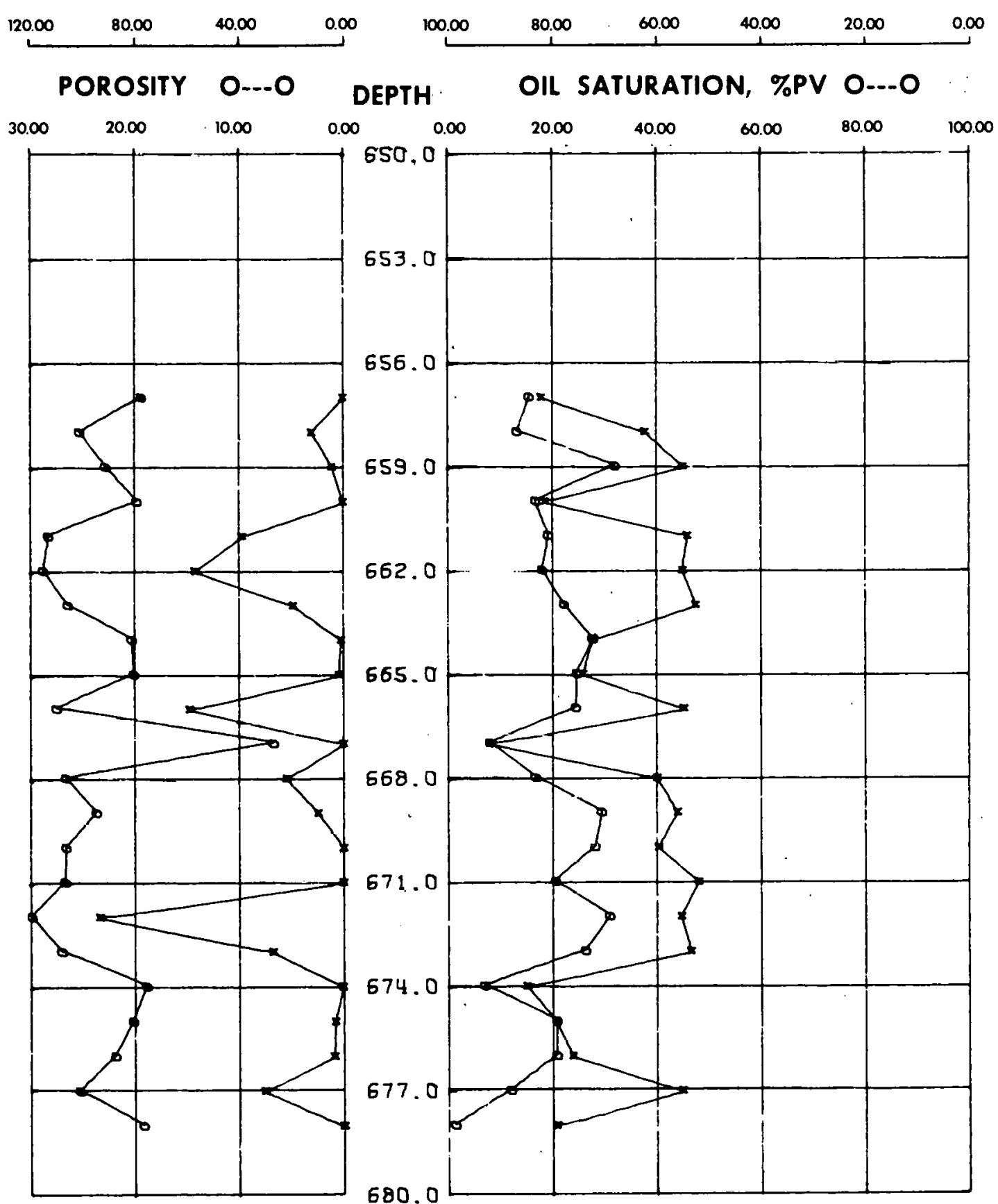
FIGURE B-21

COIITOUR HIAP OF PERIIEABILITY (FRORI CORE

AIIALYSES) FOR EL DORADO PROJECT AREA

101

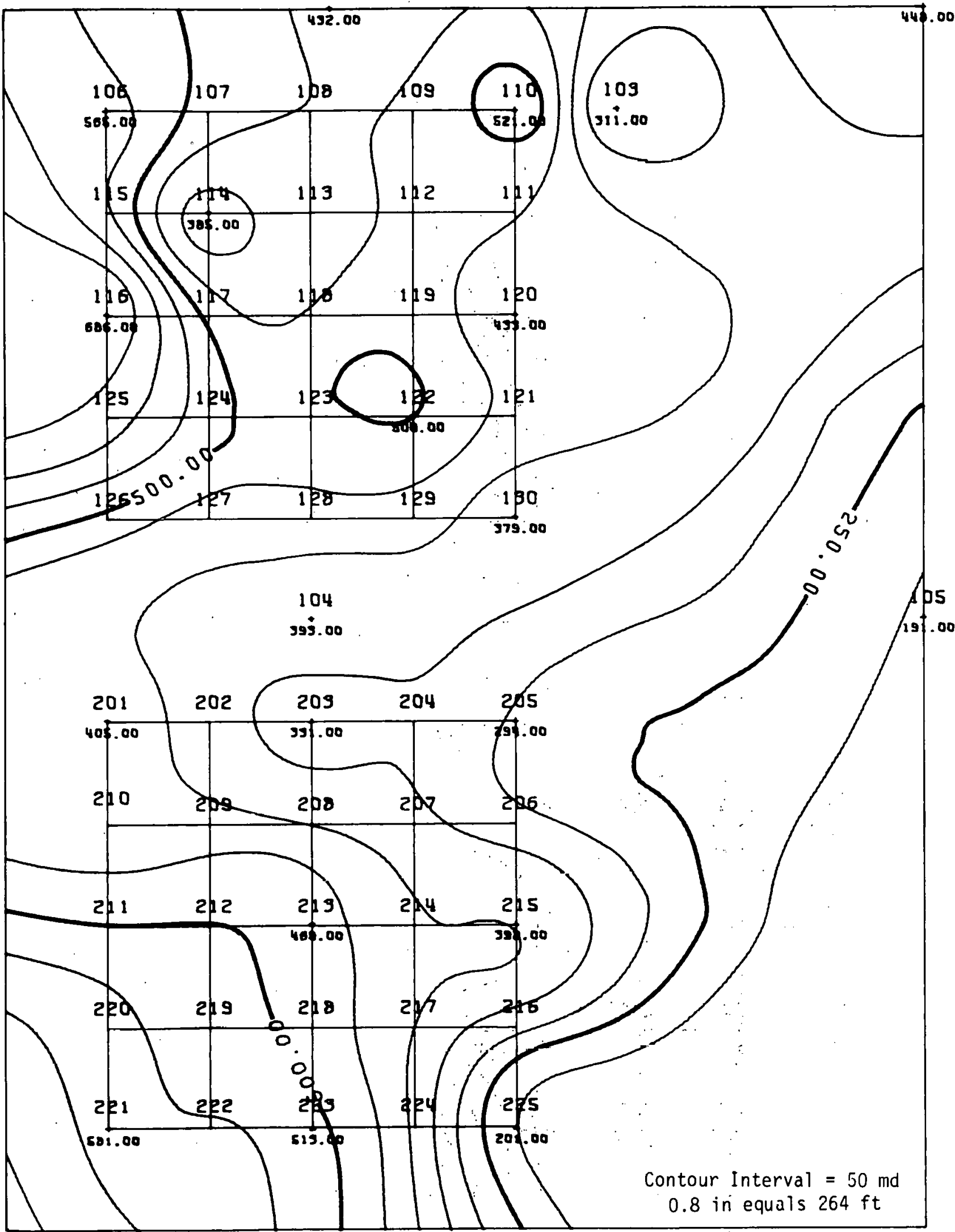


FIGURE B-22

COIITOLR IIAP OF POROSITY (FROH CORE AIAALYSES)

FOR:"El LORAdO PROJECT AREA

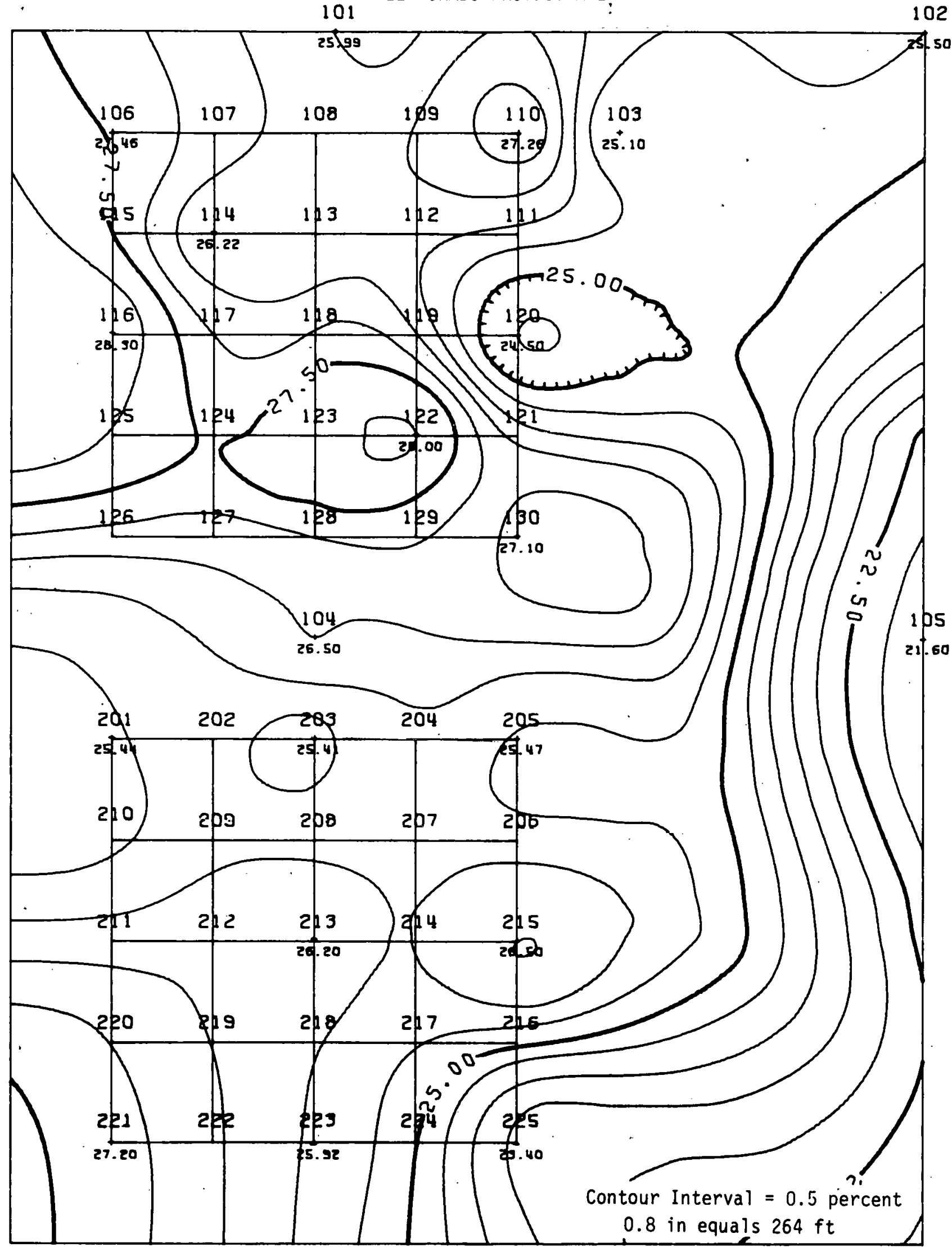


FIGURE B-23

COITTOUR IIAP OF OIL SATURATIOII (FROH CORE

AIIALYSES) FOR EL DORADO PROJECT AREA

101

102

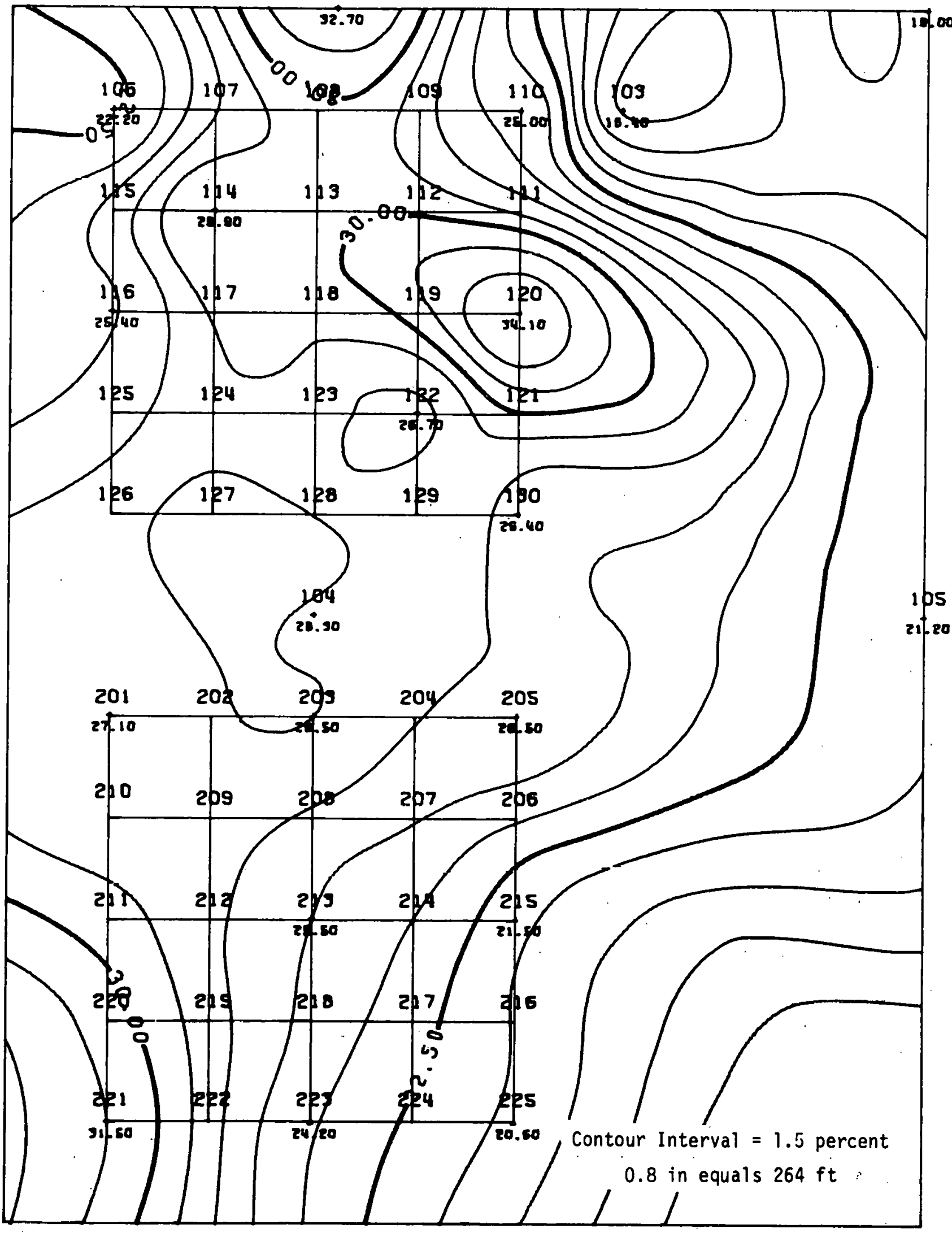


CONTOUR HIAP OF GROSS THICKIIESS (FROH: CORE AiID WELL LOGGING ANALLYSES) FOR EL DORADO

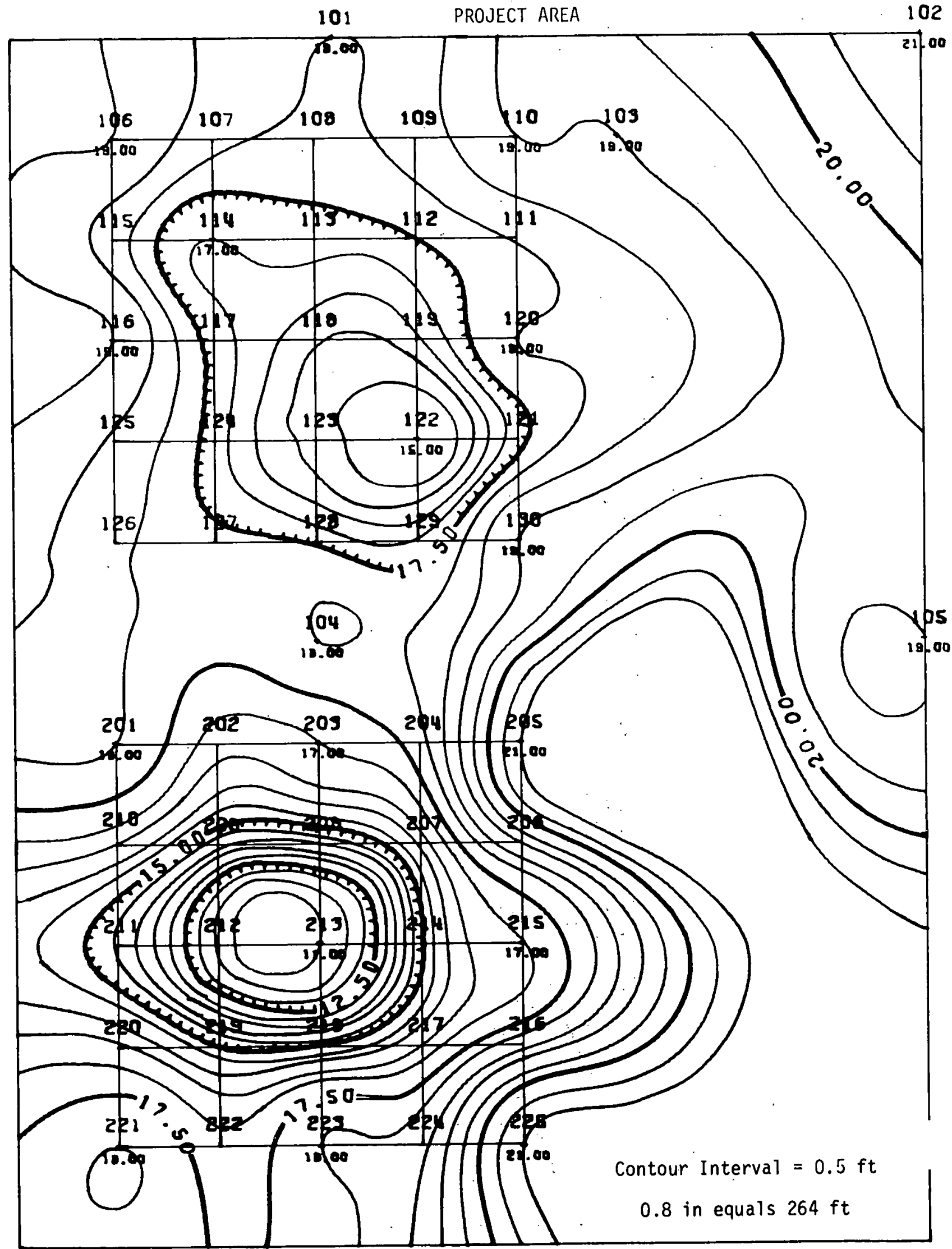


FIGURE B-25

CONTOUR IIAP OF OIL. III PLACE (FROII CORE

AIIALYSES) FOR EL UORADO PROJECT AREA

101

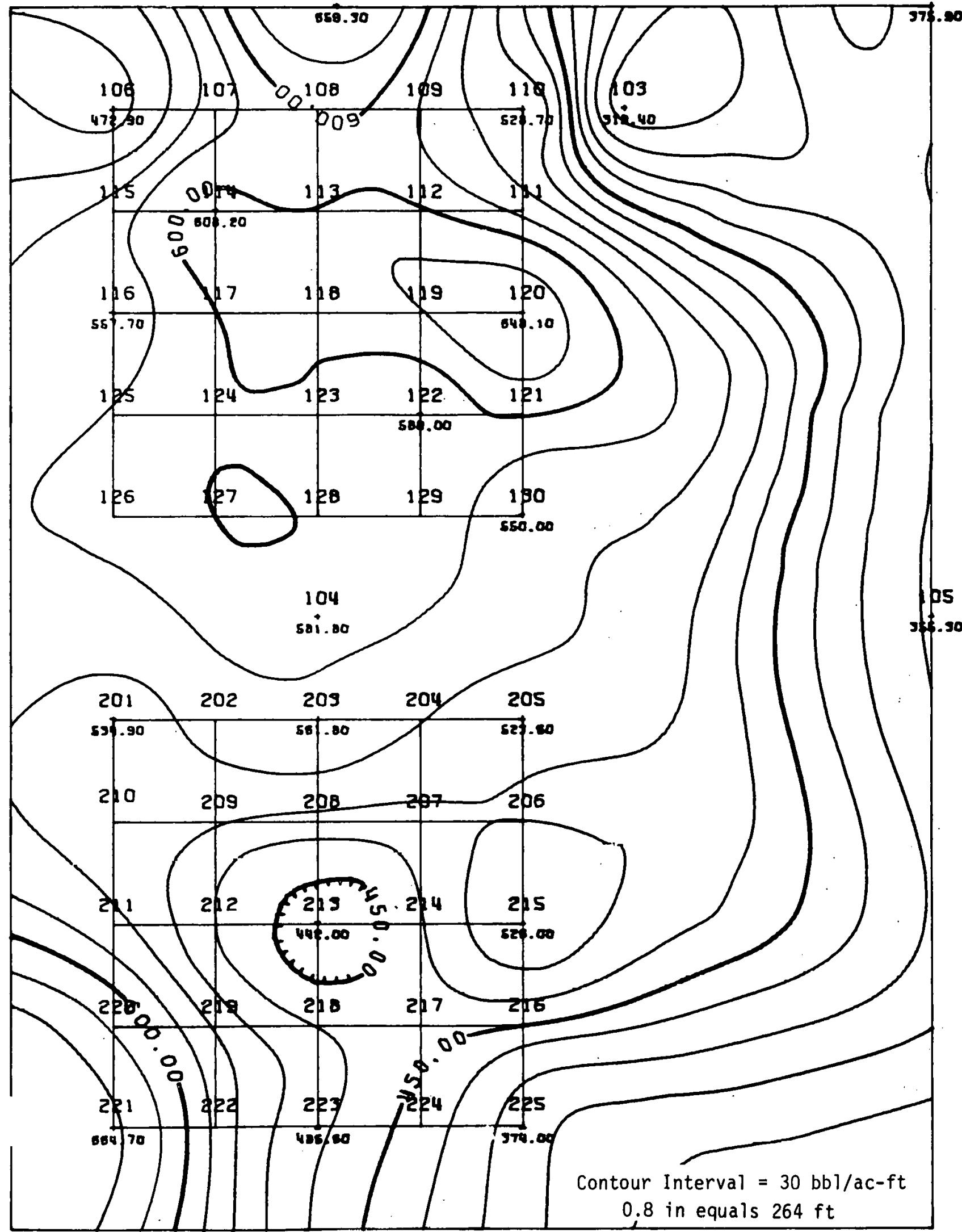


FIGURE B-26

LOGARITHM OF PERNEABILITY VS STANDARD DEVIATION

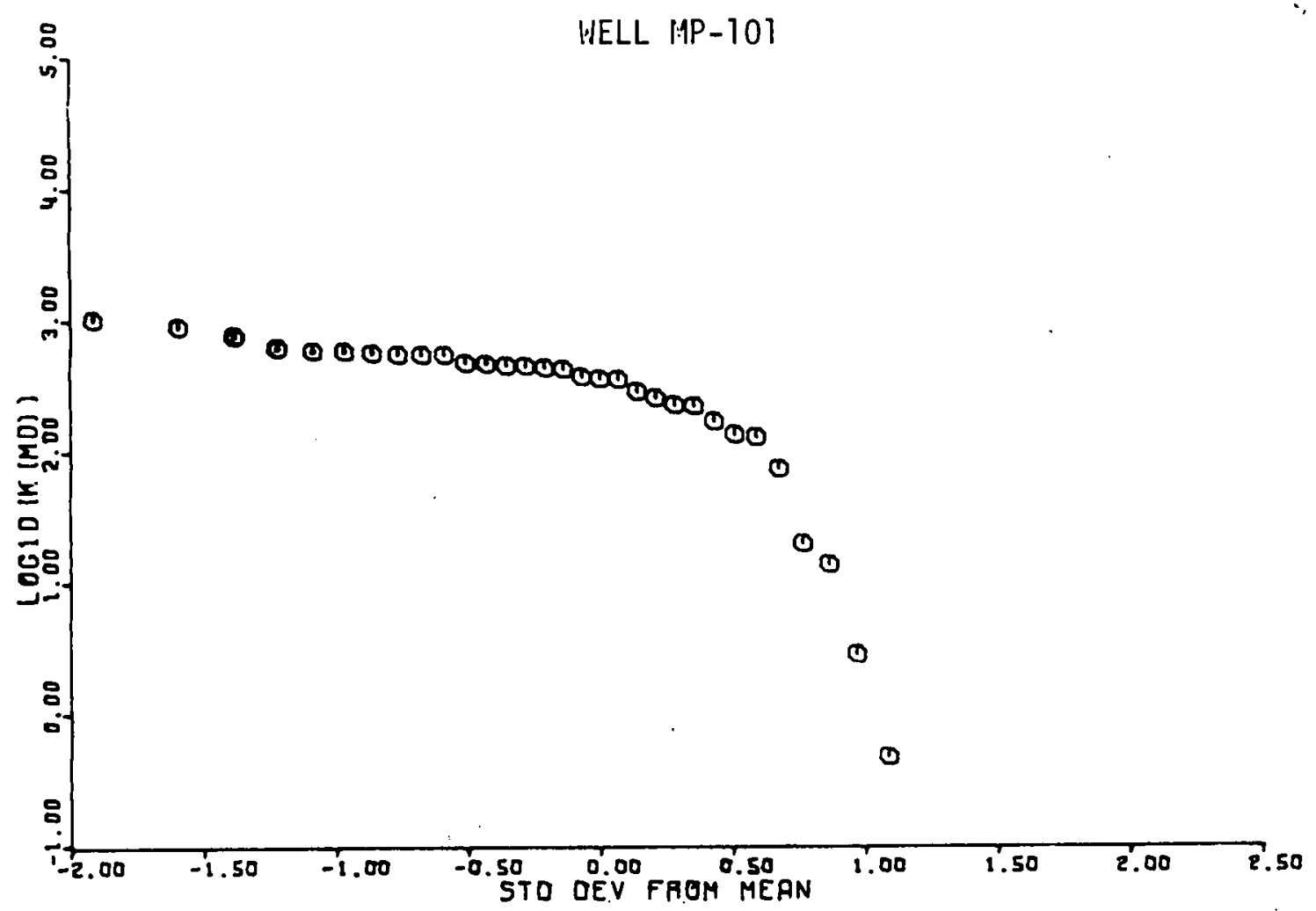

FIGURE B-27

LOGARITHM OF PERMEABILITY VS STANDARD DEVIATION

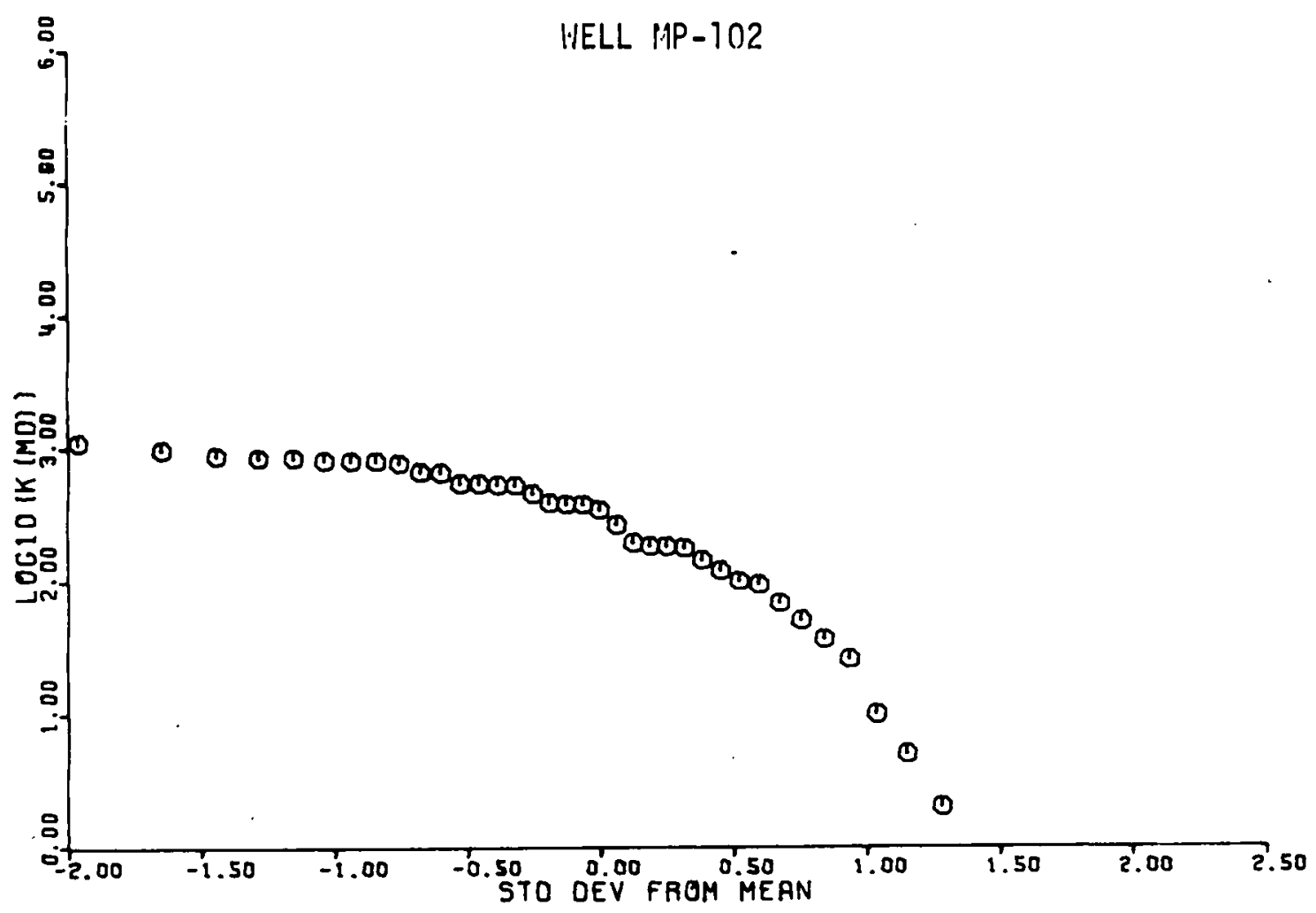


FIGURE B-28

I I -83

LOGARITHM OF PERMEABILITY VS STANDARD DEVIATION

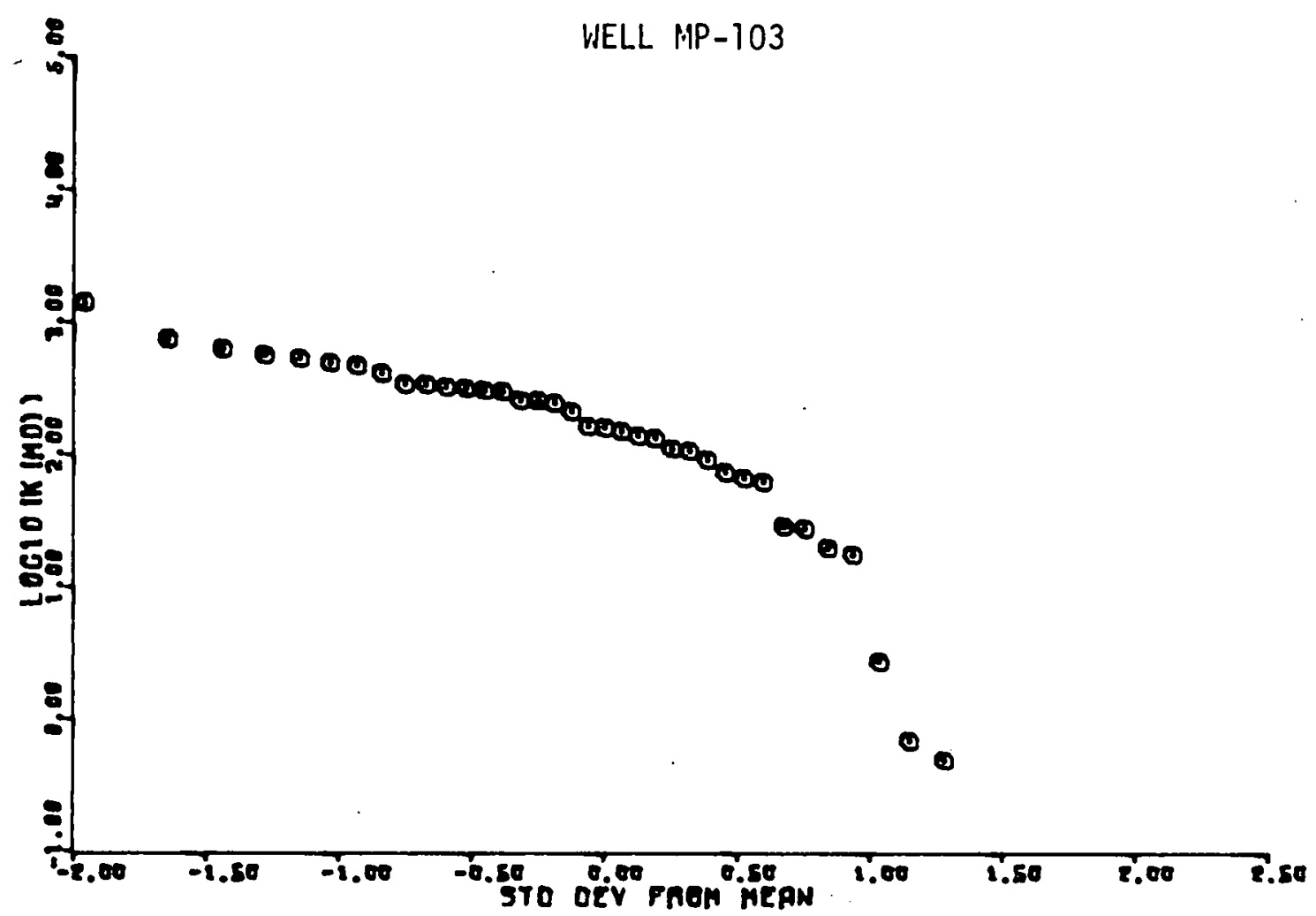

FIGURE B-29

LOGARITHP OF PERMEABILITY VS STANDARD DEVIATION

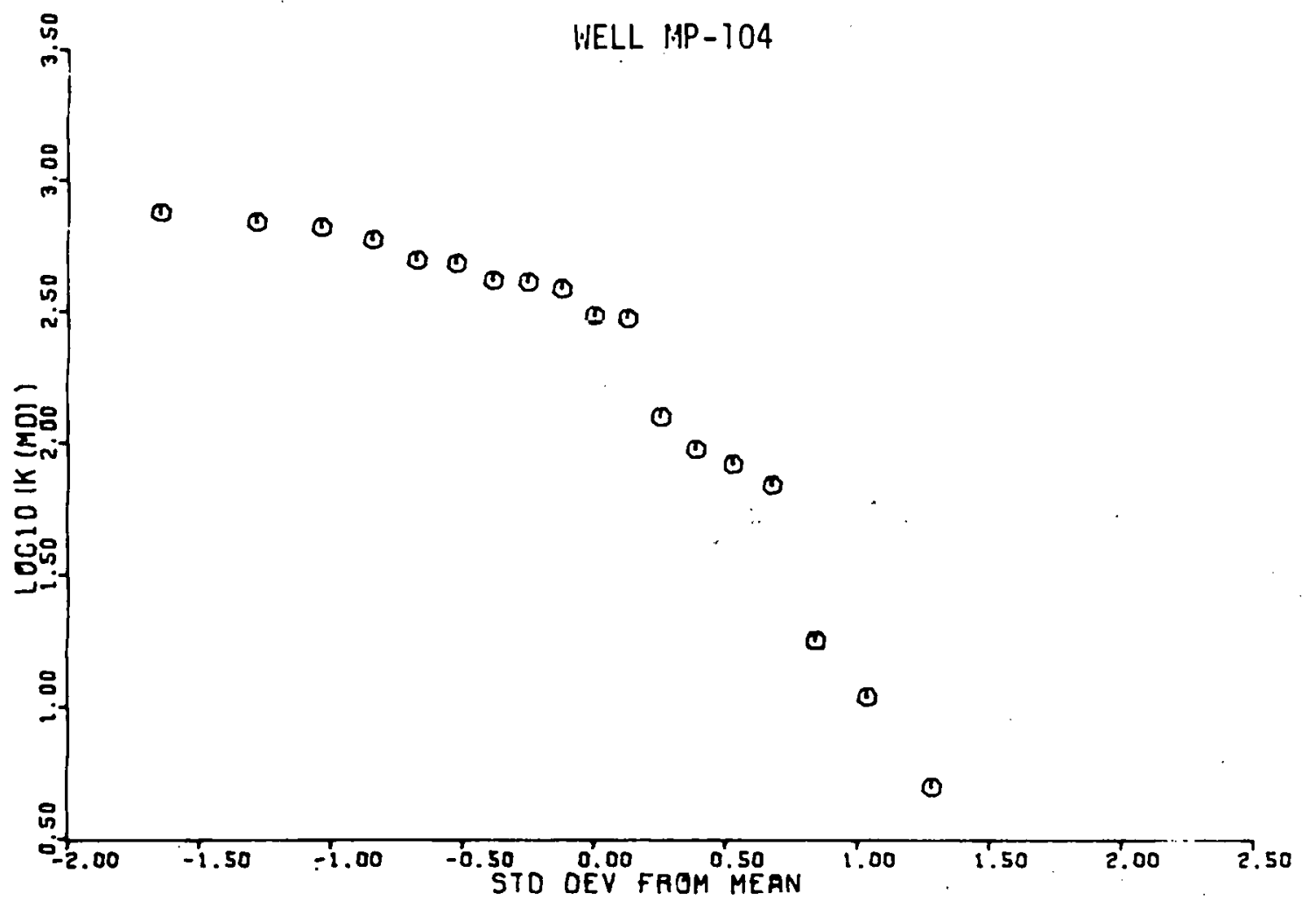


FIGURE B-30

LOGARITHM OF PERIAEABILITYY VS STANDARD DEVIATION

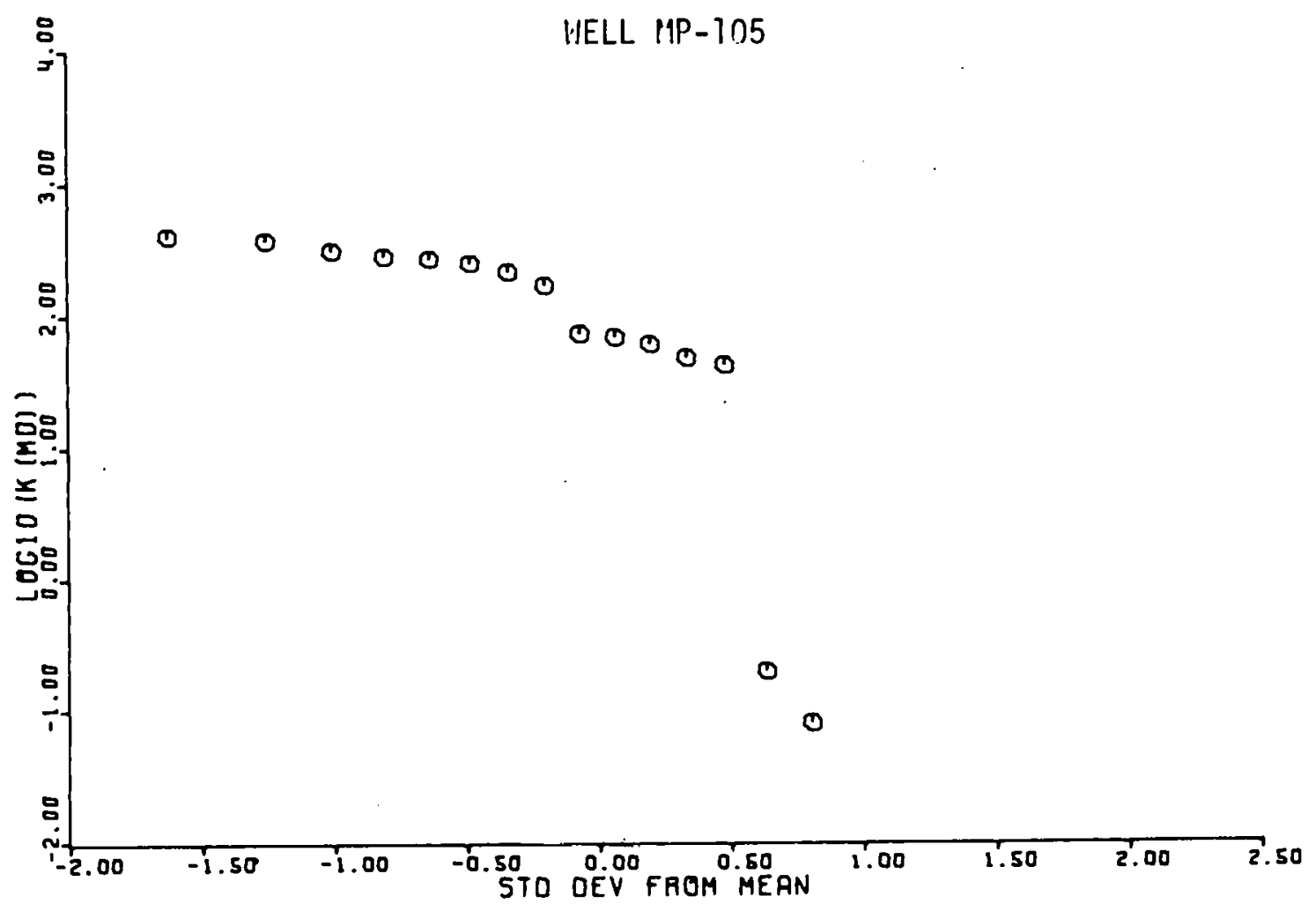

FIGURE B-31

LOGARITHM OF PERIIEABILITY VS. STANDARD DEVIATION

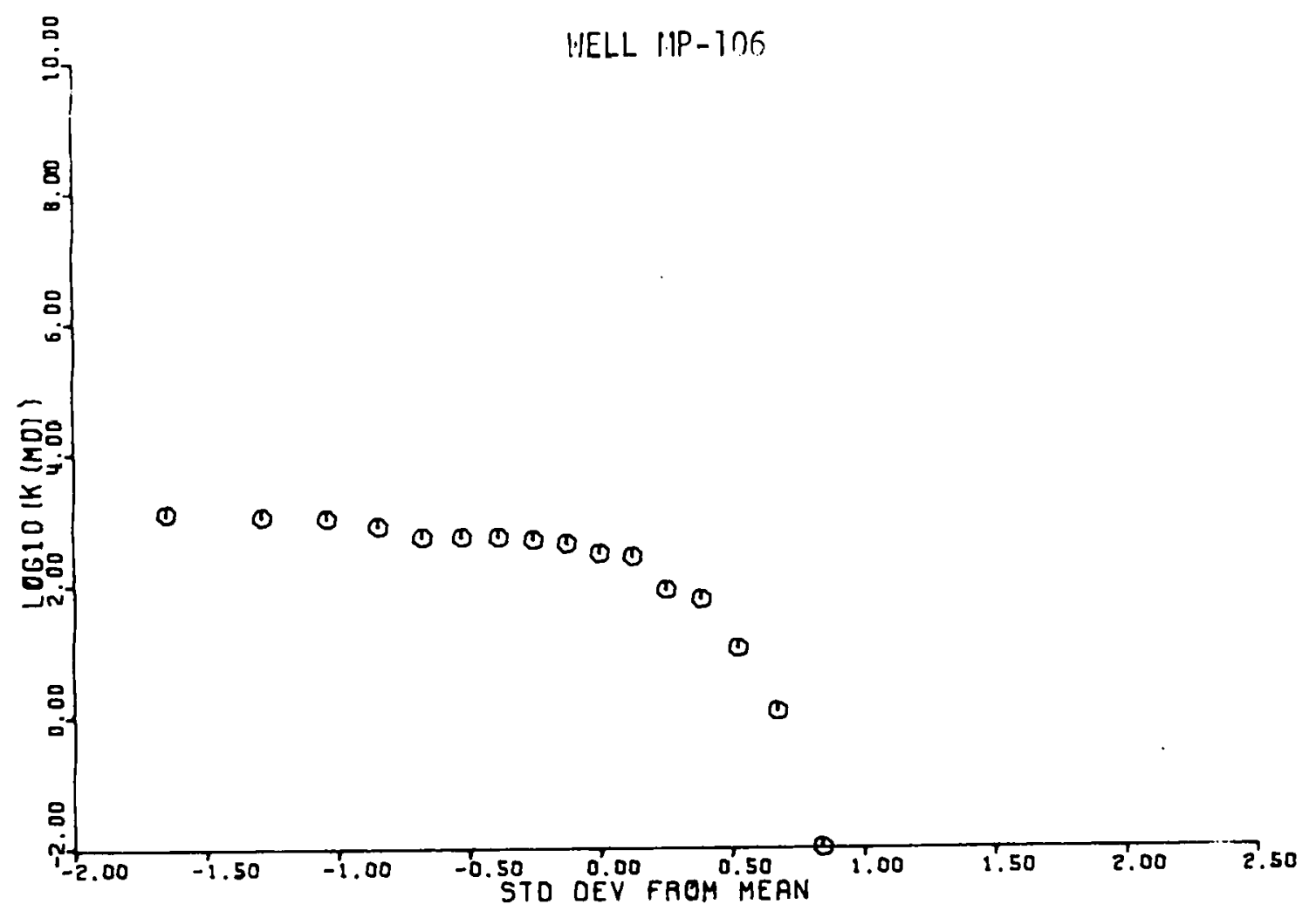


FIGURE B-32

LOGARITHM OF PERMEABILITY VS STANDARD DEVIATION

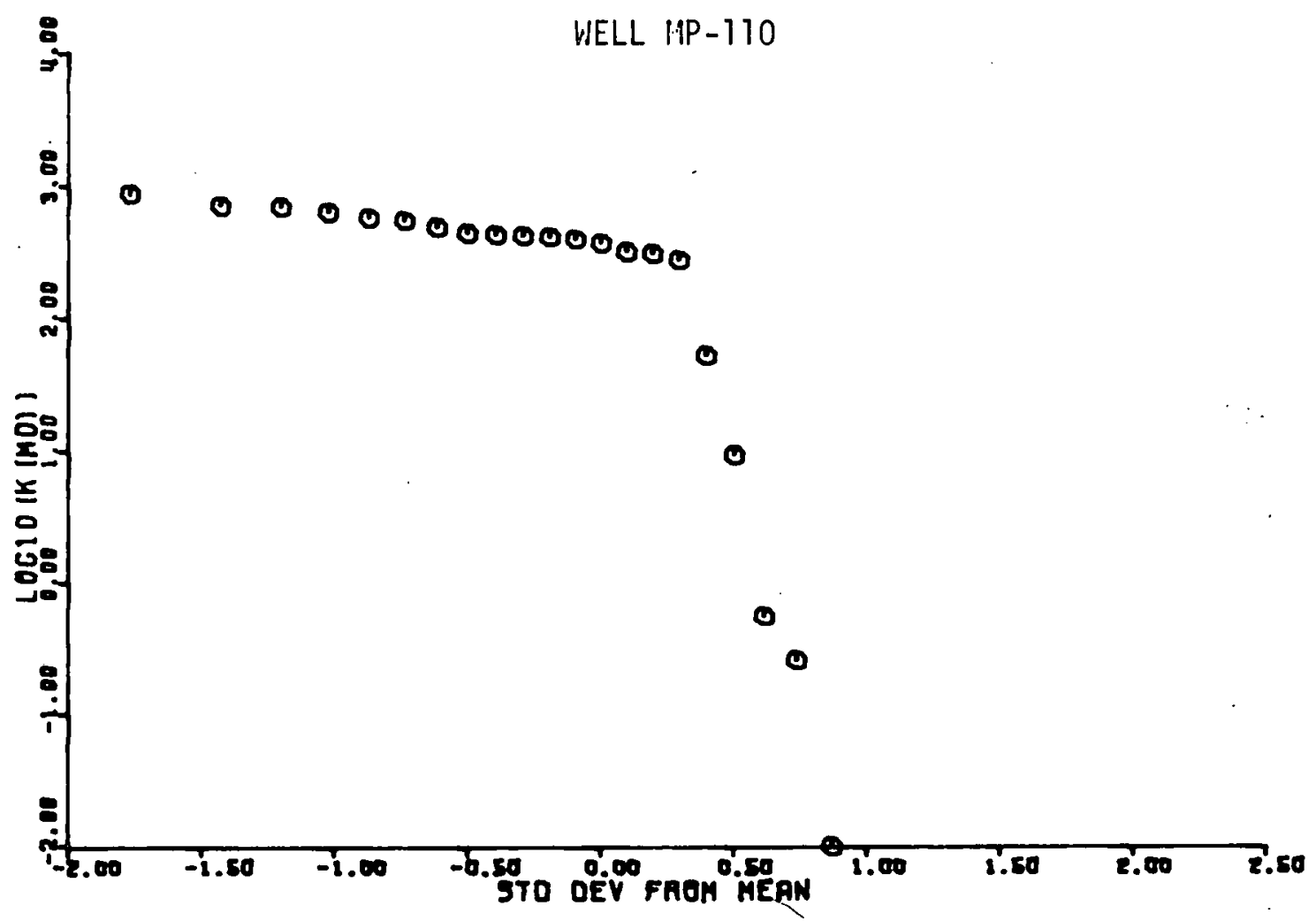

FIGURE $B-33$

LOGARITHII OF PERMEABILITY VS STANDARD DEVIATION

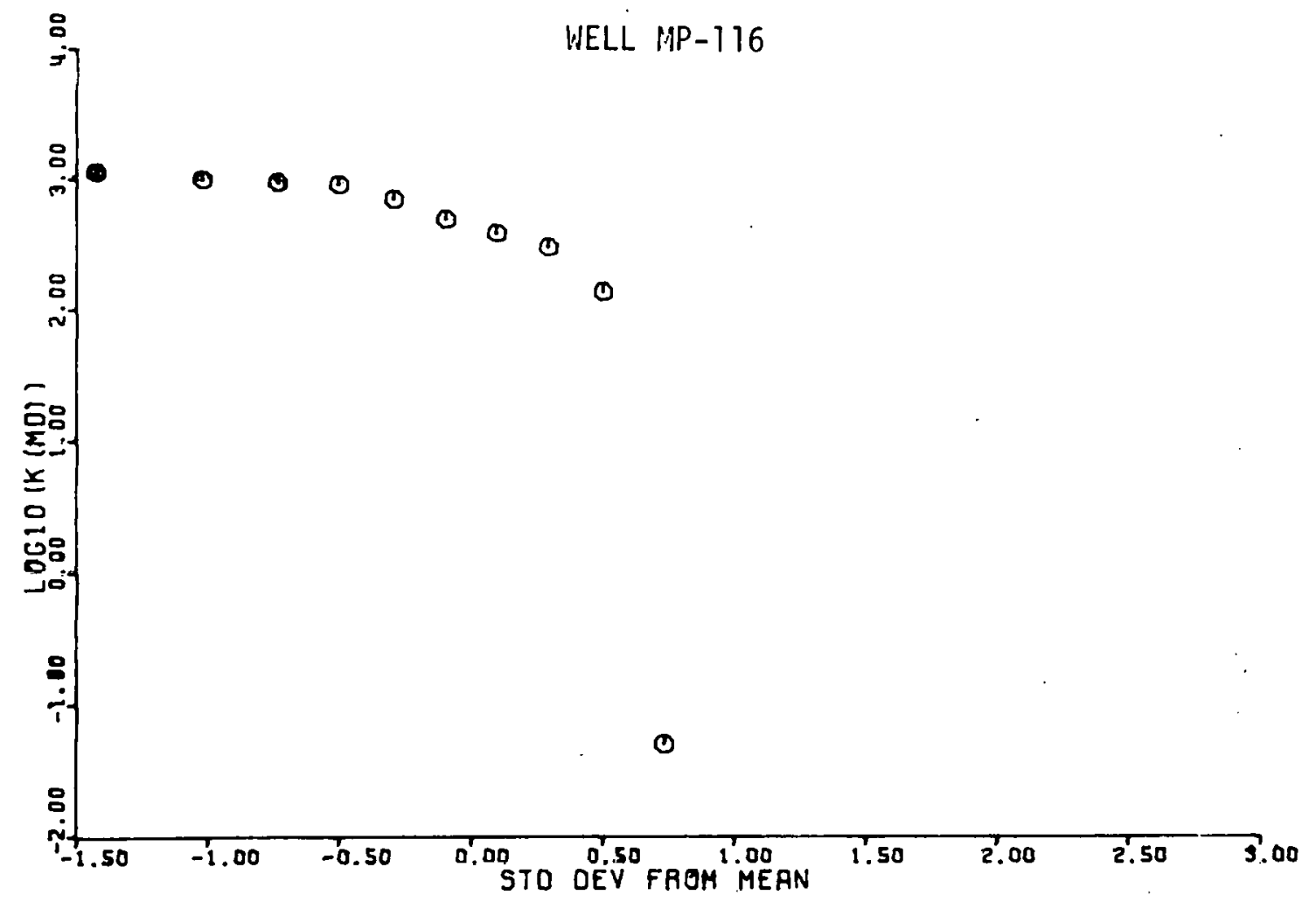


FIGURE B-34

LOGARITHI OF PER!!EABILITY VS STANDARD DEVIATION

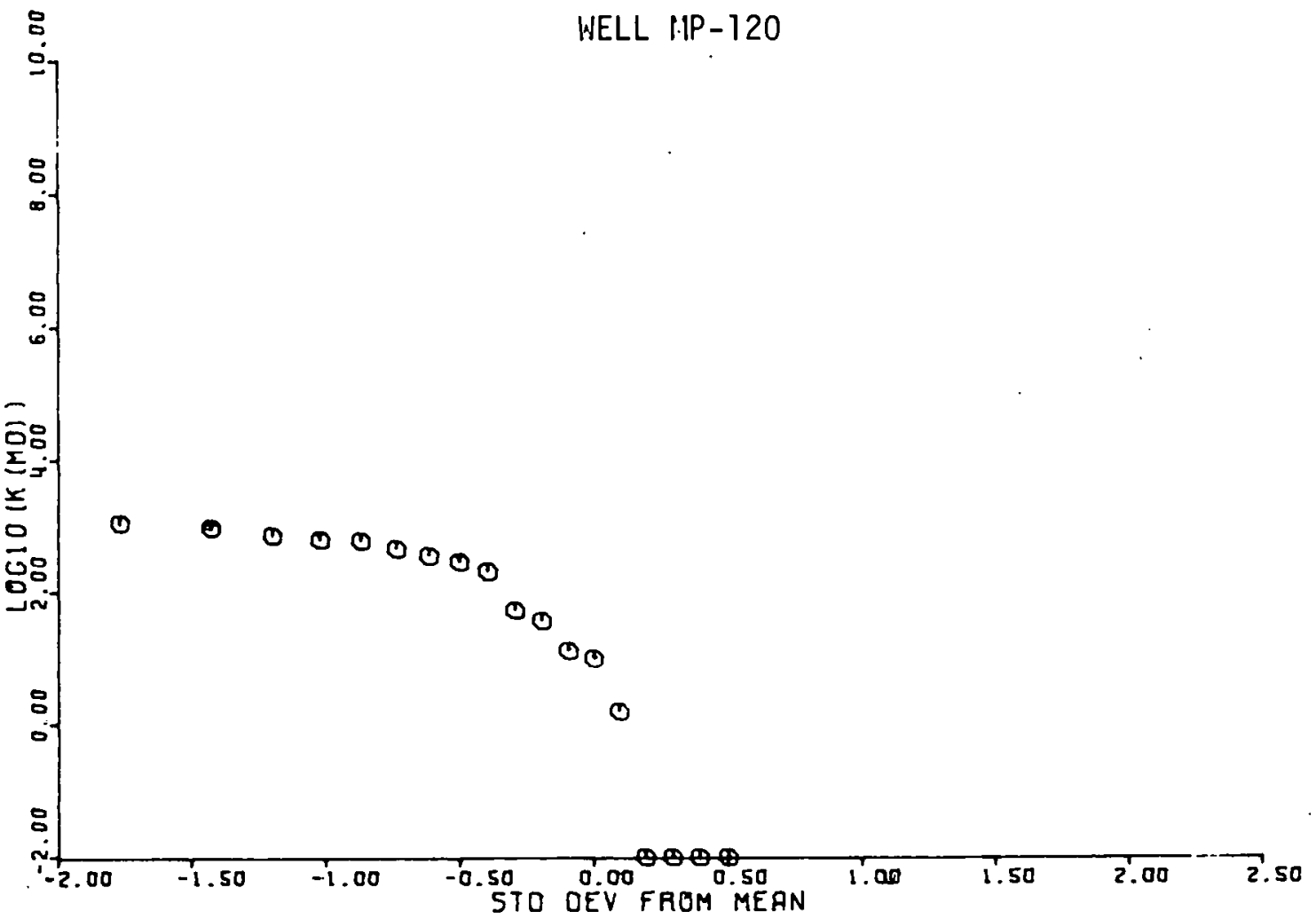

FIGURE B-35

LOGARITHII OF PERPIEABILITY VS STANDARD DEVIATION

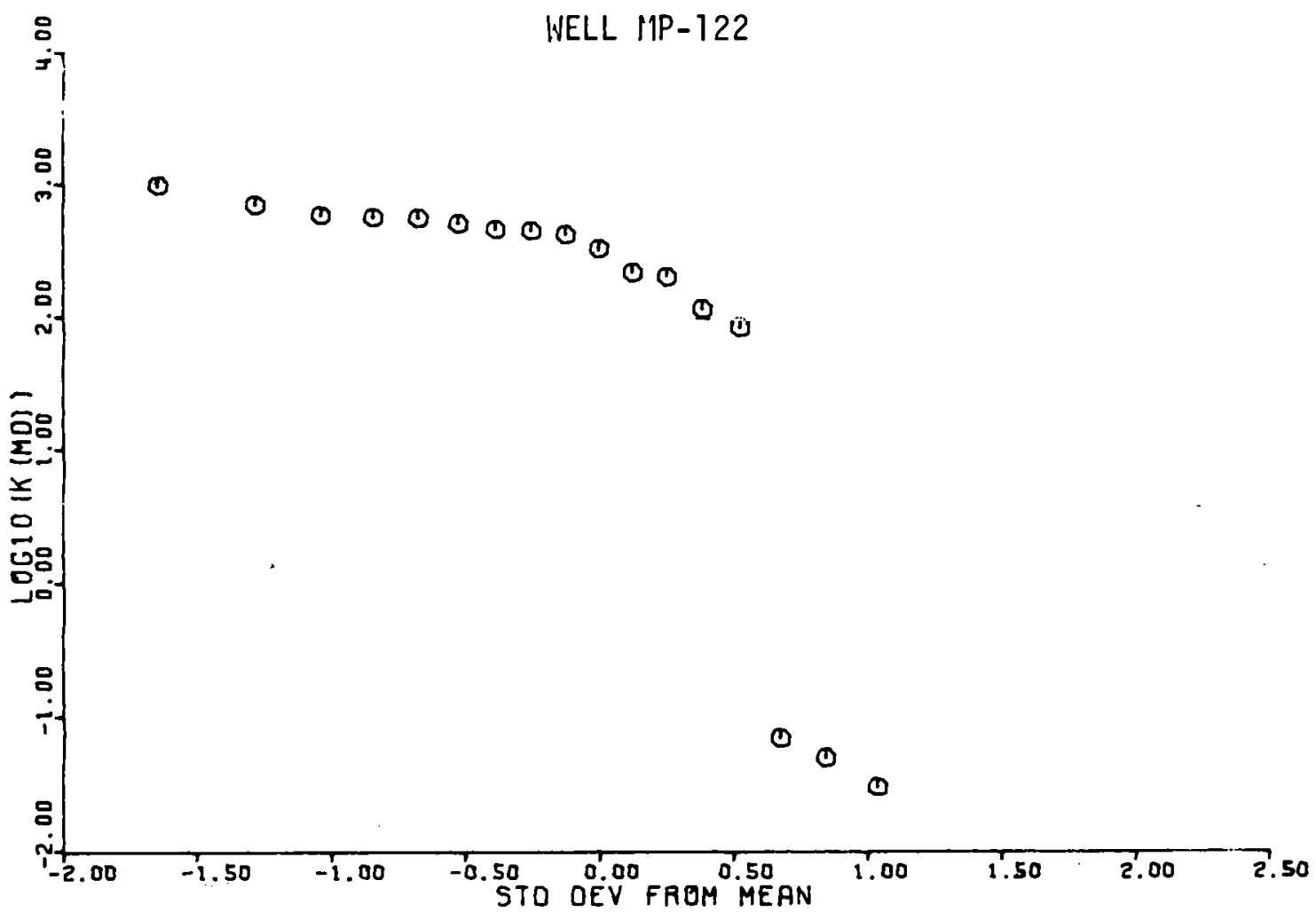


FIGURE B-36

LOGARITHM OF PERMEABILITY VS STANDARD DEVIATION

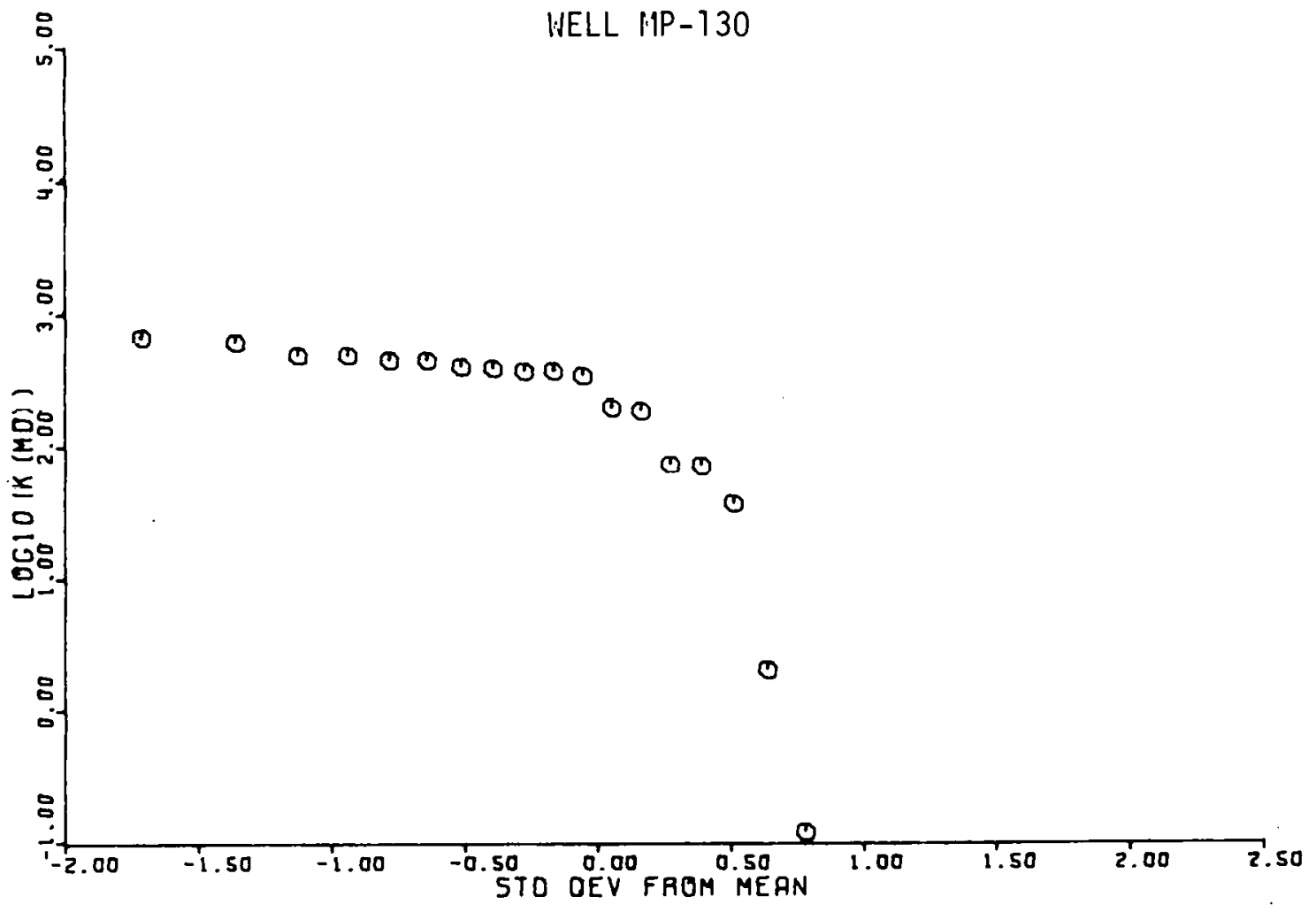

FIGURE B-37

LOGARITHM OF PERMEABILITY VS STANDARD DEVIATION

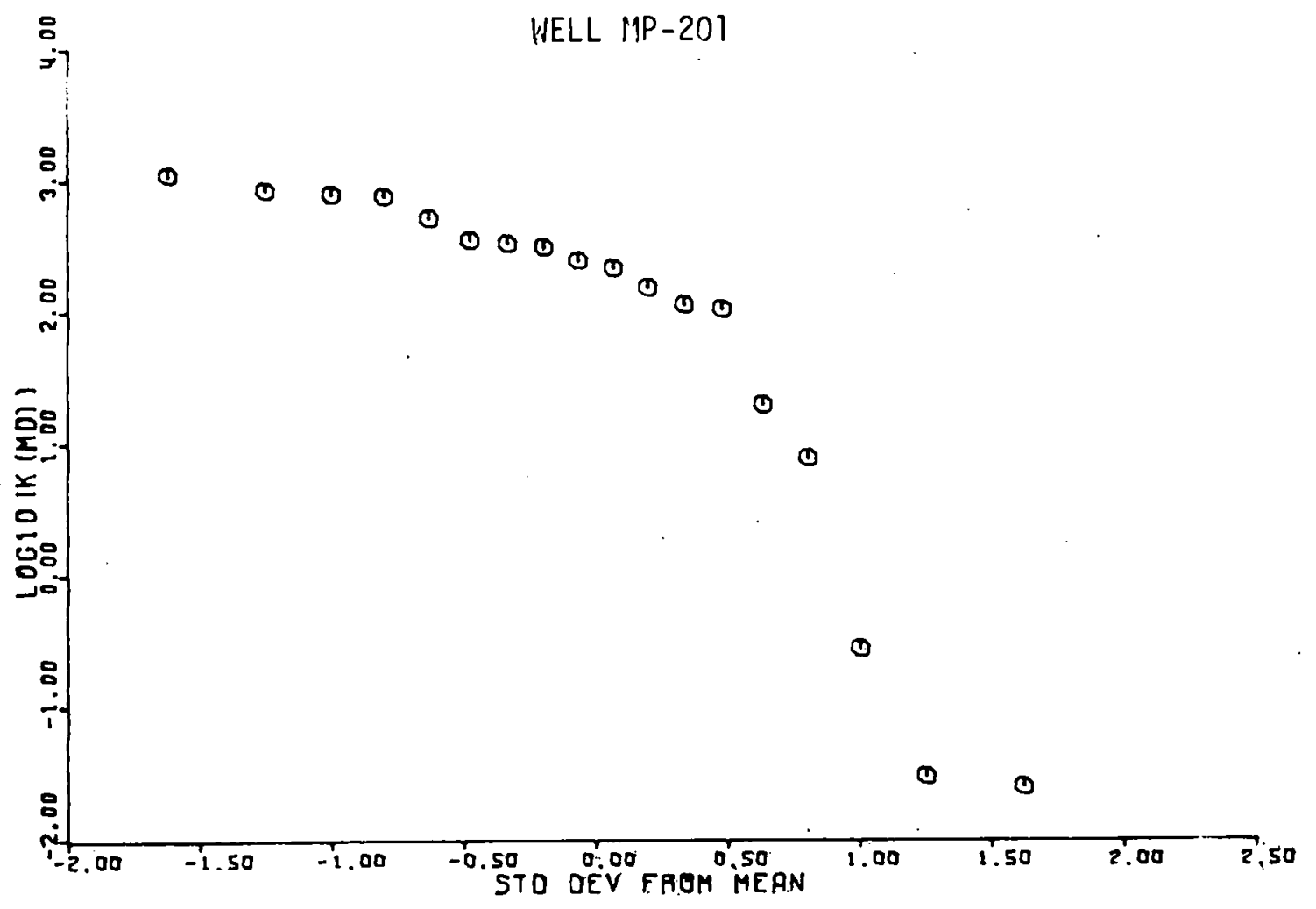


FIGURE B-38

LOGARITH:I OF PERMEABILITY VS STAFDARD DEVIATION

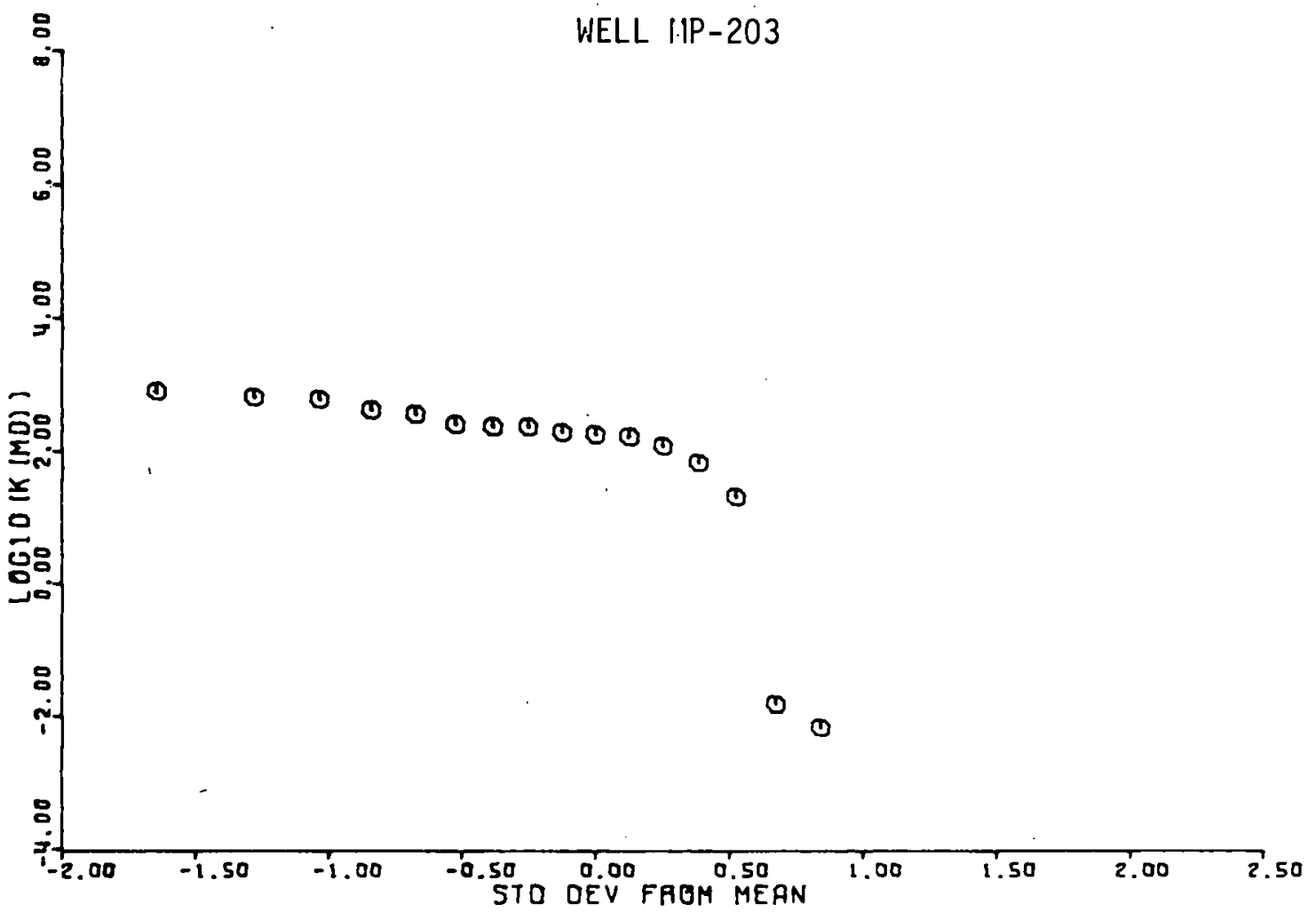

FIGURE B-39

LOGARITH:I OF PERIIEABILITY VS STANDARD .DEVIATION

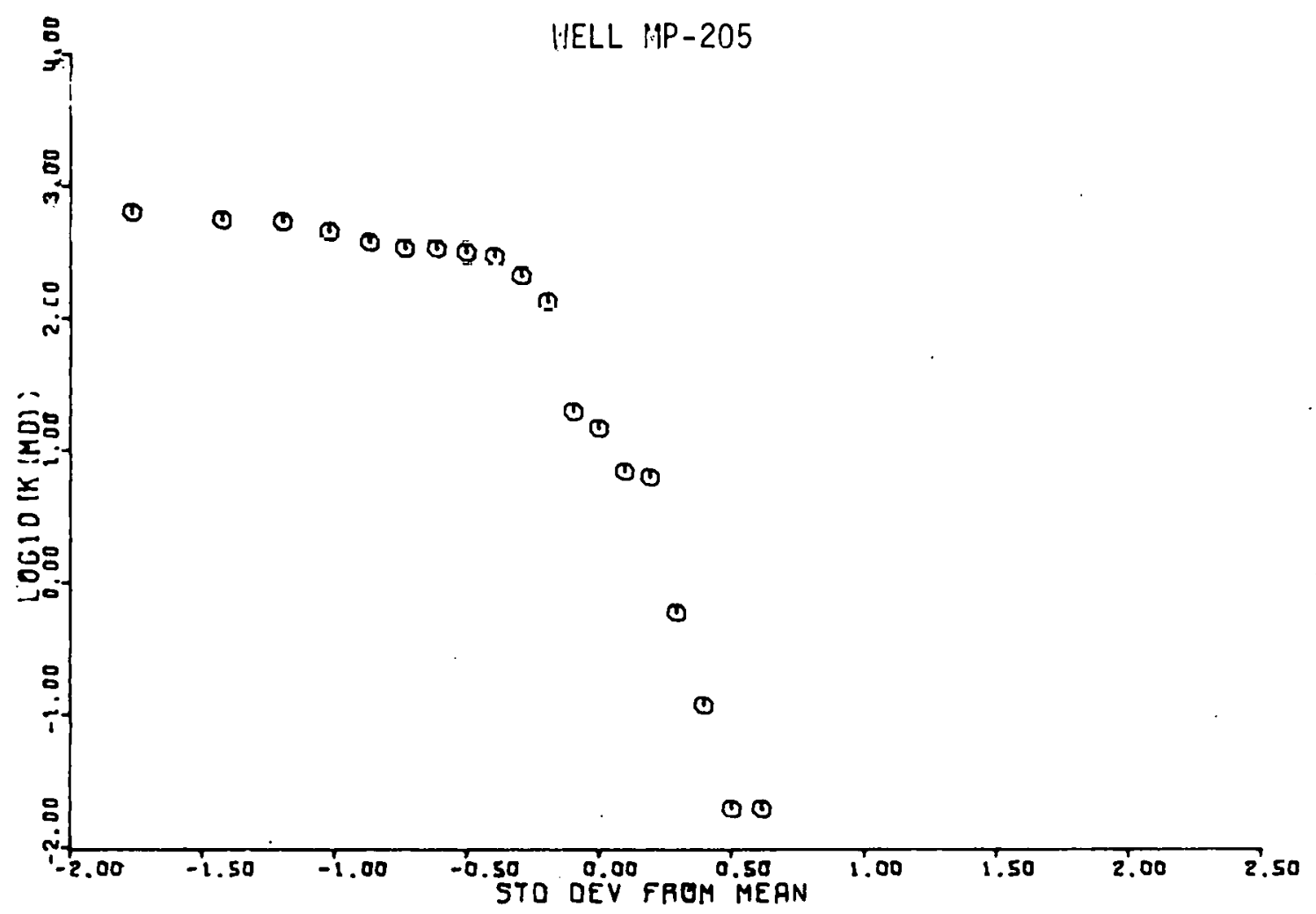


FIGURE B-40

\section{LOGARITHM OF PERIIEABILITY VS STANDARD DEVIATION}

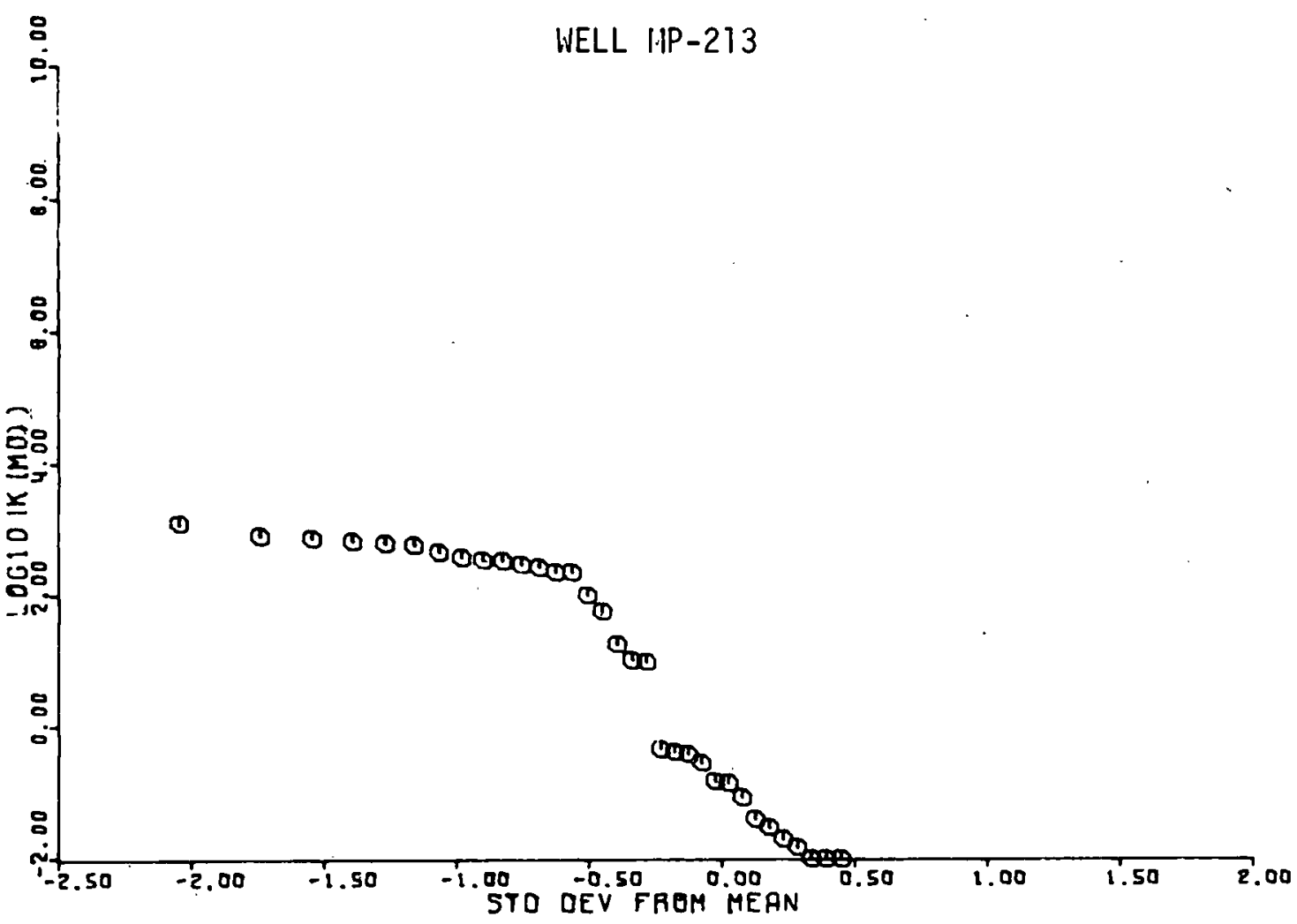

FIGURE $B-41$

LOGARITHII OF PERIIEABILITY VS STANDARD DEVIATION

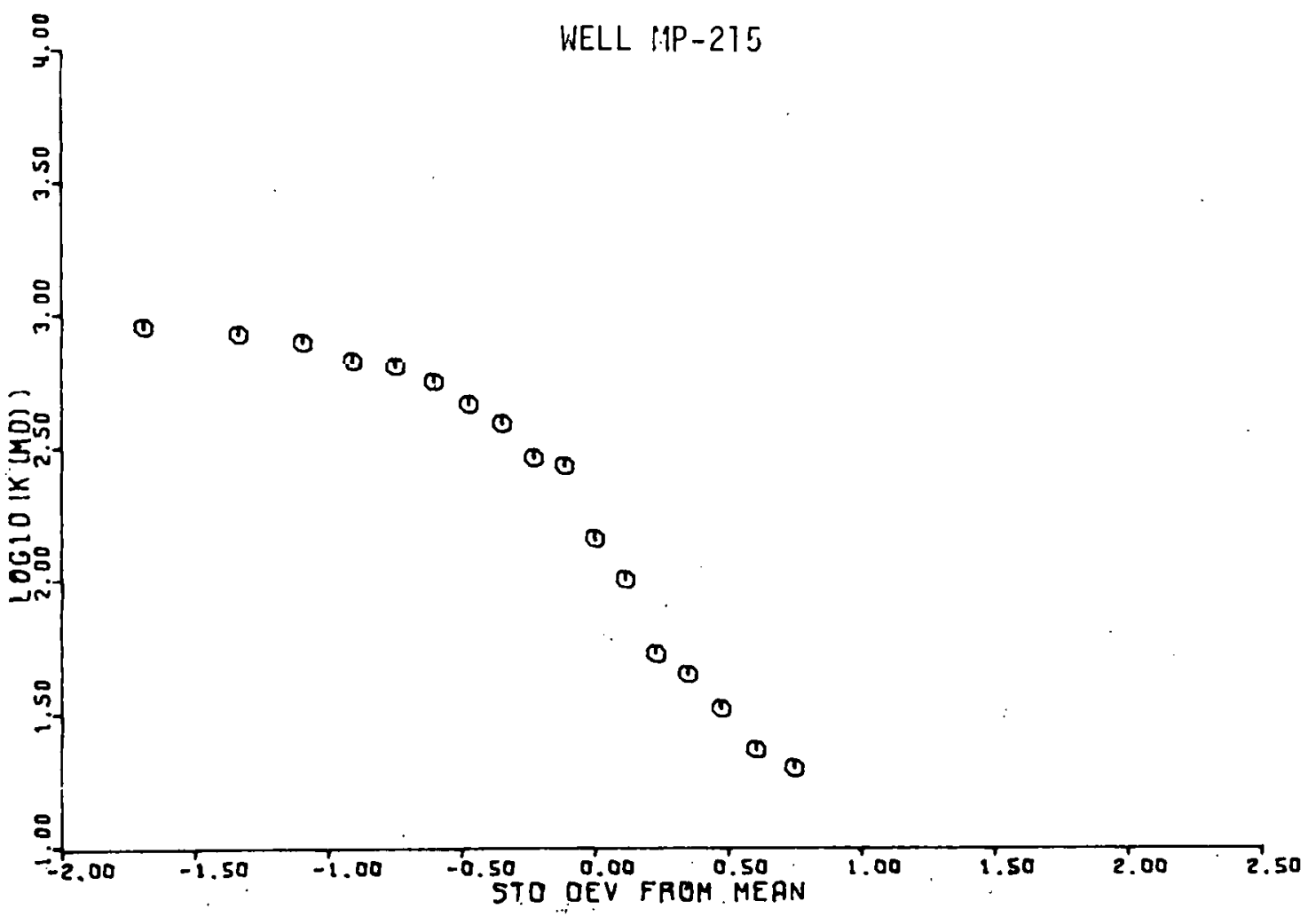




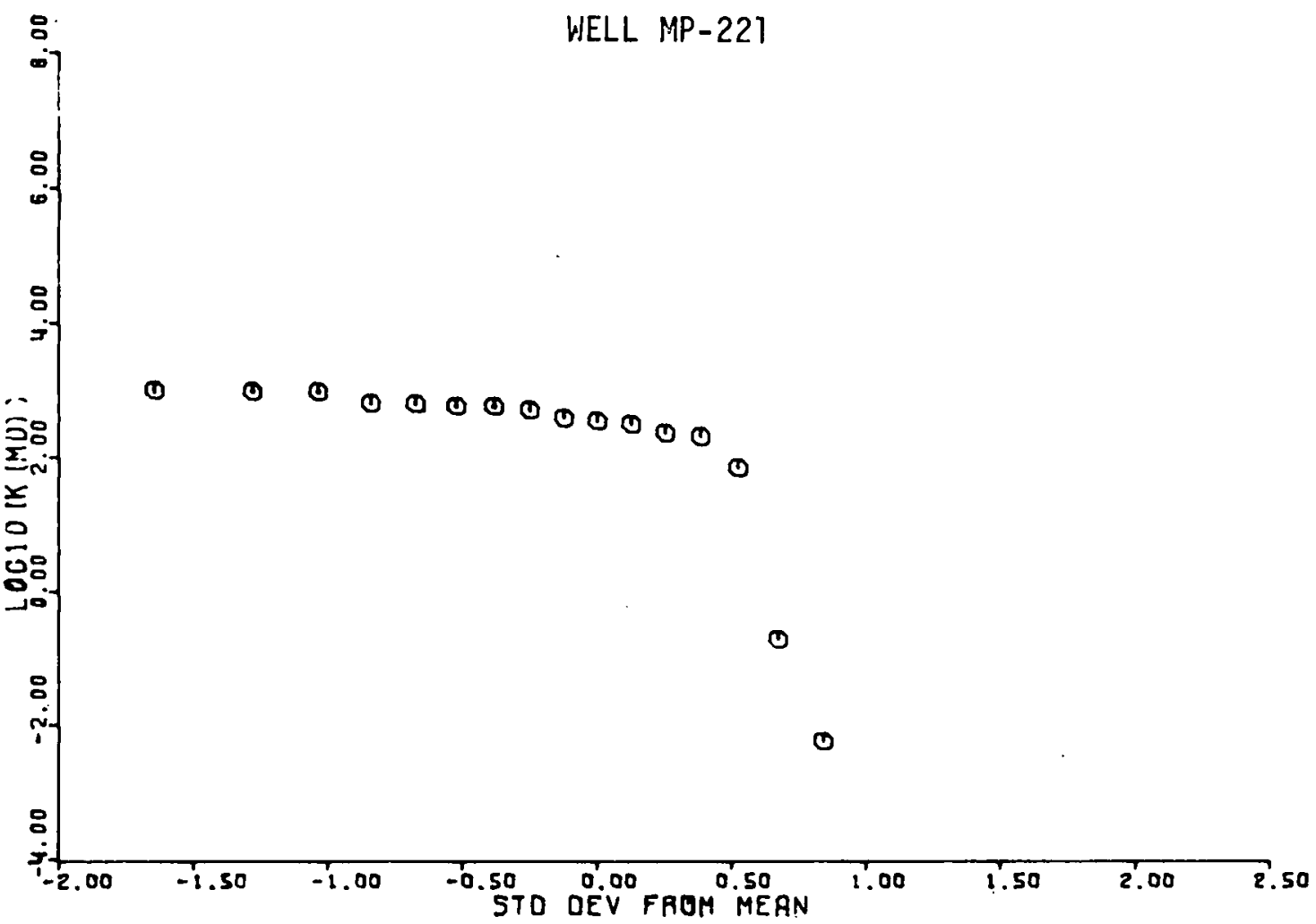

FIGURE $B-43$

LOGARITHI: OF PERIEABILITY VS STANUARD DEVIATION

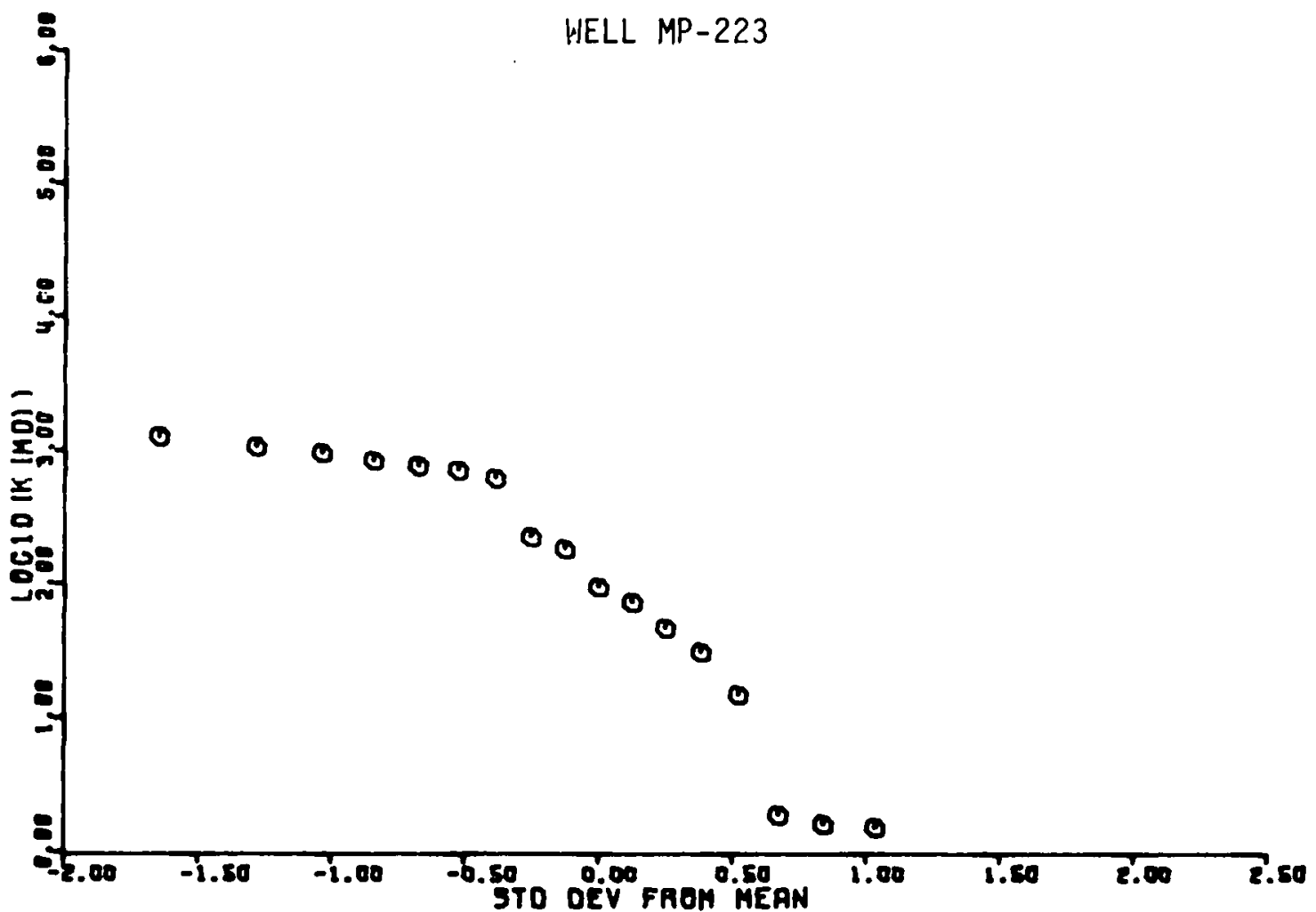


FIGURE B-AA

LOGARITHPI OF PERMEABILITY VS STAIIDARD DEVIATIOIN

MELL MP-225

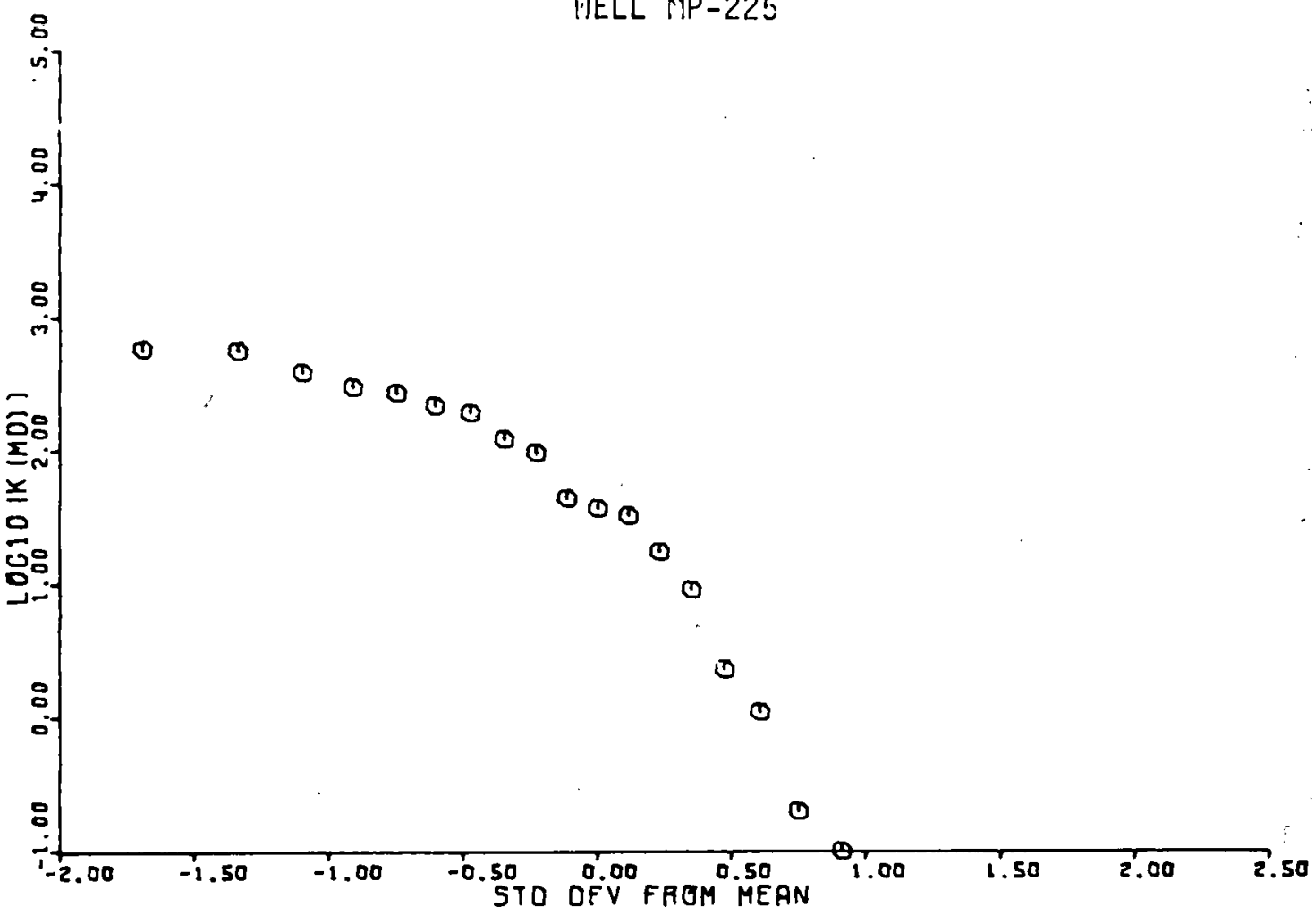


II -92

FIGURE B-45

$K_{W} / K_{0}$ vs Water Saturation

- Wel 1_IIPP-104

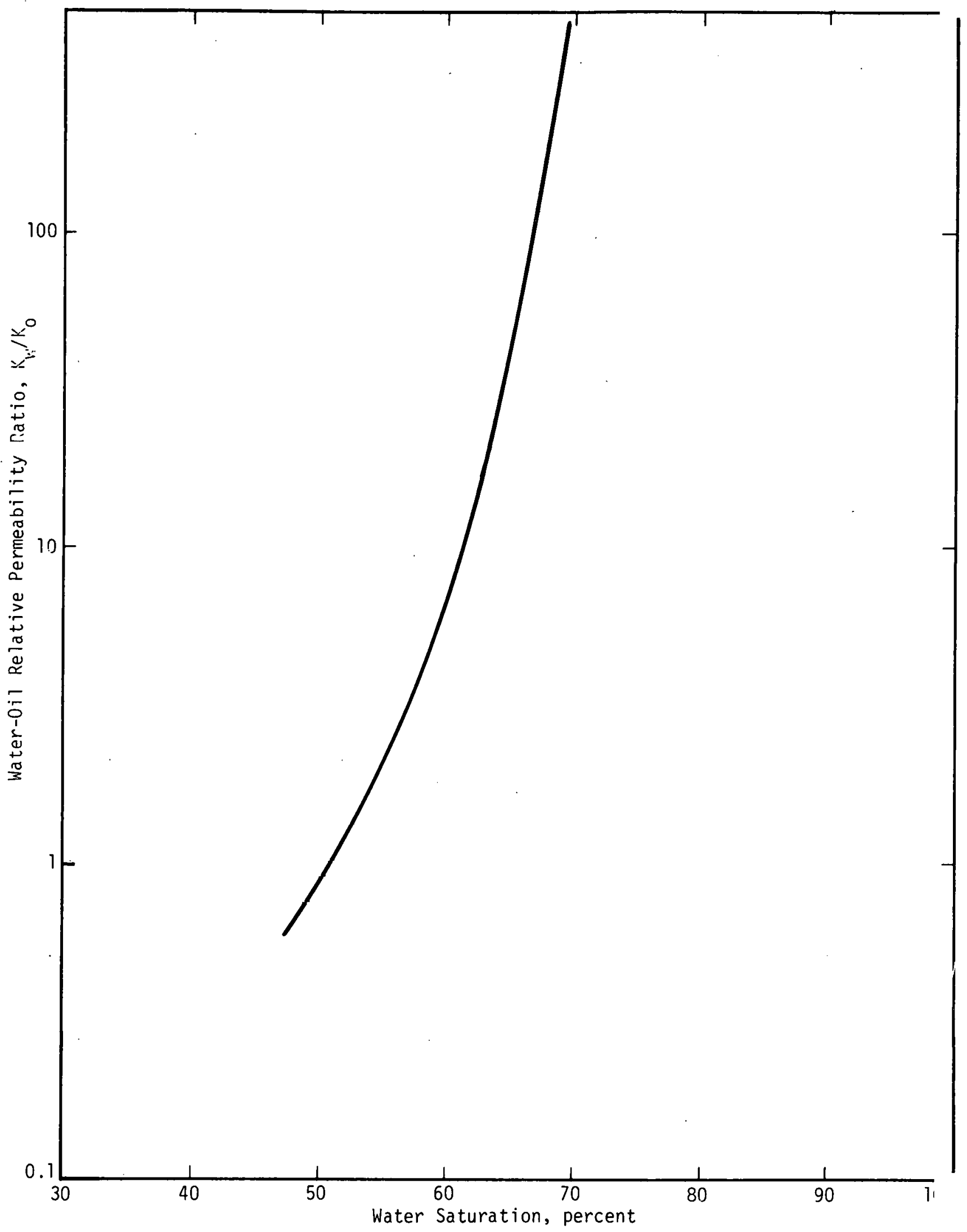


FIGURE B-46

\section{OIL-WATER RELATIVE PERMEABILITIES}

WELL MP- 104

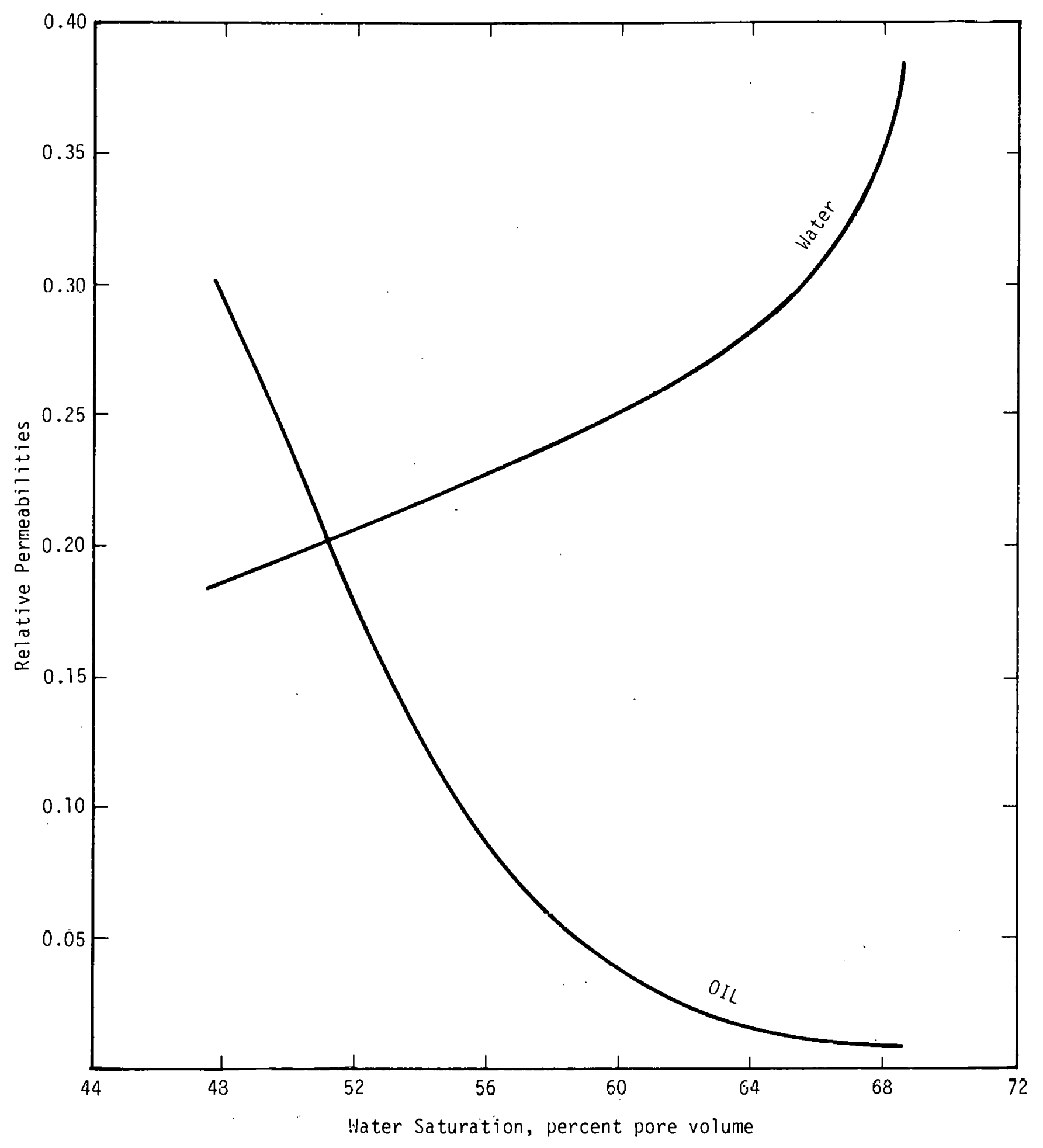


RELATIVE PERMEABILITY RATIO VS WATER. SATURATION

$\because \quad$ FOR SAMPLE 14 , WELL MP-104

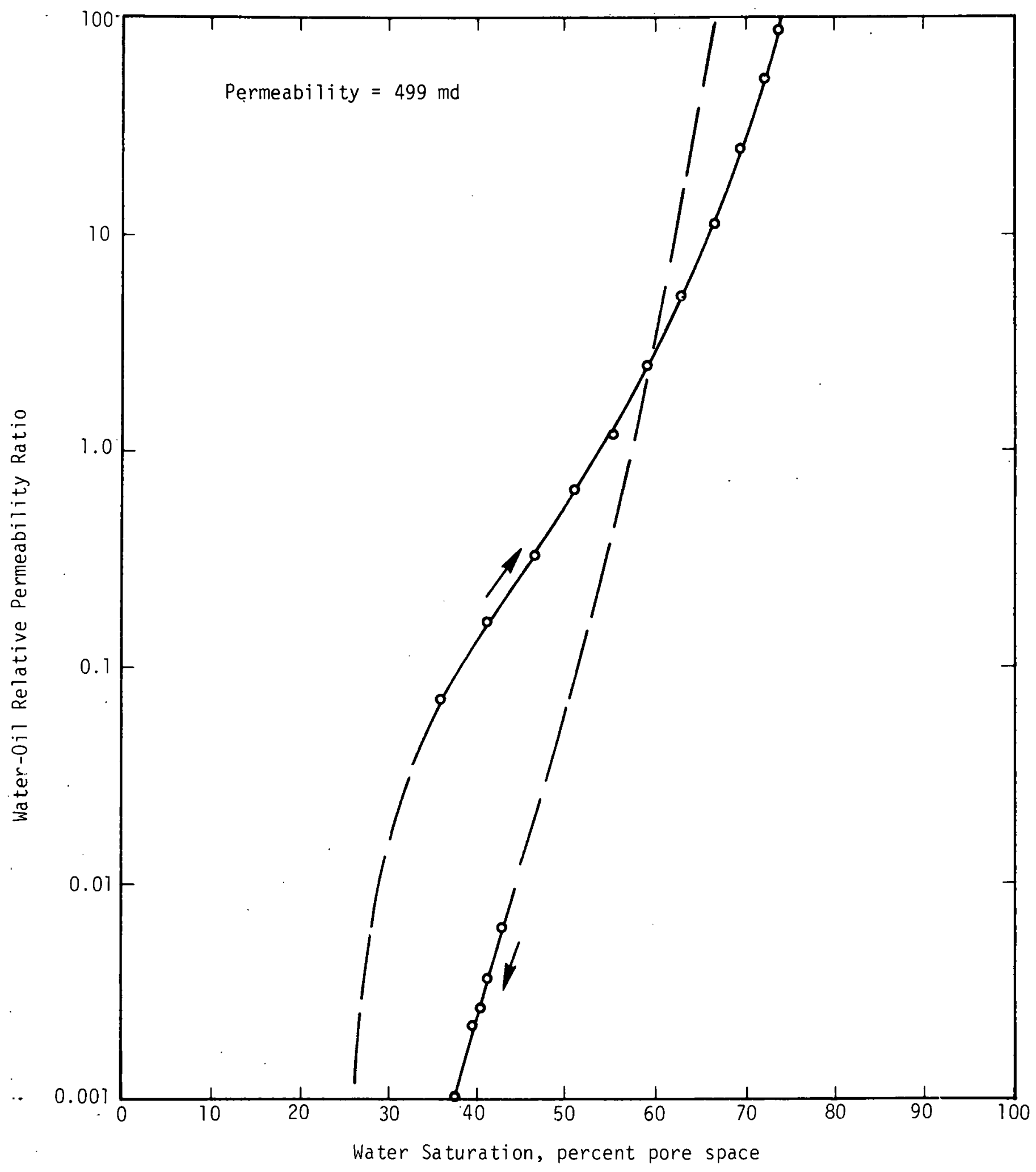


rIGURE B-48

RELATIVE PERMEABILITY FRACTION VS WATER SATURATION

FOR SAMPLE 14, WELL MP-104

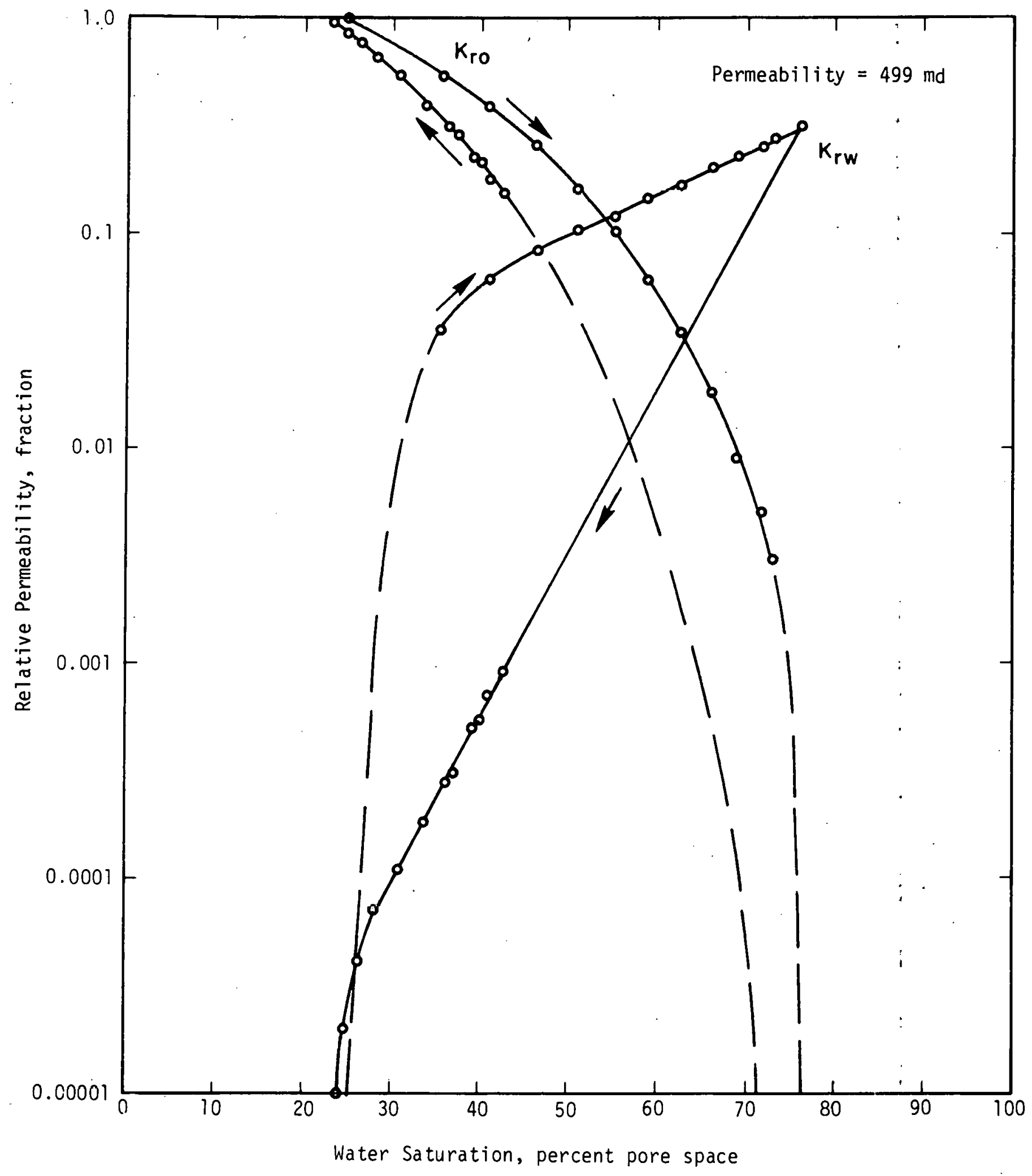


FIGURE B-49

RELATIVE PERMEABILITY RATIO VS WATER SATURATION

FOR SAMPLE 17, WELL MP-104

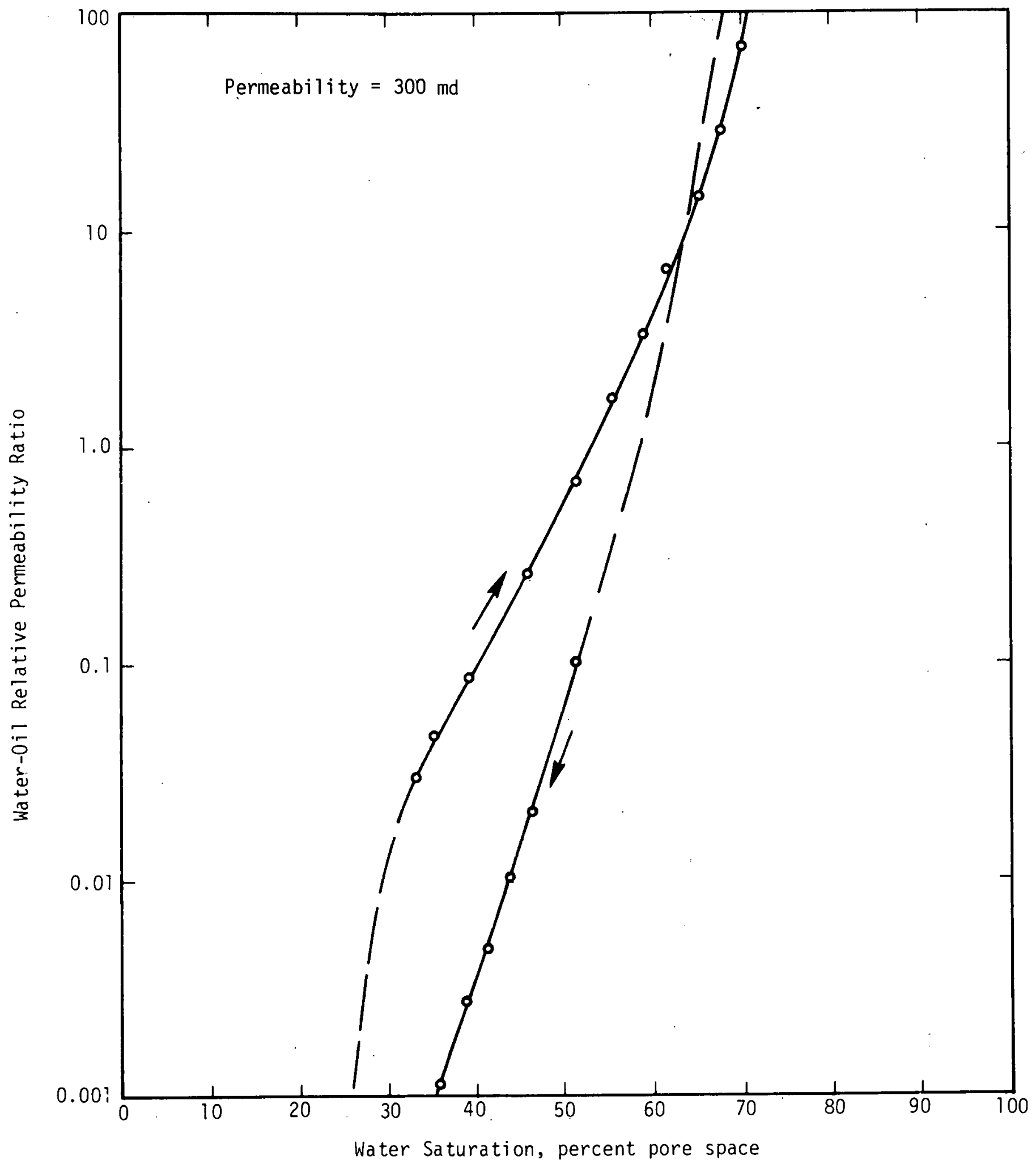


FIGURE B-50

RELATIVE PERMEABILITY FRACTION VS WATER SATURATION

FOR SAMPLE 17, WELL MP-104

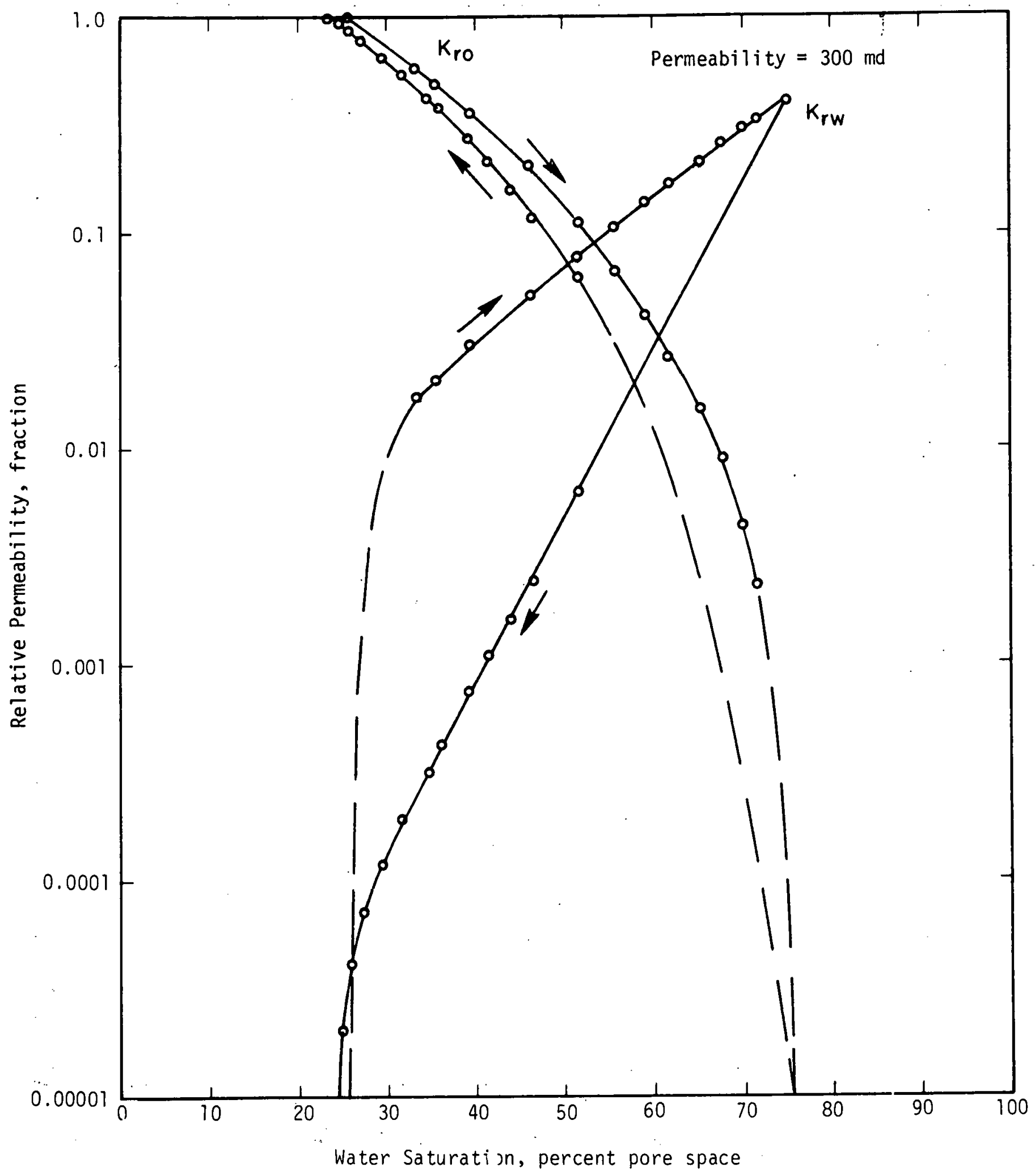


FIGURE B-51

RELATIVE PERMEABILITY RATIO VS WATER SATURATION $\therefore$ FOR SAMPLE 11, WELL MP-104

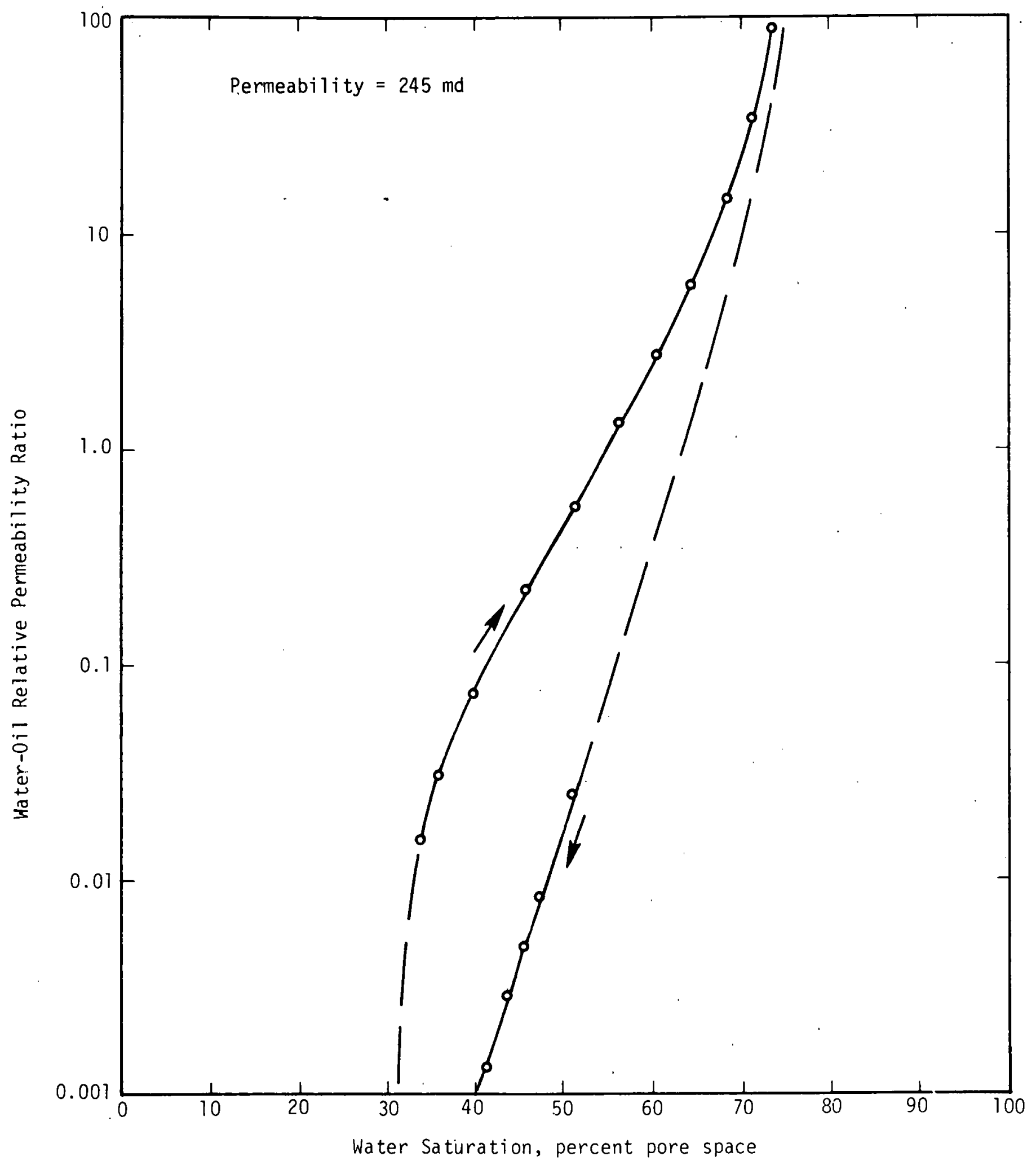




\section{FIGURE B-52}

RELATIVE PERMEABILITY FRACTION VS WATER SATURATION FOR SAMPLE 11, WELL MP-104

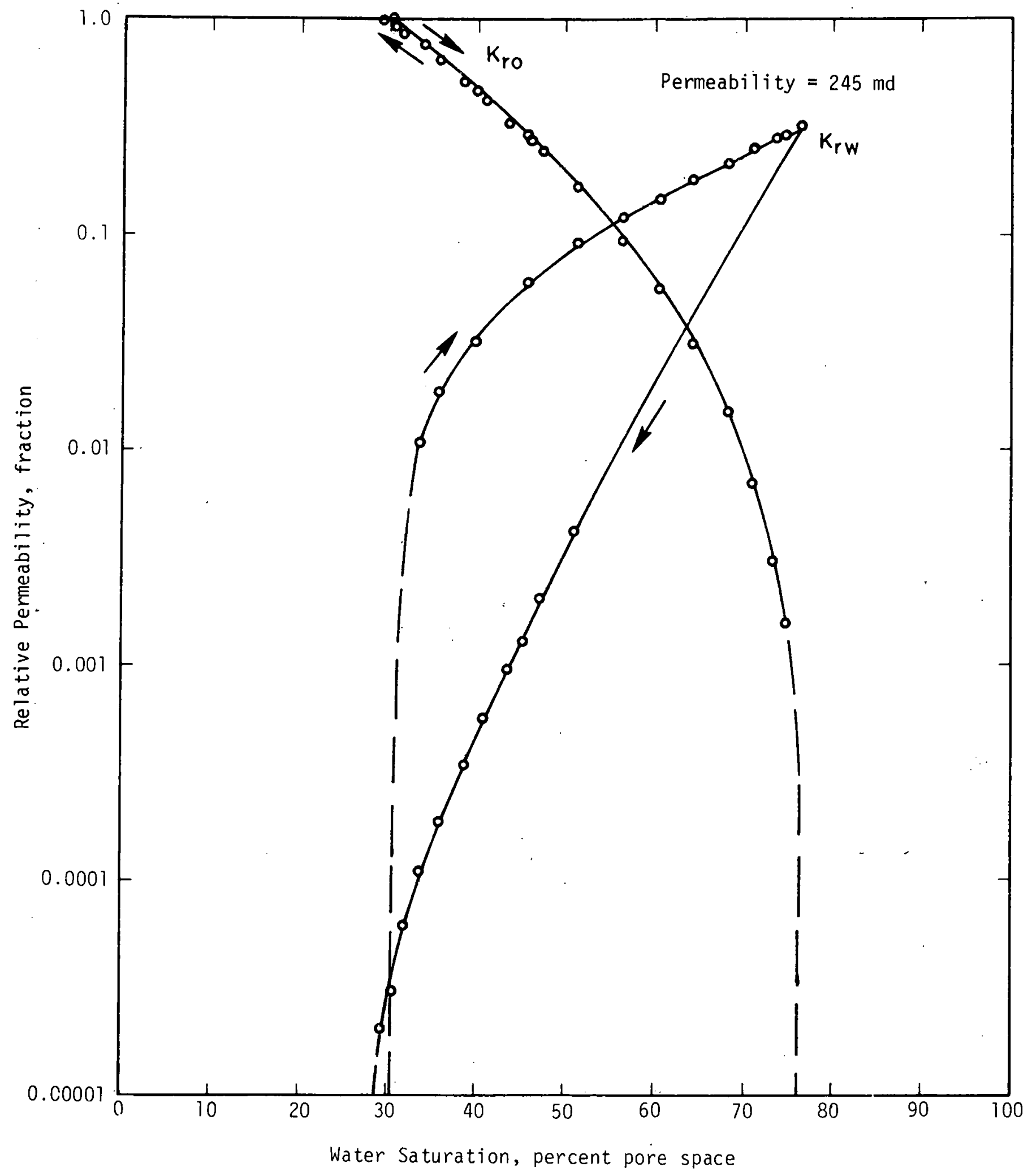


FIGURE B-53

RELATIVE PERMEABILITY RATIO VS WATER SATURATION

FOR SAMPLE 6 , WELL MP-104

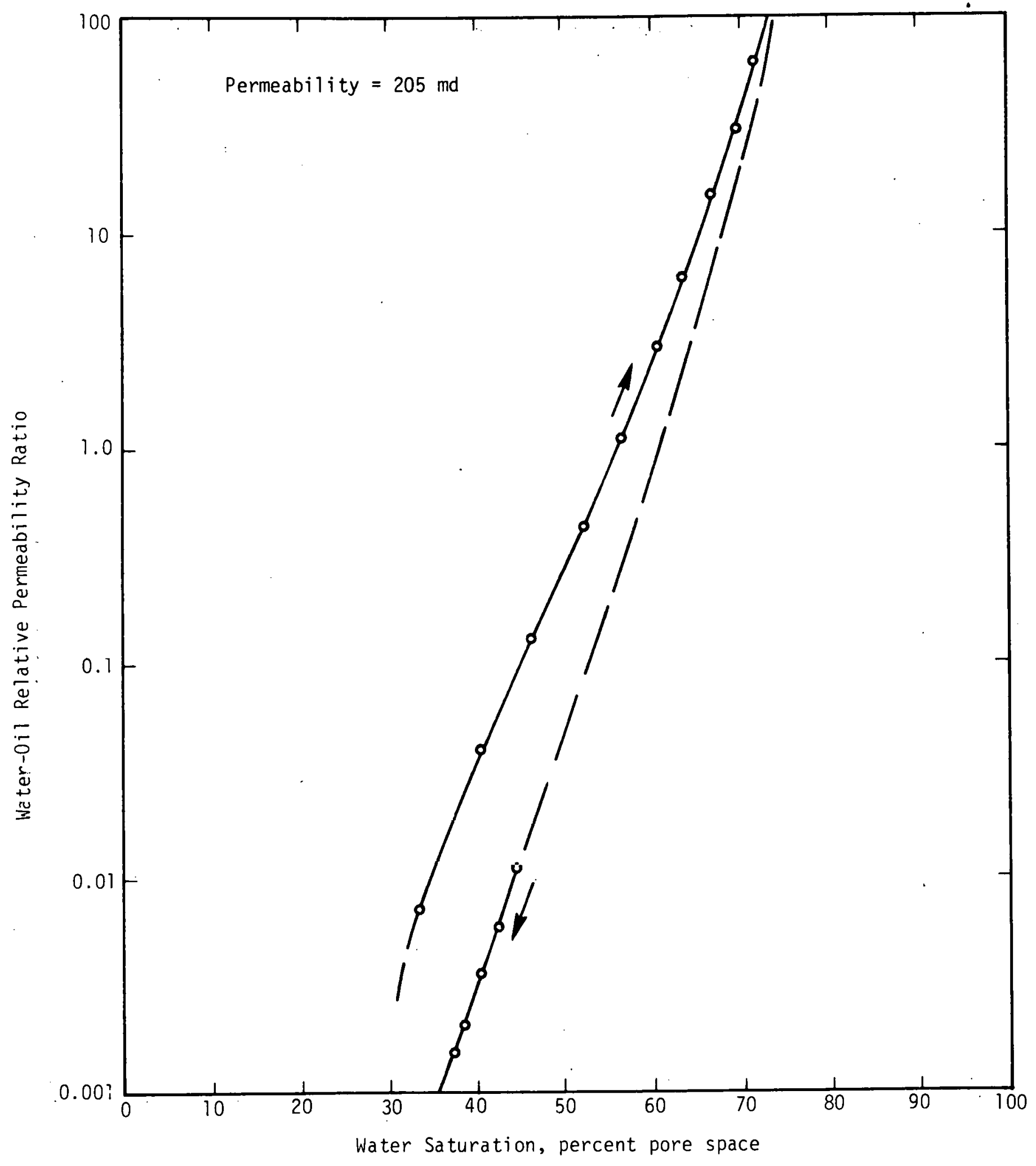


FIGURE B-54

RELATIVE PERMEABILITY FRACTION VS WATER SATURATION

FOR SAMPLE 6, WELL MP-104

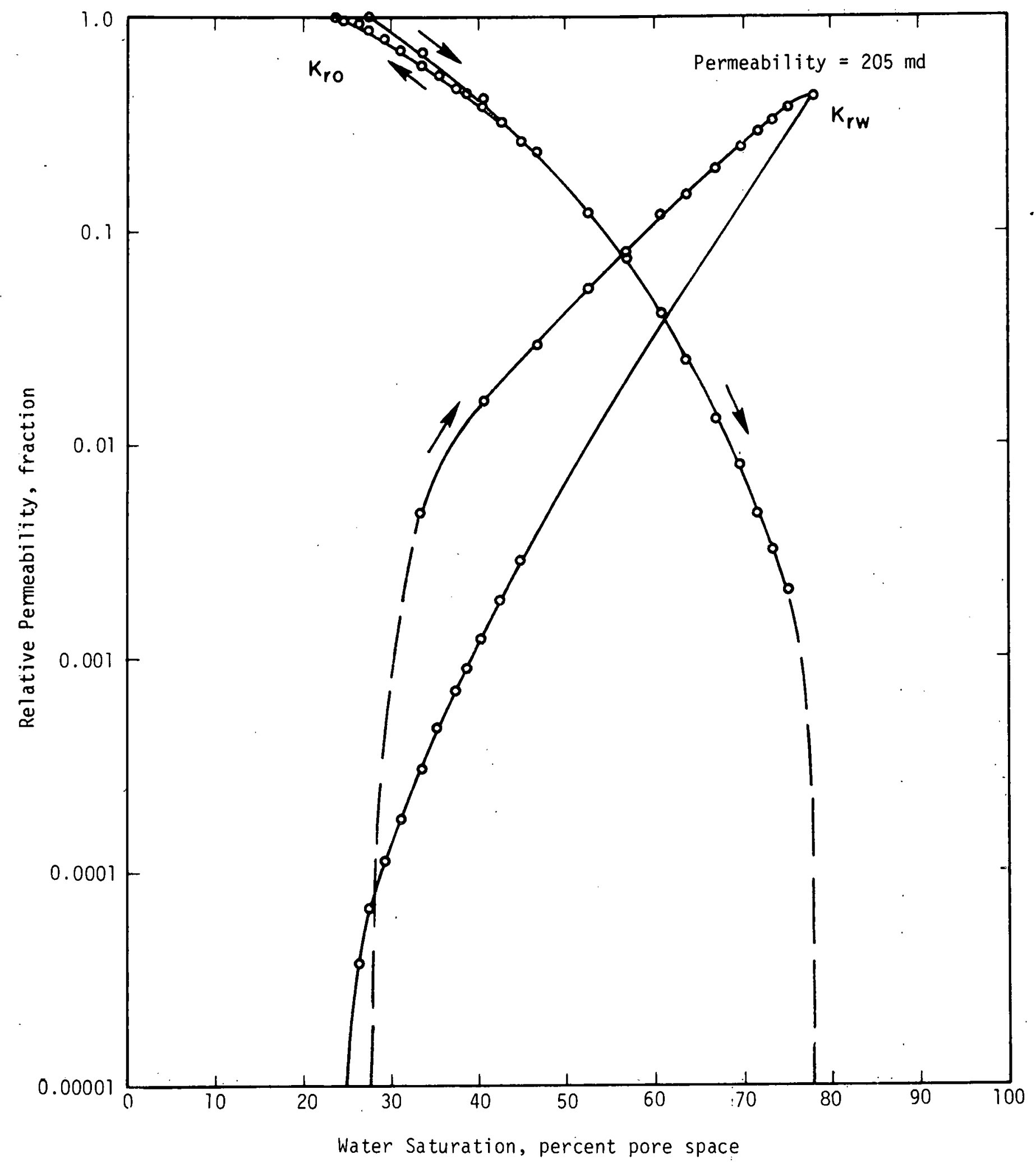


RELATIVE PERMEABILITY RATIO VS WATER SATURATION

FOR SAMPLE 21, WELL MP-104

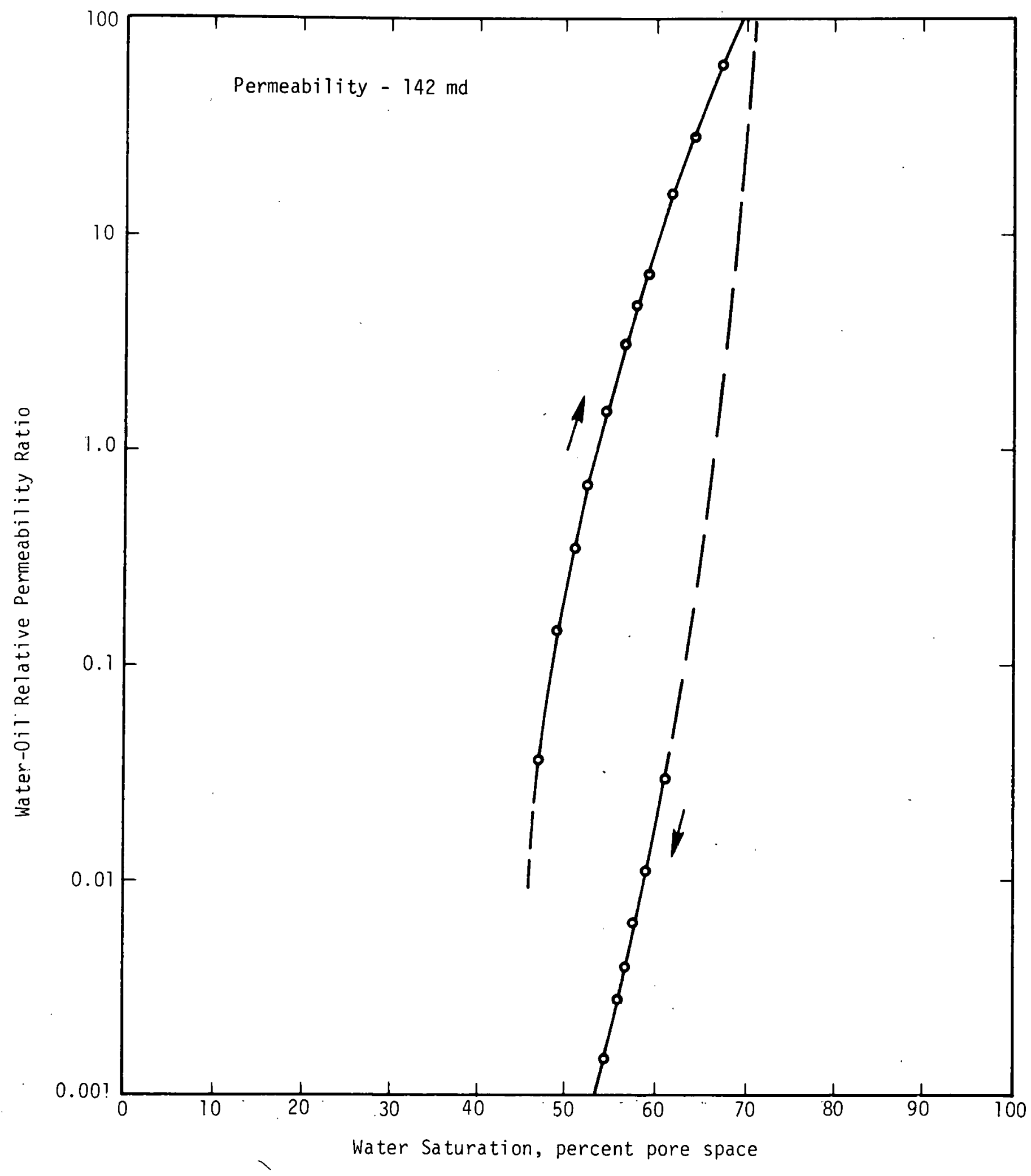


FIGURE B-56

RELATIVE PERMEABILITY. FRACTION VS WATER SATURATION

FOR SAMPLE 21, WELL MP-104

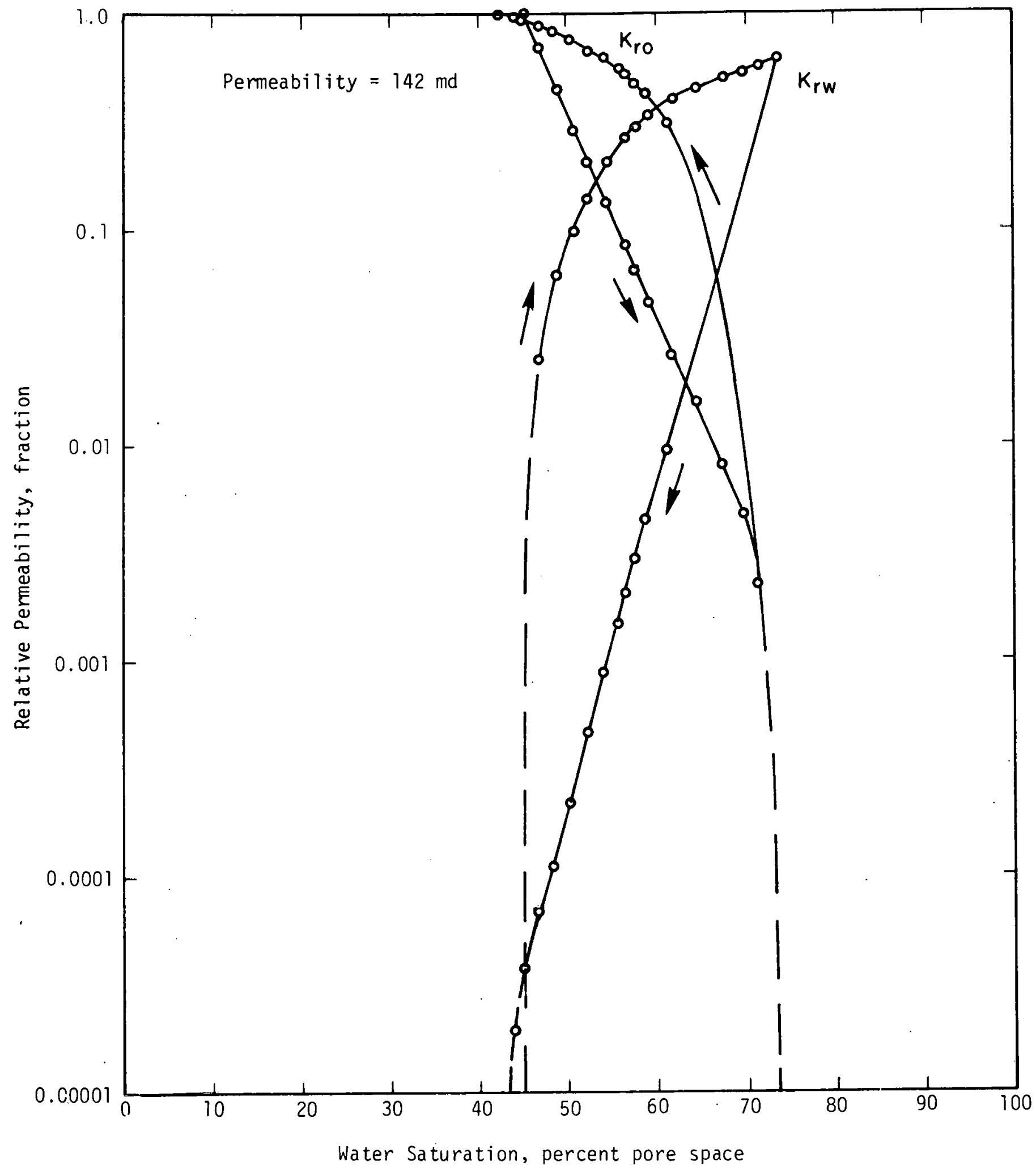


II-104

FIGURE B-57

RELATIVE PERMEABILITY RATIO VS WATER SATURATION

FOR SAMPLE 3, WELL MP-104

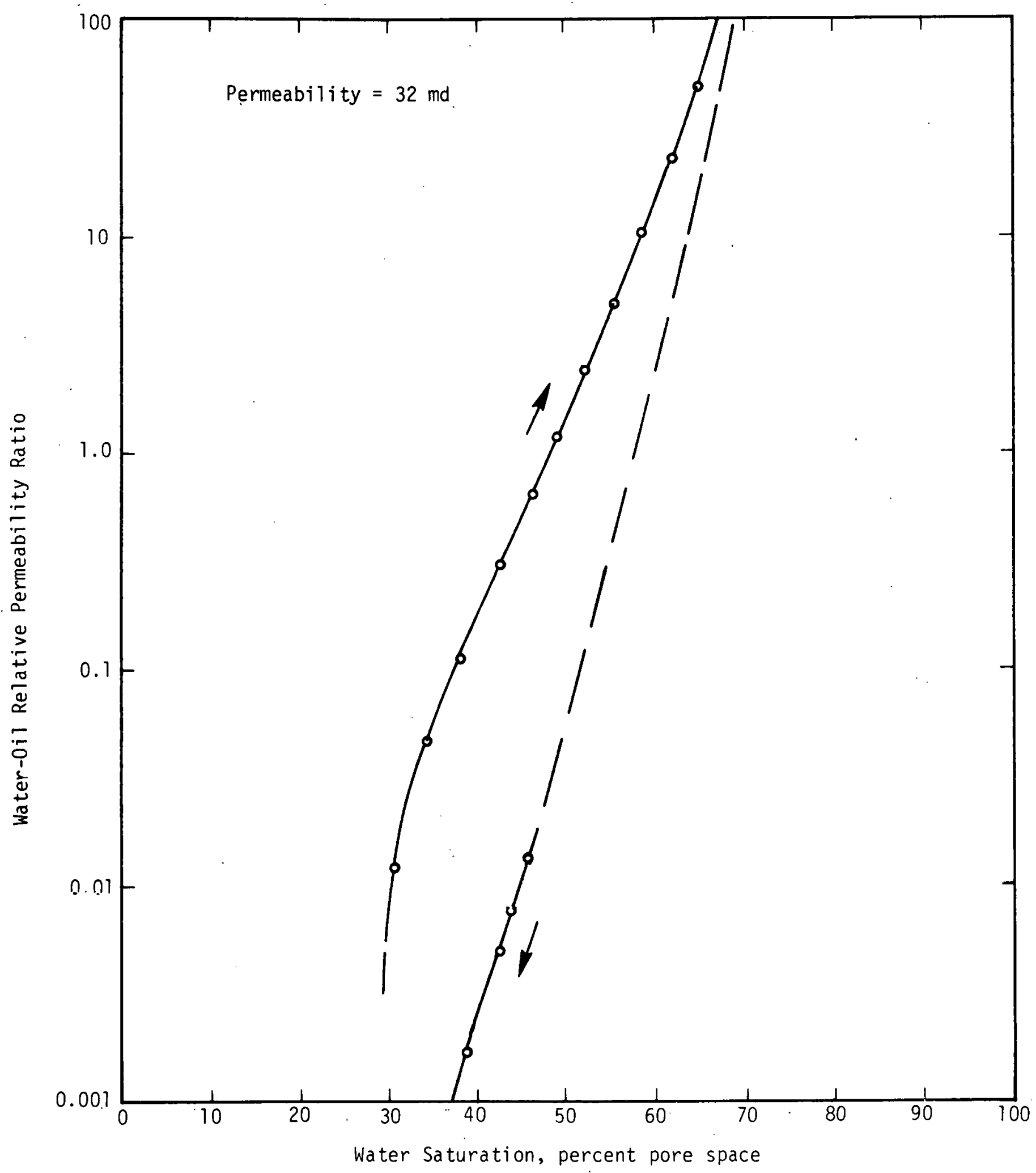


FIGURE B-58

RELATIVE PERMEABILITY FRACTION VS WATER SATURATION

FOR SAMPLE 3, WELL MP-104

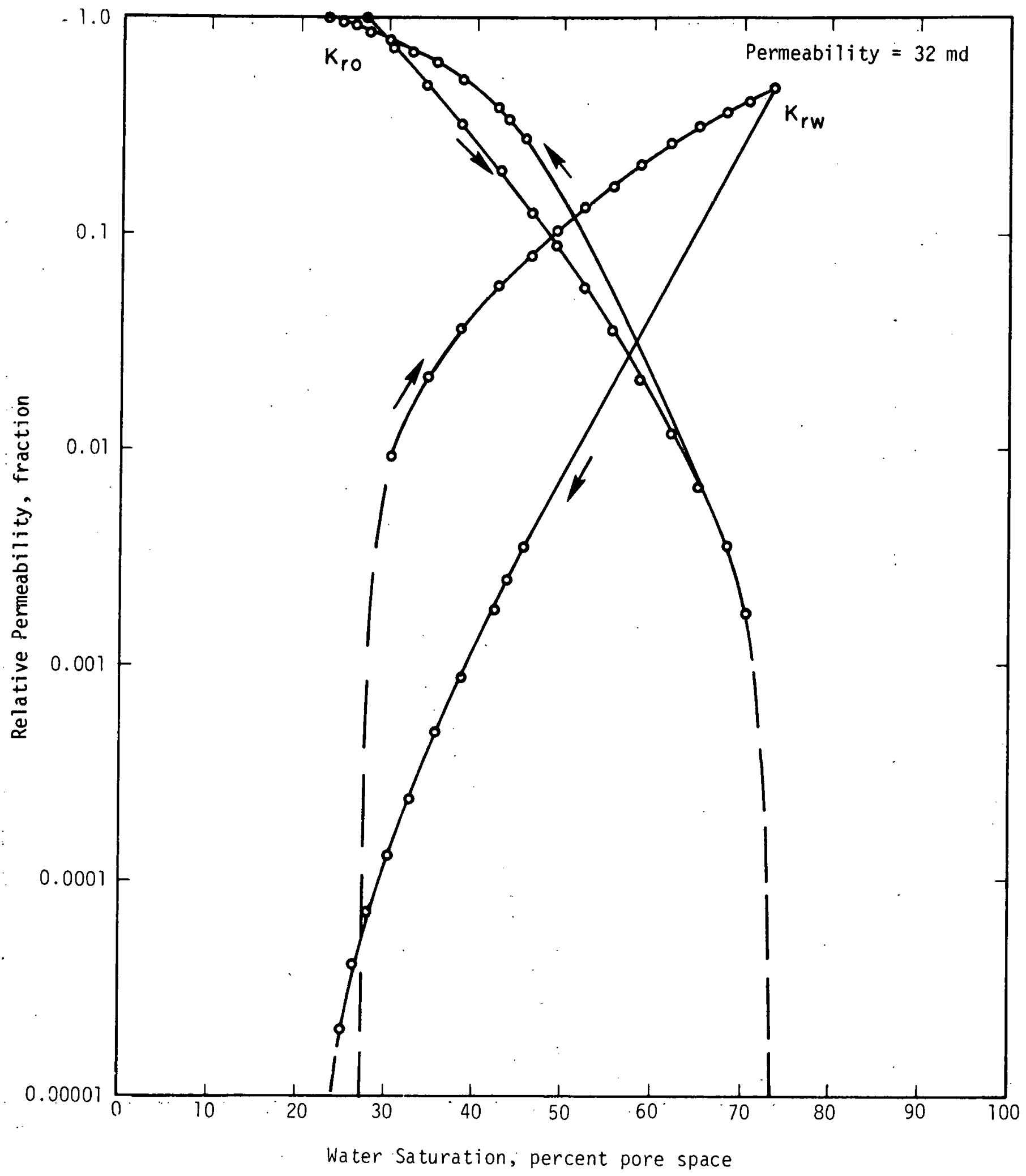


FIGURE B-59

RELATIVE PERMEABILITY RATIO VS WATER SATURATION

FOR SAMPLE 2, WELL MP-124

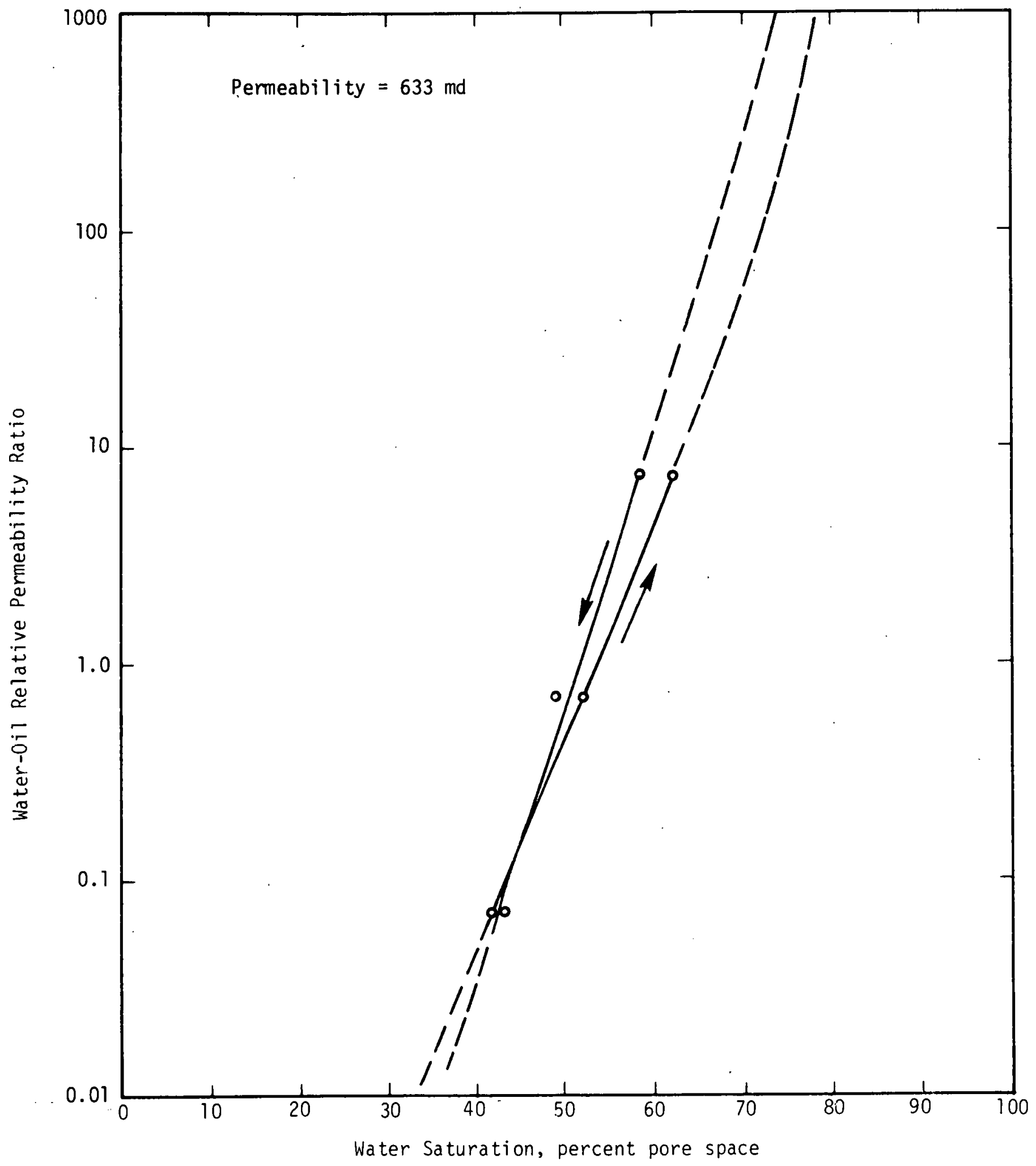


FIGURE $B-60$

RELATIVE PERMEABILITY FRACTION VS WATER SATURATION

FOR SAMPLE 2, WELL MP-124

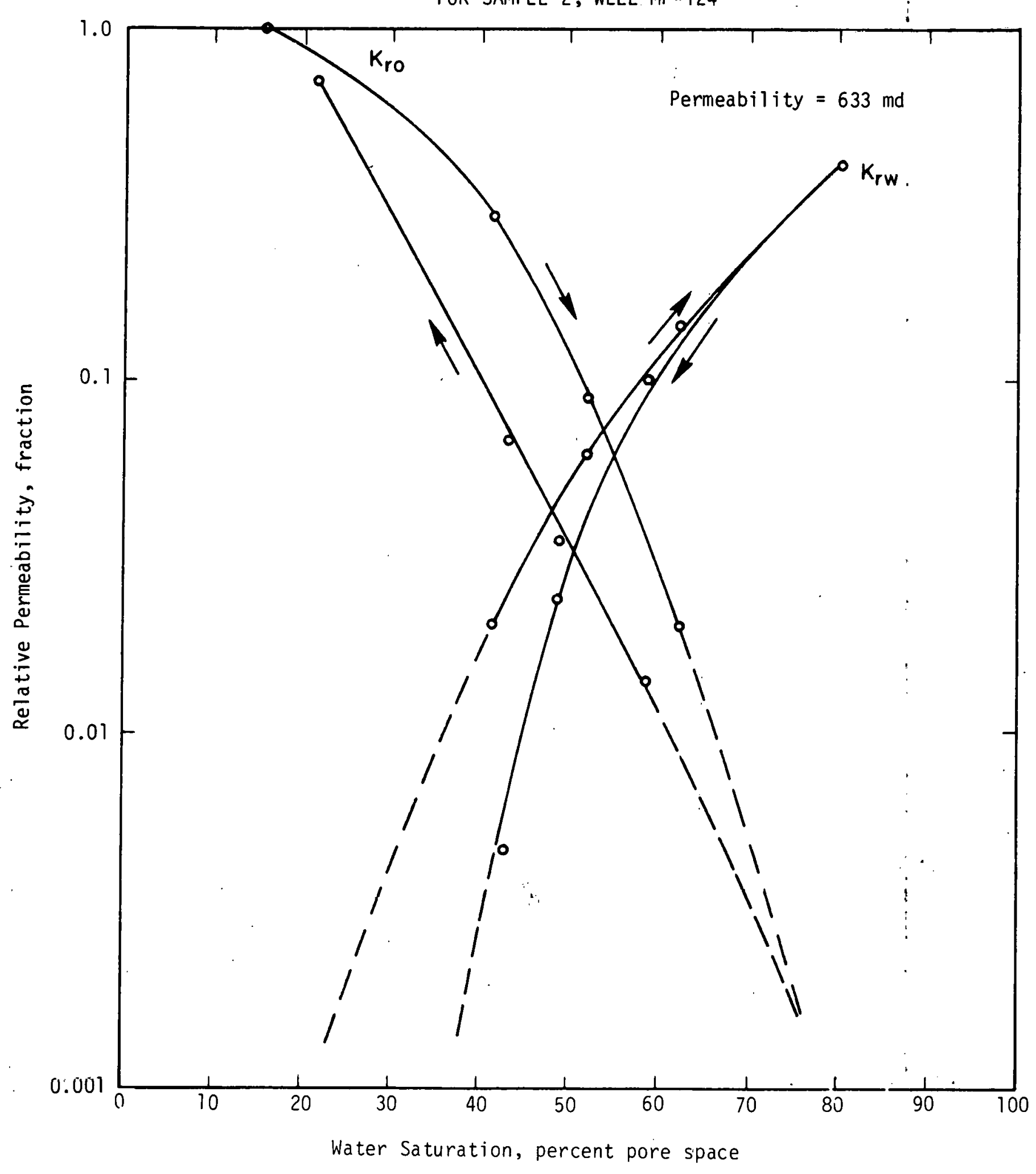




\section{THIS PAGE \\ WAS INTENTIONALLY \\ LEFT BLANK}


APPENDIX C

WELL LOGGING

Tables and Figures 
TABLE C 1

LOG ANALYSIS RESULTS FOR. THE TEST WELLS

\begin{tabular}{|c|c|c|c|c|c|c|c|}
\hline $\begin{array}{l}\text { Well } \\
\text { No. }\end{array}$ & $\begin{array}{c}\text { Net Pay, } \\
\mathrm{ft} \\
\text { (Interval) } \\
\end{array}$ & 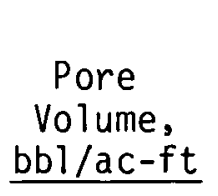 & $\begin{array}{l}\text { Average } \\
\text { Porosity, } \\
\text { percent }\end{array}$ & $\begin{array}{l}\text { Hydrocarbon } \\
\text { Pore Volume, } \\
\text { bbl/ac-ft } \\
\end{array}$ & $\begin{array}{l}\text { Average } \\
\text { Residual 0il } \\
\text { Saturation, } \\
\text { percent } \\
\end{array}$ & $\begin{array}{c}\text { Average } \\
0 i l \text { in Place, } \\
\text { percent }\end{array}$ & $\begin{array}{c}\text { Average } \\
\text { Movable } \\
0 i 1 \text {, } \\
\text { percent }\end{array}$ \\
\hline MP-101 & $\begin{array}{c}21 \\
(615-635)\end{array}$ & 1884.9 & 24.3 & 655.8 & 30.8 & 34.3 & 3.5 \\
\hline$M P-102$ & $\begin{array}{c}24 \\
(604-627)\end{array}$ & 1898.9 & 24.5 & 612.1 & 30.0 & 32.0 & 2.0 \\
\hline $\begin{array}{c}M P-103 \\
0 i 1\end{array}$ & $\begin{array}{c}10 \\
(618-627)\end{array}$ & 1892.3 & 24.4 & 580.6 & 27.7 & 31.0 & 3.2 \\
\hline $\begin{array}{c}\text { MP-103 } \\
\text { Gas }\end{array}$ & $\begin{array}{c}10 \\
(608-617)\end{array}$ & 1928.7 & 24.9 & 99.9 & 5.1 & 5.1 & 0 \\
\hline MP- 104 & $\begin{array}{c}19 \\
(652-670)\end{array}$ & 1882.4 & 24.3 & 631.2 & 31.1 & 33.5 & 2.4 \\
\hline$M P-105$ & $\begin{array}{c}20 \\
(612-631)\end{array}$ & 1708.0 & 22.0 & 485.6 & 25.3 & 27.5 & 2.2 \\
\hline MP-221 & $\begin{array}{c}20 \\
(660-679)\end{array}$ & 1908.2 & 24.6 & 671.2 & 34.2 & 34.8 & 0.6 \\
\hline MP-225 & $\begin{array}{c}21 \\
(654-674)\end{array}$ & 1727.9 & 22.3 & 489.0 & 25.5 & 27.6 & 2.1 \\
\hline $\begin{array}{l}\text { Arithmetic } \\
\text { Average }\end{array}$ & $19.1 *$ & $1843.2^{\star}$ & 23.9 & $589.4^{*}$ & $29.2^{\star}$ & $31.5^{\star}$ & $2.3^{\star}$ \\
\hline $\begin{array}{l}\text { Weighted } \\
\text { Average }\end{array}$ & & $1840.4^{*}$ & 23.8 & 590.1 * & $29.3^{*}$ & $31.6 *$ & $2.2^{\star}$ \\
\hline
\end{tabular}

*Averages do not include gas zone of MP-103. 
TABLE C2

CORE ANALYSIS RESULTS FOR THE TEST WELLS

\begin{tabular}{|c|c|c|c|c|c|}
\hline $\begin{array}{l}\text { Well } \\
\text { No. }\end{array}$ & $\begin{array}{c}\text { Net Pay, } \\
\mathrm{ft} \\
\text { (Interval) } \\
\end{array}$ & $\begin{array}{c}\text { Pore } \\
\text { Volume, } \\
\text { bb]/ac-ft }\end{array}$ & $\begin{array}{l}\text { Average } \\
\text { Porosity, } \\
\text { percent }\end{array}$ & $\begin{array}{l}\text { Hydrocarbon } \\
\text { Pore Volume, } \\
\text { bbl/ac-ft }\end{array}$ & $\begin{array}{l}\text { Average } 0 i 1 \\
\text { Saturation, } \\
\text { percent }\end{array}$ \\
\hline MP-101 & $\begin{array}{c}18 \\
(617-635)\end{array}$ & 2016.3 & 26.0 & 662.5 & 32.9 \\
\hline MP- 102 & $\begin{array}{c}20 \\
\vdots 606-625)\end{array}$ & 1978.4 & 25.5 & 375.9 & 19.0 \\
\hline $\begin{array}{c}M P-103 \\
0 i 1\end{array}$ & $\begin{array}{c}8 \\
(620-627)\end{array}$ & 1999.7 & 25.8 & 649.9 & 32.5 \\
\hline $\begin{array}{c}\text { MP-103 } \\
\text { Gas }\end{array}$ & $\begin{array}{c}12 \\
(608-619)\end{array}$ & 1908.6 & 24.6 & 108.8 & 5.7 \\
\hline MF-104 & $\begin{array}{c}19 \\
(654-672)\end{array}$ & 2017.2 & 26.0 & 554.7 & 25.7 \\
\hline MP-105 & $\begin{array}{c}19 \\
(614-632)\end{array}$ & 1674.2 & 21.6 & 374.9 & 22.4 \\
\hline MP-221 & $\begin{array}{c}19 \\
(562-680)\end{array}$ & 1941.9 & 25.7 & 611.7 & 31.5 \\
\hline MP-225 & $\begin{array}{c}21 \\
(557-678)\end{array}$ & 1818.4 & 23.4 & 376.4 & 20.7 \\
\hline $\begin{array}{c}\text { Arithmetic } \\
\text { fiverage }\end{array}$ & $17.7^{\star}$ & $1920.9 *$ & 24.8 & $515.1^{*}$ & $26.4^{\star}$ \\
\hline $\begin{array}{l}\text { Weighted } \\
\text { fiverage }\end{array}$ & & $1911.9 *$ & 24.7 & $498.6^{\star}$ & $25.5^{\star}$ \\
\hline
\end{tabular}

*Averages do not include gas zone of MP-103. 
TABLE C3

COMPARISON OF CORE- AND LOG RESULTS FOR TEST WELLS BASED ON CORE INTERVAL ONLY

\begin{tabular}{|c|c|c|c|c|c|c|c|c|c|c|c|c|}
\hline \multirow[b]{2}{*}{$\begin{array}{l}\text { Well } \\
\text { No. }\end{array}$} & \multicolumn{5}{|c|}{ Core Analysis Results } & \multicolumn{7}{|c|}{ Logging Results } \\
\hline & $\begin{array}{c}\text { Net Pay, } \\
\quad \mathrm{ft} \\
\text { (Interval) }\end{array}$ & $\begin{array}{c}\text { Pore } \\
\text { Volume, } \\
\text { bbl/ac-ft } \\
\end{array}$ & $\begin{array}{l}\text { Average } \\
\text { Porosity, } \\
\text { percent }\end{array}$ & $\begin{array}{l}\text { Hydrocarbon } \\
\text { Pore Volume, } \\
\text { bbl/ac-ft } \\
\end{array}$ & $\begin{array}{c}\text { Average } \\
0 \text { il } \\
\text { Saturation, } \\
\text { percent } \\
\end{array}$ & $\begin{array}{c}\text { Net Pay, } \\
\mathrm{ft} \\
\text { (Interval) }\end{array}$ & $\begin{array}{c}\begin{array}{c}\text { Pore } \\
\text { Volume, } \\
\text { bbl/ac-ft }\end{array} \\
\end{array}$ & $\begin{array}{l}\text { Average } \\
\text { Porosity, } \\
\text { percent }\end{array}$ & $\begin{array}{l}\text { Hydrocarbon } \\
\text { Pore Volume, } \\
\text { bbl/ac-ft }\end{array}$ & $\begin{array}{c}\text { Residual } \\
\text { oil } \\
\text { Saturation, } \\
\text { percent } \\
\end{array}$ & $\begin{array}{l}0 i 1 \\
\text { in } \\
\text { Place, } \\
\text { percent }\end{array}$ & $\begin{array}{c}\text { Movable } \\
0 \text { il, } \\
\text { percent } \\
\end{array}$ \\
\hline MP-101 & ${ }^{18}(617.5-634.5)$ & 2016.3 & 26.0 & 662.5 & 32.9 & $\begin{array}{c}18 \\
(618-635)\end{array}$ & 1931.8 & 24.9. & 668.4 & 31.2 & 34.6 & 3.4 \\
\hline MP-102 & $\begin{array}{c}13 \\
(613-625)\end{array}$ & 2006.4 & 25.9 & 517.6 & 25.8 & $\begin{array}{c}13 \\
(615-627)\end{array}$ & 1885.3 & 24.3 & 746.6 & 36.3 & 39.6 & 3.3 \\
\hline MP- 103 & $\begin{array}{c}8 \\
(620-627)\end{array}$ & 1999.7 & 25.8 & 653.8 & 32.7 & $\begin{array}{c}8 \\
(620-627)\end{array}$ & 1862.0 & 24.0 & 633.1 & 30.0 & 34.0 & 4.0 \\
\hline MP-104 & $\begin{array}{c}19 \\
(654-672)\end{array}$ & 2017.2 & 26.0 & 554.7 & 27.5 & $\begin{array}{c}19 \\
(652-670)\end{array}$ & 1882.4 & 24.3 & 630.6 & 31.1 & 33.5 & 2.4 \\
\hline MP- 105 & $\begin{array}{c}15 \\
(614-628)\end{array}$ & 1566.2 & 20.2 & 323.7 & 20.7 & $\begin{array}{c}15 \\
(612-626)\end{array}$ & 1737.9 & 22.4 & 507.5 & 27.1 & 29.2 & 2.1 \\
\hline MP-221 & $\begin{array}{c}20 \\
(662-681)\end{array}$ & 1941.0 & 25.7 & 605.8 & 30.4 & $\begin{array}{c}20 \\
(660-679)\end{array}$ & 1908.2 & 24.6 & 663.7 & 34.2 & 34.8 & 0.6 \\
\hline MP-225 & $\begin{array}{c}18 \\
(659-676)\end{array}$ & 1819.8 & 23.5 & 412.2 & 22.7 & $\begin{array}{c}18 \\
(655-672)\end{array}$ & 1776.7 & 22.9 & 543.7 & 28.4 & 30.6 & 2.2 \\
\hline $\begin{array}{l}\text { Arithmetic } \\
\text { Average }\end{array}$ & 15.9 & 1909.5 & 24.7 & 532.9 & 27.5 & 15.9 & 1854.9 & 23.9 & 627.7 & 31.2 & 33.8 & 2.6 \\
\hline $\begin{array}{l}\text { Weighted } \\
\text { Average }\end{array}$ & & 1907.8 & 24.7 & 529.9 & 27.4 & & 1857.3 & 23.9 & 625.7 & 31.2 & 33.6 & 2.4 \\
\hline
\end{tabular}


TABLE C4

ARITHHETIC AND WEIGHTED AVERAGES OF WELL LOG DETERMINED

PROPERTIES FOR FIVE TEST WELLS

\begin{tabular}{|c|c|c|c|c|c|c|c|c|c|c|c|c|c|c|}
\hline WELL & $\begin{array}{l}\text { INTERVA } \\
F T \text {. } \\
\text { FHOM }\end{array}$ & TO & $\begin{array}{l}\text { GPPSS } \\
\text { PAY } \\
\text { FT }\end{array}$ & $\begin{array}{l}\text { NEI } \\
\text { PAY } \\
\text { FT }\end{array}$ & $\begin{array}{l}\text { PURE } \\
\text { VELLNE } \\
\text { BUI./AC- FT }\end{array}$ & $\begin{array}{l}\text { AVERAGE } \\
\text { PARBSITY } \\
\text { \% }\end{array}$ & $\begin{array}{l}\text { HYDROCARBEN } \\
\text { PQRE VOLUME } \\
\text { 3AI/AC-FT }\end{array}$ & $\begin{array}{l}\text { AVERACE } \\
\text { RES. OIL } \\
\text { SAT.* }\end{array}$ & $\begin{array}{l}\text { AVERAGF } \\
\text { OIL IN } \\
\text { PLACE \% }\end{array}$ & $\begin{array}{l}\text { AVERAGE } \\
\text { MPVABLE } \\
\text { CIL } X\end{array}$ & $\begin{array}{l}\text { AVE. } \\
\text { PERME. } \\
\text { INDEX }\end{array}$ & $\begin{array}{l}\text { CLAY } \\
\text { AVE } \\
X\end{array}$ & $\begin{array}{l}\text { ELEV. } \\
\text { SEA LE } \\
\text { TOP }\end{array}$ & $\begin{array}{l}\text { FROM } \\
\text { VEL } \\
\text { BOTTOA }\end{array}$ \\
\hline $\begin{array}{l}M P=101 \\
M P=102 \\
M P=103 \\
M P=104 \\
M P=105\end{array}$ & $\begin{array}{l}615.00 \\
604.00 \\
608.00 \\
652.00 \\
612.00\end{array}$ & $\begin{array}{l}636.00 \\
628.00 \\
628.00 \\
671.00 \\
632.00\end{array}$ & $\begin{array}{l}21 \cdot 00 \\
24 \cdot 00 \\
20 \cdot 00 \\
19 \cdot 00 \\
20 \cdot 00\end{array}$ & $\begin{array}{l}21 \cdot 0 \\
24 \cdot 0 \\
16 \cdot 0 \\
19 \cdot 0 \\
19 \cdot 0\end{array}$ & $\begin{array}{l}1884.92 \\
1898.87 \\
1919.23 \\
1482.43 \\
1127.67\end{array}$ & $\begin{array}{l}24.30 \\
24.47 \\
24.74 \\
24.26 \\
22.27\end{array}$ & $\begin{array}{l}655 \cdot 81 \\
612 \cdot 12 \\
405 \cdot 49 \\
631 \cdot 19 \\
508 \cdot 08\end{array}$ & $\begin{array}{l}30.81 \\
30.00 \\
19.51 \\
31.09 \\
26.42\end{array}$ & $\begin{array}{l}34 \cdot 31 \\
32 \cdot 71 \\
21 \cdot 52 \\
33 \cdot 5.3 \\
28 \cdot 75\end{array}$ & $\begin{array}{l}3 \cdot 50 \\
? \cdot 01 \\
2 \cdot 01 \\
2 \cdot 44 \\
2 \cdot 33\end{array}$ & $\begin{array}{r}112 \cdot 19 \\
114 \cdot 12 \\
117 \cdot 12 \\
108 \cdot 42 \\
7.6 \cdot 32\end{array}$ & $\begin{array}{r}6.52 \\
11.08 \\
6.81 \\
5.95 \\
12.00\end{array}$ & $\begin{array}{l}768.00 \\
779.00 \\
783.00 \\
752.00 \\
773.00\end{array}$ & $\begin{array}{l}747.00 \\
755.00 \\
763.00 \\
733.00 \\
753.00\end{array}$ \\
\hline $\begin{array}{l}\text { ARI THME } \\
\text { WE I GHTE }\end{array}$ & $\begin{array}{l}\text { IC AVERA } \\
\text { AVERAGE }\end{array}$ & & 20.80 & 19.8 & $\begin{array}{l}1 * 62 \cdot \in 2 \\
1803 \cdot 19\end{array}$ & $\begin{array}{l}24.01 \\
24.02\end{array}$ & $\begin{array}{l}562.54 \\
571.69\end{array}$ & $\begin{array}{l}27.57 \\
28.00\end{array}$ & $\begin{array}{l}30.07 \\
30.47\end{array}$ & $\begin{array}{l}2.46 \\
2.47\end{array}$ & $\begin{array}{l}105 \cdot 64 \\
105 \cdot 85\end{array}$ & $\begin{array}{l}8.47 \\
8.62\end{array}$ & & \\
\hline
\end{tabular}


TABLE C5

GEOMETRIC MEAN OF WELL LOG DETERMINED

PROPERTIES FOR FIVE TEST WELLS

\begin{tabular}{|c|c|c|c|c|c|c|c|c|c|c|c|c|c|}
\hline WELL & $\begin{array}{l}\text { INTERV } \\
\text { FT } \\
\text { FROM }\end{array}$ & T⿱一⿻丿亅冖 & $\begin{array}{l}\text { GRESS } \\
\text { PAY } \\
\text { FT }\end{array}$ & $\begin{array}{l}\text { NET } \\
\text { PAY } \\
\text { FY }\end{array}$ & $\begin{array}{l}\text { PQRE } \\
\text { VGLUME } \\
\text { BBL/AC-FT }\end{array}$ & $\begin{array}{l}\text { G. MEAN } \\
\text { PEROSITY } \\
X\end{array}$ & $\begin{array}{l}\text { HYDRBCARBON } \\
\text { PGRE VOLUME } \\
\text { BBL /AC-FT }\end{array}$ & $\begin{array}{l}\text { G.MEAN } \\
\text { RES.OIL } \\
\text { SAT.X }\end{array}$ & $\begin{array}{l}\text { G.MEAN } \\
\text { OIL IN } \\
\text { PLACEX }\end{array}$ & $\begin{array}{l}\text { G. MEAN } \\
\text { PERME. } \\
\text { INOEX }\end{array}$ & $\begin{array}{l}\text { G. MEAN } \\
\text { CLAY } \\
X\end{array}$ & $\begin{array}{l}\text { ELEVATION } \\
\text { SEA LEVEL } \\
\text { TOP }\end{array}$ & $\begin{array}{l}\text { FROM } \\
\text { BOTTOM }\end{array}$ \\
\hline $\begin{array}{l}M P=101 \\
M P=102 \\
M P=103 \\
M P=104 \\
M P=105\end{array}$ & $\begin{array}{l}615.00 \\
604.00 \\
608.00 \\
652.00 \\
612.00\end{array}$ & $\begin{array}{l}636.00 \\
628.00 \\
628.00 \\
671.00 \\
632.00\end{array}$ & $\begin{array}{l}21.00 \\
24.00 \\
20.00 \\
19.00 \\
20.00\end{array}$ & $\begin{array}{l}21 \cdot 0 \\
24 \cdot 0 \\
16 \cdot 0 \\
19 \cdot 0 \\
19 \cdot 0\end{array}$ & $\begin{array}{l}1875.44 \\
1892.69 \\
1916.79 \\
1878.57 \\
1720.76\end{array}$ & $\begin{array}{r}24.17 \\
24.40 \\
24.71 \\
2 \quad 24.21 \\
\because \quad 22.18\end{array}$ & $\begin{array}{r}610.61 \\
571.57 \\
\quad 309.07 \\
\because \quad 626.74 \\
467.00\end{array}$ & $\begin{array}{l}29 \cdot 46 \\
28 \cdot 59 \\
15: 09 \\
30 \cdot 90 \\
25: 12\end{array}$ & $\begin{array}{l}32 \cdot 56 \\
30 \cdot 20 \\
16 \cdot 12 \\
33 \cdot 36 \\
27 \cdot 14\end{array}$ & $\begin{array}{r}103.67 \\
108.08 \\
114.37 \\
104.29 \\
71.17\end{array}$ & $\begin{array}{l}5 \cdot 56 \\
9 \cdot 32 \\
6 \cdot 18 \\
5 \cdot 68 \\
9 \cdot 63\end{array}$ & $\begin{array}{l}768.00 \\
779.00 \\
783.00 \\
752.00 \\
773.00\end{array}$ & $\begin{array}{l}747 \cdot 00 \\
755 \cdot 00 \\
763 \cdot 00 \\
733 \cdot 00 \\
753 \cdot 00\end{array}$ \\
\hline SEOMETRIC & $=$ MEAN & & 20.73 & 19.6 & 1855.49 & 23.92 & 501.02 & $25 \cdot 05$ & $27 \cdot \mathrm{cr}$ & 99.00 & 7.06 & & \\
\hline
\end{tabular}


TABLE $\mathrm{C} 6$

ARITHMETIC AND WEIGHTEO AVERAGES OF WELL LOG DETERMINED

PROPERTIES FOR CHESNEY WELLS

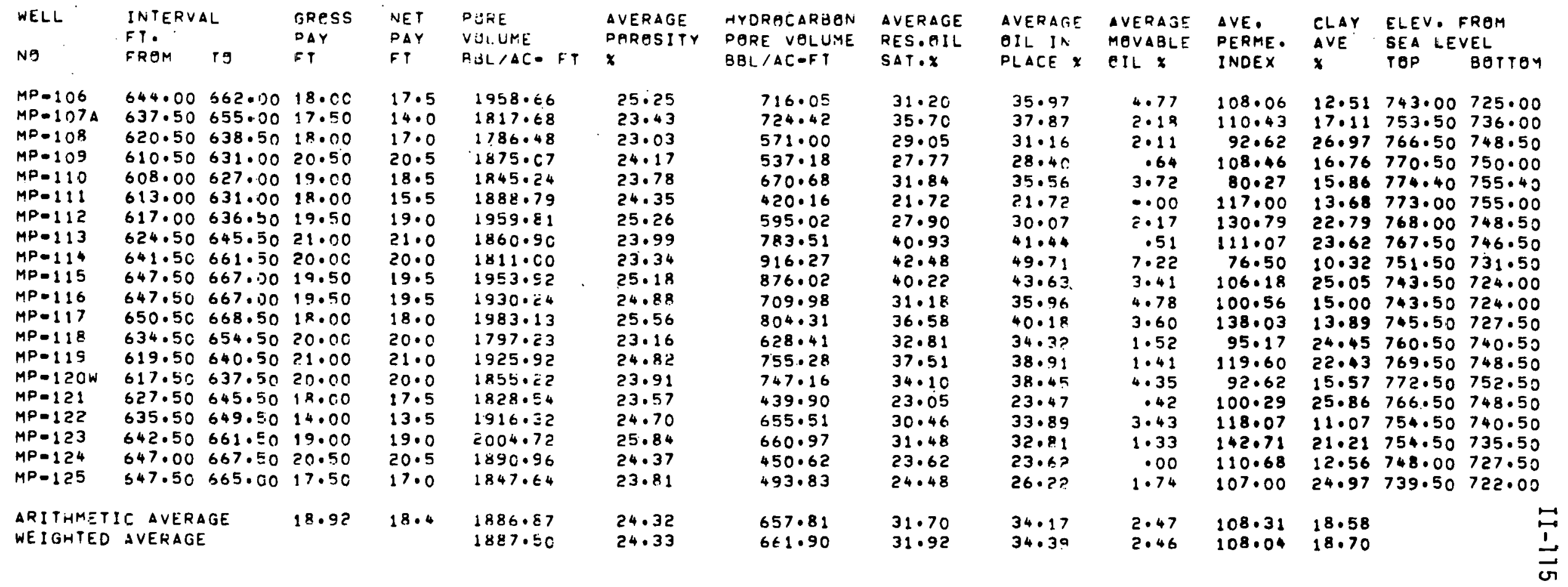


TABLE C7

GEOMETRIC MEAN OF WELL LOG DETERIMINED

PROPERTIES FOR CHESNEY WELLS

\begin{tabular}{|c|c|c|c|c|c|c|c|c|c|c|c|c|c|}
\hline WELL & $\begin{array}{l}\text { INTERVA } \\
\text { FT } \\
\text { FROM. }\end{array}$ & TO & $\begin{array}{l}\text { GRESS } \\
\text { PAY } \\
\text { FT }\end{array}$ & $\begin{array}{l}\text { NET } \\
\text { PAY } \\
\text { FT }\end{array}$ & $\begin{array}{l}\text { PEKE } \\
\text { VULUME } \\
\text { BUL / AC-FT }\end{array}$ & $\begin{array}{l}\text { T.MEAN } \\
\text { PEROSITY } \\
x\end{array}$ & $\begin{array}{l}\text { HYDROCARBEN } \\
\text { PORE VELUME } \\
\text { 3BL / AC-FT }\end{array}$ & $\begin{array}{l}\text { G.MEAN } \\
\text { RES•AIL } \\
\text { SAT•X }\end{array}$ & $\begin{array}{l}\text { GOMEAN } \\
\text { OIL IN } \\
\text { PLACEY }\end{array}$ & $\begin{array}{l}\text { G.MEAN } \\
\text { PERME. } \\
\text { INDEX }\end{array}$ & $\begin{array}{l}\text { S. MEAN } \\
\text { CLAY } \\
X\end{array}$ & $\begin{array}{l}\text { ELEVATION } \\
\text { SEA LEVEL } \\
\text { TOP }\end{array}$ & $\begin{array}{l}\text { FROM } \\
\text { BOTTOM }\end{array}$ \\
\hline $\begin{array}{l}M P=106 \\
M P=107 A \\
M P=108\end{array}$ & $\begin{array}{l}644.00 \\
637.50 \\
620 \cdot 50\end{array}$ & $\begin{array}{l}662.00 \\
655.00 \\
638.50\end{array}$ & $\begin{array}{l}18.06 \\
17.50 \\
18.00\end{array}$ & $\begin{array}{l}17.5 \\
14.0 \\
17.0\end{array}$ & $\begin{array}{l}1944.07 \\
1782.00 \\
1772.20\end{array}$ & $\begin{array}{l}25.06 \\
22.97 \\
22.84\end{array}$ & $\begin{array}{l}682 \cdot 11 \\
628 \cdot 52 \\
515 \cdot 05\end{array}$ & $\begin{array}{l}30.48 \\
33.57 \\
27.32\end{array}$ & $\begin{array}{l}35.09 \\
35.27 \\
29.06\end{array}$ & $\begin{array}{l}96.18 \\
77.67 \\
78.64\end{array}$ & $\begin{array}{l}11.07 \\
16.28 \\
24.21\end{array}$ & $\begin{array}{l}743.00 \\
753.50 \\
766.50\end{array}$ & $\begin{array}{l}725 \cdot 00 \\
736 \cdot 00 \\
748 \cdot 50\end{array}$ \\
\hline $\begin{array}{l}M P-109 \\
M P=110\end{array}$ & $\begin{array}{l}610.50 \\
608.00\end{array}$ & $\begin{array}{l}631.00 \\
627.00\end{array}$ & $\begin{array}{l}20.50 \\
19 \cdot 00\end{array}$ & $\begin{array}{l}20.5 \\
18.5\end{array}$ & $\begin{array}{l}1868.71 \\
1837.84\end{array}$ & $\begin{array}{l}24.09 \\
23.69\end{array}$ & $\begin{array}{l}52.0 \cdot 77 \\
596 \cdot 75\end{array}$ & $\begin{array}{l}27.23 \\
29.47\end{array}$ & $\begin{array}{l}27.87 \\
32.47\end{array}$ & $\begin{array}{l}95 \cdot 56 \\
76 \cdot 04\end{array}$ & $\begin{array}{l}13.63 \\
13.98\end{array}$ & $\begin{array}{l}770.50 \\
774.40\end{array}$ & $\begin{array}{l}750.00 \\
755.40\end{array}$ \\
\hline $\begin{array}{l}M P-111 \\
M P-112\end{array}$ & $\begin{array}{l}613.00 \\
617.00\end{array}$ & $\begin{array}{l}631 \cdot 00 \\
636 \cdot 50\end{array}$ & $\begin{array}{l}18.00 \\
19.50\end{array}$ & $\begin{array}{l}15.5 \\
19.0\end{array}$ & $\begin{array}{l}1880 \cdot \equiv 7 \\
1954 \cdot 41\end{array}$ & $\begin{array}{l}24.24 \\
25.19\end{array}$ & $\begin{array}{l}384.19 \\
580.77\end{array}$ & $\begin{array}{l}20.43 \\
27.59\end{array}$ & $\begin{array}{l}20.43 \\
29.72\end{array}$ & $\begin{array}{l}108 \cdot 85 \\
123.80\end{array}$ & $\begin{array}{l}12 \cdot 30 \\
20 \cdot 71\end{array}$ & $\begin{array}{l}773.00 \\
768.00\end{array}$ & $\begin{array}{l}755.00 \\
748.50\end{array}$ \\
\hline$M P=113$ & 624.50 & 645.50 & 21.00 & $21 \cdot 0$ & 1844.95 & 23.78 & 749.33 & $40 \cdot 16$ & 40.61 & 93.78 & $21 \cdot 27$ & $767 \cdot 50$ & 746.50 \\
\hline $\begin{array}{l}M P-114 \\
M P-115\end{array}$ & $\begin{array}{l}641.50 \\
647.50\end{array}$ & $\begin{array}{l}661.50 \\
667.00\end{array}$ & $\begin{array}{l}20.00 \\
19.50\end{array}$ & $\begin{array}{l}20.0 \\
19.5\end{array}$ & $\begin{array}{l}1795.23 \\
1937.06\end{array}$ & $\begin{array}{l}23.14 \\
24.97\end{array}$ & $\begin{array}{l}838.78 \\
787.74\end{array}$ & $\begin{array}{l}40 \cdot 35 \\
37.52\end{array}$ & $\begin{array}{l}46.72 \\
40.67\end{array}$ & $\begin{array}{l}66.62 \\
88.49\end{array}$ & $\begin{array}{r}9 \cdot 30 \\
24 \cdot 38\end{array}$ & $\begin{array}{l}751 \cdot 50 \\
743.50\end{array}$ & $\begin{array}{l}731.50 \\
724.00\end{array}$ \\
\hline $\begin{array}{l}M P-116 \\
M P-117\end{array}$ & $\begin{array}{l}647.50 \\
650.50\end{array}$ & $\begin{array}{l}667.00 \\
668.50\end{array}$ & $\begin{array}{l}19.50 \\
18.00\end{array}$ & $\begin{array}{l}19.5 \\
18.0\end{array}$ & $\begin{array}{l}1915.00 \\
1977.66\end{array}$ & $\begin{array}{l}24.68 \\
25.49\end{array}$ & $\begin{array}{l}661.61 \\
776.97\end{array}$ & $\begin{array}{l}30.13 \\
35.73\end{array}$ & $\begin{array}{l}34.55 \\
39.29\end{array}$ & $\begin{array}{r}85.52 \\
128.68\end{array}$ & $\begin{array}{l}12.90 \\
12.69\end{array}$ & $\begin{array}{l}743.50 \\
745.50\end{array}$ & $\begin{array}{l}724 \cdot 00 \\
727 \cdot 50\end{array}$ \\
\hline $\begin{array}{l}M P-118 \\
M P-119 \\
M P-120 W\end{array}$ & $\begin{array}{l}634.50 \\
619.50 \\
617.50\end{array}$ & $\begin{array}{l}654 \cdot 50 \\
640 \cdot 50 \\
637 \cdot 50\end{array}$ & $\begin{array}{l}20.00 \\
21.00 \\
20.00\end{array}$ & $\begin{array}{l}20 \cdot 0 \\
21 \cdot 0 \\
20 \cdot 0\end{array}$ & $\begin{array}{l}1782 \cdot 99 \\
1921 \cdot 68 \\
1827.63\end{array}$ & $\begin{array}{l}22.98 \\
24.76 \\
23.56\end{array}$ & $\begin{array}{l}593.23 \\
737.80 \\
658.18\end{array}$ & $\begin{array}{l}31 \cdot 86 \\
36.92 \\
32 \cdot 29\end{array}$ & $\begin{array}{l}33.27 \\
38.40 \\
36.01\end{array}$ & $\begin{array}{r}81.12 \\
112.41 \\
72.48\end{array}$ & $\begin{array}{l}22 \cdot 50 \\
20 \cdot 73 \\
14 \cdot 07\end{array}$ & $\begin{array}{l}760 \cdot 50 \\
769 \cdot 50 \\
772 \cdot 50\end{array}$ & $\begin{array}{l}740 \cdot 50 \\
748 \cdot 50 \\
752 \cdot 50\end{array}$ \\
\hline $\begin{array}{l}M P=121 \\
M P=122\end{array}$ & $\begin{array}{l}627.50 \\
635.50\end{array}$ & $\begin{array}{l}645.50 \\
649.50\end{array}$ & $\begin{array}{l}18.00 \\
14.00\end{array}$ & $\begin{array}{l}17.5 \\
13.5\end{array}$ & $\begin{array}{l}1817.48 \\
1910.89\end{array}$ & $\begin{array}{l}23.43 \\
24.63\end{array}$ & $\begin{array}{l}409 \cdot 43 \\
640.36\end{array}$ & $\begin{array}{l}22 \cdot 16 \\
30.03\end{array}$ & $\begin{array}{l}22.53 \\
33.51\end{array}$ & $\begin{array}{r}89.66 \\
108.66\end{array}$ & $\begin{array}{l}24 \cdot 33 \\
10 \cdot 55\end{array}$ & $\begin{array}{l}766 \cdot 50 \\
754 \cdot 50\end{array}$ & $\begin{array}{l}748 \cdot 50 \\
740.50\end{array}$ \\
\hline$M P-123$ & 642.50 & $661 \cdot 50$ & 19.00 & 19.0 & 1999.73 & 25.78 & $641 \cdot 79$ & 30.97 & 32.09 & 128.43 & 19.33 & 754.50 & $735 \cdot 50$ \\
\hline $\begin{array}{l}M P=124 \\
M P=1.25\end{array}$ & $\begin{array}{l}647.00 \\
647.50\end{array}$ & $\begin{array}{l}667.50 \\
665.00\end{array}$ & $\begin{array}{l}20.50 \\
17.50\end{array}$ & $\begin{array}{l}20.5 \\
17.0\end{array}$ & $\begin{array}{l}1886.13 \\
1832.12\end{array}$ & $\begin{array}{l}24.31 \\
23.61\end{array}$ & $\begin{array}{l}440.78 \\
459.95\end{array}$ & $\begin{array}{l}23 \cdot 37 \\
23 \cdot 56\end{array}$ & $\begin{array}{l}23 \cdot 37 \\
25 \cdot 10\end{array}$ & $\begin{array}{r}105 \cdot 55 \\
90 \cdot 3.3\end{array}$ & $\begin{array}{l}11.03 \\
23.54\end{array}$ & $\begin{array}{l}748.00 \\
739.50\end{array}$ & $\begin{array}{l}727 \cdot 50 \\
722 \cdot 00\end{array}$ \\
\hline GEQMETR & MEAN & & 18.85 & $18 \cdot 2$ & 1873.17 & $24 \cdot 14$ & $601 \cdot 39$ & 30.03 & 32.10 & 93.76 & $16 \cdot 13$ & & \\
\hline
\end{tabular}


TAELE C8

ARITHMETIC AND WEIGHTED AVERAGES OF WELL LOG DETEFMINED

PROPERTIES FOR HEGBERG WELLISS

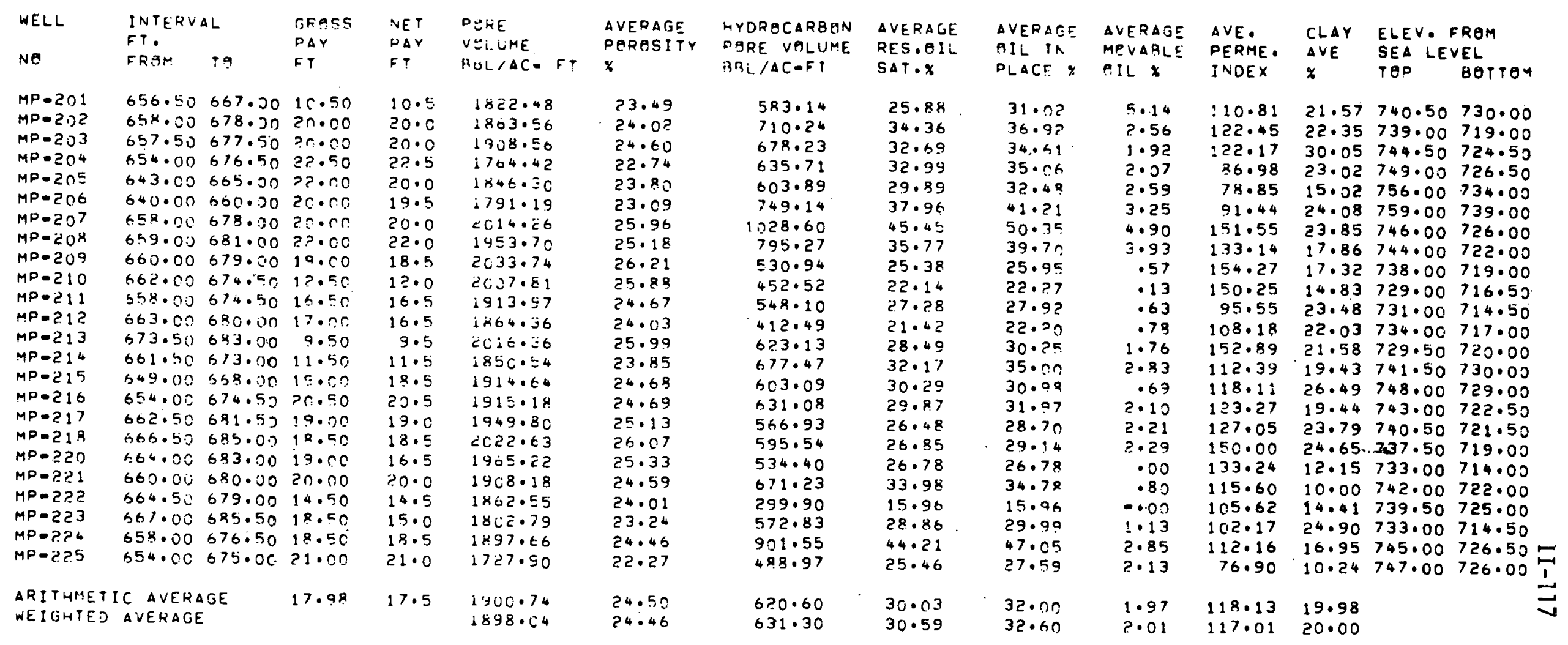


TABLE C 9

GEOMETRIC MEAN OF WELL LOG DETERMINED PROPERTIES FOR HEGBERG WELLS

\begin{tabular}{|c|c|c|c|}
\hline WELL & $\begin{array}{l}\text { INTERV } \\
\text { FT }\end{array}$ & & $\begin{array}{l}\text { GRASS } \\
\text { PAY }\end{array}$ \\
\hline NO & FROM & TO & FT \\
\hline $\begin{array}{l}M P-201 \\
M P-202\end{array}$ & $\begin{array}{l}656.50 \\
658.00\end{array}$ & $\begin{array}{l}667.00 \\
678.00\end{array}$ & $\begin{array}{l}10.50 \\
20.50\end{array}$ \\
\hline$M P=203$ & 657.50 & 677.50 & $20 \cdot 00$ \\
\hline $\begin{array}{l}M P-204 \\
M P=205\end{array}$ & $\begin{array}{l}654.00 \\
643.00\end{array}$ & $\begin{array}{l}676.50 \\
665.00\end{array}$ & $\begin{array}{l}22.50 \\
22.00\end{array}$ \\
\hline $\begin{array}{l}M P-206 \\
M P-207\end{array}$ & $\begin{array}{l}640.00 \\
658.00\end{array}$ & $\begin{array}{l}660.00 \\
678.00\end{array}$ & $\begin{array}{l}20.00 \\
20 .=0\end{array}$ \\
\hline$M P=208$ & 659.00 & 631.00 & 32.00 \\
\hline $\begin{array}{l}M P-209 \\
M P-210 \\
M P=29\end{array}$ & $\begin{array}{l}600 \cdot 00 \\
662.00\end{array}$ & $\begin{array}{l}679.00 \\
674.50\end{array}$ & $\begin{array}{l}19 \cdot 00 \\
12.50\end{array}$ \\
\hline$M P-211$ & 658.00 & 674.50 & 16.50 \\
\hline$M P-212$ & 663.00 & 680.00 & 17.00 \\
\hline$M P-213$ & 673.50 & 683.00 & 9.50 \\
\hline$p-214$ & 661.50 & 673.00 & 11.50 \\
\hline$P-215$ & 649.00 & 668.00 & $19 \cdot \mathrm{CO}$ \\
\hline MP - & 654.00 & 674.50 & 50 \\
\hline$M P-217$ & 662.50 & $681 \cdot 50$ & $19 \cdot 00$ \\
\hline $4 P-218$ & 666.50 & 6.85 .00 & 18.50 \\
\hline$P=220$ & 664.00 & 683.00 & 19.00 \\
\hline & 660.00 & 680.00 & $2 c \cdot c 0$ \\
\hline & 50 & & 14.50 \\
\hline$A P-$ & 667.00 & 635.50 & 18.50 \\
\hline & 658.00 & 676.50 & 18.50 \\
\hline & 54.00 & 00 & 21.00 \\
\hline
\end{tabular}

NET PERE

PAY VOLUME

$10.5 \quad 1793.79$

$20.0 \quad 1840.37$

$20.0 \quad 1893.40$

$22.5 \quad 1752.17$

1841.7

$19.5 \quad 1182.87$

$22.0 \quad 1942 . \mathrm{C}$

$18.5 \quad 2027.89$

$12.0 \quad 1996 . \hat{6} 3$

$16.5 \quad 1902.69$

$16.5 \quad 1853.86$

9.52009 .4

$11.5 \quad 1829.57$

$18.5 \quad 190 \% .16$

20.51898 .30

$19.0 \quad 1944.69$

$18.5 \quad 2016.96$

16.51938 .20

$20.0 \quad 1903.56$

$14.5 \quad 1856.70$

$18.5 \quad 1892.05$

$21.0 \quad 1719.79$
G. MEAN

PERESITY

23.12
23.72
24.40
22.59
23.74
22.98
25.80
25.03
26.14
25.73
24.52
23.89
25.90
23.58
24.58
24.47
25.07
26.00
25.24
24.54
23.93
22.94
24.39
22.17
24.33

HYDRGCARBEN PQRE VOLUME SAL /AC -FT

G.MEAN

526.78
651.48
618.07
591.51
584.60
720.94
980.43
734.60
519.01
424.62
502.13
389.86
560.51
594.83
574.98
571.17
537.14
573.73
500.94
638.39
282.12
463.58
873.69
436.84
559.48
RES.OIL SAT. $\%$

G. MEAN QIL IN PLACEX

$24 \cdot 74$

$33 \cdot 12$

30.79

31.71

29.20

$37 \cdot 16$

44.42

33.96

25.11

21.18

25.87

$20 \cdot 57$

26.43

30.25

29.50

38.21

25.55

26.29

25.58

32.87

$15 \cdot 19$

25.21

$43 \cdot 38$

23.51

28.01$$
39.37
$$$$
32.64
$$$$
33.76
$$$$
31.74
$$$$
40.44
$$

48.98

37.82

25.59

21.27

26.39

$21 \cdot 03$

27.89

32.52

30.15

30.09

27.62

28.44

25.58

33.54

15.19

26.05

46.18

$25.4 \mathrm{C}$

$29 \cdot 64$
G.REAN PERME. INDEX

G. MEAN CLAY $x$

ELEVATION FROM SEA LEVEL

75.57

$102 \cdot 30$

106.25

$75 \cdot 60$

75.63

9.4. 0 ?

137.63

120.94

$144 \cdot 90$

134.58

$85 \cdot 18$

95.77

$147 \cdot 0.3$

91.94

$109 \cdot 12$

106.57

119.81

138.16

124.44

$110 \cdot 75$

07.60

105.70

165.70
71.02

20.63

18.46
29.23

29.23

$21 \cdot 95$

$12 \cdot 22$
$22 \cdot 19$

22.12

16.50

15.91

13.00

20.97

19.61

19.83

18.08

25.02

16.03

21.69

23.67

11.19

0.43

23.72

15.77

8.58

TEP

BotTom

$740 \cdot 50$

739.00

744.50

749.00

756.00

759.00

746.00

744.00

738.00

729.00

731.00

734.00

729.50

741.50

$748: 00$

743.00

740.50

737.50

733.00

742.00

$739 \cdot 50$

733.00

745.00

747.00

730.00

719.00

$724 \cdot 50$

$726 \cdot 50$

$734 \cdot 00$

739.00

$726 \cdot 00$

$722 \cdot 00$

716.50

714.50

717.00

$720 \cdot 00$

$730 \cdot 00$

$729 \cdot 00$

$722 \cdot 50$

$721 \cdot 50$

719.00

$714 \cdot 00$

722.00

714.50

$714 \cdot 50$
726.50

726.50

GEOMETRIC MEAN

17.54

103.43 
TABLE C10

ARITHMETIC AND WEIGHTED AVERAGES OF WELL LOG DETERMINED

PROPERTIES FOR CHESNEY AND HEGBERG HELLS

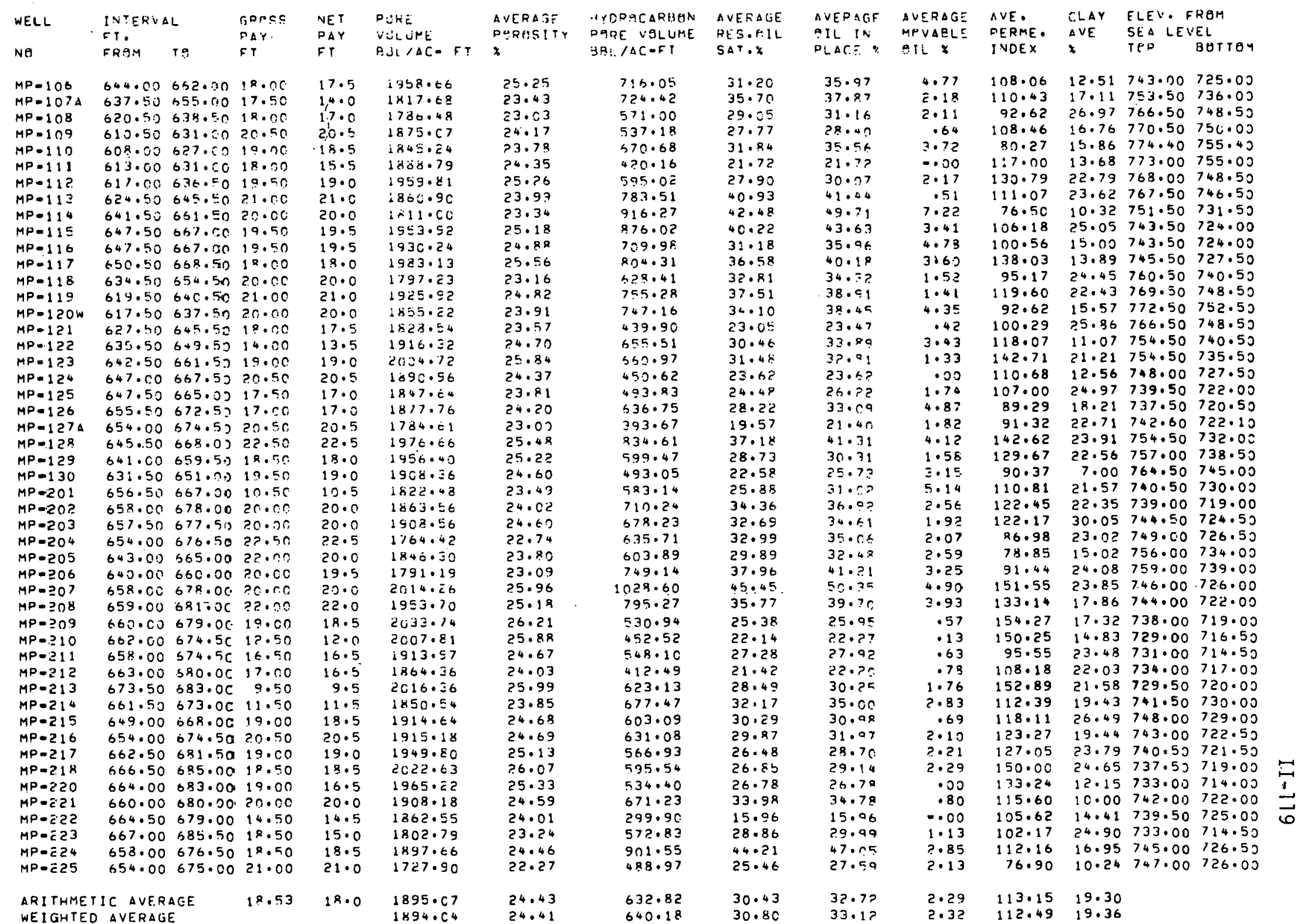


TABLE CII

GEOMETRIC MEAN OF WELL LOG DETERMINED

PROPERTIES FOR CHESNEY AND HEGBERG WELLS

\begin{tabular}{|c|c|c|c|}
\hline WELL & $\begin{array}{l}\text { INTERVA } \\
\text { FT }\end{array}$ & & $\begin{array}{l}\text { GRPSS } \\
\text { PAY }\end{array}$ \\
\hline NE & FROM & פ & FT \\
\hline$P-106$ & 644.00 & $662 \cdot 00$ & 19.00 \\
\hline$M P=107 A$ & 637.50 & 655.00 & 17.50 \\
\hline$M P=108$ & 620.50 & 638.50 & 18.00 \\
\hline$M P-109$ & 610.50 & 631.00 & 20.50 \\
\hline$M P-110$ & 608.00 & 627.00 & 19.00 \\
\hline $\begin{array}{l}M P-111 \\
M P=112\end{array}$ & $\begin{array}{l}613.00 \\
617.00\end{array}$ & $\begin{array}{l}631.00 \\
636.50\end{array}$ & $\begin{array}{l}12.00 \\
19.50\end{array}$ \\
\hline$M P=113$ & 624.50 & 645.50 & 21.00 \\
\hline$M P=114$ & 641.50 & 661.50 & 20.00 \\
\hline$M P-115$ & 647.50 & 667.00 & 19.50 \\
\hline$M P=116$ & 647.50 & 667.00 & 19.50 \\
\hline$M P-117$ & 650.50 & 668.50 & 12.00 \\
\hline$M P=118$ & 634.50 & 654.50 & 20.00 \\
\hline$M P-119$ & 619.50 & 640.50 & 21.00 \\
\hline$M P=120 W$ & 617.50 & 637.50 & 20000 \\
\hline$M P-121$ & 627.50 & 645.50 & 18.00 \\
\hline$M P=122$ & 635.50 & 649.50 & 14.00 \\
\hline$M P=123$ & 642.50 & 661.50 & 19.00 \\
\hline$M P-124$ & 647.00 & 367.50 & 20.50 \\
\hline$M P=125$ & 647.50 & 665.00 & 17.50 \\
\hline$M P=126$ & 655.50 & 672.50 & 17.00 \\
\hline$M P=127 A$ & 654.00 & 674.50 & 20.50 \\
\hline$M P=128$ & 645.50 & 668.00 & 22.50 \\
\hline$M P=123$ & 641.00 & 659.50 & 18.50 \\
\hline$M P=130$ & 631.50 & 651.00 & 19.50 \\
\hline$M P=201$ & 656.50 & 667.00 & 10.50 \\
\hline$M P=202$ & 658.00 & 678.00 & 20.00 \\
\hline$M P=203$ & 657.50 & 677.50 & 20.00 \\
\hline$M P=204$ & 654.00 & 676.50 & 22.50 \\
\hline$M P=205$ & 643.00 & 665.00 & 22.00 \\
\hline$M F-206$ & 640.00 & 660.00 & 20.00 \\
\hline$M P=207$ & 658.00 & 678.00 & 20.00 \\
\hline$M P-208$ & 659.00 & 681.00 & 22.00 \\
\hline$M P=209$ & 660.00 & 679.00 & 19.00 \\
\hline MP. & $\begin{array}{l}662.00 \\
658.00\end{array}$ & $\begin{array}{l}674.50 \\
674.50\end{array}$ & $\begin{array}{l}17.50 \\
16.50\end{array}$ \\
\hline$M P-212$ & 663.00 & 680.00 & 17.00 \\
\hline$M P=213$ & 673.50 & 683.00 & 9.50 \\
\hline$M P=214$ & 661.50 & 673.00 & 11.50 \\
\hline $\begin{array}{l}M P-215 \\
M P=216\end{array}$ & $\begin{array}{l}649.00 \\
654.00\end{array}$ & $\begin{array}{l}668.00 \\
674.50\end{array}$ & $\begin{array}{l}19.00 \\
20.50\end{array}$ \\
\hline$M P=217$ & 662.50 & 681.50 & 19.00 \\
\hline$M P-218$ & 666.50 & 685.00 & 18.50 \\
\hline$M P=220$ & 664.00 & 683.00 & 19.00 \\
\hline & 660.00 & 680.00 & 20.00 \\
\hline$M P-222$ & 664.50 & 679.00 & 14.50 \\
\hline$M P=223$ & 667.00 & $685.5 n$ & 18.50 \\
\hline$M P=224$ & 658.00 & 676.50 & 19.50 \\
\hline$M P=225$ & 654.00 & 675.00 & 21.00 \\
\hline
\end{tabular}

NET PLRE
PAY VELLME

FT BEL/ACFF

$\begin{array}{ll}17.5 & 1944 . C^{27}\end{array}$

$14.0 \quad 1782.00$

$20.5 \quad 1 \times 62.71$

$18.5 \quad 1837.84$

$15.5 \quad 1880.37$

$19.0 \quad 1954.4$

$21.0 \quad 1344.55$

$19.5 \quad 1937.06$

$19.5 \quad 1915.00$

$18.0 \quad 1977.66$

$20.0 \quad 1782.99$

$21.0 \quad 1921.68$

$20.0 \quad 1827.63$

$17.5 \quad 1817.48$

$13.5 \quad 1910.8$

$20.5 \quad 1886.13$

$17.0 \quad 1832.12$

$17.0 \quad 1863.52$

$20.5 \quad 1172.31$

$22.5 \quad 1959.74$

$\begin{array}{ll}18.0 & 1948 . \pm 2 \\ 19.0 & 1904.87\end{array}$

$10.5 \quad 1793.79$

$20.0 \quad 184 \mathrm{C} .37$

$20.0 \quad 1893.40$

1752.17

1841.74

1782.87

2001.53

$18.5 \quad 2027.89$

$12.0 \quad 1996.03$

$\begin{array}{ll}12.0 & 1996 . \hat{c} \\ 16.5 & 1902.69\end{array}$

$16.5 \quad 1853.86$

$9.5 \quad 2009.44$

11.51829 .3

$18.5 \quad 1907.16$

20.51898 .30

$18.5 \quad 1944.69$

$16.5 \quad 1958.20$

$20.0 \quad 1903.56$

$14.5 \quad 1856.70$

$15.0 \quad 1779.60$

$\begin{array}{ll}18.5 & 1892.05 \\ 21.0 & 1719.79\end{array}$
G. MEAN

25.06

22.97

22.84
24.09

23.69

24.24

25.19
23.78

23.14

24.97

24.68
25.49

?.2. 98

24.76

23.56
23.43

24.63
25.78

24.31

23.61

24.02

22.84

25.26

25.11
24.55

23.12

23.72

24.40
22.59

23.74

22.98

25.80
25.03

26.14
25.73

25.73

23.89

25.90

24.58

24.58
24.47

25.07

26.00

25.24

24.54

23.93

22.94

22.39
22.17

24.25
HYDRACARBON

682.11

628.52

515.05

520.77

384.19

580.77

749.33

838.78

787.74

661.61
776.97

593.23

737.80

658.18
409.43

640.36

641.79

440.78

459.95

565.00

349.24
778.62

581.61

526.78
651.48

618.07

591.51

584.60

720.94

980.43

734.60

519.01

424.62

389.86

560.51

594.83

574.98

571.17

537.14
573.73

500.94

638.39

282.12

463.58

873.69
436.84

573.16
G. MEAN RES. OIL
SAT. $X$

30.48

33.57
27.32

27.23
27.23

29.47

20.43
27.59

40.16

40.35

30.13

35.73

31.86

32.29

22.16

30.03

23.37

23.56

26.58
18.40

35.91

28.33

21.84

33.12

30.79

29.20

37.16

33.96

$25 \cdot 11$

21.18

20.57

$30 \cdot 25$

29.50

$28 \cdot 21$

26.29

25.58

32.87

$15 \cdot 19$

45.21
43.38

23.51

28.55
G.ME

G.MEAN

PERME

$35 \cdot \mathrm{Ca}^{\circ}$
$35 \cdot$ ? 7

96.18
77.67

77.67
78.64

$\begin{array}{ll}35.06 & 78.6 \\ 27.27 & 95.56\end{array}$

32.47

20.43

95.56
76.04

108.85

$29.72 \quad 123.80$

$46.72 \quad 66.62$

$40.67 \quad 28.49$

34.55
35.52

$33.27 \quad 81.12$

$38.4 \mathrm{C} \quad 112.41$

$22.53 \quad 89.66$

$33.51 \quad 108.66$

$23.37 \quad 105.55$

$25.10 \quad 90.33$

$19.70 \quad 80.34$

$39.73 \quad 123.39$

$\begin{array}{rr}29.85 & 118.39\end{array}$

$29.37 \quad 75.57$

$35.40 \quad 102.30$

$33.76 \quad 75.60$

$31.74 \quad 75.63$

$40.44 \quad 84.02$

$48.98 \quad 137.63$

$25.59 \quad 144.90$

$21 . ? 7 \quad 134.58$

$26.39 \quad 85.18$

$21.03 \quad 95.77$
27.89

$32.52 \quad 91.9$

$30.15 \quad 109.1$

$30.09 \quad 106.57$

138.16

$15 \cdot 19$

46.18
25.40

124.44

97.60

81.9

CLAY

ELEVATISN FROM

SEA LEVEL

11.07

743.00
753.50

16.28

19.21
13.63

13.63

12.30

12.30
20.71
21.27

9.30
64.38

$9 \cdot 30$
24.38

12.90

12.69

22.50
20.73

14.07

10.55

19.33

12.03
23.54

15.19

$19 \cdot 04$
22.37

$21 \cdot 30$

6.52
20.63

20.63

18.46
29.23

12.22

22.19

22.12

16.50

5.91

20.97

19.61

8.08

25.02

16.03

21.69
23.67

23.67
11.19

4.43

12.90
23.72
15.72

15.77
8.58

30.46

98.52

16.58

$774.40 \quad 755.40$

$768.00 \quad 748.50$

$767.50 \quad 746.50$

743.50
724.00

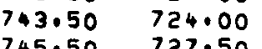

$760.50 \quad 740.50$

$769.50 \quad 748.50$

$772.50 \quad 752.50$

$754.50 \quad 740.50$

$\begin{array}{ll}754.50 & 735.50 \\ 748.00 & 727.50\end{array}$

$739.50 \quad 722.00$

$737.50-720.50$

742.60
754.50
7322.10

$757.00 \quad 738.50$

$764.50 \quad 745.00$ 
TABLE $\mathrm{C} 12$

WELL L.OG DETERMINED PROPERTIES

VS DEPTH FOR WELL MP-101

WELL LOCATION: 2848 FSL 2590 FEL 21255 5E

KELLY BUSHING ELEVATION FROM SEA LEVEL $=1383$

\begin{tabular}{|c|c|c|c|c|c|c|c|c|c|c|c|c|c|c|}
\hline DEPTH * & GR & POR & RT & $R \times \theta$ & $5 \times 0$ & ROS & SW & PER & CLAY & so & mo & PHV & HPV & ELSE \\
\hline $\begin{array}{l}615000 \\
616.00\end{array}$ & $\begin{array}{l}99.50 \\
96.20\end{array}$ & $\begin{array}{l}18 \cdot 30 \\
21.30\end{array}$ & $\begin{array}{l}4.10 \\
4.60\end{array}$ & $\begin{array}{l}3.80 \\
3.60\end{array}$ & $\begin{array}{l}78.90 \\
63.40\end{array}$ & $\begin{array}{l}21 \cdot 10 \\
36.60\end{array}$ & $\begin{array}{l}78.90 \\
63.40\end{array}$ & $\begin{array}{l}31 \cdot 00 \\
59 \cdot 00\end{array}$ & $\begin{array}{l}17.00 \\
10.00\end{array}$ & $\begin{array}{l}21.10 \\
36.60\end{array}$ & $\begin{array}{l}.00 \\
.00\end{array}$ & $\begin{array}{l}1419.79 \\
1652.54\end{array}$ & $\begin{array}{l}299.57 \\
604.83\end{array}$ & $\begin{array}{l}768.00 \\
767.00\end{array}$ \\
\hline $\begin{array}{l}617.00 \\
618.00\end{array}$ & $\begin{array}{l}86.80 \\
82.40\end{array}$ & $\begin{array}{l}22.10 \\
22.90\end{array}$ & $\begin{array}{l}4.70 \\
4.50\end{array}$ & $\begin{array}{l}4.70 \\
5.00\end{array}$ & $\begin{array}{l}71.60 \\
\epsilon 6.90\end{array}$ & $\begin{array}{l}28 \cdot 40 \\
33.10\end{array}$ & $\begin{array}{l}59.70 \\
58.80\end{array}$ & $\begin{array}{l}70.00 \\
83.00\end{array}$ & $\begin{array}{l}5.00 \\
4.00\end{array}$ & $\begin{array}{l}40.30 \\
41.20\end{array}$ & $\begin{array}{r}11.90 \\
8.10\end{array}$ & $\begin{array}{l}1714.61 \\
1776.67\end{array}$ & $\begin{array}{l}690.99 \\
731.99\end{array}$ & $\begin{array}{l}766.00 \\
765.00\end{array}$ \\
\hline $\begin{array}{l}618.00 \\
619.00\end{array}$ & $\begin{array}{l}82.40 \\
78.70\end{array}$ & $\begin{array}{l}22.90 \\
24.30\end{array}$ & $\begin{array}{l}4.50 \\
3.90\end{array}$ & $\begin{array}{l}5.00 \\
4.40\end{array}$ & $\begin{array}{l}66.90 \\
66.80\end{array}$ & $\begin{array}{l}33 \cdot 10 \\
33 \cdot 20\end{array}$ & $\begin{array}{l}58.80 \\
59.20\end{array}$ & $\begin{array}{r}83 \cdot 00 \\
106.00\end{array}$ & $\begin{array}{l}3000 \\
3: 00\end{array}$ & $\begin{array}{l}41.20 \\
40.80\end{array}$ & $\begin{array}{r}8.10 \\
7.60\end{array}$ & $\begin{array}{l}1776.067 \\
1885.29\end{array}$ & $\begin{array}{r}731.99 \\
769.20\end{array}$ & $\begin{array}{l}765.00 \\
764.00\end{array}$ \\
\hline 620.00 & 76.80 & 25.40 & $3 \cdot 20$ & 3.90 & 67.50 & $32 \cdot 50$ & 62.60 & $128 \cdot 00$ & 3.00 & 37.40 & 4.90 & 1970.63 & 737.02 & 763.00 \\
\hline 621.00 & 78.90 & 25.80 & $2 \cdot 80$ & 3.60 & $69 \cdot 10$ & 30.90 & 66.30 & 139.00 & 3.00 & 33.70 & 2.80 & 2001.67 & 674.56 & 762.00 \\
\hline 622.00 & 79.40 & 25.50 & $2 \cdot 50$ & 3.60 & $70 \cdot 40$ & 29.60 & 69.90 & 131.00 & 3.00 & $30 \cdot 10$ & .50 & $1978 \cdot 39$ & 595.50 & 1.00 \\
\hline 623.00 & $8 B .00$ & 25.70 & $2 \cdot 50$ & $3 \cdot 40$ & $71 \cdot 80$ & $28 \cdot 20$ & 69.60 & $135 \cdot 00$ & 4.00 & $30 \cdot 40$ & $2 \cdot 20$ & 1993.91 & $606 \cdot 15$ & 760.00 \\
\hline 624.00 & $92 \cdot 10$ & 25.90 & $2 \cdot 90$ & 3.50 & 69.80 & 30.20 & 64.60 & 141.00 & 5.00 & $35 \cdot 40$ & 5.20 & 2009.42 & 711.34 & 759.00 \\
\hline 625.00 & 91.60 & 25.80 & $3 \cdot 10$ & 4.20 & $64 \cdot 50$ & 35.50 & 62.10 & $138 \cdot 00$ & 5.00 & 37.90 & $2 \cdot 40$ & 2001.67 & 758.63 & 758.00 \\
\hline 626.00 & $90 \cdot 90$ & 26.10 & $3 \cdot 20$ & 4.00 & $E 4.90$ & $35 \cdot 10$ & 60.60 & 146.00 & 5.00 & 39.40 & 4.30 & 2024.94 & 797.83 & 757.00 \\
\hline 627.00 & 88.70 & 26.00 & $3 \cdot 30$ & 4.30 & 63.20 & 36.80 & 60.10 & 142.00 & 5.00 & 39.90 & $3 \cdot 10$ & 2017.18 & 804.86 & 756.00 \\
\hline $\begin{array}{l}628.00 \\
629.00\end{array}$ & $\begin{array}{l}90.00 \\
91.20\end{array}$ & $\begin{array}{l}25.70 \\
25.90\end{array}$ & $\begin{array}{l}3 \cdot 30 \\
3 \cdot 20\end{array}$ & $\begin{array}{l}3.90 \\
4.10\end{array}$ & $\begin{array}{l}66.90 \\
64.40\end{array}$ & $\begin{array}{l}33 \cdot 10 \\
35.60\end{array}$ & $\begin{array}{l}61.00 \\
61.30\end{array}$ & $\begin{array}{l}136.00 \\
140.00\end{array}$ & $\begin{array}{l}5.00 \\
5.00\end{array}$ & $\begin{array}{l}39.00 \\
38.70\end{array}$ & $\begin{array}{l}5.90 \\
3.10\end{array}$ & $\begin{array}{l}1993.91 \\
2009.42\end{array}$ & $\begin{array}{l}777.62 \\
777.65\end{array}$ & $\begin{array}{r}755.00 \\
754.00\end{array}$ \\
\hline $\begin{array}{l}630.00 \\
631.00\end{array}$ & $\begin{array}{r}96 \cdot 20 \\
100.50\end{array}$ & $\begin{array}{l}25.00 \\
25.00\end{array}$ & $\begin{array}{l}3 \cdot 20 \\
3.10\end{array}$ & $\begin{array}{l}4.00 \\
4.20\end{array}$ & $\begin{array}{l}67.80 \\
66.00\end{array}$ & $\begin{array}{l}32.20 \\
34.00\end{array}$ & $\begin{array}{l}64.10 \\
64.30\end{array}$ & $\begin{array}{l}119.00 \\
120.00\end{array}$ & $\begin{array}{l}6.00 \\
6.00\end{array}$ & $\begin{array}{l}35.90 \\
35.70\end{array}$ & $\begin{array}{l}3.70 \\
1.70\end{array}$ & $\begin{array}{l}1939.60 \\
1939.60\end{array}$ & $\begin{array}{r}696.32 \\
692.44\end{array}$ & $\begin{array}{l}753.00 \\
752.00\end{array}$ \\
\hline $\begin{array}{l}632.00 \\
633.00\end{array}$ & $\begin{array}{l}98 \cdot 90 \\
97.20\end{array}$ & $\begin{array}{l}25.30 \\
25.30\end{array}$ & $\begin{array}{l}3.00 \\
2.90\end{array}$ & $\begin{array}{l}4.00 \\
3.80\end{array}$ & $\begin{array}{l}67.30 \\
68.90\end{array}$ & $\begin{array}{l}32.70 \\
31.10\end{array}$ & $\begin{array}{l}64.50 \\
65.60\end{array}$ & $\begin{array}{l}126.00 \\
126.00\end{array}$ & $\begin{array}{l}6.00 \\
6.00\end{array}$ & $\begin{array}{l}35 \cdot 50 \\
34.40\end{array}$ & $\begin{array}{l}2 \cdot 80 \\
3.30\end{array}$ & $\begin{array}{l}1962.87 \\
1962.87\end{array}$ & $\begin{array}{l}696 \cdot 82 \\
675.23\end{array}$ & $\begin{array}{l}751.00 \\
750.00\end{array}$ \\
\hline $\begin{array}{l}634.00 \\
635.00\end{array}$ & $\begin{array}{r}96 \cdot 40 \\
11: .90\end{array}$ & $\begin{array}{l}24.40 \\
18.50\end{array}$ & $\begin{array}{l}2.80 \\
2 \cdot 80\end{array}$ & $\begin{array}{l}5.10 \\
3.70\end{array}$ & $\begin{array}{l}69.20 \\
93.70\end{array}$ & $\begin{array}{r}30.80 \\
6.30\end{array}$ & $\begin{array}{l}69.20 \\
93.70\end{array}$ & $\begin{array}{r}108 \cdot 00 \\
32.00\end{array}$ & $\begin{array}{r}9.00 \\
22.00\end{array}$ & $\begin{array}{r}30 \cdot 80 \\
6.30\end{array}$ & $\begin{array}{l}.00 \\
.00\end{array}$ & $\begin{array}{l}1893 \cdot 05 \\
1435 \cdot 30\end{array}$ & $\begin{array}{r}583.06 \\
90.42\end{array}$ & $\begin{array}{l}749.00 \\
748.00\end{array}$ \\
\hline $\begin{array}{l}\text { A.AVE. } \\
\text { G.MEAN }\end{array}$ & $\begin{array}{l}91.06 \\
90.66\end{array}$ & $\begin{array}{l}24.30 \\
24.17\end{array}$ & $\begin{array}{l}3.31 \\
3.26\end{array}$ & $\begin{array}{l}4.04 \\
4.01\end{array}$ & $\begin{array}{l}69.19 \\
68.93\end{array}$ & $\begin{array}{l}30.81 \\
29.46\end{array}$ & $\begin{array}{l}65.69 \\
65.30\end{array}$ & $\begin{array}{l}112.19 \\
103.67\end{array}$ & $\begin{array}{l}6.52 \\
5.56\end{array}$ & $\begin{array}{l}34.31 \\
32.56\end{array}$ & 3.50 & $\begin{array}{l}1884.92 \\
1875.44\end{array}$ & $\begin{array}{l}655.81 \\
610.61\end{array}$ & \\
\hline
\end{tabular}

*NOTATION--Depth, ft; GR-Gamma Ray Index, API Units; POR-Porosity, $\%$; RT-True Resistivity, Ohms-m²/m; Rx:0-Flushed Zone Resistivity, Ohms-m²/m; SX0-Flushed Zone Saturation, \%; ROS-Residual Oil Saturation, \&; SW-Water Saturation, \%; PER-Permeability Index; Clay-Clay Content, \%; SO-0il

Saturation, : $:$ MO-Mbvable $0 i 1, \%$; PHV-Pore Volume, bbl/ac-ft; HPV-Hydrocarbon Pore Volume, bbl/ac-ft; ELSE-Elevation From Sea Level, ft 
TABLE C13

WELL. LOG: DETERUINED PROPERTIES

VIS DEPTH FOR WELL MP-102

WELL LOCATION: 1848 FSL IC56 FEL 21255 5E

KELLY BUSHING ELEVATION FROM SEA LEVEL $=1,383$

\begin{tabular}{|c|c|c|c|c|c|c|c|c|c|c|c|c|c|c|}
\hline DEPTH * & $G R$ & POR & RT & $R \times e$ & $s \times 0$ & ROS & SW & PER & CLAY & so & mo & PHV & HPV & ELSE \\
\hline $\begin{array}{l}604 \cdot 00 \\
605 \cdot 00\end{array}$ & $\begin{array}{l}90 \cdot 10 \\
99.70\end{array}$ & $\begin{array}{l}21.40 \\
22.30\end{array}$ & $\begin{array}{l}3.20 \\
2.80\end{array}$ & $\begin{array}{l}4.30 \\
4.20\end{array}$ & $\begin{array}{l}79.50 \\
77.30\end{array}$ & $\begin{array}{l}20 \cdot 50 \\
22.70\end{array}$ & $\begin{array}{l}75 \cdot 30 \\
76.50\end{array}$ & $\begin{array}{l}61.00 \\
73.00\end{array}$ & $\begin{array}{r}3.00 \\
13.00\end{array}$ & $\begin{array}{l}24.70 \\
23.50\end{array}$ & $\begin{array}{r}4.20 \\
.80\end{array}$ & $\begin{array}{l}1660 \cdot 30 \\
1730 \cdot 12\end{array}$ & $\begin{array}{l}410.09 \\
406.58\end{array}$ & $\begin{array}{r}779.00 \\
778.00\end{array}$ \\
\hline 606.00 & 88.60 & $24 \cdot 40$ & 2.60 & $4 \cdot b c$ & 72.20 & 27.80 & 72.20 & 107.00 & 6.00 & 27.80 & .00 & 1893.05 & 526.27 & 777.00 \\
\hline $607 \cdot 00$ & 84.60 & 24.50 & 2.50 & $5 \cdot 10$ & $74 \cdot 00$ & $25 \cdot 60$ & 70.40 & $109 \cdot 00$ & 5.00 & 25.60 & .00 & 1900.81 & 486.61 & 776.00 \\
\hline $608 \cdot 00$ & 84.00 & $24.8 \mathrm{C}$ & $2 \cdot 20$ & 4.40 & 77.00 & 23.00 & 77.00 & 116.00 & 5.00 & 23.00 & .00 & 1924.08 & 442.54 & 775.00 \\
\hline $609 \cdot 00$ & $86 \cdot 80$ & 25.10 & $2 \cdot 10$ & 4.96 & $79 \cdot 10$ & 20.90 & 79.10 & $123 \cdot 00$ & 6.00 & 20.90 & .00 & $1947 \cdot 36$ & 407.00 & 774.00 \\
\hline $610 \cdot 00$ & $90 \cdot 10$ & $25 \cdot 20$ & 1.80 & 3.90 & 83.00 & 17.00 & 83.00 & $125.00^{\circ}$ & .7 .00 & 17.00 & .00 & $1955 \cdot 12$ & 332.37 & 773.00 \\
\hline $\begin{array}{l}611.00 \\
612.00\end{array}$ & $\begin{array}{l}83.10 \\
79.70\end{array}$ & $\begin{array}{l}25.50 \\
26.70\end{array}$ & $\begin{array}{l}1.70 \\
1.80\end{array}$ & $\begin{array}{l}3.80 \\
3.50\end{array}$ & $\begin{array}{l}84.80 \\
78.60\end{array}$ & $\begin{array}{l}15.20 \\
21.40\end{array}$ & $\begin{array}{l}84.80 \\
78.60\end{array}$ & $\begin{array}{l}130.00 \\
161.00\end{array}$ & $\begin{array}{l}6.00 \\
8.00\end{array}$ & $\begin{array}{l}15.20 \\
21.40\end{array}$ & $\begin{array}{l}.00 \\
.00\end{array}$ & $\begin{array}{l}1978.39 \\
2071.49\end{array}$ & $\begin{array}{l}300 \cdot 72 \\
443.30\end{array}$ & $\begin{array}{l}772.00 \\
771.00\end{array}$ \\
\hline 613.00 & $75 \cdot 50$ & 26.50 & 2.00 & Avo & $75 \cdot 90$ & 24.10 & 75.90 & 156.00 & 7.00 & 24.10 & .00 & 2055.97 & 495.49 & 770.00 \\
\hline 4.00 & 74.50 & 25.60 & $2 \cdot 50$ & 5.20 & .70 .00 & 30.00 & 70.00 & 134.00 & 5.00 & 30.00 & .00 & $1986 \cdot 15$ & 595.84 & 769.00 \\
\hline $615 \cdot 00$ & 78.00 & 24.80 & $3 \cdot 00$ & $5 \cdot 40$ & $\epsilon 6.70$ & $33 \cdot 30$ & 66.70 & 117.00 & 6.00 & 33.30 & .00 & 1924008 & 640.72 & 768.00 \\
\hline 616.00 & 88.80 & 25.00 & 4.00 & $5.6 \mathrm{C}$ & 59.60 & 40.40 & 56.80 & 120.00 & 11.00 & 43.20 & 2.80 & 1939.60 & 837.91 & 767.00 \\
\hline $617 \cdot 00$ & $81 \cdot 10$ & 24.90 & 4.30 & 6.00 & $57 \cdot 60$ & 42.40 & 54.80 & 119.00 & 9.00 & 45.20 & 2.80 & 1931.84 & 873.19 & 766.00 \\
\hline $\begin{array}{l}618.00 \\
619.00\end{array}$ & $\begin{array}{l}83.00 \\
89.10\end{array}$ & $\begin{array}{l}24.40 \\
24.20\end{array}$ & $\begin{array}{l}4.80 \\
4.50\end{array}$ & $\begin{array}{l}6.10 \\
5.10\end{array}$ & $\begin{array}{l}58.40 \\
60.70\end{array}$ & $\begin{array}{l}41.60 \\
39.30\end{array}$ & $\begin{array}{l}53.50 \\
55.30\end{array}$ & $\begin{array}{l}108.00 \\
104.00\end{array}$ & $\begin{array}{r}8.00 \\
15.00\end{array}$ & $\begin{array}{l}46.50: \\
4.70\end{array}$ & $\begin{array}{l}4.90 \\
5.40\end{array}$ & $\begin{array}{l}1893.05 \\
1877.53\end{array}$ & $\begin{array}{l}880.27 \\
839.26\end{array}$ & $\begin{array}{l}765.00 \\
764.00\end{array}$ \\
\hline $620 \cdot 00$ & 83.60 & 25.40 & 4.20 & 5.00 & $61 \cdot 30$ & 38.70 & 54.50 & 130.00 & 10.00 & 45.50 & 6.80 & 1970.63 & 896.64 & 763.00 \\
\hline 621.00 & 77.90 & 26.50 & 3.70 & 4.80 & \pm 0.40 & 39.60 & 55.40 & 155.00 & 10.00 & 44.60 & 5.00 & 2055.97 & 916.96 & 762.00 \\
\hline $622 \cdot 00$ & 79.50 & 26.90 & $3 \cdot 70$ & 4.90 & $58 \cdot 40$ & 41.60 & 55.00 & 165.00 & 11.00 & 45.00 & $3 \cdot 40$ & 2087.01 & 939.15 & 761.00 \\
\hline 623.00 & $83 \cdot 10$ & 26.10 & $3 \cdot 70$ & $5 \cdot 20$ & 58.90 & $41 \cdot 10$ & 56.70 & 146.00 & 15.00 & $43 \cdot 30$ & $2 \cdot 20$ & $2024 \cdot 94$ & 876.80 & 760.00 \\
\hline 624.00 & $102 \cdot 50$ & 23.90 & $3 \cdot 70$ & $5 \cdot 40$ & $E 3.80$ & $36 \cdot 20$ & 62.00 & 98.00 & 27.00 & 38.00 & 1.80 & 1854.26 & 704.62 & 759.00 \\
\hline $\begin{array}{l}625.00 \\
626.00\end{array}$ & 104.00 & 22.90 & 3.80 & $5 \cdot 30$ & 67.00 & 33.00 & $64 \cdot 30$ & $82 \cdot 00$ & 28.00 & 35.70 & 2.70 & 1776.67 & 634.27 & 758.00 \\
\hline $\begin{array}{l}626.00 \\
627.00\end{array}$ & $\begin{array}{l}98 \cdot 20 \\
98 \cdot 30\end{array}$ & 21.90 & 3.70 & $4 \cdot 90$ & $73 \cdot 20$ & 26.80 & 67.70 & 68.00 & 21.00 & $32 \cdot 30$ & $5 \cdot 50$ & 1699.09 & 548.81 & 757.00 \\
\hline & $98 \cdot 30$ & 18.50 & 3.60. & $4 \cdot 30$ & $82 \cdot 20$ & 17.80 & 82.20 & 32.00 & 24.00 & $17 \cdot 80$ & .00 & $1435 \cdot 30$ & 255.48 & 756.00 \\
\hline $\begin{array}{l}\text { A.AVE, } \\
\text { G.MEAN }\end{array}$ & $\begin{array}{l}86.82 \\
86.44\end{array}$ & $\begin{array}{l}24.47 \\
24.40\end{array}$ & $\begin{array}{l}3.16 \\
3.02\end{array}$ & $\begin{array}{l}4.85 \\
4.80\end{array}$ & $\begin{array}{l}70.00 \\
69.42\end{array}$ & $\begin{array}{l}30.00 \\
28.59\end{array}$ & $\begin{array}{l}67.99 \\
67.17\end{array}$ & $\begin{array}{l}114.12 \\
108.08\end{array}$ & $\begin{array}{r}11.08 \\
9.32\end{array}$ & $\begin{array}{l}32.01 \\
30.20\end{array}$ & 2.01 & $\begin{array}{l}1898.87 \\
1892.69\end{array}$ & $\begin{array}{l}612.12 \\
571.57\end{array}$ & . \\
\hline
\end{tabular}

*NOTATION--Depth, ft; GR-Gamma Ray Index, API Units; POR-Porosity, $\%$; RT-True Resistivity, Ohms-m²/m; RXO-Flushed Zone Resistivity, Ohms-m²/m; SX0-Flushed Zone Saturation, \%; ROS-Residual 0il Saturation, \%; SW-Water Saturation, \%; PER-Permeability Index; Clay-Clay Content, \%; SO-0il Saturation, \%; M0-Movable 0il, \%; PHV-Pore Volure, bbl/ac-ft; HPV-Hydrocarbon Pore Volume, bbl/ac-ft; ELSE-Elevation From Sea Level, ft 
TABLE C14

WELL LOG OETERMINED PROPERTIES

VS DEPTH FOR WELL MP-103

WELL LOCATION: 1584 FSC 1848 FEL $212555 F$

KELLY BUSHING ELEVATION FROM SEA LEVEL $=1391$

\begin{tabular}{|c|c|c|c|c|c|c|c|c|c|c|c|c|c|c|}
\hline EPTH * & GR & $P \theta R$ & RT & $R \times \theta$ & $5 \times 0$ & ROS & SW & PER & CLAY & so & mo & PHV & HPV & ELSE \\
\hline $\begin{array}{l}608.00 \\
609.00\end{array}$ & $\begin{array}{l}77.90 \\
80.90\end{array}$ & $\begin{array}{l}20.20 \\
23.00\end{array}$ & $\begin{array}{l}2.20 \\
1.70\end{array}$ & $\begin{array}{l}5.30 \\
3.20\end{array}$ & $\begin{array}{l}97.00 \\
54.70\end{array}$ & $\begin{array}{l}3.00 \\
5.30\end{array}$ & $\begin{array}{l}97.00 \\
94.70\end{array}$ & $\begin{array}{l}47.00 \\
84.00\end{array}$ & $\begin{array}{l}2.00 \\
3.00\end{array}$ & $\begin{array}{l}3 \cdot 00 \\
5 \cdot 30\end{array}$ & $\begin{array}{l}.00 \\
.00\end{array}$ & $\begin{array}{l}1567.20 \\
1784.43\end{array}$ & $\begin{array}{l}47.02 \\
94.57\end{array}$ & $\begin{array}{l}783.00 \\
782.00\end{array}$ \\
\hline $\begin{array}{l}610.00 \\
611.00\end{array}$ & $\begin{array}{l}86.70 \\
78.80\end{array}$ & $\begin{array}{l}24.00 \\
25.10\end{array}$ & $\begin{array}{l}1.60 \\
1.50\end{array}$ & $\begin{array}{l}3.00 \\
3.10\end{array}$ & $\begin{array}{l}\$ 5.10 \\
53.40\end{array}$ & $\begin{array}{l}4.90 \\
6.60\end{array}$ & $\begin{array}{l}95.10 \\
93.40\end{array}$ & $\begin{array}{l}101.00 \\
123.00\end{array}$ & $\begin{array}{l}4.00 \\
.00\end{array}$ & $\begin{array}{l}1.90 \\
6.60\end{array}$ & .00 & $\begin{array}{l}1862.02 \\
1947.36\end{array}$ & $\begin{array}{r}91.24 \\
128.53\end{array}$ & $\begin{array}{l}781.00 \\
780.00\end{array}$ \\
\hline $\begin{array}{l}612.00 \\
613.00\end{array}$ & $\begin{array}{l}77.50 \\
86.00\end{array}$ & $\begin{array}{l}25.80 \\
26.00\end{array}$ & $\begin{array}{l}1 \cdot 40 \\
1.30\end{array}$ & $\begin{array}{l}2.30 \\
2.10\end{array}$ & $\begin{array}{l}93 \cdot 20 \\
94.50\end{array}$ & $\begin{array}{l}6.80 \\
5.50\end{array}$ & $\begin{array}{l}93.20 \\
94: 50\end{array}$ & $\begin{array}{l}138 \cdot 00 \\
142 \cdot 00\end{array}$ & $\begin{array}{l}4.00 \\
5.00\end{array}$ & $\begin{array}{l}6.80 \\
5.50\end{array}$ & $\begin{array}{l}.00 \\
.00\end{array}$ & $\begin{array}{l}2001.67 \\
2017.18\end{array}$ & $\begin{array}{l}136.11 \\
110.95\end{array}$ & $\begin{array}{l}779.00 \\
778.00\end{array}$ \\
\hline $\begin{array}{l}b 14.00 \\
b 15.00\end{array}$ & $\begin{array}{l}79.20 \\
80.60\end{array}$ & $\begin{array}{l}26.10 \\
26.40\end{array}$ & $\begin{array}{l}1 \cdot 30 \\
1 \cdot 30\end{array}$ & $\begin{array}{l}2.20 \\
2.30\end{array}$ & $\begin{array}{l}95.30 \\
55.90\end{array}$ & $\begin{array}{l}4.70 \\
4.10\end{array}$ & $\begin{array}{l}95.30 \\
95.90\end{array}$ & $\begin{array}{l}146.00 \\
152.00\end{array}$ & $\begin{array}{l}5.00 \\
5.00\end{array}$ & $\begin{array}{l}1.70 \\
1.10 \\
5.30\end{array}$ & $\begin{array}{l}.00 \\
.00\end{array}$ & $\begin{array}{l}2024.94 \\
2048.22 \\
2040.46\end{array}$ & $\begin{array}{r}95.17 \\
83.98 \\
108.98\end{array}$ & $\begin{array}{l}777.00 \\
776.00 \\
775.00\end{array}$ \\
\hline $\begin{array}{l}616.00 \\
617.00\end{array}$ & $\begin{array}{l}78.80 \\
76.00\end{array}$ & $\begin{array}{l}26.30 \\
25.70\end{array}$ & $\begin{array}{l}1.30 \\
1.40\end{array}$ & $\begin{array}{l}2.30 \\
2.70\end{array}$ & $\begin{array}{l}54.70 \\
94.80\end{array}$ & $\begin{array}{l}5 \cdot 30 \\
5 \cdot 20\end{array}$ & $\begin{array}{l}94.70 \\
94.80\end{array}$ & $\begin{array}{l}151.00 \\
137.00\end{array}$ & 50 & $\begin{array}{l}5.30 \\
5.20\end{array}$ & $\begin{array}{l}.00 \\
.00\end{array}$ & $\begin{array}{l}2040.46 \\
1993.91\end{array}$ & 68 & $\begin{array}{l}5.00 \\
1.00\end{array}$ \\
\hline & & $\begin{array}{l}25.90 \\
26.00\end{array}$ & $\begin{array}{l}1.70 \\
2.00\end{array}$ & $\begin{array}{l}3.30 \\
3.00\end{array}$ & & & $\begin{array}{l}84.80 \\
77.80\end{array}$ & $\begin{array}{l}141.00 \\
143.00\end{array}$ & $\begin{array}{l}4.00 \\
5.00\end{array}$ & $\begin{array}{l}15.20 \\
22.20\end{array}$ & $\begin{array}{l}.00 \\
.00\end{array}$ & $\begin{array}{l}2009.42 \\
2017.18\end{array}$ & & $\begin{array}{l}773.00 \\
772.00\end{array}$ \\
\hline $\begin{array}{l}620.00 \\
621.00\end{array}$ & $\begin{array}{l}95 \cdot 10 \\
93.90\end{array}$ & $\begin{array}{l}25.80 \\
25.10\end{array}$ & $\begin{array}{l}2 \cdot 30 \\
2.70\end{array}$ & $\begin{array}{l}3.30 \\
3.80\end{array}$ & $\begin{array}{l}74.80 \\
72.20\end{array}$ & $\begin{array}{l}25 \cdot 20 \\
27.80\end{array}$ & $\begin{array}{l}72.90 \\
69.40\end{array}$ & $\begin{array}{l}139 \cdot 00 \\
122.00\end{array}$ & $\begin{array}{l}7.00 \\
6.00\end{array}$ & $\begin{array}{l}27.10 \\
30.60\end{array}$ & $\begin{array}{l}1.90 \\
2.80\end{array}$ & $\begin{array}{l}2001.67 \\
1947.36\end{array}$ & $\begin{array}{l}542 \cdot 45 \\
595.89\end{array}$ & $\begin{array}{l}771.00 \\
770.00\end{array}$ \\
\hline $\begin{array}{l}622.00 \\
623.00\end{array}$ & $\begin{array}{l}93.60 \\
98.30\end{array}$ & $\begin{array}{l}24.20 \\
24.00\end{array}$ & $\begin{array}{l}3.10 \\
3.60\end{array}$ & $\begin{array}{l}3.50 \\
4.50\end{array}$ & $\begin{array}{l}78 \cdot 30 \\
69 \cdot 30\end{array}$ & $\begin{array}{l}21.70 \\
30.70\end{array}$ & $\begin{array}{l}67.40 \\
62.90\end{array}$ & $\begin{array}{l}104.00 \\
101.00\end{array}$ & $\begin{array}{l}8.00 \\
9.00\end{array}$ & $\begin{array}{l}32.60 \\
37.10\end{array}$ & $\begin{array}{r}10.90 \\
6.40\end{array}$ & $\begin{array}{l}1877.53 \\
1862.02\end{array}$ & $\begin{array}{l}612.08 \\
690.81\end{array}$ & $\begin{array}{l}769.00 \\
768.00\end{array}$ \\
\hline $\begin{array}{l}624.00 \\
625.00\end{array}$ & $\begin{array}{r}98.60 \\
101.50\end{array}$ & $\begin{array}{l}23 \cdot 70 \\
23 \cdot 30\end{array}$ & $\begin{array}{l}3.70 \\
3.80\end{array}$ & $\begin{array}{l}5.70 \\
5.00\end{array}$ & $\begin{array}{l}62.80 \\
67.40\end{array}$ & $\begin{array}{l}37.20 \\
32.60\end{array}$ & $\begin{array}{l}62.80 \\
63.00\end{array}$ & $\begin{array}{l}94.00 \\
89.00\end{array}$ & $\begin{array}{l}10.00 \\
13.00\end{array}$ & $\begin{array}{l}37.20 \\
37.00\end{array}$ & $\begin{array}{r}.00 \\
\therefore \quad 40\end{array}$ & $\begin{array}{l}1838.74 \\
1807.71\end{array}$ & $\begin{array}{l}684.01 \\
668.85\end{array}$ & $\begin{array}{l}767.00 \\
766.00\end{array}$ \\
\hline $\begin{array}{l}626.00 \\
627.00\end{array}$ & $\begin{array}{r}99 \cdot 80 \\
102.30\end{array}$ & $\begin{array}{l}23.50 \\
22.40\end{array}$ & $\begin{array}{l}3.70 \\
3.70\end{array}$ & $\begin{array}{l}4.90 \\
5.50\end{array}$ & $\begin{array}{l}67.80 \\
67.40\end{array}$ & $\begin{array}{l}32 \cdot 20 \\
32 \cdot 60\end{array}$ & $\begin{array}{l}62.90 \\
66.50\end{array}$ & $\begin{array}{l}92 \cdot 00 \\
74 \cdot 00\end{array}$ & $\begin{array}{l}10.00 \\
12.00\end{array}$ & $\begin{array}{l}37 \cdot 10 \\
33 \cdot 50\end{array}$ & $\begin{array}{r}.90 \\
.90\end{array}$ & $\begin{array}{l}1823.22 \\
1737.88\end{array}$ & $\begin{array}{l}676.42 \\
582.19\end{array}$ & $\begin{array}{l}765.00 \\
764.00\end{array}$ \\
\hline $\begin{array}{l}\text { - AVE. } \\
\text { - MEAN }\end{array}$ & $\begin{array}{l}88.84 \\
88.32\end{array}$ & $\begin{array}{l}24.74 \\
24.71\end{array}$ & $\begin{array}{l}2.43 \\
2.23\end{array}$ & $\begin{array}{l}3.71 \\
3.56\end{array}$ & $\begin{array}{l}80.49 \\
79.63\end{array}$ & $\begin{array}{l}19.51 \\
15.09\end{array}$ & $\begin{array}{l}78.48 \\
77.34\end{array}$ & $\begin{array}{l}117 \cdot 12 \\
114 \cdot 37\end{array}$ & $\begin{array}{l}6.81 \\
6.18\end{array}$ & $\begin{array}{l}21.52 \\
16.12\end{array}$ & 01 & $\begin{array}{l}1919.23 \\
1916.79\end{array}$ & $\begin{array}{r}405.49 \\
309.07\end{array}$ & \\
\hline
\end{tabular}

*NOTATION--Depth, it; GR-Gamma Ray Index, API Units; POR-Porosity, $\%$; RT-True Resistivity, Ohms-m²/m; RX0-Flushed Zone Resistivity, Ohms-m²/m; SX0-Flushed Zone Saturation, \%; ROS-Residual $0 i 1$ Saturation, \%; SW-Water Saturation, \%; PER-Permeability Index; Clay-Clay Content, \%; SO-0il

Saturation, \%; M0-Movable 0ii, \%; PHV-Pore Volume, bbl/ac-ft; HPV-Hydrocarbon Pore Volumé, bbl/ac-ft; ELSE-Elevation From Sea Level, ft 
TABLE C15

WELL LOG OETERMINEO PROPERTIES

VS DEPTH FOR WELL MP-104

WELL LOCATION: 264 FSL 1640 FEL $212555 E$

KELLY BUSHING ELEVATION FROM SEA LEVEL $=1404$

\begin{tabular}{|c|c|c|c|c|c|c|c|c|c|c|c|c|c|c|}
\hline DEPTH * & GR & $P E R$ & $R T$ & $R \times 0$ & $5 \times 0$ & Res & SW & PER & CLAY & so & ro & PHV & HPV & ELSE \\
\hline $\begin{array}{l}652.00 \\
653.00\end{array}$ & $\begin{array}{l}97.00 \\
91.60\end{array}$ & $\begin{array}{l}21.00 \\
22.90\end{array}$ & $\begin{array}{l}4 \cdot 30 \\
4 \cdot 30\end{array}$ & $\begin{array}{l}5.90 \\
3.10\end{array}$ & $\begin{array}{l}+6.00 \\
72.80\end{array}$ & $\begin{array}{l}34.00 \\
27.20\end{array}$ & $\begin{array}{l}66.00 \\
60.60\end{array}$ & $\begin{array}{l}56.00 \\
82.00\end{array}$ & $\begin{array}{r}7.00 \\
10.00\end{array}$ & $\begin{array}{l}34.00 \\
39.40\end{array}$ & $\begin{array}{r}.00 \\
12.20\end{array}$ & $\begin{array}{l}1629.26 \\
1776.67\end{array}$ & $\begin{array}{l}553.95 \\
700.01\end{array}$ & $\begin{array}{l}752.00 \\
751.00\end{array}$ \\
\hline 654.00 & 84.90 & 23.60 & $4 \cdot 10$ & 4.50 & $\in 3 \cdot 30$ & 36.70 & 59.90 & 93.00 & 7.00 & 40.10 & 3.40 & 1830.98 & 734.22 & 750.00 \\
\hline 655.00 & 83.40 & 23.00 & 3.90 & 3.70 & $71 \cdot 80$ & $28 \cdot 20$ & 63.20 & 84.00 & 5.00 & 36.20 & 8.60 & $1784 \cdot 43$ & 656.67 & 749.00 \\
\hline 656.00 & 79.10 & 23.10 & 3.60 & $4 \cdot 30$ & 66.80 & 33.20 & 65.30 & 84.00 & 3.00 & 34.70 & 1.50 & 1792.19 & 621.89 & 748.00 \\
\hline 657.00 & $82 \cdot 90$ & 23.80 & $3 \cdot 50$ & 4.60 & $64 \cdot 30$ & 35.70 & $64 \cdot 30$ & 97.00 & 4.00 & 35.70 & .00 & 1846.50 & 659.20 & 747.00 \\
\hline $658 \cdot 00$ & 84.70 & 24.50 & 3.20 & $3 \cdot 30$ & 70.90 & $29 \cdot 10$ & 64.80 & 110.00 & 4.00 & $35 \cdot ? 0$ & 6.10 & 1900.81 & 669.08 & 746.00 \\
\hline 659.00 & 86.20 & 24.50 & 3.00 & 3.30 & $.71 \cdot 50$ & 28.50 & 67.70 & 109.00 & 4.00 & $32 \cdot 3 n$ & $3 \cdot 80$ & $1900 \cdot 81$ & 613.96 & 745.00 \\
\hline 660.00 & 90.00 & 24.70 & 2.80 & $3 \cdot 20$ & 31.20 & 28.80 & 68.90 & 114.00 & 5.00 & 31.10 & $2 \cdot 30$ & $1916 \cdot 32$ & 595.98 & 744.00 \\
\hline 661.00 & $97 \cdot 10$ & 25.30 & 2.70 & 3.50 & $68 \cdot 30$ & $31 \cdot 70$ & 68.30 & 126.00 & 7.00 & 31.70 & .00 & 1962.87 & 622.23 & 743.00 \\
\hline 662.00 & $88 \cdot 20$ & 26.40 & 2.90 & -3.80 & 63.20 & $36 \cdot 80$ & 63.20 & 152.00 & 6.00 & 36.80 & .00 & $2048 \cdot 22$ & 753.74 & 742.00 \\
\hline 663.00 & $82 \cdot 60$ & 27.10 & $2 \cdot 80$ & 4.00 & $\epsilon 2 \cdot 80$ & $37 \cdot 20$ & 62.80 & 170.00 & 6.00 & $37 \cdot 20$ & .00 & 2102.53 & 782.14 & 741.00 \\
\hline 664.00 & 81.80 & 26.60 & 2.60 & 3040 & $66 \cdot 30$ & 33.70 & $66 \cdot 30$ & 159.00 & 5.00 & 33.70 & .00 & 2063.73 & 695.48 & 740.00 \\
\hline 665.00 & 86.40 & 25.80 & 2.50 & $2 \cdot 80$ & $72 \cdot 70$ & 27.30 & 69.90 & 137.00 & 5.00 & $30 \cdot 10$ & 2.80 & 2001.67 & $602 \cdot 50$ & 739.00 \\
\hline $666 \cdot 00$ & $92 \cdot 90$ & $25 \cdot 6 \mathrm{C}$ & 2.50 & 2.80 & $73 \cdot 10$ & 26.90 & 70.30 & 134.00 & 6.00 & 29.70 & 2.80 & 1986.15 & 589.89 & 738.00 \\
\hline $667 \cdot 00$ & 101.50 & $24.4 \mathrm{C}$ & $2 \cdot 80$ & 3.50 & $70 \cdot 30$ & 29.70 & $70 \cdot 30$ & $108 \cdot 00$ & 6.00 & 29.70 & .00 & 1893.05 & 562.24 & 737.00 \\
\hline $668 \cdot 00$ & 97.70 & 23.40 & 3.00 & 3.80 & $70 \cdot 30$ & $29 \cdot 70$ & $70 \cdot 30$ & 89.00 & 5.00 & $29 \cdot 70$ & .00 & $1815 \cdot 46$ & 539.19 & $7,36 \cdot 00$ \\
\hline 669.00 & $100 \cdot 60$ & 22.70 & 3.20 & 3.60 & 73.40 & 26.60 & 70.60 & 79.00 & 8.00 & 29.40 & 2.80 & 1761.16 & 517.78 & 735.00 \\
\hline 670.00 & $102 \cdot 50$ & 22.60 & $3 \cdot 30$ & $4 \cdot 00$ & $70 \cdot 20$ & 29.80 & 70.20 & 77.00 & 10.00 & 29.80 & .00 & 1753.40 & 522.51 & 734.00 \\
\hline $\begin{array}{l}\text { A.AVE. } \\
\text { G.MEAN }\end{array}$ & $\begin{array}{l}90 \cdot 06 \\
89.77\end{array}$ & 24.26 & 3.16 & $\begin{array}{l}3.78 \\
3.72\end{array}$ & $\begin{array}{l}68.91 \\
68.81\end{array}$ & 30.90 & $\begin{array}{l}66.47 \\
66.38\end{array}$ & $\begin{array}{l}108.42 \\
104.29\end{array}$ & $\begin{array}{l}5.95 \\
5.68\end{array}$ & $\begin{array}{l}33.53 \\
33.36\end{array}$ & 2.44 & $\begin{array}{l}1882.43 \\
1878.57\end{array}$ & $\begin{array}{l}631.19 \\
626.74\end{array}$ & \\
\hline
\end{tabular}

*NOTATIOH--Depth, ft; GR-Gamma Ray Index, API Units; POR-Porosity, $\%$; RT-True Resistivity, Ohms-m²/m; RX0-Flushed Zone Resistivity, Ohms-m²/m; SX0-Flushed Zone Saturation, \%; ROS-Residual $0 i 1$ Saturation, \%; SW-Water Saturation, $\%$; PER-Permeability Index; Clay-Clay Content, \%; SO-0il

Saturation, \%; MO-Movable 0il, \%; PHV-Pore Volume, bbl/ac-ft; HPV-Hydrocarbon Pore Volume, bbl/ac-ft; ELSE-Elevation From Sea Level, ft 
TABLE C16

WELL LOG DETERMINED PROPERTIES

VS DEPTH FOR WELL MP-105

WELL LOCATION: 265 FSL 1056 FEL $212555 E$

KELLY BUSHING ELEVATION FROII SEA LEVEL $=1385$

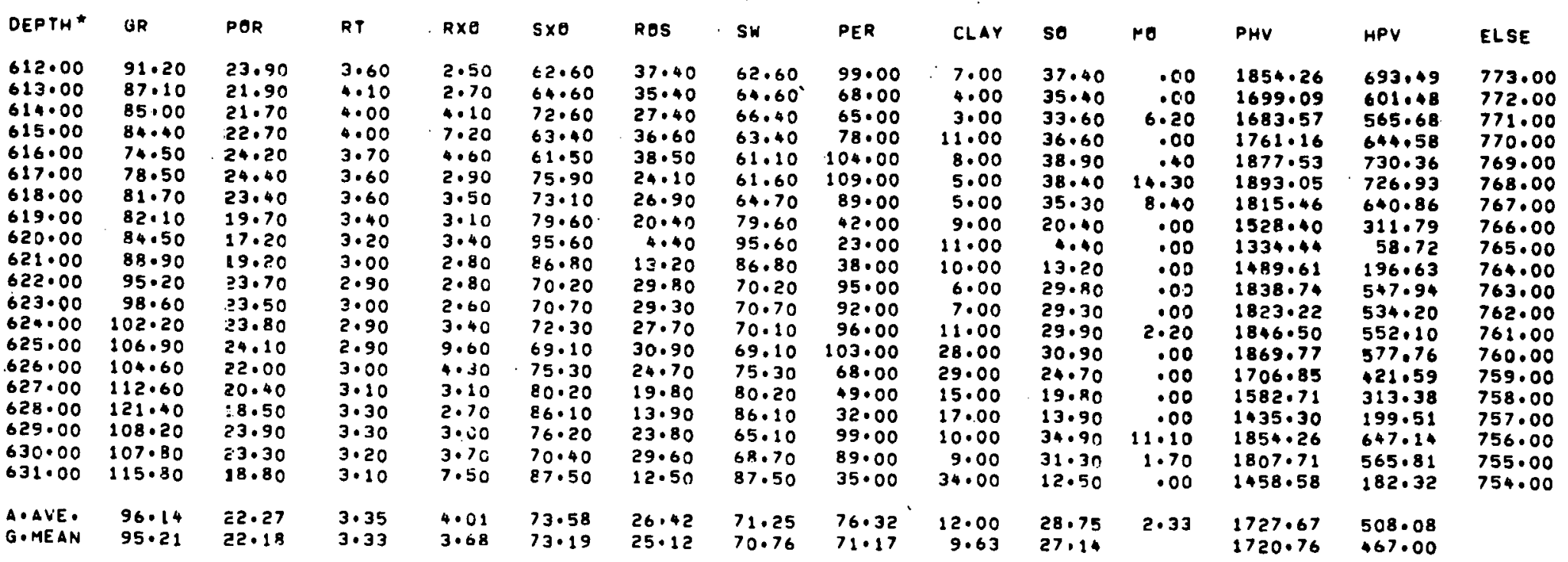

*NOTATION--Depth, ft; GR-Gamna Ray Index, API Units; POR-Porosity, $\%$; RT-True Resistivity, Ohms-m²/m; RXO-Flushed Zone Resistivity, Ohms-m²/m; SXO-Flushed Zone Saturation, \%; ROS-Residual Oil Saturation, \%; SW-Water Saturation, \%; PER-Permeability Index; Clay-Clay Content, \%; SO-0il

Saturation, \%; MO-Movable 0il, \%; PHV-Pore Volume, bbl/ac-ft; HPV-Hydrocarbon Pore Volume, bbl/ac-ft; ELSE-Elevation From Sea Level, ft 
TABLE $\mathrm{C} 17$

WELL LOG DETEPMINED PROPERTIES

VS DEPTH FOR WELL 106

WELL LOCATION: 2112 FWL 1584 FSL 21255 5E

KELLY BUSHING ELEVATION FROH SEA LEVEL $=1387$

\begin{tabular}{|c|c|c|c|c|c|c|c|c|c|c|c|c|c|c|}
\hline DEPTH * & GR & $P A R$ & ET & $R \times \theta$ & $s \times e$ & ROS & SW & PER & CLAY & sg & Me & PHV & HPV & ELSE \\
\hline $\begin{array}{l}644.00 \\
644.50\end{array}$ & 73.80 & $140^{\prime 2} 20$ & $\therefore .60$ & 3.70 & $56 \cdot 70$ & $\begin{array}{r}3 \cdot 30 \\
18.90\end{array}$ & 96.70 & $\begin{array}{r}8.00 \\
16.00\end{array}$ & $\begin{array}{l}25.00 \\
20.00\end{array}$ & & $\begin{array}{l}.00 \\
.00\end{array}$ & $\begin{array}{l}1101.69 \\
1227.89\end{array}$ & $\begin{array}{r}36.36 \\
243.41\end{array}$ & $\begin{array}{l}743.00 \\
742.50\end{array}$ \\
\hline $\begin{array}{l}644.50 \\
645.00\end{array}$ & $\begin{array}{l}69.30 \\
67.20\end{array}$ & $\begin{array}{l}16.60 \\
19.10\end{array}$ & $\begin{array}{l}4.70 \\
4.60\end{array}$ & $\begin{array}{l}3 \cdot 90 \\
3 \cdot 90\end{array}$ & $\begin{array}{l}81.10 \\
71.10\end{array}$ & $\begin{array}{l}18.90 \\
28.90\end{array}$ & $\begin{array}{l}81.10 \\
71.10\end{array}$ & $\begin{array}{l}16.00 \\
29.00\end{array}$ & $\begin{array}{l}20.00 \\
16.00\end{array}$ & $\begin{array}{l}18.90 \\
28.90\end{array}$ & $\begin{array}{l}.00 \\
.00\end{array}$ & $\begin{array}{l}1287.89 \\
1491.85\end{array}$ & $\begin{array}{l}243.41 \\
428.26\end{array}$ & $\begin{array}{l}742.50 \\
742.00\end{array}$ \\
\hline 645.50 & 65.20 & 21.10 & 4.40 & $3.8 \mathrm{C}$ & 77.30 & 22.70 & 64.70 & 45.00 & 13.00 & $35 \cdot 3 n$ & 12.60 & 1637.02 & 577.87 & 741.50 \\
\hline 646.00 & 63.10 & 22.10 & 4.30 & 3.10 & 74.50 & 25.50 & 62.70 & 56.00 & 10.00 & 37.30 & 11.80 & 1714.61 & 639.55 & 741.00 \\
\hline 646.50 & $61 \cdot 10$ & 22.20 & 4.10 & 3.00 & $15 \cdot 30$ & 24.70 & 63.70 & $56 \cdot 0.0$ & $10 \cdot 00$ & $36 \cdot 30$ & 11.60 & $17 ? 2 \cdot 36$ & 625.22 & 740.50 \\
\hline 647.00 & $60 \cdot 80$ & $23.4 n$ & 4.00 & $3 \cdot 40$ & $73 \cdot 10$ & $26 \cdot 90$ & 61.40 & 71.00 & 9.00 & $38 \cdot 60$ & 11.70 & $1815 \cdot 46$ & 700.77 & 740.00 \\
\hline 647.50 & $61 \cdot 10$ & $24.6 C$ & 3.70 & $3 \cdot 40$ & $70 \cdot 30$ & 29.70 & $60 \cdot 30$ & 88.00 & 8.00 & $39.7 \mathrm{C}$ & 10.00 & 1908.57 & 757.70 & 739.50 \\
\hline 648.00 & 61.40 & 25.50 & $3 \cdot 40$ & $3 \cdot 30$ & $\in 8 \cdot 20$ & 31.80 & 59.60 & $106 \cdot 00$ & 7.00 & 40.40 & 8.60 & $1986 \cdot 15$ & $802 \cdot 40$ & $\begin{array}{l}739.00 \\
738.50\end{array}$ \\
\hline 643.50 & 61.60 & $27.3 c$ & .20 & 3.20 & $\epsilon 4.60$ & 35.40 & 57.80 & 141.00 & 6.00 & $42 \cdot ? 0$ & 6.50 & $2118 \cdot 04$ & $\begin{array}{l}893.81 \\
879.99\end{array}$ & $\begin{array}{l}738.50 \\
738.00\end{array}$ \\
\hline $\begin{array}{l}649.00 \\
649.50\end{array}$ & $\begin{array}{l}61.60 \\
61.50\end{array}$ & $\begin{array}{l}27.80 \\
27.80\end{array}$ & $\begin{array}{l}2.90 \\
2.70\end{array}$ & $\begin{array}{l}3.10 \\
3.00\end{array}$ & $\begin{array}{l}\epsilon 4.50 \\
\epsilon 5.50\end{array}$ & $\begin{array}{l}35.50 \\
34.50\end{array}$ & $\begin{array}{l}59 \cdot 20 \\
61.30\end{array}$ & $\begin{array}{l}151.00 \\
152.00\end{array}$ & $\begin{array}{l}5.00 \\
5.00\end{array}$ & $\begin{array}{l}40.80 \\
38.70\end{array}$ & $\begin{array}{l}5.30 \\
4.20\end{array}$ & $\begin{array}{l}2156.83 \\
2156.83\end{array}$ & $\begin{array}{l}879.99 \\
834.69\end{array}$ & $\begin{array}{l}738.00 \\
737.50\end{array}$ \\
\hline $\begin{array}{l}650.00 \\
650.50\end{array}$ & $\begin{array}{l}61.30 \\
61.20\end{array}$ & $\begin{array}{l}27.90 \\
27.90\end{array}$ & $\begin{array}{l}2.70 \\
2.60\end{array}$ & $\begin{array}{l}2.90 \\
2.90\end{array}$ & $\begin{array}{l}=6.50 \\
65.80\end{array}$ & $\begin{array}{l}33.50 \\
34.20\end{array}$ & $\begin{array}{l}52.00 \\
62.70\end{array}$ & $\begin{array}{l}153.00 \\
154.00\end{array}$ & $\begin{array}{l}5.00 \\
5.00\end{array}$ & $\begin{array}{l}38.00 \\
37.30\end{array}$ & $\begin{array}{l}4.50 \\
3.10\end{array}$ & $\begin{array}{l}2164.59 \\
2164.59\end{array}$ & $\begin{array}{l}822.54 \\
807.39\end{array}$ & $\begin{array}{l}737.00 \\
736.50\end{array}$ \\
\hline 651.00 & 64.00 & 27.90 & 2.60 & $3 \cdot ن 0$ & 65.20 & $34 \cdot 30$ & 62.20 & 154.00 & 6.00 & 37.80 & 3.00 & 2164.59 & 818.22 & 736.00 \\
\hline 651.50 & 66.90 & & .70 & 200 & $E 5.10$ & 34.90 & 62.30 & 149.00 & 8. & 37.70 & 2.80 & 2149.08 & 810.20 & .50 \\
\hline 652.00 & 68.50 & $27.4 \mathrm{C}$ & 2.70 & 3.10 & $<5.10$ & 34.90 & 62.50 & 143.00 & 9.00 & 37.50 & 2.60 & $2125 \cdot 80$ & 797.17 & 735.00 \\
\hline 652.50 & 68.00 & 27.20 & 1.70 & 3.10 & $65 \cdot 30$ & 34.70 & 62.70 & 137.00 & 9.00 & 37.30 & 2.60 & $2110 \cdot 28$ & 787.14 & 734.50 \\
\hline 653.00 & 67.60 & 27.10 & 2.80 & $3 \cdot 20$ & $64 \cdot 90$ & $35 \cdot 10$ & 62.30 & 135.00 & 9.00 & 37.70 & 2.60 & $2102 \cdot 53$ & 792.65 & $734 \cdot 0.0$ \\
\hline 653.50 & 67.20 & 27.10 & 2.80 & $3 \cdot 20$ & $\in 4.90$ & $35 \cdot 10$ & 61.70 & $136 \cdot 00$ & 8.00 & $38 \cdot 3 n$ & 3.20 & 2102.53 & 805.27 & 733.50 \\
\hline 654.00 & 68.70 & 27.10 & 2.90 & .10 & $\epsilon 5.20$ & 34.80 & 61.20 & 136.00 & 8.00 & 38.20 & 4.00 & 2102.53 & 815.78 & 733.00 \\
\hline 654.50 & $72 \cdot 40$ & 27.10 & 2.90 & $3 \cdot 1 \mathrm{C}$ & $=5.60$ & $34 \cdot 40$ & 60.80 & 136.00 & 9.00 & $39.2 n$ & 4.80 & 2102.53 & 824.19 & 732.50 \\
\hline 655.00 & 74.70 & 27.10 & 3.00 & 3.20 & 65.20 & $34 \cdot 80$ & $60 \cdot 30$ & 136.00 & 9.0 & 39.70 & 4.90 & 2102.53 & 834.70 & 732.00 \\
\hline 655.50 & 77.10 & 26.80 & $3 \cdot 00$ & 3.20 & $=5 \cdot 40$ & 34.60 & 60.50 & 130.00 & 12.00 & 39.50 & $4 \cdot 90$ & $2079 \cdot 25$ & $821 \cdot 30$ & 731.50 \\
\hline 656.00 & 78.60 & 26.50 & 3.00 & $3 \cdot: 0$ & $\leftarrow 5.70$ & $34 \cdot 30$ & 61.10 & $123 \cdot 00$ & 15.00 & 38.90 & 4.60 & 2055.97 & 799.77 & 731.00 \\
\hline 656.50 & 79.30 & 26.50 & $3 \cdot 10$ & 3.30 & $t 5 \cdot 10$ & 34.90 & 60.90 & 124.00 & 17.00 & 39.10 & $4 \cdot 20$ & 2055.97 & 803.89 & 730.50 \\
\hline 657.00 & $80 \cdot 10$ & 26.60 & $3 \cdot 10$ & $3 \cdot 4 C$ & 64.00 & 36.00 & 60.70 & 124.00 & 19.00 & $39 \cdot 3 c$ & $3 \cdot 30$ & 2063.73 & 811.05 & 730.00 \\
\hline 657.50 & $80 \cdot 30$ & 26.20 & $3 \cdot 10$ & $3 \cdot 0 \pi$ & $\in 4.50$ & $35 \cdot 50$ & 61.60 & 116.00 & 19.00 & 38.40 & 2.90 & $2032.7 i_{i}$ & 780.56 & 729.50 \\
\hline 658.00 & 80.90 & 26.00 & 3.10 & 3.40 & +5.20 & 34.80 & 61.80 & 114.00 & 17.00 & $38.2 n$ & 3.40 & 2017.18 & 770 & 729.00 \\
\hline $658 \cdot 50$ & $80 \cdot 40$ & 26.20 & $3 \cdot 10$ & $3 \cdot+0$ & $\in 5 \cdot 20$ & $34 \cdot R_{0}$ & $61 \cdot 30$ & 118.00 & 16.00 & 38.70 & 3.90 & 2032.70 & 786.65 & 728.50 \\
\hline 659.00 & 19.90 & 26.30 & 3.10 & $3 \cdot 30$ & $\in 5 \cdot 30$ & 34.70 & 60.90 & 120.00 & & 39.10 & 4.40 & 2040.46 & 797 & 728.00 \\
\hline 659.50 & 81.00 & 25.90 & 3.10 & 3.30 & 67.20 & 32.80 & 62.50 & $110 \cdot 00$ & 16.00 & $37.5 \mathrm{C}$ & 4.70 & 2009.42 & 753.53 & 727.50 \\
\hline 660.00 & 84.70 & 24.10 & 3.00 & 3.30 & $72 \cdot 70$ & $27 \cdot 30$ & 67.80 & $81 \cdot 00$ & & $32 \cdot ? 0$ & 4.90 & .77 & 602.07 & 727.00 \\
\hline 660 & 97.50 & 22.80 & 3.00 & 3.20 & 17.20 & $22 \cdot 80$ & 72.20 & 64.00 & 22.00 & 27.80 & 5.00 & 1768.91 & 491.76 & 726.50 \\
\hline 661.00 & $90 \cdot 20$ & 21.50 & 3.00 & 3.20 & & 22.40 & 77.60 & $49 \cdot 00$ & 25.00 & 22.40 & .00 & 1668.06 & 373.64 & $726.00^{\circ}$ \\
\hline 661 & 92.90 & 19.10 & 2.90 & $3 \cdot 20$ & $88 \cdot 50$ & $11 \cdot 50$ & 88.50 & 29.00 & 31.00 & 11.50 & .00 & 1481.85 & 170.41 & 725.50 \\
\hline$V E$ & 1.64 & .25 & $\because$ & - 2 & 63.80 & $31 \cdot 20$ & 64.03 & 108.06 & 12.51 & 35.97 & 4.77 & 1958.66 & 716 & \\
\hline O. MEAN & 79.04 & 25.06 & 3.15 & $3.2 \pi$ & 63.57 & 30.48 & 63.75 & $96 \cdot 18$ & 11.07 & 35.09 & & 1944.07 & 682.11 & \\
\hline
\end{tabular}

*NOTAT!I!l--Depth, ft; GR-Gamma Ray Index, API Units; POR-Porosity, \%; RT-True Resistivity, Ohms-m²/m; RXO-Flushed Zone Resistivity, Ohms-m²/m; SXO-Flushed Zone Saturation, \%; ROS-Residual 0il Saturation, \%; SW-Water Saturation, \%; PER-Permeability Index; Clay-Clay Content, \%; SO-0il Saturation, \%; MO-Movable 0il, \%; PHV-Pore Volume, bbl/ac-ft; HPV-Hydrocarbon Pore Volume, bbl/ac-ft; ELSE-Elevation From Sea Level, ft 
TABLE C18

WELL LOG DETERMINED PROPERTIES

VS DEPTH FOR WELL MP-107

WELL LOCATION: 2366 FWL 15.84 FSL $21255 \quad 5 E$

KELLY BUSHING ELEVATION FROM SEA LEVEL $=1391$

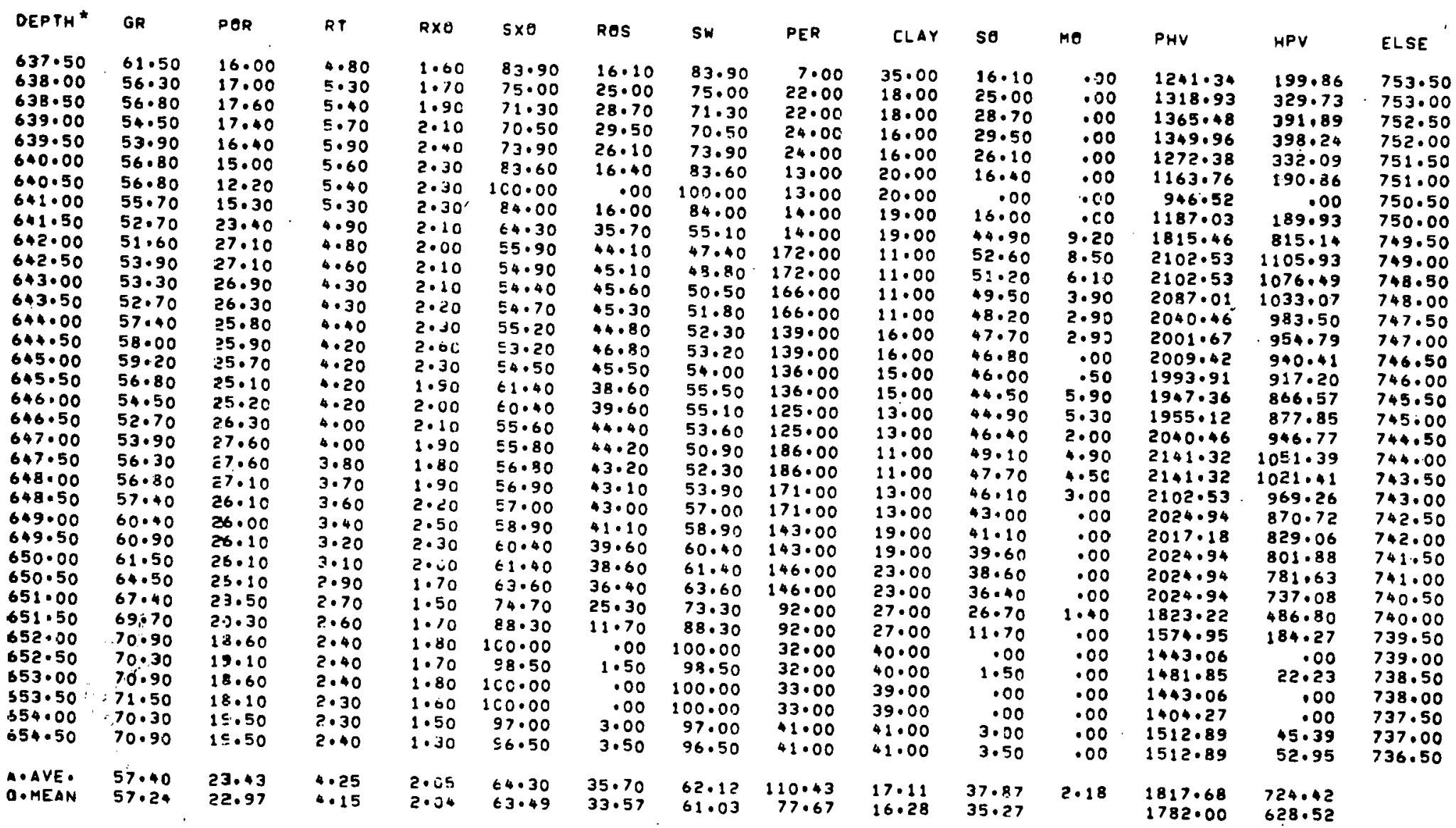

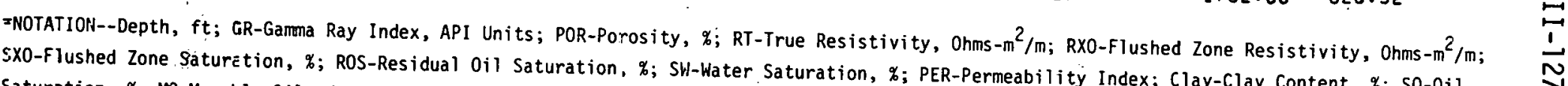
Saturation, \%; Mo-Movatle 0il, \%; PHV-Pore Volume, bbl/ac-ft; HPV-Hydror Saturation, \%; PER-Permeability Index; Clay-Clay Content, \%; SO-0il 
VS DEPTH FOR WELL MP-108

WELL LOCATION: 2640 FEL 1584 FSL $212555 E$

KELLY BUSHING ELEVATION FROH SEA LEVEL $=1387$

\begin{tabular}{|c|c|c|c|c|c|c|c|c|c|c|c|c|c|c|}
\hline DEPTH * & GR & $P O R$ & RT & $R \times \theta$ & $5 \times 0$ & RAS & Sw & PER & CLAY & so & Mo & PHV & HPV & ELSE \\
\hline $\begin{array}{l}621.00 \\
621.50\end{array}$ & $\begin{array}{l}63 \cdot 50 \\
61 \cdot 10\end{array}$ & $\begin{array}{l}20.80 \\
21.50\end{array}$ & $\begin{array}{l}4.80 \\
4.70\end{array}$ & $\begin{array}{l}1.90 \\
1.50\end{array}$ & $\begin{array}{l}63.20 \\
61.60\end{array}$ & 38.40 & 61.60 & $\begin{array}{l}53 \cdot 00 \\
53 \cdot 00\end{array}$ & $\begin{array}{l}27.00 \\
27.00\end{array}$ & $\begin{array}{l}36.80 \\
38.40\end{array}$ & $\begin{array}{l}.00 \\
.00\end{array}$ & 1668.06 & 640.53 & $\begin{array}{l}766.00 \\
765.50\end{array}$ \\
\hline 622.00 & 61.40 & 21.50 & 4.80 & $2 \cdot 00$ & 61.20 & $38 \cdot 80$ & 61.20 & 62.00 & 23.00 & 38.80 & .00 & 1668.06 & 647.21 & 765.00 \\
\hline $\begin{array}{l}622.50 \\
623.00\end{array}$ & $\begin{array}{l}61.60 \\
60.10\end{array}$ & 22.00 & 4.70 & $2 \cdot 20$ & 76.20 & 23.80 & 60.00 & $62 \cdot 00$ & 23.00 & 40.00 & $16 \cdot 20$ & 1706.85 & 682.74 & 764.50 \\
\hline $\begin{array}{l}623.50 \\
624.00\end{array}$ & $\begin{array}{l}58.40 \\
56.70\end{array}$ & $\begin{array}{l}24.20 \\
25.20\end{array}$ & $\begin{array}{l}3.70 \\
3.40\end{array}$ & $\begin{array}{l}3.10 \\
2.70\end{array}$ & $\begin{array}{l}t 0.70 \\
\epsilon 1.40\end{array}$ & $\begin{array}{l}39 \cdot 30 \\
38.60\end{array}$ & $\begin{array}{l}60.70 \\
61.40\end{array}$ & $\begin{array}{r}84.00 \\
125.00\end{array}$ & $\begin{array}{l}19.00 \\
10.00\end{array}$ & $\begin{array}{l}39.30 \\
38.60\end{array}$ & $\begin{array}{l}.00 \\
.00\end{array}$ & $\begin{array}{l}1877 \cdot 53 \\
1955 \cdot 12\end{array}$ & $\begin{array}{l}737.87 \\
754.67\end{array}$ & $\begin{array}{l}763.50 \\
763.00\end{array}$ \\
\hline $\begin{array}{l}624.50 \\
625.00\end{array}$ & 56.00 & 25.80 & $3 \cdot 20$ & $2 \cdot 40$ & $62 \cdot 40$ & 37.60 & 61.60 & 125.00 & 10.00 & 38.40 & .80 & 2001.67 & 768.64 & 762.50 \\
\hline $\begin{array}{l}625 \cdot 50 \\
626.00\end{array}$ & $\begin{array}{l}54 \cdot 80 \\
54 \cdot 90\end{array}$ & $\begin{array}{l}27.10 \\
26.80\end{array}$ & $\begin{array}{l}2.80 \\
2.60\end{array}$ & $\begin{array}{l}1.90 \\
1.70\end{array}$ & $\begin{array}{l}E 5.80 \\
\epsilon 9.60\end{array}$ & $\begin{array}{l}34.70 \\
30.40\end{array}$ & $\begin{array}{l}62.80 \\
65.40\end{array}$ & $\begin{array}{l}158.00 \\
164.00\end{array}$ & $\begin{array}{r}9.00 \\
11.00\end{array}$ & $\begin{array}{l}37.20 \\
34.60\end{array}$ & $\begin{array}{l}2.50 \\
4.20\end{array}$ & $\begin{array}{l}2102 \cdot 53 \\
2079.25\end{array}$ & $\begin{array}{l}782.14 \\
719.42\end{array}$ & $\begin{array}{l}761.50 \\
761.00\end{array}$ \\
\hline $\begin{array}{l}626 \cdot 00 \\
626 \cdot 50\end{array}$ & $\begin{array}{l}54.90 \\
57.60\end{array}$ & $26 \cdot 40$ & 2.40 & 1.80 & 69.40 & 30.60 & 68.60 & $\begin{array}{l}164 \cdot 00 \\
164.00\end{array}$ & $\begin{array}{l}11.00 \\
11.00\end{array}$ & $\begin{array}{r}14.60 \\
31.40\end{array}$ & $\begin{array}{r}.80 \\
.80\end{array}$ & 2048.22 & 643.14 & 760.50 \\
\hline 627.00 & $58 \cdot 10$ & 26.20 & $2 \cdot 30$ & 1.80 & 70.80 & 29.20 & 70.80 & 148.00 & 18.00 & 29.20 & .00 & $2032 \cdot 70$ & 593.55 & $\begin{array}{l}760.00 \\
759.50\end{array}$ \\
\hline $\begin{array}{l}627.50 \\
628.00\end{array}$ & $59 \cdot 50$ & $25 \cdot 70$ & $2 \cdot 30$ & 1.70 & $73 \cdot 30$ & 26.70 & 73.20 & 148.00 & 18.00 & 26.80 & $\cdot 10$ & $1993 \cdot 91$ & 534.37 & $\begin{array}{l}759.50 \\
759.00\end{array}$ \\
\hline $\begin{array}{l}628.00 \\
628.50\end{array}$ & 61.50 & 25.00 & $\begin{array}{l}2.40 \\
2.50\end{array}$ & $\begin{array}{l}1.90 \\
2.00\end{array}$ & $\begin{array}{l}73.90 \\
73.80\end{array}$ & $\begin{array}{l}26.10 \\
26.20\end{array}$ & $\begin{array}{l}73.90 \\
73.80\end{array}$ & 121.00 & 24.00 & $26 \cdot 10$ & .00 & $\begin{array}{l}1939.60 \\
1900.81\end{array}$ & $\begin{array}{l}506.24 \\
498.01\end{array}$ & $\begin{array}{l}759.00 \\
758.50\end{array}$ \\
\hline $\begin{array}{l}629.00 \\
629.50\end{array}$ & $\begin{array}{l}66.80 \\
69.40\end{array}$ & $\begin{array}{l}22 \cdot 50 \\
20.30\end{array}$ & $2 \cdot 60$ & $\begin{array}{l}1.70 \\
1.00\end{array}$ & $\begin{array}{l}79.10 \\
85.80 .\end{array}$ & $\begin{array}{l}20.90 \\
14.20\end{array}$ & $\begin{array}{l}79.10 \\
85.80\end{array}$ & $\begin{array}{l}75 \cdot 00 \\
75 \cdot 00\end{array}$ & $\begin{array}{l}33.00 \\
33.00\end{array}$ & 14.20 & .00 & 1574.95 & 223.64 & 757.50 \\
\hline $\begin{array}{l}629.50 \\
630 \cdot 00\end{array}$ & 71.00 & $\begin{array}{l}20.30 \\
19.10\end{array}$ & 2.90 & 1.70 & 88.90 & 11.10 & 88.90 . & 37.00 & 43.00 & $11 \cdot 10$ & .00 & 1481.85 & 164.49 & 757.00 \\
\hline 630.50 & 69.00 & 21.50 & $3 \cdot 10$ & 1.60 & $75 \cdot 50$ & $24 \cdot 50$ & 75.50 & 37.00 & 43.00 & 24.50 & .00 & 1668.06 & 408.67 & $\begin{array}{l}756.50 \\
756.00\end{array}$ \\
\hline $631 \cdot 00$ & $64 \cdot 30$ & 24.00 & $3 \cdot 40$ & 1.00 & $\epsilon 4.60$ & 35.40 & 64.60 & $101 \cdot 00$ & 29.00 & 35.40 & .00 & 1862.02 & 659.15 & $\begin{array}{l}756.00 \\
755.50\end{array}$ \\
\hline 631.50 & $62 \cdot 30$ & 24.70 & 3.60 & 1.30 & $75 \cdot 40$ & $24 \cdot 60$ & 60.80 & 101.00 & 29.00 & 39.20 & 14.60 & $1916 \cdot 32$ & 751.20 & $\begin{array}{l}755.50 \\
755.00\end{array}$ \\
\hline 632.00 & 62.60 & $25 \cdot 30$ & 3.80 & $2 \cdot 10$ & 67.10 & $32 \cdot 90$ & 57.50 & 126.00 & 23.00 & $42 \cdot 50$ & 9.60 & 1962.87 & 834.22 & $\begin{array}{l}755.0 \\
754.5\end{array}$ \\
\hline 632.50 & 64.90 & 25.40 & 3.90 & $2 \cdot 30$ & 63.90 & $36 \cdot 10$ & 56.90 & 126.00 & 23.00 & $43 \cdot 10$ & 7.00 & 1970.63 & 849.34 & $\begin{array}{l}754.5 \\
754.0\end{array}$ \\
\hline 633.00 & 67.10 & $25 \cdot 30$ & $3 \cdot 9$ & $2 \cdot 60$ & 60.70 & $39 \cdot 30$ & 57.10 & 127.00 & 27.00 & $42 \cdot 90$ & 3.60 & 1962.87 & 842.07 & $\begin{array}{l}754.00 \\
753.50\end{array}$ \\
\hline 633.50 & 67.10 & 25.20 & $3 \cdot 90$ & 2.80 & 59.00 & 41.00 & 57.30 & 127.00 & 27.00 & 42.70 & 1.70 & $1955 \cdot 12$ & 834.83 & $\begin{array}{l}753.50 \\
753.00\end{array}$ \\
\hline 634.00 & 69.50 & 23.10 & $3 \cdot 80$ & 2.60 & 67.20 & 32.80 & 63.60 & 85.00 & 32.00 & $36 \cdot 40$ & 3.60 & $1792 \cdot 19$ & $652 \cdot 36$ & 753.00 \\
\hline 634.50 & 73.60 & 19.40 & 3.70 & $2 \cdot 30$ & 77.60 & $22 \cdot 40$ & 77.60 & 85.00 & 32.00 & $22 \cdot 40$ & .00 & $1505 \cdot 13$ & $337 \cdot 15$ & $\begin{array}{l}752.50 \\
752.00\end{array}$ \\
\hline 635.00 & 75.60 & 16.80 & 3.60 & $2 \cdot 30$ & $5_{1} \cdot 40$ & 8.50 & 91.40 & 21.00 & & 8.60 & .10 & 1303.41 & 112.09 & $\begin{array}{l}752.00 \\
751.50\end{array}$ \\
\hline 635.50 & 76.10 & 15.90 & $3 \cdot 50$ & $2 \cdot 30$ & 58.40 & 1.60 & 98.40 & 21.00 & 47.00 & 1.60 & .00 & $1233 \cdot 58$ & 19.74 & 751.50 \\
\hline 636.00 & 76.00 & 15.60 & 3.50 & $2 \cdot 10$ & 100.00 & .00 & 100.00 & 16.00 & 45.00 & .00 & .00 & 1210.31 & .00 & $\begin{array}{l}751.00 \\
750.50\end{array}$ \\
\hline $636 \cdot 50$ & $73 \cdot 80$ & 17.50 & 3.50 & 1.80 & 89.10 & $10 \cdot 90$ & 89.10 & 26.00 & 45.00 & $10 \cdot 90$ & .00 & 1357.72 & 147.99 & $\begin{array}{l}750.5 \\
750.0\end{array}$ \\
\hline 637.00 & $70 \cdot 10$ & 20.80 & 3.50 & 1.80 & $74 \cdot 20$ & $25 \cdot 80$ & 74.20 & $54 \cdot 00$ & 41.00 & 25.80 & .00 & 1613.75 & $416 \cdot 35$ & $\begin{array}{l}750.0 \\
749.5\end{array}$ \\
\hline $\begin{array}{l}637.50 \\
638.00\end{array}$ & $\begin{array}{l}70.70 \\
71.60\end{array}$ & $\begin{array}{l}20.80 \\
20.70\end{array}$ & 3.50 & $2 \cdot 00$ & $\begin{array}{l}74 \cdot 20 \\
74.40\end{array}$ & $\begin{array}{l}25.80 \\
25.60\end{array}$ & $\begin{array}{l}74.20 \\
74.40\end{array}$ & $\begin{array}{l}54.00 \\
53.00\end{array}$ & $\begin{array}{l}41.00 \\
45.00\end{array}$ & $\begin{array}{l}25.80 \\
25.60\end{array}$ & $\begin{array}{l}.00 \\
.00\end{array}$ & $\begin{array}{l}1613.75 \\
1605.99\end{array}$ & $\begin{array}{r}416.35 \\
411.13\end{array}$ & $\begin{array}{l}749.50 \\
749.00\end{array}$ \\
\hline & & & & & & & & & & & & & & \\
\hline & & & 7 & & & & 68. & 92 & 26.97 & $31 \cdot 16$ & $2 \cdot 11$ & $1786 \cdot 48$ & 571.00 & \\
\hline G. MEAN & 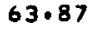 & & & & & & $0.1=$ & 78.64 & 24.21 & 99.06 & & $1772 \cdot 20$ & $515 \cdot 05$ & \\
\hline
\end{tabular}

*NOTATIOH--Depth, ft; GR-Gamma Ray Index, API Units; POR-Porosity, $\%$; RT-True Resistivity, Ohms-m ${ }^{2} / m ;$ RX0-Flushed Zone Resistivity, Ohms-m ${ }^{2} / m$; SX0-Flushed Zone Saturation, $\%$; ROS-Residual 0il Saturation, $\%$; SW-Water Saturation, \%; PER-Permeability Index; Clay-Clay Content, $\%$; SO-0il Saturation, \%; MO-Movable 0il, \%; PHV-Pore Volume, bbl/ac-ft; HPV-Hydrocarbon Pore Volume, bbl/ac-ft; ELSE-Elevation From Sea Level, ft 
WELL LOCATION: 1584 FSL 2376 FEL $212555 E$

KELLY BUSHING ELEVATION FROM SEA LEVEL $=1381$

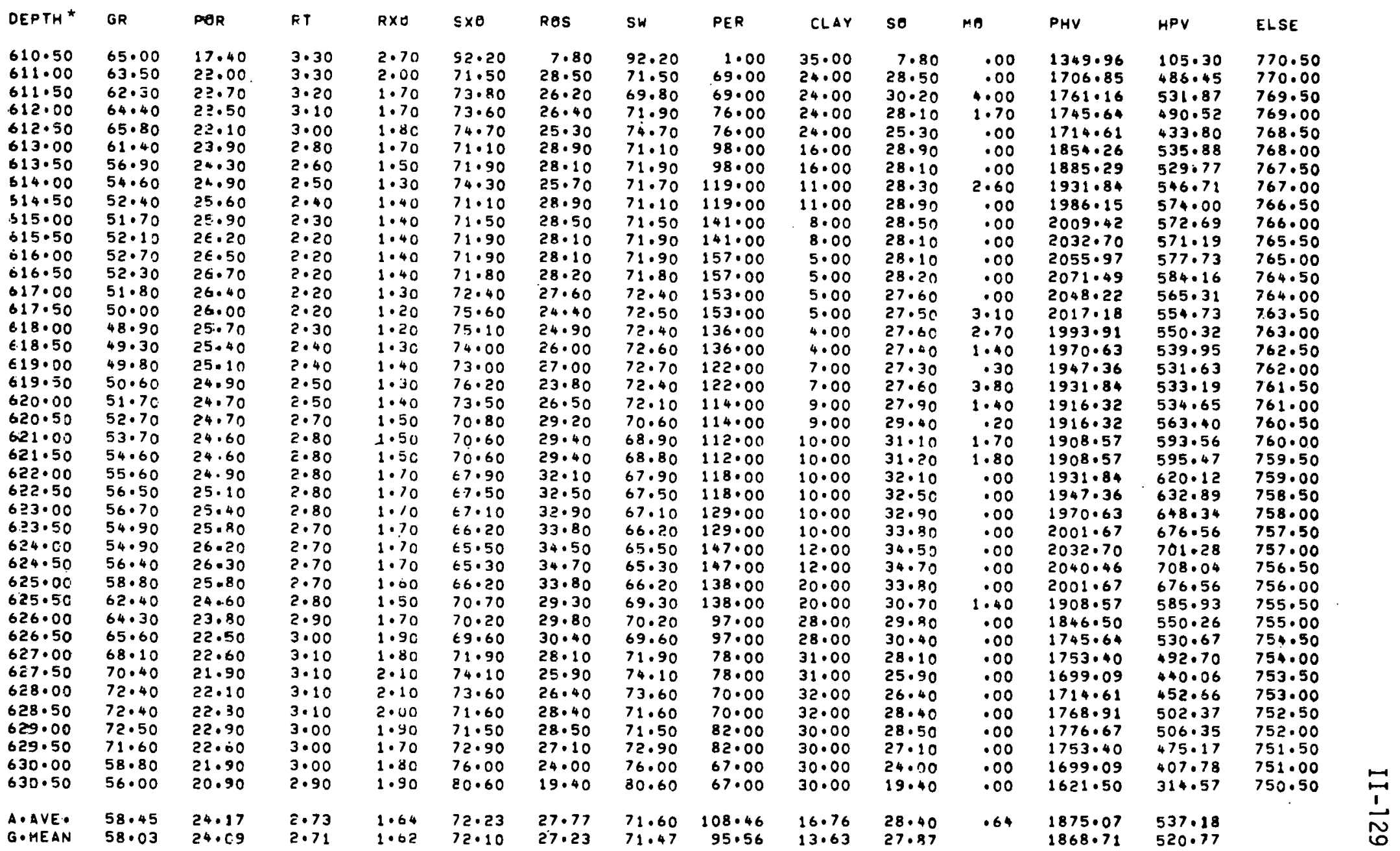

*NJTATION--Depth, ft; GR-Gamma Ray Index, API Units; POR-Porosity, $\%$; RT-True Resistivity; Ohms-m²/m; RXO-Flushed Zone Resistivity, Ohms-m²/m;

SX0-Flushed Zone Saturation, $\%$; ROS-Residual $0 i 1$ Saturation, \%; SW-Water Saturation, \%; PER-Permeabili.ty Index; Clay-Clay Content, \%; SO-0il

Saturation, \%; MO-Movable 0il, \%; PHV-Pore Volume, bbl/ac-ft; HPV-Hydrocarbon Pore Volume, bbl/ac-ft; ELSE-Elevation From Sea Level, ft 
WELL LOCATION: 2112 FEL 1584 FSL 212555

KELLY BUSHING ELEVVATION FROM SEA LEVEL $=1382$

\begin{tabular}{|c|c|c|c|c|c|c|c|c|c|c|c|c|c|c|}
\hline OEPTH * & GR & $P \theta R$ & ET & $R \times E$ & $\leqslant \times 0$ & RES & SW & PER & CLAY & so & Mo & PHV & HPV & ELSE \\
\hline $\begin{array}{l}608 \cdot 00 \\
608 \cdot 50 \\
609.00 \\
609.50 \\
610.00\end{array}$ & $\begin{array}{l}64 \cdot 00 \\
58.80 \\
57.10 \\
56 \cdot 70 \\
56.10\end{array}$ & $\begin{array}{l}21.60 \\
22.40 \\
22.60 \\
22.80 \\
22.50\end{array}$ & $\begin{array}{l}2.90 \\
2 \cdot 00 \\
2 \cdot 10 \\
2.20 \\
2.40\end{array}$ & $\begin{array}{l}1.10 \\
1.30 \\
1.20 \\
.90 \\
.20\end{array}$ & $\begin{array}{l}95.90 \\
89.40 \\
86.60 \\
24.00 \\
82.00\end{array}$ & $\begin{array}{l}4.10 \\
10.60 \\
13.40 \\
16.00 \\
18.00\end{array}$ & $\begin{array}{l}95.90 \\
89.40 \\
86.60 \\
84.00 \\
82.00\end{array}$ & $\begin{array}{l}50 \cdot 00 \\
50.00 \\
61.00 \\
61.00 \\
60.00\end{array}$ & $\begin{array}{r}13.00 \\
13.00 \\
8.00 \\
8.00 \\
7.00\end{array}$ & $\begin{array}{l}40.10 \\
10.60 \\
13.40 \\
16.00 \\
18.00\end{array}$ & $\begin{array}{l}.00 \\
.00 \\
.00 \\
.00 \\
.00\end{array}$ & $\begin{array}{l}1675.81 \\
1737.88 \\
1753.40 \\
1768.91 \\
1745.64\end{array}$ & $\begin{array}{r}68.71 \\
184.22 \\
234.96 \\
283.03 \\
31.021\end{array}$ & $\begin{array}{l}774.40 \\
773.90 \\
773.40 \\
772.90 \\
772.40\end{array}$ \\
\hline $\begin{array}{l}610.50 \\
611.00\end{array}$ & $\begin{array}{l}55 \cdot 30 \\
55 \cdot 10\end{array}$ & $\begin{array}{l}22.20 \\
21.90\end{array}$ & $\begin{array}{l}2.50 \\
2.60\end{array}$ & $\begin{aligned} .80 \\
1.00\end{aligned}$ & $\begin{array}{l}81.60 \\
81.30\end{array}$ & $\begin{array}{l}18.40 \\
18.70\end{array}$ & $\begin{array}{l}81.60 \\
81.30\end{array}$ & $\begin{array}{l}60.00 \\
60.00 \\
53.00\end{array}$ & $\begin{array}{l}7.00 \\
7.00 \\
7.00\end{array}$ & $\begin{array}{l}18.00 \\
18.40 \\
18.70\end{array}$ & $\begin{array}{l}.00 \\
.00 \\
.00\end{array}$ & $\begin{array}{l}1722.36 \\
1699.09\end{array}$ & $\begin{array}{l}316.91 \\
317.73\end{array}$ & $\begin{array}{l}771.90 \\
771.40\end{array}$ \\
\hline $611 \cdot 50$ & $5 \cdot 10$ & 22.80 & 2.70 & 1.10 & 77.00 & 23.00 & 76.70 & 53.00 & 7.00 & $23 \cdot 30$ & - 30 & $1768 \cdot 91$ & 112,16 & $\begin{array}{l}770.90 \\
770.40\end{array}$ \\
\hline $\begin{array}{l}612.00 \\
612.50\end{array}$ & $\begin{array}{l}55 \cdot 10 \\
55 \cdot 10\end{array}$ & $\begin{array}{l}23.70 \\
24.30\end{array}$ & $\begin{array}{l}2.80 \\
6.90\end{array}$ & $\begin{array}{l}1 \cdot 3 \mathrm{C} \\
1 \cdot 4 \mathrm{C}\end{array}$ & $\begin{array}{l}72 \cdot 20 \\
68 \cdot 80\end{array}$ & $\begin{array}{l}27.80 \\
31.20\end{array}$ & $\begin{array}{l}72.20 \\
68.80\end{array}$ & $\begin{array}{l}75.00 \\
75.00\end{array}$ & $\begin{array}{l}6.00 \\
6.00\end{array}$ & $\begin{array}{l}27.80 \\
31.20\end{array}$ & $\begin{array}{l}.00 \\
.00\end{array}$ & $\begin{array}{l}1838.74 \\
1885.29\end{array}$ & $\begin{array}{l}511.17 \\
588.21\end{array}$ & $\begin{array}{l}770.40 \\
769.90\end{array}$ \\
\hline $\begin{array}{l}613.00 \\
613.50\end{array}$ & $\begin{array}{l}55.20 \\
55.20\end{array}$ & $\begin{array}{l}24.60 \\
24.70\end{array}$ & $\begin{array}{l}3.00 \\
3.10\end{array}$ & $\begin{array}{l}1 \cdot b n \\
1 \cdot+0\end{array}$ & $\begin{array}{l}E 6 \cdot 40 \\
64.90\end{array}$ & $\begin{array}{l}33.60 \\
35.10\end{array}$ & $\begin{array}{l}66.40 \\
64.90\end{array}$ & $\begin{array}{l}88.00 \\
88.00\end{array}$ & $\begin{array}{l}7.00 \\
7.00\end{array}$ & $\begin{array}{l}33.60 \\
35.10\end{array}$ & $\begin{array}{l}.00 \\
.00\end{array}$ & $\begin{array}{l}1908.57 \\
1916.32\end{array}$ & $\begin{array}{l}641.28 \\
672.63\end{array}$ & $\begin{array}{l}769.40 \\
768.90\end{array}$ \\
\hline 614.00 & 56.50 & 24.60 & $3 \cdot 30$ & 1.20 & $E 6.80$ & 33.20 & 63.70 & 88.00 & 10.00 & $36 \cdot 30$ & $3 \cdot 10$ & $1908 \cdot 57$ & 692.81 & 768.90 \\
\hline 614.50 & 57.80 & 24.40 & 3.40 & 1.10 & $\in 9.80$ & 30.20 & 63.00 & 88.00 & 10.00 & 37.00 & 6.80 & 1893.05 & $700 \cdot 43$ & $\begin{array}{l}767.9 \\
767.4\end{array}$ \\
\hline $615 \cdot 50$ & 60.50. & 24.40 & $3 \cdot 70$ & $1 \cdot 10$ & 68.80 & $31 \cdot 20$ & $60 \cdot 70$ & 85.00 & 12.00 & $39 \cdot 30$ & 8.10 & $\begin{array}{l}1893.05 \\
1900.81\end{array}$ & $\begin{array}{l}743.97 \\
771.73\end{array}$ & $\begin{array}{l}766.90 \\
766.40\end{array}$ \\
\hline $\begin{array}{l}616.50 \\
617.00\end{array}$ & $\begin{array}{l}63 \cdot 00 \\
64.00\end{array}$ & $\begin{array}{l}24.50 \\
24.60\end{array}$ & $\begin{array}{l}4.00 \\
4.10\end{array}$ & $\begin{array}{l}1 \cdot 2 c \\
1 \cdot 3 c\end{array}$ & $\begin{array}{l}66.60 \\
\epsilon 4.60\end{array}$ & $\begin{array}{l}33.40 \\
35.40\end{array}$ & $\begin{array}{l}58.20 \\
56.90\end{array}$ & $\begin{array}{l}87.00 \\
89.00\end{array}$ & $\begin{array}{l}14.00 \\
15.00\end{array}$ & $\begin{array}{r}41.80 \\
43.10\end{array}$ & $\begin{array}{l}8.40 \\
7.70\end{array}$ & $\begin{array}{l}1900.81 \\
1908.57\end{array}$ & 822.59 & $\begin{array}{l}763.90 \\
765.40\end{array}$ \\
\hline 617.50 & 64.80 & 24.40 & $4 \cdot 30$ & $1 \cdot j c$ & $\epsilon 3.60$ & 36.40 & $56 \cdot 30$ & 89.00 & 15.00 & 43.70 & $7 \cdot 30$ & 1893.05 & 827.26 & 764.90 \\
\hline $618 \cdot 00$ & 65.90 & 24.10 & $4 \cdot 30$ & 1.40 & $\in 2.40$ & 37.60 & $56 \cdot 50$ & 81.00 & 17.00 & $43 \cdot 50$ & $5 \cdot 90$ & 1869.77 & $813 \cdot 35$ & $\begin{array}{l}764.40 \\
763.90\end{array}$ \\
\hline $618 \cdot 50$ & $66 \cdot 10$ & $23.7 \mathrm{C}$ & 4.40 & 1.60 & $\in 0.80$ & 39.20 & 57.00 & $81 \cdot 00$ & 17.00 & .3 .00 & 3.80 & 1838.74 & 790.66 & $\begin{array}{l}763.90 \\
763.40\end{array}$ \\
\hline 619.00 & $66 \cdot 40$ & 23.50 & 4.50 & 1.40 & $04 \cdot 80$ & $35 \cdot 20$ & 57.10 & $73 \cdot 00$ & 18.00 & $42 \cdot 90$ & 7.70 & 1823.22 & $\begin{array}{l}782.16 \\
773.70\end{array}$ & $\begin{array}{l}63.40 \\
62.90\end{array}$ \\
\hline 619.50 & $66 \cdot 60$ & $23 \cdot 30$ & 4.60 & 1.30 & 67.50 & $32 \cdot 50$ & 57.20 & 73.00 & 18.00 & $42 \cdot 90$ & $10 \cdot 30$ & $\begin{array}{r}1807.71 \\
1815.46\end{array}$ & $\begin{array}{l}773.70 \\
784.28\end{array}$ & $\begin{array}{l}762.90 \\
762.40\end{array}$ \\
\hline 620.00 & $67 \cdot 10$ & 23.40 & 4.60 & 1.40 & $66 \cdot 10$ & 33.90 & 56.80 & $71 \cdot 00$ & 19.00 & 43.20 & $9 \cdot 30$ & $\begin{array}{l}1815.46 \\
1838.74\end{array}$ & $\begin{array}{l}784.28 \\
810.88\end{array}$ & 762.00 \\
\hline $\begin{array}{l}620 \cdot 50 \\
621.00\end{array}$ & 68.00 & 23.70 & 4.60 & 1.40 & $63 \cdot 70$ & $36 \cdot 30$ & 55.90 & 71.00 & & $44 \cdot 10$ & $\begin{array}{l}7.80 \\
6.80\end{array}$ & $\begin{array}{l}1838.74 \\
1862.02\end{array}$ & 837.91 & $\begin{array}{l}761.90 \\
761.40\end{array}$ \\
\hline 621.00 & 69.30 & 24.00 & 4.60 & 1.50 & 61.80 & 38.20 & 55.00 & 79.00 & $\begin{array}{l}22.00 \\
22.00\end{array}$ & $\begin{array}{l}45.00 \\
45.70\end{array}$ & $\begin{array}{l}6.80 \\
5.70\end{array}$ & 1885.29 & 861.58 & 760.90 \\
\hline 621.50 & 69.90 & $24 \cdot 3 C$ & 4.60 & 1.50 & $\leqslant 0.00$ & 40.00 & $54 \cdot 30$ & 79.00 & $\begin{array}{l}22.00 \\
22.00\end{array}$ & $\begin{array}{l}45.70 \\
47.70\end{array}$ & $\begin{array}{l}5.70 \\
2.40\end{array}$ & 1947.36 & 919.15 & 760.40 \\
\hline $\begin{array}{l}622.00 \\
622.50\end{array}$ & $70 \cdot 50$ & $25 \cdot 10$ & $\begin{array}{l}4.60 \\
4.50\end{array}$ & $\begin{array}{l}1.70 \\
1.70\end{array}$ & $\begin{array}{l}55 \cdot 20 \\
=2.20\end{array}$ & $\begin{array}{l}44.80 \\
47.80\end{array}$ & $\begin{array}{l}52.80 \\
51.40\end{array}$ & bo & $\begin{array}{l}22.00 \\
22.00\end{array}$ & $48.6 n$ & $\begin{array}{r}2.80 \\
.80\end{array}$ & 2009.42 & 976.58 & 759.90 \\
\hline & 71.00 & 25.90 & $\begin{array}{l}4.50 \\
4.40\end{array}$ & $\begin{array}{l}1.70 \\
1.70\end{array}$ & $\begin{array}{l}E 2.20 \\
51.70\end{array}$ & $\begin{array}{l}47.80 \\
48.30\end{array}$ & $\begin{array}{l}51.40 \\
50.10\end{array}$ & 127.00 & 20.00 & 49.90 & 1.60 & 2071.49 & 1033.67 & 759.40 \\
\hline $\begin{array}{l}623.00 \\
623.50\end{array}$ & $\begin{array}{l}71.50 \\
72.10\end{array}$ & $\begin{array}{l}26.70 \\
26.90\end{array}$ & $\begin{array}{l}4.40 \\
4.30\end{array}$ & 1.00 & $E_{1.90}$ & $48 \cdot 10$ & 50.30 & 127.00 & 20.00 & 49.70 & 1.60 & 2087.01 & 1037.24 & 758.90 \\
\hline 624.00 & 72.40 & 27.20 & 4.20 & 1.60 & 52.20 & 47.80 & $50 \cdot 30$ & 138.00 & 19.00 & 49.70 & 1.90 & 2110.28 & 1048.81 & .758 .4 \\
\hline 624.50 & 71.70 & 25.80 & 4.20 & $1 \cdot b 0$ & 56.90 & $43 \cdot 10$ & 53.80 & 138.00 & 19.00 & 46.20 & $3 \cdot 10$ & 2001.67 & 924.77 & 757.90 \\
\hline 625.00 & 72.50 & 24.20 & $4 \cdot 10$ & $1 \cdot 40$ & $63 \cdot 10$ & $36 \cdot 90$ & 58.20 & $82 \cdot 00$ & 25.00 & 41.80 & 4.90 & 1877.53 & 784.81 & 757.4 \\
\hline $625 \cdot 50$ & 73.70 & 23.30 & 4.00 & $1 \cdot 30$ & 66.60 & 33.40 & 61.10 & 82.00 & 25.00 & $38.9 n$ & $5 \cdot 50$ & 7.71 & 703.20 & 756.90 \\
\hline $\begin{array}{l}626 \cdot 00 \\
626.50\end{array}$ & $\begin{array}{l}81.80 \\
85.60\end{array}$ & $\begin{array}{l}18.60 \\
16.00\end{array}$ & $\begin{array}{l}3.90 \\
3.90\end{array}$ & $\begin{array}{l}1 \cdot 50 \\
1 \cdot 30\end{array}$ & $\begin{array}{l}78.90 \\
53.50\end{array}$ & $\begin{array}{r}21 \cdot 10 \\
6.50\end{array}$ & $\begin{array}{l}78.90 \\
93.50\end{array}$ & $\begin{array}{l}26.00 \\
26.00\end{array}$ & $\begin{array}{l}39.00 \\
39.00\end{array}$ & $\begin{array}{r}21.10 \\
6.50\end{array}$ & $\begin{array}{l}.00 \\
.00\end{array}$ & $\begin{array}{l}1443.06 \\
1241.34\end{array}$ & $\begin{array}{r}304.49 \\
80.69\end{array}$ & $\begin{array}{l}756.40 \\
755.90\end{array}$ \\
\hline & 1.95 & 3.78 & 069 & & $8 \cdot 16$ & 31.84 & 64.44 & 80.27 & $15 \cdot 86$ & 35.56 & 3.72 & $1845 \cdot 2$ & 670.68 & \\
\hline & 63.99 & 239.69 & . 59 & 29 & 67.38 & 29.47 & 63.38 & 76.04 & 13.98 & 32.47 & & 1837.84 & 596.75 & \\
\hline
\end{tabular}

*NOTATIDN--Depth, ft; GR-Gamma Ray Index, API Units; POR-Porosity, $\%$; RT-True Resistivity, Ohms-m²/m; RXo-Flushed Zone Resistivity, Ohms-m²/m; SXO-Flushed Zone Saturation, \%; ROS-Residual $0 i 1$ Saturation, \%; SH-Water Saturation, \%; PER-Permeability Index; Clay-Clay Content, \%; S0-0il Saturation, \%; MO-Movable Dil, \%; PHV-Pore Volume, bbl/ac-ft; HPV-Hydrocarbon Pore Volume, bbl/ac-ft; ELSE-Elevation From Sea Level, ft 
TABLE C22

WELL LOG DETERMINED PROPERTIES

VS DEPTH FOR WELL MP-111

WELL LOCATION: 2112 FEL 1320 FSL 21255 5E

KELLY BUSHING ELEVATION FROH SEA LEVEL $=1386$

\begin{tabular}{|c|c|c|c|c|c|c|c|c|c|c|c|c|c|c|}
\hline DEPTH * & $G R$ & $P Q R$ & RT & $R \times i$ & $5 \times 0$ & ROS & sw & PER & CLAY & 50 & re & PhV & HPV & ELSE \\
\hline 613.00 & 65.50 & 22.70 & 1.60 & 1.00 & 58.60 & 1.40 & 98.60 & 79.00 & 28.00 & 1.40 & .00 & $1761 \cdot 16$ & 24.66 & 773.00 \\
\hline 613.50 & 64.90 & 22.70 & 1.60 & 1.10 & 99.00 & 1.00 & 99.00 & 79.00 & 28.00 & 1.00 & .00 & $1761 \cdot 16$ & 17.61 & 772.50 \\
\hline 614.00 & 64.00 & 22.90 & 1.60 & 1.00 & 59.00 & 1.00 & 99.00 & 81.00 & 24.00 & 1.00 & .00 & 1776.67 & 17.77 & 772.00 \\
\hline 614.50 & $60 \cdot 70$ & 22.70 & 1.60 & 1.40 & 99.70 & .30 & 99.70 & $81 \cdot 00$ & 24.00 & - 3n & .00 & $1761 \cdot 16$ & $5 \cdot 28$ & 771.50 \\
\hline $615 \cdot 00$ & $57 \cdot 30$ & 23.40 & 1.60 & 1.60 & $95 \cdot 90$ & $4 \cdot 10$ & 95.90 & $91 \cdot 00$ & 16.00 & $4 \cdot 10$ & .00 & $1815 \cdot 46$ & $\begin{array}{r}74.43 \\
200.72\end{array}$ & 771.00 \\
\hline $615 \cdot 50$ & $53 \cdot 90$ & $\bar{c} 4.80$ & 1.60 & 1.40 & $89 \cdot 10$ & $10 \cdot 90$ & 89.10 & $91 \cdot 00$ & 16.00 & $10 \cdot 90$ & .00 & 1924.08 & 269.72 & 770.50 \\
\hline 616.00 & $50 \cdot 30$ & $=5.90$ & 1.70 & 1.30 & $84 \cdot 10$ & $15 \cdot 90$ & 84.10 & 141.00 & 8.00 & $15 \cdot 90$ & .00 & $2009 \cdot 42$ & 319.50 & 770.00 \\
\hline 616.50 & 47.70 & ¿5.60 & 1.70 & $1 \cdot 30$ & $84 \cdot 00$ & $16 \cdot 00$ & 84.00 & 141.00 & 8.00 & 16.00 & .00 & $1986 \cdot 15$ & 317.78 & 769.50 \\
\hline $\begin{array}{l}617.00 \\
617.50\end{array}$ & $\begin{array}{l}46.30 \\
47.30\end{array}$ & $\begin{array}{l}E 5.40 \\
=5.50\end{array}$ & $\begin{array}{l}1.80 \\
1.80\end{array}$ & $\begin{array}{l}1.20 \\
1.20\end{array}$ & $\begin{array}{l}83.30 \\
81.60\end{array}$ & $\begin{array}{l}16.70 \\
18.40\end{array}$ & $\begin{array}{l}83.30 \\
81.60\end{array}$ & $\begin{array}{l}130 \cdot 00 \\
130 \cdot 00\end{array}$ & $\begin{array}{l}6.00 \\
6.00\end{array}$ & $\begin{array}{l}16.70 \\
18.40\end{array}$ & $\begin{array}{l}.00 \\
.00\end{array}$ & $\begin{array}{l}1970.63 \\
1978.39\end{array}$ & $\begin{array}{l}329.10 \\
364.02\end{array}$ & $\begin{array}{l}769.00 \\
768.50\end{array}$ \\
\hline $\begin{array}{l}618.00 \\
618.50\end{array}$ & $\begin{array}{l}49.60 \\
50.90\end{array}$ & $\begin{array}{l}=5.60 \\
=5.70\end{array}$ & $\begin{array}{l}1.90 \\
2.00\end{array}$ & $\begin{array}{l}1.40 \\
1.50\end{array}$ & $\begin{array}{l}80.10 \\
78.60\end{array}$ & $\begin{array}{l}19.90 \\
21.40\end{array}$ & $\begin{array}{l}80.10 \\
78.60\end{array}$ & $\begin{array}{l}135.00 \\
135.00\end{array}$ & $\begin{array}{l}8.00 \\
8.00\end{array}$ & $\begin{array}{l}19.90 \\
21.40\end{array}$ & $\begin{array}{l}.00 \\
.00\end{array}$ & $\begin{array}{l}1986.15 \\
1993.91\end{array}$ & $\begin{array}{r}395.24 \\
+26.70\end{array}$ & $\begin{array}{l}768.00 \\
767.50\end{array}$ \\
\hline $\begin{array}{l}619.00 \\
619.50\end{array}$ & $\begin{array}{l}51.80 \\
52.70\end{array}$ & $\begin{array}{l}24.50 \\
24.50\end{array}$ & $\begin{array}{l}2 \cdot 00 \\
2.10\end{array}$ & $\begin{array}{l}1.50 \\
1.00\end{array}$ & $\begin{array}{l}81.90 \\
80.60\end{array}$ & $\begin{array}{l}18.10 \\
19.40\end{array}$ & $\begin{array}{l}81.90 \\
80.60\end{array}$ & $\begin{array}{l}109.00 \\
109.00\end{array}$ & $\begin{array}{l}11.00 \\
11.00\end{array}$ & $\begin{array}{l}18.10 \\
19.40\end{array}$ & $\begin{array}{l}.00 \\
.00\end{array}$ & $\begin{array}{l}1900.81 \\
1900.81\end{array}$ & $\begin{array}{l}344.05 \\
368.76\end{array}$ & $\begin{array}{l}767.00 \\
766.50\end{array}$ \\
\hline $\begin{array}{l}620.00 \\
620.50\end{array}$ & $\begin{array}{l}53.40 \\
53.70\end{array}$ & $\begin{array}{l}24.90 \\
25.2 .0\end{array}$ & $\begin{array}{l}2.10 \\
2.20\end{array}$ & $\begin{array}{l}1.00 \\
1.10\end{array}$ & $\begin{array}{l}78.50 \\
76.60\end{array}$ & $\begin{array}{l}21.50 \\
23.40\end{array}$ & $\begin{array}{l}78.50 \\
76.60\end{array}$ & $\begin{array}{l}117.00 \\
117.00\end{array}$ & $\begin{array}{l}12.00 \\
12.00\end{array}$ & $\begin{array}{l}21.50 \\
23.40\end{array}$ & $\begin{array}{l}.00 \\
.00\end{array}$ & $\begin{array}{l}1931.84 \\
1955.12\end{array}$ & $\begin{array}{r}415.35 \\
457.50\end{array}$ & $\begin{array}{l}766.00 \\
765.50\end{array}$ \\
\hline 621.00 & 52.70 & 25.60 & 2.20 & 1.70 & 74.90 & $25 \cdot 10$ & 74.90 & 133.00 & 11.00 & $25 \cdot 10$ & .00 & 1986.15 & .998 .52 & 765.00 \\
\hline $621 \cdot 50$ & 49.60 & 26.00 & $2 \cdot 30$ & 1.70 & $72 \cdot 40$ & 27.60 & 72.40 & 133.00 & 11.00 & 27.50 & .00 & 2017.18 & 556.74 & 764.50 \\
\hline 622.00 & 49.40 & 26.10 & $2 \cdot 30$ & 1.00 & 71.50 & 28.50 & 71.50 & 145.00 & 10.00 & 28.50 & .00 & 2024.94 & 577.11 & 764.00 \\
\hline $\begin{array}{l}622.50 \\
623.00\end{array}$ & $\begin{array}{r}49.20 \\
49.00\end{array}$ & $\begin{array}{l}25.20 \\
23.90\end{array}$ & $\begin{array}{l}2 \cdot 30 \\
2.40\end{array}$ & $\begin{array}{l}1.60 \\
1.60\end{array}$ & $\begin{array}{l}73.60 \\
77.00\end{array}$ & $\begin{array}{l}26.40 \\
23.00\end{array}$ & $\begin{array}{l}73.60 \\
77.00\end{array}$ & $\begin{array}{r}145.00 \\
90.00\end{array}$ & $\begin{array}{l}10.00 \\
13.00\end{array}$ & $\begin{array}{l}26.40 \\
23.00\end{array}$ & .00 & 1955.12 & 516.15 & 763.50 \\
\hline $\begin{array}{l}623.00 \\
623.50\end{array}$ & $\begin{array}{r}49.00 \\
49.30\end{array}$ & $\begin{array}{l}23.90 \\
23.20\end{array}$ & $\begin{array}{l}2.40 \\
2.40\end{array}$ & $\begin{array}{l}1.60 \\
1.70\end{array}$ & $\begin{array}{l}77.00 \\
78.50\end{array}$ & $\begin{array}{l}23.00 \\
21.50\end{array}$ & $\begin{array}{l}77.00 \\
78.50\end{array}$ & $\begin{array}{l}90.00 \\
90.00\end{array}$ & $\begin{array}{l}13.00 \\
13.00\end{array}$ & $\begin{array}{l}23.00 \\
21.50\end{array}$ & $\begin{array}{l}.00 \\
.00\end{array}$ & $\begin{array}{l}1854.26 \\
1799.95\end{array}$ & $\begin{array}{r}426.48 \\
386.99\end{array}$ & $\begin{array}{l}763.00 \\
762.50\end{array}$ \\
\hline 624.00 & 51.50 & 22.80 & 2.50 & $\begin{array}{l}1.76 \\
1.80\end{array}$ & 79.40 & 20.60 & 79.40 & 80.00 & 15.00 & 20.60 & .00 & 1768.91 & 364.40 & $\begin{array}{l}762.50 \\
762.00\end{array}$ \\
\hline 624.50 & 57.10 & 22.50 & 2.50 & 1.80 & 80.10 & 19.90 & 80.10 & 80.00 & 15.00 & 19.90 & .00 & 1745.64 & 347.38 & 761.50 \\
\hline 625.00 & 59.10 & 22.10 & 2.60 & 1.80 & 80.90 & 19.10 & 80.90 & 70.00 & 23.00 & 19.10 & .00 & 1714.61 & 327.49 & 761.00 \\
\hline 625.50 & 60.20 & 21.20 & 2.60 & 1.80 & 84.20 & $15 \cdot 80$ & 84.20 & 70.00 & 23.00 & 15.80 & .00 & 1644.78 & 259.87 & 760.50 \\
\hline $\begin{array}{l}626.00 \\
626.50\end{array}$ & $\begin{array}{l}61 \cdot 20 \\
62.20\end{array}$ & $\begin{array}{l}20.00 \\
20.60\end{array}$ & $\begin{array}{l}2.60 \\
2.70\end{array}$ & $\begin{array}{l}1.90 \\
2.10\end{array}$ & $\begin{array}{l}88.90 \\
86.00\end{array}$ & $\begin{array}{l}11.10 \\
14.00\end{array}$ & $\begin{array}{l}88.90 \\
86.00\end{array}$ & $\begin{array}{r}45.00 \\
45.00\end{array}$ & $\begin{array}{l}26.00 \\
26.00\end{array}$ & $\begin{array}{l}11.10 \\
14.00\end{array}$ & $\begin{array}{l}.00 \\
.00\end{array}$ & $\begin{array}{l}1551.68 \\
1598.23\end{array}$ & $\begin{array}{l}172.24 \\
223.75\end{array}$ & $\begin{array}{l}760.00 \\
759.50\end{array}$ \\
\hline $\begin{array}{l}627.00 \\
627.50\end{array}$ & $\begin{array}{l}62.50 \\
59.00\end{array}$ & $\begin{array}{l}20.90 \\
21.50\end{array}$ & $\begin{array}{l}2.60 \\
2.60\end{array}$ & $\begin{array}{l}2.00 \\
2.00\end{array}$ & $\begin{array}{l}84 \cdot 50 \\
82.10\end{array}$ & $\begin{array}{l}15.50 \\
17.90\end{array}$ & $\begin{array}{l}84.50 \\
82.10\end{array}$ & $\begin{array}{l}55.00 \\
55.00\end{array}$ & $\begin{array}{l}25.00 \\
25.00\end{array}$ & $\begin{array}{l}15.50 \\
17: 90\end{array}$ & $\begin{array}{l}.00 \\
.00\end{array}$ & $\begin{array}{l}1621.50 \\
1668.06\end{array}$ & $\begin{array}{l}251.33 \\
29.8 .58\end{array}$ & $\begin{array}{l}759.00 \\
758.50\end{array}$ \\
\hline 628.00 & 54.70 & 25.30 & 2.60 & $2 \cdot 30$ & 66.60 & 33.40 & 66.60 & 149.00 & 10.00 & 33.40 & .00 & 2040.46 & 681.51 & 758.00 \\
\hline $628 \cdot 50$ & $50 \cdot 80$ & 27.40 & 2.60 & $2 \cdot 10$ & 63.70 & $36 \cdot 30$ & 63.70 & 149.00 & 10.00 & 36.30 & .00 & 2125.80 & 771.67 & 757.50 \\
\hline 629.00 & 50.80 & 23.40 & 2.60 & 1.90 & $\in 1 \cdot 50$ & $38 \cdot 50$ & 61.50 & 212.00 & 6.00 & 38.50 & .00 & $2203 \cdot 38$ & $848 \cdot 30$ & 757.00 \\
\hline 629.50 & 57.50 & 27.40 & 2.60 & 1.90 & $\epsilon 3.80$ & $36 \cdot 20$ & 63.80 & $212 \cdot 00$ & 6.00 & $36 \cdot ? 0$ & .00 & 2125.80 & 769.54 & 756.50 \\
\hline 630.00 & 63.10 & 25.20 & 2.60 & 1.90 & $E 7.10$ & 32.90 & 67.10 & $148 \cdot 00$ & $20 \cdot 00$ & 32.90 & .00 & 2032.70 & 668.76 & 756.00 \\
\hline 630.50 & $70 \cdot 50$ & 13.80 & $2 \cdot 50$ & 2.00 & $\varsigma_{1.50}$ & $8 \cdot 50$ & 91.50 & $148 \cdot 00$ & 20.00 & $8 \cdot 50$ & .00 & $2536 \cdot 16$ & 130.57 & 755.50 \\
\hline A.AVE & 54.13 & 24.35 & $2 \cdot 29$ & $1 \cdot 71$ & $78 \cdot 28$ & 21.72 & $78.2 R$ & 117.00 & 13.68 & 21.72 & .00 & $\begin{array}{l}1888.79 \\
1880.37\end{array}$ & $420 \cdot 16$ & \\
\hline G・MEAN & 53.85 & 24.24 & $2 \cdot 26$ & 1068 & $77 \cdot 89$ & 20.43 & 77.89 & $108 \cdot 85$ & $12 \cdot 30$ & 20.43 & & 1880.37 & 384.19 & \\
\hline
\end{tabular}

*NOTATION--Depth, $\mathrm{ft}$; GR-Gamma Ray Index, API Units; POR-Porosity, $\%$; RT-True Resistivity, Ohms-m²/m; RXO-Flushed Zone Resistivity, Ohms-m²/m; SXO-Flushed Zone Satıration, \%; ROS-Residual $0 i 1$ Saturation, \%; SW-Water Saturation, \%; PER-Permeability Index; Clay-Clay Content, \%; SO-0il

Saturation, \%; MO-Morable 0il, \%; PHV-Pore Volume, bbl/ac-ft; HPV-Hydrocarbon Pore Volume, bbl/ac-ft; ELSE-Elevation From Sea Level, ft 
WELL LOCATION: 2366 FEL $1330 \mathrm{FSL} 21$ ?55 $5 E$

KELLY BiSSHING ELEVATION FROH SEA LEVEL $=1335$

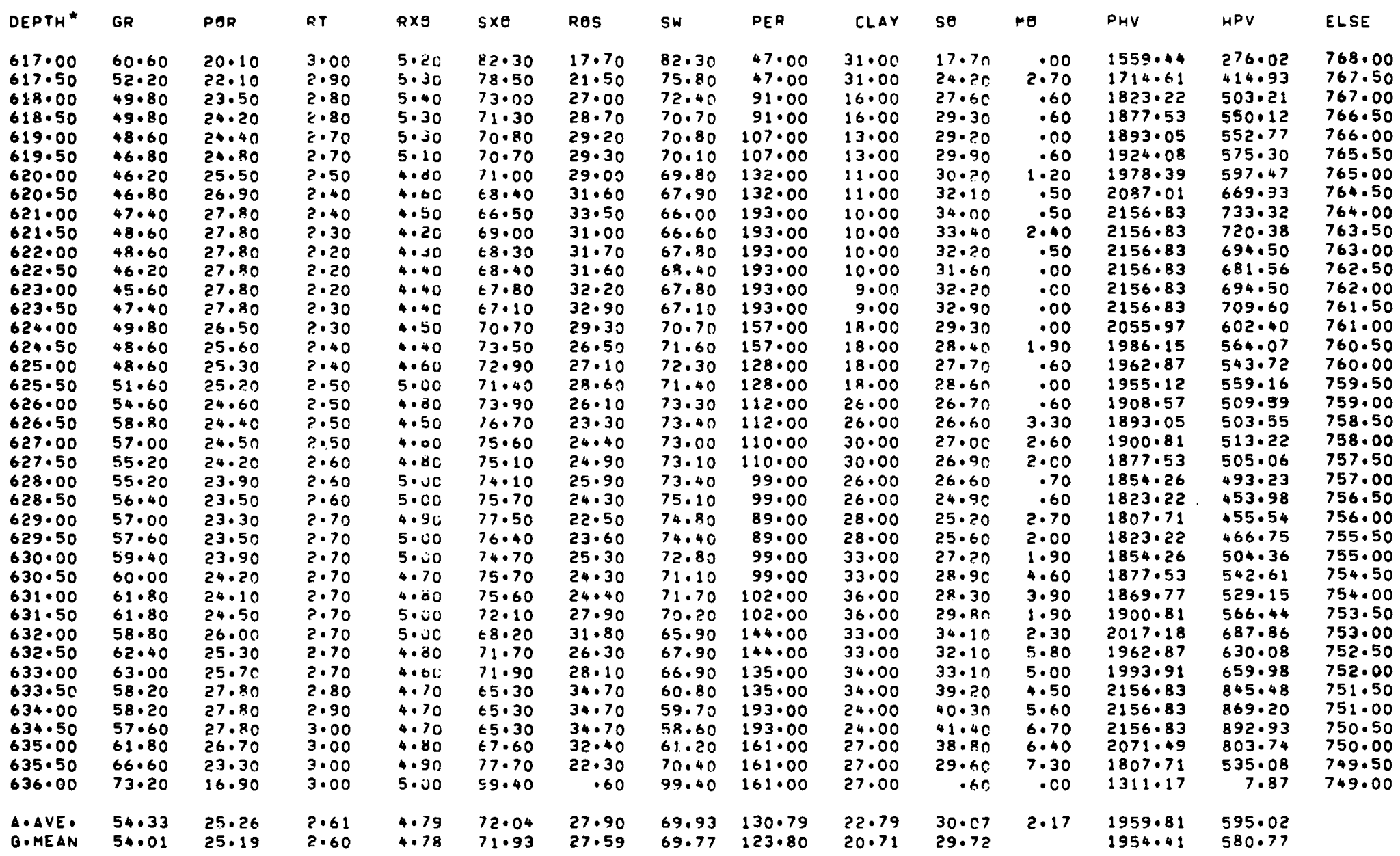

*NOTATJON--Depth, ft; GR-Satma Ray Index, API Units; POR-Porosity, $\%$; RT-True Resistivity, Ohms-m²/m; RXO-Flushed Zone Resistivity, Ohms-m²/m; SXO-Flushed Zone Saturation, \%; ROS-Residual $0 i 1$ Saturation, \%; SW-Water Saturation, \%; PER-Permeability Index; Clay-Clay Content, \%; SO-0il

Saturation, \%; MO-Movable Oil, \%; PHV-Pore Volume, bbl/ac-ft; HPV-Hydrocarbon Pore Volume, bbl/ac-ft; ELSE-Elevation From Sea Level, ft 
TABLE C24

WELL LOG DETERMINED PROPERTIES

VS OEPTH FOR WELL HAP-113

WELL LOCATION: $2640 \mathrm{FEL} 1320 \mathrm{FSL} 21255$ 5E

KELLY BUSHING ELEVATION FROM SEA LEVEL $=1392$

\begin{tabular}{|c|c|c|c|c|c|c|c|c|c|c|c|c|c|c|}
\hline DEPTH* & GF & $P \theta R$ & $R T$ & $R \times E$ & $5 \times 0$ & RES & Sw & PER & CLAY & se & re & PHV & HPV & ELSE \\
\hline 624.50 & $68 \cdot 70$ & 15.60 & $5 \cdot 20$ & . so & 82. & 17.60 & $8 ? .40$ & 8.00 & 30.00 & 17.60 & .00 & $1210 \cdot 31$ & $2 \div 3.01$ & 767.50 \\
\hline $625 \cdot 00$ & 71.10 & 16.60 & $5 \cdot 30$ & $1 \cdot 50$ & 76.90 & $23 \cdot 10$ & 76.90 & $20 \cdot 00$ & 37.00 & $23 \cdot 10$ & .00 & 1287.89 & 297.50 & 767.00 \\
\hline 625.50 & $69 \cdot 30$ & 19.00 & $5 \cdot 20$ & $2.2 c$ & 66.60 & 33.40 & 66.60 & $20 \cdot 00$ & 37.00 & 33.40 & .00 & 1474.10 & $492 \cdot 35$ & 766.50 \\
\hline 626.00 & 65.20 & 20.80 & 5.10 & $2 \cdot 40$ & $=1 \cdot 30$ & 38.70 & 61.30 & 54.00 & 25.00 & 38.70 & .00 & 1613.75 & 624.52 & 766.00 \\
\hline 626.50 & 58.90 & 20.50 & 4.90 & 1.20 & $\in 3.70$ & $36 \cdot 30$ & 63.70 & 54.00 & 25.00 & 36.30 & .00 & 1590.47 & 577.34 & 765.50 \\
\hline 627.00 & 56.10 & 20.10 & 4.80 & .90 & $E 5 \cdot 30$ & 34.70 & 65.30 & 46.00 & 15.00 & 34.70 & .00 & 1559.44 & 541.12 & 765.00 \\
\hline 627.50 & 55.10 & 21.50 & 4.70 & 1.00 & $\epsilon 1.50$ & $38 \cdot 50$ & 61.50 & $46 \cdot 00$ & 15.00 & 38.50 & .00 & 1668.06 & 642.20 & 764.50 \\
\hline 628.00 & 55.50 & 24.70 & 4.60 & 1.10 & $53 \cdot 30$ & 46.70 & 53.30 & 115.00 & 9.00 & 46.70 & .00 & 1916.32 & 394.92 & 764.00 \\
\hline 628.50 & 54.30 & 25.60 & $4 \cdot 30$ & $i .10$ & $53 \cdot 30$ & 46.70 & 53.30 & 115.00 & 9.00 & 46.70 & .00 & 1986.15 & 927.53 & 763.50 \\
\hline 629.00 & $52 \cdot 40$ & 26.30 & 4.00 & $1 \cdot v 0$ & 53.60 & $46 \cdot 40$ & 53.60 & 150.00 & 9.00 & 46.40 & .00 & 2040.46 & 946.77 & 763.00 \\
\hline 629.50 & .49 .40 & 26.60 & 3.60 & $.9 c$ & 56.20 & 43.80 & $56 . ? 0$ & 150.00 & 9.00 & 43.80 & .00 & 2063.73 & 903.92 & 762.50 \\
\hline 630.00 & 48.90 & 26.80 & $3 \cdot 30$ & .90 & $E 7.70$ & $42 \cdot 30$ & 57.70 & 162.00 & 8.00 & 42.30 & .00 & 2079.25 & 879.52 & 762.00 \\
\hline 630.50 & 49.70 & 26.90 & 3.20 & $1 \cdot 20$ & 58.90 & 41.10 & 58.90 & 162.00 & 8.00 & 41.10 & .00 & 2087.01 & 857.16 & 761.50 \\
\hline 631.00 & 50.00 & 26.90 & 3.00 & .90 & 60.60 & 39.40 & 60.60 & 167.00 & 10.00 & $39.4 \mathrm{c}$ & .00 & 2087.01 & 822.28 & 761.00 \\
\hline 631.50 & 50.10 & $26.8 \mathrm{C}$ & 2.80 & .90 & $t 3 \cdot 10$ & 36.90 & 63.10 & 167.00 & 10.00 & $36 \cdot 9.0$ & .00 & 2079.25 & 767.24 & 760.50 \\
\hline 632.00 & 51.90 & 26.60 & 2.70 & .90 & 64.80 & 35.20 & 64.80 & 158.00 & 13.00 & $35 . ? 0$ & .00 & 2063.73 & 726.43 & 760.00 \\
\hline 632.50 & $54 \cdot 10$ & 26.10 & 2.70 & .90 & 65.60 & 34.40 & 65.60 & 158.00 & 13.00 & 34.40 & .00 & 2024.94 & 696.58 & 759.50 \\
\hline 633.00 & 56.40 & 25.60 & 2.80 & $.7 \mathrm{c}$ & $\in 5.70$ & 34.30 & 65.70 & 134.00 & 20.00 & 34.30 & .00 & 1986.15 & 681.25 & 759.00 \\
\hline 633.50 & $58 \cdot 10$ & $24.8 \mathrm{C}$ & 3.10 & $.0 \mathrm{C}$ & 65.50 & 34.50 & 65.50 & 134.00 & 20.00 & 34.50 & .00 & 1924.08 & 663.81 & 758.50 \\
\hline 634.00 & $59=90$ & 24.00 & 3.40 & $.0 \mathrm{C}$ & +3.90 & 36.10 & 63.90 & 101.00 & 26.00 & 36.10 & .00 & 1862.02 & 672.19 & 758.00 \\
\hline 634.50 & 63.00 & 23.50 & 3.90 & .10 & $\neq 1.10$ & 38.90 & 61.10 & 101.00 & 26.00 & 38.90 & .00 & 1823.22 & 709.23 & 757.50 \\
\hline $635.0 \mathrm{C}$ & 65.60 & 23.80 & 4.20 & .70 & $58 \cdot 30$ & 41.70 & 58.30 & 97.00 & 33.00 & 41.70 & .00 & 1846.50 & 769.99 & 757.00 \\
\hline 635.50 & 66.10 & 24.50 & 4.50 & .70 & 57.20 & 42.80 & 55.00 & 97.00 & 33.00 & 45.00 & 2.20 & $1900 \cdot 81$ & $855 \cdot 36$ & 756.50 \\
\hline 636.00 & 64.50 & 25.00 & 4.70 & $.0 \mathrm{C}$ & 58.60 & 41.40 & 52.40 & 121.00 & 32.00 & 47.60 & 6.20 & 1939.60 & 923.25 & 756.00 \\
\hline 636.50 & $62 \cdot 30$ & 25.10 & 5.00 & .00 & 56.70 & 43.30 & 50.40 & 121.00 & 32.00 & 49.60 & 6.30 & 1947.36 & 965.89 & 755.50 \\
\hline 637.00 & 60.90 & 24.90 & $5 \cdot 30$ & .70 & 55.50 & 44.50 & 49.50 & 118.00 & 27.00 & $50 \cdot 50$ & 6.00 & 1931.84 & 975.58 & 755.00 \\
\hline 637.50 & 61.80 & 24.30 & 5.60 & - $\dot{y c}$ & 50.50 & 49.50 & 49.60 & 118.00 & 27.00 & 50.40 & .90 & 1895.29 & 950.19 & 754.50 \\
\hline 638.00 & $61 \cdot 10$ & 23.00 & 5.60 & $1 \cdot 30$ & $E 2.50$ & 47.40 & 52.60 & 83.00 & 29.00 & 47.40 & .00 & 1784.43 & 845.82 & 754.00 \\
\hline 638.50 & 60.80 & 21.70 & $5 \cdot 40$ & i. & 56.60 & 43.40 & 56.60 & 83.50 & 29.00 & 43.40 & .00 & 1683.57 & 730.67 & 753.50 \\
\hline 639.00 & 62.80 & 21.10 & $5 \cdot 30$ & $1 \cdot j i$ & $\equiv 9.20$ & 40.80 & 59.20 & 57.00 & 31.00 & 40.80 &.$=0$ & 1637.02 & 667.90 & 753.00 \\
\hline 639.50 & $64 \cdot 30$ & 20.90 & 5.20 & $1 \cdot 35$ & $t 0.30$ & 39.70 & 60.30 & 57.30 & 31.00 & 39.70 & .20 & 1621.50 & 643.74 & 752.50 \\
\hline 640.00 & 65.30 & 22.00 & 5.10 & 1.30 & 57.70 & 42.30 & 57.70 & $68 \cdot .00$ & 34.00 & $42.3 n$ & .30 & 1706.85 & 722.00 & 752.00 \\
\hline 640.50 & 65.20 & 22.50 & 4.90 & $1 \cdot 3 c$ & $=7.50$ & 42.50 & 57.50 & 68.00 & 34.00 & 42.50 & .00 & 1745.64 & 741.90 & 751.50 \\
\hline 641.00 & 62.90 & 23.40 & 4.30 & 1.20 & 55.50 & 44.50 & 55.50 & $90 \cdot 00$ & 31.00 & 44.50 & .100 & 1815.46 & 807.88 & 751.00 \\
\hline 641.50 & 61.80 & 26.60 & 4.70 & $1 \cdot \mathrm{dC}$ & 48.90 & $51 \cdot 10$ & 48.90 & 90.00 & 31.00 & 51.10 & .00 & 2063.73 & 1054.57 & 750.50 \\
\hline 642.00 & 62.30 & 28.50 & 4.50 & 1.30 & 46.00 & 54.00 & 46.00 & 213.00 & 29.00 & 54.00 & .00 & 2211.14 & 1194.02 & 750.00 \\
\hline 642.50 & 57.70 & 28.50 & 4.50 & i.jo & 46.60 & 53.40 & 46.60 & 213.00 & 29.00 & 53.40 & .00 & $2211 \cdot 14$ & 1180.75 & 749.50 \\
\hline 643.00 & 53.10 & 28.40 & 4.30 & $1 \cdot 20$ & 47.50 & $52 \cdot 50$ & 47.50 & $212 \cdot 00$ & 19.00 & 52.50 & .00 & $2203 \cdot 38$ & 1156.78 & 749.00 \\
\hline 643.50 & 55.70 & 27.30 & 4.20 & 1.10 & 50.50 & 49.50 & $50 \cdot 50$ & 212.00 & 19.00 & -9.50 & .00 & 2118.04 & $10+8.43$ & 748.50 \\
\hline 644.00 & $58 \cdot 50$ & 25.60 & 4.00 & 1.20. & 55.10 & 44.90 & 55.10 & 133.00 & 25.00 & 44.90 & .00 & 1986.15 & 891.78 & 748.00 \\
\hline 644.50 & $62 \cdot 50$ & 23.70 & 3.90 & $1.20^{\circ}$ & 60.50 & 39.50 & 60.50 & 133.00 & 25.00 & 39.50 & .00 & 1838.74 & 726.30 & 747.50 \\
\hline 645.00 & 69.30 & $\geqslant 1.30$ & 3.80 & 1.20 & 68.70 & 31.30 & 68.70 & 59.00 & 38.00 & 31.30 & .00 & $1652 \cdot 54$ & $5: 7.24$ & 747.00 \\
\hline $\begin{array}{l}\text { A.AVE. } \\
\text { G.MEAN }\end{array}$ & $\begin{array}{l}59 \cdot .20 \\
59.30\end{array}$ & $\begin{array}{l}23.99 \\
23.79\end{array}$ & $\begin{array}{l}4.34 \\
4.25\end{array}$ & $\begin{array}{l}1.08 \\
1.03\end{array}$ & $\begin{array}{l}59.07 \\
58.64\end{array}$ & $\begin{array}{l}40.93 \\
40.16\end{array}$ & $\begin{array}{l}58.56 \\
58.08\end{array}$ & $\begin{array}{r}111.07 \\
93.78\end{array}$ & $\begin{array}{l}23.62 \\
21.27\end{array}$ & $\begin{array}{l}21.44 \\
20.61\end{array}$ & .51 & $\begin{array}{l}1860.90 \\
1844.95\end{array}$ & $\begin{array}{l}783.51 \\
749.33\end{array}$ & \\
\hline
\end{tabular}

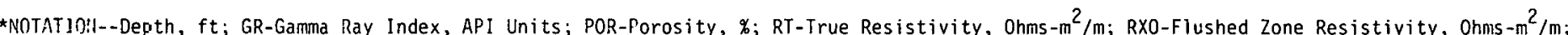

SXO-Flushed Zene Saturation, \%; ROS-Residual 0 il Saturation, \%; SH-Hater Saturation, \%; PER-Permeability Index; Clay-Clay Content, \%; SO-Oil

Saturation, \%: MO-Movable 0il, \%; PHV-Pore Volure, bbl/ac-ft; HPV-Hydrocarbon Pore Volume, bbl/ac-ft; ELSE-Elevation From Sea Level, ft 
TABLE C25

WELL LOG DETERMINEO PROPERTIES

VS OEPTH FOR WELL HAP- 114

WELL LOCATION: $2366 \mathrm{FWL} 1330 \mathrm{FSL} 2125558$

KELLY BUSHING ELEVATION FROM SEA LEVEL $=1393$

\begin{tabular}{|c|c|c|c|c|c|c|c|c|c|c|c|c|c|c|}
\hline DEPTH * & GR & $P Q R$ & $R T$ & $R \times U$ & $\leqslant \times 0$ & ROS & Sw & PER & CLAY & so & me & PHV & $H^{P V}$ & LSE \\
\hline $\begin{array}{l}641.50 \\
642.00\end{array}$ & & $\begin{array}{l}11.70 \\
20.80\end{array}$ & R.80 & $\begin{array}{r}8.40 \\
11.70\end{array}$ & 86.90 & $13 \cdot 10$ & 86.90 & $3 \cdot 00$ & 30.00 & $13 \cdot 10$ &.$c 0$ & 907.73 & 118.91 & 751.50 \\
\hline $\begin{array}{l}642.00 \\
6.2 .50\end{array}$ & $\begin{array}{l}94.50 \\
98.80\end{array}$ & $\begin{array}{l}20.80 \\
19.40\end{array}$ & $\begin{array}{r}9.90 \\
11.00\end{array}$ & $\begin{array}{l}11.70 \\
12.50\end{array}$ & $\begin{array}{l}51.80 \\
53.90\end{array}$ & 48.20 & $44 \cdot 10$ & 31.00 & 16.00 & 55.90 & 7.70 & 1613.75 & 08 & 751.00 \\
\hline 643.00 & 97.70 & $\begin{array}{l}19.40 \\
19.90\end{array}$ & $\begin{array}{l}11.00 \\
12.10\end{array}$ & $\begin{array}{l}12.50 \\
12.50\end{array}$ & $\begin{array}{l}53 \cdot 90 \\
52 \cdot 20\end{array}$ & $\begin{array}{r}46.10 \\
47.80\end{array}$ & $\begin{array}{l}45.00 \\
41.70\end{array}$ & 31.00 & 16.00 & 55.00 & 8.90 & $1505 \cdot 13$ & 827,82 & 750.50 \\
\hline 643.50 & 93.90 & 21.00 & 11.30 & 12.40 & 49.60 & 50.40 & $\begin{array}{l}41.70 \\
40.00\end{array}$ & $\begin{array}{l}44.00 \\
44.00\end{array}$ & $\begin{array}{l}15.00 \\
15.00\end{array}$ & $58 \cdot 30$ & $\begin{array}{r}10.50 \\
9.60\end{array}$ & $\begin{array}{l}1543.92 \\
1629.26\end{array}$ & 900.11 & 750.00 \\
\hline 644.00 & $90 \cdot 20$ & $21.4 \mathrm{C}$ & $11 \cdot 20$ & 11.60 & 50.00 & 50.00 & 40.10 & 53.00 & $\begin{array}{r}15.00 \\
9.00\end{array}$ & $\begin{array}{l}60.00 \\
59.90\end{array}$ & $\begin{array}{l}9.60 \\
9.90\end{array}$ & $\begin{array}{l}1629.26 \\
1660.30\end{array}$ & $\begin{array}{l}977.56 \\
994.52\end{array}$ & 749.50 \\
\hline 644.50 & $85 \cdot 80$ & 21.80 & $10 \cdot 20$ & 9.60 & 54.00 & 46.00 & 41.20 & 53.00 & 9.00 & 58.80 & 12.80 & 1691.33 & $\begin{array}{l}994.52 \\
994.50\end{array}$ & $\begin{array}{l}749.00 \\
748.50\end{array}$ \\
\hline 645.00 & $82 \cdot 50$ & 21.50 & $9 \cdot 30$ & $8.1 \mathrm{C}$ & $59 \cdot 70$ & 40.30 & 43.70 & $47 \cdot 00$ & 8.00 & 56.30 & 16.00 & 1668.06 & $\begin{array}{l}994.50 \\
939.11\end{array}$ & $\begin{array}{l}748.50 \\
748.00\end{array}$ \\
\hline 645.50 & 80.80 & $21.3 c$ & 8.40 & 6.00 & 66.80 & 33.20 & 46.40 & 47.00 & B.00 & 53.60 & 20.40 & 1652.54 & $\begin{array}{l}939.11 \\
885.76\end{array}$ & 748.00 \\
\hline 646.00 & 81.00 & 21.90 & 7.60 & $6 \cdot 30$ & $\epsilon 6.60$ & 33.40 & 47.70 & 71.00 & 6.00 & $52 \cdot 30$ & 18.90 & 1699.09 & $885 \cdot 76$ & 747.50 \\
\hline $646 \cdot 50$ & 81.00 & $23 \cdot 40$ & 6.70 & 6.00 & $63 \cdot 10$ & 36.90 & 47.10 & 71.00 & 6.00 & 52.96 & 16.00 & 181 & 888.62 & 747.00 \\
\hline $6+7.00$ & 80.90 & $25.1 \mathrm{C}$ & 6.10 & $6 \cdot 40$ & $58 \cdot 70$ & $41 \cdot 30$ & 46.10 & 121.00 & 6.00 & 53.90 & 10.00 & $1815 \cdot 46$ & $960 \cdot 38$ & 746.50 \\
\hline & 80.70 & $26.4 \mathrm{c}$ & 5.90 & 6.40 & 53.90 & 46.10 & 44.20 & & & 53.90 & $12 \cdot 60$ & $1947 \cdot 36$ & 1049.63 & 746.00 \\
\hline 648.00 & 78.20 & 25.90 & 5.70 & 6.70 & 53.60 & 46.40 & 45.80 & 107.00 & $\begin{array}{l}6.00 \\
4.00\end{array}$ & $\begin{array}{l}55.80 \\
54.20\end{array}$ & 9.70 & $2048 \cdot 22$ & 1142.90 & .50 \\
\hline $648 \cdot 50$ & $75 \cdot 90$ & 25.70 & $5 \cdot 50$ & 7.10 & 52.80 & 47.20 & .7 .00 & 107.00 & $\begin{array}{l}4.00 \\
4.00\end{array}$ & 54.20 & 7.80 & 2009.42 & 11 & 745.00 \\
\hline 649.00 & 76.20 & 25.80 & 5.50 & 7.40 & $51 \cdot 30$ & 48.70 & 46.70 & 113.00 & $\begin{array}{l}4.00 \\
5.00\end{array}$ & $53.0 n$ & $5 \cdot 80$ & 1993.91 & 1056.77 & 744.50 \\
\hline 649.50 & 77.20 & 26.00 & 6.20 & 7.70 & 50.00 & 50.00 & 43. & 1. 130 & 5.00 & $53 \cdot 30$ & $4 \cdot 60$ & 2001.67 & 1066.89 & 744,00 \\
\hline 650.00 & 78.20 & 26.20 & 6.80 & 7.80 & 49.20 & 50. & & $113 \cdot 00$ & 5.00 & $56 \cdot 20$ & 6.20 & 2017.18 & 1133.66 & 743.50 \\
\hline $650 \cdot 50$ & 79.20 & 26.60 & 7.40 & 7.90 & 48.40 & 51.60 & $\begin{array}{l}41.60 \\
39.22\end{array}$ & .00 & 7.00 & 58.40 & 7.60 & 2032.70 & 1187.10 & 743.00 \\
\hline 651.00 & 79.20 & 25.70 & 8.00 & $8.7 c$ & 47.60 & 52.40 & $\begin{array}{l}39.22 \\
39.10\end{array}$ & 124.00 & 7.00 & $60 \cdot 20$ & 9.20 & 2063.73 & 1254.75 & 742.50 \\
\hline 651.50 & 78.60 & 24.70 & $8 \cdot 30$ & $9.5 \mathrm{c}$ & 47.50 & 52 & & $90 \cdot 00$ & 6.00 & $60 \cdot 90$ & 8.50 & 1993.91 & 1214.29 & 742.00 \\
\hline $652 \cdot 00$ & 77.90 & 24.40 & 8.50 & $9.8 \mathrm{C}$ & 47.40 & 52.60 & $\begin{array}{l}40.100 \\
40.10\end{array}$ & 90.00 & 6.00 & 60.00 & 7.50 & - 32 & 1149.79 & 741.50 \\
\hline 652.50 & 81.90 & 24.60 & 8.60 & $9.6 \mathrm{C}$ & 47.50 & $52 \cdot 50$ & $\begin{array}{l}40.10 \\
39.40\end{array}$ & $\begin{array}{l}88.00 \\
88.00\end{array}$ & 7.00 & $59 \cdot 90$ & $7 \cdot 30$ & 1893.05 & 1133.94 & 741.00 \\
\hline 653.00 & 86.30 & 25.20 & $8 \cdot 30$ & 9.50 & 46.60 & 53.40 & 38.00 & $\begin{array}{r}88.00 \\
107.00\end{array}$ & 7.00 & 60.60 & $8 \cdot 10$ & 1908.57 & 1156.59 & 740.50 \\
\hline 653.50 & 87.70 & 25.70 & 8.20 & 9.80 & 44.90 & $55 \cdot 10$ & $\begin{array}{l}38.00 \\
38.60\end{array}$ & $\begin{array}{l}107.00 \\
107.00\end{array}$ & $15 \cdot 00$ & $62 \cdot 00$ & 8.60 & $1955 \cdot 12$ & 1212.17 & 740.00 \\
\hline 654.00 & 85.90 & $25.4 \mathrm{C}$ & 7.60 & $10 \cdot 10$ & 44.50 & 55.40 & 40.30 & $\begin{array}{r}107.00 \\
94.00\end{array}$ & & 61.40 & & • 91 & $1224 \cdot 26$ & 739.50 \\
\hline 654.50 & 83.60 & 24.90 & 7.10 & $9 \cdot 50$ & $47 \cdot 10$ & 52.90 & 42.80 & $\begin{array}{l}94 \cdot 00 \\
94.00\end{array}$ & 11.00 & 59.70 & $4 \cdot 30$ & 1970.63 & 1176.47 & 739.00 \\
\hline 655.00 & 84.00 & 24.70 & 6.50 & 8.00 & 50.10 & 49. & 45.20 & & 11.00 & $57 \cdot 20$ & $4 \cdot 30$ & 1931.84 & 1105.01 & 738.50 \\
\hline 655.50 & $85 \cdot 10$ & 24.40 & 5.90 & 7.70 & $=3$. & 46 & 45.20 & 86.00 & 13.00 & & 4.90 & 1916.32 & 1050.14 & 738.00 \\
\hline 656.00 & 86.20 & 24.00 & $5 \cdot 30$ & 6.70 & $58 \cdot 30$ & 41.70 & $\begin{array}{l}47.70 \\
51.50\end{array}$ & $86 \cdot 00$ & 13.00 & $52 \cdot 30$ & 5.80 & 1893.05 & 990.06 & 737.50 \\
\hline 656.50 & 87.30 & 23.90 & 5.00 & $6 \cdot c c$ & $t 2 \cdot 20$ & 37.80 & $\begin{array}{l}51.50 \\
53.50\end{array}$ & 78.00 & $13 \cdot 00$ & $48.5 c$ & 6.80 & 1862.02 & 903.08 & 737.00 \\
\hline 657.00 & 88.40 & $23.7 \mathrm{C}$ & 4.50 & 5 . & $\epsilon 3.80$ & 36.20 & $\begin{array}{l}53.50 \\
55.30\end{array}$ & 78.00 & 13.00 & $46.5 n$ & 8.70 & 1854.26 & 862.23 & 736.50 \\
\hline 657.50 & 89.40 & 23.70 & $4 \cdot 30$ & 6.00 & $\epsilon 2.50$ & & $55 \cdot 30$ & 75.00 & & $44.2 \mathrm{C}$ & 8.00 & $1838 \cdot 74$ & 812.72 & 736.00 \\
\hline 658.00 & 89.40 & 24.10 & 4.00 & 6.20 & 60.40 & $\begin{array}{l}37.50 \\
39.60\end{array}$ & $\begin{array}{l}58 \cdot 10 \\
59.20\end{array}$ & $75 \cdot 00$ & $12 \cdot 00$ & $41 \cdot 90$ & 4.40 & 1838.74 & 770.43 & 735.50 \\
\hline 658.50 & 89.40 & 24.00 & $3 \cdot 70$ & 6.30 & $E 1.40$ & 38.600 & $\begin{array}{l}59.20 \\
61.40\end{array}$ & $\begin{array}{l}80.00 \\
80.00\end{array}$ & 9.00 & $40.8 \mathrm{C}$ & 1.20 & 1869.77 & 762.87 & 735.00 \\
\hline 659.00 & $92 \cdot 80$ & 24.00 & 3.50 & 5.90 & $\in 3.80$ & $36 . \geq 0$ & $\begin{array}{l}61.40 \\
63.80\end{array}$ & $80 \cdot 00$ & 9.00 & 38.60 & .00 & 1862.02 & 718.74 & 734.50 \\
\hline 659.50 & 96.30 & 23.50 & $3 \cdot 10$ & $5 \cdot 50$ & 68.80 & 31.20 & $\begin{array}{l}63.80 \\
68.80\end{array}$ & & & & .00 & 1862.02 & 674.05 & 734.00 \\
\hline 660.00 & 101.60 & 21.10 & $3 \cdot 0$ & $5 \cdot$ & 78.90 & 21.20 & 78.80 & $\begin{array}{l}72.00 \\
37.00\end{array}$ & 9.00 & $31 \cdot 20$ & .00 & 1823.22 & 568.85 & 733.50 \\
\hline $660 \cdot 50$ & 102.60 & 20.20 & 2.90 & 4.00 & 84.20 & 15.80 & 84.20 & $\begin{array}{l}37.00 \\
37.00\end{array}$ & $\begin{array}{l}9.00 \\
9.00\end{array}$ & $\begin{array}{l}21.20 \\
15.80\end{array}$ & .00 & 1637.02 & 347.05 & \\
\hline 661.00 & 101.00 & $20.0 \mathrm{C}$ & 2.70 & $4 \cdot 20$ & 87.10 & 12.90 & 87.10 & $21 \cdot 50$ & 23.00 & $\begin{array}{l}12 \cdot \times 0 \\
12 \cdot 90\end{array}$ & $\begin{array}{l}.00 \\
.00\end{array}$ & $\begin{array}{l}1567.20 \\
1551.68\end{array}$ & & \\
\hline AVE. & 86.58 & s & $6 \cdot !$ & 7.5 & 57.5 & 42.48 & & & & & & & & \\
\hline & $1<6$ & $23 \cdot 14$ & 6.39 & & 56.57 & 40.35 & $\begin{array}{l}50.29 \\
48.83\end{array}$ & $\begin{array}{l}76.50 \\
66.62\end{array}$ & $\begin{array}{r}10.32 \\
9.30\end{array}$ & $\begin{array}{l}49.71 \\
46.72\end{array}$ & 22 & $\begin{array}{l}1811.00 \\
1795.23\end{array}$ & 838.7 & \\
\hline
\end{tabular}

*NOTATION--Depth. *t; GR-Gamma Ray Index, API Units; POR-Porosity, $\%$; RT-True Resistivity, Ohms- $\mathrm{m}^{2} / \mathrm{m} ;$ RX0-Flushed Zone Resistivity, Ohms- $\mathrm{m}^{2} / \mathrm{m}$;

Saturation, \%; MI-Hovable 0il, \%; PHV-Pore Volume, bbl/ac-ft; HPV-Hydrocarbon Pore Vo: Ume, bbl/ac-ft; ELSE-Elevation From Sea Level, ft 
TABLE C26

WELL LOG DETERHINED PROPERTIES

VS DEPTH FOR WELL MP-115

WELL LOCATION: 2112 FWL $1 \cong 20$ FSL $212555 \mathrm{E}$

KELLY BUSHING ELEVATION FROM SEA LEVEL $=1391$

\begin{tabular}{|c|c|c|c|c|c|c|c|c|c|c|c|c|c|c|}
\hline PTH* & GR & $P G R$ & RT & $R \times C$ & $5 \times 0$ & ReS & SW & PER & CLAY & se & me & PHV & HPV & ELSE \\
\hline $\begin{array}{l}647.50 \\
648.00\end{array}$ & $\begin{array}{l}91.00 \\
86.60\end{array}$ & $\begin{array}{l}15.00 \\
17.60\end{array}$ & $\begin{array}{l}4.50 \\
4.60\end{array}$ & $\begin{array}{l}1.30 \\
1.4 c\end{array}$ & $\begin{array}{l}52.60 \\
76.90\end{array}$ & $\begin{array}{r}7.40 \\
23.10\end{array}$ & $\begin{array}{l}92.60 \\
76.90\end{array}$ & $\begin{array}{r}1.00 \\
20.00\end{array}$ & $\begin{array}{l}37.00 \\
32.00\end{array}$ & $\begin{array}{r}7.40 \\
23.10\end{array}$ & $\begin{array}{l}.00 \\
.00\end{array}$ & $\begin{array}{l}1163.76 \\
1365.48\end{array}$ & $\begin{array}{r}86.12 \\
315.43\end{array}$ & $\begin{array}{l}743.50 \\
743.00\end{array}$ \\
\hline $\begin{array}{l}648.00 \\
648.50\end{array}$ & $\begin{array}{l}86.60 \\
82.30\end{array}$ & $\begin{array}{l}17.60 \\
19.8 \mathrm{C}\end{array}$ & $\begin{array}{l}4.60 \\
4.50\end{array}$ & $\begin{array}{l}1.46 \\
1.70\end{array}$ & $\begin{array}{l}76.90 \\
77.90\end{array}$ & $\begin{array}{l}23.10 \\
22.10\end{array}$ & $\begin{array}{l}76.90 \\
66.50\end{array}$ & $\begin{array}{l}20.00 \\
20.00\end{array}$ & $\begin{array}{l}32.00 \\
32.00\end{array}$ & $\begin{array}{l}23.10 \\
33.50\end{array}$ & 11.40 & $\begin{array}{l}1355 \cdot 48 \\
1536.16\end{array}$ & $\begin{array}{l}315.43 \\
514.61\end{array}$ & 742.50 \\
\hline 649.00 & 77.80 & 20.90 & 4.90 & $1.5 \mathrm{C}$ & 70.50 & 29.50 & 61.90 & 44.00 & 30.00 & 38.10 & 8.60 & 1621.50 & 617.79 & 742.00 \\
\hline 649.50 & $76 \cdot 10$ & 22.40 & 5.00 & 1.80 & $\epsilon 5.40$ & 34.60 & 56.90 & 44.00 & 30.00 & 43.10 & 8.50 & 1737.88 & 749.03 & 741.50 \\
\hline 650.00 & 74.40 & 23.60 & 5.20 & 1.80 & 62.80 & 37.20 & $5 ? .90$ & 74.00 & 28.00 & 47.10 & 9.90 & 1830.98 & 862.39 & 741.00 \\
\hline 650.50 & 72.70 & 24.40 & $5 . ? 0$ & 1.00 & $t 3.10$ & 36.90 & 50.30 & 74.00 & 28.00 & $49 . ? 0$ & 12.30 & 1893.05 & 931.38 & 740.50 \\
\hline 651.00 & 72.50 & 24.80 & 5.30 & $1 \cdot 3 c$ & $\epsilon 3.90$ & 36.10 & $\begin{array}{l}49.30 \\
0\end{array}$ & 92.00 & 25.20 & 50.80 & 14.10 & 1924.08 & 965.89 & 740.00 \\
\hline 651.50 & 72.70 & 26.00 & $5 \cdot 30$ & 1.50 & $=1.60$ & 38.40 & 47.20 & 92.00 & 25.00 & 52.80 & 14.40 & 2017.18 & 1065.07 & 739.50 \\
\hline 652.00 & 72.90 & 27.00 & 5.20 & $1.4 \mathrm{c}$ & 9.90 & 40.10 & 45.70 & 133.00 & 23.00 & 54.30 & 14.20 & 2094.77 & 1137.46 & 739.00 \\
\hline 652.50 & 70.90 & 27.80 & 5.20 & 1.70 & 2.90 & 47.10 & 44.40 & 133.00 & 23.00 & 55.60 & 8.50 & 2156.83 & 1199.20 & 738.50 \\
\hline 653.00 & $68 \cdot 90$ & $28.5 \mathrm{C}$ & 5.20 & $2 \cdot 00$ & 7.50 & $52 \cdot 50$ & 43.40 & 168.50 & 17.00 & 56.60 & 4.10 & 2211.14 & 1251.51 & 738.00 \\
\hline 653.50 & $68 \cdot 20$ & 28.40 & 5.10 & 2.40 & 4.50 & 55.50 & 43.70 & 168 & 17.00 & $56.3 r$ & .30 & 3.38 & $12+0.50$ & \\
\hline 654.00 & $69+90$ & $28.1 \mathrm{C}$ & 5.30 & 2.40 & $4 \cdot 30$ & 55.70 & 43.60 & 158.00 & 19.00 & 56.40 & .70 & 2180.11 & 1229.58 & 737.00 \\
\hline 654.50 & 70.50 & 27.60 & 5.50 & $2.0 \mathrm{C}$ & 16.90 & 53.10 & 43.40 & 158.00 & 19.00 & 56.60 & 3.50 & 2141.32 & 1211.99 & 736.50 \\
\hline & 70.50 & 27.00 & 5.80 & 2.20 & 8.10 & 51. & 43.40 & 134 & & & 4. & 14.77 & 1135.64 & 736.00 \\
\hline 655.50 & 70.40 & 26.60 & 5.60 & $2 \cdot 30$ & 8.60 & 51.40 & 44.70 & 134.00 & 20.00 & 55.30 & 3.90 & $\$ 3.73$ & $11+1 \cdot 24$ & .50 \\
\hline 656.00 & 70.20 & 26.60 & 5.50 & $2 \cdot 3 \mathrm{c}$ & 8.70 & $51 \cdot 30$ & 45.30 & 126.00 & 19.00 & 54.70 & 3.40 & 2063.73 & 1128.86 & 735.00 \\
\hline 656.50 & 70.00 & $26.7 \mathrm{C}$ & 5.30 & 2.20 & 8.80 & 51.20 & 45.80 & 126.00 & 19.00 & 54.20 & 3.00 & 2071.49 & 1122.75 & 734.50 \\
\hline & 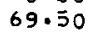 & 26 & $5 \cdot 10$ & 2.20 & 8. & & 46.40 & 129 & & 53 & 2 . & & & .00 \\
\hline 657.50 & 69.30 & $26.9 \mathrm{C}$ & 4.90 & $2 \cdot 3 \mathrm{C}$ & 48.10 & 51.90 & 47.60 & 129.00 & 18.00 & 52.40 & .50 & 2087.01 & 1093.59 & 733.50 \\
\hline 658.00 & 68.50 & 26.90 & 4.60 & 2.30 & 48.80 & 51.20 & 48.80 & 131.00 & 18.00 & $51 . ? ?$ & .00 & 2087.01 & 1068.55 & 733.00 \\
\hline 658.50 & 68.50 & 26.90 & 4.40 & 2.10 & 50.10 & 49.90 & 50.10 & $131 \cdot c 0$ & 18.00 & 49.90 & .00 & 2087.01 & $10<1.42$ & 732.50 \\
\hline 659.00 & 69.30 & 26.00 & 4.10 & 2.00 & 52.40 & 47.60 & 52.40 & 125.00 & 21.00 & 47.50 & .00 & 2063.73 & 34 & .00 \\
\hline 659.50 & 70.30 & 26.20 & 1.80 & 1.80 & $=5.40$ & 44.60 & 55.40 & 125.00 & 21 & 44.60 & .00 & 2.70 & 58 & 731.50 \\
\hline 660.00 & $71 \cdot 30$ & 25.80 & 1.70 & 1.70 & 58.20 & 41.80 & 57.10 & 109 & & 42.90 & 1.10 & 1.67 & 71 & 731.00 \\
\hline & 7 & & 0 & 1.00 & $\in 0$ & 39 & & $\begin{array}{l}109 \\
\end{array}$ & & 41.20 & 1.30 & 0.63 & & . 50 \\
\hline 661.00 & $75 \cdot 30$ & 25.00 & 3.50 & 1.60 & $\epsilon 1.10$ & 38.90 & 60.70 & 96.00 & 25.00 & 39.30 & .40 & 1939.60 & 26 & 730.00 \\
\hline $661 \cdot 50$ & 77.80 & $25.1 \mathrm{C}$ & 1.40 & 1.70 & $t 1.50$ & 38.50 & 61.50 & & & & .0 & 7.36 & & .50 \\
\hline 662.00 & 79.00 & 25.40 & $3 \cdot 30$ & $1.7 \mathrm{C}$ & ti. & 38 . & 61.50 & 103 & 29 & 38 & .00 & 1970.63 & 75 & 729.00 \\
\hline & $81 . \equiv 0$ & $25.8 \mathrm{C}$ & 3.20 & 1.00 & & 38. & 61.50 & 103.00 & 29 & 38 & .0 & 1.67 & & 728.50 \\
\hline 663.00 & $81 . \varepsilon_{0}$ & 26.10 & 3.10 & 1.50 & 61.70 & $38 \cdot 30$ & 61.70 & 115.00 & 30.00 & 38.30 & .0 & 2024.94 & 775.55 & 728.00 \\
\hline 663.50 & 82.40 & 26. & 3.00 & $1 \cdot b c$ & 62.50 & 37.50 & 62.50 & 115 & 30 & 37.50 & .00 & 2032.70 & & 727.50 \\
\hline 664.00 & $83 \cdot 50$ & 26.10 & 2.90 & 1.50 & $E 3.60$ & 36.40 & 63.60 & 115.00 & 30.00 & 36.40 & .05 & 2024.94 & 73 & 727.00 \\
\hline 664.50 & 84.30 & 26.40 & 2.90 & 1.40 & 62.70 & 37.30 & 62.70 & 115.00 & 30.00 & 37.30 & .00 & 2048.22 & 76 & 726.50 \\
\hline 665. & $84 \cdot 30$ & 27 & & 1. & 61 & & & & & & .00 & & & 726.00 \\
\hline 665.50 & $84 \cdot 30$ & 27.60 & 2.90 & 1.30 & 61.20 & 38.80 & 59.80 & 134.00 & 26.00 & 4.0 .20 & 1.40 & 2141.32 & 860 & 725.50 \\
\hline 666.00 & 89.40 & 22.90 & 2.90 & 1.40 & 73.20 & 26.80 & 73.20 & 64.00 & 38.00 & 26.80 & .00 & 1776.6 & & \\
\hline 666.50 & 94.50 & 18.30 & 2.90 & 1.00 & 52.90 & 7.10 & 92.90 & 64.00 & 38.00 & 7.10 & .00 & 1419.7 & 100.80 & 724.50 \\
\hline & & & $\cdot 3$ & & & & 56 & & & & 3.41 & 19 & & \\
\hline & & & 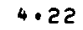 & & & & 55.23 & & & 67 & & , & & \\
\hline
\end{tabular}

*NOTATIDH--Depth, ft; GR-Gamma Ray Index, API Units; POR-Porosity, \%; RT-True Resistivity, Ohms-m²/m; RXo-Flushed Zone Resistivity, Ohms-m²/m; SXO-Flushed Zo 1 Saturation, \%; ROS-Residual 0il Saturation, \%; SW-Water Saturation, \%; PER-Permeability Index; Clay-Clay Content, \%; SO-0il Saturation, \&; M0-ilovable 0il, \%; PHV-Pore Volume, bbl/ac-ft; HPV-Hydrocarbon Pore Volume, bbl/ac-ft; ELSE-Elevation From Seá Level, ft 
TABLE C27

WELL LOG DETERMINED PROPERTIES

VS DEPTH FOR WELL MP-116

WELL LOCATION: 2112 FWL 1 C56 FSL 21255 5E

KELLY BUSHING ELEVATION FROM SEA LEVEL $=1391$

\begin{tabular}{|c|c|c|c|c|c|c|c|c|c|c|c|c|c|c|}
\hline DEPTH * & GR & $P \theta P$ & FT & $R \times U$ & $5 \times 0$ & ROS & SW & PER & CLAY & so & mo & PHV & HPV & ELSE \\
\hline $\begin{array}{l}647.50 \\
648.00\end{array}$ & $\begin{array}{l}63.70 \\
60.00\end{array}$ & $\begin{array}{l}14.90 \\
19.60\end{array}$ & $\begin{array}{l}4.70 \\
4.50\end{array}$ & $\begin{array}{l}5.90 \\
5.90\end{array}$ & $51 \cdot 30$ & $\begin{array}{r}8 \cdot 70 \\
30.70\end{array}$ & $91 \cdot 30$ & $2 \cdot 00$ & $\begin{array}{l}37.00 \\
24.00\end{array}$ & $\begin{array}{r}8.70 \\
30.70\end{array}$ & .00 & $\begin{array}{r}1156.00 \\
1520.65\end{array}$ & $\begin{array}{l}100.57 \\
466.8\end{array}$ & $\begin{array}{l}743.50 \\
743.00\end{array}$ \\
\hline $\begin{array}{l}648.00 \\
648.50\end{array}$ & $\begin{array}{l}60 \cdot 00 \\
56 \cdot 30\end{array}$ & $\begin{array}{l}19.60 \\
22.70\end{array}$ & $\begin{array}{l}\because 50 \\
4.30\end{array}$ & $\begin{array}{l}5.90 \\
5.30\end{array}$ & $\begin{array}{l}69.30 \\
70.30\end{array}$ & $\begin{array}{l}30.70 \\
29.70\end{array}$ & $\begin{array}{l}69.30 \\
60.40\end{array}$ & $\begin{array}{l}33.00 \\
33.00\end{array}$ & $\begin{array}{l}24.00 \\
24.00\end{array}$ & $\begin{array}{l}30.70 \\
39.60\end{array}$ & $\begin{array}{r}.00 \\
9.90\end{array}$ & $\begin{array}{l}1520.65 \\
1761.16\end{array}$ & $\begin{array}{l}466.84 \\
697.42\end{array}$ & $\begin{array}{l}743.00 \\
742.50\end{array}$ \\
\hline 649.00 & $54 \cdot 40$ & 24.50 & 4.20 & .10 & $65 \cdot 10$ & 34.90 & 56.80 & 87.00 & 10.00 & 43.20 & 8.30 & 1900.81 & 821.15 & 742.00 \\
\hline 649.50 & 53.50 & 24.90 & 3.80 & 1.40 & $\in 5.90$ & $34 \cdot 10$ & $58 \cdot 30$ & 87.00 & 10.00 & 41.70 & 7.60 & 1931.84 & 805.58 & 741.50 \\
\hline 650.00 & 51.60 & 25.30 & 3.60 & $5 \cdot 10$ & 66.70 & $33 \cdot 30$ & 58.90 & 100.00 & 6.00 & 41.10 & 7.80 & 1962.87 & 806.74 & 741.00 \\
\hline 650.50 & $50 \cdot 40$ & 25.50 & $3 \cdot 40$ & 4.80 & 68.00 & 32.00 & 60.00 & $100 \cdot 00$ & 6.00 & 40.00 & 8.00 & 1978.39 & 791.36 & 740.50 \\
\hline 651.00 & 49.10 & 25.60 & 3.20 & 1.50 & 69.70 & $30 \cdot 30$ & 61.50 & 106.00 & 5.00 & 38.50 & 8.20 & 1986.15 & 764.67 & 740.00 \\
\hline 651.50 & $48 \cdot 90$ & 25.80 & 3.00 & $4 \cdot 30$ & 71.40 & 28.60 & 62.90 & 106.00 & 5.00 & $37 \cdot 10$ & 8.50 & 2001.67 & 742.82 & 739.50 \\
\hline 652.00 & 49.00 & 26.00 & 2.80 & $4 \cdot 30$ & 70.50 & 29.50 & 64.40 & 114.00 & 6.00 & 35.60 & $6 \cdot 10$ & 2017.18 & 718.12 & 739.00 \\
\hline $652 \cdot 50$ & $50 \cdot 50$ & 26.20 & $2 \cdot 80$ & 4.30 & $=9.80$ & $30 \cdot 20$ & 64.90 & 114.00 & 6.00 & $35 \cdot 10$ & 4.90 & 2032.70 & 713.48 & 738.50 \\
\hline 653.00 & $51 \cdot 90$ & $26 \cdot 30$ & 2.80 & .30 & $\in 9.30$ & 30.70 & 64.60 & 119.00 & 8.00 & 35.40 & 4.70 & 2040.46 & $722 \cdot 32$ & 738.00 \\
\hline 653.50 & $53 \cdot 30$ & 26.50 & 2.70 & .40 & 68.60 & 31.40 & 64.30 & 119.00 & 8.00 & 35.70 & 4.30 & 2055.97 & 733.98 & 737.50 \\
\hline 654.00 & 54.60 & 26.70 & $2 \cdot 70$ & .40 & 67.90 & 32 & 63.80 & 12 & 9.00 & 36.20 & 4.10 & 49 & $7+9.88$ & 37.00 \\
\hline 654.50 & 56.00 & 27.00 & 2.70 & . & 65.70 & $34 \cdot 30$ & 63.30 & 127.00 & 9.00 & 36.70 & 2.40 & 2094.77 & 768.78 & 736.50 \\
\hline & 56.60 & 27.20 & 2.80 & 4.80 & $E 3.70$ & $36 \cdot 30$ & 62.10 & 138.00 & 12.00 & $37.9 n$ & 1.60 & 2110.28 & 799.80 & 736.00 \\
\hline 65 & 57 & 27 & 3 & 5.00 & 62.50 & 77 & 61.50 & 138.00 & 12.00 & 38.50 & 1.00 & 53 & & 735.50 \\
\hline 656.00 & $57 \cdot 30$ & 27.00 & 2.90 & $5 \cdot 20$ & $61 \cdot 70$ & $38 \cdot 30$ & 61.20 & 133.00 & 12.00 & $38 \cdot 8 n$ & .50 & 2094.77 & 812.77 & 735.00 \\
\hline 656.50 & 0 & 26. & 3.00 & 5.20 & $\in 1.80$ & 38.20 & 60.80 & 133.00 & 12. & $39 .>0$ & 1.00 & 9.25 & 8 & 734.50 \\
\hline & $56 \cdot 80$ & 26.90 & 3.00 & 5.10 & $\in 2.50$ & 37 & $60 \cdot 10$ & 131 & & 39.90 & 2. & 1 & & 734.00 \\
\hline & 55.70 & 26.90 & 3.10 & 5.00 & 63.20 & 36.80 & 59.40 & 131.00 & 12.00 & 40.60 & 3.80 & 2087.01 & 847.32 & 733.50 \\
\hline 658.00 & 54.60 & 26.90 & $3 \cdot 20$ & 4.50 & $\epsilon 4.00$ & 36.00 & 58.80 & 131.00 & 10.00 & $41 \cdot ? 0$ & 5.20 & 2087.01 & 859.85 & 733.00 \\
\hline 658 & 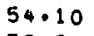 & 26. & 0 & 1.80 & $E 4 \cdot 30$ & & 58.40 & 131.00 & & 41.60 & 5 . & 51 & & 2.50 \\
\hline 659.00 & 53.90 & 27.0 & $3 \cdot 3$ & o do & $E 3.90$ & 36 & 57.90 & 132.00 & 10.00 & $42 \cdot 10$ & 6.00 & 2094.77 & 881.90 & 732.00 \\
\hline 659.50 & 53.80 & 27.00 & $3 \cdot 30$ & $5 \cdot 00$ & $E 2.60$ & 37. & 57.40 & 132.00 & 10 & $42.6 C$ & 5.20 & 2094.77 & 37 & 731.50 \\
\hline 660.00 & 53.60 & 27. & $3 \cdot 30$ & $5 \cdot 1$ & 62.40 & 37 & 57.40 & 132.00 & & 42.62 & 5.00 & 2094 & & 731.00 \\
\hline 660 & 53.80 & 26. & 3.40 & 5.00 & $E 3.10$ & $36 \cdot 90$ & 57.50 & 132.00 & & $42 \cdot 50$ & 5.60 & 2079.25 & 883 & 730.50 \\
\hline 661 & 57.00 & 26.60 & 3.40 & $5 \cdot 00$ & $\varepsilon 3.70$ & $36 \cdot 30$ & 57.50 & 126.00 & 14.00 & 42.50 & 6.20 & 2063.73 & 977.09 & 730.00 \\
\hline $661 \cdot 50$ & 61. & 26. & $3 \cdot$ & 5 . & $E 4.40$ & & 57.60 & 126 & 14 & & 6.80 & & & 729.50 \\
\hline 662.00 & $65 \cdot 30$ & 24. & 3.40 & 5.00 & $\in 9.90$ & 30 & 63.30 & 84.00 & 22.00 & 36.70 & 6.60 & 1885.29 & 69 & 729.00 \\
\hline & $70 \cdot 30$ & 20. & 3.40 & 5.20 & $75 \cdot 80$ & 24. & 75.80 & 84.00 & & & .0 & 1598 & & 728.50 \\
\hline & 73. & 18. & 3.40 & 5.30 & 87.20 & 12. & 87.20 & 23.00 & 32 & 12.20 & .00 & 1412.03 & 18 & 728.00 \\
\hline 663.50 & $70 \cdot 90$ & & & & $79 \cdot 10$ & 20 & 79.10 & 23.00 & & & .00 & 1551.68 & & 727.50 \\
\hline 664 & 68.00 & 21.90 & $3 \cdot 30$ & 4.90 & 79.40 & 20. & 71.90 & 53.00 & 28.00 & 28.10 & 7.50 & 1699.09 & 47 & 727.00 \\
\hline 664.50 & $65 \cdot 20$ & 23.90 & $3 \cdot 30$ & $4.7 \mathrm{C}$ & 73.80 & 26.20 & 65.60 & 53.00 & 28 & 34.40 & 8.20 & 1854.26 & 63 & .50 \\
\hline & 62 & & 3. & 4.70 & $\in 8$ & & & 105 & & 38.80 & 7.70 & 1986.15 & & \\
\hline $665 \cdot 50$ & $61 \cdot 30$ & 26. & & 4.60 & 67.10 & 32 & 59.60 & 105 & & 40.40 & 7.50 & 2040 & & \\
\hline 666.00 & $65 \cdot 20$ & 24.50 & $3 \cdot 20$ & 4.00 & 72.70 & 27.3 & 64.60 & 86.00 & 20.00 & 35.40 & 8.10 & 1900.81 & 672.89 & 725.00 \\
\hline 666.50 & 70.00 & 20.90 & 3.20 & 4.60 & $76 \cdot 90$ & 23.10 & 76.90 & 86.00 & 20.00 & 23.10 & .00 & 1621.50 & 374.57 & 724.50 \\
\hline & .57 & & & & 68.53 & & & 85.52 & 12.99 & 34.55 & 78 & 1930.24 & & \\
\hline & 7.57 & 4. & . 26 & 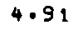 & 68.53 & 30 & 3.61 & $85 \cdot 52$ & $12 \cdot 90$ & 34.55 & & 1915.00 & 661 & \\
\hline
\end{tabular}

*NOTATIOH--Depth, ft; GR-Gamma Ray Index, API Units; POR-Porosity, $\%$; RT-True Resistivity, Ohms-m²/m; RXo-Flushed Zone Resistivity, Ohms-m²/m; SX0-Flushed Zone Saturation, \%; ROS-Residual 0il Saturation, \%; SW-Water Saturation, \%; PER-Permeability Index; Clay-Clay Content, \%; SO-0il

Saturation, \%; MO-Movable 0il, \%; PHV-Pore Volume, bbl/ac-ft; HPV-Hydrocarbon Pore Volume, bbl/ac-ft; ELSE-Elevation From Sea Level, ft 
TABLE C28

WELL LOG DETERMINED PROPERTIES

VS DEPTH FOR WELL MP-117

WELL LOCATION: 2376 FWL 1 C56 FSL $212555 E$

KELLY BUSHING ELEVATION FROM SEA LEVEL $=1396$

\begin{tabular}{|c|c|c|c|c|c|c|c|c|c|c|c|c|c|c|}
\hline DEPTH * & $G R$ & $P B R$ & $R T$ & $R \times 0$ & $5 \times 0$ & ROS & Sw & PER & CLAY & so & MO & PHV & HAPV & ELSE \\
\hline $\begin{array}{l}650.50 \\
651.00\end{array}$ & $\begin{array}{l}60.50 \\
58.70\end{array}$ & $\begin{array}{l}19.30 \\
23.90\end{array}$ & $\begin{array}{l}2.80 \\
2.70\end{array}$ & $\begin{array}{l}1.90 \\
2.10\end{array}$ & $\begin{array}{l}88.90 \\
72.20\end{array}$ & $\begin{array}{l}11.10 \\
27.80\end{array}$ & $\begin{array}{l}88.90 \\
72.20\end{array}$ & $\begin{array}{l}14.00 \\
98.00\end{array}$ & $\begin{array}{r}18.00 \\
7.00\end{array}$ & $\begin{array}{l}11.1 \mathrm{C} \\
27.8 \mathrm{C}\end{array}$ & $\begin{array}{l}.00 \\
.00\end{array}$ & $\begin{array}{l}1497.37 \\
1854.26\end{array}$ & $\begin{array}{l}166.21 \\
515.48\end{array}$ & $\begin{array}{l}745.50 \\
745.00\end{array}$ \\
\hline $651 \cdot 50$ & 58.00 & 26.00 & 2.80 & 2.30 & $t 5.40$ & 34.60 & 65.40 & 98.00 & 7.00 & 34.60 & .00 & 2017.18 & 697.94 & 744.50 \\
\hline $652 \cdot 00$ & $57 \cdot 20$ & 26.60 & 2.80 & 1.90 & $63 \cdot 40$ & 36.60 & 63.40 & 158.00 & 5.00 & 36.60 & .00 & 2063.73 & 755.33 & 744.00 \\
\hline $652 \cdot 50$ & 6.40 & 27.00 & 2.80 & .70 & 65.70 & $34 \cdot 30$ & 62.10 & 158.00 & 5.00 & 37.90 & 3.60 & 2094.77 & $793: 92$ & 743.50 \\
\hline .00 & 9.20 & 6.80 & .90 & .70 & $66 \cdot 10$ & 3.90 & 1.40 & 164.00 & 6.00 & 38.60 & 4.70 & 2079.25 & 802.59 & 743.00 \\
\hline $653 \cdot 50$ & 58.60 & $26 \cdot 70$ & 3.00 & 1.70 & 66.50 & 33.50 & 61.00 & $164 \cdot 00$ & 6.00 & 39.00 & 5.50 & 2071.49 & 807.88 & 742.50 \\
\hline 654.00 & 60.00 & 26.50 & 3.20 & 1.40 & $72 \cdot 30$ & 27.70 & 59.70 & 156.00 & 8.00 & $40 \cdot 30$ & 12.60 & 2055.97 & 828.56 & 742.00 \\
\hline 654.50 & 61.90 & 26.40 & $3 \cdot 30$ & 1.40 & 73.50 & 26.50 & 58.90 & 156.00 & 8.00 & $41 \cdot 10$ & 14.60 & 2048.22 & 841.82 & 741.50 \\
\hline 655.00 & 63.20 & 26.10 & 3.40 & 1.70 & $68 \cdot 30$ & 31.70 & 58.70 & 145.00 & 12.00 & $41 \cdot 30$ & 9.60 & 2024.94 & 836.30 & 741.00 \\
\hline $655.5 c$ & 64.80 & 25.80 & 3.50 & $2 \cdot 20$ & $60 \cdot 30$ & 39.70 & 58.40 & 145.00 & 12.00 & 41.60 & 1.90 & 2001.67 & 832.69 & 740.50 \\
\hline $656.0 \mathrm{C}$ & 65.40 & 5.50 & 1.60 & 2.50 & $58 \cdot 30$ & 41.70 & 58.30 & 131.00 & 17.00 & 41.70 & .00 & 1978.39 & 824.99 & 740.00 \\
\hline & & 25.10 & 3.70 & $\cdot 70$ & & 41.70 & $58 \cdot 30$ & & & & .00 & 1947.36 & & 739.50 \\
\hline 65 & 66.60 & 25. & 3.80 & .70 & 67. & 43.00 & 57.00 & 131.00 & 17.00 & 43.00 & .00 & 1978.39 & 85 & 739.00 \\
\hline 657.50 & 67.40 & 26.10 & 3.80 & 2.40 & $57 \cdot 30$ & $42 \cdot 70$ & 55.30 & 131.00 & 17.00 & 44.70 & 2.00 & 2024.94 & 905.15 & 738.50 \\
\hline 658.00 & 65.70 & 26.10 & 3.80 & $2 \cdot 20$ & 59. & 40.30 & 55.20 & 146. & 13.00 & 44.80 & 4.50 & 4.94 & & 738.00 \\
\hline $658 \cdot 50$ & 64.10 & 25. & 3.90 & $2 \cdot 2$ & 60. & 39. & 55.90 & 146 & & & 4.3 & 2001.67 & 882.73 & 17.50 \\
\hline 659.00 & 62.40 & 25.40 & 3.90 & 2.20 & 61.00 & 39.00 & 56.50 & $130 \cdot .00$ & 9.00 & 43.50 & 4.50 & 1970.63 & 857.22 & 737.00 \\
\hline 659.50 & 63.90 & $25 \cdot 30$ & $3 \cdot 90$ & $2 \cdot 20$ & $61 \cdot 50$ & $38 \cdot 50$ & 56.60 & 130 & 9.00 & 43.40 & 4.90 & 1962.87 & 851.89 & 736.50 \\
\hline 660.00 & 64.80 & $25 \cdot 30$ & 3.90 & -2.20 & 61.70 & 38.30 & 56.60 & 126. & 13.00 & 43.40 & $5 \cdot 10$ & 1962.87 & 851.89 & 736.00 \\
\hline 660 & 65.180 & 25. & 4.00 & 2.20 & & & $56 \cdot 40$ & 126 & 13 & 43.60 & $5 \cdot 30$ & 19 & & . 50 \\
\hline $661 \cdot 00$ & 67.80 & 25.20 & 4.00 & $2 \cdot 20$ & 61.70 & $38 \cdot 30$ & $56 \cdot 30$ & 124.00 & 16.00 & 43.70 & $5 \cdot 40$ & 1955.12 & 854.38 & 735.00 \\
\hline 661.50 & 68.50 & 25.10 & $4 \cdot$. & $2 \cdot c$ & 61. & & 56.40 & 124 & 16. & 43.60 & 5.20 & 1947.36 & & 734.50 \\
\hline 662 & 66.40 & 24. & 4.00 & 2.40 & $\epsilon 1.80$ & 38.20 & 58.40 & 107.00 & 17.00 & $\$ 1.60$ & 3.40 & 1893.05 & 737.51 & 734.00 \\
\hline $662 \cdot 50$ & 70.50 & 23.90 & 4.00 & 2.60 & 60.10 & 39.90 & 59.80 & 107.00 & 17.00 & 40.20 & .30 & 1854.26 & $7+5.41$ & 733.50 \\
\hline 663.00 & $75 \cdot 10$ & 24. & $3 \cdot$ & $2 \cdot$ & 58 & & 58.60 & & & & .00 & 1908.57 & & 7.33 .00 \\
\hline $663 \cdot 50$ & 80.10 & 22 & & 2. & 66.60 & 33.40 & 66.60 & 113.00 & 24.00 & 33.40 & .00 & 1714.61 & $5 ? 2.68$ & 732.50 \\
\hline 664.00 & $77 \cdot 90$ & 23.80 & 3.70 & $2 \cdot 40$ & $\in 3.40$ & $36 \cdot 60$ & 62.10 & 98.00 & 25.00 & 37.90 & $1 \cdot 30$ & 1846.50 & 699.82 & 732.00 \\
\hline $664 \cdot 50$ & 74.30 & 26.30 & 3.60 & $2 \cdot 20$ & 58.90 & 41.10 & 56.80 & 98.00 & 25.00 & 43.20 & $2 \cdot 10$ & 2040.46 & 881.48 & 731.50 \\
\hline 665.00 & $70 \cdot 70$ & 28.10 & 3.50 & 2.10 & $56 \cdot 80$ & 43.20 & 53.10 & $202 \cdot c 0$ & 17.00 & 46.90 & 3.70 & 2180.11 & 1022.47 & 731.00 \\
\hline 665.50 & 68.20 & 28.50 & 3.50 & 1.90 & $58 \cdot 10$ & 41.90 & 52.70 & $202 \cdot c 0$ & 17.00 & 47.30 & 5.40 & 2211.14 & 1065.87 & 730.50 \\
\hline 666.00 & 65.00 & 29.00 & 3.50 & 1. & 57.90 & $42 \cdot 10$ & $52 \cdot 10$ & $23 c \cdot 00$ & 12.00 & 47.90 & 5.80 & 2249.93 & 1037.72 & 730.00 \\
\hline 666.50 & 64.20 & 28 & & & 58 & & & & & & 4.10 & 2195.63 & 1009.99 & \\
\hline 667.00 & $68 \cdot 000$ & 27.50 & $3 \cdot 30$ & 2.00 & 58.50 & $41 \cdot 50$ & 56.10 & $182 \cdot 00$ & 13.00 & 43.90 & 2.40 & 2133.56 & 926.63 & 729.00 \\
\hline & $74 . \overline{c 0}$ & 24.70 & $3 \cdot 30$ & $2 \cdot 00$ & $\epsilon 6 \cdot 10$ & 33.90 & 63.20 & $182 \cdot 00$ & 13.00 & 36.80 & 2.90 & 1916.32 & $7 C 5.21$ & 728.50 \\
\hline 668.00 & $77 \cdot 60$ & $22 \cdot 30$ & $3 \cdot 30$ & 1.90 & $75 \cdot 60$ & 24.40 & 71.00 & 73.00 & 20.00 & 29.00 & 4.60 & 1730.12 & 501.74 & 728.00 \\
\hline$A V E$ & $6 . c 3$ & 25.56 & 3.51 & $2 \cdot 12$ & 63.42 & 36.58 & 59.82 & 138.03 & 13.89 & 40.18 & .60 & 1983.13 & 304.31 & \\
\hline
\end{tabular}

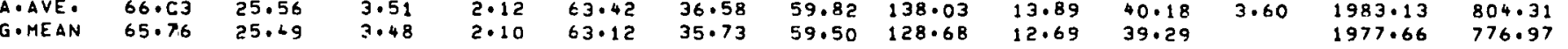

SX0-Flushed Zone Saturation, $\%$; ROS-Residual 0il Saturation, $\%$, SW-hiater Saturation

Saturation, \%; MO-Movable 0il, \%; PHV-Pore Volume, bbl/ac-ft; HPV-Hydrocarbon Pore Volume, bbl/ac-ft; ELSE-Elevation From Sea Le\%el, ft 
TABLE C29

WELL LOG DETERMINED PROPERTIES

VS DEPTH FOR WELL MP- 118

WELL LOCATION: $1086 \mathrm{FSL} 2640 \mathrm{FEL} 212555 \mathrm{~S}$

KELLY EUSHING ELEVATION FROM SEA LEVEL $=1395$

\begin{tabular}{|c|c|c|c|c|c|c|c|c|c|c|c|c|c|c|}
\hline OEPTH * & $G_{R}$ & PQR & RT & $R \times E$ & $s \times e$ & ROS & SW & PER & CLAY & se & me & PHV & HPV & ELSE \\
\hline $634 \cdot 50$ & $65 \cdot 10$ & 16.40 & 5.00 & $3.2 \mathrm{C}$ & 80.00 & 20.00 & 90.00 & 7.00 & 40.00 & 20.00 &.$c 0$ & 1272.38 & 254.48 & 760.50 \\
\hline 635.00 & $3 \cdot 30$ & 18.10. & 5.00 & $3.2 \mathrm{C}$ & 71.80 & 28.20 & 71.80 & 29.00 & 37.00 & 38 &.$c 0$ & 1404.27 & 396.00 & 760.00 \\
\hline $635 \cdot 50$ & $59 \cdot 80$ & 20.90 & 5.00 & $3 \cdot 30$ & 70.60 & 29.40 & 51.40 & 29.00 & 37.00 & $38 \cdot 60$ & 9.20 & $\begin{array}{l}1621.50 \\
1823.22\end{array}$ & 625.90 & 759.50 \\
\hline 636.00 & $56 \cdot 20$ & 23.50 & 4.80 & $3 \cdot 30$ & $\epsilon 1 \cdot 50$ & 38.50 & 55.30 & 92.00 & 23.00 & 44.70 & 6.20 & 1823.22 & & 759.00 \\
\hline 636.50 & $54 \cdot 30$ & 24.10 & .50 & $3 \cdot 40$ & 59.60 & 40.40 & 55.90 & $92 \cdot 00$ & 23.00 & 44.10 & 3.70 & 1869.77 & 824.57 & 758.50 \\
\hline $637 \cdot 00$ & $55 \cdot 40$ & $24 \cdot 30$ & - 30 & $3 \cdot 40$ & $59 \cdot 30$ & 40.70 & 56.60 & 106.00 & 22.00 & $43.4 \mathrm{C}$ & $2 \cdot 70$ & 1885.29 & 818.22 & 758.00 \\
\hline $637 \cdot 50$ & $58 \cdot 10$ & 23.80 & .10 & 3.30 & $61 \cdot 10$ & $38 \cdot 90$ & 59.10 & 106.00 & 22.00 & 40.90 & 2.00 & 1846.50 & 755.22 & 757.50 \\
\hline 638.00 & 57.50 & $24 \cdot 20$ & 3.90 & 3.20 & 60.60 & 39.40 & 59.40 & 105.00 & 25.00 & 40.60 & 1.20 & 1877.53 & 762.28 & 757.00 \\
\hline 638.50 & $5 \cdot 30$ & 4.80 & 1.70 & 3.10 & 60.80 & 39.20 & 59.60 & 105.00 & 25.00 & 40.40 & 1.20 & $192+.08$ & 777.33 & 756.50 \\
\hline & $3 \cdot 20$ & 25.40 & .20 & $2 \cdot 30$ & $\in 2.00$ & 38.00 & 62.00 & 129.00 & 16.00 & 38.00 & .00 & 1970.63 & 748.84 & 756.00 \\
\hline $639.5 c$ & 51.00 & 25.80 & 2.80 & 2.80 & $\in 5 \cdot 30$ & $4 \cdot 70$ & 65.30 & 129.00 & 16.00 & 34.70 & .00 & 2001.67 & 694.58 & 755.50 \\
\hline $6+0.00$ & $48 \cdot 90$ & 27.30 & $2 \cdot 80$ & 2.70 & $t 2.20$ & 37.80 & $62 . ? 0$ & 178.00 & 12.00 & 37.80 & .00 & 2118.04 & 800.62 & 755.00 \\
\hline 640.50 & 48.50 & 27.60 & .70 & 2.50 & $\epsilon 2.40$ & 37.60 & 62.40 & 178.00 & 12.00 & 37.60 & .00 & $2141 \cdot 32$ & 14 & 754.50 \\
\hline 641.00 & 48.10 & 27.40 & 2.60 & $2 \cdot 50$ & $\in 3 \cdot 30$ & 36.70 & $63.3 \%$ & 181.00 & 14.00 & 36.70 & .00 & 80 & 17 & 754.00 \\
\hline $641 \cdot 50$ & 47.60 & 27.30 & 2.60 & $2 \cdot 50$ & $\in 3.60$ & 36.40 & 53.60 & $181 \cdot 00$ & 14.00 & 36.40 & .00 & 2118.04 & 770.97 & 753.50 \\
\hline $642 \cdot 00$ & 47.50 & 26.80 & .60 & $2 \cdot 40$ & 65.00 & 35.00 & 65.00 & 163.00 & $15 \cdot Q 0$ & 35.00 & .00 & 2079.25 & 727.74 & 753.00 \\
\hline $642 \cdot 50$ & $46 \cdot 70$ & 26.20 & .80 & $2 \cdot 00$ & $64 \cdot 90$ & 35 . & 64.90. & 163 & 15. & & .0 & & & .50 \\
\hline $643 \cdot 00$ & $45 \cdot 80$ & 25. & $2 \cdot 90$ & 2.70 & $\in 5 \cdot 50$ & 34.50 & 65.50 & $132 \cdot 00$ & 14.00 & 34.50 & .00 & 1978.39 & 682.54 & 752.00 \\
\hline $643 \cdot 50$ & 45.00 & 25.00 & .00 & 2.80 & 65.50 & 34.50 & 65.50 & 132.00 & 14.00 & 34.50 & .00 & 1939.60 & 669.16 & 751.50 \\
\hline 644.00 & 44.20 & 24.60 & .10 & 3.00 & $\epsilon 5.50$ & 14.50 & 65.50 & 111.00 & 13.00 & & .00 & & 45 & 751.00 \\
\hline 644. & 43.40 & 23.90 & . 30 & $3 \cdot 20$ & $\in 5.70$ & 30 & 65.70 & 111 & 13. & 34 & .00 & & 636.01 & 750.50 \\
\hline $645 \cdot 00$ & $44 \cdot 70$ & 23.60 & 1.40 & $3 \cdot 40$ & 65.50 & 34.50 & 65.50 & 93.00 & 14.00 & 34.50 & .00 & 1830.98 & 631.69 & 750.00 \\
\hline $645 \cdot 50$ & 46.20 & 23.70 & 0 & 3. & $\in 3.90$ & $36 \cdot 10$ & 63.90 & & & & .00 & 1838.74 & & 749.50 \\
\hline $646 \cdot 00$ & $48 \cdot 90$ & 23.60 & 0 & 3.50 & $\in 3.00$ & 37 & 63.00 & 93. & 18.00 & & .00 & 1830.98 & & 749.00 \\
\hline & 0.60 & 23. & & 3. & & & 62. & 93.00 & 18.00 & & .00 & 1815.46 & & 748.50 \\
\hline 647.00 & $52 \cdot 30$ & 23.00 & 1.90 & $3 \cdot 00$ & $\in 6.50$ & 33.50 & 62.90 & 84.00 & 22.00 & 37.10 & 3.60 & 1784.43 & 02 & 748.00 \\
\hline $647 \cdot 50$ & 53.80 & 22. & . & $2 \cdot$ & 69.50 & & 63. & & 22 & & 6.50 & 1776.67 & & \\
\hline 648.00 & $54 \cdot 30$ & 22.80 & 3.90 & 2.90 & $\in 8 \cdot 50$ & 31. & 63. & 80 & 27.00 & & 4.60 & 1768.91 & 638.58 & 747.00 \\
\hline & $55 \cdot 10$ & 22.8 & 3.80 & 3.00 & 66.80 & 33.20 & 64.30 & 80.00 & 27.00 & 35.70 & 2.50 & 17.68 .91 & 631.50 & 746.50 \\
\hline 649.00 & $55 \cdot 30$ & 22.80 & $3 \cdot$ & 3. & $\in 6 \cdot 30$ & 33.70 & 63.90 & 80 & 30. & & 2.40 & 1768.91 & 638.58 & 746.00 \\
\hline $649 \cdot 50$ & $55 \cdot 40$ & 22 . & 3.90 & 3.10 & 66.00 & 34.00 & 63.60 & 80.00 & 30.00 & 36.40 & 2.40 & 1768.91 & 643 & 745.50 \\
\hline 650.00 & 55.40 & 23.10 & $3 \cdot 90$ & $3 \cdot 10$ & $\in 5 \cdot 10$ & $34 \cdot 90$ & 62.60 & 85.00 & 31. & 37.40 & 2.50 & 2.19 & 670 & 745.00 \\
\hline & 57.10 & $23 \cdot 3$ & 3.90 & $3 \cdot 10$ & $64 \cdot 20$ & $35 \cdot 80$ & 61.80 & 85.00 & 31.00 & 38 & $2 \cdot 40$ & 1807.71 & 690.5 & 744.50 \\
\hline 651.00 & $58 \cdot 80$ & 22. & 4.00 & 3. & 66.80 & 33.20 & $64 \cdot 30$ & 72.00 & 37.00 & $35 \cdot 70$ & 2.50 & $1722 \cdot 36$ & 614.88 & 744.00 \\
\hline $651 \cdot 50$ & 60.50 & 20.80 & 4.00 & 3.20 & 71.00 & 29.00 & 69.00 & 72.00 & 37.00 & 31.00 & 2.00 & 1613.75 & 500.26 & 743.50 \\
\hline 652.00 & $62 \cdot 20$ & 19.50 & 4.00 & 3.3 & $75 \cdot 40$ & & 74.40 & 40.00 & 41. & 25. & 1.00 & 1512.89 & 387 & 743.00 \\
\hline 552.50 & 62.50 & 19. & $3 \cdot$ & 3. & 78 & 21 & & & & & 1.50 & & & \\
\hline 653.00 & 62.40 & 18. & $3 \cdot$ & 3. & & 20.70 & 78.80 & 35.00 & 39.00 & 21.20 & .50 & 1466.34 & 310 & 742.00 \\
\hline 653. & 62.40 & 18.1 .0 & 3.60 & 3.20 & $24 \cdot 20$ & 15.80 & 84.20 & 35.00 & 39.00 & 15.80 & .00 & 1404.27 & 221 & 741.50 \\
\hline 654.00 & 62.40 & $17.3 n$ & 3.60 & 3.10 & 89.00 & 11.00 & 89.00 & 24.00 & 38.00 & 11.00 & .00 & 1342.20 & 147.64 & 741.00 \\
\hline $\begin{array}{l}\text { - AVE, } \\
\text { - MEAN }\end{array}$ & $\begin{array}{l}53.88 \\
53.53\end{array}$ & $\begin{array}{l}23.16 \\
22.99\end{array}$ & $\begin{array}{l}3.67 \\
3.62\end{array}$ & 3.04 & $\begin{array}{l}67.19 \\
\epsilon 6.88\end{array}$ & $\begin{array}{l}32.81 \\
31.86\end{array}$ & $\begin{array}{l}65.67 \\
65.32\end{array}$ & $\begin{array}{l}95 \cdot 17 \\
81.12\end{array}$ & 22.50 & 33.27 & & $\begin{array}{l}1797.23 \\
1782.99\end{array}$ & $\begin{array}{l}628.41 \\
593.23\end{array}$ & \\
\hline
\end{tabular}

*NOTATIOH--Depth, ft; GR-Gamma Ray Index, API Units; POR-forosity, $\%$; RT-True Resistivity, 0hms-m²/m; RX0-Flushed Zone Resistivity, Ohms-m² $/ m$; SX0-Flushed Zone Saturation, \%; ROS-Residual 0il Saturation, \%; SW-Water Saturation, \%; PER-Permeability Index; Clay-Clay Content, \%; SO-0il

Saturation, \%; MO-Movable 0il, \%; PHV-Pore Volume, bbl/ac-ft; HPV-Hydrocarbon Pore Volume, bbl/ac-ft; ELSE-Elevation From Sea Level, ft 
TABLE C30

WELL LOG DETERMINED PROPERTIES

VS DEPTH FOR WELL MP-119

WELL LOCATION: 2376 FEL 1 C56 FSL 2125 S $5 E$

KELLY BUSHING ELEVATION FROM SEA LEVEL $=1389$

\begin{tabular}{|c|c|c|c|c|c|c|c|c|c|c|c|c|c|c|}
\hline$D E$ & GR & PQR & RT & $R \times B$ & $5 \times 0$ & ROS & Sw & PER & \multirow{2}{*}{$\begin{array}{l}\text { CLAY } \\
39.00 \\
20.00\end{array}$} & \multirow{2}{*}{$\begin{array}{l}\text { so } \\
30.20 \\
37.30\end{array}$} & \multirow{2}{*}{$\begin{array}{l}\text { Mo } \\
.50 \\
.00\end{array}$} & \multirow{3}{*}{$\begin{array}{l}\text { PHV } \\
1737.88 \\
1893.05\end{array}$} & \multirow{2}{*}{$\begin{array}{l}\text { HPV } \\
524.84 \\
706.11\end{array}$} & ELSE \\
\hline $\begin{array}{l}619.50 \\
620.00\end{array}$ & $\begin{array}{l}67 \cdot 40 \\
65 \cdot 30\end{array}$ & $\begin{array}{l}22 \cdot 40 \\
24.40\end{array}$ & $\begin{array}{l}3 \cdot 30 \\
3 \cdot 50\end{array}$ & $\begin{array}{l}4 \cdot 40 \\
5 \cdot 10\end{array}$ & $\begin{array}{l}70 \cdot 30 \\
62.70\end{array}$ & $\begin{array}{l}29.70 \\
37.30\end{array}$ & $\begin{array}{l}69.80 \\
62.70\end{array}$ & $\begin{array}{r}18 \cdot 00 \\
108 \cdot 00\end{array}$ & & & & & & $\begin{array}{l}769.50 \\
769.00\end{array}$ \\
\hline 620.50 & 63.50 & 24.60 & 3.50 & 5.70 & 61.40 & 38.60 & 61.40 & 108.00 & 20.00 & 38.60 & .00 & & 736.71 & 768.50 \\
\hline $621 \cdot 00$ & 63.50 & 24.70 & 3.70 & $5 \cdot 00$ & 59.70 & 40.30 & 59.70 & $11 \cdot 00$ & $10 \cdot 00$ & 0.30 & .00 & 1916.32 & 772.28 & 768.00 \\
\hline $621 \cdot 50$ & $65 \cdot .00$ & 24.80 & 3.90 & 3.90 & $67 \cdot 30$ & 32.70 & 58.40 & $11+000$ & 10.00 & .1 .60 & 8.00 & 1924.08 & 800.42 & 767.50 \\
\hline $622 \cdot 00$ & $65 \cdot 80$ & 24.10 & 3.90 & $3 \cdot 60$ & 71.50 & 28.50 & 59.50 & $102 \cdot 00$ & $32 \cdot 00$ & $40.5 \mathrm{C}$ & $12 \cdot 00$ & 1869.77 & 757.26 & 767.00 \\
\hline 622.50 & 63.80 & $24 \cdot 30$ & 4.00 & 3.70 & $70 \cdot 00$ & 30.00 & $58 \cdot 30$ & $102 \cdot 00$ & 32.00 & 41.70 & 11.70 & $1885 \cdot 29$ & 786.17 & 766.50 \\
\hline $\begin{array}{l}623 \cdot 00 \\
623.50\end{array}$ & $\begin{array}{l}60.90 \\
59.50\end{array}$ & $\begin{array}{l}24.40 \\
24.80\end{array}$ & $\begin{array}{l}410 \\
410\end{array}$ & $\begin{array}{l}4.50 \\
5.20\end{array}$ & $\begin{array}{l}63.30 \\
57.90\end{array}$ & $\begin{array}{l}36.70 \\
42.10\end{array}$ & 57.80 & 109.00 & 26.00 & $42 \cdot 20$ & 5.50 & $\begin{array}{l}1893.05 \\
1924.08\end{array}$ & $\begin{array}{l}798.87 \\
831.20\end{array}$ & $\begin{array}{l}766.00 \\
765.50\end{array}$ \\
\hline 624.00 & $58 \cdot: 0$ & 25.20 & 4.00 & $5 \cdot 30$ & 56.50 & $\begin{array}{r}2.10 \\
43.50\end{array}$ & $\begin{array}{l}56.80 \\
55.80\end{array}$ & $\begin{array}{l}109.00 \\
125.00\end{array}$ & $\begin{array}{l}26.00 \\
18.00\end{array}$ & $\begin{array}{l}43.20 \\
44.20\end{array}$ & $\begin{array}{r}1.10 \\
.70\end{array}$ & 1955.12 & $864 \cdot 16$ & 765.00 \\
\hline $\begin{array}{l}624.50 \\
625.00\end{array}$ & $56 \cdot: 0$ & 25.60 & 4.10 & $5 \cdot 30$ & $55 \cdot 20$ & 44.80 & 54.80 & 125.00 & 18.00 & 45.20 & .40 & $1986 \cdot 15$ & 897.74 & 764.50 \\
\hline $\begin{array}{l}625 \cdot 00 \\
625.50\end{array}$ & $56 \cdot 30$ & 26.90 & $4 \cdot 10$ & $\begin{array}{l}5.50 \\
5.70\end{array}$ & $\begin{array}{l}51 \cdot 80 \\
50 \cdot 30\end{array}$ & $48 \cdot 20$ & 51.80 & 166.00 & 14.00 & 48.20 & .00 & 2087.01 & $1005 \cdot 94$ & 764.00 \\
\hline $625 \cdot 50$ & $55 \cdot 10$ & 27.60 & 4.10 & $\begin{array}{l}5.70 \\
5.80\end{array}$ & $\begin{array}{l}50 \cdot 30 \\
52 \cdot 50\end{array}$ & 49.70 & $50 \cdot 30$ & $166 \cdot 00$ & 14.00 & 49.70 & .00 & $21+1 \cdot 32$ & 1064.23 & 763.50 \\
\hline 626.00 & $54 \cdot 010$ & 27.10 & $3 \cdot 90$ & 5.80 & 52.50 & 47.50 & $52 \cdot 50$ & $172 \cdot 00$ & $12 \cdot 00$ & 47.50 & .00 & $2102 \cdot 53$ & 998.70 & 763.00 \\
\hline 626.50 & $52 \cdot 50$ & 26.70 & & $5 \cdot 50$ & 54.70 & $45 \cdot 30$ & 54.70 & 172.00 & 12.00 & $45 \cdot 30$ & .00 & 2071.49 & $938 \cdot 39$ & 762.50 \\
\hline 627.00 & $51 \cdot: 0$ & 26.20 & $3 \cdot 50$ & $5 \cdot 20$ & 57.50 & $42 \cdot 50$ & 57.50 & 148.00 & 13.00 & $42 \cdot 50$ & .00 & $2032 \cdot 70$ & 863.90 & 762.00 \\
\hline 627.50 & $50 \cdot \in 0$ & 25.70 & $3 \cdot 30$ & $5 \cdot c 0$ & $\in 0.30$ & 39.70 & $60 \cdot 30$ & $148 \cdot 00$ & 13.00 & 39.70 & .00 & 1993.91 & 791.58 & 761.50 \\
\hline 628.00 & 49.70 & 25.50 & $3 \cdot 20$ & 4.70 & 61.70 & $38 \cdot 30$ & 61.70 & 130.00 & 12.00 & $38 \cdot 30$ & .00 & 1978.39 & 757.72 & 761.00 \\
\hline $628 \cdot 50$ & $50 \cdot 70$ & 25.20 & $3 \cdot 10$ & 4.60 & $\in 3 \cdot 90$ & $36 \cdot 10$ & 63.90 & 130.05 & 12.00 & 36.10 & .00 & 1955.12 & 705.80 & 760.50 \\
\hline 629.00 & 51.70 & 24.90 & 3.00 & $4 \cdot 40$ & 66.00 & 34.00 & 66.00 & 119.00 & 16.00 & 34.00 & .00 & 1931.84 & 656.83 & 760.00 \\
\hline 629.50 & $53 \cdot 10$ & $25 \cdot 10$ & $2 \cdot 80$ & $4 \cdot 30$ & $\epsilon 7.20$ & 32.80 & 67.20 & 119.00 & 16.00 & $32 \cdot 80$ & .00 & 1947.36 & 638.73 & 759.50 \\
\hline 630.00 & $53 \cdot 50$ & 25.40 & 2.80 & $4 \cdot 30$ & 66.90 & 33.10 & 66.90 & 129.00 & 18.00 & $33 \cdot 10$ & .02 & 1970.63 & 652.28 & 759.00 \\
\hline 630.50 & $53 \cdot 90$ & 25.90 & 2.80 & 4040 & $\epsilon 4.80$ & $35 \cdot 20$ & 64.80 & 129.00 & 18.00 & 35.20 & .00 & 2009.42 & $707 \cdot 32$ & 758.50 \\
\hline 631.00 & $54 \cdot 30$ & 26.30 & $2 \cdot 90$ & .050 & 62.60 & $37 \cdot 40$ & 62.60 & $151 \cdot 00$ & $20 \cdot 00$ & 37.40 & .00 & 2040.46 & 763.13 & 758.00 \\
\hline 631.50 & $54 \cdot 70$ & 26.50 & 3.00 & 4.40 & $61 \cdot 20$ & 38.80 & 61.20 & $151 \cdot 00$ & 20.00 & $38 \cdot 80$ & .00 & 2055.97 & 797.72 & 757.50 \\
\hline 632.00 & 54.70 & 26.30 & 3.10 & .070 & 60.60 & 39.40 & 60.60 & $150 \cdot 00$ & 20.00 & 39.40 & .00 & 2040.46 & 803.94 & 757.00 \\
\hline 632.50 & $54 \cdot 40$ & $26 \cdot 10$ & 3.20 & 5.10 & 60.20 & 39.80 & 60.20 & 150.00 & 20.00 & 39.80 & .00 & 2024.94 & 805.93 & 756.50 \\
\hline 633.00 & $54 \cdot 10$ & 25.90 & $3 \cdot 40$ & 5.60 & 59.40 & 40.60 & 59.40 & 141.00 & 19.00 & 40.60 & .00 & 2009.42 & 815.83 & 756.00 \\
\hline 633.50 & 53.80 & 25.60 & 3.50 & 5.50 & 59.40 & & 59.40 & $141 \cdot 00$ & 19.00 & 40.60 & .00 & 1986.15 & $806 \cdot 38$ & 755.50 \\
\hline 634.00 & 55.43 & 25.40 & 3.60 & $5 \cdot 40$ & $58 \cdot 90$ & 41.10 & 58.90 & 129.00 & 19.00 & $41 \cdot 10$ & . no & 1970.63 & 809.93 & 755.00 \\
\hline 634.50 & 56.93 & 25.00 & $3 \cdot 60$ & $5 \cdot 30$ & $59 \cdot 40$ & 40.60 & 59.40 & 129.00 & 19.00 & 40.60 & .00 & 1939.60 & $787 \cdot 48$ & 754.50 \\
\hline 635.00 & 60.23 & 24.90 & 3.70 & $5 \cdot 10$ & $59 \cdot 30$ & 40.70 & 59.30 & 118.00 & 25.00 & $\$ 0.70$ & .00 & 1931.84 & 786.26 & 754.00 \\
\hline 635.50 & 62.43 & 24.70 & 3.70 & 4.90 & $\epsilon 0.00$ & 40.00 & 59.50 & 118.00 & 25.00 & +0.50 & .50 & 1916.32 & 776.11 & 753.50 \\
\hline 636.00 & 64.60 & 24.80 & 3.70 & .170 & 60.90 & 39.10 & 59.40 & $116.0 \mathrm{C}$ & & 40.60 & 1.50 & 1924.08 & 781.18 & 753.00 \\
\hline $636 \cdot 50$ & $66 \cdot 21)$ & 23.90 & $3 \cdot 70$ & 4.50 & $64 \cdot 50$ & $35 \cdot 50$ & 61.80 & 116.00 & 32.00 & $38 \cdot ? 0$ & 2.70 & 1854.26 & $708 \cdot 33$ & $152 \cdot 50$ \\
\hline 637.00 & $65 \cdot 50$ & 23.90 & 3.70 & 4.60 & $63 \cdot 80$ & 36.20 & 61.80 & $98 \cdot 00$ & 36.00 & $38 \cdot ? 0$ & 2.00 & 1854.26 & $708 \cdot 33$ & 752.00 \\
\hline 637.50 & $64 \cdot 50$ & 23.70 & 3.70 & 4.80 & $E 3 \cdot 40$ & 36.60 & 62.30 & $98 \cdot 00$ & 36.00 & 37.70 & 1.10 & 1838.74 & $\begin{array}{l}693.20 \\
703.52\end{array}$ & 751.50 \\
\hline 638.00 & 63.60 & 23.80 & 3.70 & 4.70 & 63.90 & $36 \cdot 10$ & $61 \cdot 90$ & 97.00 & 33.00 & $38 \cdot 10$ & $2.0 \mathrm{C}$ & $\begin{array}{l}1846.50 \\
1792.19\end{array}$ & $\begin{array}{l}703.52 \\
652.36\end{array}$ & $\begin{array}{l}751.00 \\
750.50\end{array}$ \\
\hline 638.50 & $65 \cdot 10$ & 23.10 & $3 \cdot 80$ & 4.50 & $67 \cdot 10$ & $32 \cdot 90$ & 63.60 & 97.00 & 33.00 & $36 \cdot 40$ & 3.56 & $\begin{array}{l}1792.19 \\
1691.33\end{array}$ & 546.30 & $\begin{array}{l}750.50 \\
750.00\end{array}$ \\
\hline 639.00 & 66.00 & 21.80 & 3.80 & 4.50 & $71 \cdot 20$ & $28 \cdot 80$ & 67.70 & $\begin{array}{l}66.00 \\
66.00\end{array}$ & $\begin{array}{l}36.00 \\
36.00\end{array}$ & $\begin{array}{l}32.30 \\
25.70\end{array}$ & $\begin{array}{l}3.50 \\
1.50\end{array}$ & $\begin{array}{l}1691.33 \\
1644.78\end{array}$ & 488.50 & $\begin{array}{l}750.00 \\
749.50\end{array}$ \\
\hline $\begin{array}{l}639.50 \\
640.00\end{array}$ & $\begin{array}{l}65.50 \\
68.60\end{array}$ & $\begin{array}{l}21.20 \\
18.20\end{array}$ & $\begin{array}{l}3.70 \\
3.70\end{array}$ & $\begin{array}{l}4.80 \\
5.00\end{array}$ & $\begin{array}{l}71.80 \\
83.70\end{array}$ & $\begin{array}{l}28 \cdot 20 \\
16 \cdot 30\end{array}$ & $\begin{array}{l}70.30 \\
83.70\end{array}$ & $\begin{array}{l}66.00 \\
29.00\end{array}$ & $\begin{array}{l}36.00 \\
41.00\end{array}$ & $\begin{array}{l}25.70 \\
16.30\end{array}$ & $\begin{array}{r}1.50 \\
.00\end{array}$ & $\begin{array}{l}164.78 \\
1412.03\end{array}$ & $23 c \cdot 16$ & 749.00 \\
\hline & & & & • 85 & 62.49 & 37.51 & 6 & $119 \cdot 60$ & & 38.5 & 1.41 & 1925.92 & 755.28 & \\
\hline $\begin{array}{l}\text { - AVE. } \\
\text { - MEAN }\end{array}$ & $\begin{array}{l}58.85 \\
58.62\end{array}$ & 24.76 & 3.54 & .82 & 62.20 & 36.92 & 60.84 & 112.41 & 20.73 & 38.40 & & 1921.08 & 737.80 & \\
\hline
\end{tabular}

*NOTATION--Deptr, ft; GR-Ganma Ray Index, API Units; POR-Porosity, $\%$; RT-True Resistivity, Ohms-m²/m; RXo-Flushed Zone Resistivity, Ohms-m²/m;

SXO-Flushed Zone Saturation, \%; ROS-Residual 0il Saturation, \%; SW-Water Saturation, \%; PER-Permeability Index; Clay-Clay Content, \%; SO-0il

Saturation, $\%$; M-Movable 0il, \%; PHV-Pore Volume, bbl/ac-ft; HPV-Hydrocarbon Pore Volume, bbl/ac-ft; ELSE-Elevation From Sea Level, ft 
TABLE C 31

WELL LOG DETERMINED PROPERTIES

VS DEPTH FOR WELL MP-120

WELL LOCATION: 1056 FSL 2112 FEL 21255 5E

KELLY BUSHING ELEVATION FROM SEA LEVEL $=1390$

\begin{tabular}{|c|c|c|c|c|c|c|c|c|c|c|c|c|c|c|}
\hline CEPTH * & $G R$ & $P A R$ & RT & $R \times \theta$ & $s \times 0$ & RES & SW & PER & CLAY & so & re & PHV & HPV & ELSE \\
\hline 617.50 & 70.60 & 16.30 & 4.10 & $5 \cdot 40$ & 29.30 & 10.70 & $89 \cdot 30$ & 6.00 & 22.00 & 10.70 & .00 & 1264.62 & 135.31 & 772.50 \\
\hline 618.00 & 71.00 & 19.90 & 4.10 & 4.90 & 71.40 & 28.60 & 71.40 & 35.00 & 20.00 & 28.60 & .00 & 1543.92 & 441.56 & 772.00 \\
\hline 618.50 & $71 \cdot 30$ & 19.40 & 4.20 & $5 \cdot 30$ & 72.60 & $27 \cdot 40$ & 72.60 & $35 \cdot 00$ & $20 \cdot 00$ & 27.40 & .00 & 1505.13 & 412.41 & 771.50 \\
\hline 619.00 & 69.40 & 18.60 & $4 \cdot 30$ & $5 \cdot 90$ & $75 \cdot 40$ & $24 \cdot 60$ & 75.40 & 26.00 & 19.00 & 24.60 & .00 & 1443.06 & 354.99 & 771.00 \\
\hline 019.50 & 67.30 & 18.20 & $4 \cdot 30$ & 6.20 & 76.60 & 23.40 & 76.60 & 26.00 & 19.00 & 23.40 & .00 & 1412.03 & 330.41 & 770.50 \\
\hline 620.00 & $65 \cdot 30$ & 17.80 & 4.40 & 6.10 & 77.80 & 22.20 & 77.80 & 21.00 & 17.00 & 22.20 & .00 & 1380.99 & 306.58 & 770.00 \\
\hline $620 \cdot 50$ & 63.20 & 17.40 & 4.50 & $6.8 \mathrm{C}$ & $79 \cdot 10$ & $20 \cdot 90$ & 79.10 & 21.00 & 17.00 & 20.90 & .00 & 1349.96 & 282.14 & 769.50 \\
\hline 621.00 & 60.90 & 16.60 & $4 \cdot 50$ & $7 \cdot 40$ & 83.10 & $16 \cdot 90$ & 83.10 & $1 \epsilon .00$ & 13.00 & 16.90 & .00 & 1287.89 & 217.65 & 769.00 \\
\hline 621.50 & 59.90 & 15.70 & 4.40 & 7.80 & 88.50 & 11.50 & 88.50 & 16.00 & 13.00 & 11.50 & .00 & 1218.07 & 140.08 & 768.50 \\
\hline $32 c .00$ & 58.90 & 19.90 & 4.40 & 7.10 & $73 \cdot 30$ & 26.70 & 69.40 & 35.00 & 11.00 & 30.60 & 3.90 & 1543.92 & 472.44 & 768.00 \\
\hline 522.50 & 58.00 & 24.00 & $4 \cdot 30$ & $6 \cdot 4 \mathrm{C}$ & $\in 3.00$ & 37.00 & 57.10 & 35.00 & 11.00 & 42.90 & 5.90 & 1862.02 & 798.80 & 767.50 \\
\hline 523.00 & 57.00 & 26.60 & $4 \cdot 10$ & 5.90 & 59.00 & 41.00 & 52.10 & 125.00 & 5.00 & 47.90 & 6.90 & 2063.73 & 988.53 & 767.00 \\
\hline 623.50 & 58.80 & 26.80 & 4.00 & 5.80 & 58.80 & 41.20 & 52.50 & 125.00 & 6.00 & 47.50 & 6.30 & 2079.25 & 987.64 & 766.50 \\
\hline 624.00 & 60.50 & 26.90 & 3.90 & 5.80 & 58.80 & 41.20 & 53.10 & 131.00 & 7.00 & 46.90 & 5.70 & 2087.01 & 978.81 & 766.00 \\
\hline 624.50 & $62 \cdot 30$ & 27.10 & $3 \cdot 80$ & 5.10 & $58 \cdot 70$ & $41 \cdot 30$ & 53.50 & 131.00 & 7.00 & 46.50 & $5 \cdot 20$ & 2102.53 & 977.67 & 765.50 \\
\hline $625 \cdot 00$ & 64.40 & 27.30 & 3.70 & 5.70 & $58 \cdot 20$ & 41.80 & 53.90 & 140.00 & 7.00 & 46.10 & 4.30 & 2118.04 & 976.42 & 765.00 \\
\hline 625.50 & 64.40 & 27.70 & 3.50 & 5.70 & $=7.40$ & 42.60 & 54.00 & 140.00 & 7.0 & 46.0 & 3.40 & 2149.08 & 57 & 764.50 \\
\hline 626.00 & $63 \cdot 50$ & 27.80 & $3 \cdot 50$ & $5 \cdot 60$ & 57.20 & 42.80 & 53.80 & 153.00 & 8.00 & $46 . ? 0$ & 3.40 & 2156.83 & 996.46 & 764.00 \\
\hline 626.50 & 63.10 & 27.90 & 3.60 & $5 \cdot 60$ & 57.00 & 43.00 & 53.30 & 153.00 & 8.00 & 46.70 & 3.70 & 2164.59 & $1010 \cdot 86$ & 763.50 \\
\hline 627.00 & 63.30 & 27.70 & 3.60 & 5.00 & 57.50 & 42.50 & 53.40 & 150.00 & 9.00 & 46.60 & $4 \cdot 10$ & 2149.08 & 1001.47 & 763.00 \\
\hline 627.50 & 63.60 & 27.50 & 3.60 & 5.60 & 58.00 & 42.00 & 53.60 & 150.00 & 9.00 & 46.40 & 4.40 & 2133.56 & 97 & 762.50 \\
\hline $628 \cdot 00$ & 63.90 & 27.20 & 3.70 & $5 \cdot 10$ & $58 \cdot 50$ & 41.50 & 53.90 & 138.00 & $10 \cdot 00$ & $46 \cdot 1 n$ & 4.60 & 2110.28 & 972.84 & 762.00 \\
\hline 628. & 66.80 & 26.90 & 3.70 & 5.70 & $59 \cdot 10$ & 40.90 & 54.20 & 138.00 & 10.00 & 45.80 & 4.90 & 2087.01 & 955.85 & 761.50 \\
\hline 629.00 & 69.60 & 26. & $3 \cdot 80$ & 5.70 & 60. & 39 & & 118. & 13 & 44.60 & 5.20 & 20 & & .00 \\
\hline 629.50 & 71.40 & 25.80 & 3.80 & $5 \cdot 70$ & $\epsilon 1.80$ & $38 \cdot 20$ & 56.30 & 118.00 & 13.00 & 43.70 & 5.50 & 2001.67 & & .50 \\
\hline 630.00 & 73.30 & 25.10 & 3.80 & $5 \cdot 70$ & $63 \cdot 30$ & 36.70 & 57.50 & 93.00 & 19.00 & 42.50 & $5 \cdot 80$ & 1947.36 & 827.63 & 760.00 \\
\hline 630.50 & $75 \cdot 10$ & 24.50 & $3 \cdot 90$ & 5.00 & 65.70 & 34 & 58.60 & 98.00 & 19 & 41.40 & 7.10 & 1900 & 93 & 759.50 \\
\hline 631.00 & 76.80 & $24 \cdot 30$ & 3.90 & $5 \cdot 50$ & $\epsilon$ & & 59.00 & 84.00 & 24.00 & .1 .00 & 8.20 & 5.29 & 772.97 & 759.00 \\
\hline 631.50 & 78.60 & 211.60 & 4.00 & $5 \cdot 90$ & $64 \cdot 10$ & 35.90 & 57.90 & 84.00 & 24.00 & $42 \cdot 10$ & 6.20 & 1908.57 & $803 \cdot 51$ & $\begin{array}{l}758.50 \\
758.00\end{array}$ \\
\hline 632 & 78 & & & 5.90 & $\in 3$ & & & & & & & & & 758.00 \\
\hline 632.50 & 77.00 & 25.20 & $4 \cdot 10$ & $5 \cdot 90$ & $\epsilon 2 \cdot 10$ & 37.90 & 55.70 & 93.00 & 24.00 & $44 \cdot 30$ & 6.40 & 1955.12 & 866.12 & 757.50 \\
\hline 633.00 & 75.60 & 26.20 & 4.10 & 6.00 & 59.30 & $40 \cdot 70$ & 53.00 & 118.00 & 20.00 & 47.00 & $6 \cdot 30$ & 2032.70 & 955.37 & 757.00 \\
\hline 633.50 & $74 \cdot 20$ & 27.00 & 4.20 & $6 \cdot 10$ & $56 \cdot$ & $43 \cdot 3$ & 51.20 & 118.00 & 20.00 & 48.80 & $5 \cdot 50$ & 2094.77 & 1022.25 & 756.50 \\
\hline 634.00 & 72.70 & 27.50 & $4 \cdot 20$ & 6.30 & $54.80^{\circ}$ & 45.20 & 49.90 & 145.00 & 17.00 & $50 \cdot 10$ & 4.90 & 2133.56 & 1068.91 & 756.00 \\
\hline 634.50 & 71.50 & 27.00 & $4 \cdot 10$ & 6.00 & 57.50 & 42.50 & 51.00 & 145000 & 17.00 & 48.60 & 6.10 & 2094.77 & 1018.06 & 755.50 \\
\hline 635.00 & $70 \cdot 30$ & 26.40 & 4.00 & $5 \cdot 90$ & 59.40 & 40.60 & 53.20 & 121.00 & 15.00 & 46.80 & 6.20 & 2048.22 & & 755.00 \\
\hline $635 \cdot 50$ & $75 \cdot 30$ & 25. & $3 \cdot 90$ & $5 \cdot 50$ & 63.00 & 37. & $55.4 \mathrm{C}$ & 121.00 & 15. & 44.60 & 7.60 & 1993 & & \\
\hline 636.00 & $80 \cdot 30$ & 24.70 & $3 \cdot 80$ & $5 \cdot 30$ & 67.40 & 32.60 & 53.60 & 90.00 & 23.00 & 41.40 & 8.80 & 1916.32 & $793 \cdot 36$ & 754.00 \\
\hline 636.50 & 85.40 & 21.70 & 3.70 & 5.00 & 79.40 & 20.60 & 68.20 & 90.00 & 23.00 & 31.80 & 11.20 & 1683.57 & $535 \cdot 38$ & 753.50 \\
\hline 637.00 & 90.80 & 18.40 & 3.60 & 4.70 & 82.40 & 17.60 & 82.40 & 25.00 & 37.00 & 17.60 & .00 & 1427.54 & $251 \cdot 25$ & 753.00 \\
\hline $\begin{array}{l}\text { A.AV } \\
\text { G.MEE }\end{array}$ & $\begin{array}{l}69.09 \\
68.68\end{array}$ & $\begin{array}{l}23.91 \\
23.56\end{array}$ & $\begin{array}{l}3.98 \\
3.97\end{array}$ & $\begin{array}{l}5.86 \\
5.83\end{array}$ & 65 & & $\begin{array}{l}55 \\
60\end{array}$ & & & $\begin{array}{l}45 \\
01\end{array}$ & 5 & & & \\
\hline & & & & & & & & & & & & & & \\
\hline
\end{tabular}

*NOTATION--Depth, ft; GR-Gamma Zay Index, API Units; POR-Porosity, $\%$; RT-True Resistivity, Ohms-m²/m; RXo-Flushed Zone Resistivity, Ohms-m²/m;

SXO-Flushed Zone Saturation, \%; ROS-Residual 0 il Saturation, \%; SW-Water Saturation, \%; PER-Permeability Index; Clay-Clay Content, \%; SO-0il

Saturation, \%; MO-Movable 0il, \%; PHV-Pore Volume, bbl/ac-ft; HPV-Hydrocarbon Pore Volume, bbl/ac-ft; ELSE-Elevation From Sea Level, ft 
TABLE C32

WELL LOG DETERAINED PROPERTIES

VS DEPTH FOR WELL MP-121

JELL LOCATION: 792 FSL 2112 FEL $2125 \mathrm{~S} 5 \mathrm{EE}$

«ELLY BUSHING ELEVATION FROM SEA LEVEL $=1394$

\begin{tabular}{|c|c|c|c|c|c|c|c|c|c|c|c|c|c|c|}
\hline JEPTH * & GR & DSR & RT & $R \times y$ & $s \times e$ & Ros & Sw & PER & CLAY & so & Mo & PHV & HPV & ELSE \\
\hline 527.50 & 58.70 & $1 \in .00$ & 3.50 & 2.10 & 58.50 & 1.50 & 98.50 & 11.00 & 31.00 & 1.50 & .00 & 1241.34 & 18.62 & 266.50 \\
\hline 628.00 & 54.60 & $1 \subseteq .0 \mathrm{C}$ & 3.40 & $2 \cdot 10$ & $\varepsilon 8 \cdot 10$ & 11.90 & 88.10 & 28.00 & 19.00 & $11 \cdot 70$ & .00 & 1396.51 & 166.18 & .766 .00 \\
\hline $628 \cdot 50$ & 54.00 & 19.00 & $3 \cdot 30$ & 2.10 & $84 \cdot 30$ & 15.70 & 84.30 & 28.00 & 19.00 & 15.70 & .00 & 1474.10 & 231.43 & 765.50 \\
\hline 629.00 & $55 \cdot 10$ & 26.00 & 3.20 & $2 \cdot 1 \mathrm{c}$ & 81.20 & 18.80 & 81.20 & 45.00 & 18.00 & 18.80 & .00 & 1551.68 & 291.72 & 765.00 \\
\hline 629.50 & 55.20 & 20.00 & 3.00 & 1.90 & 83.20 & 16.80 & 83.20 & 45.00 & 18.00 & 16.80 & .00 & 1551.68 & 260.68 & 764.50 \\
\hline $630 \cdot 00$ & $54 \cdot 40$ & $20 \cdot 30$ & 2.80 & 1.30 & 84.00 & 16.00 & 84.00 & 49.00 & 17.00 & 16.00 & .00 & 1574.95 & 251.99 & 764.00 \\
\hline 630.50 & 53.60 & 21.70 & 2.70 & 1.70 & 80.80 & $19 \cdot 20$ & 80.80 & 49.00 & 17.00 & 19.20 & .00 & 1683.57 & 323.25 & 763.50 \\
\hline 631.00 & 52.50 & 231.40 & 2.60 & 1.50 & 77.70 & 22.30 & 76.30 & 89.00 & 15.00 & 23.70 & 1.40 & 1815.46 & 430.26 & 763.00 \\
\hline 6.31 .50 & $51 \cdot 30$ & 25.00 & 2.50 & 1.50 & $73 \cdot 30$ & 26.70 & 72.20 & 89.00 & 15.00 & 27.80 & 1.10 & 1939.60 & 539.21 & 762.50 \\
\hline$E .32 .00$ & 50.80 & 26.20 & 2.40 & 1.00 & $70 \cdot 10$ & 29.90 & $70 \cdot 10$ & 149.00 & 15.00 & $29.9 n$ & .00 & 2032.70 & 607.78 & 762.00 \\
\hline$\epsilon 32.50$ & $52 \cdot 30$ & 27.30 & $2 \cdot 30$ & 1.40 & 68.70 & $31 \cdot 30$ & 68.70 & 149.00 & 15.00 & 31.30 & .00 & 2118.04 & 662.95 & 761.50 \\
\hline 633.00 & 5.3 .60 & 27.40 & $2 \cdot 20$ & 1.00 & 68.90 & $31 \cdot 10$ & 68.90 & 181.00 & 17.00 & $31 \cdot 10$ & .00 & 2125.80 & 661.12 & 761.00 \\
\hline $63.3 \cdot 50$ & 54.00 & 26.70 & 2.20 & 1.90 & 70.70 & 29.30 & 70.70 & 181.00 & 17.00 & 29.30 & .00 & 2071.49 & 606.95 & 760.50 \\
\hline 634.00 & 54.50 & 25.90 & 2.20 & $2 \cdot 10$ & 73.10 & 26.90 & 73.10 & 142.00 & 20.00 & 26.90 & .00 & 2009.42 & 540.53 & 760.00 \\
\hline 634.50 & $54.9 C$ & 24.70 & 2.20 & 1.90 & $77 \cdot 10$ & $22 \cdot 90$ & 77.10 & 142.00 & 20.00 & 22.90 & .00 & 1916.32 & 438.84 & 759.50 \\
\hline 635.00 & 55.40 & 23.60 & 2.20 & 1.00 & 81.00 & 19.00 & 81.00 & 93.00 & 21.00 & 19.00 & .00 & 1830.98 & 347.89 & 759.00 \\
\hline $6: 35 \cdot 5.0$ & 55.80 & 24.00 & $\ddot{c} \cdot 20$ & 1.40 & $19 \cdot 30$ & 20.70 & 79.30 & 93.00 & 21.00 & 20.76 & .00 & 1862.02 & 385.44 & 758.50 \\
\hline 636.00 & 56.30 & $24 . .80$ & $\therefore .20$ & $1 \cdots 0$ & 76.50 & 23.50 & 76.50 & 116.00 & 23.00 & 23.50 & .00 & 1924.08 & 452.16 & 758.00 \\
\hline 636.50 & 56.80 & 5.30 & $2 \cdot 30$ & 1.40 & 74.50 & 25.50 & 74.50 & 116.00 & 23.00 & 25.50 & .00 & 1962.87 & 500.53 & 757.50 \\
\hline $63 \% .00$ & 57.90 & 25.80 & $2 \cdot 30$ & 1.40 & 73.00 & 27.00 & 72.60 & 138.00 & 24.00 & 27.40 & .40 & 2001.67 & 548.46 & 757.00 \\
\hline 637.50 & 59.00 & 20.00 & $2 \cdot 30$ & 1.40 & 72.40 & 27.60 & 71.80 & 138.00 & 24.00 & 28.20 & .60 & 2017.18 & 568.85 & 756.50 \\
\hline $63 x .00$ & 60.00 & 25.70 & $2 \cdot 30$ & $1 \cdot 40$ & 74.50 & $25 \cdot 50$ & 72.20 & 135.00 & 25.00 & 27.80 & $2 \cdot 30$ & 1993.91 & 554.31 & 756.00 \\
\hline $638 \cdot 50$ & 61.10 & 25.30 & 2.40 & 1.40 & 74.60 & 25.40 & 73.00 & 135.00 & 25.00 & 27.00 & 1.60 & 2.87 & 529.98 & 755.50 \\
\hline 6.39 .00 & $62 \cdot 20$ & 25.30 & 2.40 & 1.00 & 72.50 & 27.50 & 72.50 & $12 \% .00$ & 31.00 & 27.50 & .00 & 1962.87 & 539.79 & 755.00 \\
\hline 639.50 & 64.80 & $25.6 C$ & 2.40 & 1.150 & 71.40 & 28.60 & 71.40 & 127.00 & 31.00 & 28.60 & .00 & 1986.15 & 568.04 & 754.50 \\
\hline $6+0 \cdot 00$ & 67.40 & 23.90 & 2.40 & 1.00 & $76 \cdot 30$ & 23.70 & 76.30 & 99.00 & 35.00 & 23.70 & .00 & 1854.26 & 439.46 & 754.00 \\
\hline 640.50 & 69.90 & $22 \cdot 00$ & 2.50 & 1.00 & 82.90 & 17.10 & 82.90 & 99.00 & 35.00 & 17.10 & .00 & 1706.85 & 291.87 & 753.50 \\
\hline $6 \cdot 1 \cdot 00$ & $70 \cdot 40$ & 21.30 & 2.50 & 1.70 & 84.50 & 15. & $84.5 n$ & 60.00 & 42.00 & & .00 & 1652.5 & 256.14 & 753.00 \\
\hline $641.5 c$ & 69.00 & 21.80 & 2.60 & 1.00 & $\varepsilon_{1} \cdot 50$ & 18.50 & 81.50 & 60.00 & 42.00 & 18.50 & .00 & 1691.33 & 312.90 & 752.50 \\
\hline 642.00 & 67.50 & $22 \cdot 30$ & 2.70 & 1.50 & $78: 80$ & 21.20 & 78.80 & 73.00 & 39.00 & $21 .=0$ & .00 & 1730.12 & 366.79 & 752.00 \\
\hline 642.50 & $66 \cdot 10$ & 23.00 & 2.70 & 1.50 & 73.80 & 21.20 & 75.40 & 73.00 & 39.00 & 24.60 & 3.40 & 1784.43 & 438.97 & 751.50 \\
\hline 643.00 & 64.70 & 24.00 & 2.80 & 1.00 & 73.70 & $26 \cdot 30$ & 71.10 & 100.00 & 34.00 & 28.90 & 2.60 & 1862.02 & 538.12 & 751.00 \\
\hline 643.50 & 65.00 & 24.70 & 2.80 & 1.70 & 69.40 & 30.60 & 68.00 & 100.00 & 34.00 & 32.00 & 1.40 & 1916.32 & 613.22 & 750.50 \\
\hline 644.00 & $66 \cdot 50$ & 24.80 & 2.90 & 1.90 & 67.40 & 32.60 & 67.40 & 116.00. & 34.00 & 32.60 & .00 & 1924.08 & 627.25 & 750.00 \\
\hline 644.50 & 71.70 & & 2.90 & 1.90 & 76.70 & 23.30 & 76.70 & 116.00 & 34.00 & 23.30 & .00 & 1691.33 & 394.08 & 749.50 \\
\hline 645.00 & 76.90 & 18.30 & $3.0 \mathrm{C}$ & 1.80 & 52.30 & 7.70 & $92 \cdot 30$ & 30.00 & 47.00 & 7.70 & .00 & 1419.79 & 109.32 & 749.00 \\
\hline AVE. & 6 & .57 & 2.57 & 1.0 & 76 & 5 & 76 & 29 & & 23.47 & .42 & $8 \cdot 54$ & & \\
\hline G.MEA & 59.49 & 23.13 & 2.55 & 1.04 & 76.59 & $22 \cdot 16$ & 76.29 & 89.66 & 24.33 & 22.53 & & 1817.48 & 409.43 & \\
\hline
\end{tabular}

*NOTATIOH--Depth, ft; GR-Gamma Fay Index, API Units; POR-Porosity, \%; RT-True Resistivity, Ohms-m²/m; RXO-Flushed Zone Resistivity, Ohms-m²/m; SX0-Flushed Zone Saturation, \%; ROS-Residual 0il Saturation, \%; SW-Water Saturation, \%; PER-Permeability Index; Clay-Clay Content, \%; SO-0il

Saturation, \%; MO-Movable 0il, \%; PHV-Pore Volume, bbl/ac-ft; HPV-Hydrocarbon Pore Volume, bbl/ac-ft; ELSE-Elevation From Sea Level, ft 
TABLE C33

WELL LOG DETERMINED PROPERTIES

VS DEPTH FOR WELL MP-122

WELL LOCATION: 2366 FEL 782 FSL 21255 5E

KELLY BUSHING ELEVATIOH FROM SEA LEVEL $=1390$

\begin{tabular}{|c|c|c|c|c|c|c|c|c|c|c|c|c|c|c|}
\hline DEPTH* & GR & POR & $R T$ & $R \times \theta$ & $5 \times 0$ & ROS & SW & PER & CLAY & so & MO & PHV & HPV & ELSE \\
\hline $\begin{array}{l}635.50 \\
636.00\end{array}$ & $\begin{array}{l}70.30 \\
65.50\end{array}$ & $\begin{array}{l}21.30 \\
22.60\end{array}$ & $\begin{array}{l}3.60 \\
3.50\end{array}$ & $\begin{array}{l}5 \cdot 10 \\
5 \cdot 10\end{array}$ & $\begin{array}{l}76 \cdot 20 \\
71 \cdot 30\end{array}$ & $\begin{array}{l}23.80 \\
28.70\end{array}$ & $\begin{array}{l}71.50 \\
67.60\end{array}$ & $\begin{array}{l}16.00 \\
77.00\end{array}$ & $\begin{array}{l}27.00 \\
12.00\end{array}$ & $\begin{array}{l}28.50 \\
32.40\end{array}$ & $\begin{array}{l}4.70 \\
3.70\end{array}$ & $\begin{array}{l}1652.54 \\
1753.40\end{array}$ & $\begin{array}{r}470.97 \\
568.10\end{array}$ & $\begin{array}{l}754 . \\
754 .\end{array}$ \\
\hline $\begin{array}{l}636.50 \\
637.00\end{array}$ & $\begin{array}{l}60.80 \\
57.90\end{array}$ & $\begin{array}{l}25.20 \\
26.00\end{array}$ & $\begin{array}{l}3.50 \\
3.30\end{array}$ & $\begin{array}{l}4.90 \\
4.70\end{array}$ & $\begin{array}{l}64 \cdot 60 \\
64.20\end{array}$ & $\begin{array}{l}35.40 \\
35.80\end{array}$ & $\begin{array}{l}60.30 \\
59.70\end{array}$ & $\begin{array}{r}77.00 \\
142.00\end{array}$ & $\begin{array}{r}12.00 \\
7.00\end{array}$ & $\begin{array}{l}39.70 \\
40.30\end{array}$ & $\begin{array}{r}430 \\
4.50\end{array}$ & $\begin{array}{l}1955.12 \\
2017.18\end{array}$ & $\begin{array}{l}776.18 \\
812.92\end{array}$ & $\begin{array}{l}753.5 \\
753.0\end{array}$ \\
\hline $\begin{array}{l}637.50 \\
638.00\end{array}$ & $\begin{array}{l}57.60 \\
58.50\end{array}$ & $\begin{array}{l}26.10 \\
26.10\end{array}$ & $\begin{array}{l}3.20 \\
3.10\end{array}$ & $\begin{array}{l}4.40 \\
4.30\end{array}$ & $\begin{array}{l}65.90 \\
66.50\end{array}$ & $\begin{array}{l}34.10 \\
33.50\end{array}$ & $\begin{array}{l}60.20 \\
61.20\end{array}$ & $\begin{array}{l}142.00 \\
146.00\end{array}$ & $\begin{array}{l}7.00 \\
9.00\end{array}$ & $\begin{array}{l}39.80 \\
38.80\end{array}$ & $\begin{array}{l}5.70 \\
5.30\end{array}$ & $\begin{array}{l}2024.94 \\
2024.94\end{array}$ & $\begin{array}{l}805.93 \\
785.68\end{array}$ & $\begin{array}{l}752.5 \\
752.0\end{array}$ \\
\hline $\begin{array}{l}638.50 \\
639.00\end{array}$ & $\begin{array}{l}60.90 \\
62.70\end{array}$ & $\begin{array}{l}25.90 \\
25.80\end{array}$ & $\begin{array}{l}3.00 \\
2.90 \\
2.80\end{array}$ & $\begin{array}{l}4.30 \\
4.30\end{array}$ & $\begin{array}{l}66.90 \\
67.20\end{array}$ & $\begin{array}{l}33.10 \\
32.80\end{array}$ & $\begin{array}{l}62.70 \\
64.10\end{array}$ & $\begin{array}{l}146.00 \\
138.00\end{array}$ & $\begin{array}{r}9.00 \\
10.00\end{array}$ & $\begin{array}{l}37.30 \\
35.90\end{array}$ & $\begin{array}{l}4.20 \\
3.10\end{array}$ & $\begin{array}{l}2009.42 \\
2001.67 \\
1993.91\end{array}$ & $\begin{array}{l}749.51 \\
718.60 \\
689.89\end{array}$ & $\begin{array}{l}751.5 \\
751.0\end{array}$ \\
\hline $639 \cdot 50$ & 64.40 & 25.70 & 2.80 & $4 \cdot 30$ & $67 \cdot 30$ & 32.70 & 65.40 & $138 \cdot 00$ & $10 \cdot 00$ & 34.60 & 1.90 & 1993.91 & 689.89 & \\
\hline $\begin{array}{l}640 \cdot 00 \\
640.50\end{array}$ & $\begin{array}{l}64.20 \\
63.20\end{array}$ & $\begin{array}{l}26.00 \\
26.60\end{array}$ & $\begin{array}{l}2.80 \\
2.80\end{array}$ & $\begin{array}{l}4.40 \\
4.30\end{array}$ & $\begin{array}{l}66.30 \\
64.70\end{array}$ & $\begin{array}{l}33.70 \\
35.30\end{array}$ & $\begin{array}{l}65.40 \\
63.70\end{array}$ & $\begin{array}{l}143.00 \\
143.00\end{array}$ & 10.00 & $\begin{array}{l}34.60 \\
36.30\end{array}$ & $\begin{array}{r}90 \\
1.00\end{array}$ & $\begin{array}{l}2017.18 \\
2063.73\end{array}$ & 697.94 & \\
\hline 641.00 & 62.20 & 27.20 & 2.80 & 4.30 & 63.60 & 36.40 & 62.30 & 173.00 & 7.00 & 37.70 & $\begin{array}{l}1.00 \\
1.30\end{array}$ & 2110.28 & 58 & 0 \\
\hline $641 \cdot 50$ & $61 \cdot 10$ & 27.50 & .80 & $4 \cdot 30$ & $t 2 \cdot 80$ & 37.20 & 61.20 & 173.00 & 7.00 & 38.80 & 1.60 & 2133.56 & 827.82 & \\
\hline $\begin{array}{l}642 \cdot 00 \\
642 \cdot 50\end{array}$ & $\begin{array}{l}62 \cdot 50 \\
65 \cdot 10\end{array}$ & $\begin{array}{l}27.60 \\
26.00\end{array}$ & $\begin{array}{l}2.80 \\
2.80\end{array}$ & $\begin{array}{l}4 \cdot 30 \\
4.30\end{array}$ & $\begin{array}{l}62.60 \\
67.10\end{array}$ & $\begin{array}{l}37 \cdot 40 \\
32 \cdot 90\end{array}$ & $\begin{array}{l}60.80 \\
64.70\end{array}$ & $\begin{array}{l}187.00 \\
187.00\end{array}$ & $\begin{array}{l}7.00 \\
7.00\end{array}$ & $\begin{array}{l}39 \cdot 20 \\
35 \cdot 30\end{array}$ & $\begin{array}{l}1.80 \\
2.40\end{array}$ & $\begin{array}{l}2141.32 \\
2017.18\end{array}$ & $\begin{array}{l}839.40 \\
712.07\end{array}$ & $\begin{array}{l}748.0 \\
747.5\end{array}$ \\
\hline $643 \cdot 00$ & 67.20 & 24.70 & 2.90 & $4 \cdot 30$ & $70 \cdot 80$ & 29.20 & 68.00 & 114.00 & 11.00 & $32 \cdot 00$ & 2.80 & $1916 \cdot 32$ & 613.22 & 747.0 \\
\hline $\begin{array}{l}643.50 \\
644.00\end{array}$ & $\begin{array}{l}69.40 \\
74.10\end{array}$ & $\begin{array}{l}24.30 \\
22.80\end{array}$ & $\begin{array}{l}2.90 \\
2.90\end{array}$ & $\begin{array}{l}4.30 \\
4.20\end{array}$ & $\begin{array}{l}72.00 \\
77.70\end{array}$ & $\begin{array}{l}28.00 \\
22.30\end{array}$ & $\begin{array}{l}68.80 \\
73.40\end{array}$ & $\begin{array}{r}114.00 \\
80.00\end{array}$ & $\begin{array}{l}11.00 \\
14.00\end{array}$ & $\begin{array}{l}31.20 \\
26.60\end{array}$ & $\begin{array}{l}3.20 \\
4.30\end{array}$ & $\begin{array}{l}1885.29 \\
1768.91\end{array}$ & $\begin{array}{l}588.21 \\
470.53\end{array}$ & $\begin{array}{l}746.5 \\
746.5\end{array}$ \\
\hline $\begin{array}{l}644.50 \\
645.00\end{array}$ & $\begin{array}{l}77.30 \\
75.20\end{array}$ & $\begin{array}{l}21.00 \\
22.60\end{array}$ & $\begin{array}{l}3.00 \\
3.00\end{array}$ & $\begin{array}{l}4.30 \\
40.40\end{array}$ & $\begin{array}{l}79.70 \\
77.10\end{array}$ & $\begin{array}{l}20 \cdot 30 \\
22.90\end{array}$ & $\begin{array}{l}79.70 \\
72.80\end{array}$ & $\begin{array}{l}80.00 \\
77.00\end{array}$ & $\begin{array}{l}14.00 \\
14.00\end{array}$ & $\begin{array}{l}20.30 \\
27.20\end{array}$ & $\begin{array}{r}.00 \\
.030\end{array}$ & $\begin{array}{l}1629.26 \\
1753.40\end{array}$ & $\begin{array}{l}330.74 \\
476.92\end{array}$ & $\begin{array}{l}745.5 \\
745.0\end{array}$ \\
\hline $\begin{array}{l}645.50 \\
646.00\end{array}$ & $\begin{array}{l}73.10 \\
74.30\end{array}$ & $\begin{array}{l}24.30 \\
23.70\end{array}$ & $\begin{array}{l}3 \cdot 10 \\
3.10\end{array}$ & $\begin{array}{l}4.50 \\
4.50\end{array}$ & $\begin{array}{l}70.70 \\
71.90\end{array}$ & $\begin{array}{l}29 \cdot 30 \\
28 \cdot 10\end{array}$ & $\begin{array}{l}66.70 \\
67.90\end{array}$ & $\begin{array}{l}77.00 \\
95.00\end{array}$ & $\begin{array}{l}14.00 \\
13.00\end{array}$ & $\begin{array}{l}33 \cdot 30 \\
32 \cdot 10\end{array}$ & $\begin{array}{l}1.00 \\
4.00\end{array}$ & $\begin{array}{l}1885.29 \\
1838.74\end{array}$ & $\begin{array}{l}627.80 \\
590.24\end{array}$ & 744.0 \\
\hline $646 \cdot 50$ & $75 \cdot 40$ & 23.10 & 3.20 & $4 \cdot 00$ & $73 \cdot 30$ & $26 \cdot 70$ & 68.90 & 95.00 & 13.00 & $31 \cdot 10$ & 1.40 & $1792 \cdot 19$ & 557.37 & 743. \\
\hline $647 \cdot 00$ & 74.80 & & $3 \cdot 2$ & 4.60 & 71. & 28. & 67.20 & 94.00 & $11 \cdot 0$ & & 4.50 & $1830 \cdot 98$ & $600 \cdot 56$ & 743 \\
\hline $647 \cdot 50$ & 74.00 & $24 \cdot 50$ & $3 \cdot 20$ & 4.60 & $69 \cdot 10$ & 30.90 & 64.50 & 94.00 & 11.00 & $35 \cdot 50$ & 4.60 & $1900 \cdot 81$ & & 742. \\
\hline $648 \cdot 00$ & $73 \cdot 30$ & 25.00 & 3.20 & 4.50 & $E 7.80$ & $32 \cdot 20$ & 63.10 & $120 \cdot 00$ & 11.00 & 36.90 & 4.70 & 1939.60 & 715.71 & 742. \\
\hline & $77 \cdot 50$ & 21.70 & & $4 \cdot 60$ & 78.60 & $21 \cdot 40$ & $73 \cdot 10$ & $120 \cdot 00$ & 11.00 & $26 \cdot 90$ & 5.50 & & & \\
\hline $649 \cdot 00$ & 83.80 & 6.80 & 20 & .60 & 97.50 & & 97.50 & 21.00 & 24. & 50 & .00 & 1303.41 & 32.59 & \\
\hline MEAV & 6720 & $246=$ & 30 & ( & +0.37 & 30.46 & 66.11 & 1080 & 11.07 & 33.89 & 3.43 & $1916 \cdot 32$ & 655.51 & \\
\hline
\end{tabular}

*NOTATIOH--Depth, ft; GR-Ganma Ray Index, API Units; POR-Porosity, $\%$; RT-True Resistivity, Ohms-m²/m; RXO-Flushed Zone Resistivity, Ohms-m²/m; SXO-Flushed Zone Saturation, \%; ROS-Residual 0 il Saturation, \%; SW-Water Saturation, \%; PER-Permeability Index; Clay-Clay Content, \%; SO-0il Saturation, \%; MO-Movable 0il, \%; PHV-Pore Volume, bbl/ac-ft; HPV-Hydrocarbon Pore Volume, bbl/ac-ft; ELSE-Elevation From Sea Level, ft 
TABLE C34

WELL LOG DETERMINED PROPERTIES

VS DEPTH FOR WELL. MP-123

WELL LOC.4TION: 2640 FEL 792 FSL $212555 E$

KELLY BUSHING ELEVATION FROM SEA LEVEL $=1397$

\begin{tabular}{|c|c|c|c|c|c|c|c|c|c|c|c|c|c|c|}
\hline DEPTH* & GR & PYR & PT & $R \times B$ & $5 \times 0$ & ROS & SW & PER & CLAY & so & mo & PHV & HPV & ELSE \\
\hline $\begin{array}{l}642.50 \\
643.00\end{array}$ & $\begin{array}{l}84.40 \\
79.60\end{array}$ & $\begin{array}{l}21.10 \\
21.60\end{array}$ & $\begin{array}{l}4 \cdot 50 \\
4.30\end{array}$ & $\begin{array}{l}1.30 \\
1.30\end{array}$ & $\begin{array}{l}t 4.00 \\
t 8.50\end{array}$ & $\begin{array}{l}36.00 \\
31.50\end{array}$ & $\begin{array}{l}64.00 \\
55.40\end{array}$ & $\begin{array}{r}3.00 \\
112.00\end{array}$ & $\begin{array}{l}43.00 \\
22.00\end{array}$ & $\begin{array}{l}36.00 \\
44.60\end{array}$ & $\begin{array}{r}.00 \\
13.10\end{array}$ & $\begin{array}{l}1637.02 \\
1908.57\end{array}$ & $\begin{array}{l}589.33 \\
851.22\end{array}$ & $\begin{array}{l}754.50 \\
754.00\end{array}$ \\
\hline 643.50 & 4.70 & 5.00 & .10 & 1.50 & 64.00 & 36.00 . & 55.80 & 112.00 & 22.00 & 44.20 & 8.20 & 1939.60 & 857.30 & 753.50 \\
\hline 44.00 & 0.80 & 5.50 & . 80 & 1.40 & 65.00 & 35.00 & 56.80 & 130.00 & 12.00 & 43.20 & 8.20 & 1978.39 & 854.66 & 753.00 \\
\hline 34.50 & 0.00 & 5.70 & .60 & 0 & $6 \cdot 30$ & 3.70 & 8.40 & 130.00 & 2.00 & 41.60 & 7.90 & 1993.91 & $829 \cdot+7$ & 752.50 \\
\hline 645.00 & 8.10 & 5.80 & .30 & 1.20 & 9.00 & 1.00 & 0.70 & 138.00 & 1.00 & 39.30 & $8 \cdot 30$ & 2001.67 & 786.65 & $\begin{array}{l}752.00 \\
751.50\end{array}$ \\
\hline 346.00 & 40 & $\begin{array}{l}25.60 \\
25.50\end{array}$ & $\begin{array}{l}3.00 \\
2.70\end{array}$ & $\begin{array}{l}1.40 \\
1.50\end{array}$ & $\begin{array}{l}64.10 \\
67.50\end{array}$ & $\begin{array}{l}5 \cdot 90 \\
2.50\end{array}$ & $\begin{array}{l}64.00 \\
67.50\end{array}$ & $\begin{array}{l}138.00 \\
130.00\end{array}$ & $\begin{array}{l}11.00 \\
10.00\end{array}$ & $\begin{array}{l}36.00 \\
32.50\end{array}$ & $\begin{array}{l}.10 \\
.00\end{array}$ & $\begin{array}{l}1986.15 \\
1978.39\end{array}$ & $\begin{array}{l}15.01 \\
42.98\end{array}$ & $\begin{array}{l}51.50 \\
51.00\end{array}$ \\
\hline 546.50 & 7.00 & 25.80 & .50 & 2.10 & 0.60 & 29.40 & 0.60 & 130.00 & 0.00 & 29.40 & .00 & 2001.67 & 588.49 & 50.50 \\
\hline 647.00 & 68.50 & 29.10 & $2 \cdot 40$ & 2.00 & $72 \cdot 80$ & 27.20 & 72.80 & 123.00 & 10. & 27.20 & .00 & 1947.36 & 529.68 & 750.00 \\
\hline 647.50 & 69.60 & 25.00 & 2.30 & 1.30 & 75.00 & 25.00 & 75.00 & 123.00 & 10.00 & 25.00 & .00 & 1939.60 & 484.90 & 749.50 \\
\hline $\begin{array}{l}648.00 \\
648.50\end{array}$ & $\begin{array}{l}70.80 \\
72.60\end{array}$ & $\begin{array}{l}2 \epsilon .60 \\
27.20\end{array}$ & $\begin{array}{l}2.20 \\
2.20\end{array}$ & $\begin{array}{l}1.20 \\
1.00\end{array}$ & $\begin{array}{l}71.00 \\
70.50\end{array}$ & $\begin{array}{l}29.00 \\
29.50\end{array}$ & $\begin{array}{l}71.00 \\
70.30\end{array}$ & $\begin{array}{l}158.00 \\
158.00\end{array}$ & $\begin{array}{l}13.00 \\
13.00\end{array}$ & $\begin{array}{l}29.00 \\
29.70\end{array}$ & $\begin{array}{l}.00 \\
.20\end{array}$ & $\begin{array}{l}2063.73 \\
2110.28\end{array}$ & $\begin{array}{l}598.48 \\
626.75\end{array}$ & $\begin{array}{l}749.00 \\
748.50\end{array}$ \\
\hline 049.00 & 75.10 & 27.40 & 10 & 1.20 & $70 \cdot 80$ & 29 & 80 & 181.00 & 15.00 & 29.20 & .00 & 21 & 73 & 748.00 \\
\hline 649.50 & 77.80 & 27.50 & .10 & 1.30 & 70.70 & 29.30 & 70.70 & 181.00 & 5.00 & 29.30 & .00 & 3.56 & 25.13 & 747.50 \\
\hline $\begin{array}{l}650.00 \\
850.50\end{array}$ & $\begin{array}{l}80.80 \\
82.70\end{array}$ & $\begin{array}{l}27.50 \\
26.70\end{array}$ & $\begin{array}{l}2 \cdot 10 \\
2.20\end{array}$ & $\begin{array}{l}1.10 \\
1.30\end{array}$ & $\begin{array}{l}70.00 \\
71.60\end{array}$ & $\begin{array}{l}30.00 \\
28.40\end{array}$ & $\begin{array}{l}70.00 \\
71.60\end{array}$ & $\begin{array}{l}183.00 \\
183.00\end{array}$ & $\begin{array}{l}16.00 \\
16.00\end{array}$ & $\begin{array}{l}30.00 \\
28.40\end{array}$ & $\begin{array}{l}.00 \\
.00\end{array}$ & $\begin{array}{l}2133.56 \\
2071.49\end{array}$ & $\begin{array}{l}640.07 \\
588.30\end{array}$ & $\begin{array}{l}747.00 \\
746.50\end{array}$ \\
\hline$E 51.00$ & 83.80 & 25.90 & 2.20 & 1.20 & $73 \cdot 30$ & $26 \cdot 70$ & 73.30 & 140.00 & 24.00 & 26.70 & .00 & 2009.42 & 536.52 & 746.00 \\
\hline 1.50 & 3.60 & 25.60 & 0 & 1.20 & 73.00 & 27.00 & 73.00 & 140.00 & 24.00 & 27.00 & .00 & .15 & 536.26 & 745.50 \\
\hline 652.00 & $81 \cdot 40$ & 25 & $2 \cdot 30$ & 1.70 & 71.70 & $28 \cdot 30$ & 71.70 & 137.00 & 23. & 28 & .00 & 20 & 566.47 & 745.00 \\
\hline 652.50 & 75.60 & 2! & 0 & 1.70 & 71.20 & 28 & 20 & 137.00 & 23 & 28 & .00 & & & 744.50 \\
\hline 653.00 & 74.80 & 26,00 & $2 \cdot 50$ & 1.50 & 68.40 & 31.60 & 68.40 & 144.00 & 18.00 & 31.60 & .00 & 2017.18 & 637.43 & 744.00 \\
\hline 653.50 & 75.20 & 26.60 & 6 & 1. & 66.10 & 33 & .10 & 144.00 & & & .00 & & 699.61 & 743.50 \\
\hline 4.00 & 76.00 & $27 \cdot 30$ & $2 \cdot 60$ & 1.20 & $64 \cdot 80$ & $35 \cdot 20$ & 63.90 & 177.00 & 15.00 & 36.10 & .90 & 2118.04 & 764.61 & 743.00 \\
\hline $654 \cdot 50$ & 77.20 & 27.60 & 2.60 & 1. & 64.50 & 35 & & 177.00 & & & 1.70 & & & 2.50 \\
\hline (j5.00 & 78.70 & 27.70 & 2.60 & 1.30 & $62 \cdot 50$ & 37.50 & 50 & 188.00 & & 37.50 & .00 & 2149.08 & 805.90 & 742.00 \\
\hline & 81.40 & 27.80 & 2.60 & 1.50 & $62 \cdot 30$ & 37.70 & 62.30 & 188.00 & 19. & 37.70 & .00 & 2156.83 & 813.13 & 741.50 \\
\hline 656.00 & 83.30 & 26.30 & 2.60 & 1.50 & 66.00 & 34.00 & 66.00 & 151.00 & & 34.00 & .00 & 2040.46 & & $7 \$ 1.00$ \\
\hline 656.50 & 84.60 & 25.10 & $2 \cdot 60$ & 1.60 & 69.40 & 30.60 & 69.40 & 151.00 & 28.00 & 30.60 & .00 & 1947.36 & 595.89 & 740.50 \\
\hline 657.00 & $85 \cdot 40$ & 24.50 & 2.60 & 1.80 & $71 \cdot 30$ & 28.70 & 71.30 & 110.00 & 36.00 & 28.70 & .00 & 1900.81 & 545.53 & 740.00 \\
\hline 6.57 .5 & & & & 1.60 & 64.90 & 35.10 & 64.90 & 110.00 & & $35 \cdot 10$ & .00 & 20 & 729.82 & 739.50 \\
\hline 658.00 & 80.40 & 27.20 & $2 \cdot 70$ & 1.30 & 63.40 & 36.60 & 63.40 & $175 \cdot 00$ & 29.00 & 36.60 & .00 & .28 & $772 \cdot 36$ & 739.00 \\
\hline 658.50 & 83.70 & 26.60 & 2.70 & 1. & 66.60 & & & & & & 1.90 & & & \\
\hline 659.00 & 84.90 & 25.70 & $2 \cdot 70$ & 1.60 & 66.70 & $33 \cdot 30$ & 66.70 & 136.00 & 35.00 & $33 \cdot 30$ & .00 & 1993.91 & 663.97 & 738.00 \\
\hline 659.50 & $81 \cdot 60$ & 27.00 & & 1.60 & $63 \cdot 10$ & 36.90 & 63.10 & 136.00 & $35 \cdot 00$ & $36.9 n$ & .00 & $4 \cdot 77$ & 772.97 & 737.50 \\
\hline $660 \cdot 00$ & 79.70 & 27.30 & $2 \cdot 80$ & 1.50 & 61.40 & 38.60 & & 278.00 & & & .00 & & & \\
\hline & 90.5 & 22.8 & & 1.50 & 73. & 26. & & 178 & & & .00 & & 46 & 736.50 \\
\hline & & 19.20 & $2 \cdot 90$ & 1.50 & $88 \cdot 20$ & $11 \cdot 80$ & 88.20 & 38.00 & +1.00 & 11.80 & .00 & 1489.61 & 175.77 & 736.00 \\
\hline MEAN & 77.55 & 25.78 & 2,68 & 1.40 & 68.36 & 30.97 & 67.19 & 128.43 & 19.33 & 32.09 & 3 & 1999,73 & 641.79 & \\
\hline
\end{tabular}

*NOTATION--Depth, ft; GR-Gamma Ray Index, API Units; POR-Porosity, $\%$; RT-True Resistivity, Ohms-m²/m; RX0-Flushed Zone Resistivity, Ohms-m²/m;

SX0-Flushed Zone Saturation, \%; ROS-Residual 0il Saturation, \%; SW-Water Saturation, \%; PER-Permeability Index; Ciay-Clay Content, $\%$; SO-0il 
TABLE C35

WELL LOG DETERMINED PROPERTIES

VS DEPTH FOR WELL MP-124

WELL LOCATION: 2366 FWL 72 FSL $212555 E$

KELLY BUSHING ELEVATION FROM SEA LEVEL=1395

\begin{tabular}{|c|c|c|c|c|c|c|c|c|c|c|c|c|c|c|}
\hline DEPTH * & GR & $P O R$ & FT & $R \times U$ & $5 \times 0$ & RES & SW & PER & CLAY & so & ro & PhV & HPV & ELSE \\
\hline $\begin{array}{l}647.00 \\
647.50\end{array}$ & 78.00 & $\begin{array}{l}21.10 \\
22.60\end{array}$ & $\begin{array}{l}2.90 \\
2.80\end{array}$ & $\begin{array}{l}3.90 \\
3.60\end{array}$ & $\begin{array}{l}80.60 \\
76.00\end{array}$ & $\begin{array}{l}19 \cdot 40 \\
24.00\end{array}$ & $\begin{array}{l}80.60 \\
76.00\end{array}$ & $\begin{array}{l}57.00 \\
57.00\end{array}$ & $\begin{array}{l}17.00 \\
17.00\end{array}$ & $\begin{array}{l}19.40 \\
24.00\end{array}$ & $\begin{array}{l}.00 \\
.00\end{array}$ & $\begin{array}{l}1637.02 \\
1753.40\end{array}$ & $\begin{array}{l}317.58 \\
420.82\end{array}$ & $\begin{array}{l}748.00 \\
747.50\end{array}$ \\
\hline $\begin{array}{l}647.50 \\
648.00\end{array}$ & $\begin{array}{l}79.20 \\
76.60\end{array}$ & $\begin{array}{l}22.60 \\
23.70\end{array}$ & $\begin{array}{l}2.80 \\
2.60\end{array}$ & $\begin{array}{l}3.60 \\
3.50\end{array}$ & $\begin{array}{l}76.00 \\
74.90\end{array}$ & $\begin{array}{l}24.00 \\
25.10\end{array}$ & $\begin{array}{l}76.00 \\
74.90\end{array}$ & 95.00 & 23.00 & $\begin{array}{l}24.00 \\
25.10\end{array}$ & .00 & $\begin{array}{l}1753.00 \\
1838.74\end{array}$ & $\begin{array}{l}420.82 \\
461.52\end{array}$ & $\begin{array}{l}747.50 \\
747.00\end{array}$ \\
\hline $648 \cdot 50$ & 71.30 & 25.00 & 2.40 & 3.40 & $73 \cdot 10$ & 26.90 & 73.10 & 95.00 & 23.00 & 26.90 & .00 & 1939.60 & 521.75 & 746.50 \\
\hline 649.00 & 67.30 & 24.90 & $2 \cdot 20$ & 3.50 & $76 \cdot 70$ & $23 \cdot 30$ & 16.70 & 119.00 & 6.00 & 23.30 & .00 & 1931.84 & 450.12 & 46.00 \\
\hline 649.50 & $65 \cdot 30$ & 24.90 & $2 \cdot 20$ & 3.60 & 77.40 & 22.60 & 77.40 & 119.00 & 6.00 & 22.60 & .00 & 1931.84 & 436.60 & 745.50 \\
\hline 650.00 & 63.60 & 24.80 & $2 \cdot 20$ & 3.50 & 77.80 & $22 \cdot 20$ & 77.80 & 116.00 & $5 \cdot 00$ & $22 \cdot 20$ & .00 & 1924.08 & 427.15 & 745.00 \\
\hline 650.50 & $62 \cdot 30$ & 25.60 & $2 \cdot 20$ & 3.40 & $75 \cdot 50$ & 24.50 & 75.50 & 116.00 & 5.00 & $24 \cdot 50$ & .00 & 1986.15 & 486.61 & 744.50 \\
\hline 651.00 & $62 \cdot 30$ & $26 \cdot 30$ & $2 \cdot 20$ & 3.30 & $73 \cdot 20$ & 26.80 & 73.20 & 151.00 & 8.00 & 26.80 & .00 & 2040.46 & 546.84 & 744.00 \\
\hline 651.50 & $62 \cdot 40$ & $27.0 n$ & $2 \cdot 10$ & $3 \cdot 30$ & $71 \cdot 30$ & $28 \cdot 70$ & 71.30 & $151 \cdot 00$ & 8.00 & 28.70 & .00 & 2094.77 & 601.20 & 743.50 \\
\hline 652.00 & $62 \cdot 40$ & 26.90 & $2 \cdot 10$ & $3 \cdot 30$ & 71.80 & 28.20 & 71.80 & 166.00 & 10.00 & 28.20 & .00 & 2087.01 & 588.5 & 743.00 \\
\hline $652 \cdot 50$ & 62.80 & 26.70 & $2 \cdot 10$ & $3 \cdot 40$ & $72 \cdot 40$ & 27.60 & 72.40 & $166 \cdot 00$ & 10.00 & 27.60 & .00 & 2071.49 & 571.73 & 742.50 \\
\hline 653.00 & 64.40 & 26.60 & $2 \cdot 10$ & 3.40 & 73.00 & 27.00 & 73.00 & $157 \cdot 00$ & 9.00 & 27.00 & .00 & 2063.73 & 557.21 & 742.00 \\
\hline 653.50 & $65 \cdot 80$ & 26.50 & $2 \cdot 10$ & 3.40 & 73.40 & 26.60 & 73.40 & 157.00 & 9.00 & 26.60 & .00 & $2055 \cdot 97$ & 546.89 & 741.50 \\
\hline 654.00 & 67.20 & 26.30 & $2 \cdot 20$ & $3 \cdot 40$ & $73 \cdot 30$ & $26 \cdot 70$ & 73.30 & 150.00 & 10.00 & $26 \cdot 70$ & .00 & 2040.46 & 544.80 & 741.00 \\
\hline $654 \cdot 50$ & 67.80 & 26.10 & $2 \cdot 20$ & $3 \cdot 4 \mathrm{C}$ & $73 \cdot 10$ & $26 \cdot 90$ & 73.10 & $150 \cdot 00$ & 10.00 & 26.90 & .00 & $2024 \cdot 94$ & 544.71 & 740.50 \\
\hline 655.00 & 67.80 & 26.00 & $2 \cdot 20$ & $3 \cdot 40$ & 73.00 & 27.00 & 73.00 & 142.00 & 9.00 & 27.00 & .00 & $2017 \cdot 18$ & 594.64 & 740.00 \\
\hline $655 \cdot 50$ & 67.90 & 25.90 & $2 \cdot 30$ & 3.40 & 71.70 & 28. & 71.70 & 142.00 & 9.00 & $28 \cdot 30$ & .00 & 2009.42 & 568.67 & 739.50 \\
\hline 656.00 & 67.90 & 25.10 & $2 \cdot 40$ & 3.60 & 73.00 & 27.00 & 73.00 & 122.00 & 7.00 & 27.00 & .00 & 36 & 525.79 & 739.00 \\
\hline $656 \cdot 50$ & $69 \cdot 10$ & 24.30 & $2 \cdot 50$ & 3.80 & $74 \cdot 40$ & $25 \cdot 60$ & 74.40 & $122 \cdot 00$ & 7.00 & 25.60 & .00 & $1885 \cdot 29$ & 482.63 & .50 \\
\hline 657.00 & $70 \cdot 30$ & 22. & 2.60 & 4.000 & 79.00 & 21.00 & 79.00 & 78.00 & 11.00 & 21.00 & .00 & $1761 \cdot 16$ & 369.84 & 738.00 \\
\hline 657.50 & 71.40 & $22 \cdot 10$ & 2.60 & $4 \cdot 20$ & $80 \cdot 10$ & 19.90 & 80.10 & 78.00 & 11 & & .00 & 1714.61 & 341.21 & 737.50 \\
\hline 658.00 & 71.50 & 21.20 & 1.70 & $4 \cdot 40$ & $82 \cdot 30$ & $17 \cdot 70$ & $82 \cdot 30$ & 59.00 & $10 \cdot 00$ & $17 \cdot 70$ & .00 & 1644.78 & 291.13 & 737.00 \\
\hline $658 \cdot 50$ & 71.00 & $20 \cdot 30$ & 2.80 & 4.10 & $85 \cdot 50$ & 14.50 & 85.50 & 59.00 & 10.00 & $14 \cdot 50$ & .00 & 1574.95 & 228.37 & 736.50 \\
\hline 659.00 & 71.70 & 21.60 & 2.70 & 3.70 & $81 \cdot 20$ & $18 \cdot 80$ & 81.20 & 63.00 & 4.00 & 18.80 & .00 & 1675.81 & 315.05 & 736.00 \\
\hline 659.50 & 72.40 & 23.20 & 2.60 & 3.50 & 76.50 & 23.50 & 76.50 & 63.00 & 4.00 & 23.50 & .00 & 1799.95 & 422.99 & 735.50 \\
\hline 660.00 & 73.10 & 24.40 & $2 \cdot 50$ & $3 \cdot 50$ & 73.60 & $26 \cdot 40$ & 73.60 & 109.00 & 9.00 & $26 \cdot 40$ & .00 & 1893.05 & 499.76 & 5.00 \\
\hline $660 \cdot 50$ & $74 \cdot 20$ & 24.10 & $2 \cdot 40$ & 3.00 & 76.20 & 23.80 & 76.20 & 109.00 & 9.00 & & .00 & 1869.77 & 445.01 & 734.50 \\
\hline $661 \cdot 00$ & $75 \cdot 10$ & 23.90 & $2 \cdot 30$ & 3.70 & 78.00 & 22.00 & 78.00 & 99.00 & 19.00 & 22.00 & .00 & $1854 \cdot 26$ & 407.94 & 734.00 \\
\hline $661 \cdot 50$ & $75 \cdot 70$ & 23.80 & $2 \cdot 30$ & 3.70 & $79 \cdot 20$ & $20 \cdot 80$ & 79.20 & 99.00 & 19.00 & 20.80 & .00 & 1846.50 & 384.07 & 733.50 \\
\hline 662.00 & $76 \cdot 30$ & 24.40 & 2.30 & 3.60 & $77 \cdot 70$ & $22 \cdot 30$ & 77.70 & 108.00 & 16.00 & & .00 & 1893.05 & 422.15 & 733.00 \\
\hline 662.50 & 76.60 & 24.60 & $2 \cdot 20$ & 3.00 & 77.50 & $22 \cdot 50$ & 77.50 & 108.00 & 16.00 & 22.50 & .00 & & & 732.50 \\
\hline 663.00 & $76 \cdot 80$ & 25.1 & $2 \cdot 20$ & 3.00 & $76 \cdot 70$ & $23 \cdot 30$ & 76.70 & 122.00 & 10.00 & 23.30 & .00 & 1947.36 & 453.73 & 732.00 \\
\hline 663.50 & 77.00 & 25.20 & $2 \cdot 2$ & 3.60 & $75 \cdot 90$ & $24 \cdot 10$ & 75.90 & 122.00. & 10.00 & 24.10 & .00 & 1955.12 & 471.18 & 731.50 \\
\hline 664.00 & $77 \cdot 70$ & $25 \cdot 30$ & 2.20 & 3.00 & $75 \cdot 10$ & $24 \cdot 90$ & $75 \cdot 10$ & 127.00 & 13.00 & 24.90 & .00 & 1962.87 & 488.76 & 731.00 \\
\hline 664.50 & $78 \cdot 30$ & 25.20 & $2 \cdot 20$ & 3.60 & $75 \cdot 20$ & $24 \cdot 80$ & 75.20 & 127.00 & 13.00 & 24.80 & .00 & 195 & 484 & .50 \\
\hline 665.00 & 77.90 & 24.80 & 2. & 3.50 & $76 \cdot 10$ & 23.90 & 76.10 & 116.00 & 19.00 & 23.90 & .00 & 1924 & 459 & 73 \\
\hline & 77.50 & 22.90 & $2 \cdot 30$ & 3.50 & 81. & $18 \cdot 50$ & $81 \cdot 50$ & 116.00 & & & .00 & 1776.67 & 328.68 & 729.50 \\
\hline 666.00 & 78.90 & 22.70 & $2 \cdot 40$ & 3.50 & 80.80 & 19.20 & 80.80 & 79 & & & .00 & & $338 \cdot 14$ & .00 \\
\hline 666.50 & 80.60 & 23.50 & 2.50 & 3.70 & $76 \cdot 70$ & $23 \cdot 30$ & 76.70 & 79.00 & 25.00 & $23 \cdot 3 n$ & .00 & & & .50 \\
\hline 667.00 & .70 & 22.00 & 2.60 & 3.30 & $81 \cdot 10$ & 18.90 & $81 \cdot 10$ & 68.00 & 35.00 & $18 \cdot 90$ & .00 & 1706.85 & 322.59 & 728.00 \\
\hline & & & $2 \cdot:$ & $\begin{array}{l}3.60 \\
3.59\end{array}$ & $\begin{array}{l}76 \cdot 38 \\
76 \cdot 31\end{array}$ & $\begin{array}{l}23.62 \\
23.37\end{array}$ & $\begin{array}{l}76 \cdot 38 \\
76 \cdot 31\end{array}$ & $\begin{array}{l}110.68 \\
105.55\end{array}$ & $\begin{array}{l}12.56 \\
11.03\end{array}$ & $\begin{array}{l}23.62 \\
23.37\end{array}$ & .00 & $\begin{array}{l}1890 \cdot 96 \\
1886.13\end{array}$ & $\begin{array}{l}450.62 \\
440.78\end{array}$ & \\
\hline
\end{tabular}

*NOTATION--Depth, ft; GR-Gamma Ray Index, API Units; POR-Porosity, $\%$; RT-True Resistivity, Ohms-m² $/ m$; RXo-flushed Zone Resistivity, Ohms-m²/m; SXO-Flushed Zone Saturation, $\%$; ROS-Residual $0 i 1$ Saturation, $\%$; SW-Water Saturation, \%; PER-Permeability Index; Clay-Clay Content, z; SO-0il

Saturation, \%; MO-Movable 0il, \%; PHV-Pore Volume, bbl/ac-ft; HPV-Hydrocarbon Pore Volume, bbl/ac-ft; ELSE-Elevation From Sea Level, ft 
TABLE C36

WELL LOG DETERMINED PROPERTIES

VS DEPTH FOR WELL MP-125

WELL LOCATION: 2112 FWL 792 FSL $212555 E$

KELLY BUSAING ELEVATION FROM SEA LEVEL $=1387$

\begin{tabular}{|c|c|c|c|c|c|c|c|c|c|c|c|c|c|c|}
\hline DEPTH* & $G R$ & POP & קT & $R \times \theta$ & $5 \times 0$ & ROS & SW & PER & CLAY & so & mo & PHV & HPV & ELSE \\
\hline $\begin{array}{l}647.50 \\
648.00\end{array}$ & $\begin{array}{l}63.40 \\
61.30\end{array}$ & $\begin{array}{l}15.20 \\
19.70\end{array}$ & $\begin{array}{r}+50 \\
+50\end{array}$ & $\begin{array}{l}2.20 \\
2.30\end{array}$ & $\begin{array}{l}21.10 \\
78.80\end{array}$ & $\begin{array}{r}8 \cdot 90 \\
21.20\end{array}$ & $\begin{array}{l}91.10 \\
70.70\end{array}$ & $\begin{array}{r}1.00 \\
42.00\end{array}$ & $\begin{array}{l}38.00 \\
32.00\end{array}$ & $\begin{array}{r}8.90 \\
29.30\end{array}$ & $\begin{array}{l}\because .00 \\
8 \cdot 10\end{array}$ & $\begin{array}{l}1179.28 \\
1528.40\end{array}$ & $\begin{array}{l}104.96 \\
47.82\end{array}$ & $\begin{array}{l}739.50 \\
739.00\end{array}$ \\
\hline 648.50 & 59.30 & $20 \cdot 30$ & .10 & 2.30 & 75.90 & 24.10 & 70.30 & 42.00 & 32.00 & 29.70 & 5.60 & 1574.95 & 467.76 & 738.50 \\
\hline 649.00 & 54.00 & 20.40 & 3.90 & .20 & 77.90 & 22.10 & 1.50 & 49.00 & 21.00 & 28.50 & 6.40 & 1582.71 & 451.07 & 738.00 \\
\hline $\begin{array}{l}649.50 \\
650.00\end{array}$ & $\begin{array}{l}48.50 \\
47.00\end{array}$ & $\begin{array}{l}20.50 \\
21.50\end{array}$ & $\begin{array}{l}3.70 \\
3.60\end{array}$ & $\begin{array}{l}2.10 \\
2.00\end{array}$ & $\begin{array}{l}72.90 \\
77.10\end{array}$ & $\begin{array}{l}27.10 \\
22.90\end{array}$ & $\begin{array}{l}72.90 \\
70.90\end{array}$ & $\begin{array}{l}49.00 \\
61.00\end{array}$ & $\begin{array}{l}21.00 \\
15.00\end{array}$ & $\begin{array}{l}27.10 \\
29.10\end{array}$ & $\begin{array}{l}.00 \\
6.20\end{array}$ & $\begin{array}{l}1590.47 \\
1668.06\end{array}$ & $\begin{array}{l}431.02 \\
485.40\end{array}$ & $\begin{array}{l}737.50 \\
737.00\end{array}$ \\
\hline 650.50 & $45 \cdot 30$ & 22.40 & $\equiv .40$ & 2.00 & 74.50 & $25 \cdot 50$ & 69.00 & 61.00 & 15.00 & 31.00 & 5.50 & 1737.88 & 538.74 & 736.50 \\
\hline 651.00 & 44.90 & 23.50 & 3.20 & 1.90 & 72.50 & 27.50 & 68.20 & 92.00 & 15.00 & 31.80 & 4.30 & 1823.22 & 579.78 & 736.00 \\
\hline 651.50 & 44.60 & 25.40 & $\bar{c} .90$ & 1.80 & 67.70 & $32 \cdot 30$ & 66.20 & 92.00 & 15.00 & 33.80 & 1.50 & 1970.63 & 666.07 & 735.50 \\
\hline 652.00 & 46.40 & 26.50 & 2.70 & 1.60 & $t 9.10$ & 30.90 & 64.70 & 156.00 & 17.00 & $35 \cdot 30$ & 4.40 & 2055.97 & 725.76 & 735.0 \\
\hline 652.50 & 48.30 & 26.90 & 2.60 & 1.40 & $72 \cdot 70$ & 27.30 & 65.50 & 156.00 & 17.00 & 34.50 & 7.20 & 2087.01 & 720.02 & 734.5 \\
\hline $\begin{array}{l}653.00 \\
653.50\end{array}$ & $\begin{array}{l}49.70 \\
50.70\end{array}$ & $\begin{array}{l}26.90 \\
26.90\end{array}$ & $\begin{array}{l}2 \cdot 50 \\
2.40\end{array}$ & $\begin{array}{l}1.50 \\
1.50\end{array}$ & $\begin{array}{l}69.00 \\
\epsilon 9.30\end{array}$ & $\begin{array}{l}31.00 \\
30.70\end{array}$ & $\begin{array}{l}66.80 \\
67.90\end{array}$ & $\begin{array}{l}167.00 \\
167.00\end{array}$ & $\begin{array}{l}19.00 \\
19.00\end{array}$ & $\begin{array}{l}33 . ? 0 \\
32.10\end{array}$ & $\begin{array}{l}2.20 \\
1.40\end{array}$ & $\begin{array}{l}2087.01 \\
2087.01\end{array}$ & $\begin{array}{l}692.89 \\
669.93\end{array}$ & $\begin{array}{l}734.00 \\
733.50\end{array}$ \\
\hline 654.00 & $52 \cdot 30$ & 26.80 & 2.30 & 1.50 & 69.80 & 30.20 & 69.20 & 163.00 & 21.00 & 30.80 & .60 & 2079.25 & 640.41 & 733.000 \\
\hline 654.50 & $52 \cdot 90$ & $26.7 \mathrm{C}$ & 2.30 & 1.60 & 70.50 & 29.50 & 70.50 & 163.00 & 21.00 & 29.50 & .00 & 2071.49 & 611.09 & 732.5 \\
\hline 655.00 & 53.60 & 26.60 & 2.20 & 1.00 & 71.80 & 28.20 & 71.80 & 158.00 & 20.00 & 28.20 & .00 & 2063.73 & 581.97 & 732.0 \\
\hline 655.50 & 54.00 & 26.50 & $2 \cdot 20$ & 1.10 & $72 \cdot 50$ & 27.50 & 72.50 & 158.00 & 20.00 & 27.50 & .00 & 2055.97 & $565 \cdot 39$ & 731.50 \\
\hline 656.00 & 54.00 & 26.40 & 2.20 & 1.60 & 72.90 & 27.10 & 72.90 & 153.00 & 19.00 & 27.10 & .00 & 2048.22 & 555.07 & 731.00 \\
\hline 656.50 & 54.00 & 26.40 & $2 \cdot 10$ & 1.50 & $73 \cdot 30$ & 26.70 & 73.30 & 153.00 & 19.00 & 26.70 & .00 & 2048.22 & 546.87 & 730.5 \\
\hline 657.00 & 53.00 & $26 \cdot 30$ & 2.10 & 1.50 & $73 \cdot 70$ & $26 \cdot 30$ & 73.70 & 151.00 & 18.00 & 26.30 & .00 & 2040.46 & 536.64 & 730.0 \\
\hline 657.50 & 52.00 & 26.00 & 2.10 & 1.40 & $75 \cdot 70$ & $24 \cdot 30$ & $75 \cdot 30$ & 151.00 & 18.00 & 24.70 & .40 & 2017.18 & 498.24 & 729.5 \\
\hline & $51 \cdot 80$ & 25.70 & 2 . & 1.30 & 79.20 & $20 \cdot 80$ & 75.00 & 137.00 & 19.00 & 25.00 & 4.20 & 1993.91 & 498.48 & .0 \\
\hline & 53.30 & 25.50 & 2.20 & 1.40 & 76.00 & 24.00 & 74.80 & 137.00 & 19.00 & 25.20 & 1.20 & 1978.39 & 498.55 & 728.5 \\
\hline 659.00 & 55.00 & 25.10 & $2 \cdot 30$ & 1.60 & $75 \cdot 10$ & 24.90 & 75.10 & 121.00 & 25.00 & 24.90 & .00 & 1947.36 & 484.89 & 728.0 \\
\hline & 56.70 & 24.70 & $2 \cdot 30$ & 1.60 & $75 \cdot 30$ & 24.70 & $75 \cdot 30$ & 121.00 & 25.00 & 24.70 & .00 & 1916.32 & 473.33 & 727.5 \\
\hline $660 \cdot 00$ & $58.6 \mathrm{C}$ & 24.50 & $2 \cdot 40$ & 1.70 & $74 \cdot 20$ & 25.80 & 74.20 & 110.00 & 29.00 & 25.80 & .00 & 1900.81 & 490.41 & .727 .0 \\
\hline $660 \cdot 50$ & 60.20 & 24.60 & 2.50 & 1.70 & 73.00 & 27.00 & 73.00 & 110.00 & 29.00 & 27.00 & .00 & 1908.57 & 515.31 & 726.5 \\
\hline 661.00 & 6.2 .80 & 24.10 & 2.60 & 1.70 & 73.60 & $26 \cdot 40$ & 73.60 & 103.00 & 34.00 & 26.40 & .00 & 1869.77 & 493.62 & 726.0 \\
\hline 661.50 & $E 4.00$ & 23.60 & 2.50 & 1.80 & $74 \cdot 40$ & 25.60 & 74.40 & 103.00 & 34.00 & 25.60 & .00 & 1830.98 & 468.73 & 725.5 \\
\hline & 65.10 & 23.00 & 2.70 & 1.80 & 75.80 & 24.20 & 75.80 & 83.00 & 36.00 & 24.20 & .00 & 1784.43 & 431.83 & 725.0 \\
\hline 662.50 & 67.20 & 21.70 & 2.70 & 1.80 & $80 \cdot 10$ & 19.90 & 80.10 & 83.00 & 36.00 & 19.90 & .00 & 1683.57 & 335.03 & 726.5 \\
\hline 663.00 & $58 \cdot 60$ & 20.80 & $2 \cdot 30$ & 1.70 & 83.30 & $16 \cdot 70$ & 83.30 & 53.00 & 42.00 & 16.70 & .00 & 1613.75 & 269.50 & 724.0 \\
\hline 663. & 70.20 & 19.60 & $2 \cdot 30$ & 1.70 & 28.00 & 12.00 & 88.00 & 53.00 & .2 .00 & 12.00 & .00 & 1520.65 & 182.48 & 723.5 \\
\hline 664.00 & 71.00 & 19.00 & 2.80 & 1.80 & $\varsigma_{1.10}$ & 8.90 & 91.10 & 36.00 & 47.00 & 8.90 & .00 & 1474.10 & 131.19 & 723.0 \\
\hline 664 & 72.30 & 17.90 & 2.80 & 1.80 & 97.80 & 2.20 & 97.80 & 36.00 & 47.00 & 2.20 & .00 & 138.75 & 30.55 & 722.5 \\
\hline & 70 & 3.81 & $\because 7$ & 1.73 & $73 \cdot 46$ & 24.48 & 73.78 & 107.00 & 24.97 & 26.22 & 1.74 & 1847.64 & $\$ 93.83$ & \\
\hline & .97 & 3.61 & 2.70 & 1.11 & $72 \cdot 15$ & 23.56 & 73.53 & $90 \cdot 33$ & 23.54 & 25.10 & & 1832.12 & 459.95 & \\
\hline
\end{tabular}

*NOTATION--Depth, ft; GR-Gamma Ras Index, API Units; POR-Porosity, $\%$; RT-True Resistivity, Ohms-m²/m; RXo-Flushed Zone Resistivity, Ohms-m²/m; SXO-Flushed Zone Saturation, \%; RCS-Residual 0il Saturation, \%; SW-Water Saturation, \%; PER-Permeability Index; Clay-Clay Content, \%; SO-0il

Saturation, \%; MO-Movable 0il, \%; PHV-Pore Volume, bbl/ac-ft; HPV-Hydrocarbon Pore Volume, bbi/ac-ft; ELSE-Elevation From Sea Level, ft 
VS DEPTH FOR WELL MP-126

WELL LOCA'ION: 2112 FWL 528 FSL 21255 5E

KELLY BUS:IING ELEVATION FROI SEA LEVEL $=1393$

\begin{tabular}{|c|c|c|c|c|c|c|c|c|c|c|c|c|c|c|}
\hline DEPTH* & GR & $P Q R$ & $R T$ & $R \times E$ & $\leqslant x \theta$ & ReS & sw & PER & CLAY & se & ro & PHV & HPV & ELSE \\
\hline $\begin{array}{l}655.50 \\
656.00\end{array}$ & $\begin{array}{l}78 \cdot 50 \\
74.10\end{array}$ & $\begin{array}{l}16.80 \\
21.60\end{array}$ & $\begin{array}{l}6.10 \\
6.10\end{array}$ & $\begin{array}{l}4.80 \\
4.90\end{array}$ & $\begin{array}{l}70.20 \\
71.80\end{array}$ & $\begin{array}{l}29.80 \\
28.20\end{array}$ & $\begin{array}{l}70.20 \\
53.60\end{array}$ & $\begin{array}{r}2.00 \\
50.00\end{array}$ & $\begin{array}{l}30.00 \\
24.00\end{array}$ & $\begin{array}{l}29.80 \\
46.40\end{array}$ & $\begin{array}{r}.00 \\
18.20\end{array}$ & $\begin{array}{l}1303.41 \\
1675.81\end{array}$ & $\begin{array}{r}388.42 \\
777.58\end{array}$ & $\begin{array}{l}737.50 \\
737.00\end{array}$ \\
\hline $\begin{array}{l}656.50 \\
657.00\end{array}$ & $\begin{array}{l}69.70 \\
66.80\end{array}$ & $\begin{array}{l}25.00 \\
25.30\end{array}$ & $\begin{array}{l}6.20 \\
5.80\end{array}$ & $\begin{array}{l}4.90 \\
4.90\end{array}$ & $\begin{array}{l}61.00 \\
60.60\end{array}$ & $\begin{array}{l}39.00 \\
39.40\end{array}$ & $\begin{array}{l}45.70 \\
46.40\end{array}$ & $\begin{array}{r}50.00 \\
101.00\end{array}$ & $\begin{array}{l}24.00 \\
13.00\end{array}$ & $\begin{array}{l}54.30 \\
53.60\end{array}$ & $\begin{array}{l}15.30 \\
14.20\end{array}$ & $\begin{array}{l}1939.60 \\
1962.87\end{array}$ & $\begin{array}{l}1053.20 \\
1052.10\end{array}$ & $\begin{array}{l}736.50 \\
736.00\end{array}$ \\
\hline 657.50 & 65.70 & 25.10 & 5.40 & 4.70 & 62.00 & 38.00 & 48.40 & 101.00 & 13.00 & 51.60 & 13.60 & 1.947 .36 & $1004 \cdot 84$ & 735.50 \\
\hline 658.00 & 64.50 & 25.10 & $5 \cdot 10$ & $4.0 \mathrm{C}$ & $\leqslant 3.00$ & 37.00 & 50.20 & 97.00 & 9.00 & 49.80 & $12 \cdot 80$ & $1947 \cdot 36$ & 969.78 & 735.00 \\
\hline 658.50 & $6.3 \cdot 30$ & 25.20 & 4.70 & $4 \cdot 50$ & $\in 3.80$ & $36 \cdot 20$ & 52.10 & 97.00 & 9.00 & 47.90 & 11.70 & 1955.12 & 936.50 & 734.50 \\
\hline 659.00 & $62 \cdot 10$ & 25.70 & $4 \cdot 10$ & $4.2 \mathrm{C}$ & 64.60 & 35.40 & 54.70 & 108.00 & 8.00 & $45 \cdot 30$ & 9.90 & 1993.91 & 903.24 & 734.00 \\
\hline 659.50 & 61.90 & 26.20 & 3.80 & 4.00 & $E 4.60$ & 35.40 & $55 \cdot 30$ & 108.00 & 8.00 & 44.70 & $9 \cdot 30$ & 2032.70 & 908.62 & 733.50 \\
\hline 660.00 & 62.60 & 26.80 & 3.50 & $3 \cdot \Delta 0$ & $\epsilon 4.70$ & $35 \cdot 30$ & 56.00 & 128.00 & 7.00 & 44.00 & 8.70 & $2079 \cdot 25$ & 914.87 & 733.00 \\
\hline 660.50 & 63.10 & 26.90 & $3 \cdot 30$ & $3 \cdot 00$ & 66.00 & 34.00 & 57.90 & 128.00 & 7.00 & $42 \cdot 10$ & $8 \cdot 10$ & 2087.01 & 878.63 & $732 \cdot 50$ \\
\hline 661.00 & 63.70 & $26.8 \mathrm{C}$ & 3.00 & $3 \cdot 4 \mathrm{C}$ & $\in 7 \cdot 80$ & 32.20 & 60.40 & 130.00 & 7.00 & $39.6 \mathrm{C}$ & $7 \cdot 40$ & 2079.25 & 823.38 & 732.00 \\
\hline $661 \cdot 50$ & 64.20 & 26.80 & 2.80 & $3 \cdot 30$ & $\in 9.00$ & $31 \cdot 00$ & 63.20 & 130.00 & 7.00 & 36.890 & $5 \cdot 80$ & 2079.25 & $765 \cdot 16$ & 731.50 \\
\hline 662.00 & 54.70 & 26.90 & 2.50 & $3 \cdot 40$ & $\in 8 \cdot 40$ & 31.60 & 66.30 & $130 \cdot 00$ & 7.00 & 33.70 & $2 \cdot 10$ & 2087.01 & 703.32 & 731.00 \\
\hline $662 \cdot 50$ & $55 \cdot 30$ & 26.90 & 2.50 & $3 \cdot 40$ & 67.60 & $32 \cdot 40$ & 66.50 & 130.00 & 7.00 & 33.50 & 1.10 & 2087.01 & 699.15 & 730.50 \\
\hline 663.00 & 66.00 & 26.70 & 2.40 & $3 \cdot 40$ & 68.60 & $31 \cdot 40$ & 67.70 & 126.00 & 11.00 & $32 \cdot 30$ & .90 & 2071.49 & 669.09 & 730.00 \\
\hline 663.50 & $66 \cdot 40$ & 26.40 & 2.40 & 3.30 & 70.00 & 30.00 & 69.10 & 126.00 & 11.00 & 30.90 & .90 & 2048.22 & 632.90 & 729.50 \\
\hline 664.00 & 06.80 & 26.10 & 2.40 & $3 \cdot 30$ & $71 \cdot 30$ & 28.70 & 70.50 & 125.00 & $10 \cdot 00$ & 29.50 & .80 & 2024.94 & $597 \cdot 36$ & 729.00 \\
\hline 664.50 & $67 \cdot 10$ & 25.80 & 2.40 & $3 \cdot 30$ & 72.00 & 28.00 & 71.10 & 115.00 & 10.00 & 28.90 & .90 & 2001.67 & 578.48 & 728.50 \\
\hline $665 \cdot 00$ & 68.00 & 25.60 & 8.40 & $3 \cdot 40$ & 12.00 & 28.00 & 70.90 & 106.00 & 12.00 & 29.10 & 1.10 & 1986.15 & 577.97 & 728.00 \\
\hline $665 \cdot 50$ & $70 \cdot 10$ & $25 \cdot 50$ & 2.50 & $3 \cdot 40$ & 71.90 & 28.20 & 70.50 & 106.00 & $12 \cdot 00$ & 29.50 & $1 \cdot 30$ & 1978.39 & 583.63 & 727.50 \\
\hline 666.00 & 71.50 & 25.40 & 2.50 & 3.50 & 71.60 & 28.40 & 70.20 & 102.00 & 16.00 & 29.80 & 1.40 & 1970.63 & 587.25 & 727.00 \\
\hline 666.50 & 73.00 & 25.10 & 2.60 & $3 \cdot 50$ & $72 \cdot 10$ & 27.90 & 70.60 & 102.00 & 16.00 & 29.40 & 1.50 & 1947.36 & 572.52 & 726.50 \\
\hline 667.00 & 74.50 & 24.80 & 2.60 & 3.00 & $72 \cdot 30$ & 27.70 & 73.70 & 92.00 & 21.00 & $29 \cdot 30$ & 1.60 & 1924.08 & 563.76 & 726.00 \\
\hline 667.50 & $75 \cdot 90$ & 24.10 & 8.70 & 3.50 & $74 \cdot 30$ & 25.70 & 72.40 & 92.00 & 21.00 & $27.6 n$ & 1.90 & 1869.77 & 516.06 & 725.50 \\
\hline 668.00 & 77.50 & 23.30 & $2 \cdot 70$ & 3.00 & $76 \cdot 80$ & 23.20 & 74.50 & 69.00 & 27.00 & 25.50 & $2 \cdot 30$ & 1807.71 & $460 \cdot 97$ & 725.00 \\
\hline 668.50 & 78.00 & 23.10 & 2.80 & 3.10 & 77.20 & 22.80 & 74.30 & 69.00 & 27.00 & 25.70 & 2.90 & $1792 \cdot 19$ & 460.59 & 724.50 \\
\hline 669.00 & 78.50 & 22.90 & $2 \cdot 80$ & $3 \cdot 7 c$ & 77.70 & $22 \cdot 30$ & 74.40 & 64.00 & 28.00 & 25.60 & & 1776.67 & 454.83 & 724.00 \\
\hline 669.50 & 79.10 & 22.70 & $2 \cdot 90$ & 3.80 & 77.70 & 22.30 & $74 \cdot 30$ & 64.00 & 28.00 & 25.70 & 3.40 & 1761.16 & 452.62 & 723.50 \\
\hline 670.00 & 79.60 & $22 \cdot 30$ & 2.90 & $3 \cdot 80$ & $78 \cdot 30$ & $21 \cdot 70$ & 75.10 & 57.00 & 33.00 & 24.90 & 3.20 & $1730 \cdot 12$ & $430 \cdot 80$ & 723.00 \\
\hline 670.50 & 81.90 & $20.6 c$ & 3.00 & 3.90 & 81.00 & 19.00 & 81.00 & 57.00 & 33.00 & 19.00 & .00 & 1598.23 & 303.66 & $722 \cdot 50$ \\
\hline 671.00 & 83.40 & 19.50 & 3.00 & $3 \cdot 90$ & 85.40 & $14 \cdot 60$ & 85.40 & 32.00 & 39.00 & 14.60 & .00 & 1512.89 & 220.88 & 722.00 \\
\hline 671.50 & 84.90 & 18.40 & 3.00 & 3.10 & 50.50 & 9.50 & 90.50 & 32.00 & 39.00 & 9.50 & .00 & .54 & 135.62 & 721.50 \\
\hline 672.00 & $86 \cdot 20$ & 17.50 & 3.10 & 3.90 & 54.70 & $5 \cdot 30$ & 94.70 & 20.00 & $45: 00$ & $5 \cdot 30$ & .00 & 1357.72 & 71.96 & 721.00 \\
\hline & 77 & 0 & Z & 3 & 71.78 & $28 \cdot 22$ & $\begin{array}{l}66.91 \\
65.84\end{array}$ & 89.29 & $\begin{array}{l}18.21 \\
15.19\end{array}$ & 30.31 & 4.87 & $\begin{array}{r}1877.76 \\
1863.92\end{array}$ & $\begin{array}{l}636.75 \\
565.00\end{array}$ & \\
\hline
\end{tabular}

*NOTATION--Depth, ft; GR-Gamma Ray .Index, API Units; POR-Porosity, $\%$; RT-True Resistivity, Ghms- $\mathrm{m}^{2} / \mathrm{m}$; RX0-Flushed Zone Resistivity, Ohms-m²/m; SX0-Flushed Zone Saturation, \%; ROS-Residual 0il Saturation, \%; SH-Water Saturation, \%; PER-Permeability Index; Clay-Clay Content, \%; SO-0il

Saturation, \%; MO-Movable $0 i 1$, \%; PHV-Pore Volume, bbl/ac-ft; HPV-Hydrocarbon Pore Volume, bbl/ac-ft; ELSE-Elevation From Sea Level, ft 
TABLE C38

WELL LOG DETERHINED PROPERTIES

VS DEPTH FOR WELL MP-127

WELL LOCATION: 2386 FWL 528 FSL $2125 S 5 E$

KELLY BUSHING ELEVATION FROM SEA LEVEL $=1396$

\begin{tabular}{|c|c|c|c|c|c|c|c|c|c|c|c|c|c|c|}
\hline$P T H^{*}$ & GR & POF & RT & $R \times U$ & $5 \times \theta$ & ReS & SW & PER & CLAY & se & MO & PHV & HPV & ELSE \\
\hline $\begin{array}{l}654 \cdot 00 \\
654 \cdot 50 \\
655.00\end{array}$ & $\begin{array}{l}71 \cdot 90 \\
68.70 \\
65.30\end{array}$ & $\begin{array}{l}16.40 \\
19.50 \\
21.50\end{array}$ & $\begin{array}{l}3.70 \\
3.60 \\
3.50\end{array}$ & $\begin{array}{l}4.80 \\
4.00 \\
4.50\end{array}$ & $\begin{array}{l}\$ 2.90 \\
77.70 \\
79.40\end{array}$ & $\begin{array}{r}7 \cdot 10 \\
22 \cdot 30 \\
20.60\end{array}$ & $\begin{array}{l}92.90 \\
77.70 \\
70.80\end{array}$ & $\begin{array}{l}19.00 \\
19.00 \\
64.00\end{array}$ & $\begin{array}{l}28.00 \\
28.00 \\
12.00\end{array}$ & $\begin{array}{r}7.10 \\
22.30 \\
29.70\end{array}$ & $\begin{array}{r}.00 \\
.00 \\
8.60\end{array}$ & $\begin{array}{l}1272.38 \\
1520.65 \\
1675.81\end{array}$ & $\begin{array}{r}90.34 \\
339.10 \\
499.34\end{array}$ & $\begin{array}{l}742.60 \\
742.10 \\
741.60\end{array}$ \\
\hline $\begin{array}{l}655.00 \\
655.50\end{array}$ & 62.50 & $\begin{array}{l}21.60 \\
23.20\end{array}$ & 3.40 & 4.40 & $\begin{array}{l}79.40 \\
74.50\end{array}$ & $\begin{array}{l}20.60 \\
25.50\end{array}$ & $\begin{array}{l}70.80 \\
66.50\end{array}$ & $\begin{array}{l}64 \cdot 00 \\
64.00\end{array}$ & $\begin{array}{l}12.00 \\
12.00\end{array}$ & $\begin{array}{l}29.20 \\
33.50\end{array}$ & $\begin{array}{l}8.60 \\
8.00\end{array}$ & $\begin{array}{l}1675.81 \\
1799.95\end{array}$ & $\begin{array}{l}4.9 .34 \\
602.98\end{array}$ & $\begin{array}{l}741.60 \\
741.10\end{array}$ \\
\hline 656.00 & $58 \cdot 20$ & 23.30 & $3 \cdot 20$ & $4 \cdot 3 \mathrm{C}$ & $75 \cdot 10$ & $24 \cdot 90$ & 68.80 & 89.00 & 8.00 & 31.20 & $6 \cdot 30$ & 1807.71 & 564.00 & 740.60 \\
\hline $\begin{array}{l}656.50 \\
657.00\end{array}$ & $\begin{array}{l}56.50 \\
54.80\end{array}$ & $\begin{array}{l}23.40 \\
23.7 \mathrm{C}\end{array}$ & $\begin{array}{l}3.00 \\
2.80\end{array}$ & $\begin{array}{l}4.10 \\
4.000\end{array}$ & $\begin{array}{l}76 \cdot 20 \\
76.30\end{array}$ & $\begin{array}{l}23.80 \\
23.70\end{array}$ & $\begin{array}{l}70.40 \\
71.80\end{array}$ & $\begin{array}{l}89 \cdot 00 \\
95.00\end{array}$ & $\begin{array}{l}8.00 \\
6.00\end{array}$ & 29.60 & 5.80 & 1815.46 & $537 \cdot 38$ & $740 \cdot 10$ \\
\hline 657.50 & 53.10 & 23.70 & 2.60 & 3.90 & $\begin{array}{l}76 \cdot 30 \\
77 \cdot 10\end{array}$ & $\begin{array}{l}23.70 \\
22.90\end{array}$ & $\begin{array}{l}71.80 \\
74.00\end{array}$ & $\begin{array}{l}95.00 \\
95.00\end{array}$ & $\begin{array}{l}6.00 \\
6.00\end{array}$ & $\begin{array}{l}28.20 \\
26.00\end{array}$ & $\begin{array}{l}4.50 \\
3.10\end{array}$ & $\begin{array}{l}1838.74 \\
1838.74\end{array}$ & $\begin{array}{l}518.52 \\
478.07\end{array}$ & $\begin{array}{l}739.60 \\
739.10\end{array}$ \\
\hline 658.00 & $51 \cdot \varepsilon 0$ & $23.5 \mathrm{C}$ & $2 \cdot 40$ & $3 \cdot 5 \mathrm{c}$ & $78 \cdot 30$ & 21.70 & 78.30 & 92.00 & 5.00 & 21.70 & .00 & 1823.22 & 395.64 & 738.60 \\
\hline $658 \cdot 50$ & $52 \cdot=0$ & 24.70 & $2 \cdot 30$ & 3.10 & $75 \cdot 60$ & 24.40 & 75.60 & 92.00 & 5.00 & 24.40 & .0 .5 & $1916 \cdot 32$ & 467.58 & 738.10 \\
\hline 659.00 & $52 \cdot 50$ & 26.10 & $2 \cdot 20$ & 2.80 & 12.60 & 27.40 & 72.60 & 145.00 & 11.00 & 27.40 & .00 & 2024.94 & 554.83 & 737.60 \\
\hline 659.50 & $54 \cdot 10$ & $26 \cdot 30$ & 2.10 & $2 \cdot 90$ & $73 \cdot 40$ & 26.60 & 13.40 & $145 \cdot 00$ & 11.00 & 2.5 .60 & .00 & 2040.46 & 542.76 & 737.10 \\
\hline $660 \cdot 00$ & 55.60 & 26.20 & $2 \cdot 10$ & 3.10 & 78.60 & 21.40 & 74.60 & 147.00 & 14.00 & 25.40 & 4.00 & 2032.70 & $516 \cdot 31$ & 736.60 \\
\hline $660 \cdot 50$ & $57 \cdot \infty 0$ & 26.00 & $2 \cdot 10$ & 3.20 & 77.40 & $22 \cdot 60$ & 75.40 & $147 \cdot 00$ & 14.00 & 24.60 & 2.00 & $2017 \cdot 18$ & 496.23 & 736.10 \\
\hline $661 \cdot 00$ & 58.40 & 25.80 & $2 \cdot 10$ & 3.40 & $76 \cdot 10$ & 23.90 & 76.10 & 138.00 & 14.00 & 23.90 & .00 & 2001.67 & 478.40 & 735.60 \\
\hline 661.50 & $59 \cdot 90$ & 25.60 & $2 \cdot 10$ & 3.50 & 76.90 & $23 \cdot 10$ & 76.90 & 138.00 & 14.00 & 23.10 & .00 & 1986.15 & 458.80 & 335.10 \\
\hline 662.00 & 62.00 & 25.50 & $2 \cdot 10$ & $3 \cdot 70$ & $77 \cdot 20$ & $22 \cdot 80$ & 77.20 & $131 \cdot 00$ & 16.00 & $22 \cdot 80$ & .00 & 1978.39 & 451.07 & 734.60 \\
\hline 662.50 & 62.85 & 25.40 & $? \cdot 10$ & 3.60 & 77.60 & $22 \cdot 40$ & 77.60 & 131.00 & 16.00 & $22 \cdot 40$ & .00 & 1970.63 & 441.42 & 734.10 \\
\hline 663.00 & 63.25 & 25.20 & $2 \cdot 10$ & $3 \cdot 40$ & $77 \cdot 90$ & $22 \cdot 10$ & 77.90 & 126.00 & 18.00 & $22 \cdot 10$ & .00 & 1955.12 & 432.08 & 733.60 \\
\hline 663.50 & 62.92 & 25.20 & $2 \cdot 10$ & 3.20 & 80.00 & 20.00 & 77.50 & 126.00 & 18.00 & 22.50 & $2.5 \mathrm{C}$ & 1955.12 & 439.90 & 733.10 \\
\hline $664 \cdot 00$ & 62.53 & $25 \cdot 10$ & $2 \cdot 10$ & 3.00 & 77.50 & 22.50 & 77.50 & 123.00 & 14.00 & 22.50 & .00 & $1947 \cdot 36$ & $438 \cdot 16$ & 732.60 \\
\hline 664.50 & $62 \cdot 20$ & 25.00 & $2 \cdot 10$ & $3 \cdot 00$ & $77 \cdot 40$ & 22.60 & $77 \cdot 40$ & 123.00 & 14.00 & 22.60 & .00 & 1939.60 & 439.35 & $732 \cdot 10$ \\
\hline 665.00 & $61 \cdot 90$ & 24.90 & $2 \cdot 20$ & $3 \cdot 10$ & $77 \cdot 40$ & $22 \cdot 60$ & 77.40 & 119.00 & 17.00 & $22 \cdot 6 n$ & .00 & 1931.84 & 436.60 & 731.60 \\
\hline 665.50 & 63.00 & 24.40 & 2.20 & 3.20 & $78 \cdot 90$ & $21 \cdot 10$ & 78.90 & 119.00 & 17.00 & $21 \cdot 10$ & .00 & 1893.05 & 399.43 & 731.10 \\
\hline $666 \cdot 00$ & $64 \cdot 30$ & 23.10 & $2 \cdot 20$ & $3 \cdot 30$ & $\varepsilon 3 \cdot 20$ & $16 \cdot 80$ & 83.20 & 85.00 & 27.00 & 16.80 & .00 & 1792.19 & 301.09 & 730.60 \\
\hline 666.50 & 65.50 & 22.00 & $2 \cdot 30$ & 3.50 & 85.40 & 14.60 & 85.40 & 85.00 & 27.00 & 14.60 & .00 & 1706.85 & 249.20 & 730.10 \\
\hline 667.00 & 66.80 & 20.80 & $2 \cdot 40$ & 3.70 & 88.60 & 11.40 & 88.60 & 53.00 & 32.00 & 11.40 & .00 & 1613.75 & 183.97 & 729.60 \\
\hline 667.50 & $67.7 C$ & 19.90 & $2 \cdot 60$ & $3 \cdot 90$ & 50.50 & $9 \cdot 50$ & 90.50 & 53.00 & 32.00 & $9 \cdot 50$ & .00 & 1543.92 & 146.67 & 729.10 \\
\hline 668.00 & $68.4 \mathrm{C}$ & 19.70 & 2.70 & $4 \cdot 00$ & 89.70 & $10 \cdot 30$ & 89.70 & $42 \cdot 00$ & 36.00 & $10 \cdot 30$ & .00 & 1528.40 & 157.43 & 728.60 \\
\hline 668.50 & $69.1 C$ & 19.40 & $2 \cdot 80$ & 4.00 & 89.80 & $10 \cdot 20$ & 89.80 & $42 \cdot 00$ & 36.00 & 10.20 & .00 & $1505 \cdot 13$ & 153.52 & $728 \cdot 10$ \\
\hline 669.00 & $69.7 \mathrm{C}$ & 18.90 & $2 \cdot 80$ & $4 \cdot 10$ & $52 \cdot 10$ & 7.90 & $92 \cdot 10$ & 35.00 & 38.00 & 7.90 & .00 & $1466 \cdot 34$ & 115.34 & 727.60 \\
\hline 669.50 & 69.90 & $19.4 \mathrm{C}$ & $2 \cdot 80$ & $4 \cdot 10$ & $89 \cdot 10$ & $10 \cdot 90$ & 89.10 & $35 \cdot 00$ & 38.00 & 10.90 & .00 & 1505.13 & 164.06 & 727.10 \\
\hline 670.00 & $69.6 a$ & 20.10 & $2 \cdot 80$ & $4 \cdot 00$ & $\varepsilon 5 \cdot 20$ & $14 \cdot 80$ & 85.20 & 46.00 & 38.00 & 14.80 & .00 & 1559.44 & 230.80 & 726.60 \\
\hline 670.50 & $70 \cdot 90$ & 19.60 & 2.80 & 4.00 & 87.30 & 12.70 & 87.30 & 46.00 & 38.00 & 12.70 & .00 & 1520.65 & 193.12 & $726 \cdot 10$ \\
\hline 671.00 & $69 \cdot 30$ & 21.70 & $2 \cdot 90$ & 3.90 & 78.00 & 22.00 & 78.00 & 65.00 & 40.00 & 22.00 & .00 & 1683.57 & 370.39 & 725.60 \\
\hline 671.50 & 67.70 & 23.70 & $2 \cdot 90$ & 3.80 & 78.50 & $21 \cdot 50$ & 71.20 & 65.00 & 40.00 & 28.80 & 7.30 & 1838.74 & 529.56 & 725.10 \\
\hline 672.00 & $66 \cdot 10$ & $24 \cdot 90$ & $2 \cdot 80$ & 3.70 & $75 \cdot 30$ & 24.70 & 67.60 & 117.00 & 37.00 & 32.40 & 7.70 & 1931.84 & 625.92 & 724.60 \\
\hline 672.50 & $64 \cdot 20$ & 25.50 & 2.80 & 3.70 & 73.40 & 26.60 & 65.80 & 117.00 & 37.00 & $34 \cdot 20$ & 7.60 & 1978.39 & 676.61 & $724 \cdot 10$ \\
\hline 673.00 & $62 \cdot 40$ & $25 \cdot 20$ & $2 \cdot 80$ & 3.70 & $74 \cdot 30$ & $25 \cdot 70$ & 66.90 & 124.00 & 33.00 & 33.10 & 7.40 & 1955.12 & 647.14 & $7 \approx 3.60$ \\
\hline 673.50 & $65 \cdot 90$ & $20 \cdot 70$ & 2.80 & 3.70 & 82.80 & 17.20 & 82.80 & 124.00 & 33.00 & $17 . ? 0$ & .00 & 1605.99 & 276.23 & 723.10 \\
\hline 674.00 & 69.60 & 18.70 & 2.80 & $3 . \%$ & $52 \cdot 30$ & 7.70 & $92 \cdot 30$ & 34.00 & 43.00 & $7 \cdot 70$ & .00 & 1450.82 & 111.7 & 722.60 \\
\hline & & & $\begin{array}{l}2.59 \\
2.55\end{array}$ & & $\begin{array}{l}80.43 \\
80.22\end{array}$ & $\begin{array}{l}19.57 \\
18.40\end{array}$ & $\begin{array}{l}79.60 \\
79.23\end{array}$ & $\begin{array}{l}91 \cdot 32 \\
80 \cdot 34\end{array}$ & $\begin{array}{l}22.71 \\
19.04\end{array}$ & $\begin{array}{l}21.40 \\
19.70\end{array}$ & 1.82 & $\begin{array}{l}1784.61 \\
1772.31\end{array}$ & $\begin{array}{l}393.67 \\
349.24\end{array}$ & \\
\hline
\end{tabular}

*NOTATION--Depth. ft; GR-Gamna Ray Index, API Units; POR-Porosity, $\%$; RT-True Resistivity, Ohms-m $\mathrm{m}^{2} / \mathrm{m}$; RXO-Flushed Zone Resistivity, OhmIs-m²/m;

SXO-Flushed Zone Saturation, \%; ROS-Residual 0il Saturation, \%; SW-Water Saturation, \%; PER-Permeability Index; :lay-Clay Content, $;$; SO-0il

Saturation, \%; MC-Movable 0il, \%; PHV-Pore Volume, bbl/ac-ft; HPV-Hydrocarbon Pore Volume, bbl/ac-ft; ELSE-Elevation From Sea Level. ft 
TABLE C39

WELL LOG DETERMINED PROPERTIES

VS DEPTH FOR WELL MP-128

WELL LOCATION: 2640 FWL $5 \check{8} 8$ FSL $2125 S$ SE

KELLY BUSHING ELEVATION FROA SEA LEVEL $=1400$

\begin{tabular}{|c|c|c|c|c|c|c|c|c|c|c|c|c|c|c|}
\hline DEPTH* & $G R$ & $F Q R$ & RT & $R \times O$ & $s \times 0$ & RAS & sw & PER & CLAY & se & No & PHV & HPV & ELSE \\
\hline $\begin{array}{l}645.50 \\
646.00\end{array}$ & $\begin{array}{l}85.20 \\
83.00\end{array}$ & $\begin{array}{l}15.70 \\
18.00\end{array}$ & $\begin{array}{l}4.10 \\
4.30\end{array}$ & $\begin{array}{l}5.90 \\
7.20\end{array}$ & $\begin{array}{l}52 \cdot 70 \\
78.30\end{array}$ & $\begin{array}{r}7.30 \\
21.70\end{array}$ & $92 \cdot 70$ & 8.00 & 42.00 & 7.30 & .00 & 1218.07 & 88.92 & 754.50 \\
\hline $\begin{array}{l}646.00 \\
646.50\end{array}$ & $\begin{array}{l}83.00 \\
81.70\end{array}$ & $\begin{array}{l}18.00 \\
19.30\end{array}$ & $\begin{array}{l}4.30 \\
4.50\end{array}$ & $\begin{array}{l}7.20 \\
7.06\end{array}$ & $\begin{array}{l}78.30 \\
70.50\end{array}$ & $\begin{array}{l}21.70 \\
29.50\end{array}$ & $\begin{array}{l}78.30 \\
70.50\end{array}$ & $\begin{array}{l}28.00 \\
28.00\end{array}$ & $\begin{array}{l}30.00 \\
30.00\end{array}$ & $\begin{array}{l}21.70 \\
29.50\end{array}$ & $\begin{array}{l}.00 \\
.00\end{array}$ & $\begin{array}{l}1396.51 \\
1497.37\end{array}$ & $\begin{array}{l}303.04 \\
441.72\end{array}$ & $\begin{array}{l}754.00 \\
753.50\end{array}$ \\
\hline 647.00 & 82.60 & 20.30 & 4.70 & 5.90 & 67.60 & 32.40 & 66.00 & 48.00 & 32.00 & 34.00 & 1.60 & 1574.95 & 535.48 & $\begin{array}{l}753.00 \\
753.00\end{array}$ \\
\hline 647.50 & 2.00 & 22.00 & 4.80 & 4.70 & 69.50 & 30.50 & 59.60 & 48.00 & 32.00 & 40.40 & 9.90 & 1706.85 & 689.57 & 752.50 \\
\hline 648.00 & 3.70 & 24.10 & 4.80 & 4.40 & 65.00 & 35.00 & 54.10 & 102.00 & 32.00 & 45.90 & 10.90 & 1869.77 & 858.23 & 752.00 \\
\hline $648 \cdot 50$ & 84.20 & 24.10 & 4.70 & 4.50 & $64 \cdot 10$ & 35.90 & 54.80 & 102.00 & 32.00 & $45 . ? 0$ & 9.30 & 1869.77 & 845.14 & 751.50 \\
\hline 649.00 & $3 \cdot 90$ & 24.40 & 4.50 & 4.70 & 61.70 & $38 \cdot 30$ & 54.90 & 108.00 & 29.00 & $.45 \cdot 10$ & 6.80 & 1893.05 & 853.76 & 751.00 \\
\hline 649.50 & 6.50 & 26.80 & $4 \cdot 30$ & 4.70 & $56 \cdot 10$ & $43 \cdot 90$ & 50.60 & 108.00 & 29.00 & 49.40 & 5.50 & 2079.25 & 1027.15 & 750.50 \\
\hline 650.00 & 0.30 & 26.90 & .20 & 4.50 & 56.80 & 43.20 & 11.40 & 167.00 & 14.00 & 48.60 & 5.40 & 2087.01 & 1014.29 & 750.00 \\
\hline $650 \cdot 50$ & 8.30 & 27.00 & .90 & $4 \cdot 40$ & 7.50 & $42 \cdot 50$ & 52.90 & 167.00 & 14.00 & 47.10 & 4.60 & 2094.77 & 986.63 & 749.50 \\
\hline 651.00 & $8 \cdot 30$ & 27.20 & 1.80 & $4 \cdot \mathrm{jo}$ & 57.60 & 42.40 & 53.30 & 173.00 & 13.00 & 46.70 & 4.30 & 2110.28 & 985.50 & 749.00 \\
\hline 651.50 & 7.40 & 27.30 & 1.60 & 4.30 & 57.70 & $42 \cdot 30$ & $54 \cdot 10$ & 173.00 & 13.00 & 45.90 & 3.60 & 2118.04 & 972.18 & 748.50 \\
\hline 652.00 & 6.50 & 27.50 & .50 & 4.00 & 55.40 & 44.60 & 54.40 & 183.00 & 11.00 & 45.50 & 1.00 & 2133.56 & 972.90 & 748.00 \\
\hline 652.50 & 5.60 & 27.40 & 0 & 4.10 & 56.60 & 43.40 & 56.60 & 183.00 & 11.00 & 43.40 & .00 & 2125.80 & 922.60 & 747.50 \\
\hline 653.00 & $6 \cdot 10$ & 27 & 0 & 4.00 & 60.30 & 39.70 & 59.10 & 171.00 & 13 & 40.90 & 1.20 & 2102.53 & 859.93 & 747.00 \\
\hline 653.50 & 6.90 & 27.30 & .00 & 3.70 & $\epsilon 1.90$ & 38.10 & 59.20 & 171000 & 13 & 40.80 & 2.70 & 2118.04 & 864.16 & 746.50 \\
\hline 554.00 & 7.70 & 27.60 & .90 & 3.10 & 61.40 & 38.60 & 59.40 & 187.00 & 16.00 & 40.60 & 2.00 & 2141.32 & 869.37 & 746.00 \\
\hline 554.50 & 0.10 & & 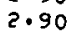 & 3.70 & 60.70 & 39.30 & 59.10 & 187.00 & & & 1.60 & 2164.59 & $885 \cdot 32$ & 745.50 \\
\hline 655.00 & 72.40 & 28.10 & .90 & 3.00 & $t c .80$ & 39.20 & 59.30 & $200 \cdot 00$ & 20 & 40.70 & 1.50 & 2180.11 & 887.30 & 745.00 \\
\hline 655.50 & 5.40 & 3.20 & 90 & 1.60 & $\epsilon 0.90$ & 39.10 & 58.50 & 200.00 & 20.00 & 41.50 & 2.40 & 3187 & 907 & 4.50 \\
\hline & 6.50 & 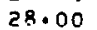 & 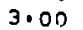 & 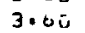 & 61.30 & 38.70 & 8.10 & 199.00 & 24.00 & 41.90 & 3.20 & $2172 \cdot 35$ & 910.21 & 744.00 \\
\hline 656.50 & 77.60 & & 10 & & & & 57.60 & 199.00 & & 42.40 & 3.70 & 2164.59 & 917.79 & 743.50 \\
\hline 657.00 & 8.70 & 27.90 & .20 & 3.90 & 59.10 & 40.90 & 56.10 & 194.00 & 23.00 & 43.90 & 3.00 & & .26 & 743.00 \\
\hline 657.50 & 8.90 & 27.50 & .40 & 4.20 & 57.80 & $42 \cdot 20$ & 5.70 & 194.00 & 23.00 & $44 \cdot 30$ & 2.10 & 2133.56 & 945.17 & 742.50 \\
\hline 658.00 & .70 & & 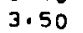 & 4. & 58 & 41.40 & 55.50 & 174.00 & & 44.50 & 3.10 & 2110.28 & 939.08 & 742.00 \\
\hline 658.50 & $6 \cdot 80$ & 27.20 & 70 & 4.20 & 58.80 & 41.20 & 54.00 & 174.00 & & & & & & .50 \\
\hline 659.00 & $5 \cdot 10$ & 27.50 & .80 & $4 \cdot 10$ & $58 \cdot 20$ & 41.80 & 52.70 & 183.00 & 20.00 & 47.30 & 5.50 & 2133.56 & .17 & 00 \\
\hline 659.50 & 2.60 & 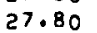 & 90 & 4.10 & 57.60 & 42.40 & 1.40 & 183.00 & $20 \cdot 0$ & 48.60 & 6.20 & 6.83 & 1048.22 & 740.50 \\
\hline 660.00 & 0.90 & 27.80 & .90 & .1 & & & & & & & 6.50 & 2156.83 & 1052.53 & 740.00 \\
\hline 660.50 & 70.50 & 27.60 & 1.90 & 4.10 & 58.20 & 41.80 & 51.50 & 00 & 17.00 & 50 & 6.70 & & 54 & .50 \\
\hline 661.00 & 70.90 & 27.20 & م0. & $4 \cdot 10$ & $58 \cdot 90$ & $41 \cdot 10$ & 52.00 & 175 & & 48.00 & 6.90 & 2110.28 & 1012.94 & 739.00 \\
\hline 661 & 1.30 & 2 & .00 & $4 \cdot$ & & +1.10 & & & & & & & 80 & .50 \\
\hline 662.00 & 72.70 & 26.80 & .0 & 4.2 & 59.00 & 41.00 & 52.80 & 165.00 & 18.00 & 47.20 & 6.20 & 25 & 981.41 & 738.00 \\
\hline 662.50 & 74.70 & 26.60 & 3.9 & $4 \cdot 3$ & 59.00 & 41.00 & 53.40 & 165.00 & 18. & 46.60 & 5.60 & & & \\
\hline 663.00 & 76.40 & 26.10 & 3. & $4 \cdot 3$ & 60 & 40.00 & 54.70 & 146.00 & 24.00 & $45 \cdot 30$ & $5 \cdot 30$ & 2024.94 & 30 & 737.00 \\
\hline 663.50 & 76.70 & 25.80 & 3.5 & 4. & 60 & 39.80 & 55.60 & 146.00 & & & & & & 736.50 \\
\hline 664 & 77 & 26. & $3 \cdot$ & 4. & 58. & $41 \cdot 20$ & 90 & $i+7.00$ & 27.00 & $45 \cdot 10$ & 3.90 & 20 & 916.75 & 736.00 \\
\hline 664.50 & 77.30 & 26.10 & 3. & 4. & 59.60 & & 55.20 & 147.00 & 27.00 & 44.80 & 4.40 & 20 & 17 & 735.50 \\
\hline & & 25.60 & 3. & & $\epsilon 1.70$ & & & & & & & & 03 & 735.00 \\
\hline 665.50 & 82.10 & 23.80 & 3. & 4.20 & 67.50 & $32 \cdot 50$ & $61.90^{\circ}$ & 133.00 & 32 . & 38 & 5.60 & 184 & & 734.50 \\
\hline 666. & 83.90 & 22.70 & 3.70 & 4.20 & 71.30 & 28.70 & 65.60 & 78.00 & 38 & & 5.70 & 1761 & & .00 \\
\hline 666.50 & 84.50 & 22.40 & 3.60 & $4 \cdot 30$ & 71.20 & 28.80 & 67.10 & 78.00 & 38.00 & 32.90 & 4.10 & 173 & 571 & 733.50 \\
\hline 667.00 & $86 \cdot 30$ & 20.70 & 3.60 & 4.40 & 76.40 & 23.60 & 73.50 & 53.00 & & 26.50 & 2.90 & & & 733.00 \\
\hline 667.50 & 88.00 & 18.80 & 3.50 & 4.40 & 82.50 & 17. & 82.50 & 53.00 & 41.00 & 17.50 & .0 & & 255 & 732.50 \\
\hline$A \cdot A$ & 88 & & & 37 & $\begin{array}{l}62.82 \\
62.42\end{array}$ & $\begin{array}{l}37 \cdot 18 \\
35.91\end{array}$ & $\begin{array}{l}58.69 \\
58.15\end{array}$ & $\begin{array}{l}142.62 \\
123.39\end{array}$ & $\begin{array}{l}23.91 \\
22.37\end{array}$ & $\begin{array}{l}41.31 \\
39.73\end{array}$ & 12 & $\begin{array}{l}1976.66 \\
1959.74\end{array}$ & $\begin{array}{l}834.61 \\
778.62\end{array}$ & \\
\hline & 7 & 26 & & & & & & & & & & & & \\
\hline
\end{tabular}

*NOTATION--Depth, ft; GR-Gamma Ray Index, API Units; POR-Porosity, $\%$; RT-True Resistivity, Ohms-m²/m; RXo-Flushed Zone Resistivity; Ohms-m²/m; SXO-Flushed Zone Saturation, $\%$; ROS-Residual 0il Saturation, $\%$; SH-Water Saturation, \%; PER-Permeability Index; Clay-Clay Content, $\%$; SO-0il

Saturation, \%; MO-Movable 0il, \%; PHV-Pore Volume, bbl/ac-ft; HPV-Hydrocarbon Pore Volume, bbl/ac-ft; ELSE-Elevation From Sea Level, ft 
TABLE C40

WELL LOG DETERMINED PROPERTIES

VS DEPTH FOR WELL MPP-1 129

WELL LOCATION: 23376 FEL $5 a ̂ 8$ FSL 21 25S 5E

KELLY BUSHING ELEVATION FROM SEA LEVEL $=1398$

\begin{tabular}{|c|c|c|c|c|c|c|c|c|c|c|c|c|c|c|}
\hline DEPTH* & $G R$ & $P 9 H$ & RT & $R \times U$ & $\leqslant x \theta$ & ROS & Sw & PER & CLAY & so & $r e$ & PHV & HPV & ELSE \\
\hline $\begin{array}{l}641.00 \\
641.50\end{array}$ & $\begin{array}{l}89.0 C \\
85.1 C\end{array}$ & $\begin{array}{l}15.7 c \\
18.50\end{array}$ & $\begin{array}{l}3.70 \\
3.50\end{array}$ & $\begin{array}{l}1 \cdot 30 \\
1.30\end{array}$ & $\begin{array}{l}57.00 \\
83.80\end{array}$ & & 83.80 & $16 \cdot 00$ & 31.00 & $2 \cdot 40$ & .00 & 1218.07 & 29.23 & 757.00 \\
\hline $642 \cdot 00$ & $81.7 C$ & 20.00 & $3 \cdot 30$ & 1.20 & $\begin{array}{l}83.80 \\
78.90\end{array}$ & $\begin{array}{l}16 \cdot 20 \\
21 \cdot 10\end{array}$ & $\begin{array}{l}83.80 \\
78.90\end{array}$ & $\begin{array}{l}16.00 \\
45.00\end{array}$ & $\begin{array}{l}31.00 \\
21.00\end{array}$ & 16.20 & .00 & $1435 \cdot 30$ & 232.52 & $\begin{array}{l}756.50 \\
756.00\end{array}$ \\
\hline $642 \cdot 50$ & $77.8 \mathrm{C}$ & 22.80 & $3 \cdot 20$ & 1.40 & 74.70 & $25 \cdot 30$ & 70.60 & 45.00 & $\begin{array}{l}21.00 \\
21.00\end{array}$ & $\begin{array}{l}21.10 \\
29.40\end{array}$ & $\begin{array}{r}.00 \\
4.10\end{array}$ & $\begin{array}{l}1551.68 \\
1768.91\end{array}$ & 327.40 & $\begin{array}{l}756.00 \\
755.50\end{array}$ \\
\hline 643.00 & 77.40 & $22.8 \mathrm{C}$ & 3.00 & $1 \cdot+0$ & $75 \cdot 30$ & 24.70 & 72.30 & 81.00 & 15.00 & 27.70 & 3.00 & $\begin{array}{l}1768.91 \\
1768.91\end{array}$ & $\begin{array}{l}520.06 \\
489.99\end{array}$ & $\begin{array}{l}755.50 \\
755.00\end{array}$ \\
\hline $643 \cdot 50$ & 78.50 & $23.0 \mathrm{C}$ & $2 \cdot 80$ & 1.50 & $74 \cdot 10$ & 25.90 & $74 \cdot 10$ & 81.00 & 15.00 & $25.9 \mathrm{C}$ & .00 & 1784.43 & 462.17 & 754.50 \\
\hline $644 \cdot 00$ & 79.90 & 23.60 & $2 \cdot 70$ & 1.40 & $73 \cdot 80$ & $26 \cdot 20$ & 73.80 & 94.00 & 17.00 & $26 \cdot 20$ & .00 & $1830 \cdot 98$ & 479.72 & 754.00 \\
\hline $644 \cdot 50$ & $81 \cdot 30$ & $24 \cdot ? 0$ & $2 \cdot 50$ & $1 \cdot 20$ & $74 \cdot 80$ & 25.20 & 74.40 & 94.00 & 17.00 & 25.60 & .40 & 1877.53 & 480.65 & 753.50 \\
\hline $645 \cdot 00$ & $82 \cdot 30$ & $24.7 \mathrm{C}$ & 2.40 & $1 \cdot 30$ & $74 \cdot 90$ & 25.20 & 74.80 & 114.00 & 17.00 & $25 \cdot ? n$ & .00 & 1916.32 & 482.91 & 753.00 \\
\hline $645 \cdot 50$ & $82 \cdot 20$ & 25.40 & $2 \cdot 30$ & $1 \cdot<0$ & 73.70 & $26 \cdot 3 n$ & 73.70 & 114.00 & 17.00 & $26 \cdot 30$ & .00 & 1970.63 & 518.28 & 752.50 \\
\hline 646.00 & $82 \cdot 00$ & 25.90 & $2 \cdot 20$ & 1.00 & $15 \cdot 30$ & 24.70 & 73.20 & 140.00 & 14.00 & 26.80 & 2.10 & 2009.42 & 538.53 & .00 \\
\hline 646.50 & 81.80 & 26.50 & $2 \cdot 20$ & $1 \cdot 00$ & $76 \cdot 30$ & 23.70 & 72.40 & 140.00 & $14 \cdot 00$ & 27.60 & 3.90 & 97 & 567.45 & 751.50 \\
\hline $647 \cdot 00$ & $81 \cdot 10$ & 27.00 & $2 \cdot 20$ & $1 \cdot 00$ & 73.00 & 27.00 & 71.00 & 167.00 & 13.00 & n & 2.00 & 2094.77 & 607.48 & 751.00 \\
\hline $647 \cdot 50$ & 82.00 & 27.30 & $2 \cdot 20$ & $1 \cdot 20$ & $70 \cdot 10$ & 29.90 & $70 \cdot 10$ & 167.00 & 13.00 & 29.90 & .00 & 2118.04 & 633.29 & 750.50 \\
\hline 648.00 & $83 \cdot 40$ & 27.10 & $2 \cdot 20$ & 1.20 & $70 \cdot 10$ & 29.90 & 70.10 & $172 \cdot 00$ & 19.00 & 29.90 & .00 & 2102.53 & & \\
\hline $648 \cdot 50$ & 84.80 & 26.90 & $2 \cdot 20$ & 1.10 & $71 \cdot 30$ & 28.70 & $70 \cdot 10$ & 172.00 & $19 \cdot 00$ & & 1.20 & 01 & 624 & 749.50 \\
\hline 649.00 & 84.60 & 27.00 & $2 \cdot 30$ & 1.20 & 69.40 & 30.60 & 69.40 & $169 \cdot 00$ & 23.00 & 30.60 & .00 & 2094.77 & $6+1.00$ & 749.00 \\
\hline $649 \cdot 50$ & $83 \cdot 20$ & 27.10 & $2 \cdot 30$ & $1 \cdot 30$ & 68.70 & $31 \cdot 30$ & 68.70 & $169 \cdot 00$ & 23.00 & $31 \cdot 30$ & .00 & 2102.53 & 658.09 & 748.50 \\
\hline 650.00 & $83 \cdot 30$ & 27.20 & $2 \cdot 30$ & $1 \cdot 30$ & $\in 8 \cdot 10$ & 31.90 & 68.10 & 174.00 & & 31.90 & & & & \\
\hline 650.50 & $84 \cdot 30$ & 27.20 & 2.40 & $1 \cdot 20$ & 66.60 & 33.40 & 66.60 & 174.00 & 22 & & .00 & 2110.28 & 704.83 & 747.50 \\
\hline 651.00 & 83.00 & 27.20 & $2 \cdot 50$ & $1 \cdot 10$ & 69.90 & 30.10 & 65 & 175.00 & 21.00 & $34 \cdot 30$ & 4.20 & 2110.28 & 723.83 & 747.00 \\
\hline $651 \cdot 50$ & $31 \cdot 30$ & 27.10 & 2. & $1 \cdot 10$ & $70 \cdot 40$ & 29.60 & 65.80 & 175.00 & 21.00 & $34 \cdot 20$ & 4.60 & 2102.53 & 719.06 & 740.50 \\
\hline $652 \cdot 00$ & 79.70 & 26.80 & $2 \cdot 50$ & $1 \cdot 10$ & 69.50 & 30.50 & 65.40 & 163.00 & 18.00 & 33.60 & 3.10 & & & 46.00 \\
\hline $.552 \cdot 50$ & $77 \cdot 20$ & 26.70 & $2 \cdot 50$ & $1 \cdot 20$ & 67. & 32.10 & 66.40 & 163.00 & & BO & 1.50 & 2071.49 & 696.02 & 745.50 \\
\hline 653.00 & 76.40 & 26.60 & 2.60 & $1 \cdot 20$ & 67 & $32 \cdot 20$ & 66.30 & $159 \cdot 00$ & 15.00 & $33 \cdot>0$ & 1.50 & 2063.73 & 695.48 & 745.00 \\
\hline 653.50 & 78.20 & 26.10 & $2 \cdot 60$ & 1.00 & $E 9.00$ & 31.00 & 66.70 & 159.00 & 15.00 & $33 \cdot 30$ & $2 \cdot 30$ & 202 & 674.31 & 744.50 \\
\hline 654.00 & 80.00 & 25.60 & 2.80 & $1 \cdot 20$ & $70 \cdot 20$ & 29.80 & 56.40 & 133.00 & 19.00 & $33 . \leqslant 0$ & 3.80 & $1986 \cdot 15$ & 667.35 & 744.00 \\
\hline 654.50 & 86.50 & 24.80 & $2 \cdot 80$ & 1.40 & $\in 8.00$ & 32 . & 68.00 & 133.00 & 19.00 & $32 \cdot \mathrm{co}$ & .00 & .08 & 615.71 & 743.50 \\
\hline $655 \cdot 00$ & $92 \cdot 20$ & 23.40 & $2 \cdot 90$ & 1.70 & & 28 & 71.60 & 90.00 & 34.00 & 28.40 & .0 & & & 743.00 \\
\hline 655.50 & 96.30 & 21.70 & $2 \cdot 90$ & $1 \cdot 40$ & 79.50 & $20 \cdot 50$ & 77.30 & 90.00 & 34.00 & 22.70 & 2.20 & 1683.57 & 382.17 & 742.50 \\
\hline 656.00 & 93.60 & 23.90 & $2 \cdot 90$ & $1 \cdot 30$ & 73.00 & 27. & 69.30 & 99.00 & 35. & 30.70 & 3.70 & 185 & 569.26 & 742.00 \\
\hline 656.50 & $90 \cdot 50$ & 26.50 & 3.00 & $1 \cdot 5 c$ & $\in 1$ & & 61.70 & 99.00 & 35.00 & $38 \cdot 30$ & .00 & 97 & 787 & 741.50 \\
\hline 657.00 & 91.60 & 26.80 & 3.00 & $1 \cdot 70$ & 60.50 & $39 \cdot 50$ & 60.50 & 164.00 & 33.00 & 39 & .00 & $2079 \cdot 25$ & $821 \cdot 30$ & 7.1 .00 \\
\hline 657.50 & 91.90 & 26.90 & 3.00 & $1 \cdot 3 \mathrm{C}$ & $E 5$ & & 60. & 164.00 & 33.00 & 39.80 & 5.40 & 2087.01 & 830 & 740.50 \\
\hline 658.00 & $92 \cdot 30$ & $26 \cdot 30$ & $3 \cdot 10$ & $1 \cdot 30$ & 66.60 & 33. & 61.40 & 151.00 & 34.00 & 38.60 & 5.20 & 204 & 787.62 & 740.00 \\
\hline $\begin{array}{l}658.50 \\
659.00\end{array}$ & $\begin{array}{r}95 \cdot 60 \\
10.0 .50\end{array}$ & $\begin{array}{l}25.00 \\
22.20\end{array}$ & $\begin{array}{l}3.00 \\
3.00\end{array}$ & $\begin{array}{l}1.40 \\
1.50\end{array}$ & $\begin{array}{l}67.20 \\
74.40\end{array}$ & $\begin{array}{l}32.80 \\
25.60\end{array}$ & $\begin{array}{l}65.10 \\
73.90\end{array}$ & $\begin{array}{r}151.00 \\
72.00\end{array}$ & $\begin{array}{l}34.00 \\
41.00\end{array}$ & $\begin{array}{l}34.90 \\
26.10\end{array}$ & $\begin{array}{r}2.10 \\
.50\end{array}$ & $\begin{array}{l}1939.60 \\
1722.36\end{array}$ & $\begin{array}{l}676.92 \\
449.54\end{array}$ & $\begin{array}{l}739.50 \\
739.00\end{array}$ \\
\hline & & & & & & & & 129 & 22 & & .58 & & & \\
\hline & & & & $20<6$ & $71 \cdot 11$ & 28.33 & 59.51 & 118.39 & $21 \cdot 30$ & & & 32 & .61 & \\
\hline
\end{tabular}

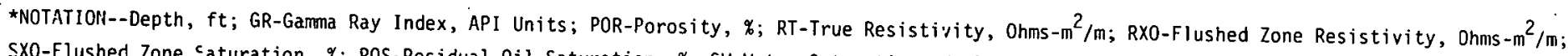
SX0-Flushed Zone Saturation, $\%$; ROS-Residual 0 il Saturation, $\%$; SW-Water Saturation, $\%$; PER-Permeability Index; Clay-Clay Content, \%; S0-0il 
TABLE C41

WELL LOG DETERMINED PROPERTIES

VS DEPTH FOR WELL MP-130

WELL LOCATION: $2112 \mathrm{FEL} 528$ FSL 21 255 $5 E$

KELLY BUSHING ELEVATIOII FROII SEA LEVEL $=1396$

\begin{tabular}{|c|c|c|c|c|c|c|c|c|c|c|c|c|c|c|}
\hline DEPTH & GR & PQR & RT & $R \times E$ & $5 \times 0$ & ROS & sw & PER & CLAY & so & mo & PHV & HPV & ELSE \\
\hline $\begin{array}{l}631.50 \\
632.00\end{array}$ & $\begin{array}{l}80.80 \\
74.90\end{array}$ & $\begin{array}{l}16.40 \\
21.00\end{array}$ & $\begin{array}{l}3.40 \\
3.40\end{array}$ & $\begin{array}{l}3.80 \\
3.50\end{array}$ & $\begin{array}{l}96.80 \\
74.70\end{array}$ & 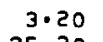 & 96.80 & $2 \cdot 00$ & 24.00 & 3.20 & .00 & $\begin{array}{l}1272 \cdot 38 \\
1629.26\end{array}$ & $\begin{array}{r}40.72 \\
+12.20\end{array}$ & $\begin{array}{l}764.50 \\
764.00\end{array}$ \\
\hline 632.50 & 70.50 & 2.20 & $3 \cdot 30$ & $\begin{array}{l}3.50 \\
3.30\end{array}$ & $\begin{array}{l}74.70 \\
70 \cdot 90\end{array}$ & $\begin{array}{l}25.30 \\
29.10\end{array}$ & $\begin{array}{l}74.70 \\
70.90\end{array}$ & $\begin{array}{l}44.00 \\
44.00\end{array}$ & $\begin{array}{l}12.00 \\
12.00\end{array}$ & $\begin{array}{l}25 \cdot 36 \\
29.10\end{array}$ & $\begin{array}{l}.00 \\
.00\end{array}$ & 1722.36 & 501.21 & $\begin{array}{l}764.00 \\
763.50\end{array}$ \\
\hline 633.00 & $69 \cdot 10$ & 23.10 & $3 \cdot \geq 0$ & $3 \cdot 2 c$ & 79.90 & 20.10 & 68.10 & 67.00 & 11.00 & 31.90 & 11.80 & 1792.19 & 571.71 & $\begin{array}{l}3.00 \\
3.00\end{array}$ \\
\hline $633 \cdot 50$ & $68 \cdot 20$ & 3.30 & 1.20 & 1.10 & 79.90 & 20.10 & 68.50 & 67.00 & 11.00 & 31.50 & 11.40 & 1807.71 & 569.43 & 762.50 \\
\hline $\begin{array}{l}634.00 \\
634.50\end{array}$ & 67.20 & 23.40 & .10 & . 0 & $t 9.20$ & 30.80 & 69.20 & 71.00 & 8.00 & 30.90 & .00 & 1815.46 & $559 \cdot 16$ & 762.00 \\
\hline $\begin{array}{l}634.50 \\
635.00\end{array}$ & 66.60 & $23.6 n$ & 3.00 & - 90 & $\in 9.40$ & 30.60 & 69.40 & 71.00 & 8.00 & $30.6 n$ & .00 & 1830.98 & 560.28 & 761.50. \\
\hline $\begin{array}{l}635 \cdot 00 \\
635 \cdot 50\end{array}$ & 66.10 & 23.80 & $2 \cdot 90$ & .80 & $70 \cdot 30$ & 29.70 & 70.30 & 77.00 & 6.00 & 29.70 & .00 & 1846.50 & $548.4 !$ & 761.00 \\
\hline $\begin{array}{l}635 \cdot 50 \\
636.00\end{array}$ & $64 \cdot 30$ & $24 \cdot 20$ & $2 \cdot 80$ & $2 \cdot 80$ & $70 \cdot 30$ & 29.70 & $70 \cdot 30$ & 77.00 & 6.20 & 29.70 & .00 & 1877.53 & 557.63 & 760.50 \\
\hline $\begin{array}{l}636.00 \\
636.50\end{array}$ & 63.20 & 25.00 & $2 \cdot 70$ & $2 \cdot ة 0$ & 77.60 & 22.40 & 69.30 & 96.00 & 4.00 & 30.70 & $8 \cdot 30$ & 1939.60 & 595.46 & 760.00 \\
\hline & 63.40 & $25.7 \mathrm{C}$ & $2 \cdot 60$ & 2.90 & $75 \cdot 10$ & 24.90 & 68.70 & 96.00 & 4.00 & $31 \cdot 30$ & 6.40 & 1993.91 & 624.09 & 759.50 \\
\hline 637.00 & 63.50 & 26.10 & $2 \cdot 40$ & 2.80 & $74 \cdot 70$ & $25 \cdot 30$ & $70 \cdot 10$ & 114.00 & 4.00 & 29.90 & 4.60 & 2024.94 & $605 \cdot 46$ & 759.00 \\
\hline 637.50 & 63.60 & 26.10 & $2 \cdot 30$ & 2.80 & $75 \cdot 40$ & 24.60 & 71.50 & 114.00 & 4.00 & 50 & 3.90 & 2024.94 & 577.11 & 758.50 \\
\hline 538.00 & 63.60 & 26 & 3.2 & 2.70 & $76 \cdot 90$ & $23 \cdot 10$ & 73.70 & 114.00 & .00 & $26 \cdot 30$ & $3 \cdot 20$ & 4.94 & 532.56 & 758.00 \\
\hline 538.50 & 63.60 & 26.10 & $2 \cdot 10$ & $2 \cdot 0 c$ & 77 & $22 \cdot 30$ & 74.90 & 114.00 & 4.00 & 25.10 & $2 \cdot 80$ & 2024.94 & 26 & 50 \\
\hline 639.00 & 65.10 & 25.10 & .00 & $2 \cdot 50$ & $78 \cdot 60$ & $21 \cdot 4 \mathrm{C}$ & 76.10 & 114 & 4.00 & 23.90 & 2.50 & 2024.94 & 483.96 & 757.00 \\
\hline $\begin{array}{l}639.50 \\
640.00\end{array}$ & 0 & 26.10 & 3.00 & $2 \cdot 50$ & $79 \cdot 60$ & $20 \cdot 40$ & 77.00 & 114.00 & 4.00 & 00 & 2.60 & 2024.94 & 465.74 & 756.50 \\
\hline $\begin{array}{l}640.00 \\
640.50\end{array}$ & 69.10 & 26.10 & 0 & ว. & & 21.00 & $77.3 n$ & 114.00 & 5.00 & 22.70 & 1.70 & 94 & & 756.00 \\
\hline $640 \cdot 50$ & $70 \cdot 40$ & 26.00 & $2 \cdot 00$ & $2 \cdot 50$ & & & 76.70 & $114 \cdot 00$ & 5.00 & $23 \cdot 30$ & 2.00 & $2017 \cdot 18$ & 470.00 & 50 \\
\hline $641 \cdot 00$ & 70.90 & $25 \cdot 5 c$ & .00 & $2 \cdot 0 \mathrm{C}$ & 0.00 & 20.00 & 77.70 & 103 & Do & & $2 \cdot 30$ & $1978 \cdot 39$ & $441 \cdot 18$ & 755.00 \\
\hline $\begin{array}{l}641.50 \\
642.00\end{array}$ & 70.90 & 25 & 10 & 2.00 & 0 & $21 \cdot 10$ & 75.70 & $103 \cdot 00$ & 6.00 & $24 \cdot 30$ & 3.20 & & 482.63 & .50 \\
\hline $\begin{array}{l}642 \cdot 00 \\
642.50\end{array}$ & 70.90 & 25.70 & $2 \cdot 20$ & $2 \cdot 00$ & 78.20 & 21.80 & 74.60 & 108.00 & $5 \cdot 00$ & $25 \cdot 40$ & 3.60 & 1993.91 & 506.45 & 4.00 \\
\hline $642 \cdot 50$ & 70.90 & 25.80 & $\cdot 20$ & 2.70 & 77.70 & $22 \cdot 30$ & 73.60 & 108 & 5. & 26.40 & 4.10 & 2001.67 & 528.44 & 3.50 \\
\hline $64.3 \cdot 00$ & 72.70 & $25.8 \mathrm{C}$ & - 30 & $2.7 c$ & 77.70 & $22 \cdot 30$ & 73.00 & 108.00 & 5.00 & & 4.70 & 67 & 540.45 & 753.00 \\
\hline 643.50 & 74.00 & 25.60 & 0 & 70 & 0 & 22.50 & 72.70 & 108.00 & 5.00 & 27.30 & 4.80 & & 22 & 752.50 \\
\hline 644.00 & $75 \cdot 20$ & 25.20 & $c .50$ & 2.76 & 78.50 & 21.50 & 71.70 & 98.00 & 5.00 & $28 \cdot 30$ & 6.80 & 1955.12 & 553.30 & 2.00 \\
\hline $644 \cdot 50$ & 77.50 & 24.80 & +50 & 2.80 & .20 & 20.90 & 72.70 & 98. & 5. & 27.30 & 6.50 & 1924.08 & 525.27 & . 50 \\
\hline $645 \cdot 0$ & 78.80 & 24.60 & $2 \cdot 50$ & 2.80 & $9 \cdot 30$ & 20.70 & 73.20 & 88.00 & 9.00 & 26.20 & 6.10 & & 511.50 & 751.00 \\
\hline 645.50 & 77.70 & 24.50 & $2 \cdot 50$ & 2. & & 26. & 73.40 & 88.00 & 9.00 & $26.6 \mathrm{C}$ & .00 & 190 & & \\
\hline 646.00 & 76.00 & 24.50 & $2 \cdot 50$ & 2.80 & $73 \cdot 30$ & 26.70 & 73.30 & 87.00 & 10.00 & 26.70 & .00 & 1900.81 & 507.52 & 0.00 \\
\hline & 77.80 & 24.60 & 2.50 & $2 \cdot 80$ & $79 \cdot 10$ & $20 \cdot 90$ & 73.20 & 87.00 & & & & 1908.57 & 511.50 & . 50 \\
\hline 647.00 & $80 \cdot 30$ & 24.80 & 2.40 & $2 \cdot 80$ & 78.60 & 21.40 & 73.20 & 92.00 & 11.00 & 26.80 & 5.40 & 192 & 515 & .00 \\
\hline 647.50 & 82.90 & 24.60 & $\bar{c} \cdot 40$ & $2 \cdot 80$ & & $20 \cdot 50$ & 74.50 & 92.00 & & $25 \cdot 50$ & $5 \cdot 00$ & & & \\
\hline 648.00 & $80 \cdot 70$ & 24.20 & $2 \cdot 40$ & 2.80 & 16.40 & 23.60 & $76.4 \mathrm{C}$ & 82.00 & 7.00 & 23.60 & .00 & 1877.53 & 443.10 & 748.00 \\
\hline & 81.20 & 24.50 & $2 \cdot 30$ & 2.70 & $76 \cdot 10$ & 23.90 & 76.10 & $8 \overline{2} \cdot 00$ & 7.00 & 23.90 & .00 & 1900 & 454 & .50 \\
\hline 649. & 84.50 & 24.60 & $2 \cdot 30$ & 2.70 & 76.20 & 23. & 76.20 & 89.00 & 7.0 & 23.80 & .00 & 1908 & 454 & \\
\hline 649.50 & 86.60 & 23.60 & $2 \cdot 20$ & $2.7 c$ & 80 & 19. & 80.30 & 89.00 & 0 & 19.70 & .00 & 98 & & \\
\hline 650.00 & $90 \cdot 90$ & 21.70 & $2 \cdot 20$ & 2.80 & 89.20 & $10 \cdot 80$ & 89.20 & 51.00 & $10 \cdot 00$ & $10 \cdot 80$ & .00 & 1683.57 & 181.83 & 46.00 \\
\hline 65 & 93.90 & 20.50 & $2 \cdot 20$ & 2.80 & 54.90 & $5 \cdot 10$ & 94.90 & 51.00 & 10.0 & 5.10 & .00 & & & 45.5 \\
\hline & 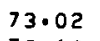 & & 2.47 & $2 \cdot 19$ & $77 \cdot 42$ & & & 0.37 & & $\begin{array}{l}25.73 \\
24.83\end{array}$ & 3.15 & 36 & 25 & \\
\hline & & & 2.44 & .78 & .28 & & & & & & & 87 & & \\
\hline
\end{tabular}

*NOTATIO!--Depth, ft; GR-Gamma Ray Index, API Units; POR-Porosity, $\%$; RT-True Resistivity, Ohms-m ${ }^{2} / m ;$ RXo-Flushed Zone Resistivity, Ohms-m²/m; SxO-Flusher Zone Saturation, $\%$; ROS-Residual $0 i 1$ Saturation, $\%$; SW-Water Saturation, \%; PER-Permeability Index; Clay-Clay Content, $\%$; S0-0il

Saturation, \%; MG-Movable 0il, \%; PHV-Pore Volume, bbl/ac-ft; HPV-Hydrocarbon Pore Volume, bbl/ac-ft; ELSE-Elevation From Sea Level, ft 
HELL LOCATION: 2112 FWL O FNL $282555 E$

FELLYY BUSHING ELEVATION FROM SEA LEVEL $=1397$

\begin{tabular}{|c|c|c|c|c|c|c|c|c|c|c|c|c|c|c|}
\hline DEPTK* & $G R$ & PER & RT & $8 \times 0$ & $5 \times \theta$ & ROS & SW & PER & CLAY & SA & mo & PHV & HPV & ELSE \\
\hline $\begin{array}{l}656.50 \\
657.00\end{array}$ & $\begin{array}{l}61 \cdot 50 \\
63.30\end{array}$ & $\begin{array}{l}16.00 \\
20.60\end{array}$ & $\begin{array}{l}3.90 \\
4.00\end{array}$ & $\begin{array}{l}4.70 \\
4.20\end{array}$ & $\begin{array}{l}52.60 \\
69.70\end{array}$ & $\begin{array}{r}7.40 \\
30 \cdot 30\end{array}$ & $\begin{array}{l}92.60 \\
69.70\end{array}$ & $\begin{array}{r}1 \cdot 00 \\
51 \cdot 00\end{array}$ & $\begin{array}{l}31.00 \\
36.00\end{array}$ & $\begin{array}{r}7.40 \\
30 \cdot 30\end{array}$ & $\begin{array}{l}.00 \\
.00\end{array}$ & $\begin{array}{l}1241 \cdot 34 \\
1598 \cdot 23\end{array}$ & $\begin{array}{r}91.86 \\
484.26\end{array}$ & $\begin{array}{l}740.50 \\
740.00\end{array}$ \\
\hline $\begin{array}{l}\epsilon .57 .50 \\
\epsilon 58.00\end{array}$ & $\begin{array}{l}63.80 \\
61.10\end{array}$ & $\begin{array}{l}20.10 \\
22.10\end{array}$ & $\begin{array}{l}4.10 \\
4.20\end{array}$ & $\begin{array}{l}4.20 \\
4.30\end{array}$ & $\begin{array}{l}70.70 \\
79.40\end{array}$ & $\begin{array}{l}29.30 \\
20.60\end{array}$ & $\begin{array}{l}70.70 \\
63.30\end{array}$ & $\begin{array}{l}51.00 \\
70.00\end{array}$ & $\begin{array}{l}36.00 \\
30.00\end{array}$ & $\begin{array}{l}29.30 \\
36.70\end{array}$ & $\begin{array}{r}.00 \\
16.10\end{array}$ & $\begin{array}{l}1559.40 \\
1714.61\end{array}$ & $\begin{array}{l}456,92 \\
629.26\end{array}$ & $\begin{array}{l}739.50 \\
739.00\end{array}$ \\
\hline$\epsilon 58.50$ & 59.00 & 22.20 & $4 \cdot 30$ & 4.40 & $78 \cdot 30$ & 21.70 & 62.00 & 70.00 & 30.00 & $38 \cdot 00$ & 16.30 & 1722.36 & 654,50 & $738 \cdot 50$ \\
\hline 659.00 & 56.80 & 21.00 & 4.40 & 4.40 & 65.30 & 34.70 & 65.30 & 56.00 & 23.00 & 34.70 & .00 & $1629 \cdot 26$ & 565.35 & 738.00 \\
\hline 659.50 & 54.60 & 19.60 & 4.50 & $5 \cdot 1 \mathrm{C}$ & $\epsilon 9.30$ & 30.70 & .69 .30 & 56.00 & 23.00 & 30.70 & .00 & 1520.65 & 466.84 & 737.50 \\
\hline 660.00 & 51.50 & 18.00 & 4.40 & 5.50 & $76 \cdot 80$ & 23.20 & 76.80 & 29.00 & 16.00 & 23.20 & .00 & 1396.51 & 323.99 & 737.00 \\
\hline 660.50 & 50.40 & 16.90 & 4.00 & $5 \cdot O C$ & $86 \cdot 10$ & 13.00 & $86 \cdot 10$ & 29.00 & 16.00 & $13 \cdot 90$ & .90 & $131.1 \cdot 17$ & 182.25 & 736.50 \\
\hline 661.00 & 51.20 & 20.30 & 3.80 & 5.30 & 78.70 & 21.30 & 72.40 & 48.00 & 15.00 & 27.60 & 6.30 & 1574.95 & 434.69 & 736.00 \\
\hline 661.50 & 52.20 & 22.50 & 3.60 & 4.70 & 74.50 & 25.50 & 67.50 & 48.00 & 15.00 & 32.50 & 7.00 & 1745.64 & 567.33 & 735.50 \\
\hline 662.00 & 53.60 & 26.00 & 3.20 & $4.1 \mathrm{C}$ & $\epsilon 8.40$ & 31.60 & 61.10 & 144.00 & 17.00 & 38.90 & $7 \cdot 30$ & 2017.18 & 784.68 & 735.00 \\
\hline $662 \cdot 50$ & 54.60 & $27-10$ & 3.00 & $3 \cdot 50$ & $70 \cdot 60$ & 29.40 & 60.50 & 244000 & 17.00 & 39.50 & 10.10 & 2102.53 & 830.50 & 734.50 \\
\hline 653.00 & 55.60 & 27.80 & 2.50 & $3 \cdot 20$ & 71.50 & 28.50 & 60.90 & 192.00 & 20.00 & 39.10 & 10.60 & 2156.83 & 843.32 & 734.00 \\
\hline 653.50 & 56.50 & 28.30 & 2.60 & 3.20 & 70.50 & 29.50 & $61 \cdot 30$ & 192.00 & 20.00 & 38.70 & 9.20 & 2195.63 & 849.71 & 733.50 \\
\hline 654.00 & 57.50 & 28.40 & 2.40 & $3 \cdot 20$ & 70.50 & 29.50 & 63.50 & 210.00 & 22.00 & 36.50 & 7.00 & $2203 \cdot 38$ & $804 \cdot 23$ & 733.00 \\
\hline $6.54 \cdot 50$ & 57.60 & 28.50 & $P .30$ & 3.20 & 70.50 & 29.50 & 65.50 & 210.00 & 22.00 & 34.50 & 5.00 & $2211 \cdot 14$ & 762.84 & 732.50 \\
\hline 655.00 & 57.00 & 28.00 & $2 \cdot 10$ & $3 \cdot 20$ & 71.50 & 28.50 & 69.00 & 198.00 & 17.00 & $31 \cdot 00$ & 2.50 & $2172 \cdot 35$ & 673.43 & 732.00 \\
\hline 605.50 & 36.50 & 27.50 & 2.10 & $3 \cdot 30$ & $72 \cdot 40$ & 27.60 & 70.10 & 198.00 & 17.00 & 29.90 & $2 \cdot 30$ & 2133.56 & 637.93 & 731.50 \\
\hline $606 \cdot c 0$ & 56.00 & 26.80 & $2 \cdot 30$ & $3 \cdot 3 \mathrm{c}$ & 73.60 & $26 \cdot 40$ & 69.60 & 165.00 & 15.00 & 30.40 & 4.00 & 2079.25 & 632.09 & $\begin{array}{l}731.00 \\
730.50\end{array}$ \\
\hline 666.50 & 55.40 & 25.60 & 2.40 & 3.50 & 74.70 & $25 \cdot 30$ & 71.30 & 165.00 & 15.00 & 28.70 & 3.40 & 1986.15 & 570.02 & 730.50 \\
\hline A.AV & 56.46 & 230.49 & 3.34 & c & 74.08 & 25.88 & 68.98 & 110.81 & 21.57 & 31.02 & $5 \cdot 14$ & $1822 \cdot 48$ & 583.14 & \\
\hline MEL & $56 \cdot 34$ & 1.12 & .22 & $4 \cdot 12$ & 73.84 & 24.74 & 68.56 & 75.57 & 20.63 & 29.37 & & $1793 \cdot 79$ & & \\
\hline
\end{tabular}

*NOTATID:I--Depth, ft; GR-Gamma Ray Index, API Units; POR-Porosity, $\%$; RT-True Resistivity, Ohms-m² $/ m$; RXO-Flushed Zone Resistivity, Ohmis-m² $/ m$; SXO-Flusher Zone Saturation, \%; ROS-Residual 0il Saturation, \%; SW-Water Saturation, \%; PER-Permeability Index; Clay-Clay Content, \%; SO-Cil Saturation, \%; MO-Movable 0il, \%; PHV-Pore Volume, bbl/ac-ft; HPV-Hydrocarbon Pore Volume, bbl/ac-ft; ELSE-Elevetion From Sea Level, ft 
TABLE C43

WELL LOG DETERMINED PROPERTIES

VS DEPTH FOR WELL IMP-202

WELL LOCATION: $2376 \mathrm{FmL}$ O FNL 28255 5E

〈ELLY BUSHING ELEVATION FROM SEA LEVEL $=1397$

\begin{tabular}{|c|c|c|c|c|c|c|c|c|c|c|c|c|c|c|}
\hline DEPTH* & $G R$ & PgR & $R^{T}$ & $R \times C$ & $s \times \theta$ & RES & sw & PER & CLAY & so & ro & PHV & HPV & ELSE \\
\hline $\begin{array}{l}658.00 \\
658.50\end{array}$ & $\begin{array}{l}92 \cdot 80 \\
91.20\end{array}$ & $\begin{array}{l}17.00 \\
17.70\end{array}$ & $\begin{array}{l}4.40 \\
4.70\end{array}$ & $\begin{array}{l}1.50 \\
1.80\end{array}$ & $\begin{array}{l}81.40 \\
76.30\end{array}$ & $\begin{array}{l}18.60 \\
23.70\end{array}$ & $\begin{array}{l}81.40 \\
76.30\end{array}$ & $\begin{array}{l}22 \cdot 00 \\
22.00\end{array}$ & $\begin{array}{l}46.00 \\
46.00\end{array}$ & $\begin{array}{l}18.60 \\
23.70\end{array}$ & $\begin{array}{l}.00 \\
.00\end{array}$ & $\begin{array}{l}1318.93 \\
1373.24\end{array}$ & $\begin{array}{l}245.32 \\
325.46\end{array}$ & $\begin{array}{l}739.00 \\
738.50\end{array}$ \\
\hline $\begin{array}{l}659.00 \\
659.50\end{array}$ & $\begin{array}{l}85.50 \\
76.10\end{array}$ & $\begin{array}{l}21.4 \mathrm{C} \\
23.60\end{array}$ & $\begin{array}{l}4.90 \\
5.00\end{array}$ & $\begin{array}{l}1.00 \\
2.20\end{array}$ & $\begin{array}{l}73.60 \\
56.40\end{array}$ & $\begin{array}{l}26.40 \\
43.60\end{array}$ & $\begin{array}{l}60.80 \\
53.80\end{array}$ & $\begin{array}{l}61.00 \\
61.00\end{array}$ & $\begin{array}{l}28.00 \\
28.00\end{array}$ & $\begin{array}{r}39.20 \\
46.20\end{array}$ & $\begin{array}{r}12.80 \\
2.60\end{array}$ & $\begin{array}{l}1660.30 \\
1830.98\end{array}$ & $\begin{array}{l}650.84 \\
845.91\end{array}$ & $\begin{array}{l}738.00 \\
737.50\end{array}$ \\
\hline 660.00 & 81.40 & 23.20 & $5 \cdot 10$ & & $\begin{array}{l}54.50 \\
t 5.50\end{array}$ & $\begin{array}{l}45.50 \\
34.50\end{array}$ & $\begin{array}{l}54.50 \\
65.50\end{array}$ & $\begin{array}{l}87.00 \\
87.00\end{array}$ & $\begin{array}{l}17.00 \\
17.00\end{array}$ & $\begin{array}{r}45.50 \\
34.50\end{array}$ & $\begin{array}{l}.00 \\
.00\end{array}$ & $\begin{array}{l}1799.95 \\
1536.16\end{array}$ & $\begin{array}{l}818.98 \\
529.98\end{array}$ & \\
\hline $\begin{array}{l}660.50 \\
661.00\end{array}$ & $\begin{array}{l}86 \cdot 70 \\
88.20\end{array}$ & $\begin{array}{l}19.80 \\
19.20\end{array}$ & $\begin{array}{l}5 \cdot 00 \\
4.80\end{array}$ & $\begin{array}{r}3.30 \\
.3 .20\end{array}$ & $\begin{array}{l}65.50 \\
68.90\end{array}$ & $\begin{array}{l}34.50 \\
31 \cdot 10\end{array}$ & $\begin{array}{l}65.50 \\
68.90\end{array}$ & 87.00 & 23.00 & 31.10 & .00 & $\begin{array}{l}1536.16 \\
1489.61\end{array}$ & 463.27 & $\begin{array}{l}736.50 \\
736.00\end{array}$ \\
\hline 661.50 & $85 \cdot 10$ & $=1.00$ & 4.60 & 2.20 & 63.80 & 36.20 & 63.70 & 87.00 & 23.00 & 36.30 & .10 & 1629.26 & 591.42 & 735.50 \\
\hline 602.00 & $78 \cdot 30$ & 3.90 & 4.40 & 1.70 & $\epsilon 2.40$ & 37.60 & 56.70 & 98.00 & 24.00 & 43.30 & 5.70 & $1854 \cdot 26$ & 802.89 & 735.00 \\
\hline 662.50 & 73.40 & $i: 5.20$ & $4 \cdot 30$ & $1.0 \mathrm{C}$ & $\epsilon 2.00$ & 38.00 & 54.40 & 98.00 & 24.00 & 45.60 & 7.60 & 1955.12 & 891.53 & 734.50 \\
\hline 663.00 & 70.50 & 26.60 & $4 \cdot 10$ & 1.60 & $58 \cdot 60$ & 41.40 & 52.50 & 158.00 & 10.00 & $47 \cdot 50$ & $6 \cdot 10$ & 2063.73 & 980.27 & 734.00 \\
\hline 653.50 & 68.20 & $27.1 \mathrm{C}$ & 3.90 & 1.00 & 57.50 & 42.50 & 52.70 & 158.00 & 10.00 & 47.30 & 4.80 & 2102.53 & 994.49 & 733.50 \\
\hline 664.00 & 67.80 & 27.60 & 3.70 & $1 \cdot 50$ & 57.00 & 43.00 & 53.00 & 186.00 & 8.00 & 47.00 & 4.00 & $2141 \cdot 32$ & 1006.42 & 733.00 \\
\hline 664.50 & 68.00 & 27.50 & $3 \cdot 50$ & 1.00 & $55 \cdot 40$ & 44.60 & 54.80 & 186.00 & 8.00 & 45.20 & .60 & 2133.56 & 964.37 & 732.50 \\
\hline $665 \cdot 00$ & 66.90 & 27.40 & $3 \cdot 30$ & $1 \cdot 10$ & $56 \cdot 20$ & 43.80 & 56.20 & 179.00 & 6.00 & 43.80 & .00 & $2125 \cdot 80$ & 931.10 & 732.00 \\
\hline $665 \cdot 50$ & $64 \cdot 90$ & 37.50 & $3 \cdot 50$ & 1.70 & 57.30 & 42.70 & 57.30 & 179.00 & 6.00 & $42.7 n$ & .00 & 2133.56 & 911.03 & 731.50 \\
\hline 666.00 & 65.00 & 27.60 & 3.10 & 1.10 & 58.20 & 41.80 & 58.20 & 187.00 & 5.00 & 41.20 & .00 & 2141.32 & 895.07 & 731.00 \\
\hline 666.50 & 66.90 & 27.40 & 3.00 & 1.70 & 59.70 & 40.30 & 59.70 & 187.00 & 5.00 & 40.30 & .00 & 2125.80 & 856.70 & 730.50 \\
\hline 667.00 & 69.70 & 27.00 & 2.90 & 1.00 & $\epsilon 1.50$ & $3 R \cdot 50$ & 61.50 & 170.00 & 9.00 & 38.50 & .00 & 2094.77 & 806.48 & 730.00 \\
\hline 667.50 & 69.90 & 27.00 & 2.80 & 1.40 & $\in 2.20$ & 37.80 & 62.20 & 170.00 & 9.00 & 37.80 & .00 & 2094.77 & 791.82 & 729.50 \\
\hline 668.00 & 71.10 & 27.70 & $2 \cdot 80$ & 1.40 & $t 1 \cdot 10$ & 38.90 & 61.10 & $189 . Q 0$ & 14.00 & 38.90 & .00 & 2149.08 & 835.99 & 729.00 \\
\hline 668.50 & 72.90 & 27.40 & 2.80 & 1.30 & $\in 2 \cdot 20$ & 37.80 & 61.20 & 189.00 & 14.00 & 38.20 & 1.00 & 2125.80 & 824.81 & 728.50 \\
\hline 669.00 & 74.40 & 26.70 & $2 \cdot 90$ & $1 \cdot 30$ & 63.70 & $36 \cdot 30$ & 62.10 & 161.00 & 19.00 & 37.90 & 1.60 & 2071.49 & 785.09 & 728.00 \\
\hline 669.50 & 76.20 & 26.20 & $3 \cdot 30$ & 1.30 & $65 \cdot 30$ & 34.70 & 62.70 & 161.00 & 19.00 & 37.30 & 2.60 & 2032.70 & 758.20 & 727.50 \\
\hline $\begin{array}{l}670.00 \\
670.50\end{array}$ & $\begin{array}{l}77.90 \\
76.30\end{array}$ & $\begin{array}{l}25.90 \\
25.80\end{array}$ & $\begin{array}{l}3 \cdot 00 \\
3.10\end{array}$ & $\begin{array}{l}1 \cdot 30 \\
1.30\end{array}$ & $\begin{array}{l}67 \cdot 30 \\
66 \cdot 10\end{array}$ & 32.70 & 62.80 & 140.00 & 24.00 & $37 \cdot 20$ & 4.50 & 2009.42 & $\begin{array}{l}747.51 \\
746.62\end{array}$ & $\begin{array}{l}727.00 \\
726.50\end{array}$ \\
\hline $\begin{array}{l}670.50 \\
671.00\end{array}$ & $\begin{array}{l}76.30 \\
73.80\end{array}$ & $\begin{array}{l}25.80 \\
25.70\end{array}$ & $\begin{array}{l}3.10 \\
3.20\end{array}$ & $\begin{array}{l}1.30 \\
1.50\end{array}$ & $\begin{array}{l}\in 6.10 \\
62.70\end{array}$ & $\begin{array}{l}33 \cdot 90 \\
37.30\end{array}$ & $\begin{array}{l}62.70 \\
61.30\end{array}$ & $\begin{array}{l}140.00 \\
136.00\end{array}$ & $\begin{array}{l}24.00 \\
19.00\end{array}$ & $\begin{array}{l}37.30 \\
38.70\end{array}$ & $\begin{array}{l}3.40 \\
1.40\end{array}$ & $\begin{array}{l}2001.67 \\
1993.91\end{array}$ & 771.64 & $\begin{array}{l}726.50 \\
726.00\end{array}$ \\
\hline $\begin{array}{l}371.60 \\
371.50\end{array}$ & 71.70 & 26.00 & 3.40 & 1.40 & $\begin{array}{l}62.70 \\
\epsilon 2.40\end{array}$ & $\begin{array}{l}37.30 \\
37.60\end{array}$ & 59.20 & $\begin{array}{l}136.00 \\
136.00\end{array}$ & $\begin{array}{l}19.00 \\
19.00\end{array}$ & $\begin{array}{l}38.70 \\
40.80\end{array}$ & $\begin{array}{l}1.40 \\
3.20\end{array}$ & 2017.18 & 823.01 & 725.50 \\
\hline 572.00 & 71.40 & 26.30 & $3 \cdot 50$ & 1.40 & 61.80 & 38.20 & 57.20 & 191.00 & 15.00 & 42.80 & 4.60 & 2040.46 & 873.32 & 725.00 \\
\hline 672.50 & 73.20 & 26.20 & 3.60 & 1.40 & $\epsilon 3.60$ & 36.40 & 56.50 & 191.00 & 15.00 & 43.50 & $7 \cdot 10$ & 2032.70 & 884.22 & 724.50 \\
\hline 673.00 & 74.90 & 26.40 & 3.60 & 1.30 & $\in 3.70$ & 36.30 & 56.10 & 152.00 & 20.00 & 43.90 & 7.60 & $2048 \cdot 22$ & 899.17 & 724.00 \\
\hline 673.50 & 79.70 & 26.90 & 3.70 & 1.40 & $\epsilon 1.80$ & 38.20 & 54.40 & 152.00 & 20.00 & 45.60 & 7.40 & 2087.01 & 951.68 & 723.50 \\
\hline 674.00 & $88 \cdot 10$ & 25.80 & $3 \cdot 80$ & 1.40 & 63.30 & 36.70 & 56.30 & 139.00 & 25.00 & 43.70 & 7.00 & 2001.67 & 874.73 & 723.00 \\
\hline 674.50 & 87.70 & 22.10 & $3 \cdot 80$ & $1 \cdot 50$ & 73.00 & 27.00 & 66.40 & 139.00 & 25.00 & 33.60 & 6.60 & 1714.61 & 576.11 & 722.50 \\
\hline $675 \cdot 00$ & 89.60 & 20.00 & 3.80 & 1.50 & 73.50 & 26.50 & 73.50 & 45.00 & 38.00 & 26.50 & .00 & 1551.68 & $\begin{array}{r}411.19 \\
327.25\end{array}$ & 722.00 \\
\hline $675 \cdot 50$ & $90 \cdot 10$ & 19.00 & 3.80 & $1 \cdot 50$ & 77.80 & $22 \cdot 20$ & 77.80 & $45 \cdot 00$ & & & .00 & $1474 \cdot 10$ & & \\
\hline 676.00 & 91.40 & 17.50 & 3.80 & 1.50 & 84.70 & $15 \cdot 30$ & 84.70 & $25 \cdot 00$ & 50.00 & $15 \cdot 30$ & .00 & 1357.72 & 207.73 & 721.00 \\
\hline & 92.80 & 17.30 & 3.90 & 1.00 & $85 \cdot 30$ & 14.70 & & 25.00 & & & .00 & $13+2 \cdot 20$ & 197.30 & 720.50 \\
\hline 677.00 & $92 \cdot 40$ & $19 \cdot 10$ & 3.90 & 1.00 & 76.70 & $23 \cdot 30$ & 76.70 & $36 \cdot 00$ & 47.00 & $23 \cdot 30$ & .00 & 1481.85 & 345.27 & 720.00 \\
\hline 677.50 & 93.20 & $18 \cdot 1 C$ & $3 \cdot 90$ & $1 \cdot 50$ & $81 \cdot 10$ & 18.90 & $81 \cdot 10$ & 36.00 & 47.0 & 18. & .00 & 1404.27 & 265.41 & 19.50 \\
\hline- & 38 & 2 & 3.75 & .06 & 65.64 & $34 \cdot 36$ & 63.08 & $122 \cdot 45$ & $22 \cdot 35$ & 36.92 & 2.56 & $\begin{array}{l}1863.56 \\
1840.37\end{array}$ & $\begin{array}{l}710.24 \\
651.48\end{array}$ & \\
\hline & 77.86 & 23.72 & 3.69 & 1.61 & $65 \cdot 15$ & 33.12 & 62.46 & $102 \cdot 30$ & 18.46 & 35.40 & & & & \\
\hline
\end{tabular}

*NOTATIOH--Depth, ft; GR-Gamma Ray Index, API Units; POR-Porosity, $\%$; RT-True Resistivity, 0hms-m² $/ m$; RXO-Flushed Zone Resistivity, Ohnis-m²/m;

Sx0-Flushed Zone jaturation, \%; ROS-Residual 0il Saturation, \%; SW-Water Saturation, \%; PER-Permeability Index; Clay-Clay Content, \%; SO-0il

Saturation, \%; Ma-Movable 0il, \%; PHV-Pore Volume, bbl/ac-ft; HPV-Hydrocarbon Pore Volume, bbl/ac-ft; ELSE-Elevation From Sea Level, ft 
TABLE C44

WELL LOG DETERMINED PROPERTIES

VS DEPTH FOR WELL MP-203

WELL LOCATION: $2640 \mathrm{FWL}-C-F N L 282555 E$

KELLY BUSHING ELEVATION FROM SEA LEVEL $=1402$

\begin{tabular}{|c|c|c|c|c|c|c|c|c|c|c|c|c|c|c|}
\hline DEPTH* & GR & PQD & RT & $R \times O$ & $5 \times \theta$ & RES & SW & PER & CLAY & so & mo & PHV & HPV & ELSE \\
\hline $\begin{array}{l}657.50 \\
658.00\end{array}$ & $\begin{array}{l}72.40 \\
39.90\end{array}$ & $\begin{array}{l}16.80 \\
18.70\end{array}$ & $\begin{array}{r}3.80 \\
3.80\end{array}$ & $\begin{array}{l}5 \cdot 30 \\
4 \cdot 10\end{array}$ & $\begin{array}{l}89.30 \\
79.70\end{array}$ & $\begin{array}{l}10 \cdot 70 \\
20 \cdot 30\end{array}$ & $\begin{array}{l}89.30 \\
79.70\end{array}$ & $\begin{array}{l}12.00 \\
33.00\end{array}$ & $\begin{array}{l}37.00 \\
27.00\end{array}$ & $\begin{array}{l}10.70 \\
20.30\end{array}$ & .00 & $\begin{array}{l}1303.11 \\
1450.82\end{array}$ & $\begin{array}{l}139.46 \\
294.52\end{array}$ & $\begin{array}{l}744.50 \\
744.00\end{array}$ \\
\hline 658.50 & 39.10 & 21.40 & 3.80 & 3.50 & 76.50 & 23.50 & 69.00 & 33.00 & 27.00 & 31.00 & 7.50 & $1660 \cdot 30$ & 514.69 & 743.50 \\
\hline 659.00 & 00.70 & $22 \cdot 30$ & 3.70 & $3 \cdot 40$ & $74 \cdot 60$ & $25 \cdot 40$ & 66.40 & 73.00 & 31.00 & 33.60 & 8.20 & 1730.12 & 581.32 & 743.00 \\
\hline 659.50 & 1.80 & 22.50 & 3.70 & 3.50 & 72.90 & 27.10 & 66.50 & 73.00 & 31.00 & 33.50 & 6.40 & 1745.64 & 584.79 & 742.50 \\
\hline 660.00 & $: 3.00$ & 22.60 & 3.50 & 3.60 & 70.70 & 29.30 & 67.30 & 78.00 & 39.00 & 32.70 & 3.40 & 1753.40 & 573.36 & 742.00 \\
\hline $660 \cdot 50$ & 89.60 & 24.60 & 3.40 & 3.80 & 63.10 & 36.90 & $62 \cdot 5.0$ & 78.00 & 39.00 & 37.50 & .60 & 1908.57 & 715.71 & 741.50 \\
\hline 661.00 & 5.50 & 25.20 & 3.20 & 3.80 & 62.80 & 37.20 & 62.80 & 124.00 & 27.00 & 37.20 & .00 & 1955.12 & 727.30 & 741.00 \\
\hline 661.50 & 6.2 .50 & 25.00 & 3.10 & 3.60 & 64.30 & 35.70 & 64.30 & 124.00 & 27.00 & 35.70 & .00 & 1939.60 & 692.44 & 740.50 \\
\hline 662.00 & $\epsilon 0.90$ & 24.60 & 3.00 & 3.50 & 66.60 & 33.40 & 66.60 & 112.00 & 21.00 & 33.40 & .00 & 1908.57 & 637.46 & 740.00 \\
\hline 662.50 & 59.30 & 24.80 & $2 \cdot 80$ & $3 \cdot 30$ & 68.00 & 32.00 & 68.00 & 112.00 & 21.00 & 32.00 & .00 & 1924.08 & 615.71 & 739.50 \\
\hline 663.00 & 57.70 & 25.80 & 2.80 & $3 \cdot 20$ & 66.00 & 34.00 & 65.90 & 138.00 & 18.00 & $34 \cdot 10$ & .10 & 2001.67 & 682.57 & 739.00 \\
\hline 663.50 & 56.70 & 26.50 & 2.70 & 3.10 & $\notin 5.00$ & 35.00 & 64.50 & 138.00 & 18.00 & 35.50 & .50 & 2055.97 & 729.87 & 738.50 \\
\hline 664.00 & $57 \cdot 10$ & 27.30 & 2.70 & 3.00 & $\epsilon 4.00$ & 36.00 & 63.10 & 177.00 & 18. & 36.90 & .9 & & 781.56 & 738.00 \\
\hline $664 \cdot 50$ & 59.20 & 27.50 & 2.60 & 3.10 & 63.00 & 37.00 & 63.00 & 177.00 & 18.00 & 37.00 & .00 & 2133.56 & 789.42 & 737.50 \\
\hline $665 \cdot 00$ & 60.90 & 27.40 & 2.70 & 3.10 & 63.10 & 36.90 & 63.10 & 180.00 & 24.00 & 36.90 & .00 & 2125.80 & $78+.42$ & 737.00 \\
\hline $665 \cdot 50$ & 62.60 & 27.30 & 2.70 & 3.20 & 62.90 & 37.10 & 62.90 & 180.00 & 24.00 & 37.10 & .00 & 2118.04 & 785.79 & 736.50 \\
\hline 666.00 & $64 \cdot 30$ & 27.40 & $\bar{c} .70$ & 3.30 & $\in 2 \cdot 30$ & 37.70 & 62.30 & 180.00 & 28.00 & 37.70 & .00 & & 801.43 & 736.00 \\
\hline 666.50 & $65 \cdot 10$ & 27.50 & 2.80 & 3.30 & 61.70 & $38 \cdot 30$ & 61.70 & 180.00 & 28.00 & 38.30 & .00 & 2133.56 & 817.15 & 735.50 \\
\hline 667.00 & 65.40 & 27.60 & 2.90 & 3.30 & 60.50 & 39.50 & 60.50 & 185.00 & 30.00 & 39.50 & .00 & $21+1 \cdot 32$ & 845.82 & 735.00 \\
\hline 667.50 & 6.2 .70 & 27.90 & 2.90 & 3.30 & 59.60 & 40.40 & 58.80 & 185.00 & 30.00 & 41.20 & .80 & 2164.59 & 891.81 & 734.50 \\
\hline 668.00 & $6^{7} .00$ & 27.60 & 3.00 & 3.30 & 60.40 & 39.60 & 58.60 & 185.00 & 31.00 & 41.40 & 1.80 & 2141.32 & 886.50 & 734.00 \\
\hline 668.50 & 67.30 & 27.20 & 3.20 & 3.40 & 60.30 & 39.70 & 58.20 & 185.00 & 31.00 & 41.80 & 2.10 & 2110.28 & 882.10 & 733.50 \\
\hline 669.00 & 66.50 & 26.80 & 3.30 & 3.50 & 59.70 & 40.30 & 57.90 & 164.00 & 30.00 & 42.10 & 1.80 & 2079.25 & 875.36 & 733.00 \\
\hline 669.50 & 65.40 & $26 \cdot 30$ & 3.40 & 3.70 & 59.80 & 40.20 & 58.00 & 164.00 & 30.00 & 42.00 & 1.80 & 2040.46 & 856.99 & 732.50 \\
\hline 670.00 & 64.40 & 25.80 & 3.50 & 3.80 & 59.90 & 40.10 & 58.60 & 137.00 & 28.00 & 41.40 & 1.30 & 2001.67 & 828.69 & 32.00 \\
\hline 670.50 & $6 \equiv .70$ & 25.70 & 3.50 & 4.00 & 58.90 & 41.10 & 58.70 & $137.0 \mathrm{C}$ & 28.00 & 41.30 & .20 & 1993.91 & 823.48 & 731.50 \\
\hline 671.00 & $64 \cdot 30$ & 25.90 & 3.50 & 4000 & 58.50 & 41.50 & 53.20 & 141.00 & 28.00 & 41.80 & .30 & 2009.42 & 339.94 & 731.00 \\
\hline 671.50 & 65.30 & 26.10 & 3.50 & 3.80 & 59.20 & 40.80 & 57.60 & 141.00 & 28.00 & 42.40 & 1.60 & 24.94 & 858 & 730.50 \\
\hline 672.00 & $66 \cdot 30$ & 26.00 & 3.50 & 3.40 & 62.70 & 37.30 & 57.90 & 144.00 & 30.00 & 42.10 & 4.80 & 2017.18 & 849.2 .3 & 730.00 \\
\hline $672 \cdot 50$ & $67 \cdot 30$ & 25.80 & 3.50 & 3.40 & 63.60 & 36.40 & 58.50 & 144 & 30 & 41.50 & $5 \cdot 10$ & 2001.67 & 830 & 729.50 \\
\hline 673.00 & 68.30 & 25.50 & 3.50 & 3.40 & 64.40 & 35.60 & 59.40 & 132.00 & 32.00 & 40.60 & 5.00 & 1978.39 & 803.23 & 729.00 \\
\hline 673.50 & $69 \cdot 10$ & 25.20 & 3.50 & 3.40 & $65 \cdot 30$ & 34.70 & 60.50 & 132.00 & 32.00 & 39.50 & 4.80 & 1955.12 & 772.27 & 728.50 \\
\hline 674.00 & 71.50 & 24.30 & 3.40 & 3.40 & 67.90 & $32 \cdot 10$ & 63.30 & 106.00 & 37.00 & 36.70 & 4.60 & 1885.29 & 691.90 & 728.00 \\
\hline 674.50 & 72.80 & 23.00 & 3.40 & 3.40 & 71.50 & 28.50 & 67.40 & 106.00 & 37.00 & 32.60 & 4.10 & 1784.43 & 581.72 & 727.50 \\
\hline 675.00 & 72.30 & 23.00 & 3.40 & 3.50 & $71 \cdot 30$ & 28.70 & 67.80 & 83.00 & 38.00 & 32.20 & 3.50 & 1784.43 & 574.59 & 727.00 \\
\hline 675.50 & 71.70 & 22.60 & 3.30 & 3.50 & $72 \cdot 30$ & 27.70 & 69.30 & 83.00 & 38.00 & 30.70 & 3.00 & 1753.40 & $538 \cdot 29$ & 726.50 \\
\hline 676.50 & 78.40 & 18.50 & $3 \cdot$ & 3. & 86.50 & 13 & 86.50 & 54. & 41.00 & 13.50 & & & & \\
\hline 676.00 & 74.60 & 20.80 & 3.3 & 3.50 & 78.50 & 21.50 & 76.00 & 54. & 41.00 & 24.00 & 2.50 & 1613.75 & 387.30 & 726.00 \\
\hline 677.00 & 79.90 & 17.20 & $3 \cdot 20$ & $3 \cdot 70$ & 94.90 & $5 \cdot 10$ & 94.90 & 23.00 & 49.00 & 5.10 & .00 & 1334.44 & 68.06 & 725.00 \\
\hline $\begin{array}{l}\text { A.AVE. } \\
\text { G.MEAN }\end{array}$ & $\begin{array}{l}66.96 \\
66.75\end{array}$ & $\begin{array}{l}24.60 \\
24.40\end{array}$ & $\begin{array}{l}3.24 \\
3.22\end{array}$ & $\begin{array}{l}3.52 \\
3.51\end{array}$ & $\begin{array}{l}67.30 \\
66.81\end{array}$ & $\begin{array}{l}32.69 \\
30.79\end{array}$ & $\begin{array}{l}65.39 \\
64.89\end{array}$ & $\begin{array}{l}122.17 \\
106.25\end{array}$ & $\begin{array}{l}30.05 \\
29.23\end{array}$ & $\begin{array}{l}34.61 \\
32.64\end{array}$ & 2 & $\begin{array}{l}1908.56 \\
1893.40\end{array}$ & $\begin{array}{l}678.23 \\
618.07\end{array}$ & \\
\hline
\end{tabular}

*NOTATION--Dejth, ft; GR-Gamma Ray Index, API Units; POR-Porosity, \%; RT-True Resistivity, Ohms- $\mathrm{n}^{2} / \mathrm{m}$; RXO-Flushed Zone Resistivity, Ohms-m²/m;

SXO-Flushed Zone Saturation, \%; ROS-Residual 0i1 Saturation, \%; SW-Water Saturation, \%; PER-Permeability Index; Clay-Clay Content, \%; SO-0il

Saturation, \%. MO-Movable 0il, \%; PHV-Pore Volume, bbl/ac-ft; HPV-Hydrocarbon Pore Volume, bbl/ac-ft; ELSE-Elevation From Sea Level, ft 
TABLE C45

WELL LOG DETERMINEO PROPERTIES

VS DEPTH FOR WELL MP-204

WELL LOCATION: 2376 FEL O FNL $282555 E$

KELLY BUSHING ELEVATION FROM SEA LEVEL $=1403$

\begin{tabular}{|c|c|c|c|c|c|c|c|c|c|c|c|c|c|c|}
\hline DEPTH* & GR & $P O R$ & RT & $R \times 0$ & $5 \times \theta$ & RAS & sw & PER & CLAY & so & me & PHV & HPV & ELSE \\
\hline $\begin{array}{l}654 \cdot 00 \\
654.50\end{array}$ & $\begin{array}{l}55.20 \\
56.70\end{array}$ & $\begin{array}{l}16.60 \\
19.20\end{array}$ & $\begin{array}{l}5.20 \\
5.20\end{array}$ & $\begin{array}{l}2.60 \\
2.10\end{array}$ & $\begin{array}{l}77.20 \\
73.40\end{array}$ & $\begin{array}{l}22.80 \\
26.60\end{array}$ & $\begin{array}{l}77.20 \\
66.00\end{array}$ & $\begin{array}{l}20.00 \\
20.00\end{array}$ & $\begin{array}{l}21.00 \\
21.00\end{array}$ & $\begin{array}{l}22.80 \\
34.00\end{array}$ & $\begin{array}{r}.00 \\
7.40\end{array}$ & $\begin{array}{l}1287.89 \\
1489.61\end{array}$ & $\begin{array}{l}293.64 \\
506.47\end{array}$ & $\begin{array}{l}749.00 \\
748.50\end{array}$ \\
\hline 655.00 & 55.90 & 19.50 & $5 \cdot 10$ & 1.90 & 75.90 & $24 \cdot 10$ & 65.50 & 41.00 & 22.00 & 34.50 & 10.40 & 1512.89 & 521.95 & 748.00 \\
\hline 655.50 & $62 \cdot 20$ & 20.80 & 4.90 & 1.90 & 71.00 & 29.00 & 62.60 & 41.00 & 22.00 & 37.40 & $8 \cdot 40$ & 1613.75 & $603 \cdot 54$ & 747.50 \\
\hline 656.00 & $64 \cdot 10$ & 21.00 & .170 & 1.80 & 71.20 & 28.80 & $63 \cdot 30$ & $56 \cdot 00$ & $32 \cdot 00$ & 36.70 & 7.90 & 1629.26 & 597.94 & 747.00 \\
\hline & $64 \cdot 10$ & 22.20 & 4.40 & 1.60 & 72.00 & 28.00 & 61.40 & 56.00 & 32.00 & 38.60 & $10 \cdot 60$ & $1722 \cdot 36$ & 664.83 & \\
\hline 657.00 & $62 \cdot 40$ & 23.00 & $4 \cdot 20$ & 1.50 & 70.60 & 29.40 & 50.80 & 84.00 & 27.00 & $49 . ? 0$ & 19.80 & 1784.43 & 877.94 & 746.00 \\
\hline 657.50 & 59.50 & 23.10 & 3.90 & 1.70 & $\epsilon 6.80$ & 33.20 & $62 \cdot 30$ & 84.00 & 27.00 & 37.70 & 4.50 & $1792 \cdot 19$ & 675.65 & 745.50 \\
\hline 658.00 & 56.70 & 23.30 & $3 \cdot 90$ & $2 \cdot 10$ & 62.20 & 37.80 & 62.20 & 88.00 & 18.00 & 37.80 & .00 & 1807.71 & 683.31 & 745.00 \\
\hline $658 \cdot 50$ & $54 \cdot 70$ & $22 \cdot 70$ & $3 \cdot 80$ & $2 \cdot 30$ & 64.70 & $35 \cdot 30$ & 64.70 & 88.00 & 18.00 & $35 \cdot 30$ & .00 & $1761 \cdot 16$ & 621.69 & 744.50 \\
\hline 659.00 & $52 \cdot 70$ & 21.70 & 3.70 & 2.20 & $68 \cdot 30$ & 31.70 & $68 \cdot 30$ & 65.00 & 15.00 & 31.70 & .00 & 1683.57 & 533.69 & 744.00 \\
\hline 659.50 & 49.00 & $21 \cdot 30$ & 3.70 & 1.70 & 71.70 & $28 \cdot 30$ & 69.60 & 65.00 & 15.00 & $30 \cdot 40$ & 2.10 & 1652.54 & $502 \cdot 37$ & 743.50 \\
\hline $660 \cdot 00$ & 48.00 & 20.90 & $3 \cdot 80$ & 1.70 & $74 \cdot 30$ & $25 \cdot 70$ & 70.40 & $55 \cdot 00$ & 12.00 & 29.60 & 3.90 & 1621.50 & 479.97 & 743.00 \\
\hline 660.50 & $50 \cdot 00$ & 20.70 & 3.90 & $2 \cdot 20$ & 70.50 & 29.50 & 70.50 & 55.00 & 12.00 & 29.50 & .00 & 1605.99 & 473.77 & 742.50 \\
\hline 661.00 & $51 \cdot 10$ & 20.90 & 4.00 & $2 \cdot 6 \mathrm{C}$ & $\in 8.50$ & 31.50 & 68.50 & 55.00 & 13.00 & 31.50 & .00 & $1621 \cdot 50$ & 510.77 & 742.00 \\
\hline 661.50 & 53.50 & 21.80 & $4 \cdot 20$ & $2 . \%$ & $64 \cdot 00$ & 36.00 & 64.00 & 55.00 & 13.00 & 36.00 & .00 & $1691 \cdot 33$ & 608.88 & 741.50 \\
\hline 662.00 & 53.90 & $22 \cdot 90$ & 4.40 & 2.30 & $59 \cdot 40$ & 40.60 & 59.40 & 82.00 & 15.00 & 40.60 & .00 & 1776.67 & 721.33 & 741.00 \\
\hline 662.50 & $55 \cdot 90$ & 27.50 & 4.60 & $2 \cdot 40$ & 47.70 & $52 \cdot 30$ & 47.70 & 82.00 & 15.00 & $52 \cdot 30$ & .00 & 2133.56 & 1115.85 & 740.50 \\
\hline $663 \cdot 00$ & $\begin{array}{l}58.00 \\
59.20\end{array}$ & 27.70 & 4.70 & 2.50 & 46.70 & $53 \cdot 30$ & 46.70 & $189 \cdot 00$ & 18.00 & 53.30 & .00 & 2149.08 & 1145.46 & 740.00 \\
\hline 663.50 & $\begin{array}{l}59.20 \\
59.30\end{array}$ & 27.70 & 4.90 & 2.70 & 46.00 & $54 \cdot 00$ & 46.00 & 189.00 & 18.00 & $54 \cdot 00$ & .00 & 2149.08 & 1160.50 & 739.50 \\
\hline 664.00 & $59 \cdot 30$ & 27.60 & $4 \cdot 90$ & 2.60 & 46.20 & 53.80 & 46.20 & $186 \cdot 00$ & 20.00 & 53.80 & .00 & $21+1 \cdot 32$ & 1152.03 & 739.00 \\
\hline $664 \cdot 50$ & 58.60 & 27.60 & $4 \cdot 80$ & 2.60 & 46.80 & $53 \cdot 20$ & 46.80 & 186.00 & 20.00 & 53.20 & .00 & $2141 \cdot 32$ & 1139.18 & 738.50 \\
\hline $665 \cdot 00$ & 57.70 & 27.60 & $4 \cdot 30$ & $2 \cdot 60$ & $49 \cdot 20$ & $50 \cdot 80$ & 49.20 & 185.00 & 17.00 & $50 \cdot 80$ & .00 & $2141 \cdot 32$ & 1087.79 & 738.00 \\
\hline $665 \cdot 50$ & $56 \cdot 80$ & 27.10 & 4.00 & 2.20 & 52.00 & 48.00 & 52.00 & 185.00 & 17.00 & $4 B .00$ & .00 & 2102.53 & 1009.21 & 737.50 \\
\hline 666.00 & 56.40 & 26.10 & 3.60 & 1.90 & 57.10 & $42 \cdot 90$ & 57.10 & 145.00 & 18.00 & $42 \cdot 90$ & .00 & 2024.94 & 868.70 & 737.00 \\
\hline 666.50 & $56 \cdot 40$ & 25.00 & 3.40 & 1.50 & $\in 4.50$ & $35 \cdot 50$ & 61.70 & $145 \cdot 00$ & 18.00 & 38.30 & 2.80 & 1939.60 & 742.87 & 736.50 \\
\hline $\begin{array}{l}667.00 \\
667.50\end{array}$ & $\begin{array}{l}56.90 \\
57.40\end{array}$ & $\begin{array}{l}24.90 \\
24.80\end{array}$ & $\begin{array}{l}3 \cdot 20 \\
3 \cdot 10\end{array}$ & $\begin{array}{l}1.40 \\
1.40\end{array}$ & $\begin{array}{l}66.90 \\
68.60\end{array}$ & $\begin{array}{l}33 \cdot 10 \\
31.40\end{array}$ & $\begin{array}{l}63.40 \\
64.70\end{array}$ & $\begin{array}{l}118.00 \\
118.00\end{array}$ & $\begin{array}{l}20.00 \\
20.00\end{array}$ & $\begin{array}{l}36.60 \\
35.30\end{array}$ & $\begin{array}{l}3.50 \\
3.90\end{array}$ & $\begin{array}{l}1931.84 \\
1924.08\end{array}$ & $\begin{array}{l}707.05 \\
679.20\end{array}$ & $\begin{array}{l}736.00 \\
735.50\end{array}$ \\
\hline 668.00 & 58.80 & $24 \cdot 30$ & 3.10 & 1.40 & 68.90 & $31 \cdot 10$ & 66.60 & 107.00 & 23.00 & 33.40 & 2.30 & 1885.29 & 629.69 & 735.00 \\
\hline $668 \cdot 50$ & 60.00 & 23.20 & 3.10 & 1.10 & $70 \cdot 20$ & $29 \cdot 80$ & 70.20 & 107.00 & 23.00 & $29 \cdot 80$ & .00 & $1799 \cdot 95$ & 536.38 & 734.50 \\
\hline $\begin{array}{l}669 \cdot 00 \\
669.50\end{array}$ & $\begin{array}{l}61.00 \\
62.70\end{array}$ & $\begin{array}{l}22.60 \\
22.30\end{array}$ & $\begin{array}{l}3.10 \\
3.20\end{array}$ & $\begin{array}{l}1.60 \\
1.80\end{array}$ & $\begin{array}{l}71.60 \\
71.40\end{array}$ & $\begin{array}{l}28.40 \\
28.60\end{array}$ & $\begin{array}{l}71.60 \\
71.40\end{array}$ & $\begin{array}{l}78.00 \\
78.00\end{array}$ & $\begin{array}{l}26.00 \\
26.00\end{array}$ & $\begin{array}{l}28.40 \\
28.60\end{array}$ & $\begin{array}{l}.00 \\
.00\end{array}$ & $\begin{array}{l}1753 \cdot 40 \\
1730.12\end{array}$ & $\begin{array}{l}497.96 \\
494.81\end{array}$ & $\begin{array}{l}734.00 \\
733.50\end{array}$ \\
\hline 670.00 & $\begin{array}{l}65.80 \\
67.00\end{array}$ & $\begin{array}{l}20.9 c \\
20.20\end{array}$ & $\begin{array}{l}3.30 \\
3.40\end{array}$ & $\begin{array}{l}1.80 \\
1.80\end{array}$ & $\begin{array}{l}75 \cdot 70 \\
77.30\end{array}$ & $\begin{array}{l}24.30 \\
22.70\end{array}$ & $\begin{array}{l}75.70 \\
77.30\end{array}$ & 54.00 & 33.00 & $24 \cdot 30$ & .00 & 1613.75 & 392.14 & 733.00 \\
\hline $670 \cdot 50$ & $\begin{array}{l}67.00 \\
66.40\end{array}$ & 20.50 & & $\begin{array}{l}1.80 \\
1.80\end{array}$ & 75.40 & $\begin{array}{l}22.70 \\
24.60\end{array}$ & $77 \cdot 30$ & 54.00 & 33.00 & $22 \cdot 70$ & .00 & 1567.20 & 355.75 & 732.50 \\
\hline 671.00 & $\begin{array}{l}66.40 \\
65.00\end{array}$ & $\begin{array}{l}20.50 \\
21.10\end{array}$ & 3.50 & $\begin{array}{l}1.80 \\
1.80\end{array}$ & $\begin{array}{l}75.40 \\
73.10\end{array}$ & $\begin{array}{l}24.60 \\
26.90\end{array}$ & $\begin{array}{l}75.40 \\
73.10\end{array}$ & $50 \cdot 00$ & $35 \cdot 00$ & $24 \cdot 60$ & .00 & 1590.47 & 391.26 & 732.00 \\
\hline $\begin{array}{l}671.50 \\
672.00\end{array}$ & $\begin{array}{l}65 \cdot 00 \\
64.70\end{array}$ & 21.50 & 3.50 & 1.90 & 71.60 & 28.40 & $\begin{array}{l}73.10 \\
71.60\end{array}$ & $\begin{array}{l}50.00 \\
62.00\end{array}$ & $\begin{array}{l}35.00 \\
32.00\end{array}$ & $\begin{array}{l}26.90 \\
28.40\end{array}$ & .00 & $\begin{array}{l}1637.02 \\
1668.06\end{array}$ & $\begin{array}{l}440.36 \\
473.73\end{array}$ & $\begin{array}{l}731.50 \\
731.00\end{array}$ \\
\hline $672 \cdot 50$ & $64 \cdot 10$ & $22 \cdot 30$ & $3 \cdot 50$ & 1.80 & 69.00 & 31.00 & 69.00 & 62.00 & 32.00 & 31.00 & .00 & 1730.12 & 536.34 & 730.50 \\
\hline 673.00 & & 23.20 & $3 \cdot 50$ & 1.70 & 67.10 & $32 \cdot 90$ & 65.80 & & 26.00 & 34.20 & 1.30 & 1799.95 & 615.58 & 730.00 \\
\hline 673.50 & 61.70 & $23 \cdot 10$ & $3 \cdot 50$ & 1.60 & $68 \cdot 70$ & $31 \cdot 30$ & 66.40 & $86 \cdot 00$ & $26 \cdot 00$ & 33.60 & $2 \cdot 30$ & 1792.19 & $602 \cdot 18$ & 729.50 \\
\hline 674.00 & $61 \cdot 30$ & 22.60 & $3 \cdot 50$ & 1.60 & $70 \cdot 00$ & 30.00 & 68.00 & 77.00 & $25 \cdot 00$ & 32.00 & 2.00 & 1753.40 & 561.09 & 729.00 \\
\hline $674 \cdot 50$ & $63 \cdot 70$ & 21.40 & $3 \cdot 40$ & 2.00 & $72 \cdot 40$ & 27.60 & 72.40 & 77.00 & 25.00 & 27.60 & .00 & $1660 \cdot 30$ & 458.24 & 728.50 \\
\hline $675 \cdot 00$ & $64 \cdot 80$ & 21.20 & 3.40 & 1.70 & $73 \cdot 50$ & 26.50 & 73.50 & $58 \cdot 00$ & 30.00 & 26.50 & .00 & 1644.78 & 435.87 & 728.00 \\
\hline $675 \cdot 50$ & 68.60 & 19.40 & $3 \cdot 40$ & 1.60 & $80 \cdot 90$ & $19 \cdot 10$ & 80.90 & $58 \cdot 00$ & $30 \cdot 00$ & $19 \cdot 10$ & .00 & $1505 \cdot 13$ & 287.48 & 727.50 \\
\hline 676.00 & 69.40 & 18.50 & $3 \cdot 40$ & 1.60 & $85 \cdot 20$ & 14.80 & 85.20 & $32 \cdot 00$ & 40.00 & 14.80 & .00 & $1435 \cdot 30$ & 212.42 & 727.00 \\
\hline $\begin{array}{l}\text { A.AVE. } \\
\text { G.MEAN }\end{array}$ & $\begin{array}{l}59 \cdot 32 \\
59 \cdot 10\end{array}$ & $\begin{array}{l}22.74 \\
22.59\end{array}$ & $\begin{array}{l}3.91 \\
3.86\end{array}$ & $\begin{array}{l}1.95 \\
1.92\end{array}$ & $\begin{array}{l}67.01 \\
66.23\end{array}$ & $\begin{array}{l}32.99 \\
31.71\end{array}$ & $\begin{array}{l}64.94 \\
64.18\end{array}$ & $\begin{array}{l}86.98 \\
75.60\end{array}$ & $\begin{array}{l}23.02 \\
21.95\end{array}$ & $\begin{array}{l}35.06 \\
33.76\end{array}$ & 2.07 & $\begin{array}{l}1764.42 \\
1752.17\end{array}$ & $\begin{array}{l}635.71 \\
591.51\end{array}$ & \\
\hline
\end{tabular}

*NOTATION--Depth, ft; GR-Gamma Ray Index, API Units; POR-Porosity, $\%$; RT-True Resistivity, Ohms-m² $/ m$; RX0-Flushed Zone Resistivity, Ohms-m²/m; SX0-Flushed Zone Saturation, \%; ROS-Residual $0 i 1$ Saturation, \%; SW-Water Saturation, \%; PER-Permeability Index; Clay-Clay Content, \%; Sn-nil

Saturation, \%; Mo-Movable 0il, \%; PHV-Pore Volume, bbl/ac-ft; HPV-Hydrocarbon Pore Volume, bbl/ac-ft; ELSE-Elevation From Sea Level, : 
TABLE C46

WELL LOG DETERMINED PROPERTIES

VS DEPTH FOR WELL MP-205

WELL LOCATION: 2112 FEL C FNL $282555 E$

KELLY BUSHING ELEVATION FFOM SEA LEVEL $=1399$

\begin{tabular}{|c|c|c|c|c|c|c|c|c|c|c|c|c|c|c|}
\hline DEPTH * & $G R$ & $P B R$ & RT & $R \times i$ & $\varepsilon \times \theta$ & RPS & sw & PER & CLAY & so & reg & PHV & HPV & ELSE \\
\hline $643 \cdot 00$ & 97.60 & 18.80 & 4.70 & 1.00 & 70.70 & $29 \cdot 30$ & 70.70 & 27.00 & $18.0 \mathrm{C}$ & $29 \cdot 3 n$ & .00 & $\begin{array}{r}1458.58 \\
1598.23\end{array}$ & $\begin{array}{l}427.36 \\
584.95\end{array}$ & \\
\hline $\begin{array}{l}643.50 \\
644.00\end{array}$ & $93 \cdot 30$ & 20.60 & 4.90 & 1.20 & $E 3.40$ & 36.60 & 63.40 & 40.00 & 17.00 & 36.60 & $\begin{array}{r}.00 \\
100\end{array}$ & & $\begin{array}{l}584.95 \\
792.25\end{array}$ & $\begin{array}{l}755.50 \\
755.00\end{array}$ \\
\hline $\begin{array}{l}644.00 \\
64.50\end{array}$ & $\begin{array}{l}86.60 \\
82.20\end{array}$ & 23.00 & 5.00 & 1.50 & 73.10 & 26.90 & 55.60 & 66.00 & 10.00 & 44.40 & 17.50 & $\begin{array}{l}1784.43 \\
1869.77\end{array}$ & 802.13 & \\
\hline $\begin{array}{l}64.50 \\
645.00\end{array}$ & $\begin{array}{l}82.20 \\
77.00\end{array}$ & $\begin{array}{l}24.10 \\
24.10\end{array}$ & $\begin{array}{l}4 \cdot 30 \\
4.10\end{array}$ & $\begin{array}{l}1.70 \\
1.90\end{array}$ & $\begin{array}{l}\epsilon 6 \cdot 30 \\
\epsilon 2.20\end{array}$ & $\begin{array}{l}33.70 \\
37.80\end{array}$ & 57.10 & 81.00 & 6.00 & & $\begin{array}{l}9.20 \\
4.10\end{array}$ & $\begin{array}{l}1869.77 \\
1869.77\end{array}$ & 783.43 & $\begin{array}{l}754.50 \\
754.00\end{array}$ \\
\hline $6+5 \cdot 50$ & $\begin{array}{l}77.00 \\
75.90\end{array}$ & $\begin{array}{l}24.10 \\
24.10\end{array}$ & $\begin{array}{l}4.10 \\
4.00\end{array}$ & $\begin{array}{l}1.90 \\
2.00\end{array}$ & $\begin{array}{l}\epsilon 2 \cdot 20 \\
\epsilon 0.30\end{array}$ & $\begin{array}{l}37.80 \\
39.70\end{array}$ & 58.10 & $81 \cdot 00$ & 4.00 & $\begin{array}{l}41.96 \\
40.70\end{array}$ & 1.00 & 1869.77 & 761.00 & $\begin{array}{r}754 \cdot 00 \\
753.50\end{array}$ \\
\hline 646.00 & $\begin{array}{l}75 \cdot 90 \\
74.30\end{array}$ & $\begin{array}{l}24.10 \\
24.00\end{array}$ & $3.8 \mathrm{C}$ & $1.3 \mathrm{c}$ & $\begin{array}{l}60.30 \\
\epsilon 2.50\end{array}$ & $\begin{array}{l}39.70 \\
37.50\end{array}$ & $\begin{array}{l}59.30 \\
60.70\end{array}$ & $\begin{array}{l}80.00 \\
79.00\end{array}$ & 3.00 & $\begin{array}{l}40.70 \\
39.30\end{array}$ & 1.80 & 1862.02 & 731.77 & $\begin{array}{l}753.50 \\
753.00\end{array}$ \\
\hline 646.50 & 75.50 & .23 .80 & 3.60 & $1.7 \mathrm{C}$ & $\begin{array}{l}\epsilon 2.50 \\
\epsilon 6.40\end{array}$ & $\begin{array}{l}37.50 \\
33.60\end{array}$ & $\begin{array}{l}60.70 \\
63.40\end{array}$ & $\begin{array}{l}79.00 \\
76.00\end{array}$ & $\begin{array}{l}2.00 \\
2.00\end{array}$ & 36.60 & 3.00 & 1846.50 & 675.82 & 752.50 \\
\hline 647.00 & 76.40 & 23.80 & 3.40 & 1.50 & 69.80 & $30.2 \mathrm{c}$ & 64.90 & 77.00 & $\begin{array}{l}2.00 \\
3.00\end{array}$ & $35 \cdot 10$ & 4.90 & 1846.50 & 648.12 & 752.00 \\
\hline 647.50 & 76.60 & 23.30 & 3.10 & 1.50 & 72.20 & 27.80 & 69.20 & 69.00 & 3.00 & 30.20 & 3.00 & 1807.71 & 556.77 & 751.50 \\
\hline 648.00 & 78.80 & 22.80 & 3.00 & 1.30 & 79.00 & 21.00 & 72.00 & 63.00 & 4.00 & 28.00 & 7.00 & 1768.91 & $495 \cdot 30$ & 751.00 \\
\hline 648.50 & 81.40 & 22.20 & 2.90 & 1.30 & 75.60 & 24.40 & 75.60 & 56.00 & 6.00 & $24.4 \pi$ & .00 & $1722 \cdot 36$ & & 750.50 \\
\hline 669.00 & 83.40 & $19.9 \mathrm{C}$ & 2.80 & $1 \cdot 30$ & 86.50 & $13 \cdot 50$ & 86.50 & $35 \cdot 00$ & 11.00 & 13.50 & .00 & 1543.92 & 208.43 & 750.00 \\
\hline 649.50 & 85.40 & $17.8 \mathrm{C}$ & 2.70 & 1.20 & 59.40 & .60 & 99.40 & 21.00 & 14.00 & .60 & .00 & 1380.99 & $8 \cdot 29$ & 749.50 \\
\hline 650.00 & $88 \cdot 50$ & $16.6 \mathrm{C}$ & 2.80 & 1.20 & 160.00 & .00 & 100.00 & 16.00 & 17.00 & . no & .00 & 1287.89 & .00 & 749.00 \\
\hline 650.50 & 91.50 & 16.20 & 2.90 & $1.3 \mathrm{C}$ & 160.00 & .00 & 100.00 & 14.00 & 18.00 & .00 & .00 & 1256.86 & .00 & 748.50 \\
\hline 651.00 & 94.50 & 19.50 & 3.00 & 1.50 & 85.40 & $14 \cdot 60$ & 85.40 & 32.00 & 19.00 & 14.60 & .00 & 1512.89 & 220.88 & 748.00 \\
\hline 651.50 & 97.50 & $23.1 \mathrm{C}$ & 3.20 & 1.50 & $73 \cdot 50$ & $26 \cdot 50$ & 69.50 & 67.00 & 20.00 & 30.50 & 4.00 & 1792.19 & 546.62 & 747.50 \\
\hline 652.00 & $100 \cdot 10$ & $23.9 \mathrm{C}$ & 3.30 & 1.00 & 67.60 & $32 \cdot 40$ & 55.60 & 78.00 & 24.00 & 34.40 & 2.00 & 1854.26 & 637.86 & 747.00 \\
\hline 652.50 & 101.30 & 24.80 & 3.30 & 1.90 & $\epsilon 3.00$ & 37 & 63.00 & 91 & 23.00 & 37.00 & .00 & 1924.08 & 711.91 & 746.50 \\
\hline 653.00 & 98.90 & $25.4 \mathrm{C}$ & $3 \cdot 30$ & 1.90 & 61.70 & $38 \cdot 30$ & 61.70 & $102 \cdot 00$ & 19.00 & 38.30 & .00 & 1970.63 & 754.75 & 746.00 \\
\hline 653.50 & 96.50 & $25.4 \mathrm{C}$ & $3 \cdot 20$ & 1.50 & 62.00 & 38.00 & 62.00 & 102.00 & 16.00 & 38.00 & .00 & 1970.63 & $748 \cdot 84$ & 745.50 \\
\hline 654.00 & 96.60 & 25.40 & 3.10 & 1.30 & $\in 3.20$ & 36.80 & 63.20 & 102.00 & 14.00 & 36.90 & .00 & 1970.63 & 725.19 & 745.00 \\
\hline 654.50 & 96.80 & 25.40 & 3.00 & 1.00 & $E 4.70$ & $35 \cdot 30$ & 64.40 & 102.00 & 12.00 & 35.60 & .30 & 1970.63 & $701 \cdot 54$ & 744.50 \\
\hline 655.00 & 97.00 & 25.30 & 2.90 & 1.30 & 70.70 & 29.30 & 65.70 & $\$ 01.00$ & 11.00 & $34 \cdot ? 0$ & .00 & 1962.87 & 673.27 & 744.00 \\
\hline 655.50 & 96.20 & 25.40 & 2.80 & 1.20 & 74.30 & 25.70 & 66.90 & 101.00 & 11.00 & 33.10 & 7.40 & 1970.63 & 652.28 & 743.50 \\
\hline 655.00 & 95.40 & 25.40 & 2.70 & 1.10 & 77.80 & $22 \cdot 20$ & 67.80 & 102.00 & 11.00 & $32.2 n$ & 10.00 & 1970.63 & 634.54 & 743.00 \\
\hline 655.50 & 95.00 & 24.90 & 2.70 & 1.20 & 76.60 & 23.40 & 69.60 & 93.00 & 16.00 & 30.40 & 7.00 & 1931.84 & 587.28 & 742.50 \\
\hline 657.00 & 96.50 & 24.60 & 2.70 & 1.40 & 71.50 & 28.50 & 70.00 & 89.00 & 19.00 & 30.00 & 1.50 & 1908.57 & 572.57 & 742.00 \\
\hline 657.50 & 97.80 & 2.4 .50 & 2.70 & 1.60 & $70 \cdot 10$ & 29.30 & 70.10 & 86.00 & 21.00 & $29.9 n$ & .00 & 1900.81 & 568.34 & 741.50 \\
\hline 658.00 & 98.50 & 24.20 & 2.80 & 1.50 & 70.50 & 29.50 & 70.50 & 82.00 & 22.00 & 29.50 & .00 & 1877.53 & 553.87 & 741.00 \\
\hline $65 B \cdot 50$ & 98.70 & 24.10 & 2.80 & 1.50 & 70.50 & 20.50 & 70.50 & 81.00 & 24.00 & 29.50 & .00 & 1869.77 & 551.58 & 740.50 \\
\hline 659.00 & 98.40 & 24.20 & 2.30 & 1.00 & 09.80 & 30.20 & 69.80 & 82.00 & 23.00 & $30.2 n$ & .00 & 1877.53 & 567.01 & 740.00 \\
\hline 659.50 & 98.30 & $24.3 n$ & 2.90 & 1.00 & $\epsilon 9.00$ & 31.00 & 69.10 & 84.00 & 23.00 & $31 \cdot 0 n$ & .00 & 1885.29 & 584.44 & 739.50 \\
\hline 660.00 & $98 \cdot 30$ & 24.20 & 2.90 & 1.00 & $68 \cdot 80$ & $31 \cdot 20$ & 68.80 & 83.00 & 23.00 & $31 \cdot ? 0$ & .00 & 1877.53 & 585.79 & 739.00 \\
\hline 660.50 & $98 \cdot 30$ & 24.10 & 2.90 & $1 \cdot 50$ & 70.00 & 30.00 & 68.80 & 81.00 & 24.00 & $31 . ? 0$ & 1.20 & 1869.77 & 583.37 & 738.50 \\
\hline 661.00 & 97.70 & 24.40 & 3.00 & 1.50 & $\epsilon 8.50$ & 31.50 & 67.60 & 85.00 & 21.00 & 32.40 & .90 & 1893.05 & 613.35 & 738.00 \\
\hline 661.50 & $: 00.30$ & 24.80 & 3.00 & 1.50 & 68.40 & 31.60 & 66.30 & 92.00 & 18.00 & 33.70 & 2.10 & 1924 & 648.42 & \\
\hline 662.00 & 102.90 & 25.20 & 3.00 & 1.40 & $\epsilon 7.70$ & $32 \cdot 30$ & 65.40 & 99.00 & 18.00 & 34.60 & c. 30 & 1955.12 & 676.47 & 737.00 \\
\hline 662.50 & 105.50 & 24.60 & 2.90 & 1.40 & 70.50 & 29.50 & 67.40 & 89.00 & 18.00 & $32.6 n$ & $\equiv .10$ & 1908.57 & 622.19 & 736.50 \\
\hline 663.00 & 106.60 & 24.20 & 2.90 & 1.40 & 70.90 & 29.10 & 68.80 & 83.00 & 17.00 & $31 \cdot ? 0$ & a. 10 & 1877.53 & 585.79 & 736.00 \\
\hline 663.50 & & 23.80 & 2.90 & 1.40 & 73.80 & $26 \cdot 20$ & 70.50 & 77.00 & 20.00 & 29.50 & 3.30 & 1846.50 & 544.72 & \\
\hline 664.00 & 111.10 & 22.30 & 2.80 & 1.30 & 76.00 & 24.00 & 76.00 & 57.00 & 25.00 & 24.00 & .00 & 1730.12 & 415.23 & 735.00 \\
\hline 664.50 & 118.60 & $17.6 \mathrm{C}$ & 2.80 & 1.20 & $\$ 8.60$ & 1.40 & 98.60 & $20 \cdot 00$ & 38.00 & 1.40 & .0 & 1365.48 & 19.12 & 734 \\
\hline$\because A V E$ & 9 & $\begin{array}{l}23.80 \\
23.74\end{array}$ & $\begin{array}{l}3.23 \\
3.18\end{array}$ & $\begin{array}{l}1.53 \\
1.52\end{array}$ & $\begin{array}{l}11 \\
.87\end{array}$ & $\begin{array}{l}29.89 \\
29.20\end{array}$ & $\begin{array}{l}67.52 \\
67.25\end{array}$ & $\begin{array}{l}78.85 \\
75.63\end{array}$ & 12.22 & $\begin{array}{l}32.49 \\
31.74\end{array}$ & .59 & $\begin{array}{l}1846 \cdot 30 \\
1841,74\end{array}$ & $\begin{array}{l}603.89 \\
584.60\end{array}$ & \\
\hline
\end{tabular}

*NOTATION--Depth, ft; GR-Gamma Ray Index, API Units; POR-Porosity, \%; RT-True Resistivity, Ohms-m²/m; RXo-Flushed Zone Resistivity, Ohnis-m²/m; SXO-Flushe: Zone Saturation, \%; ROS-Residual 0il Saturation, \%; SH-Hater Saturation, \%; PER-Perneability Index; Clay-Clay Content, \%; SCI-0il

Saturation, \%; M0-Movable 0il, \%; PHV-Pore Volume, bbl/ac-ft; HPV-Hydrocarbon Pore Volume, bbl/ac-ft; ELSE-Elevation From Sea Level, ft 
TABLE C47

WELL LOG DETERMINED PROPERTIES

VS DEPTH FOR WELL MP-206

WELL LOCATION: 2112 FEL 264 FNL $282555 E$

KELLY BUSHING ELEVATION FROI SEA LEVEL $=1399$

\begin{tabular}{|c|c|c|c|c|c|c|c|c|c|c|c|c|c|c|}
\hline DEPTH * & $G R$ & $P Q R$ & RT & $R \times t$ & $5 \times \theta$ & ReS & Sw & PER & CLAY & se & 0 & PHV & HPV & ELSE \\
\hline 640.00 & 61.70 & $18.0 \mathrm{C}$ & $4 \cdot 60$ & $8 \cdot v C$ & $25 \cdot 90$ & $24 \cdot 10$ & 75.60 & 28.00 & 17.00 & $24 \cdot 40$ & $\cdot 30$ & 1396.51 & 340.75 & 759.00 \\
\hline 640.50 & 60.80 & 20.20 & 4.70 & 7.00 & 71.70 & 28.30 & 66.00 & 28.00 & 17.00 & 34.00 & 5.70 & 1567.20 & 532.85 & 758.50 \\
\hline 641.00 & $63 \cdot 30$ & 22.50 & 4.90 & 6.10 & 68.00 & $32 \cdot 00$ & $57 \cdot 30$ & 76.00 & 22.00 & 42.70 & $10 \cdot 70$ & 1745.64 & $745 \cdot 39$ & 758.00 \\
\hline 641.50 & 63.80 & $23.3 \mathrm{C}$ & 4.90 & 6.30 & $\in 2 \cdot 20$ & 37.80 & 55.50 & 76.00 & 22.00 & 44.50 & 6.70 & 1807.71 & $804 \cdot 43$ & 757.50 \\
\hline 642.00 & 00.40 & 23.50 & $4 \cdot 80$ & $6 \cdot 9 C$ & $t 1.50$ & $38 \cdot 50$ & 55.50 & 92.00 & 16.00 & 44.50 & 6.00 & 1823.22 & 811.33 & 757.00 \\
\hline 642.50 & 55.60 & 23.80 & 4.70 & 6.70 & $\epsilon 1.50$ & 38.50 & 55.30 & 92.00 & 16.00 & 44.70 & 6.20 & 1846.50 & $825 \cdot 38$ & 756.50 \\
\hline 643.00 & 53.60 & $24.1 \mathrm{C}$ & 4.60 & 6.70 & $\in 0.70$ & 39.30 & 55.10 & 102.00 & 9.00 & 44.90 & 5.60 & 1869.77 & 839.53 & 756.00 \\
\hline $643 \cdot 50$ & $54 \cdot 20$ & 24.20 & 4.50 & $6.9 \mathrm{C}$ & 59.40 & 40.60 & $5.5 \cdot 30$ & $102 \cdot 00$ & 9.00 & 44.70 & $4 \cdot 10$ & 1877.53 & 839.26 & 755.50 \\
\hline 644.00 & 56.60 & 24.70 & 4.50 & 7.20 & $56 \cdot 70$ & $43 \cdot 30$ & 54.00 & 115.00 & 13.00 & 46.00 & 2.70 & 1916.32 & 881.51 & 755.00 \\
\hline 644.50 & $59 \cdot 00$ & $25.2 \mathrm{C}$ & 4.50 & 7.90 & 53.00 & 47.00 & 52.60 & 115.00 & 13.00 & 47.40 & .40 & $2955 \cdot 12$ & 926.72 & 754.50 \\
\hline 645.00 & $59 \cdot 80$ & $26.0 \mathrm{C}$ & 4.50 & 8.90 & $51 \cdot 20$ & 48.80 & 51.20 & 142.00 & $.18 \cdot 00$ & 48.80 & .00 & 2017.18 & 984.38 & 754.00 \\
\hline $645 \cdot 50$ & 60.50 & $26.6 \mathrm{C}$ & 4.50 & 9.90 & 50.10 & 49.90 & 50.10 & 142.00 & 18.00 & 49.90 & .00 & 2063.73 & 1029.80 & 753.50 \\
\hline 646.00 & 55.00 & $27.8 \mathrm{C}$ & 4.40 & 8.10 & $48 \cdot 60$ & $51 \cdot 40$ & 48.60 & 191.00 & 13.00 & 51.40 & .00 & 2156.83 & 1108.61 & 753.00 \\
\hline 646.50 & $55 \cdot 40$ & 27.00 & 4.10 & 6.80 & $53 \cdot 30$ & 46.70 & $51 \cdot 30$ & 191.00 & 13.00 & 48.70 & 2.00 & 2094.77 & $1020 \cdot 15$ & 752.50 \\
\hline 647.00 & 50.00 & 24.60 & $4 \cdot 10$ & $6 \cdot 10$ & $\epsilon 2 \cdot 20$ & 37.80 & 57.50 & 111.00 & 12.00 & 42.50 & 4.70 & 1908.57 & 811.14 & 752.00 \\
\hline 647.50 & $57 \cdot 30$ & $24.0 \mathrm{C}$ & 3.90 & 5.80 & $E 5.60$ & 34.40 & 59.80 & 111.00 & 12.00 & $40 \cdot 20$ & 5.80 & 1862.02 & 748.53 & 751.50 \\
\hline 648.00 & 59.80 & $23.5 c$ & 3.90 & $5 \cdot 30$ & 70.10 & 29.90 & 61.10 & $92 \cdot 00$ & 22.00 & 38.90 & 9.00 & 1823.22 & 709.23 & 751.00 \\
\hline 648.50 & $62 \cdot 90$ & 23.00 & 4.00 & 6.20 & 66.20 & 33.80 & 61.90 & 92.00 & 22.00 & $38 \cdot 10$ & $4 \cdot 30$ & 1784.43 & 679.87 & 750.50 \\
\hline 649.00 & 67.00 & 22.90 & $4 \cdot 20$ & $7 \cdot 20$ & $t 2.40$ & 37.60 & 60.90 & 82.00 & 31.00 & 39.10 & 1.50 & 1776.67 & 694.68 & 750.00 \\
\hline 649.50 & $74 \cdot 40$ & 19.10 & $4 \cdot 30$ & 7.70 & 73.00 & 27.00 & 73.00 & $82 \cdot 00$ & 31.00 & $27 \cdot n n$ & .00 & $1481 \cdot 85$ & $400 \cdot 10$ & 749.50 \\
\hline 650.00 & $75 \cdot 80$ & 19.10 & 4.50 & $8 \cdot 30$ & $71 \cdot 30$ & 28.70 & $71 \cdot 30$ & 37.00 & 47.00 & 28.70 & .00 & 1481.85 & 425.29 & 749.00 \\
\hline 650.50 & 74.60 & 20.50 & $4 \cdot 70$ & $9 \cdot 10$ & 64.70 & $35 \cdot 30$ & 64.70 & 37.00 & 47.00 & $35 \cdot 3 n$ & .00 & 1590.47 & 561.44 & 748.50 \\
\hline 651.00 & $71 \cdot 30$ & $23.0 \mathrm{C}$ & $5 \cdot 10$ & $9 \cdot 00$ & $55 \cdot 20$ & 44.80 & 55.10 & 83.00 & 29.00 & 44.90 & .10 & 1784.43 & 801.21 & 748.00 \\
\hline $651 \cdot 50$ & 70.70 & 23.50 & $5 \cdot 30$ & 9.10 & $53 \cdot 30$ & 46.70 & 52.80 & 83.00 & 29.00 & 47.20 & .50 & 1823.22 & 860.56 & 747.50 \\
\hline 652.00 & $70 \cdot 40$ & 23.78 & 5.40 & $9 \cdot 30$ & 52.40 & 47.60 & 51.90 & 95.00 & 31.00 & $48 \cdot 10$ & .50 & 1838.74 & $884 \cdot 43$ & 747.00 \\
\hline $652 \cdot 50$ & $70 \cdot 30$ & 23.30 & 5.40 & 9.00 & $54 \cdot 30$ & 45.70 & 52.50 & $95 \cdot 00$ & 31.00 & 47.50 & 1.80 & 1807.71 & 858.66 & 746.50 \\
\hline 653.00 & 68.80 & 23.70 & $5 \cdot 30$ & 8.20 & 55.90 & $44 \cdot 10$ & $52 \cdot 30$ & 94.00 & 28.00 & 47.70 & 3.60 & 1838.74 & 877.08 & 746.00 \\
\hline 653.50 & $67 \cdot 10$ & 24.00 & 5.00 & 7.10 & $59 \cdot 10$ & 40.90 & 53.00 & 94.00 & 28.00 & 47.00 & 6.10 & 1862.02 & 875.15 & 745.50 \\
\hline 654.00 & $68 \cdot 70$ & 23.20 & $4 \cdot 80$ & 6.40 & $64 \cdot 70$ & $35 \cdot 30$ & $56 \cdot 10$ & 87.00 & 24.00 & 43.90 & 8.60 & 1799.95 & 790.18 & 745.00 \\
\hline $654 \cdot 50$ & $69 \cdot 90$ & 22.50 & 4.70 & 6. JC & 67.60 & 32.40 & 59.00 & 87.00 & 24.00 & 41.00 & 8.60 & 1745.64 & 715.71 & 744.50 \\
\hline 655.00 & $69 \cdot 30$ & $23.0 \mathrm{C}$ & 4.60 & 7.00 & 59.60 & $40: 40$ & 58.00 & 84.00 & 35.00 & 42.00 & 1.60 & 1784.43 & 749.46 & 744.00 \\
\hline $655 \cdot 50$ & 68.60 & 23.60 & 4.50 & $8 \cdot c 0$ & 57.10 & $42 \cdot 90$ & 57.10 & 84.00 & 35.00 & 42.90 & .00 & 1830.98 & 785.49 & 743.50 \\
\hline 656.00 & 67.60 & 24.00 & 4.40 & 7.40 & 57.80 & $42 \cdot 20$ & 56.80 & 101.00 & 27.00 & $43 \cdot ? 0$ & 1.00 & 1862.02 & 804.39 & 743.00 \\
\hline 656.50 & 69.10 & 23.00 & $4 \cdot 30$ & 6.10 & E 3.90 & 36.10 & 60.00 & 101.00 & 27.00 & 40.00 & 3.90 & 1784.43 & 713.77 & 742.50 \\
\hline 657.00 & 69.70 & $22 \cdot 5 c$ & $4 \cdot 20$ & $6 \cdot 40$ & $\in 6 \cdot 50$ & $33 \cdot 50$ & $62 \cdot 30$ & 76.00 & 28.00 & 37.70 & $4 \cdot 20$ & 1745.64 & 658.11 & 742.00 \\
\hline 657.50 & 69.90 & 22.20 & 4.20 & $6 \cdot 50$ & $\epsilon 7.10$ & 32.90 & 63.30 & 76.00 & 28.00 & 36.70 & 3.80 & 1722.36 & 632.11 & 741.50 \\
\hline 658.00 & $68 \cdot 20$ & 22.90 & $4 \cdot 20$ & 6.00 & $\in 4 \cdot 50$ & $35 \cdot 50$ & 61.10 & 82.00 & 29.00 & & 3.40 & 1776.67 & 691.12 & 741.00 \\
\hline $658 \cdot 50$ & 70.40 & 21.10 & 4.20 & 6.10 & $70 \cdot 20$ & 29.80 & 66.90 & 82.00 & 29.00 & 33.10 & 3.30 & 1637.02 & 541.85 & 740.50 \\
\hline 659.00 & 74.80 & 17.60 & 4.20 & 6.70 & $81 \cdot 20$ & 18.80 & 81.20 & 26.00 & 37.00 & 18.80 & .00 & $1365 \cdot 48$ & 256.71 & 740.00 \\
\hline 659.50 & 78.40 & 15.10 & 4.20 & 6.70 & $55 \cdot 50$ & 4.50 & 95.50 & 26.00 & 37.00 & 4.50 & .00 & 1171.52 & 52.72 & 739.50 \\
\hline A.AVE. & 64.78 & 23 & 4.56 & $7 \cdot 34$ & $\epsilon 2 \cdot 04$ & 37.96 & 58.79 & 91.44 & 24.08 & $41 \cdot 21$ & 3.25 & 17 & 14 & \\
\hline G.ME $\triangle N$ & $64 \cdot 41$ & 22.98 & 4.54 & 7.27 & 61.60 & $37 \cdot 16$ & 58.39 & 84.02 & $22 \cdot 19$ & 40.44 & & 1782.87 & 720.94 & \\
\hline
\end{tabular}

*NOTATIOH--Depth, ft; GR-Gamma Ray Index, API Units; POR-Porosity, \%; RT-True Resistivity, Ohms-m²/m; RXO-Flushed Zone Resistivity, Ohmis-m²/m; SX0-Flushed Zone Saturation, \%; ROS-Residual $0 i 1$ Saturation, \%; SW-Water Saturation, \%; PER-Permeability Index; Clay-Clay Content, \%; S0-0il

Saturation, \%; M0-Hovable 0il, \%; PHV-Pore Volume, bbl/ac-ft; HPV-Hydrocarbon Pore Volume, bbl/ac-ft; ELSE-Elevation From Sea Level, ft 
TABLE C48

WELL LOG DETERMINED PROPERTIES

VS DEPTH FOR WELL MP-207

WELL LOCATION: 264 FNL 2376 FEL 28 2.5S $5 E$

KELLY BUSHING ELEVATION FROM SEA LEVEL $=1404$

\begin{tabular}{|c|c|c|c|c|c|c|c|c|c|c|c|c|c|c|}
\hline DEPTH * & GR & $P \theta R$ & RT & $R \times e$ & $5 \times 0$ & RoS & sw & PER & CLAY & so & mo & PHV & HPV & ELSE \\
\hline $\begin{array}{l}658.00 \\
658.50\end{array}$ & $\begin{array}{l}71.20 \\
71.90\end{array}$ & $\begin{array}{l}16.00 \\
17.10\end{array}$ & $\begin{array}{l}5 \cdot 30 \\
5 \cdot 70\end{array}$ & & $\begin{array}{l}80.00 \\
71.90\end{array}$ & $\begin{array}{l}20.00 \\
28.10\end{array}$ & $\begin{array}{l}80.00 \\
71.90\end{array}$ & $\begin{array}{l}17.00 \\
17.00\end{array}$ & $\begin{array}{l}37.00 \\
37.00\end{array}$ & $\begin{array}{l}20.00 \\
28.10\end{array}$ & $\begin{array}{l}.00 \\
.00\end{array}$ & $\begin{array}{l}1241.34 \\
1326.69\end{array}$ & $\begin{array}{l}248.27 \\
372.80\end{array}$ & $\begin{array}{l}746.00 \\
745.50\end{array}$ \\
\hline $\begin{array}{l}658 \cdot 50 \\
659.00\end{array}$ & $\begin{array}{l}71.90 \\
66.60\end{array}$ & $\begin{array}{l}17.10 \\
21.40\end{array}$ & $\begin{array}{l}5 \cdot 70 \\
6 \cdot 10\end{array}$ & $\begin{array}{l}6.60 \\
7.50\end{array}$ & $\begin{array}{l}71 \cdot 90 \\
t 2 \cdot 10\end{array}$ & $\begin{array}{l}28.10 \\
37.90\end{array}$ & $\begin{array}{l}71.90 \\
54.40\end{array}$ & $\begin{array}{l}17.00 \\
61.00\end{array}$ & $\begin{array}{l}37.00 \\
34.00\end{array}$ & $\begin{array}{l}28.10 \\
45.60\end{array}$ & $\begin{array}{r}.00 \\
7.70\end{array}$ & $\begin{array}{l}1326.69 \\
1660.30\end{array}$ & $\begin{array}{r}372.80 \\
757.09\end{array}$ & $\begin{array}{l}745.50 \\
745.00\end{array}$ \\
\hline 659.50 & 59.40 & $24 \cdot 30$ & 6.50 & 8.50 & 51.00 & 49.00 & 46.00 & 61.00 & 34.00 & 54.00 & 5.00 & 1885.29 & 1018.06 & 744.50 \\
\hline 650.00 & 53.90 & $27 \cdot 30$ & $6 \cdot 90$ & 9.50 & $42 \cdot 70$ & 57.30 & 39.40 & 178.00 & $15 \cdot 00$ & $60 \cdot 60$ & 3. 30 & 2118.04 & 1283.53 & 744.00 \\
\hline 660.50 & 53.00 & 27.30 & 7.20 & $9 \cdot 30$ & 43.00 & 57.00 & 38.40 & 178.00 & 15.00 & 61.60 & 4.60 & 2118.04 & 1304.71 & 743.50 \\
\hline $661 \cdot 00$ & 52.80 & 26.90 & 7.70 & 8.80 & $45 \cdot 20$ & $54 \cdot 80$ & 37.90 & $165 \cdot 00$ & 12.00 & $62 \cdot 10$ & $7 \cdot 30$ & 2087.01 & 1296.03 & 743.00 \\
\hline $661 \cdot 50$ & 52.60 & 26.60 & 8.00 & 8.20 & 47.10 & $52 \cdot 90$ & 37.50 & $165 \cdot 00$ & 12.00 & $62 \cdot 50$ & 9.60 & 2063.73 & 1289.83 & 742.50 \\
\hline $\begin{array}{l}662 \cdot 00 \\
662 \cdot 50\end{array}$ & $\begin{array}{l}52 \cdot 00 \\
51 \cdot 30\end{array}$ & $\begin{array}{l}26.60 \\
26.50\end{array}$ & $\begin{array}{l}8 \cdot 30 \\
8 \cdot 30\end{array}$ & $\begin{array}{l}8.20 \\
8.40\end{array}$ & $\begin{array}{l}47.20 \\
47.00\end{array}$ & $\begin{array}{l}52 \cdot 80 \\
53.00\end{array}$ & $\begin{array}{l}37.00 \\
37.00\end{array}$ & $\begin{array}{l}157.00 \\
157.00\end{array}$ & $\begin{array}{l}11.00 \\
11.00\end{array}$ & $\begin{array}{l}63.00 \\
63.00\end{array}$ & $\begin{array}{l}10.20 \\
10.00\end{array}$ & $\begin{array}{l}2063.73 \\
2055.97\end{array}$ & $\begin{array}{l}1300.15 \\
1295.26\end{array}$ & $\begin{array}{l}742.00 \\
741.50\end{array}$ \\
\hline 663.00 & 50.60 & 26.50 & 8.00 & 8.50 & 46.50 & 53.50 & 37.60 & 156.00 & 10.00 & 62.40 & 8.90 & 2055.97 & 1282.93 & $7+1.00$ \\
\hline 663.50 & 51.40 & 26.60 & 7.80 & 8.40 & 46.70 & 53.30 & 38.20 & 156.00 & 10.00 & 61.80 & 8.50 & 2063.73 & 1275.39 & 740.50 \\
\hline 664.00 & $52 \cdot 60$ & 26.60 & 7.50 & $8 \cdot 20$ & 47.00 & 53.00 & 38.70 & 159.00 & 12.00 & $61 \cdot 30$ & 8.30 & 2063.73 & 1265.07 & 740.00 \\
\hline $664 \cdot 50$ & $54 \cdot 60$ & 26.80 & 7.20 & $8 \cdot 10$ & 47.20 & 52.80 & 39.20 & 159.00 & 12.00 & $60 \cdot 80$ & 8.00 & 2079.25 & 1264.18 & 739.50 \\
\hline 665.00 & $55 \cdot 70$ & 26.90 & 7.00 & 7.90 & $47 \cdot 50$ & $52 \cdot 50$ & 39.80 & 166.00 & 17.00 & 60.20 & 7.70 & 2087.01 & $1256 \cdot 38$ & 739.00 \\
\hline $665 \cdot 50$ & $55 \cdot 80$ & 27.00 & $6 \cdot 70$ & 7.80 & $47 \cdot 70$ & $52 \cdot 30$ & 40.40 & 166.00 & 17.00 & 59.60 & $7 \cdot 30$ & 2094.77 & $1248 \cdot 48$ & 738.50 \\
\hline 666.00 & $55 \cdot 90$ & $27 \cdot 30$ & 6.40 & 7.60 & 47.60 & $52 \cdot 40$ & 40.70 & 178.00 & 19.00 & $59 \cdot 30$ & 6.90 & 2118.04 & 1256.00 & 738.00 \\
\hline $666 \cdot 50$ & $57 \cdot 10$ & 27.60 & 6.20 & 7.60 & $47 \cdot 20$ & $52 \cdot 80$ & $41 \cdot 10$ & 178.00 & 19.00 & $58 \cdot 90$ & 6.10 & $2141 \cdot 32$ & 1261.24 & 737.50 \\
\hline $\begin{array}{l}667 \cdot 00 \\
667 \cdot 50\end{array}$ & $\begin{array}{l}58.00 \\
59.00\end{array}$ & $\begin{array}{l}27.30 \\
26.70\end{array}$ & $\begin{array}{l}5.80 \\
5.60\end{array}$ & $\begin{array}{l}7.50 \\
7.50\end{array}$ & $\begin{array}{r}47.90 \\
49.40\end{array}$ & $\begin{array}{l}52 \cdot 10 \\
50.60\end{array}$ & $\begin{array}{l}42.90 \\
45.00\end{array}$ & $\begin{array}{l}178.00 \\
178.00\end{array}$ & $\begin{array}{l}23.00 \\
23.00\end{array}$ & $\begin{array}{l}57.10 \\
55.00\end{array}$ & $\begin{array}{l}5.00 \\
. .40\end{array}$ & $\begin{array}{l}2118.04 \\
2071.49\end{array}$ & $\begin{array}{l}1209.40 \\
1139.32\end{array}$ & $\begin{array}{l}737.00 \\
736.50\end{array}$ \\
\hline $\begin{array}{l}668 \cdot 00 \\
668 \cdot 50\end{array}$ & $\begin{array}{l}60.00 \\
59 \cdot 10\end{array}$ & $\begin{array}{l}26.30 \\
26.00\end{array}$ & $\begin{array}{l}5 \cdot 30 \\
5 \cdot 00\end{array}$ & $\begin{array}{l}7.40 \\
7.20\end{array}$ & $\begin{array}{l}50.10 \\
51.50\end{array}$ & $\begin{array}{l}49.90 \\
48.50\end{array}$ & $\begin{array}{l}46.70 \\
48.40\end{array}$ & $\begin{array}{l}151 \cdot 00 \\
151 \cdot 00\end{array}$ & $\begin{array}{l}27.00 \\
27.00\end{array}$ & $\begin{array}{l}53.30 \\
51.60\end{array}$ & $\begin{array}{l}3.40 \\
3.10\end{array}$ & $\begin{array}{l}2040.46 \\
2017.18\end{array}$ & $\begin{array}{l}1087.56 \\
1040.87\end{array}$ & $\begin{array}{l}736.00 \\
735.50\end{array}$ \\
\hline $\begin{array}{l}669 \cdot 00 \\
669.50\end{array}$ & $\begin{array}{l}58.00 \\
59.20\end{array}$ & $\begin{array}{l}25.70 \\
25.80\end{array}$ & $\begin{array}{l}4.80 \\
4.50\end{array}$ & $\begin{array}{l}7.10 \\
6.90\end{array}$ & $\begin{array}{l}52.70 \\
53.20\end{array}$ & $\begin{array}{l}47.30 \\
46.80\end{array}$ & $\begin{array}{l}50.30 \\
51.60\end{array}$ & $\begin{array}{l}136.00 \\
136.00\end{array}$ & $\begin{array}{l}24.00 \\
24.00\end{array}$ & $\begin{array}{l}49.70 \\
48.40\end{array}$ & $\begin{array}{l}2.40 \\
1.60\end{array}$ & $\begin{array}{l}1993.91 \\
2001.67\end{array}$ & $\begin{array}{l}990.97 \\
968.81\end{array}$ & $\begin{array}{l}735.00 \\
734.50\end{array}$ \\
\hline 670.00 & $60 \cdot 60$ & 26.00 & $4 \cdot 30$ & 6.50 & 54.40 & 45.60 & 52.50 & 144.00 & 27.00 & 47.50 & 1.90 & 2017.18 & 958.16 & 734.00 \\
\hline 670.50 & $62 \cdot 00$ & $26 \cdot 30$ & 4.00 & $6 \cdot 10$ & 55.60 & $44 \cdot 40$ & 53.60 & 144.00 & 27.00 & 46.40 & 2.00 & 2040.46 & 946.77 & 733.50 \\
\hline $671 \cdot 00$ & $63 \cdot 40$ & 26.20 & 3.80 & $5 \cdot 70$ & 57.60 & $42 \cdot 40$ & 55.40 & $149 \cdot 00$ & 28.00 & 44.60 & 2.20 & $2032 \cdot 70$ & 906.58 & 733.00 \\
\hline 671.50 & 63.90 & 25.90 & 3.70 & $5 \cdot 30$ & 60.60 & 39.40 & 56.60 & 149.00 & 28.00 & 43.40 & 4.00 & 2009.42 & 872.09 & $732.50^{\circ}$ \\
\hline 672.00 & 64.00 & 25.60 & $3 \cdot 70$ & $5 \cdot 00$ & 63.00 & 37.00 & 57.70 & 134.00 & 31.00 & $42 \cdot 30$ & $5 \cdot 30$ & 1986.15 & $B 40.14$ & 732.00 \\
\hline $672 \cdot 50$ & 63.40 & $26 \cdot 10$ & 3.60 & $5 \cdot 00$ & 61.90 & $38 \cdot 10$ & 56.90 & 134.00 & 31.00 & $43 \cdot 10$ & 5.00 & 2024.94 & 872.75 & 731.50 \\
\hline 673.00 & 62.60 & 26.40 & 3.60 & 4.90 & $\in 1 \cdot 20$ & 38.80 & $56 \cdot 40$ & $154 \cdot 00$ & 27.00 & 43.60 & $\triangle B O$ & $2048 \cdot 22$ & .393 .02 & 731.00 \\
\hline $673 \cdot 50$ & 62.90 & 27.70 & $3 \cdot 50$ & $4 \cdot 90$ & 58.40 & $41 \cdot 60$ & 54.10 & 154.00 & 27.00 & 45.90 & $4 \cdot 30$ & 2149.08 & 986.42 & 730.50 \\
\hline 674.00 & $64 \cdot 00$ & 27.60 & 3.50 & 4.90 & $58 \cdot 90$ & $41 \cdot 10$ & 54.60 & $185 \cdot 00$ & 28.00 & 45.40 & 4.30 & 2141.32 & $972 \cdot 16$ & 730.00 \\
\hline 674.50 & 63.60 & 27.90 & $3 \cdot 50$ & 4.90 & $58 \cdot 20$ & $41 \cdot 80$ & 53.80 & $185 \cdot 00$ & 28.00 & $46 \cdot 20$ & $4+40$ & 2164.59 & $1000 \cdot 04$ & 729.50 \\
\hline 675.00 & 63.00 & 28.80 & 3.50 & $4 \cdot 80$ & $56 \cdot 50$ & $43 \cdot 50$ & 52.50 & $224 \cdot 00$ & 28.00 & 47.50 & 4.00 & 2234.42 & 1061.35 & 729.00 \\
\hline 675.50 & 61.90 & 28.90 & 3.40 & 4.80 & 56.60 & 43.40 & 52.70 & 224.00 & 28.00 & 47.30 & 3.90 & 2242.18 & $1060 \cdot 55$ & 728.50 \\
\hline $\begin{array}{l}676.00 \\
676.50\end{array}$ & $\begin{array}{l}62.70 \\
65.30\end{array}$ & $\begin{array}{l}28.90 \\
27.20\end{array}$ & $\begin{array}{l}3.40 \\
3.30\end{array}$ & $\begin{array}{l}4.80 \\
4.90\end{array}$ & $\begin{array}{l}56.20 \\
59.70\end{array}$ & $\begin{array}{l}43.80 \\
40.30\end{array}$ & $\begin{array}{l}53.00 \\
57.10\end{array}$ & $\begin{array}{l}228.00 \\
228.00\end{array}$ & $\begin{array}{l}29.00 \\
29.00\end{array}$ & $\begin{array}{r}47.00 \\
42.90\end{array}$ & $\begin{array}{l}3.20 \\
2.60\end{array}$ & $\begin{array}{l}2242.18 \\
2110.28\end{array}$ & $\begin{array}{r}1053.82 \\
905.31\end{array}$ & $\begin{array}{l}728.00 \\
727.50\end{array}$ \\
\hline 677.00 & $68 \cdot 50$ & 24.50 & $3 \cdot 30$ & 4.90 & 66.50 & 33.50 & 64.10 & 111.00 & 38.00 & 35.90 & 2.40 & 1900.81 & 682.39 & 727.00 \\
\hline 677 & 71.90 & 21.40 & $3 \cdot 20$ & 4.90 & $77 \cdot 10$ & $22 \cdot 90$ & 74.70 & 111.00 & 38.00 & $25 \cdot 30$ & $2 \cdot 40$ & $1660 \cdot 30$ & 420.05 & 726.50 \\
\hline $\begin{array}{l}\text { A.AVE. } \\
\text { O,MEAN }\end{array}$ & $\begin{array}{l}59.88 \\
59.61\end{array}$ & $\begin{array}{l}25.96 \\
25.80\end{array}$ & $\begin{array}{l}5 \cdot 38 \\
5 \cdot 10\end{array}$ & $\begin{array}{l}6.78 \\
6.61\end{array}$ & $\begin{array}{l}54.54 \\
53.90\end{array}$ & $\begin{array}{l}45.45 \\
44.42\end{array}$ & $\begin{array}{l}49.64 \\
48.60\end{array}$ & $\begin{array}{l}151.55 \\
137.63\end{array}$ & $\begin{array}{l}23.85 \\
22.12\end{array}$ & $\begin{array}{l}50.35 \\
48.98\end{array}$ & 4.90 & $\begin{array}{l}2014.26 \\
2001.53\end{array}$ & $\begin{array}{r}1028.60 \\
980.43\end{array}$ & \\
\hline
\end{tabular}

*NOTATION--Depth, ft; GR-Gamma Ray Index, API Units; POR-Porosity, $\%$; RT-True Resistivity, Ohms-m²/m; RXo-Flushed Zone Resistivity, Ohms-m²/m; SXO-Flushed Zone Saturation, \%; ROS-Residual 0il Saturation, \%; SW-Water Saturation, \%; PER-Permeability Index; Clay-Clay Content, \%; Si-0il

Saturation, .6 ; M0-Movable 0il, $\%$; PHV-Pore Volume, bbl/ac-ft; HPV-Hydrocarbon Pore Volume, bbl/ac-ft; ELSE-Elevation From Sea Level, ft 
TABLE C49

WELL LOG DETERMINED PROPERTIES

VS DEPTH FOR WELL MP-208

WELL LOCATION: 2640 FWL 264 FNL $282555 E$

KELLY BUSHING ELEVATION FROM SEA LEVEL $=1403$

\begin{tabular}{|c|c|c|c|c|c|c|c|c|c|c|c|c|c|c|}
\hline DEPTH ${ }^{*}$ & GR & $P Q R$ & RT & $R \times \theta$ & $5 \times \theta$ & ROS & sw & PER & CLAY & so & mo & PHV & HPV & ELSE \\
\hline $\begin{array}{l}659.00 \\
659.50\end{array}$ & $\begin{array}{l}65.60 \\
61.60\end{array}$ & $\begin{array}{l}18.20 \\
20.20\end{array}$ & $\begin{array}{l}3.20 \\
3.40\end{array}$ & $\begin{array}{l}1.50 \\
1.40\end{array}$ & $\begin{array}{r}89.20 \\
77.50\end{array}$ & $\begin{array}{l}10.80 \\
22.50\end{array}$ & $\begin{array}{l}89.20 \\
77.50\end{array}$ & $\begin{array}{l}30.00 \\
30.00\end{array}$ & $\begin{array}{l}26.00 \\
26.00\end{array}$ & $\begin{array}{l}10.80 \\
22.50\end{array}$ & .00 & $\begin{array}{l}1412.03 \\
1567.20\end{array}$ & $\begin{array}{l}152.50 \\
352.62\end{array}$ & $\begin{array}{l}744.00 \\
743.50\end{array}$ \\
\hline 660.00 & $56 \cdot 10$ & 21.70 & 3.50 & 1.40 & $70 \cdot 30$ & 29.70 & 70.30 & 65.00 & 20.00 & 29.70 & .00 & 1683.57 & 500.02 & 743.00 \\
\hline 660.50 & 52.00 & 4.60 & 3.60 & 2.30 & 73.50 & 26.50 & 60.40 & 65.00 & 20.00 & 39.60 & 13.10 & 1908.57 & 755.79 & 742.50 \\
\hline 661.00 & 49.60 & 6.60 & 3.70 & 4.80 & 54.90 & 45.10 & 54.90 & 158.00 & 12.00 & 45.10 & .00 & 2063.73 & 930.74 & 742.00 \\
\hline 661.50 & 51.00 & 26.70 & 3.80 & 5.90 & 53.80 & 46.20 & 53.80 & 158.00 & 12.00 & 46.20 & .00 & 2071.49 & 957.03 & 741.50 \\
\hline 662.00 & 52.90 & 25.90 & 3.90 & $2 \cdot 40$ & 69.30 & 30.70 & 55.30 & 141.00 & 10.00 & $44.70^{\circ}$ & 14.00 & 2009.42 & 898.21 & 741.00 \\
\hline $662 \cdot 50$ & 55.50 & 5.60 & $3 \cdot 80$ & $2 \cdot 10$ & 74.70 & $25 \cdot 30$ & 57.00 & 141.00 & 10.00 & 43.00 & 17.70 & 1986.15 & 854.04 & 740.50 \\
\hline 663.00 & $56 \cdot 30$ & 25.30 & 3.60 & 2.40 & 70.70 & 29.30 & 59.10 & 127.00 & 12.00 & 40.90 & 11.60 & 1962.87 & 802.82 & 740.00 \\
\hline 663.50 & 52.60 & 24.80 & 3.40 & 2.60 & $t 9.10$ & 30.90 & 62.00 & 127.00 & 12.00 & 38.00 & 7.10 & 1924.08 & 731.15 & 739.50 \\
\hline 664.00 & 49.80 & 24.40 & 3.20 & 2.60 & 70.90 & $29 \cdot 10$ & 64.60 & 108.00 & 9.00 & 35.40 & 6.30 & 1893.05 & 670.14 & 739.00 \\
\hline 664.50 & 48.00 & 24.60 & 3.20 & 2.60 & 69.60 & 30.40 & 64.40 & 108.00 & 9.00 & 35.60 & 5.20 & 1908.57 & 679.45 & 738.50 \\
\hline & 48.90 & 5.10 & 3.20 & 2.60 & 67.80 & & 62.60 & 123.00 & 10.00 & 37.40 & $5 \cdot 20$ & 1947.36 & 728.31 & 738.00 \\
\hline 665 & $49 \cdot 10$ & 25.80 & 1.30 & 2.50 & 67.10 & 32.90 & 60.10 & 123.00 & 10.00 & 39.90 & 7.00 & 2001.67 & 798.66 & 737.50 \\
\hline 666.00 & $49 \cdot 10$ & 26.70 & 3.40 & 2.70 & $63 \cdot 10$ & 36.90 & 57.20 & 162.00 & 10.00 & 42.80 & 5.90 & 2071.49 & 886.60 & 737.00 \\
\hline 666.50 & 49.00 & 27.20 & 3.50 & 3.00 & $58 \cdot 30$ & 41.70 & 55.50 & 162.00 & 10.00 & 44.50 & 2.80 & 2110.28 & 939.08 & 736.50 \\
\hline 667.00 & 48.70 & 27.70 & 3.60 & 3.30 & $=50.80$ & 45.20 & 53.90 & 188.00 & 10.00 & 46.10 & .90 & 2149.08 & 990 & .00 \\
\hline 667.50 & 49.00 & 27.40 & 3.60 & 3.20 & $56 \cdot 10$ & 43.90 & 54.00 & 188.00 & 10.00 & 46.00 & 2.10 & 2125.80 & 977.87 & 735.50 \\
\hline 668.00 & 49.60 & 26.90 & $3 \cdot 70$ & 3.00 & 58.80 & 41.20 & 54.20 & 167.00 & 11.00 & 45.80 & 0.60 & 2087.01 & 955.85 & 735.00 \\
\hline 668.50 & 51.00 & 27.00 & 3.90 & 3.40 & 55.10 & 44.90 & 53.00 & 167.00 & 0 & 47.00 & 2.10 & & & .50 \\
\hline 669.00 & 52.70 & 27.00 & 3.90 & 3.90 & 52.40 & .7 .60 & 52.40 & 179.00 & 14.00 & 47.60 & .00 & 2125.80 & 1011.88 & 734.00 \\
\hline 669.50 & 55.20 & 27.60 & 3.80 & 3.70 & 52.00 & 48.00 & 52.00 & 179.00 & 14.00 & 48.00 & .00 & 2141.32 & 1027.83 & 733.50 \\
\hline 670.00 & 57.70 & 27.70 & 3.80 & 3.00 & 57.50 & 42.50 & 51.90 & 189.00 & 18.00 & 48.10 & 5.60 & 2149.08 & 1033.70 & 733.00 \\
\hline 670.50 & 59.60 & 27.60 & 3.80 & 3.10 & 56.10 & 43.90 & 52.10 & 189.00 & 18.00 & 90 & 4.00 & 2141.32 & 1025.69 & .50 \\
\hline 671.00 & 59.90 & 27.40 & 3.80 & $3 \cdot 30$ & $55 \cdot 30$ & 44.70 & 52.60 & 180.00 & 22.00 & 47.40 & 2.70 & 2125.80 & 1007.63 & 732.00 \\
\hline 671.50 & 60.30 & 27.20 & 3.80 & 3.30 & 55.80 & 44.20 & 53.10 & 180.00 & 22 & 46.90 & 2.70 & 2110.28 & & \\
\hline 672.00 & 61.20 & 27.10 & 3.80 & 2.90 & 59.70 & 40.30 & 53.20 & 173.00 & 22.00 & 46.80 & 6.50 & 2102.53 & 983.98 & .00 \\
\hline 672.50 & & & 4.00 & 2.90 & to. & 0 & 52.60 & 173.00 & 00 & 47.40 & & 2079.25 & 985.56 & 50 \\
\hline 673.00 & 62.00 & 26.80 & 4.10 & 3.20 & 57.00 & 43.00 & 51.70 & 165.00 & 18.00 & $48 \cdot 30$ & 5.30 & 2079.25 & 1004.28 & 730.00 \\
\hline 673.50 & 60.90 & 26.80 & 4.20 & 3.00 & E9. & 40 & 51.40 & 165 & & & 8. & & & \\
\hline 674.0 & 59.60 & 26.70 & 4.10 & 3.20 & 57.40 & 42.60 & 52.00 & 160.00 & 18.00 & 48.00 & 5.00 & 2071.49 & 994.32 & 729.00 \\
\hline 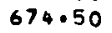 & 58.30 & 26.60 & 4.10 & .00 & 52.70 & 47.30 & 52.70 & 160.00 & 18.00 & 47.30 & .00 & 2063.73 & 976.15 & 728.50 \\
\hline 675.00 & 59.30 & 26.50 & 4.00 & 4.20 & 53.40 & 46. & 53.40 & 155.00 & 19 & 46.60 & .00 & 2055.97 & 958.08 & 728.00 \\
\hline 675.50 & 59.60 & 26.60 & 3.80 & 3.90 & $54 \cdot 10$ & 45. & 54.10 & 155 & & 45.90 & .00 & 3 & 947 & .50 \\
\hline $07<$ & 59.90 & 26.40 & 3.60 & 3.40 & 56.90 & 43.10 & 56.10 & 153.00 & 17.00 & 43.90 & .80 & 2048.22 & 899.17 & 727.00 \\
\hline 676.50 & 59.90 & 26.00 & 3.40 & 3.10 & $60 \cdot 00$ & 40.00 & 58.50 & 153.00 & 17.00 & 41.50 & 1.50 & 2017.18 & 837.13 & 726.50 \\
\hline 677.00 & $59 \cdot$ & 24 & $3 \cdot$ & 3.40 & 63.10 & 36 & 63.10 & 113.00 & 22 & 36.90 & .00 & 1908.57 & 70 & \\
\hline 677 & & 23 & & 3.10 & 66.10 & 33 & 65.90 & 113.00 & 0 & 34.10 & .20 & 1846.50 & & \\
\hline 678.00 & 60.80 & 22.80 & 3.30 & 2.90 & $.72 \cdot 10$ & 27.90 & 69.80 & 81.00 & 27.00 & 31.20 & 3.30 & 1768.91 & 551.90 & 725.00 \\
\hline 678.50 & 61.70 & 22.40 & $3 \cdot 30$ & 2.70 & $75 \cdot 90$ & & & 81 & & & 5.80 & & & .50 \\
\hline & $62 \cdot 80$ & $22 \cdot 20$ & $3 \cdot 30$ & 2.60 & $78 \cdot 30$ & 21. & & 71.00 & 30. & & 7.40 & 1722.36 & & \\
\hline 679.50 & $64 \cdot 30$ & 21.10 & $3 \cdot 30$ & 2.60 & 74.80 & $25 \cdot 20$ & 74.80 & $? 1.00$ & 30.00 & 25.20 & .00 & 1637.02 & 412.53 & 723.50 \\
\hline $680 \cdot 00$ & 66.20 & 19.60 & $3 \cdot 30$ & 2.50 & $80 \cdot 80$ & 19.20 & 80.80 & 41.00 & 36.00 & $19 \cdot 20$ & .00 & 1520.65 & 291.96 & 723.00 \\
\hline & $680^{\circ}$ & & $3 \cdot 3$ & $2 \cdot 5$ & & & & & & & & & 5 & \\
\hline $\begin{array}{l}\text { A.AVE. } \\
\text { G.MEAN }\end{array}$ & $56 \cdot 76$ & 25.18 & 01 & 300 & 4.22 & 33.96 & 10 & 133.14 & & 39. & 3.93 & 70 & 27. & \\
\hline & & & & & & & & & & & & & & \\
\hline
\end{tabular}

*NOTATION--Depth, ft; GR-Gamma Ray Index, API Units; POR-Porosity, $\%$; RT-True Resistivity, Ohms-m²/m; RX0-Flushed Zone Resistivity, Ohms-m²/m; SX0-Flushed Zone Saturation, \%; ROS-Residual 0il Saturation, \%; SW-Water Saturation, \%; PER-Permeability Index; Clay-Clay Content, \%; SO-0il .

Saturation, \%; MD-Movable 0il, q; PHV-Pore Volume, bbl/ac-ft; HPV-Hydrocarbon Pore Volume, bbl/ac-ft; ELSE-Elevation From Sea Level, ft 
TABLE C50

WELL LOG DETERMINED PROPERTIES

VS OEPTH FOR WELL MP-209

WELL LOCATION: 2366 FWL 254 FNL 28 25S $5 E$

KELLY BUSHING ELEVATION FROM SEA. LEVEL $=1398$

\begin{tabular}{|c|c|c|c|c|c|c|c|c|c|c|c|c|c|c|}
\hline DEPTH * & GR & POR & 81 & $R \times U$ & $s \times \theta$ & ReS & SW & PER & CLAY & se & mo & PHV & HPV & ELSE \\
\hline $\begin{array}{l}660 \cdot 00 \\
660 \cdot 50 \\
661 \cdot 00\end{array}$ & $\begin{array}{l}63.20 \\
60.30 \\
57.70\end{array}$ & $\begin{array}{l}17.00 \\
19.9 \mathrm{C} \\
22.50\end{array}$ & $\begin{array}{l}3 \cdot 20 \\
3.10 \\
2.90\end{array}$ & $\begin{array}{l}3.50 \\
3.50 \\
3.40\end{array}$ & $\begin{array}{l}55.60 \\
82.80 \\
74.20\end{array}$ & $\begin{array}{r}4.40 \\
17.20 \\
25.30\end{array}$ & $\begin{array}{l}95.60 \\
82.80 \\
74.20\end{array}$ & $\begin{array}{l}22.00 \\
22.00 \\
76.00\end{array}$ & $\begin{array}{l}27.00 \\
27.00 \\
15.00\end{array}$ & $\begin{array}{r}4.40 \\
17.20 \\
25.80\end{array}$ & $\begin{array}{l}.00 \\
.00\end{array}$ & $\begin{array}{l}1318.93 \\
1543.92 \\
1745.64\end{array}$ & $\begin{array}{r}58.03 \\
265.55\end{array}$ & $\begin{array}{l}738.00 \\
737.50 \\
737.00\end{array}$ \\
\hline $\begin{array}{l}661.50 \\
662 \cdot 00\end{array}$ & $\begin{array}{l}56.60 \\
57.80\end{array}$ & $\begin{array}{l}25.10 \\
26.80\end{array}$ & $\begin{array}{l}2.70 \\
2.60\end{array}$ & $\begin{array}{l}3.30 \\
3.20\end{array}$ & $\begin{array}{l}72 \cdot 30 \\
68 \cdot 40\end{array}$ & $\begin{array}{l}27.70 \\
31.60\end{array}$ & $\begin{array}{l}74.20 \\
68.50 \\
65.00\end{array}$ & $\begin{array}{r}76.00 \\
76.00 \\
163.00\end{array}$ & $\begin{array}{l}15.00 \\
15.00 \\
16.00\end{array}$ & $\begin{array}{l}31.50 \\
35.00\end{array}$ & $\begin{array}{l}3.80 \\
3.40\end{array}$ & $\begin{array}{l}1947.36 \\
2079 \cdot 25\end{array}$ & $\begin{array}{l}613.42 \\
727.74\end{array}$ & $\begin{array}{l}737.00 \\
736.50 \\
736.00\end{array}$ \\
\hline $\begin{array}{l}662 \cdot 50 \\
663.00\end{array}$ & $\begin{array}{l}58.60 \\
56.40\end{array}$ & $\begin{array}{l}26.50 \\
26.80\end{array}$ & $\begin{array}{l}2.60 \\
2.50\end{array}$ & $\begin{array}{l}3.10 \\
3.10\end{array}$ & $\begin{array}{l}70.00 \\
69.50\end{array}$ & $\begin{array}{l}30.00 \\
30.50\end{array}$ & $\begin{array}{l}66.90 \\
67.20\end{array}$ & $\begin{array}{l}163.00 \\
162.00\end{array}$ & $\begin{array}{l}16.00 \\
15.00\end{array}$ & $\begin{array}{l}33.10 \\
32.80\end{array}$ & $\begin{array}{l}3.10 \\
2.30\end{array}$ & $\begin{array}{l}2055.97 \\
2079.25\end{array}$ & $\begin{array}{l}680.53 \\
681.99\end{array}$ & $\begin{array}{l}736.00 \\
735.50 \\
735.00\end{array}$ \\
\hline 663.50 & 53.90 & 27.30 & 2.40 & $3 \cdot 10$ & 67.80 & 32.20 & 66.80 & 162.00 & 15.00 & 33.20 & 1.00 & 2118.04 & 703.19 & $\begin{array}{l}735.00 \\
734.50\end{array}$ \\
\hline 664.00 & $50 \cdot 60$ & 27.10 & $2 \cdot 30$ & 3.10 & $68 \cdot 30$ & 31.70 & 68.20 & 171.00 & 8.00 & 31.80 & .10 & $2102 \cdot 53$ & 668.60 & 734.00 \\
\hline $664 \cdot 50$ & 42.00 & 26.60 & $2 \cdot 30$ & $3 \cdot 10$ & $70 \cdot 00$ & 30.00 & 70.00 & $171 \cdot 00$ & 8.00 & 30.00 & .00 & 2063.73 & 619.12 & 733.50 \\
\hline $665 \cdot 00$ & 50.60 & 26.10 & $2 \cdot 30$ & $3 \cdot 20$ & $72 \cdot 10$ & 27.90 & 72.10 & 146.00 & 8.00 & 27.90 & .00 & 2024.94 & 564.96 & 733.0 \\
\hline $\begin{array}{l}665.50 \\
666.00\end{array}$ & $\begin{array}{l}51 \cdot 30 \\
51.90\end{array}$ & $\begin{array}{l}25.00 \\
25.20\end{array}$ & $\begin{array}{l}2.20 \\
2.10\end{array}$ & $\begin{array}{l}3.30 \\
3.20\end{array}$ & $\begin{array}{l}74.20 \\
77.40\end{array}$ & $\begin{array}{l}25 \cdot 30 \\
22.60\end{array}$ & $\begin{array}{l}74.20 \\
77.40\end{array}$ & $\begin{array}{l}146.00 \\
129.00\end{array}$ & $\begin{array}{r}8.00 \\
11.00\end{array}$ & $\begin{array}{l}25.80 \\
22.60\end{array}$ & $\begin{array}{l}.50 \\
.00\end{array}$ & $\begin{array}{l}1939.60 \\
1955.12\end{array}$ & $\begin{array}{l}500 \cdot 42 \\
441.86\end{array}$ & $\begin{array}{l}732.50 \\
732.00\end{array}$ \\
\hline 666.50 & 52.30 & 25.00 & 2.00 & 3.00 & 79.40 & $20 \cdot 60$ & 79.40 & $129 \cdot 00$ & 11.00 & 20.60 & .00 & 1939.60 & 399.56 & 731.50 \\
\hline $667 \cdot 00$ & 52.60 & 25.30 & $2 \cdot 00$ & 2.90 & $76 \cdot 50$ & 23.50 & 76.50 & $151 \cdot 00$ & 13.00 & $23 \cdot 50$ & .00 & 2040.46 & 479.51 & 731.0 \\
\hline 667.50 & 53.60 & 27.80 & 1.90 & 2.80 & 73.60 & 26.40 & 73.60 & $151 \cdot 00$ & 13.00 & 26.40 & .00 & 2156.83 & 569.40 & $\begin{array}{l}730.50 \\
730.00\end{array}$ \\
\hline 668.00 & $54 \cdot 30$ & 23.20 & 1.80 & 2.70 & $74 \cdot 20$ & 25.80 & 74.20 & $204 \cdot 00$ & 14.00 & 25.80 & .00 & 2187.87 & 564.47 & $\begin{array}{l}730.0 \\
729.5\end{array}$ \\
\hline 668.50 & $54 \cdot 60$ & 2.3 .60 & 1.70 & $2 \cdot 60$ & $74 \cdot 90$ & $25 \cdot 10$ & 74.90 & $204 \cdot 00$ & 14.00 & 25.10 & .00 & $2218 \cdot 90$ & 556.94 & 729.5 \\
\hline 669.00 & $54 \cdot 90$ & 23.90 & 1.70 & $2 \cdot 60$ & 75.00 & 25.00 & 75.00 & $229 \cdot 00$ & 15.00 & $25 \cdot 0 n$ & .00 & $\begin{array}{l}2242.18 \\
2226.66\end{array}$ & $\begin{array}{l}560.54 \\
536.63\end{array}$ & $\begin{array}{l}729.0 \\
728.5\end{array}$ \\
\hline $\begin{array}{l}669.50 \\
670.00\end{array}$ & $\begin{array}{l}55.20 \\
53.70\end{array}$ & $\begin{array}{l}23.70 \\
23.4 \mathrm{C}\end{array}$ & $\begin{array}{l}1.70 \\
1.70\end{array}$ & $\begin{array}{l}2.60 \\
2.00\end{array}$ & $\begin{array}{l}75 \cdot 90 \\
75.30\end{array}$ & $\begin{array}{l}24 \cdot 10 \\
24.70\end{array}$ & $\begin{array}{l}75.90 \\
75.30\end{array}$ & $\begin{array}{l}229.00 \\
212.00\end{array}$ & $\begin{array}{l}15.00 \\
13.00\end{array}$ & $\begin{array}{l}24.10 \\
24.70\end{array}$ & .00 & $\begin{array}{l}2226.66 \\
2203.38\end{array}$ & $\begin{array}{l}536.63 \\
544.24\end{array}$ & $\begin{array}{l}728.50 \\
728.00\end{array}$ \\
\hline $\begin{array}{l}670.00 \\
670.50\end{array}$ & $\begin{array}{l}53.70 \\
53.40\end{array}$ & 28.16 & $\begin{array}{l}1.70 \\
1.80\end{array}$ & $\begin{array}{l}2.00 \\
2.00\end{array}$ & $\begin{array}{l}75 \cdot 30 \\
74 \cdot 80\end{array}$ & $\begin{array}{l}24.70 \\
25.20\end{array}$ & $\begin{array}{l}75.30 \\
74.80\end{array}$ & 212.00 & $\begin{array}{l}13.00 \\
13.00\end{array}$ & $\begin{array}{l}24.70 \\
25.20\end{array}$ & .00 & $2180 \cdot 11$ & 549.39 & $\begin{array}{l}728.00 \\
727.50\end{array}$ \\
\hline 571.00 & 53.40 & 28.00 & 1.90 & 2.60 & 73.80 & 26.20 & 73.80 & 199.00 & 14.00 & 26.20 & .00 & $2172 \cdot 35$ & 569.16 & 727.00 \\
\hline 571.50 & 53.40 & 28.00 & $1 \cdot 90$ & 2.60 & $72 \cdot 70$ & 27.30 & 72.70 & $199 \cdot 00$ & 14.00 & $27 \cdot 30$ & .00 & $2172 \cdot 35$ & 593.05 & 726.5 \\
\hline $572: 00$ & 53.00 & $2 \because \cdot 7 c$ & 2.00 & 2.70 & 72.20 & $27 \cdot 80$ & 72.20 & $190 \cdot 00$ & 14.00 & 27.80 & .00 & 2149.08 & 597.44 & 726.0 \\
\hline $572 \cdot 50$ & $52 \cdot 60$ & $2: 10$ & $2 \cdot 00$ & $2 \cdot 70$ & 13.00 & $27 \cdot 00$ & 73.00 & $190 \cdot 00$ & 14.00 & 27.00 & .00 & 2102.53 & 567.68 & $\begin{array}{l}725.5 \\
725.0\end{array}$ \\
\hline 673.00 & $52 \cdot 20$ & $2 \epsilon .60$ & 2.00 & $2 \cdot 80$ & $74 \cdot 20$ & 25.80 & 74.20 & 159.00 & 15.00 & 25.80 & .00 & 2063.73 & 532.44 & 725.00 \\
\hline 673.50 & $\begin{array}{l}52 \cdot 10 \\
53.60\end{array}$ & $\begin{array}{l}2 E .20 \\
2 E .90\end{array}$ & $\begin{array}{l}2.10 \\
2.10\end{array}$ & $\begin{array}{l}2.90 \\
3.00\end{array}$ & $\begin{array}{l}75 \cdot 50 \\
76 \cdot 10\end{array}$ & 24.50 & $\begin{array}{l}75.50 \\
70.10\end{array}$ & $159 \cdot 00$ & 15.00 & 24.50 & .00 & 2032.70 & 498.01 & $\begin{array}{l}724.50 \\
724.00\end{array}$ \\
\hline 674.00 & 53.60 & 25.90 & $2 \cdot 10$ & 3.00 & $76 \cdot 10$ & 23.90 & 70.10 & 141.00 & 17.00 & 29.90 & 6.00 & $2009 \cdot 42$ & 600.82 & $\begin{array}{l}724.00 \\
723.50\end{array}$ \\
\hline 674.50 & $56 \cdot 30$ & 26.00 & $2 \cdot 10$ & $3 \cdot 00$ & $75 \cdot 50$ & $24 \cdot 50$ & 75.50 & $141 \cdot 00$ & 17.00 & $24 \cdot 50$ & .00 & $2017 \cdot 18$ & 494.21 & 723.50 \\
\hline $\begin{array}{l}675 \cdot 00 \\
675.50\end{array}$ & $\begin{array}{l}58 \cdot 10 \\
59.80\end{array}$ & $\begin{array}{l}26.20 \\
26.30\end{array}$ & $\begin{array}{l}2.10 \\
2.10\end{array}$ & $\begin{array}{l}2 \cdot 90 \\
2.90\end{array}$ & $\begin{array}{l}75.00 \\
14.50\end{array}$ & $\begin{array}{l}25.00 \\
25.50\end{array}$ & $\begin{array}{l}75.00 \\
74.50\end{array}$ & $\begin{array}{l}147.00 \\
147.00\end{array}$ & $\begin{array}{l}22.00 \\
22.00\end{array}$ & $\begin{array}{l}25.00 \\
25.50\end{array}$ & $\begin{array}{l}.00 \\
.00\end{array}$ & $\begin{array}{l}2032.70 \\
2040.46\end{array}$ & $\begin{array}{l}508.17 \\
520.32\end{array}$ & $\begin{array}{l}723.0 \\
722.5\end{array}$ \\
\hline 676.00 & $62 \cdot 30$ & 26.40 & 2.10 & 2.80 & 74.20 & 25.80 & 74.20 & 152.00 & 28.00 & 25.80 & .00 & 2048.22 & 528.44 & 722.00 \\
\hline $6.76 \cdot 50$ & $63 \cdot 50$ & 25.60 & $2 \cdot 10$ & $2 \cdot 80$ & 77.00 & 23.00 & 76.70 & $152 \cdot 00$ & 28.00 & $23 \cdot 30$ & . 30 & $1986 \cdot 15$ & & $721 \cdot 5$ \\
\hline 677.00 & $64 \cdot 60$ & 24.80 & $2 \cdot 20$ & 2.70 & $77 \cdot 90$ & $22 \cdot 10$ & 77.90 & 117.00 & 32.00 & $22 \cdot 10$ & .00 & 1924.08 & .425 .22 & 721.00 \\
\hline 677.50 & $65 \cdot 50$ & 24.60 & $2 \cdot 20$ & $2 \cdot 70$ & 77.40 & 22.60 & 77.40 & 117.00 & $32 \cdot 00$ & 22.60 & .00 & 1908.57 & $431 \cdot 34$ & 720.5 \\
\hline 678.00 & $66 \cdot 30$ & 23.70 & $2 \cdot 30$ & $2 \cdot 80$ & $79 \cdot 20$ & $20 \cdot 80$ & 79.20 & 95.00 & 37.00 & 20.90 & .00 & 1838.74 & 382.46 & 720.0 \\
\hline 678.50 & 67.50 & 21.90 & $2 \cdot 40$ & $2 \cdot 80$ & 85.00 & 15.00 & 85.00 & 95.00 & 37.00 & $15 \cdot \mathrm{co}$ & .00 & 1699.09 & 254.86 & 719. \\
\hline A.AVE- & & & 2.16 & an & 50 & & 5 & 27 & & 95 & .57 & 2033.74 & & \\
\hline & 55.62 & 26.14 & $2 \cdot 14$ & • 90 & $74 \cdot 50$ & $25 \cdot 11$ & 73.93 & 144.90 & 15.91 & 5.59 & & 2027.89 & 519.01 & \\
\hline
\end{tabular}

*:NOTATIOH--Depth, $\mathrm{ft}$; GR-Gamma Ray Index, API Units; POR-Porosity, \%; RT-True Resistivity, Ohms-m²/m; RX0-Flushed Zone Resistivity, Ohms-m²/m; SxO-Flushed Zone Satura:ion, \%; ROS-Residual 0il Saturation, \%; SH-Water Saturation, \%; PER-Permeability Index; Clay-Clay Content, $\%$; SO-0il

Saturation, \%; MO-Movabe 0il, \%; PHV-Pore Volume, bbl/ac-ft; HPV-Hydrocarbon Pore Volume, bbl/ac-ft; ELSE-Elevation From Sea Level, ft 
TABLE C51

WELL LOG DETERMINED PROPERTIES

VS DEPTH FOR WELL MP-210

WELL LOCATION: 2112 FWL E64 FNL 28 25S $5 E$

KELL.Y BUSHIi!G ELEVATION FROH SEA LEVEL $=1391$

\begin{tabular}{|c|c|c|c|c|c|c|c|c|c|c|c|c|c|c|}
\hline DEPTH* & GR & PQF & $R T$ & $R \times C$ & $s \times e$ & ROS & SW & PER & CLAY & so & ro & PHV & HPV & ELSE \\
\hline 62.00 & 68.00 & 19.80 & 2.90 & $1.8 \mathrm{C}$ & 84.90 & $15 \cdot 10$ & 84.90 & 4.00 & 32.00 & $15 \cdot 10$ & .00 & $1536 \cdot 16$ & $231 \cdot 96$ & 0 \\
\hline 662.50 & 66.30 & 22.00 & 3.00 & $1.9 \mathrm{C}$ & 75.80 & 24.20 & 75.30 & 4.00 & 32.00 & 24.70 & .50 & 1706.85 & 421.59 & 728.50 \\
\hline 663.00 & 65.90 & 24.50 & 3.00 & 1.90 & $\in 7.50$ & $32 \cdot 50$ & 66.50 & 110.00 & 26.00 & 33.50 & 1.00 & $1900 \cdot 81$ & 636.77 & 728.00 \\
\hline 663.50 & 63.80 & 24.60 & 3.10 & $1.0 \ddot{0}$ & $E 7.60$ & 32.40 & 66.00 & 110.00 & 26.00 & $34.0 n$ & 1.60 & 1908.57 & 648.91 & 727.50 \\
\hline 664.00 & 60.00 & 24.40 & 2.70 & 1.96 & $70 \cdot 20$ & 29.80 & 70.20 & 108.00 & 20.00 & 29.80 & .00 & 1893.05 & $564 \cdot 13$ & 727.00 \\
\hline 664.50 & 55.50 & 23.90 & 2.50 & $2 \cdot 00$ & $74 \cdot 90$ & $25 \cdot 10$ & 74.90 & 108.00 & 20.00 & $25 \cdot 10$ & .00 & $1854 \cdot 26$ & 465.42 & 726.50 \\
\hline 665.00 & 53.60 & 22.90 & 2.30 & $2 \cdot 4 \mathrm{C}$ & $22 \cdot 20$ & 17.80 & 82.20 & 81.00 & 14.00 & 17.80 & .00 & 1776.67 & $316 \cdot 25$ & 726.00 \\
\hline 665.50 & 55.60 & 21.40 & 2.20 & 2.40 & 91.20 & 8.80 & 91.20 & 81.00 & 14.00 & 8.80 & .00 & $1660 \cdot 30$ & 146.11 & 725.50 \\
\hline 666.00 & 55.60 & 22.50 & 2.00 & 2.00 & 89.50 & $10 \cdot 50$ & 89.50 & 76.00 & 13.00 & $10 \cdot 5 c$ & .00 & 1745.64 & 183.29 & 725.00 \\
\hline 666.50 & 54.80 & $24.4 \mathrm{C}$ & 1.90 & 1.50 & 84.90 & 15.10 & 84.90 & 76.00 & 13.00 & 15.10 & .00 & 1893.05 & 285.85 & 724.50 \\
\hline 667.00 & 54.00 & 26.90 & 1.80 & 1.40 & 77.70 & $22 \cdot 30$ & 77.70 & 167.00 & 6.00 & $22 \cdot 30$ & .00 & 2087.01 & 465.40 & 724.00 \\
\hline 667.50 & 55.80 & 27.10 & 1.80 & $1 \cdot 3 c$ & $78 \cdot 30$ & 21.70 & 78.30 & 167.00 & 6.00 & 21.70 & .00 & 2102.53 & $456 \cdot 25$ & 723.50 \\
\hline 668.00 & 57.60 & 26.90 & 1.70 & $1.2 \mathrm{G}$ & 79.80 & $20 \cdot 10$ & 79.90 & 167.00 & 11.00 & $20 \cdot 10$ & .00 & 2087.01 & .19 .49 & 723.00 \\
\hline 668.50 & 59.50 & 26.70 & 1.70 & $1 \cdot 30$ & 81.40 & 18.60 & 81.40 & 167.00 & 11.00 & 18.60 & .00 & 2071.49 & $385 \cdot 30$ & 722.50 \\
\hline 669.00 & 59.80 & 27.00 & 1.60 & 1.60 & 81.50 & 18.50 & 81.50 & 169.00 & 14.00 & 18.50 & .00 & 2094.77 & 387.53 & 722.00 \\
\hline 669.50 & 57.80 & $27.3 \mathrm{c}$ & 1.60 & $1.7 c$ & 80.80 & 19.20 & 80.80 & 169.00 & 14.00 & $19 . ? 0$ & .00 & 2118.04 & 406.66 & 721.50 \\
\hline 670.00 & $55 \cdot 80$ & $28.0 \mathrm{C}$ & 1.60 & 1.10 & $78 \cdot 30$ & 21.70 & 78.30 & 198.00 & 6.00 & 21.70 & .00 & $2172 \cdot 35$ & 471.40 & 721.00 \\
\hline 670.50 & $54 \cdot 90$ & 28.60 & 1.70 & 1.00 & $16 \cdot 30$ & 23.70 & 76.30 & $198 \cdot 00$ & 6.00 & 23.70 & .00 & 2218.90 & 525.88 & 720.50 \\
\hline 671.00 & 55.90 & 28.8 & 1.70 & 1.40 & $75 \cdot 30$ & 24.70 & 75.30 & 223.00 & 7.00 & 24.70 & .00 & $2234 \cdot 42$ & $551 \cdot 90$ & 720.00 \\
\hline 671.50 & 59.20 & 29.00 & 1.70 & $1 \cdot 30$ & $74 \cdot 40$ & $25 \cdot 60$ & 74.40 & 223.00 & 7.00 & $25 \cdot 60$ & .00 & 2249.93 & 575.98 & 719.50 \\
\hline 672.00 & 62.50 & 29. & 1.70 & 1.40 & 73.00 & 27.00 & 73.00 & 243.00 & 11.00 & & .00 & 2273.21 & 613.77 & 719.00 \\
\hline 672.50 & 65.80 . & 29.80 & 1.70 & 1.50 & $71 \cdot 30$ & 28.70 & $71 \cdot 30$ & 243.00 & 11.00 & 28.70 & .00 & 2312.00 & 663.54 & 718.50 \\
\hline 673.00 & 69.00 & 28.60 & 1.80 & 1.00 & 73.70 & $26 \cdot 30$ & 73.70 & 217.00 & 18.00 & $26 \cdot 30$ & .00 & 2218.90 & 583.57 & 718.00 \\
\hline 673.50 & 71.00 & 26.70 & 1.80 & $1 \cdot 50$ & $78 \cdot 10$ & 21.90 & 78.10 & 217.00 & 18.00 & 21.90 & .00 & 2071.49 & 453.66 & 717.50 \\
\hline 674.00 & 73.00 & 21.90 & 1.90 & $1 \cdot 5 \mathrm{C}$ & $55 \cdot 70$ & $4 \cdot 30$ & 95.70 & 68.00 & 33.00 & 4.30 & .00 & 1699.09 & 73.06 & 717.00 \\
\hline$A \cdot A$ & 59 & 25.8 & 2.06 & 1 . & 77 & 14 & 77.73 & 150 & 14.83 & 22.27 & .13 & 2007.81 & 52 & \\
\hline & & 25. & 2.01 & & $7 \%$ & $21 \cdot 18$ & & 134 & 13.00 & 21.27 & & $1996 \cdot 23$ & 424.62 & \\
\hline
\end{tabular}

*NOTATID:1--Depth, ft; GR-Gamma Ray Index, API Units; POR-Porosity, $\%$; RT-True Resistivity, Ohms-m²/m; RX0-Flushed Zone Resistivity, Ohms-m² ${ }^{2}$; SX0-Flushed Zone Saturation, $\%$; ROS-Residual $0 i 1$ Saturation, $\%$; SW-Water Saturation, \%; PER-Permeability Index; Clay-Clay-Content, \%; SO-0il

Saturation, \%; MO-Movable 0il, \%; PHV-Pore Volume, bbl/ac-ft; HPV-Hydrocarbon Pore Volume, bbl/ac-ft; ELSE-Elevation From Sea Level, ft 
TABLE C52

WELL LOG DETERHINEO PROPERTIES

VS DEPTH FOR WELL MP-211

WELL LOCATION: 2112 FWL 752 FNL 282555

KELLY BUSHING ELEVATION FROI SEA LEVEL $=1389$

\begin{tabular}{|c|c|c|c|c|c|c|c|c|c|c|c|c|c|c|}
\hline DEPTH * & GR & PQF & RT & $R \times U$ & $\leq \times A$ & RAS & SW & PER & CLAY & so & mo & PMV & HPV & ELSE \\
\hline $\begin{array}{l}658 \cdot 60 \\
658 \cdot 50\end{array}$ & $\begin{array}{l}65 \cdot 50 \\
64.30\end{array}$ & $\begin{array}{l}19.00 \\
26.30\end{array}$ & $\begin{array}{l}3.60 \\
3.50\end{array}$ & $\begin{array}{l}4.30 \\
4.60\end{array}$ & $\begin{array}{l}80.30 \\
62.00\end{array}$ & $\begin{array}{l}19.70 \\
38.00\end{array}$ & $\begin{array}{l}80.30 \\
57.70\end{array}$ & $\begin{array}{l}29.00 \\
29.00\end{array}$ & $\begin{array}{l}27.00 \\
27.00\end{array}$ & $\begin{array}{l}19 \cdot 70 \\
42.30\end{array}$ & $\begin{array}{r}.00 \\
4.30\end{array}$ & $\begin{array}{l}1474.10 \\
2040.46\end{array}$ & $\begin{array}{l}290.40 \\
863.11\end{array}$ & $\begin{array}{l}731.00 \\
730.50\end{array}$ \\
\hline $659 \cdot 00$ & 68.50 & 26.60 & 3.20 & 4.50 & $t 1.70$ & $38 \cdot 30$ & 59.00 & $126 \cdot 00$ & $20 \cdot 00$ & 41.00 & 2.70 & 2063.73 & 846.13 & 730.00 \\
\hline 659.50 & 69.60 & 25.80 & 3.20 & 4.30 & $\epsilon 5.80$ & 34.20 & 61.80 & 126.00 & 20.00 & 38.20 & 4.00 & 2001.67 & 764.64 & 729.50 \\
\hline $\begin{array}{l}660.50 \\
661.00 \\
661.50\end{array}$ & $\begin{array}{l}61 \cdot 30 \\
58 \cdot 90\end{array}$ & $\begin{array}{l}25.70 \\
25.10\end{array}$ & $\begin{array}{l}2.80 \\
2.80\end{array}$ & $\begin{array}{l}4.30 \\
4.10\end{array}$ & $\begin{array}{l}65.40 \\
67.70\end{array}$ & $\begin{array}{l}34 \cdot 60 \\
32 \cdot 30\end{array}$ & $\begin{array}{l}65.40 \\
67.70\end{array}$ & $\begin{array}{r}118.00 \\
97.00\end{array}$ & $\begin{array}{l}14.00 \\
12.00\end{array}$ & $32 \cdot 30$ & .00 & 1947.36 & 629.00 & 728.00 \\
\hline $661 \cdot 50$ & $56 \cdot 50$ & $\begin{array}{l}24.20 \\
23.20\end{array}$ & $\begin{array}{l}2.70 \\
2.50\end{array}$ & $5 \cdot 2 \mathrm{C}$ & $71 \cdot 80$ & 28.20 & 71.80 & 97.00 & 12.00 & $28 \cdot 20$ & .00 & $\begin{array}{l}1877.53 \\
1799.95\end{array}$ & $\begin{array}{l}529.46 \\
408.59\end{array}$ & $\begin{array}{l}727.50 \\
727.00\end{array}$ \\
\hline $\begin{array}{l}662 \cdot 00 \\
662.50\end{array}$ & $\begin{array}{l}54.80 \\
53.60\end{array}$ & $\begin{array}{l}23.20 \\
22.10\end{array}$ & $\begin{array}{l}2.50 \\
2.40\end{array}$ & $\begin{array}{l}5 \cdot 10 \\
4.30\end{array}$ & $\begin{array}{l}77 \cdot 30 \\
82.90\end{array}$ & $\begin{array}{l}22.70 \\
17.10\end{array}$ & $\begin{array}{l}77.30 \\
82.90\end{array}$ & $\begin{array}{l}68 \cdot 00 \\
68.00\end{array}$ & $\begin{array}{l}11.00 \\
11.00\end{array}$ & $\begin{array}{l}22.70 \\
17.10\end{array}$ & $\begin{array}{l}.00 \\
.00\end{array}$ & $\begin{array}{l}1799.95 \\
1714.61\end{array}$ & 293.20 & 726.50 \\
\hline 663.00 & 54.20 & 21.60 & 2.40 & 3.90 & 86.50 & $13 \cdot 50$ & 86.50 & $50 \cdot 00$ & 12.00 & 13.50 & .00 & $1675 \cdot 81$ & $\begin{array}{l}226.23 \\
186.02\end{array}$ & $\begin{array}{l}726.00 \\
725.50\end{array}$ \\
\hline $663 \cdot 50$ & $\begin{array}{l}56 \cdot 00 \\
56 \cdot 50\end{array}$ & $\begin{array}{l}21.60 \\
23.30\end{array}$ & $2 \cdot 20$ & $3 \cdot 90$ & $88 \cdot 90$ & $\begin{array}{l}11.10 \\
17.50\end{array}$ & $\begin{array}{l}88.90 \\
82.50\end{array}$ & $\begin{array}{l}50.00 \\
70.00\end{array}$ & $\begin{array}{l}12.00 \\
14.00\end{array}$ & $\begin{array}{l}11.10 \\
17.50\end{array}$ & .00 & $\begin{array}{l}1675.81 \\
1807.71\end{array}$ & $\begin{array}{l}186.02 \\
316.35\end{array}$ & $\begin{array}{l}725.50 \\
725.00\end{array}$ \\
\hline $\begin{array}{l}664 \cdot 00 \\
66.4 \cdot 50\end{array}$ & $\begin{array}{l}56 \cdot 50 \\
57.10\end{array}$ & $\begin{array}{l}23.30 \\
26.00\end{array}$ & $\begin{array}{l}2.20 \\
2.20\end{array}$ & $\begin{array}{l}3.90 \\
3.70\end{array}$ & $\begin{array}{l}82 \cdot 50 \\
74 \cdot 10\end{array}$ & $\begin{array}{l}17 \cdot 50 \\
25 \cdot 90\end{array}$ & $\begin{array}{l}82.50 \\
74.10\end{array}$ & $\begin{array}{l}70.00 \\
70.00\end{array}$ & $\begin{array}{l}14.00 \\
14.00\end{array}$ & 25.90 & .00 & 2017.18 & 522.45 & 724.50 \\
\hline $66.5 \cdot 00$ & $57 \cdot 10$ & 27.30 & 2.10 & 3.00 & 71.50 & 28.50 & 71.50 & 141.00 & $\$ 1.00$ & 28.50 & .00 & 2118.04 & 603.64 & 724.00 \\
\hline $\begin{array}{l}6 E 6.50 \\
6 E 7.00\end{array}$ & $\begin{array}{l}64 \cdot 30 \\
67.30\end{array}$ & $\begin{array}{l}27.30 \\
27.70\end{array}$ & $\begin{array}{l}2 \cdot 20 \\
2 \cdot 30\end{array}$ & $\begin{array}{l}3.00 \\
3.70\end{array}$ & $\begin{array}{l}70.30 \\
67.40\end{array}$ & $\begin{array}{l}29.70 \\
32.60\end{array}$ & $\begin{array}{l}70.30 \\
67.40\end{array}$ & $\begin{array}{l}140.00 \\
148.00\end{array}$ & 22.00 & 32.60 & .00 & 2149.08 & 700.60 & 722.00 \\
\hline $\begin{array}{l}668 \cdot 00 \\
668 \cdot 50\end{array}$ & & $\begin{array}{l}27.10 \\
26 . \geqslant 0\end{array}$ & $\begin{array}{l}2 \cdot 40 \\
2 \cdot 50\end{array}$ & $\begin{array}{l}4 \cdot C 0 \\
3 \cdot 90\end{array}$ & $\begin{array}{l}t 7 \cdot 20 \\
66 \cdot 30\end{array}$ & $\begin{array}{l}32.80 \\
33.70\end{array}$ & $\begin{array}{l}67.20 \\
66.30\end{array}$ & $\begin{array}{l}135.00 \\
135.00\end{array}$ & $\begin{array}{l}27.00 \\
27.00\end{array}$ & 33.70 & .00 & 2087.01 & $703 \cdot 32$ & 720.50 \\
\hline $\begin{array}{l}668.50 \\
669.00\end{array}$ & 67.90 & $\begin{array}{l}26.30 \\
26.20\end{array}$ & 2.60 & $\begin{array}{l}3.90 \\
3.90\end{array}$ & 65.40 & 34.60 & 65.40 & 129.00 & 29.00 & $34.6 \mathrm{C}$ & .00 & 2079.25 & 719.42 & 720.00 \\
\hline $\begin{array}{l}669 \cdot 00 \\
669.50\end{array}$ & 67.90 & $26.50^{\circ}$ & 2.60 & $3 \cdot 90$ & $\in 6 \cdot 30$ & 33.70 & 66.30 & 129.00 & 29.00 & $33.7 \mathrm{C}$ & .00 & 2055.97 & 692.86 & 719.50 \\
\hline 670.00 & 68.50 & $26 \cdot 30$ & 2.60 & $4 \cdot 00$ & $\in 6.10$. & 33.90 & 66.10 & 119.00 & 30.00 & 33.90 & .00 & 2040.46 & 691.71 & 719.00 \\
\hline 670.50 & 69.60 & $26 \cdot 30$ & 2.70 & 4.00 & 67.00 & 33.00 & 66.50 & 119.00 & 30.00 & 33.50 & .50 & $2017 \cdot 18$ & 675.76 & 718.50 \\
\hline 671.00 & 72.00 & $25 \cdot .00$ & 2.70 & $3 \cdot 9 \mathrm{C}$ & 70.40 & 29.60 & 68.60 & $95 \cdot 00$ & 34.00 & 31.40 & 1.80 & 1939.60 & 609.03 & 718.00 \\
\hline $671 \cdot 50$ & 75.00 & 23.50 & 2.80 & 4.00 & $74 \cdot 40$ & $25 \cdot 60$ & 72.50 & $95 \cdot 00$ & 34.00 & $27 \cdot 50$ & 1.90 & $1830 \cdot 98$ & 503.52 & 717.50 \\
\hline 672.00 & $78 \cdot 50$ & 21.10 & $2 \cdot 90$ & $3 \cdot 90$ & $79 \cdot 90$ & $20 \cdot 10$ & 79.90 & $45 \cdot 00$ & 42.00 & $20 \cdot 10$ & .00 & 1637.02 & 329.04 & $\begin{array}{l}717.00 \\
716.50\end{array}$ \\
\hline 672.50 & $78 \cdot 50$ & 21.00 & 2.80 & 4.00 & $\varepsilon_{1} \cdot 40$ & $18 \cdot 60$ & 81.40 & $45 \cdot 00$ & 42.00 & 18.60 & .00 & 1629.26 & 303.04 & 716.50 \\
\hline 673.00 & $76 \cdot 80$ & 21.90 & $2 \cdot 90$ & $4 \cdot 10$ & $79 \cdot 80$ & $20 \cdot 20$ & 76.30 & 53.00 & 42.00 & 23.70 & 3.50 & 1699.09 & 402.68 & 716.00 \\
\hline 673.50 & 76.20 & 21.70 & 2.90 & $4 \cdot 10$ & $77 \cdot 10$ & 22.90 & 77.10 & 53.00 & 42.00 & 22.90 & .00 & 1683.57 & 385.54 & 715.50 \\
\hline 674.00 & $80 \cdot 30$ & $18.2 \mathrm{C}$ & 2.90 & 4.20 & 50.50 & 9.50 & 90.50 & 27.00 & 49.00 & $9 \cdot 50$ & .00 & 1458.58 & 138.56 & 715.00 \\
\hline AVE. & $\begin{array}{l}65.53 \\
65.09\end{array}$ & .67 & $2 \cdot 65$ & و & 7 & 27.28 & 2.08 & 95.55 & & 39 & .63 & $\begin{array}{l}1913.97 \\
1902.69\end{array}$ & $\begin{array}{l}548.10 \\
502.13\end{array}$ & \\
\hline - MEAR & 65.09 & .52 & .62 & 4.07 & $72 \cdot 31$ & $25 \cdot 87$ & 1.62 & & & & & & & \\
\hline
\end{tabular}

*NOTATION--Depth, $\mathrm{ft}$; GR-Gamma Ray Index, API Units; POR-Porosity, $\%$; RT-True Resistivity, Ohms-m²/m; RXO-Flushed Zone Resistivity, Ohms-m²/m; S:XO-Flushed Zone Saturction, \%; ROS-Residual 0il Saturation, \%; SW-Water Saturation, \%; PER-Permeability Index; Clay-Clay Content, \%; SO-0il

saturation, \%; MD-Movatie 0il, s; PHV-Pore Volume, bbl/ac-ft; HPV-Hydrocarbon Pore Volume, bbl/ac-ft; ELSE-Elevation From Sea Level, ft 
TABLE C53

WELL LOG DETERMINED PROPERTIES

VS DEPTH FOR WELL MP-212

WELL LOCATION: 528 FNL 2376 FWL 28255 5E

KELLY BUISHING ELEVATION FROM SEA LEVEL $=1397$

\begin{tabular}{|c|c|c|c|c|c|c|c|c|c|c|c|c|c|c|}
\hline DEPTH* & GR & $P Q R$ & RT & $R \times O$ & $5 \times 0$ & ROS & $S W$ & PER & CLAY & se & mo & PHV & HPV & ELSE \\
\hline $\begin{array}{l}663.00 \\
663.50\end{array}$ & $\begin{array}{l}78.80 \\
70.30\end{array}$ & $\begin{array}{l}18.50 \\
22.80\end{array}$ & $\begin{array}{l}4.40 \\
4.40\end{array}$ & $\begin{array}{l}2 \cdot 50 \\
2 \cdot 80\end{array}$ & $\begin{array}{l}74.40 \\
65.70\end{array}$ & $\begin{array}{l}25 \cdot 60 \\
34 \cdot 30\end{array}$ & $\begin{array}{l}74.40 \\
59.80\end{array}$ & $\begin{array}{l}32 \cdot 00 \\
32 \cdot 00\end{array}$ & $\begin{array}{l}39.00 \\
39.00\end{array}$ & $\begin{array}{l}25.60 \\
40.20\end{array}$ & $\begin{array}{r}.00 \\
5.90\end{array}$ & $\begin{array}{l}1435.30 \\
1768.91\end{array}$ & $\begin{array}{l}367.44 \\
711.10\end{array}$ & $\begin{array}{l}734.00 \\
733.50\end{array}$ \\
\hline $\begin{array}{l}664 \cdot 00 \\
664 \cdot 50\end{array}$ & $\begin{array}{l}63.80 \\
62.30\end{array}$ & $\begin{array}{l}22.80 \\
22.65\end{array}$ & $\begin{array}{l}4.40 \\
4.30\end{array}$ & $\begin{array}{l}2.60 \\
2.50\end{array}$ & $\begin{array}{l}67.70 \\
69.50\end{array}$ & $\begin{array}{l}32.30 \\
30.50\end{array}$ & $\begin{array}{l}59.90 \\
61.10\end{array}$ & $\begin{array}{l}81.00 \\
81.00\end{array}$ & $\begin{array}{l}17.00 \\
17.00\end{array}$ & $\begin{array}{l}40.10 \\
38.90\end{array}$ & $\begin{array}{l}7.80 \\
8.40\end{array}$ & $\begin{array}{l}1768.91 \\
1753.40\end{array}$ & $\begin{array}{l}709.33 \\
682.07\end{array}$ & $\begin{array}{l}733.00 \\
732.50\end{array}$ \\
\hline $\begin{array}{l}665 \cdot 50 \\
666.00\end{array}$ & $\begin{array}{l}61.00 \\
58.40\end{array}$ & $\begin{array}{l}2.1 .80 \\
21.70\end{array}$ & $\begin{array}{l}3 \cdot 70 \\
3 \cdot 30\end{array}$ & $\begin{array}{l}3 \cdot 20 \\
3.20\end{array}$ & $\begin{array}{l}68.40 \\
72.40\end{array}$ & $\begin{array}{l}31.60 \\
27.60\end{array}$ & $\begin{array}{l}68.40 \\
72.40\end{array}$ & $\begin{array}{l}68.00 \\
64.00\end{array}$ & $\begin{array}{l}16.00 \\
12.00\end{array}$ & $\begin{array}{l}31.60 \\
27.60\end{array}$ & $\begin{array}{l}.00 \\
.00\end{array}$ & 1683.57 & 464.67 & \\
\hline 667.50 & $\begin{array}{l}56.70 \\
57.80\end{array}$ & $\begin{array}{l}21.90 \\
24.00\end{array}$ & $\begin{array}{l}2.60 \\
2.40\end{array}$ & $\begin{array}{l}2.80 \\
2.00\end{array}$ & $\begin{array}{l}80.60 \\
77.00\end{array}$ & 23.00 & $\begin{array}{l}80.60 \\
77.00\end{array}$ & $\begin{array}{l}68.00 \\
68.00\end{array}$ & $\begin{array}{l}11.00 \\
11.00\end{array}$ & $\begin{array}{l}19.40 \\
23.00\end{array}$ & .00 & 1862.02 & 428.26 & 729.50 \\
\hline $\begin{array}{l}668 \cdot 00 \\
668.50\end{array}$ & $59 \cdot 20$ & $25 \cdot 4 n$ & $2 \cdot 10$ & $2 \cdot 30$ & $77 \cdot 50$ & 22.50 & 77.50 & 128.00 & 12.00 & $22 \cdot 50$ & .00 & $\begin{array}{l}1970.63 \\
2017.18\end{array}$ & $\begin{array}{r}443.39 \\
391.33\end{array}$ & $\begin{array}{l}729.00 \\
728.50\end{array}$ \\
\hline $\begin{array}{l}668.50 \\
669.00\end{array}$ & $\begin{array}{l}60 \cdot 90 \\
62.60\end{array}$ & $\begin{array}{l}26.00 \\
26.90\end{array}$ & $\begin{array}{l}1.80 \\
1.70\end{array}$ & $\begin{array}{l}2.20 \\
2.00\end{array}$ & $\begin{array}{l}80 \cdot 60 \\
80.30\end{array}$ & $\begin{array}{l}19 \cdot 40 \\
19.70\end{array}$ & $\begin{array}{l}80.60 \\
80.30\end{array}$ & $\begin{array}{l}128.00 \\
166.00\end{array}$ & $\begin{array}{l}12.00 \\
13.00\end{array}$ & $\begin{array}{l}19.40 \\
19.70\end{array}$ & .00 & $\begin{array}{l}2017.18 \\
2087.01\end{array}$ & 411.14 & 728.00 \\
\hline $\begin{array}{l}669.50 \\
670.50\end{array}$ & $\begin{array}{l}62.90 \\
62.80\end{array}$ & 27.20 & 1.60 & 1.80 & $82 \cdot 10$ & $17 \cdot 90$ & $82 \cdot 10$ & $166 \cdot 00$ & 13.00 & 17.90 & .00 & 2110.28 & $\begin{array}{l}377.74 \\
364.30\end{array}$ & $\begin{array}{l}727.50 \\
726.50\end{array}$ \\
\hline $\begin{array}{l}670.50 \\
670.00\end{array}$ & $\begin{array}{l}62 \cdot 80 \\
63 \cdot 20\end{array}$ & $\begin{array}{l}27 \cdot 30 \\
27.30\end{array}$ & $\begin{array}{l}1.60 \\
1.50\end{array}$ & $\begin{array}{l}1.70 \\
1.70\end{array}$ & $\begin{array}{l}82.80 \\
83.00\end{array}$ & $\begin{array}{l}17.20 \\
17.00\end{array}$ & $\begin{array}{l}82.80 \\
83.00\end{array}$ & $\begin{array}{l}176.00 \\
176.00\end{array}$ & $\begin{array}{l}12.00 \\
12.00\end{array}$ & $\begin{array}{l}17.20 \\
17.00\end{array}$ & $\begin{array}{l}.00 \\
.00\end{array}$ & $\begin{array}{l}2118.04 \\
2118.04\end{array}$ & $\begin{array}{l}364.30 \\
360.07\end{array}$ & 727.00 \\
\hline $\begin{array}{l}670.00 \\
671.00\end{array}$ & 62.20 & 27.10 & $\begin{array}{l}1.50 \\
1.60\end{array}$ & $\begin{array}{l}1.70 \\
1.70\end{array}$ & $\begin{array}{l}83 \cdot 00 \\
83.00\end{array}$ & 17.00 & $\begin{array}{l}83.00 \\
83.00\end{array}$ & $\begin{array}{l}176.00 \\
172.00\end{array}$ & 13.00 & 17.00 & .00 & 2102.53 & 357.43 & 726.00 \\
\hline 671.50 & 61.30 & 26.80 & 1.60 & $1.7 \mathrm{C}$ & 82.70 & $17 \cdot 30$ & 82.70 & 172.00 & 13.00 & $17 \cdot 30$ & .00 & 2079.25 & 359.71 & $725 \cdot 50$ \\
\hline 672.00 & $61 \cdot 50$ & 26.40 & 1.70 & $1 \cdot 8 \mathrm{c}$ & $22 \cdot 00$ & 18.00 & 82.00 & 153.00 & $15 \cdot 00$ & 18.00 & .00 & $2048 \cdot 22$ & 368.68 & $725 \cdot 00$ \\
\hline 672.50 & 63.80 & 26.00 & .80 & $1.8 \mathrm{C}$ & 81.80 & $18 \cdot 20$ & 81.80 & 153.00 & $15 \cdot 00$ & $18 \cdot 20$ & .00 & $2017 \cdot 18$ & 367.13 & 724.50 \\
\hline $\begin{array}{l}673 \cdot 00 \\
673.50\end{array}$ & $65 \cdot 50$ & 26.60 & $1 \cdot 80$ & $1 \cdot 90$ & 78.20 & 21.80 & 78.20 & 157.00 & $20 \cdot 00$ & 21.80 & .00 & $\begin{array}{l}2063.73 \\
2079.25\end{array}$ & $\begin{array}{r}449.89 \\
496.94\end{array}$ & $\begin{array}{l}724 \cdot 00 \\
723.50\end{array}$ \\
\hline 674.00 & $\begin{array}{l}66 \cdot 80 \\
68 \cdot 10\end{array}$ & $\begin{array}{l}26.80 \\
26.80\end{array}$ & $\begin{array}{l}1.90 \\
2.00\end{array}$ & $\begin{array}{l}2 \cdot 00 \\
2.00\end{array}$ & $\begin{array}{l}76 \cdot 10 \\
74.00\end{array}$ & $\begin{array}{l}23.90 \\
26.00\end{array}$ & $\begin{array}{l}76.10 \\
74.00\end{array}$ & $\begin{array}{l}157.00 \\
163.00\end{array}$ & $\begin{array}{l}20.00 \\
22.00\end{array}$ & $\begin{array}{l}23.90 \\
26.00\end{array}$ & $\begin{array}{l}.00 \\
.00\end{array}$ & $\begin{array}{l}2079.25 \\
2079.25\end{array}$ & 540.60 & 723.00 \\
\hline $\begin{array}{l}674.00 \\
674.50\end{array}$ & $\begin{array}{l}68.10 \\
70 \cdot 10\end{array}$ & 26.50 & $2 \cdot 10$ & $2 \cdot 10$ & 72.90 & 27.10 & 72.90 & 163.00 & 22.00 & 27.10 & .00 & $2055 \cdot 97$ & $\begin{array}{l}557.17 \\
536.52\end{array}$ & $\begin{array}{l}722.50 \\
722.00\end{array}$ \\
\hline 675.00 & $72 \cdot 10$ & 25.90 & $2 \cdot 20$ & $2 \cdot 20$ & $73 \cdot 30$ & $26 \cdot 70$ & $73 \cdot 30$ & $1+1 \cdot 00$ & 27.00 & $26 \cdot 7 n$ & $\begin{array}{l}.00 \\
.00\end{array}$ & $\begin{array}{l}2009.42 \\
1838.74\end{array}$ & $\begin{array}{l}536.52 \\
371.43\end{array}$ & 721.50 \\
\hline $\begin{array}{l}676.00 \\
676.50\end{array}$ & $\begin{array}{l}76.80 \\
77.90\end{array}$ & $\begin{array}{l}22.30 \\
21.90\end{array}$ & $\begin{array}{l}2.30 \\
2.40\end{array}$ & $\begin{array}{l}2 \cdot 30 \\
2 \cdot 20\end{array}$ & $\begin{array}{l}83 \cdot 90 \\
84.80\end{array}$ & $\begin{array}{l}16 \cdot 10 \\
15 \cdot 20\end{array}$ & 84.80 & 73.00 & 37.00 & $15 \cdot 20$ & .00 & 1699.09 & 258.26 & $720 \cdot 50$ \\
\hline 677.00 & 80.00 & 20.90 & 2.40 & 2.30 & 88.50 & $11 \cdot 50$ & 88.50 & 55.00 & 41.00 & 11.50 & .00 & 1621.50 & 186.47 & 720.00 \\
\hline $677 \cdot 50$ & $80 \cdot 30$ & 20.90 & $2 \cdot 50$ & $2 \cdot 20$ & $87 \cdot 70$ & $12 \cdot 30$ & 87.70 & $55 \cdot 00$ & 41.00 & $12 \cdot 30$ & .00 & $\begin{array}{l}1621.50 \\
1668.06\end{array}$ & 199.45 & $\begin{array}{l}719.50 \\
719.00\end{array}$ \\
\hline 678.00 & 79.90 & 21.50 & $2 \cdot 50$ & $2 \cdot 10$ & $85 \cdot 10$ & 14.90 & 85.10 & 61.00 & 39.00 & 14.90 & $\begin{array}{l}.00 \\
.00\end{array}$ & $\begin{array}{l}1668.06 \\
1714.61\end{array}$ & $\begin{array}{l}248.54 \\
298.34\end{array}$ & 718.50 \\
\hline $\begin{array}{l}678.50 \\
679.00\end{array}$ & $\begin{array}{l}78 \cdot 30 \\
78.10\end{array}$ & $\begin{array}{l}22.10 \\
20.90\end{array}$ & $\begin{array}{l}2.50 \\
2.50\end{array}$ & $\begin{array}{l}2 \cdot 10 \\
2 \cdot 20\end{array}$ & $\begin{array}{l}82.60 \\
87.80\end{array}$ & $\begin{array}{l}17.40 \\
12.20\end{array}$ & $\begin{array}{l}82.60 \\
87.80\end{array}$ & 54.00 & $\begin{array}{l}39.00 \\
35.00\end{array}$ & $\begin{array}{l}17.40 \\
12.20\end{array}$ & .00 & 1621.50 & 197.82 & 718.00 \\
\hline $679 \cdot 50$ & $78 \cdot 40$ & $19 \cdot 20$ & $2 \cdot 50$ & $2 \cdot 50$ & $95 \cdot 40$ & 4.60 & 95.40 & $54 \cdot 00$ & 35.00 & 4.6 & .0 & 1489.61 & 68.52 & $717 \cdot 50$ \\
\hline & 70 & 80 & 37 & $\begin{array}{l}2.25 \\
2.21\end{array}$ & $\begin{array}{l}78.58 \\
78.34\end{array}$ & $\begin{array}{l}21.42 \\
20.57\end{array}$ & $\begin{array}{l}77.80 \\
77.39\end{array}$ & $\begin{array}{r}108.18 \\
95.77\end{array}$ & $\begin{array}{l}22.03 \\
19.61\end{array}$ & $\begin{array}{l}22.20 \\
21.03\end{array}$ & .78 & $\begin{array}{l}1864.36 \\
1853.86\end{array}$ & $\begin{array}{r}412.49 \\
389.86\end{array}$ & \\
\hline
\end{tabular}

*NOTATION--Depth, $\mathrm{ft}$; GR-Gamma Ray Index, API Units; POR-Porosity, $\%$; RT-True Resistivity, Ohms-m²/m; RXO-Flushed Zone Resistivity; Ohms-m²/m; SXO-Flushed Zone Saturatio 7 \%; ROS-Residual 0il Saturation, \%; SW-Water Saturation, \%; PER-Permeability Index; Clay-Clay Content, \%; SO-0il Saturction, \%; MO-Movable Jil, \%; PHV-Pore Volume, bbl/ac-ft; HPV-Hydrocarbon Pore Volume, bbl/ac-ft; ELSE-Elevation From Sea Level, ft 
TABLE C54

WELL LOG DETERMINED PROPERTIES

VS DEPTH FOR WELL MP-213

WELL LOCATION: $2640 \mathrm{FWL} 5 \overline{8} \mathbf{F N L} 28$ 25S 5E

KELLY BUSHING ELEVATION FROM SEA LEVEL=1403

\begin{tabular}{|c|c|c|c|c|c|c|c|c|c|c|c|c|c|c|}
\hline DEPTH* & $G R$ & PAR & RT & $R \times O$ & $s \times \theta$ & RES & SW & PER & CLAY & se & mo & PHV & HPV & ELSE \\
\hline $\begin{array}{l}673.50 \\
674.00\end{array}$ & $\begin{array}{l}90 \cdot 80 \\
87.20\end{array}$ & $\begin{array}{l}19.90 \\
24.00\end{array}$ & $\begin{array}{l}2.60 \\
2.60\end{array}$ & $\begin{array}{l}3 \cdot 30 \\
3.30\end{array}$ & $\begin{array}{l}89.90 \\
78.00\end{array}$ & $\begin{array}{l}10.10 \\
22.00\end{array}$ & $\begin{array}{l}89.90 \\
73.00\end{array}$ & $\begin{array}{l}91.00 \\
91.00\end{array}$ & $\begin{array}{l}38.00 \\
38.00\end{array}$ & $\begin{array}{l}10.10 \\
27.00\end{array}$ & $\begin{array}{r}.00 \\
5.00\end{array}$ & $\begin{array}{l}1543.92 \\
1862.02\end{array}$ & $\begin{array}{l}155.94 \\
502.74\end{array}$ & $\begin{array}{l}729.50 \\
729.00\end{array}$ \\
\hline $674 \cdot 50$ & 83.60 & 26.90 & 2.70 & 3.30 & 88.60 & 31.40 & 64.00 & 91.00 & 38.00 & 36.00 & 4.60 & 2087.01 & $751 \cdot 32$ & 728.50 \\
\hline $\begin{array}{l}675 \cdot 00 \\
675 \cdot 50\end{array}$ & $80 \cdot 90$ & $27 \cdot 20$ & $2 \cdot 70$ & $3 \cdot 40$ & 67.50 & $32 \cdot 50$ & $63 \cdot 10$ & $177 \cdot 00$ & 28.00 & $36 \cdot 90$ & 4.40 & 2110.28 & 778.69 & $\begin{array}{l}728.00 \\
727.50\end{array}$ \\
\hline $\begin{array}{l}676.00 \\
676.50\end{array}$ & $\begin{array}{l}75.20 \\
73.40\end{array}$ & $\begin{array}{l}27.20 \\
27.20\end{array}$ & 2.70 & 3.40 & $\begin{array}{l}66.90 \\
66.60\end{array}$ & $\begin{array}{l}33.10 \\
33.40\end{array}$ & $\begin{array}{l}63.40 \\
63.50\end{array}$ & $\begin{array}{l}210.00 \\
210.00\end{array}$ & $\begin{array}{l}17.00 \\
17.00\end{array}$ & $\begin{array}{l}36 \cdot 60 \\
36 \cdot 50\end{array}$ & $3 \cdot 10$ & 2110.28 & 770.25 & 726.50 \\
\hline $\begin{array}{l}677 \cdot 0.0 \\
677 \cdot 50\end{array}$ & 73.20 & 27.20 & $2 \cdot 60$ & 3.50 & $66 \cdot 30$ & 33.70 & 63.60 & 201.00 & 13.00 & $36 \cdot 40$ & 2.70 & $\begin{array}{l}2110.28 \\
2110.28\end{array}$ & $\begin{array}{l}768.14 \\
763.92\end{array}$ & $\begin{array}{l}726.00 \\
725.50\end{array}$ \\
\hline $\begin{array}{l}677 \cdot 50 \\
678 \cdot 0.0\end{array}$ & $\begin{array}{l}73.00 \\
73.00\end{array}$ & $\begin{array}{l}27.20 \\
27.20\end{array}$ & $\begin{array}{l}2.60 \\
2.60\end{array}$ & $\begin{array}{l}3.50 \\
3.50\end{array}$ & $\begin{array}{l}66.00 \\
65.80\end{array}$ & $\begin{array}{l}34 \cdot 00 \\
34 \cdot 20\end{array}$ & $\begin{array}{l}63.80 \\
63.90\end{array}$ & $\begin{array}{l}201 \cdot 00 \\
186 \cdot 00\end{array}$ & $\begin{array}{l}13.00 \\
12.00\end{array}$ & $\begin{array}{l}36 \cdot 20 \\
36.10\end{array}$ & $\begin{array}{l}2 \cdot 20 \\
1.90\end{array}$ & $\begin{array}{l}2110.28 \\
2110.28\end{array}$ & $761 \cdot 81$ & 725.00 \\
\hline $678 \cdot 50$ & 73.00 & 27.20 & 2.60 & $3.5 \mathrm{C}$ & 65.60 & 34.40 & 64.00 & 186.00 & 12.00 & 36.00 & 1.60 & 2110.28 & 759.70 & $\begin{array}{l}724.50 \\
724.00\end{array}$ \\
\hline $\begin{array}{l}679.00 \\
679.50\end{array}$ & $71 \cdot 20$ & 27.20 & $2 \cdot 50$ & 3.60 & 65.40 & $34 \cdot 60$ & 65.00 & $162 \cdot 00$ & 14.00 & $35 \cdot 00$ & .40 & 2110.28 & 738.60 & $\begin{array}{l}724.00 \\
723.50\end{array}$ \\
\hline $\begin{array}{l}680.00 \\
680.50\end{array}$ & $\begin{array}{l}73.40 \\
75.00\end{array}$ & $\begin{array}{l}26.40 \\
26.30\end{array}$ & $\begin{array}{l}2 \cdot 40 \\
2 \cdot 30\end{array}$ & $\begin{array}{l}3.60 \\
3.50\end{array}$ & $\begin{array}{l}69.10 \\
70.70\end{array}$ & $\begin{array}{l}30 \cdot 90 \\
29 \cdot 30\end{array}$ & $\begin{array}{l}69.10 \\
70.70\end{array}$ & $\begin{array}{l}140.00 \\
140.00\end{array}$ & $\begin{array}{l}17.00 \\
17.00\end{array}$ & $\begin{array}{l}30 \cdot 90 \\
29.30\end{array}$ & .00 & $\begin{array}{l}2048 \cdot 22 \\
2040 \cdot 46\end{array}$ & $\begin{array}{l}632 \cdot 90 \\
597.85\end{array}$ & $\begin{array}{l}723.00 \\
722.50 \\
722.00\end{array}$ \\
\hline $\begin{array}{l}681 \cdot 00 \\
681 \cdot 50\end{array}$ & $\begin{array}{l}72.00 \\
75.80\end{array}$ & $\begin{array}{l}26.20 \\
26.10\end{array}$ & $\begin{array}{l}2 \cdot 20 \\
2 \cdot 20\end{array}$ & $\begin{array}{l}3 \cdot 50 \\
3 \cdot 40\end{array}$ & $\begin{array}{l}72.00 \\
73.40\end{array}$ & $\begin{array}{l}28 \cdot 00 \\
26.60\end{array}$ & $\begin{array}{l}72.00 \\
73.40\end{array}$ & $\begin{array}{l}133.00 \\
133.00\end{array}$ & $\begin{array}{l}17.00 \\
17.00\end{array}$ & $\begin{array}{l}28 \cdot 00 \\
26.60\end{array}$ & $\begin{array}{l}.00 \\
.00\end{array}$ & $\begin{array}{l}2032 \cdot 70 \\
2024 \cdot 94\end{array}$ & $\begin{array}{l}569.16 \\
538.63\end{array}$ & $\begin{array}{l}722.00 \\
721.50\end{array}$ \\
\hline $\begin{array}{l}682 \cdot 00 \\
682.50\end{array}$ & $\begin{array}{l}83.70 \\
88.40\end{array}$ & $\begin{array}{l}24.90 \\
21.40\end{array}$ & $\begin{array}{l}2 \cdot 10 \\
2.00\end{array}$ & $\begin{array}{l}3.30 \\
3.20\end{array}$ & $\begin{array}{l}78 \cdot 60 \\
94.30\end{array}$ & $\begin{array}{r}21.40 \\
5.70\end{array}$ & $\begin{array}{l}78.60 \\
94.30\end{array}$ & $\begin{array}{l}107.00 \\
107.00\end{array}$ & $\begin{array}{l}30.00 \\
30.00\end{array}$ & $\begin{array}{r}21.4 n \\
5.70\end{array}$ & .00 & $\begin{array}{l}1931.84 \\
1660.30\end{array}$ & $\begin{array}{r}413.41 \\
94.64\end{array}$ & $\begin{array}{l}721.00 \\
720.50\end{array}$ \\
\hline & 7.44 & 25.99 & $\begin{array}{l}2.49 \\
2.48\end{array}$ & $\begin{array}{l}3.43 \\
3.43\end{array}$ & $\begin{array}{l}71.51 \\
71.10\end{array}$ & $\begin{array}{l}28.49 \\
26.43\end{array}$ & $\begin{array}{l}69.75 \\
69.25\end{array}$ & $\begin{array}{l}152.89 \\
147.03\end{array}$ & $\begin{array}{l}21.58 \\
19.83\end{array}$ & $\begin{array}{l}30.25 \\
27.89\end{array}$ & .76 & $\begin{array}{l}2016 \cdot 36 \\
2009 \cdot 44\end{array}$ & $\begin{array}{l}623.13 \\
560.51\end{array}$ & \\
\hline
\end{tabular}

^NOTATIOH--Depth, ft; GR-Gamma Ray Index, API Units; POR-Porosity, 6 ; RT-True Resistivity, Ohms-m²/m; RXO-flusied Zone Resistivity, Ohmis-m²/m; SXO-Flushed Zone Saturation, \%; ROS-Residual 0il Saturation, \%; SW-Water Saturation, \%; PER-Permeability Index; Clay-Clay Content, \%; SO-0il

Saturation, \%; MO-Mowable 0il, \%; PHV-Pore Volume, bbl/ac-ft; HPV-Hydrocarbon Pore Volume, bbl/ac-ft; ELSE-Elevation From Sea Level, ft 
TABLE C55

WELL LOG DETERMINED PROPERTIES

VS DEPTH FOR WELL PAP-214

WELL LOCATION: 2376 FEL 528 FNL $282555 E$

KELLY BUSHING ELEVATION FROM SEA LEVEL $=1403$

\begin{tabular}{|c|c|c|c|c|c|c|c|c|c|c|c|c|c|c|}
\hline DEPTH* & $G R$ & $P Q R$ & RY & $R \times E$ & $\leqslant \times 0$ & ROS & SW & PER & CLAY & so & mo & PHV & HPV & ELSE \\
\hline $\begin{array}{l}661.50 \\
662.00\end{array}$ & $\begin{array}{l}77.10 \\
70.50\end{array}$ & $\begin{array}{l}17.00 \\
18.50\end{array}$ & $\begin{array}{l}3.60 \\
3.70\end{array}$ & $\begin{array}{l}1.40 \\
1.60\end{array}$ & $\begin{array}{l}89.90 \\
81.00\end{array}$ & $\begin{array}{l}10.10 \\
19.00\end{array}$ & $\begin{array}{l}89.90 \\
81.00\end{array}$ & $\begin{array}{l}11.00 \\
32.00\end{array}$ & $\begin{array}{l}49.00 \\
29.00\end{array}$ & $\begin{array}{l}10.10 \\
19.00\end{array}$ & $\begin{array}{l}.00 \\
.00\end{array}$ & $\begin{array}{l}1318.93 \\
1435.30\end{array}$ & $\begin{array}{l}133.21 \\
272.71\end{array}$ & $\begin{array}{l}741.50 \\
741.00\end{array}$ \\
\hline 662.50 & $66 \cdot 70$ & 18.50 & 3.70 & 2.50 & 21.00 & 19.00 & 81.00 & 32.00 & 29.00 & 19.00 & .00 & 1435.30 & 272.71 & 740.50 \\
\hline 663.00 & 63.00 & 18.50 & 3.80 & $3.8 \mathrm{C}$ & $80 \cdot 70$ & $19 \cdot 30$ & 80.70 & 32.00 & 18.00 & $19 \cdot 30$ & .00 & $1435 \cdot 30$ & 277.01 & 740.00 \\
\hline 663.50 & 62.50 & 18.70 & 3.80 & $3 \cdot 0$ & 79.50 & 20.50 & 79.50 & 32.00 & 18.00 & 20.50 & $\begin{array}{r}.00 \\
6.30\end{array}$ & 1450.82 & 297.42 & 739.50 \\
\hline $\begin{array}{l}664.00 \\
664.50\end{array}$ & $\begin{array}{l}63.90 \\
62.50\end{array}$ & $\begin{array}{l}22.70 \\
24.70\end{array}$ & $\begin{array}{l}3.80 \\
3.80\end{array}$ & $\begin{array}{l}2.00 \\
1.90\end{array}$ & $\begin{array}{l}70.80 \\
65.30\end{array}$ & $\begin{array}{l}29 \cdot 20 \\
34.70\end{array}$ & $\begin{array}{l}64.50 \\
58.80\end{array}$ & $\begin{array}{l}78.00 \\
78.00\end{array}$ & $\begin{array}{l}19.00 \\
19.00\end{array}$ & $\begin{array}{l}35.50 \\
41.20\end{array}$ & $\begin{array}{l}6.30 \\
6.50\end{array}$ & $\begin{array}{l}1761 \cdot 16 \\
1916 \cdot 32\end{array}$ & $\begin{array}{l}625.21 \\
789.52\end{array}$ & 739.00 \\
\hline 665.00 & 61.40 & 26.80 & 3.80 & 1.90 & 59.60 & 40.40 & 53.90 & 163.00 & 15.00 & 46.10 & 5.70 & 2079.25 & 958.53 & 738.00 \\
\hline 665.50 & 60.80 & 26.40 & 3.80 & 1.90 & $60 \cdot 40$ & 39.60 & 54.80 & 163.00 & 15.00 & 45.20 & 5.60 & 2048.22 & 925.79 & 737.50 \\
\hline 666.00 & 63.40 & 25.80 & 3.80 & 2.00 & 61.40 & 38.60 & 56.10 & 137.00 & 15.00 & 43.90 & $5 \cdot 30$ & 2001.67 & 878.73 & 737.00 \\
\hline 666.50 & 64.60 & 25.40 & 3.80 & 2.10 & 61.40 & 38.60 & 57.10 & 137.00 & 15.00 & 42.90 & 4.30 & 1970.63 & 845.40 & 736.50 \\
\hline 667.00 & 64.50 & 26.30 & 3.70 & 2.00 & $59 \cdot 30$ & 40.70 & 55.60 & 150.00 & 16.00 & 4.0 .00 & 3.70 & 2040.46 & 905.96 & 736.00 \\
\hline 667.50 & 62.50 & 27.20 & 3.70 & 2.00 & $58 \cdot 10$ & 41.90 & 54.20 & 150.00 & 16.00 & 45.80 & 3.90 & 2110.28 & 966.51 & 735.50 \\
\hline 668.00 & 63.10 & 26.90 & 3.60 & 1.90 & 59.50 & 40.50 & 55.50 & 166.00 & 18.00 & 44.50 & $\$ .00$ & 2087.01 & 928.72 & 735.00 \\
\hline 668.50 & 61.30 & 27.00 & 3.50 & 1.90 & 60.10 & 39.90 & 55.60 & 166.00 & 18.00 & 44.40 & $\$ .50$ & 2094.77 & 930.08 & 734.50 \\
\hline 669.00 & 59.80 & 27.30 & 3.40 & 1.80 & 60.00 & 40.00 & 55.80 & 177.00 & 12.00 & 44.20 & 4.20 & 2118.04 & 936.17 & 734.00 \\
\hline 669.50 & 59.10 & 27.00 & 3.00 & 1.90 & $60 \cdot 30$ & 39.70 & 56.90 & 177.00 & 12.00 & 13.10 & 3.40 & 2094.77 & 902.84 & 733.50 \\
\hline 670.00 & 59.40 & 26.50 & $3 \cdot 30$ & 1.80 & 62.50 & 37.50 & 58.80 & 157.00 & 10.00 & 41.20 & 3.70 & 2055.97 & 847.06 & 733.00 \\
\hline 670.50 & 65.60 & 26.10 & 3.20 & 2.10 & $60 \cdot 30$ & 39.70 & 60.30 & 157.00 & 10.00 & 39.70 & .00 & 2024.94 & 803.90 & 732.50 \\
\hline 671.00 & 70.40 & 24.60 & 3.20 & 2.10 & 65.00 & 35.00 & 65.00 & 112.00 & 19.00 & 35.00 & .00 & 1908.57 & 668.00 & 732.00 \\
\hline 671.50 & 73.10 & 24.20 & 3.20 & 1.90 & 66.60 & 33.40 & 66.30 & 112.00 & 19.00 & & .30 & 1877.53 & 632.73 & 731.50 \\
\hline 672.00 & $75 \cdot 30$ & ?. 3.00 & 3.20 & 1.80 & 73.60 & 26.40 & 69.90 & 83.00 & 28.00 & 30.10 & 3.70 & 1784.43 & 537.11 & 731.00 \\
\hline & 79 & 13 & $=$ & 1.60 & $\varepsilon$ & 1 & & 83 & 2 & & .00 & 1512.89 & 246.60 & 730.50 \\
\hline A.AVE. & 65.63 & 23.85 & 57 & .04 & 67.83 & 32.17 & 65.00 & 112.39 & $19 \cdot 43$ & 35.00 & 2.83 & 1850.54 & 677.47 & \\
\hline G.MEAN & 65.40 & 23.58 & 56 & 99 & 67.18 & 30.25 & 64.08 & 91.94 & 18.08 & $32.5 ?$ & & 1829.37 & 594.83 & \\
\hline
\end{tabular}

*NOTATICH--Depth, ft; GR-Gamma Ray Index, API Units; POR-Porosity, \%; RT-True Resistivity, Ohms-m²/m; RXo-Flushed Zone Resistivity, Ohnis-m²/m; SX0-Flushed Zone Saturation, \%; ROS-Residual 0 il Saturation, \%; SW-Water Saturation, \%; PER-Permeability Index; Clay-Clay Content, \%; S0-0il Saturation, \%; MO-Movable Dil, \%; PHV-Pore Volume, bbl/ac-ft; HPV-Hydrocarbon Pore Volume, bbl/ac-ft; ELSE-Elevation From Sea Level, ft 
WELL LOG DETERHINED PROPERTIES

VS DEPTH FOR WELL MP- 215

WELL LOCATION: 2112 FEL $5 \overline{8} 8$ FNL 28 25S 5E

KELLY BUSHING ELEVATION FROM SEA LEVEL $=1397$

\begin{tabular}{|c|c|c|c|c|c|c|c|c|c|c|c|c|c|c|}
\hline DEPTH * & $G R$ & PJR & $R T$ & $R \times E$ & $5 \times 0$ & ROS & Sw & PER & CLAY & so & ro & PHV & HPV & ELSE \\
\hline 649.00 & $60 \cdot 20$ & 13.80 & 3.40 & 4.40 & 83.10 & 16.90 & $83 \cdot 10$ & 35.00 & 28.00 & 16.90 & .00 & 1458.58 & 246.50 & 748.00 \\
\hline 649.50 & 54.90 & $2+.20$ & 3.40 & $5 \cdot 00$ & 67.00 & 33.00 & 64.00 & 35.00 & 28.00 & 36.00 & 3.00 & 1877.53 & 675.91 & 747.50 \\
\hline 650.00 & $52 \cdot 60$ & 2.000 & 3.20 & 5 (5) & 67.70 & $32 \cdot 30$ & 66.50 & $100 \cdot 00$ & 15.00 & 33.50 & $\begin{array}{l}1.20 \\
2.60\end{array}$ & 1862.02 & 623.78 & 747.00 \\
\hline .650 .50 & 50.80 & 23.70 & 3.10 & 4.10 & $70 \cdot 40$ & 29.60 & 67.80 & $100 \cdot 00$ & 15.00 & 32.20 & 2.60 & 1838.74 & 592.07 & 746.50 \\
\hline 651.00 & 50.20 & 23.70 & $3 \cdot 10$ & 4.00 & 71.00 & 29.00 & 67.80 & 96.00 & 14.00 & $32 \cdot ? 0$ & 3.20 & 1838.74 & 592.07 & 746.00 \\
\hline 651.50 & 51.40 & $2+.00$ & 3.00 & 4.00 & 70.30 & $29.7 n$ & 68.30 & 96.00 & 14.00 & 31.70 & 2.00 & $1862: 02$ & 590.26 & 745.50 \\
\hline 652.00 & 51.40 & $2+.10$ & 2.90 & 4.00 & 69.90 & 30.10 & 59.80 & 103.00 & 15.00 & 30.20 & .10 & 1869.77 & 564.57 & 745.00 \\
\hline 652.50 & 49.00 & $2+.20$ & $2 \cdot 80$ & 4.10 & $70 \cdot 10$ & 29.90 & 70.10 & 103.00 & 15.00 & 29.90 & .00 & 1877.53 & $561 \cdot 38$ & 744.50 \\
\hline 653.00 & 49.00 & $2+.40$ & 2.70 & $4 \cdot 40$ & 71.60 & 28.40 & 71.60 & 107.00 & 14.00 & 28.40 & .00 & 1893.05 & 537.63 & 744.00 \\
\hline 553.50 & 49.00 & $2 . .40$ & 2.60 & 4.10 & $72 \cdot 30$ & 27.70 & $72 \cdot 30$ & 107.00 & 14.00 & 27.70 & .00 & 1893.05 & 524.37 & $7+3.50$ \\
\hline 554.00 & 50.80 & 24.50 & 2.60 & 4.70 & $71 \cdot 90$ & $28 \cdot 10$ & 71.90 & 110.00 & 18.00 & 28.10 & .00 & 1900.81 & 534.13 & 743.00 \\
\hline 354.50 & 53.70 & 24.20 & 2.60 & 4.60 & 72.60 & 27.40 & 72.60 & 110.00 & 18.00 & 27.40 & .00 & 1877.53 & 514.44 & 742.50 \\
\hline 555.00 & $57 \cdot 30$ & $24.7 n$ & 2.70 & 4.00 & 70.50 & $29 \cdot 50$ & 70.50 & 115.00 & 24.00 & 29.50 & .00 & 1916.32 & 565.32 & 742.00 \\
\hline 555.50 & 60.80 & 24.90 & $2 \cdot 80$ & 4.00 & $68 \cdot 70$ & $31 \cdot 30$ & 68.70 & $115 \cdot 00$ & 24.00 & $31 \cdot 30$ & .00 & 1931.84 & 604.67 & 741.50 \\
\hline 556.00 & 65.00 & $2 \hat{\varepsilon} \cdot R 0$ & 2.90 & 4.50 & $74 \cdot 90$ & $25 \cdot 10$ & 74.10 & 80.00 & 39.00 & 25.90 & .80 & 1768.91 & 458.15 & 741.00 \\
\hline .556 .50 & 69.10 & $1 \subseteq .50$ & 2.80 & 4.70 & 87.80 & 12.20 & 87.80 & 80.00 & 39.00 & $12 \cdot 20$ & .00 & 1520.65 & 185.52 & 740.50 \\
\hline 557.00 & 66.10 & 21.50 & $2 \cdot 90$ & $5 \cdot: 0$ & $78 \cdot 10$ & $21 \cdot 90$ & 78.10 & 63.00 & 41.00 & 21.90 & .00 & 1668.06 & 365.30 & 740.00 \\
\hline 057.50 & 52.60 & 24.90 & 2.90 & $5 \cdot 3+\mathrm{c}$ & $\epsilon 6.80$ & 33.20 & 66.80 & 63.00 & 41.00 & 33.20 & .00 & 1931.84 & $641 \cdot 37$ & 739.50 \\
\hline 658.00 & $60 \cdot 8.0$ & $2 \epsilon \cdot 30$ & $2 \cdot 90$ & $5 \cdot 20$ & $\epsilon 3.50$ & $36 \cdot 50$ & 63.50 & 151.00 & 30.00 & 36.50 & .00 & 2040.46 & 744.77 & 739.00 \\
\hline 658.50 & 59.60 & 27.10 & 2.90 & $5 \cdot<0$ & $61 \cdot 10$ & $38 \cdot 90$ & 61.10 & 151.00 & 30.00 & $38 \cdot 90$ & .00 & 2102.53 & 817.88 & 738.50 \\
\hline 659.00 & $60 \cdot 20$ & $2 \epsilon .90$ & 2.90 & 4.80 & 61.40 & 38.60 & 61.40 & 167.00 & 29.00 & 38.60 & .00 & 2087.01 & 805.58 & 738.00 \\
\hline$\$ 59.50$ & 61.40 & $2 \epsilon .50$ & 2.90 & $4 \cdot 2 C$ & $66 \cdot 20$ & 33.80 & 63.20 & 167.00 & 29.00 & 36.80 & 3.00 & 2055.97 & 756.60 & 737.50 \\
\hline 060.00 & 61.40 & 26.10 & 2 & $4 \cdot 40$ & 65.90 & 34.10 & 64.70 & 145.00 & 28.00 & $35 \cdot 30$ & 1.20 & 2024.94 & 714.80 & 737.00 \\
\hline $660 \cdot 50$ & $60 \cdot 80$ & 25.50 & 2.80 & $4 \cdot 30$ & 68.20 & 31.80 & 67.00 & 145.00 & 28.00 & 33.00 & 1.20 & 1978.39 & 652.87 & 736.50 \\
\hline 061.00 & 61.40 & 24.80 & 2.70 & 4.10 & 72.40 & 27.60 & 69.70 & 115.00 & 28.00 & $30 \cdot 30$ & 2.70 & 1924.08 & 583.00 & 736.00 \\
\hline 661.50 & 62.60 & 24.40 & 2.70 & $4 \cdot 10$ & 73.00 & 27.00 & 71.60 & 115.00 & 28.00 & 28.40 & 1.40 & 1893.05 & 537.63 & 735.50 \\
\hline $062 \cdot 00$ & 63.20 & 23.70 & 2.60 & $4 \cdot 40$ & $75 \cdot 10$ & 24.90 & 75.10 & 95.00 & 31.00 & 24.90 & .00 & 1838.74 & 457.85 & 735.00 \\
\hline 662.50 & 61.40 & 24.10 & $2 \cdot 50$ & $4 \cdot 50$ & $74 \cdot 30$ & 25.70 & $74 \cdot 30$ & 95.00 & 31.00 & 25.70 & .00 & 1869.77 & 480.53 & 734.50 \\
\hline 6.63 .00 & $59 \cdot 60$ & 25.10 & 2.60 & $4 \cdot 40$ & $70 \cdot 10$ & 29.90 & 70.10 & 121.00 & 24.00 & 29.90 & .00 & 1947.36 & $582 \cdot 26$ & 734.00 \\
\hline 6.63 .50 & 60.80 & 26.30 & 2.60 & 4.50 & 66.40 & 33.60 & 66.40 & 121.00 & 24.00 & 33.60 & .00 & 2040.46 & 685.59 & 733.50 \\
\hline 664.00 & $60 \cdot 80$ & 26.80 & 2.70 & $4 \cdot 30$ & 64.60 & 35.40 & 64.60 & 164.00 & 27.00 & 35.40 & .00 & 2079.25 & 736.05 & 733.00 \\
\hline 664.50 & $57 \cdot 90$ & 27.30 & $2 \cdot 80$ & 4.50 & $62 \cdot 20$ & 37.8 & $6 ? .20$ & 164.00 & 27.00 & 37.80 & .00 & 2118.04 & 800.62 & 732.50 \\
\hline 665.00 & $57 \cdot 90$ & 27.80 & 2.80 & $4 \cdot 50$ & 60.50 & $\begin{array}{l}39.50 \\
39.50\end{array}$ & 60.40 & 192.00 & 27.00 & 39.60 & .10 & 2156.83 & 854.11 & 732.00 \\
\hline $\begin{array}{l}665 \cdot 50 \\
666.30\end{array}$ & $\begin{array}{l}59.60 \\
62.00\end{array}$ & $\begin{array}{l}28.00 \\
28.10 .\end{array}$ & 2.80 & $\begin{array}{l}4.40 \\
4.50\end{array}$ & Ec. 50 & 39.50 & $60 \cdot 40$ & 192.00 & 27.00 & 39.60 & .10 & $2172 \cdot 35$ & 860 & 731.50 \\
\hline $666 \cdot 00$ & $62 \cdot 00$ & 28.10. & 2.90 & $4 \cdot 50$ & $59 \cdot 70$ & 40.30 & 59.10 & 202.00 & 33.00 & 40.90 & .60 & 2180.11 & 891.66 & 731.00 \\
\hline & $66 \cdot 10$ & 25.40 & 3.00 & 4.40 & $67 \cdot 10$ & $32 \cdot 90$ & 64.60 & 202.00 & 33.00 & 35.40 & 2.50 & 1970.63 & 697.60 & 730.50 \\
\hline 667.00 & 71.50 & $20 \cdot 30$ & 3.00 & 4.40 & 82.40 & 17.60 & 82.40 & 48.00 & $50 \cdot 00$ & 17.60 & .00 & 1574.95 & 277.19 & 730.00 \\
\hline 667.50 & $75.0 \mathrm{C}$ & 16.90 & 3.00 & $4.4 \mathrm{C}$ & $59 \cdot 70$ & $\cdot 30$ & 99.70 & 48.00 & 50.00 & .20 & .00 & 1311.17 & 3,93 & 729.50 \\
\hline A.AVE, & 58.73 & 24.68 & 2.85 & 4.59 & $\in 9.71$ & 30.29 & 69.02 & 118.11 & 26.49 & $30 \cdot 98$ & .69 & 1914.64 & 603.09 & \\
\hline
\end{tabular}

*NOTITIOH--Depth, ft; ìR-Gamma Ray Index, API Units; POR-Porosity, $\%$; RT-True Resistivity, Ohms-m ${ }^{2} / m$; RX0-Flushed Zone Resistivity, Ohns-m ${ }^{2} / m$;

jXO-Flushed Zone Saturation, \%; ROS-Residual 0il Saturation, \%; SW-Water Saturation, \%; PER-Permeability Index; Clay-Clay Content, \%; SO-0il

saturation, z; Mo-Movable 0il, z; PHV-Pore Volume, bbl/ac-ft; HPV-Hydrocarbon Pore Volume, bbl/ac-ft; ELSE-Elevazion From Sea Levei, ft 
TABLE C57

WELL LOG DETERAINED PROPERTIES

VS DEPTH FOR WELL MP-216

WELL LOCATION: 3168 FEL $792 \mathrm{FNL} 282555$

KELLY BUSHING ELEVATION FROM SEA LEVEL $=1397$

\begin{tabular}{|c|c|c|c|c|c|c|c|c|c|c|c|c|c|c|}
\hline DEPTH ${ }^{\star}$ & GR & $P Q R$ & $R T$ & $R \times C$ & $5 \times 8$ & RES & SW & PER & CLAY & so & re & PHV & HPV & ELSE \\
\hline $\begin{array}{l}654.00 \\
654.50\end{array}$ & $\begin{array}{l}62 \cdot 20 \\
66.90\end{array}$ & $\begin{array}{l}19.80 \\
22.70\end{array}$ & $\begin{array}{l}4.00 \\
4.10\end{array}$ & $\begin{array}{l}6.20 \\
4.40\end{array}$ & $\begin{array}{l}72 \cdot 90 \\
73.80\end{array}$ & $\begin{array}{l}27.10 \\
26.20\end{array}$ & $\begin{array}{l}72.90 \\
62.10\end{array}$ & $\begin{array}{l}43.00 \\
43.00\end{array}$ & $\begin{array}{l}6.00 \\
6.00\end{array}$ & $\begin{array}{l}27.10 \\
37.90\end{array}$ & 11.00 & $\begin{array}{l}1536 \cdot 16 \\
1761 \cdot 16\end{array}$ & $\begin{array}{l}416.30 \\
667.48\end{array}$ & $\begin{array}{l}743.00 \\
742.50\end{array}$ \\
\hline $\begin{array}{l}655.00 \\
655.50\end{array}$ & $\begin{array}{l}81.40 \\
83.70\end{array}$ & $\begin{array}{l}17.00 \\
15.60\end{array}$ & $\begin{array}{l}4 \cdot 20 \\
4 \cdot 10\end{array}$ & $\begin{array}{l}4.10 \\
5.00\end{array}$ & $\begin{array}{l}84.00 \\
\$ 3.60\end{array}$ & $\begin{array}{r}16.00 \\
6.40\end{array}$ & $\begin{array}{l}84.00 \\
93.60\end{array}$ & $\begin{array}{l}23.00 \\
16.00\end{array}$ & $\begin{array}{l}6.00 \\
6.00\end{array}$ & $\begin{array}{r}16.00 \\
6.40\end{array}$ & $\begin{array}{l}.00 \\
.00\end{array}$ & $\begin{array}{l}1318.93 \\
1210.31\end{array}$ & $\begin{array}{r}211.03 \\
77.46\end{array}$ & $\begin{array}{l}742.00 \\
741.50\end{array}$ \\
\hline 656.00 & 77.90 & 22.20 & 3.80 & 5.00 & $\begin{array}{l}71.60 \\
59.50\end{array}$ & $\begin{array}{l}28.40 \\
40.50\end{array}$ & $\begin{array}{l}65.80 \\
54.90\end{array}$ & $\begin{array}{l}71.00 \\
71.00\end{array}$ & $\begin{array}{l}43.00 \\
43.00\end{array}$ & $\begin{array}{l}34.2 C \\
45.10\end{array}$ & $\begin{array}{l}5.80 \\
4.60\end{array}$ & $\begin{array}{l}1722.36 \\
2087.01\end{array}$ & $\begin{array}{l}589.05 \\
941.24\end{array}$ & $\begin{array}{l}741.00 \\
740.50\end{array}$ \\
\hline $\begin{array}{l}656 \cdot 50 \\
657.00\end{array}$ & $\begin{array}{l}69.60 \\
66.40\end{array}$ & $\begin{array}{l}26.90 \\
26.90\end{array}$ & $\begin{array}{l}3.60 \\
3.50\end{array}$ & $\begin{array}{l}4.70 \\
4.40\end{array}$ & $\begin{array}{l}59.50 \\
\epsilon 1.40\end{array}$ & $\begin{array}{l}40.50 \\
38.60\end{array}$ & 56.30 & 166.00 & 10.00 & 43.70 & 5.10 & 2087.01 & 912.02 & $\begin{array}{l}740.50 \\
740.00\end{array}$ \\
\hline 657.50 & 65.80 & 26.80 & $3 \cdot 30$ & 4.10 & 63.90 & 36.10 & 58.30 & 166.00 & 10.00 & 41.70 & 5.60 & 2079.25 & 867.05 & 739.50 \\
\hline 658.00 & $63 \cdot 30$ & 26.70 & 3.00 & 4.10 & $64 \cdot 70$ & $35 \cdot 30$ & 60.80 & 161.00 & 8.00 & 39.20 & $3 \cdot 90$ & 2071.49 & 812.02 & 739.00 \\
\hline $658 \cdot 50$ & 59.70 & 26.80 & 2.90 & $4 \cdot 10$ & $64 \cdot 10$ & 35.90 & 62.20 & 161.00 & 8.00 & 37.80 & 1.90 & 2079.25 & 785.96 & 738.50 \\
\hline 659.00 & 60.20 & 27.20 & 2.80 & $4 \cdot 10$ & $\in 3 \cdot 10$ & 36.90 & 62.10 & 176.00 & 8.00 & 37.90 & 1.00 & 2110.28 & 799.80 & 738.00 \\
\hline 659.50 & 59.90 & 27.10 & 2.70 & 3.70 & 66.80 & 33.20 & 63.20 & 176.00 & 8.00 & 36.80 & 3.60 & 2102.53 & 773.73 & 737.50 \\
\hline $660 \cdot 00$ & $60 \cdot 10$ & 26.30 & 2.70 & $3 \cdot 30$ & $73 \cdot 30$ & 26.70 & 65.90 & 151.00 & 8.00 & $34 \cdot 10$ & 7.40 & 2040.46 & 695.80 & 737.00 \\
\hline 660.50 & 61.50 & 24.10 & 2.70 & $3 \cdot 30$ & 79.40 & $20 \cdot 60$ & 72.30 & 151.00 & 8.00 & 27.70 & 7.10 & 1869.77 & 517.93 & 736.50 \\
\hline 661.00 & 62.90 & 23.60 & 2.70 & 3.70 & $77 \cdot 10$ & 22.90 & 73.70 & 93.00 & 17.00 & $26 \cdot 30$ & 3.40 & 1830.98 & 481.55 & 736.00 \\
\hline 661.50 & 64.50 & 23.40 & 2.70 & $4 \cdot 10$ & $73 \cdot 90$ & $26 \cdot 10$ & 73.80 & 93.00 & 17.00 & 26.20 & .10 & 1815.46 & 475.65 & 735.50 \\
\hline 662.00 & $70 \cdot 60$ & 23.60 & 2.80 & 4.50 & $72 \cdot 40$ & 27.60 & 72.40 & 93.00 & 27.00 & 27.60 & .00 & $1830 \cdot 98$ & $505 \cdot 35$ & 735.00 \\
\hline 662.50 & 71.10 & 24.60 & 2.90 & 4.40 & $68 \cdot 30$ & 31.70 & $68 \cdot 30$ & 93.00 & 27.00 & 31.70 & .00 & 1908.57 & 605.02 & 734.50 \\
\hline 663.20 & 80.60 & 18.60 & 2.90 & $4 \cdot 30$ & 91.20 & 8.80 & 91.20 & 33.00 & 40.00 & 8.80 & .00 & 1443.06 & 126.99 & 734.00 \\
\hline 663.50 & 82.10 & 19.20 & 3.00 & 4.10 & 87.70 & $12 \cdot 30$ & 87.70 & 33.00 & 40.00 & $12 \cdot 30$ & .00 & 1489.61 & 183.22 & 733.50 \\
\hline 664.00 & 76.00 & 25.30 & 3.00 & 4.10 & $68 \cdot 10$ & 31.90 & 65.20 & 126.00 & 31.00 & 34.80 & 2.90 & 1962.87 & 683.08 & 733.00 \\
\hline 664.50 & $72 \cdot 10$ & $23.4 n$ & 2.90 & 4.00 & 61.30 & 39.70 & 57.80 & 126.00 & 31.00 & 42.20 & 3.50 & $2203 \cdot 38$ & 929.83 & 732.50 \\
\hline 665.00 & 69.30 & 27.70 & $2 \cdot 90$ & $3 \cdot 90$ & 63.40 & 36.60 & 59.70 & 190.00 & 27.00 & 40.30 & 3.70 & 2149.08 & 866.08 & 732.00 \\
\hline 665.50 & 64.10 & 26.40 & 2.80 & 3.80 & 67.40 & 32.60 & 63.90 & 190.00 & 27.00 & 36.10 & 3.50 & 2048.22 & 739.41 & 731.50 \\
\hline 660.00 & 61.30 & 25.90 & 2.70 & 3.80 & 69.10 & 30.90 & 66.30 & 140.00 & 15.00 & 33.70 & 2.80 & 2009.42 & 677.18 & 731.00 \\
\hline 666.50 & 54.90 & 25.90 & 2.70 & 3.90 & $\in 8.40$ & 31.50 & 66.40 & 140.00 & 15.00 & 33.60 & 2.00 & 2009.42 & 675.17 & 730.50 \\
\hline 667.00 & $59 \cdot 30$ & 26.70 & 2.70 & $4 \cdot c 0$ & $\in 5.40$ & 34.60 & 64.50 & 161.00 & 10.00 & 35.50 & .90 & 2071.49 & 735.38 & 730.00 \\
\hline 667.50 & 64.70 & 26.90 & 2.60 & 4.10 & 64.60 & $35 \cdot 40$ & 64.60 & 161.00 & 10.00 & 35.40 & .00 & 2087.01 & 738.80 & 729.50 \\
\hline 668.00 & 67.20 & 26.40 & 2.60 & 4.00 & $65 \cdot 80$ & 34.20 & 65.80 & 153.00 & 46.00 & 34.20 & .00 & 2048.22 & 700.49 & 729.00 \\
\hline 668.50 & 65.00 & 26.60 & 2.70 & 3.90 & 65.90 & $34 \cdot 10$ & 64.90 & 153.00 & 46.00 & 35.10 & 1.00 & 2063.73 & 724.37 & 728.50 \\
\hline 669.00 & 58.00 & 26.90 & 2.60 & 4.00 & 64.60 & 35.40 & 64.40 & 167.00 & 14.00 & 35.60 & .20 & 2087.01 & 742.97 & 728.00 \\
\hline 669.50 & $61 \cdot 90$ & 27.50 & 2.60 & $4 \cdot 10$ & $\epsilon 3.20$ & 36.80 & 63.20 & 167.00 & 14.00 & $36.8 \mathrm{C}$ & .00 & 2133.56 & 785.15 & 727.50 \\
\hline 670.00 & 64.40 & 27.70 & 2.70 & 4.20 & $62 \cdot 10$ & 37.90 & 62.10 & 190.00 & 20.00 & 37.90 & .00 & 2149.08 & 814.50 & 727.00 \\
\hline 670.50 & 64.00 & 27.80 & 2.70 & $4 \cdot 30$ & $\epsilon 1.80$ & 38.20 & 61.80 & 190.00 & 20.00 & 38.20 & .00 & 2156.83 & 823.91 & 726.50 \\
\hline 671.00 & 63.70 & 27.30 & 2.70 & 4.20 & 62.70 & $37 \cdot 30$ & 62.70 & 178.00 & 15.00 & 37.30 & .00 & 2118.04 & 790.03 & 726.00 \\
\hline $\begin{array}{l}671.50 \\
672.00\end{array}$ & $\begin{array}{l}62.70 \\
59.30\end{array}$ & $\begin{array}{l}24.70 \\
23.90\end{array}$ & $\begin{array}{l}2.80 \\
2.80\end{array}$ & $\begin{array}{l}4.30 \\
4.40\end{array}$ & $\begin{array}{l}69.10 \\
70.70\end{array}$ & $\begin{array}{l}30.90 \\
29.30\end{array}$ & $\begin{array}{l}69.10 \\
70.70\end{array}$ & $\begin{array}{r}178.00 \\
98.00\end{array}$ & $\begin{array}{l}15.00 \\
13.00\end{array}$ & $\begin{array}{l}30.90 \\
29.30\end{array}$ & .00 & $\begin{array}{l}1916.32 \\
1854.26\end{array}$ & $\begin{array}{l}592.14 \\
543.30\end{array}$ & $\begin{array}{l}725.50 \\
725.00\end{array}$ \\
\hline 672.50 & $\begin{array}{l}60.90 \\
64.30\end{array}$ & $\begin{array}{l}23.90 \\
23.60 \\
23.20\end{array}$ & $\begin{array}{l}2.80 \\
2.90 \\
2.90\end{array}$ & $\begin{array}{l}4.40 \\
4.40 \\
4.30\end{array}$ & $\begin{array}{l}70.70 \\
71.00\end{array}$ & $\begin{array}{l}29.30 \\
29.00\end{array}$ & 70.30 & $\begin{array}{l}98.00 \\
87.00\end{array}$ & 13.00 & 29.70 & .70 & 1830.98 & 543.80 & 724.50 \\
\hline 673.00 & & 23.20 & 2.90 & & 73.00 & & & & & & 1.30 & & $\begin{array}{l}509.39 \\
459.66\end{array}$ & $\begin{array}{l}724.00 \\
723.50\end{array}$ \\
\hline $\begin{array}{l}673.50 \\
674.00\end{array}$ & $\begin{array}{l}66.10 \\
69.50\end{array}$ & $\begin{array}{l}22.70 \\
21.50\end{array}$ & $\begin{array}{l}2.90 \\
2.90\end{array}$ & $\begin{array}{l}4.10 \\
4.00\end{array}$ & $\begin{array}{l}76.30 \\
78.70\end{array}$ & $\begin{array}{l}23.70 \\
21.30\end{array}$ & $\begin{array}{l}73.90 \\
78.70\end{array}$ & $\begin{array}{l}87.00 \\
61.00\end{array}$ & $\begin{array}{l}21.00 \\
27.00\end{array}$ & $\begin{array}{l}26.10 \\
21.30\end{array}$ & $\begin{array}{r}2.40 \\
.00\end{array}$ & $\begin{array}{l}1761.16 \\
1668.06\end{array}$ & $\begin{array}{l}459.66 \\
355.30\end{array}$ & $\begin{array}{l}723.50 \\
723.00\end{array}$ \\
\hline A.AVE. & 66.32 & 24.69 & 2.99 & 4.20 & 70.13 & 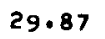 & 68.03 & 123.27 & 19.44 & 31.97 & 2.10 & .18 & 631.08 & \\
\hline G.MEAN & 65.98 & 24.47 & 2.96 & .17 & 69.70 & 8.21 & 67.52 & 106.57 & 16.03 & 30.09 & & $1898 \cdot 30$ & 571.17 & \\
\hline
\end{tabular}

*NOTATJOH--Depth, ft; GR-Ganma Ray Index, API Units; POR-Porosity, $\%$; RT-True Resistivity, Ohms-m²/m; RXO-Fiushed Zone Resistivity, Ohnis-m ${ }^{2} / m$; SXO-Flushed Zone Saturation, \%; ROS-Residual $0 i 1$ Saturation, \%; SW-Water Saturation, \%; PER-Permeability Index; Clay-Clay Content, \%; SO-0il

Saturation, \%; M0-Movable 0il, \%; PHV-Pore Volume, bbl/ac-ft; HPV-Hydrocarbon Pore Volume, bbl/ac-ft; ELSE-Elevation From Sea Level, ft 
TABLE C58

WELL LOG DETERMINED PROPERTIES

VS DEPTH FOR WELL MPP-217

WELL LOCATION: 2366 FEL $8 C 2$ FNL 28255 5E

KELLY BUSHING ELEVATION FROII SEA LEVEL $=1403$

\begin{tabular}{|c|c|c|c|c|c|c|c|c|c|c|c|c|c|c|}
\hline DEPTH ${ }^{\star}$ & GR & $P .9 R$ & RT & $8 \times 0$ & $s \times \theta$ & RBS & SW & PER & CLAY & so & MO & PHV & HPV & ELSE \\
\hline $662 \cdot 50$ & $61 \cdot 10$ & 20.20 & $3 \cdot 20$ & 3.70 & 80.20 & $19 \cdot 80$ & 80.20 & 17.00 & 27.00 & 19.80 & $\cdot c 0$ & 1567.20 & $310 \cdot 30$ & 740.50 \\
\hline 663.00 & 59.00 & 24.70 & 3.00 & $3 \cdot 40$ & 77.10 & $22 \cdot 90$ & 65.90 & 115.00 & 22.00 & $34 \cdot 10$ & 11.20 & $1916 \cdot 32$ & 653.47 & 740.00 \\
\hline 663.50 & $56 \cdot 80$ & 25.60 & 2.90 & 3.60 & 71.90 & 28.10 & 65.00 & 115.00 & 22.00 & 35.00 & 6.90 & 1986.15 & 695.15 & 739.50 \\
\hline 664.00 & $54 \cdot 70$ & 25.70 & 2.80 & 3.70 & 70.50 & 29.50 & 66.20 & 135.00 & 14.00 & 33.80 & $4 \cdot 30$ & 1993.91 & 673.94 & 739.00 \\
\hline 664.50 & 53.10 & $26 \cdot 1 \cdot 0$ & 2.70 & 3.00 & 10.70 & 29.30 & 66.40 & 135.00 & 14000 & 33.60 & $4 \cdot 30$ & 2024.94 & 680.38 & 738.50 \\
\hline 655.00 & $54 \cdot 80$ & $26 \cdot 30$ & 2.60 & 3.50 & $71 \cdot 30$ & 28.70 & 67.20 & 150.00 & 13.00 & 32.80 & 4.10 & $2040 \cdot 46$ & 669.27 & 738.00 \\
\hline 665.50 & 54.00 & 26.50 & 2.50 & 3.60 & $t 9.60$ & 30.40 & 67.30 & 150.00 & 13.00 & 32.70 & $2 \cdot 30$ & $2055 \cdot 97$ & 672.30 & 737.50 \\
\hline 666.00 & $54 \cdot 30$ & 26.70 & $2 \cdot 50$ & 3.60 & $68 \cdot 70$ & $31 \cdot 30$ & 67.00 & 162.00 & $12 \cdot 00$ & $33 \cdot 00$ & 1.70 & 2071.49 & 683.59 & 737.00 \\
\hline 666.50 & 53.00 & 26.90 & 2.50 & 3.50 & E 9.40 & 30.60 & 66.90 & 162.00 & 12.00 & 33.10 & 2.50 & 2087.01 & 690.80 & 736.50 \\
\hline 667.00 & 51.00 & 26.70 & 2.40 & $3 \cdot 40$ & 71.40 & 28.60 & 68.00 & 160.00 & 7.00 & 32.00 & $3 \cdot 40$ & 2071.49 & 662.88 & 736.00 \\
\hline 667.50 & $49 \cdot 10$ & 25.70 & 2.40 & 3.60 & 71.50 & $28 \cdot 50$ & 71.10 & $160 \cdot 0 \mathrm{C}$ & 7.00 & 28.90 & .40 & 1993.91 & 576.24 & 735.50 \\
\hline 66.3 .00 & $51 \cdot 20$ & 24.90 & 2.40 & 3.80 & 74.00 & 26.00 & 74.00 & $117.0 \mathrm{C}$ & 11.00 & 26.00 & .00 & 1931.84 & $502 \cdot 28$ & 735.00 \\
\hline 608.50 & 54.00 & 24.10 & $2 \cdot 40$ & $3 \cdot 90$ & $76 \cdot 70$ & 23.30 & 76.70 & 117.00 & 11.00 & 23.30 & .00 & 1869.77 & 435.66 & 734.50 \\
\hline 669.00 & $56 \cdot 70$ & 25.00 & 2.40 & 3.00 & 73.90 & $26 \cdot 10$ & 73.90 & 119.00 & 16.00 & $26 \cdot 10$ & .00 & 1939.60 & 506.24 & 734.00 \\
\hline 669.50 & $59 \cdot 40$ & $25 \cdot 40$ & $2 \cdot 40$ & $3 \cdot 20$ & 77.50 & 22.50 & $72 \cdot 30$ & 119.00 & 16.00 & 27.70 & $5 \cdot 20$ & 1970.63 & 545.86 & 733.50 \\
\hline 670.00 & 63.70 & 25.80 & 2.40 & 3.20 & $75 \cdot 40$ & 24.60 & 70.70 & 137.00 & 26.00 & $29 \cdot 30$ & 4.70 & 2001.67 & 586.49 & 733.00 \\
\hline 670.50 & $66 \cdot 30$ & 25.10 & $2 \cdot 50$ & $3 \cdot 40$ & $75 \cdot 70$ & $24 \cdot 30$ & $72 \cdot 30$ & 137.00 & 26.00 & $27.7 n$ & 3.40 & $1947 \cdot 36$ & 539.42 & 732.50 \\
\hline 671.00 & $68 \cdot 00$ & 24.50 & 2.50 & 3.60 & $75 \cdot 90$ & $24 \cdot 10$ & 73.80 & 110.00 & 33.00 & 26.20 & $2 \cdot 10$ & $1900 \cdot 81$ & 498.01 & 732.00 \\
\hline 671.50 & 65.90 & 26.00 & $2 \cdot 50$ & 3.70 & $70 \cdot 20$ & 29.80 & 68.80 & 110.00 & 33.00 & $31 \cdot ? \mathrm{C}$ & 1.40 & 2017.18 & 629.36 & 731.50 \\
\hline 672.00 & 63.90 & 26.70 & $2 \cdot 50$ & 3.10 & $68 \cdot 30$ & 31.70 & 66.70 & 160.00 & $25 \cdot 00$ & $33 \cdot 3 n$ & $1 \cdot \in 0$ & 2071.49 & 689.81 & 731.00 \\
\hline 672.50 & 64.00 & 26.40 & 2.50 & 3.70 & 68.90 & 31.10 & 67.30 & 160.00 & 25.00 & 32.70 & 1.60 & $2048 \cdot 22$ & 669.77 & 730.50 \\
\hline 673.00 & $64 \cdot 80$ & 25.80 & 2.60 & 3.80 & $70 \cdot 10$ & 29.90 & 68.80 & 137.00 & 28.00 & $31 \cdot ? 0$ & $1 \cdot 30$ & 2001.67 & 624.52 & 730.00 \\
\hline 673.50 & $65 \cdot 60$ & 25.50 & 2.60 & 3.80 & $t 9.90$ & $30 \cdot 10$ & 69.20 & 137.00 & 28.00 & 30.80 & .70 & $1986 \cdot 15$ & 611.73 & 729.50 \\
\hline 674.00 & 64.60 & 26.60 & 2.60 & 3.80 & 67.60 & 32.40 & 66.60 & 158.00 & 27.00 & 33.40 & 1.00 & 2063.73 & 689.29 & 729.00 \\
\hline 674.50 & 63.50 & 26.80 & $2 \cdot 50$ & 3.70 & 67.70 & $32 \cdot 30$ & 66.00 & 158.00 & 27.00 & 34.00 & 1.70 & 2079.25 & 706.94 & 728.50 \\
\hline 675.00 & 63.70 & 26.40 & 2.50 & 3.60 & 69.80 & 30.20 & 67.40 & 152.00 & 23.00 & 32.60 & 2.40 & 2048.22 & 667.72 & 728.00 \\
\hline 675.50 & 65.90 & 24.70 & $2 \cdot 50$ & $3 \cdot 5 n$ & $75 \cdot 80$ & $24 \cdot 20$ & 72.40 & $152 \cdot 00$ & 23.00 & 27.60 & 3.40 & $1916 \cdot 32$ & 528.91 & 727.50 \\
\hline 676.00 & $67 \cdot 80$ & 23.40 & 2.50 & $3 \cdot 50$ & $76 \cdot 80$ & 23.20 & 76.80 & 89.00 & 33.00 & $23 . ? 0$ & .00 & 1815.46 & 421.19 & 727.00 \\
\hline 676.50 & $68 \cdot 20$ & 23.60 & $2 \cdot 50$ & $3 \cdot 50$ & $79 \cdot 70$ & $20 \cdot 30$ & 76.20 & 89.00 & 33.00 & 23.80 & $3 \cdot 50$ & 1830.98 & 435.77 & 726.50 \\
\hline 677.00 & 67.40 & 24.20 & 2.50 & 3.60 & $77 \cdot 10$ & 22.90 & 74.10 & 104.00 & $34 \cdot 00$ & 25.90 & 3.00 & 1877.53 & 486.28 & 726.00 \\
\hline & 66.70 & 24.80 & 2.50 & 3.70 & $74 \cdot 20$ & 25.80 & 72.20 & 104.00 & 34.00 & 27.80 & 2.05 & 1924.08 & 534.89 & 725.50 \\
\hline 678.00 & $65 \cdot 50$ & 25.70 & 2.50 & 3.70 & 70.80 & $29 \cdot 20$ & 69.60 & 135.00 & $32 \cdot 00$ & $30 \cdot 40$ & 1.23 & 1993.91 & $606 \cdot 15$ & 725.00 \\
\hline 678.50 & $64 \cdot 30$ & 26.30 & 2.50 & 3.80 & 68.40 & 31.60 & 67.90 & 135.00 & 32.00 & $32 \cdot 10$ & .50 & 2040.46 & 654.99 & 724.50 \\
\hline 679.00 & 63.60 & 25.30 & 2.50 & 3.80 & 68.00 & 32.00 & 68.00 & 149.00 & 26.00 & 32.00 & .00 & 2040.46 & 652.95 & 724.00 \\
\hline 679.50 & & 25.60 & 2.50 & 3.80 & 70.40 & 2.9 .60 & 69.80 & 143.00 & 26.00 & $3 c \cdot 20$ & .60 & $1986 \cdot 15$ & 599.82 & 723.50 \\
\hline 680.00 & $67 \cdot 50$ & 23.80 & 2.50 & 3.70 & 77.30 & 22.70 & 75.60 & 97.00 & 34.00 & 24.40 & 1.70 & 1846.50 & 450.55 & 723.00 \\
\hline 680.50 & $71 \cdot 30$ & $21 \cdot 10$ & 2.50 & 3.50 & 86.40 & 13.60 & 86.40 & 97.00 & 34.00 & $1 \equiv \cdot 60$ & .00 & 1637.02 & 222.64 & 722.50 \\
\hline 681.00 & 73.20 & 19.30 & $2 \cdot 50$ & $3 \cdot 40$ & $54 \cdot 80$ & $5 \cdot 20$ & 94.80 & 39.00 & 45.00 & 5.20 & .00 & 1497.37 & 77.86 & 722.00 \\
\hline A.AVE. & 61.67 & 25.13 & 2.55 & 01 & 73.52 & 26.48 & $71 \cdot 30$ & 127.05 & 23.79 & 28.70 & 2.21 & $19+9 \cdot 80$ & 566.93 & \\
\hline G.MEAN & $61 \cdot 34$ & 25.07 & $2 \cdot 54$ & 01 & 73.33 & $25 \cdot 55$ & 71.08 & 119.81 & 21.69 & $27.6 ?$ & & 1944.69 & $53 \% \cdot 14$ & \\
\hline
\end{tabular}

*NOTAIID!1--Depth, :t; GR-Gamma Ray Index, API Units; POR-Porosity, $\%$; RT-True Resistivity, Ohms-m²/m; RXO-Flus ${ }^{2}$ ed Zone Resistivity, Ohnls-m²/m;

SXO-Flushed Zone Saturation, \%; ROS-Residual $0 i 1$ Saturation, \%; SW-Water Saturation, \%; PER-Permeatility Index; Clay-Clay Content, \%; SO-0il

Saturation, \%; MO-NoJable 0il, \%; PHV-Pore Volume, bbl/ac-ft; HPV-Hydrocarbon Pore Volume, bbl/ac-ft; ELSE-Eleration From Sea Level, ft 
TABLE C 59

WELL LOG DETERMINED PROPERTIES

VS DEPTH FOR WELL MP-218

WELL LCICATION: 2640 FEL 792 FNL $282555 E$

KELLY BUSHING ELEVATION FROM SEA LEVEL $=1404$

\begin{tabular}{|c|c|c|c|c|c|c|c|c|c|c|c|c|c|c|}
\hline DEPTH* & GR & $P A R$ & $R T$ & $R \times U$ & $5 \times 0$ & ROS & SW & PER & CLAY & so & ro & PHV & HPV & ELSE \\
\hline 666.50 & $62 \cdot 10$ & 18.70 & $3 \cdot 30$ & 4.30 & 85.40 & 14.60 & 85.40 & 6.00 & 14.00 & 14.60 & .00 & 1450.82 & 211.82 & 737.50 \\
\hline 667.00 & $64 \cdot 50$ & $25 \cdot 10$ & $3 \cdot 20$ & 6.00 & $65 \cdot 20$ & $34 \cdot 80$ & 63.60 & $121 \cdot 00$ & 20.00 & 36.40 & 1.60 & 1947.36 & 708.84 & 737.00 \\
\hline 667.50 & 67.50 & 25.30 & 3.00 & 4.30 & 76.20 & 23.80 & 65.20 & 121.00 & 20.00 & 34.80 & 11.00 & 1962.87 & 683.08 & 736.50 \\
\hline 668.00 & 70.40 & 25.20 & 2.90 & 2.30 & 66.50 & 33.50 & 66.50 & 126.00 & 25.00 & 33.50 & .00 & 1955.12 & 654.96 & 736.00 \\
\hline 668.50 & 69.60 & 24.90 & 2.70 & $2 \cdot 30$ & $\epsilon 8.60$ & 31.40 & 68.60 & 126.00 & 25.00 & 31.40 & .00 & 1931.84 & 606.60 & 735.50 \\
\hline 669.00 & 66.50 & 24.70 & $2 \cdot 70$ & 3.50 & $70 \cdot 50$ & 29.50 & $70 \cdot 50$ & 114.00 & 20.00 & 29.50 & .00 & 1916.32 & $565 \cdot 32$ & 735.00 \\
\hline 669.50 & 66.70 & 24.60 & 2.60 & 3.90 & $71 \cdot 90$ & $28 \cdot 10$ & 71.90 & 114.00 & 20.00 & $28 \cdot 10$ & .00 & 1908.57 & 536.31 & 734.50 \\
\hline 670.00 & 68.80 & 25.00 & 2.50 & 3.40 & 71.60 & 28.40 & 71.60 & 121.00 & 25.00 & 28.40 & .00 & 1939.60 & 550.85 & 734.00 \\
\hline 670.50 & $71 \cdot 10$ & 25.80 & 2.40 & 3.10 & 70.50 & 29.50 & $70 \cdot 50$ & 121.00 & 25.00 & 29.50 & .00 & 2001.67 & 590.49 & 733.50 \\
\hline 671.00 & $.72 \cdot 70$ & 25.80 & 2.40 & $3.1 \mathrm{c}$ & $71 \cdot 30$ & $28 \cdot 70$ & 71.30 & 139.00 & 31.00 & $28 \cdot 70$ & .00 & 2001.67 & 574.48 & 733.00 \\
\hline 671.50 & 73.00 & 25.40 & $2 \cdot 30$ & 3.00 & 73.40 & 26.60 & 73.40 & 139.00 & 31.00 & 26.60 & .00 & 1970.63 & 524.19 & 732.50 \\
\hline 672.00 & 69.40 & 25.50 & $2 \cdot 30$ & $3 \cdot 40$ & 73.90 & $26 \cdot 10$ & 73.90 & $132 \cdot 00$ & 25.00 & 26.10 & .00 & 1978.39 & 516.36 & 732.00 \\
\hline 672.50 & 63.80 & $25 . ? 0$ & 2.20 & $4 \cdot 00$ & $79 \cdot ? 0$ & $20 \cdot 80$ & 75.60 & 132.00 & 25.00 & $24 \cdot 40$ & 3.60 & 1955.12 & 477.05 & 731.50 \\
\hline 673.00 & 61.60 & $2.5 \cdot 30$ & 2.20 & 4.60 & $76 \cdot 10$ & 23.90 & 76.10 & 126.00 & 15.00 & 23.90 & .00 & 1962.87 & 469.13 & 731.00 \\
\hline 673.50 & $61 \cdot 10$ & $25 \cdot 30$ & $2 \cdot 10$ & $5 \cdot 10$ & $76 \cdot 50$ & $23 \cdot 50$ & 76.50 & 126.00 & 15.00 & 23.50 & .00 & 1962.87 & 461.28 & 730.50 \\
\hline 674.00 & 63.90 & 26.40 & $2 \cdot 10$ & 4.80 & $73 \cdot 10$ & $26 \cdot 90$ & 73.10 & 154.00 & 18.00 & 26.90 & .00 & 2048.22 & 550.97 & 730.00 \\
\hline 674.50 & 67.50 & 27.40 & 2.20 & 3.90 & 73.70 & $26 \cdot 30$ & 70.10 & 154.00 & 18.00 & 29.90 & 3.60 & 2125.80 & 635.61 & 729.50 \\
\hline 675.00 & 70.40 & 27.20 & $2 \cdot 20$ & 3.40 & 79.60 & $20 \cdot 40$ & 70.50 & 173.00 & 25.00 & 29.50 & 9.10 & 2110.28 & 622.53 & 729.00 \\
\hline $675 \cdot 50$ & 67.50 & 26.80 & 2.20 & $3 \cdot 30$ & $71 \cdot 50$ & $28 \cdot 50$ & 71.50 & 173.00 & 25.00 & 28.50 & .00 & 2079.25 & 592.59 & 728.50 \\
\hline 676.00 & 62.40 & 26.90 & $2 \cdot 20$ & 4.10 & $73 \cdot 10$ & $26 \cdot 90$ & $71 \cdot 30$ & $165 \cdot 00$ & 14.00 & 28.70 & 1.80 & 2087.01 & 598.97 & 728.00 \\
\hline 676.50 & $60 \cdot 60$ & 27.20 & $2 \cdot 20$ & 4.50 & $70 \cdot 30$ & 29.70 & $70 \cdot 30$ & 165.00 & 14.00 & $29 \cdot 70$ & .00 & 2110.28 & 626.75 & 727.50 \\
\hline 677.00 & $61 \cdot 50$ & 27.60 & $2 \cdot 20$ & $4 \cdot 30$ & $\epsilon 9.60$ & 30.40 & 68.80 & $185 \cdot 00$ & 16.00 & $31 \cdot 20$ & .80 & $2141 \cdot 32$ & 668.09 & 727.00 \\
\hline 677.50 & $66 \cdot 50$ & 27.80 & $2 \cdot 20$ & 4.20 & $70 \cdot 10$ & 29.90 & 67.40 & $185 \cdot 00$ & 16.00 & $32 \cdot 60$ & 2.70 & 2156.63 & 703.13 & 726.50 \\
\hline 678.00 & 67.70 & 28.10 & $2 \cdot 30$ & 4.00 & $70 \cdot 70$ & $29 \cdot 30$ & $66 \cdot 10$ & 201.00 & 25.00 & $33 \cdot 90$ & 4.60 & $2180 \cdot 11$ & 739.06 & 726.00 \\
\hline 678.50 & $68 \cdot 80$ & 27.90 & $2 \cdot 30$ & 3.90 & $71 \cdot 80$ & $28 \cdot 20$ & 65.80 & 201.00 & 25.00 & $34 \cdot ? 0$ & 6.00 & 2164.59 & 740.29 & 725.50 \\
\hline 679.00 & $69 \cdot 50$ & 27.60 & 2.40 & $4 \cdot 60$ & 72.00 & 28.00 & 66.60 & 185.00 & 27.00 & 33.40 & $5 \cdot 40$ & $2141 \cdot 32$ & 715.20 & 725.00 \\
\hline 679.50 & $70 \cdot 20$ & 26.80 & 2.40 & 4.50 & 70.50 & $29.5 n$ & 68.40 & 185.00 & 27.00 & 31.60 & 2.10 & 2079.25 & 657.04 & 724.50 \\
\hline 680.00 & 70.80 & 26.80 & 2.40 & 4.60 & $\epsilon 9.70$ & $30 \cdot 30$ & 68.20 & 163.00 & 30.00 & 31.80 & 1.50 & 2079.25 & 661.20 & 724.00 \\
\hline 680.50 & $70 \cdot 40$ & $27.2 c$ & 2.40 & 5.00 & $\epsilon 6.90$ & 33.10 & 66.90 & 163.00 & 30.00 & 33.10 & $\therefore 00$ & 2110.28 & $\begin{array}{r}698.50 \\
727.54\end{array}$ & 723.50 \\
\hline 681.00 & $70 \cdot 40$ & 27.50 & 2.40 & 4.50 & 68.00 & $32 \cdot 00$ & 65.90 & $183 \cdot 00$ & 30.00 & $34 \cdot 10$ & $2 \cdot 10$ & 2133.56 & 727.54 & 723.00 \\
\hline 681.50 & $72 \cdot 10$ & $27.8 \mathrm{C}$ & 2.40 & $3 \cdot 80$ & $73 \cdot 60$ & $26 \cdot 40$ & 65.40 & 183.00 & 30.00 & 34.60 & $8 \cdot 20$ & 2156.83 & 746.26 & 722.50 \\
\hline 682.00 & 71.50 & 28.30 & $2 \cdot 30$ & 3.60 & 73.80 & $26 \cdot 20$ & 65.00 & 207.00 & 31.00 & 35.00 & 8.80 & 2195.63 & 768.47 & 722.00 \\
\hline $682 \cdot 50$ & $72 \cdot 70$ & 28.70 & $2 \cdot 30$ & 3.90 & 69.60 & $30 \cdot 40$ & 64.60 & 207.00 & 31.00 & 35.40 & 5.00 & 2226.66 & 788.24 & 721.50 \\
\hline 683.00 & 74.70 & $27.3 \mathrm{C}$ & 2.20 & 3.70 & 76.00 & 24.00 & 69.10 & 176.00 & 33.00 & 30.90 & 6.90 & 2118.04 & 654.47 & 721.00 \\
\hline 683.50 & 76.90 & 25.20 & $2 \cdot 20$ & 3.40 & 76.20 & 23.80 & 76.20 & 176.00 & 33.00 & 23.80 & .00 & 1955.12 & 465.32 & 720.50 \\
\hline 684.00 & $76 \cdot 30$ & 24.00 & 2.10 & 3.20 & $81 \cdot 10$ & 18.90 & $81 \cdot 10$ & 101.00 & 39.00 & 18.90 & .00 & 1852.02 & 351.92 & 720.00 \\
\hline 684.50 & 76.40 & $22 \cdot 30$ & $2 \cdot 10$ & 3.30 & 88.90 & $11 \cdot 10$ & 88.90 & 101.00 & 39.00 & 11.10 & .00 & 1730.12 & 192.04 & 719.50 \\
\hline $\begin{array}{l}\text { A. AVE. } \\
\text { G.MEAN }\end{array}$ & $\begin{array}{l}68 \cdot 58 \\
68.44\end{array}$ & $\begin{array}{l}26.07 \\
26.00\end{array}$ & $\begin{array}{l}2 \cdot 39 \\
2.38\end{array}$ & $\begin{array}{l}3.91 \\
3.84\end{array}$ & $\begin{array}{l}73.15 \\
73.00\end{array}$ & $\begin{array}{l}26.85 \\
26.29\end{array}$ & $\begin{array}{l}70.86 \\
70.66\end{array}$ & $\begin{array}{l}150 \cdot 00 \\
138.16\end{array}$ & $\begin{array}{l}24.65 \\
23.67\end{array}$ & $\begin{array}{l}29.14 \\
28.44\end{array}$ & 2.29 & $\begin{array}{l}2022.63 \\
2016.96\end{array}$ & 595.54 & \\
\hline G. MEAN & & & $2 \cdot 38$ & 3.84 & 73.00 & $26 \cdot 29$ & 70.66 & $138 \cdot 16$ & 23.67 & 28.44 & & 2016.96 & 573.73 & \\
\hline
\end{tabular}

*NOTATIDIl--Depth, ft; GR-Garma Ray Index, API Units; POR-Porosity, $\%$; RT-True Resistivity, Ohms-m²/m; RX0-Flushed Zone Resistivity, Ohms-m²/m; SX0-Flushed Zone Saturaticn: \%; ROS-Residual 0il Saturation, \%; SW-Water Saturation, \%; PER-Permeability Index; Clay-Clay Content, \%; S0-0il

Saturation, \%; M0-Movable 0-1, \%; PHV-Pore Volume, bbl/ac-ft; HPV-Hydrocarbon Pore Volume, bb1/ac-ft; ELSE-Elevation From Sea Level, ft 
TABLE C60

WELL LOG DETERMINED PROPERTIES

VS DEPTH FOR HELL MAP-220

WELL LOCATION: 792 FNL 2112 FWL 28 25S $5 E$

KELLLY BUSHING ELEVATION FROM SEA LEVEL $=1397$

\begin{tabular}{|c|c|c|c|c|c|c|c|c|c|c|c|c|c|c|}
\hline JEPTH * & $G R$ & PQR & RT & $R \times \theta$ & $s \times \theta$ & RES & Sw & PER & CLAY & so & סי & PHV & HPV & ELSE \\
\hline $\begin{array}{l}.564 .00 \\
564.50\end{array}$ & $\begin{array}{l}71.70 \\
64.70\end{array}$ & $\begin{array}{l}19.50 \\
2 \bar{c} .00\end{array}$ & $\begin{array}{l}3.00 \\
2.90\end{array}$ & $\begin{array}{l}2.50 \\
2.00\end{array}$ & $\begin{array}{l}85 \cdot 30 \\
76.70\end{array}$ & $\begin{array}{l}14.70 \\
23.30\end{array}$ & $\begin{array}{l}85.30 \\
76.70\end{array}$ & $\begin{array}{l}40.00 \\
40.00\end{array}$ & $\begin{array}{l}17.00 \\
17.00\end{array}$ & $\begin{array}{l}14.70 \\
23.30\end{array}$ & $\begin{array}{l}.00 \\
.00\end{array}$ & $\begin{array}{l}1512.89 \\
1706.85\end{array}$ & $\begin{array}{l}222.39 \\
397.70\end{array}$ & $\begin{array}{r}733.00 \\
732.50\end{array}$ \\
\hline 565.00 & 62.70 & 21.40 & 2.70 & 3.30 & 81.80 & 18.20 & 81.80 & 61.00 & 9.00 & 18.20 & .00 & 1660.30 & 302.17 & 732.00 \\
\hline $\begin{array}{l}065.50 \\
066.00\end{array}$ & $\begin{array}{l}67.30 \\
70.20\end{array}$ & $\begin{array}{l}25.00 \\
1 \varepsilon .00\end{array}$ & $\begin{array}{l}2.50 \\
2.40\end{array}$ & $\begin{array}{l}3.60 \\
3.40\end{array}$ & $\begin{array}{r}91.90 \\
100.00\end{array}$ & $\begin{array}{r}8.10 \\
.00\end{array}$ & $\begin{array}{r}91.90 \\
100.00\end{array}$ & $\begin{array}{l}61.00 \\
28.00\end{array}$ & $\begin{array}{r}9.00 \\
17.00\end{array}$ & $\begin{array}{r}8.10 \\
.00\end{array}$ & $\begin{array}{l}.00 \\
.00\end{array}$ & $\begin{array}{l}1551.68 \\
1396.51\end{array}$ & $\begin{array}{r}125.69 \\
.00\end{array}$ & $\begin{array}{l}731.50 \\
731.00\end{array}$ \\
\hline $\begin{array}{l}666.50 \\
667.00\end{array}$ & $\begin{array}{l}69 \cdot 10 \\
64 \cdot 10\end{array}$ & $\begin{array}{l}1 \epsilon .90 \\
1 \epsilon .6 C\end{array}$ & $\begin{array}{l}2.30 \\
2.20\end{array}$ & $\begin{array}{l}2.30 \\
2.20\end{array}$ & $\begin{array}{l}100.00 \\
160.00\end{array}$ & $\begin{array}{l}.00 \\
.00\end{array}$ & $\begin{array}{l}100.00 \\
100.00\end{array}$ & $\begin{array}{l}28.00 \\
20.00\end{array}$ & $\begin{array}{l}17.00 \\
15.00\end{array}$ & .00 & $\begin{array}{l}.00 \\
.00\end{array}$ & $\begin{array}{l}1311.17 \\
1287.89\end{array}$ & $\begin{array}{l}.00 \\
.00\end{array}$ & $\begin{array}{l}730.50 \\
730.00\end{array}$ \\
\hline 667.50 & 61.20 & $1 \varepsilon . ? 0$ & 2.10 & 2.00 & 100.00 & .00 & 100.00 & $20 \cdot 00$ & 15.00 & . on & .00 & 1412.03 & .00 & 329.50 \\
\hline 668.00 & 58.50 & 26.90 & 2.00 & 1.90 & $96 \cdot 30$ & 3.70 & $96 \cdot 30$ & 55.00 & 11.00 & 3.70 & .00 & 1621.50 & 60.00 & 729.00 \\
\hline 668.50 & $54 \cdot 90$ & $2 \bar{z} .50$ & 2.00 & 1.80 & 50.20 & 9.80 & 90.20 & 55.00 & 11.00 & 9.70 & .00 & 1745.64 & 171.07 & 728.50 \\
\hline 069.00 & 55.90 & $2 E .40$ & 1.90 & 1.90 & 80.20 & 19.80 & 80.20 & 128.00 & 8.00 & 19.30 & .00 & 1970.63 & 390.19 & 728.00 \\
\hline 669.50 & 57.40 & 25.90 & 1.90 & 1.90 & 78.90 & 21.10 & 78.90 & 128.00 & 8.00 & 21.10 & .00 & 2009.42 & 423.99 & 727.50 \\
\hline 670.00 & $59 \cdot 10$ & 26.50 & 1.90 & 1.80 & 77.50 & $22 \cdot 50$ & 77.50 & 156.00 & 6.00 & 22.50 & .00 & 2055.97 & 462.59 & 727.00 \\
\hline 670.50 & $60 \cdot 90$ & 27.00 & 1.80 & 1.70 & 77.20 & 22.80 & 77.20 & 156.00 & 6.00 & 22.80 & .00 & 2094.77 & 477.61 & 726.50 \\
\hline 671.00 & 63.20 & 27.30 & 1.90 & 1.00 & 74.70 & $25 \cdot 30$ & 74.70 & 178.00 & 5.00 & $25 \cdot 30$ & .00 & 2118.04 & 535.86 & 726.00 \\
\hline 671.50 & $65 \cdot 50$ & 27.50 & 2.00 & 1.70 & $72 \cdot 10$ & 27.90 & 72.10 & 178.00 & 5.00 & 27.90 & .00 & 2133.56 & 595.26 & 725.50 \\
\hline 6.72 .00 & $64 \cdot 80$ & 27.30 & 2.10 & 2.10 & 70.50 & 29.50 & 70.50 & 178.00 & 5.00 & 29.50 & .00 & 2118.04 & 624.82 & 725.00 \\
\hline 6.72 .50 & 62.50 & 26.40 & 2.30 & 2.40 & 71.20 & 28.80 & 71.20 & 178.00 & 5.00 & 28.80 & .00 & 2048.22 & 589.89 & 724.50 \\
\hline 6.73 .00 & 61.90 & 25.80 & 2.40 & 2.90 & $71 \cdot 10$ & 28.90 & 71.10 & 138.00 & 9.00 & 28.90 & .00 & 2001.67 & 578.48 & 724.00 \\
\hline 6.73 .50 & 63.00 & 25.40 & 2.50 & $3 \cdot 30$ & 70.50 & 29.50 & 70.50 & 138.00 & 9.00 & 29.50 & .00 & 1970.63 & $581 \cdot 34$ & 723.50 \\
\hline 674.00 & 63.70 & 25.00 & 2.60 & 2.90 & $70 \cdot 10$ & 29.90 & 70.10 & 120.00 & 12.00 & 29.90 & .00 & 1939.60 & 579.94 & 723.00 \\
\hline 674.50 & 65.50 & 24.90 & 2.70 & 2.20 & 69.20 & 30.80 & 69.20 & 120.00 & 12.00 & 30.80 & .00 & 1931.84 & 595.01 & 722.50 \\
\hline 675.00 & $67 \cdot 30$ & 25.00 & 2.80 & 2.20 & 68.20 & 31.80 & 68.20 & 121.00 & 15.00 & 31.80 & .00 & 1939.60 & 616.79 & 722.00 \\
\hline 675.50 & 69.80 & 25.60 & 2.70 & 2.00 & 66.80 & 33.20 & 66.80 & 121.00 & 15.00 & 33.20 & .00 & & 659.40 & 721.50 \\
\hline 676.00 & 70.00 & 26.30 & 2.70 & 1.90 & $t 5.60$ & 34.40 & 65.60 & 152.00 & 13.00 & 34.40 & .00 & 2040.46 & 701.92 & 721.00 \\
\hline 676.50 & 70.20 & 27.00 & 2.60 & 2.00 & 64.90 & 35.10 & 64.90 & 152.00 & 13.00 & 35.10 & .00 & 2094.77 & 735,26 & 720.50 \\
\hline 677.00 & 72.00 & 27.40 & 2.50 & 2.00 & 64.70 & $35 \cdot 30$ & 64.70 & 181.00 & 12.00 & $35 \cdot 30$ & .00 & 2125.80 & 750.41 & $? 20.00$ \\
\hline 677.50 & 76.80 & 27.40 & 2.50 & 2.00 & $\epsilon 5.60$ & 34.40 & 65.60 & 181.00 & 12.00 & 34.40 & .00 & 2125.80 & 731.27 & 719.50 \\
\hline 678.00 & 78.60 & 27.30 & 2.40 & $2 \cdot c 0$ & 66.70 & $33 \cdot 30$ & 66.70 & 176.00 & 16.00 & 33.30 & .00 & 2118.04 & 705.31 & 719.00 \\
\hline 678.50 & $80 \cdot 10$ & 26.50 & 2.40 & 1.90 & 68.60 & 31.40 & 68.60 & 176.00 & 16.00 & 31.40 & .00 & 2055.97 & 645,58 & 718.50 \\
\hline 679.00 & $81 \cdot 16$ & 25.60 & 2.40 & 1.50 & $70 \cdot 90$ & 29.10 & 70.90 & 133.00 & 20.00 & 29.10 & .00 & $1986 \cdot 15$ & 577.97 & 718.00 \\
\hline 679.50 & 78.50 & 26.40 & 2.40 & 2.00 & 68.70 & $31 \cdot 30$ & 68.70 & 133.00 & 20.00 & 31.30 & .00 & $2048 \cdot 22$ & $6+1.09$ & 717.50 \\
\hline 680.00 & $80 \cdot 10$ & 26.50 & 2.40 & 2.60 & 68.20 & 31.80 & 68.20 & 156.00 & 17.00 & 31.90 & .00 & 2055.97 & 653.80 & 717.00 \\
\hline 6880.50 & 82.40 & 25.40 & 2.40 & 2.00 & 71.70 & $28 \cdot 30$ & 71.70 & 156.00 & 17.00 & 28.30 & .00 & 1970.63 & 557.69 & 716.50 \\
\hline 6881.00 & 83.20 & 25.40 & 2.40 & $2 \cdot 60$ & 71.90 & 28.10 & 71.90 & 129.00 & 16.00 & 28.10 & .00 & 1970.63 & 553.75 & 716.00 \\
\hline $681 \cdot 50$ & 83.20 & $25 \cdot 30$ & 2.40 & $2 \cdot 10$ & 72.70 & 27.30 & 72.70 & 129.00 & 16.00 & 27.30 & .00 & 1962.87 & 535.86 & 715.50 \\
\hline $\begin{array}{l}682 \cdot 00 \\
682.50\end{array}$ & $\begin{array}{l}81.70 \\
80.70\end{array}$ & $\begin{array}{l}25.20 \\
23.80\end{array}$ & $\begin{array}{l}2 \cdot 30 \\
2 \cdot 30\end{array}$ & $\begin{array}{l}2.00 \\
1.90\end{array}$ & $\begin{array}{l}73 \cdot 50 \\
78.50\end{array}$ & $\begin{array}{l}26.50 \\
21.50\end{array}$ & $\begin{array}{l}73.50 \\
78.50\end{array}$ & $\begin{array}{l}124.00 \\
124.00\end{array}$ & $\begin{array}{l}15.00 \\
15.00\end{array}$ & $\begin{array}{l}26.50 \\
21.50\end{array}$ & $\begin{array}{l}.00 \\
.00\end{array}$ & $\begin{array}{l}1955.12 \\
1846.50\end{array}$ & $\begin{array}{l}518.11 \\
397.00\end{array}$ & $\begin{array}{l}715.00 \\
714.50\end{array}$ \\
\hline A.AVE. & 69.71 & 25.33 & 2.38 & $2 \cdot 1$ & $73 \cdot 22$ & 26.78 & 73.22 & 133.24 & 12.15 & 26.78 & .00 & 1965.22 & 534.40 & \\
\hline$G \cdot M E \triangle N$ & 69.17 & 25.24 & $\overline{2} \cdot 36$ & $2 \cdot 14$ & 72.93 & 25.58 & 72.93 & 124.44 & $11 \cdot 19$ & 25.58 & & 1958.20 & 500.34 & \\
\hline
\end{tabular}

*NOTATIOH--Depth, ft; iR-Gamma Ray Index, API Units; POR-Porosity, $\%$; RT-True Resistivity, Ohms-m²/m; RXO-Flushej Zone Resistivity, Ohnis-m²/m;

¿XO-Flusher Zone Saturation, \%; ROS-Residual 0il Saturation, \%; SW-Water Saturation, \%; PER-Permeability Index; Clay-Clay Content, \%; SO-0il

jaturation, \%; MO-Movasle 0il, \%; PHV-Pore Volume, bbl/ac-ft; HPV-Hydrocarbon Pore Volume, bbl/ac-ft; ELSE-Elevation From Sea Level, ft 
TABLE C61

HELL LOG DETERHINED PROPERTIES

VS DEPTH FOR WELL PAP-221

WELL LOCATION: 1056 FNL $2112 \mathrm{FWL} 28$ 25S $5 E$

KELLY BUSHING ELEVATION FROM SEA LEVEL $=1402$

\begin{tabular}{|c|c|c|c|c|c|c|c|c|c|c|c|c|c|c|}
\hline DEPTH * & GR & PAK & RT & $R \times E$ & $s \times 0$ & ROS & sw & PER & CLAY & se & ro & PHV & HPV & ELSE \\
\hline 660.00 & 82.60 & 23.90 & 4.50 & $7 \cdot 90$ & 56.40 & 43.60 & 56.40 & 99.00 & 2.00 & 43.60 &.$c 0$ & 1854.26 & $808 \cdot 46$ & $\begin{array}{l}742.00 \\
741.00\end{array}$ \\
\hline 661.00 & 82.40 & 25.30 & 4.70 & 5.70 & 52.50 & 47.50 & 52.10 & 127.00 & 4.00 & 47.90 & .40 & 1962.87 & 940.22 & 741.00 \\
\hline $\begin{array}{l}662.00 \\
663.00\end{array}$ & $76 \cdot 20$ & $\begin{array}{l}25 \cdot 30 \\
25.10\end{array}$ & $\begin{array}{l}4.50 \\
4.30\end{array}$ & 4.70 & 57.80 & $\begin{array}{r}42.20 \\
42.80\end{array}$ & 52.80 & 127.00 & 3.00 & 47.20 & 5.00 & 1962.87 & 926.48 & $\begin{array}{l}740.00 \\
739.00\end{array}$ \\
\hline $\begin{array}{l}663.00 \\
664.00\end{array}$ & $\begin{array}{l}73.40 \\
73.80\end{array}$ & $\begin{array}{l}25.10 \\
24.80\end{array}$ & $\begin{array}{l}4.30 \\
3.90\end{array}$ & $\begin{array}{l}4.90 \\
4.30\end{array}$ & $\begin{array}{l}57 \cdot 20 \\
\epsilon 1 \cdot 30\end{array}$ & $\begin{array}{r}2.80 \\
38.70\end{array}$ & $\begin{array}{l}54.50 \\
57.70\end{array}$ & $\begin{array}{l}122.00 \\
117.00\end{array}$ & $\begin{array}{l}2.00 \\
2.00\end{array}$ & $\begin{array}{l}5.50 \\
4.30\end{array}$ & $\begin{array}{l}2.70 \\
3.60\end{array}$ & $\begin{array}{l}1947.36 \\
1924.08\end{array}$ & $\begin{array}{l}886.05 \\
813.89\end{array}$ & $\begin{array}{l}739.00 \\
738.00\end{array}$ \\
\hline 665.00 & 78.70 & $25 . ? 0$ & 3.60 & $4 \cdot 30$ & $E 0.80$ & 39.20 & 59.50 & 125.00 & 2.00 & 40.50 & $1 \cdot 30$ & 1955.12 & 791.82 & 737.00 \\
\hline 666.00 & 85.10 & 26.10 & 3.40 & 4.30 & 58.60 & 41.40 & 58.60 & 145.00 & 3.00 & 41.40 & .00 & 2024.94 & $838 \cdot 33$ & 736.00 \\
\hline 667.00 & 91.50 & 26.80 & $3 \cdot 30$ & 4.00 & $59 \cdot 10$ & .0 .90 & 58.20 & 163.00 & 5.00 & 11.80 & .90 & 2079.25 & 869.13 & 735.00 \\
\hline 668.00 & 89.50 & 27.00 & 3.00 & $3 \cdot 80$ & 60.80 & $39 \cdot 20$ & 50.80 & $168 \cdot 00$ & 4.00 & 39.20 & .00 & 2094.77 & $921 \cdot 15$ & 734.00 \\
\hline 669.00 & 89.40 & 26.60 & 2.70 & 3.40 & 64.60 & $35 \cdot 40$ & 64.60 & 159.00 & 4.00 & 35.40 & .00 & 2063.73 & 730.56 & 733.00 \\
\hline 670.00 & $88 \cdot 30$ & 26.10 & $2 \cdot 40$ & $3 \cdot 30$ & 69.50 & 30.50 & 69.50 & 146.00 & 4.00 & 30.50 & .00 & 2024.94 & 617.61 & 732.00 \\
\hline 671.00 & 89.90 & 25.10 & 2.40 & $3 \cdot 70$ & $72 \cdot 70$ & $27 \cdot 30$ & 72.70 & 123.00 & 5.00 & $27 \cdot 30$ & .00 & 1947.36 & 531.63 & 731.00 \\
\hline 672.00 & SC.5O & 24.10 & $2 \cdot 50$ & 3.60 & $75 \cdot 20$ & $24 \cdot 30$ & 75.20 & 102.00 & 8.00 & 24.80 & .50 & 1869.77 & .63 .70 & 730.00 \\
\hline 673.00 & 95.50 & $24 \cdot 10$ & 2.70 & $4 \cdot 00$ & $72 \cdot 30$ & 27.70 & $72 \cdot 30$ & $102 \cdot 00$ & 15.00 & 27.70 & .00 & 1869.77 & 517.93 & 729.00 \\
\hline 674.00 & $100 \cdot 10$ & 24.00 & $2 \cdot 90$ & 4.00 & 70.30 & 29.70 & $70 \cdot 30$ & $100 \cdot 00$ & 22.00 & 29.70 & .00 & 1862.02 & 553.02 & 728.00 \\
\hline 675.00 & $100 \cdot 40$ & 23.80 & 3.00 & 3.90 & $\in 8.80$ & $31 \cdot 20$ & 68.80 & 96.00 & 22.00 & 31.20 & .00 & $1846 \cdot 50$ & 576.11 & 727.00 \\
\hline 676.00 & 99.40 & 23.40 & 3.00 & 3.90 & 70.20 & 29.30 & 70.20 & 89.00 & 20.00 & 29.80 & .50 & 1815.46 & 541.01 & 726.00 \\
\hline 677.00 & $100 \cdot 40$ & 22.90 & 3.00 & 4.00 & 71.70 & $29 \cdot 30$ & 71.70 & 83.00 & 20.00 & 28.30 & .0 & 1776.67 & 502 & 725.00 \\
\hline 678.00 & 104.50 & 22.60 & 3.00 & 4.00 & $73 \cdot 10$ & $25 \cdot 90$ & 73.10 & 77.00 & 23.00 & 26.90 & 1.00 & 1753.40 & 471.66 & 724.00 \\
\hline 679.00 & 109.40 & 19.70 & 0.0 & 4.00 & 85.40 & 14.60 & 85.40 & $42 \cdot 00$ & 30.00 & $14 \cdot 60$ & .00 & 1528.40 & 223.15 & 723.00 \\
\hline & 9 & 2 & 3.29 & $4 \cdot 28$ & $t 5.91$ & 33.98 & 65.22 & 115.60 & 10. & 34.78 & .80 & 18 & & \\
\hline$A N$ & 8 & 24.54 & $3 \cdot 22$ & 4.20 & 65.44 & 32.87 & 64.64 & & 6.43 & 33.54 & & 1903.56 & 638.39 & \\
\hline
\end{tabular}

*NOTATIO:I--Depth, ft; GR-Gamma Ray Index, API Units; POR-Porosity, $\%$; RT-True Resistivity, Ohms-m²/m; Rxo-Flushed Zone Resistivity, Ohnis-m²/m; SXO-Flushed Zone Saturation, \%; ROS-Residual 0il Saturation, \%; SW-Water Saturation, \%; PER-Permeability Index; Clay-Clay Content, \%; SO-0il Saturation, \%; HO-Hovable Dil, \%; PHV-Pore Volume, bbl/ac-ft; HPV-Hydrocarbon Pore Volume, bbl/ac-ft; ELSE-Elevation From Sea Level, ft 
TABLE C62

WELL LOG DETERMINED PROPERTIES

VS DEPTH FOR WELL MP-222

WELL LOCATION: 2376 FWL 1 C56 FNL $282555 E$

KELLY BUSHING ELEVATION FROM SEA LEVEL $=1404$

\begin{tabular}{|c|c|c|c|c|c|c|c|c|c|c|c|c|c|c|}
\hline DEPT $\mu^{*}$ & $G R$ & PIOP & $R T$ & $R \times E$ & $5 \times \theta$ & RAS & Sw & PER & CLAY & 50 & mo & PHV & HPV & ELSE \\
\hline $\begin{array}{l}665.00 \\
665.50\end{array}$ & $\begin{array}{l}68 \cdot 00 \\
65.90\end{array}$ & $\begin{array}{l}23.30 \\
23.40\end{array}$ & $\begin{array}{l}2.90 \\
2.70\end{array}$ & 1.60 & 73.50 & 26.50 & 73.50 & 89.00 & 23.00 & & .00 & 1815.46 & 481.10 & 738.50 \\
\hline 666.00 & $63 . c 0$ & 23.30 & 2.60 & 1.40 & $75 \cdot 30$ & $24 \cdot 70$ & $75 \cdot 30$ & 89.00 & 16.00 & 24.70 & .00 & 1807.71 & 446.50 & 738.00 \\
\hline $\begin{array}{l}666.50 \\
667.00\end{array}$ & $60 \cdot 50$ & 23.20 & 2.50 & $1 \cdot 30$ & 77.80 & $22 \cdot 20$ & 77.80 & 89.00 & 16.00 & 22.20 & .00 & 1799.95 & 399.59 & 737.50 \\
\hline 667.00 & $58 \cdot 20$ & 23.20 & $2 \cdot 30$ & 1.30 & $80 \cdot 70$ & $19 \cdot 30$ & 80.70 & 87.00 & $15 \cdot 00$ & $19 \cdot 30$ & .00 & 1799.95 & 347.39 & 737.00 \\
\hline 667.50 & $56 \cdot 70$ & 23.20 & $2 \cdot 20$ & 1.40 & 83.80 & $16 \cdot 20$ & 83.80 & 87.00 & 15.00 & 16.20 & .00 & 1799.95 & 291.59 & 736.50 \\
\hline $668 \cdot 00$ & $56 \cdot 70$ & 23.20 & $2 \cdot 00$ & $1.4 \mathrm{C}$ & 85.90 & $14 \cdot 10$ & 35.90 & 87.00 & 12.00 & $14 \cdot 10$ & .00 & 1799.95 & 253.79 & 736.00 \\
\hline 668.50 & $55 \cdot 80$ & 23.30 & 2.00 & 1.40 & 87.50 & 12.50 & 87.50 & 87.00 & 12.00 & $12 \cdot 50$ & .00 & $1807.7 i$ & 225.96 & 735.50 \\
\hline 669.00 & $54 \cdot \in 0$ & 24.20 & 1.90 & 1.60 & $86 \cdot 10$ & 13.90 & 86.10 & 104.00 & 8.00 & 13.90 & .00 & 1877.53 & 260.98 & 735.00 \\
\hline $\begin{array}{l}669.50 \\
670.00\end{array}$ & $53 \cdot 70$ & 25.50 & 1.80 & 1.50 & 82.90 & $17 \cdot 10$ & 82.90 & 104.00 & 8.00 & 17.10 & .00 & 1978.39 & $338 \cdot 30$ & 734.50 \\
\hline $\begin{array}{l}670.00 \\
670.50\end{array}$ & $53 \cdot 30$ & 25.80 & 1.70 & 1.20 & 80.20 & 19.80 & 30.20 & $163 \cdot 00$ & 4.00 & 19.80 & .00 & 2079.25 & 411.69 & 734.00 \\
\hline 670.50 & $58 \cdot 50$ & 27.30 & 1.70 & $1 \cdot 0$ & 79.90 & $20 \cdot 10$ & 79.90 & 163.00 & 4.00 & 20.10 & .00 & 2118.04 & 425.73 & 733.50 \\
\hline 671.30 & $63 \cdot 10$ & 37.20 & 2.60 & 1.40 & $81 \cdot 30$ & 18.70 & 81.30 & 175.00 & 7.00 & $18 \cdot 70$ & .00 & 2110.28 & $\begin{array}{l}394.62 \\
372.87\end{array}$ & 733.00 \\
\hline 671.50 & 65.50 & 27.00 & 1.60 & 1.40 & $82 \cdot 20$ & 17.80 & 82.20 & $175 \cdot 00$ & 7.00 & 17.80 & .00 & 2094.77 & $\begin{array}{r}372.81 \\
336.39\end{array}$ & 732.50 \\
\hline 672.00 & 6.90 & 25.60 & 2.60 & 1.40 & 83.70 & $16 \cdot 30$ & 83.70 & 159.00 & 7.00 & $16 \cdot 30$ & .00 & 2063.73 & $336 \cdot 39$ & 732.00 \\
\hline 672.50 & 66.30 & 25.20 & 1.70 & 1.40 & $84 \cdot 00$ & 16.00 & 84.00 & 159.00 & 7.00 & 16.00 & .00 & 2032.70 & 325.23 & 731.50 \\
\hline 673.00 & $66 \cdot 30$ & 25.20 & 1.70 & $1 \cdot 40$ & 86.90 & 13.40 & 86.60 & 125.00 & 16.00 & 13.40 & .00 & 1955.12 & 261.99 & 731.00 \\
\hline 673.50 & 66.30 & $24 \cdot 30$ & 1.70 & $1 \cdot 50$ & 88.60 & 11.40 & 88.60 & 125.00 & 16.00 & 11.40 & .00 & 1885.29 & 214.92 & 730.50 \\
\hline 674.00 & 66.80 & 23.60 & 1.80 & 1.00 & 89.90 & 10.10 & 89.90 & 93.00 & 21.00 & 10.10 & .00 & 1830.98 & 184.93 & 730.00 \\
\hline 674.50 & $67 .<0$ & $24 \cdot 3 c$ & 1.90 & 1.80 & $\varepsilon 5.60$ & 14.40 & 85.50 & 93.00 & 21.00 & 14.40 & .00 & 1885.29 & 271.48 & 729.50 \\
\hline 675.00 & 69.30 & 24.80 & 1.90 & 2.00 & $\varepsilon 2.60$ & 17.40 & 82.60 & 116.00 & 14.00 & 17.40 & .00 & 1924.08 & 334.79 & 729.00 \\
\hline 675.50 & $71 \cdot 30$ & 24.00 & 2.00 & 1.00 & $24 \cdot 30$ & 15.70 & 24.30 & 116.00 & 14.00 & 15.70 & .00 & 1862.02 & 292.34 & 728.50 \\
\hline 676.00 & 73.20 & 22.80 & 2.00 & 1.00 & 88.40 & 11.60 & 38.40 & 80.00 & 22.00 & 11.60 & .00 & 1768.91 & 205.19 & 728.00 \\
\hline 676.50 & 74.50 & 21.90 & $2 \cdot 10$ & $1 \cdot 30$ & 50.60 & 9.40 & 90.60 & 80.00 & 22.00 & 9.40 & .00 & 1699.09 & 159.71 & 727.50 \\
\hline 677.00 & 74.70 & 21.70 & $2 \cdot 10$ & $2 \cdot 20$ & 51.10 & 8.90 & 91.10 & 64.00 & 20.00 & 8.90 & .00 & 1683.57 & 149.84 & 727.00 \\
\hline 677.50 & 73.30 & 22.60 & $2 \cdot 10$ & 2.20 & 87.40 & 12.60 & 87.40 & 64.00 & 20.00 & $12.6 n$ & .00 & 1753.40 & 220.93 & 726.50 \\
\hline 678.00 & 70.60 & 23.50 & $2 \cdot 10$ & $1.9 \mathrm{C}$ & 83.90 & $16 \cdot 10$ & 83.90 & 93.00 & 12.00 & 16.10 & .00 & $1830 \cdot 98$ & .79 & 726.00 \\
\hline 678.50 & 73.50 & 21.50 & $2 \cdot 10$ & 1.70 & 52.90 & 7.20 & 92.90 & 93.00 & 12.00 & 7.20 & .00 & 1668.06 & 120.10 & 725.50 \\
\hline A AVE. & 64.44 & 23.93 & 2.04 & $\begin{array}{l}1.01 \\
1.58\end{array}$ & $\begin{array}{l}84.04 \\
83.89\end{array}$ & $\begin{array}{l}15.96 \\
15.19\end{array}$ & $\begin{array}{l}84.04 \\
83.89\end{array}$ & $\begin{array}{r}105.62 \\
97.60\end{array}$ & $\begin{array}{l}14 \cdot 41 \\
12 \cdot 90\end{array}$ & $\begin{array}{l}15.96 \\
15.19\end{array}$ & .00 & $\begin{array}{l}1862.55 \\
1856.70\end{array}$ & $\begin{array}{l}299.90 \\
282.12\end{array}$ & \\
\hline
\end{tabular}

*NOTATIO:l--Depth, ft. GR-Gamma Ray Index, API Units; POR-Porosity, $\%$; RT-True Resistivity, Ohms-m²/n; RX0-Flushed Zone Resistivity, Ohms-m²/m; \$XO-Flusher Zone Saturation, \%; ROS-Residual 0il Saturation, \%; SW-Water Saturation, \%; PER-Permeability [ndex; Clay-Clay Content, \%; SO-0il

Saturation, \%; M0-110rable 0il, \%; PHV-Pore Volume, bbl/ac-ft; HPV-Hydrocarbon Pore Volume, bbl/ac-ft; ELSE-Elevation From Sea Level, f.t 
TABLE C63

WELL LOG DETERMINED PROPERTIES

VS DEPTH FOR WELL H.PP-223

WELL LODATION: 2640 FEL 1 C56 FNL 28 255 SE

KELLY BUSHING ELEVATION FROM SEA LEVEL $=1400$

\begin{tabular}{|c|c|c|c|c|c|c|c|c|c|c|c|c|c|c|}
\hline DEPTH* & $G R$ & $P O R$ & RT & $R \times U$ & $5 \times \theta$ & ROS & sw & PER & CLAY & so & mo & PHV & HPV & ELSE \\
\hline $\begin{array}{l}667.00 \\
667.50\end{array}$ & $\begin{array}{l}65.00 \\
66.80\end{array}$ & $\begin{array}{l}18.20 \\
20.40\end{array}$ & $\begin{array}{l}* 20 \\
3 \cdot 90\end{array}$ & $\begin{array}{l}5 \cdot 20 \\
5 \cdot 50\end{array}$ & $\begin{array}{l}78.00 \\
76.60\end{array}$ & $\begin{array}{l}22.00 \\
23.40\end{array}$ & $\begin{array}{l}78.00 \\
71.90\end{array}$ & $\begin{array}{l}30.00 \\
30.00\end{array}$ & $\begin{array}{l}24.00 \\
24.00\end{array}$ & $\begin{array}{l}22 \cdot 00 \\
28.10\end{array}$ & $\begin{array}{r}.00 \\
4.70\end{array}$ & $\begin{array}{l}1412.03 \\
1582.71\end{array}$ & $\begin{array}{r}310.65 \\
444.74\end{array}$ & $\begin{array}{l}733.00 \\
732.50\end{array}$ \\
\hline 668.00 & 63.30 & 19.70 & $3 \cdot 70$ & $\begin{array}{l}5.20 \\
5.30\end{array}$ & 76.60 & $\begin{array}{l}23.40 \\
15.90\end{array}$ & $\begin{array}{l}76.60 \\
84.10\end{array}$ & $\begin{array}{r}42.00 \\
42.00\end{array}$ & $\begin{array}{l}20.00 \\
20.00\end{array}$ & $\begin{array}{l}23.40 \\
15.90\end{array}$ & $\begin{array}{l}.80 \\
.00\end{array}$ & $\begin{array}{l}1528.40 \\
1427.54\end{array}$ & $\begin{array}{l}357.65 \\
226.98\end{array}$ & $\begin{array}{l}732.00 \\
731.50\end{array}$ \\
\hline $\begin{array}{l}668.50 \\
669.00\end{array}$ & $\begin{array}{l}59.20 \\
58.60\end{array}$ & $\begin{array}{l}18.40 \\
17.60\end{array}$ & $\begin{array}{l}3 \cdot 50 \\
3 \cdot 30\end{array}$ & $\begin{array}{l}5 \cdot 30 \\
5 \cdot 40\end{array}$ & $\begin{array}{l}84.10 \\
91.00\end{array}$ & $\begin{array}{r}15.90 \\
9.00\end{array}$ & $\begin{array}{l}84.10 \\
91.00\end{array}$ & 26.00 & 16.00 & 9.50 & .00 & $\begin{array}{l}1427.54 \\
1365.48\end{array}$ & 122.89 & $\begin{array}{l}731.50 \\
731.00\end{array}$ \\
\hline $669 \cdot 50$ & 59.80 & 17.00 & $3 \cdot 20$ & 5.50 & $56 \cdot 30$ & 3.70 & 96.30 & 26.00 & 16.00 & 3.70 & .00 & 1318.93 & 48.80 & 730.50 \\
\hline 670.00 & $56 \cdot 30$ & 15.90 & $3 \cdot 20$ & 5.10 & 100.00 & $.0 n$ & 100.00 & 17.00 & 13.00 & .00 & .00 & $12.33 \cdot 58$ & .00 & 730.00 \\
\hline 670.50 & $55 \cdot 70$ & 15.00 & 3.00 & $5 \cdot 50$ & 100.00 & .00 & 100.00 & 17.00 & 13.00 & . On & .00 & 1163.76 & .00 & 729.50 \\
\hline 671.00 & 56.80 & 14.50 & 2.90 & 4.80 & 160.00 & .00 & 100.00 & 11.00 & 16.00 & .00 & .00 & 1124.97 & .00 & 729.00 \\
\hline 671.50 & 59.20 & 15.90 & $2 \cdot 90$ & 4.30 & 100.00 & .00 & 100.00 & 11.00 & 16.00 & .00 & .00 & 1233.58 & .00 & 728.50 \\
\hline 672.00 & 62.70 & 22.80 & 2.80 & 4.10 & $79 . \geq 0$ & $20 \cdot 80$ & 74.30 & $80 \cdot 00$ & 19.00 & 25.70 & 4.90 & 1768.91 & 454.61 & 728.00 \\
\hline 672.50 & 66.80 & 25.10 & 2.90 & 4.10 & 70.60 & 29.40 & 66.30 & 80.00 & 19.00 & 33.70 & 4.30 & $1947 \cdot 36$ & 656.26 & 727.50 \\
\hline 673.00 & 68.60 & 25.20 & 3.00 & 4.00 & 66.60 & 33.40 & 64.80 & 126.00 & 23.00 & $35 \cdot 20$ & 1.80 & 1955.12 & 688.20 & 727.00 \\
\hline 673.50 & 69.10 & 23.50 & $3 \cdot 10$ & $5 \cdot$ ن & 69.30 & 30.70 & $69 \cdot 30$ & 126.00 & 23.00 & 30.70 & .00 & 1823.22 & 559.73 & 726.50 \\
\hline 674.00 & $73 \cdot 80$ & 20.50 & 3.10 & $5 \cdot 20$ & 79.50 & 20.50 & 79.50 & $50 \cdot 00$ & 36.00 & $20 \cdot 50$ & .00 & 1590.47 & 326.05 & 726.00 \\
\hline 674.50 & 78.50 & 17.40 & $2 \cdot 30$ & $5 \cdot 30$ & 52.30 & 7.70 & $9 ? \cdot 30$ & $50 \cdot 00$ & 36.00 & 7.70 & .00 & 1349.96 & 103.95 & 725.50 \\
\hline 675.00 & 83.80 & 14.80 & 3.50 & $5 \cdot 20$ & 160.00 & .00 & 100.00 & 12.00 & 49.00 & .00 & .00 & 1148.24 & .00 & 725.00 \\
\hline $675 \cdot 50$ & $85 \cdot 50$ & 13.70 & 3.70 & $5 \cdot 20$ & 100.00 & .00 & 100.00 & 12.00 & .9 .00 & .00 & .00 & 1062.90 & .00 & 724.50 \\
\hline 676.00 & 82.00 & 15.60 & 3.90 & $5 \cdot 40$ & 54.80 & 5.20 & 94.80 & 15.00 & .6 .00 & $5 \cdot 20$ & .00 & $1210 \cdot 31$ & 62.94 & 724.00 \\
\hline 676.50 & 76.80 & 13.60 & 4.00 & $5 \cdot 10$ & 77.90 & $22 \cdot 10$ & 77.90 & 15.00 & 46.00 & $22 \cdot 10$ & .00 & 1443.06 & 318.92 & 723.50 \\
\hline 677.00 & 73.20 & 23.40 & $4 \cdot 10$ & $6 \cdot 20$ & $62 \cdot 30$ & 37.70 & 60.10 & 91.00 & 31.00 & 39.90 & $2 \cdot 20$ & 1815.46 & 724.37 & 723.00 \\
\hline 677.50 & 70.30 & 26.70 & $4 \cdot 20$ & 6.20 & $54 \cdot 10$ & 45.90 & 51.70 & $.91 \cdot 00$ & 31.00 & 48.30 & $2 \cdot 40$ & 2071.49 & 1000.53 & 722.50 \\
\hline 678.00 & 67.40 & 27.80 & $4 \cdot 10$ & 5.00 & $54 \cdot 30$ & 45.70 & 50.00 & $192 \cdot 00$ & 18.00 & 50.00 & 4.30 & 2156.83 & 1078.42 & 722.00 \\
\hline 678.50 & 66.80 & 27.80 & 4.00 & 5.60 & 54.20 & 45.80 & 50.40 & 192.00 & 18.00 & 49.60 & 3.80 & 2156.83 & 1069.79 & 721.50 \\
\hline 679.00 & 66.80 & 27.30 & 4.00 & 5.80 & $54 \cdot 30$ & 45.70 & 51.40 & 178.00 & 17.00 & 48.60 & 2.90 & 2118.04 & 1029.37 & 721.00 \\
\hline 679.50 & 68.00 & 26.20 & $3 \cdot 80$ & 6.00 & $55 \cdot 80$ & 44.20 & 55.30 & 178.00 & 17.00 & 44.70 & .50 & 2032.70 & 908.62 & 720.50 \\
\hline 680.00 & 67.40 & 25.70 & 3.60 & 5.00 & 59.00 & 41.00 & 58.00 & 136.00 & 21.00 & $42 \cdot 00$ & 1.00 & 1993.91 & 837.44 & 720.00 \\
\hline $680 \cdot 50$ & 67.40 & 25.50 & $3 \cdot 30$ & $5 \cdot 20$ & $\epsilon 1 \cdot 30$ & 38.20 & 60.70 & $136 \cdot 00$ & 21.00 & $39 \cdot 30$ & $1 \cdot 10$ & $1978 \cdot 39$ & 777.51 & 719.50 \\
\hline 681.00 & 70.90 & 25.50 & $3 \cdot 10$ & 5.10 & $\epsilon 3.00$ & 37.00 & 63.00 & 131.00 & 24.00 & 37.00 & .00 & 1978.39 & 732.00 & 719.00 \\
\hline $681 \cdot 50$ & 72.70 & 25.20 & 3.00 & 4.90 & $64 \cdot 80$ & 35.20 & 64.80 & 131.00 & 24.00 & 35.20 & .00 & 1955.12 & 688.20 & 718.50 \\
\hline 682.00 & 68.00 & 26.30 & $2 \cdot 80$ & 4.60 & $\epsilon 3 \cdot 50$ & $36 \cdot 40$ & 63.60 & 151.00 & 22.00 & 36.40 & .00 & 2040.46 & 742.73 & 718.00 \\
\hline 682.50 & 64.50 & 26.80 & 2.70 & 4.60 & $t+\cdot 10$ & 35.90 & 64.10 & 151.00 & 22.00 & 35.90 & .00 & 2079.25 & 746.45 & 717.50 \\
\hline 683.00 & 65.00 & 26.70 & $2 \cdot 50$ & 4.60 & 66.20 & 33.80 & 66.20 & 160.00 & 17.00 & 33.90 & .00 & 2071.49 & $700 \cdot 16$ & 717.00 \\
\hline $683 \cdot 50$ & 68.60 & 26.30 & 2.50 & 4.60 & 68.40 & 31.60 & 68.40 & 160.00 & 17.00 & 31.60 & .00 & 2040.46 & 644.78 & 716.50 \\
\hline 684.00 & 73.80 & 24.80 & $2 \cdot 40$ & $4 \cdot 40$ & 74.40 & 25.60 & 74.40 & 115.00 & 26.00 & 25.60 & .00 & 1924.08 & 492.56 & 716.00 \\
\hline 684.50 & 77.90 & 22.10 & 2.40 & 4.40 & 83.90 & 16.10 & 83.90 & 115.00 & 26.00 & $16 \cdot 10$ & .00 & 1714.61 & 276.05 & 715.50 \\
\hline 685.00 & $82 \cdot 60$ & 20.00 & 2.40 & 4.40 & 53.40 & 6.60 & 93.40 & $45 \cdot 00$ & 43.00 & 6.60 & .00 & 1551.68 & 102.41 & 715.00 \\
\hline $\begin{array}{l}\text { A.AVE. } \\
\text { G.MEAN }\end{array}$ & $\begin{array}{l}69.42 \\
69.18\end{array}$ & $\begin{array}{l}23.24 \\
22.94\end{array}$ & $\begin{array}{l}3.32 \\
3.27\end{array}$ & $\begin{array}{l}5.13 \\
5.09\end{array}$ & $\begin{array}{l}71.14 \\
70.11\end{array}$ & $\begin{array}{l}28.86 \\
25.21\end{array}$ & $\begin{array}{l}70.01 \\
68.83\end{array}$ & $\begin{array}{r}102.17 \\
81.91\end{array}$ & $\begin{array}{l}24.90 \\
23.72\end{array}$ & $\begin{array}{l}29.99 \\
26.05\end{array}$ & $1 \cdot 13$ & $\begin{array}{l}1802.79 \\
1779.60\end{array}$ & $\begin{array}{l}572.83 \\
463.58\end{array}$ & \\
\hline
\end{tabular}

*NOTATION--Depth, ft; GR-Gamma Ray Index, API Units; POR-Porosity, $\%$; RT-True Resistivity, Ohms-m²/m; RX0-Flushed Zone Resistivity, OhnIs-m²/m; SX0-Flushed Zone Saturation, \%; ROS-Residual 0il Saturation, \%; SW-Water Saturation, \%; PER-Permeability Index; Clay-Clay Content, \%; SO-0il

Saturztion, \%; MO-Movable Jil, \%; PHV-Pore Volume, bbl/ac-ft; HPV-Hydrocarbon Pore Volume, bbl/ac-ft; ELSE-Elevation From Sea Level, ft 
TABLE C64

WELL LOG DETERMINED PROPERTIES

VS DEPTH FOR HELL IIPP-224

WELL LOCATION: 2376 FEL 1 C56 FNL $212555 E$

KELLY BUSHING ELEVATION FROM SEA LEVEL $=1403$

\begin{tabular}{|c|c|c|c|c|c|c|c|c|c|c|c|c|c|c|}
\hline$D \equiv P T H^{*}$ & GR & PQP. & $R T$ & RXE & $s \times \theta$ & $\operatorname{RES}$ & Sw & PER & CLAY & so & ro & PHV & HPV & ELSE \\
\hline $\begin{array}{l}658.00 \\
658.50\end{array}$ & $\begin{array}{l}76.20 \\
75.10\end{array}$ & $\begin{array}{l}18.70 \\
24.30\end{array}$ & $\begin{array}{l}5 \cdot 00 \\
4.80\end{array}$ & $\begin{array}{l}7.20 \\
4.90\end{array}$ & $\begin{array}{l}70.00 \\
56.60\end{array}$ & $\begin{array}{r}30.00 \\
43.40\end{array}$ & $\begin{array}{l}70.00 \\
53.60\end{array}$ & $\begin{array}{l}33.00 \\
33.00\end{array}$ & $\begin{array}{l}14.00 \\
14.00\end{array}$ & $\begin{array}{l}30.00 \\
46.40\end{array}$ & $\begin{array}{r}.00 \\
3.00\end{array}$ & $\begin{array}{l}1450.82 \\
1885.29\end{array}$ & $\begin{array}{l}435.25 \\
874.77\end{array}$ & $\begin{array}{l}745.00 \\
744.50\end{array}$ \\
\hline 659.00 & 72.80 & 24.50 & 4.50 & 4.60 & 57.60 & 42.40 & 54.40 & 111.00 & 13.00 & 45.60 & 3.20 & 1900.81 & 866.77 & 744.00 \\
\hline 659.50 & 72.00 & 24.50 & 4.40 & $4 \cdot 30$ & 59.50 & 40.50 & 55.20 & 111.00 & 13.00 & 44.80 & 4.30 & 1900.81 & 851.56 & 743.50 \\
\hline 650.00 & 76.00 & 24.70 & 4.40 & 4.30 & 59.80 & 40.20 & 55.30 & 113.00 & 13.00 & 14.70 &. .50 & $1916 \cdot 32$ & 856.60 & 743.00 \\
\hline 650.50 & 80.50 & 24.80 & 4.30 & 4.20 & 59.70 & 40.30 & 55.30 & 113.00 & 13.00 & 4.70 &. .40 & 1924.08 & 860.06 & 7.2 .50 \\
\hline 651.00 & 84.00 & 24.70 & $4 \cdot 30$ & 4.20 & 59.90 & 40.10 & 55.60 & 115.00 & 27.00 & 44.40 & 4.30 & 1916.32 & 850.85 & 742.00 \\
\hline 651.50 & 76.90 & $24 \cdot 30$ & 4.90 & 4.00 & 58.50 & 41.50 & 53.00 & 115.00 & 27.00 & 47.00 & 5.50 & $1885 \cdot 29$ & 886.09 & 741.50 \\
\hline 652.00 & $75 \cdot 50$ & 24.00 & 5.00 & $5 \cdot 26$ & $55 \cdot 50$ & 44.50 & 52.90 & 101.00 & 13.00 & 47.10 & 2.60 & 1862.02 & 877.01 & 741.00 \\
\hline 652.50 & 74.30 & 24.50 & 5.10 & $6 \cdot \cup 0$ & 51.50 & 48.50 & 51.50 & 101.00 & 13.00 & 48.50 & $\cdot \mathrm{CO}$ & $1900 \cdot 81$ & 921.89 & 740.50 \\
\hline $653 \cdot 00$ & $73 \cdot 80$ & 24.70 & $5 \cdot 10$ & 6.00 & 50.90 & $49 \cdot 10$ & 50.90 & 114.00 & 8.00 & 49.10 & .00 & $1916 \cdot 32$ & 940.91 & $\begin{array}{l}740.00 \\
739.50\end{array}$ \\
\hline $\begin{array}{l}653.50 \\
634.00\end{array}$ & $\begin{array}{l}73.60 \\
73.20\end{array}$ & 25.00 & $\begin{array}{l}5.20 \\
5.30\end{array}$ & $6 \cdot 4 \mathrm{C}$ & 49.80 & 50.20 & 49.80 & 114.00 & 8.00 & 50.20 & .00 & 1939.60 & 68 & $\begin{array}{l}739.50 \\
739.00\end{array}$ \\
\hline $\begin{array}{l}654.00 \\
654.50\end{array}$ & $\begin{array}{l}73.20 \\
74.00\end{array}$ & $\begin{array}{l}25.40 \\
25.40\end{array}$ & $\begin{array}{l}5 \cdot 30 \\
5 \cdot 30\end{array}$ & $\begin{array}{l}6.60 \\
5.80\end{array}$ & $\begin{array}{l}48 \cdot 60 \\
45 \cdot 60\end{array}$ & $\begin{array}{l}51.40 \\
50.40\end{array}$ & $\begin{array}{l}48.60 \\
48.30\end{array}$ & $\begin{array}{l}129.00 \\
129.00\end{array}$ & $\begin{array}{l}8.00 \\
8.00\end{array}$ & $\begin{array}{l}51 \cdot 40 \\
51.70\end{array}$ & $\begin{array}{r}.00 \\
1.30\end{array}$ & $\begin{array}{l}1970.63 \\
1970.63\end{array}$ & $\begin{array}{l}1012.90 \\
1018.82\end{array}$ & $\begin{array}{l}739.00 \\
738.50\end{array}$ \\
\hline 655.00 & $75 \cdot 30$ & 25. & $5 \cdot 40$ & $4 \cdot 50$ & 56.50 & 43.50 & 48.50 & 127.00 & 15.00 & 51.50 & 8.00 & 1962.87 & 1010.88 & 738.00 \\
\hline 635.50 & 76.90 & $25 \cdot 20$ & 5.40 & $4 \cdot 30$ & $58 \cdot 10$ & 41.90 & 48.60 & 127.00 & 15.00 & $51 \cdot 40$ & 9.50 & 1955.12 & 1004.93 & 737.50 \\
\hline $6.56 \cdot 00$ & 78.70 & 25.20 & $5 \cdot 40$ & .60 & $56 \cdot 30$ & 43.70 & 48.70 & $125 \cdot 00$ & 21.00 & $51 \cdot 30$ & 7.60 & $1955 \cdot 12$ & 1002.97 & 737.00 \\
\hline $6.56 \cdot 50$ & 78.90 & 25.20 & $5 \cdot 30$ & $5 \cdot 10$ & $53 \cdot 20$ & $46 \cdot 80$ & 48.80 & $125 \cdot 00$ & 21.00 & $51 \cdot 20$ & 1.40 & 1955.12 & 1001.02 & 736.50 \\
\hline 657.00 & 78.50 & $25 \cdot ? 0$ & $5 \cdot 30$ & $5 \cdot 40$ & 52.00 & 48.00 & 49.80 & 125.00 & 23.00 & $51 \cdot 20$ & 3.20 & 1955.12 & 1001.02 & 736.00 \\
\hline 6.57 .50 & 78.40 & 25.20 & $5 \cdot 30$ & $5 \cdot 50$ & $E_{1} \cdot 30$ & 48.70 & 48.90 & $125 \cdot 00$ & 23.00 & $51 \cdot 10$ & 2.40 & 1955.12 & 999.06 & 735.50 \\
\hline $658 \cdot 00$ & $78 \cdot 10$ & 25.20 & $5 \cdot 30$ & $5 \cdot 80$ & 49.80 & $50 \cdot 20$ & 48.90 & 125.00 & 17.00 & $5: \cdot: 0$ & .90 & $1955 \cdot 12$ & 999.06 & 735.00 \\
\hline 658. & 77.80 & 25.20 & 5.40 & -1L & $48 \cdot 90$ & $51 \cdot 10$ & 48.70 & $125 \cdot 00$ & 17.00 & $51 \cdot 30$ & .20 & 1955.12 & 1002.97 & 734.50 \\
\hline 609.00 & 77.10 & 25.10 & $5 \cdot 30$ & $6 \cdot 30$ & 49.00 & 51.00 & 49.00 & $123 \cdot 00$ & 14.00 & 51.00 & .00 & $1947 \cdot 36$ & $993 \cdot 15$ & 734.00 \\
\hline 659.50 & 70.10 & 25.00 & $5 \cdot 20$ & .40 & $2 \cdot 10$ & 47.90 & 49.80 & 123.00 & 14.00 & 50.20 & $2 \cdot 30$ & 1939.60 & 973.68 & 733.50 \\
\hline 670.00 & $78 \cdot 90$ & 24.80 & $5 \cdot 10$ & $5 \cdot 60$ & 54.80 & 45.70 & $50 \cdot 60$ & 117.00 & 17.00 & 49.40 & 4.20 & 1924.08 & 950.50 & 733.00 \\
\hline 670.50 & 81.90 & 24.50 & $5 \cdot 10$ & .00 & 57.70 & $42 \cdot 30$ & $51 \cdot 30$ & 117.00 & 17.00 & 48.70 & 6.40 & 1908.57 & 929,47 & 732.50 \\
\hline 671.00 & 85.70 & 23.50 & $5 \cdot 10$ & .00 & 61.60 & 38.40 & $54 \cdot 10$ & 91.00 & 26.00 & 45.90 & 7.50 & 1323.22 & 836.86 & 732.00 \\
\hline $671 \cdot 50$ & 83.10 & 24.10 & $5 \cdot 10$ & 10 & $55 \cdot 80$ & $44 \cdot 20$ & 52.60 & 91.00 & 26.00 & 47.40 & 3.20 & 1869.77 & 886.27 & 731.50 \\
\hline $672 \cdot 00$ & 79.80 & 24.10 & 5.10 & • 3c & $54 \cdot 70$ & $45 \cdot 30$ & 52.60 & $102 \cdot 00$ & 19.00 & 47.40 & $2 \cdot 10$ & 1869.77 & 886.27 & 731.00 \\
\hline $672 \cdot 50$ & $78 \cdot 60$ & 24.00 & 5.10 & 4.90 & 57.30 & 42.70 & $52 \cdot 30$ & $102 \cdot 00$ & 19.00 & 47.7 .0 & 5.00 & 1362.02 & 888.18 & 730.50 \\
\hline $673 \cdot c 0$ & 77.60 & 24.10 & $5 \cdot 10$ & $5 \cdot 10$ & 56.00 & $44 \cdot n 0$ & 52.10 & $103 \cdot 00$ & 13.00 & 47.92 & 3.90 & 1869.77 & 895.62 & 730.00 \\
\hline 673.50 & 77.10 & 24.70 & 5.00 & $5 \cdot 50$ & $\leq 2.60$ & 47.40 & 51.20 & 103.00 & 13.00 & 48.80 & $1 \cdot 40$ & $1916 \cdot 32$ & 935.17 & 729.50 \\
\hline 674.00 & 77.10 & 25.80 & 4.80 & $5 \cdot 50$ & 50.10 & 49.90 & 50.10 & 138.00 & 11.00 & 49.90 & .00 & 2001.67 & 998.83 & 729.00 \\
\hline 674.50 & $78 \cdot 30$ & 26.90 & $4 . \leqslant 0$ & $5 \cdot 30$ & $49 \cdot 20$ & $50 \cdot 30$ & 49.20 & $13 R \cdot 00$ & 11.00 & 50.90 & .00 & 2087.01 & $1060 \cdot 20$ & 728.50 \\
\hline $675 \cdot 00$ & 83.00 & 27.10 & $4 \cdot 30$ & 4.90 & 50.40 & 49.60 & 50.40 & 171.00 & 20.00 & 49.60 & .00 & 2102.53 & 1042.85 & 728.00 \\
\hline 675.50 & 89.00 & 22.70 & $3 \cdot 90$ & 4.50 & $t 3.40$ & 35.60 & 33.40 & $: 71.00$ & 20.00 & 36.60 & .00 & $1761 \cdot 16$ & 644.58 & 727.50 \\
\hline $6^{2} 6.00$ & $92 \cdot 90$ & 17.30 & 3.90 & 4.40 & 86.10 & 13.90 & 86.10 & 24.00 & 43.00 & 13.90 & .00 & $1342 \cdot 20$ & 186.57 & 727.00 \\
\hline$\therefore$ & $\begin{array}{l}78 \cdot 37 \\
78.25\end{array}$ & 6 & .35 & 120 & & & 95 & & & 47.05 & $2 \cdot 85$ & & & \\
\hline & & & & & & & & & & & & & & \\
\hline
\end{tabular}

*NOTATID:S-Dept'́n, ft; GR-Gamma Ray Index, API Units; POR-Porosity, $\%$; RT-True Resistivity, Ohms-m²/m; Rxo-Flushed Zone Resistivj.ty, Ohms-m²/m; SXO-Flushed Zone Saturation, \%; ROS-Residual 0 il Saturation, \%; SW-Water Saturation, $z$; PER-Permeability Index; Clay-Clay Content, $\%$; SO-0il

Saturation, \%; M0-Movable 0il, \%; PHV-Pore Volume, bbl/ac-ft; HPV-Hydrocarbon Pore Volume, bbl/ac-ft; ELSE-Elevation From Sea Level, ft 
TABLE C65

WELL LOG DETERMINED PROPERTIES

VS DEPTH FOR WELL MP-225

WELL LOCATION: 1056 FNL 2112 FEL 28 25S $5 E$

KELLY BUSHING ELEVATION FROM SEA LEVEL $=1401$

\begin{tabular}{|c|c|c|c|c|c|c|c|c|c|c|c|c|c|c|}
\hline DEPTH * & GR & PAR & RT & $R \times E$ & $s \times \theta$ & ROS & SW & PER & CLAY & so & ro & PHV & HPV & ELSE \\
\hline $\begin{array}{l}654.00 \\
655.00\end{array}$ & $\begin{array}{l}88.80 \\
85.20\end{array}$ & 16.70 & $\begin{array}{l}3.90 \\
3.90\end{array}$ & $\begin{array}{l}7.80 \\
5.20\end{array}$ & $\begin{array}{l}88.40 \\
72.60\end{array}$ & $\begin{array}{l}11.60 \\
27.40\end{array}$ & $\begin{array}{l}88.40 \\
67.90\end{array}$ & $\begin{array}{l}20.00 \\
61.00\end{array}$ & $\begin{array}{l}12.00 \\
10.00\end{array}$ & $\begin{array}{l}11.60 \\
32.10\end{array}$ & $\begin{array}{r}.00 \\
. .70\end{array}$ & $\begin{array}{l}1295.65 \\
1660.30\end{array}$ & $\begin{array}{l}150 \cdot 30 \\
532 \cdot 96\end{array}$ & $\begin{array}{l}747.00 \\
746.00\end{array}$ \\
\hline 656.00 & 92.40 & 22.70 & 3.60 & 2.70 & 66.50 & 33.50 & 66.50 & 79.00 & 6.00 & 33.50 & .00 & 1761.16 & 589.99 & $\begin{array}{l}746.00 \\
745.00\end{array}$ \\
\hline 657.00 & $91 \cdot 50$ & 22.80 & $3 \cdot 30$ & 2.80 & $\in 9 \cdot 30$ & 30.70 & 69.30 & 80.00 & 8.00 & 30.70 & .00 & 1768.91 & 543.06 & 744.0 \\
\hline 658.00 & 87.80 & 22.80 & 3.00 & 2.70 & 72.60 & 27.40 & 72.60 & 81.00 & 6.00 & 27.40 & .00 & 1768.91 & 484.68 & 743.0 \\
\hline 659.00 & 89.10 & 22.40 & 3.00 & 3.10 & 74.20 & 25.80 & 74.20 & 75.00 & 5.00 & 25.80 & .00 & 1737.88 & 448.37 & 742.0 \\
\hline $\begin{array}{l}660.00 \\
661.00\end{array}$ & $\begin{array}{l}89 \cdot 10 \\
95.30\end{array}$ & $\begin{array}{l}23.26 \\
23.70\end{array}$ & $\begin{array}{l}3.00 \\
3.50\end{array}$ & $\begin{array}{l}2 \cdot 30 \\
2.50\end{array}$ & $\begin{array}{l}71.60 \\
\epsilon 4.60\end{array}$ & $\begin{array}{l}28.40 \\
35.40\end{array}$ & $\begin{array}{l}7.1 .60 \\
64.60\end{array}$ & $\begin{array}{l}86.00 \\
96.00\end{array}$ & $\begin{array}{r}6.00 \\
13.00\end{array}$ & $\begin{array}{l}28.40 \\
35.40\end{array}$ & $\begin{array}{l}.00 \\
.00\end{array}$ & $\begin{array}{l}1799.95 \\
1838.74\end{array}$ & $\begin{array}{l}511.19 \\
650.91\end{array}$ & $\begin{array}{l}741.00 \\
740.00\end{array}$ \\
\hline 662.00 & 103.30 & 23.80 & 3.90 & 3.20 & 61.20 & 38.80 & 61.20 & 96.00 & 12.00 & 38.90 & .00 & $1846 \cdot 50$ & 716.44 & $\begin{array}{l}739.00 \\
738.00\end{array}$ \\
\hline $\begin{array}{l}663.00 \\
664.00\end{array}$ & 97.40 & $\begin{array}{l}23.50 \\
22.60\end{array}$ & 3.80 & 4000 & 74.70 & $25 \cdot 30$ & 61.90 & 92.00 & 7.00 & $38 \cdot 10$ & 12.80 & $\begin{array}{l}1823.22 \\
1753.40\end{array}$ & $\begin{array}{l}694.65 \\
597.91\end{array}$ & $\begin{array}{l}738.00 \\
737.00\end{array}$ \\
\hline $\begin{array}{l}664.00 \\
665.00\end{array}$ & $\begin{array}{l}91.40 \\
91.40\end{array}$ & $\begin{array}{l}22.60 \\
20.20\end{array}$ & $\begin{array}{l}3 \cdot 70 \\
3.50\end{array}$ & $\begin{array}{l}3.90 \\
4.90\end{array}$ & $\begin{array}{l}79 \cdot 40 \\
79.50\end{array}$ & $\begin{array}{l}20.60 \\
20.50\end{array}$ & $\begin{array}{l}65.90 \\
76.10\end{array}$ & $\begin{array}{l}78.00 \\
47.00\end{array}$ & $\begin{array}{l}4.00 \\
6.00\end{array}$ & $\begin{array}{l}34.10 \\
23.90\end{array}$ & $\begin{array}{r}13.50 \\
3.40\end{array}$ & $\begin{array}{l}1753.40 \\
1567.20\end{array}$ & $\begin{array}{l}597.91 \\
374.56\end{array}$ & $\begin{array}{l}737.00 \\
736.00\end{array}$ \\
\hline 666.00 & 90.80 & 22.40 & 3.30 & 4.30 & 76.10 & 23.90 & 70.70 & 74.00 & 6.00 & 29.30 & 5.40 & 1737.88 & 509.20 & 735.00 \\
\hline $\begin{array}{l}667.00 \\
668.00\end{array}$ & $\begin{array}{l}95.80 \\
95.00\end{array}$ & 23.50 & $\begin{array}{l}3.10 \\
2.90\end{array}$ & $\begin{array}{l}3.10 \\
3.30\end{array}$ & $\begin{array}{l}69.50 \\
68.50\end{array}$ & $\begin{array}{l}30.50 \\
31.50\end{array}$ & $\begin{array}{l}69.50 \\
68.50\end{array}$ & $\begin{array}{r}92.00 \\
105.00\end{array}$ & $\begin{array}{l}4.00 \\
5.00\end{array}$ & 30.50 & $\begin{array}{l}.00 \\
.00\end{array}$ & $\begin{array}{l}1823.22 \\
1885.29\end{array}$ & $\begin{array}{l}556.08 \\
593.87\end{array}$ & $\begin{array}{l}734.00 \\
733.00\end{array}$ \\
\hline 669.00 & 93.50 & 24.60 & 2.90 & 2.90 & $\epsilon 7.60$ & 32.40 & 67.60 & 113.00 & 7.00 & 32.40 & .00 & 1908.57 & 618.38 & $\begin{array}{l}733000 \\
732.00\end{array}$ \\
\hline 670.00 & 92.70 & 25.30 & 2.90 & 2.80 & $\in 5.90$ & $34 \cdot 10$ & 65.90 & 128.00 & 8.00 & $34 \cdot 10$ & .00 & 1962.87 & 669.34 & 731.0 \\
\hline 671.00 & 97.00 & 23.40 & 2.90 & 3.20 & 71.80 & 23.20 & 71.80 & $90 \cdot 00$ & 13.00 & 28.20 & 5.00 & 1815.46 & 511.96 & 730.00 \\
\hline 672.00 & 103.50 & 20.40 & 2.90 & 2.70 & 83.20 & $16 \cdot 80$ & 83.20 & 49.00 & 23.00 & 16.80 & .00 & 1582.71 & 265.90 & 729. \\
\hline 673.00 & 107.30 & $19 \cdot 30$ & $2 \cdot 80$ & 2.90 & 89.40 & $10 \cdot 60$ & 89.40 & 39.00 & 27.00 & 10.60 & .00 & 1497.37 & 158.72 & 728.0 \\
\hline 674.00 & 107.60 & 18.70 & 2.70 & 2.70 & 93.80 & $6 \cdot 20$ & 93.80 & 34.00 & 27.00 & 6.20 & .00 & 1450.82 & 89.95 & 27. \\
\hline A.AVE. & 94.57 & 22.27 & 3.26 & $\begin{array}{l}3.48 \\
3.32\end{array}$ & $\begin{array}{l}74 \cdot 30 \\
73.85\end{array}$ & $\begin{array}{l}25.46 \\
23.51\end{array}$ & $72 \cdot 41$ & $\begin{array}{l}76.90 \\
71.02\end{array}$ & $\begin{array}{r}10.24 \\
8.58\end{array}$ & $\begin{array}{l}27.59 \\
25.40\end{array}$ & $2 \cdot 13$ & $\begin{array}{l}1727.90 \\
1719.79\end{array}$ & $\begin{array}{l}488.97 \\
436.84\end{array}$ & \\
\hline
\end{tabular}

*NOTATIDH--Depth, ft; GR-Gamma Ray Index, API Units; POR-Porosity, $\%$; RT-True Resistivity, Ohms-m²/m; RXO-Flushed Zone Resistivity, Ohms-m²/m; SX0-Flushed Zone Saturation. \%; ROS-Residual 0il Saturation, \%; SW-Water Saturation, \%; PER-Permeability Index; Clay-Clay Content, \%; SO-0il

Saturation, \%; MO-Movable 0il, \%; PHV-Pore Volume, bbl/ac-ft; HPV-Hydrocarbon Pore Volume, bbl/ac-ft; ELSE-Elevation From Sea Level, ft 
FIGURE C-1

DUNL INDUCTION FOCUSED LOG FOR UELL IMP-208

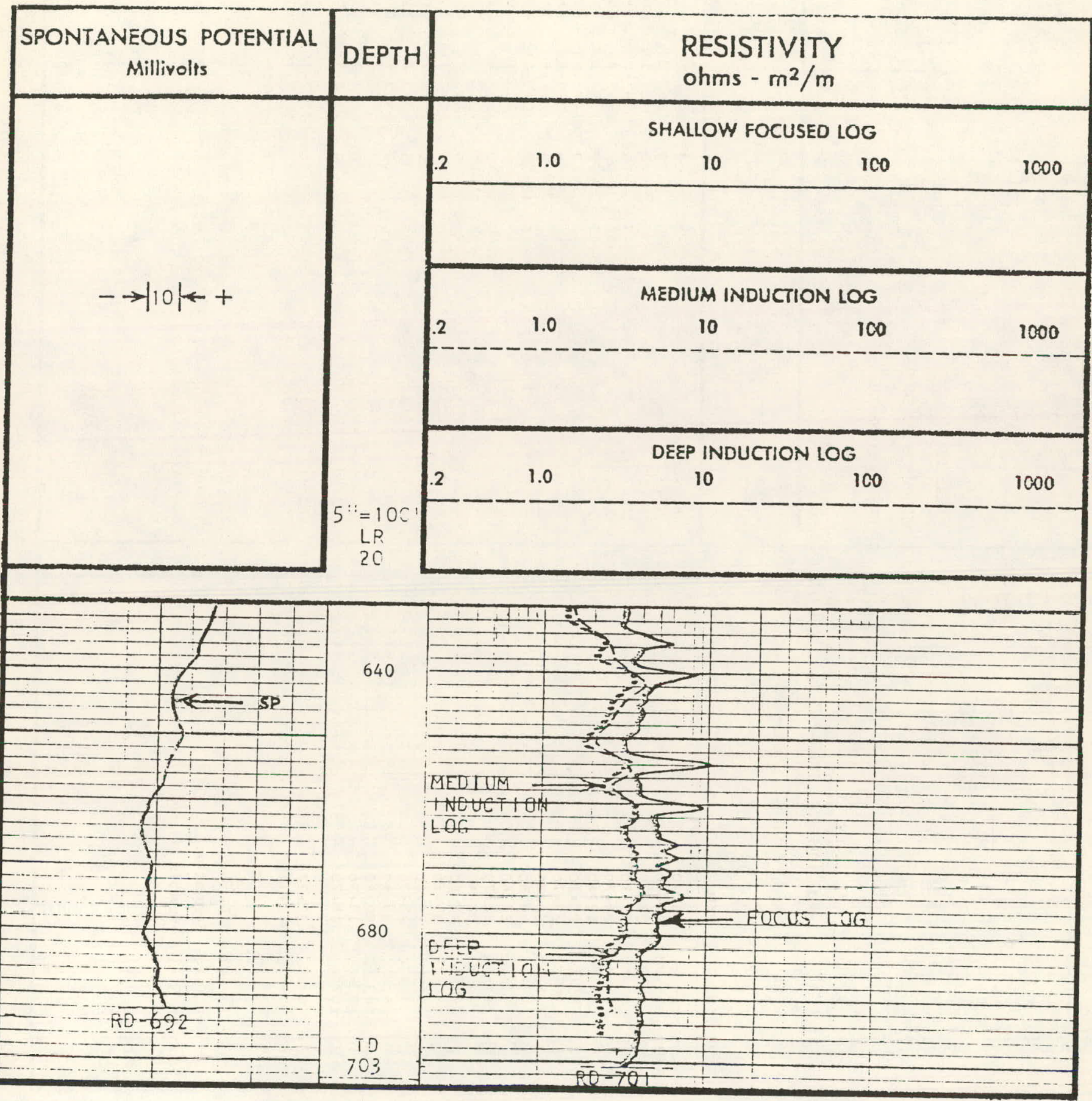


II-176

FIGURE $\mathrm{C}-2$

FROXIMITY LOG-IIIIILOG HITH CALIPER

FOR WELL IIP-2NO

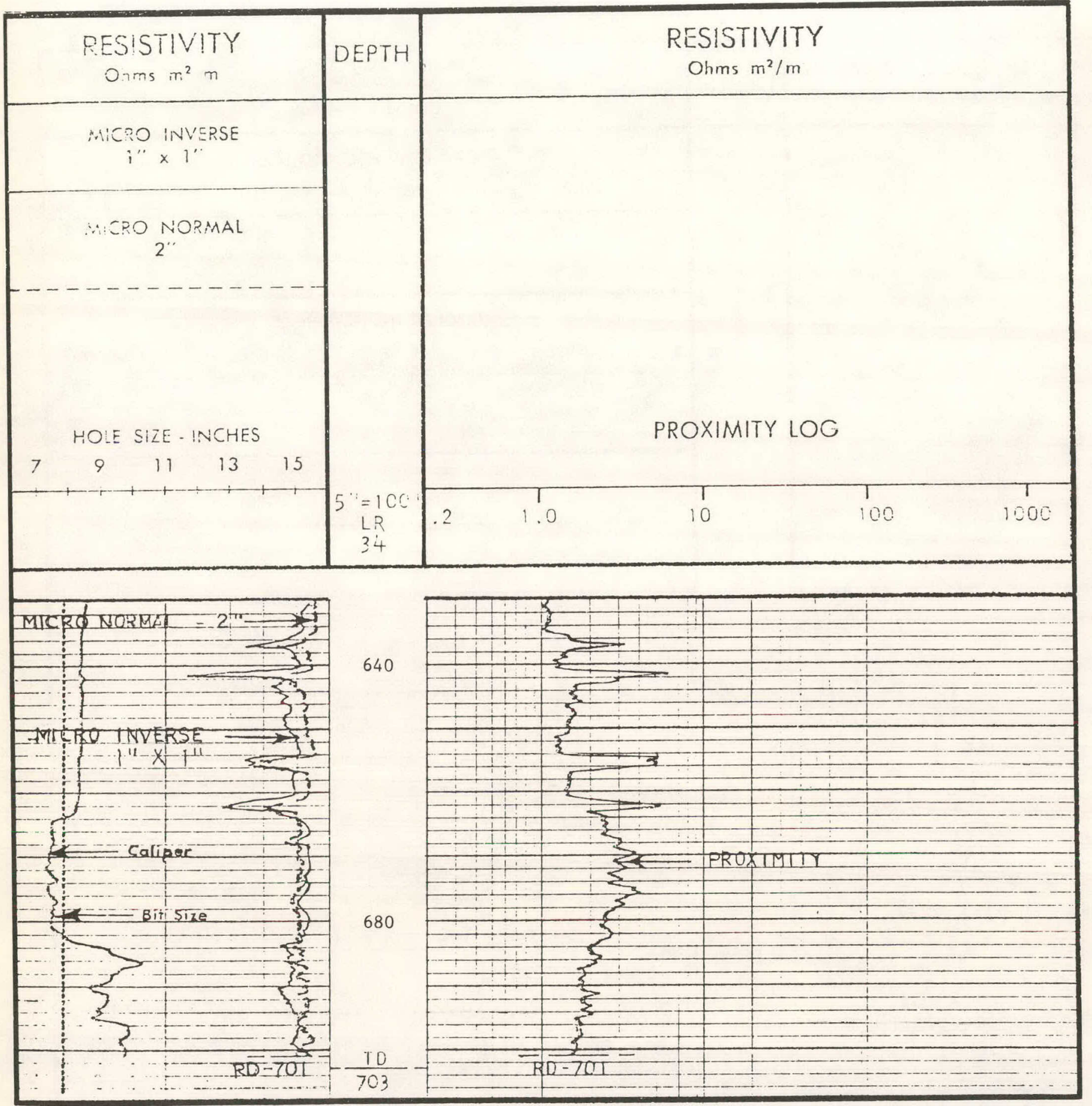


FIGURE C-3

COIPENSATED DENSILOG FOR WELL MP-208

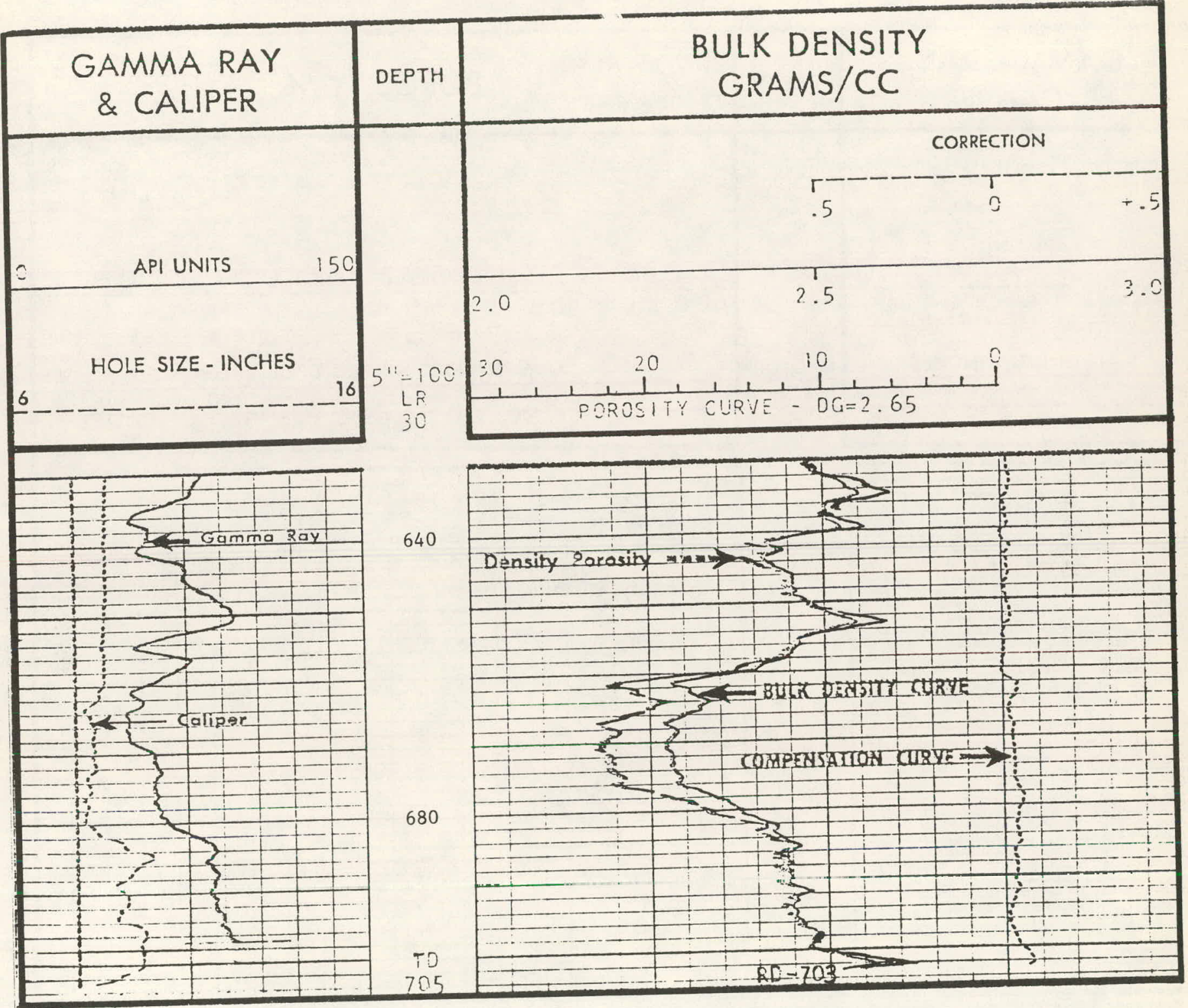


II-178

FIGURE C-4

COMPENSATED NEUTRON LOG FOR HELL IMP-203

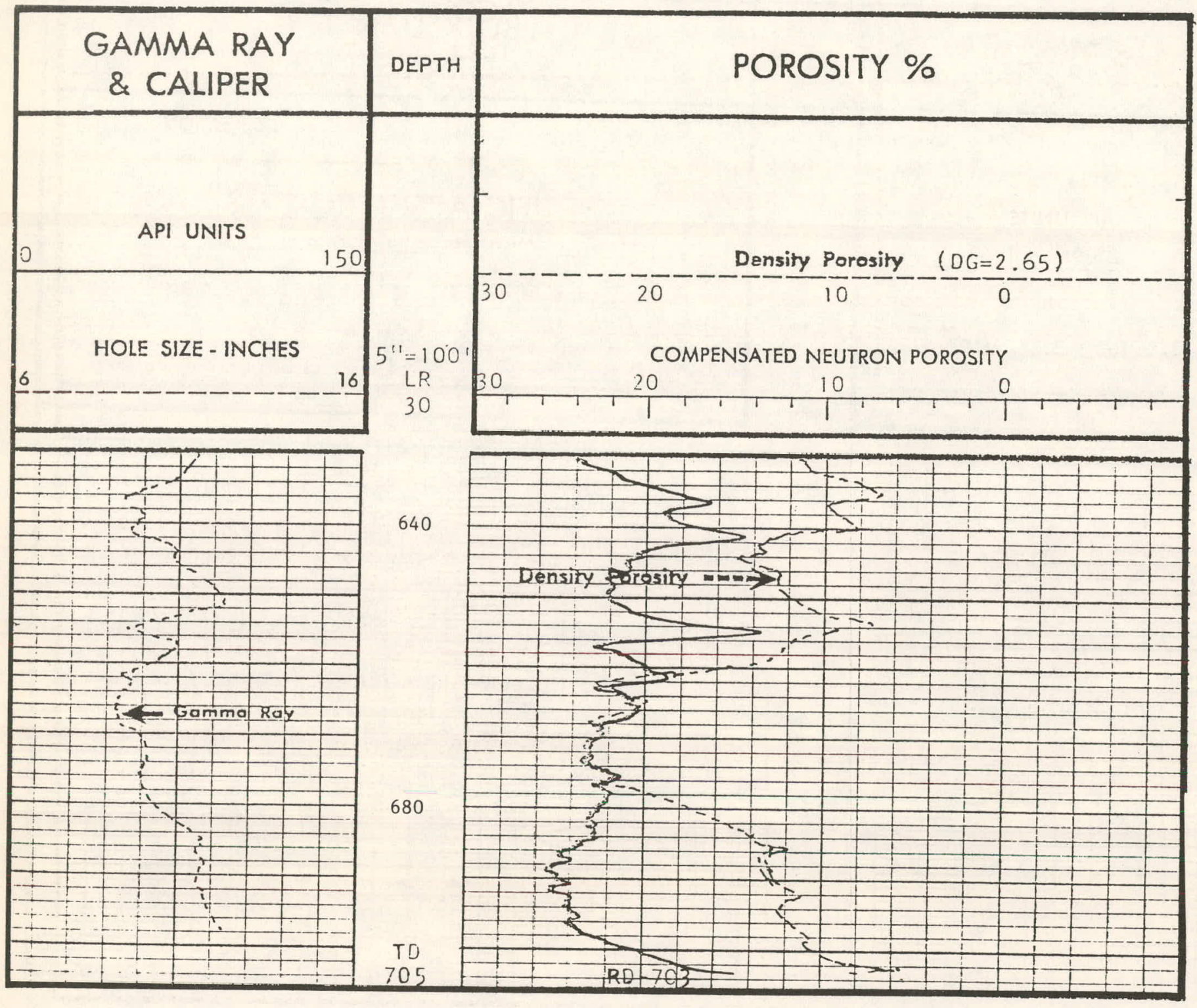


FIGURE $\mathrm{C}-5$

COMPENSATED ACOUSTILOG FOR WELL IIP-208

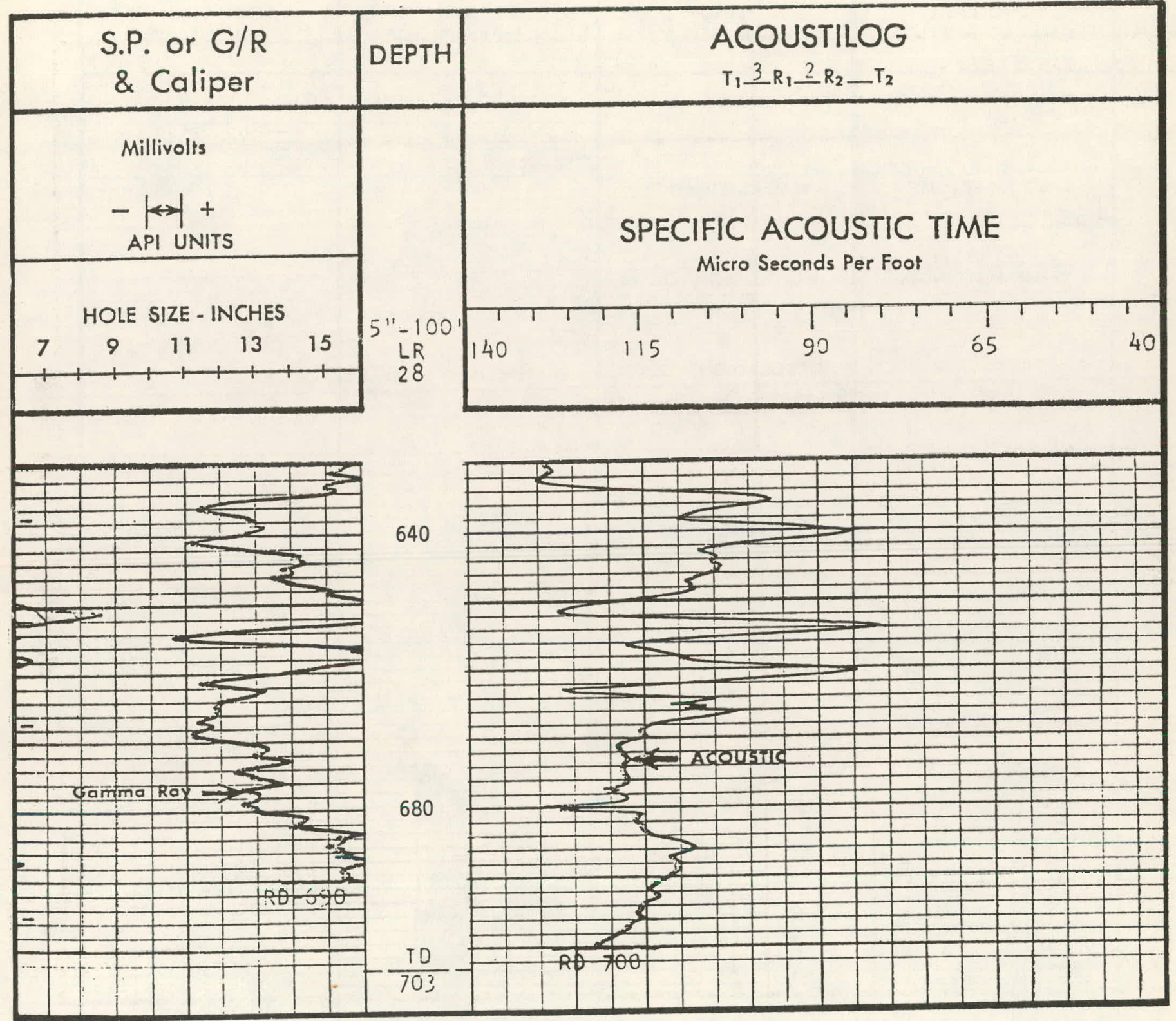


FIGURE C-6

EXAIIPLE EPILOG

(FOR WELL MP-208)

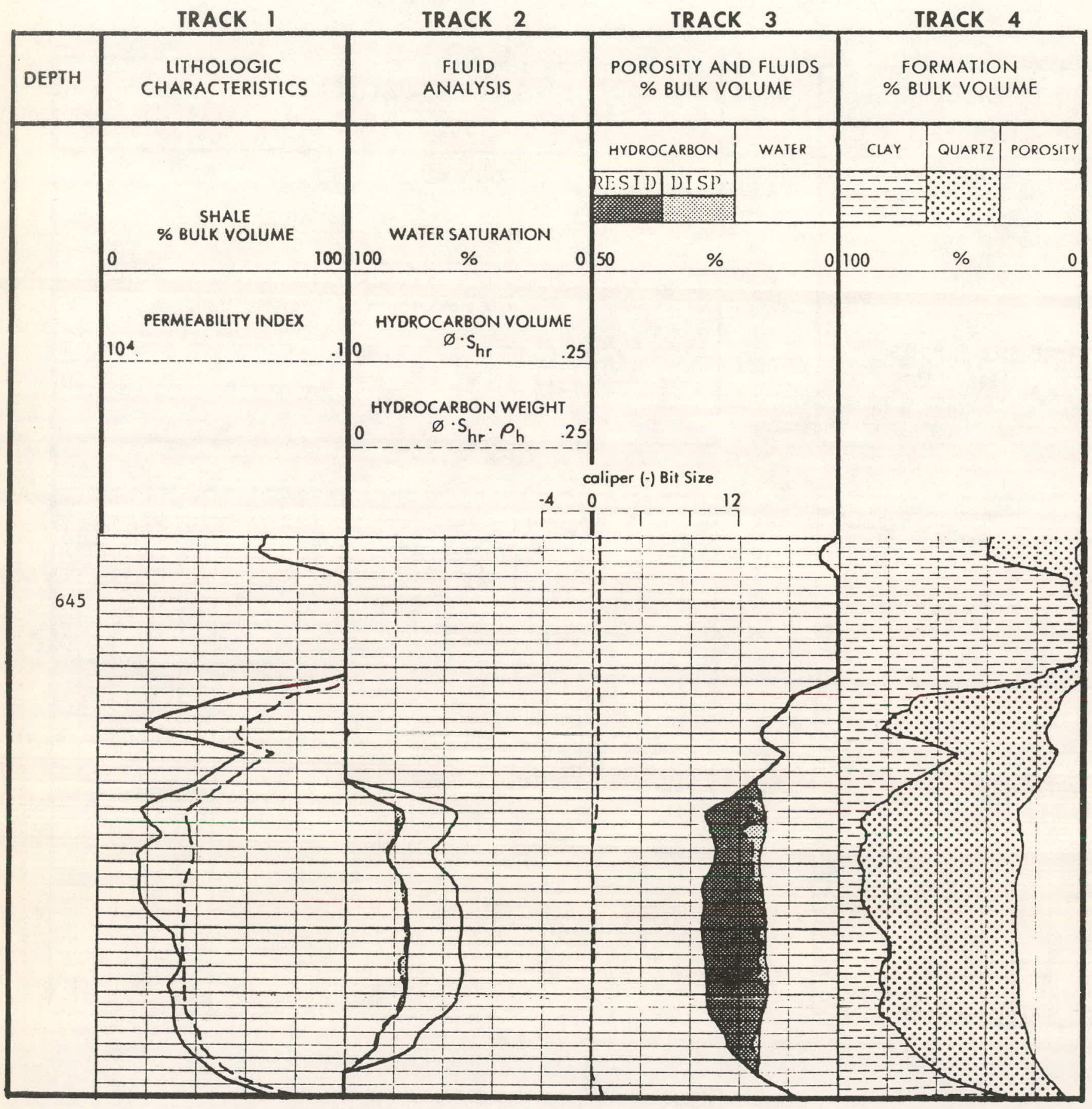


FIGURE $\quad$ - -7

EPILOG FOR WELL MP-101

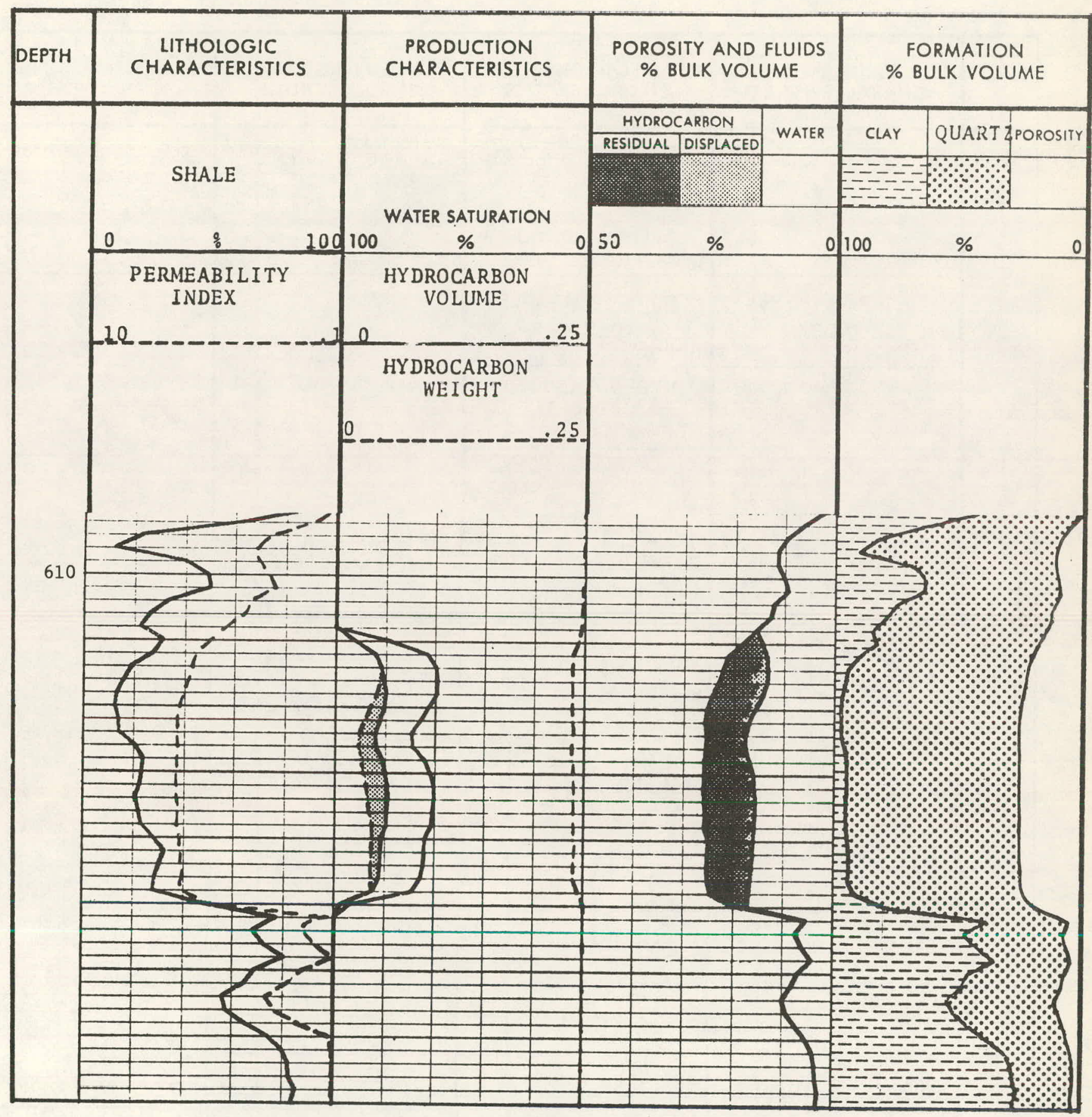


II-182

FIGURE C-8

EPILOG FDR WELL MP-102

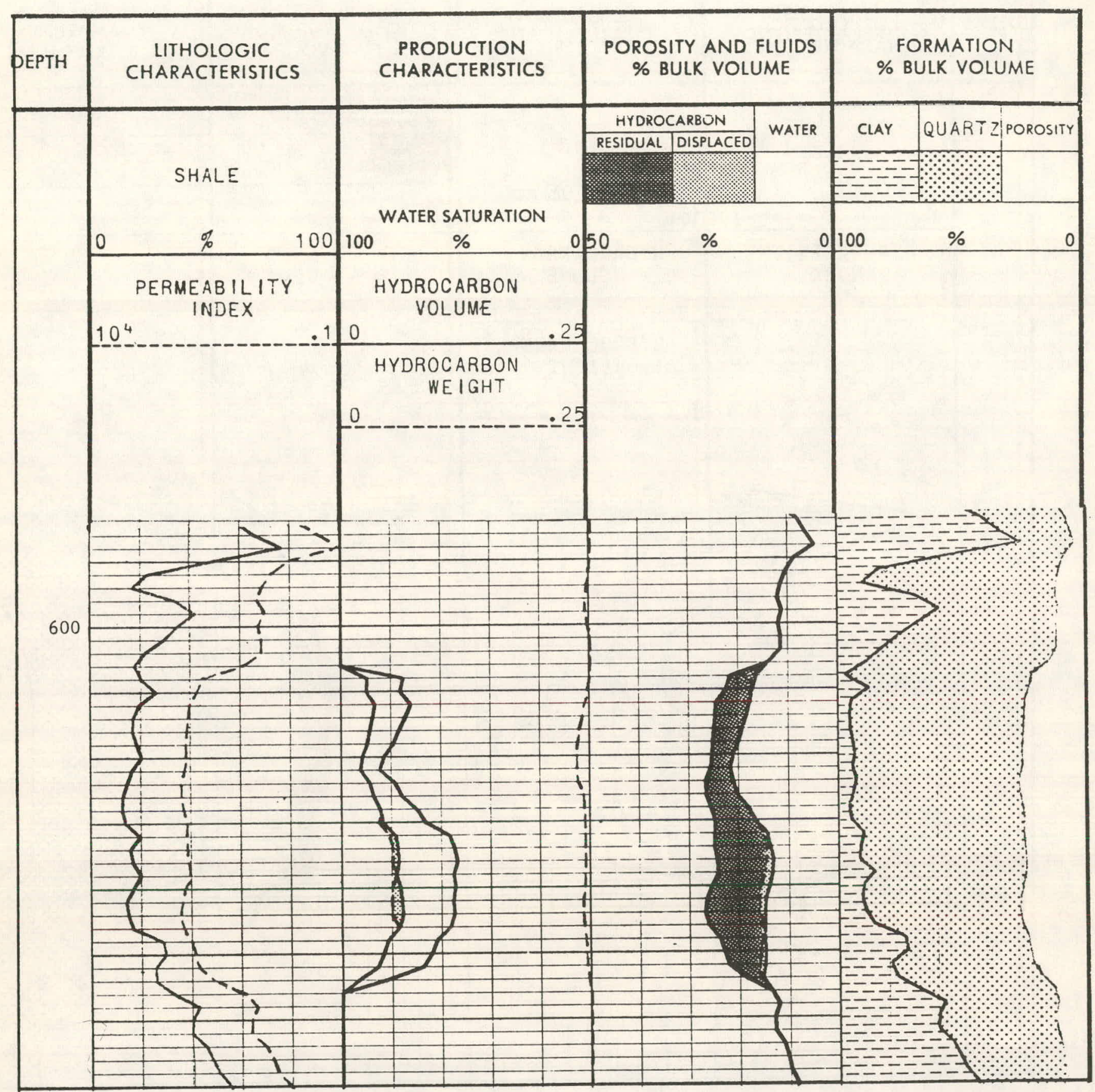


FIGURE C-9

EPILOG FOR IJELL IIP-103

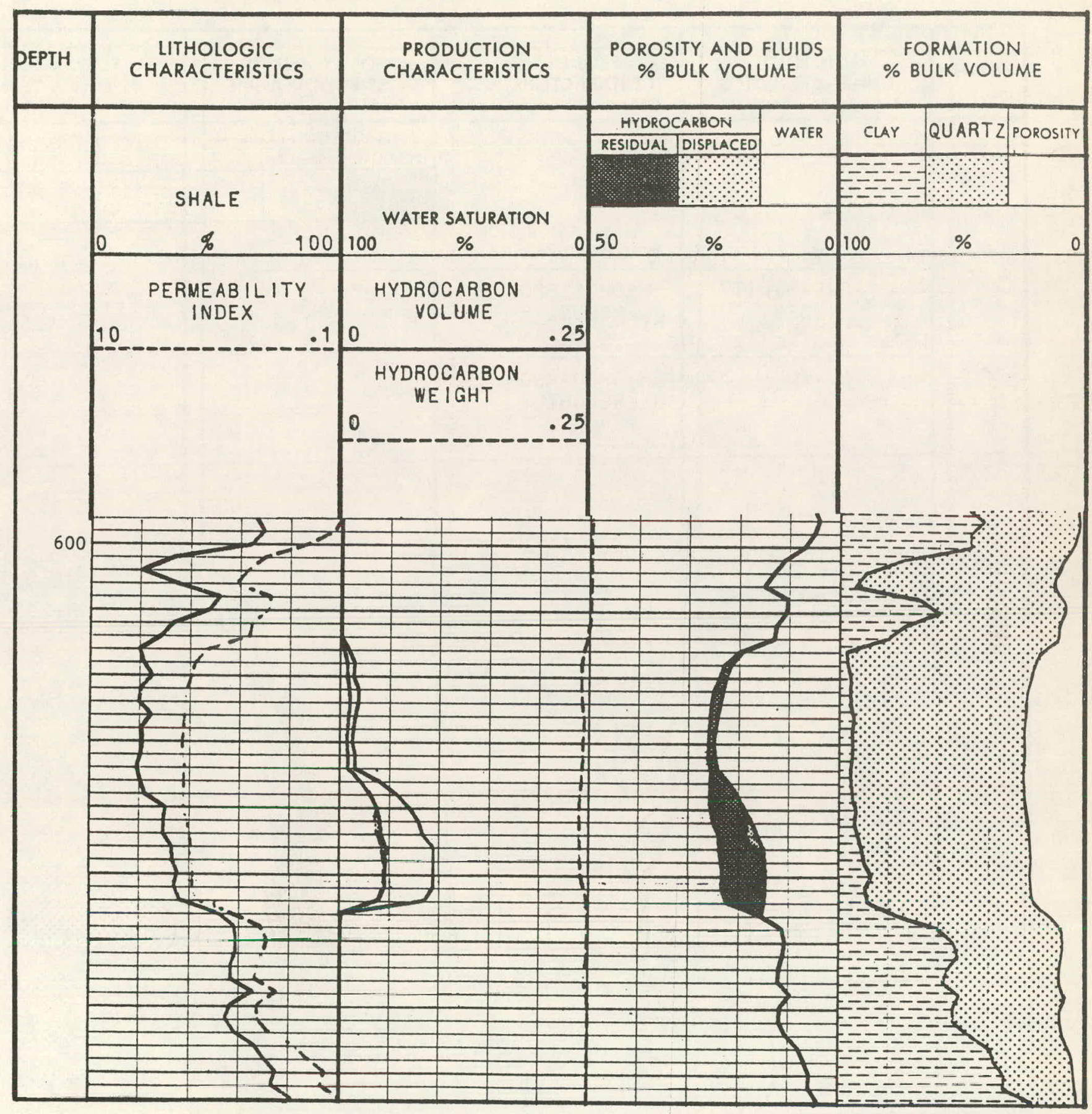


II - 184

FIGURE C-10

EPILOG FOR WELL [1P-104

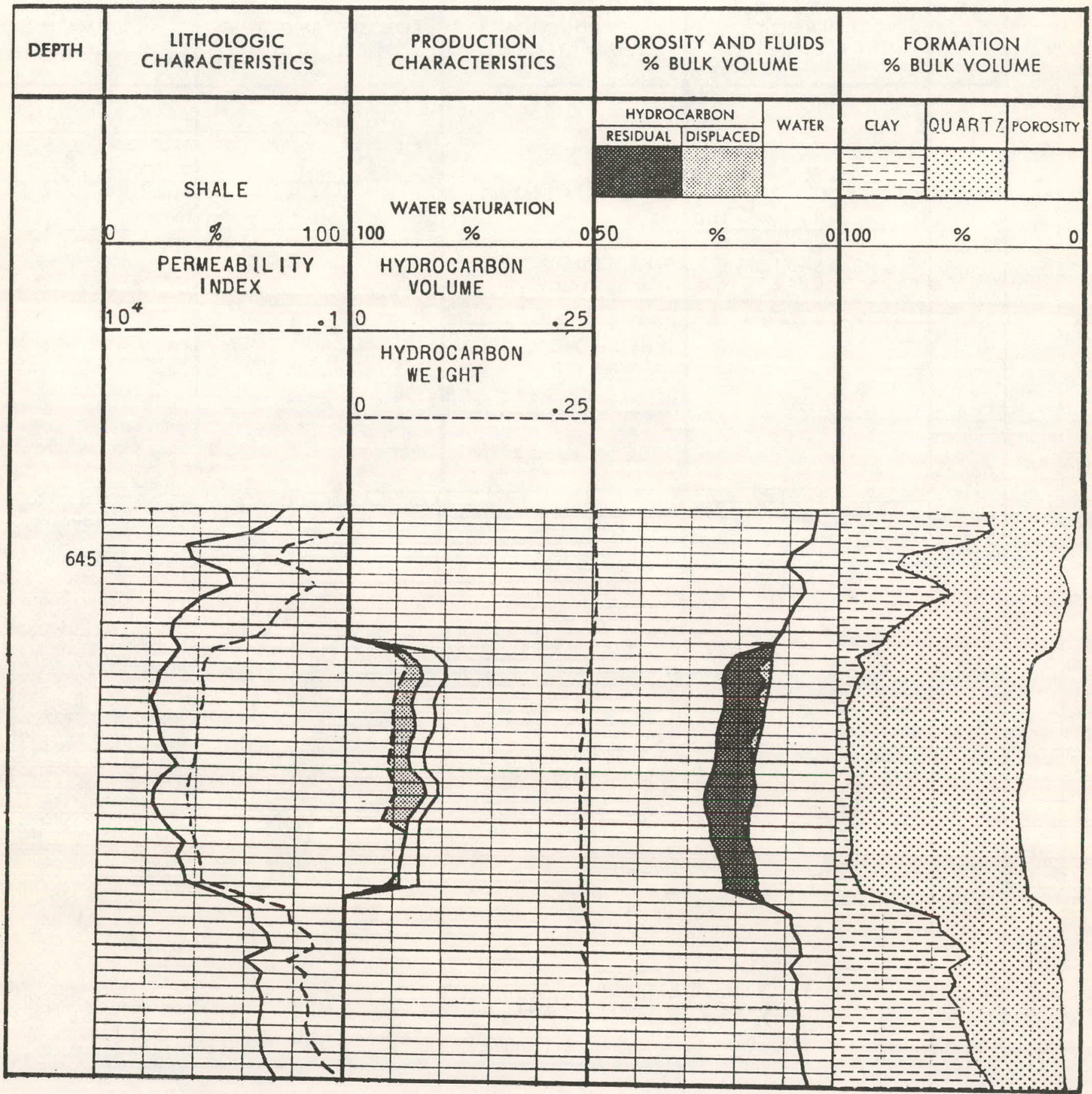


FIGURE C-11

EPILOG FOR HELL MP-105

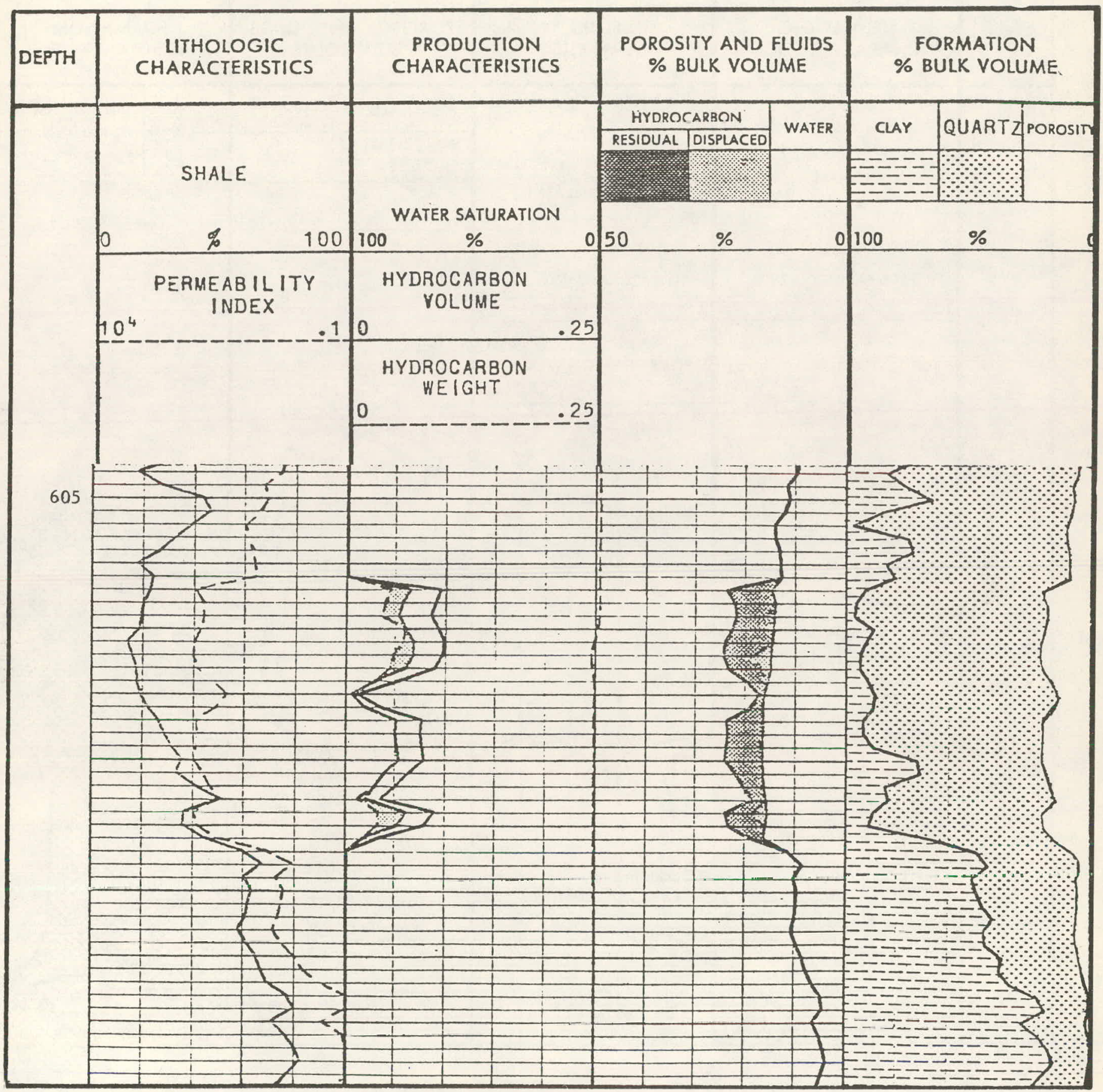


II-186

FIGURE C-12

EPILOG FOR WELL IIP-106

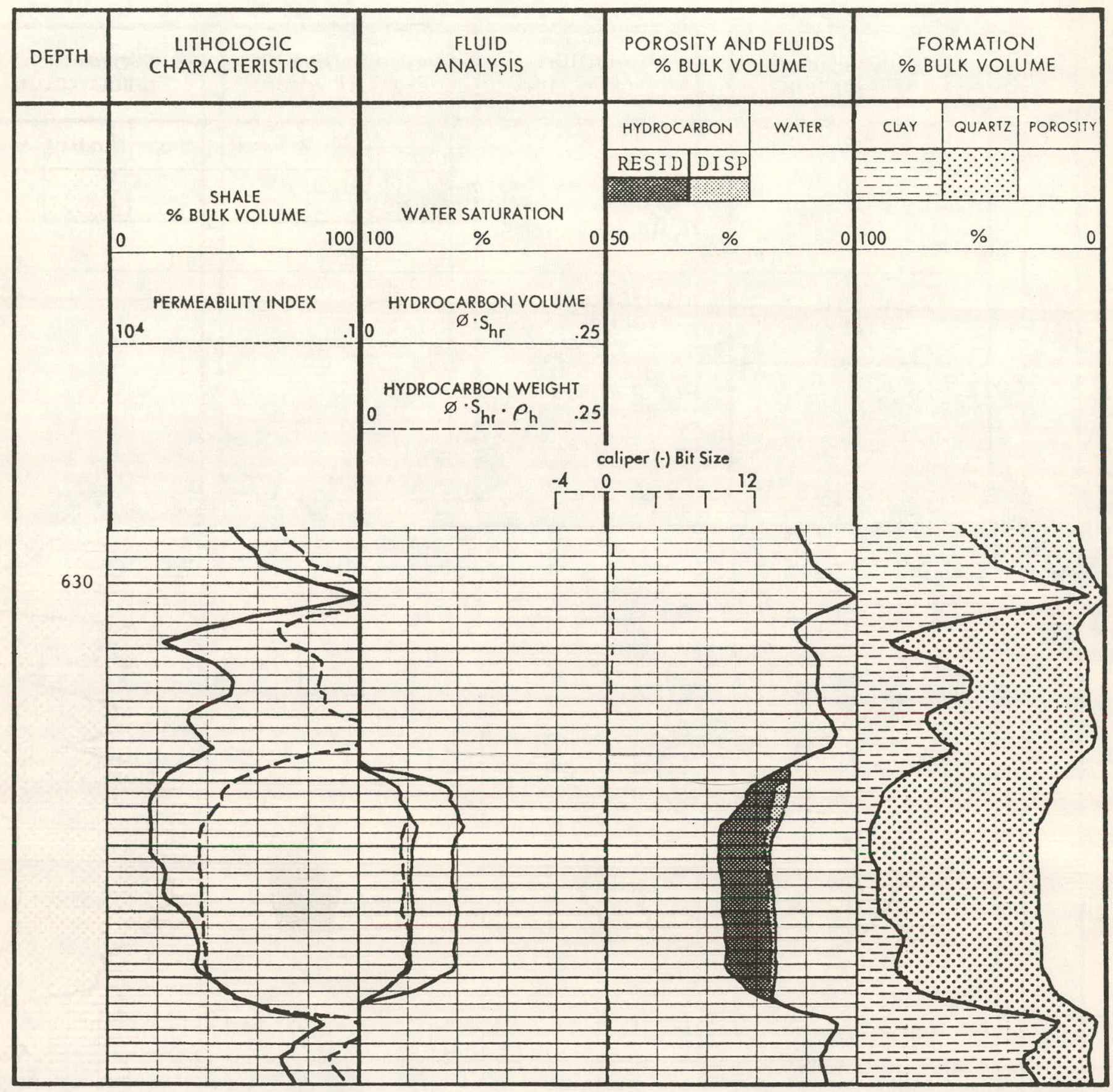


FIGURE C-13

EPILOG FOR WELL IIP-107

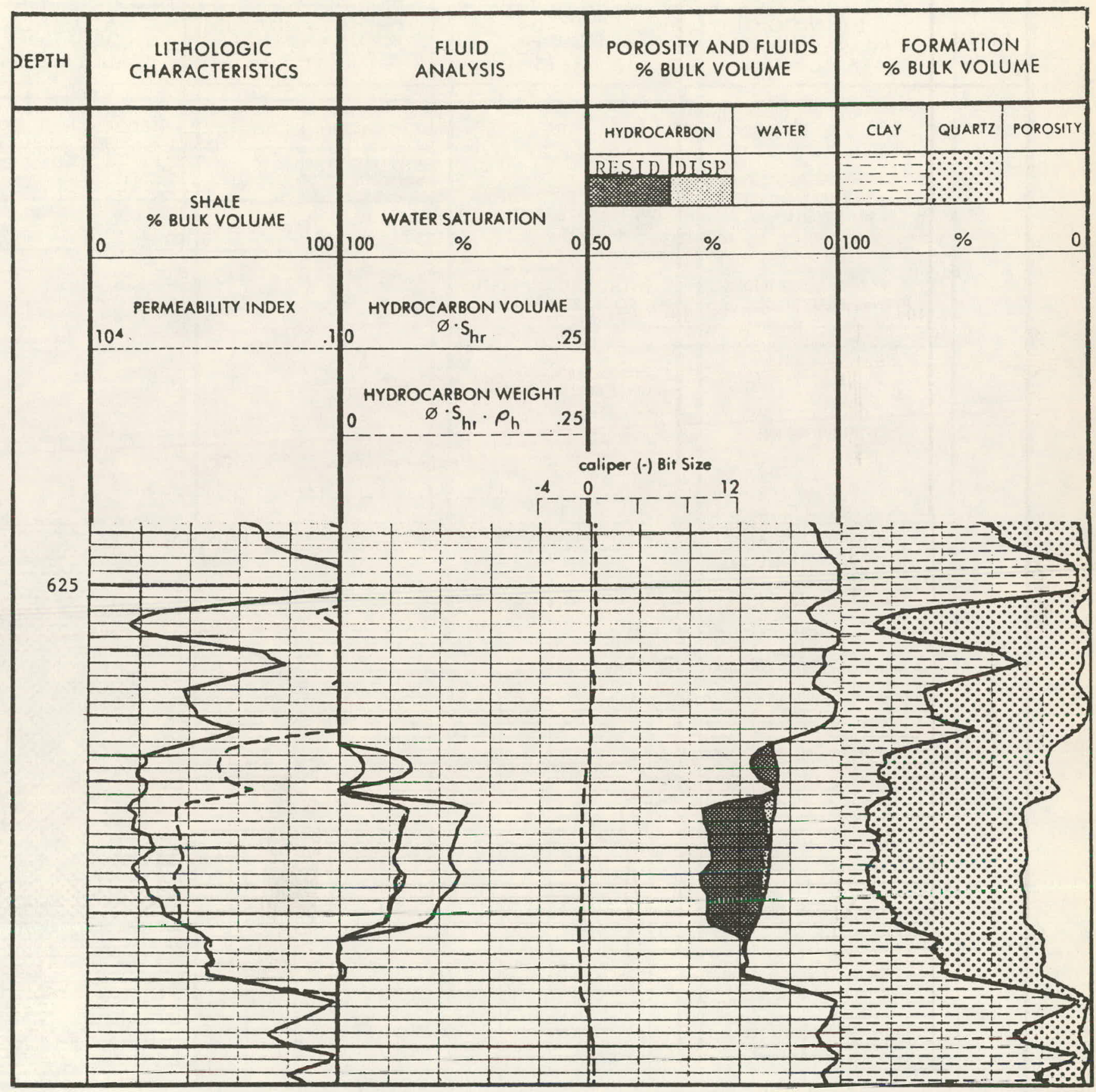


FIGURE C-14

EPILOG FOR WELL IIP-108

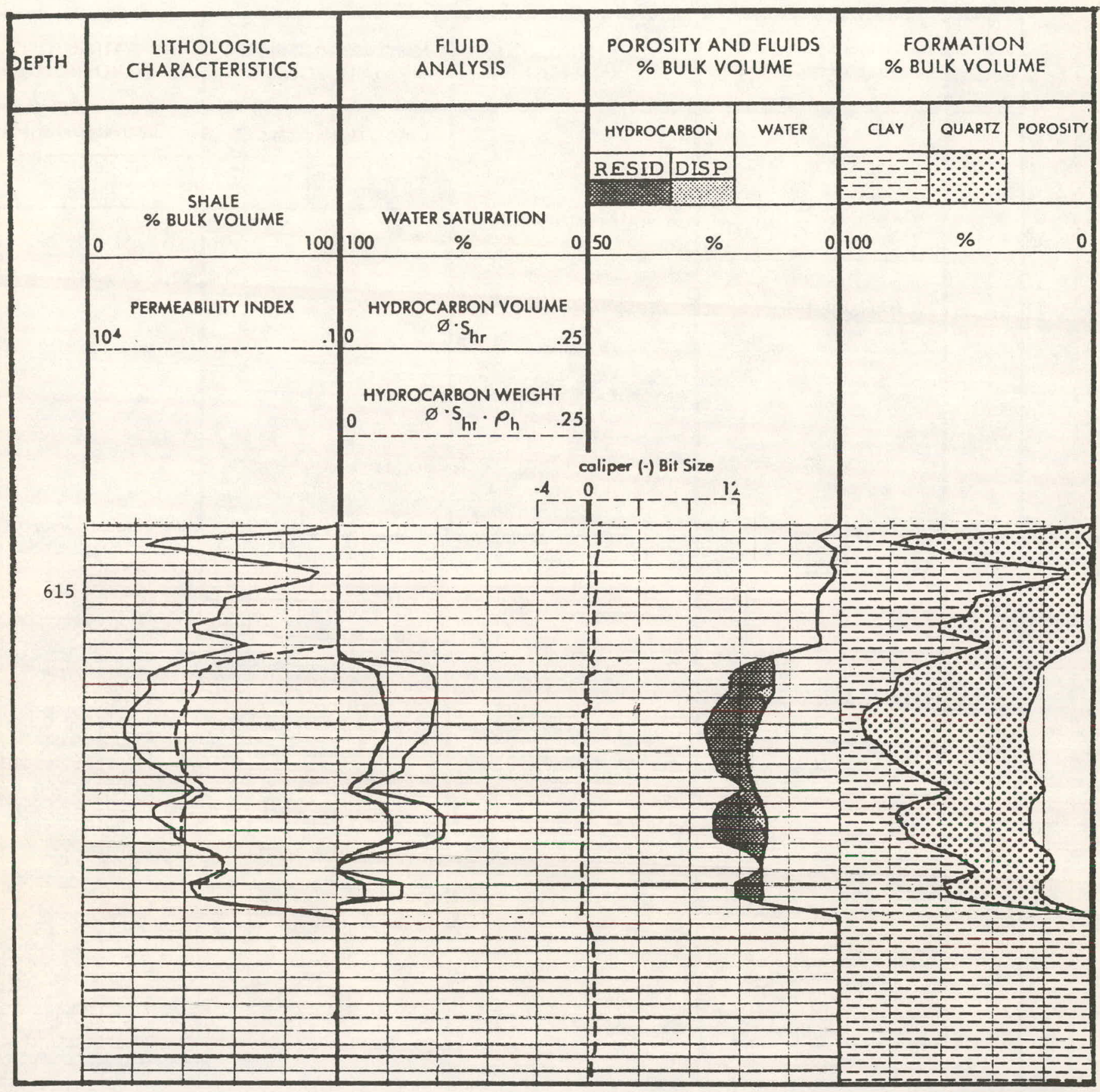


FIGURE C-15

EPILOG FOR WELL IIP-109

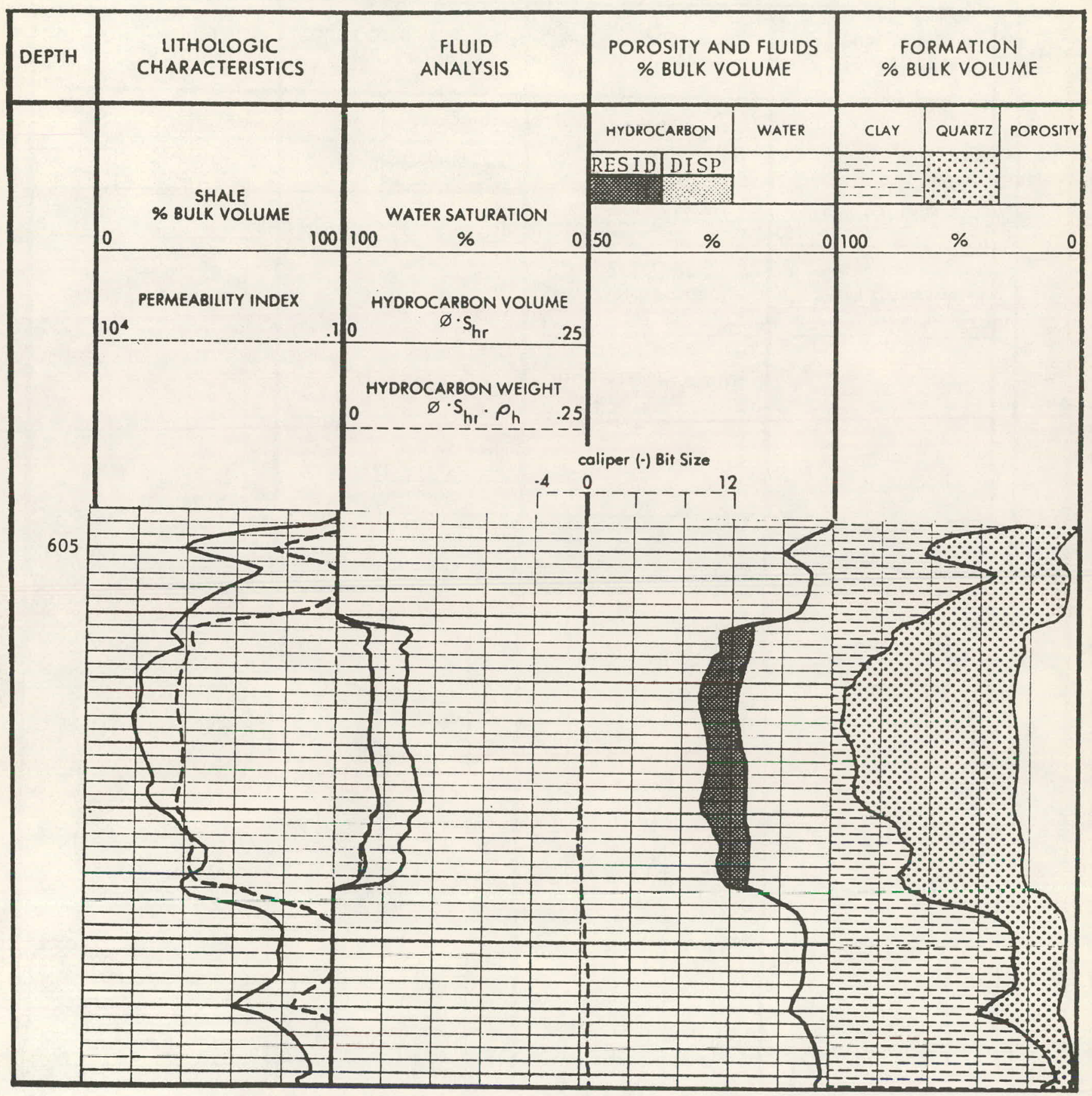


II-190

FIGURE C $-\cdots 16$

EPILOG FOR WELL IIP-110

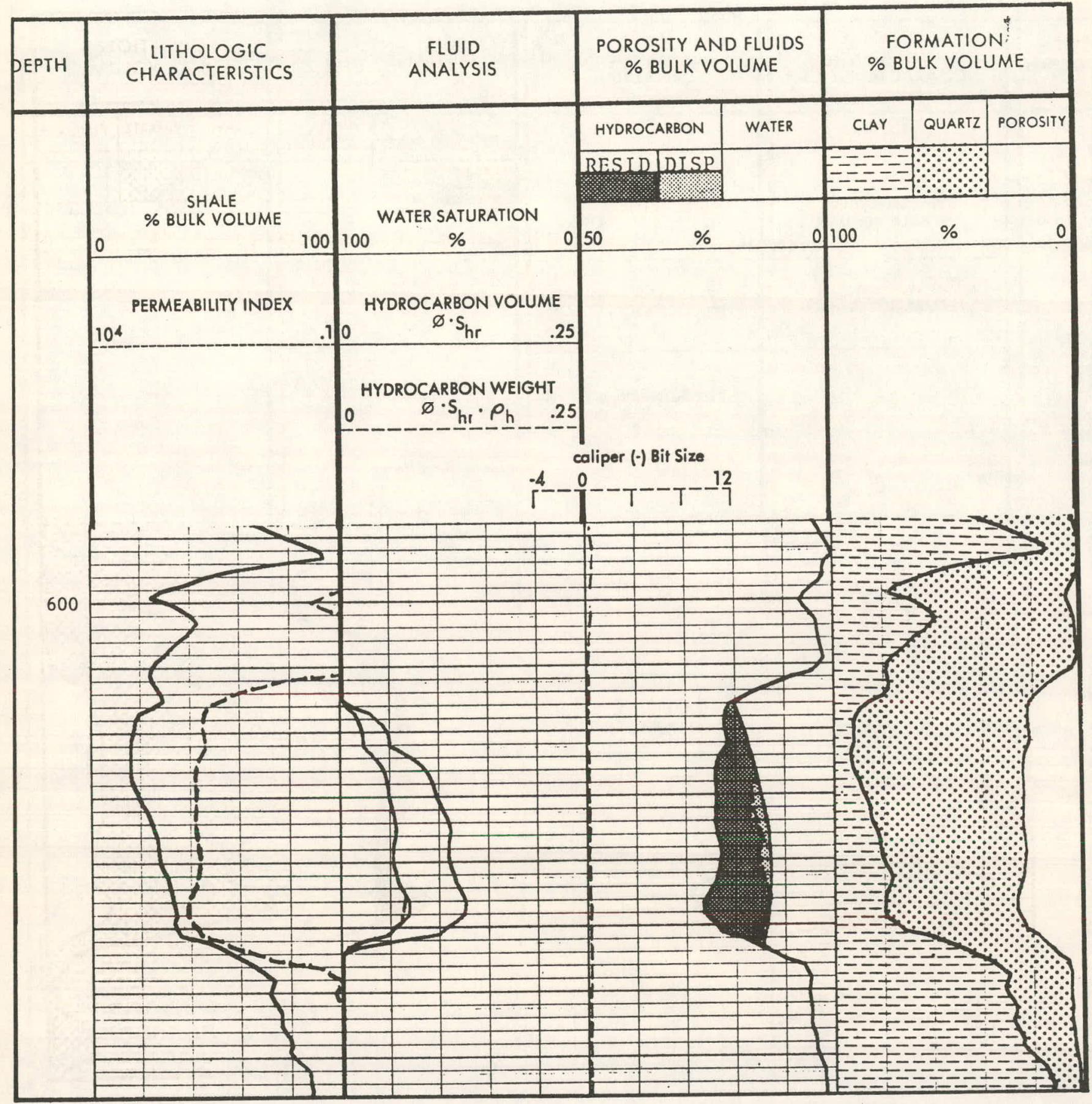


FIGURE $\mathrm{C}-17$

EPILOG FOR WELL MP-111

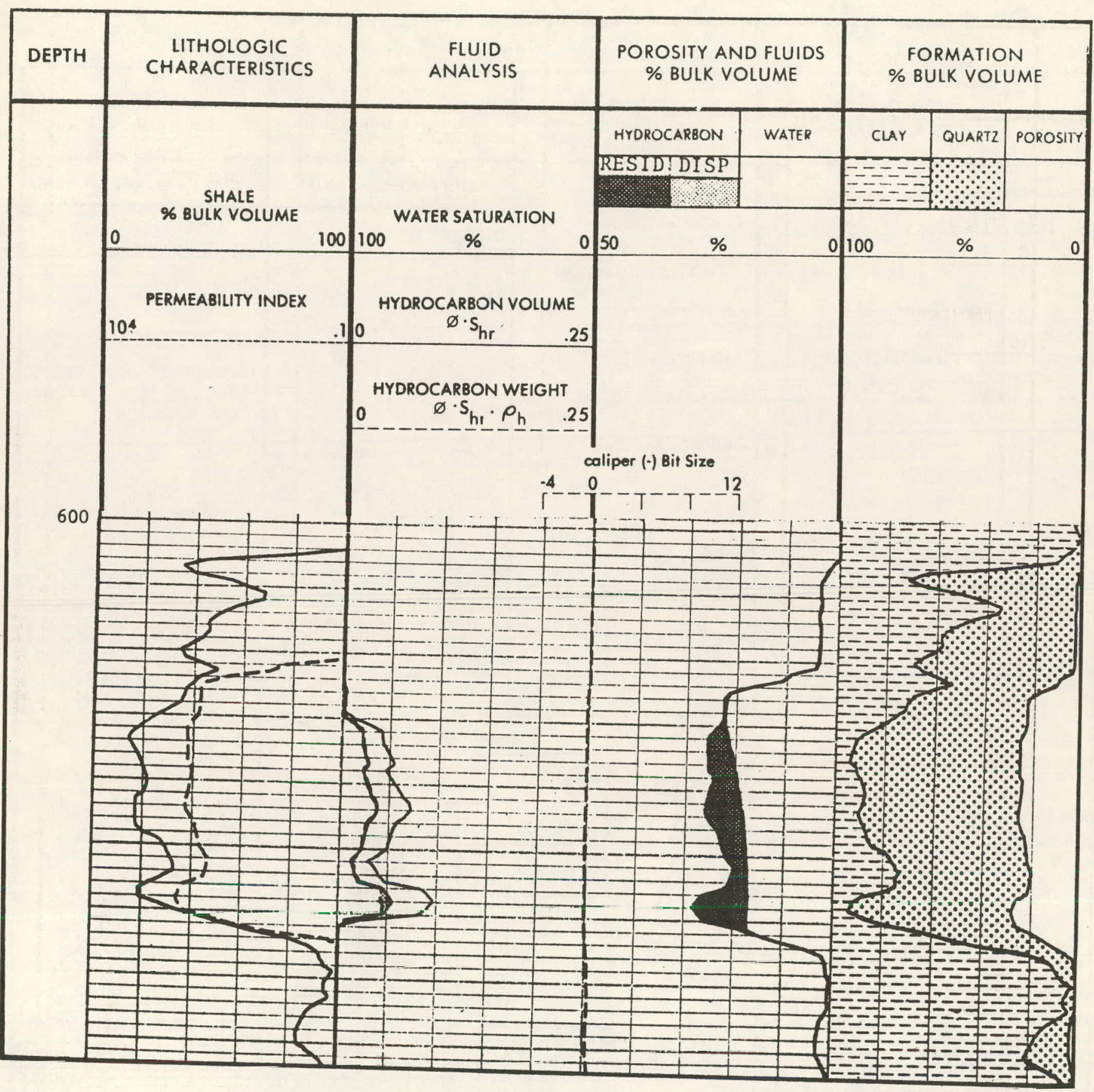


II-192

FIGURE $\mathrm{C}-18$

EPILOG FOR VIELL IIP-112

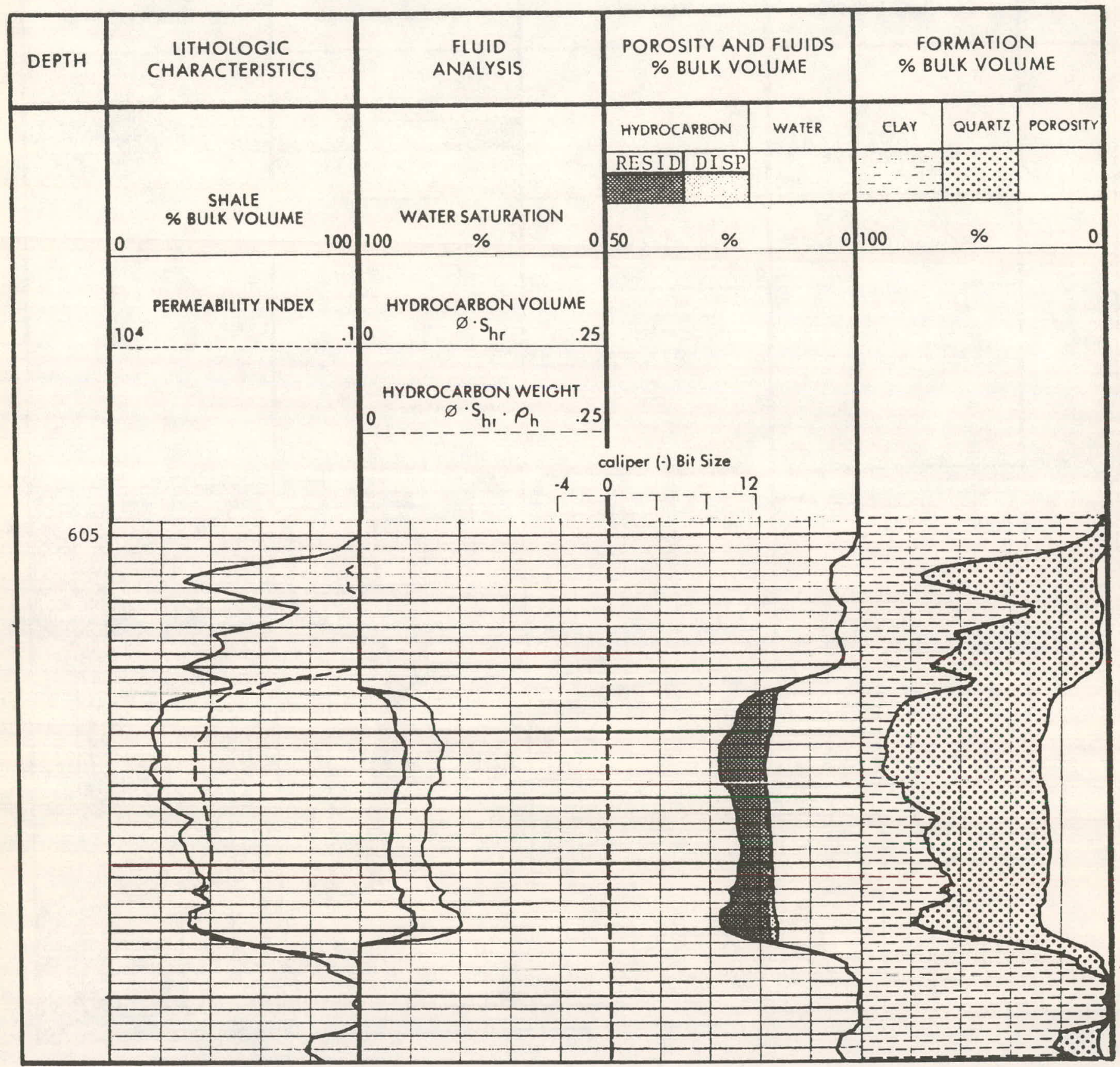


FIGURE C-19

EPILOG FOR WELL IIP-113

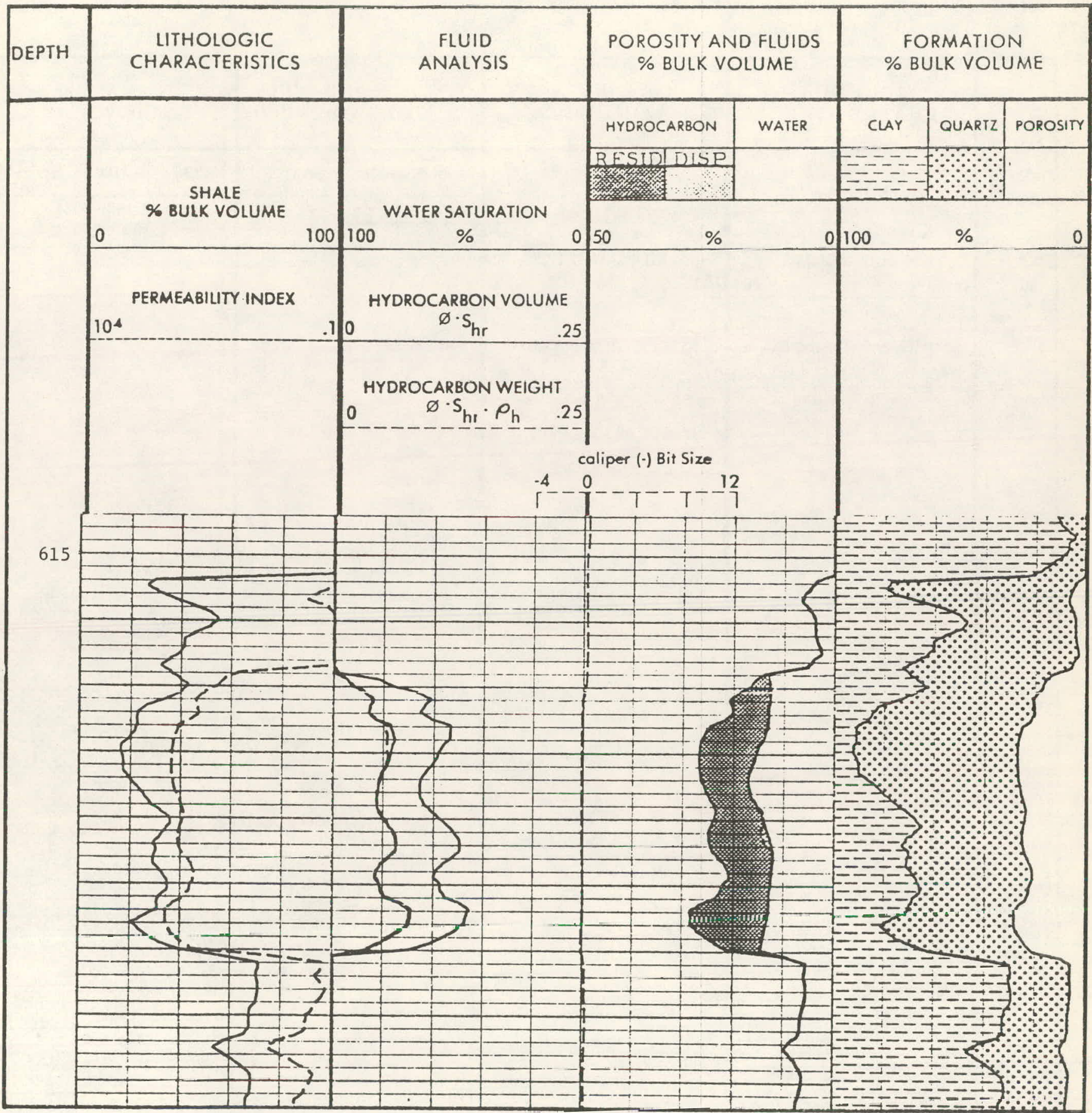


II-194

FIGURE $C-20$

EPILOG FOR WELL MP-114

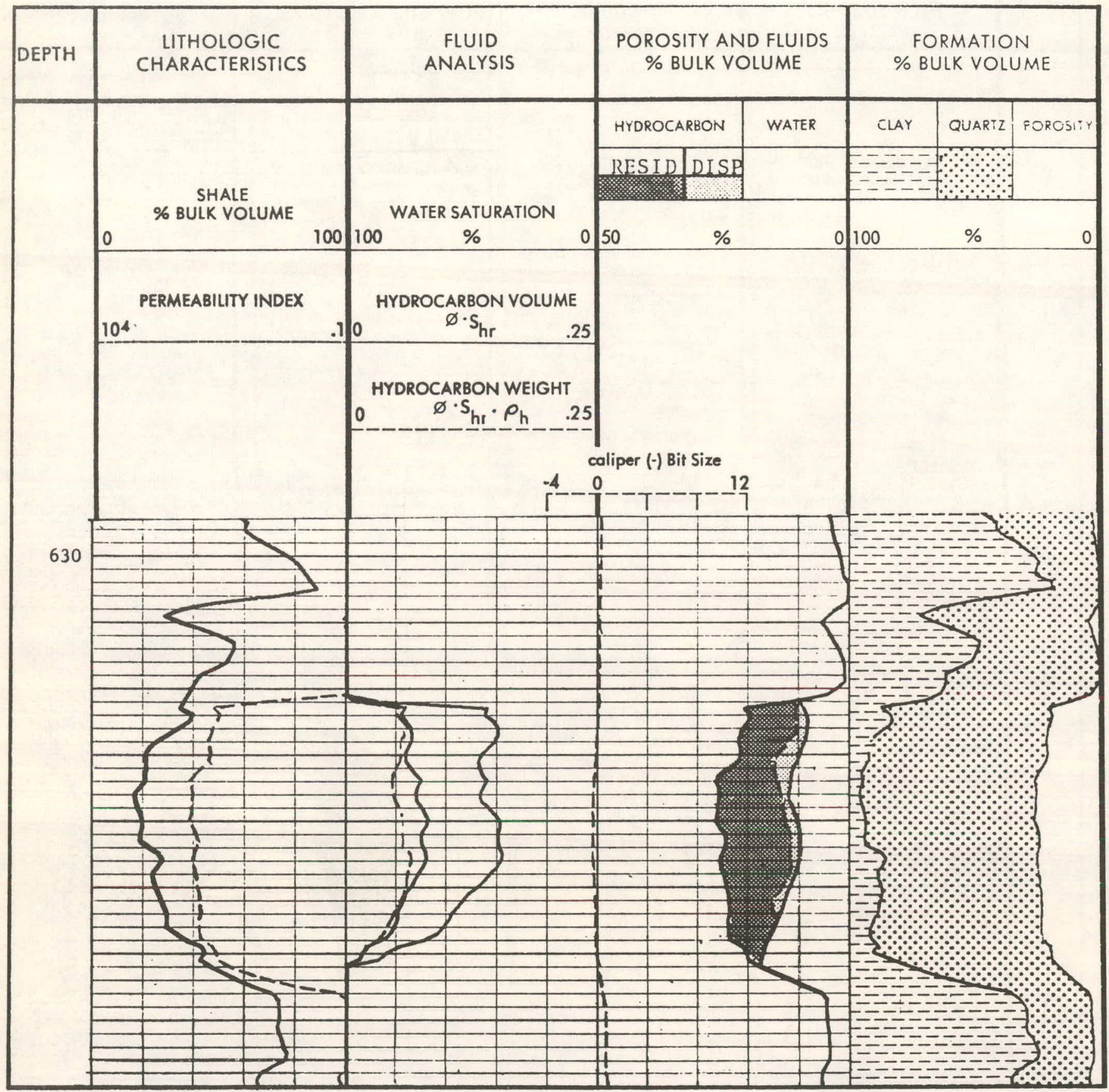


FIGURE C-21

EPILOG FOR HELL MP-115

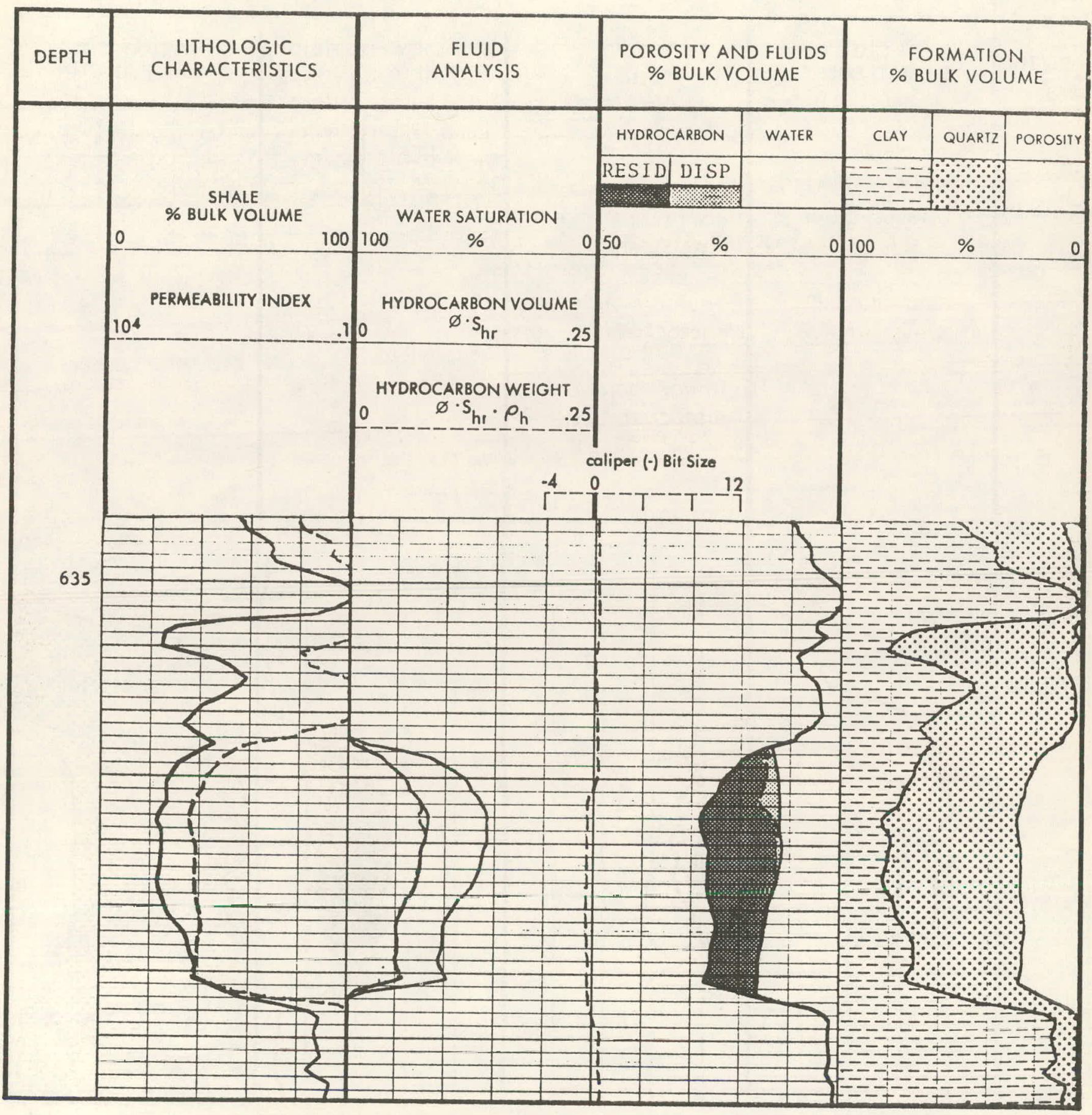


FIGURE C-22

EPILOG FOR HELL I!P-116

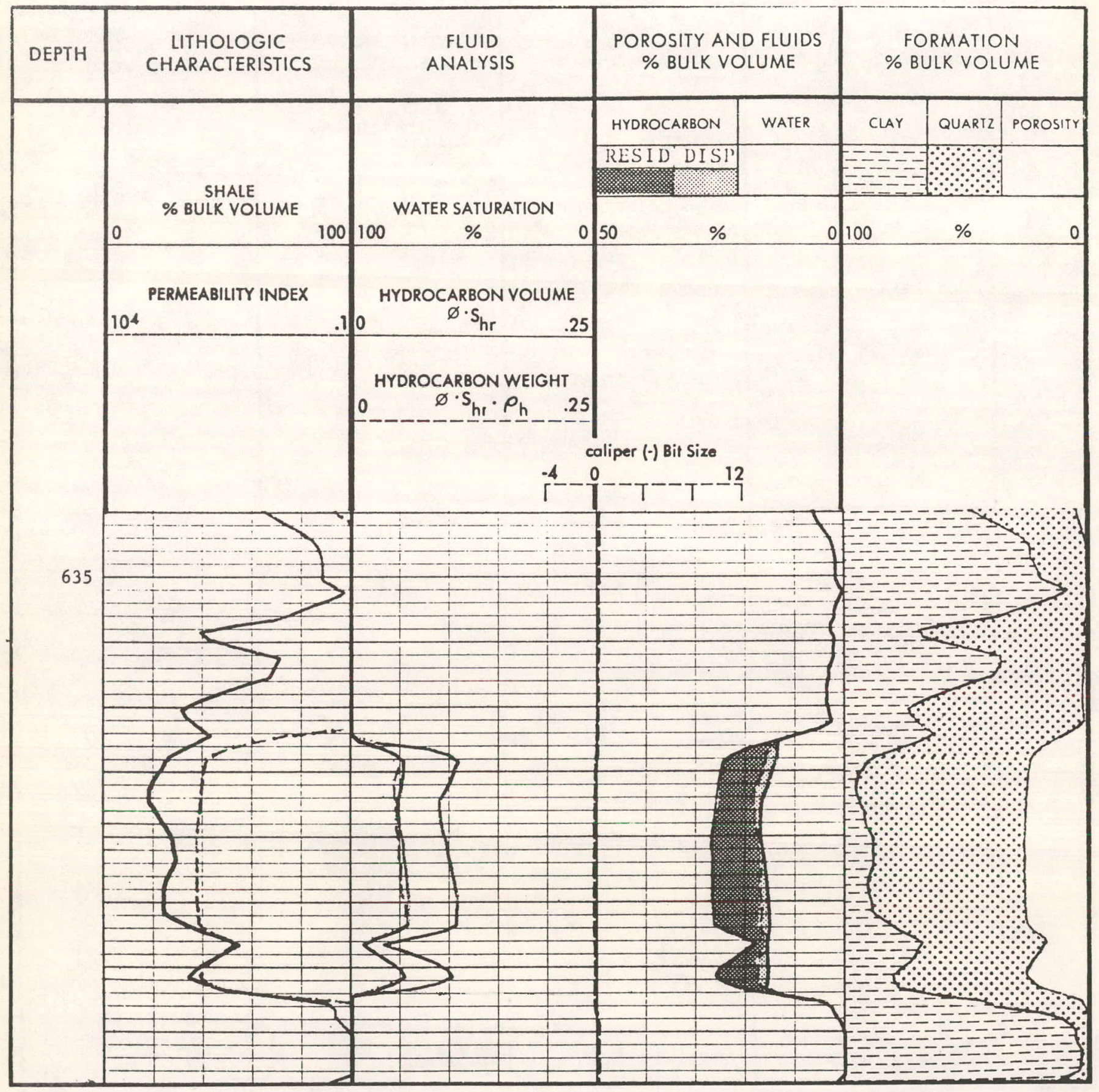


FIGURE C-23

EPILOG FOR WELL IIP-117

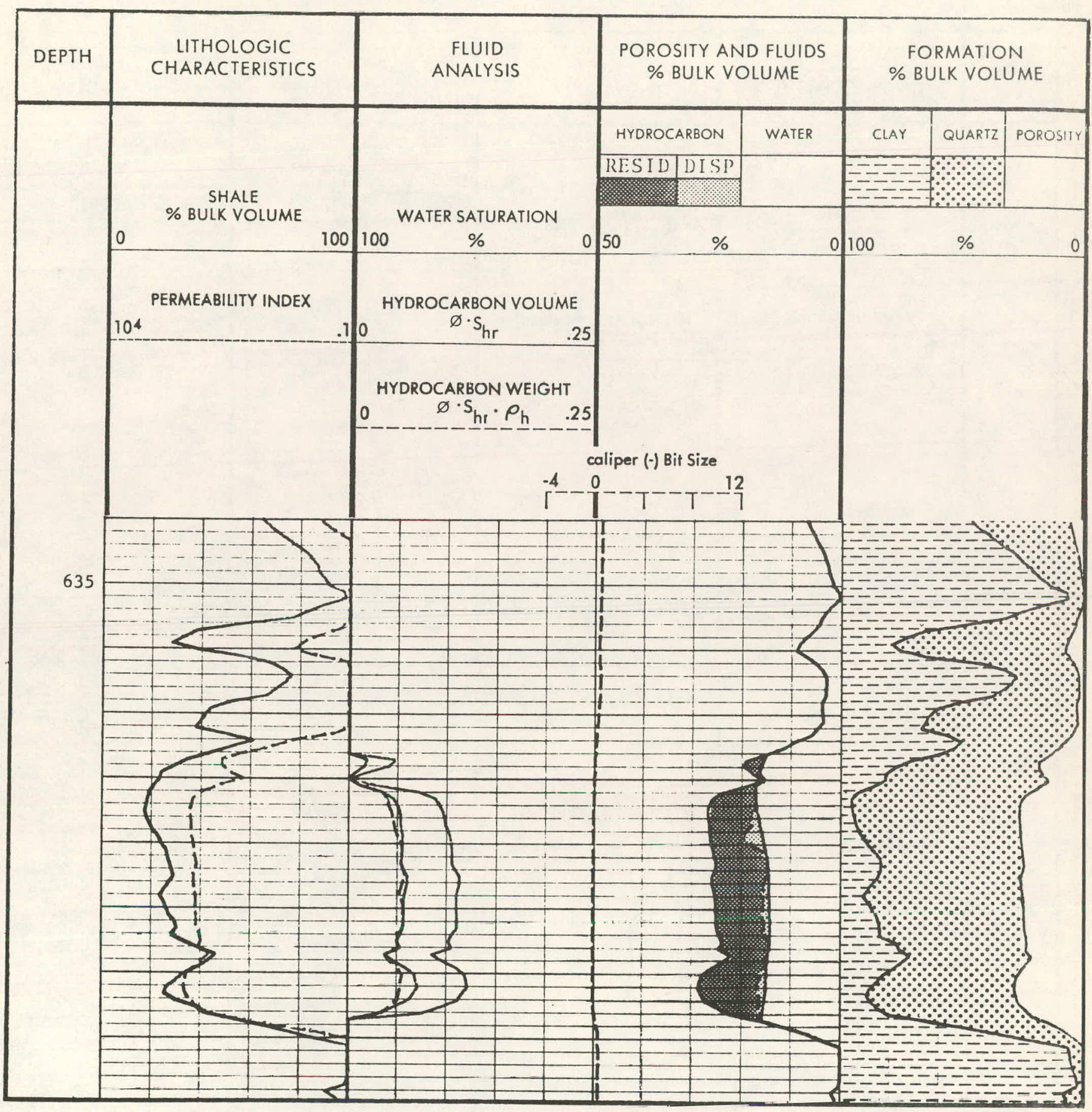


FIGURE C-24

EPILOG FOR HELL MP-118

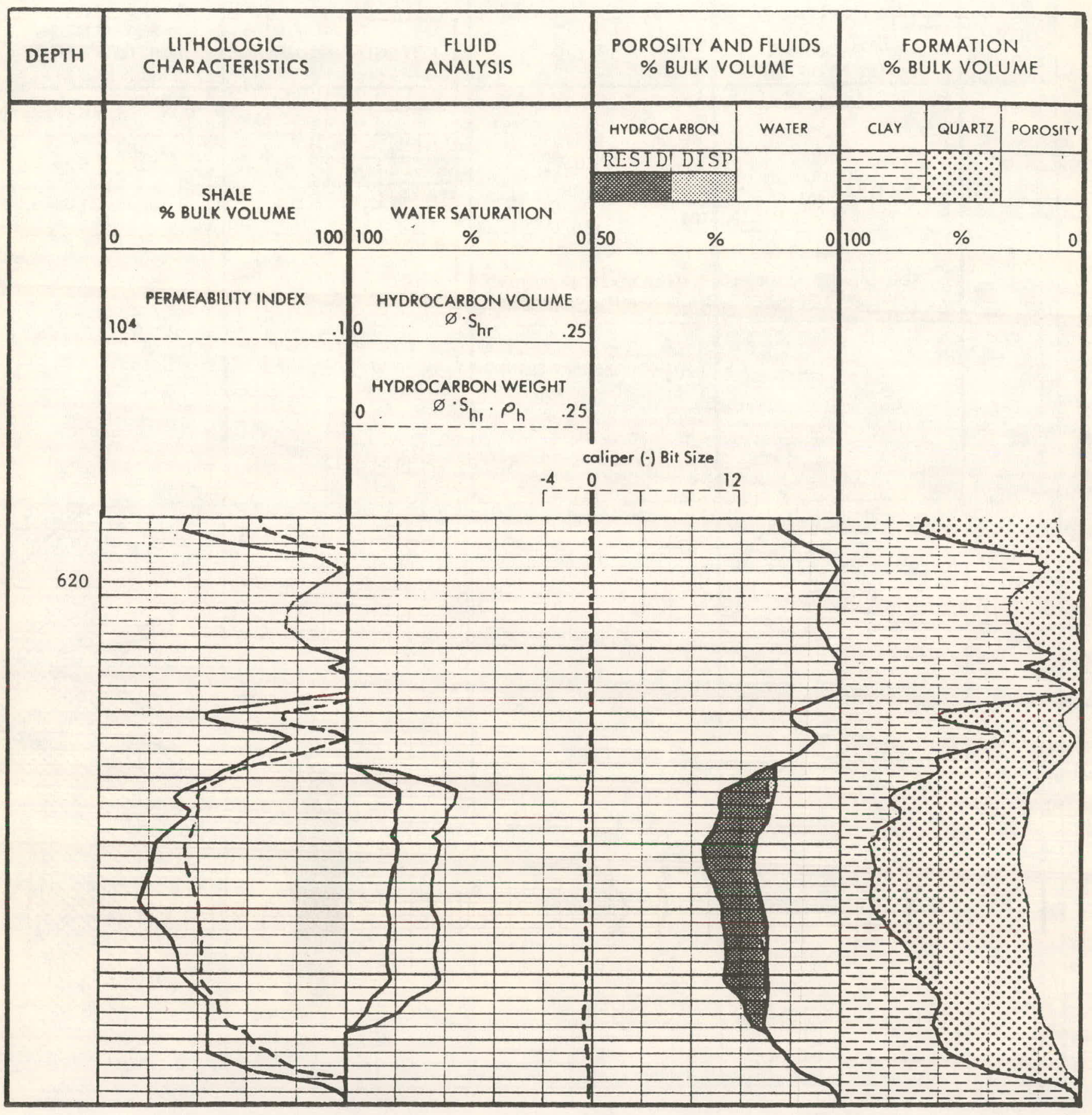


FIGURE C-25

EPILOG FOR WELL MP-119

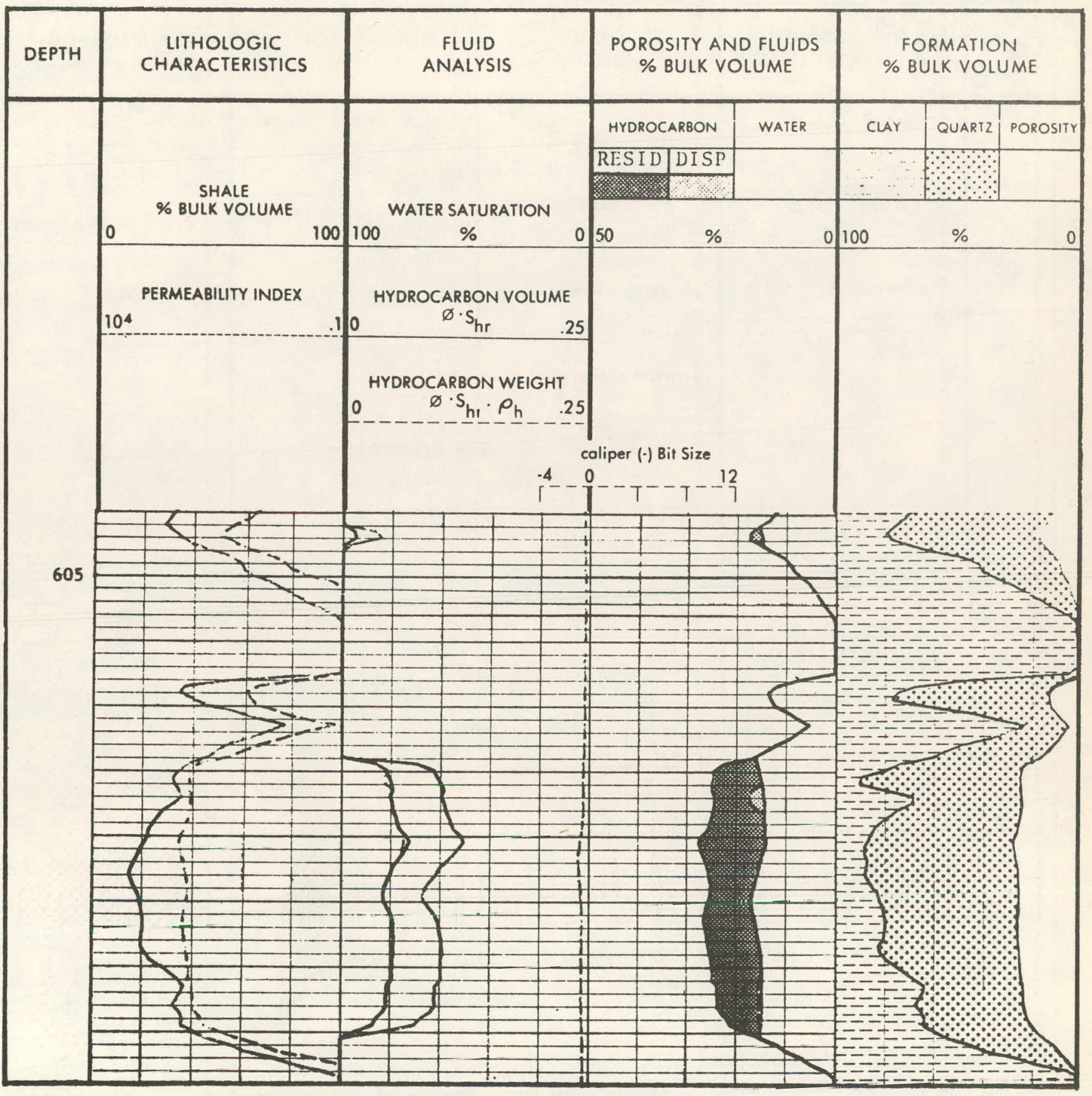


FIGURE C-26

EPILOG FOR WELL MP-120

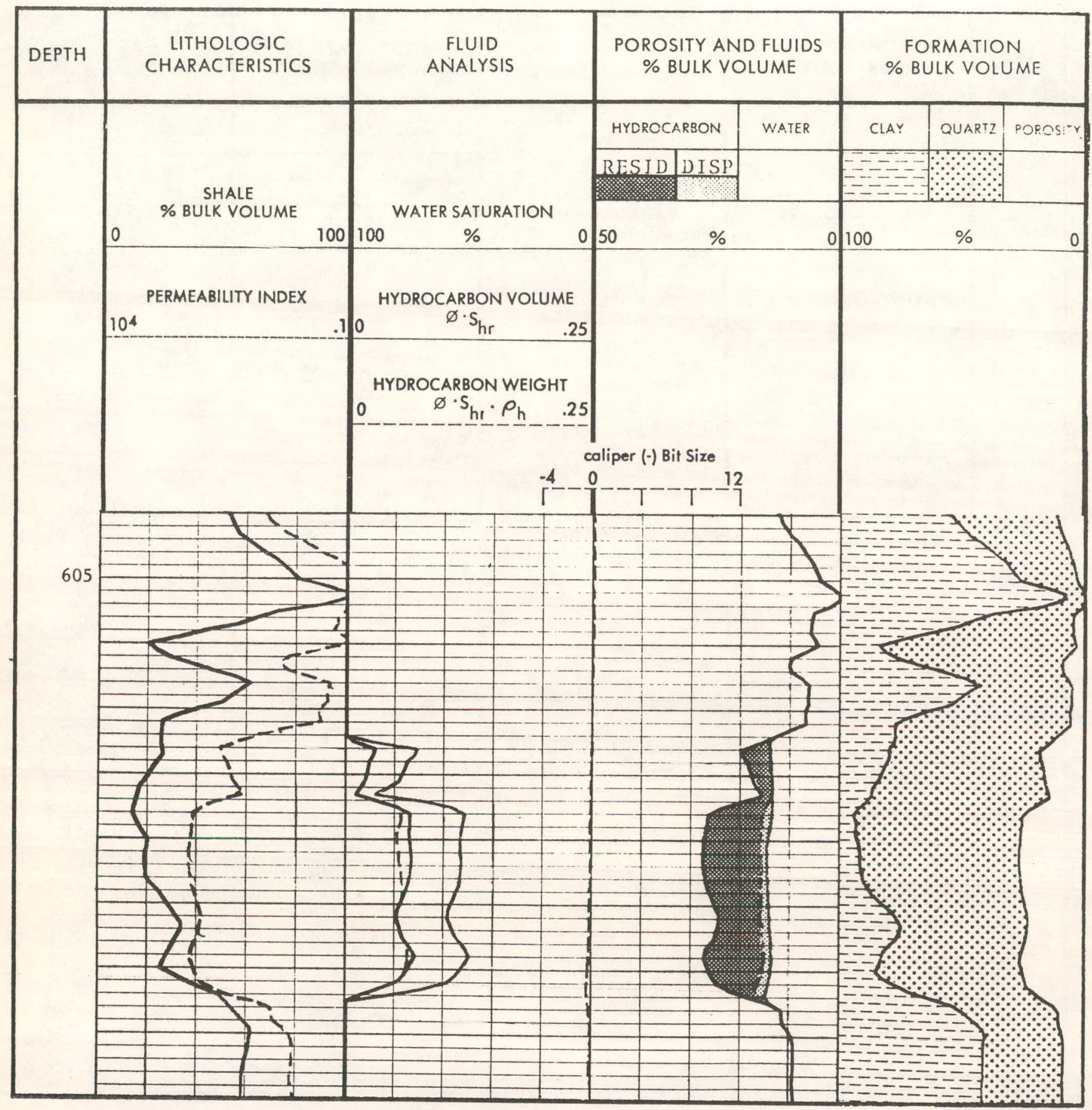


FIGURE $\quad$ - -27

EPILOG FOR WELL IIP-121

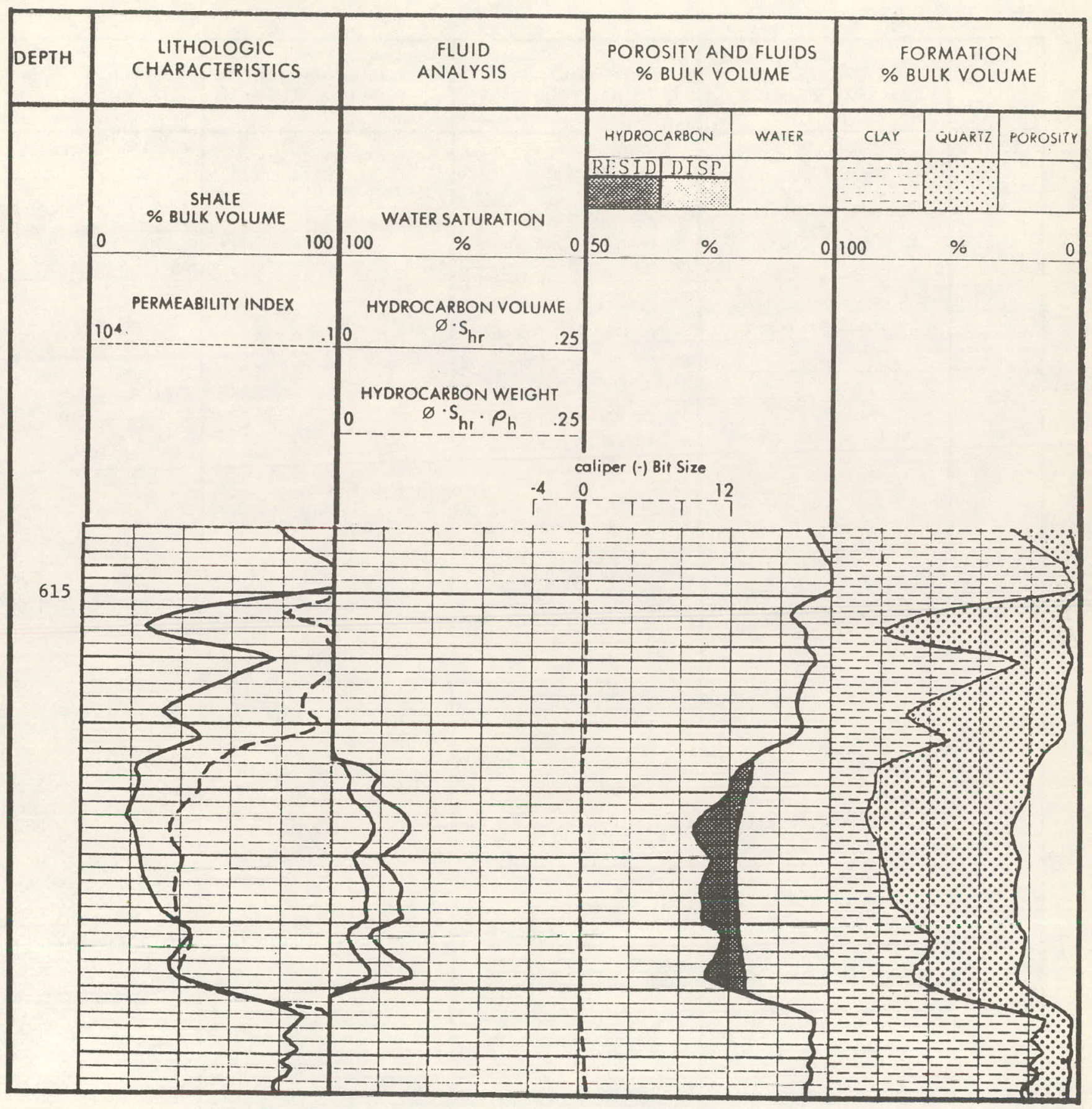




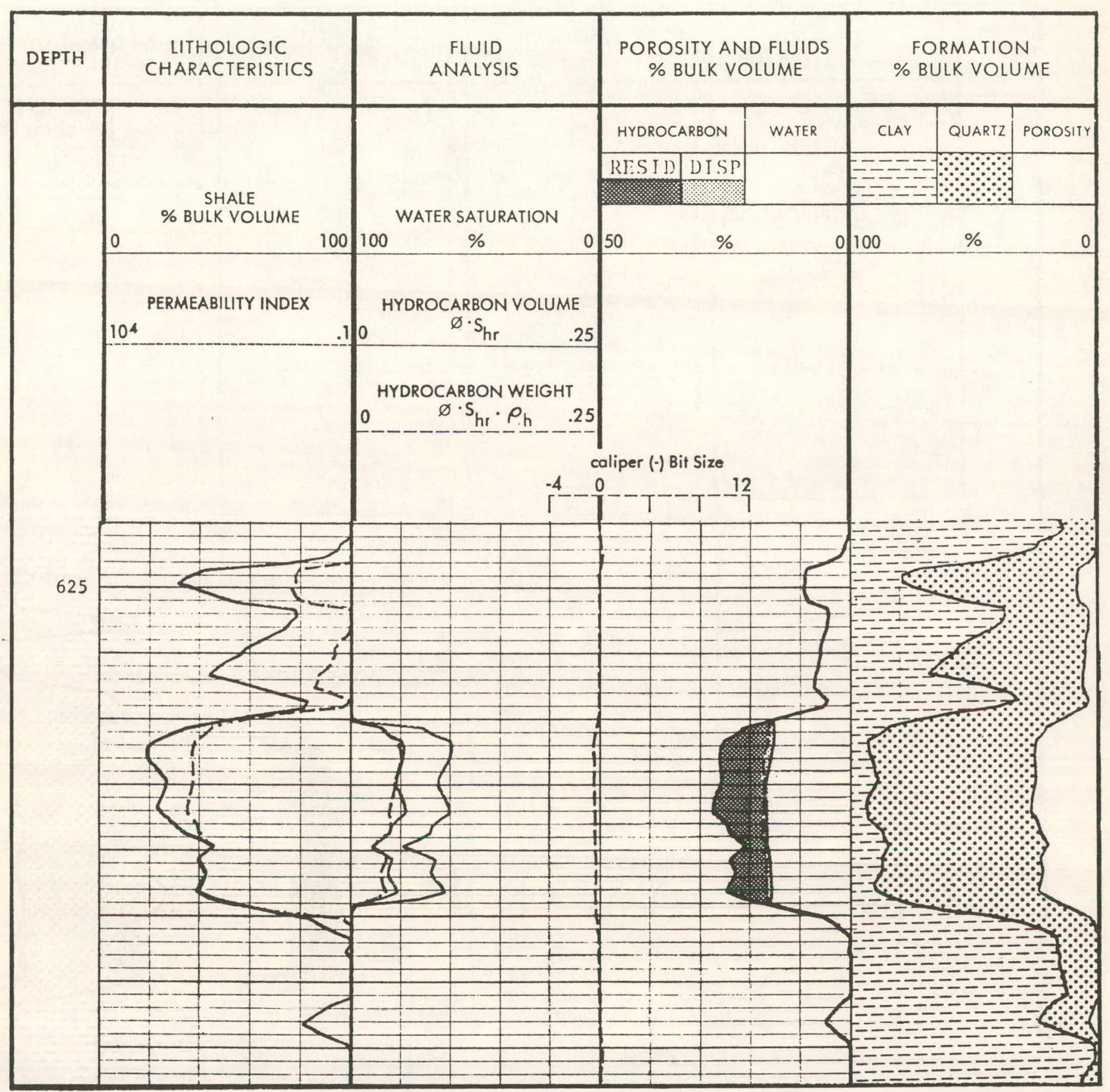


FIGURE C-29

EPILOG FOR WELL IIP-123

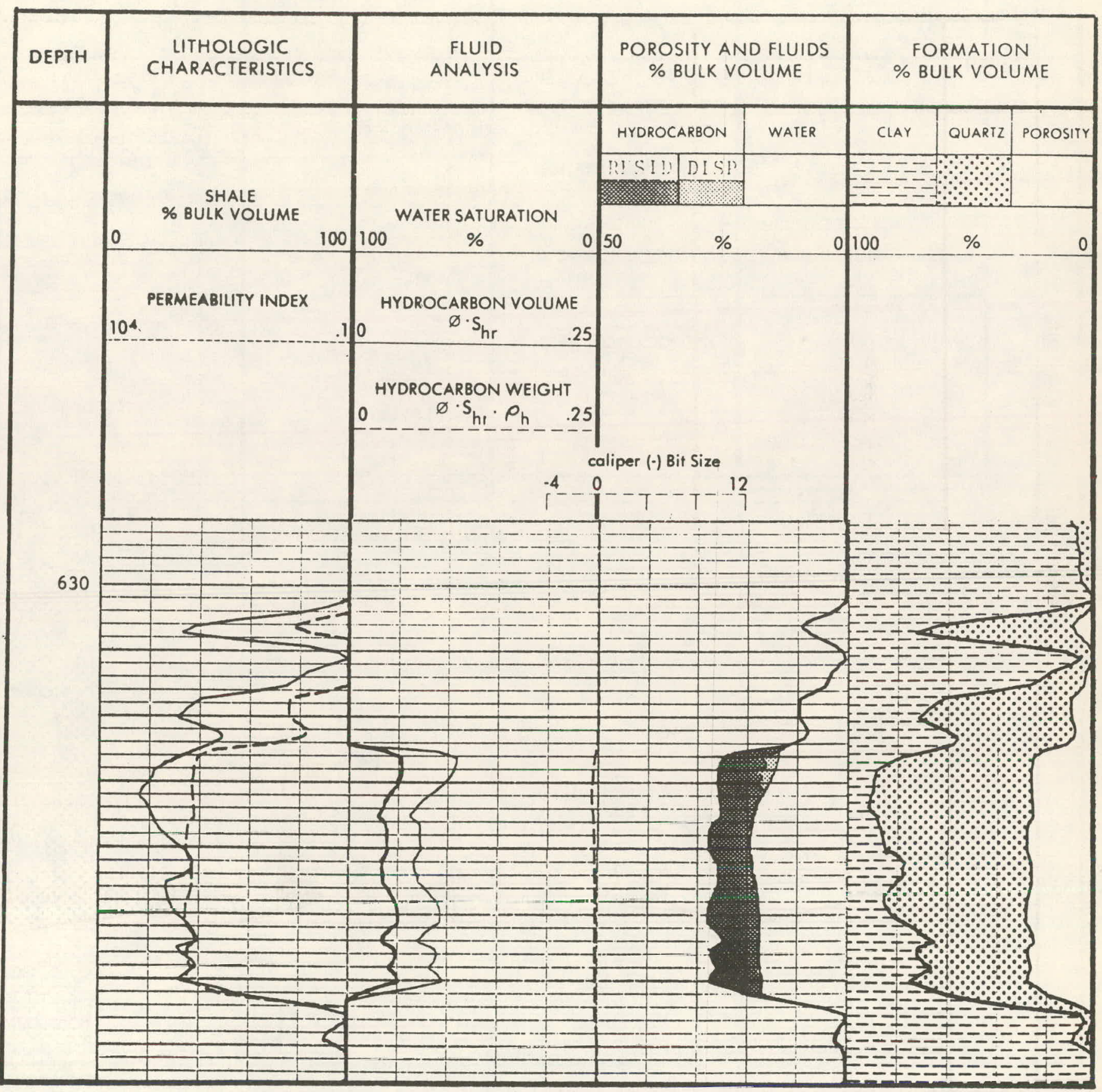


I I-204

FIGURE $\mathrm{C}-30$

EPILOG FOR WELL IIP-12.4

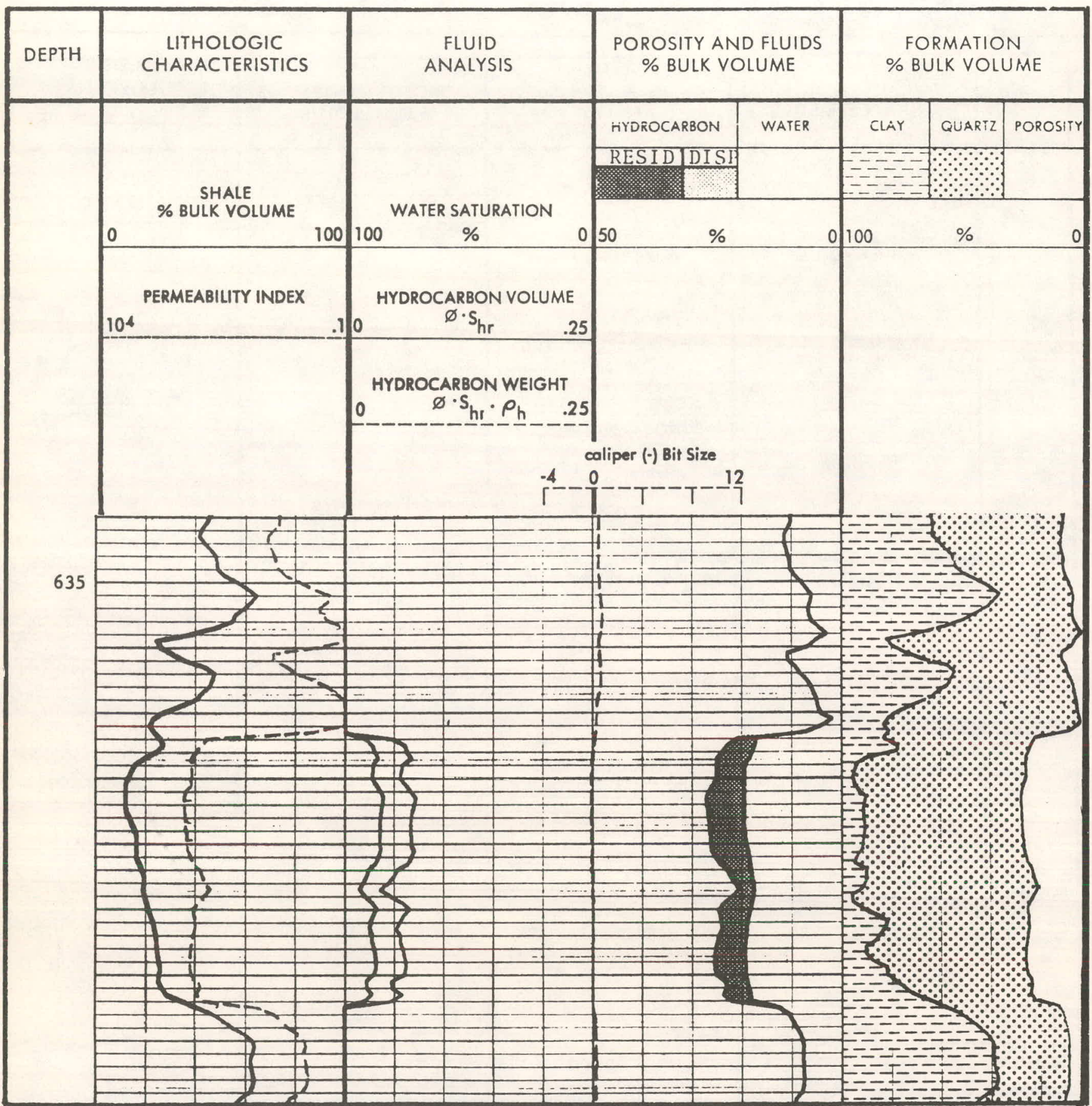


FITUURE C-31

EPILOG FOR WELL IIP-125

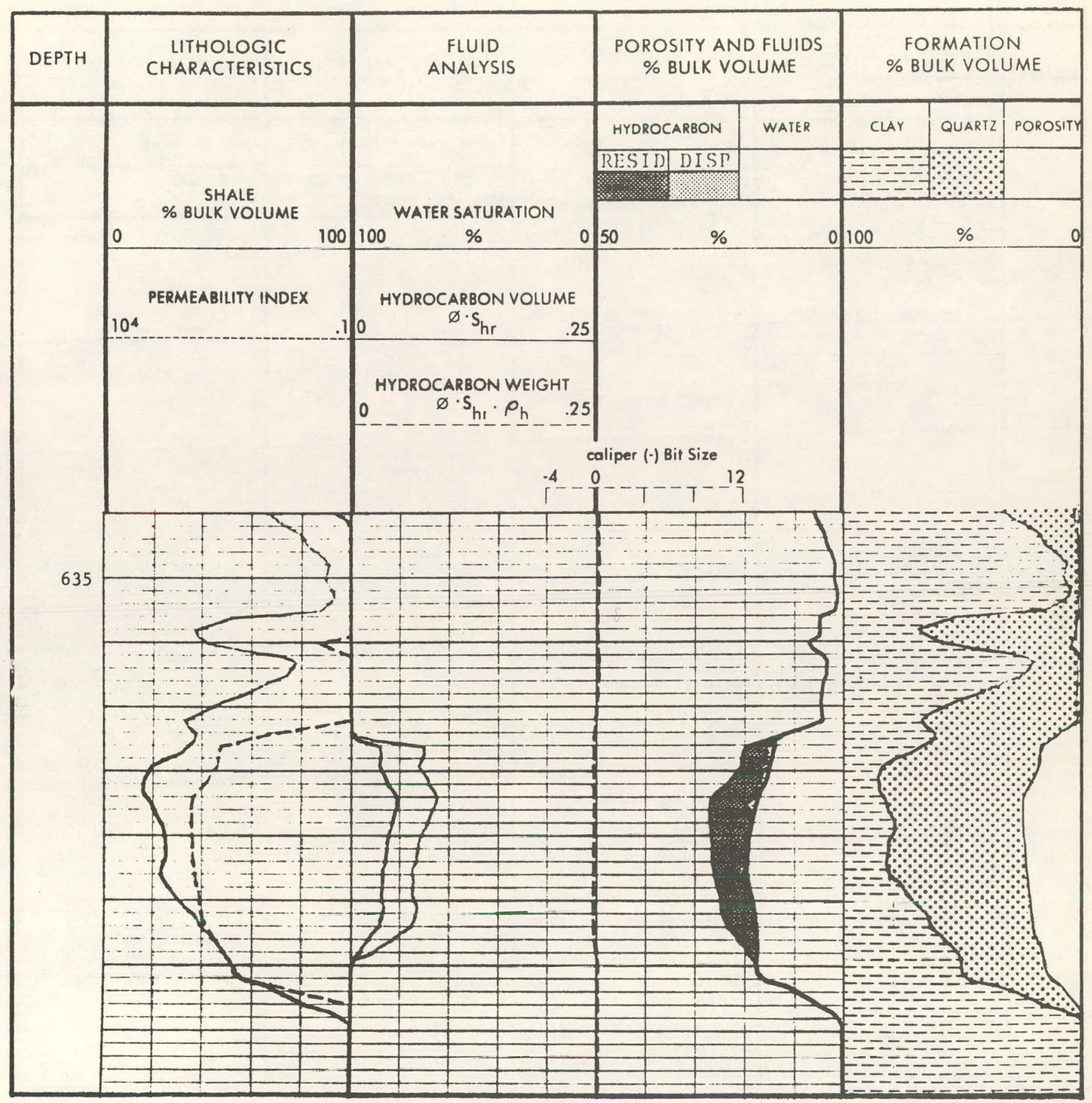


II-206

FIGURE C-32

EPILOG FOR WELL IIP-126

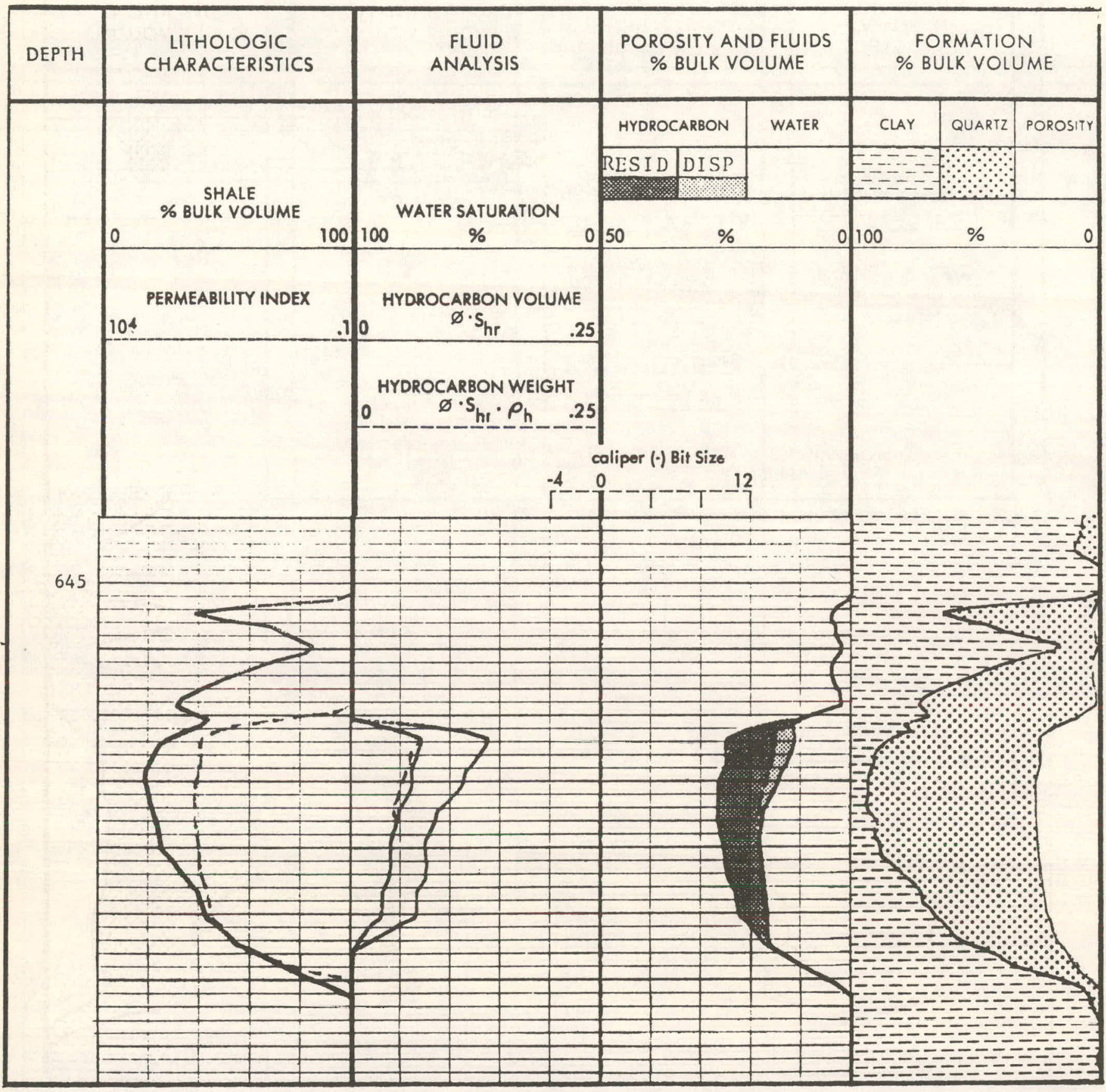


FIGURE $\mathrm{C}-33$

EPILOG FOR WELL IIP-127

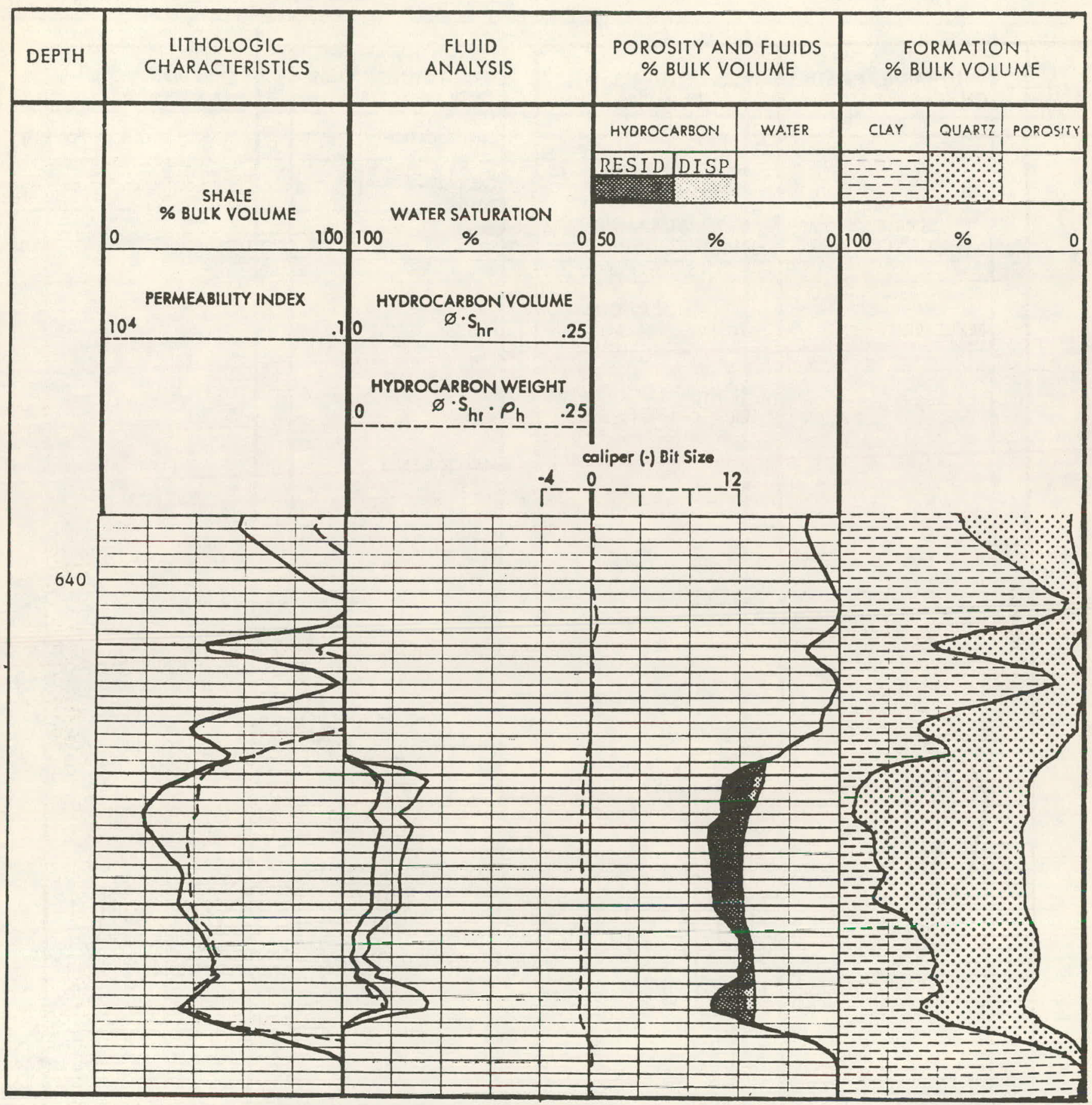


II -208

FIGURE C-34

EPILOG FOR WELL MP-128

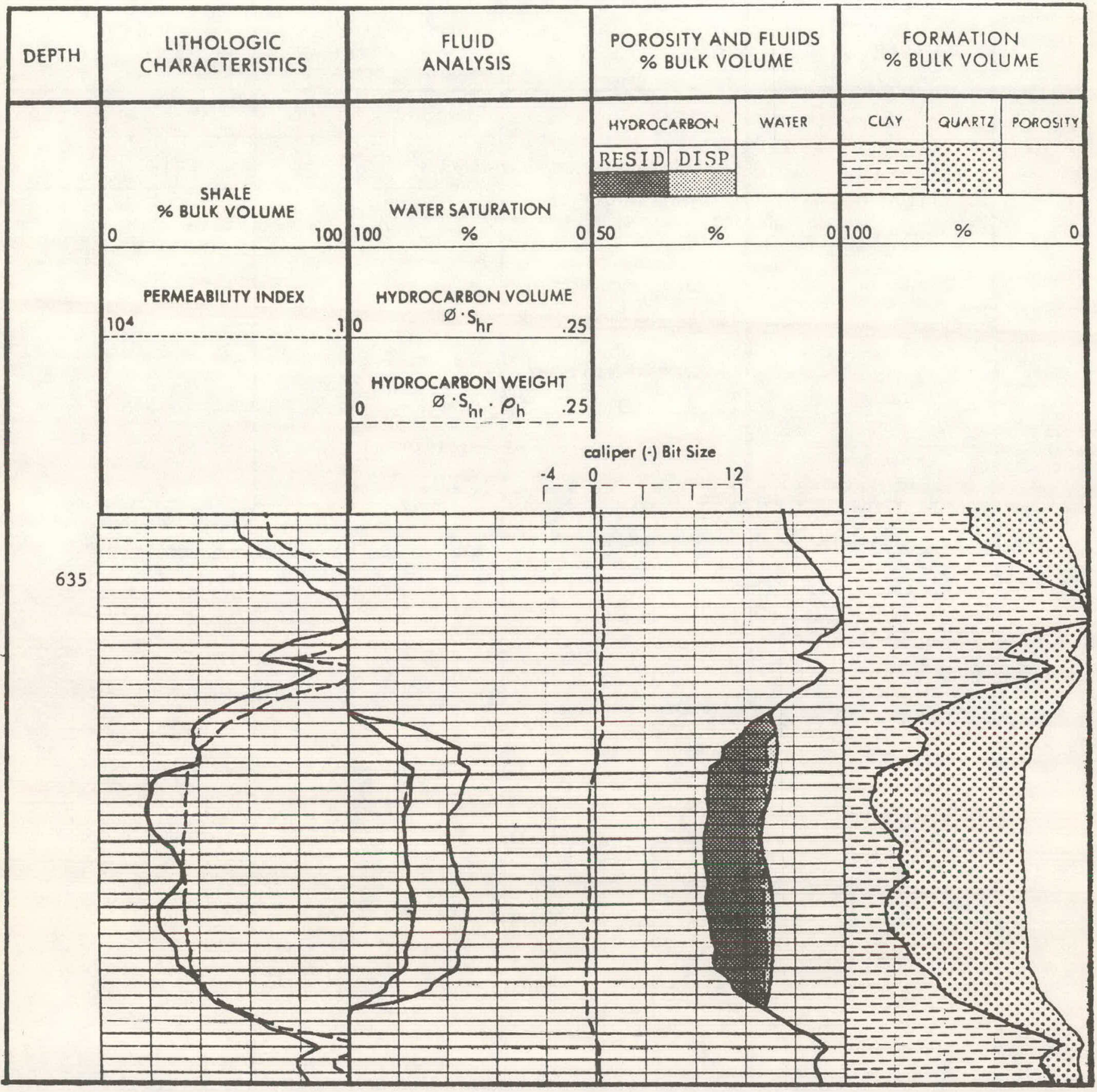


FIGURE C-35

EPILOG FOR HELL IIP-12.9

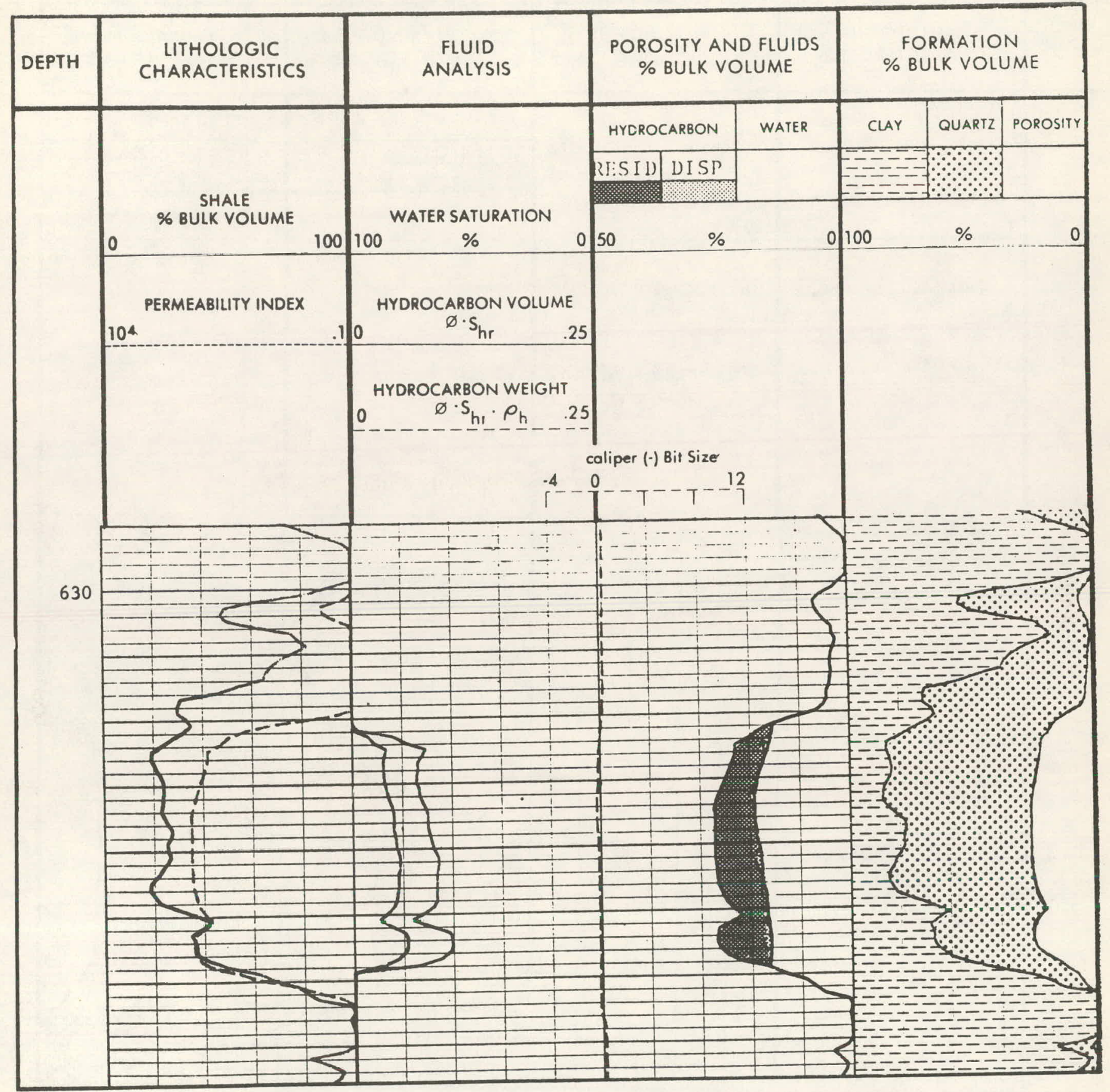


II-210

FIGURE C-36

EPILOG FOR WELL IIP-130

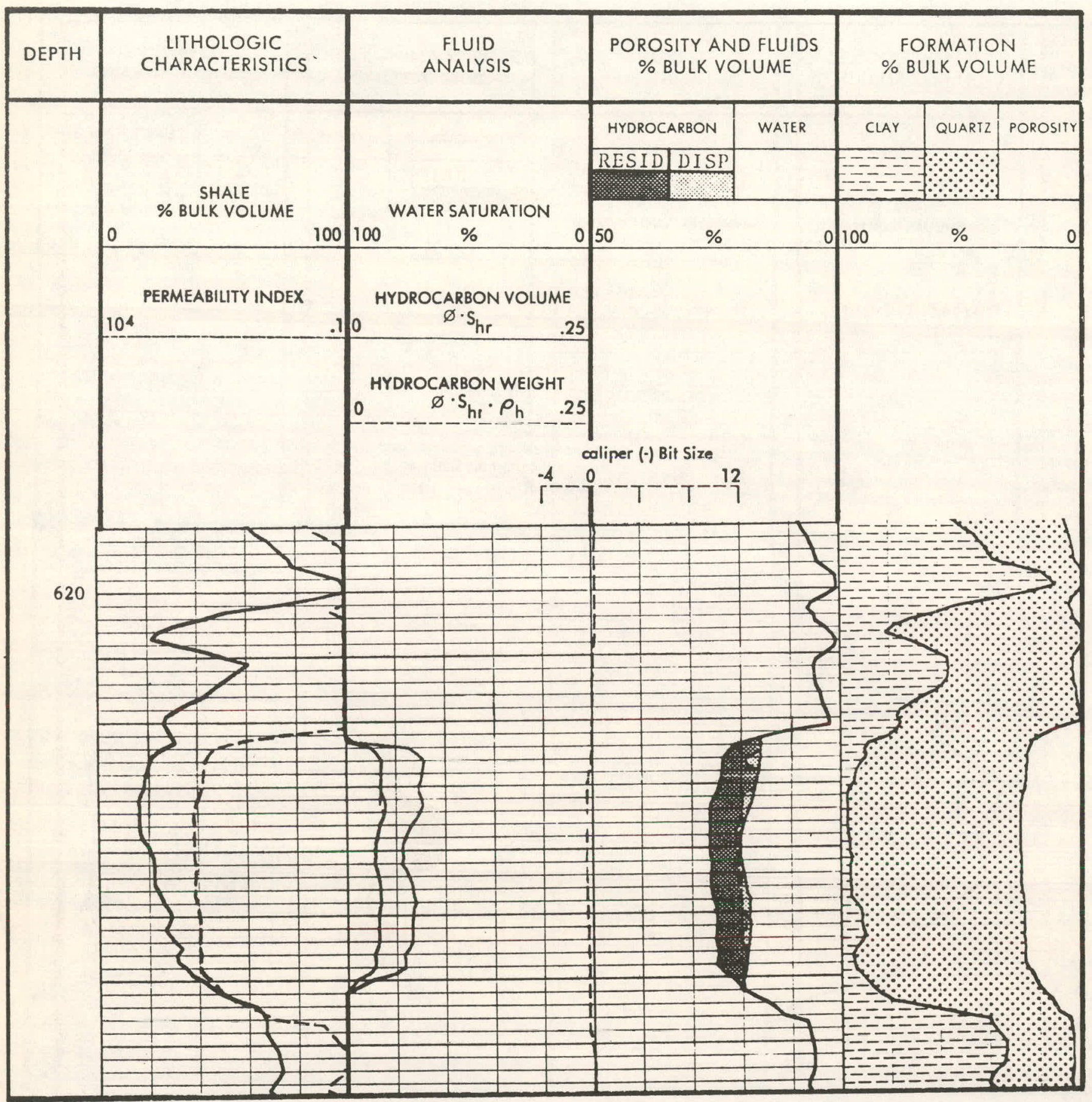


FIGURE C-37

EPILOG FOR IIELL IIP-201

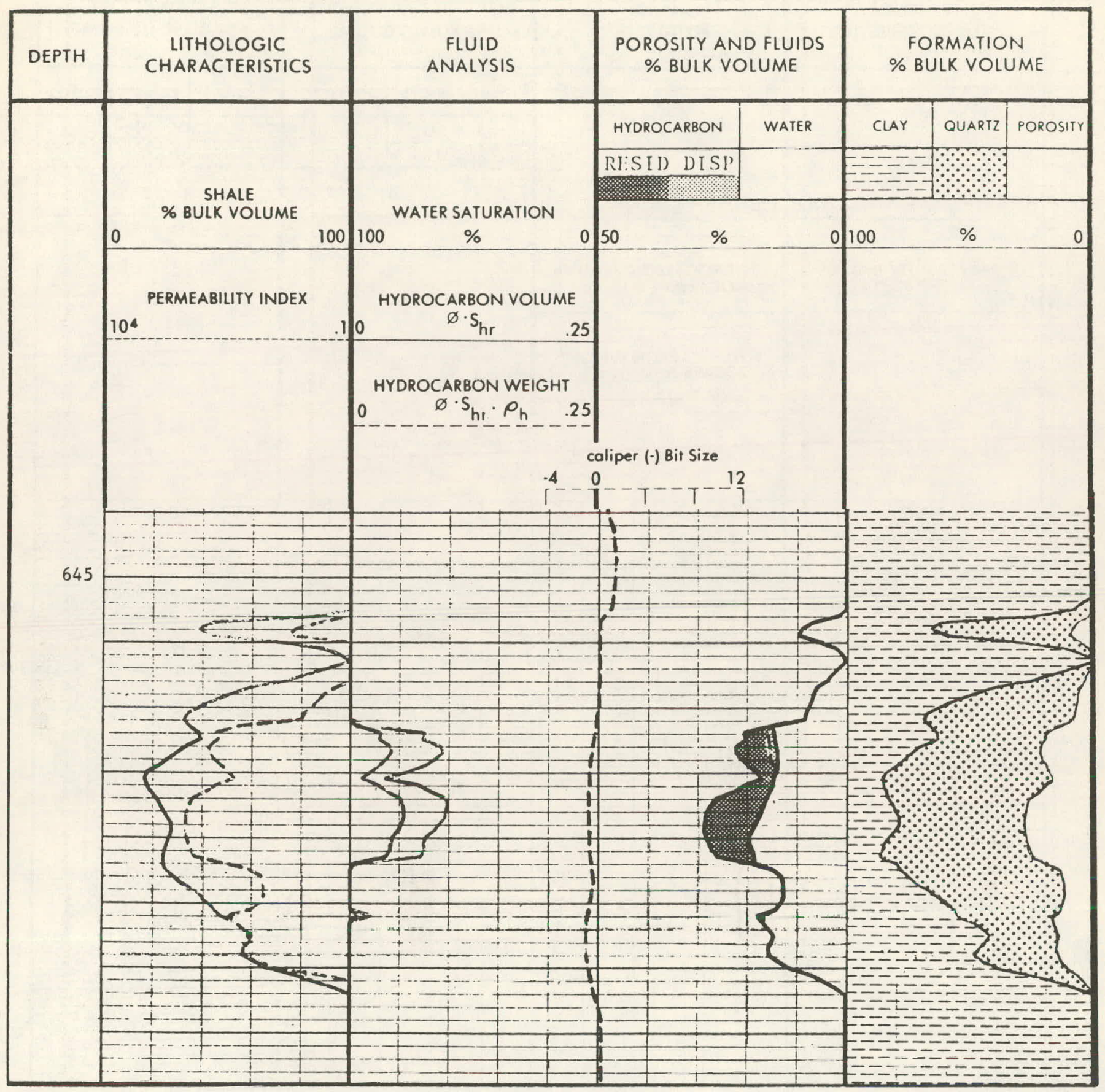


FIGURE C-38

EPILOG FOR HELL MP-202

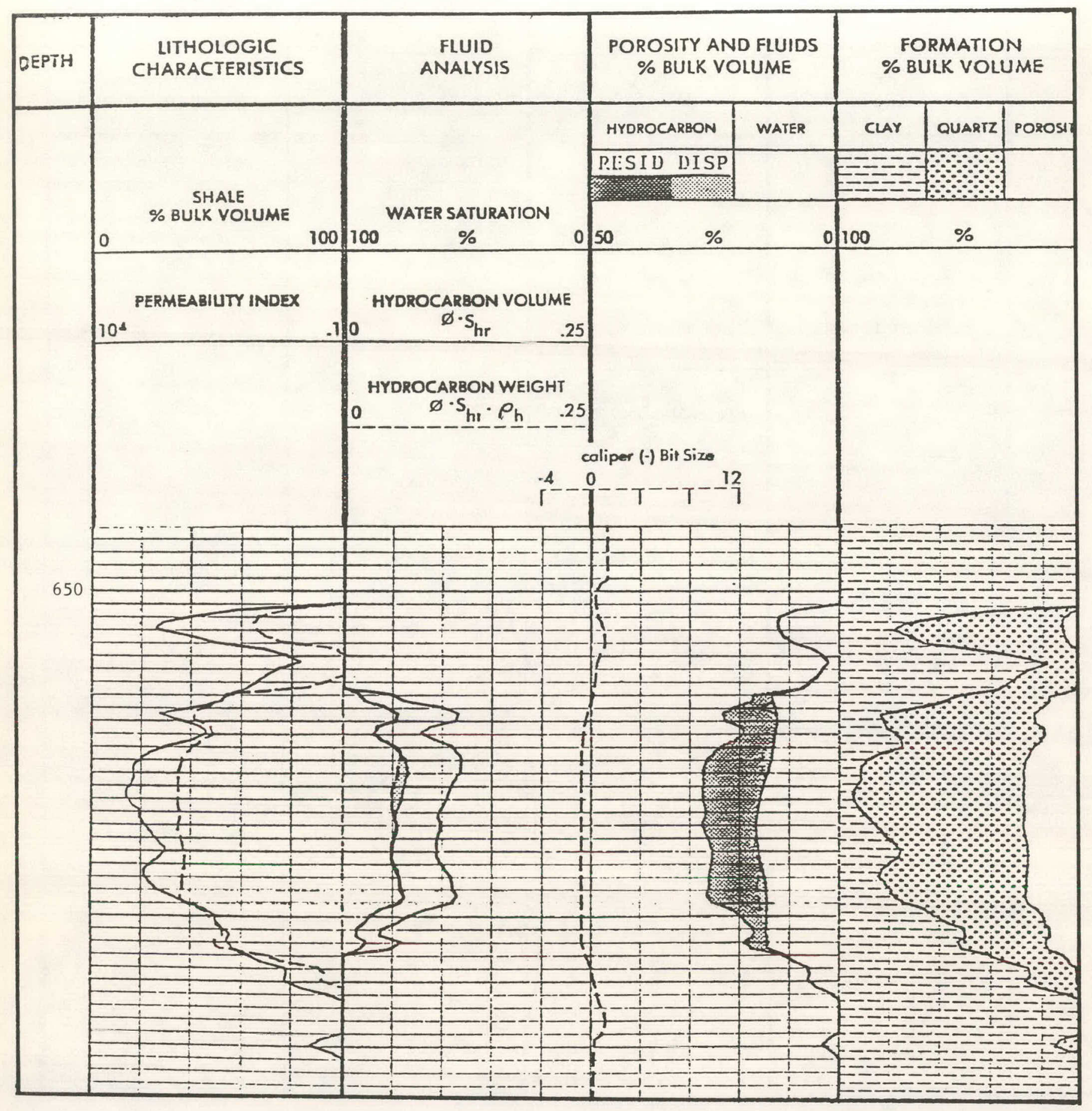


FIGURE C.3?

EPILOG FOR HELL IIP-203

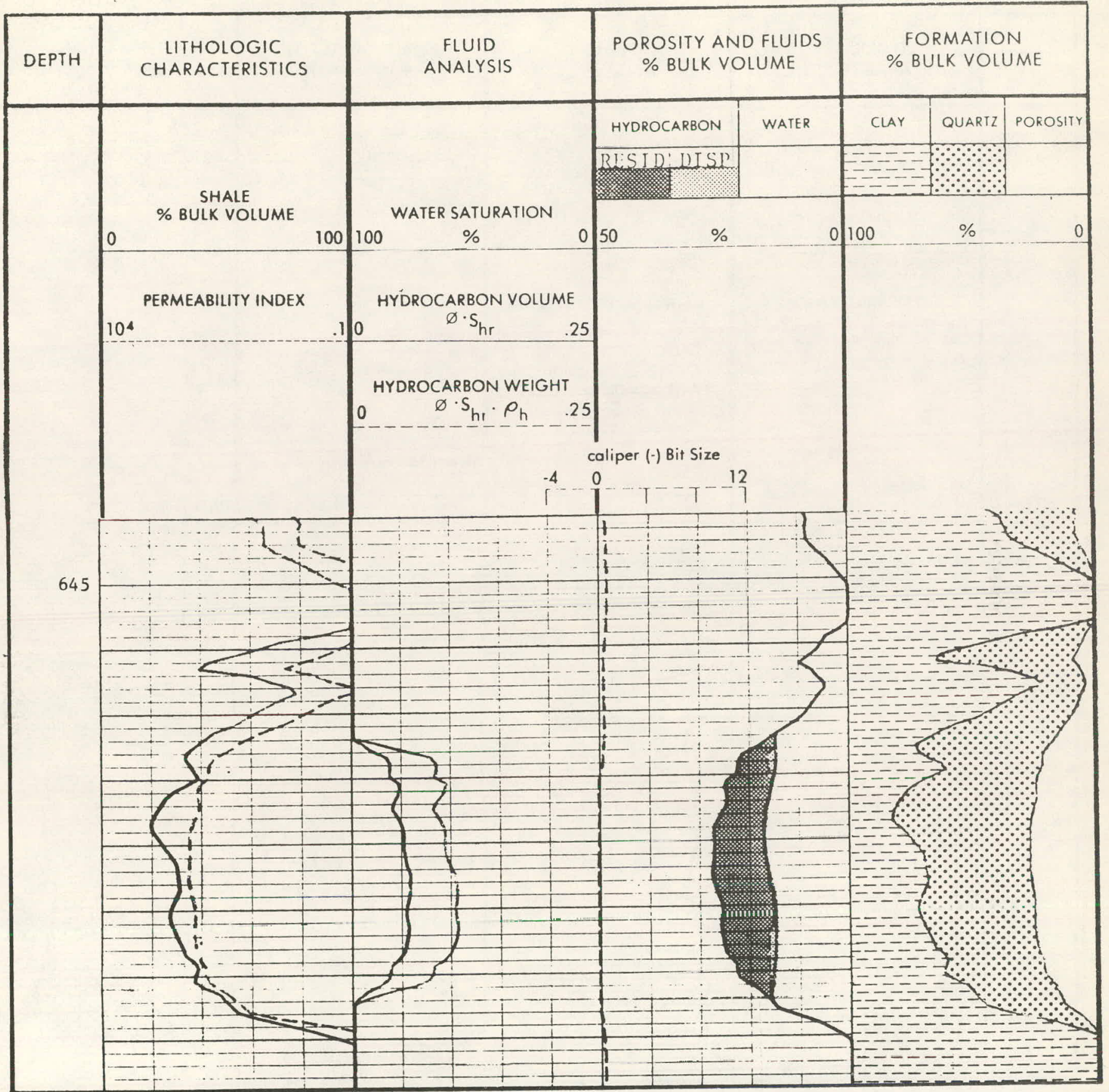


FIGURE C-40

EPILOG FOR MELL IIP-204

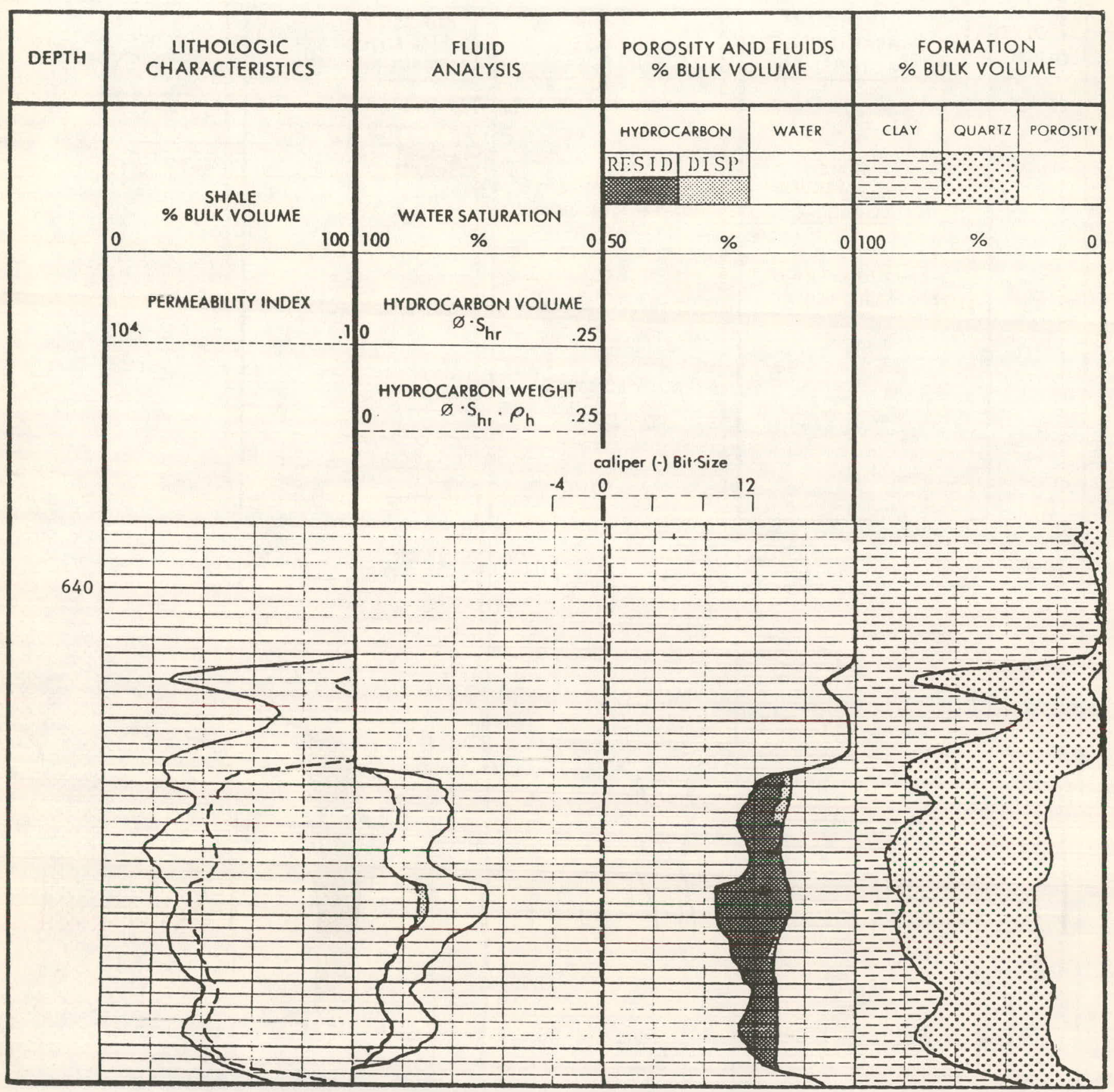


FIGURE C-41

EPILOG FOR HELL MP-205

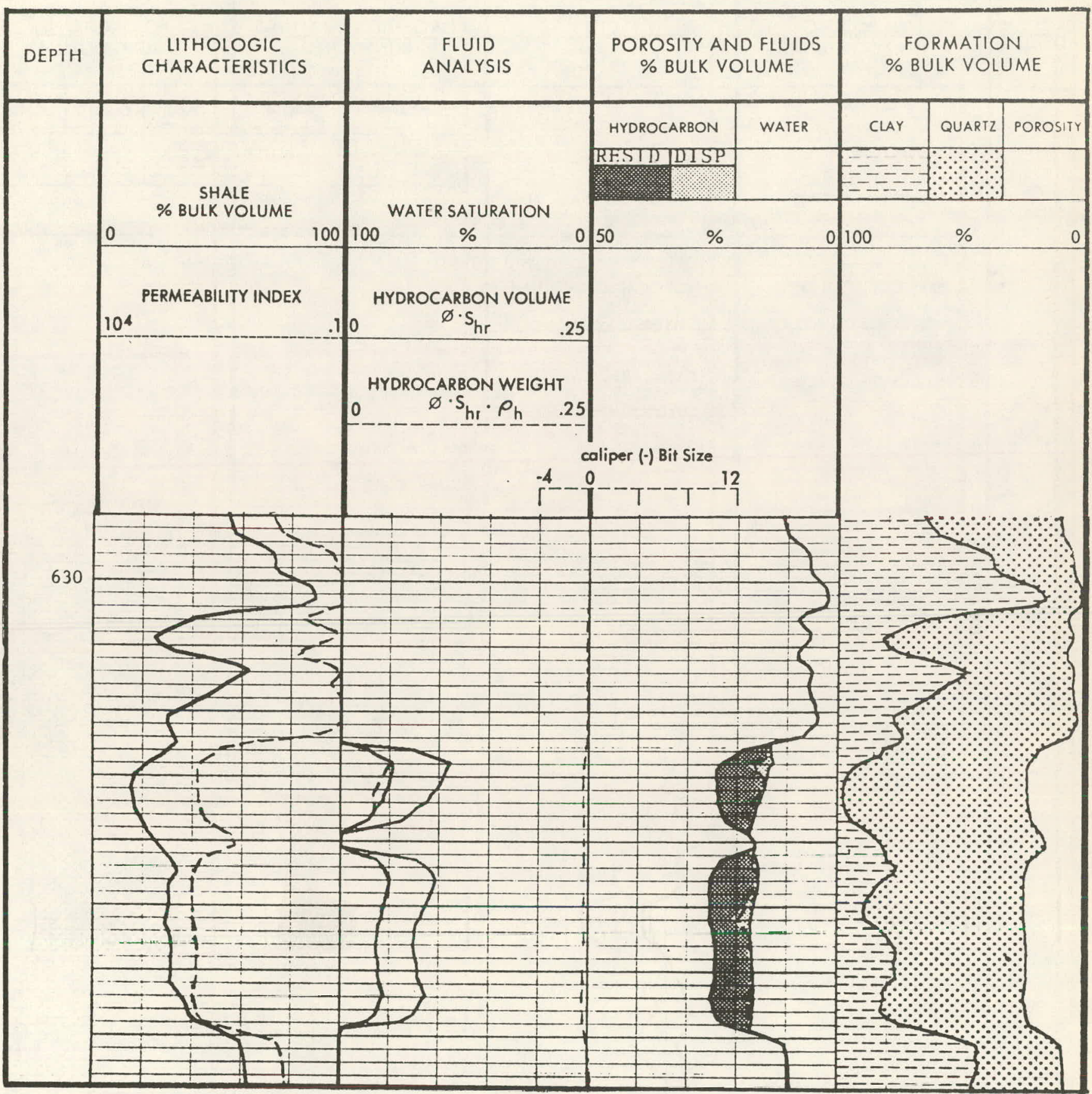


II-216

FIGURE $\quad C-42$

EPILOG FOR UELL IIP-200

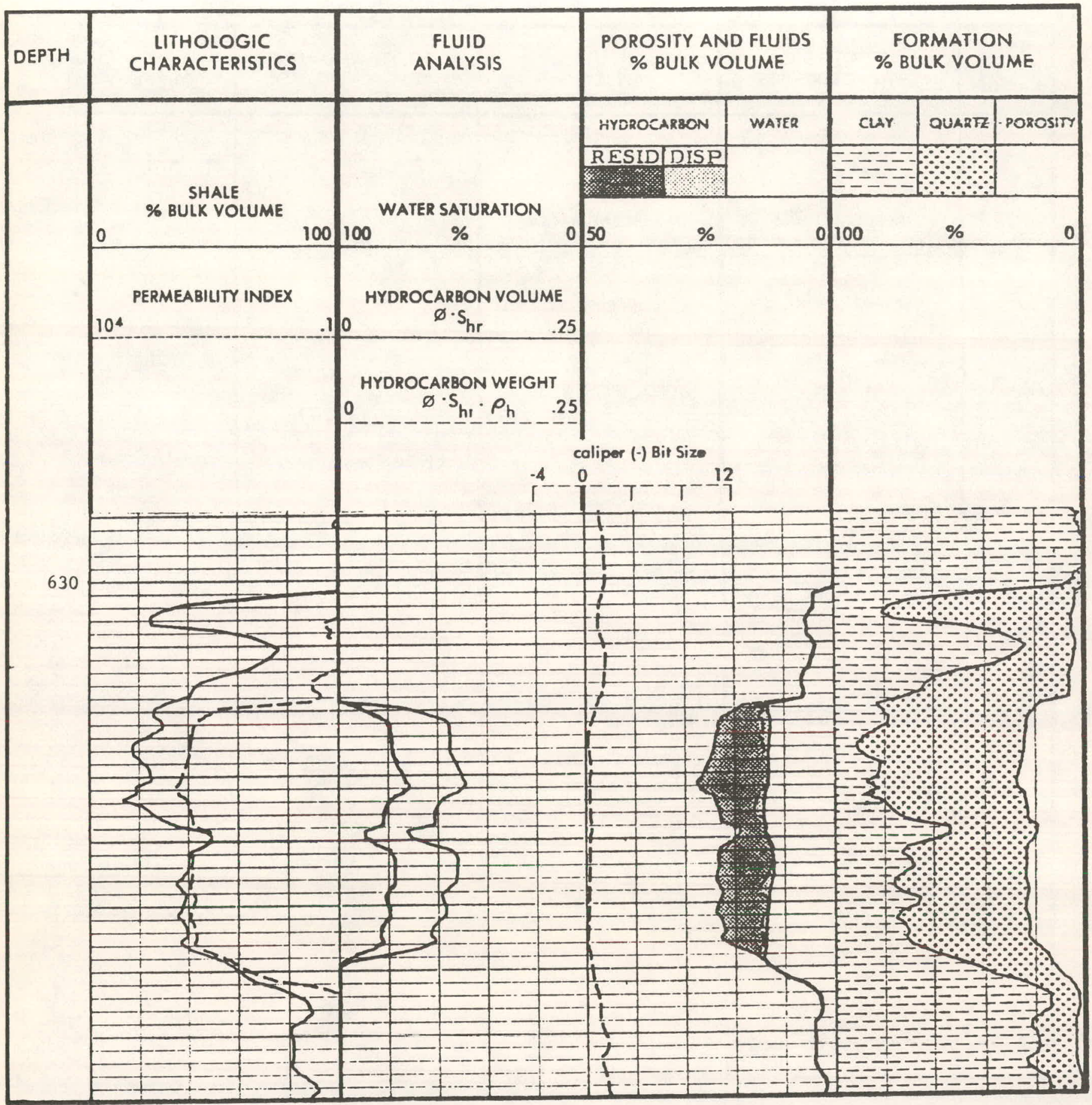


FIGURE $\mathrm{C}-43$

EPILOG FOR WELL MP-207

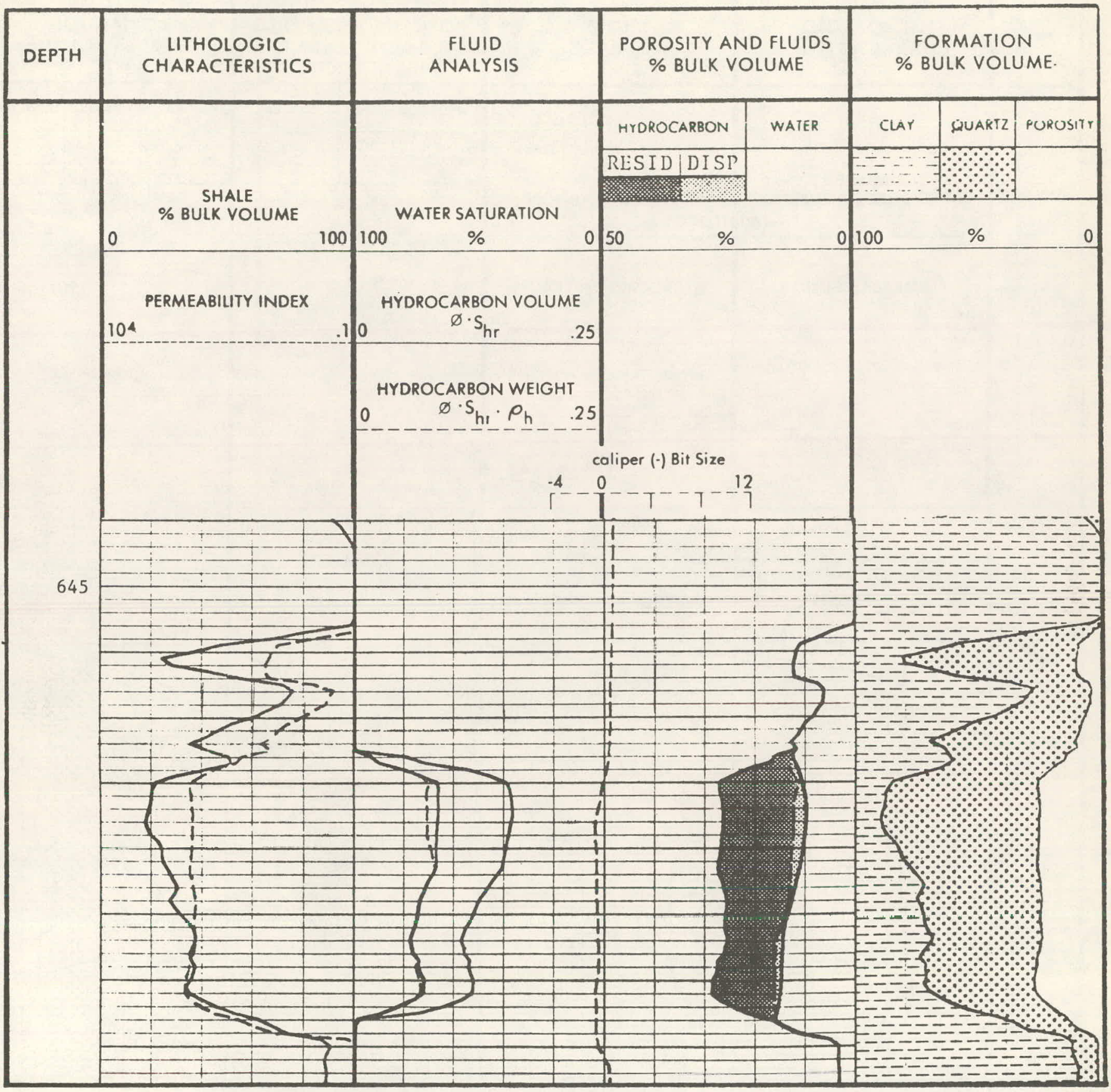


FIGURE C-SA

EPILOG FOR WELL MP-208

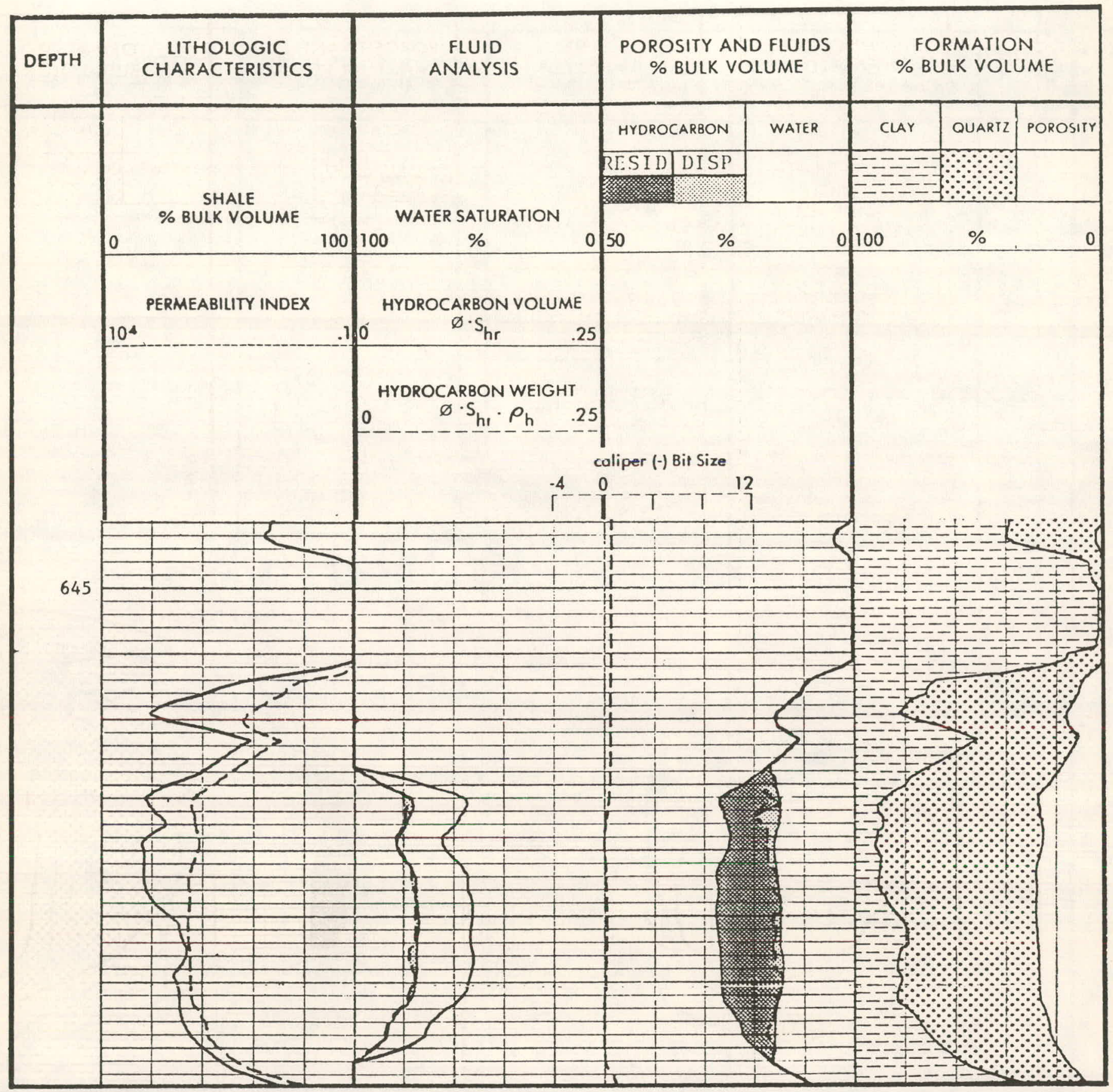


FIGURE $\mathrm{C}-45$

EPILOG FOR HELL IIP-209

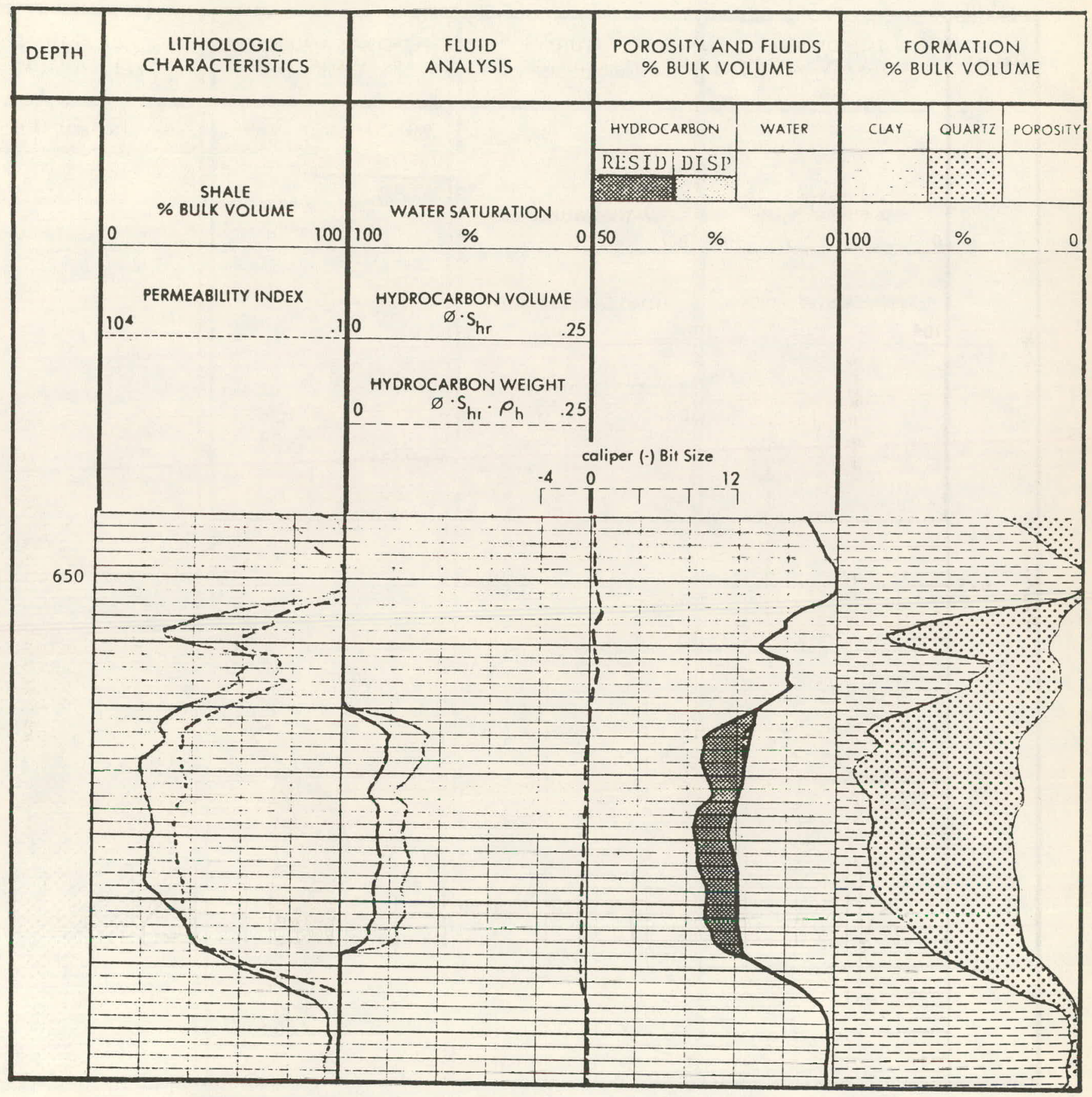


FIGURE C.MG

EPILOG FOR WELL IIP-210

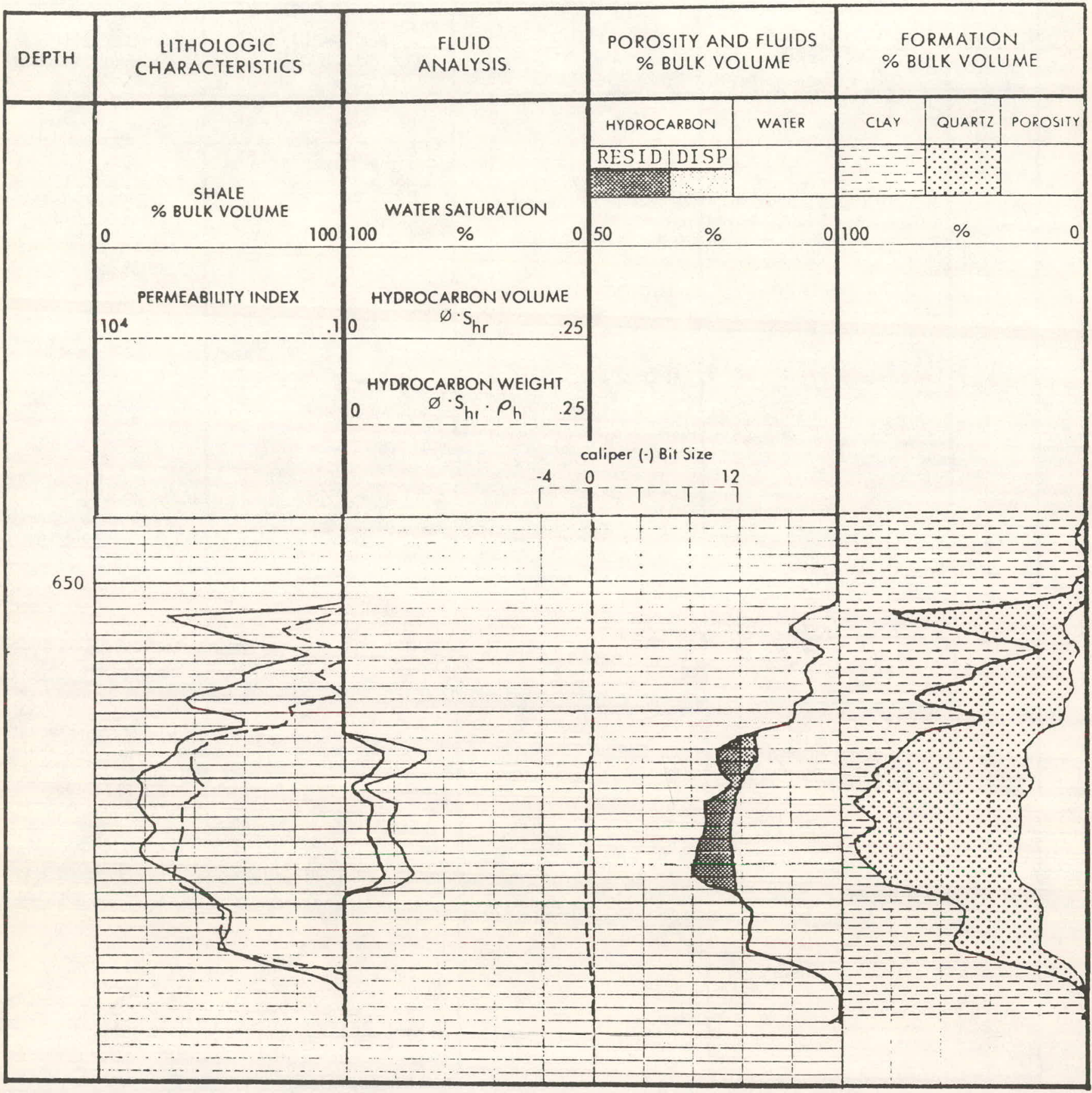


FIGLURE $\quad-17$

EPILOG FOR HELL [IP-211

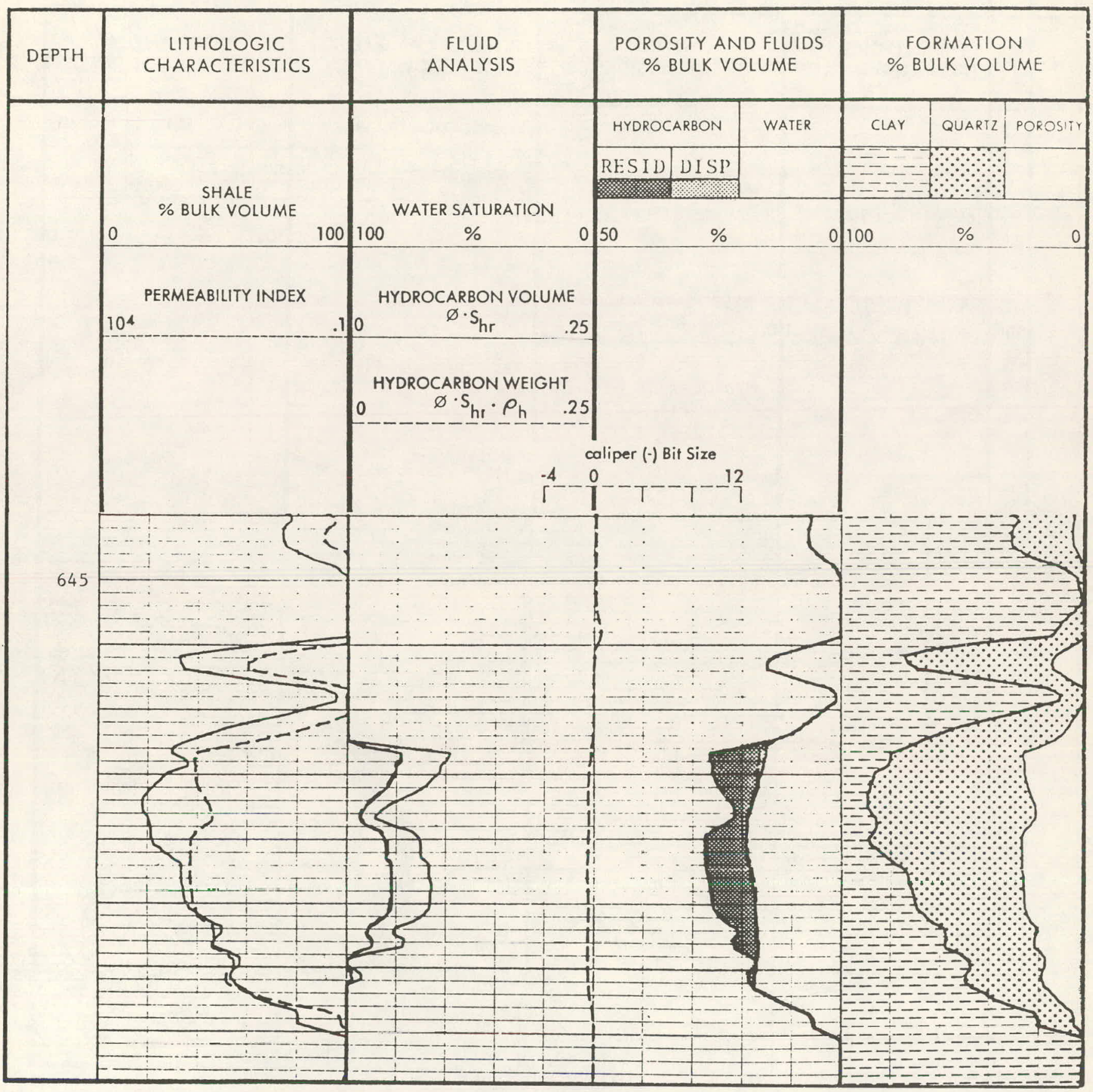


II-222

FIGURE C-48

EPILOG FOR IJELL IIP-212

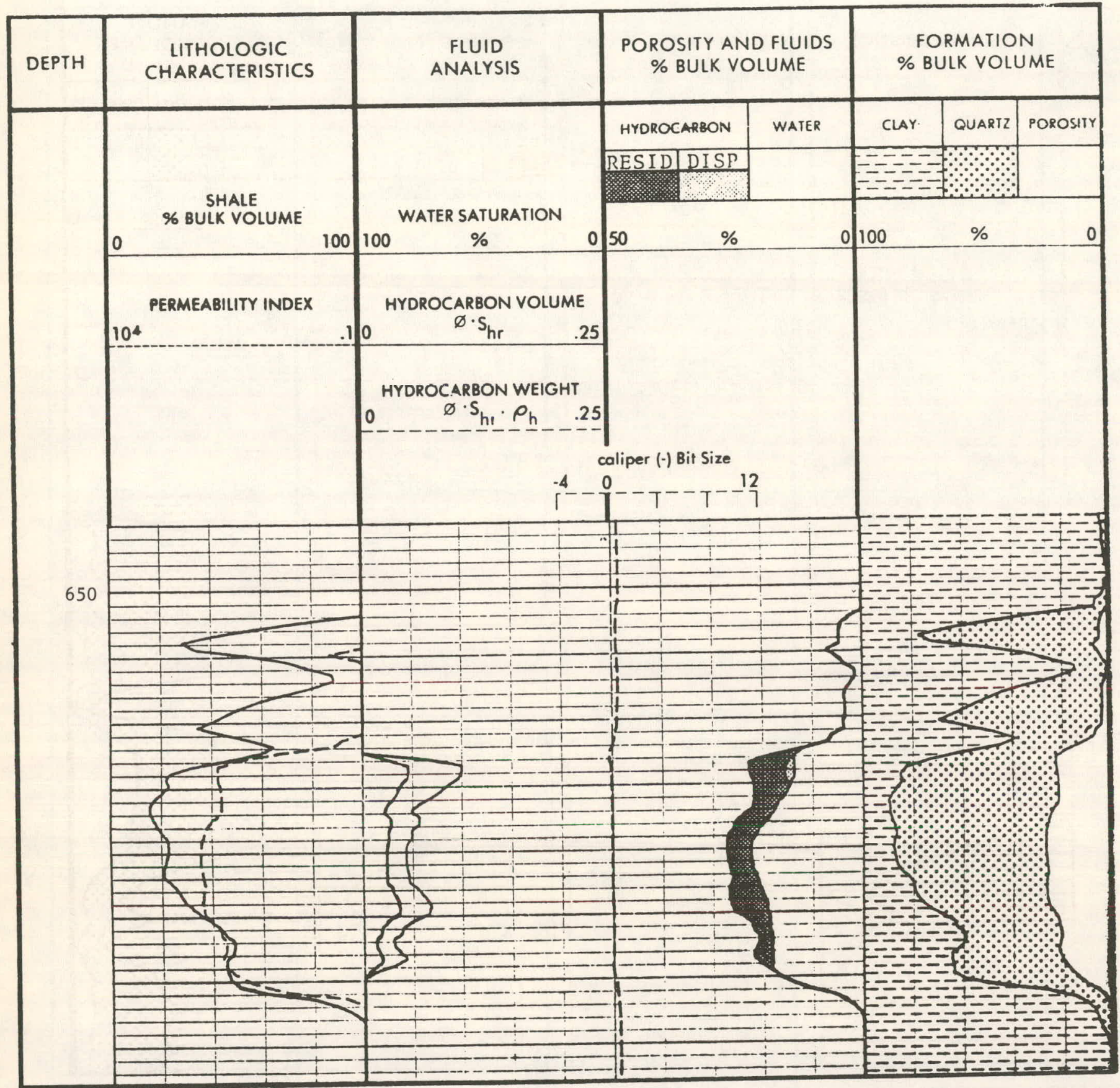


FIGURE $\quad$ C-49

EPILOG FOR WELL MP-213

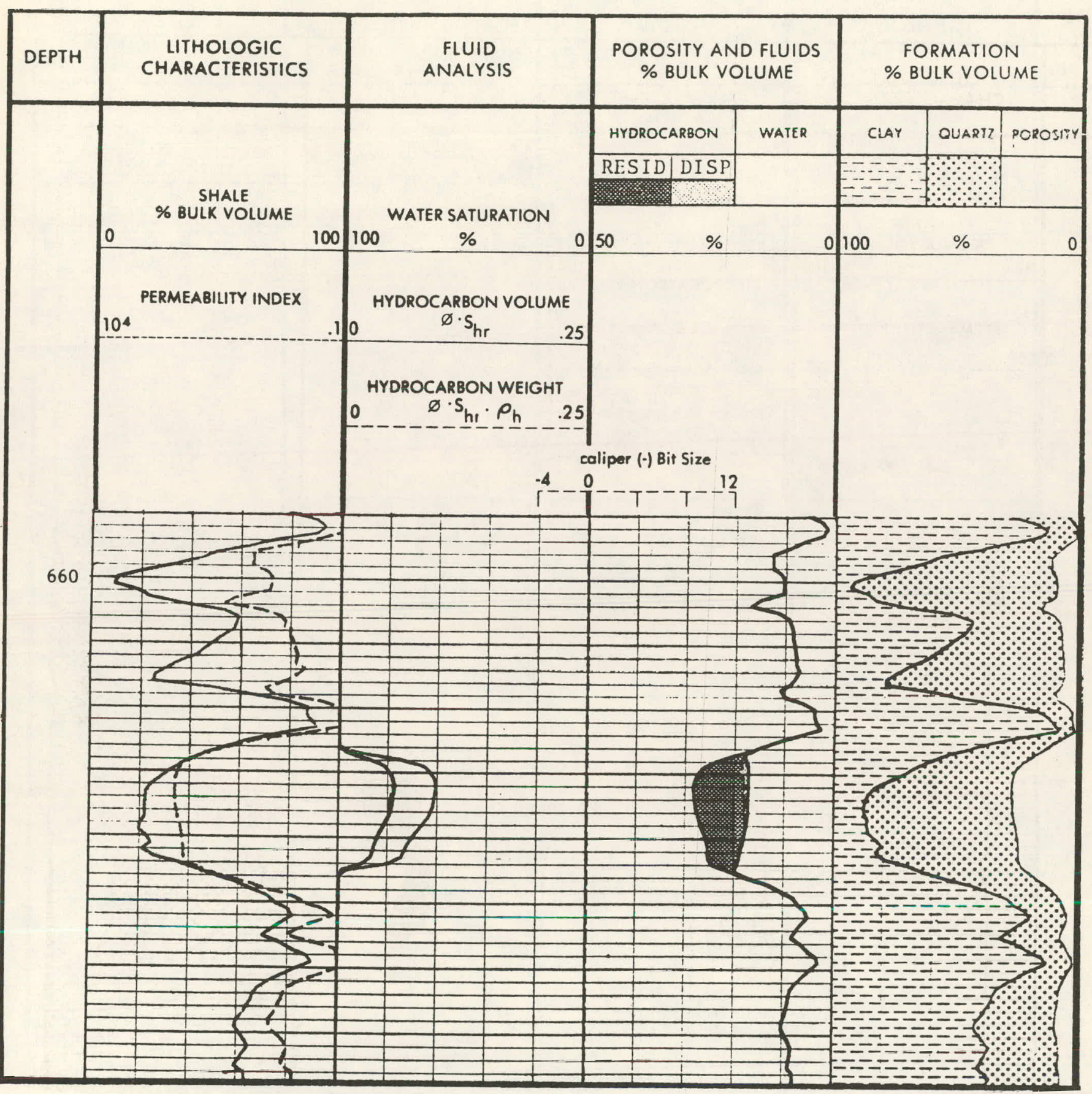


EPILOG FOR HELL IIP-214

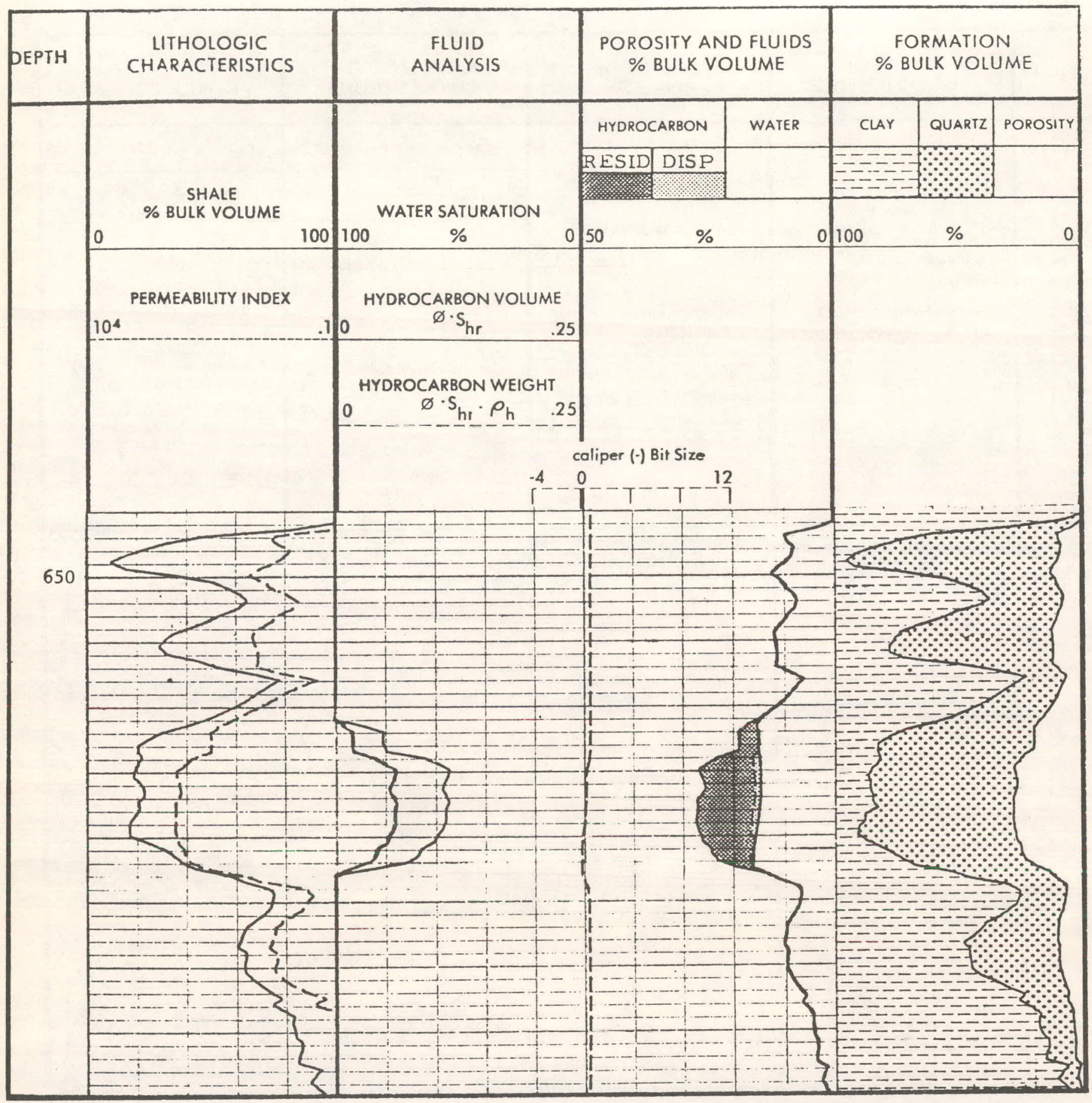


FIGURE C-51

EPILOG FOR WELL IIP-215

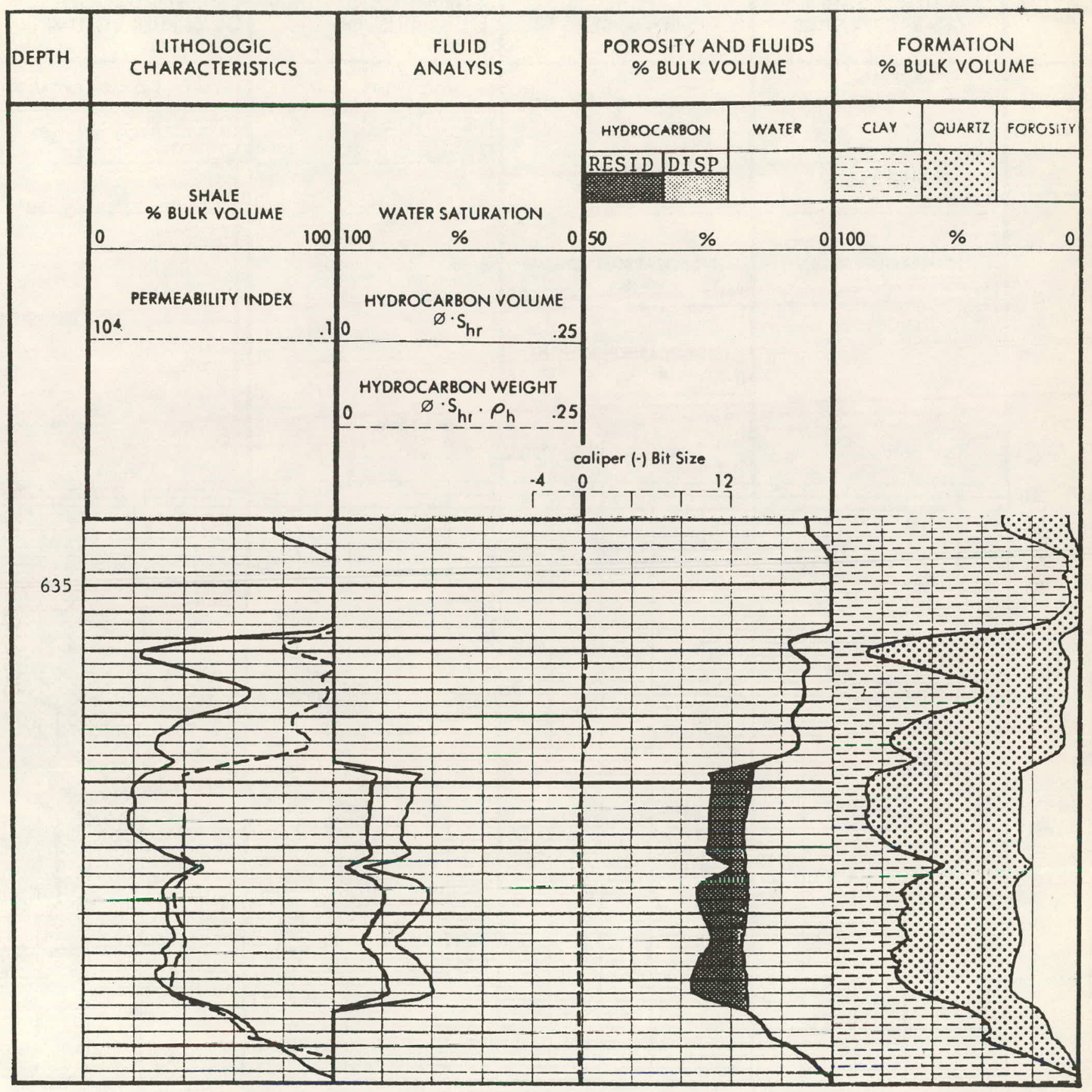


FIGURE C-52

EPILOG FOR WELL MP-216

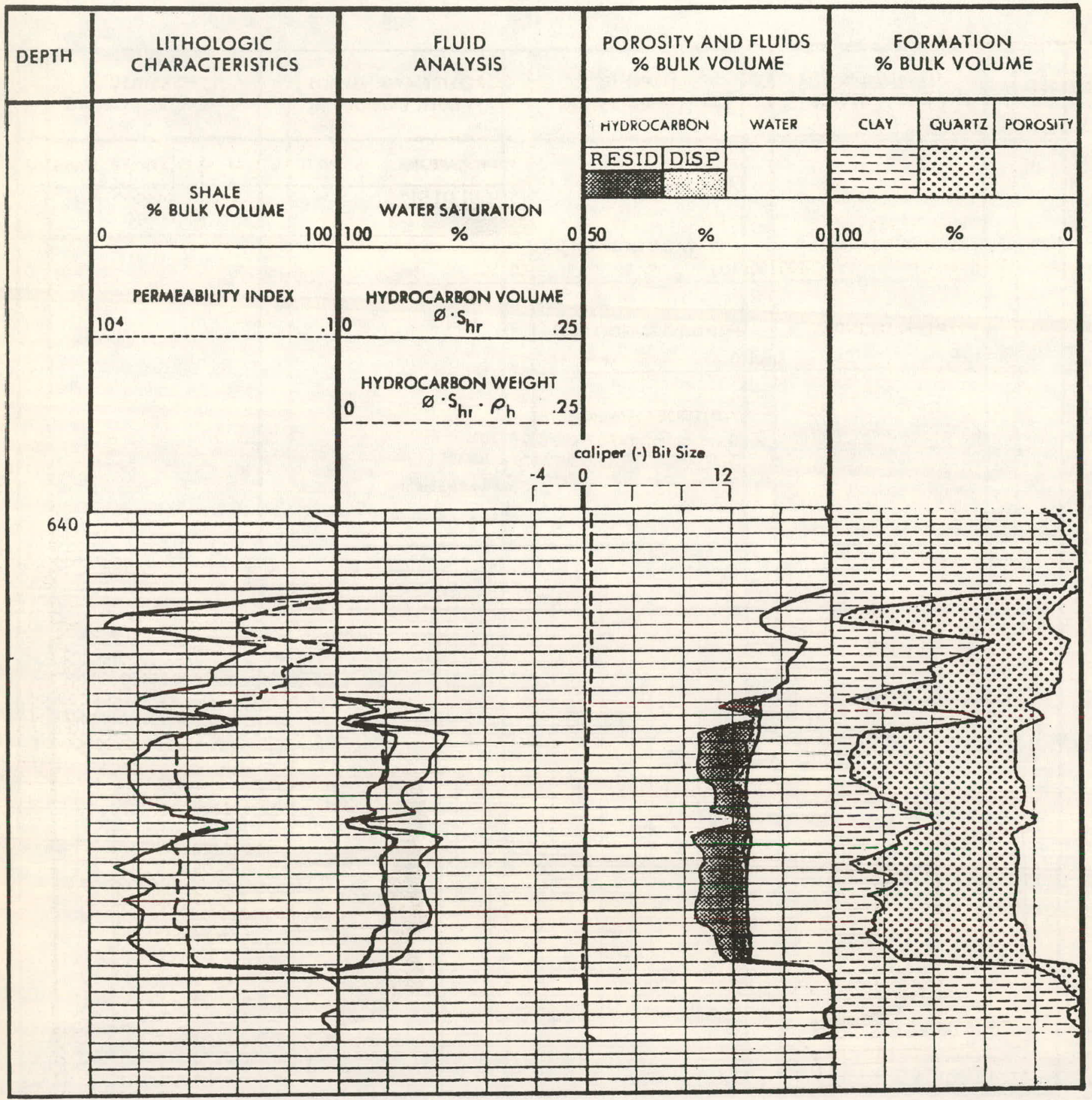


FIGURE C-53

EPILOG FOR WELL MP-217

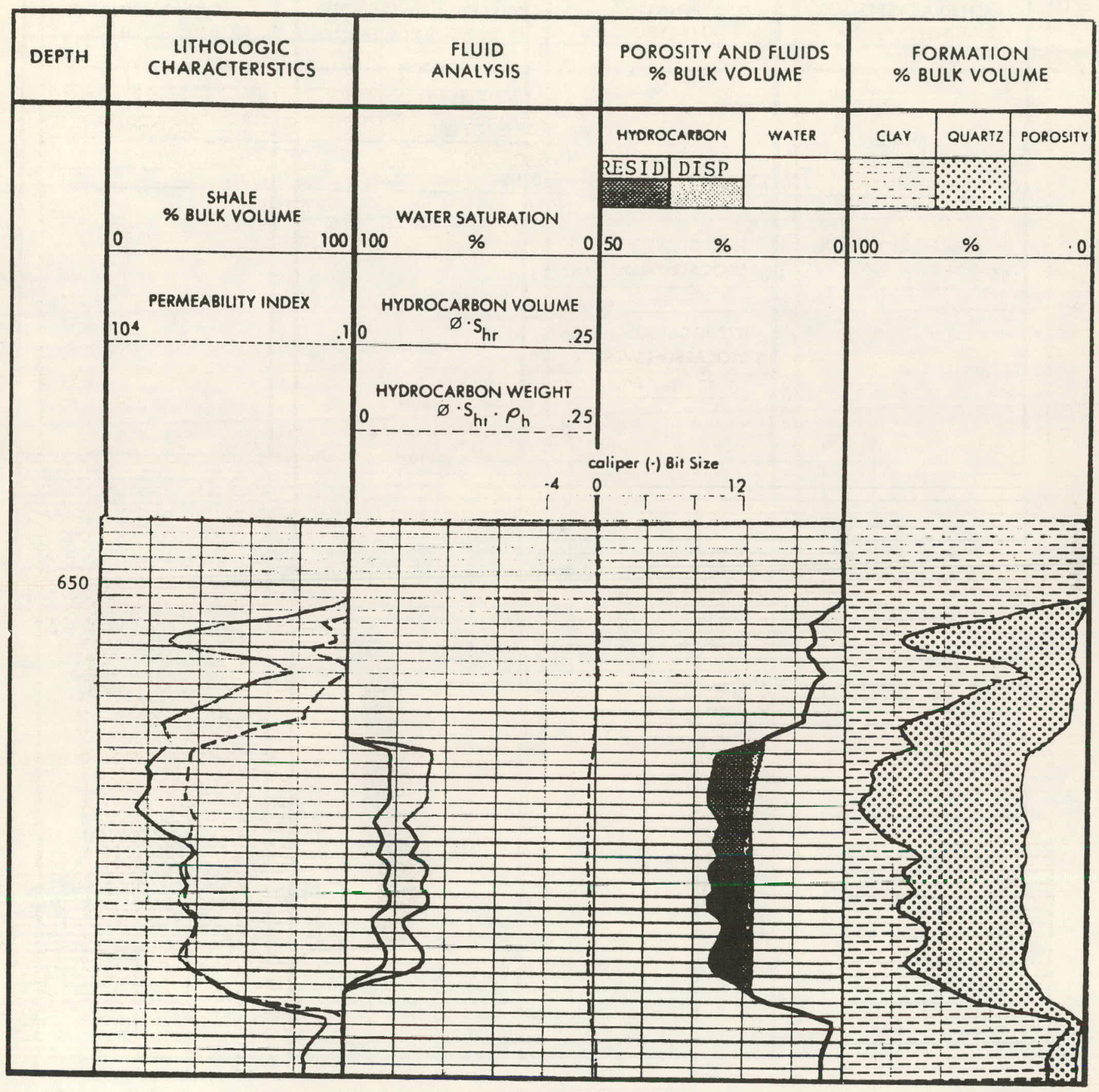


I I-228

FIGURE C-54

EPILOG FOR WELL IIP-218

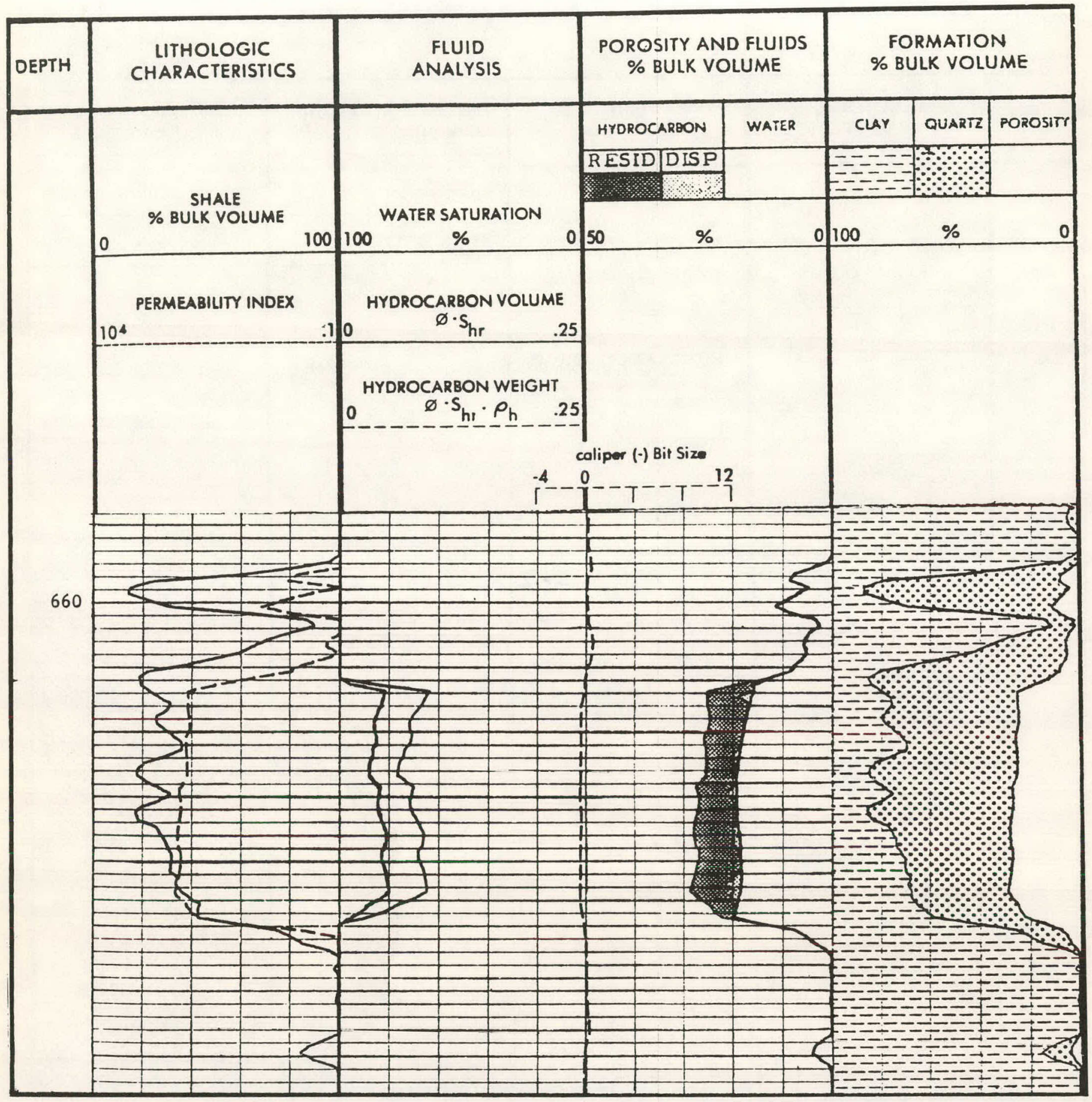


FIGURE C-55

EPILOG FOR WELL IIP-220

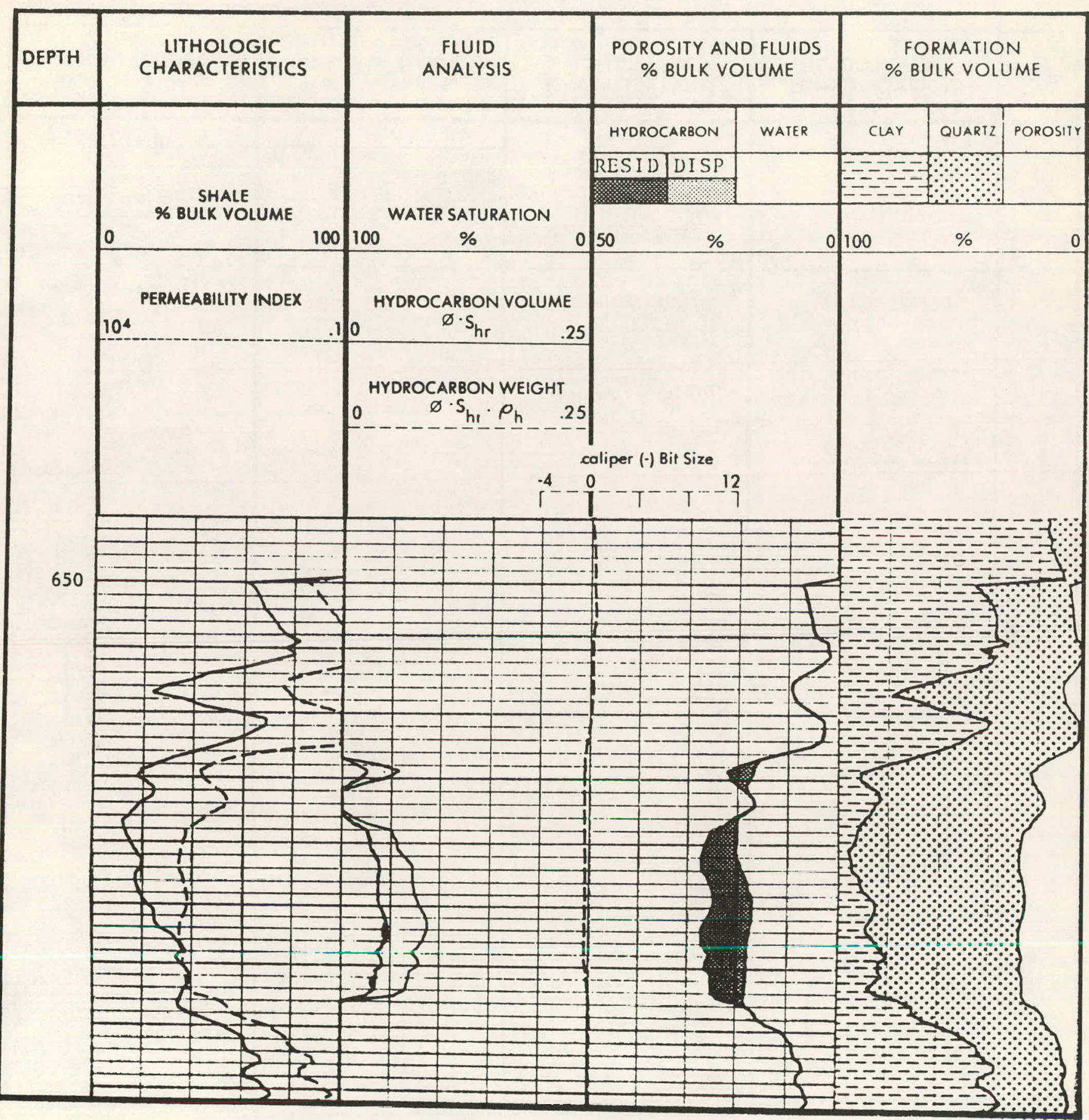


FIGURE C-56

EPILOG FOR WELL MP-221

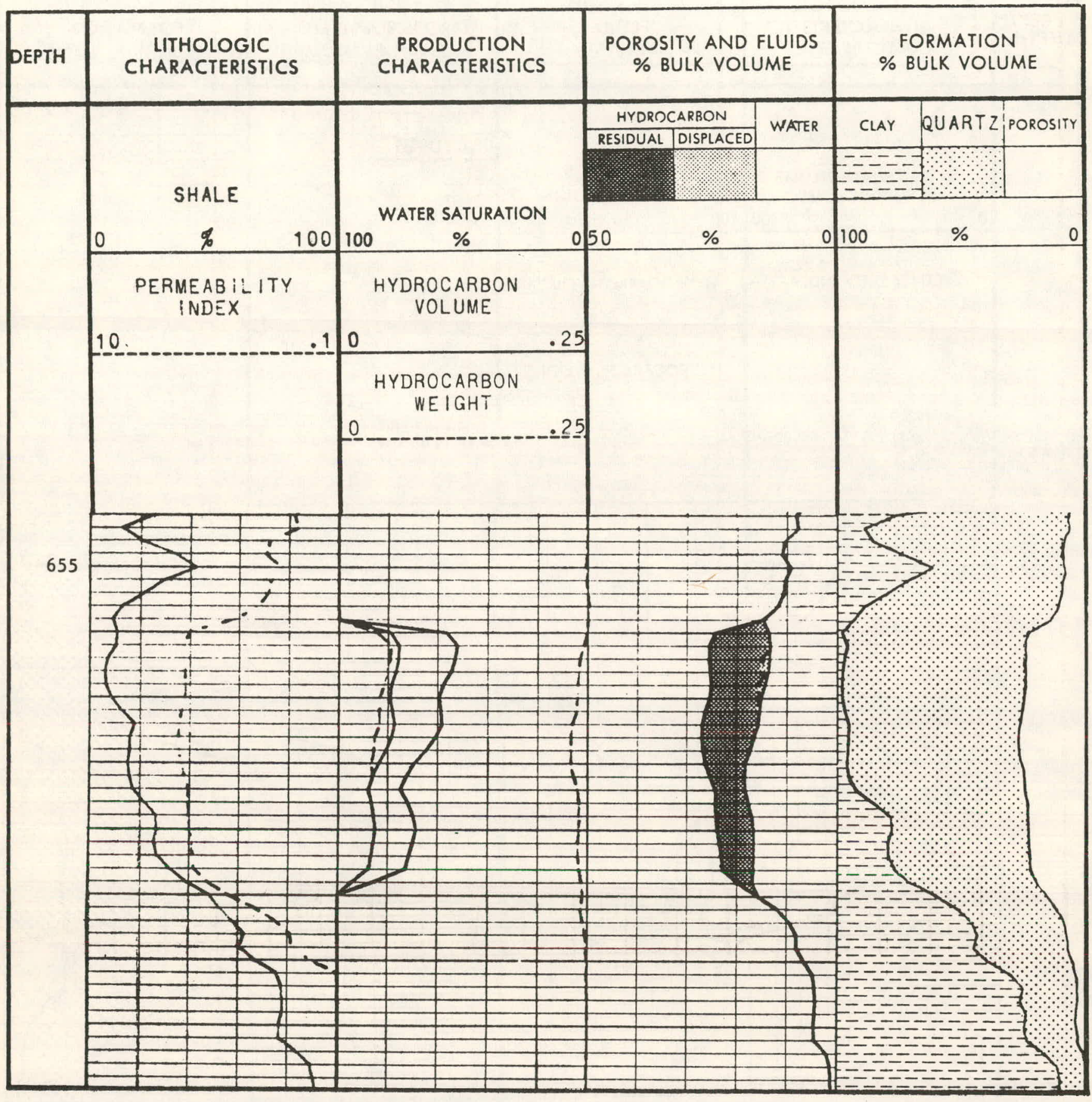


FIGURE C-57

EPILOG FOR WELL IIP-222

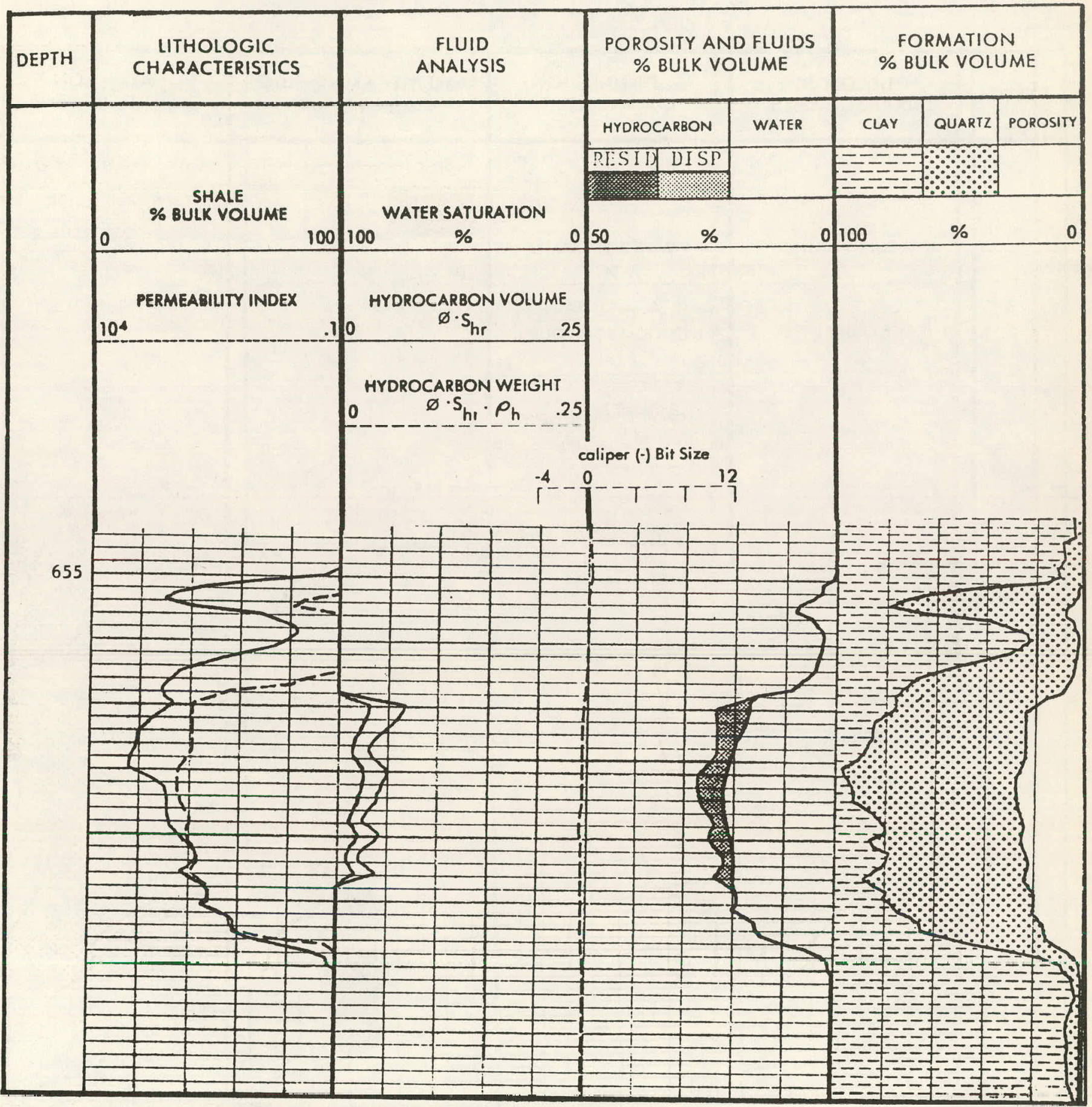


FIGURE C-58

EPILOG FOR WELL IIP-223

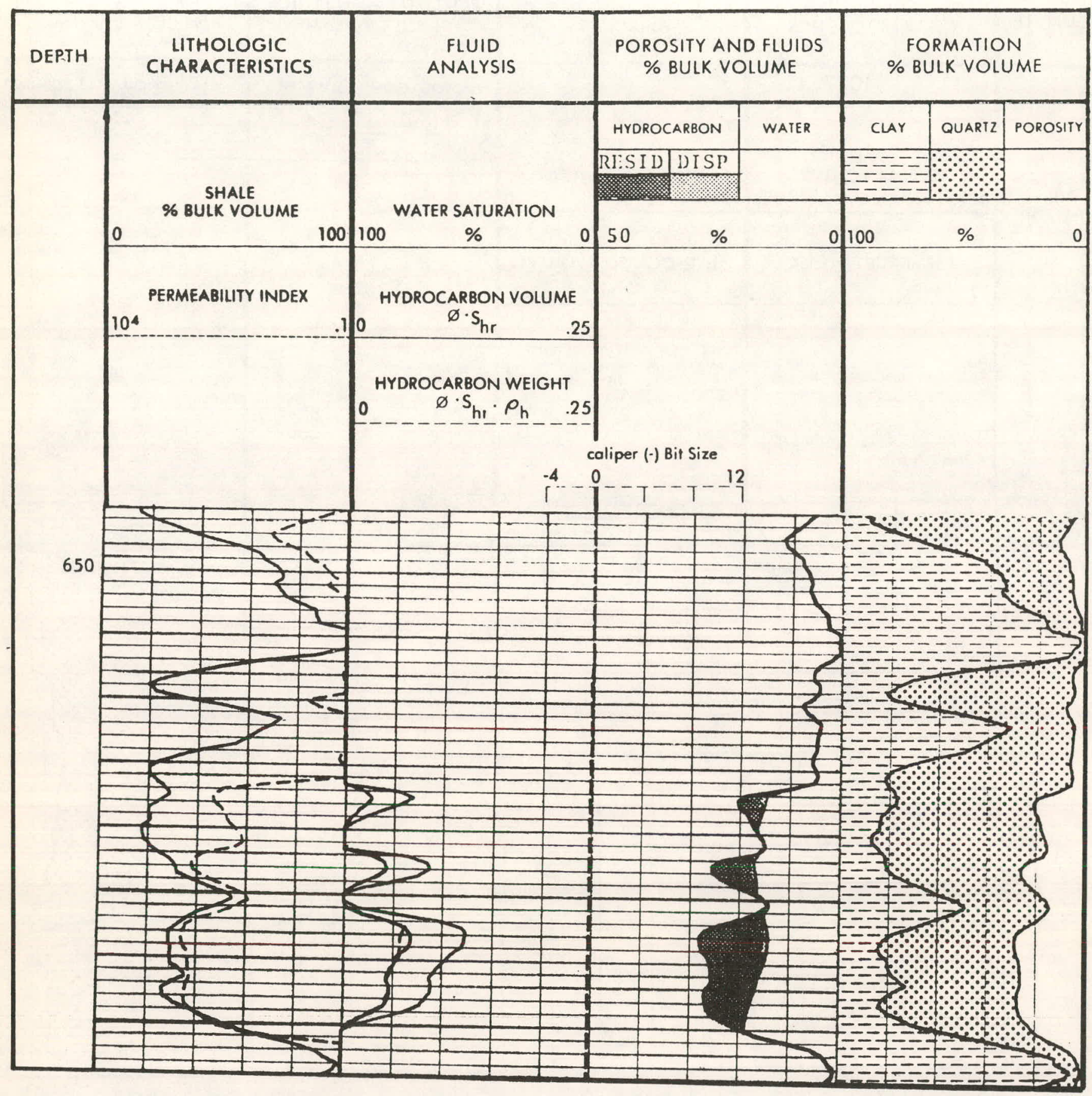


FIGURE C-59

EPILOG FOR HELL IIP-224

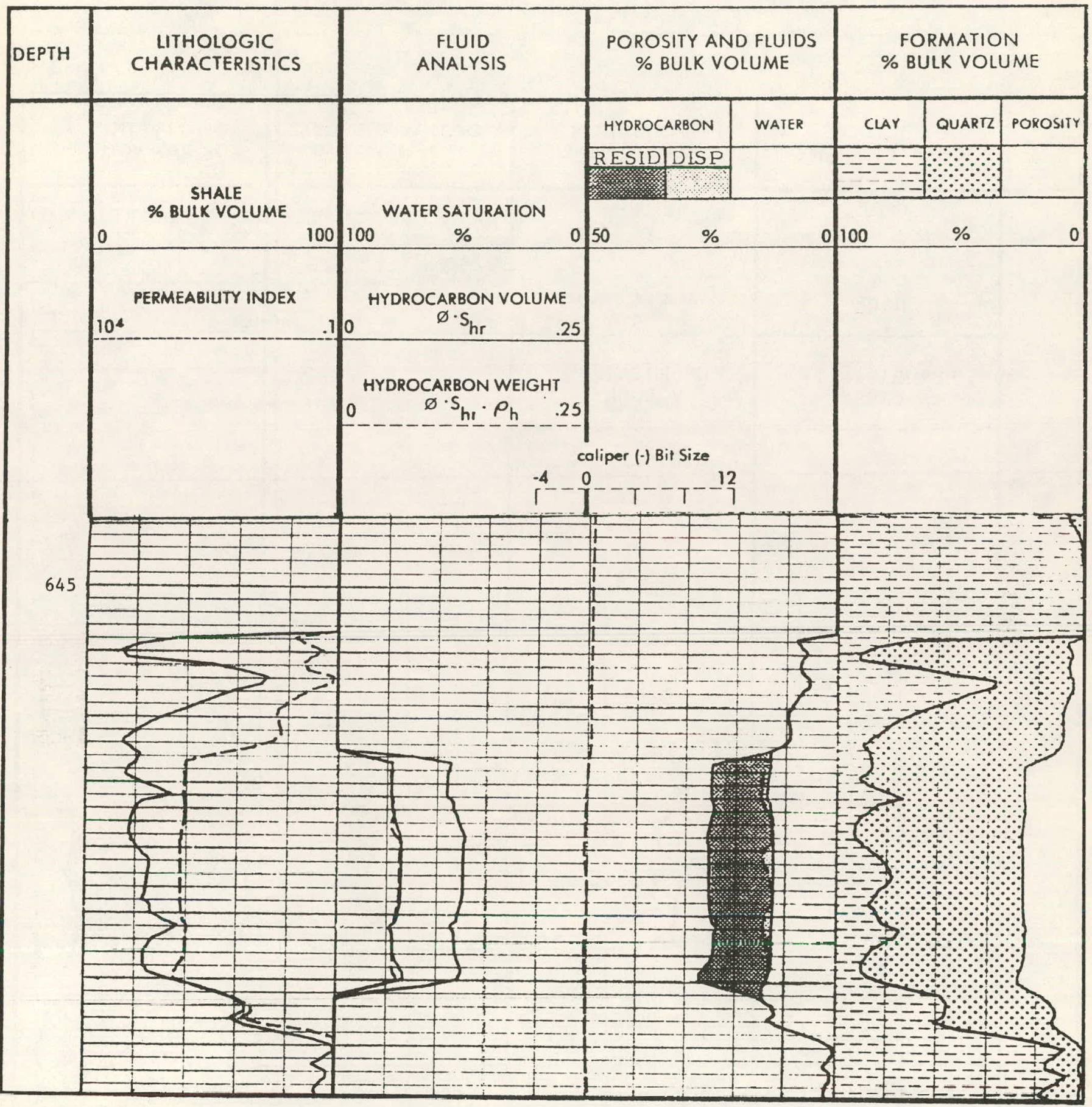


FIGURE $\quad C-60$

EPILOG FOR WELL IIP-225

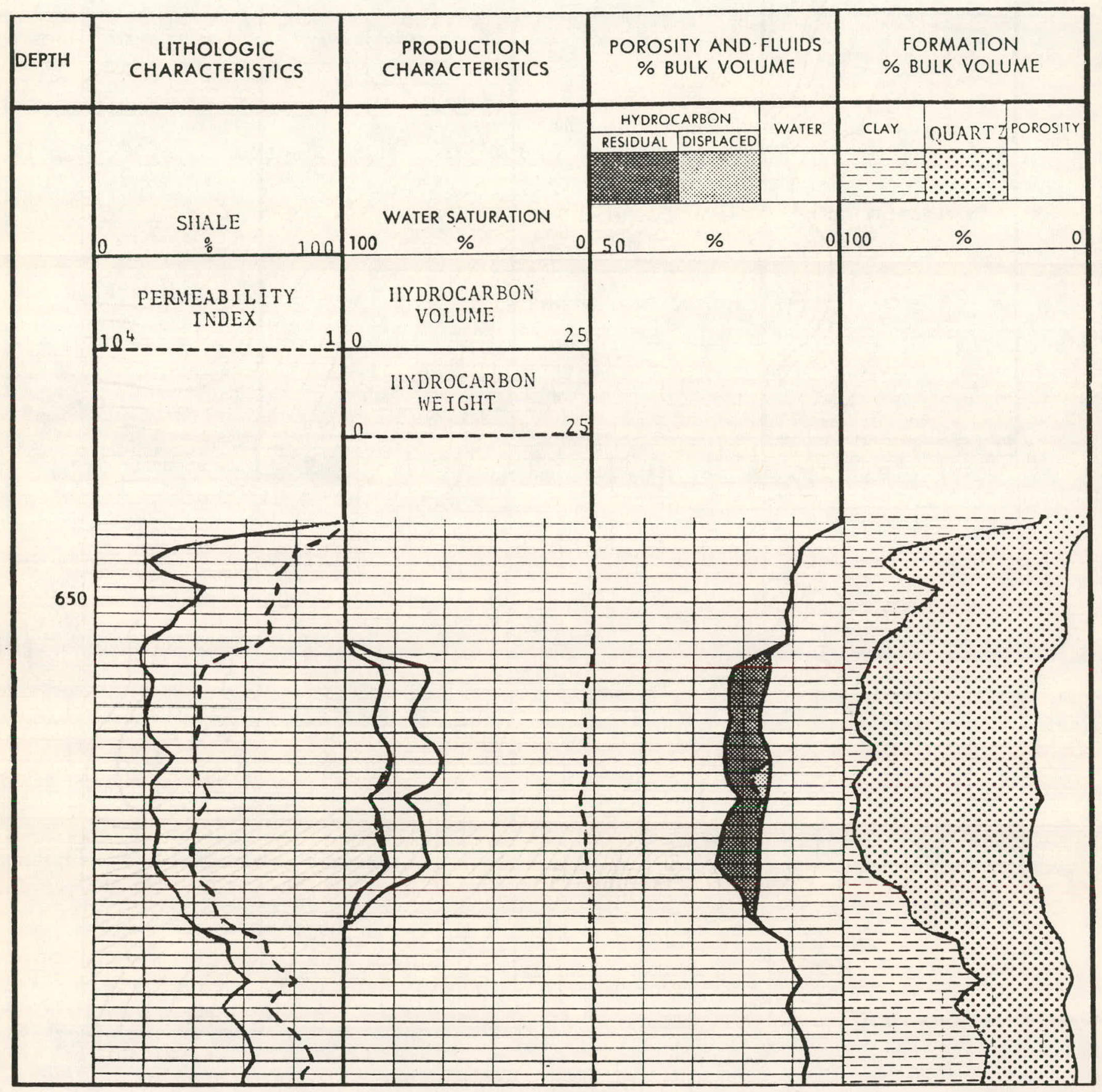




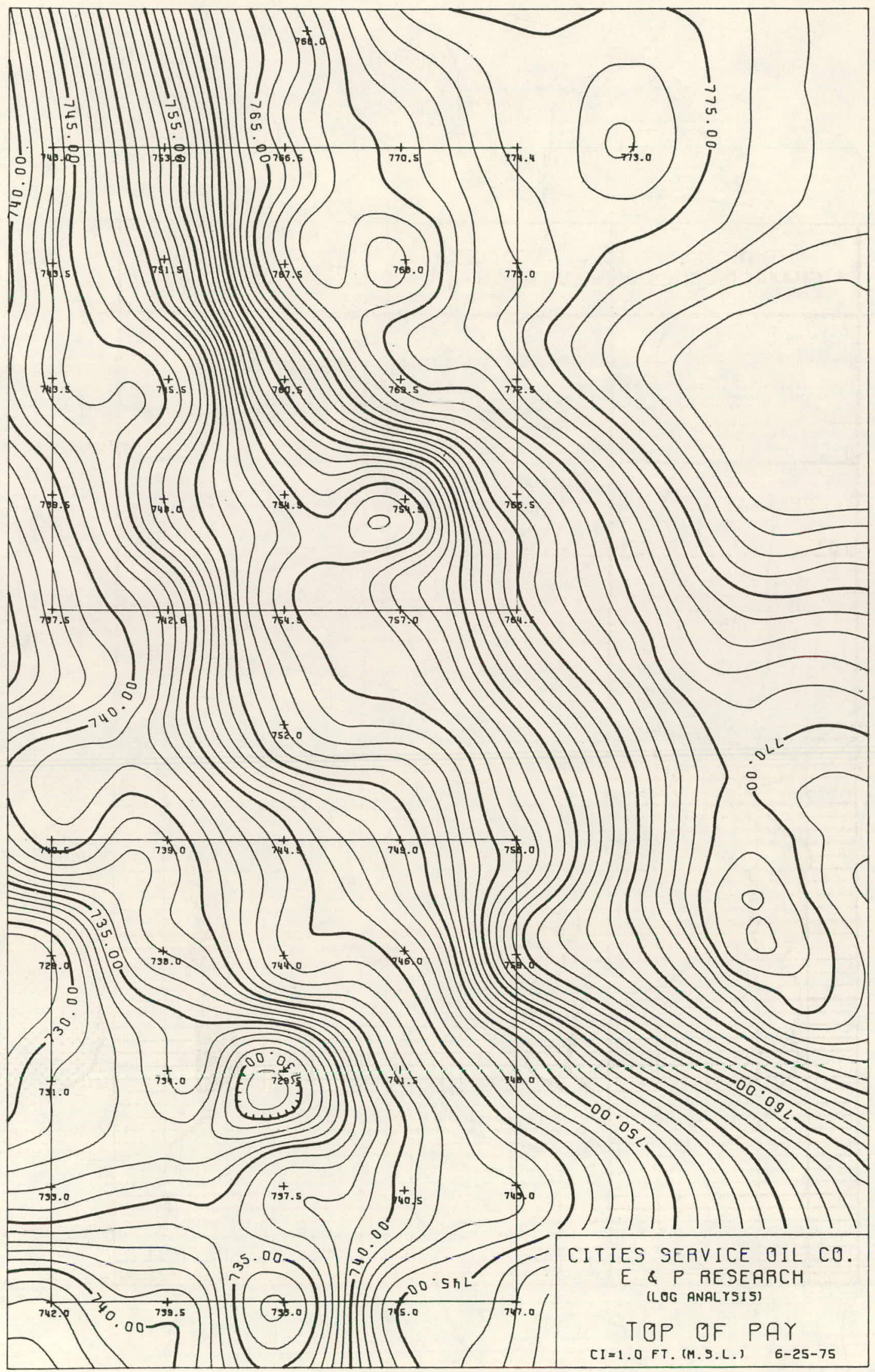

FIGURE C-61 


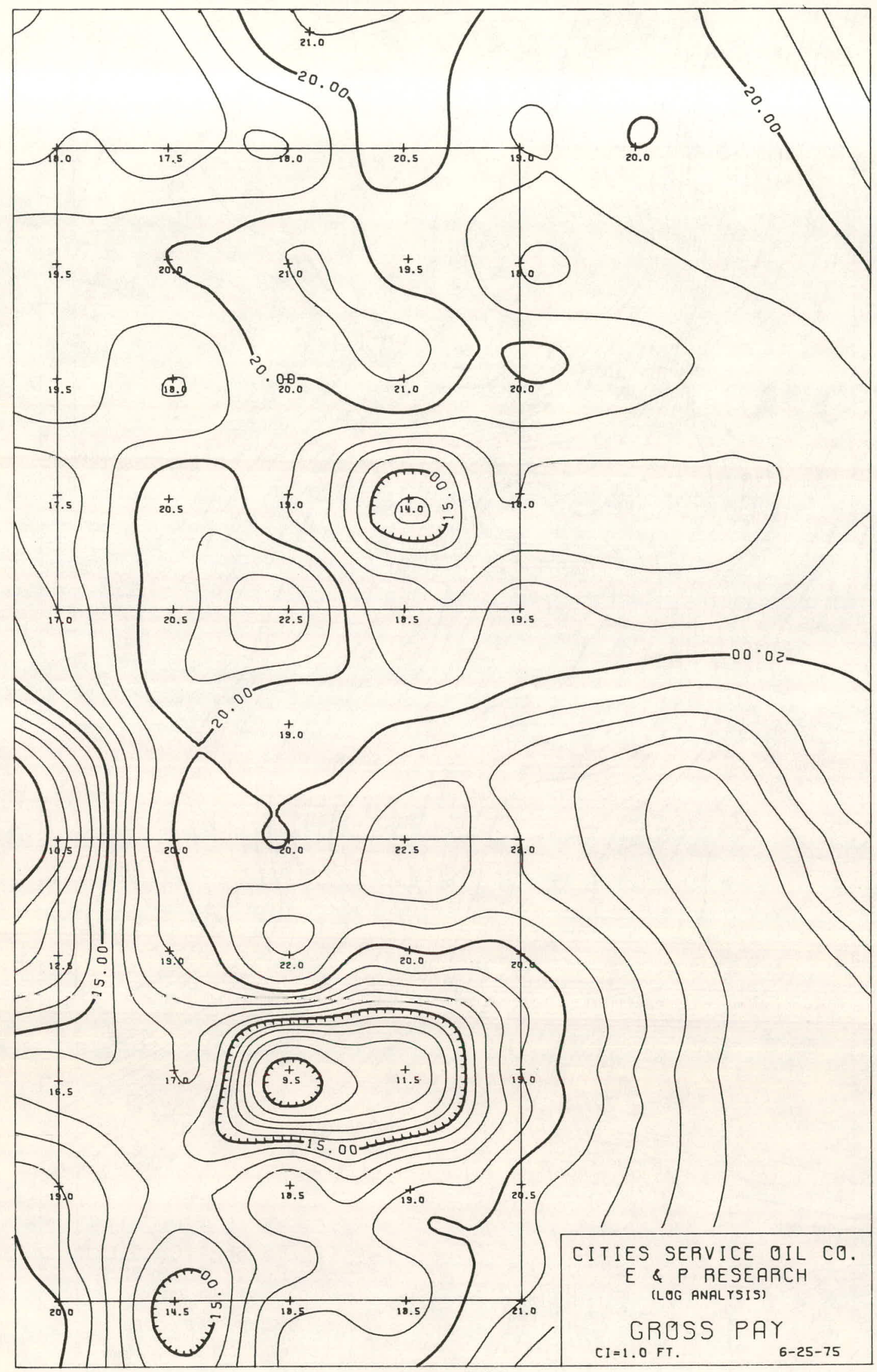

FIGURE C-62 


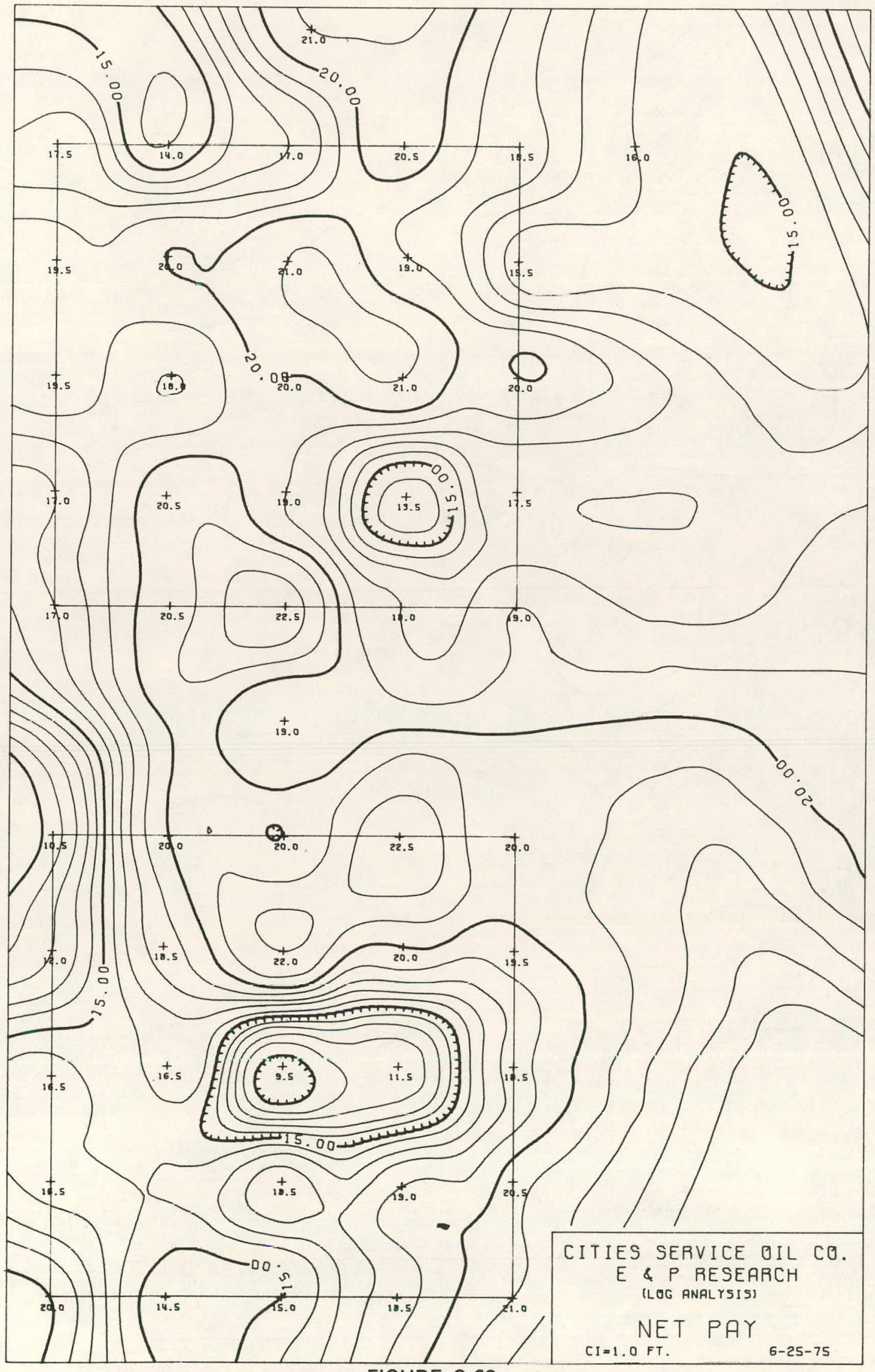

FIGURE C-63 


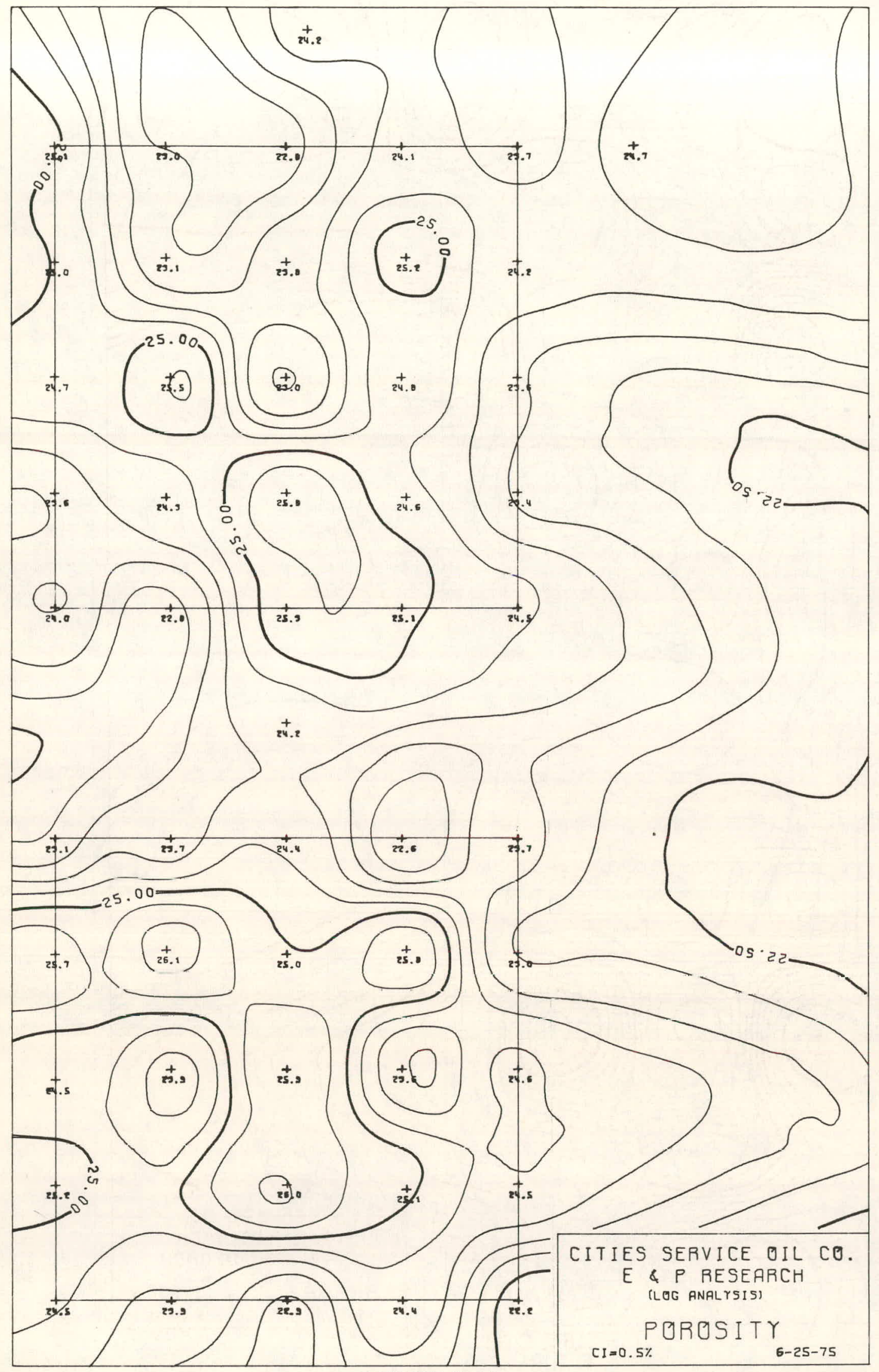




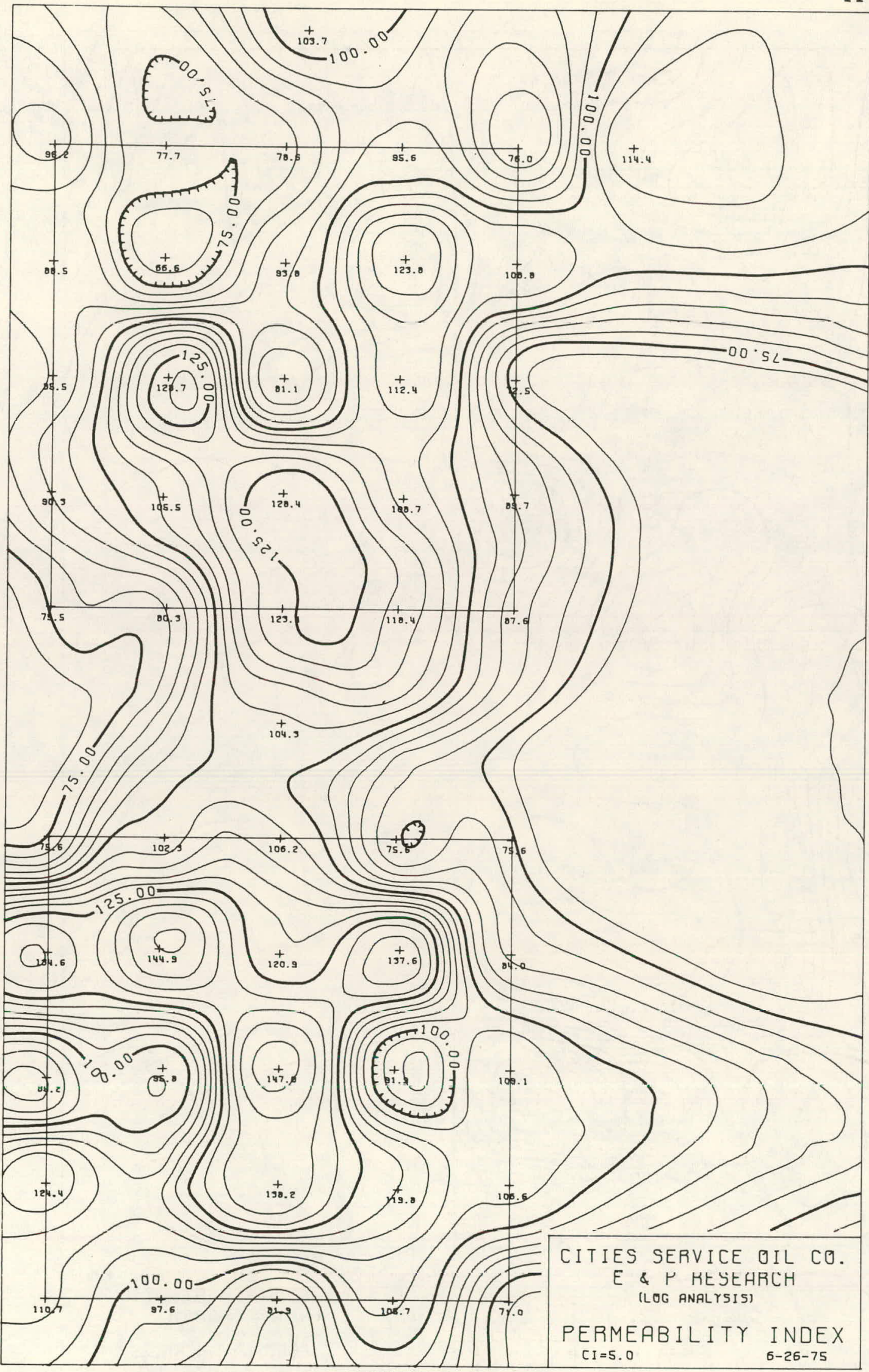

FIGURE C-65 


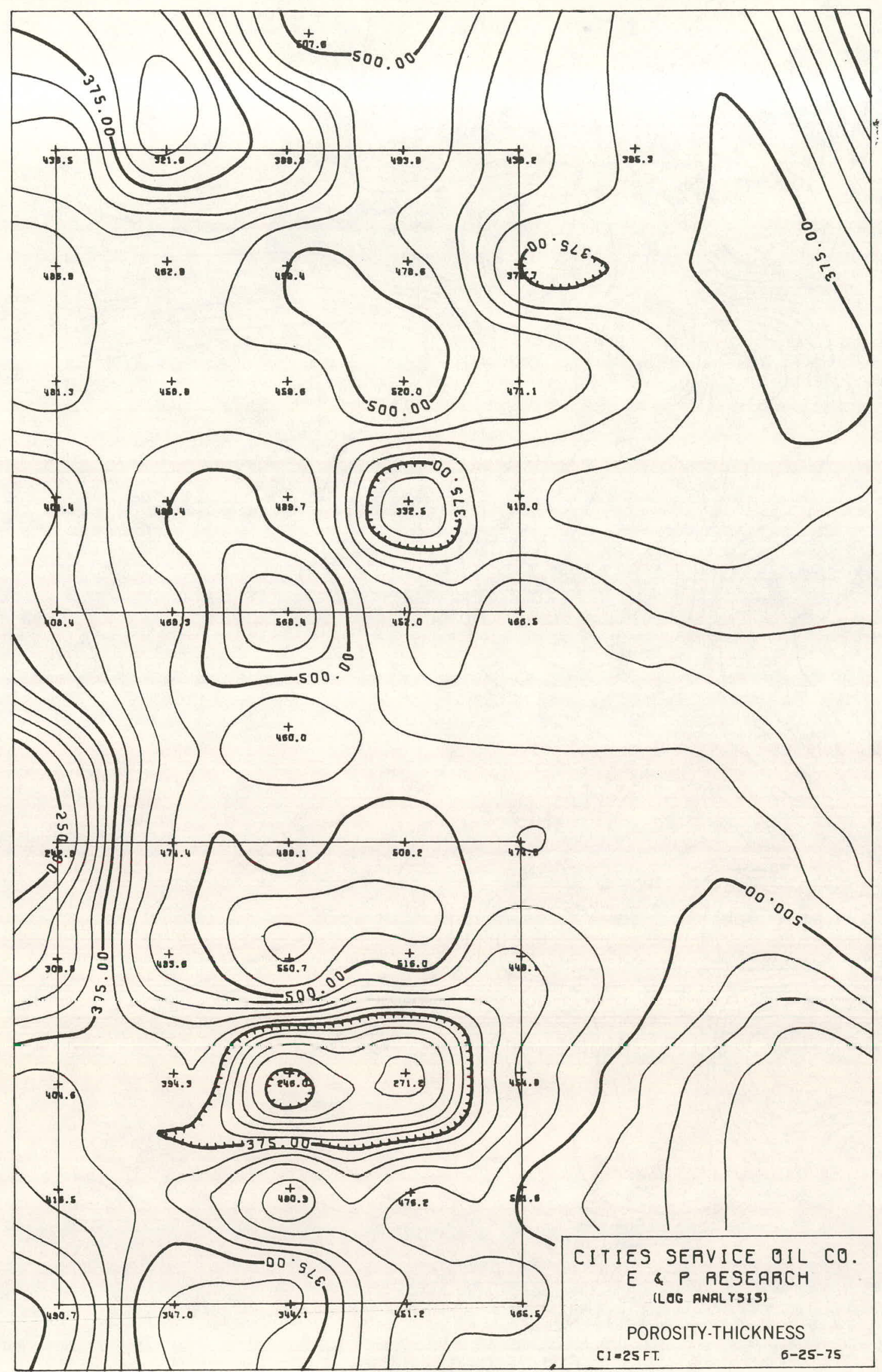

FIGURE C-66 


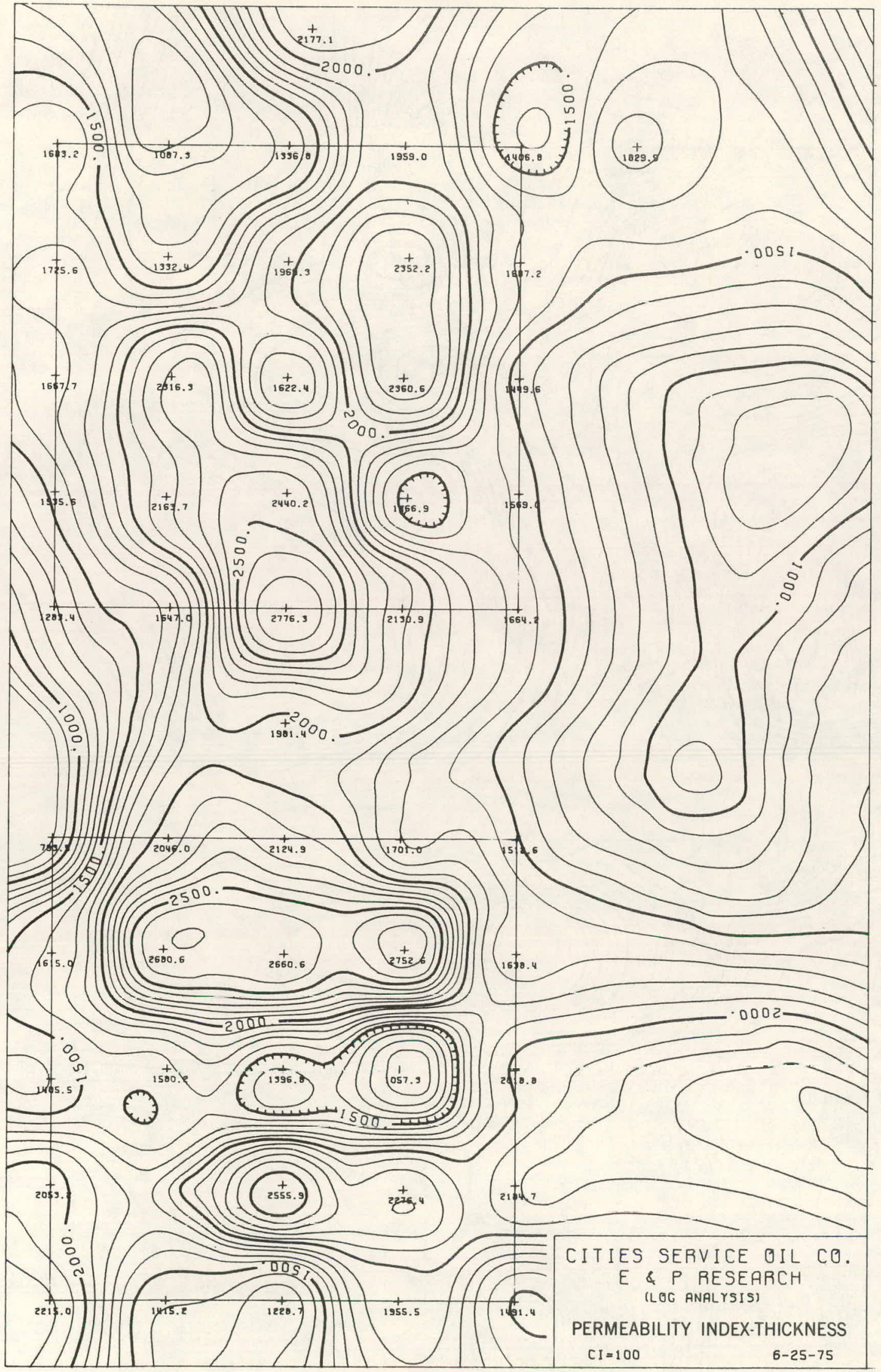

FIGURE C-67 


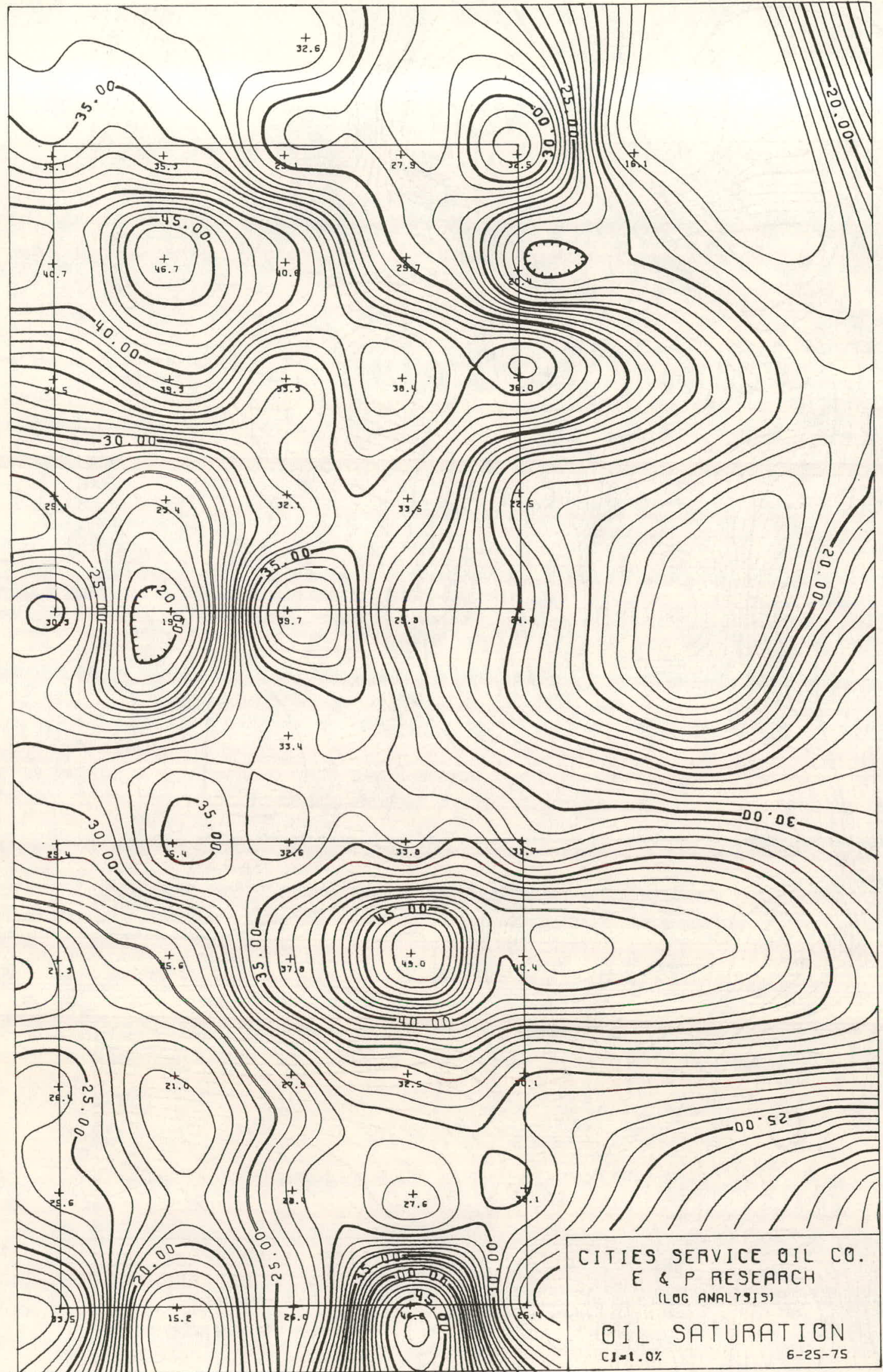

FIGURE C-68 


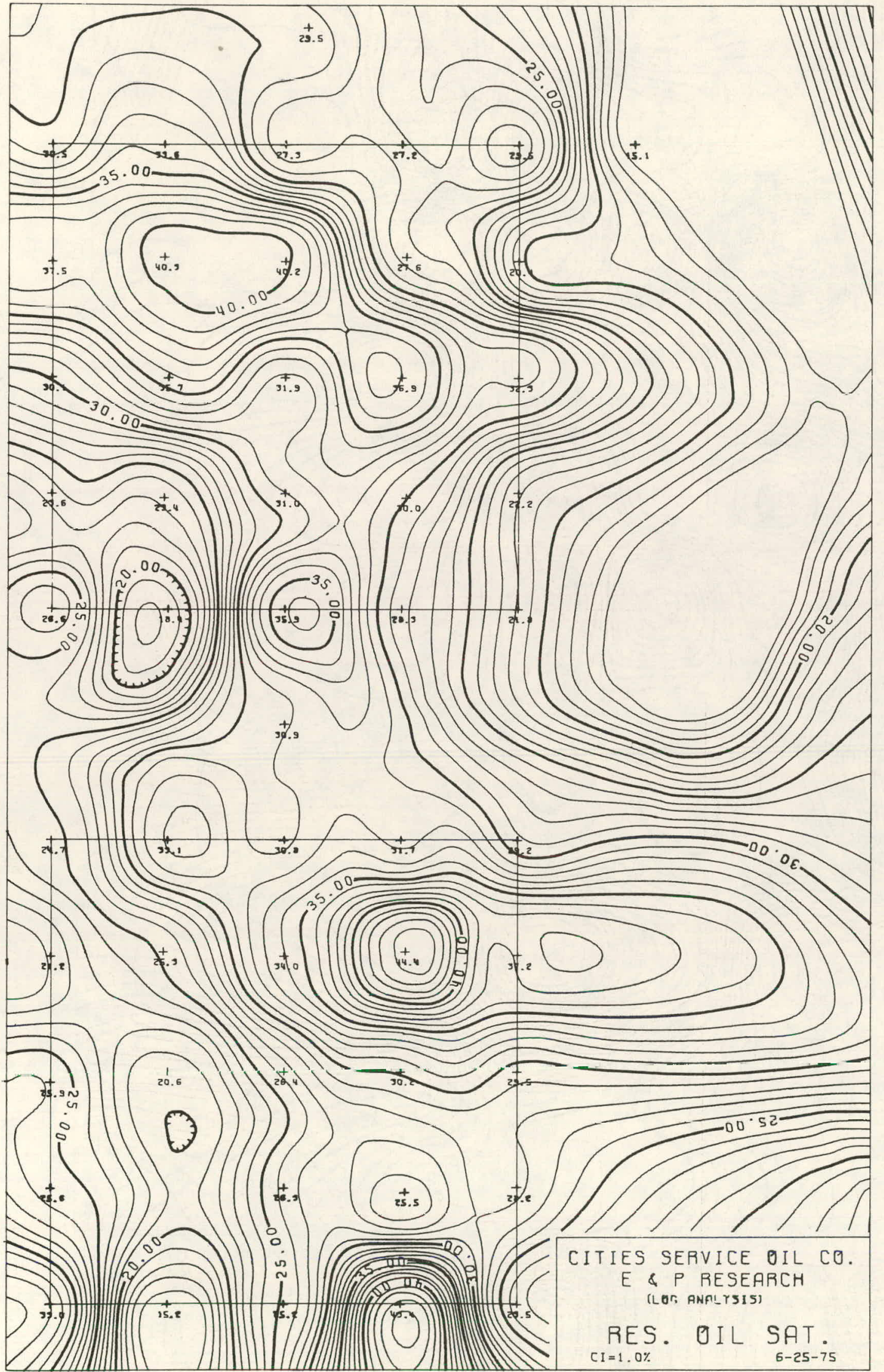

FIGURE C-69 


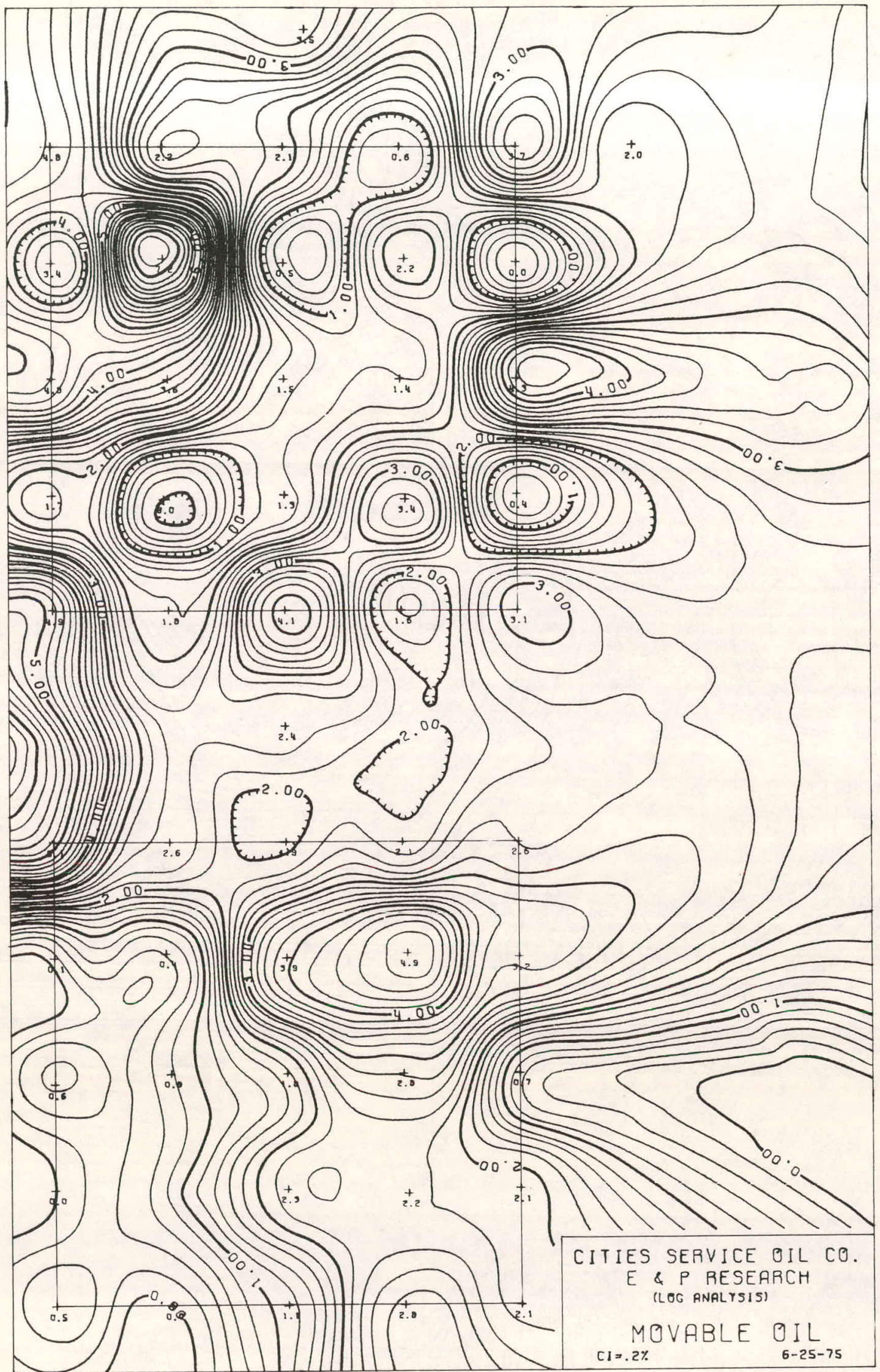

FIGURE C-70 


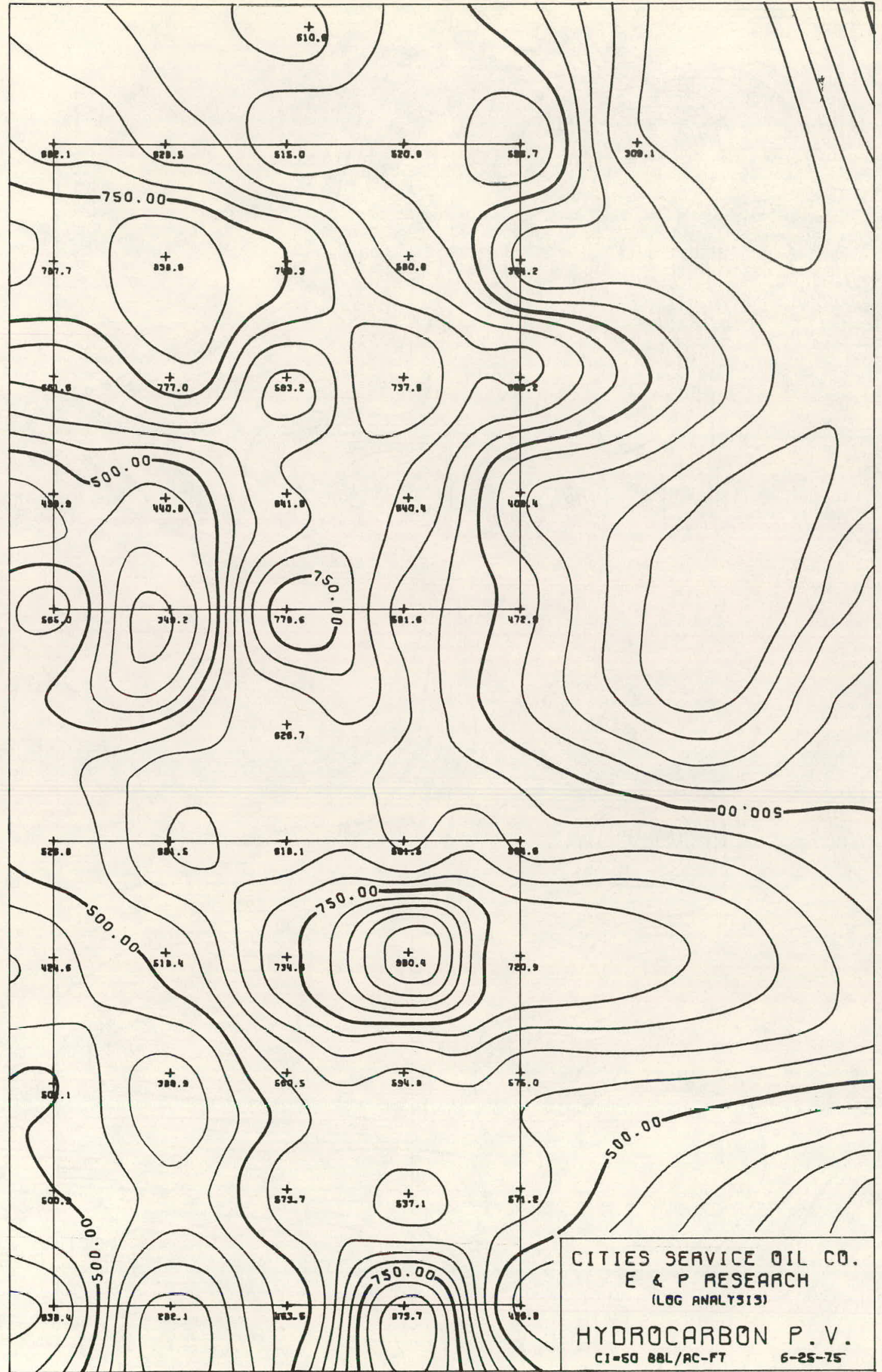

FIGURE C-71 


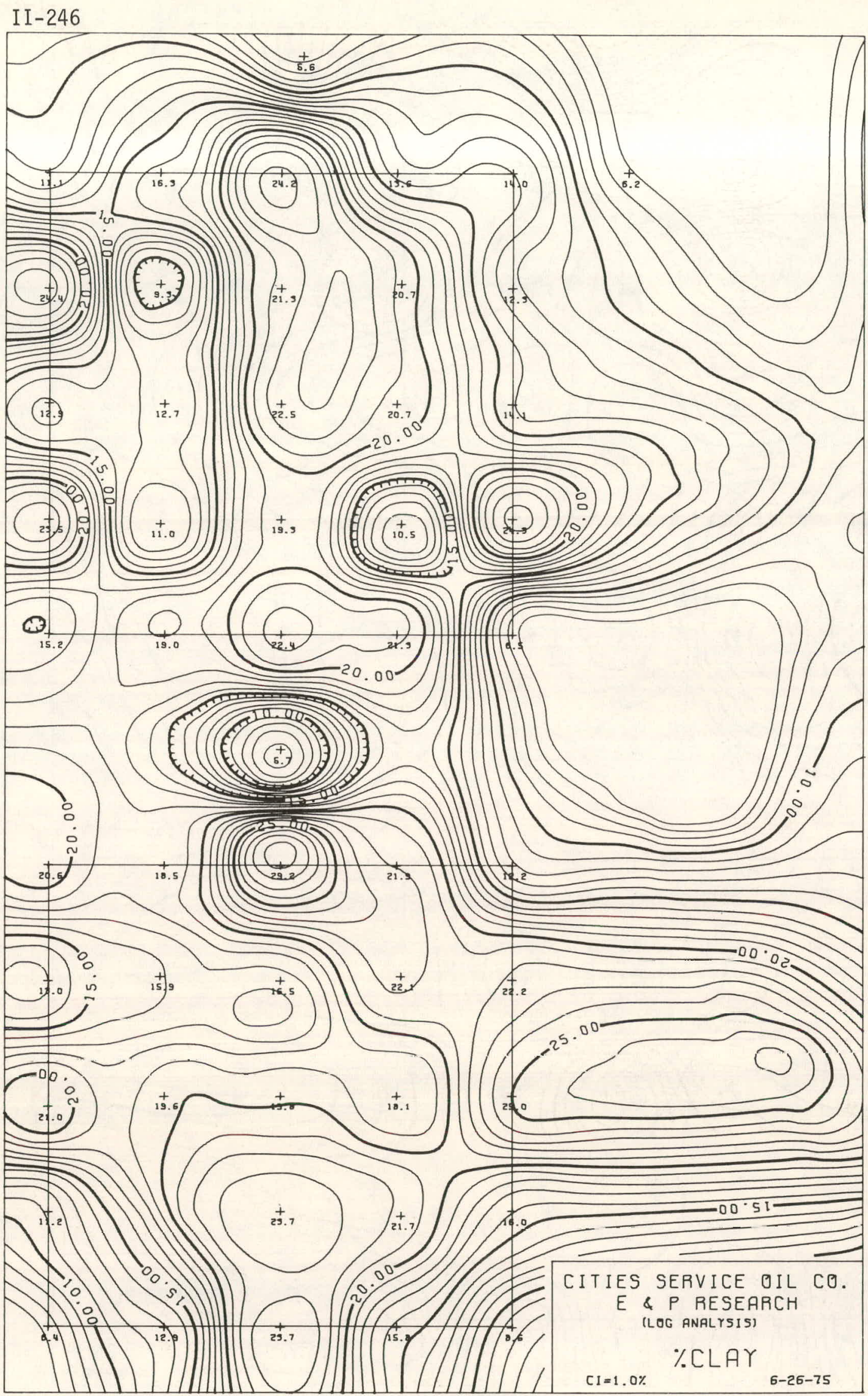

FIGURE C-72 


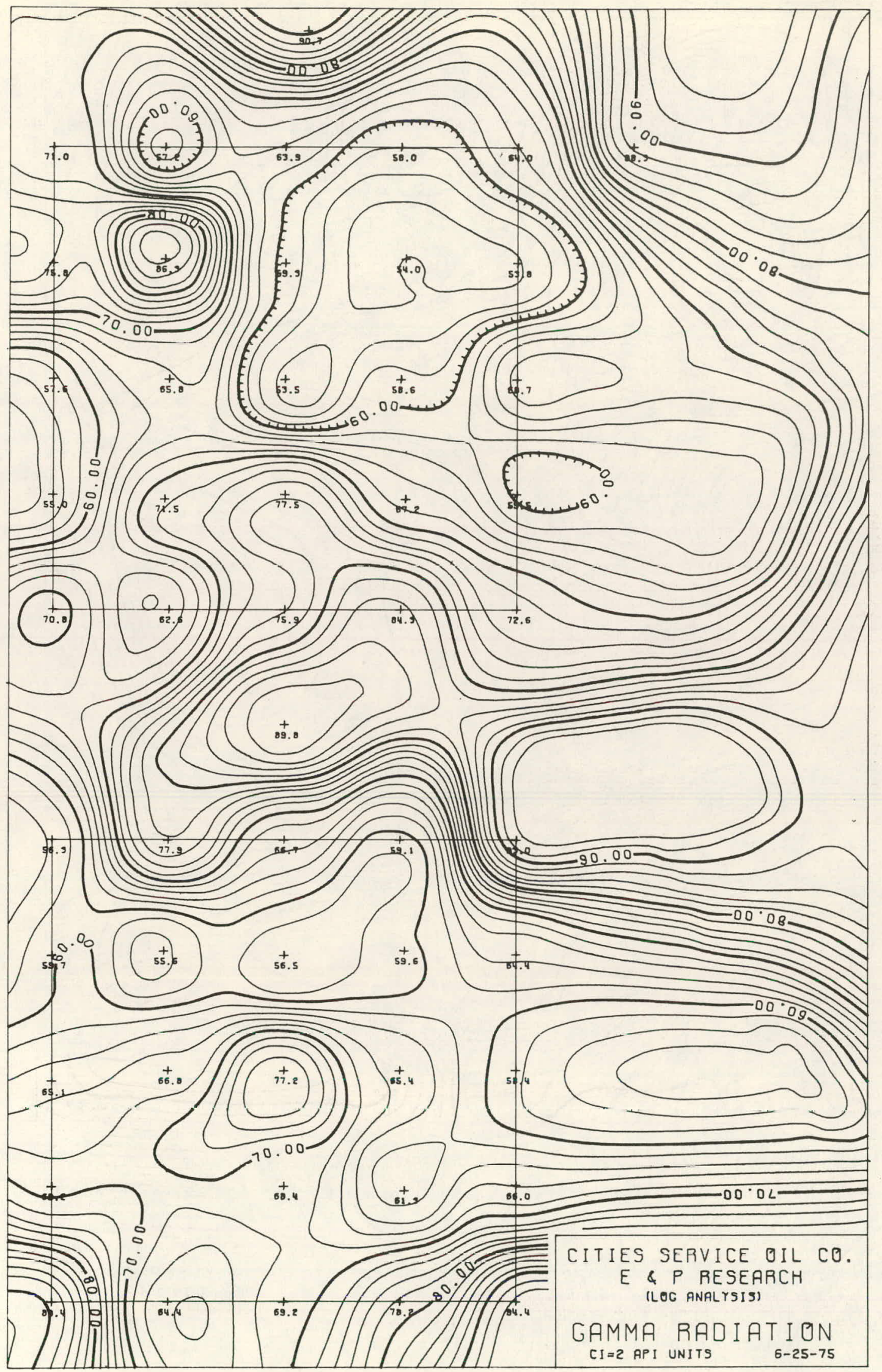

FIGURE C-73 


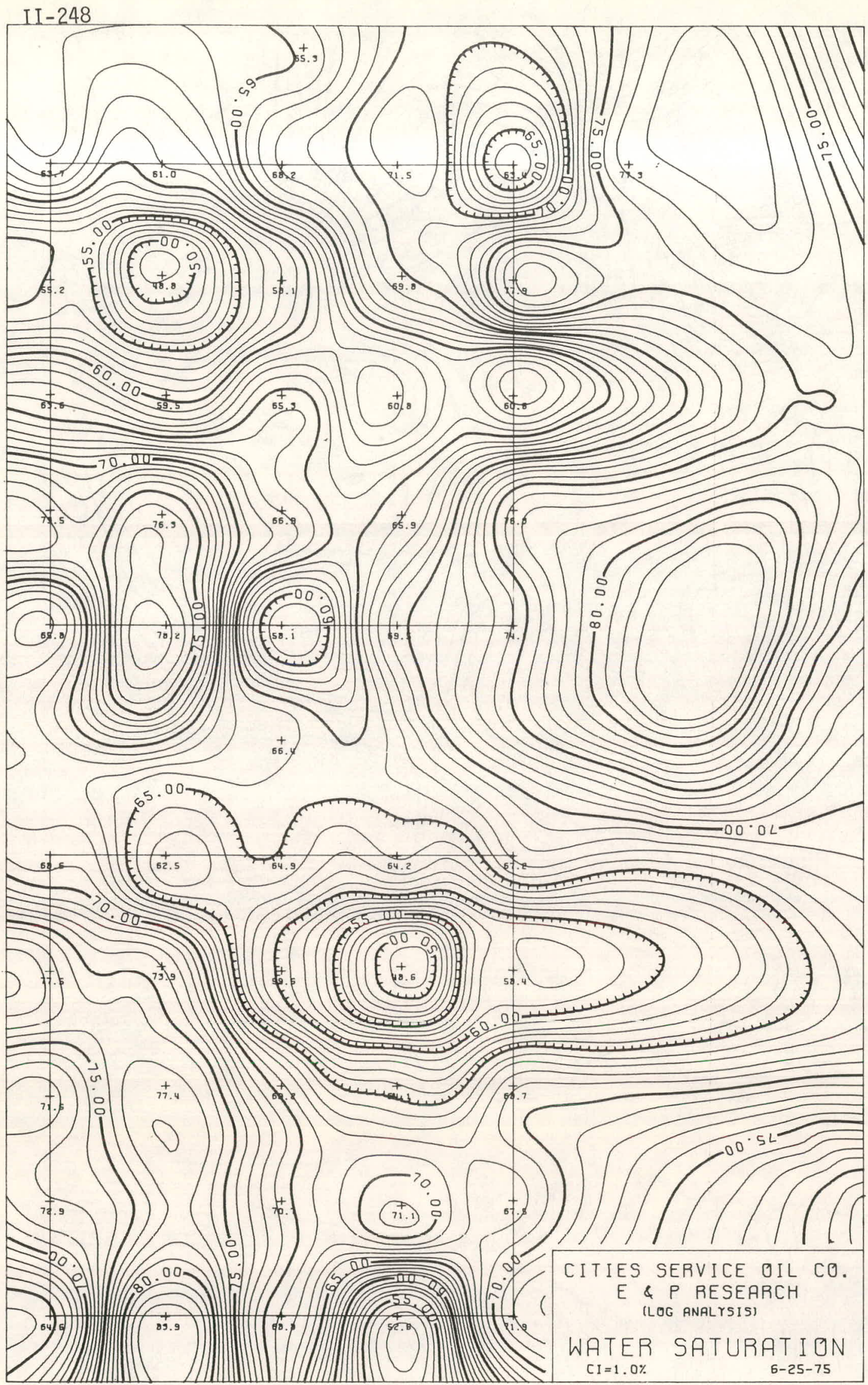

FIGURE C-74 


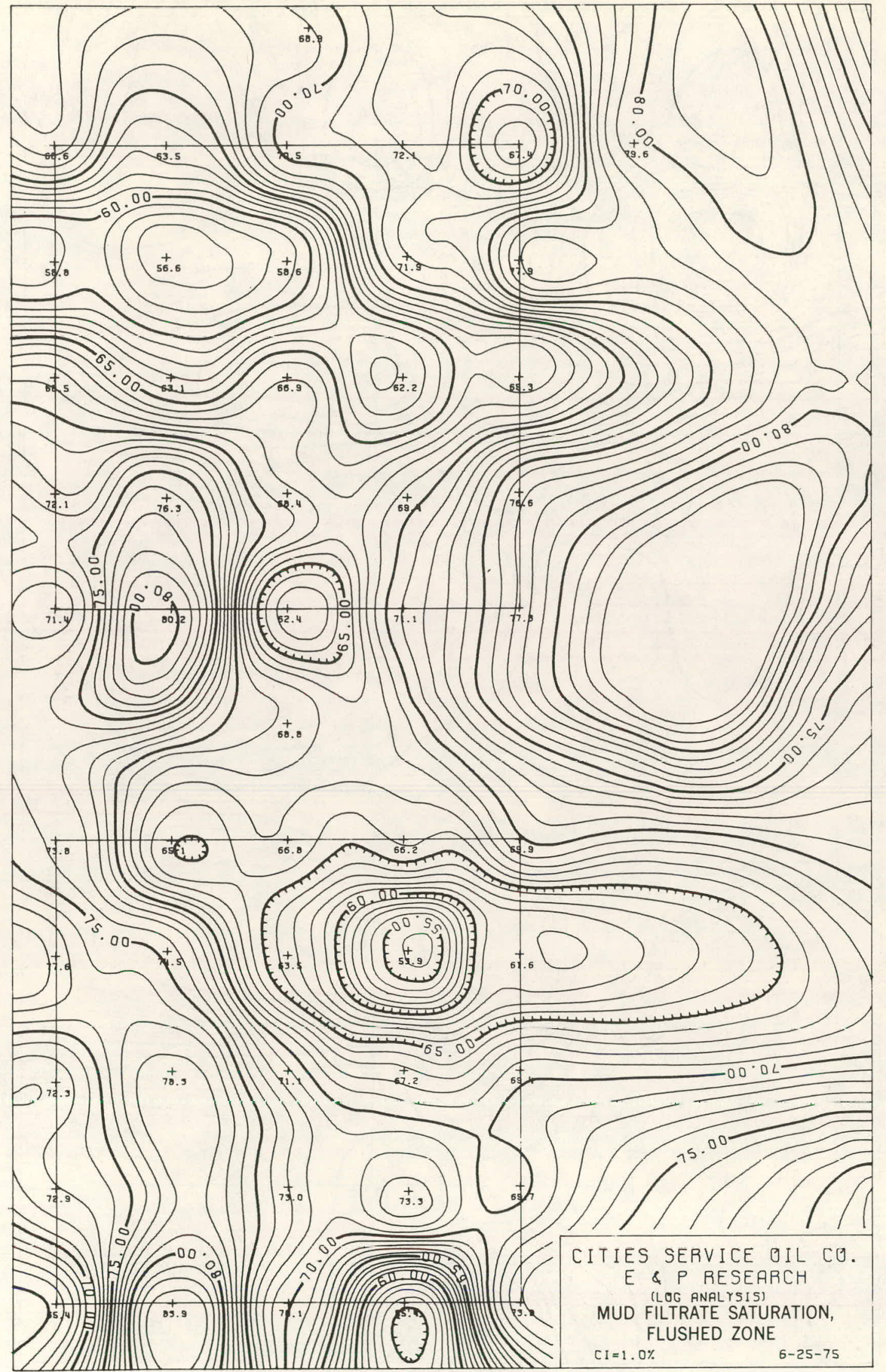

FIGURE C-75 


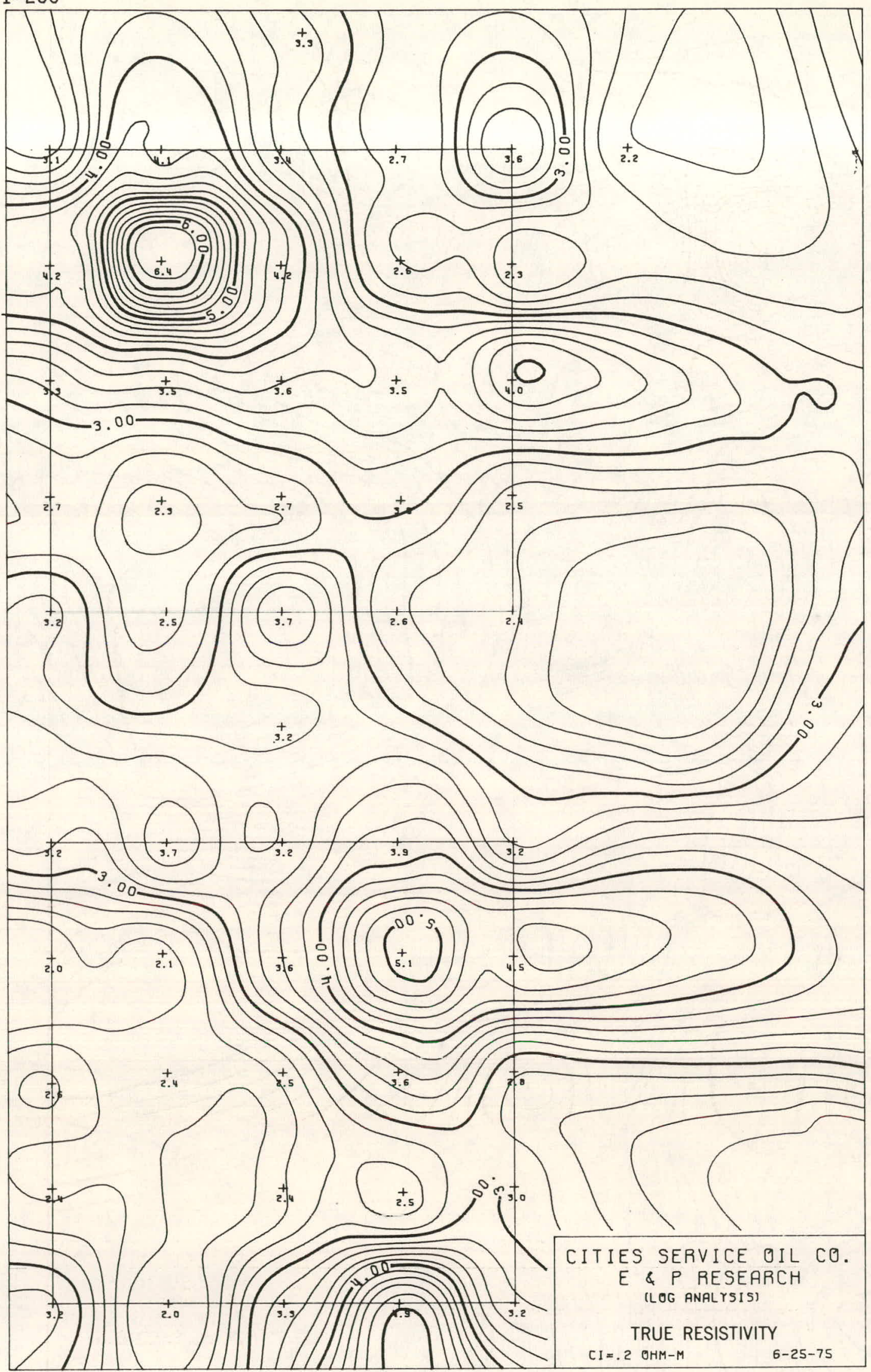

FIGURE C-76 


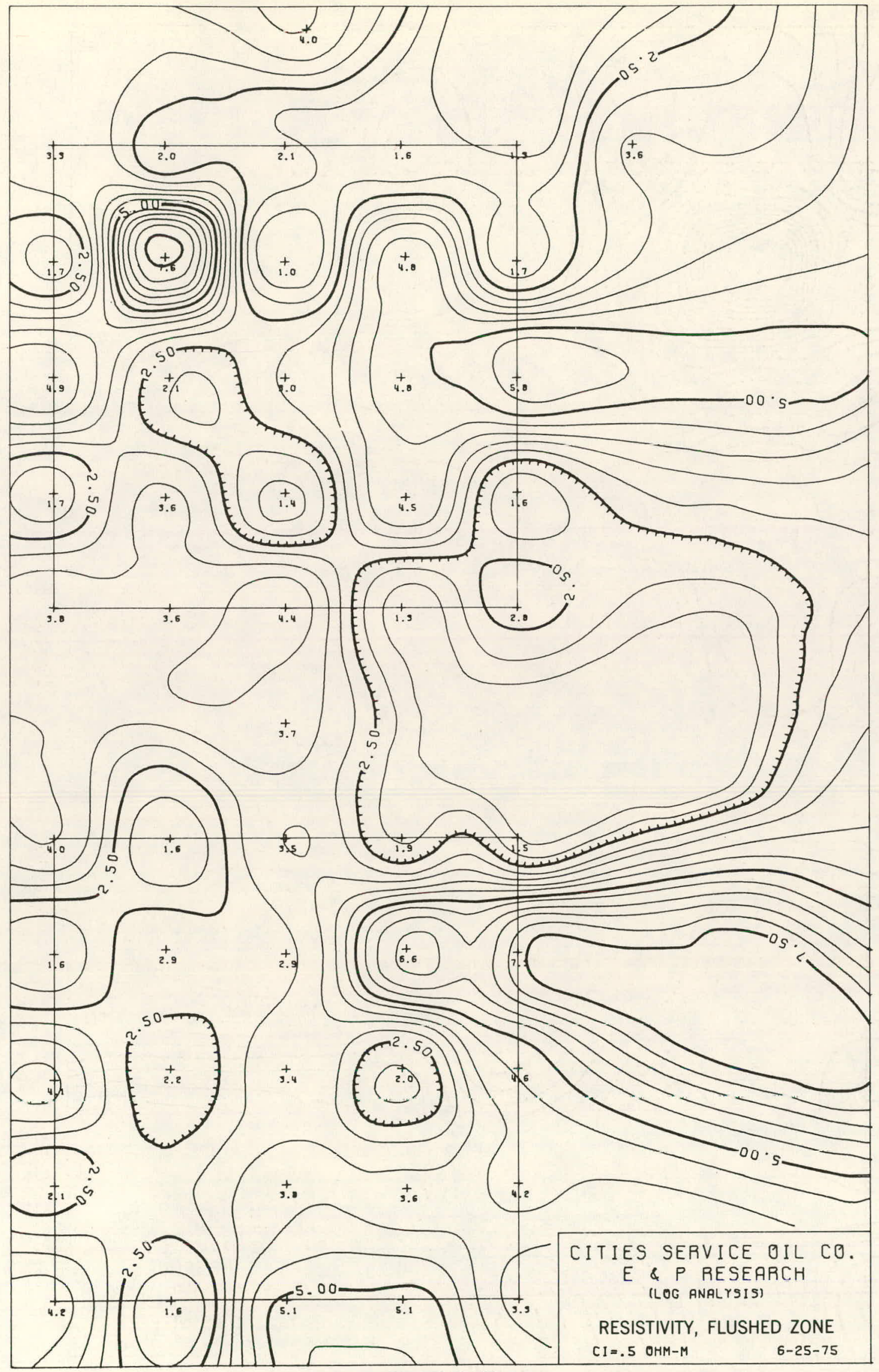

FIGURE C-77 


\section{THIS PAGE \\ WAS INTENTIONALLY \\ LEFT BLANK}


APPENDIX D

FORMATION INJECTIVITY

Tables and Figures 


\section{TABLE D1}

STATIC PRESSURE COMPARISON FOR

EL DORADO TEST WELLS

AND CHESNEY WELL 190

\begin{tabular}{|c|c|c|c|c|c|c|}
\hline Well & Pressure ${ }^{1}$ & $\begin{array}{c}\text { Depth } \\
\text { ft above MSL* }\end{array}$ & $\begin{array}{l}\Delta \mathrm{h} \\
\mathrm{ft}\end{array}$ & $\begin{array}{l}\Delta p_{h} \\
\text { psi }\end{array}$ & $\begin{array}{c}\Delta \mathrm{p}_{\text {barometer }}{ }^{2} \\
\text { psia } \\
\end{array}$ & $\begin{array}{c}\text { Pressure } \\
\text { at } 700 \text { feet } \\
\text { Above MSL*, psia } \\
\end{array}$ \\
\hline$M P-101$ & 197.7 psia & 700 & 0 & 0 & 0 & 197.7 \\
\hline$M P-103$ & $2.7 \mathrm{psig}$ & 1086 & 386 & $174.9^{3}$ & 13.9 & 191.5 \\
\hline MP- 104 & $220.0 \mathrm{psig}$ & 683 & -17 & $-7.7^{3}$ & 13.9 & 226.2 \\
\hline$C-190$ & 180.0 psig & 753 & 53 & $25.9^{4}$ & 13.9 & 219.8 \\
\hline$M P-221$ & 225.4 psia & 681 & -19 & $-8.6^{3}$ & 0 & 216.8 \\
\hline$M P-225$ & 207.1 psig & 683 & -17 & $-7.7^{3}$ & 13.9 & 213.3 \\
\hline
\end{tabular}

*MSL $=$ Mean Sea Leve1

1. Measured (or interpolated) at an elapsed time of $850 \mathrm{hr}(1000 \mathrm{hr}$, Aug. 5, 1974).

2: $P_{\text {barometer }}=P_{\text {standard }} \exp \left[\frac{-\mathrm{elev}(\mathrm{ft})}{25000}\right]=14.65 \exp \left[\frac{-1400}{25000}\right]=13.9 \mathrm{psia}$

3. Measured gradient of boric acid solution in well MP-225 $=0.453 \mathrm{psi} / \mathrm{ft}$ (assumed in all wells except $(-190)$.

4. Measured gradient of native brine in Chesney we11 C-190 $=0.488 \mathrm{psi} / \mathrm{ft}$.

5. Compares reasonably well to average of Chesney wellis C-174, C-190, and C-195 which was determined in Apri1, 1974. 164.2 psig at 750 feet MSL $\rightarrow 164.2+$ $13.7+0.488(750-700)=202.5$ psia at 700 feet MSL. 
TABLE D2

SUMMARY OF INJECTIVITY FOR TEST USING WELL MP-104

\begin{tabular}{|c|}
\hline $\begin{array}{l}\text { Type Fluid } \\
\text { Injected }\end{array}$ \\
\hline $\begin{array}{l}\text { Pretreatment } \\
\text { Dilute Preflush } \\
\text { Concentrated Preflust with } \\
\text { Powdered Oxygen Scavenger }\end{array}$ \\
\hline $\begin{array}{l}\text { Concentrated Preflush with } \\
\text { Liquid Oxygen Scavenger }\end{array}$ \\
\hline $\begin{array}{l}\text { Micellar Water } \\
\text { Soluble 0il }\end{array}$ \\
\hline $\begin{array}{l}\text { Dilute Polymer } \\
\text { Concentrated Polymer } \\
\text { Drive Water }\end{array}$ \\
\hline $\begin{array}{l}\text { Concentrated Preflush } \\
\text { Follow-up Test (Dec., 1974) } \\
\text { Immediately Preceding Breakdown }\end{array}$ \\
\hline
\end{tabular}

\begin{abstract}
Yolume
Injected,

barrels
\end{abstract}

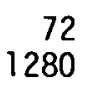

192

944

259

182

139

646

1321

200

\begin{tabular}{c} 
Time \\
Injected, \\
hours \\
\hline
\end{tabular}

Average

Flow Rate,

Average

bbl/day

16

16
427

108

64

72

72

232

98

130

121

74

74

172

184

30

160

570

505
Average

\begin{tabular}{c} 
Injectivity, \\
$\mathrm{BPD} / \mathrm{psi}$ \\
\hline
\end{tabular}

0.30

0.17

0.17

0.23

0.44

0.32

0.31

0.29

0.49

0.53

505

0.53
Stabilized Conditions

\begin{tabular}{ccc}
\hline Rate, \\
bbl/day
\end{tabular} $\begin{gathered}\text { Pressure, } \\
\text { psi }\end{gathered} \quad \begin{gathered}\text { Injectivity, } \\
\text { BPD/psi }\end{gathered}$

---n----- None

------- None

None

None

485

0.64

0.44

0.30

0.28

0.49 
TABLE D3

SUMMARY OF FALLOFF TESTS FOR WELL MP-104

\begin{tabular}{|c|c|c|c|c|}
\hline Date (1974) & Injection Fluid & $\begin{array}{c}\text { Injection } \\
\text { Rate, } \\
\text { bbl/day } \\
\end{array}$ & $\begin{array}{l}\text { Effective } \\
\text { Injection } \\
\text { Time, hr } \\
\end{array}$ & Skin Factor \\
\hline $\begin{array}{l}\text { July } 8 \\
\text { July } 12 \\
\text { Aug. } 15 \\
\text { Aug. } 25 \\
\text { Aug. } 29 \\
\text { Sept. } 9 \\
\text { Sept. } 15 \\
\text { Sept. } 28 \\
\text { Oct. } 8 \\
\text { Dec. } 17 \\
\text { Dec. } 21\end{array}$ & $\begin{array}{l}1500 \mathrm{ppm} \mathrm{NaCl} \\
1500 \mathrm{ppm} \mathrm{NaCl} \\
\text { Dilute Preflush } \\
\text { Dilute Preflush } \\
\text { Dilute Preflush } \\
\text { Conc. Preflush } \\
\text { Micellar Fluids } \\
\text { Polymer } \\
\text { Drive Water } \\
\text { Conc. Preflush } \\
\text { City Water }\end{array}$ & $\begin{array}{r}288 \\
192 \\
55 \\
90 \\
110 \\
96.5 \\
151.2 \\
73.0 \\
772.0 \\
160.0 \\
1007.0\end{array}$ & $\begin{array}{r}7.75 \\
37.75 \\
253.09 \\
147.96 \\
46.42 \\
237.68 \\
69.90 \\
257.92 \\
184.33 \\
30.00 \\
4.98\end{array}$ & $\begin{array}{r}-2.46 \\
-1.62 \\
33.66 \\
17.33 \\
14.08 \\
15.02 \\
1.37 \\
9.85 \\
2.00 \\
2.34 \\
-5.12\end{array}$ \\
\hline
\end{tabular}

Reservoir and Fluid Properties Used:
$k=42.2 \mathrm{md}$
$\phi=0.26$
$\mu=1 \mathrm{cp}$
$h=18 \mathrm{ft}$
$\beta=1.0$
$c_{t}=7.9 \times 10^{-6} \mathrm{psi}^{-1}$ 
FIGURE D-1

SURMMARY OF INTERFERENCE

TEST DATA

C-195

$$
\begin{aligned}
\text { ASSUMPTIOHS: } & h=0.1 \mathrm{MILE} \\
\phi & =18 \mathrm{ft} \\
\phi & =0.26 \\
\beta & =1.0 \\
\mu & =1 \mathrm{cp}
\end{aligned}
$$

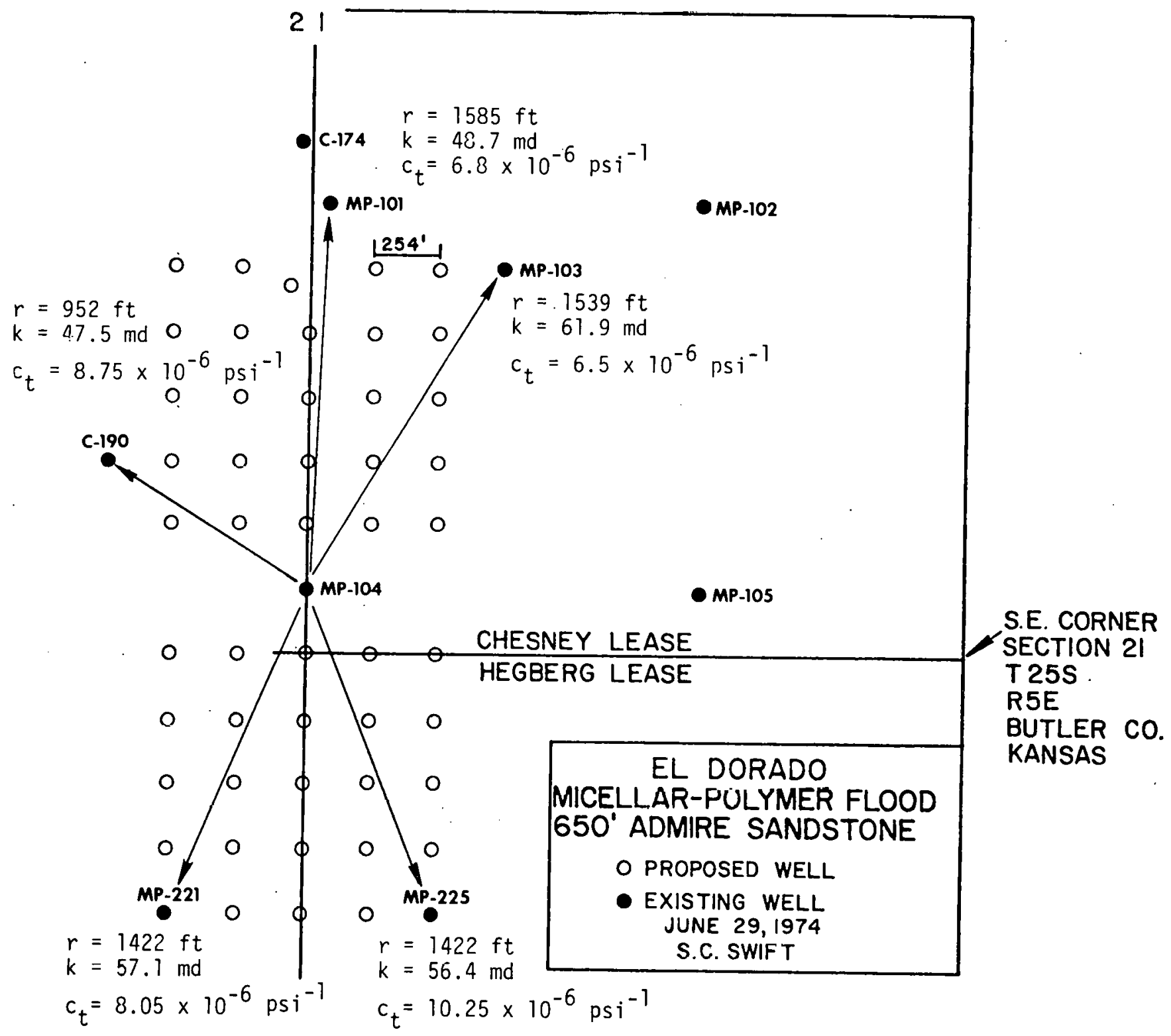




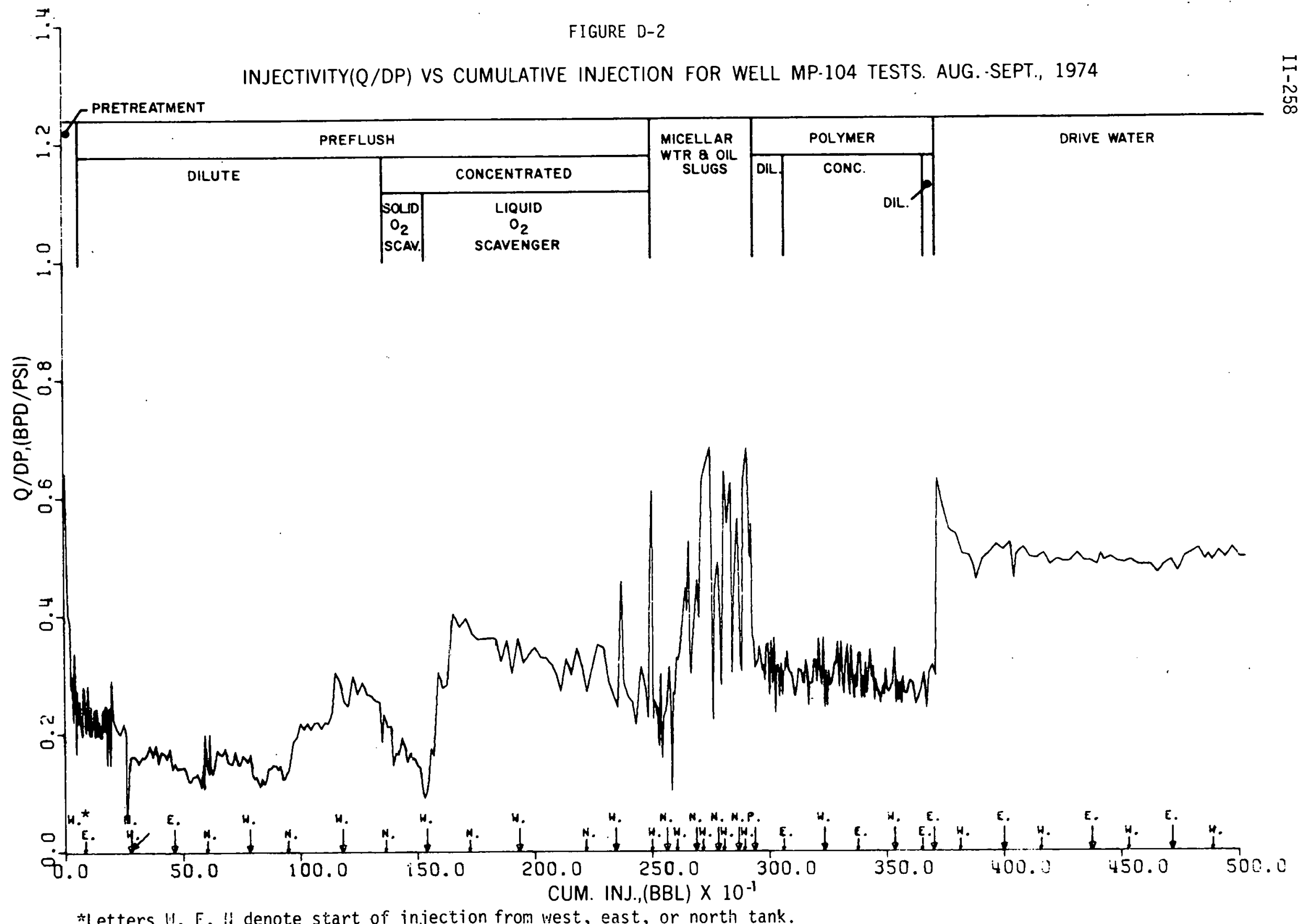

*Letters $U, E, \|$ denote start of injection from west, east, or north tank. 


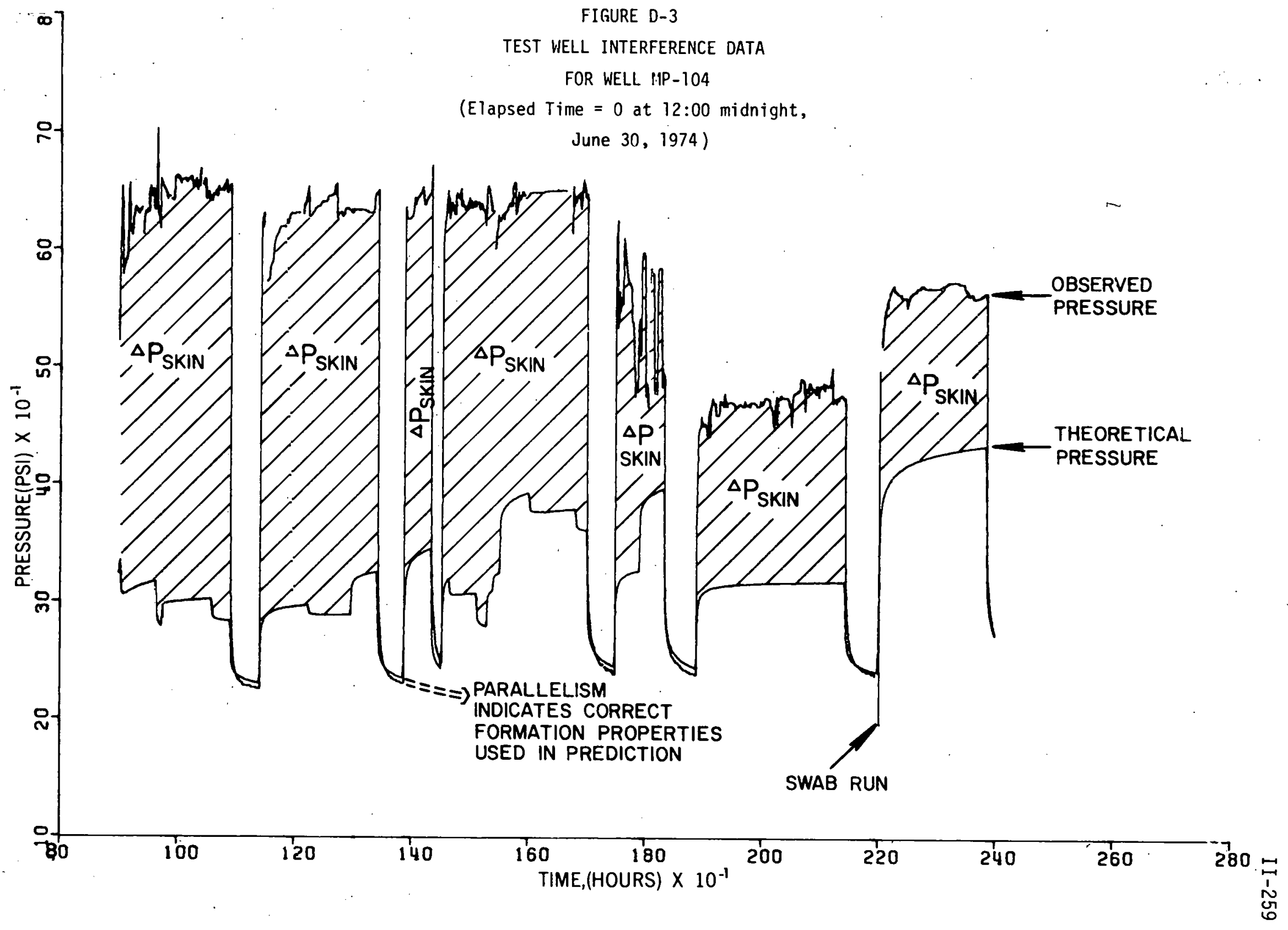


FIGURE D-4

MULTI-RATE INJECTION TESTS

FOR WELL MP-104

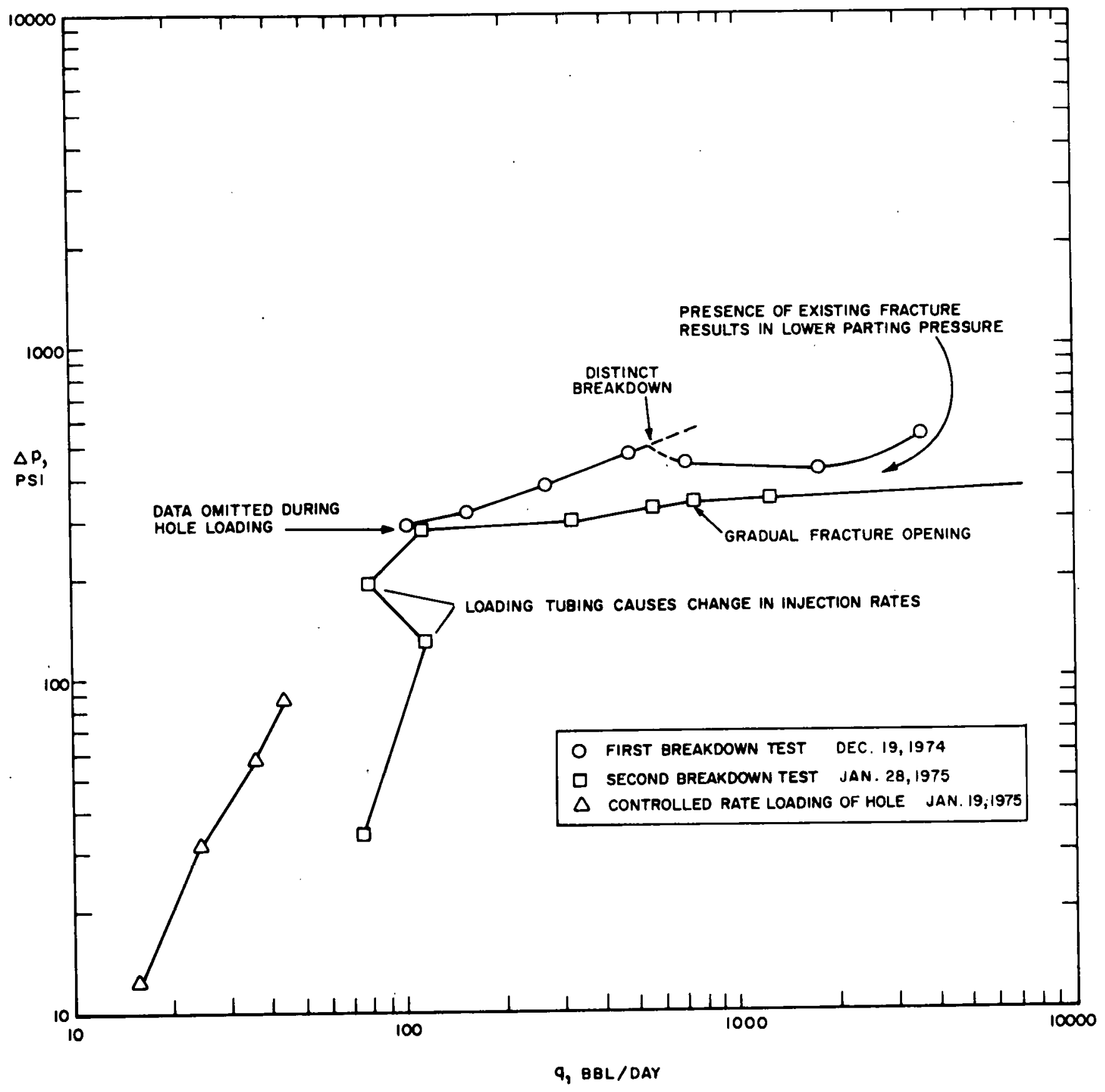


FIGURE D-5

PULTI-RATE INJECTION TESTS

FOR HELLS I.PP-101 AND IIP-103

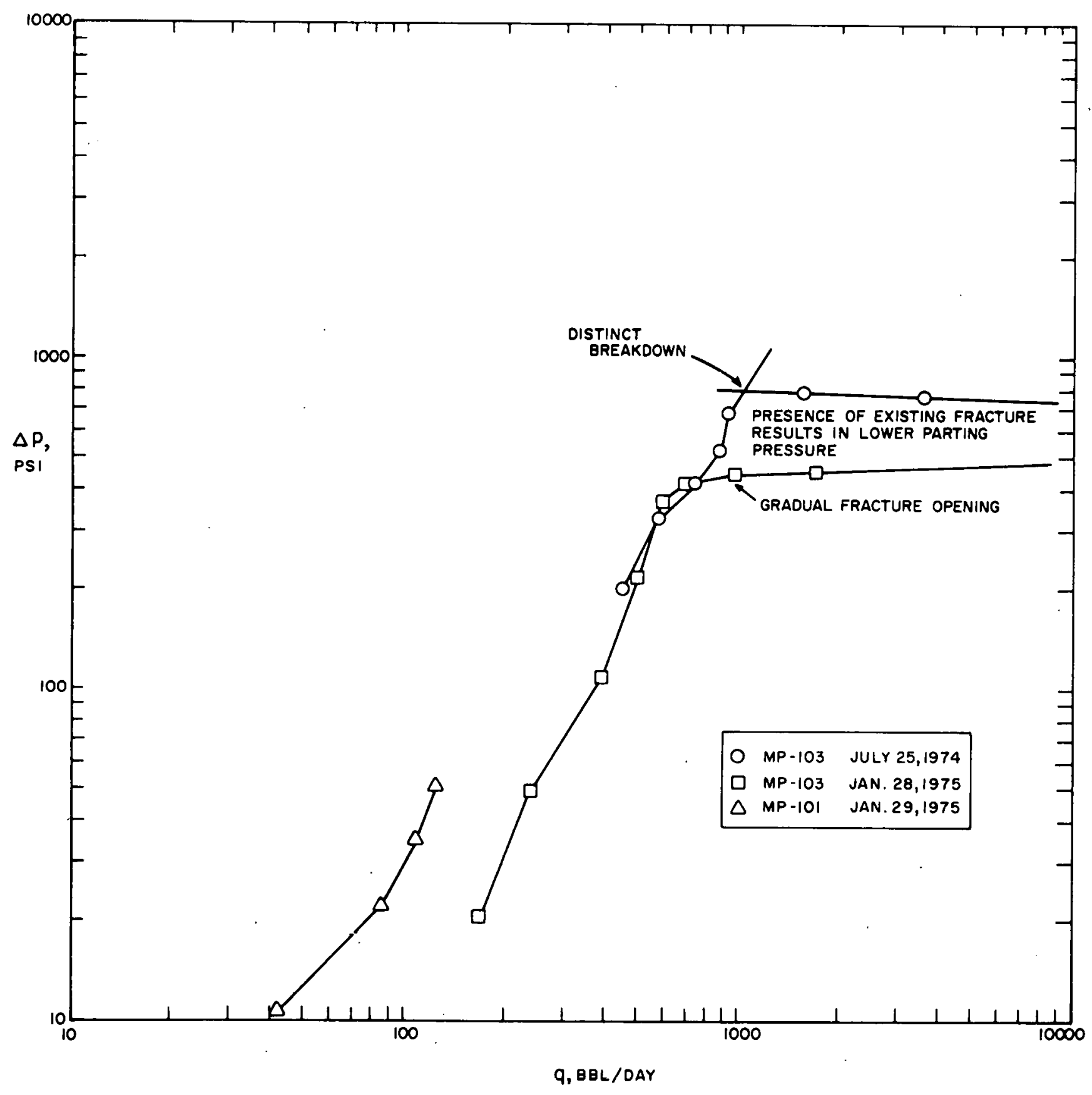


THIS PAGE

WAS INTENTIONALLY

LEFT BLANK 
APPENDIX E

LITERATURE WORK

Table and Figures 
TABLE EI

\author{
DISCLOSURE KEYS FOR ANALYSIS OF THE \\ SCIENTIFIC AND TECHNICAL CONTENTS OF A PATENT
}

Key

Number

1

2

3

4

5

6

7

8

9

10

11
Definitions of Key Values

0 patent not relevant to recovery of oil (ROO) by micellarpolymer flooding

1 only peripheral disclosure to ROO

2 disclosures of intermediate importance to R0O

3 very important disclosures to ROO

1 if process claim, 0 if not

1 if composition claim, 0 if not

Preflush Slug (PS)

0 if PS is not disclosed

1 if PS is not required by disclosure

2 if PS is optional

3 if PS is required

4 if cannot tell

1 if PS formulation is disclosed, 0 if not

1 if precipitating agent in PS is disclosed, 0 if not

1. if sacrificial adsorbant in PS is disclosed, 0 if not

1 if any other unusual feature of PS, 0 if not

Surfactant Slug (SS)

I SS injected as surh

2 alternate injection of SS components

3 alkaline waterflood

4 SS created in situ by injecting reactive chemicals

5 none of the above

6 several of above

7 cannot tell

8 not disclosed

1 if salinity tolerance of SS is disclosed, 0 if not

1 if hardness tolerance of SS disclosed, 0 if not 
DISCLOSURE KEYS FOR ANALYSIS OF THE

SCIENTIFIC AND TECHNICAL CONTENTS OF A PATENT

(continued)

Key

Number

12

13

14

15

16

17

18

19

20

\section{Definitions of Key Values}

1 if Ca+t tolerance of SS disclosed, 0 if not

Total number of surfactants used if surfactant composition is disclosed, 0 if not

Total number of cosurfactants used if cosurfactant composition of SS is disclosed, 0 if not

Number of other chemicals disclosed, 0 if not

1 if injected slug is an oil external micellar solution or swollen micellar solution (MS or SMS)

2 if injected slug is a water external MS or SMS

3 if injected slug is an MS or SMS of undetermined external phase

4 if injected slug is not an MS or SMS

5 cannot tell what the slug is from the patent

6 slug not disclosed

1 if any other unusual feature of SS, 0 if not

Mobility Buffer and/or Drive Water (MB-DW)

1 if MB-DW is disclosed, 0 if not

1 no thickening agent in MB-DW

2 partially hydrolyzed polyacrylaminde in MB-DW

3 polysaccharide in MB-DW

4 other thickening agent in MB-DW

5 several of above thickening agents in MB-DW

6 cannot tell

1 If any other unusual chemical in MB-DW, 0 if not 
FIGURE E-1

VISCOSITY BEHAVIOR OF HYDROLYZED POLYACRYLAMIDE SOLUTIONS -500 PPM (AFTER JENNINGS, ET $A L^{2}$ )

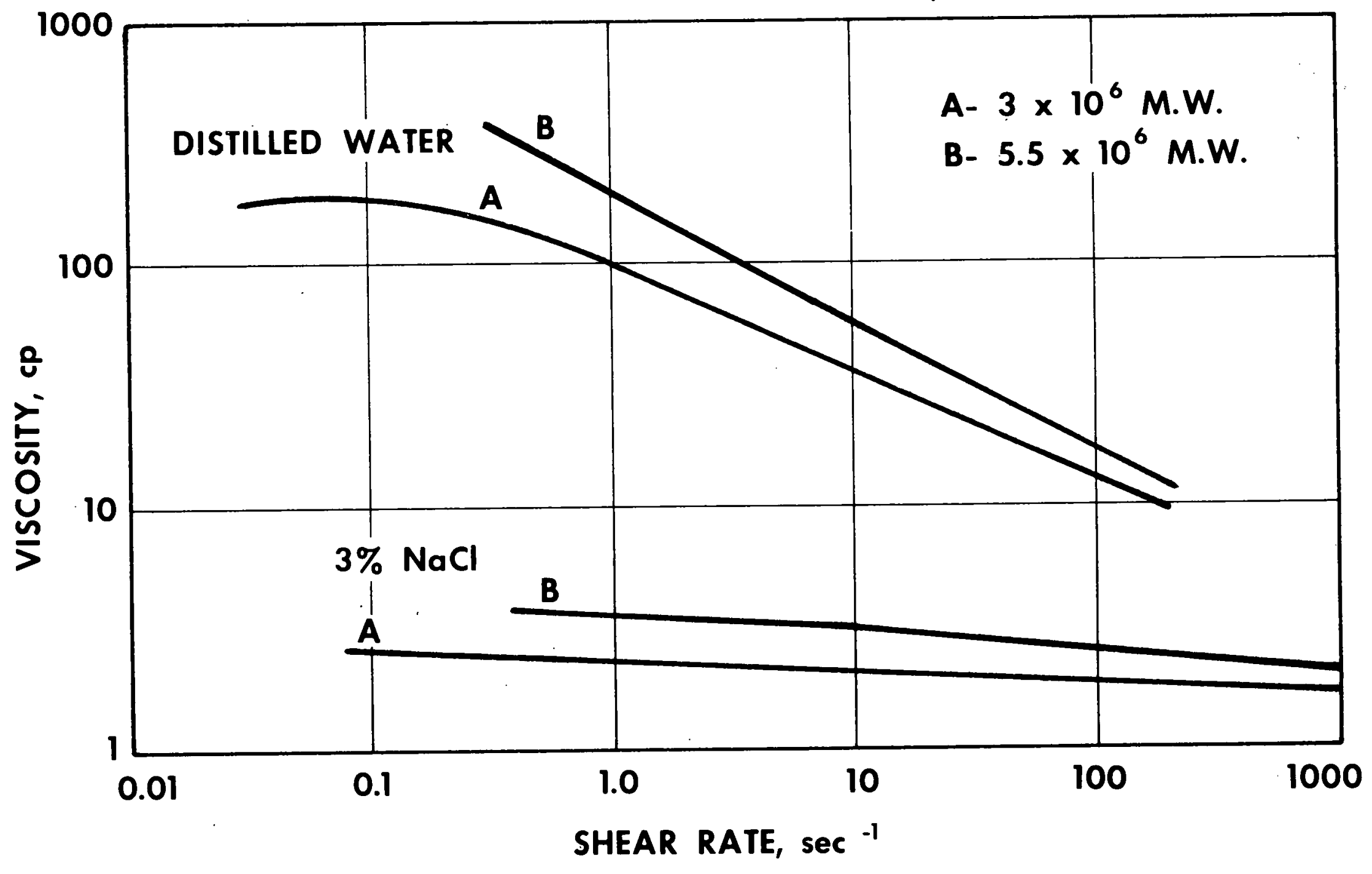


FIGURE E-2

VISCOSITY BEHAVIOR OF XANTHOMONAS POLYSACCHARIDE -500 PPM

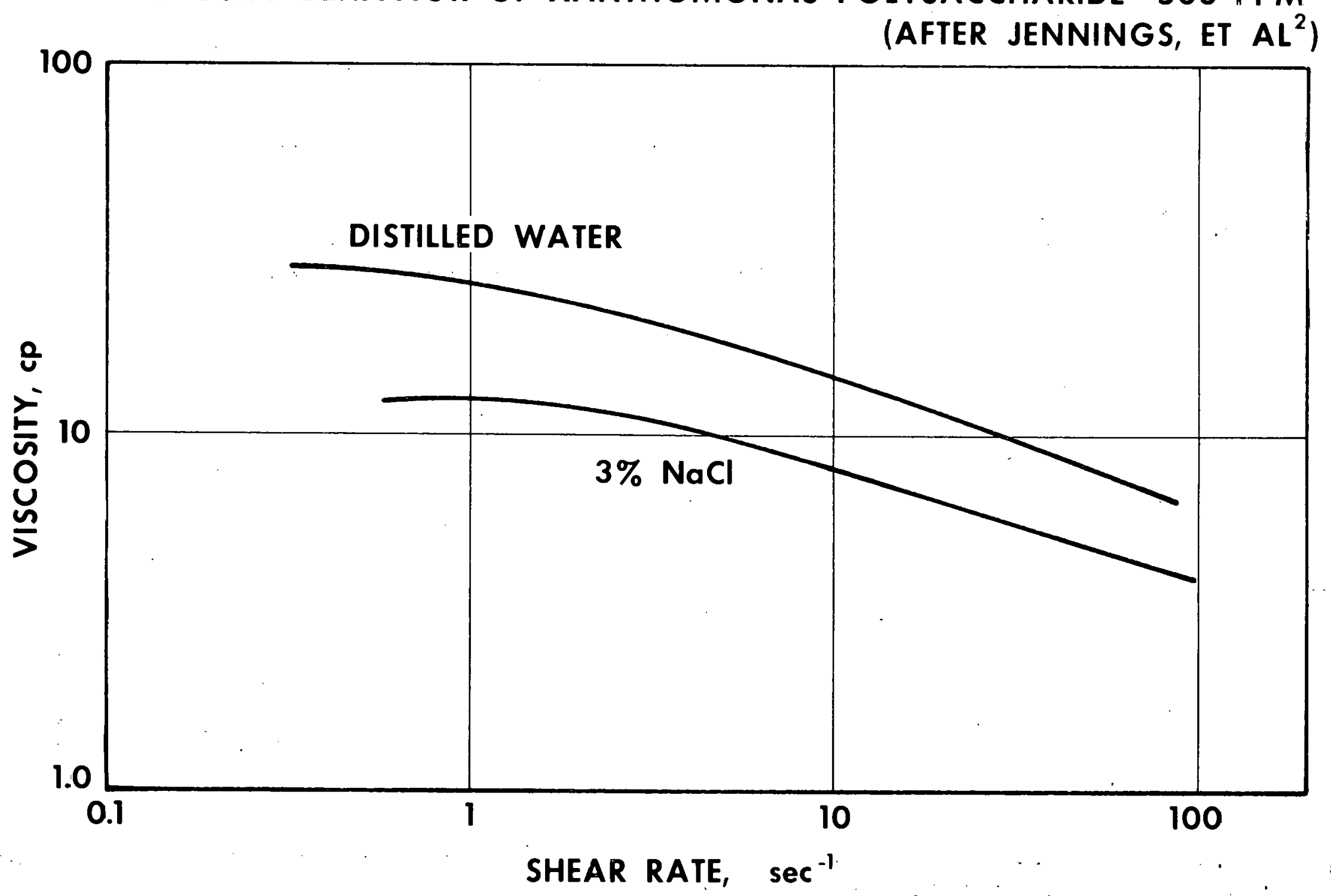




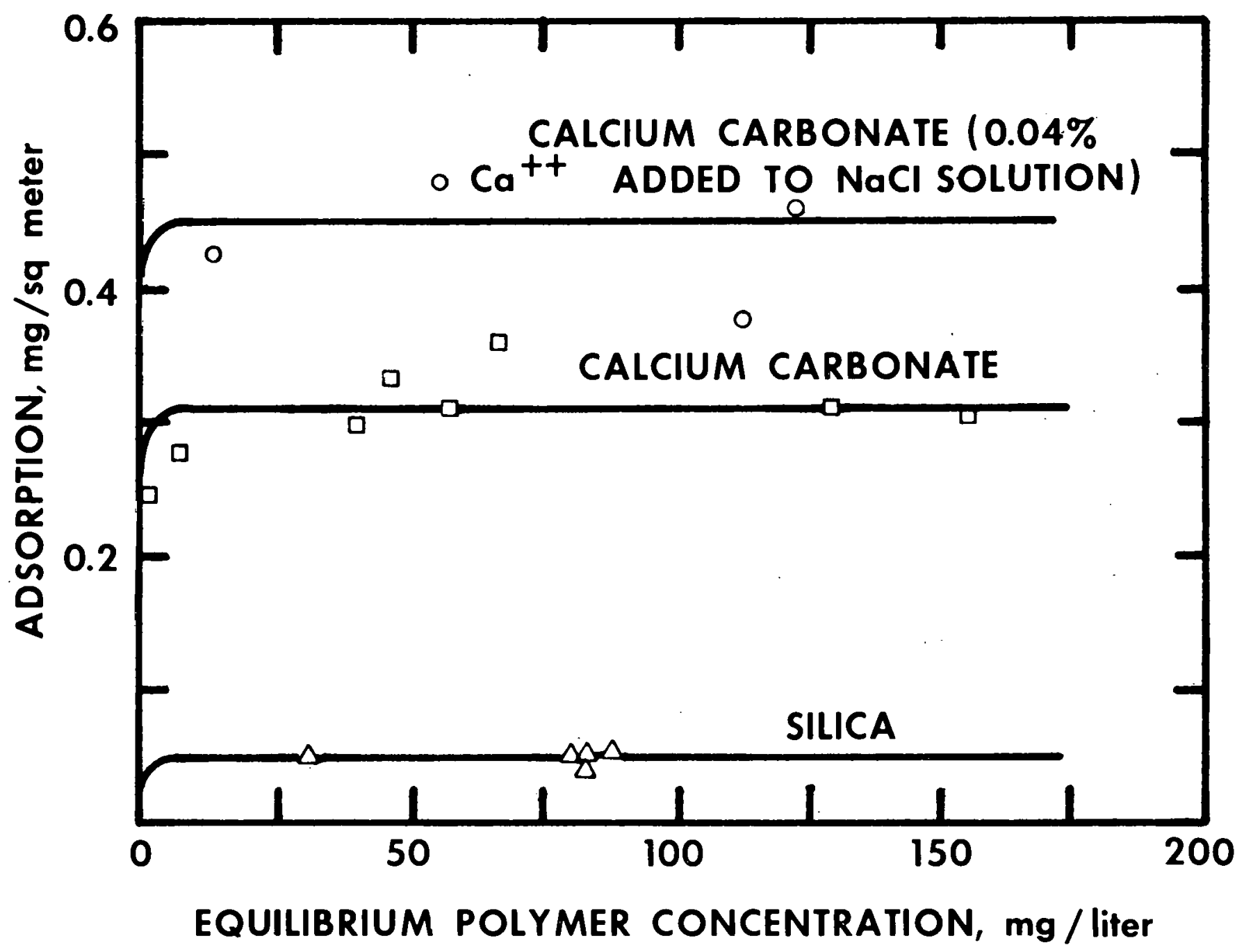

FIGURE E-3

ADSORPIION OF POLYMER FROM 10 PERCENT

SODIUM CHLORIDE SOLUTION

(FROM SMITH ${ }^{4}$ ) 


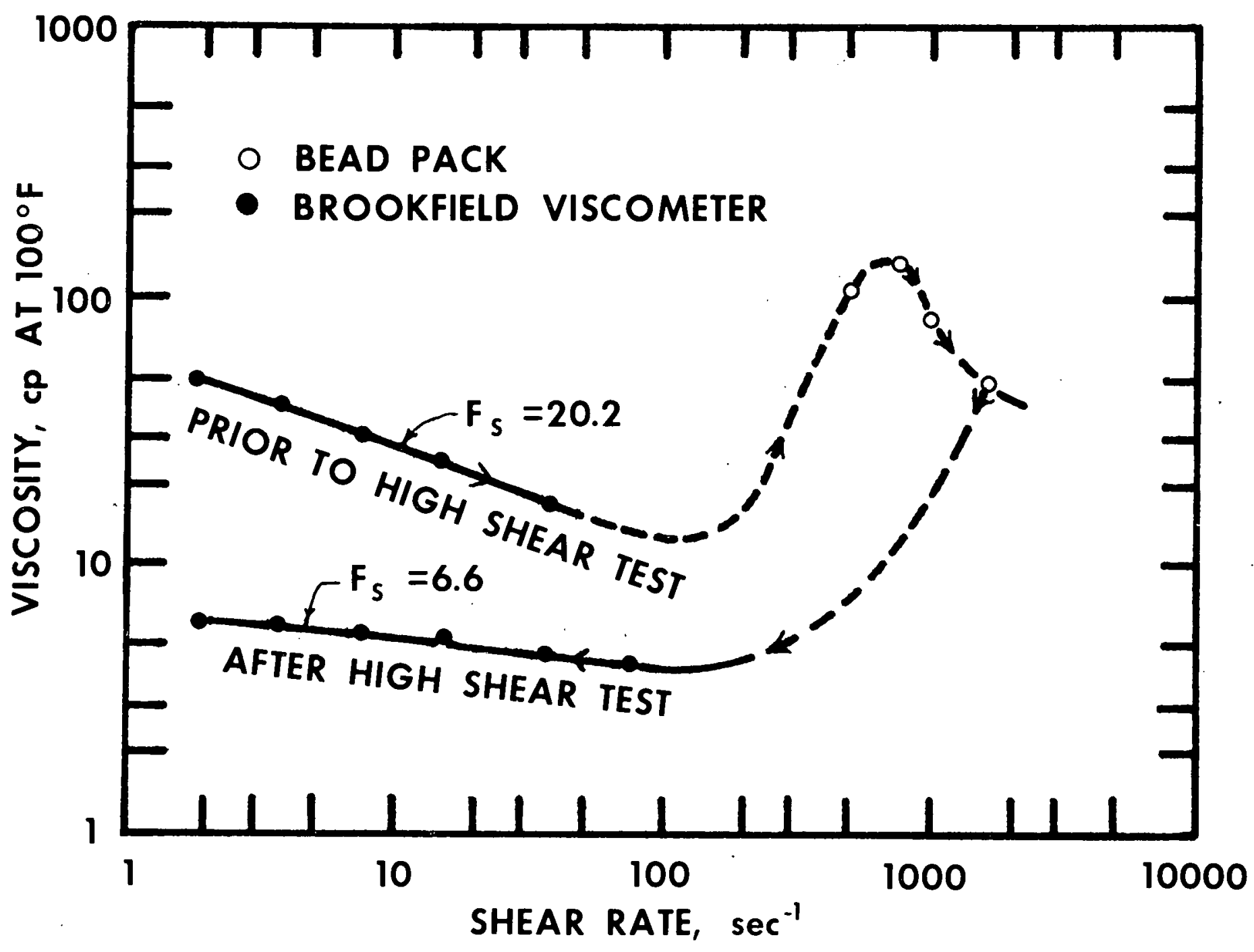

FIGURE E-4

VISCOSITY VS SHEAR RATE, 600 PPM POLYACRYLAMIDE IN 300.PPM NaCl SOLUTION.

(FROM HILL, ET $A L^{\prime}$ ) 


\section{THIS PAGE \\ WAS INTENTIONALLY \\ LEFT BLANK}


APPENDIX $F$

PERFORMANCE PREDICTION

Tables and Figures 
TABLE FI

BREAKTHROUGH SWEEP EFFICIENCY

AT VARIOUS RESERVOIR CONDITIONS

Reservoir Type

1. Homogeneous

2. Two layers, $k_{2} / k_{1}=2$

3. Directional permeability, $k_{x} / k_{y}=7$

4. Directional permeability, $k_{x} / k_{y}=2.7$

*Gas saturation at beginning of waterflood.
Breakthrough Sweep Efficiency

$S_{g}=0.16$

$$
\mathrm{S}_{\mathrm{g}}=0
$$

at Start*

at Start*

0.68

0.69

0.63

0.65

0.33

0.34

0.47

0.52 
TABLE F2

DATA USED IN PREFLUSH SIMULATION

\begin{tabular}{|c|c|c|}
\hline Fluid and Reservoir Properties & $\begin{array}{l}\text { Hegberg } \\
\text { Pattern } \\
\end{array}$ & $\begin{array}{l}\text { Chesney } \\
\text { Pattern } \\
\end{array}$ \\
\hline Water Density, $\mathrm{lb} / \mathrm{cu} \mathrm{ft}$ & 62.150 & 62.150 \\
\hline Water Furmation Volume Factor, RB/STB & 1.000 & 1.000 \\
\hline Water Viscosity, $c p$ & 1.070 & 1.070 \\
\hline Water Compressibility, $\mathrm{psi}^{-1}$ & 0.000003 & 0.000003 \\
\hline Rock Compressibility, $\mathrm{psi}^{-1}$ & 0.000003 & 0.000003 \\
\hline Saline Water Formation Volume Factor, RB/STB & 1.000 & 1.000 \\
\hline Saline Water Density, $1 \mathrm{~b} / \mathrm{cu} \mathrm{ft}$ & 62.150 & 62.150 \\
\hline Saline Water Viscosity, $\mathrm{cP}$ & 1.070 & 1.070 \\
\hline Saline Hater Compressibility, $\mathrm{psi}^{-1}$ & 0.0000035 & 0.000003 \\
\hline Permeability in $x$ Direction, md & 36.0 & 58.8 \\
\hline Permeability in $Y$ Direction, md & 36.0 & 58.8 \\
\hline Permeability in $Z$ Direction, md & 0 & 0 \\
\hline Thickness, ft & 20 & 27 \\
\hline Porosity (adjusted for immobile oil) & 0.2038 & 0.1686 \\
\hline Dip in $X$ Direction, degrees & 0 & 0 \\
\hline Dip in $Y$ Direction, degrees & 0 & 0 \\
\hline Initial Pressure, psia. & 200 & 200 \\
\hline Initial Saline Water & 1.0 & 1.0 \\
\hline
\end{tabular}

\section{Model Parameters}

Number of Blocks in. X'Direction 23

Number of Blocks in Y Direction 
TABLE F3

LINEAR DISPLACEIIENT DATA

Relative Permeability Data'

Connate water saturation, $S_{W C}$

Residual oil saturation, $S_{\text {or }}$

Relative perm. to oil at $S_{W C}$

Relative perm. to water at $S_{\text {or }}$

Water viscosity, $c p-14$

$0 i 1$ viscosity, $c p-1.60$

Displacement Data ${ }^{2}$

Initial water satu. in slug, $S_{w m m}$

Final water satu. in slug, $S_{\text {wmf }}$

$0 i 1$ satu. in $0 i 1$ bank, $S_{\text {om }}$

$0 i 1$ cut in $0 i 1$ bank, fom 0.82

$0 i 1$ satu. in oil + water bank, $S_{\text {of }}$

$0 i l$ cut in $0 i l+$ water bank, fof

\section{Calculated Displacement Efficiency}

Breakthrough of specified bank at total pore volumes injected.

$$
\begin{aligned}
& \frac{\text { Slug Size }}{4 \% \mathrm{PV}} \\
& 0 i 1+\text { water bank - } 0.402 \\
& \text { Micellar slug - }
\end{aligned}
$$

TFrom Cities Service inter-office letter by R. V. Maul to J. 0. Scott, September 11, 1964.

${ }^{2}$ For calculation method of J. A. Davis, Jr. and S. C. Jones, Journal of Petroleum Technology, 20:1415, December, 1968.

${ }^{3}$ Assumed value, same as used by Davis and Jones. 
TABLE F4

INJECTION RATES AND SLUG. SIZES

EL DORADO MICELLAR-POLYM'ER PROJECT

\section{Chesney Pattern}

Injection slug size, PV

Salinity Adjustment

Micellar

Polymer

Drive Water

0.15

0.04

0.60

$\frac{0.80}{1.59}$

140

150

100

150

Drive Water

Total injection wells

Effective injection wells

Pore Volume, bbl

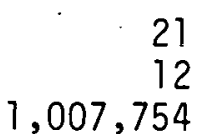

$1,007,754$

Hegberg Pattern

Effective pattern injection rates $\mathrm{PV} / \mathrm{yr}$

Salinity Adjustment

Micellar

0.6085

0.6519

0.7197

Polymer

0.4346

0.7197

0.4798

Drive Water

0.6519 


\title{
TABLE F5 \\ PARAMETERS USED IN \\ SIMULATION FOR PATTERN COMPARISON
}

\author{
Number of Cells Between Unlike Wells 22 \\ Densities, $\mathrm{lb} / \mathrm{cu} \mathrm{ft}$ \\ 62.150 \\ Formation volume Factors, RB/STB \\ 1.00 \\ Compressibilities

$$
\begin{aligned}
& \text { Water, } \mathrm{psi}^{-1} \\
& \text { Rock, } \mathrm{psi}^{-1}
\end{aligned}
$$$$
0.000004
$$$$
0.000004
$$ \\ Viscosities, $c p$$$
1.070
$$ \\ Permeability, md \\ 58.8 \\ Porosity \\ 0.1686 \\ Initial Reservoir Pressure, psig \\ 200.0
}


TABLE F6

PORE VOLUMES INJECTED AT BREAKTHROUGH FOR

EL DORADO MICELLAR-POL YMER FLOOD

MOBILITY RATIO $=1$

Pattern

Constant Pressure (P),

or Constant Injection Rate (Q)

Pore Volumes

Type

$\mathrm{P}$

0.39

9-Spot

P, with 90 day delay in side wells

0.53

9-Spot

P, with 150 day delay in side wells

0.65

5-Spot

P

0.70

9-Spot

Q, 5:1 rate ratio

0.49

9-Spot

Q, 5:1 rate ratio with 90 day delay

0.62

5-Spot

Q

0.77 
TABLE F7

ESTIMATED STABILIZED INJECTION RATES FOR

EL DORADO MICELLAR POLYMER FLOOD

Type of Operation

Rate, STB/day

\begin{tabular}{ccccccc}
\hline Well & Wells & Wells & Wells & We11 & Pattern \\
1 & $2 \& 3$ & $4 \& 5$ & $6 \& 7$ & 8 & Total \\
\hline
\end{tabular}

Nine-Spot

$\begin{array}{lrrrrrr}\text { Constant Pressure } & 120 & 120 & 72 & 71 & 41 & 404 \\ \text { Constant Pressure with 90 day delay } & 120 & 120 & 72 & 71 & 41 & 404 \\ \text { Constant Flow Rate with 5:1 rate ratio } & & 180 & & 80 & \end{array}$

Five-Spot

Constant Pressure 284

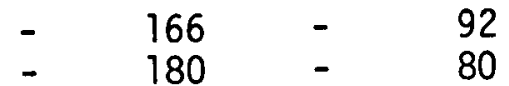

Constant Flow Rate

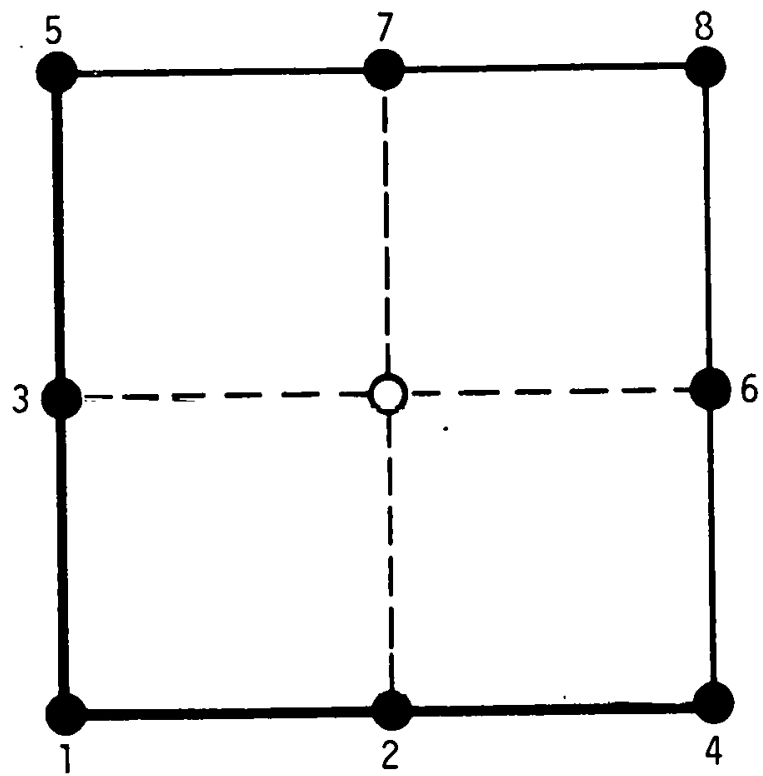

O PRODUCTION WELL

- INJECTION WELL

- NO-FLOW BOUNDARY

- CROSS-FLOW PATTERN BOUNDARY 
TABLE F8

COMPARISON OF 6.4 ACRE AND 3.2 ACRE FIVE-SPOTS AND 6.4 ACRE NINE-SPOTS

\begin{tabular}{lllllllll}
\hline \multicolumn{10}{c}{ Pattern Type } \\
Four 6.4 & Five & Six & Seven & Eight & Five 3.2 & Five 3.2 & Five 3.2 & Four 6.4 \\
Acre & 3.2 & 3.2 & 3.2 & 3.2 & Acre +2 & Acre +3 & Acre +4 & Acre \\
5-Spots & Acre & Acre & Acre & Acre & Producers & Producers & Producers & 9 -Spots \\
\hline
\end{tabular}

Pattern Injection Rate Equals Pattern Production Rate. (Stabilized Rates)

Production wells in pattern (number of wells)

Injection wells in pattern (number of wells)

$\begin{array}{rrrrrrrrr}4 & 5 & 6 & 7 & 8 & 7 & 8 & 9 & 4 \\ 9 & 12 & 13 & 14 & 15 & 12 & 12 & 12 & 21 \\ 148 & 153 & 152 & 151 & 150 & 158 & 163 & 161 & 157 \\ 66 & 64 & 70 & 75 & 80 & 92 & 109 & 121 & 30 \\ 86 & 79 & 95 & 97 & 103 & 111 & 126 & 134 & 41 \\ 58 & 56 & 60 & 63 & 66 & 77 & 86 & 115 & 22 \\ 30 & 30 & 30 & 30 & 30 & 30 & 30 & 30 & 30 \\ 253 & 247 & 252 & 255 & 259 & 276 & 295 & 303 & 219 \\ 217 & 280 & 332 & 383 & 438 & 403 & 477 & 530 & 230 \\ 1241 & 639 & 596 & 604 & 594 & 542 & 511 & 517 & 780 \\ 20.2 & 13.3 & 14.9 & 17.3 & 19.5 & 16.4 & 18.3 & 20.4 & 13.4 \\ 17.8 & 19.3 & 22.6 & 25.7 & 28.9 & 25.6 & 28.7 & 31.3 & 17.7\end{array}$

Average production rate per well, bbl/day

Average injection rate per well, bbl/day

Maximum injection rate per well, bbl/day

Minimum injecticn rate per well, t.bl/day

Production well pressure, psia

Injection well pressure, psia

Injection rate, $M S T B / y r$

Earliest breakthrough time, days

Area swept at breakthrough, acres

Area swept at 3 jears, acres

Note: Total rates and areas are fcr one lease (25.6 acre) 
TABLE F9

FOUR 6.4 ACRE FIVE-SPOT PATTERNS WITH OVERINJECTION

Part 1: 400 psi Injection Pressure*

\begin{tabular}{|c|c|c|c|}
\hline $\begin{array}{l}\text { Time, } \\
\text { days }\end{array}$ & $\begin{array}{c}\text { Injection } \\
\text { Rate, } \\
\text { STB } \\
\text { day } \\
\end{array}$ & $\begin{array}{l}\text { Percent } \\
\text { Increase } \\
\text { Over } \\
\text { Base Case }\end{array}$ & $\begin{array}{c}\text { Production } \\
\text { Rate, } \\
\text { STB } \\
\text { day }\end{array}$ \\
\hline $\begin{array}{l}10 \\
30 \\
60 \\
90\end{array}$ & $\begin{array}{l}1261 \\
1172 \\
1142 \\
1128\end{array}$ & $\begin{array}{r}113 \\
98 \\
93 \\
90\end{array}$ & $\begin{array}{l}912 \\
942 \\
952 \\
956\end{array}$ \\
\hline $\begin{array}{l}180 \\
365 \\
730\end{array}$ & $\begin{array}{l}1110 \\
1097 \\
1086\end{array}$ & $\begin{array}{l}88 \\
85 \\
84\end{array}$ & $\begin{array}{l}962 \\
966 \\
969\end{array}$ \\
\hline $\begin{array}{l}1095 \\
1460 \\
1825\end{array}$ & $\begin{array}{l}1081 \\
1078 \\
1075\end{array}$ & $\begin{array}{l}83 \\
8 ? \\
82\end{array}$ & $\begin{array}{l}970 \\
972 \\
972\end{array}$ \\
\hline
\end{tabular}

\begin{tabular}{|c|c|c|}
\hline $\begin{array}{l}\text { Percent } \\
\text { Increase } \\
\text { Over } \\
\text { Base Case }\end{array}$ & $\frac{\text { Injection Rate }}{\text { Production Rate }}$ & $\begin{array}{c}\text { Cumulative } \\
\text { Injection, } \\
\text { MSTB }\end{array}$ \\
\hline $\begin{array}{l}54 \\
59 \\
61 \\
62\end{array}$ & $\begin{array}{l}1.38 \\
1.24 \\
1.20 \\
1.18\end{array}$ & $\begin{array}{r}12 \\
36 \\
70 \\
104\end{array}$ \\
\hline $\begin{array}{l}62 \\
63 \\
64\end{array}$ & $\begin{array}{l}1.15 \\
1.14 \\
1.12\end{array}$ & $\begin{array}{l}204 \\
407 \\
804\end{array}$ \\
\hline $\begin{array}{l}64 \\
64 \\
64\end{array}$ & $\begin{array}{l}1.11 \\
1.11 \\
1.11\end{array}$ & $\begin{array}{l}1198 \\
1592 \\
1984\end{array}$ \\
\hline
\end{tabular}

Percent

Over

Base Case

113
103
97
95
91
88
86
85
84
84

Percent

Cumulative Increase

Production, Over

MSTB

9

28
56

85

172
350

350
704

1058

1413

1768
Base Case.

Cumulative Injection

Cumulative Production

$\begin{array}{ll}52 & 1.38 \\ 58 & 1.29 \\ 59 & 1.25 \\ 60 & 1.22 \\ 61 & 1.18 \\ 62 & 1.16 \\ 63 & 1.14 \\ 63 & 1.13 \\ 63 & 1.12 \\ 64 & 1.12\end{array}$

*The base case of stabilized rates gave in injection rate of 592 STB/day.

Part 2: 500 psi Injection Pressure*

$\begin{array}{rlll}10 & 1712 & 189 & 1124 \\ 30 & 1560 & 164 & 1175 \\ 60 & 1508 & 155 & 1192 \\ 90 & 1486 & 151 & 1199 \\ 180 & 1457 & 146 & 1208 \\ 365 & 1434 & 142 & 1214 \\ 730 & 1416 & 139 & 1220 \\ 1095 & 1408 & 138 & 1222 \\ 1460 & 1402 & 137 & 1224 \\ 1825 & 1397 & \vdots 36 & 1226\end{array}$

*The base case of stabilized rates gave an injection rate of $592 \mathrm{STB} /$ day.

$\begin{array}{lr}1.52 & 17 \\ 1.33 & 49 \\ 1.27 & 94 \\ 1.24 & 138 \\ 1.21 & 270 \\ 1.18 & 534 \\ 1.16 & 1052 \\ 1.15 & 1566 \\ 1.14 & 2077 \\ 1.14 & 2586\end{array}$

189
173
163
159
153
147
143
142
140
139

11
34
70
106

215
440
885
1332
1778
2226

90
94
98
100

102
104
105
105
106
106

1.52
1.41
1.33
1.30
1.25
1.21
1.19
1.18
1.17
1.16


TABLE F10

TWINNED CENTRAL INJECTION WELLS

FOUR 6.4 ACRE FIVE-SPOT PATTERNS

\begin{tabular}{|c|c|c|c|c|}
\hline $\begin{array}{l}\text { Distance } \\
\text { Between } \\
\text { Twinned } \\
\text { Wells, } \\
\mathrm{ft} \\
\end{array}$ & $\begin{array}{c}\text { Injection } \\
\text { Well } \\
\text { Pressure, } \\
\text { psi } \\
\end{array}$ & $\begin{array}{c}\text { Total } \\
\text { Rate of } \\
\text { Twinned } \\
\text { Wells, } \\
\text { STB/day } \\
\end{array}$ & $\begin{array}{l}\text { Percent } \\
\text { Increase } \\
\text { in Rate } \\
\end{array}$ & $\begin{array}{c}\text { Percent } \\
\text { Increase } \\
\text { in } \\
\text { Injectivity } \\
\end{array}$ \\
\hline Base Case & 253.3 & 85.6 & - & - \\
\hline 5 & 252.0 & 103.4 & 20.8 & 23.8 \\
\hline 10 & 251.5 & 109.5 & 27.9 & 32.4 \\
\hline 15 & 251.3 & 112.9 & 31.9 & 37.0 \\
\hline 20 & 251.0 & 115.7 & 35.2 & 41.3 \\
\hline
\end{tabular}




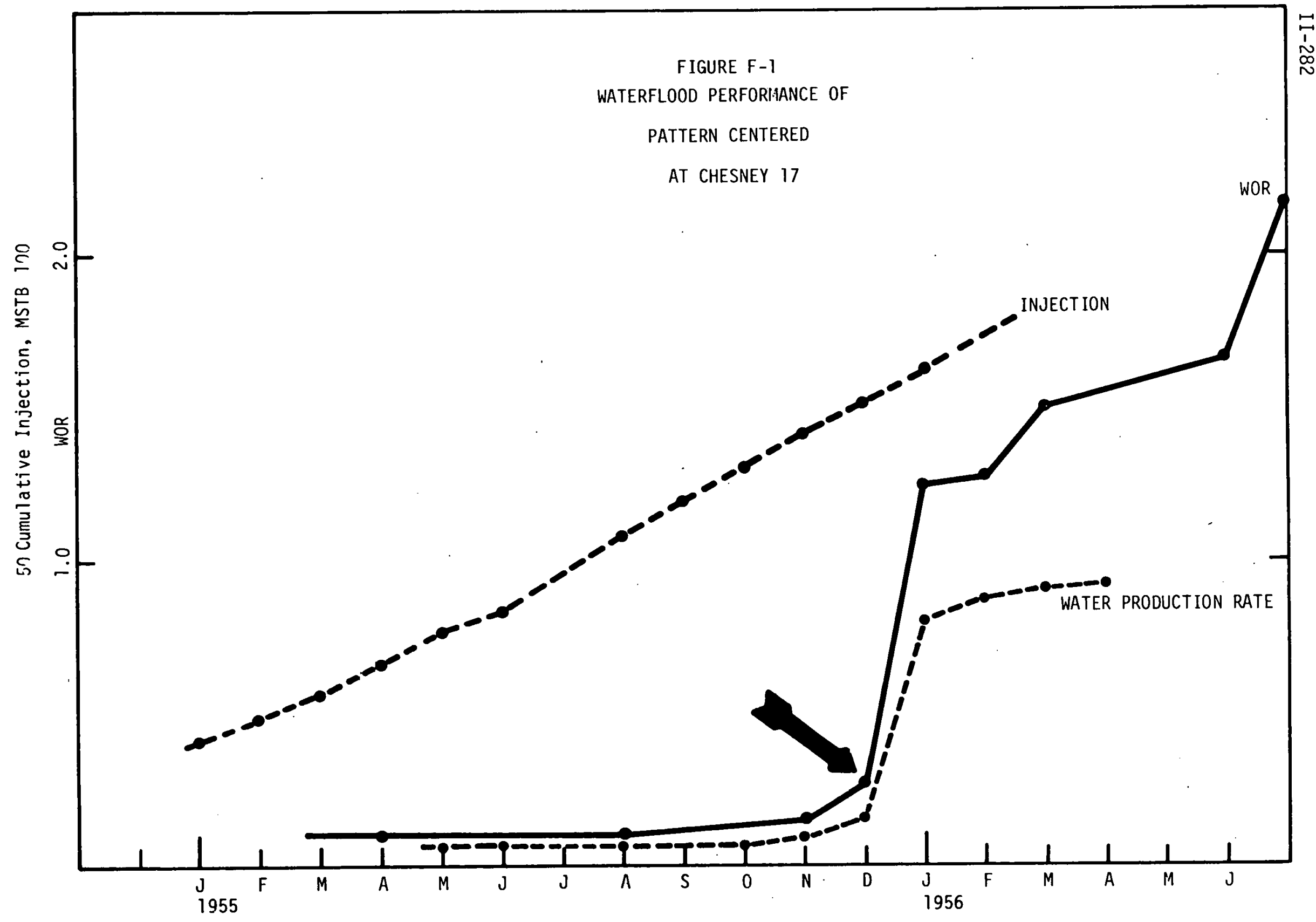




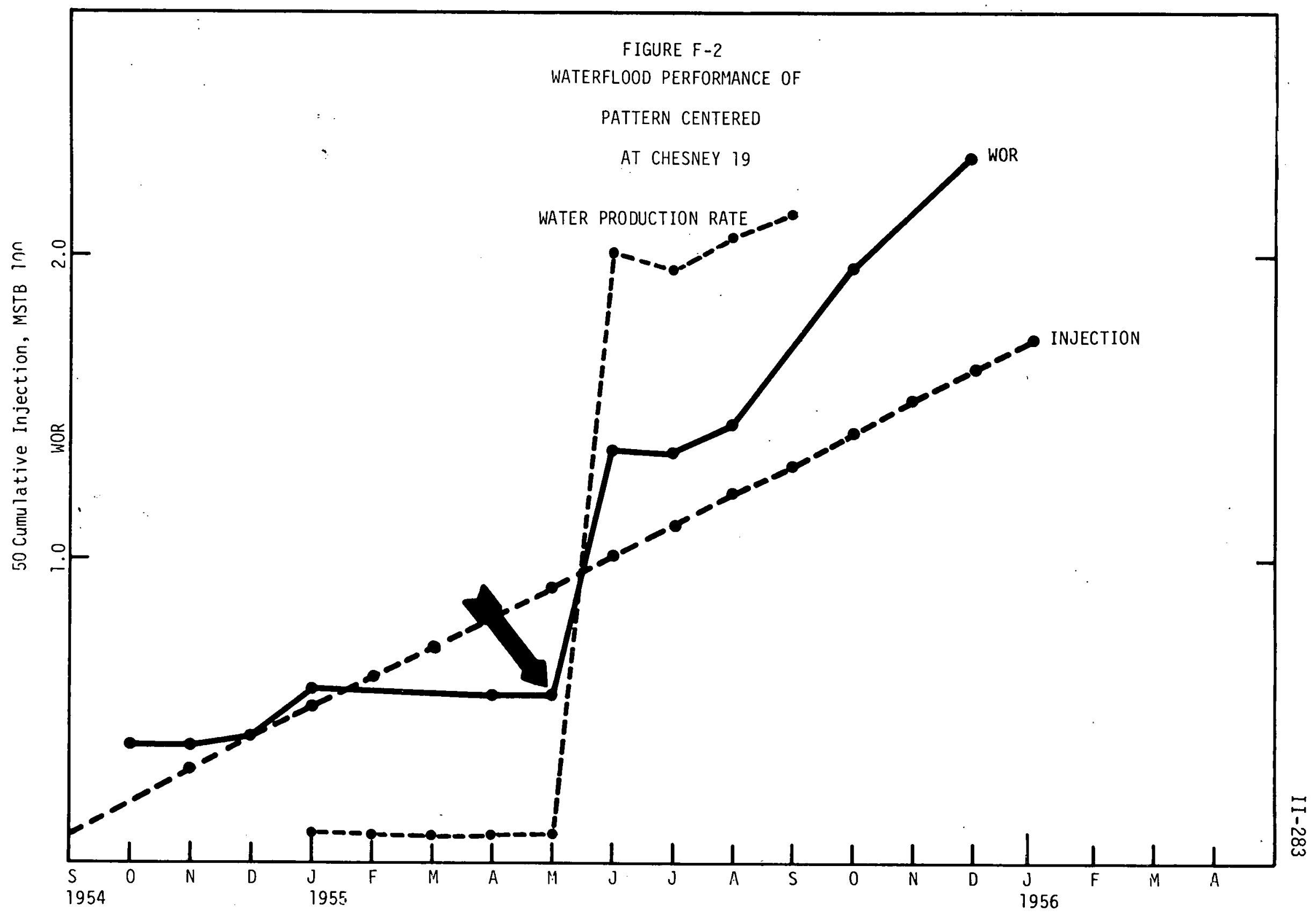




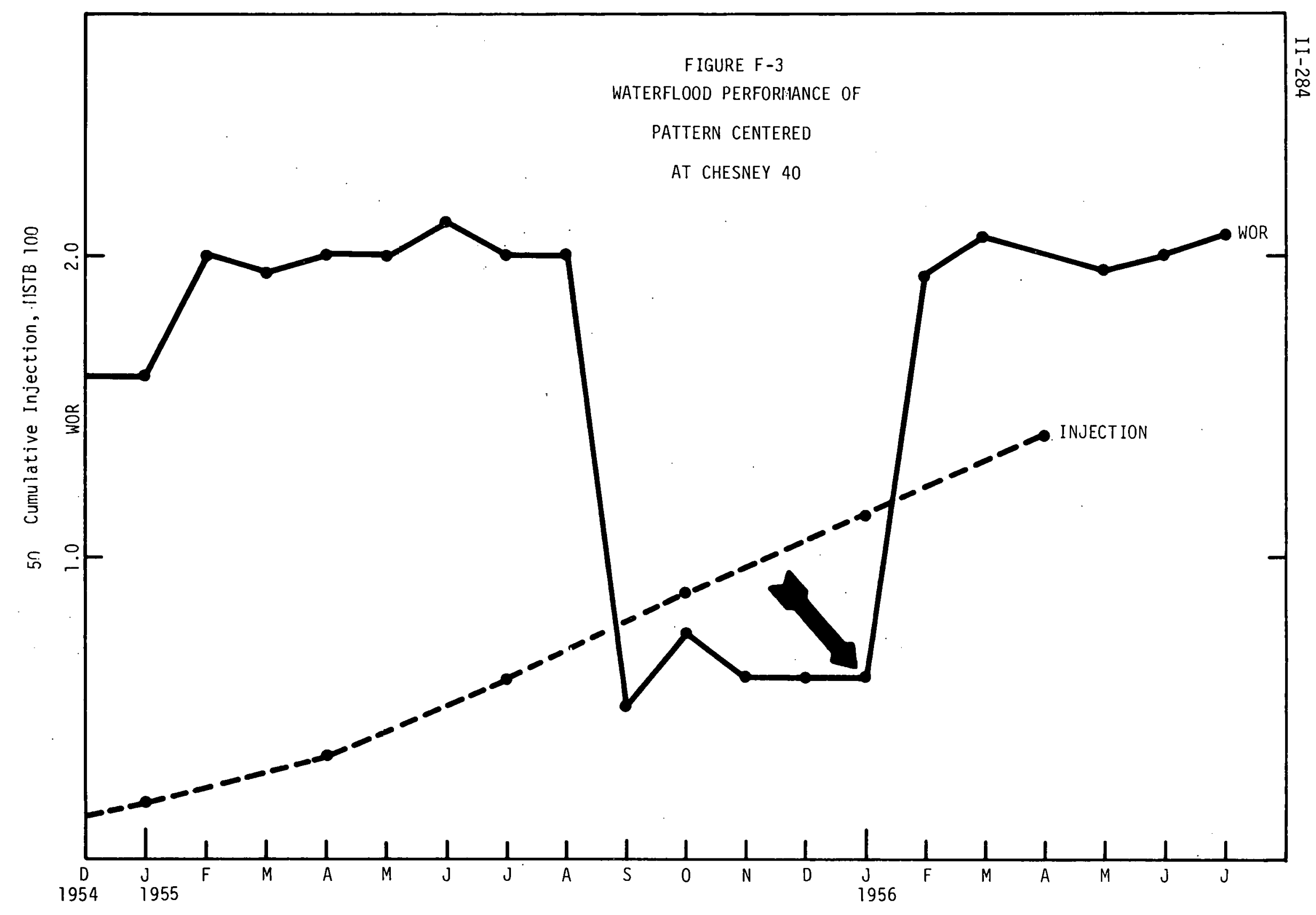


FIGURE F-4

RELATIVE PERMEABILITY AND CAPILLARY PRESSURE

FOR PREFLUSH CALCULATIONS

$M=1$

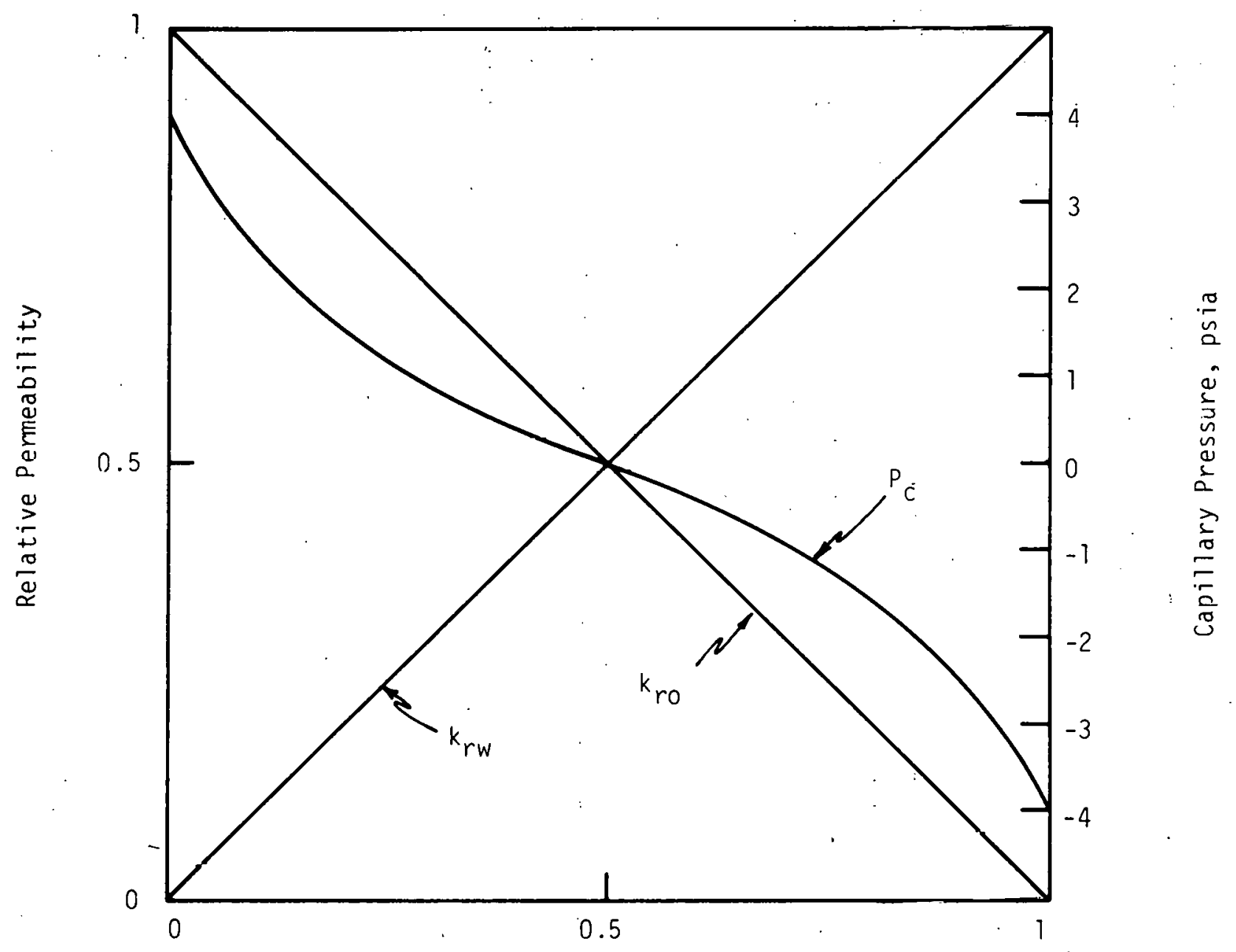

Injected Water Saturation 
FIGURL F-5

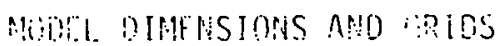

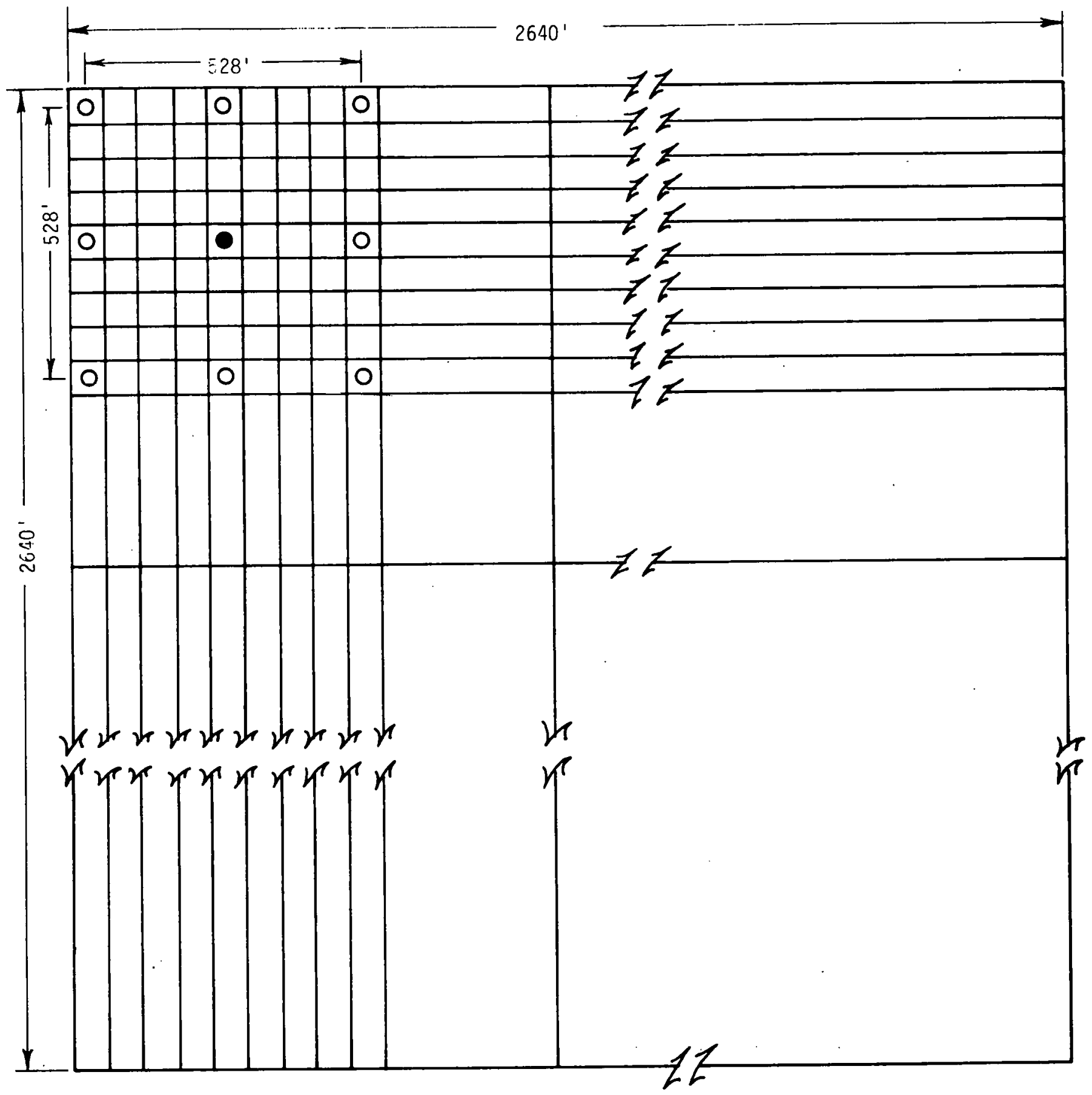

- Production Wells

O - Injection Wells 
FIGURE F-6

DISPLACEMENT OF BRINE WITH FRESH WATER IN A CORE

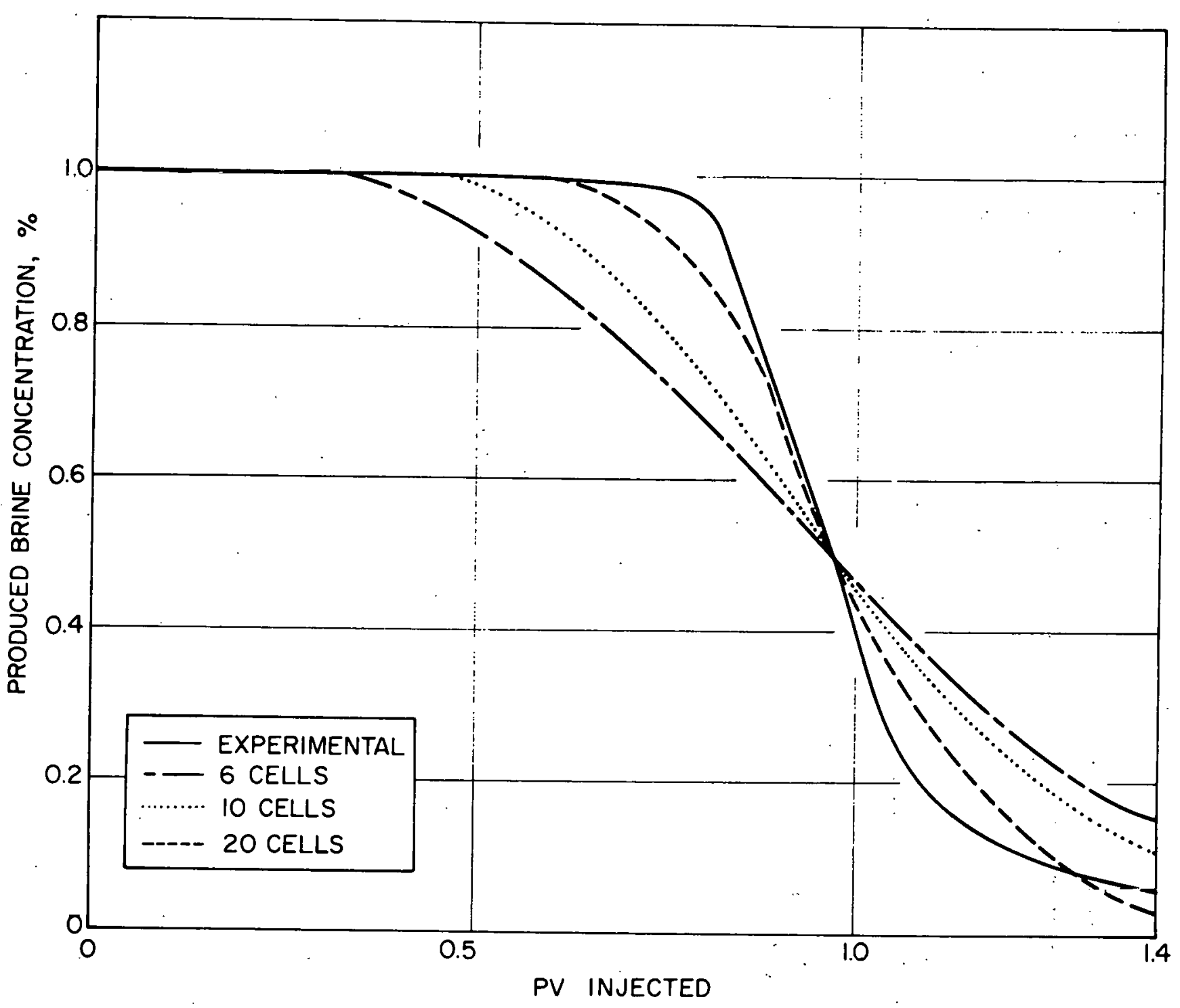


FIGURE F-7

CHESNEY PATTERN

ESTIMATED PREFLUSH INJECTION VS TIME

Case 1: Side and corner wells put on injection simultaneously with and without stimulation of producer

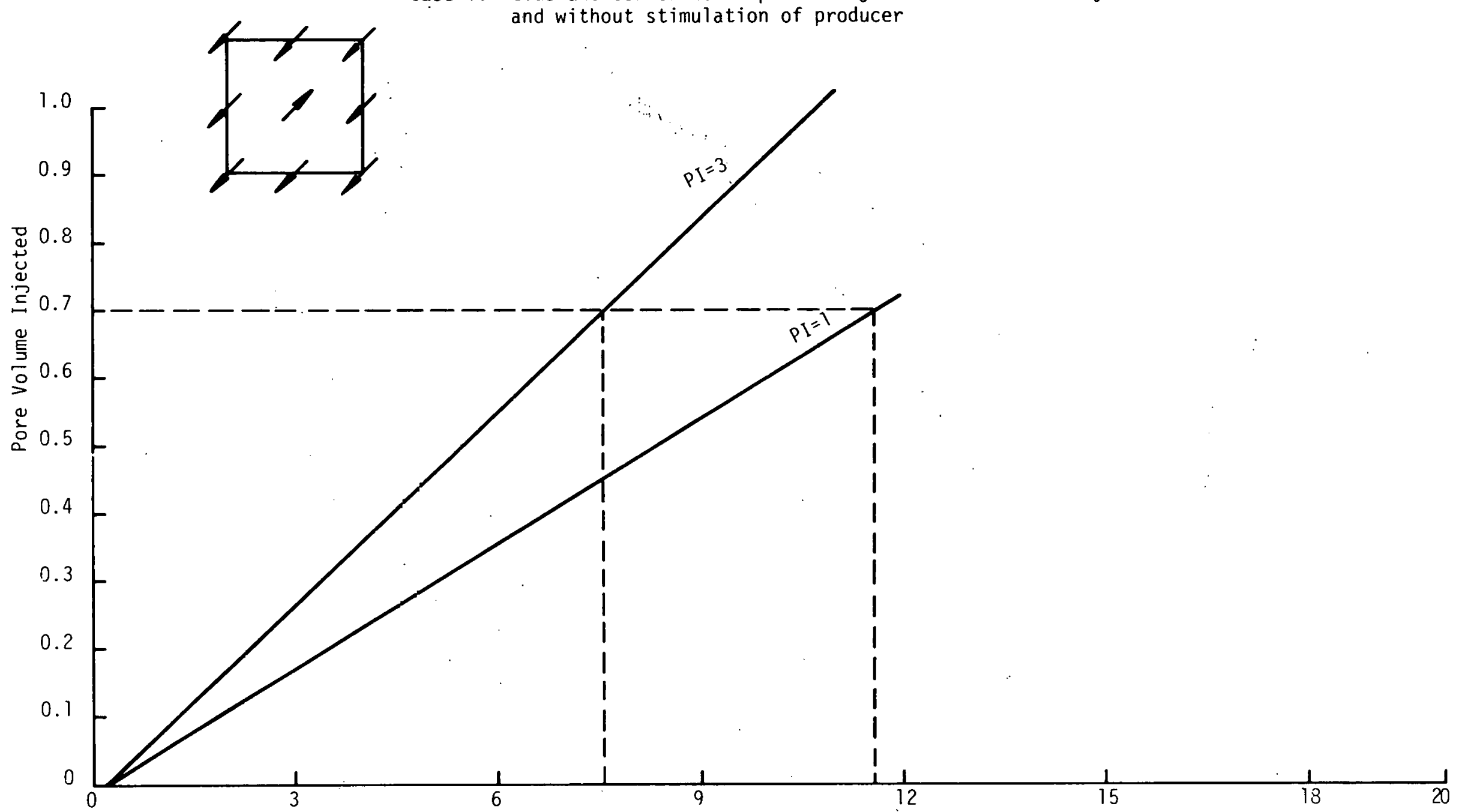

Time Since. Start of Injection, i.lonths 
FIGURE F-8

CHESNEY PATTERN

EST IMATED PREFLUSH INJECTION VS TIME:

Case 2: Side wells put on injection after 30 or 90 days

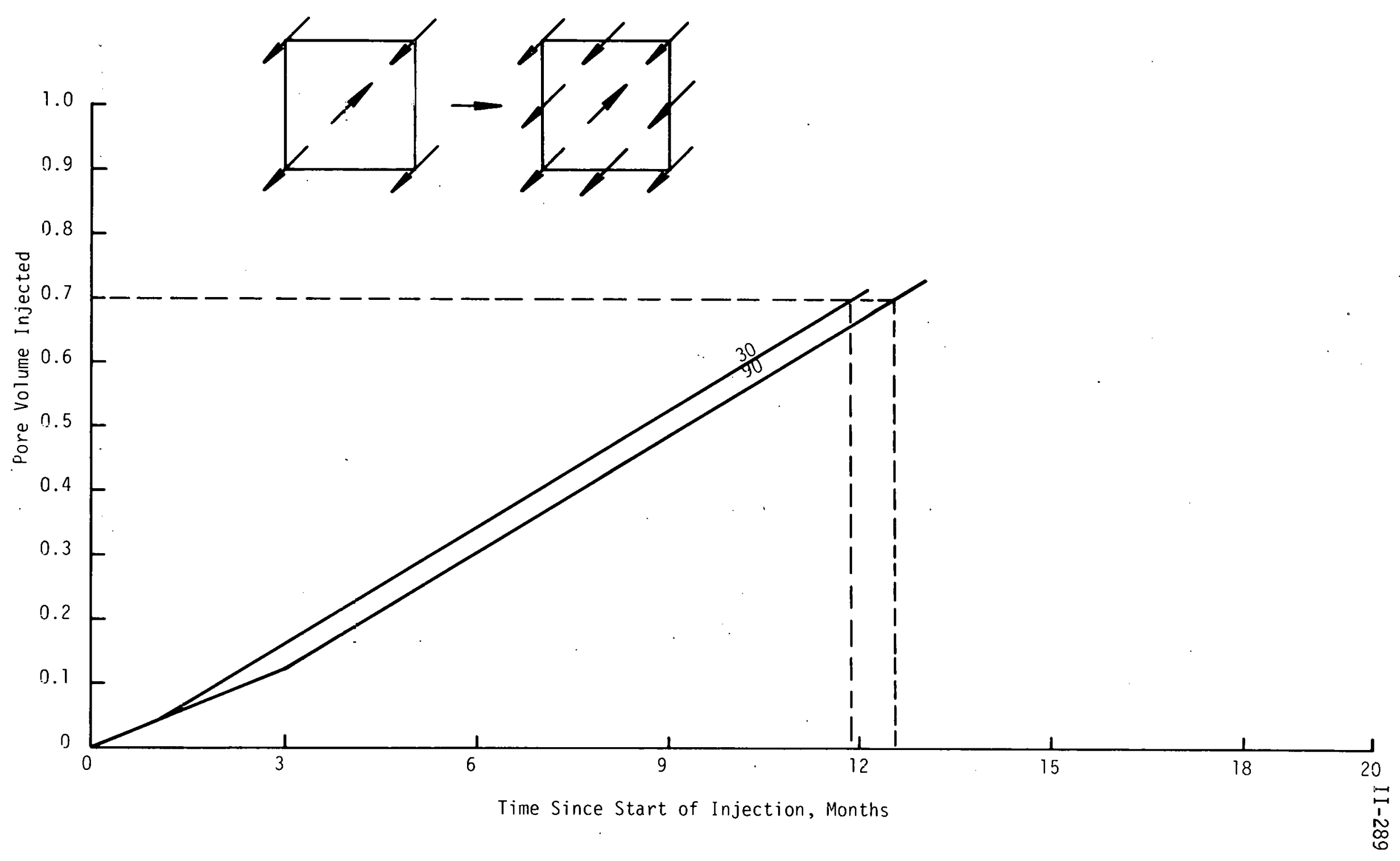


FIGURE F-9

CHESNEY PATTERN

EST IMATED PREFLUSH INJECTION VS TIME

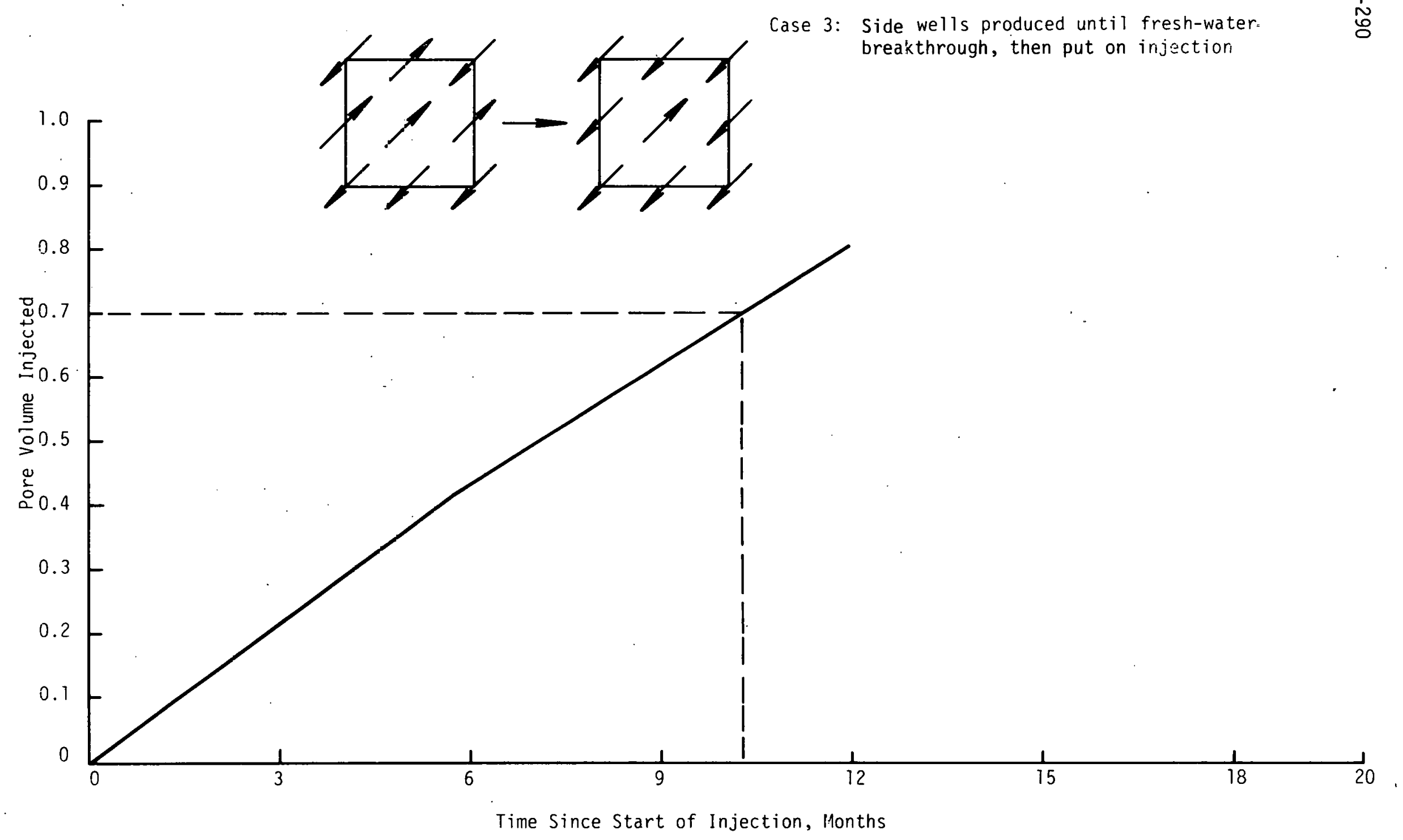


HEGBERG PATTERN

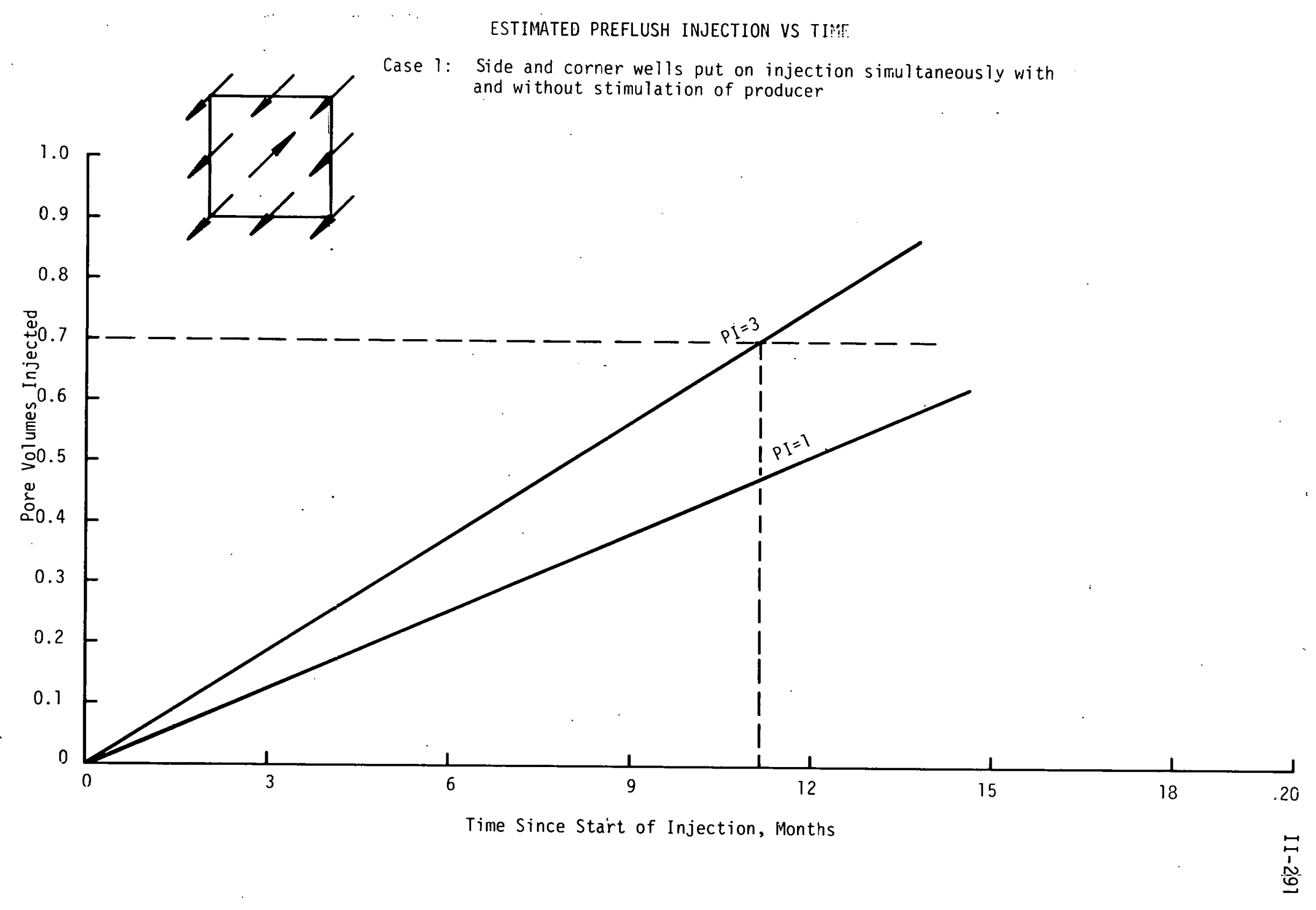


FIGURE F-11

HEGBERG PATTERN

ESTIMATED PREFLUSH INJECTION VS TIME

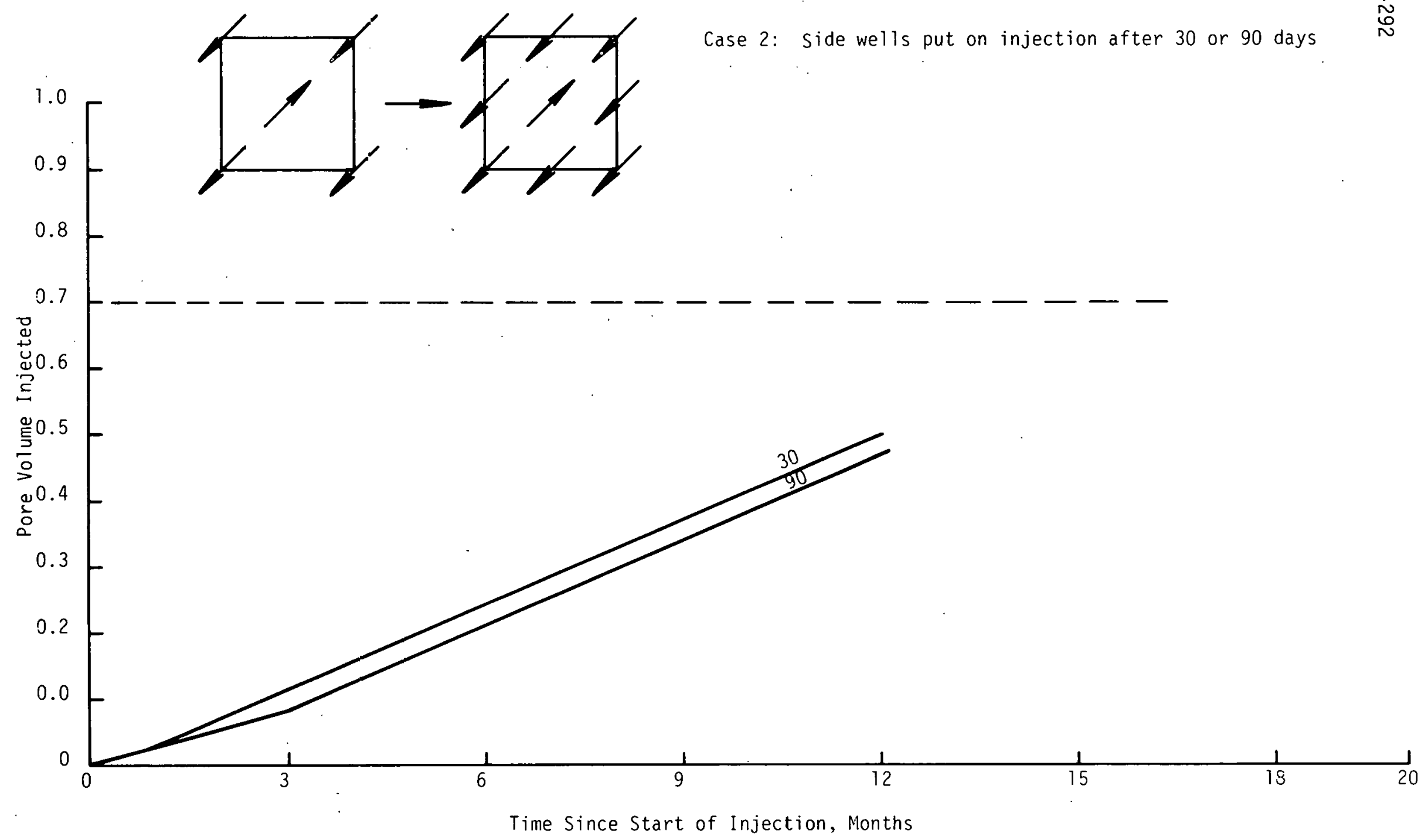


FIGURE $F-12$

HEGBERG PATTERN

EST IMATED PREFLUSH INJECTION VS TIME

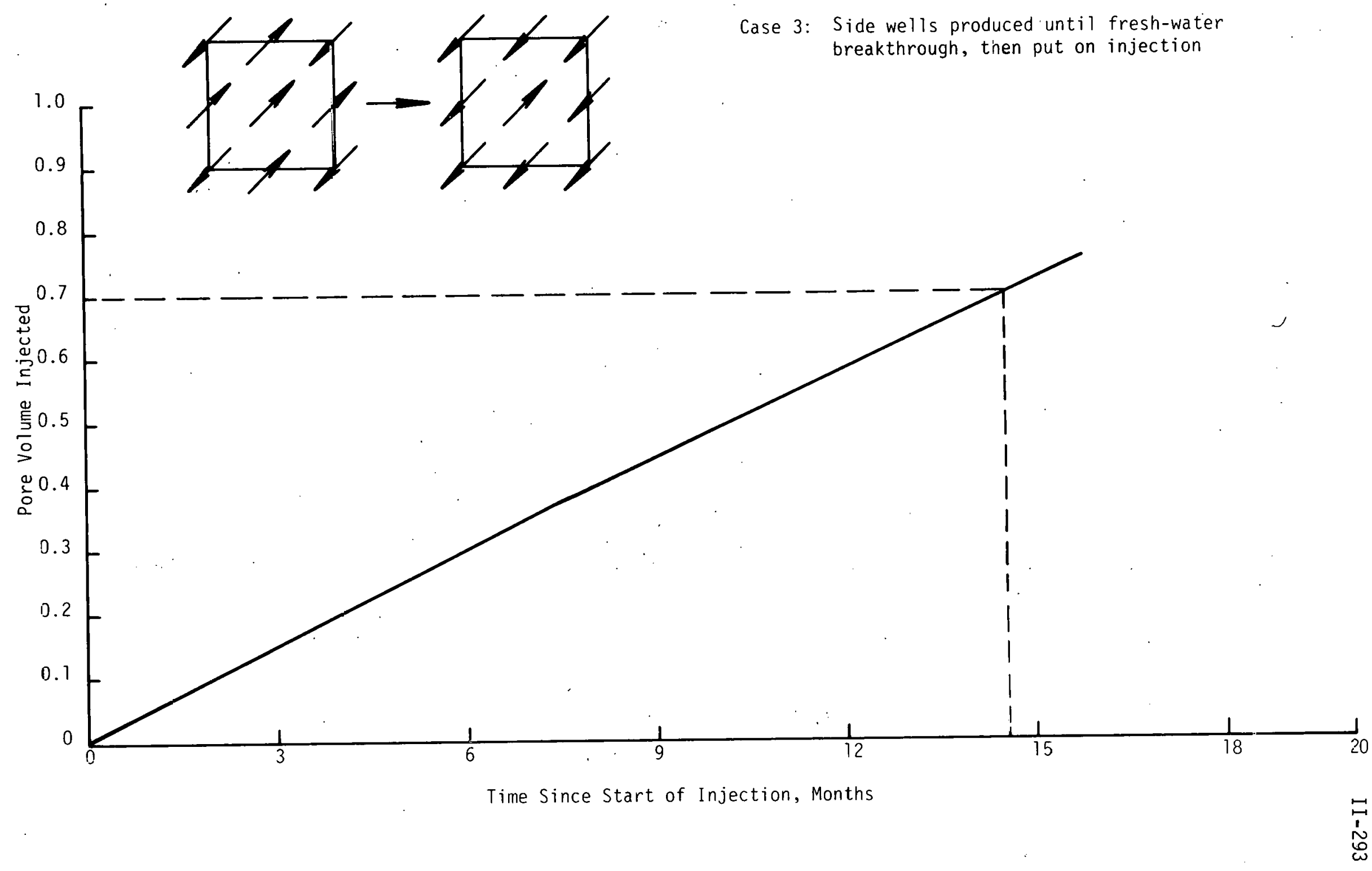


FISUURE F-13

HEGBERG PATTERN

FRESH WATER SATURATION IAAPS

FOR QUARTER OF A NINE-SPOT

TIIIE $=10$ IDNTHS

Case 1: Ho stimulation,

simultaneous injection Case 2b: Delay injection in side wells
for 90 days
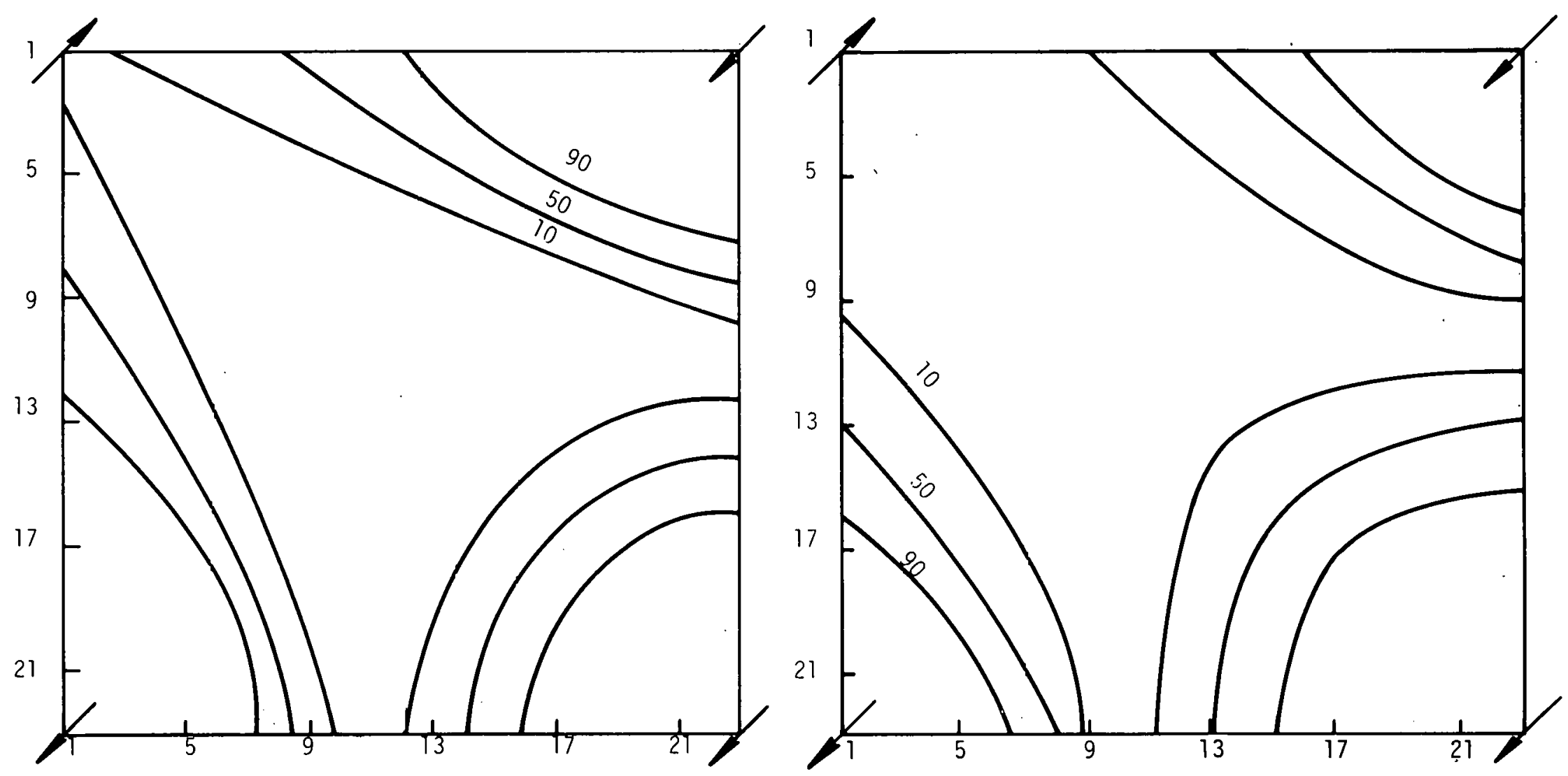
FIGURE $F-14$

HEŔBERG PATTER'N

FRESH HATER SATURATION IIAFS

FOR QUARTER OF A NINE-SPOT

TII.IE = 10 IIONTHS

Case 3: Side wells produced until

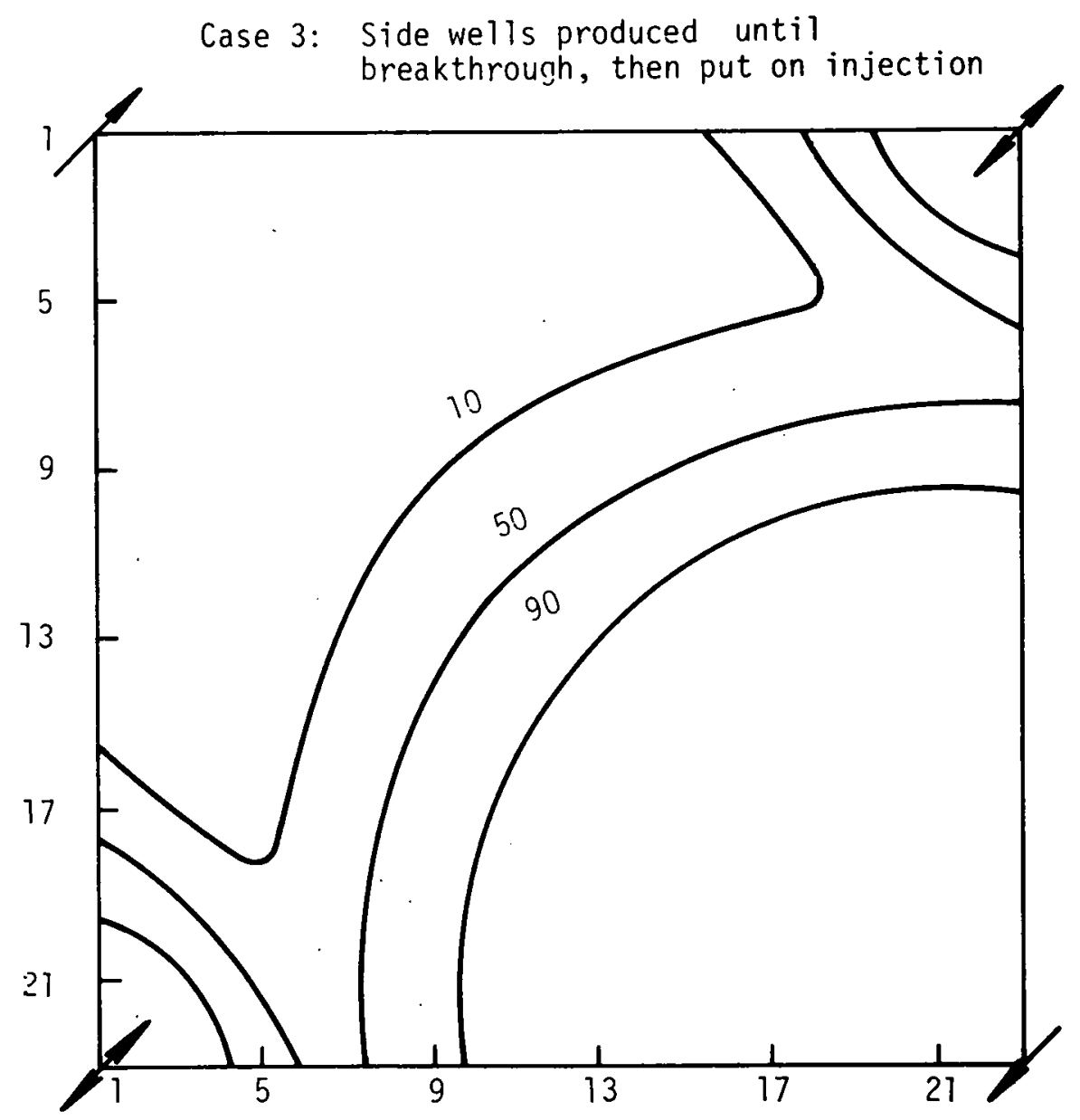




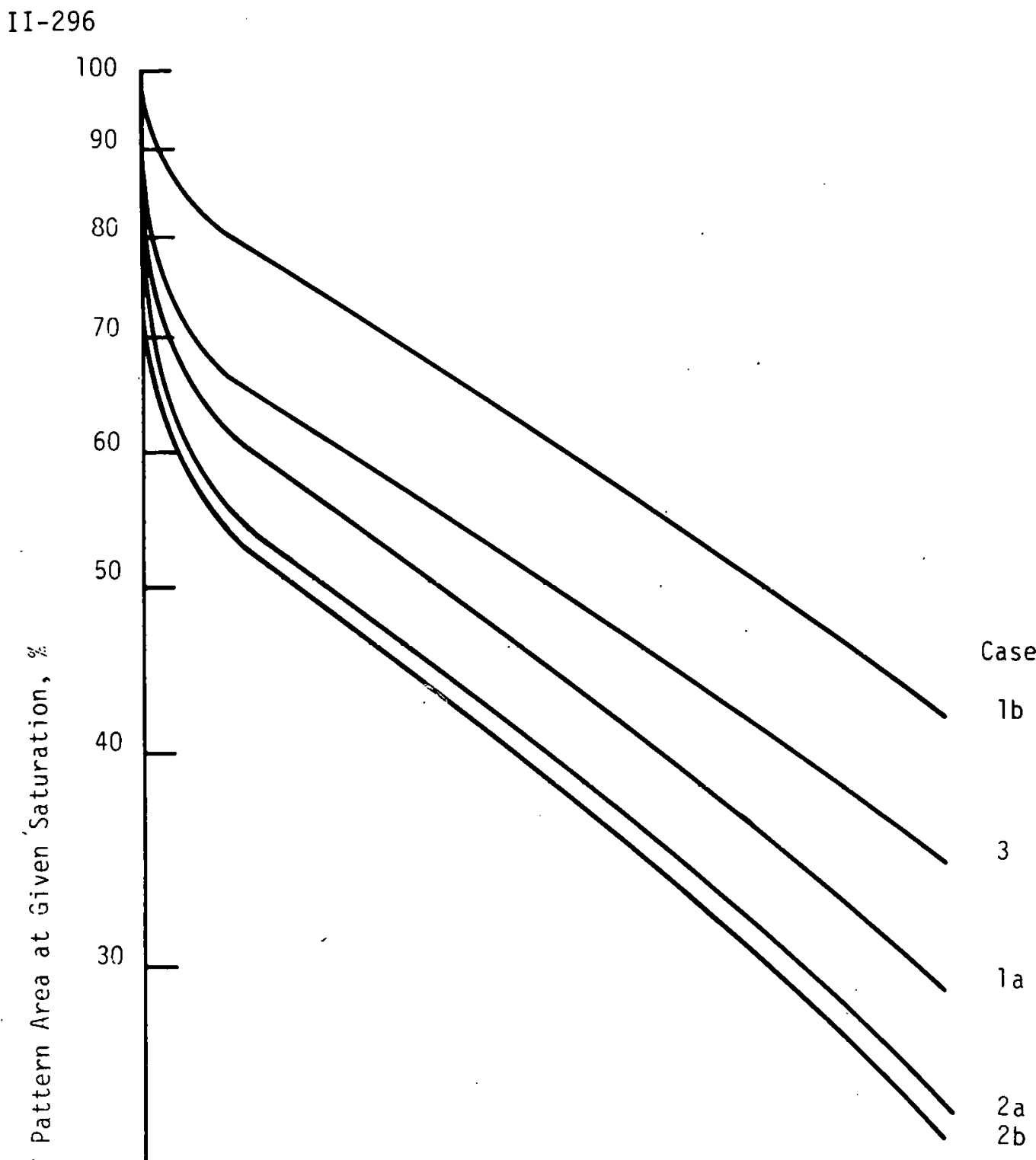

FIGURE $F-15$

HEGBERG PATTERN

r.MPARISON OF DISPLACEMENT EFFICIENCIES

10

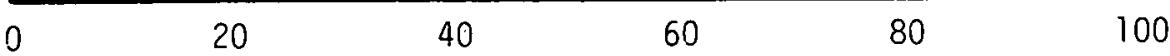

Injected Water Saturation Above Value Shown, \% 


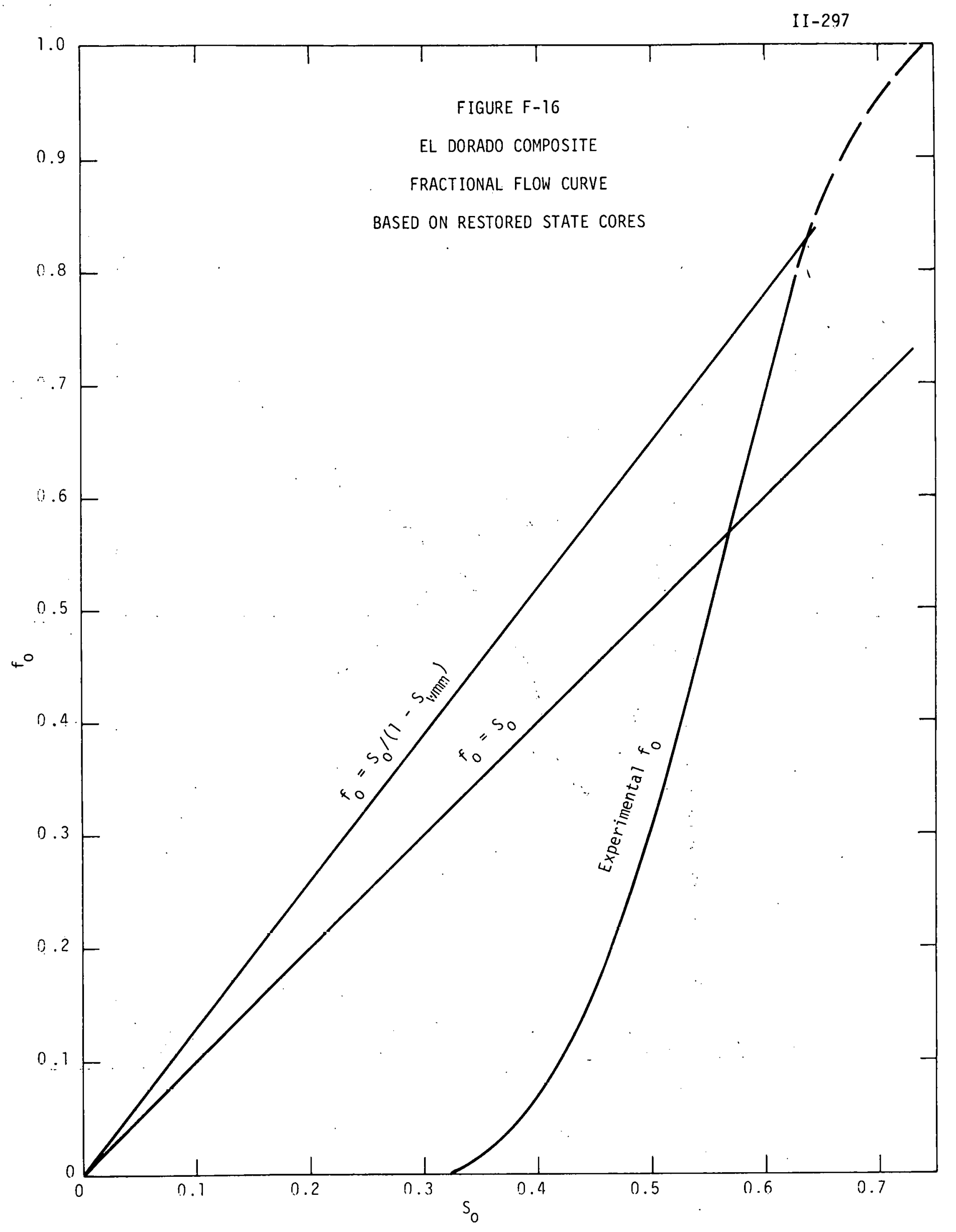




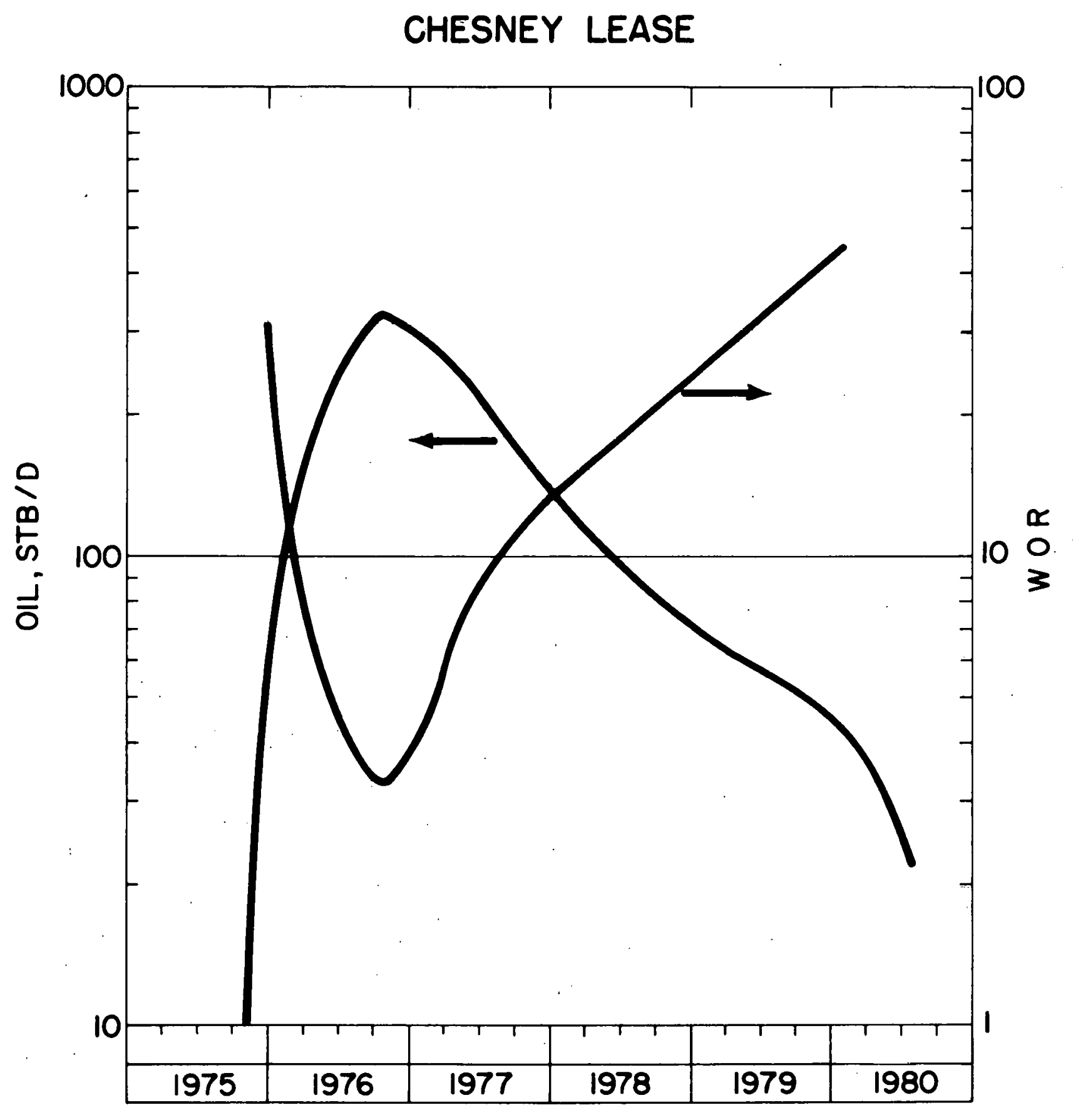

FIGURE $F-17$

ESTIMATED PRODUCTION SCHEDULE 
HEGBERG LEASE

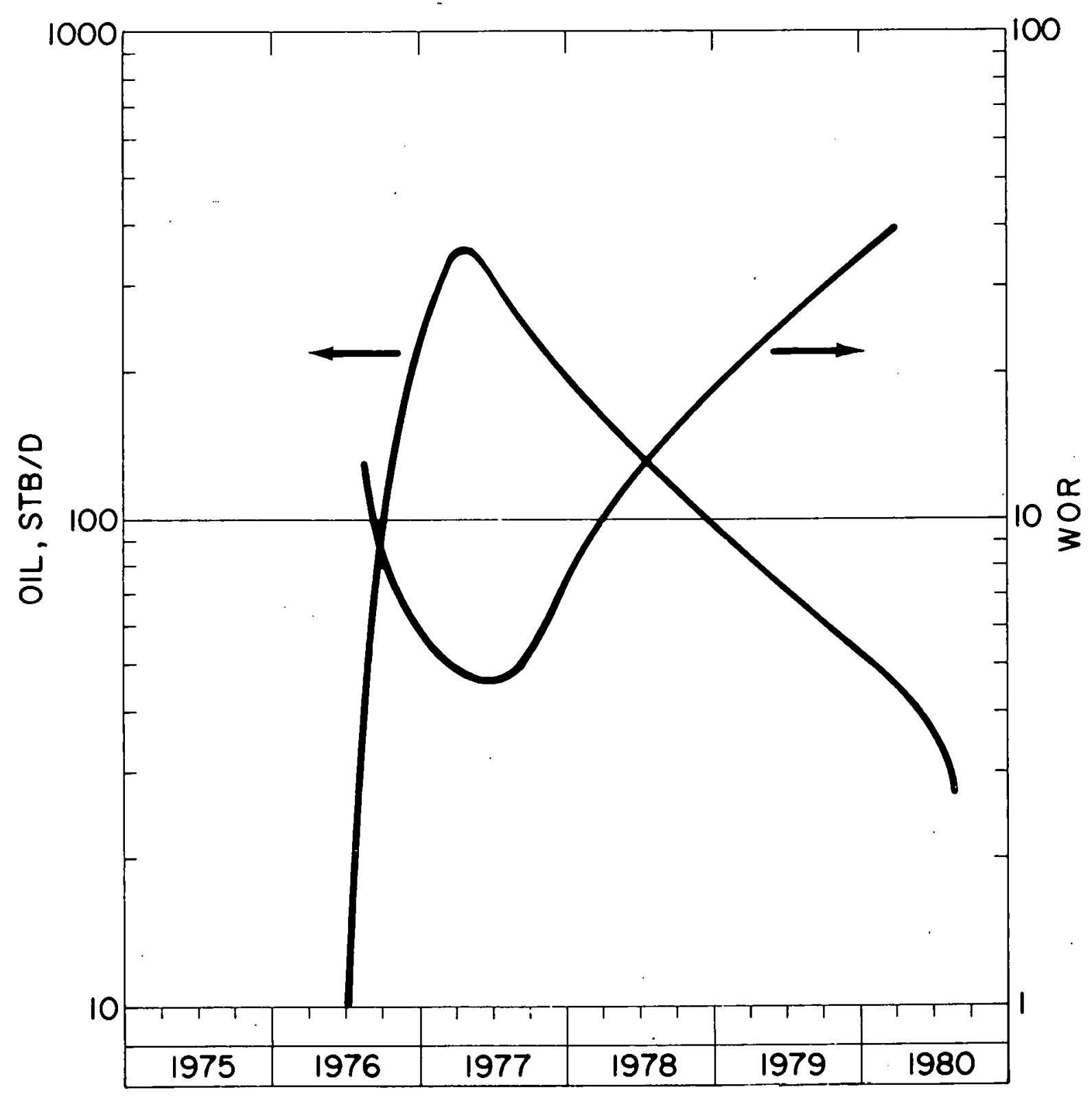

FICULLE F..10

ESTIIMTED PROOLCTIOH SCHEDULE 


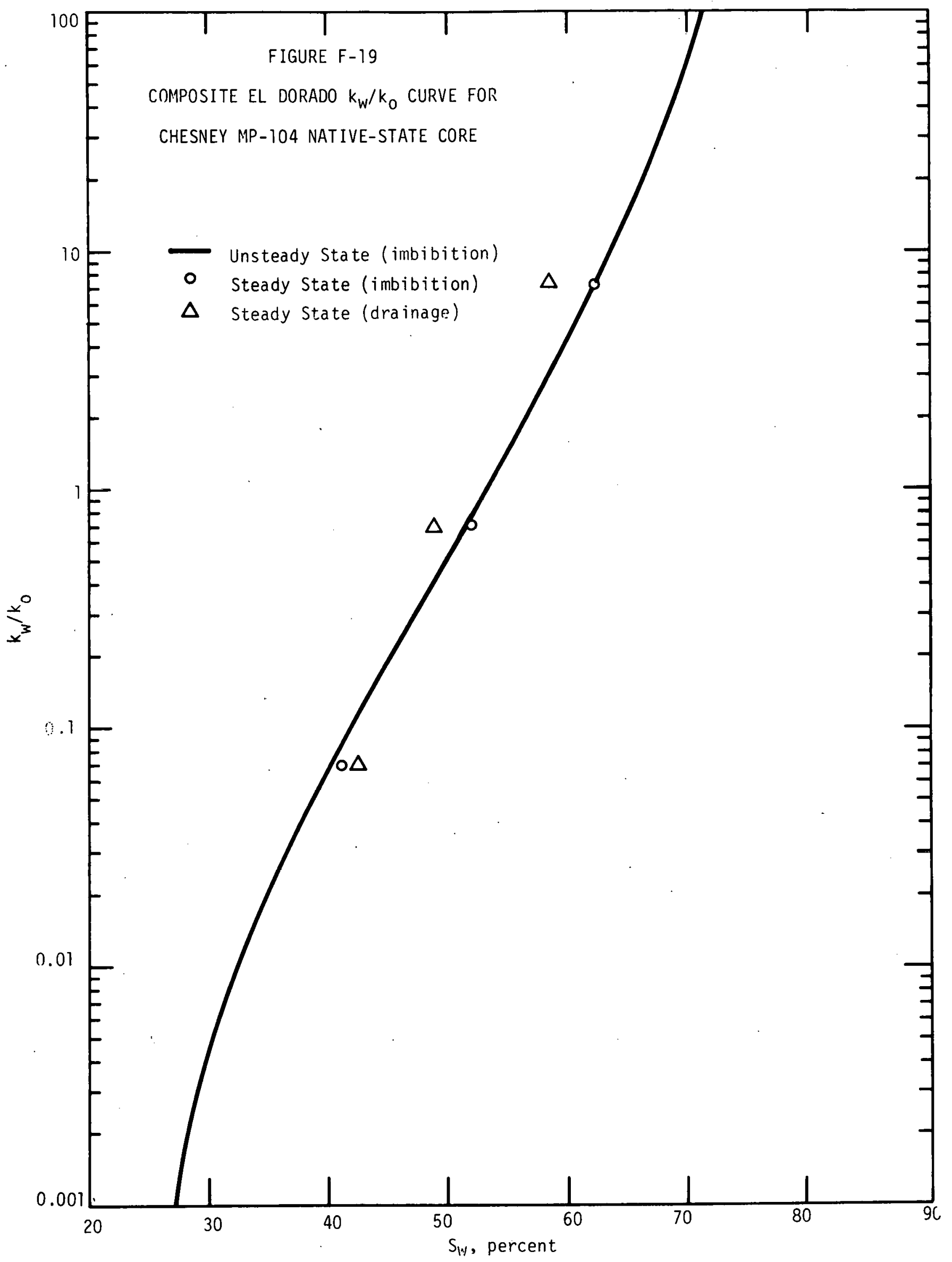


II -30 I

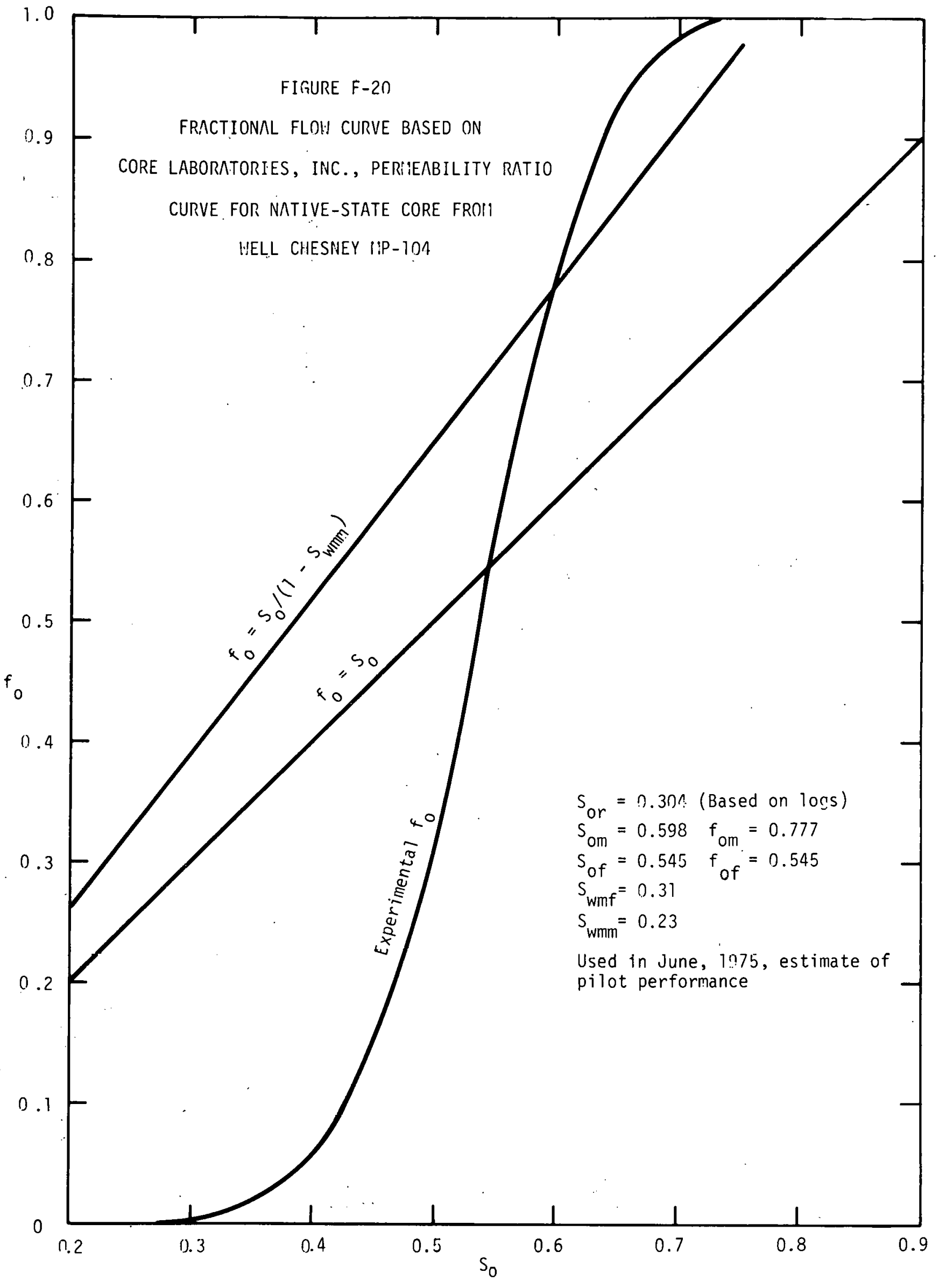


OIL RECOVERY VS CUMULATIVE INJECTION

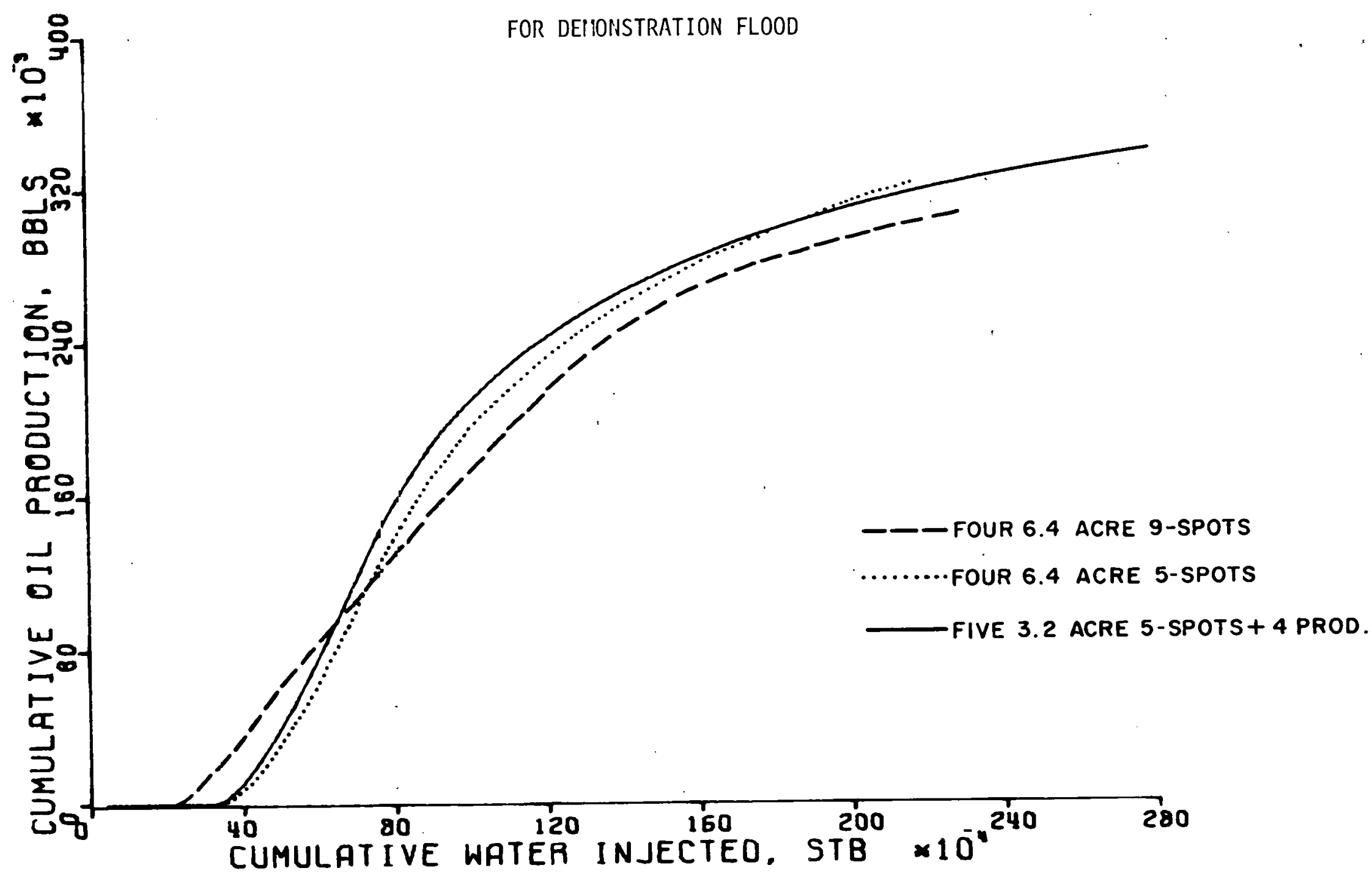


FIGURE F-22

HOR VS CURIULATIVE INJECTION

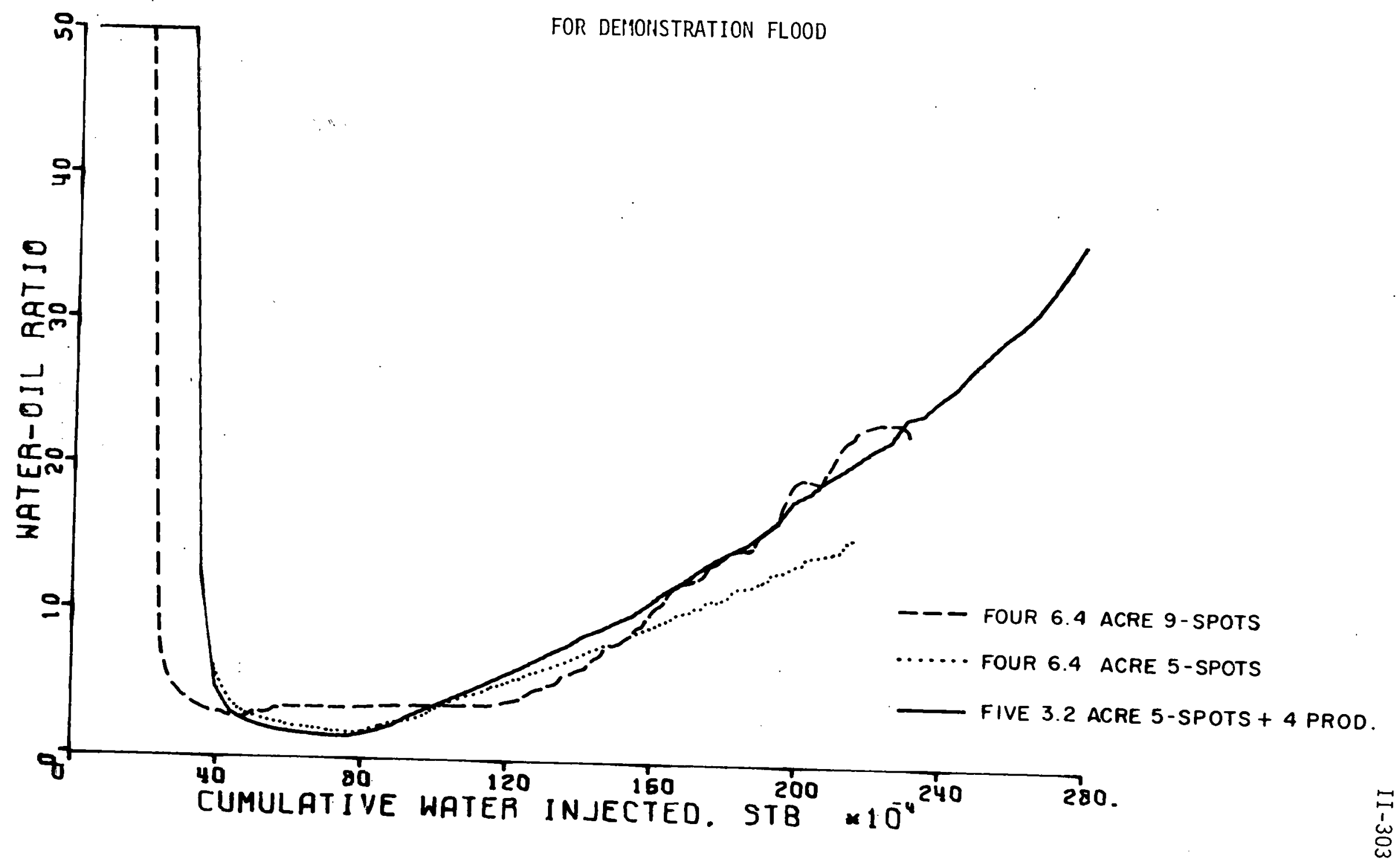


FIGURE F-23

OIL RECOVERY VS TIME FOR DEMONSTRATION FLOOD

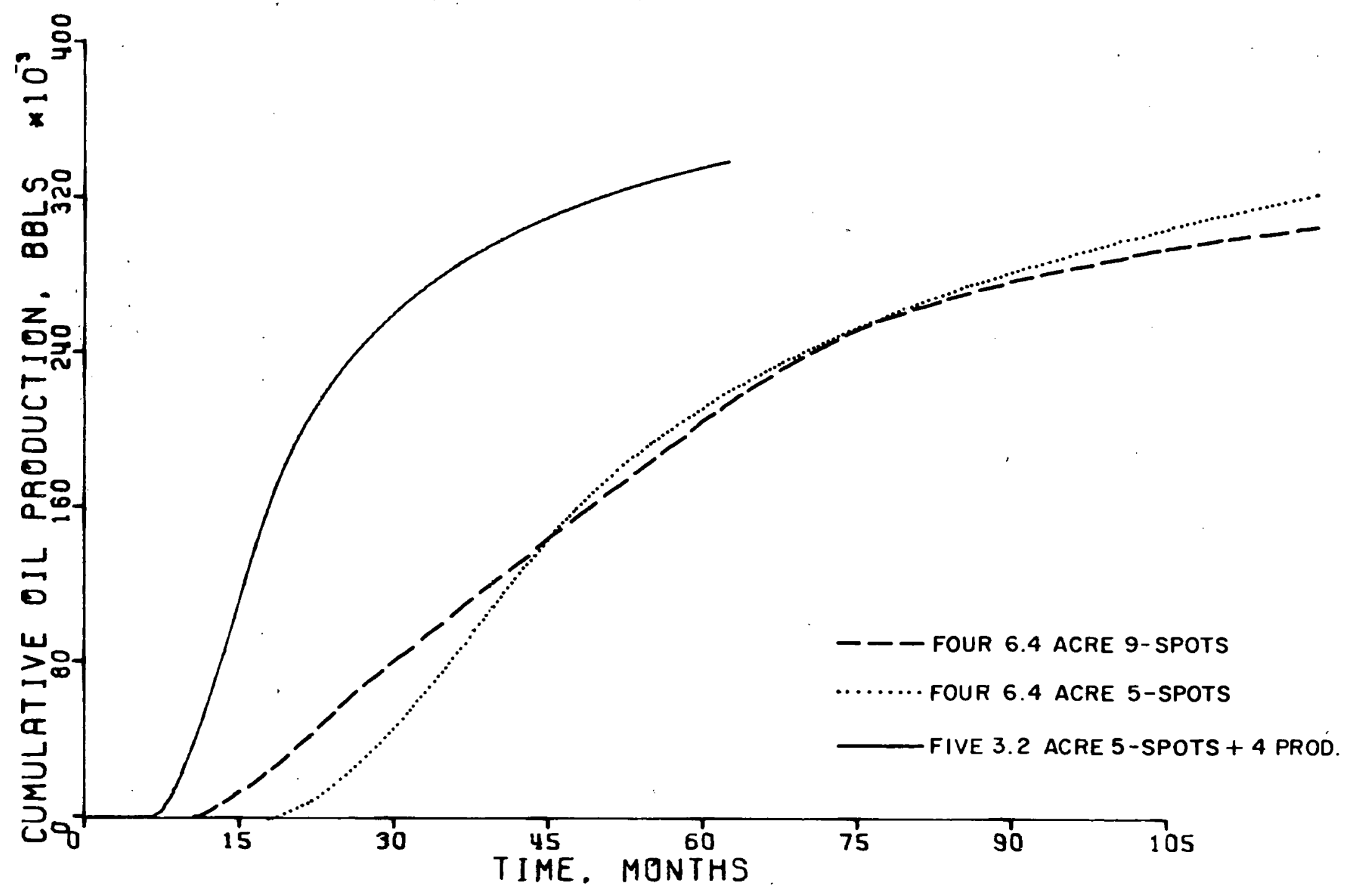


FIGURE F-24

WOR VS TIME FOR DEMONSTRATION FLOOD

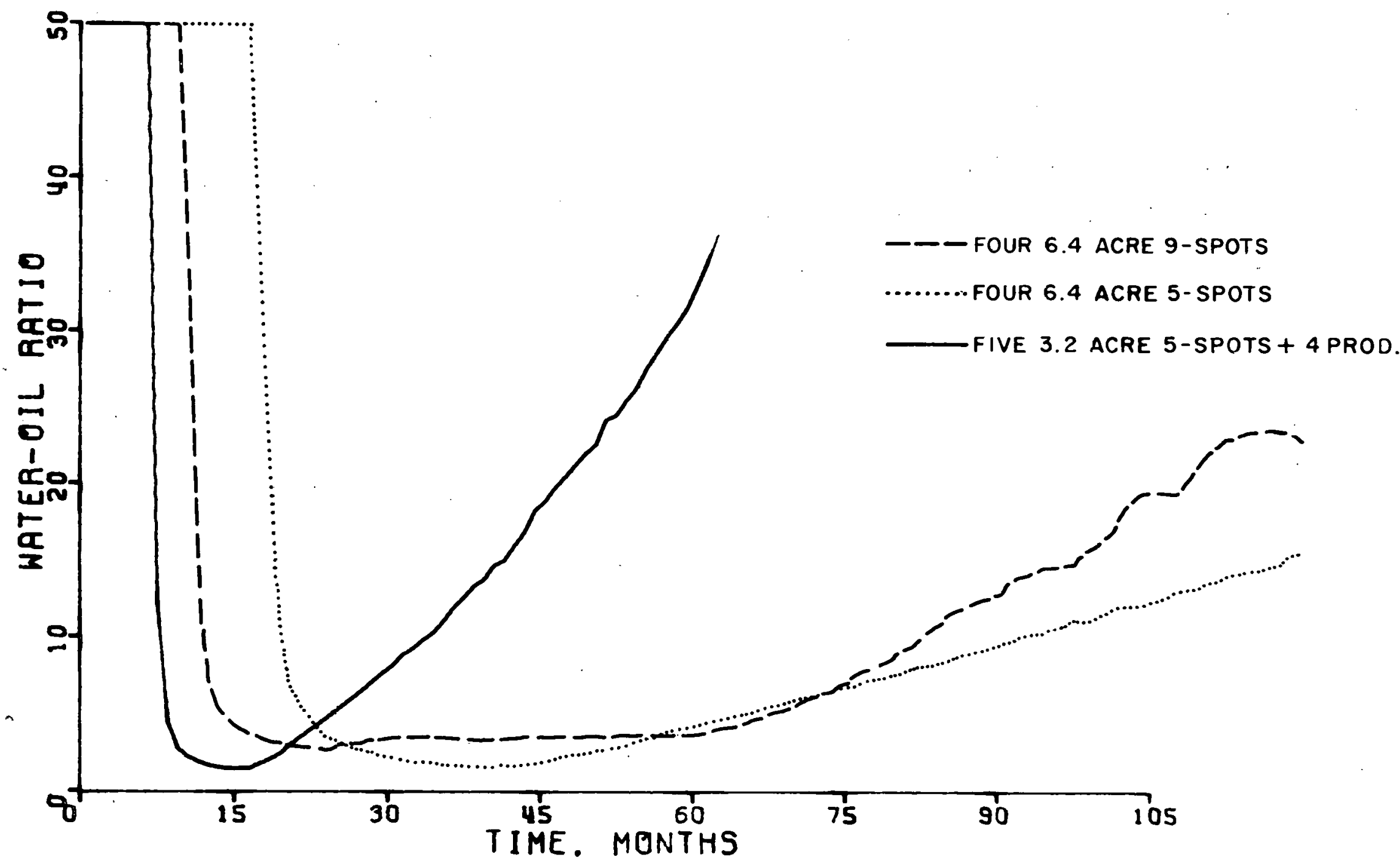




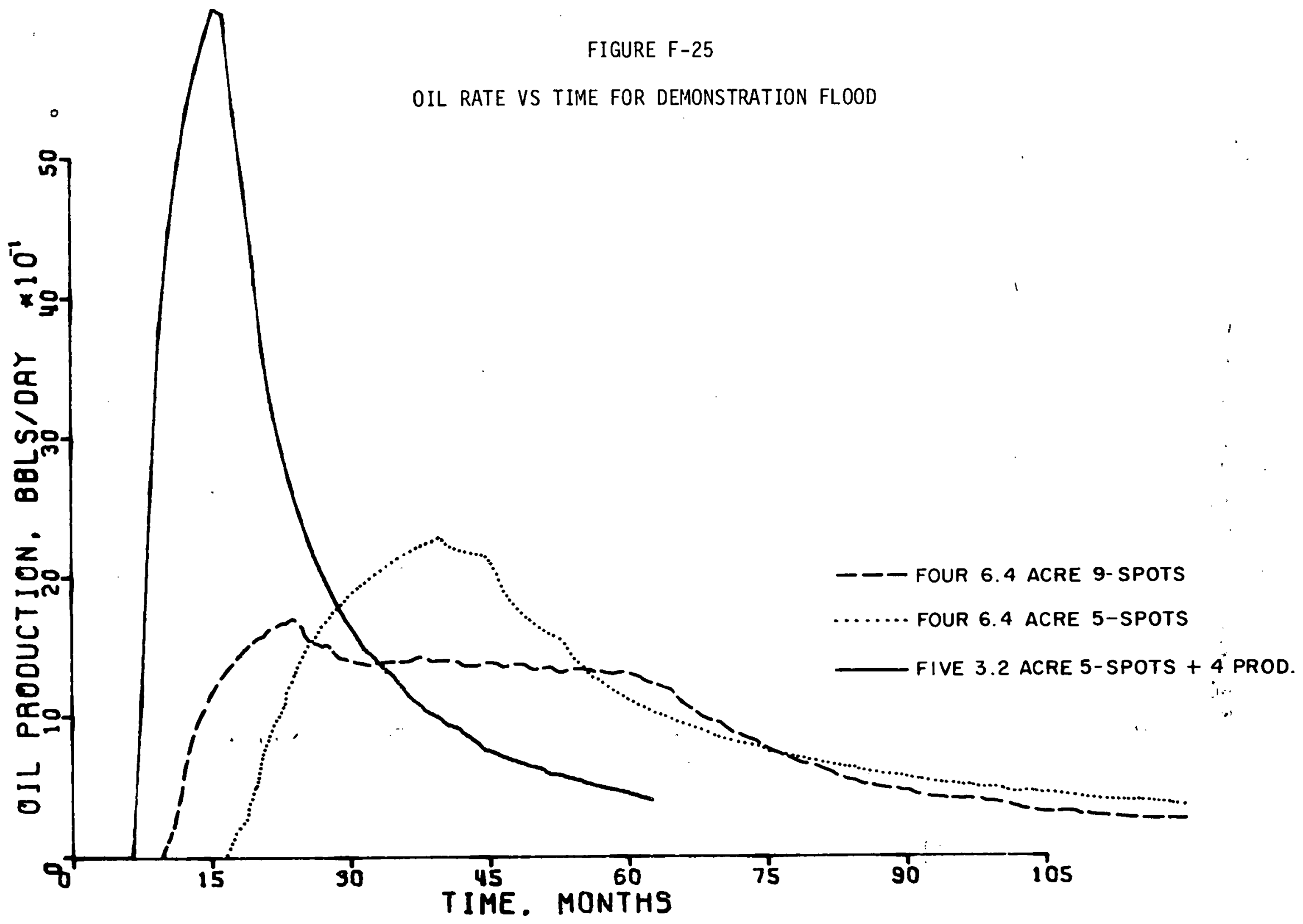




\section{FIGURE F-26 \\ PATTERN NOMENCLATURE}

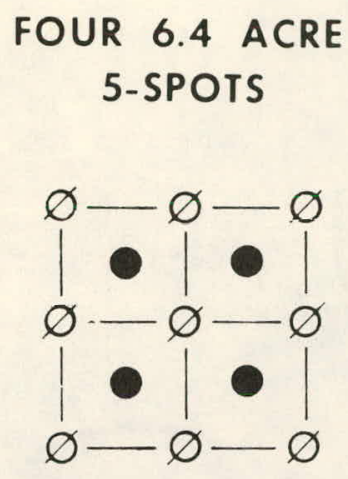

$$
\begin{aligned}
& \text { SIX } 3.2 \text { ACRE } \\
& \text { 5-SPOTS } \\
& +\varnothing \varnothing+ \\
& \varnothing^{\prime}, \varnothing^{\prime} \circ \varnothing
\end{aligned}
$$

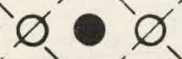

$$
\begin{aligned}
& \varnothing \cdot \varnothing^{\prime} \varnothing \\
& +\varnothing_{\varnothing} \varnothing+
\end{aligned}
$$

FIVE 3.2 ACRE 5-SPOTS PLUS TWO PRODUCERS

$+\varnothing \varnothing+$

$\gamma^{\prime} \varnothing^{\prime}-\varnothing^{\prime}$

- $\varnothing$ - $\varnothing$ '

$\varnothing^{\prime} \varnothing^{\prime} \varnothing \varnothing$

$+\varnothing^{\prime} \varnothing^{\prime}+$
FOUR 6.4 ACRE

9-SPOTS

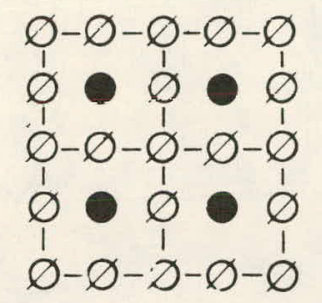

SEVEN 3.2 ACRE

$$
\text { 5-SPOTS }
$$

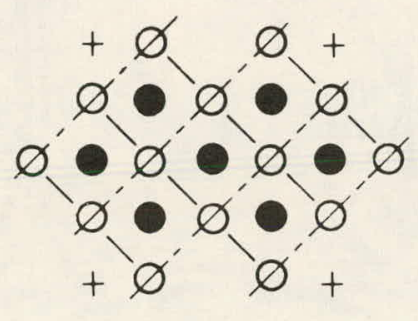

FIVE 3.2 ACRE

5-SPOTS PLUS

THREE PRODUCERS

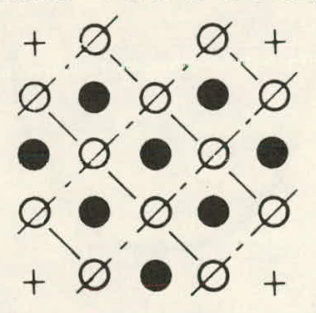

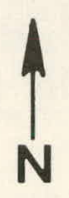

FIVE 3.2 ACRE

5-SPOTS

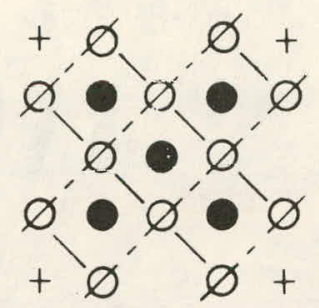

EIGHT 3.2 ACRE

5-SPOTS

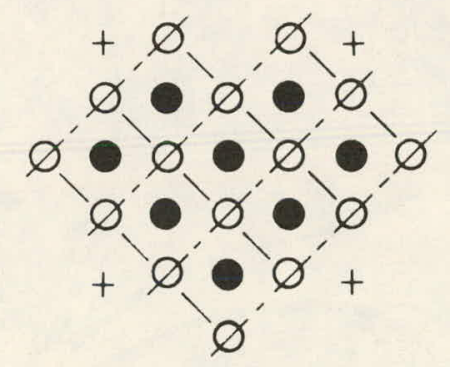

FIVE 3.2 ACRE 5-SPOTS PLUS FOUR PRODUCERS

$$
\begin{aligned}
& +\varnothing \odot \sigma+ \\
& \varnothing^{\prime} \varnothing^{\prime} \varnothing \\
& \text { - } \varnothing \text { ' } \varnothing \text { '. }
\end{aligned}
$$

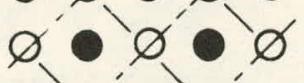

$$
\begin{aligned}
& +\varnothing \odot \varnothing+
\end{aligned}
$$

PRODUCTION WELL

$\varnothing$ INJECTION WeLl

+ CORNERS OF ORIGINAL PATTERN 
FIGURE F-27

FOUR 6.4 ACRE NINE-SPOTS ON EACH LEASE

(30 PSI BHP IN PRODUCERS, BALANCED INJECTION WITH EQUAL BHP IN ALL INJECTORS)

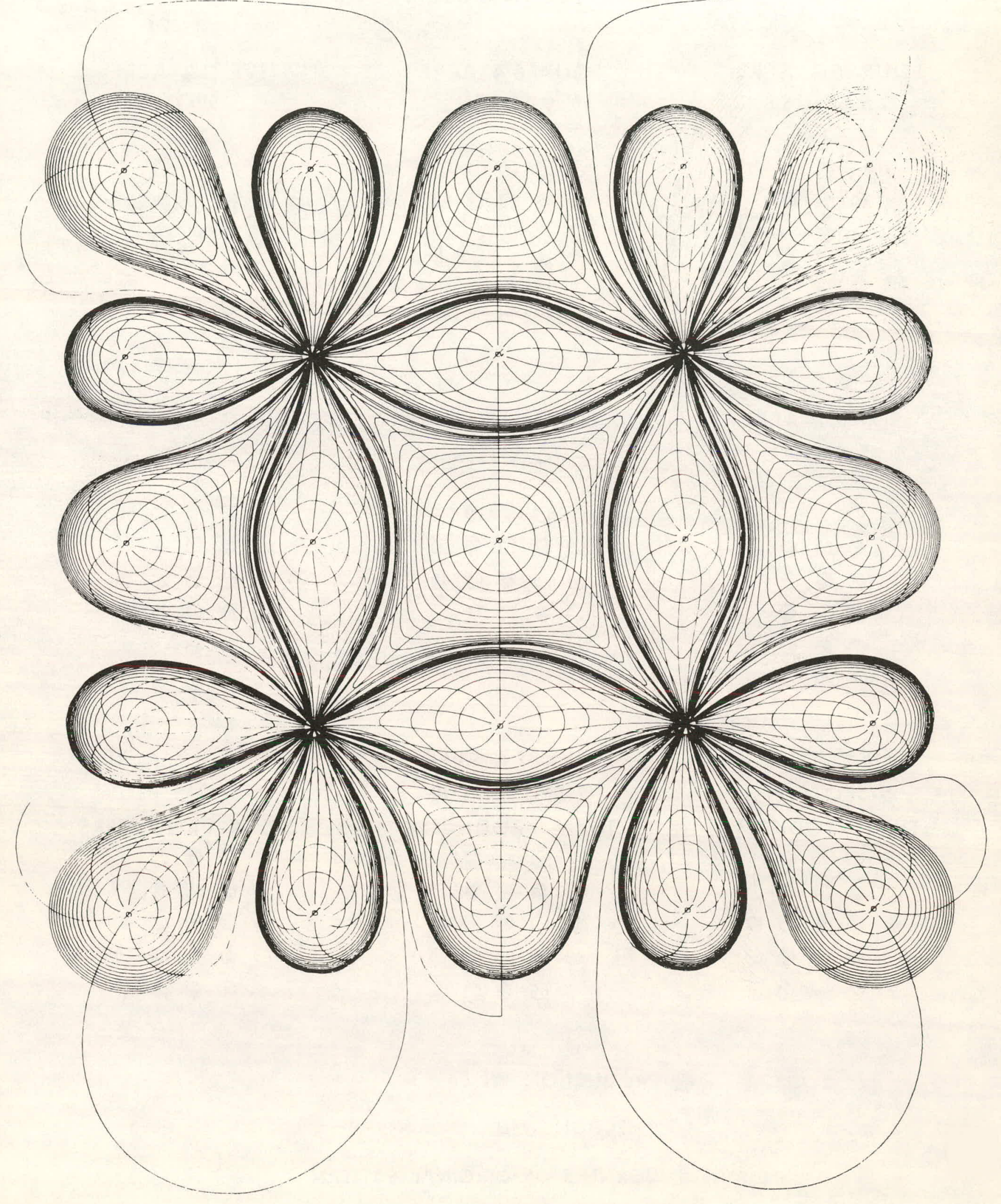


II -309

FIGURE F-28

FOUR 6.4 ACRE FIVE-SPOTS ON EACH LEASE

(30 PSI BHP IN PRODUCERS, BALANCED INJECTION WITH EQUAL BHP IN ALL INJECTORS)

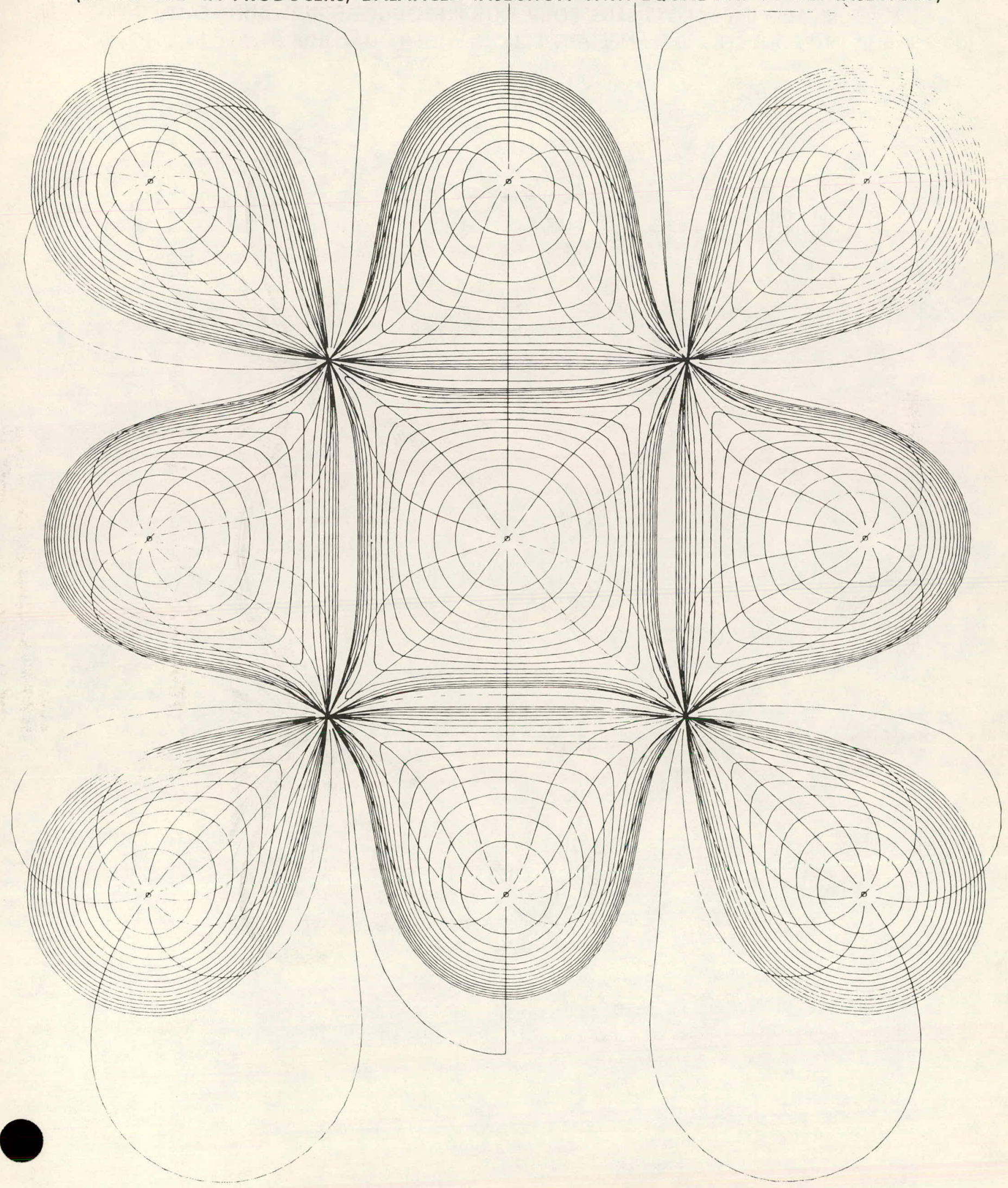




\section{FIGURE F-29}

FIVE 3.2 ACRE FIVE-SPOTS PLUS FOUR EXTRA PRODUCERS ON EACH LEASE (30 PSI BHP IN PRODUCERS, BALANCED INJECTION WITH EQUAL BHP IN ALL INJECTORS)

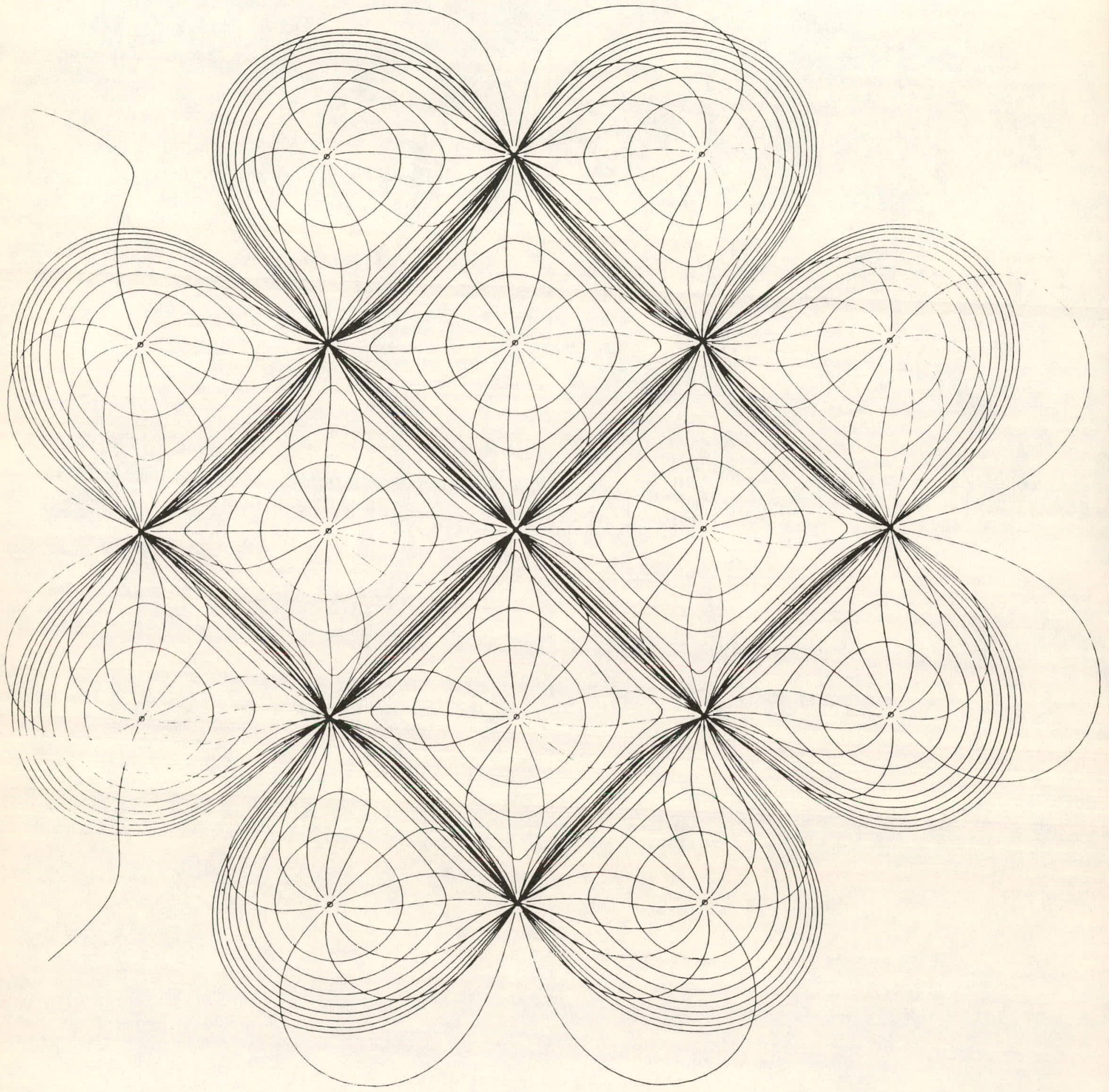


APPENDIX G

ENGINEERING AND OPERATIONS

Tables and Figure 
TABLE GI

CHESNEY-HEGBERG UHIT MP WELL DATA (SERIES 100 WELLS)

\begin{tabular}{|c|c|c|c|c|c|}
\hline \multirow[b]{2}{*}{$\begin{array}{l}\text { Well } \\
\text { No. } \\
\end{array}$} & \multicolumn{2}{|c|}{$\begin{array}{l}\text { Surface Casing } \\
(85 / 8 \text { in })\end{array}$} & \multicolumn{2}{|c|}{$\begin{array}{l}\text { Prodsction } \\
\text { Casing }\end{array}$} & \multirow[b]{2}{*}{$\begin{array}{l}\text { Casing } \\
\text { Size, } \\
\text { in } \\
\end{array}$} \\
\hline & $\begin{array}{l}\text { Depth, } \\
\mathrm{ft} \\
\end{array}$ & $\begin{array}{l}\text { Cement, } \\
\text { yd or } \\
\text { sacks } \\
\end{array}$ & $\begin{array}{l}\text { Depth, } \\
\mathrm{ft}\end{array}$ & $\begin{array}{l}\text { Cement, } \\
\text { No. of } \\
\text { sacks } \\
\end{array}$ & \\
\hline MP- 101 & 30 & $15 \mathrm{sx}$ & 688 & 150 & $51 / 2$ \\
\hline :IP- 102 & 31 & $15 \mathrm{sx}$ & 688 & 125 & $51 / 2$ \\
\hline MP-"103 & 30 & $15 \mathrm{sx}$ & 689 & 150 & $51 / 2$ \\
\hline MP- 104 & 31 & $155 x$ & 729 & 150 & $51 / 2$ \\
\hline MP- 105 & 27 & $15 \mathrm{sx}$ & $689^{\prime}$ & 150 & $51 / 2$ \\
\hline MP- 106 & 28 & $155 x$ & 644 & 175 & $41 / 2$ \\
\hline UP- 107A & 33 & $1 \mathrm{yd}$ & 637 & 175 & $41 / 2$ \\
\hline MP- 108 & 27 & $2 / 3$ yd & 623 & 175 & $41 / 2$ \\
\hline MP- 109 & 29 & $2 / 3$ yd & 611 & 175 & $41 / 2$ \\
\hline$M P-110$ & 29 & $2 / 3 y d$ & 614 & 225 & $41 / 2$ \\
\hline MP- 111 & 22 & $2 / 3$ yd & 615 & 175 & $41 / 2$ \\
\hline MP- 112 & 33 & $2 / 3$ yd & 675 & 175 & $51 / 2$ \\
\hline MP- 113 & 29 & $2 / 3$ yd & 625 & 175 & $41 / 2$ \\
\hline MP- 114 & 29 & $1 / 2$ yd & 704 & 150 & $51 / 2$ \\
\hline$M P-115$ & 28 & $2 / 3$ yd & 651 & 175 & $41 / 2$ \\
\hline MP- 116 & 29 & $2 / 3$ yd & 649 & 175 & $41 / 2$ \\
\hline MP- -117 & 30 & $2 / 3$ yd & 653 & 175 & $41 / 2$ \\
\hline MP- 118 & 30 & $2 / 3 \mathrm{yd}$ & 635 & 175 & $41 / 2$ \\
\hline MP- 119 & 27 & $2 / 3$ yd & 623 & 175 & $41 / 2$ \\
\hline PAP- 120 & 30 & $1 / 2 y d$ & 619 & 175 & $41 / 2$ \\
\hline MP- 121 & 22 & $2 / 3 \mathrm{yd}$ & 627 & 175 & $41 / 2$ \\
\hline : $: P-122$ & 35 & 1 yd & 693 & 175 & $51 / 2$ \\
\hline MP-123 & 28 & $2 / 3$ yd & 643 & 175 & $41 / 2$ \\
\hline MP- 124 & 27 & $l y d$ & 709 & 150 & $51 / 2$ \\
\hline$M P-125$ & 28 & $2 / 3 \mathrm{yd}$ & 647 & 175 & $41 / 2$ \\
\hline$M P-126$ & 30 & $2 / 3$ yd & $65 \varepsilon$ & 175 & $41 / 2$ \\
\hline$A P-127 A$ & 28 & $1 / 2$ yd & 655 & 200 & $41 / 2$ \\
\hline$M P-128$ & 35 & $2 / 3 y d$ & 648 & 175 & $41 / 2$ \\
\hline$P P-129$ & 29 & $2 / 3 \mathrm{yd}$ & $64^{-}$ & 175 & $41 / 2$ \\
\hline MP- 130 & 29 & $1 / 2$ yd & 632 & 185 & $41 / 2$ \\
\hline
\end{tabular}

$\frac{\text { Location }}{1848^{\prime} \text { FSL, 2590' FEL Sec. 21-255-5E }}$

$1846^{\prime}$ FSL, 1056' FEL Sec. 27-255-5E

$1564^{\prime}$ FSL, 1848' FEL Sec. 21-25S-5E

264' FSL, 2640' FEL Sec. 21-25S-5E 264' FSL, 1056' FEL Sec. 21-25S-5E 584' FSL, 2112' FWL Sec. 21-255-5E $1584^{\prime}$ FSL, 1595' FWL Sec. 21-25S-5E $1584^{\prime}$ FSL, 2640' FEL Sec. 21-25S-5E 2376' FEL, 1584' FSL Sec. 21-25S-5E $2112^{\prime} \mathrm{FEL}, 1584^{\prime} \mathrm{FSL}$ Sec. 21-25S-5E 2112' FEL, 1320' FSL Sec. 21-25S-5E $2366^{\prime} \mathrm{FEL}, 1330^{\circ} \mathrm{FSL}$ Sec. 21-25S-5E 1320 FSL, 2640' FEL Sec. 21-25S-5E $2366^{\prime}$ FWL, 1330' FSL Sec. 21-25S-5E $1320^{\prime} \mathrm{FSL}, 2112^{\prime}$ FWL Sec. 21-25S-5E 1056' FSL, 2112' FWL Sec. 21-255-5E 2376' FWL, 1056' FSL Sec. 21-25S-5E $1056^{\prime} \mathrm{FSL}, 2640^{\circ} \mathrm{FEL} \mathrm{Sec}$. 21-255-5E $1056^{\prime}$ FSL, 2376' FEL Sec. 21-25S-5E 2112' FEL, 1056' FSL Sec. 21-25S-5E $2112^{\prime} \mathrm{FEL}, 795^{\prime} \mathrm{FSL} \mathrm{Sec}$. 21-25S-5E 2376' FEL, 792' FSL Sec. 21-25S-5E 792' FSL, 2640' FEL Sec. 21-25S-5E 2366' FWL, 792' FSL Sec. 21-25S-5E 212' FWL, 792' FSL Sec. 21-25S-5E 2120' FWL, 528' FSL Sec. 21-25S-5E $2386^{\prime} \mathrm{FWL}, 528^{\prime} \mathrm{FSL}$ Sec. 21-25S-5E $528^{\circ} \mathrm{FSL}, 2640^{\circ}$ FEL Sec. 21-25S-5E 528' FSL, 2376' FEL Sec. 21-25S-5E $2112^{\prime}$ FEL, 528' FSL Sec. 21-25S-5E

\begin{tabular}{|c|c|c|c|c|c|c|c|}
\hline $\begin{array}{c}\text { Completion } \\
\text { Date } \\
\end{array}$ & $\begin{array}{l}\text { Top of } \\
\text { Sand, } \mathrm{ft} \\
\end{array}$ & $\begin{array}{l}\text { Datum* } \\
\quad \mathrm{ft} \\
\end{array}$ & $\begin{array}{c}\text { Gross } \\
\text { Thickness, } \\
\mathrm{ft} \\
\end{array}$ & $\begin{array}{l}\text { Total } \\
\text { Depth, } \\
\mathrm{ft} \\
\end{array}$ & $\begin{array}{l}\text { Plugged } \\
\text { Back Total } \\
\text { Depth, ft. }\end{array}$ & \multirow{2}{*}{\multicolumn{2}{|c|}{$\begin{array}{l}\text { Completion } \\
\text { Interval }, \mathrm{ft} \\
\mathrm{PF} \star \star 6] \in-635\end{array}$}} \\
\hline $7 / 2 / 74$ & 616 & 757 & 19 & 696 & 689 & & \\
\hline $5 / 28 / 74$ & 605 & 778 & 25 & 692 & --- & $-\cdot-$ & - \\
\hline $7 / 2 / 74$ & 610 & 781 & 17 & 690 & 686 & $\mathrm{PF}$ & $610-628$ \\
\hline $7 / 2 / 74$ & 653 & 751 & 20 & 730 & 720 & PF & $653-671$ \\
\hline $5 / 28 / 74$ & 610 & 773 & 21 & 690 & $\cdots$ & --- & 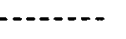 \\
\hline $4 / 16 / 75$ & 644 & 743 & 18 & 682 & 659 & $\mathrm{OH}$ & $644-59$ \\
\hline $4 / 15 / 75$ & 636 & 755 & 19 & 690 & 652 & $\mathrm{OH}$ & $637-52$ \\
\hline $5 / 30 / 75$ & 622 & 765 & 18 & 675 & 637 & $\mathrm{OH}$ & $623-637$ \\
\hline $4 / 17 / 75$ & 610 & $? 71$ & 20 & 655 & 626 & $\mathrm{OH}$ & $611-626$ \\
\hline $4 / 18 / 75$ & 608 & 774 & 19 & 645 & 624 & $\mathrm{OH}$ & $614-624$ \\
\hline $4 / 21 / 75$ & 611 & 775 & 19 & 660 & 627 & $\mathrm{OH}$ & $615-627$ \\
\hline $3 / 27 / 75$ & 615 & 770 & 19 & 676 & 674 & PF & $617-35$ \\
\hline $5 / 23 / 75$ & 624 & 768 & 20 & 670 & 641 & $\mathrm{OH}$ & $625-641$ \\
\hline $3 / 27 / 75$ & 640 & 753 & 18 & 705 & 687 & PF & $6: 41-658$ \\
\hline $4 / 14 / 75$ & 649 & 742 & 18 & 690 & 664 & $\mathrm{OH}$ & $651-64$ \\
\hline $4 / 14 / 75$ & 648 & 743 & 20 & 686 & 660 & $\mathrm{OH}$ & $649-656$ \\
\hline $4 / 10 / 75$ & 652 & 744 & 18 & 685 & 666 & $\mathrm{OH}$ & $653-666$ \\
\hline $4 / 8 / 75$ & 634 & 761 & 20 & 680 & $6 \dot{7} 7$ & $\mathrm{OH}$ & $635-651$ \\
\hline $4 / 18 / 75$ & 621 & 768 & 20 & 660 & $63 \varepsilon$ & $\mathrm{OH}$ & $623-633$ \\
\hline $4 / 12 / 75$ & 617 & 773 & 19 & 655 & $€ 35$ & $\mathrm{OH}$ & $619-635$ \\
\hline $4 / 22 / 75$ & 626 & 768 & 19 & 672 & $64 \hat{i}$ & $\mathrm{OH}$ & $627-642$ \\
\hline $4 / 2 / 75$ & 636 & 754 & 14 & 696 & 685 & PF & $636=650$ \\
\hline $4 / 5 / 75$ & 642 & $75 \%$ & 20 & 683 & 665 & $\mathrm{OH}$ & $643-65$ \\
\hline $4 / 2 / 75$ & 647 & 748 & 19 & 710 & 701 & $\mathrm{PF}$ & $648-665$ \\
\hline $4 / 14 / 75$ & 646 & 741 & 20 & 700 & $6 ศ 3$ & $\mathrm{OH}$ & $647-663$ \\
\hline $4 / 10 / 75$ & 655 & 738 & 19 & 690 & 670 & $\mathrm{OH}$ & $.656-670$ \\
\hline $4 / 5 / 75$ & 654 & 741 & 20 & 695 & 669 & $\mathrm{OH}$ & $655-669$ \\
\hline $4 / 4 / 75$ & 647 & 753 & 20 & 690 & 666 & $\mathrm{OH}$ & $648-666$ \\
\hline $4 / 21 / 75$ & 639 & 759 & 21 & 683 & 656 & $\mathrm{OH}$ & $64.1-656$ \\
\hline $4 / 21 / 75$ & 630 & 766 & 21 & 670 & 649 & $\mathrm{OH}$ & $632-649$ \\
\hline
\end{tabular}

*Datum above mean sea level

** $\mathrm{PF}=$ Perforated Completion, $\mathrm{OH}=$ Open Hole Comfletion 


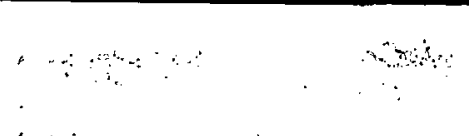

TABLE G2

CHESNEY-HEGBERG UNIT MP NELL DATA (SERIES 200 WELLS)

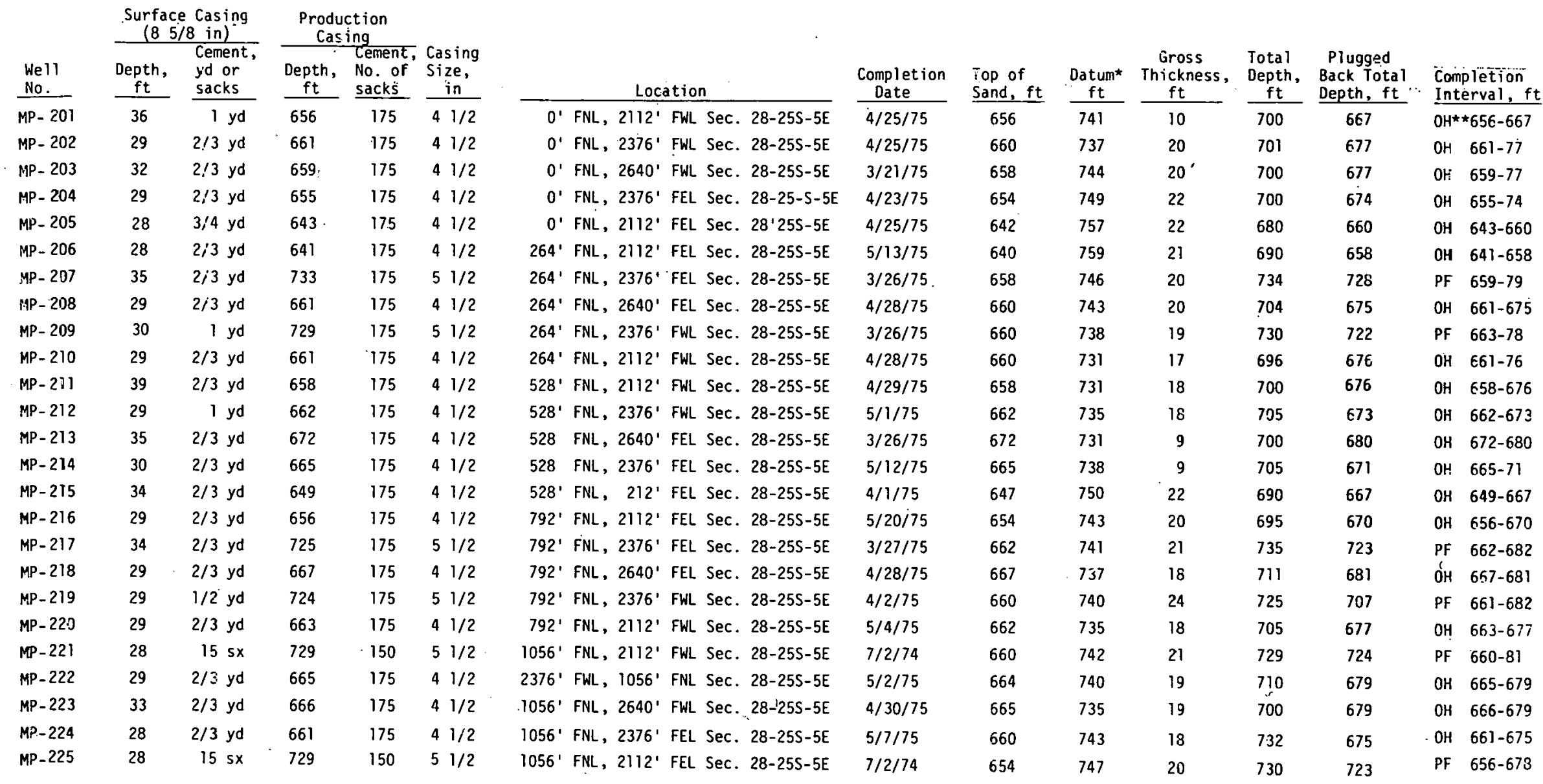

*Datum above mean sea leve

$\star \star \mathrm{PF}=$ Perforated Completion, $\mathrm{OH}=$ Open HCle Completion 

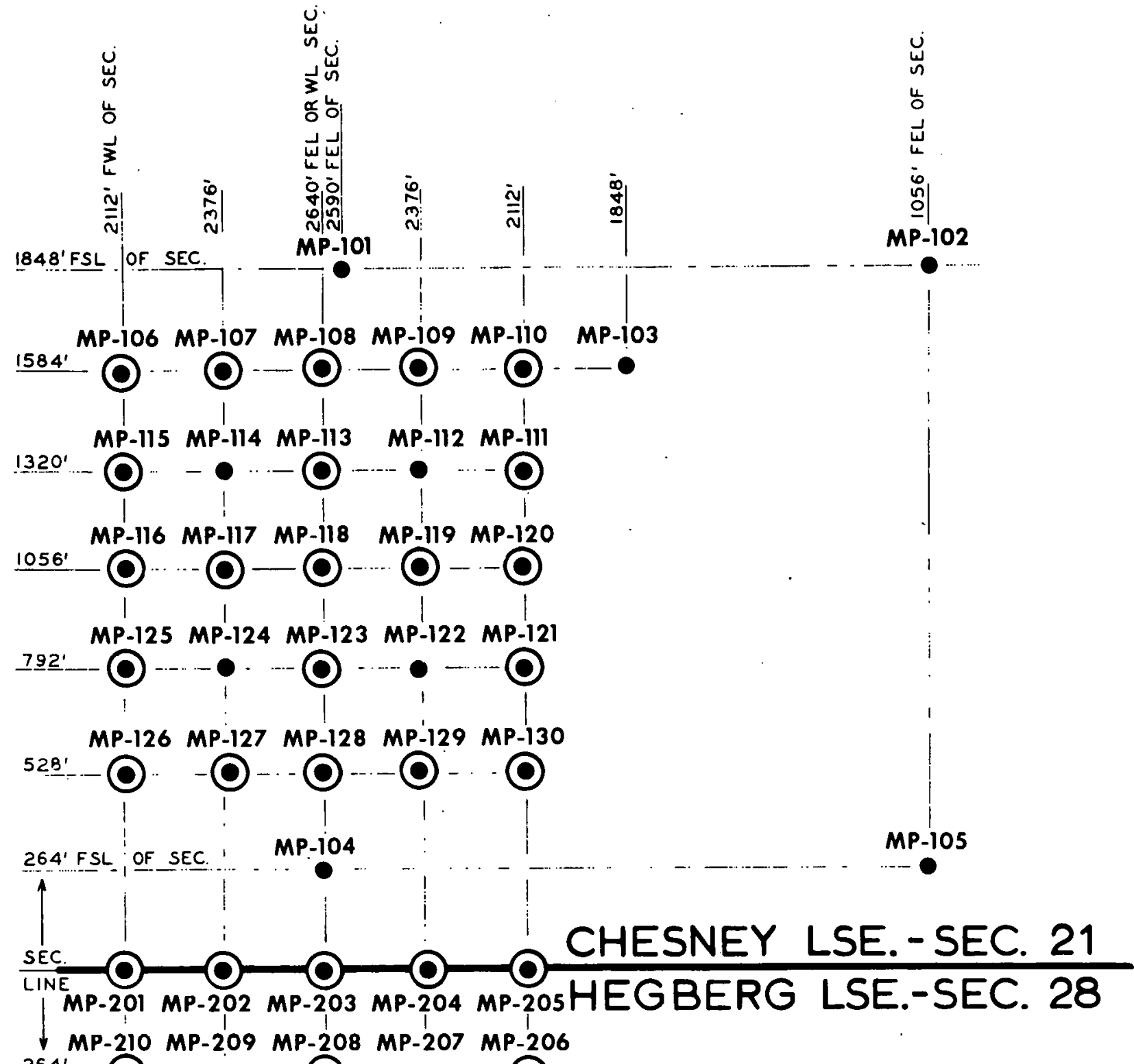

$264^{M} \bigcirc \cdot \cdots \cdot(-)$

MP-211 MP-212 MP-213 MP-214 MP-215

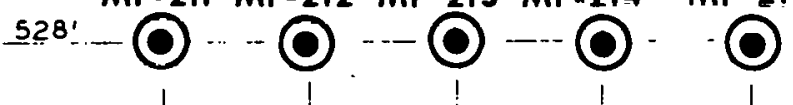

FIGURE G-1

MF MP-220 MP-219 MP-218 MP-217 MP-216 $792^{\prime}$

(.) - - (

MP-221 MP-222 MP-223 MP-224 MP-225

$1056^{\prime}-\mathbf{Q}-\mathbf{Q}-\mathbf{Q}-\mathbf{O} \cdots-\mathbf{O}$

OF SEC.

PROJECT LAYOUT

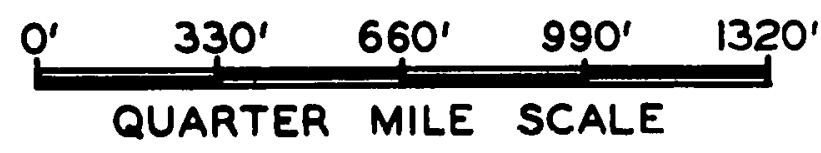


APPENDIX $H$

CONSTRUCTION

Figures 
wryovia mote asisildwis

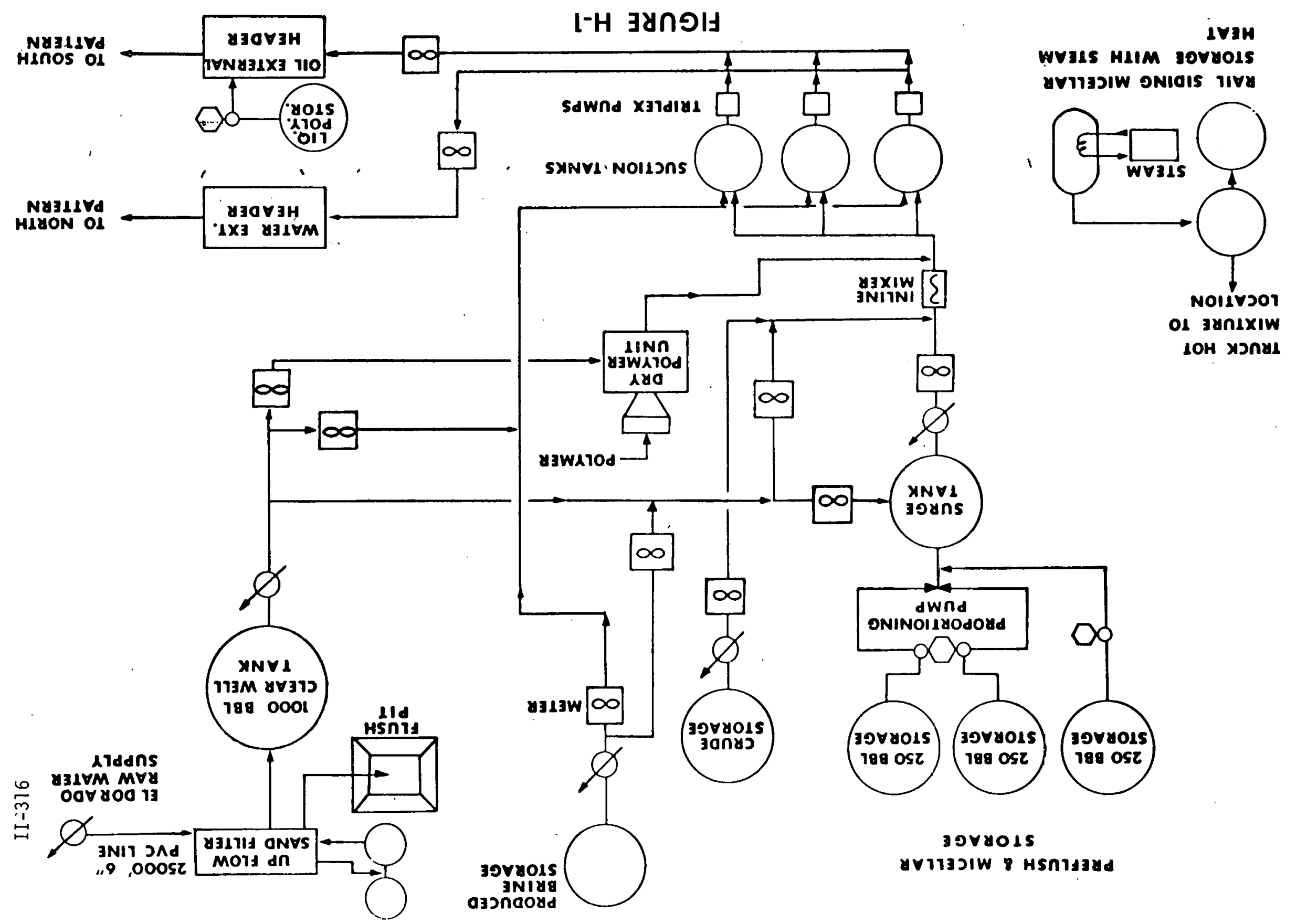




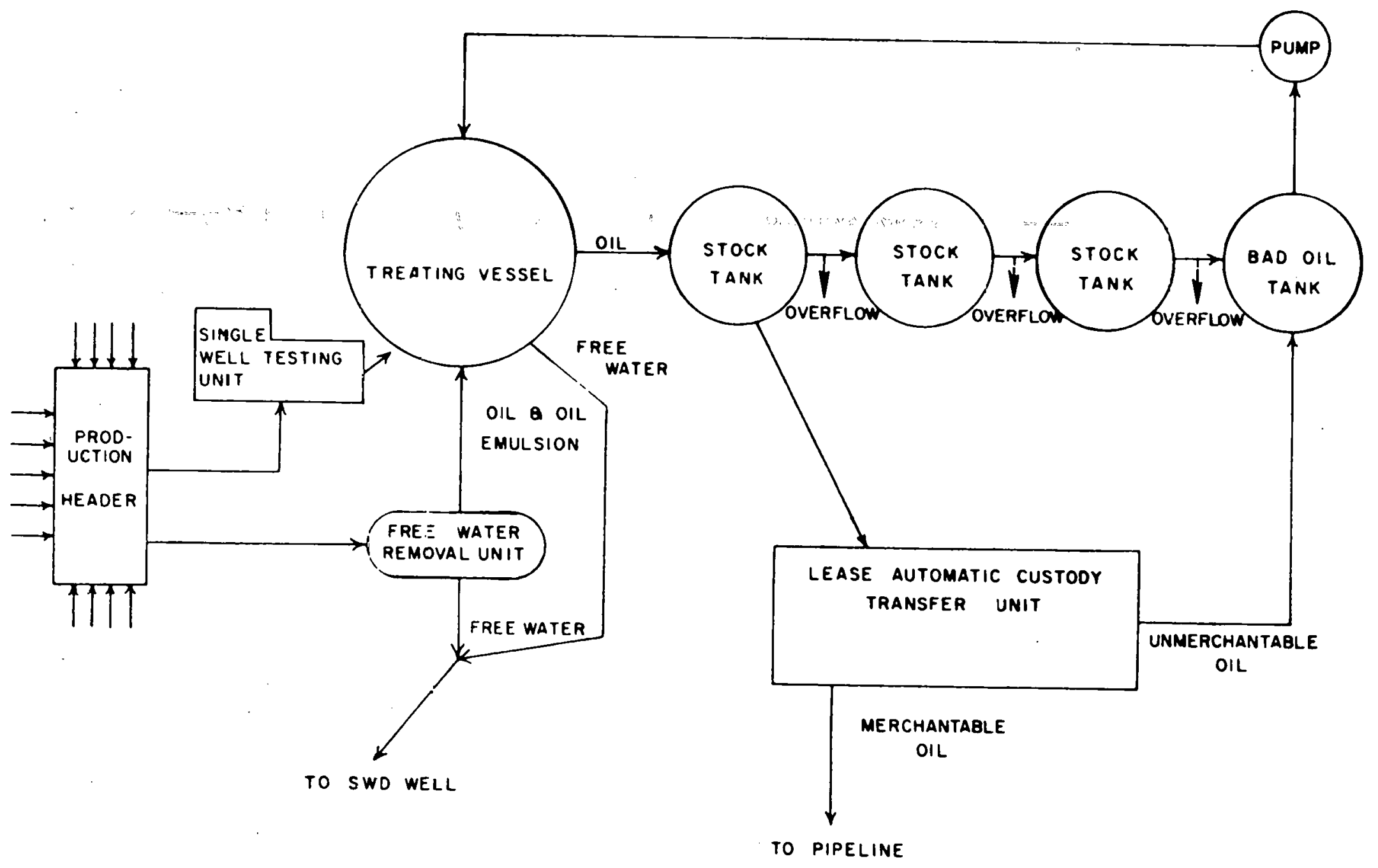

FIGURE H-2

BATTERY FACILITIES 